DOC. 20041108.0005

QA: QA

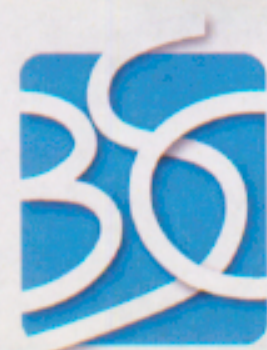

BECHTEL SAIC companvuc

MDL-MGR-MD-000001 REV 01

November 2004

\title{
Biosphere Model Report
}

Prepared for:

U.S. Department of Energy

Office of Civilian Radioactive Waste Management

Office of Repository Development

1551 Hillshire Drive

Las Vegas, Nevada 89134-6321

Prepared by:

Bechtel SAIC Company, LLC

1180 Town Center Drive

Las Vegas, Nevada 89144

Under Contract Number

DE-AC28-01RW12101 


\section{DISCLAIMER}

This report was prepared as an account of work sponsored by an agency of the United States Government. Neither the United States Government nor any agency thereof, nor any of their employees, nor any of their contractors, subcontractors or their employees, makes any warranty, express or implied, or assumes any legal liability or responsibility for the accuracy, completeness, or any third party's use or the results of such use of any information, apparatus, product, or process disclosed, or represents that its use would not infringe privately owned rights. Reference herein to any specific commercial product, process, or service by trade name, trademark, manufacturer, or otherwise, does not necessarily constitute or imply its endorsement, recommendation, or favoring by the United States Government or any agency thereof or its contractors or subcontractors. The views and opinions of authors expressed herein do not necessarily state or reflect those of the United States Government or any agency thereof. 
QA: QA

Biosphere Model Report

MDL-MGR-MD-000001 REV 01

November 2004 


\section{OCRWM}

\section{Model Signature Page/Change History}

2. Type of Mathematical Model

$\bigotimes$ Process Model

Abstraction Model

System Model

Describe Intended Use of Model

The biosphere process model is to evaluate radionuclide transport in the biosphere and assess associated human radiation dose.

The process model is to support the TSPA-LA

3. Title

Biosphere Model Report

4. DI (including Rev. No., if applicable):

MDL-MGR-MD-000001 REV 01

5. Total Appendices

Five

6. No. of Pages in Each Appendix

$A=4$ pages, $B=8$ pages, $C=4$ pages, $D=16$ pages, $E=6$ pages

\begin{tabular}{|c|c|c|c|}
\hline & Printed Name & Signature & Date \\
\hline 7. Originator & $\begin{array}{l}\text { D.W. Wu } \\
\text { A. J. Smith }\end{array}$ & & \\
\hline 8. Independent Technical Reviewer & R.L. Howard & & \\
\hline 9. Checker & K. Rautenstrauch & & \\
\hline 10. QER & K. O. Gilkerson & & \\
\hline 11. Responsible Manager/Lead & M. Wasiolek & & \\
\hline 12. Responsible Manager & M. Zhu & & \\
\hline
\end{tabular}

This report addresses errata generated as a result of Condition Report No. 980 and TER-03-0039.

D.W. Wu was the author of Revision 0 of this report. A.J. Smith is the originator of this revision (Revision 1). The name of the original author was retained on the report because the conceptual and mathematical model and its implementation and validation were not affected by this revision. The revision primarily concerns the documentation of the model.

\section{Change History}

\begin{tabular}{|l|l|}
\hline \multicolumn{1}{|c|}{ 14. Revision No. } & \multicolumn{1}{c|}{ 15. Description of Change } \\
\hline Rev 00 & Initial issue \\
\hline \multirow{3}{*}{ Rev 01} & $\begin{array}{l}\text { Revised to incorporate Regulatory Integration Team evaluation comments, to provide better } \\
\text { transparency and traceability, include specific items from the YMRP, revise citations to reflect most } \\
\text { recent documentation, and comply to revisions in procedures. No changes were made to the product } \\
\text { output (the model) but changes were too extensive to use step 5.8(f)(1) per AP-SIII.10Q Rev 2 } \\
\text { ICN 7. }\end{array}$ \\
\hline
\end{tabular}




\section{INTENTIONALLY LEFT BLANK}




\section{CONTENTS}

Page

ACRONYMS AND ABBREVIATIONS ........................................................................ xvii

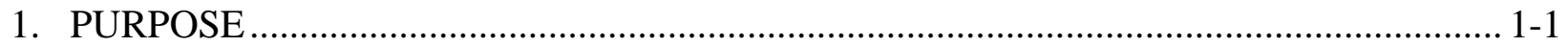

2. QUALITY ASSURANCE ............................................................................. 2-1

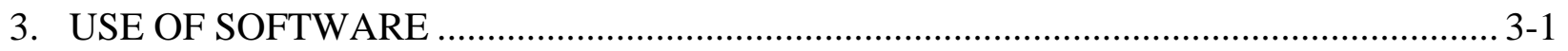

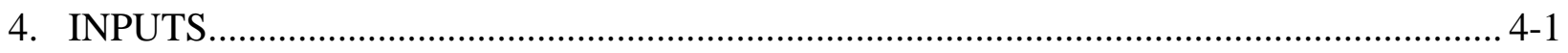

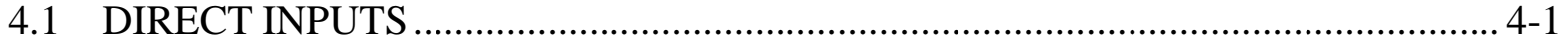

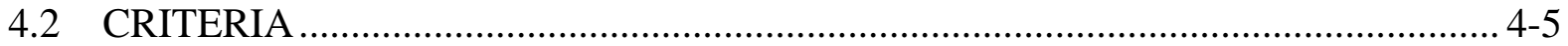

4.3 CODES, STANDARDS, AND REGULATIONS.............................................. 4-10

5. ASSUMPTIONS ................................................................................................. 5 -1

6. MODEL DISCUSSION ......................................................................................... $6-1$

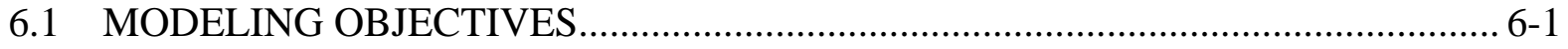

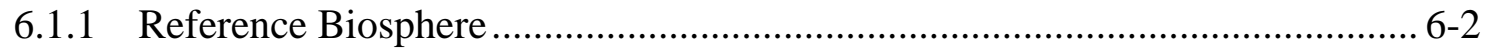

6.1.1.1 Geography, Geology, and Physiography …................................6-2

6.1.1.2 Climate, Flora, and Fauna ..................................................... 6-6

6.1.1.3 Groundwater, Human Activities, and Agriculture.......................... 6-6

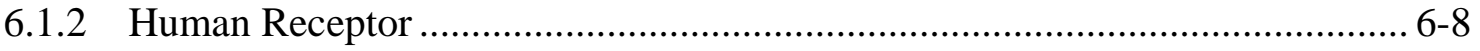

6.1.3 Exposure Scenarios and Radionuclides of Interest.................................... 6-10

6.2 BIOSPHERE FEATURES, EVENTS, AND PROCESSES ................................ 6-11

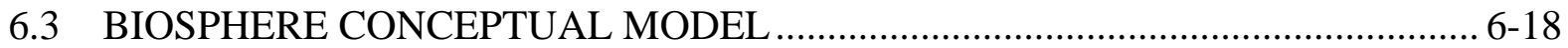

6.3.1 Conceptual Model for the Groundwater Exposure Scenario ......................... 6-18

6.3.1.1 Scenario Description.............................................................. 6-19

6.3.1.2 Identification of Biosphere Model Components......................... 6-21

6.3.1.3 Radionuclide Transfer Interaction Matrix .................................. 6-22

6.3.1.4 Conceptual Model Approximations ............................................. 6-23

6.3.1.5 Submodels for the Groundwater Scenario .................................. 6-29

6.3.1.6 Description of Conceptual Model for the Groundwater

Scenario................................................................................ 6-30

6.3.2 Conceptual Model for the Volcanic Ash Exposure Scenario ......................... 6-37

6.3.2.1 Scenario Description.............................................................. 6-37

6.3.2.2 Identification of Biosphere Model Components......................... 6-40

6.3.2.3 Radionuclide Transfer Interaction Matrix ................................. 6-41

6.3.2.4 Conceptual Model Approximations ............................................ 6-41

6.3.2.5 Submodels for the Volcanic Ash Scenario ................................... 6-46

6.3.2.6 Description of Conceptual Model for the Volcanic Ash

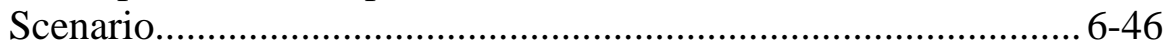

6.3.3 Alternative Conceptual Models ................................................................... 6-50

6.3.4 FEPs Considered in the Biosphere Conceptual Model................................. 6-54

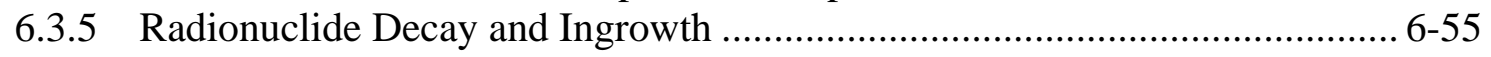




\section{CONTENTS (Continued)}

6.4 MATHEMATICAL MODEL FOR THE GROUNDWATER SCENARIO............... 6-58

6.4.1 Surface Soil Submodel.............................................................................. 6-59

6.4.1.1 Primary Radionuclides in the Surface Soil .................................. 6-59

6.4.1.2 Radionuclide Decay and Ingrowth in the Surface Soil.................. 6-62

6.4.1.3 Radionuclide Leaching from the Surface Soil .............................. 6-65

6.4.1.4 Surface Soil Erosion .................................................................. 6-66

6.4.2 Air Submodel................................................................................. 6-66

6.4.2.1 Resuspended Particles from Surface Soil ...................................... 6-66

6.4.2.2 Aerosols from Evaporative Cooler Operation ................................ 6-69

6.4.2.3 Radon Exhalation from Surface Soil .......................................... 6-70

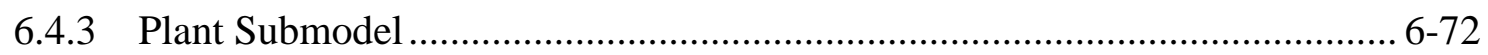

6.4.3.1 Root Uptake .............................................................................6-74

6.4.3.2 Uptake Following Foliar Interception of Irrigation Water ............ 6-74

6.4.3.3 Uptake by Foliar Interception of Airborne Particulates................. 6-78

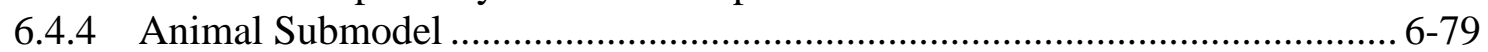

6.4.4.1 Animal Feed..................................................................... 6-80

6.4.4.2 Animal Drinking Water ................................................................ 6-81

6.4.4.3 Animal Soil Ingestion ................................................................6-82

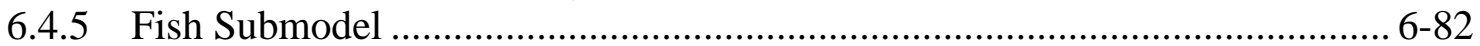

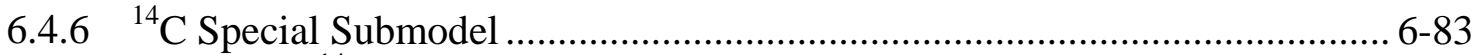

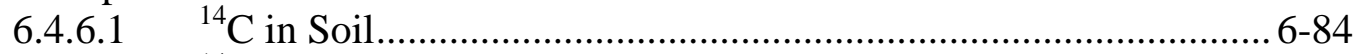

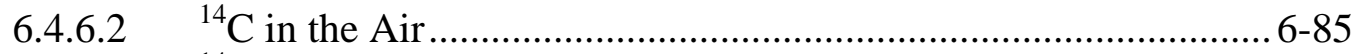

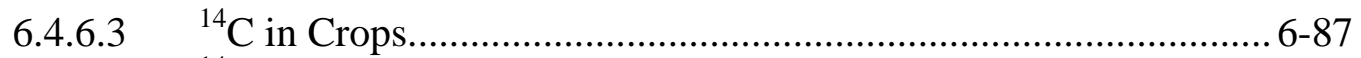

6.4.6.4 ${ }^{14} \mathrm{C}$ in Animal Products .............................................................. 6-88

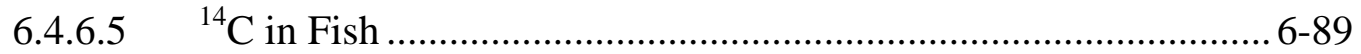

6.4.7 External Exposure Submodel........................................................................ 6-89

6.4.7.1 Exposure to Contaminated Soil ..................................................... 6-89

6.4.7.2 Effective Dose Coefficients for Exposure to Contaminated

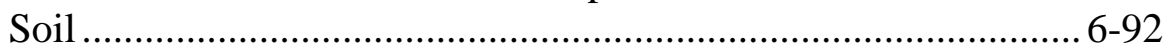

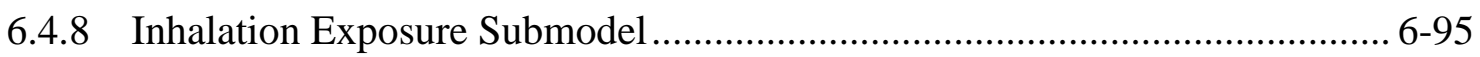

6.4.8.1 Inhalation of Resuspended Particles .............................................6-95

6.4.8.2 Inhalation of Aerosols from Evaporative Coolers ......................... 6-97

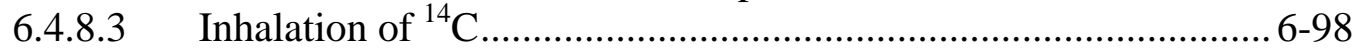

6.4.8.4 Inhalation of Radon Decay Products ........................................... 6-98

6.4.8.5 Effective Dose Conversion Factor for Inhalation ........................ 6-100

6.4.9 Ingestion Exposure Submodel ................................................................... 6-103

6.4.9.1 Ingestion of Drinking Water .................................................... 6-104

6.4.9.2 Ingestion of Crop Foodstuffs ...................................................... 6-104

6.4.9.3 Ingestion of Animal Products ...................................................... 6-105

6.4.9.4 Ingestion of Fish ................................................................... 6-106

6.4.9.5 Inadvertent Soil Ingestion ..........................................................6-106

6.4.9.6 Effective Dose Conversion Factor for Ingestion ......................... 6-107 


\section{CONTENTS (Continued)}

6.4.10 All-Pathway Dose and Biosphere Dose Conversion Factor Calculations .... 6-110

6.4.10.1 All-Pathway Doses...................................................................... 6-110

6.4.10.2 Biosphere Dose Conversion Factors for the Groundwater

Scenario................................................................................ 6-110

6.4.10.3 Pathway Contribution to Dose …………………........................ 6-112

6.4.10.4 Use of BDCFs in the Total System Performance Assessment

Model ................................................................................... 6-113

6.5 MATHEMATICAL MODEL FOR THE VOLCANIC ASH SCENARIO............... 6-114

6.5.1 Surface Soil Submodel........................................................................ 6-114

6.5.1.1 Cultivated Land..................................................................... 6-115

6.5.1.2 Noncultivated Land.............................................................. 6-116

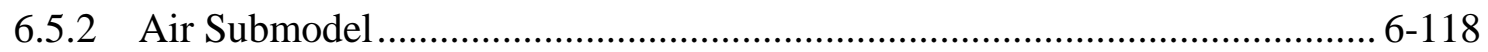

6.5.2.1 Resuspension of Volcanic Ash or a Mixture of Soil and Ash .... 6-118

6.5.2.2 Exhalation of Radon from the Ground Surface ........................... 6-120

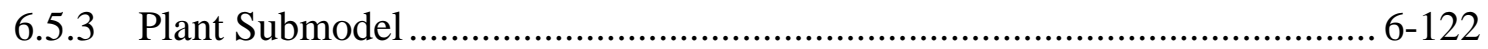

6.5.3.1 Root Uptake ............................................................................ 6-123

6.5.3.2 Uptake from Resuspended Soil.................................................... 6-123

6.5.4 Animal Submodel ......................................................................... 6-125

6.5.4.1 Animal Feed......................................................................... 6-125

6.5.4.2 Animal Soil Ingestion ............................................................. 6-126

6.5.5 External Exposure Submodel.................................................................... 6-126

6.5.5.1 External Exposure to Contaminated Ground Surface................... 6-126

6.5.5.2 Effective Dose Coefficients for Contaminated Ground

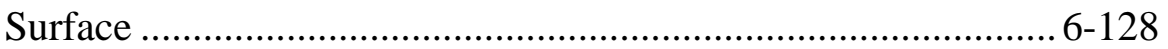

6.5.6 Inhalation Submodel ................................................................................ 6-130

6.5.6.1 Inhalation of Resuspended Particles ............................................ 6-131

6.5.6.2 Inhalation of Radon Decay Products .......................................... 6-132

6.5.7 Ingestion Submodel .............................................................................. 6-133

6.5.7.1 Ingestion of Crop Foodstuffs ....................................................... 6-133

6.5.7.2 Ingestion of Animal Products .................................................... 6-134

6.5.7.3 Inadvertent Soil Ingestion ............................................................ 6-134

6.5.8 All-Pathway Dose and Biosphere Dose Conversion Factor Calculation....... 6-135

6.5.8.1 All-Pathway Dose …………………………............................ 6-135

6.5.8.2 Biosphere Dose Conversion Factors for the Volcanic Ash

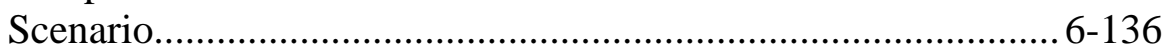

6.5.8.3 Use of BDCFs in the Total System Performance Assessment

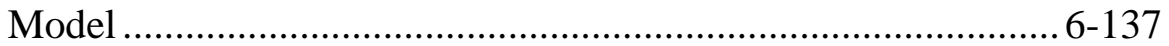

6.6 CONSIDERATION OF MODEL UNCERTAINTY ………………………......... 6-138

6.6.1 Conceptual Model Uncertainty .................................................................. 6-138

6.6.2 Mathematical Model Uncertainty .............................................................. 6-139 


\section{CONTENTS (Continued)}

6.6.3 Input Parameter Uncertainty .................................................................... 6-143

6.6.3.1 List of Input Parameters........................................................... 6-143

6.6.3.2 Consideration of Parameter Uncertainty..................................... 6-148

6.6.3.3 Summary of Parameter Uncertainty............................................ 6-149

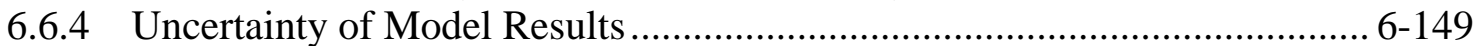

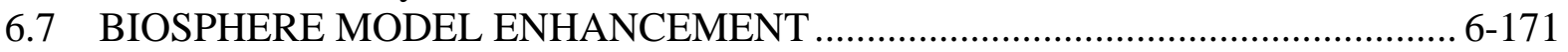

6.7.1 Disposition of FEPs within Biosphere Mathematical Model ........................ 6-171

6.7.2 Comparison of Biosphere Models ........................................................... 6-180

6.7.3 Software Selection ................................................................................... 6-183

6.8 GOLDSIM IMPLEMENTATION OF THE BIOSPHERE MODEL FOR

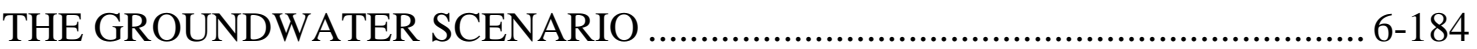

6.8.1 Nuclide Database ............................................................................. 6-186

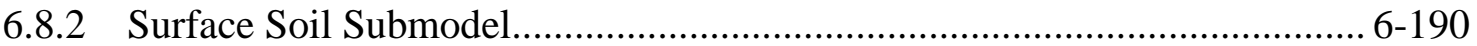

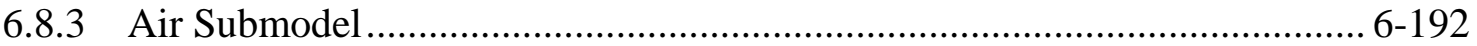

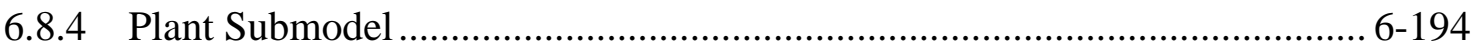

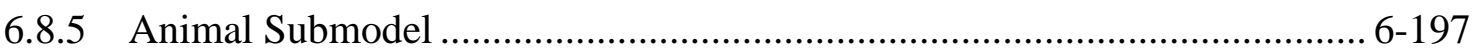

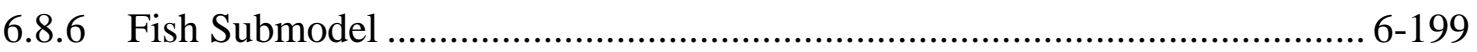

6.8.7 External Exposure Submodel.............................................................. 6-200

6.8.8 Inhalation Submodel .................................................................. 6-202

6.8.9 Ingestion Submodel ........................................................................... 6-205

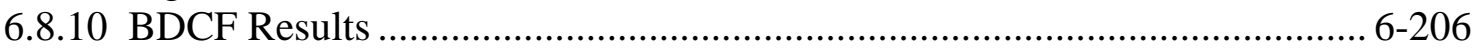

6.9 GOLDSIM IMPLEMENTATION OF THE BIOSPHERE MODEL FOR

THE VOLCANIC ASH SCENARIO ……………...................................... 6-208

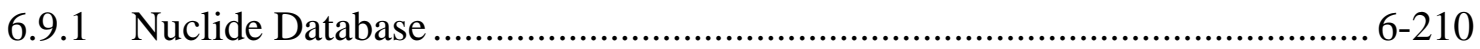

6.9.2 Surface Soil Submodel..................................................................... 6-211

6.9.3 Air Submodel.............................................................................. 6-212

6.9.4 Plant Submodel ........................................................................... 6-214

6.9.5 Animal Submodel .......................................................................... 6-215

6.9.6 External Exposure Submodel.............................................................. 6-216

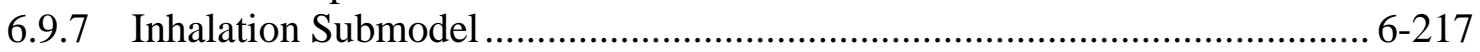

6.9.8 Ingestion Submodel ....................................................................... 6-218

6.9.9 BDCF Results ................................................................................... 6-219

6.10 VERIFICATION OF THE ERMYN IMPLEMENTATION IN GOLDSIM............ 6-220

6.10.1 Verification of the Groundwater Scenario ................................................... 6-221

6.10.1.1 Verification of Deterministic Calculations ................................ 6-221

6.10.1.2 Verification of Stochastic Calculations .................................... 6-232

6.10.2 Verification of the Volcanic Ash Scenario ................................................... 6-232

6.10.2.1 Verification of Deterministic Calculations .................................. 6-232

6.10.2.2 Verification of Stochastic Calculations ..................................... 6-239 


\section{CONTENTS (Continued)}

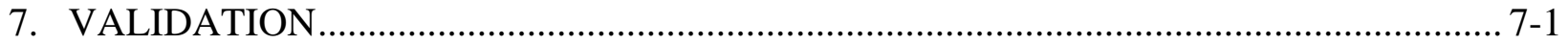

7.1 VALIDATION APPROACH ..................................................................................

7.1.1 Confidence Building During Model Development to Establish the Scientific Basis and Accuracy for Intended Use .............................................. 7-4

7.1.2 Post-Development Model Validation to Support the Scientific Basis of the Model ................................................................................................ 7-5

7.1.3 Selection of Supporting Information ............................................................. 7-8

7.2 COMPARISON OF BIOSPHERE CONCEPTUAL MODELS ............................... 7-10

7.2.1 Review of Biosphere Assessment Context .................................................... 7-10

7.2.2 Consideration of Scenarios, Submodels, and Pathways ................................. 7-13

7.3 COMPARISON OF BIOSPHERE MATHEMATICAL SUBMODELS.................... 7-14

7.3.1 Comparison of Surface Soil Submodels ……………................................... 7-16

7.3.1.1 Comparison of Surface Soil Submodels for the Groundwater Scenario............................................................................... 7-16

7.3.1.2 Comparison of Surface Soil Submodels for the Volcanic Ash

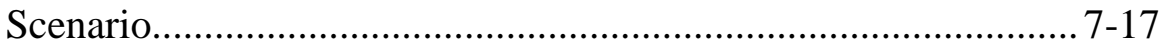

7.3.2 Validation of the Air Submodel........................................................ 7-23

7.3.2.1 Particle Resuspension from Surface Soil........................................ 7-23

7.3.2.2 Radon from Radium-Contaminated Soil ....................................... 7-28

7.3.2.3 Contaminated Aerosols from the Operation of Evaporative

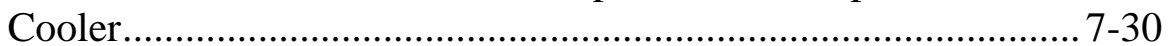

7.3.3 Validation of the Plant Submodel......................................................... 7-30

7.3.3.1 Root Uptake ............................................................................ 7-30

7.3.3.2 Uptake from Irrigation Water ................................................... 7-34

7.3.3.3 Uptake from Resuspended Soil.................................................... 7-43

7.3.4 Validation of the Animal Submodel ................................................................ 7-45

7.3.4.1 Animal Feed...................................................................... 7-45

7.3.4.2 Drinking Water ...................................................................... 7-50

7.3.4.3 Soil Ingestion ........................................................................ 7-50

7.3.4.4 Dust Inhalation................................................................... 7-50

7.3.5 Validation of the Fish Submodel ................................................................. 7-56

7.3.6 Validation of the ${ }^{14} \mathrm{C}$ Special Submodel ........................................................ 7-58

7.3.7 Validation of the External Exposure Submodel ............................................. 7-69

7.3.8 Validation of the Inhalation Submodel ........................................................ 7-73

7.3.9 Validation of the Ingestion Submodel ...................................................... 7-77

7.4 NUMERICAL COMPARISON OF MODEL RESULTS ...................................... 7-84

7.4.1 Radionuclide Decay and Ingrowth ........................................................... 7-84

7.4.2 Surface Soil Submodel................................................................................ 7-88

7.4.2.1 Time Required to Establish Equilibrium Condition ...................... 7-88

7.4.2.2 Comparison of Radionuclide Decay Chains in the GENII-S Model and ERMYN ................................................................. 7-89

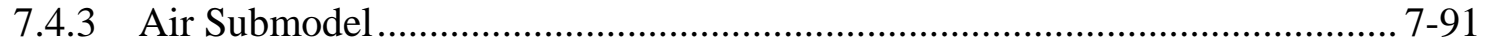

7.4.3.1 Radon Pathway ……………………………………………... 7-91

7.4.3.2 Evaporative Cooler …………………….................................. 7-94 


\section{CONTENTS (Continued)}

Page

7.4.4 Plant Uptake Submodel ........................................................................ 7-94

7.4.4.1 Direct Deposition of Irrigation Water on Crop Surfaces ............... 7-94

7.4.4.2 Crop Interception Fraction for Irrigation Water ............................ 7-95

7.4.4.3 Direct Deposition of Resuspended Soil on Crop Surfaces ........... 7-96

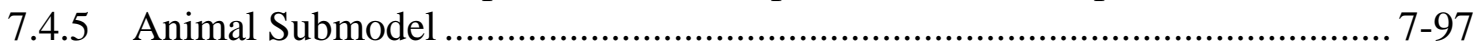

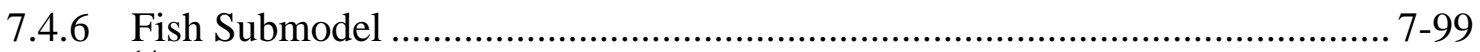

7.4.7 $\quad{ }^{14} \mathrm{C}$ Special Submodel ............................................................................. 7-99

7.4.8 External Exposure Submodel..................................................................... 7-100

7.4.8.1 Air Submersion .................................................................... 7-100

7.4.8.2 Water Immersion .................................................................. 7-104

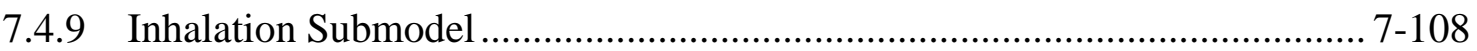

7.4.10 Ingestion Submodel .................................................................................. 7-109

7.5 VALIDATION OF THE GOLDSIM OUTPUT .................................................. 7-109

7.5.1 Validation of the GoldSim Output for the Groundwater Scenario ................ 7-110

7.5.2 Validation of the GoldSim Output for the Volcanic Ash Scenario ................ 7-110

7.6 MODEL VALIDATION RANGE......................................................................... 7-110

7.7 EXTERNAL REVIEW OF THE ERMYN ...................................................... 7-112

7.8 SUMMARY OF MODEL VALIDATION.......................................................... 7-112

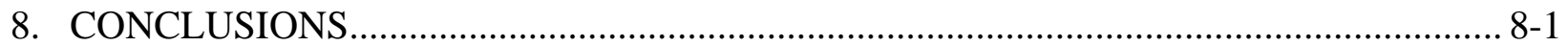

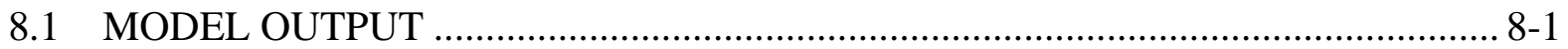

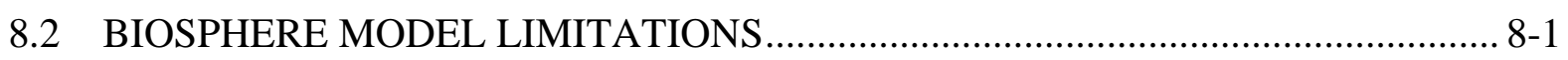

8.3 HOW ACCEPTANCE CRITERIA WERE ADDRESSED ........................................ 8-2

9. INPUTS AND REFERENCES ............................................................................ 9-1

9.1 DOCUMENTS CITED …………………………............................................... 9-1

9.2 CODES, STANDARDS, REGULATIONS, AND PROCEDURES.......................... 9-13

9.3 SOURCE DATA, LISTED BY DATA TRACKING NUMBER ............................. 9-14

9.4 OUTPUT DATA, LISTED BY DATA TRACKING NUMBER ………................. 9-14

9.5 SOFTWARE CODES …………………………............................................ 9-14

APPENDIX A - ELECTRONIC FILES USED FOR THE MODEL REPORT ……............... A-1

APPENDIX B - ANALYTICAL SOLUTION FOR RADON CONCENTRATIONS IN

THE AIR DUE TO RADIUM CONTAMINATED SOIL ..............................

APPENDIX C - CALCULATION OF ABSOLUTE HUMIDITY FROM RELATIVE

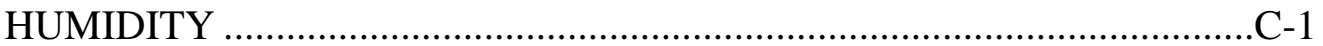

APPENDIX D - EVAPORATIVE COOLER PATHWAYS ……………………….............. D-1

APPENDIX E - FISH INGESTION DOSE IN THE VOLCANIC SCENARIO ........................ 


\section{FIGURES}

Page

1-1. Overview of the Yucca Mountain Biosphere Model Documentation ........................... 1-3

6.1-1. Yucca Mountain in Relation to the Great Basin ....................................................... 6-3

6.1-2. Regional Map of Yucca Mountain and the Amargosa Valley ....................................... 6-5

6.3-1. Graphical Representation of the Biosphere System for the Groundwater

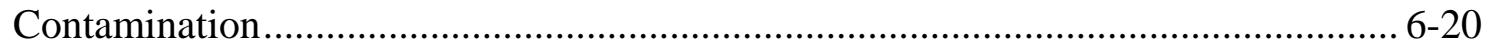

6.3-2. Relationship Among Biosphere Submodels for the Groundwater Scenario................ 6-30

6.3-3. Representation of a Volcanic Eruption Intersecting the Repository............................ 6-39

6.3-4. Relationship Between the Biosphere Submodels for the Volcanic Ash Scenario ...... 6-47

6.7-1. Basic Elements in the GoldSim Environment ........................................................ 6-184

6.8-1. Cover Page for the ERMYN_GW Model in GoldSim ............................................ 6-185

6.8-2. Graphical Representation of the Groundwater Scenario in GoldSim ........................ 6-186

6.8-3. Nuclide Database Container .................................................................................... 6-186

6.8-4. Input Parameters for Crop Transfer Factors ............................................................... 6-187

6.8-5. Radionuclide-Specific Input Parameter Selection .................................................. 6-188

6.8-6. Calculation of Effective Dose Coefficients and Dose Conversion Factors ............... 6-188

6.8-7. Primary_Rn Container and Selection of the Second Decay Product.......................... 6-189

6.8-8. Soil Submodel Container ....................................................................................... 6-191

6.8-9. Input Parameter Container (SoilModel_Input) for the Soil Submodel ...................... 6-191

6.8-10. Air Submodel Container ............................................................................... 6-193

6.8-11. Input Parameter Container (AirModel_Input) for the Air Submodel ........................ 6-193

6.8-12. Plant Submodel Container .................................................................................. 6-195

6.8-13. Input Parameter Container (PlantModel_Input) for the Plant Submodel .................. 6-195

6.8-14. Animal Submodel Container................................................................................. 6-198

6.8-15. Input Parameter Container (AnimalModel_Input) for the Animal Submodel........... 6-198

6.8-16. Fish Submodel Container.................................................................................... 6-200

6.8-17. External Exposure Submodel Container..................................................................... 6-200

6.8-18. Input Parameter Container (External_Input) for the External Exposure

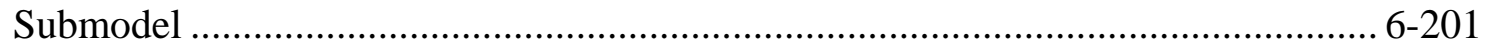

6.8-19. Inhalation Submodel Container …………….................................................... 6-203

6.8-20. Input Parameter Container (Inhalation_Input) for the Inhalation Submodel............. 6-203

6.8-21. Ingestion Submodel Container....................................................................... 6-205

6.8-22. Input Parameter Container (Ingestion_Input) for the Ingestion Submodel ............... 6-205

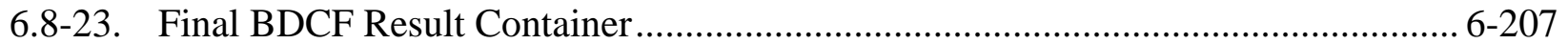

6.9-1. Cover Page for the ERMYN_VA Model in GoldSim ........................................... 6-208

6.9-2. Biosphere Model for the Volcanic Ash Scenario ..................................................... 6-209

6.9-3. Nuclide Database Container and Radionuclide List ................................................. 6-210

6.9-4. Surface Soil Submodel Container .......................................................................... 6-212

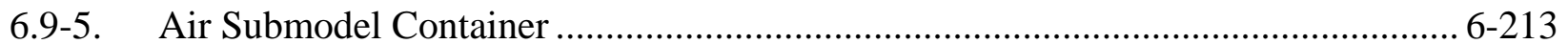

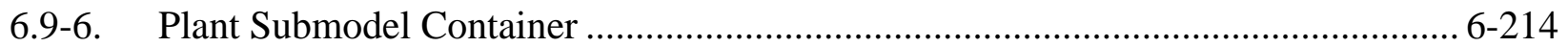

6.9-7. Animal Submodel Container................................................................................ 6-215

6.9-8. External Exposure Submodel Container ................................................................. 6-216

6.9-9. Inhalation Submodel Container …………...................................................... 6-218 


\section{FIGURES (Continued)}

Page

6.9-10. Ingestion Submodel Container.

6.9-11. Final BDCF Result Container.

6-220 


\section{TABLES}

4-1. $\quad$ Additional Sources of Equations Used as a Technical Product Input .......................... 4-2

4.2-1. Requirements Applicable to Biosphere Modeling.......................................................... 4-6

6.1-1. Radionuclides of Interest for the TSPA-LA ………................................................ 6-11

6.2-1. Description and Consideration of FEPs in the ERMYN ........................................... 6-12

6.3-1. Exposure Pathways for the Groundwater Scenario ..................................................... 6-19

6.3-2. Radionuclide Transfer Interaction Matrix for the Groundwater Scenario................... 6-22

6.3-3. Exposure Pathways for the Volcanic Ash Scenario.................................................. 6-40

6.3-4. Radionuclide Transfer Interaction Matrix for the Volcanic Ash Scenario .................. 6-42

6.3-5. Alternative Conceptual Models Considered ................................................................ 6-53

6.3-6. Mapping of FEPs to Interaction Matrices and Relevant Submodel............................. 6-54

6.3-7. Radionuclides of Interest and Their Decay Products................................................ 6-56

6.4-1. The Primary Radionuclides and Their Decay Chains for Soil................................... 6-65

6.4-2. Effective Dose Coefficients for Exposure to Soil Contaminated to an Infinite

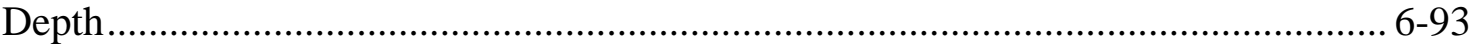

6.4-3. Effective Dose Conversion Factors for Inhalation................................................. 6-101

6.4-4. Effective Dose Conversion Factors for Ingestion..................................................... 6-108

6.4-5. Summary of the Biosphere Submodels for the Groundwater Scenario ...................... 6-111

6.5-1. Effective Dose Coefficients for Exposure to Contaminated Ground Surface ........... 6-128

6.5-2. Summary of the Biosphere Submodels for the Volcanic Ash Scenario ..................... 6-136

6.6-1. Uncertainty Considerations for All Approximations Used in the Model .................. 6-141

6.6-2. Summary of Parameters Used in the ERMYN for the Two Scenarios...................... 6-144

6.6-3. Summary of Representative Input Parameter Values and Their Distributions.......... 6-150

6.7-1. Disposition of the Included FEPs within the Biosphere Mathematical Model.......... 6-171

6.7-2. Comparison of Biosphere Models for the TSPA-LA and TSPA-SR........................ 6-180

6.8-1. Radionuclide-Related Input Parameters in the Nuclide_Database Container ........... 6-189

6.8-2. Parameters in the Surface Soil Submodel......................................................... 6-192

6.8-3. Parameters in the Air Submodel ................................................................... 6-194

6.8-4. Parameters in the Plant Submodel ...................................................................... 6-196

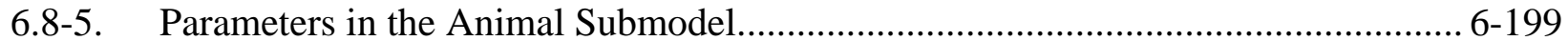

6.8-6. Parameters in the Fish Submodel......................................................................... 6-200

6.8-7. Parameters in the External Exposure Submodel.................................................... 6-201

6.8-8. Parameters in the Inhalation Submodel .............................................................. 6-204

6.8-9. Parameters in the Ingestion Submodel.......................................................... 6-206

6.8-10. Parameters in the Final BDCF Results ............................................................ 6-207

6.9-1. Radionuclide Related Input Parameters .................................................................. 6-210

6.9-2. Parameters in the Surface Soil Submodel.......................................................... 6-212

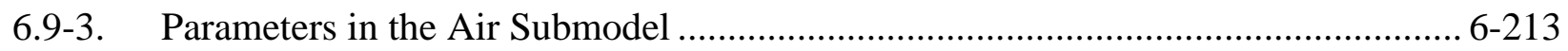

6.9-4. Parameters in the Plant Submodel ..................................................................... 6-214

6.9-5. Parameters in the Animal Submodel.............................................................. 6-216

6.9-6. Parameters in the External Exposure Submodel..................................................... 6-217

6.9-7. Parameters in the Inhalation Submodel .......................................................... 6-218

6.9-8. Parameters in the Ingestion Submodel............................................................. 6-219

$\begin{array}{lll}\text { MDL-MGR-MD-000001 REV } 01 \quad \text { xiii } & \text { November } 2004\end{array}$ 


\section{TABLES (Continued)}

6.9-9. Parameters in the Final BDCF Results

6.10-1. Verification of ERMYN in GoldSim for the Groundwater Scenario (Case $1,{ }^{239} \mathrm{Pu}$ )

6.10-2. Verification of ERMYN in GoldSim for the Groundwater Scenario (Case $2,{ }^{14} \mathrm{C}$ )

6.10-3. Verification of ERMYN in GoldSim for the Groundwater Scenario (Case $3,{ }^{226} \mathrm{Ra}$ ).

6.10-4. Results from Deterministic and Stochastic Runs for the Groundwater Scenario.

6.10-5. Verification of ERMYN in GoldSim for the Volcanic Ash Scenario (Case $1,{ }^{239} \mathrm{Pu}$ )

6.10-6. Verification of ERMYN in GoldSim for the Volcanic Ash Scenario (Case $2,{ }^{226} \mathrm{Ra}$ )

6.10-7. Results from the Deterministic and Stochastic Runs for the Volcanic Ash Scenario.

7.2-1. Assessment Context for the ERMYN, BIOMASS ERB2A, and EPRI-YM

Biosphere Models for Groundwater Contamination

7.2-2. $\quad$ Pathways and Submodels in Seven Biosphere Models......................................... 7-13

7.3-1. Summary of Mathematical Model Comparisons .................................................. 7-14

7.3-2. Comparison of Radionuclide Concentration in Surface Soil................................... 7-18

7.3-3. Comparison of Removal Coefficients for Surface Soil .......................................... 7-20

7.3-4. Soil Resuspension in the Air Submodel............................................................. 7-26

7.3-5. Comparison of Radon Release from Radium Contaminated Soil ........................... 7-29

7.3-6. Comparison of Radionuclide Concentration in Indoor Air from the Operation of an Evaporative Cooler..................................................................................... 7-31

7.3-7. Comparison of Crop Contamination Due to Root Uptake...................................... 7-32

7.3-8. $\quad$ Comparison of Direct Deposition on Leaf Surfaces Due to the Interception of

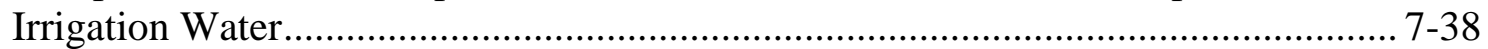

7.3-9. Comparison of Irrigation Deposition Rate........................................................ 7-40

7.3-10. Comparison of the Intercept Fraction for Irrigation ............................................ 7-42

7.3-11. Comparison of Direct Deposition on Leaf Surface Due to the Interception of

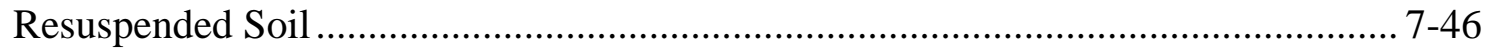

7.3-12. Comparison of the Dust Deposition Rate ............................................................ 7-47

7.3-13. Comparison of Interception Fraction for Resuspended Soil................................... 7-48

7.3-14. Comparison of Animal Product Contamination Due to Animal Feed....................... 7-51

7.3-15. Comparison of Animal Product Contamination Due to Drinking Water ................. 7-53

7.3-16. Comparison of Animal Product Contamination Due to Soil Ingestion ..................... 7-54

7.3-17. Comparison of Animal Product Contamination Due to Dust Inhalation................... 7-55

7.3-18. Comparison of Fish Contamination Due to Fishpond Water................................... 7-57

7.3-19. Comparison of ${ }^{14} \mathrm{C}$ Special Model for Soil Contamination ...................................... 7-60

7.3-20. Comparison of ${ }^{14} \mathrm{C}$ Special Model for Air Contamination ...................................... 7-61

7.3-21. Comparison of ${ }^{14} \mathrm{C}$ Special Model for Plant Contamination .................................. 7-63 


\section{TABLES (Continued)}

7.3-22. Comparison of ${ }^{14} \mathrm{C}$ Special Model for Animal Product Contamination ...................... 7-67

7.3-23. Comparison of External Exposure to Contaminated Soil ........................................... 7-71

7.3-24. Comparison of Inhalation Pathway........................................................................... 7-75

7.3-25. Comparison of Water Ingestion Pathway ................................................................ 7-78

7.3-26. Comparison of Crop Ingestion Pathway ............................................................ 7-79

7.3-27. Comparison of Animal Product Ingestion Pathway................................................. 7-80

7.3-28. Comparison of Fish Ingestion Pathway ………........................................................ 7-81

7.3-29. Comparison of Soil Ingestion Pathway.................................................................... 7-82

7.4-1. Effective Dose Coefficients for Exposure to Soil Contaminated to an Infinite

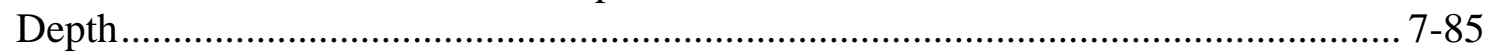

7.4-2. Effective Equivalent Conversion Factors for Inhalation .......................................... 7-86

7.4-3. Effective Dose Conversion Factors for Ingestion...................................................... 7-87

7.4-4. Time to Reach 95-Percent Equilibrium ............................................................. 7-88

7.4-5. Radionuclide Decay Chains Included in the GENII-S Model and ERMYN............... 7-90

7.4-6. Radon Contribution to the Final ${ }^{226}$ Ra Dose.............................................................. 7-91

7.4-7. Radon Release Factors Due to Radium Contaminated Soil....................................... 7-92

7.4-8. Comparison of Evaporative Cooler Model ................................................................... 7-94

7.4-9. Comparison of Direct Deposition of Irrigation Water on Crop Surfaces .................... 7-95

7.4-10. Calculated Interception Fraction for Irrigation Water ................................................ 7-96

7.4-11. Comparison of Direct Deposition of Resuspended Soil on Crop Surfaces ................. 7-96

7.4-12. Numerical Calculation of Animal Uptake Submodel ................................................. 7-98

7.4-13. Evaluation of Plant Uptake in ${ }^{14} \mathrm{C}$ Special Submodel........................................... 7-100

7.4-14. Effective Dose Coefficients for Air Submersion .................................................... 7-101

7.4-15. Comparison of Dose Coefficients for Infinite Depth Soil and Air Submersion ........ 7-103

7.4-16. Effective Dose Coefficients for Water Immersion ................................................ 7-105

7.4-17. Effective Dose Coefficients for Infinite Depth Soil and Water Immersion............... 7-107

7.4-18. Evaluation of Water Immersion Pathway ................................................................ 7-107

7.4-19. Evaluation of Population and Environmental Related Inhalation Submodel ........... 7-109

7.6-1. Unreasonable Parameters Input Values ..................................................................... 7-111

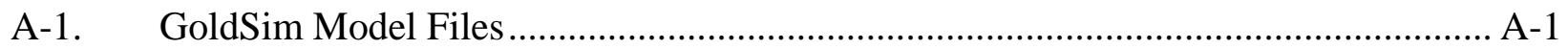

A-2. GoldSim Model Verification Files....................................................................... A-1

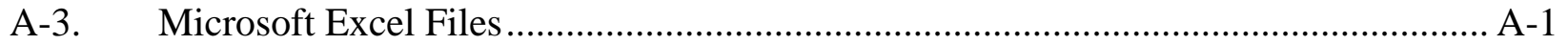

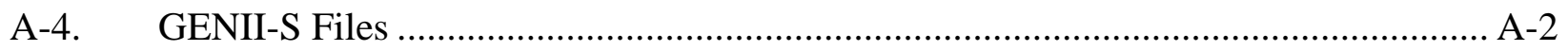

B-1. Calculation of the Radon Release Factor ................................................................

B-2. $\quad$ Radon Release Factors Due to Radium Contaminated Soil.......................................... B-5

C-1. Average Relative Humidity and Temperature at the Weather Station 9 ........................-2

D-1. Time Spent in Indoor Environments and Corresponding Breathing Rates.................. D-2

D-2. Representative Parameter Values and Their Sources ................................................... D-3 


\section{TABLES (Continued)}

D-3. Effective Dose Conversion Factors and Annual Doses for Ingestion and

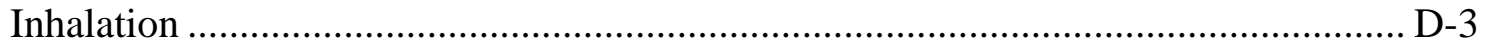

D-4. Radionuclide Half-Lives and Removal Constants ..................................................... D-5

D-5. Partition Coefficient Values Used to Determine Radionuclide Concentration in

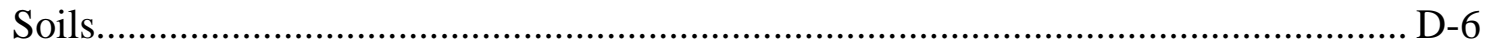

D-6. $\quad$ Other Parameter Values Used to Determine External Exposure to Soils .................... D-6

D-7. Leaching Removal Constants ............................................................................ D-6

D-8. Summary of Individual Removal Constants, Effective Removal Constants, and Activity Concentration in Surface Soil ................................................................. D-7

D-9. Estimated Annual Dose from External Exposure to Irrigated Soils ............................ D-8

D-10. Effective Specific Gamma Ray Constants for a Point Source.................................... D-9

D-11. Estimated Annual Dose from External Exposure to Evaporative Coolers ................. D-11

D-12. Estimated Annual Dose from Ground Shine and Evaporative Cooler Units for the Volcanic Scenario ……………............................................................... D-12

D-13. Annual Dose Estimates from External Exposure from Radionuclides in Soil and in Evaporative Coolers and from Drinking Water ................................................... D-13

E-1. Dimensions of the Grow-Out Ponds .........................................................................

E-2. Fish Ingestion Dose for Representative Radionuclides Resulting from Ashfall

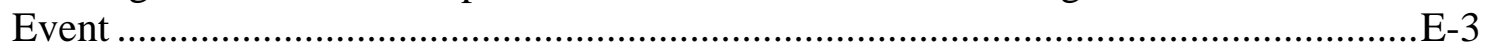

E-3. Comparison of Fish Ingestion Dose and BDCFs from Volcanic Ashfall Event............E-3 


\section{ACRONYMS AND ABBREVIATIONS}

$\begin{array}{ll}\text { ACM } & \text { alternative conceptual model } \\ \text { BDCF } & \text { biosphere dose conversion factor } \\ \text { CEDE } & \text { committed effective dose equivalent } \\ \text { DOE } & \text { U.S. Department of Energy } \\ \text { EDE } & \text { effective dose equivalent } \\ \text { ERMYN } & \text { Environmental Radiation Model for Yucca Mountain, Nevada } \\ \text { FEPs } & \text { features, events, and processes } \\ \text { FGR } & \text { Federal Guidance Report } \\ \text { IAEA } & \begin{array}{l}\text { International Atomic Energy Agency } \\ \text { ICRP }\end{array} \\ \text { International Commission on Radiological Protection } \\ \text { LAN }\end{array}$




\section{INTENTIONALLY LEFT BLANK}




\section{PURPOSE}

The purpose of this report is to document the biosphere model, the Environmental Radiation Model for Yucca Mountain, Nevada (ERMYN), which describes radionuclide transport processes in the biosphere and associated human exposure that may arise as the result of radionuclide release from the geologic repository at Yucca Mountain. The biosphere model is one of the process models that support the Yucca Mountain Project (YMP) Total System Performance Assessment (TSPA) for the license application (LA), TSPA-LA. The ERMYN provides the capability of performing human radiation dose assessments.

This report documents the biosphere model, which includes:

1. Describing the reference biosphere, human receptor, exposure scenarios, and primary radionuclides for each exposure scenario (Section 6.1)

2. Developing a biosphere conceptual model using site-specific features, events, and processes (FEPs) (Section 6.2), the reference biosphere (Section 6.1.1), the human receptor (Section 6.1.2), and approximations (Sections 6.3.1.4 and 6.3.2.4)

3. Building a mathematical model using the biosphere conceptual model (Section 6.3) and published biosphere models (Sections 6.4 and 6.5)

4. Summarizing input parameters for the mathematical model, including the uncertainty associated with input values (Section 6.6)

5. Identifying improvements in the ERMYN compared with the model used in previous biosphere modeling (Section 6.7)

6. Constructing an ERMYN implementation tool (model) based on the biosphere mathematical model using GoldSim stochastic simulation software (Sections 6.8 and 6.9)

7. Verifying the ERMYN by comparing output from the software with hand calculations to ensure that the GoldSim implementation is correct (Section 6.10)

8. Validating the ERMYN by corroborating it with published biosphere models; comparing conceptual models, mathematical models, and numerical results (Section 7).

This revision of this report was conducted in accordance with Revision 3 of the Technical Work Plan for Biosphere Modeling and Expert Support (BSC 2004 [DIRS 169573]) (TWP). This revision was produced to update references, enhance transparency and traceability of the report, and to bring it up to date with project procedures. The biosphere model validation was done during development of Revision 0 of this report (BSC 2003 [DIRS 166507]) in accordance with Revision 1 of the TWP (BSC 2003 [DIRS 163602]).

As directed by the TWP for biosphere modeling (BSC 2003 [DIRS 163602] and BSC 2004 [DIRS 169573]), the ERMYN is based on a previous biosphere model (CRWMS M\&O 2000 
[DIRS 151615]) that supported the TSPA for the site recommendation (SR), TSPA-SR (CRWMS M\&O 2000 [DIRS 153246]). The ERMYN implements capabilities to address shortcomings of the GENII-S software used for the previous biosphere model (GENII-S; Leigh et al. 1993 [DIRS 100464]), addresses site specific concerns, better incorporates the included FEPs into the conceptual model, incorporates recommendations of an International Atomic Energy Agency (IAEA) international review team (IAEA 2001 [DIRS 155188]), addresses acceptance criteria for the review of the LA (NRC 2003 [DIRS 163274]), and addresses biosphere-related issues raised by the U.S. Nuclear Regulatory Commission (NRC) (Reamer 2001 [DIRS 157090]; Reamer 2001 [DIRS 158380]; Reamer 2001 [DIRS 159894]).

The ERMYN uses relevant site-specific information, and the environmental pathways and model simplifications are specific to the required reference biosphere and human receptor (10 CFR 63.305 [DIRS 156605]). The model is used for environmental radiation dose assessments and can calculate radionuclide-specific doses or provide radionuclide-specific biosphere dose conversion factors (BDCFs) for a human receptor. A BDCF is numerically equal to an all-pathway dose that a defined human receptor receives under specific biosphere conditions when the receptor is exposed to radionuclide contamination in environmental media arising from a unit concentration of a radionuclide in a source medium. The TSPA-LA model requires radionuclide-specific BDCFs, and the use of BDCFs in the TSPA-LA model is described in Sections 6.4.10 and 6.5.8. The radionuclides for which the model is developed are those identified in the analysis Radionuclide Screening (BSC 2002 [DIRS 160059], Section 7). The ERMYN is based on biosphere pathways consistent with arid or semi-arid conditions and little or no surface water discharge or transport. Limitations of the ERMYN are discussed in Section 8.2. A principal limitation is the consideration of only chronic exposure scenarios. The model is not applicable to acute exposures, such as are expected during the period of the volcanic eruption. Because the model is valid for input parameters applicable to arid/semiarid conditions, the use of the model for high precipitation regions may produce invalid results. The BDCFs generated by the model for the groundwater scenario are applicable after long-term irrigation (for some radionuclides this may be many hundreds of years or longer). If the model is used for shorter periods of irrigation, the dose predictions made from the BDCFs generated for long irrigation periods will be conservative for radionuclides having large partition coefficients. The posteruption volcanic model is limited to cases where the radionuclides are dispersed with fine ash particles. The model is not valid for coarse (or larger) tephra particles or ash flows.

To help readers navigate the biosphere model documentation, a graphical representation of the documentation structure is presented (Figure 1-1). Figure 1-1 illustrates the interrelationships among the products (i.e., analysis and model reports) developed for biosphere modeling, and the biosphere abstraction products for TSPA, as identified in the Technical Work Plan for Biosphere Modeling and Expert Support (BSC 2004 [DIRS 169573]). The biosphere model documentation currently consists of ten documents. This Biosphere Model Report is shown in the middle of the figure. The input parameter reports shown to the right of the Biosphere Model Report contain detailed descriptions of the development of the model input parameters and their distributions. Outputs from these six reports are used in the Nominal Performance Biosphere Dose Conversion Factor Analysis and Disruptive Event Biosphere Dose Conversion Factor Analysis to generate the biosphere dose conversion factors (BDCFs), which are input parameters for the TSPA-LA model. The Biosphere Dose Conversion Factor Importance and Sensitivity Analysis analyzes the 
output of these two BDCF reports. The biosphere FEP report summarizes the FEPs in the model and provides justification for excluding FEPs.

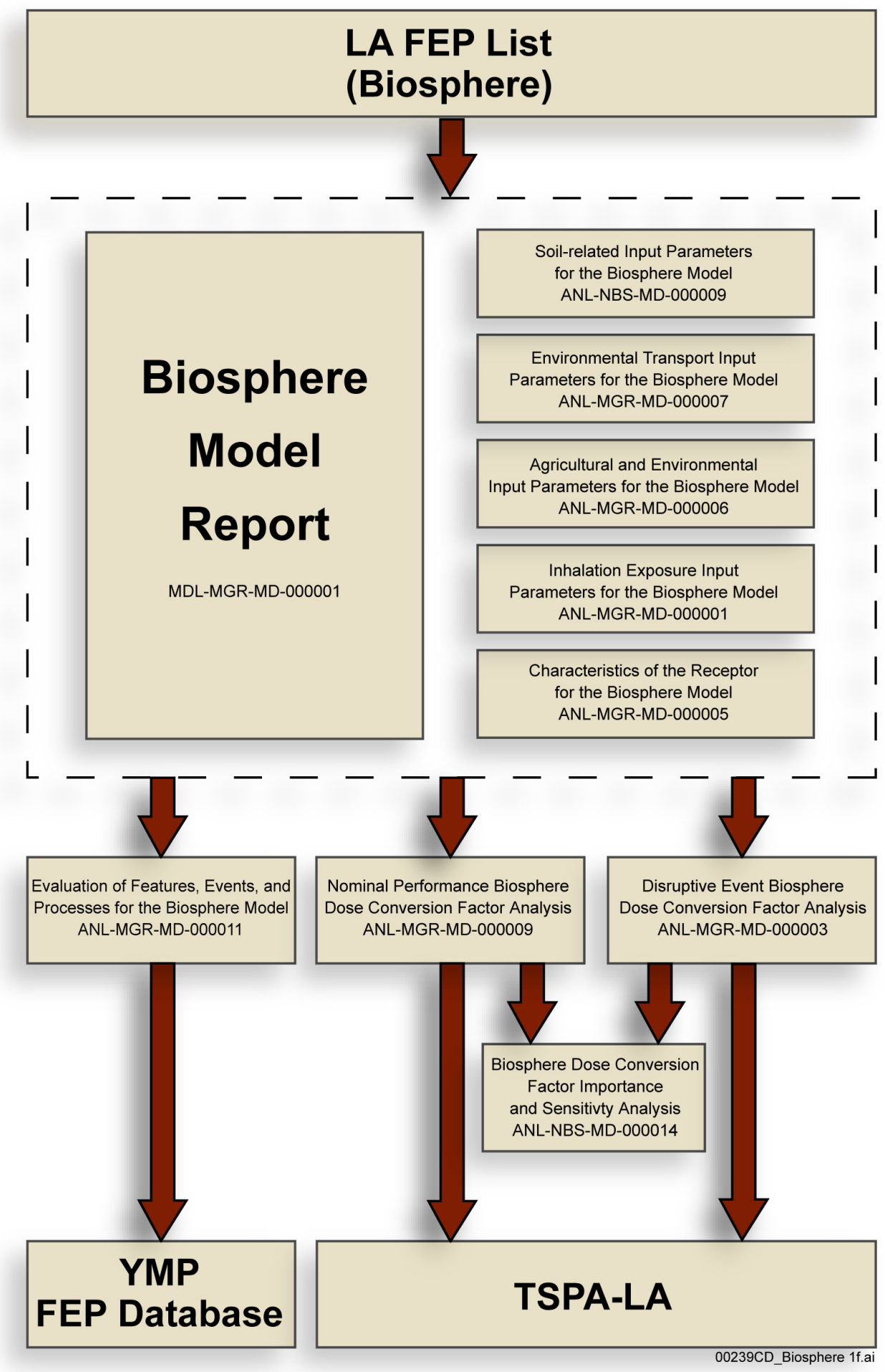

Figure 1-1. Overview of the Yucca Mountain Biosphere Model Documentation 
The FEPs considered for the biosphere model (Section 6.2) are listed in the LA FEP List (DTN: MO0407SEPFEPLA.000 [DIRS 170760]), and the majority of the biosphere-related FEPs are listed in the TWP (BSC 2004 [DIRS 169573], Table 2). Consideration of the LA FEPs List (DTN: MO0407SEPFEPLA.000 [DIRS 170760]) constitutes a deviation from the TWP (BSC 2004 [DIRS 169573]), which referred to an earlier revision of the FEPs list. Two additional included FEP are now addressed; these are FEP 2.2.08.01.0A, Chemical characteristics of groundwater in the SZ and FEP 1.3.07.02.0A, Water table rise affects SZ ( $\mathrm{SZ}=$ saturated zone).

With the exception of the parameters developed in Section 6.4.3.2, none of the parameter values used in the ERMYN mathematical model are developed in this report. This report describes the biosphere model, which is built in GoldSim software (GoldSim Technology Group 2002 [DIRS 160643]), but it does not contain the numerical results (outputs) of biosphere modeling (i.e., radionuclide-specific BDCFs for the TSPA-LA). 


\section{QUALITY ASSURANCE}

As described in the TWP (BSC 2004 [DIRS 169573]), this report documents a scientific model that supports the TSPA-LA, and, thus, it is a quality affecting activity in accordance with AP-2.27Q, Planning for Science Activities. Approved quality assurance procedures identified in the TWP (BSC 2004 [DIRS 169573], Section 4) are used to conduct and document the activities described in this report. Electronic data for this analysis are controlled in accordance with the TWP (BSC 2004 [DIRS 169573], Section 8). The governing procedure for the development of this model report was AP-SIII.10Q, Models.

The original version of this report (BSC 2003 [DIRS 164186]) was developed, validated, and approved per the TWP (BSC 2003 [DIRS 163602]) in effect at that time.

The natural barriers and items identified in the Q-List (BSC 2004 [DIRS 168361]) are not pertinent to this analysis, and a Safety Category per AP-2.22Q, Classification Analyses and Maintenance of the Q-List, is not applicable. 


\section{INTENTIONALLY LEFT BLANK}




\section{USE OF SOFTWARE}

The ERMYN is implemented using GoldSim stochastic simulation software. This software was selected because it is a graphical, object-oriented computer program for carrying out dynamic, probabilistic simulations (GoldSim Technology Group 2002 [DIRS 160643]). GoldSim allows for visually creating and manipulating data and equations, which are displayed as graphical objects (referred to as graphical elements in GoldSim). This software allows the model developer and user to perform probabilistic analyses in which multiple processes subject to parametric uncertainty are simulated with all uncertainties propagated to the required result. It, therefore, meets the biosphere model requirements of stochastic sampling, data management and manipulation, and calculation. There are no known limitations on outputs from ERMYN due to the use of this software. The use of this software for development of the ERMYN is consistent with the intended use and within the documented validation range of the software described in the Validation Test Report, GoldSim Version 7.50.100 (BSC 2002 [DIRS 163962]).

The GoldSim software (Version 7.50.100) is qualified under the Office of Civilian Radioactive Waste Management, Quality Assurance Program for use on the YMP (software tracking number: 10344-7.5.100-00; BSC 2003 [DIRS 161572]). Software Configuration Management provided a copy of the GoldSim software and installed it on a DELL Precision Workstation 530 computer (CPU\# 151555) under the Microsoft Windows 2000 operating system. GoldSim is appropriate for constructing the ERMYN, and it is used within the range of validation in accordance with procedure LP-SI.11Q-BSC, Software Management.

For comparisons between the ERMYN and other biosphere models, some calculations are performed using Microsoft Excel 97 SR-2, a commercial, off-the-shelf program, which is exempt form software qualification under LP-SI.11Q-BSC. These calculations use standard Excel functions as described in this report. All formulas, inputs, and outputs related to the use of Excel and required to reproduce the computations are documented in the applicable tables of this report or in the files in Appendix A. The results of these calculations are not dependent upon the software used. 


\section{INTENTIONALLY LEFT BLANK}




\section{INPUTS}

\subsection{DIRECT INPUTS}

This report describes the development and validation of the ERMYN. Execution of the model requires hundreds of input parameters. However, this report does not develop values for the parameters nor was the model executed to produce product output as part of this report. All of the parameter values for the ERMYN are developed in the input parameter reports (Figure 1-1). BDCF calculations and other interim calculations in this report are only for verifying and validating the model.

The ERMYN mathematical model was developed using published literature sources. The mathematical representations described in Sections 6.4 and 6.5 were based primarily on a review of published biosphere models. Documents describing the models reviewed are listed in Section 7.1, which identifies models used for, and excluded from, validation. These models are appropriate for the following reasons: (1) they include state-of-the-art methods for environmental radiation models, (2) they include a comprehensive description of the methods available to predict doses from chronic radiation exposure, and (3) at least some part of each model is applicable to the ERMYN conceptual model.

FEPs - The LA FEP List (DTN: MO0407SEPFEPLA.000. [DIRS 170760]) is the source of FEPs for developing this model. This is a comprehensive list of FEPs that may be applicable to the exposure scenarios that might result from storing spent nuclear fuel and high-level radioactive waste in a geologic repository at Yucca Mountain. This is an appropriate list from which to select FEPs for the ERMYN (Section 6.2). See the Evaluation of Features Events, and Processes (FEP) for the Biosphere Model (BSC 2004 [DIRS 169826]) for a list of FEPs screened for inclusion in this model.

Radionuclides of Interest-Although the mathematical models described in Sections 6.4 and 6.5 apply to any radionuclides, the ERMYN focuses on radionuclides considered for the TSPA-LA (BSC 2002 [DIRS 160059]). The model output, the ERMYN GoldSim model, is built for only those 28 primary radionuclides (Section 6.1.3). These radionuclides are listed in Table 6.1-1. The list includes all long-lived radionuclides that could make an important dose contribution during the first million years after establishing a repository at Yucca Mountain; therefore, it is the appropriate list for the ERMYN. Many of these primary radionuclides, once introduced into the biosphere, have short-lived decay products that can contribute to exposure to the reasonably maximally exposed individual (RMEI). The ERMYN considers these decay products, as defined in Characteristics of the Receptor for the Biosphere Model (BSC 2004 [DIRS 169671], Table 6-23) and further discussed in Section 6.3.5.

Mathematical Equations-The majority of the individual mathematical models developed in Sections 6.4 and 6.5 are based on previously published models of radionuclide transport and consequence analysis in the biosphere.

The extent to which the equations demonstrate the properties of interest was documented and the following factors were considered in the succeeding sections to evaluate the equations regarding their suitability for intended use: 
- Reliability of equations source

- Qualification of personnel or organizations generating the equations

- Prior uses of the equations

- Availability of corroborating equations, information, or data.

ERMYN is primarily based on the GENII/GENII-S code. The equations for that code are documented by Napier et al. (1988 [DIRS 157927]). The Pacific Northwest National Laboratory WEB site at http://www.pnl.gov/eshs/software/genii.html provides the following pertinent information on the GENII code. The GENII computer code was developed at Pacific Northwest National Laboratory to incorporate the internal dosimetry models recommended by the International Commission on Radiological Protection (ICRP) into updated versions of existing environmental pathway analysis models. The resulting second generation of environmental dosimetry computer codes is compiled in the Hanford Environmental Dosimetry System (Generation II or GENII). The GENII system was developed to provide a state-of-the-art, technically peer-reviewed documented set of programs for calculating radiation doses from radionuclides released to the environment. Because this is also the intent of the ERMYN, the equations match the properties of interest required for use in this model. Although the codes were developed for use at Hanford, they were designed with the flexibility to accommodate input parameters for a wide variety of generic sites. The GENII code has been used extensively for radiological assessments following radionuclide releases into the environment, most notably for the evaluation and licensing of the Waste Isolation Pilot Plant in New Mexico. Napier et al. (1988 [DIRS 157927]) is the source of all equation except those identified in Table 4-1.

Table 4-1. Additional Sources of Equations Used as a Technical Product Input

\begin{tabular}{|l|l|l|}
\hline \multicolumn{1}{|c|}{ Item } & \multicolumn{1}{|c|}{ Equation } & \multicolumn{1}{c|}{ Source } \\
\hline 1 & $6.4 .1-7$ & Lamarsh 1983 [DIRS 149069], Section 2.9 \\
\hline 2 & $6.4 .1-8$ & Lamarsh 1983 [DIRS 149069], Section 2.9 \\
\hline 3 & $6.4 .1-10$ & Baes and Sharp 1983 [DIRS 109606] \\
\hline 4 & $6.4 .2-4$ & NCRP 1999 [DIRS 155894], Section 4.3.6 \\
\hline 5 & $6.4 .2-5$ & Yu et al. 2001 [DIRS 159465], Equation C.12 \\
\hline 6 & $6.4 .3-5$ & Hoffman et al. 1989 [DIRS 124110] \\
\hline 7 & $6.4 .6-4$ & Yu et al. 2001 [DIRS 159465], p. L-18 \\
\hline
\end{tabular}

The individual equations used in the GENII code were extensively analyzed in this report and compared with corresponding equations used in other biosphere models for the same processes and properties. This comparison, documented in Sections 7.3 and 7.4, corroborates the appropriateness of the equations from the GENII code for their intended purpose. The equations from this report are, thus, considered suitable for the specific application and qualified for the intended use of developing the biosphere model by virtue of their representativeness, reliability of the source, the qualifications of the personnel and organizations generating the equations, and the prior uses of the equations.

The other equations used in the ERMYN (Table 4-1) are discussed below. 
Items 1 and 2. Lamarsh (1983 [DIRS 149069]) is a textbook for use in university nuclear engineering classes. The equations cited here are simple conservation equations. The first equates the rate of change of the number of atoms of a particular radionuclide in surface soil to rate of ingrowth from the parent radionuclide less the rate of removal by radioactive decay, leaching, and erosion. The second equation is derived from the first by normalizing to unit area and changing the variable from number of atoms to activity. Being based on the principle of conservation, these equations are considered established fact and do not require further justification.

Item 3. Baes and Sharp (1983 [DIRS 109606]) developed an approximate solution for the annual average leaching rate in soils resulting from overwatering to remove unwanted salt build-up. This equation matches the properties of interest and the intent of the leaching calculations in the soil submodel, as discussed in Section 6.4.1.3, as is, thus, considered qualified for the intended use. This equation for the leaching constant has been used in previous environmental assessment models, most notably the GENII code. Its use here is captured in the discussion above on the GENII code. This direct prior use by the originators of the GENII code satisfies the requirement to justify its use in the biosphere model.

Item 4. NCRP (1999 [DIRS 155894]) - The National Council on Radiation Protection and Measurements (NCRP) Report No. 129, Recommended Screening Limits for Contaminated Surface Soil and Review of Factors Relevant to Site-Specific Studies, provides screening approaches that can be applied to sites where the surface soil is contaminated with radionuclides to assist with impact evaluations and with making decisions regarding any necessary remediation. The report includes a description of the methods used to arrive at the values of screening factors. These screening approach methods were chosen such that they are conservative under conditions that normally apply to soil contamination, such as those modeled in this report. The description of the methods and the pertinent parameters are useful for developing parameter values for the ERMYN biosphere model. The NCRP calculation used in this model estimates the concentration of radon outdoors from the amount of radon exhaled from the soil. This matches the properties of interest and the intent of the air submodel (Section 6.4.2.3).

The NCRP is an organization that formulates and widely disseminates information, guidance, and recommendations on radiation protection and measurements, and its publications represent the consensus of leading scientific thinking on the topics presented. The NCRP was chartered by the U.S. Congress in 1964 as the National Council on Radiation Protection and Measurements. The Charter of the Council (Public Law 88-376) states that some of its objectives are to:

1. collect, analyze, develop, and disseminate in the public interest information and recommendations about (a) protection against radiation (referred to herein as radiation protection) and (b) radiation measurements, quantities, and units, particularly those concerned with radiation protection;

2. develop basic concepts about radiation quantities, units, and measurements, about the application of these concepts, and about radiation protection; 
3. cooperate with the ICRP, the Federal Radiation Council, the International Commission on Radiation Units and Measurements, and other national and international organizations, governmental and private, concerned with radiation quantities, units, and measurements and with radiation protection.

The NCRP is a nongovernmental, not-for-profit, public service organization and has status as an educational and scientific body. The main products of the NCRP are scientific reports. The recommendations promulgated by the Council provide the scientific basis for radiation protection efforts throughout the country. Because of these factors, the NCRP reports can be considered as sources of established fact equations, information, and data. The specific use of the information identified in this biosphere model (Eq. 6.4.2-4 to model the outdoor radon concentration) is, therefore, justified.

Items 5 and 7. Yu et al. (2001[DIRS 159465]) is the User's Manual for RESRAD Version 6. An overview of the code is provided in the preface to the document. This description is as follows.

In 1993, a manual on using Version 5 of the RESRAD code to implement the U.S. Department of Energy's (DOE's) residual radioactive material guidelines was released. Since then, as part of the RESRAD quality assurance (QA) program, the RESRAD code has undergone extensive review, benchmarking, verification, and validation. The manual and code have been used widely by DOE and its contractors, the NRC, U.S. Environmental Protection Agency, U.S. Army Corps of Engineers, industrial firms, universities, and foreign government agencies and institutions. New features, some in response to comments received from users, have been incorporated into the code to form RESRAD 6. These improvements have increased RESRAD's capabilities and flexibility, and enabled users to interact with the code more easily. With the improvements, the code has become more realistic in terms of the models and default parameters it uses. RESRAD 6 represents the sixth major version of the RESRAD code since it was first issued in 1989.

The equations from this reference are used in the biosphere model to calculate indoor radon concentration and ${ }^{14} \mathrm{C}$ uptake by crops through their roots. This equation matches the properties of interest and the intent of the air (Section 6.4.2.3) and ${ }^{14} \mathrm{C}$ submodels (Section 6.4.6.3). These equations are discussed, compared to other equations representing the same property, and justified for intended use in Section 7.3.2.2 and 7.3.5, for Items 5 and 7 (Table 4.1-1) respectively.

By virtue of RESRAD's pedigree discussed above (i.e., the reliability of the source of the equations and qualification of personnel or organizations generating the equations) and the prior use of the RESRAD suite of code employing the equations, it is considered suitable for the specific application and qualified for use within this report.

Item 6. Hoffman et al. (1989 [DIRS 124110]) at Oak Ridge National Laboratory performed a set of experiments involving spraying contamination on the foliage of three types of plant with five radionuclides. Three independent variables were controlled: irrigation intensity, quantity of irrigation per application, and crop standing biomass. The measured dependent variable was the interception fraction for the conditions defined. The details are further discussed in Section 6.4.3.2. The authors then fitted an empirical equation (Eq. 6.4.3-5) to their data. This 
empirical equation is used in this report with the fitted parameters for beryllium data that provided the highest (conservative) prediction for the interception fraction.

The primary reason the empirical equation is used in the ERMYN is to incorporate variation and uncertainty in irrigation rates and the types of crops grown in the Amargosa Valley. Hoffman et al. (1989 [DIRS 124110]) show that the proportion of radionuclides intercepted differs depending on the size of plants (i.e., aboveground biomass), the rate at which water is applied, the amount of water applied, and the charge carried by the chemical element. Therefore, a single value per crop type is not adequate because there are a substantial number of crops per crop type grown in the Amargosa Valley (BSC 2004 [DIRS 169673], Section 7 and Appendix A). The method proposed by Hoffman represents the properties of interest because it accounts for differences in irrigation requirements and growth forms of the crops. It also accounts for differences resulting from climate change. A comparison of the results of this equation to corroborate data summarized in Anspaugh (1987 [DIRS 123696]) is presented in Section 7.3.3.2. It is concluded that the equation results encompass the range of variation in the corroborating data. It is, thus, suitable for the application in the biosphere model and qualified for the intended use.

The use of this equation is also justified for its use in the biosphere model by the qualification of the personnel and organizations generating the equations, in addition to the extent to which the equation demonstrates the properties of interest.

Gamma Ray Exposure Data-Appendix D of this report considers contributions to exposure from various pathways associated with evaporative coolers. The biosphere model includes the exposure contribution from inhalation of radionuclides transferred from the water to the cooling air stream during the evaporation process. Appendix D demonstrates that the external exposure contribution to dose from build-up of precipitates in the cooler system is negligible to the external dose contribution from radionuclide build-up in irrigated soils. As a result, this pathway is excluded from the evaporative cooler model. This analysis required data of "specific gamma ray dose constants at one meter" for the radionuclides of concern. These data were taken from The Health Physics and Radiological Health Handbook (Shleien 1992 [DIRS 127299], Table 6.1.2). This reference handbook serves as a primary source of information for radiation protection professionals. The handbook provides an encyclopedia of radiation health information, with toxicity tables, classification of workplaces, decay schemes, nonionizing radiation, and environmental monitoring programs, as well as extensive glossaries on ionizing radiation, light and lasers, ultrasound, and radiofrequency electromagnetic fields. The data presented in this handbook are therefore considered accepted fact in the field of health physics and radiological safety. The data used from Table 6.1.2 are suitable for the screening arguments developed in Appendix D and are qualified for that purpose.

\subsection{CRITERIA}

Regulation 10 CFR Part 63 [DIRS 156605], Energy: Disposal of High-Level Radioactive Wastes in a Geologic Repository at Yucca Mountain, Nevada, contains regulatory requirements that apply to developing the biosphere model. The Project Requirements Document (Canori and Leitner 2003 [DIRS 166275], Table 2-3) contains three requirements that apply to biosphere modeling (Table 4.2-1). Also listed in Table 4.2-1 are the acceptance criteria from the Yucca 
Mountain Review Plan, Final Report (NRC 2003 [DIRS 163274]) applicable to this analysis. The list is based on meeting the requirements of 10 CFR 63.114, 10 CFR 63.305, and 10 CFR 63.312 [DIRS 156605] that relate in whole, or in part, to this analysis.

Table 4.2-1. Requirements Applicable to Biosphere Modeling

\begin{tabular}{|c|c|c|c|}
\hline $\begin{array}{c}\text { Requirement } \\
\text { Number }^{\mathrm{a}}\end{array}$ & Requirement Title $^{a}$ & 10 CFR 63 Link & $\begin{array}{l}\text { YMRP Acceptance } \\
\text { Criteria }^{\mathbf{b}}\end{array}$ \\
\hline PRD-002/T-015 & $\begin{array}{l}\text { Requirements for Performance } \\
\text { Assessment }\end{array}$ & $\begin{array}{l}10 \text { CFR } 63.114 \\
\text { (a) to }(g)\end{array}$ & \multirow[t]{2}{*}{$\begin{array}{l}\text { 2.2.1.3.13, criteria } 1 \text { to } 5 \\
2.2 \cdot 1.3 .14 \text {, criteria } 1 \text { to } 5\end{array}$} \\
\hline PRD-002/T-026 & $\begin{array}{l}\text { Required Characteristics of the Reference } \\
\text { Biosphere }\end{array}$ & 10 CFR 63.305 & \\
\hline PRD-002/T-028 & $\begin{array}{l}\text { Required Characteristics of the } \\
\text { Reasonably Maximally Exposed Individual }\end{array}$ & 10 CFR 63.312 & 2.2.1.3.14, criteria 1 to 5 \\
\hline
\end{tabular}

a from Canori and Leitner (2003 [DIRS 166275], Table 2-3).

b from NRC (2003 [DIRS 163274]).

YMRP=Yucca Mountain Review Plan

In addition to the requirements listed in Table 4.2-1, definition of terms in 10 CFR 63.2 and description of concepts in 10 CFR 63.102 [DIRS 156605] relevant to biosphere modeling are also applicable to this analysis.

The acceptance criteria identified in Sections 2.2.1.3.13.3 and 2.2.1.3.14.3 of the Yucca Mountain Review Plan (NRC 2003 [DIRS 163274] are included below. In cases where subsidiary criteria are listed in the Yucca Mountain Review Plan for a given criterion, only the subsidiary criteria addressed by this model report are listed below. Where a subcriterion includes several components, only some of those components may be addressed. How these components are addressed is summarized in Section 8.3 of this report.

\section{Acceptance Criteria from Section 2.2.1.3.13, Redistribution of Radionuclides in Soil}

\section{Acceptance Criterion 1, System Description and Model Integration are Adequate}

(1) Total system performance assessment adequately incorporates important features, physical phenomena and couplings between different models, and uses consistent and appropriate assumptions throughout the abstraction of redistribution of radionuclides in the soil abstraction process;

(2) The total system performance assessment model abstraction identifies and describes aspects of redistribution of radionuclides in soil that are important to repository performance, including the technical bases for these descriptions. For example, the abstraction should include modeling of the deposition of contaminated material in the soil and determination of the depth distribution of the deposited radionuclides;

(3) Relevant site features, events, and processes have been appropriately modeled in the abstraction of redistribution of radionuclides, from surface processes, and sufficient technical bases are provided. 


\section{Acceptance Criterion 2, Data are Sufficient for Model Justification}

(1) Behavioral, hydrological, and geochemical values used in the license application are adequately justified (e.g., irrigation and precipitation rates, erosion rates, radionuclide solubility values, etc.). Adequate descriptions of how the data were used, interpreted, and appropriately synthesized into the parameters are provide; and

\section{Acceptance Criterion 3, Data Uncertainty in Characterized and Propagated Through the Model Abstraction}

(1) Models use parameter values, assumed ranges, probability distributions, and bounding assumptions that are technically defensible, reasonably account for uncertainties and variabilities, do not result in an under-representation of the risk estimate, and are consistent with the characteristics of the reasonably maximally exposed individual in 10 CFR Part 63;

(2) The technical bases for the parameter values and ranges in the total system performance assessment abstraction are consistent with data from the Yucca Mountain region, e.g., Amargosa Valley survey, studies of surface processes in the Fortymile Wash drainage basin; applicable laboratory testings; natural analogs; or other valid sources of data. For example, soil types, crop types, plow depths, and irrigation rates should be consistent with current farming practices, and data on the airborne particulate concentration should be based on the resuspension of appropriate material in a climate and level of disturbance similar to that which is expected to be found at the location of the reasonably maximally exposed individual, during the compliance time period;

(3) Uncertainty is adequately represented in parameters for conceptual models, process models, and alternative conceptual models considered in developing the total system performance assessment abstraction of redistribution of radionuclides in soil, either through sensitivity analyses, conservative limits, or bounding values supported by data, as necessary. Correlations between input values are appropriately established in the total system performance assessment;

(4) Parameters or models that most influence repository performance based on the performance measure and time period of compliance, specified in 10 CFR Part 63, are identified; and

\section{Acceptance Criterion 4, Model Uncertainty is Characterized and Propagated Through the Model Abstraction}

(1) Alternative modeling approaches of features, events, and processes are considered and are consistent with available data, and current scientific understanding, and the results and limitations are appropriately considered in the abstraction;

(2) Sufficient evidence is provided that appropriate alternative conceptual models of features, events, and processes have been considered; that the preferred models (if any) are consistent with available data (e.g., field, laboratory, and natural analog) and current scientific understanding; and that the effect on total system performance assessment of uncertainties from these alternative conceptual models has been evaluated; and

(3) Consideration of conceptual model uncertainty is consistent with available site characterization data, laboratory experiments, field measurements, natural analog information 
and process-level modeling studies; and the treatment of conceptual model uncertainty does not result in an under-representation of the risk estimate.

\section{Acceptance Criterion 5, Evaluation Findings}

(1) Models implemented in the abstraction provide results consistent with output from detailed process-level models and/or empirical observations (e.g., laboratory testing, field measurements, and/or natural analogs).

\section{Acceptance Criterion from Section 2.2.1.3.14, Biosphere Characteristics}

\section{Acceptance Criterion 1, System Description and Model Integration are Adequate}

(1) Total system performance assessment adequately incorporates important site features, physical phenomena, and couplings, and consistent and appropriate assumptions throughout the biosphere characteristics modeling abstraction process;

(2) The total system performance assessment model abstraction identifies and describes aspects of the biosphere characteristics modeling that are important to repository performance, and includes the technical bases for these descriptions. For example, the reference biosphere should be consistent with the arid or semi-arid conditions in the vicinity of Yucca Mountain;

(3) Assumptions are consistent between the biosphere characteristics modeling and other abstractions. For example, the U.S. Department of Energy should ensure that the modeling of features, events, and processes, such as climate change, soil types, sorption coefficients, volcanic ash properties, and the physical and chemical properties of radionuclides are consistent with assumption in other total system performance assessment abstractions.

\section{Acceptance Criterion 2, Data are Sufficient for Model Justification}

(1) The parameter values used in the license application are adequately justified (e.g., behaviors and characteristics of the residents of the Town of Amargosa Valley, Nevada, characteristics of the reference biosphere, etc.) and consistent with the definition of the reasonably maximally exposed individual in 10 CFR Part 63. Adequate descriptions of how the data were used, interpreted, and appropriately synthesized into the parameters are provided;

(2) Data are sufficient to assess the degree to which features, events, and processes related to biosphere characteristics modeling have been characterized and incorporated in the abstraction. As specified in 10 CFR Part 63, the U.S. Department of Energy should demonstrate that features, events, and processes, which describe the biosphere, are consistent with present knowledge of conditions in the region, surrounding Yucca Mountain. As appropriate, the U.S. Department of Energy sensitivity and uncertainty analyses (including consideration of alternative conceptual models) are adequate for determining additional data needs, and evaluating whether additional data would provide new information that could invalidate prior modeling results and affect the sensitivity of the performance of the system to the parameter value or model. 


\section{Acceptance Criterion 3, Data Uncertainty is Characterized and Propagated Through the Model Abstraction}

(1) Models use parameter values, assumed ranges, probability distributions, and bounding assumptions that are technically defensible, reasonably account for uncertainties and variabilities, do not result in an under-representation of the risk estimate, and are consistent with the definition of the reasonably maximally exposed individual in 10 CFR Part 63;

(4) Uncertainty is adequately represented in parameter development for conceptual models and process-level models considered in developing the biosphere characteristics modeling, either through sensitivity analyses, conservative limits, or bounding values supported by data, as necessary. Correlations between input values are appropriately established in the total system performance assessment, and the implementation of the abstraction does not inappropriately bias results to a significant degree.

(6) Parameters or models that most influence repository performance, based on the performance measure and time period of compliance specified in 10 CFR Part 63, are identified.

Acceptance Criterion 4, Model Uncertainty is Characterized and Propagated Through the Model Abstraction.

(1) Alternative modeling approaches of features, events, and processes are considered and are consistent with available data and current scientific understanding, and the results and limitations of alternative modeling approaches are appropriately considered in the abstraction. Staff should evaluate alternate conceptual models of the biosphere or biosphere processes, recognizing that 10 CFR 63.305 and 63.312 place a number of constraints on both the biosphere and the characteristics of the reasonably maximally exposed individual. Alternate conceptual models focus on exploring the variability and uncertainty in the physical features, events, and processes, mindful of the regulatory constraints. Evaluation of behavior and characteristics of the reasonably maximally exposed individual emphasizes understanding the characteristics of the current residents of the Town of Amargosa Valley, and uncertainty and variability in the data used to derive mean values.

(2) Sufficient evidence is provided that existing alternative conceptual models of features and processes that are important to waste isolation, such as plant uptake of radionuclides from soil, soil resuspension, and the inhalation dose model for igneous events, have been considered; and

(3) Consideration of conceptual model uncertainty is consistent with available site characterization data, laboratory experiments, field measurements, natural analog information and process-level modeling studies; and the treatment of conceptual model uncertainty does not result in an under-representation of the risk estimate.

\section{Acceptance Criterion 5, Model Abstraction Output is Supported by Objective Comparisons}

(1) Dose calculations pertaining to this total system performance assessment abstraction provide results consistent with output from detailed process-level models and/or empirical observations (e.g., laboratory testing, field measurements, and/or natural analogs). 


\subsection{CODES, STANDARDS, AND REGULATIONS}

No codes, standards, or regulations, other than those identified in the Project Requirements Document (Canori and Leitner 2003 [DIRS 166275], Table 2-3) and in Section 4.2 and determined to be applicable, were used in this analysis. 


\section{ASSUMPTIONS}

AP-SIII.10Q defines an assumption to be "a statement or proposition that is taken to be true or representative in the absence of direct confirming data or evidence, or those estimations, approximations, and/or limitations made during model development (such as when expanding the range of variables to achieve conservatism)." Attachment 2 to AP-SIII.10Q requires that "this section (Section 5) shall include a description of the assumptions used, in the absence of direct confirming data or evidence, to perform the model activity. Other model assumptions are described in Section 6 of the model report." No assumptions are made in the absence of direct confirming data or evidence in this report, since parameter input data are developed in supporting reports (See Figure 1-1). Other model assumptions or approximations are made, discussed, and justified in Section 6. 


\section{INTENTIONALLY LEFT BLANK}




\section{MODEL DISCUSSION}

The ERMYN biosphere model was developed, validated, and documented in the initial issue of this document (BSC 2003 [DIRS 164186]). This revision corrects some typographical errors in the text, as noted in the errata sheet to BSC (2003 [DIRS 166507]), and enhances transparency and traceability of the document, while bringing it up to date with project procedures. The model developed by this effort generates the BDCFs that provide TSPA-LA with the capability to calculate annual dose from their predictions of radionuclide activity density in the environments for the groundwater and volcanic eruption release scenarios.

In this section, the biosphere model (i.e., the ERMYN) is described. The modeling objectives, including a description of the biosphere system, are discussed in Section 6.1. Model inputs and outputs, and their use in downstream models and analyses, are also discussed in Section 6.1. The ERMYN is based on as much site-specific information as possible. The FEPs considered in the ERMYN are listed in Section 6.2. Based on characteristics of the biosphere system, the included FEPs, modeling approximations, and the scenarios considered, a biosphere conceptual model is developed (Section 6.3). Alternative conceptual models (ACMs), which usually refer to other process models, are considered in the model development (Section 6.3.3). To quantify the radiation dose to a specific receptor, which in the case of the TSPA-LA is the RMEI, a series of mathematical representations are presented for the groundwater (Section 6.4) and volcanic ash scenarios (Section 6.5). All input parameters required for the mathematical model, including the uncertainty associated with them, are summarized in Section 6.6. Improvements in the ERMYN compared to the GENII-S model, used in the previous YMP biosphere modeling activities (CRWMS M\&O 2000 [DIRS 151615]; CRWMS M\&O 2001 [DIRS 152536]; CRWMS M\&O 2001 [DIRS 152539]), are identified in Section 6.7. The developed biosphere model, built in the GoldSim software (BSC 2003 [DIRS 161572]) (Section 3), produces BDCFs for the TSPA-LA model. Discussion of the model implementation is given for the groundwater (Section 6.8) and volcanic ash scenarios (Section 6.9). The GoldSim implementation of the ERMYN is verified in Section 6.10. This report does not develop model inputs or results; these are documented elsewhere as discussed in Section 1 (Figure 1-1).

\subsection{MODELING OBJECTIVES}

The objective of the ERMYN is to provide capabilities for calculating doses in the TSPA-LA model by building a biosphere model to estimate the annual human radiation dose that would result if unit concentrations of radionuclides were released from the geologic repository into the environment. In general, this biosphere model provides a method for assessing chronic radiation doses with an upper limit to radiation dose of tens of rem (Eckerman et al. 1988 [DIRS 101069], Section II). This is because the dose conversion factors and dose coefficients in the model apply to chronic intakes and low exposure conditions (continuous exposure to low level contamination), and are not appropriate for acute intakes and high exposure conditions (a few exposures to high contamination).

The radionuclides can be released into different environmental media, such as water for the groundwater scenario or soil for the volcanic ash scenario. Because the activity concentration in relevant source medium is developed from other process models, a unit activity concentration is considered as the source term in the biosphere model. This approach, using a biosphere process 
independent from the radionuclide source (BSC 2003 [DIRS 166296], Section 5.1), is evaluated in Sections 6.4.10 and 6.5.8. All biosphere model inputs are described in Sections 6.4 and 6.5 where the mathematical submodels are presented. All input parameters for generating model outputs are summarized in Section 6.6.

The biosphere model is used to calculate the sets of radionuclide-specific BDCFs that are used in the TSPA-LA model to calculate radiation dose to the RMEI. Between the radionuclide source and the BDCFs is the biosphere model, which contains a representation of radionuclide transfer mechanisms in the biosphere system, along with related approximations and simplifications, and the representation of the receptor.

It is important to first describe the biosphere system to be modeled. In general, the biosphere system includes the reference biosphere and the human receptor. Conceptually, the biosphere system is composed of a set of specific biotic and abiotic components of the accessible environment and the relationships between these components. These components are the essentials of a reference biosphere, as delineated by the final rule (10 CFR 63.305 [DIRS 156605]).

\subsubsection{Reference Biosphere}

The reference biosphere is the environment inhabited by the RMEI along with associated human radiation exposure pathways and parameters (10 CFR 63.102(i) [DIRS 156605]). The reference biosphere includes characteristics of the geography, geology, physiology, climate, hydrology, and population in the region surrounding the Yucca Mountain site. A brief overview of the reference biosphere is presented in this section. As specified by 10 CFR 63.305 [DIRS 156605], the required characteristics of the reference biosphere include FEPs that describe the reference biosphere (Section 6.2), the current conditions of society, biosphere, human biology, and human knowledge (Sections 6.1.1.1 and 6.1.1.3); predicted future conditions of geology, hydrology, and climate consistent with present knowledge (Section 6.1.1.2); and biosphere pathways consistent with arid and semi-arid conditions (Section 6.3). For developing the biosphere model, the characteristics of the reference biosphere are focused on the area around the location of the RMEI, as specified in 10 CFR 63.312 [DIRS 156605]. The regional and site information presented in this section provides an overview of the basis for selecting the FEPs and biosphere pathways considered in the ERMYN. Other biosphere characteristics, such as those identified by the IAEA international review team (IAEA 2001 [DIRS 155188], Section 4.1), are considered in the model.

\subsubsection{Geography, Geology, and Physiography}

Yucca Mountain is located in Nye County in southern Nevada, approximately $160 \mathrm{~km}$ northwest of Las Vegas, in an arid, sparsely populated region in the transition zone between the Great Basin and the Mojave deserts. Yucca Mountain and surrounding areas are in the southern-most part of the Great Basin, a subprovince of the Basin and Range Physiographic Province (Figure 6.1-1). The topography is typical of the Great Basin, which is characterized by more or less regularly spaced, north-south trending mountain ranges and intervening alluvial basins that are formed by faulting. 


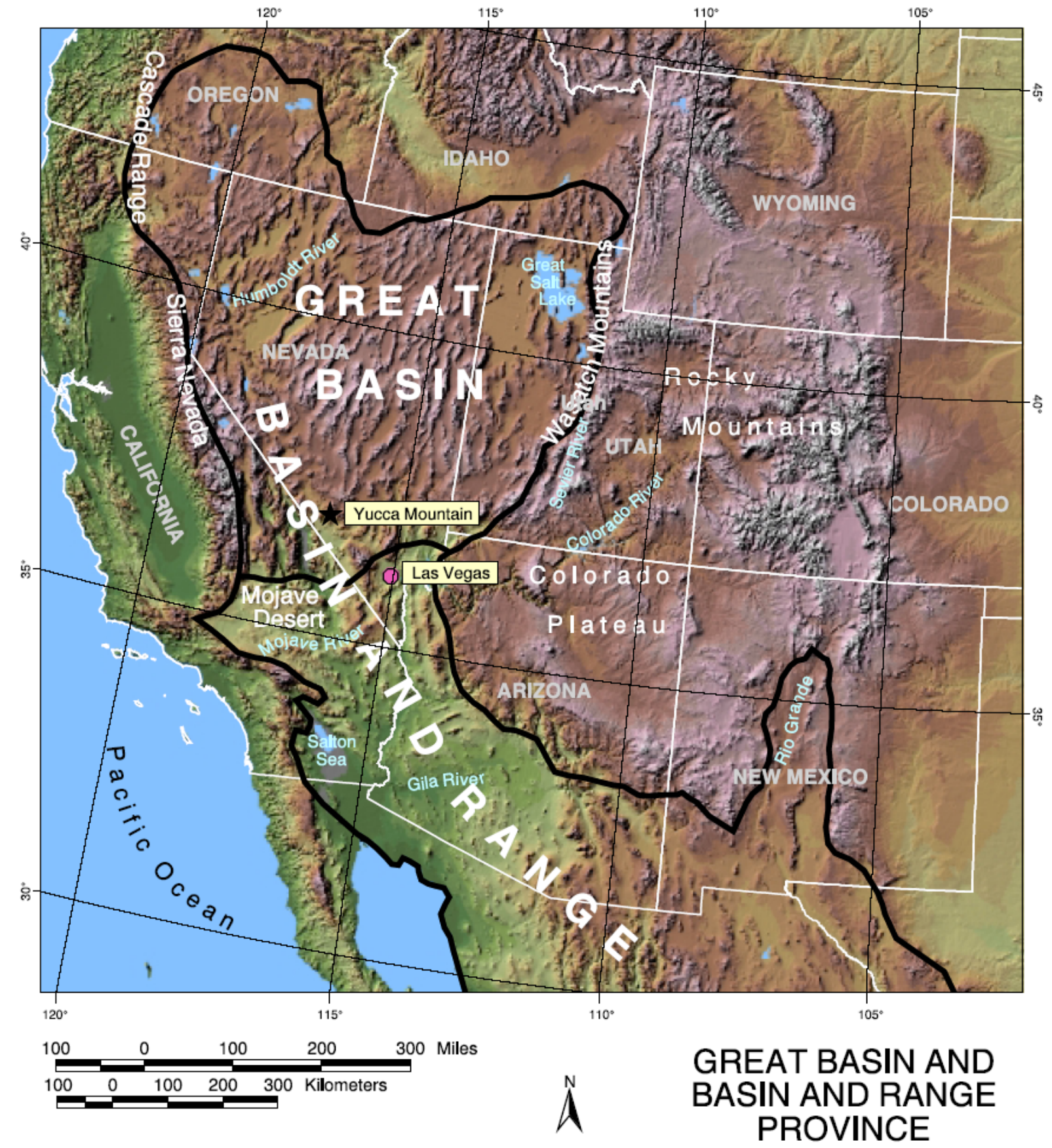

DTN MO0009YMP00093.000

NOTE: Names of specific geographical points are not necessary to understand the technical content of this document.

Figure 6.1-1. Yucca Mountain in Relation to the Great Basin 
The area surrounding Yucca Mountain can be divided into eight clearly defined physiographic landforms (BSC 2004 [DIRS 169734], Section 4.4.2.1). The four landforms most relevant to the biosphere conceptual model are Yucca Mountain, Fortymile Wash, Jackass Flats, and the Amargosa Desert (Figure 6.1-2). Yucca Mountain is an irregularly shaped upland, 6 to $10 \mathrm{~km}$ wide and about $40 \mathrm{~km}$ long. The crest of the mountain reaches elevations of 1,500 to 1,930 m, about $650 \mathrm{~m}$ higher than the floors of adjacent washes in Crater Flat and Jackass Flats. Yucca Mountain is composed of fine-grained volcanic rocks and is formed from fault blocks that tilt eastward, such that the fault-bounded west-facing slopes are generally high, steep, and straight, which contrasts with the gentler and often deeply dissected east-facing slopes. Drainage from the west flank of the mountain flows southward down narrow fault-controlled canyons and out into Crater Flat. Drainage from the east flank flows southeastward down Yucca, Drill Hole, and Dune Washes into Fortymile Wash.

There are no perennial streams at Yucca Mountain or northern Amargosa Valley. The ephemeral Amargosa River, which infrequently carries runoff from the Yucca Mountain area via Fortymile Wash, flows southeast along the western edge of the basin, eventually ending in Death Valley (Simmons 2004 [DIRS 166960], Sections 3.4.2 and 3.4.3). Fortymile Wash is a large ephemeral wash that drains an approximately $620 \mathrm{~km}^{2}$ area east and northeast of Yucca Mountain. From its headwaters northeast of Yucca Mountain, it flows southward through Fortymile Canyon and continues down the south-sloping piedmont that forms the west end of Jackass Flats. Along this latter reach, the wash cuts a nearly linear trench through the alluvial deposits, 150 to 600-m wide and up to 25-m deep. This trench gradually decreases downslope until the wash merges with the Amargosa Desert basin.

Jackass Flats is an alluvial basin, 8- to 10-km wide and nearly 20-km long, that lies east of Yucca Mountain and Fortymile Wash. Jackass Flats is formed principally by piedmonts that slope away from highlands to the north, east, and south, merge in the central basin area, and descend gradually westward and southwestward towards Fortymile Wash.

The Amargosa Desert is a broad northwest-trending basin approximately 80-km long and up to 30-km wide. The basin is one of the largest in the southern Great Basin. The basin floor slopes gently southeastward from elevations of about $975 \mathrm{~m}$ at the north end (near Beatty, Nevada) to about $600 \mathrm{~m}$ toward the south end.

The soils on alluvial fans and in stream channels in northern Amargosa Valley generally are deep and well to excessively drained. The surface soil layer generally is less than $20 \mathrm{~cm}$ (8 in.) thick and subsurface soils are up to $150 \mathrm{~cm}$ (59 in.) deep. Soil textures are very gravelly with fine sands to sandy loams. The soils are calcareous and moderately alkaline. This site-specific information is from the Natural Resource Conservation Service (Dollarhide 1999 [DIRS 159253] and CRWMS M\&O 1999 [DIRS 107736]). 


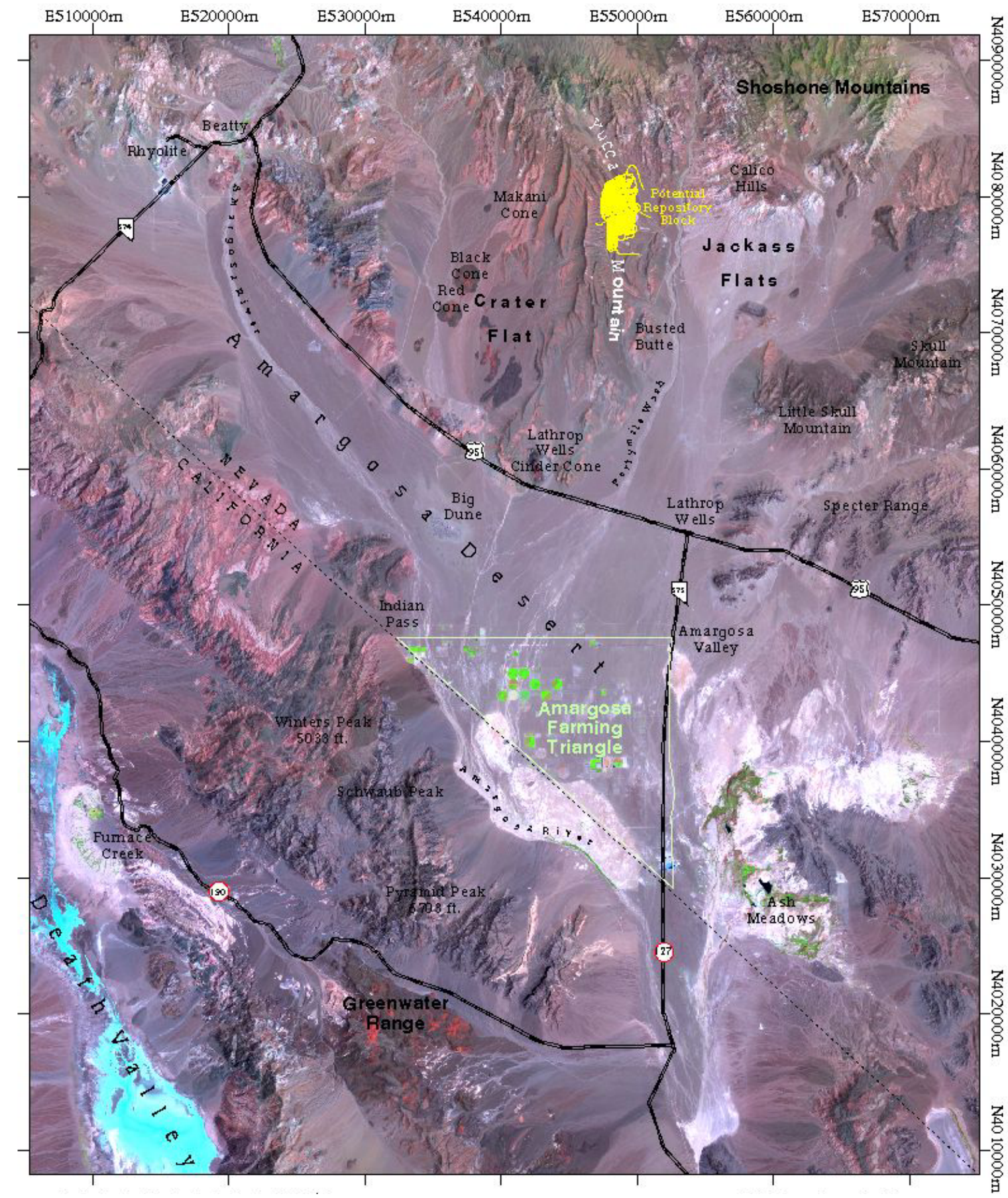

NOTE: Names of specific geographical points are not necessary to understand the technical content of this document.

Figure 6.1-2. Regional Map of Yucca Mountain and the Amargosa Valley 


\subsubsection{Climate, Flora, and Fauna}

The regional climate is characterized by low precipitation, hot summers, cool winters, and low relative humidity (BSC 2004 [DIRS 169734], Section 6.2). The Sierra Nevada mountain range, a dominant feature in the region, is a major barrier to moist air moving east from the Pacific Ocean and creates a rain shadow. Annual average precipitation in the region ranges from 100 to $200 \mathrm{~mm}$ (4 to 8 inches) and decreases from higher to lower elevations. About 50 percent of the annual precipitation is from frontal storms during November through April. Precipitation during the summer months often occurs as localized thunderstorms that may create floods and runoff. Precipitation often varies between years by a factor of two. Temperatures vary through the year. Average maximum daytime temperatures are about $35^{\circ} \mathrm{C}\left(95^{\circ} \mathrm{F}\right)$ in July and $11^{\circ} \mathrm{C}\left(52^{\circ} \mathrm{F}\right)$ in January. Although the average nighttime temperature in January is above freezing, $2^{\circ} \mathrm{C}\left(36^{\circ} \mathrm{F}\right)$, freezing temperatures occur. Low precipitation and warm temperatures keep atmospheric humidity low, with annual average relative humidity less than 20 percent.

The combination of low precipitation, warm temperatures, and low humidity results in high rates of evaporation and the loss of moisture by plants via transpiration. Shrubs adapted to periodic drought and extremes in temperatures dominate the native vegetation in the region (CRWMS M\&O 1996 [DIRS 102235]). Shrubs cover 20 to 30 percent of the ground, depending upon precipitation, and are typical of the northern Mojave Desert, such as creosote bush (Larrea tridentata) and white bursage (Ambrosia dumosa). There are no forested areas in Amargosa Valley or elsewhere south of Yucca Mountain (DOE 2002 [DIRS 155970], Section 3.1.5.1.1).

Wildlife in the Yucca Mountain region is dominated by species associated with the Mojave Desert, with some species from the Great Basin Desert at higher elevations. Game species found in the region include Gambel's quail (Callipepla gambelii), chukar (Alectoris chukar), mourning dove (Zenaida macroura), and mule deer (Odocoileus hemionus) (CRWMS M\&O 1999 [DIRS 104593]). Those species are most common in the mountains surrounding Amargosa Valley and Yucca Mountain and in areas where water is available from springs, seeps, and man-made water developments (CRWMS M\&O 1999 [DIRS 104593], Section 3.3.2.3; DOE 2002 [DIRS 155970], Section 3.1.5.1.2).

Geological and biological media provide a historical record of the types and periodicity of climate change in the Yucca Mountain region. Future climate predictions for the next 10,000 years indicate that the present-day interglacial climate at Yucca Mountain should persist for another 400 to 600 years. After that, the climate shifts to a warmer and wetter monsoon climate (lasting 900 to 1,400 years) and then to a cooler and wetter glacial transition climate for 8,000 to 8,700 years (BSC 2004 [DIRS 170002], Section 6.6).

\subsubsection{Groundwater, Human Activities, and Agriculture}

Water in the aquifers beneath Yucca Mountain generally flows from north to south (D'Agnese et al. 1997 [DIRS 100131]). Therefore, groundwater flows from the repository area south to the Amargosa Valley (Figure 6.1-2). If radionuclides are released into the groundwater or the air at Yucca Mountain, groundwater flow and wind patterns suggest that some of these radionuclides would spread south and east into the Amargosa Valley region. 
Water used for domestic, municipal, and agricultural purposes in Amargosa Valley comes from groundwater. There are no public water treatment systems in Amargosa Valley, and there is only a small, quasi-municipal water delivery system for which drinking water standards could be enforced (State of Nevada 1997 [DIRS 110951]).

The region surrounding Yucca Mountain is rural and sparsely populated. The nearest human residents to Yucca Mountain (in the direction of groundwater flow) live in the Amargosa Valley. At the time of the 2000 census, it was estimated that 1,176 people in 422 households resided in the approximately 1,300-km² Amargosa Valley Census County Division (Bureau of the Census 2001 [DIRS 156858], Tables P3 and H6). Residents living closest to the repository are located at the intersection of U.S. Highway 95 and Nevada State Route 373 (Figure 6.1-2), which is approximately 20 to $21 \mathrm{~km}$ south of Yucca Mountain (BSC 2003 [DIRS 168723], Table 1 and Figure 1). Soil conditions at this location generally are similar to those further downgradient where farming currently is practiced (CRWMS M\&O 1999 [DIRS 107736]). In 2003, an estimated 23,180 people lived within $84 \mathrm{~km}$ (52 mi) of Yucca Mountain. About 80 percent lived more than $64 \mathrm{~km}$ (40 mi) from Yucca Mountain in and near Pahrump, Nevada. About 6 percent lived in the Amargosa Valley region about 20 to $56 \mathrm{~km}$ (12 to $35 \mathrm{mi}$ ) south of Yucca Mountain (BSC 2003 [DIRS 168723], Table 1 and Figure 1). Other communities and employment centers in south-central Nevada and the approximate highway distance from the intersection of Highway 95 and State Route 373 are Beatty, 45 km (28 mi); Pahrump, 70 km (43 mi); Indian Springs, $70 \mathrm{~km}$ (43 mi); and Las Vegas, $120 \mathrm{~km}(75 \mathrm{mi})$. Part of this information was used to characterize the receptor and the size of the reference biosphere (BSC 2004 [DIRS 169671], Sections 5.1.1 and 6.1).

In 2004, government and other community and social services in Amargosa Valley included a public library, an elementary school, churches, a community center and park, a senior center, a small medical clinic, an ambulance service, and a post office. There also were small convenience or general stores; restaurants, saloons, or gambling establishments; miscellaneous retail stores; and a motel (Rasmuson 2004 [DIRS 169506], Enclosure 2). Most of the agriculture and the majority of the population are located approximately $30 \mathrm{~km}$ south of Yucca Mountain in the Amargosa farming triangle, which is bounded by the Amargosa Farm Road on the north, Nevada State Route 373 on the east, and the California-Nevada border running from northwest to southeast. Most of the major roads in the area are paved. The nearest indoor recreation (e.g., movie theatres, other restaurants), larger stores, and hospitals are in Pahrump and Las Vegas.

There is a small agricultural industry in Amargosa Valley. Agriculture mainly involves growing feed (e.g., alfalfa) for farm animals; however, gardening and animal husbandry are common (YMP 1999 [DIRS 158212]). Small grains, pistachios, grapes, orchard crops, garlic, and onions are also grown commercially. Commercial agriculture in the Amargosa Valley farming triangle includes a dairy (approximately 5,000 cows) and a fish farm (approximately 15,000 catfish and bass; YMP 1999 [DIRS 158212], Tables 9 and 11). There are approximately 1,400 acres planted in alfalfa, 300 acres in other hay, 100 acres in pistachios, 16 acres in fruit trees, and 10 acres in grapes (BSC 2004 [DIRS 169673]).

There are no naturally occurring surface waters (i.e., perennial lakes and streams) in the area. Commercial crops are irrigated with groundwater, primarily using center pivot and other 
overhead sprinkler systems. Local wells provide water for household, agriculture, horticulture, and animal husbandry. Many residences have gardens with vegetable plots and some have a few cattle, sheep, chickens, and other farm animals (CRWMS M\&O 1997 [DIRS 101090], Section 3.4; Horak and Carns 1997 [DIRS 124149], pp. 4 to 18, YMP 1999 [DIRS 158212], Section 3.4; BSC 2004 [DIRS 169673], Appendix A).

\subsubsection{Human Receptor}

Characteristics of the RMEI, the human receptor for the TSPA-LA, are specified in regulation 10 CFR 63.312 [DIRS 156605]. The RMEI:

(a) Lives in the accessible environment above the highest concentration of radionuclides in the plume of contamination.

(b) Has a diet and life style representative of the people now residing in the Amargosa Valley, Nevada. Projections based on surveys of these people are used to determine the current diets and life styles, and mean values of these factors are used in assessments conducted for 10 CFR 63.311 and 10 CFR 63.321.

(c) Uses groundwater with average concentrations of radionuclides based on an annual water demand of 3000 acre-feet.

(d) Drinks groundwater (2 L/d) from wells at the location specified in paragraph (a).

(e) Is an adult metabolically and physiologically consistent with present knowledge of adults.

These requirements are used for developing the external exposure time, inhalation exposure time, and food consumption rates (BSC 2004 [DIRS 169671], Section 6). A food consumption survey of the residents of the Amargosa Valley was conducted (DOE 1997 [DIRS 100332]). The recent census data indicates that, although the Amargosa Valley is a rural community, most of the residents do not work in the agricultural business (Bureau of the Census 2002 [DIRS 159728], Table P49). The following is a summary of information on the people residing in Amargosa Valley used to aid the development of the model.

Diet-Based on a survey of Amargosa Valley residents (DOE 1997 [DIRS 100332]), it was determined that many people in that region consume some locally produced vegetables, fruit, grain, meat, poultry, fish, eggs, and milk (DOE 1997 [DIRS 100332]). This information was used to identify the ingestion pathways in the ERMYN (BSC 2004 [DIRS 169671], Section 6.4).

Use of Evaporative Coolers - About three quarters of Amargosa Valley residents surveyed used evaporative coolers, and they used them for an average of 5 months per year (DOE 1997 [DIRS 100332], Table 2.4.2; BSC 2004 [DIRS 169671], Section 6.3.4). Therefore, the model included exposure to radionuclides resulting from the use of evaporative coolers during part of the year.

Gardens-A little under half of Amargosa Valley residents surveyed had gardens (DOE 1997 [DIRS 100332], Table 2.4.2). This and other site-specific information (e.g., Horak and Carns 
1997 [DIRS 124149], pp. 5 to 6 and Table 1; Mills et al. n.d. [DIRS 124338]) was used to select the representative crops considered during development of input parameters that characterized irrigation requirements and farming methods (BSC 2004 [DIRS 169673], Section 6 and Appendix A).

Employment-About four in ten of Amargosa Valley residents (16 or more years old) were not employed in 1999 (Bureau of the Census 2002 [DIRS 159728], Table P47). Of the residents who were employed, the largest proportion (a quarter) worked in mining and only about one in twenty worked in agriculture (Bureau of the Census 2002 [DIRS 159728], Table P49). This information was used to calculate exposure times and to determine the proportion of the population that worked indoors and outdoors in Amargosa Valley (BSC 2004 [DIRS 169671], Section 6.3).

Commute Time - About two thirds of Amargosa Valley residents (16 or more years old) who worked commuted 10 minutes or more to work one way. About a fifth commuted 35 minutes or more one way (Bureau of the Census 2002 [DIRS 159728], Table P31). This information was used to model the proportion of the Amargosa Valley population who would work in areas where radionuclides may be present and the amount of time that local workers would commute within those areas (BSC 2004 [DIRS 169671], Section 6.3).

Housing Type-About nine out of ten occupied housing units in Amargosa Valley during 2000 were mobile homes, and about the same fraction of the total population lived in mobile homes (Bureau of the Census 2002 [DIRS 159728], Tables H30, H31, and H33). This information was used to select building shielding factors for lightly constructed housing (BSC 2004 [DIRS 169671], Section 6.6) and parameters related to evaporative coolers, house ventilation rates, and equilibrium factors for ${ }^{222} \mathrm{Rn}$ decay products indoors (BSC 2004 [DIRS 169672], Sections 6.5 and 6.6).

Metabolic and Physiological Considerations - The biokinetic and dosimetric models applicable to the RMEI were based on a hypothetical 'average' adult person with the anatomical and physiological characteristics defined in the Report of the Task Group on Reference Man by the ICRP (ICRP 1975 [DIRS 101074]). Breathing rates used in the model were based on the more recent biometric results for adult persons used in the respiratory track model developed by the ICRP (ICRP 1994 [DIRS 153705]; BSC 2004 [DIRS 169671], Section 6.3.3). These characteristics are used in developing dosimetric parameters, including the dose coefficients for external exposure and dose conversion factors for inhalation and ingestion. These parameters, taken from Federal Guidance Report (FGR)-11 (Eckerman et al. 1988 [DIRS 101069]) and FGR-12 (Eckerman and Ryman 1993 [DIRS 107684]), are based on the ICRP Publication 30 series on dosimetric methodology (ICRP 1978 [DIRS 101076]; ICRP 1979 [DIRS 110386]; ICRP 1980 [DIRS 110351]; ICRP 1981 [DIRS 153056]; ICRP 1981 [DIRS 110352]; ICRP 1982 [DIRS 153057]).

Although the ERMYN is based on the requirements of 10 CFR Part 63 for the RMEI, it can be used to evaluate doses to human receptors with other dietary and lifestyle characteristics, and can use dosimetric methods other than those of ICRP Publication 30 by using appropriate data as input. 


\subsubsection{Exposure Scenarios and Radionuclides of Interest}

In the biosphere model, two human exposure scenarios are considered: the groundwater exposure scenario (groundwater scenario) and the volcanic ash exposure scenario (volcanic ash scenario). The scenarios are considered separately because the initial radionuclide source terms, the radionuclide transport mechanisms in the biosphere, and the human exposure pathways are different. TSPA scenario classes considered for the TSPA-LA include the nominal scenario class and the disruptive scenario classes (BSC 2003 [DIRS 166296], Section 4). The nominal scenario class represents the most plausible evolution of the repository system and includes favorable future conditions and potentially adverse future conditions. The disruptive event scenario classes are developed using combinations of FEPs that have a low probability of occurrence but may produce potentially adverse future conditions. The disruptive event scenario classes include the igneous scenario class, which in turn includes the igneous intrusion and volcanic eruption cases, and the seismic scenario class.

For the groundwater scenario, radionuclides enter the biosphere from wells that extracts contaminated groundwater from an aquifer. Human exposure arises from using the contaminated water for domestic and agricultural purposes. BDCFs for the groundwater scenario apply to the TSPA-LA modeling cases that consider groundwater releases of radionuclides. The nominal scenario class and some modeling cases from the disruptive scenario classes (i.e., igneous intrusion and seismic events) may result in the release of radionuclides to groundwater. Dose assessments for such releases involve BDCFs for the groundwater scenario.

The volcanic ash scenario applies to the TSPA-LA volcanic eruption modeling case of the igneous scenario class. The biosphere model for the volcanic ash scenario, and, thus, the BDCFs generated using this model, support only the volcanic eruption modeling case of the disruptive event scenario classes. The remaining disruptive event scenario classes result in radionuclide releases to groundwater, and are supported by the biosphere model for the groundwater scenario.

The radionuclides of interest (Table 6.1-1) for the biosphere model depend on the release type, as discussed in Radionuclide Screening (BSC 2002 [DIRS 160059]). Two exposure scenarios (groundwater and volcanic ash), and, therefore, two lists of radionuclides, are applicable for the TSPA-LA. Characteristics of the biosphere for the groundwater and volcanic ash scenarios are discussed in detail in the following sections. The biosphere model for the groundwater scenario and the volcanic ash scenario is the same for most radionuclides. However, two radionuclides, ${ }^{14} \mathrm{C}$ and ${ }^{222} \mathrm{Rn}$ (a decay product of ${ }^{226} \mathrm{Ra}$ ), are modeled differently because of the volatility of ${ }^{14} \mathrm{C}$, the large amount of stable carbon $\left({ }^{12} \mathrm{C}\right)$ in the environment, and the gaseous release of ${ }^{222} \mathrm{Rn}$. 
Table 6.1-1. Radionuclides of Interest for the TSPA-LA

\begin{tabular}{|c|c|c|c|c|}
\hline \multirow[b]{2}{*}{ Radionuclide } & \multicolumn{2}{|c|}{ Groundwater Scenario } & \multicolumn{2}{|c|}{ Volcanic Ash Scenario } \\
\hline & $1 \times 10^{2}$ to $2 \times 10^{4}$ yrs & $2 \times 10^{4}$ to $1 \times 10^{6} \mathrm{yrs}$ & $1 \times 10^{2}$ to $2 \times 10^{4} \mathrm{yrs}$ & $2 \times 10^{4}$ to $1 \times 10^{6} \mathrm{yrs}$ \\
\hline Carbon-14 & C-14 & C-14 & & \\
\hline Chlorine-36 & & $\mathrm{Cl}-36$ & & \\
\hline Selenium-79 & & Se-79 & & \\
\hline Strontium-90 & Sr-90 & & Sr-90 & \\
\hline Technetium-99 & Tc-99 & Tc-99 & & Tc-99 \\
\hline Tin-126 & & Sn-126 & & Sn-126 \\
\hline lodine-129 & $\mathrm{l}-129$ & $\mathrm{I}-129$ & & \\
\hline Cesium-135 & Cs-135 & Cs-135 & & \\
\hline Cesium-137 & Cs-137 & & Cs-137 & \\
\hline Lead-210 & & $\mathrm{Pb}-210$ & & $\mathrm{~Pb}-210$ \\
\hline Radium-226 & Ra-226 & Ra-226 & & Ra-226 \\
\hline Actinium-227 & Ac-227 & Ac-227 & Ac-227 & Ac-227 \\
\hline Thorium-229 & Th-229 & Th-229 & Th-229 & Th-229 \\
\hline Thorium-230 & & Th-230 & & Th-230 \\
\hline Thorium-232 & & Th-232 & & Th-232 \\
\hline Protactinium-231 & $\mathrm{Pa}-231$ & $\mathrm{~Pa}-231$ & & $\mathrm{~Pa}-231$ \\
\hline Uranium-232 & $\mathrm{U}-232$ & & $\mathrm{U}-232$ & \\
\hline Uranium-233 & $\mathrm{U}-233$ & $\mathrm{U}-233$ & $\mathrm{U}-233$ & $\mathrm{U}-233$ \\
\hline Uranium-234 & U-234 & $\mathrm{U}-234$ & U-234 & U-234 \\
\hline Uranium-236 & & $\mathrm{U}-236$ & & U-236 \\
\hline Uranium-238 & $\mathrm{U}-238$ & U-238 & & U-238 \\
\hline Neptunium-237 & $\mathrm{Np}-237$ & $\mathrm{~Np}-237$ & & $\mathrm{~Np}-237$ \\
\hline Plutonium-238 & $\mathrm{Pu}-238$ & & Pu-238 & \\
\hline Plutonium-239 & Pu-239 & Pu-239 & Pu-239 & Pu-239 \\
\hline Plutonium-240 & Pu-240 & Pu-240 & Pu-240 & Pu-240 \\
\hline Plutonium-242 & & Pu-242 & & Pu-242 \\
\hline Americium-241 & Am-241 & & Am-241 & \\
\hline Americium-243 & Am-243 & Am-243 & Am-243 & Am-243 \\
\hline $\begin{array}{l}\text { Number of } \\
\text { Radionuclides }\end{array}$ & 20 & 23 & 12 & 18 \\
\hline
\end{tabular}

Source: BSC (2002 [DIRS 160059], Section 7).

\subsection{BIOSPHERE FEATURES, EVENTS, AND PROCESSES}

Biosphere FEPs describe the reference biosphere and, along with some approximations and simplifications, are the fundamental elements used to build the biosphere conceptual model. The biosphere system and associated chemical, physical, and biological processes are characterized by appropriate FEPs.

Biosphere-related FEPs are identified based on a review of the LA FEP List (DTN: MO0407SEPFEPLA.000. [DIRS 170760]). The AMR Evaluation of Features, Events, and Processes (FEP) for the Biosphere Model (BSC 2004 [DIRS 169826]) screened out a number of the FEPs leaving 32 for inclusion in the ERMYN (Table 6.2-1). 
Table 6.2-1. Description and Consideration of FEPs in the ERMYN

\begin{tabular}{|c|c|c|c|}
\hline $\begin{array}{l}\text { LA FEP } \\
\text { Number }\end{array}$ & FEP Name & FEP Description $^{a}$ & FEP Consideration \\
\hline 1.2.04.07.0A & Ashfall & $\begin{array}{l}\text { Finely-divided waste particles are } \\
\text { carried up a volcanic vent and } \\
\text { deposited at land surface from an ash } \\
\text { cloud. }\end{array}$ & $\begin{array}{l}\text { Volcanic ash is the initial source of } \\
\text { contamination for the volcanic } \\
\text { scenario. } \\
\text { Ash characteristics and depth were } \\
\text { considered in development of the } \\
\text { input parameters for the soil and air } \\
\text { submodels. }\end{array}$ \\
\hline 1.3.01.00.0A & $\begin{array}{l}\text { Climate } \\
\text { change }\end{array}$ & $\begin{array}{l}\text { Climate change may affect the long- } \\
\text { term performance of the repository. } \\
\text { This includes the effects of long-term } \\
\text { change in global climate (e.g., } \\
\text { glacial/interglacial cycles) and shorter- } \\
\text { term change in regional and local } \\
\text { climate. Climate is typically } \\
\text { characterized by temporal variations in } \\
\text { precipitation and temperature. }\end{array}$ & $\begin{array}{l}\text { Separate distributions were developed } \\
\text { for input parameters that are direct } \\
\text { effects of climate change. Separate } \\
\text { sets of BDCFs were developed for the } \\
\text { present-day and future climate states. }\end{array}$ \\
\hline 1.3.07.02.0A & $\begin{array}{l}\text { Water table } \\
\text { rise affects SZ }\end{array}$ & $\begin{array}{l}\text { Climate change could produce } \\
\text { increased infiltration, leading to a rise } \\
\text { in the regional water table, possibly } \\
\text { affecting the release and exposure } \\
\text { from the repository by altering flow } \\
\text { and transport pathways in the SZ. A } \\
\text { regionally higher water table and } \\
\text { change in SZ flow patterns might } \\
\text { move discharge points closer to the } \\
\text { repository. }\end{array}$ & $\begin{array}{l}\text { The biosphere does not consider } \\
\text { surface discharge of groundwater } \\
\text { directly. However, the conceptual and } \\
\text { mathematical models for the } \\
\text { groundwater scenario implicitly } \\
\text { included this FEP because BDCFs } \\
\text { were calculated per unit activity } \\
\text { concentration, regardless of source } \\
\text { (e.g., surface discharge as a result of } \\
\text { water table rise), so long as the use } \\
\text { and treatment of water within the } \\
\text { reference biosphere remains } \\
\text { unchanged. }\end{array}$ \\
\hline 1.4.07.01.0A & $\begin{array}{l}\text { Water } \\
\text { management } \\
\text { activities }\end{array}$ & $\begin{array}{l}\text { Water management is accomplished } \\
\text { through a combination of dams, } \\
\text { reservoirs, canals, pipelines, and } \\
\text { collection and storage facilities. Water } \\
\text { management activities could have a } \\
\text { major influence on the behavior and } \\
\text { transport of contaminants in the } \\
\text { biosphere. }\end{array}$ & $\begin{array}{l}\text { Water management activities } \\
\text { conducted in the Yucca Mountain } \\
\text { region (e.g., irrigation, fish farming) } \\
\text { were incorporated throughout the } \\
\text { conceptual and mathematical model } \\
\text { and considered in the development of } \\
\text { parameter values for the plant and fish } \\
\text { submodels. }\end{array}$ \\
\hline 1.4.07.02.0A & Wells & $\begin{array}{l}\text { One or more wells drilled for human } \\
\text { use (e.g. drinking water, bathing) or } \\
\text { agricultural use (e.g. irrigation, animal } \\
\text { watering) may intersect the } \\
\text { contaminant plume. }\end{array}$ & $\begin{array}{l}\text { A well is the source of groundwater for } \\
\text { domestic and agricultural uses in the } \\
\text { groundwater exposure scenario. }\end{array}$ \\
\hline
\end{tabular}


Table 6.2-1. Description and Consideration of FEPs in the ERMYN (Continued)

\begin{tabular}{|c|c|c|c|}
\hline $\begin{array}{l}\text { LA FEP } \\
\text { Number }\end{array}$ & FEP Name & FEP Description ${ }^{a}$ & FEP Consideration \\
\hline 2.2.08.01.00 & $\begin{array}{l}\text { Chemical } \\
\text { characteristics } \\
\text { of groundwater } \\
\text { in the SZ }\end{array}$ & $\begin{array}{l}\text { Chemistry and other characteristics of } \\
\text { groundwater in the saturated zone } \\
\text { may affect groundwater flow and } \\
\text { radionuclide transport of dissolved and } \\
\text { colloidal species. Groundwater } \\
\text { chemistry and other characteristics, } \\
\text { including temperature, pH, Eh, ionic } \\
\text { strength, and major ionic } \\
\text { concentrations, may vary spatially } \\
\text { throughout the system as a result of } \\
\text { different rock mineralogy. }\end{array}$ & $\begin{array}{l}\text { The source of activity in the biosphere } \\
\text { groundwater scenario is the } \\
\text { groundwater pumped from wells. The } \\
\text { model considers radionuclide transport } \\
\text { within and between biosphere } \\
\text { compartments culminating in an } \\
\text { annual dose to a defined receptor. } \\
\text { The model allows the parameters } \\
\text { quantifying these transport } \\
\text { mechanisms to be distributions (that } \\
\text { are defined in supporting analyses) } \\
\text { thereby taking into account localized } \\
\text { physical and chemical properties. For } \\
\text { the parameters that are dependent on } \\
\text { chemical properties of the } \\
\text { groundwater and are fixed values } \\
\text { (such as dose coefficients for } \\
\text { radionuclide intake), the model } \\
\text { assumes parameter values such that } \\
\text { the risk to the receptor is not } \\
\text { underrepresented. }\end{array}$ \\
\hline 2.3.02.01.0A & Soil type & $\begin{array}{l}\text { Soil type is determined by many } \\
\text { different factors (e.g., formative } \\
\text { process, geology, climate, vegetation, } \\
\text { land-use). The physical and chemical } \\
\text { attributes of the surficial soils (such as } \\
\text { organic matter content, pH), may } \\
\text { influence the mobility of contaminants. }\end{array}$ & $\begin{array}{l}\text { This feature was included through the } \\
\text { consideration of the soil characteristics } \\
\text { in the reference biosphere in the } \\
\text { development of parameter values for } \\
\text { the soil, plant, and }{ }^{14} \mathrm{C} \text { submodels. }\end{array}$ \\
\hline 2.3.02.02.0A & $\begin{array}{l}\text { Radionuclide } \\
\text { accumulation } \\
\text { in soils }\end{array}$ & $\begin{array}{l}\text { Radionuclide accumulation in soils } \\
\text { may occur as a result of upwelling of } \\
\text { contaminated groundwater (leaching, } \\
\text { evaporation at discharge location), } \\
\text { deposition of contaminated water or } \\
\text { particulates (irrigation water, runoff), } \\
\text { and/or atmospheric deposition. }\end{array}$ & $\begin{array}{l}\text { Accumulation of radionuclides in soil } \\
\text { from deposition of irrigation water and } \\
\text { volcanic ash was modeled in the soil } \\
\text { submodel. }\end{array}$ \\
\hline 2.3.02.03.0A & $\begin{array}{l}\text { Soil and } \\
\text { sediment } \\
\text { transport in the } \\
\text { biosphere }\end{array}$ & $\begin{array}{l}\text { Contaminated sediments can be } \\
\text { transported to and through the } \\
\text { biosphere by surface runoff and fluvial } \\
\text { processes, and, to a lesser extent, by } \\
\text { aeolian processes and bioturbation. } \\
\text { Sediment transport and redistribution } \\
\text { may cause concentration or dilution of } \\
\text { radionuclides in the biosphere. }\end{array}$ & $\begin{array}{l}\text { Soil and sediment transport via } \\
\text { erosion were included in the soil and } \\
\text { air submodels. }\end{array}$ \\
\hline 2.3.04.01.0A & $\begin{array}{l}\text { Surface water } \\
\text { transport and } \\
\text { mixing }\end{array}$ & $\begin{array}{l}\text { Contaminants released from an } \\
\text { underground repository might enter } \\
\text { the biosphere through discharge of } \\
\text { deep groundwater into a lake or river. } \\
\text { Transport and mixing within the } \\
\text { surface water bodies affects the } \\
\text { subsequent behavior and transport of } \\
\text { contaminants in the biosphere. } \\
\text { Transport and mixing includes dilution, } \\
\text { sedimentation, aeration, streamflow, } \\
\text { and river meander. }\end{array}$ & $\begin{array}{l}\text { The groundwater scenario implicitly } \\
\text { included water transport because the } \\
\text { model applied to the use of any water } \\
\text { containing radionuclides, regardless of } \\
\text { the origin, if the reference biosphere, } \\
\text { water-use practices, and } \\
\text { characteristics of the RMEl remain } \\
\text { unchanged. The model did not } \\
\text { consider mixing of contaminated and } \\
\text { uncontaminated water. }\end{array}$ \\
\hline
\end{tabular}


Table 6.2-1. Description and Consideration of FEPs in the ERMYN (Continued)

\begin{tabular}{|c|c|c|c|}
\hline $\begin{array}{l}\text { LA FEP } \\
\text { Number }\end{array}$ & FEP Name & FEP Description $^{a}$ & FEP Consideration \\
\hline 2.3.11.01.0A & Precipitation & $\begin{array}{l}\text { Precipitation is an important control on } \\
\text { the amount of recharge. It transports } \\
\text { solutes with it as it flows downward } \\
\text { through the subsurface or escapes as } \\
\text { runoff. Precipitation influences } \\
\text { agricultural practices of the receptor. } \\
\text { The amount of precipitation depends } \\
\text { on climate. }\end{array}$ & $\begin{array}{l}\text { Levels of precipitation consistent with } \\
\text { current knowledge of reference } \\
\text { biosphere were considered in the } \\
\text { development of input parameter } \\
\text { distributions for the soil, plant, and }{ }^{14} \mathrm{C} \\
\text { submodels. }\end{array}$ \\
\hline 2.3.13.01.0A & $\begin{array}{l}\text { Biosphere } \\
\text { characteristics }\end{array}$ & $\begin{array}{l}\text { The principal components, conditions, } \\
\text { or characteristics of the biosphere } \\
\text { system can influence contaminant } \\
\text { transport and affect the long-term } \\
\text { performance of the disposal system. } \\
\text { These include the characteristics of } \\
\text { the natural environment of the } \\
\text { reference biosphere such as climate, } \\
\text { soils and microbes, flora and fauna, } \\
\text { and their influences on human } \\
\text { activities. }\end{array}$ & $\begin{array}{l}\text { The principal components, conditions, } \\
\text { and characteristics of the reference } \\
\text { biosphere that influence radionuclide } \\
\text { transport were represented in the } \\
\text { conceptual and mathematical models. } \\
\text { Current knowledge of the conditions in } \\
\text { the reference biosphere was } \\
\text { considered in the development of } \\
\text { parameter distributions for all } \\
\text { submodels. }\end{array}$ \\
\hline 2.3.13.02.0A & $\begin{array}{l}\text { Radionuclide } \\
\text { alteration } \\
\text { during } \\
\text { biosphere } \\
\text { transport }\end{array}$ & $\begin{array}{l}\text { Once in the biosphere, radionuclides } \\
\text { may be transported and transferred } \\
\text { through and between different } \\
\text { compartments of the biosphere. } \\
\text { Temporally- and spatially-dependent } \\
\text { physical and chemical environments in } \\
\text { the biosphere may lead to alteration of } \\
\text { both the physical and chemical } \\
\text { properties of the radionuclides as they } \\
\text { move through or between the different } \\
\text { compartments of the biosphere. } \\
\text { These alterations could consequently } \\
\text { control exposure to the human } \\
\text { population. }\end{array}$ & $\begin{array}{l}\text { Changes in the physical and chemical } \\
\text { form of radionuclides during transfer } \\
\text { among biosphere components were } \\
\text { incorporated throughout the } \\
\text { conceptual and mathematical models. } \\
\text { This FEP was also implicitly } \\
\text { incorporated through the use of } \\
\text { radionuclide-specific transfer factors in } \\
\text { the plant and animal submodels. }\end{array}$ \\
\hline 2.4.01.00.0A & $\begin{array}{l}\text { Human } \\
\text { characteristics } \\
\text { (physiology, } \\
\text { metabolism) }\end{array}$ & $\begin{array}{l}\text { This FEP addresses human } \\
\text { characteristics. These include } \\
\text { physiology, metabolism, and variability } \\
\text { among individual humans. }\end{array}$ & $\begin{array}{l}\text { Metabolic and physiologic } \\
\text { considerations consistent with present } \\
\text { knowledge of adults, as per } \\
10 \text { CFR } 63.312 \text {, were used in the } \\
\text { development of parameter } \\
\text { distributions for the external exposure, } \\
\text { inhalation, and ingestion submodels. }\end{array}$ \\
\hline 2.4.04.01.0A & Human lifestyle & $\begin{array}{l}\text { Human lifestyle, including everyday } \\
\text { household activities and leisure } \\
\text { activities, will influence the critical } \\
\text { exposure pathways to man. }\end{array}$ & $\begin{array}{l}\text { Activities representative of the living } \\
\text { style of the residents of the town of } \\
\text { Amargosa Valley were incorporated } \\
\text { throughout the conceptual and } \\
\text { mathematical model. The living styles } \\
\text { of Amargosa Valley residents were } \\
\text { considered in the development of } \\
\text { parameter distributions for the air, } \\
\text { external exposure, inhalation, and } \\
\text { ingestion submodels. }\end{array}$ \\
\hline
\end{tabular}


Table 6.2-1. Description and Consideration of FEPs in the ERMYN (Continued)

\begin{tabular}{|c|c|c|c|}
\hline $\begin{array}{l}\text { LA FEP } \\
\text { Number }\end{array}$ & FEP Name & FEP Description $^{a}$ & FEP Consideration \\
\hline 2.4.07.00.0A & Dwellings & $\begin{array}{l}\text { This FEP addresses human dwellings, } \\
\text { and the ways in which dwellings might } \\
\text { affect human exposures. Exposure } \\
\text { pathways might be influenced by } \\
\text { building materials and location. }\end{array}$ & $\begin{array}{l}\text { Characteristics of dwellings } \\
\text { representative of the living style of the } \\
\text { residents of the town of Amargosa } \\
\text { Valley were considered in the } \\
\text { development of input parameters for } \\
\text { the air, external exposure, and } \\
\text { inhalation submodels. }\end{array}$ \\
\hline 2.4.08.00.0A & $\begin{array}{l}\text { Wild and } \\
\text { natural land } \\
\text { and water use }\end{array}$ & $\begin{array}{l}\text { This FEP addresses human uses of } \\
\text { wild and natural lands (forests, bush, } \\
\text { coastlines) and water (lakes, rivers, } \\
\text { oceans) that may affect the long-term } \\
\text { performance of the repository. Wild } \\
\text { and natural land use will be primarily } \\
\text { controlled by natural factors } \\
\text { (topography, climate, etc.). }\end{array}$ & $\begin{array}{l}\text { Wild and natural land and water use } \\
\text { (e.g., use of natural lands, ingestion of } \\
\text { game animals) of the residents of the } \\
\text { town of Amargosa Valley was } \\
\text { incorporated into the air, external } \\
\text { exposure, and ingestion submodels. } \\
\text { These lifestyle characteristics were } \\
\text { considered in the development of } \\
\text { parameters for those submodels. }\end{array}$ \\
\hline 2.4.09.01.0B & $\begin{array}{l}\text { Agricultural } \\
\text { land use and } \\
\text { irrigation }\end{array}$ & $\begin{array}{l}\text { Agricultural areas exist near Yucca } \\
\text { Mountain, particularly in the direction } \\
\text { of groundwater flow. Current practices } \\
\text { include irrigation, plowing, fertilization, } \\
\text { crop storage, and soil modification and } \\
\text { amendment. Existing practices may } \\
\text { play a significant role in determining } \\
\text { exposure pathways and dose. }\end{array}$ & $\begin{array}{l}\text { Agricultural land use and irrigation } \\
\text { practices of the residents of the town } \\
\text { of Amargosa Valley were incorporated } \\
\text { into the soil, air, plant, animal, }{ }^{14} \mathrm{C} \text {, and } \\
\text { fish submodels. These practices were } \\
\text { considered in the development of } \\
\text { parameters for those submodels. }\end{array}$ \\
\hline 2.4.09.02.0A & $\begin{array}{l}\text { Animal farms } \\
\text { and fisheries }\end{array}$ & $\begin{array}{l}\text { Domestic livestock or fish could } \\
\text { become contaminated through the } \\
\text { intake of contaminated feed, water, or } \\
\text { soil. Such contamination would then } \\
\text { enter the foodchain. }\end{array}$ & $\begin{array}{l}\text { Animal farms and fisheries practices of } \\
\text { the residents of the town of Amargosa } \\
\text { Valley were incorporated into the } \\
\text { animal and fish submodels. These } \\
\text { practices were considered in the } \\
\text { development of parameters for those } \\
\text { submodels. }\end{array}$ \\
\hline 2.4.10.00.0A & $\begin{array}{l}\text { Urban and } \\
\text { industrial land } \\
\text { and water use }\end{array}$ & $\begin{array}{l}\text { This FEP addresses urban and } \\
\text { industrial uses of land and water } \\
\text { (industry, urban development, } \\
\text { earthworks, energy production, etc.) } \\
\text { that may affect the long-term } \\
\text { performance of the repository. Urban } \\
\text { and industrial land use will be } \\
\text { controlled by both natural factors } \\
\text { (topography, climate, etc.) and human } \\
\text { factors (economics, population } \\
\text { density, etc.). }\end{array}$ & $\begin{array}{l}\text { Land and water use in urban and } \\
\text { industrial settings of the residents of } \\
\text { the town of Amargosa Valley were } \\
\text { incorporated into the soil, air, }{ }^{14} \mathrm{C} \text {, } \\
\text { external exposure, inhalation, and } \\
\text { ingestion submodels. These lifestyle } \\
\text { characteristics were considered in the } \\
\text { development of parameters for those } \\
\text { submodels. }\end{array}$ \\
\hline
\end{tabular}


Table 6.2-1. Description and Consideration of FEPs in the ERMYN (Continued)

\begin{tabular}{|c|c|c|c|}
\hline $\begin{array}{l}\text { LA FEP } \\
\text { Number }\end{array}$ & FEP Name & FEP Description $^{a}$ & FEP Consideration \\
\hline 3.1.01.01.0A & $\begin{array}{l}\text { Radioactive } \\
\text { decay and } \\
\text { ingrowth }\end{array}$ & $\begin{array}{l}\text { Radioactivity is the spontaneous } \\
\text { disintegration of an unstable atomic } \\
\text { nucleus that results in the emission of } \\
\text { subatomic particles. Radioactive } \\
\text { isotopes are known as radionuclides. } \\
\text { Radioactive decay of the fuel in the } \\
\text { repository changes the radionuclide } \\
\text { content in the fuel with time and } \\
\text { generates heat. Radionuclide } \\
\text { quantities in the system at any time } \\
\text { are the result of the radioactive decay } \\
\text { and the growth of daughter products } \\
\text { as a consequence of that decay (i.e., } \\
\text { ingrowth). Over a 10,000-year } \\
\text { performance period, these processes } \\
\text { will produce daughter products that } \\
\text { need to be considered in order to } \\
\text { adequately evaluate the release and } \\
\text { transport of radionuclides to the } \\
\text { accessible environment. }\end{array}$ & $\begin{array}{l}\text { Radionuclide decay and ingrowth in } \\
\text { soil was included in the soil submodel. } \\
\text { In addition, the calculation of effective } \\
\text { dose coefficients and effective dose } \\
\text { conversion factors included dose } \\
\text { contributions from the decay products } \\
\text { of primary radionuclides. }\end{array}$ \\
\hline 3.2.10.00.0A & $\begin{array}{l}\text { Atmospheric } \\
\text { transport of } \\
\text { contaminants }\end{array}$ & $\begin{array}{l}\text { Atmospheric transport includes } \\
\text { radiotoxic and chemotoxic species in } \\
\text { the air as gas, vapor, particulates or } \\
\text { aerosol. Transport processes include } \\
\text { wind, plowing and irrigation, } \\
\text { degassing, saltation, and precipitation. }\end{array}$ & $\begin{array}{l}\text { The processes of atmospheric } \\
\text { transport of radionuclides from } \\
\text { resuspension of soil and ash particles, } \\
\text { gaseous emission of radionuclides } \\
\text { from soil, and generation of aerosols } \\
\text { from evaporative coolers were } \\
\text { included in the air and }{ }^{14} \mathrm{C} \text { submodels. }\end{array}$ \\
\hline 3.3.01.00.0A & $\begin{array}{l}\text { Contaminated } \\
\text { drinking water, } \\
\text { foodstuffs and } \\
\text { drugs }\end{array}$ & $\begin{array}{l}\text { This FEP addresses human diet and } \\
\text { fluid intake. Consumption of food, } \\
\text { water, soil, drugs, etc., will affect } \\
\text { human exposure to radionuclides. } \\
\text { Other influences include filtration of } \\
\text { water, dilution of diet with } \\
\text { uncontaminated food, and food } \\
\text { preparation techniques. }\end{array}$ & $\begin{array}{l}\text { Annual consumption rates of } \\
\text { contaminated water, soil, locally } \\
\text { produced crops, animal products, and } \\
\text { fish were included in the ingestion } \\
\text { submodel. Consumption rates were } \\
\text { based on the diet of the residents of } \\
\text { the town of Amargosa Valley and the } \\
\text { requirements of } 10 \text { CFR } 63.312 \text {. }\end{array}$ \\
\hline 3.3.02.01.0A & Plant uptake & $\begin{array}{l}\text { Uptake and accumulation of } \\
\text { contaminants by plants could affect } \\
\text { potential exposure pathways. Plant } \\
\text { uptake from contaminated soils and } \\
\text { irrigation water is possible. Particulate } \\
\text { deposition onto plant surfaces is also } \\
\text { possible. These plants may be used } \\
\text { as feed for livestock and/or consumed } \\
\text { directly by humans. }\end{array}$ & $\begin{array}{l}\text { The process of plant uptake of } \\
\text { radionuclides was included in the plant } \\
\text { and }{ }^{14} \mathrm{C} \text { submodels. }\end{array}$ \\
\hline 3.3.02.02.0A & Animal uptake & $\begin{array}{l}\text { Livestock may accumulate } \\
\text { radionuclides as a result of ingestion } \\
\text { of water, feed and soil/sediment and } \\
\text { inhalation of aerosols and particulates. } \\
\text { Depending on the livestock, they may } \\
\text { be used for human consumption } \\
\text { directly, or their produce (milk, eggs, } \\
\text { etc.) may be consumed. }\end{array}$ & $\begin{array}{l}\text { The animal submodel included the } \\
\text { process of radionuclide uptake by farm } \\
\text { animals. }\end{array}$ \\
\hline
\end{tabular}


Table 6.2-1. Description and Consideration of FEPs in the ERMYN (Continued)

\begin{tabular}{|c|c|c|c|}
\hline $\begin{array}{l}\text { LA FEP } \\
\text { Number }\end{array}$ & FEP Name & FEP Description ${ }^{a}$ & FEP Consideration \\
\hline 3.3.02.03.0A & Fish uptake & $\begin{array}{l}\text { Uptake and bioaccumulation of } \\
\text { contaminants in aquatic organisms } \\
\text { could affect potential exposure } \\
\text { pathways. }\end{array}$ & $\begin{array}{l}\text { The fish submodel included the } \\
\text { bioaccumulation of radionuclides in } \\
\text { fish. }\end{array}$ \\
\hline 3.3.03.01.0A & $\begin{array}{l}\text { Contaminated } \\
\text { non-food } \\
\text { products and } \\
\text { exposure }\end{array}$ & $\begin{array}{l}\text { Contaminants may be concentrated in } \\
\text { various products: clothing (e.g., hides, } \\
\text { leather, linen, wool); furniture (e.g., } \\
\text { wood, metal); building materials (e.g., } \\
\text { stone, clay for bricks, wood, dung); } \\
\text { fuel (e.g., peat), tobacco, pets. }\end{array}$ & $\begin{array}{l}\text { The external exposure submodel } \\
\text { bounded exposure to the few nonfood } \\
\text { products known to be produced in } \\
\text { Amargosa Valley that may contain } \\
\text { radionuclides by assuming that the } \\
\text { RMEl would be exposed to equilibrium } \\
\text { activity concentrations in contaminated } \\
\text { soil at all times while in the biosphere. }\end{array}$ \\
\hline 3.3.04.01.0A & Ingestion & $\begin{array}{l}\text { Ingestion is human exposure to } \\
\text { repository-derived radionuclides } \\
\text { through eating contaminated } \\
\text { foodstuffs or drinking contaminated } \\
\text { water. }\end{array}$ & $\begin{array}{l}\text { The ingestion submodel included } \\
\text { ingestion of contaminated food, } \\
\text { drinking water, and soil. }\end{array}$ \\
\hline 3.3.04.02.0A & Inhalation & $\begin{array}{l}\text { Two inhalation pathways are likely. } \\
\text { The first is inhalation of gases and } \\
\text { vapors emanating directly from the } \\
\text { ground after transport through the far- } \\
\text { field. The second is inhalation of } \\
\text { suspended, contaminated particulate } \\
\text { matter (e.g., daughter products of } \\
\text { radon, dust, smoke, pollen, and soil } \\
\text { particles). }\end{array}$ & $\begin{array}{l}\text { The inhalation submodel included } \\
\text { inhalation of contaminated } \\
\text { resuspended particles, aerosols from } \\
\text { evaporative coolers, }{ }^{14} \mathrm{C} \text {, and radon } \\
\text { decay products. }\end{array}$ \\
\hline 3.3.04.03.0A & $\begin{array}{l}\text { External } \\
\text { exposure }\end{array}$ & $\begin{array}{l}\text { External exposure is human exposure } \\
\text { to repository-derived radionuclides by } \\
\text { contact, use, or exposure to } \\
\text { contaminated materials. }\end{array}$ & $\begin{array}{l}\text { The external exposure submodel } \\
\text { included external exposure to } \\
\text { contaminated materials. }\end{array}$ \\
\hline 3.3.05.01.0A & $\begin{array}{l}\text { Radiation } \\
\text { doses }\end{array}$ & $\begin{array}{l}\text { The radiation dose is calculated from } \\
\text { exposure rates (external, inhalation, } \\
\text { and ingestion) and dose conversion } \\
\text { factors. The latter are based upon } \\
\text { radiation type, human metabolism, } \\
\text { metabolism of the element of concern } \\
\text { in the human body, duration of } \\
\text { exposure. }\end{array}$ & $\begin{array}{l}\text { Calculation of the predicted annual } \\
\text { dose as required by } 10 \text { CFR } 63.311 \\
\text { for a unit activity concentration of a } \\
\text { radionuclide (i.e., BDCF) was } \\
\text { conducted in the external exposure, } \\
\text { inhalation, and ingestion submodels. }\end{array}$ \\
\hline 3.3.08.00.0A & $\begin{array}{l}\text { Radon and } \\
\text { radon daughter } \\
\text { exposure }\end{array}$ & $\begin{array}{l}\text { This FEP addresses human exposure } \\
\text { to radon and radon decay products. } \\
{ }^{226} \mathrm{Ra} \text { occurs in nuclear fuel waste and } \\
\text { it gives rise to }{ }^{222} \mathrm{Rn} \text { gas, the } \\
\text { radioactive daughters of which can be } \\
\text { harmful to humans and animals upon } \\
\text { inhalation. }\end{array}$ & $\begin{array}{l}\text { Concentrations of }{ }^{222} \mathrm{Rn} \text { and }{ }^{222} \mathrm{Rn} \\
\text { decay products were calculated in the } \\
\text { air submodel. Exposure to }{ }^{222} \mathrm{Rn} \text { and } \\
\text { decay products was included in the } \\
\text { inhalation submodel. }\end{array}$ \\
\hline
\end{tabular}

${ }^{a}$ FEP names and descriptions are based on the LA FEP list (DTN: MO0407SEPFEPLA.000 [DIRS 170760]), which is considered to be the source of these FEPs.

$\mathrm{BDCF}=$ biosphere dose conversion factor; $\mathrm{FEP}=$ feature, event, or process; $\mathrm{LA}=$ =license application; RMEI=reasonably maximally exposed individual; $\mathrm{SZ}=$ saturated zone

The FEPs shown in Table 6.2-1 (referred to as the included FEPs) represent elements of the arid to semi-arid environment in the Yucca Mountain area and the possible processes leading to radionuclide transport and exposure in the environment. These FEPs are presented in 
Table 6.2-1 with the LA FEP number, FEP name, FEP description, and brief comments on how the FEP is incorporated into the model. Detailed information on how each FEP is incorporated into the exposure scenarios is provided in Section 6.3.4.

Screening arguments for the excluded biosphere-related FEPs (excluded from the ERMYN and the TSPA-LA) are presented in the biosphere FEPs report (BSC 2004 [DIRS 169826]). Most of these FEPs are inconsistent with the requirements of the reference biosphere or the RMEI (10 CFR Part 63 [DIRS 156605]), and some are excluded based on low probability of occurrence and/or low consequence.

Relationships among the biosphere-related FEPs, the biosphere conceptual model, and the exposure scenarios are more fully examined in Section 6.3. As a tool to illustrate the movement of radionuclides through the biosphere compartments, a radionuclide transfer interaction matrix (Tables 6.3-2 and 6.3-4) links each included FEP in the matrix for each exposure scenario. The matrices demonstrate that all included FEPs are considered in the biosphere conceptual model (Section 6.3.4).

In addition, the disposition of the included FEPs in the biosphere mathematical model, submodels, and associated equations and parameters are discussed in Section 6.7.1. Because of the relationship between the biosphere model and the TSPA-LA (Sections 6.4.10 and 6.5.8), only the results of the biosphere model (i.e., the BDCF distributions) are used for direct input to the TSPA-LA model. Therefore, the incorporation of biosphere-related FEPs in the TSPA-LA model is through the BDCFs.

\subsection{BIOSPHERE CONCEPTUAL MODEL}

In the previous section, the biosphere-related FEPs, and the methods for including them in the ERMYN, are discussed. The biosphere conceptual model is constructed by considering these FEPs for a specific exposure scenario. To make it easier to understand, the conceptual model is presented in a logical framework that relates a contamination source to a human radiation dose using all possible mechanisms for radionuclide transport in the environment and human exposure pathways. In this section, the biosphere conceptual models for the groundwater (Section 6.3.1) and volcanic ash scenarios (Section 6.3.2) are discussed. Although many transport processes and exposure pathways are the same for both scenarios, they differ because of the different environmental media that is initially contaminated (i.e., groundwater and volcanic ash). Other issues related to the conceptual model are discussed later, including ACMs (Section 6.3.3), individual FEPs applicable to specific exposure scenarios (Section 6.3.4), and short-lived decay products (Section 6.3.5).

\subsubsection{Conceptual Model for the Groundwater Exposure Scenario}

An exposure scenario is a well-defined, connected sequence of FEPs that describes characteristics of present-day and possible future biosphere where radionuclide transport occurs, and circumstances of human exposure. The reference biosphere and the human receptor (Section 6.1) are fundamental concepts of the groundwater exposure scenario, or simply groundwater scenario. The description of the groundwater scenario (Section 6.3.1.1) includes background information on the biosphere system. Based on site-specific information, 
radionuclide transport in various environmental media is examined using the radionuclide transfer interaction matrix (Table 6.3-2). These environmental media and exposure modes are considered as subsystems of the overall biosphere system.

\subsubsection{Scenario Description}

Under the groundwater exposure scenario, radionuclides would be released into the biosphere from contaminated groundwater drawn from a well. Human exposure, then, would arise when the local community, where the receptor resides, uses the contaminated water for domestic and agricultural purposes. There is no evidence to suggest the widespread use of water treatment in the Amargosa Valley, and there is only a small quasi-municipal system where a water standard could be enforced (State of Nevada 1997 [DIRS 110951]). In the model, no credit is taken for water treatment before use, and radionuclide concentrations in the well water are considered to be equal to concentrations in the groundwater, and groundwater is the source for all water needs, including drinking water, irrigation, and other domestic uses. The groundwater scenario is used to evaluate the radiological consequences of nominal performance of the geologic repository and performance under disrupted conditions (igneous intrusions and seismic events) that can lead to radionuclide releases into the groundwater (BSC 2003 [DIRS 166296], Section 4).

Environmental transport pathways are the routes by which radionuclides move from the source to the environmental media. Human exposure pathways arise when people are exposed, internally or externally, to the contaminated media (Figure 6.3-1). The environmental transport pathways, the media, and the exposure pathways are identified in the discussion of biosphere FEPs (Section 6.2). Six environmental media (water, soil, air, plants, animals, and fish) and three human exposure pathways (external exposure, inhalation, and ingestion) are considered (Table 6.3-1). These pathways are representative of a rural community in the Yucca Mountain region and consistent with arid and semi-arid conditions.

Table 6.3-1. Exposure Pathways for the Groundwater Scenario

\begin{tabular}{|c|c|c|c|}
\hline $\begin{array}{l}\text { Environmental } \\
\text { Medium }\end{array}$ & $\begin{array}{l}\text { Exposure } \\
\text { Mode }\end{array}$ & Exposure Pathways & Examples of Typical Activities \\
\hline Water & Ingestion & Water intake. & $\begin{array}{l}\text { Drinking water and water-based } \\
\text { beverages and water used in food } \\
\text { preparation. }\end{array}$ \\
\hline Soil & Ingestion & Inadvertent soil ingestion. & $\begin{array}{l}\text { Recreational activities, occupational } \\
\text { activities, gardening, and } \\
\text { consumption of fresh fruit and } \\
\text { vegetables. }\end{array}$ \\
\hline Soil & External & External radiation exposure. & $\begin{array}{l}\text { Activities on or near contaminated } \\
\text { soils. }\end{array}$ \\
\hline Air & Inhalation & $\begin{array}{l}\text { Breathing resuspended particles, gases } \\
\left({ }^{222} \mathrm{Rn} \text { and progeny, plus }{ }^{14} \mathrm{CO}_{2}\right), \text { and } \\
\text { aerosols from evaporative coolers. }\end{array}$ & $\begin{array}{l}\text { Outdoor activities, including soil- } \\
\text { disturbing activities related to work } \\
\text { and recreation. Domestic activities, } \\
\text { including sleeping. }\end{array}$ \\
\hline Plants & Ingestion & $\begin{array}{l}\text { Consumption of locally produced crops: leafy } \\
\text { vegetables, other vegetables, fruit, and grain. }\end{array}$ & Eating contaminated crop foodstuffs. \\
\hline Animals & Ingestion & $\begin{array}{l}\text { Consumption of locally produced animal } \\
\text { products: meat, poultry, milk, and eggs. }\end{array}$ & $\begin{array}{l}\text { Eating contaminated animal product } \\
\text { foodstuffs. }\end{array}$ \\
\hline Fish & Ingestion & $\begin{array}{l}\text { Consumption of locally produced freshwater } \\
\text { fish. }\end{array}$ & Eating fish. \\
\hline
\end{tabular}




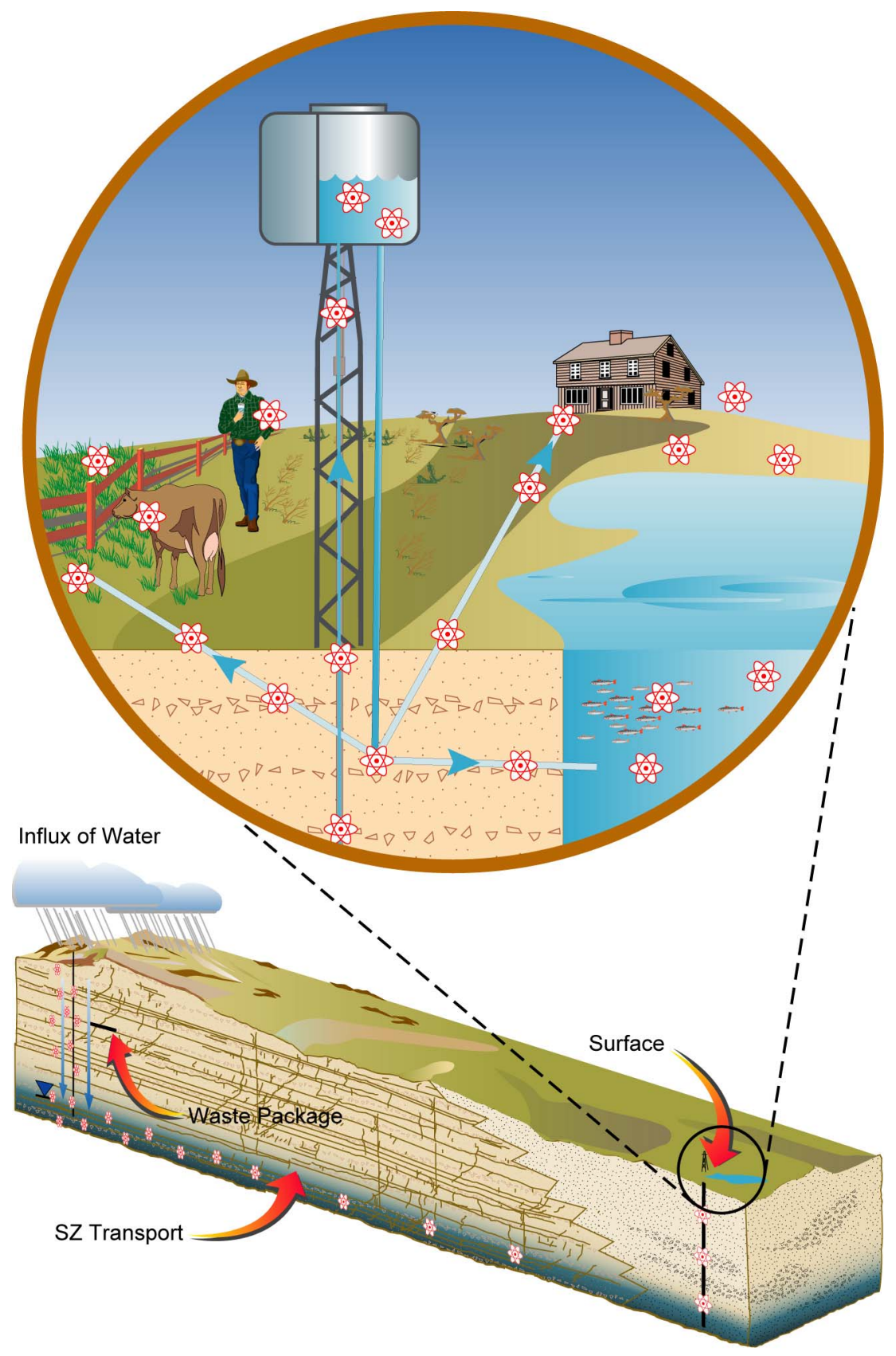

00284CD_Biosphere Groundwater Radionuclides Rev A.ai

NOTE: $S Z$ = saturated zone.

Figure 6.3-1. Graphical Representation of the Biosphere System for the Groundwater Contamination 
The future climate for the region around Yucca Mountain is predicted to be cooler and wetter (BSC 2004 [DIRS 170002]) than the present-day climate. Climate change is incorporated into the ERMYN by using different values for input parameters that are influenced by temperature and precipitation (Table 6.6-2). In addition, different sets of BDCFs are calculated for the present-day and future climate states. A wetter climate may cause the water table to rise and discharge groundwater at springs in the Yucca Mountain area. The ERMYN applies to the discharge of groundwater from springs if use of, and exposure to, water remains the same, and there is no mixing of contaminated and uncontaminated water (or other processes that would cause the radionuclide concentrations to change). There are no permanent rivers or lakes (these features would require additional pathways that are not consistent with arid to semi-arid conditions and therefore are not in the ERMYN; for example, water immersion due to swimming and external exposure due to contaminated sediments). These limitations are summarized in Section 8.2.

\subsubsection{Identification of Biosphere Model Components}

As defined in AP-SIII.10Q, a conceptual model is a set of hypotheses consisting of assumptions, simplifications, and idealizations that describe the essential aspects of a system, process, or phenomenon. The biosphere conceptual model provides a description of the biosphere system, the essential components, and the mechanisms of interaction between the biosphere components. It also presents a logical way to evaluate human radiation dose from exposure to radionuclides released from the repository at Yucca Mountain.

As described in Section 6.1, a biosphere system consists of the reference biosphere and a human receptor. The number of biosphere components depends on the radionuclide exposure scenario. For the groundwater scenario, there are seven biosphere components: six foodstuffs and the human receptor:

- Water-groundwater from a well is the source of radionuclides in the biosphere.

- Soil-cultivated soil from farmland and gardens, limited to surface soil down to the tilling depth.

- Atmosphere-including outdoor air and indoor air.

- Plants-crops for human and farm animal consumption, grown in the cultivated soil and irrigated with contaminated water.

- Animals-animal products for human consumption, raised by humans using contaminated local fodder and contaminated water.

- Fish-raised at a fish farm using contaminated groundwater.

- Human Receptor-exposed through external exposure, inhalation, and ingestion of the contaminated media listed above; for the TSPA-LA, input information for the receptor is based on the RMEI. 
The aquifer, the source of groundwater, is not a part of the biosphere; it is part of the geosphere. The biosphere-geosphere interface is through the extraction of well water. The biosphere model does not include processes related to long-range atmospheric transport and dispersion of airborne radionuclides. However, the ERMYN considers airborne activity resulting from resuspension of contaminated soil and gaseous emission of radionuclides from soil to air followed by atmospheric dilution. In the ERMYN, radionuclides are removed from the biosphere by leaching and erosion, but these transport mechanisms do not provide radionuclide sources in any subsequent model. The ERMYN includes radioactive decay.

\subsubsection{Radionuclide Transfer Interaction Matrix}

After the components of a biosphere system are defined, radionuclide transport between components is considered. A radionuclide transfer interaction matrix is constructed to identify the important processes leading to radionuclide transfer between biosphere components (Table 6.3-2).

Table 6.3-2. Radionuclide Transfer Interaction Matrix for the Groundwater Scenario

\begin{tabular}{|c|c|c|c|c|c|c|c|}
\hline $\mathrm{i}, \mathbf{j}$ & 1 & 2 & 3 & 4 & 5 & 6 & 7 \\
\hline 1 & $\begin{array}{c}\text { SOURCE } \\
\text { (groundwater) }\end{array}$ & irrigation & evaporation & $\begin{array}{l}\text { irrigation } \\
\text { interception }\end{array}$ & $\begin{array}{l}\text { ingestion of } \\
\text { water }\end{array}$ & $\begin{array}{l}\text { bio- } \\
\text { accumulation } \\
\text { (water use in } \\
\text { fisheries) }\end{array}$ & $\begin{array}{l}\text { drinking water } \\
\text { ingestion }\end{array}$ \\
\hline 2 & leaching $^{a}$ & $\begin{array}{l}\text { SURFACE } \\
\text { SOIL }\end{array}$ & $\begin{array}{l}\text { particle } \\
\text { resuspension, } \\
\text { gas release, } \\
\text { soil erosion }^{\text {a }}\end{array}$ & root uptake & soil ingestion & - & $\begin{array}{l}\text { soil ingestion, } \\
\text { external } \\
\text { exposure }\end{array}$ \\
\hline 3 & - & dust deposit & AIR & $\begin{array}{c}\text { dust } \\
\text { deposition, } \\
\text { photosynthesis }\end{array}$ & - & - & $\begin{array}{l}\text { inhalation of } \\
\text { particulates, } \\
\text { gases, and } \\
\text { aerosols }\end{array}$ \\
\hline 4 & - & $\begin{array}{l}\text { weathering, } \\
\text { harvest } \\
\text { removal }\end{array}$ & - & $\begin{array}{l}\text { PLANTS } \\
\text { (crops) }\end{array}$ & $\begin{array}{l}\text { ingestion of } \\
\text { feed }\end{array}$ & - & crop ingestion \\
\hline 5 & - & fertilization & - & - & $\begin{array}{l}\text { ANIMALS } \\
\text { (animal } \\
\text { products) }\end{array}$ & - & $\begin{array}{l}\text { animal product } \\
\text { ingestion }\end{array}$ \\
\hline 6 & - & - & - & - & - & FISH & fish ingestion \\
\hline 7 & - & - & - & - & - & - & $\begin{array}{l}\text { HUMAN } \\
\text { (receptor) }\end{array}$ \\
\hline
\end{tabular}

${ }^{a}$ Leaching and soil erosion are modeled in the soil submodel only as removal mechanisms. The possibility that the removed radionuclides could become a new source is not considered. 
The diagonal elements in the interaction matrix represent the biosphere components (features of the biosphere), and the off-diagonal elements represent the interactions between components (biosphere processes defined by the FEPs). By convention, the direction of interaction between components is clockwise. For example, in Table 6.3-2, the element in Row 2 and Column 4 (Element $[2,4]$ ), refers to the transfer of radionuclides from the surface soil to the plants via root uptake. Off-diagonal elements with a dash (-) indicate that interactions between the two components are not included in the ERMYN. For all off-diagonal elements with interactions, radionuclide transfer mechanisms are discussed in the conceptual model section and evaluated quantitatively in the mathematical model section.

\subsubsection{Conceptual Model Approximations}

The following 11 approximations are incorporated in the conceptual model for the groundwater exposure scenario. Each is presented as an approximation statement, a rationale providing the basis for the approximation, and the section or sections in this report where the approximation has been applied.

\section{Approximation 1 - Groundwater Source}

Statement-Radionuclide concentrations in the groundwater are constant through time.

Rationale-Radionuclides will accumulate in soil that is continuously irrigated with contaminated groundwater until equilibrium conditions are reached; therefore, doses calculated for a specified time will be influenced by groundwater concentrations prior to that time until radionuclide concentrations in the soil are in equilibrium. The time required for the radionuclides considered in this model to reach equilibrium conditions is shorter than the 10,000-year compliance period for the TSPA-LA (Section 7.4.2); therefore, it is assumed that concentrations in groundwater are constant at a concentration defined by the user of the model (e.g., unit concentration of $1 \mathrm{~Bq} / \mathrm{m}^{3}$ ).

In the event that equilibrium conditions for a radionuclide have not been reached and groundwater concentrations are increasing, this approximation will result in overestimating the dose for that radionuclide (Section 6.4.10.4). If concentrations in groundwater are decreasing, the dose may be underestimated. However, it is unlikely that groundwater concentrations will decrease until long after the compliance period of 10,000 years (Section 6.4.10.4).

This approximation allows separate and independent calculations of time-dependent radionuclide concentrations in a TSPA and time-independent BDCFs in the ERMYN, as required by the Total System Performance Assessment-License Application Methods and Approach (BSC 2003 [DIRS 166296], p. 72). The approximation requires no further confirmation because it is likely that equilibrium conditions will be reached in less than 10,000 years, and because the dose will not be underestimated if those conditions are not reached by that time.

Applicability-This approximation is applied to the groundwater scenario in Sections 6.3.1.6, 6.4, 6.4.1.1, and 6.4.10.4. 


\section{Approximation 2 - Consideration of Short-Lived Decay Products}

Statement-Short-lived decay products (half-life less than 180 days) are always in secular equilibrium with the long-lived primary radionuclides.

Rationale-Modeling radionuclide decay and ingrowth can be complicated if every decay product is considered as a function of time. This approximation eliminates the need to consider the dynamics of long decay chains for high atomic number (greater than or equal to 82) radionuclides. This approximation is conservative because the activity of a decay product reaches a maximum value when in equilibrium with the long-lived parent radionuclide. This approximation is reasonable because the primary radionuclides have long half-lives (Section 6.3.5), and the primary radionuclides and decay products in the groundwater and the volcanic ash are expected to be in secular equilibrium with the short-lived decay products. If the radionuclides in a decay chain are transferred to the biosphere or between biosphere components, the secular equilibrium could be perturbed because of different transfer characteristics in the biosphere and groundwater (e.g., due to different leaching rates or transfer factors). However, calculations of radionuclide transfer and doses are based on one-year average values, and a new equilibrium will be reached quickly due to the relatively short-lived decay products. This approximation also is used in the RESRAD code (Yu et al. 2001 [DIRS 159465], Section 3.1). The developed effective dose coefficients and effective dose conversion factors, which are based on this approximation, are compared with results from the RESRAD model to confirm this approximation (Section 7.4.1).

Applicability-This approximation is applied to the groundwater scenario in Sections 6.3.5, 6.4.1.2, 6.4.9, and 7.4.1.

\section{Approximation 3 - Long-Term Irrigation, Land Use, and Crop Rotation}

Statement-Current land use and irrigation practices continue throughout the period of interest on agricultural land. The average irrigation rate for all crops is appropriate for calculating radionuclide concentrations in the soil.

Rationale-Based on the present-day and predicted future climates in the Amargosa Valley (BSC 2004 [DIRS 170002]), irrigation will be required for farming and gardening during the regulatory compliance period of 10,000 years. Because irrigation rates differ among crops (BSC 2004 [DIRS 169673], Section 6.5), radionuclide concentrations in the soil will differ among fields depending on the types of crops grown. However, crop rotation is a common agricultural practice in the Amargosa Valley (Horak and Carns 1997 [DIRS 124149], Section 1.b), and crop rotation over long periods will average out the short-term differences in irrigation rates among fields, resulting in an average radionuclide concentration among fields. Therefore, the annual average irrigation rate for all crops is appropriate for calculating the concentration of radionuclides in agricultural soil. This approximation simplifies the surface soil submodel because the long-term irrigation rate does not depend on crop type. This approximation requires no further confirmation because it is based on common agricultural practices. 
Applicability-This approximation is applied to the surface soil submodel for the groundwater scenario in Sections 6.3.1.6, 6.4.1.1, and 6.4.10.4.

Approximation 4-Crop Harvest Removal and the Use of Contaminated Manure for $\underline{\text { Fertilizer }}$

Statement-Radionuclides added to the soil due to the use of manure for fertilizer replace radionuclides removed from the soil by harvesting crops.

Rationale-Harvesting crops removes radionuclides from cultivated fields, and fertilizing with contaminated fertilizer adds radionuclides. It is reasonable to assume that Amargosa Valley farmers will use manure from a dairy for fertilizer because they currently use manure from the dairy for fertilizer (Horak and Carns 1997 [DIRS 124149], p. 10). This approximation considers the removal of radionuclides from fields in animal feed and soil to be balanced by the addition of radionuclides in animal manure on fields where animal feed is grown. Intake of radionuclides by animals is from feed, soil, and water; therefore, drinking water is an additional source of radionuclides in manure not considered by this approximation. However, the contribution of radionuclides to animal intake from drinking water is low (about 3 percent; Table 7.4-12) compared to that from animal feed and soil. In addition, only a portion of the radionuclides taken in by animals is transferred to animal products. Therefore, as these two processes approximately compensate for each other (i.e., annual input to fields from use of manure as fertilizer approximately equals annual removal less radionuclides retained in animals used for human consumption) in terms of radionuclide concentration in soil, the approach is reasonable and conservative. Therefore this approximation requires no further confirmation. This approximation eliminates the need to calculate losses from crop harvest removal and gains from animal manure used as fertilizer. Applying this approximation to the entire Amargosa Valley is realistic because alfalfa is planted solely for livestock fodder, and is the major crop grown in Amargosa Valley (CRWMS M\&O 1997 [DIRS 101090], pp. 3-18 to 3-19; YMP 1999 [DIRS 158212], Tables 10 and 11).

Applicability - This approximation is applied to the surface soil submodel for the groundwater scenario in Sections 6.3.1.6, 6.4.1.1, and 7.

\section{Approximation 5 - Radionuclide Buildup in Surface Soil}

Statement-Radionuclide concentrations in irrigated soils are at equilibrium because of long-term use of contaminated groundwater to irrigate crops.

Rationale-When cultivated lands are irrigated with contaminated groundwater over long periods of time, radionuclides accumulate in the soil until an equilibrium condition is reached (Section 6.4.1). The time to reach equilibrium depends on the rates of radionuclide addition (i.e., irrigation) and removal from the soil (Equation 6.4.1-4). After equilibrium is reached, radionuclide concentrations remain constant. This is a conservative approximation because the activity concentration of a radionuclide in the soil is highest at equilibrium. It also is reasonable because the regulatory compliance period is 10,000 years, and during that period of time, most or all of the radionuclides will reach equilibrium (Section 7.4.2.1), assuming that current agricultural land use and irrigation practices continue (Approximation 3). Other biosphere 
models, such as BIOMASS ERB2A (BIOMASS 2000 [DIRS 154522], Tables 22 to 25), also assume equilibrium conditions for dose calculations. This approximation eliminates the need to consider time-dependent radionuclide concentrations in the surface soil, and it permits evaluating biosphere dose contributions separately from the groundwater source in performance assessment (Approximation 1). This approximation is further discussed in Section 6.4.10, where the total dose and BDCF calculations are described. This approximation does not require further confirmation because it is likely that equilibrium conditions will be reached in less than 10,000 years, and because the dose will not be underestimated if those conditions are not reached.

Applicability - The approximation is applied to the surface soil submodel for the groundwater scenario in Sections 6.3.1.6, 6.4.1, 6.4.9.5, 6.4.10.4, 6.6, 7.3.3.1, and 7.4.2.

\section{Approximation 6 - Crop Weathering Loss and Surface Soil Gain}

Statement-All radionuclides in irrigation water are deposited on the surface of the soil, even when overhead irrigation is used and a fraction of the irrigation water is initially intercepted and absorbed by plant leaves.

Rationale-Farmers in the Amargosa Valley, especially the larger commercial operations, irrigate using spray and overhead systems (BSC 2004 [DIRS 169673], Section 6.3.2). When plants are irrigated from above, a portion of water is intercepted and absorbed by the leaves. The total amount of contaminated water eventually deposited on the soil depends on the initial foliar interception fraction, crop weathering, and the crop growing time. This approximation is conservative because it double-counts the radionuclides remaining on the plant and absorbed through plant leaves because they are also treated as deposited on the soil. This approximation is reasonable because the amount of radioactive material on and in the plant leaves is low compared to the overall irrigation source. It is estimated that a small fraction (less than 7 percent) of the radioactivity in the irrigation water is transferred to the edible parts of the crops, while the rest is deposited on the soil or remains in the nonedible portion of the plants, and, eventually, is incorporated into the soil from the nonharvested portions of the plants or contaminated manure (Section 7.4.4.1). This approximation is used in other biosphere models such as GENII (Napier et al. 1988 [DIRS 157927], Section 4.7.4) and BIOMASS ERB2A (BIOMASS 2000 [DIRS 154522], Section 7). This approximation simplifies the mathematical representation of the weathering process, in which a fraction of the intercepted radioactive material is deposited on the soil surface. Based on above discussions, this approximation requires no further confirmation.

Applicability - This approximation is applied to the surface soil submodel for the groundwater scenario in Section 6.3.1.6. It is also discussed in the plant submodel (Sections 6.3.1.6 6.4.3.2, and 7.3.6).

\section{Approximation 7 - Crop Roots in Surface Soil}

Statement-All crop roots are in the surface soil layer down to the tilling depth.

Rationale - In the ERMYN, soil in cultivated fields and gardens is divided into two compartments (surface soil and deep soil), and only the surface layer is considered part of the 
biosphere. Although the deep soil would become contaminated due to leaching from the surface soil, radionuclide concentrations in the deep soil are not calculated directly because those radionuclides are considered lost from the biosphere. Because many crops require tilling every year, radionuclides would be uniformly distributed throughout the surface layer over the long term. Thus, the soil tilling depth fits the concept of a surface soil depth. This approximation is reasonable because 80 to 90 percent of the plant roots occur in the upper 60 to 75 percent of the root zone (Jensen et al. 1990 [DIRS 160001], p. 22]. Although crop roots can penetrate into the deep soil compartment, radionuclide concentrations would typically decrease with soil depth due to leaching. This approximation does not underestimate radionuclide concentrations in the crops because the higher radionuclide concentrations in the surface soil are used for calculating crop root uptake. Therefore, the approximation requires no further confirmation.

Applicability - This approximation applies to the plant submodel for the groundwater scenario in Sections 6.3.1.6, 6.4.1, 6.4.1.1, 6.4.3.1, and 7.3.3.1.

\section{Approximation 8 - Animal Feed}

Statement-Locally grown fresh forage is the only feed given to beef cattle and dairy cows, and locally produced grain is the only feed given to poultry and laying hens.

Rationale-Farm animals become contaminated by ingesting contaminated feed, water, and soil. Among these, animal feed is an important pathway (Section 7.4.5). In the Amargosa Valley, alfalfa and other hays are the most common crops (YMP 1999 [DIRS 158212], Tables 10 and 11), and dry hay used for livestock feed is produced locally and imported from outside the area (Horak and Carns 1997 [DIRS 124149], p. 12). Water is added to locally grown alfalfa hay and commercial feed before feeding it to animals (Horak and Carns 1997 [DIRS 124149], p. 16). It is reasonable to assume that animals are fed locally grown fresh forage rather than dry hay with water added because they are equivalent, and this approximation simplifies the mathematical model. In addition, although poultry and laying hens could be fed with other types of feed, locally produced grain is the only feed considered in the model. This approximation is conservative because it assumes that all animal feed is locally produced and contaminated; therefore, this approximation requires no further confirmation. This approximation eliminates the need to consider radionuclide concentrations in other types of feed, the fraction of those feeds, and the fraction of imported uncontaminated feed.

Applicability-The approximation applies to the plant (Sections 6.3.1.6 and 6.4.3) and the animal submodels (Sections 6.3.1.6, 6.4.4, and 7.3.4.1) for the groundwater scenarios.

\section{Approximation 9-Animal Product Type}

Statement-People consume animal products from four categories: meat, milk, poultry, and eggs. Meat includes beef, pork, lamb, and game animals; milk is from dairy cows, goats, and sheep; poultry includes chicken, turkey, duck, geese, and game hens; and eggs come from laying hens (chickens) and ducks.

Rationale-Farm animals in the Amargosa Valley include cattle, dairy cows, pigs, goats, ostriches, and poultry (YMP 1999 [DIRS 158212], Tables 8 and 9), plus sheep and ducks (Horak and Carns 1997 [DIRS 124149], Tables 5 and 6). There are more cattle than pigs and goats 
combined, and there are more dairy cows than goats (YMP 1999 [DIRS 158212], Tables 8 and 9). According to the Environmental Protection Agency Food Ingestion Factors (EPA 1997 [DIRS 152549], Table 11-9) and the U.S. Department of Agriculture Census of Agriculture, Nevada State and County Data (USDA 1999 [DIRS 158643], Tables 20, 29, 40), beef, milk from cows, chickens, and chicken eggs are the most frequently consumed products or are the most commonly raised and sold products in each category. Therefore, beef and milk from cows are considered representative of the meat and milk categories, respectively. Because it is difficult to count the number of small animals in the Amargosa Valley, poultry and chicken eggs are used as general categories in the model. These two categories match the categories in the food consumption survey (DOE 1997 [DIRS 100332], Appendix B). This approximation is reasonable because the uncertainty range of transfer coefficients for the selected animal products includes variation in transfer coefficients between selected and unselected animal products. For example, the selected transfer coefficient for meat is $1.3 \times 10^{-5} \mathrm{~d} / \mathrm{kg}$, with a range of $3.3 \times 10^{-8}$ to $4.7 \times 10^{-3} \mathrm{~d} / \mathrm{kg}$ for plutonium (Table 6.6-3), while the transfer coefficients for pork, sheep meat, and lamb are $8.0 \times 10^{-5}, 9.4 \times 10^{-5}$, and $3.1 \times 10^{-3} \mathrm{~d} / \mathrm{kg}$, respectively (IAEA 1994 [DIRS 100458], pp. 38 to 39). Therefore, this approximation requires no further confirmation. This approximation reduces the number of animal-product ingestion pathways for humans, and eliminates the need for transfer coefficients for the other types of animal products.

Applicability - This approximation applies to the animal submodel for both exposure scenarios (Sections 6.3.1.6, 6.3.2.5, 6.4.4, and 6.5.4).

\section{Approximation 10 - Dose Coefficients for Exposure to Contaminated Soil}

Statement-Dose coefficients for an infinite depth of soil are appropriate for estimating external exposure for the groundwater scenario.

Rationale-The dose coefficients for soil contaminated to an infinite depth (Eckerman and Ryman 1993 [DIRS 107684], Section II) are based on an infinite isotropic (i.e., a homogeneously contaminated infinite) source and a receptor standing at the air-ground interface (Eckerman and Ryman 1993 [DIRS 107684], p. 11). Under the groundwater scenario, it is reasonable to consider that the contaminated area is infinitely large because the size of contaminated fields is large relative to the area from which the external exposure generally is received. In addition, noncultivated lands eventually will be contaminated by surface soil transport, although the level of contamination would be lower than cultivated land. This approximation is conservative because only a small portion of Amargosa Valley is irrigated (BSC 2004 [DIRS 169458], Section 6). Regarding the source depth, using dose coefficients based on an infinite depth is reasonable because deep soil will be contaminated by leaching, although at levels lower than those for surface soil. Dose coefficients for an infinite depth and those for a 15-cm depth differ by less than 10 percent for most primary radionuclides (Eckerman and Ryman 1993 [DIRS 107684], Tables III-6 and III-7). Only radionuclides with strong gamma emissions, such as ${ }^{226} \mathrm{Ra}$ and ${ }^{137} \mathrm{Cs}$, have a relatively large difference for the two depths. Because dose coefficients for these radionuclides are greater for a source of infinite depth, this approximation is conservative. This approximation is reasonable for the groundwater scenario and does not underestimate the dose; it does not require further confirmation. 
Applicability-The approximation applies to the external exposure submodel for the groundwater scenario (Sections 6.3.1.6 and 6.4.7.1).

\section{Approximation 11 - Evaporative Cooler Use and Exposure Time}

Statement-Evaporative coolers do not cause radionuclides to build up in indoor air, radionuclide concentrations in indoor air are constant on days when coolers are used, and the contribution of contaminated aerosols to the outdoor environments is unimportant.

Rationale-This approximation is necessary to evaluate radiation doses from aerosols generated by evaporative coolers using contaminated water. To be most effective, evaporative coolers are operated with an open window or door to let air circulate, thus radionuclides do not build up in indoor air because the large volume of airflow would carry contaminated aerosols out of the house. Radionuclide concentrations in indoor air are assumed constant on days when evaporative coolers are used. Although coolers cycle on and off to maintain the temperature setting, the period when the cooler is temporarily off usually would be relatively short, and decreases in radionuclide concentrations due to decay and air exchange would be insignificant. The contribution of contaminated aerosols generated from evaporative coolers and transferred to the outdoor air is not important and thus is not further considered because the indoor air is diluted and rapidly dispersed in the large outdoor environment. Therefore, this approximation requires no further confirmation.

Applicability-This approximation applies to the air and inhalation submodels for the groundwater scenario (Sections 6.3.1.6, 6.4.2.2, and 6.4.8.2).

\subsubsection{Submodels for the Groundwater Scenario}

To illustrate radionuclide transfer among biosphere components (Figure 6.3-2), the conceptual model is divided into seven parts matching the seven biosphere components (diagonal elements) in the interaction matrix (Table 6.3-2). The human receptor component, however, is further divided into three parts that represent the three major dose pathways. All of these parts are considered as submodels, except for groundwater, which is the radionuclide source for this scenario. The final box in the figure, "Results: BDCF," is not considered a submodel, rather it represents the output of the biosphere model.

In Figure 6.3-2, arrows point in the direction of radionuclide transfer between biosphere components in the ERMYN. For example, groundwater is used for human drinking water (to ingestion submodel), animal drinking water (to animal submodel), irrigation water (to soil and plant submodels), fish pond water (to fish submodel), and evaporative cooler water (to air submodel, see Appendix D).

A special submodel is used to calculate ${ }^{14} \mathrm{C}$ concentrations in the surface soil, air, crops, and animal products because the transfer mechanisms for this radionuclide are different from the others in the model. This special submodel is an additional submodel and is discussed separately. The direction of ${ }^{14} \mathrm{C}$ transfer is the same as shown in the radionuclide transfer interaction matrix (Table 6.3-2) and in the relationships among the biosphere submodels (Figure 6.3-2). 


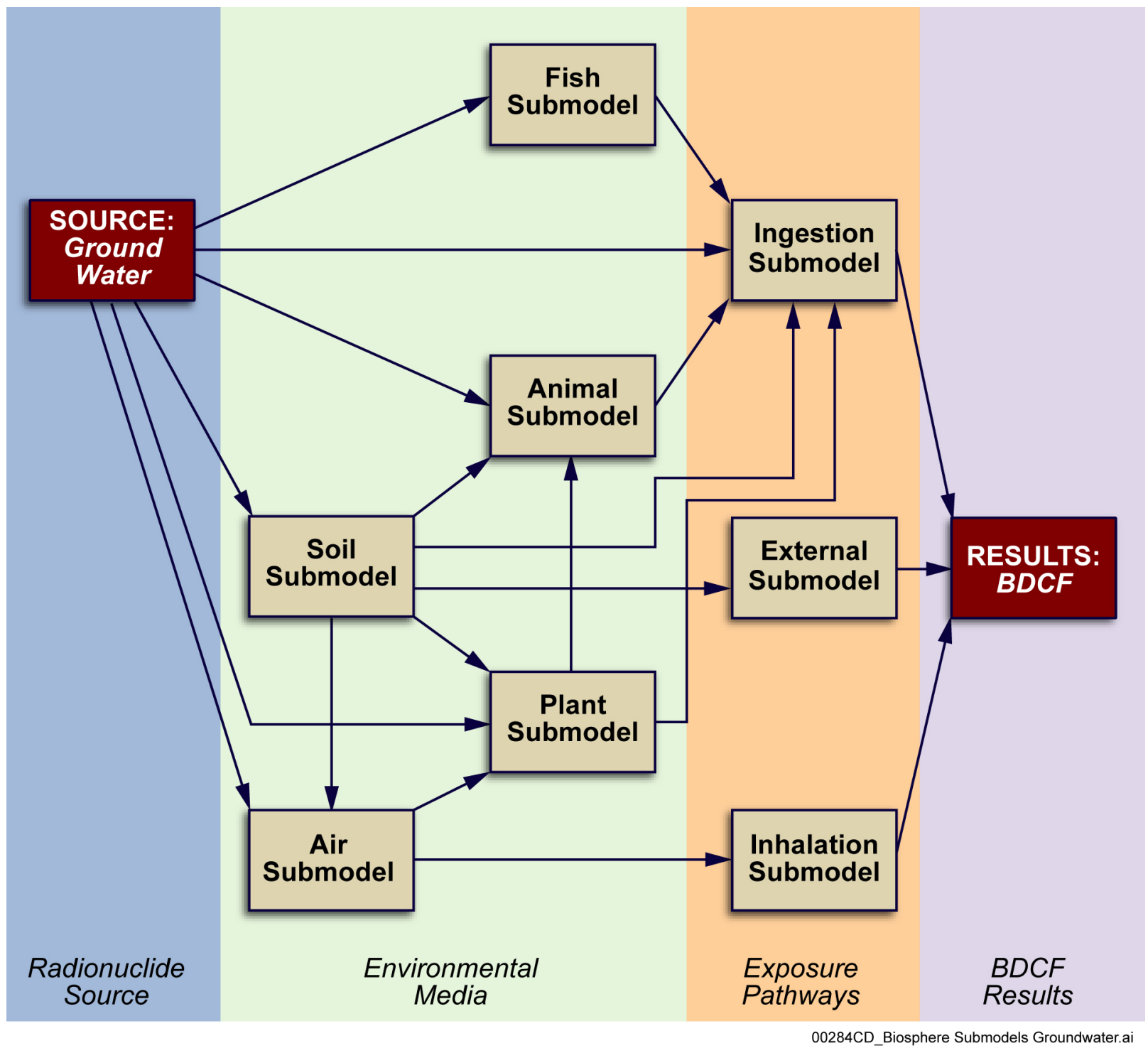

Figure 6.3-2. Relationship Among Biosphere Submodels for the Groundwater Scenario

\subsubsection{Description of Conceptual Model for the Groundwater Scenario}

The conceptual model for the groundwater scenario includes groundwater, surface soil, air, plants, animals, fish, and the human receptor. The radionuclide transfer interaction matrix (Table 6.3-2) illustrates the radionuclide transfer mechanisms among the biosphere components. Separation of this model into nine submodels makes the conceptual and mathematical models easier to comprehend.

Groundwater Source-For the groundwater scenario, the source of radionuclides is water from a well ("well water" in 10 CFR 63.312 [DIRS 156605]). The well provides the interface between the geosphere and biosphere. Radionuclide concentrations in the well water are considered to equal the groundwater concentrations (Section 6.3.1.1), so the groundwater can be considered as the source of radionuclides in the biosphere model. To generate BDCFs with the biosphere 
model, the activity concentration of a radionuclide in groundwater is assumed constant at a predetermined concentration (e.g., $1 \mathrm{~Bq} / \mathrm{m}^{3}$; Approximation 1). The groundwater source is not considered a submodel because no radionuclide transport is modeled to or from the groundwater, and it is the source term for the groundwater scenario. The FEP, surface water transport and mixing (FEP 2.3.04.01.0A), is implicitly considered in the conceptual model for the groundwater scenario because the outcome of biosphere modeling (i.e., BDCFs) is insensitive to the source of the groundwater (e.g., well or spring) as long as the reference biosphere remains unchanged. The biosphere model can use contaminated water from any source for domestic and agricultural purposes. Thus wells and springs are equivalent sources of contamination in the biosphere as long as the concentration of radionuclides in the water remains the same. Mixing of groundwater with uncontaminated surface water is not considered in the ERMYN because there are no sources of uncontaminated surface water in the reference biosphere.

Surface Soil Submodel-The purpose of the surface soil submodel, or simply the soil submodel, is to calculate the radionuclide concentration in surface soil (i.e., the root zone or tilling depth). The source of radionuclides in the surface soil is contaminated groundwater used for crop irrigation (Table 6.3-2, Element [1,2]). Based on agricultural practices in Amargosa Valley, groundwater is the only source of irrigation water. Because the objective of the postclosure dose assessment is to predict the future dose from the repository, the biosphere conceptual model assumes long-term irrigation using contaminated groundwater (Approximation 3). This results in the addition of radionuclides to cultivated lands, leading to a buildup of radionuclides in the irrigated soil. When overhead irrigation is used, radionuclides in irrigation water can be intercepted by crop leaves. However, crop weathering by wind and other mechanisms (Table 6.3-2, Element [4,2]) will displace some initially intercepted radionuclides onto the soil. Therefore, the biosphere conceptual model conservatively assumes that all radionuclides in the crop irrigation water reach the soil (Approximation 6).

Besides the groundwater source, contaminated fertilizer (animal manure and nonharvested plant residue) could contribute additional radionuclides to the surface soil (Table 6.3-2, Element [5,2]). However, over the long term (i.e., 10,000 years), most radioactivity incorporated into crops likely would be recycled in the form of animal manure or the nonedible parts of crops. Therefore, the addition of radionuclides to the surface soil due to contaminated fertilizer is considered to compensate for the removal of radionuclides from the surface soil due to crop harvest removal (Table 6.3-2, Element [4,2]). This approximation is justified in Section 6.3.1.4 (Approximation 4), and it simplifies the mathematical model.

Contaminated resuspended dust deposited on the surface of the soil is another possible radionuclide source (Table 6.3-2, Element [3,2]). However, dust deposition on the surface soil could be balanced by particle resuspension (Table 6.3-2, Element [2,3]). Thus, these two mechanisms are not numerically modeled in the surface soil submodel. Furthermore, dust could originate from nonirrigated, uncontaminated soils, such that the radioactivity in the deposited dust would be less than that in the resuspended particles. These two processes (deposition and resuspension) are associated with surface soil erosion, which is a removal mechanism in this submodel, as discussed below.

Processes in the conceptual model that result in the removal of radionuclides from the surface soil are radioactive decay, leaching to the deep soil (Table 6.3-2, Element [2,1]), surface soil 
wind erosion (Table 6.3-2, Element [2,3]), and the gaseous release (Table 6.3-2, Element [2,3]) of ${ }^{222} \mathrm{Rn}$ and ${ }^{14} \mathrm{C}$. Some published biosphere models include crop harvest as a removal mechanism, but the ERMYN does not, as discussed above. Radionuclides removed by leaching from the root zone were considered unavailable to plants. Although radionuclides eroded from cultivated fields could be deposited elsewhere in the biosphere, the biosphere model only tracked radionuclides in the surface soil of cultivated fields.

Because of the continuous addition of radionuclides to the surface soil with only fractional removals over long periods of time, the concentrations of radionuclides in surface soil eventually increase to equilibrium conditions. Under equilibrium conditions, radionuclide concentrations in the surface soil would not change with time. In the ERMYN, the soil submodel is constructed based on the approximation that equilibrium conditions already have been reached. This approximation is justified in Section 6.3.1.4 (Approximation 5), and it eliminates the need to calculate time-dependent BDCFs.

Leaching removal is a function of deep water percolation and soil characteristics, including radionuclide-specific solid-liquid partition coefficients. The initial condition is that water and soil are free of radionuclides from the repository at Yucca Mountain.

The output from the soil submodel is used in most of the remaining submodels (Figure 6.3-2) because the modeling of many environmental transport and exposure pathways depends in some way on the radionuclide concentration in surface soil.

Air Submodel-The air submodel is used to calculate radionuclide concentrations in the air. Inputs to the air submodel come from the surface soil submodel or directly from contaminated water. Three air contamination processes are considered: resuspension of contaminated soil particles (Table 6.3-2, Element [2,3]), generation of contaminated aerosols by evaporative coolers (Table 6.3-2, Element [1,3]), and the gaseous release of radionuclides from the soil (Table 6.3-2, Element [2,3]).

Resuspension of contaminated soil may be caused by natural forces (e.g., wind) or human activities (e.g., tilling). Radionuclide concentrations in the air depend on particle sizes, mineral composition of the soil particles, and the ability of the soil particles to sorb radionuclides. Resuspended particles deposit on crop leaves (Table 6.3-2, Element $[3,4]$ ) and directly on the soil surface (Table 6.3-2, Element [3,2]). Contaminated resuspended particles are a source of radionuclides for human inhalation (Table 6.3-2, Element [3,7]). In the submodel, contaminated resuspended particles come from the surface soil of irrigated land, although resuspended particles also could come from uncontaminated soil. The most important sources of resuspended particulates would be human dust-generating activities, such as farming (Chow et al. 1993 [DIRS 162999]; Chow 1999 [DIRS 145212]). Therefore, all resuspended particles are considered to come from contaminated soils. Resuspended particles, transported from the outdoors, are also considered in indoor environments.

The air submodel includes an enhancement factor, which accounts for measured differences between the activity concentration per unit mass of resuspended particles and the average activity concentration per unit mass in the surface soil available for resuspension. For crop deposition, the enhancement factor is taken to be unity, as soil is transferred to the plant by 
splashing action from overhead irrigation systems. Thus the radionuclide concentrations in airborne particles are considered equal to the concentrations in surface soil per unit of mass. For inhalation, radionuclide concentrations in resuspended particles from wind action can be higher or lower than those in the surface soil and might differ among environments. Therefore, the enhancement factor is environment specific.

Some radionuclides may be released from soil to air as gasses. This mechanism is only of concern for radionuclides that are gases, produce gaseous progeny, or form gaseous compounds (e.g., ${ }^{222} \mathrm{Rn}$ and ${ }^{14} \mathrm{C}$ ). Radon, a decay product of ${ }^{226} \mathrm{Ra}$, is a radioactive gas that leads to a chain of short-lived progeny. The release of ${ }^{222} \mathrm{Rn}$ is considered only from accumulated radium in soil because little radon would be released directly from water (Section 6.4.2.3). Radon concentrations are considered separately for indoor and outdoor environments. ${ }^{14} \mathrm{C}$ is released from soil as radioactive carbon dioxide gas $\left({ }^{14} \mathrm{CO}_{2}\right)$. In this form, the ${ }^{14} \mathrm{CO}_{2}$ could be taken up by plants during photosynthesis and could contribute to human inhalation exposure. Concentrations of gaseous species in the air are affected by atmospheric mixing and dilution. Gases released from the soil contribute to radionuclide concentrations in indoor and outdoor air.

Radionuclide concentrations in indoor air would be affected by the use of contaminated groundwater in evaporative coolers. Evaporative coolers work by forcing air through a wet, porous material (i.e., a pad), resulting in the evaporation of water and the cooling of air. When water evaporates in the coolers, some of the contaminants in the water would be released into indoor air. Radionuclide concentrations in the air would depend on the water evaporation rate, the inlet air flow rate, and the fraction of radionuclides transferred from the water to air (see Appendix D). Air leaving the house would carry the radioactive contaminants outdoors, where they would be an unimportant contribution to the outdoor inhalation dose because of atmospheric dilution (Approximation 11). Any radionuclides that are not transferred to the airflow remain in the system, where they are a source of external radiation exposure. This pathway is evaluated in Appendix D where it is shown that the increase of external exposure due to this pathway compared with that from contaminated soil is negligible.

The activity concentrations of radionuclides in the air (as particles, gases, and aerosols) are the outputs of the air submodel. These concentrations are important inputs for calculating the contribution from the inhalation pathways, and they provide inputs for the direct deposition of particles on crop leaves and carbon uptake by photosynthesis in the plant submodel (Figure 6.3-2 and Table 6.3-2).

Plant Uptake Submodel (i.e., the plant submodel)-The purpose of the plant submodel is to calculate radionuclide concentrations in crops consumed by humans and farm animals. The plant submodel receives input from the soil submodel, the air submodel, the ${ }^{14} \mathrm{C}$ submodel, and directly from the contaminated water source. The mechanisms of radionuclide transfer to crops in the submodel are root uptake (Table 6.3-2, Element $[2,4]$ ), direct deposition on crop leaves from irrigation water (Table 6.3-2, Element $[1,4]$ ), photosynthesis of carbon dioxide containing ${ }^{14} \mathrm{C}$ (Table 6.3-2, Element $[3,4]$ ) and deposition of resuspended particles (Table 6.3-2, Element $[3,4])$.

Root uptake is modeled based on a state of equilibrium between radionuclide concentrations in the soil and crops. It is assumed that plant roots grow only in the surface soil compartment 
(Approximation 7). Direct deposition is modeled as a continuous process occurring during the crop growing time, accompanied by the continuous removal of radionuclides by weathering. Two types of direct deposition, irrigation water and resuspended particles, are considered in the submodel. The fraction of irrigation water intercepted depends on irrigation practices and plant biomass. The fraction of resuspended particles intercepted is a function of plant type and biomass. These two processes are modeled using empirical equations. The activity remaining on the crops may be translocated in whole or in part to the edible portion of the plants. Radionuclides removed from crop surfaces by weathering would be eventually incorporated into the soil surface, which is not separately tracked, as discussed in the surface soil submodel.

For the groundwater scenario (Section 6.1.3), four types of crops are considered for human consumption: leafy vegetables, other vegetables, fruit, and grain. In addition, fresh forage is considered for beef cattle and dairy cow feed. The grain used for human consumption is also considered as the only feed for poultry and laying hens. It is also assumed that fresh forage for beef cattle and dairy cows would be available year around (Approximation 8). Radionuclide concentrations would differ among crop types due to different irrigation rates, growing times, and other agricultural parameters.

The output of the plant submodel, activity concentrations of radionuclides in crops, is used as input to calculate the contribution to the human ingestion pathway from consumption of crop foodstuffs, as well as the contamination of animal products via ingestion of feed (Figure 6.3-2 and Table 6.3-2).

Animal Uptake Submodel (i.e., the animal submodel)-Ingestion of contaminated crops (Table 6.3-2, Element [4,5]), water (Table 6.3-2, Element [1,5]), and soil (Table 6.3-2, Element $[2,5]$ ) may contribute to radionuclide uptake by farm animals, and the animal submodel includes these three environmental transport pathways. Radionuclide uptake by inhalation is another potential radionuclide transfer process for animals; however, this is not an important pathway (Section 7.4.5) and is excluded from the submodel.

An equilibrium approach is used to assess radionuclide concentrations in animal products, where the equilibrium is between the rate of animal activity intake and the activity concentration in an animal product. The total animal intake of radionuclides is the sum of intakes from contaminated feed, water, and soil. Four types of animal products (meat, poultry, milk, and eggs) are considered in the submodel, where meat is representative of beef, pork, and lamb; milk is representative of milk from cows and sheep; poultry is representative of chickens, turkeys, ducks, geese, and game hens; and eggs are representative of those from laying hens and ducks (Approximation 9).

The output of the submodel, radionuclide concentrations in animal products, is used as input to calculate the contribution from the consumption of animal products in the human ingestion pathways (Figure 6.3-2 and Table 6.3-2).

Fish Submodel-The fish submodel is used to calculate radionuclide concentrations in farm-raised fish. The ERMYN includes fish because there is a fish farm in the Amargosa Valley with about 15,000 catfish and bass in 1998 and 1999 (YMP 1999 [DIRS 158212], Tables 8 
and 9). Radionuclide accumulation in the fish is considered to be caused exclusively by the use of contaminated water in the fishponds.

The radionuclide transfer from water to fish is through a bioaccumulation process (Table 6.3-2, Element $[1,6]$ ) that is based on equilibrium conditions between radionuclide concentrations in the water and concentrations in the edible parts of fish. This submodel may be better applied to fish in rivers, lakes, or reservoirs where fish and fish food are in equilibrium with the contaminated water. In the Amargosa Valley, fish are given commercial feed (Roe 2002 [DIRS 160674]), which is likely to be uncontaminated because it is not produced locally. Therefore, using bioaccumulation factors results in an upper bound analysis. Resuspended radioactive particles could be deposited into fishponds, but it is shown that this additional source is small compared to the contaminated water source (Section 6.4.5).

The output for the submodel, activity concentration in fish, is used to calculate the contribution of fish consumption to the human ingestion pathway (Figure 6.3-2 and Table 6.3-2).

Carbon-14 Submodel-The environmental transport pathways of ${ }^{14} \mathrm{C}$ are different from those considered for other radionuclides. While most radionuclides are in solid form, carbon can move in the environment as a gas. Moreover, stable carbon is an abundant and ubiquitous element in the environment. As for the other radionuclides, groundwater is the source of ${ }^{14} \mathrm{C}$, and the calculation of ${ }^{14} \mathrm{C}$ concentrations in the soil are based on equilibrium conditions between ${ }^{14} \mathrm{C}$ gains and losses from surface soil. The most important process resulting in the loss of this radionuclide from surface soil, gaseous emission, is unique to gases and is not considered for other radionuclides. After it is released into the atmosphere, ${ }^{14} \mathrm{CO}_{2}$ could be incorporated into crops via photosynthesis. The predominant transport pathway to plants is foliar uptake via stomata. The uptake of ${ }^{14} \mathrm{C}$ may also occur via the root system; however, root uptake plays a smaller role than foliar uptake. Following plant uptake, ${ }^{14} \mathrm{C}$ may move into the animal food chain. Consumption of drinking water and soil are additional sources of ${ }^{14} \mathrm{C}$ intake by animals. All of these processes are incorporated into the conceptual model.

Modeling the transport of ${ }^{14} \mathrm{C}$ in the biosphere is carried out using a special ${ }^{14} \mathrm{C}$ submodel. The concentration of ${ }^{14} \mathrm{C}$ in air is calculated based on the equilibrium concentration of ${ }^{14} \mathrm{C}$ in the surface soil, with the rate of loss controlled primarily by the gaseous emission loss rate of ${ }^{14} \mathrm{CO}_{2}$ from the soil. In the air, ${ }^{14} \mathrm{C}$ is subject to mixing due to atmospheric processes, which are modeled using air movement in a mixing cell of defined dimensions. The uptake of ${ }^{14} \mathrm{C}$ by crops is modeled using the ratios of ${ }^{14} \mathrm{C}$ to stable carbon in soil and air, and the proportion of carbon in crops that is due to transport from these media. The concentration of ${ }^{14} \mathrm{C}$ in animal products is estimated from the ratio of ${ }^{14} \mathrm{C}$ to stable carbon uptake with water, soil, and feed. The bioaccumulation of ${ }^{14} \mathrm{C}$ in fish is assessed using the same method as that used for other radionuclides, which is based on the ratio of concentrations between water and the edible parts of fish. After the media concentrations of ${ }^{14} \mathrm{C}$ are calculated, the dose assessment is carried out using the same approach as is used for other radionuclides.

External Exposure Submodel-The purpose of the external exposure submodel is to calculate the dose resulting from external radiation exposure, which would occur as a result of direct exposure to radiation emitted by radioactive materials outside the human body. The annual effective dose equivalent (EDE) is calculated for this pathway. For environmental dose 
assessments, these materials typically include soil, air, and water. The corresponding exposures are referred to as ground exposure, air submersion, and water immersion, respectively. The conceptual model considers only one of these exposure pathways: exposure to emissions from radionuclides in the soil (Table 6.3-2, Element [2,7]). The ERMYN does not include air submersion or water immersion because they contribute relatively little to the annual dose (Section 7.4.8). Radiation sources of concern in the soil are radionuclides with gamma and high energy beta rays, which are penetrating and could deposit energy in human organs and tissues.

External exposure from other types of media (e.g., building material, furniture, and clothing; see FEP 3.3.03.01.0A) also is possible. However, few or no building materials, clothes, or other materials are produced in the Amargosa Valley using contaminated water. Furthermore, it is assumed that the size and depth of contaminated soils are infinite (Approximation 10), and residents are considered to be exposed to contaminated soil at all times while within the valley. Thus, the soil exposure time would be longer than for other contaminated media, and, therefore, it is reasonable to not evaluate exposures from these types of media in the ERMYN.

This submodel considers indoor and outdoor external exposure to radionuclides in the soil. For outdoor exposures, radiation doses depend on radionuclide concentrations in the soil, the duration of exposure, and the dose coefficients that convert exposure to dose. For indoor exposures, the shielding effect of dwellings reduces the level of exposure. Although the radionuclide concentrations in the soil used as input to this submodel apply to the surface soil, the dose coefficients apply to soil contaminated to an infinite depth. This choice of dose coefficients is considered appropriate because the radiation contributing to external exposure may also originate in the deep soil contaminated due to long-term radionuclide leaching from the surface soil. As discussed in the air submodel discussion on evaporative coolers, the external exposure from cooling systems in evaluated in Appendix D, where it is shown that this specific cooler pathway introduces only a small increase into the external exposure from radionuclide accumulation in continually irrigated soils.

The output of the external exposure submodel, annual dose from external exposure, contributes to the all-pathway dose, which is used to calculate BDCFs (Figure 6.3-2).

Inhalation Submodel-The purpose of the inhalation submodel is to calculate radiation doses due to the inhalation of radionuclides. The 50-year committed effective dose equivalent (CEDE) resulting from annual intake of radionuclides by inhalation is calculated for this pathway. Three sources of contamination are considered in this submodel: resuspended particles, gaseous emissions from the soil, and aerosols generated by evaporative coolers (Table 6.3-2, Element [3,7]). These sources are provided by the air submodel.

In addition to radionuclide concentrations in the air, inhalation doses depend on the duration of inhalation exposure, the breathing rate, and the intake-to-dose conversion factors. Human breathing rates and exposure times differ by activity, occupation, work location, and other factors related to the behavior of the receptor. To account for differences and uncertainty in those behaviors, breathing rates and exposure times differ among environments and among population groups (Section 6.4.7.1) that comprise the receptor (RMEI). 
The output of the inhalation submodel, annual inhalation dose, contributes to the all-pathway dose, which is used to calculate BDCFs (Figure 6.3-2).

Ingestion Submodel-The ingestion submodel is used to calculate radiation doses due to the ingestion of radionuclides. The 50-year CEDE resulting from annual intake of radionuclides by ingestion is calculated for this pathway. Inputs to the ingestion submodel are radionuclide concentrations in the groundwater (Table 6.3-2, Element [1,7]), and the outputs from the soil (Element [2,7]), plant (Element [4,7]), animal (Element [5,7]), and fish submodels (Element [6,7]).

Eleven ingestion exposure pathways are considered for the groundwater scenario, including the use of untreated, contaminated groundwater; inadvertent soil ingestion; and consumption of four types of plant foodstuffs, four types of animal products, and fish. The concentrations in these media are combined with the corresponding consumption rates and dose conversion factors, and used to produce ingestion doses.

The output of the ingestion submodel, annual ingestion dose, contributes to the all-pathway dose, which is used to calculate BDCFs (Figure 6.3-2).

BDCFs and ERMYN Results-The all-pathway dose is the sum of the radionuclide-specific annual doses from the external, inhalation, and ingestion exposure pathways. The all-pathway dose is expressed in terms of the total effective dose equivalent (TEDE) from annual intake. For purposes of assessing doses to the RMEI, the TEDE is the sum of the EDE for external exposures and the CEDE for internal exposures (10 CFR 63.2 [DIRS 156605]). The BDCFs, in units of $(\mathrm{Sv} /$ year $) /\left(\mathrm{Bq} / \mathrm{m}^{3}\right)$, are numerically equal to the all-pathway dose from a unit activity concentration in the groundwater. The calculation of each radionuclide concentration as a function of time in the groundwater is carried out in the TSPA-LA model. The total annual dose is the sum of the products of the radionuclide-specific BDCFs and the time-dependent activity concentrations of radionuclides in the groundwater.

\subsubsection{Conceptual Model for the Volcanic Ash Exposure Scenario}

Similar to the groundwater scenario, the volcanic ash exposure scenario (or simply the volcanic ash scenario) is discussed in this section. The biosphere conceptual model for the volcanic ash scenario uses the same reference biosphere and human receptor as the groundwater scenario. The major difference is the radionuclide source, which is contaminated volcanic ash deposited on the surface of the ground, rather than contaminated groundwater. In this scenario, water is uncontaminated. Because the radionuclide sources are different, some of the radionuclide transfer mechanisms between biosphere components differ.

\subsubsection{Scenario Description}

The volcanic ash scenario is used to evaluate the radiological consequences of a volcanic eruption at the repository, which is one of the igneous scenarios considered for the TSPA-LA (BSC 2003 [DIRS 166296], Section 4.2). For this scenario, volcanic ash deposited on the ground is the radionuclide source. The contaminated volcanic ash could come directly from a volcanic eruption as the initial source, or it could be resuspended and transported into the biosphere by wind (aeolian processes) or flooding (fluvial processes). These differing deposition 
processes are not considered in the ERMYN. The ERMYN is concerned with radionuclide transport in the biosphere and human exposure after the ash is deposited on the ground, regardless of the deposition process. On cultivated soils, volcanic ash would mix with surface soil and contaminate crops and animal products, which would contribute to the human ingestion dose. On noncultivated lands, the volcanic ash could be resuspended into the air, causing human inhalation doses. The volcanic ash also may cause external exposure to humans. The ERMYN uses a predefined (e.g., $1 \mathrm{~Bq} / \mathrm{m}^{2}$ ) concentration of a radionuclide deposited on the ground. A graphical representation of the volcanic ash scenario (Figure 6.3-3) shows dispersion, initial deposition, and radionuclide transfer in the biosphere.

In biosphere modeling for the TSPA-LA, volcanic eruptions are represented by three phases. The first phase, eruption and deposition, is when the ash is transported and dispersed in the atmosphere, and subsequently deposited on the ground. The second phase, a transient phase, is when the volcanic ash is resuspended into the air at concentrations higher than those experienced before the eruption. The last phase, a steady-state, is when particle concentrations in the air return to preeruption levels. This approach was used in earlier efforts for the biosphere (CRWMS M\&O 2001 [DIRS 152536]). A similar approach is followed here. The data required to estimate dose during the first phase of ash fall are developed in BSC 2004 [DIRS 167287] and are not discussed in this document. However, the evolution of the posteruption biosphere is modeled in the ERMYN through time-dependent, multicomponent BDCFs that apply to both posteruption phases.

During the eruption phase, radiation doses to humans mainly come from inhalation. Because exposure conditions depend on the characteristics of the volcanic eruption, radiation doses to the receptor accrued during the eruption phase is calculated in the TSPA-LA code using the dose factors developed for unit activity per unit volume of air (BSC 2004 [DIRS 167287], Section 7.4).

After the eruption, volcanic ash deposited on cultivated and noncultivated land is addressed differently in the ERMYN. For each radionuclide, the BDCF contains three factors. The first factor includes the ingestion dose (from volcanic ash deposited on cultivated land that contaminates foodstuffs) and external exposure (which does not depend on dust levels or ash thickness). The second factor includes the increased inhalation dose from the resuspension of volcanic ash from noncultivated lands after an eruption, which is a function of time and ash thickness. This factor decreases with time after the eruption until it becomes zero. The third factor is similar to the second, except that it remains constant over time, and represents the inhalation dose after airborne particle concentrations return to preeruption levels.

The deposition and subsequent redistribution of volcanic ash constitute a sequence of events that are relatively limited in duration. Therefore, long-term radionuclide accumulation in surface soils would not occur, and radionuclide concentrations in the surface soil would only vary with the amount of deposited and redistributed volcanic ash. Changes in radionuclide concentrations in volcanic ash and soil mixture in the biosphere due to radionuclide decay, ash redistribution, and wind erosion are not considered explicitly in the ERMYN. Instead, they are considered in the calculation of the source term (i.e., activity per unit area for soil) in the TSPA-LA model used with the BDCFs to calculate dose to the receptor. 


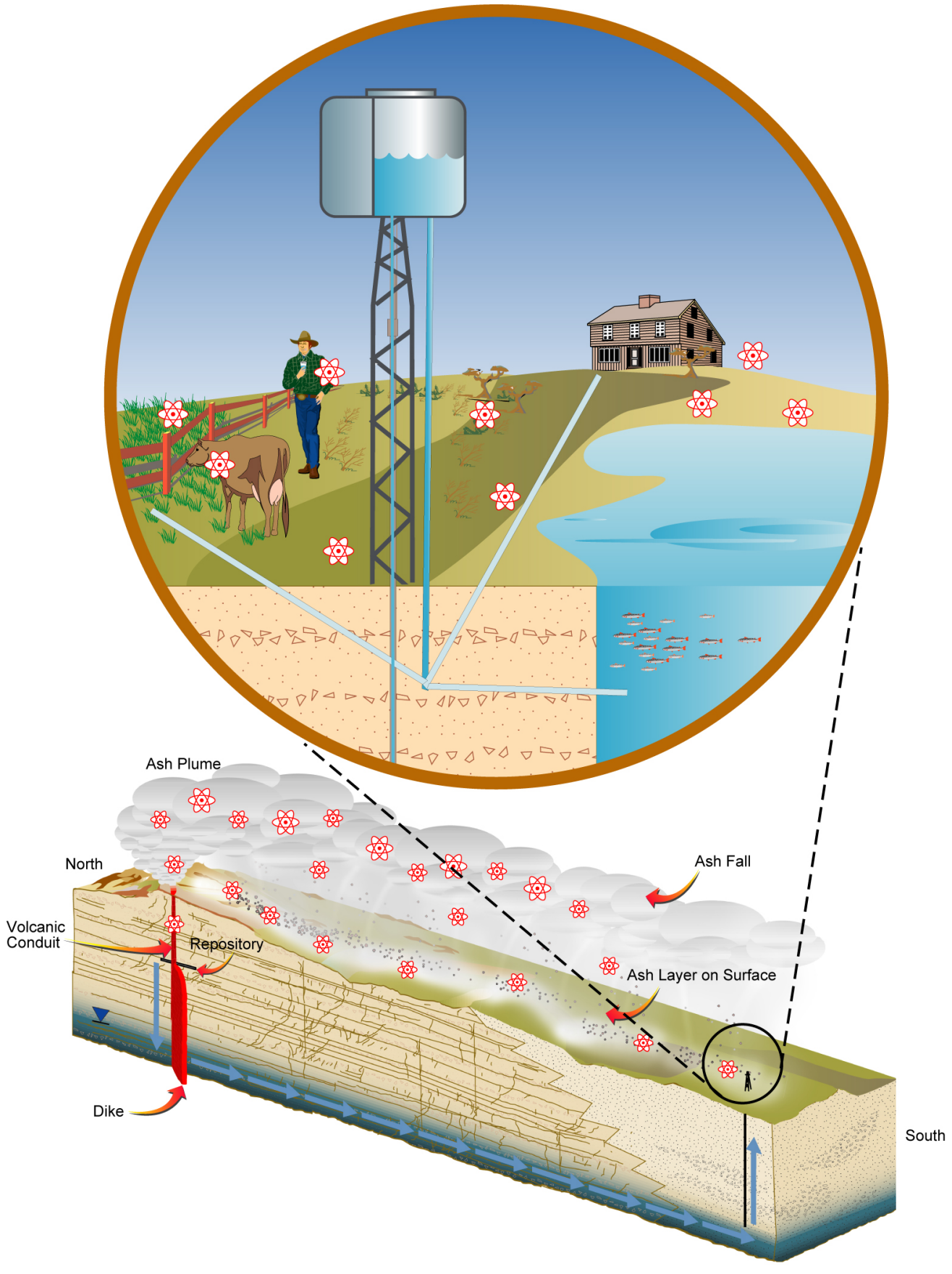

00284CD_Biosphere Volcanic Ash Radionuclides Rev A.ai

Figure 6.3-3. Representation of a Volcanic Eruption Intersecting the Repository

Similar to the groundwater scenario, human exposure for the volcanic ash scenario (Table 6.3-3) arises from the contamination of environmental media. These environmental media and exposure modes are identified from the biosphere FEPs (Section 6.2). Only four media (soil, air, plants, and animals) are considered to be contaminated due to ash deposition. Groundwater is not contaminated in this scenario, and, therefore, fish are considered to be uncontaminated. Even if contaminated ash deposits on the surface of the fish ponds, the activity is not likely to be 
available for uptake by the fish as readily as if it were dissolved in the groundwater. In addition, inhalation is the dominant pathway under this scenario (Section 6.10), and the fish contribution is likely to be unimportant. The exposure pathways in the ERMYN for the volcanic ash scenario are typical of an area affected by a volcanic eruption, and the biosphere characteristics are consistent with arid and semi-arid conditions.

In comparison to the groundwater scenario, fewer exposure pathways are considered in the volcanic ash scenario because processes and media involving radionuclides in water were only included in the groundwater exposure scenario. Three pathways are eliminated (ingestion of drinking water, ingestion of locally produced fish (Appendix E), and inhalation of indoor aerosols generated by an evaporative cooler). The pathways associated with ${ }^{14} \mathrm{C}$ gas are not considered because ${ }^{14} \mathrm{C}$ is not defined to be a significant contributor to exposure (i.e., it is not a primary radionuclide) in this scenario (Table 6.1-1). For the pathways in both scenarios, the calculation methods are similar.

Table 6.3-3. Exposure Pathways for the Volcanic Ash Scenario

\begin{tabular}{|c|c|c|c|}
\hline $\begin{array}{c}\text { Environmental } \\
\text { Medium }\end{array}$ & $\begin{array}{c}\text { Exposure } \\
\text { Mode }\end{array}$ & Exposure Pathways & Examples of Typical Activities \\
\hline Soil & Ingestion & Inadvertent soil ingestion. & $\begin{array}{l}\text { Recreational activities, occupational } \\
\text { activities, gardening, consumption of } \\
\text { fresh fruit and vegetables with attached } \\
\text { soil. }\end{array}$ \\
\hline Soil & External & External radiation exposure. & Activities on or near contaminated soils. \\
\hline Air & Inhalation & $\begin{array}{l}\text { Breathing of airborne particulates; } \\
\text { breathing of gases }\left({ }^{222} \mathrm{Rn} \text { and }\right. \\
\text { progeny). }\end{array}$ & $\begin{array}{l}\text { Outdoor activities, including soil- } \\
\text { disturbing activities related to work and } \\
\text { recreation. Domestic activities, } \\
\text { including sleeping. }\end{array}$ \\
\hline Plants & Ingestion & $\begin{array}{l}\text { Consumption of locally produced crops, } \\
\text { including leafy vegetables, other } \\
\text { vegetables, fruit, and grain. }\end{array}$ & Eating contaminated crop foodstuffs. \\
\hline Animals & Ingestion & $\begin{array}{l}\text { Consumption of locally produced } \\
\text { animal products, including meat, } \\
\text { poultry, milk, and eggs. }\end{array}$ & $\begin{array}{l}\text { Eating and drinking contaminated } \\
\text { animal product foodstuffs. }\end{array}$ \\
\hline
\end{tabular}

\subsubsection{Identification of Biosphere Model Components}

Based on Table 6.3-3, six biosphere components are considered in the conceptual model for the volcanic ash scenario:

- Volcanic ash-deposited on the surface of the soil (initial source of contamination)

- Soil-cultivated land (limited to the tilling depth), and uncultivated land (limited to the critical thickness of soil that could be readily resuspended)

- Atmosphere-including outdoor and indoor air

- Plants-crops contaminated by ash or foliar uptake from ash for use by human and farm animals; irrigated with uncontaminated water 
- Animals-animal products for human consumption; animals raised using ash-contaminated local fodder and uncontaminated water

- Human Receptor-exposed through external exposure, inhalation, and ingestion of contaminated environmental media.

Although contaminated volcanic ash is a biosphere component, it is considered to be mixed with surface soil after initial deposition, producing a mixture of contaminated ash and soil. The atmospheric transport of contaminated volcanic ash, followed by deposition and redistribution, is modeled in the TSPA-LA. However, the ERMYN considers airborne activity resulting from the resuspension of contaminated ash (or the ash-soil mixture) and gaseous emission of relevant radionuclides from ash (or the ash-soil mixture).

\subsubsection{Radionuclide Transfer Interaction Matrix}

The radionuclide transfer interaction matrix (Table 6.3-4) for the volcanic ash scenario is constructed based on the identified biosphere components, radionuclide transfer between the components, and the included FEPs (Section 6.2). An explanation of the interaction matrix concept and notation is presented in Section 6.3.1.3.

The first diagonal element is the radionuclide source, volcanic ash. Because groundwater is considered to be uncontaminated, the element for fish is not considered in the matrix (see Appendix E for a discussion of this exclusion), and there are no intersections of Column 6 with any row in the matrix (Column 6 is retained in the matrix to maintain consistency with Table 6.3-2). All major exposure pathways are considered, including ingestion of contaminated crops and animal products, inhalation of a contaminated ash-soil mixture, and external exposure from contaminated ash on the ground.

\subsubsection{Conceptual Model Approximations}

As indicated below, of the 11 approximations listed in Section 6.3.1.4 that are applicable to the conceptual model for the groundwater exposure scenario, five are also applicable to the volcanic ash exposure scenario. Five additional approximations apply exclusively to the conceptual model for the volcanic ash scenario.

\section{Approximation 1 - Groundwater Source}

This approximation does not apply to the volcanic ash scenario.

\section{Approximation 2 - Consideration of Short-Lived Decay Products}

Statement-See Section 6.3.1.4.

Rationale-See Section 6.3.1.4.

Applicability-This approximation is applied to the volcanic ash scenario in the same way as it is applied to the groundwater scenario. 
Table 6.3-4. Radionuclide Transfer Interaction Matrix for the Volcanic Ash Scenario

\begin{tabular}{|c|c|c|c|c|c|c|c|}
\hline$(i, j)$ & 1 & 2 & 3 & 4 & 5 & 6 & 7 \\
\hline 1 & $\begin{array}{c}\text { SOURCE } \\
\text { (volcanic ash) }\end{array}$ & ashfall & - & - & - & - & - \\
\hline 2 & - & $\begin{array}{l}\text { SURFACE } \\
\text { SOIL } \\
\text { (ash or ash- } \\
\text { soil mixture) }\end{array}$ & $\begin{array}{c}\text { particle } \\
\text { resuspension, } \\
\text { gas release }\end{array}$ & root uptake & soil ingestion & - & $\begin{array}{l}\text { soil ingestion, } \\
\text { ground } \\
\text { exposure }\end{array}$ \\
\hline 3 & - & $\begin{array}{c}\text { particle } \\
\text { deposition }\end{array}$ & AIR & $\begin{array}{c}\text { particle } \\
\text { deposition }\end{array}$ & - & - & $\begin{array}{l}\text { inhalation of } \\
\text { particulates } \\
\text { and gas }\end{array}$ \\
\hline 4 & - & $\begin{array}{l}\text { weathering, } \\
\text { harvest } \\
\text { removal }\end{array}$ & - & $\begin{array}{l}\text { PLANTS } \\
\text { (crops) }\end{array}$ & feed ingestion & - & crop ingestion \\
\hline 5 & - & fertilization & - & - & $\begin{array}{l}\text { ANIMALS } \\
\text { (animal } \\
\text { products) }\end{array}$ & - & $\begin{array}{l}\text { animal product } \\
\text { ingestion }\end{array}$ \\
\hline 6 & - & - & - & - & - & FISH & - \\
\hline 7 & - & - & - & - & - & - & $\begin{array}{l}\text { HUMAN } \\
\text { (receptor) }\end{array}$ \\
\hline
\end{tabular}

\section{Approximation 3 - Long-Term Irrigation, Land Use, and Crop Rotation}

This approximation does not apply to the volcanic ash scenario.

Approximation 4 - Crop Harvest Removal and the Use of Contaminated Manure for $\underline{\text { Fertilizer }}$

Statement-See Section 6.3.1.4.

Rationale-See Section 6.3.1.4.

Applicability-This approximation is applied to the volcanic ash scenario in the same way as it is applied to the groundwater scenario.

\section{Approximation 5 - Radionuclide Buildup in Surface Soil}

This approximation does not apply to the volcanic ash scenario. 


\section{Approximation 6 - Crop Weathering Loss and Surface Soil Gain}

This approximation does not apply to the volcanic ash scenario.

\section{Approximation 7 - Crop Roots in Surface Soil}

Statement-See Section 6.3.1.4.

Rationale-See Section 6.3.1.4.

Applicability-This approximation is applied to the volcanic ash scenario in the same way as it is applied to the groundwater scenario.

\section{Approximation 8 - Animal Feed}

Statement-See Section 6.3.1.4.

Rationale-See Section 6.3.1.4.

Applicability-This approximation is applied to the volcanic ash scenario in the same way as it is applied to the groundwater scenario.

\section{Approximation 9 - Animal Product Type}

Statement-See Section 6.3.1.4.

Rationale-See Section 6.3.1.4.

Applicability-This approximation is applied to the volcanic ash scenario in the same way as it is applied to the groundwater scenario.

\section{Approximation 10 - Dose Coefficients for Exposure to Contaminated Soil}

This approximation does not apply to the volcanic ash scenario.

\section{Approximation 11 - Evaporative Cooler Use and Exposure Time}

This approximation does not apply to the volcanic ash scenario.

\section{Approximation 12 - Volcanic Ash Source}

Statement-On cultivated lands, ash that falls on the ground or is fluvially transported to the area of interest is tilled into the surface soil, but the soil density does not change when mixed with ash. On noncultivated lands, ash that falls on the ground may or may not mix with the surface soil, depending on the thickness of the ash, and the ash density does not change when mixed with soil.

Rationale-Ash deposited at the location of the receptor is expected to be thin. Under normal, variable wind conditions, ash depths predicted in the TSPA-LA at a location about $18 \mathrm{~km}$ south 
of Yucca Mountain, range from zero to about $25 \mathrm{~cm}$. About 86 percent of predicted depths from 1,000 computer simulations were less than $0.1 \mathrm{~mm}$, about 93 percent were less than $1 \mathrm{~mm}$, and 97 percent were less than $10 \mathrm{~mm}$ (DTN: MO0410MWDTLSCM.000 [DIRS 172208]).

On cultivated land, plowing and irrigation would uniformly mix the ash and surface soil, and the small amount of ash would not change the soil density. Most land in Amargosa Valley is not plowed or irrigated (BSC 2004 [DIRS 169458], Section 6), and ash falling on noncultivated land would not be mixed with the surface soil by agricultural practices, although some downward migration of contamination through the soil profile will occur following initial deposition. A thin layer (i.e., a critical thickness) of ash could mix with surface soil. This critical thickness is defined as the layer of soil from which soil particles are readily resuspended into the air. If the thickness of the ash layer is less than the critical thickness, it is assumed that the ash and soil can become resuspended due to wind and other disturbing forces and mix in the process. It is assumed that the ash and soil mixing layer would be no more than the critical thickness, and that the ash density remains unchanged when soil is added. If the thickness of the ash layer on noncultivated land is greater than the critical thickness, no mixing or dilution of the ash occurs because all resuspended particles would be from the top layer of ash. These approximations are based on current Amargosa Valley land-use patterns, the arid or semiarid climate, and that they are reasonable; therefore, this approximation requires no further confirmation.

Applicability-This approximation is applied to the surface soil submodel for the volcanic ash scenario in Sections 6.3.2.6, 6.5.1.1, and 7.3.1.2.

\section{Approximation 13-Ash Resuspension}

Statement-Resuspended volcanic ash that deposits on plants is from cultivated land, while ash that contributes to the human inhalation dose is from noncultivated lands.

Rationale-After a volcanic eruption, much of the reference biosphere would be contaminated with ash, and ash available for resuspension would come from two sources: cultivated and uncultivated lands. Most of the resuspended particles that deposit on crops would come from the cultivated lands where the crops are grown; therefore cultivated lands are the appropriate source for these particles. However, for the human inhalation dose, uncultivated lands are the appropriate source because cultivated lands cover only a small fraction of Amargosa Valley (BSC 2004 [DIRS 169458], Section 6.1). This approximation does not underestimate the human inhalation dose because human inhalation is the major pathway in the volcanic ash scenario, and contaminated particles from noncultivated lands have a higher radionuclide concentration than those from cultivated land (Tables 6.10-5 and 6.10-6). This approximation may underestimate radionuclide concentrations in the crops; but in the volcanic ash scenario, the ingestion pathway contributes less to the all-pathway dose than the inhalation pathway. This approximation does not underestimate the final dose; it simplifies calculating radionuclide concentrations in the air, and it requires no further confirmation.

Applicability - This approximation is applied to the air submodel for the volcanic ash scenario in Sections 6.3.2.6, 6.5.1.2, and 6.5.2.1. 


\section{Approximation 14 - Time Dependent Mass Loading}

Statement-After a volcanic eruption, mass loading (i.e., the concentration of resuspended particulates in the air) decreases with time and eventually returns to levels similar to preeruption conditions.

Rationale-A time-dependent mass loading function is used to avoid overestimating the expected inhalation dose after a volcanic eruption that otherwise would be calculated using a constant and high mass loading value. This approximation is reasonable because levels of resuspended particulates after volcanic eruptions decrease with time (BSC 2004 [DIRS 169458], Section 6.3). The time-dependent function is developed in the ERMYN, but it is implemented in the TSPA-LA model. The time-dependent function is documented in BSC (2004 [DIRS 169458], Section 6.3). This approximation requires no further confirmation because it is based on observed changes in mass loading after volcanic eruptions.

Applicability - This approximation is applied to the air submodel for the volcanic ash scenario in Sections 6.3.2.6 and 6.5.2.1.

\section{Approximation 15 - Radon Gas Released From Volcanic Ash}

Statement-All ${ }^{222} \mathrm{Rn}$ from ${ }^{226} \mathrm{Ra}$-contaminated volcanic ash is released into the air where it mixes and is available for inhalation. In the ash/soil, no credit is taken for radon transport (i.e., the subsequent decay products are returned to their source).

Rationale - The thickness of volcanic ash deposited on the ground is anticipated to be relatively thin (Approximation 12). Following the initial deposition, mixing of the ash and soil will occur through atmospheric and mechanical processes affecting the soil surface, and through the downward migration of the contaminant into the soil profile. Because of this mixing, and because the ash thickness is not known a priori in the biosphere modeling, predicting the radium concentration profile in the soil is not a straight-forward process, and estimating the amount of radon released from such a source would require a radon diffusion submodel, for which data are not available. In the ERMYN, a simplified method is used in which all radon produced from the contaminated ash is considered to be released to the atmosphere. The relationship between the concentration of ${ }^{226} \mathrm{Ra}$ in the surface soil $\left(\mathrm{Bq} / \mathrm{m}^{2}\right)$, radon flux density from the soil $\left(\mathrm{Bq} /\left(\mathrm{m}^{2} \mathrm{~s}\right)\right)$, and the concentration of ${ }^{222} \mathrm{Rn}$ in the air $\left(\mathrm{Bq} / \mathrm{m}^{3}\right)$ is derived from available data. This approximation is conservative, as only a fraction of the ${ }^{222} \mathrm{Rn}$ would be exhaled from the soil and released into the air, and, therefore, it requires no further confirmation.

Applicability - This approximation is applied to the air submodel for the volcanic ash scenario in Sections 6.3.2.6, 6.5.2, and 7.4.3.1.

\section{Approximation 16 - External Exposure From the Ground Surface}

Statement-Radionuclides in volcanic ash layer are concentrated on the top surface of the ash deposit, and dose conversion factors for surficial contaminants are appropriate.

Rationale-The ash layer is expected to be relatively thin (Approximation 12), and it is reasonable to assume that only the ground surface would be contaminated. Therefore, the 
external exposure submodel from the groundwater scenario, which considers long-term irrigation and soil contaminated to an infinite depth, does not apply to the volcanic ash scenario. By considering that radionuclides in the ash are on the surface of the ground, external exposure can be calculated using surface contamination methods rather than volumetric methods. This approximation is reasonable because there is considerably more noncultivated land than cultivated land in the Amargosa Valley (BSC 2004 [DIRS 169458], Section 6), and the mixing of volcanic ash on noncultivated land would be limited. This approach is conservative because it ignores radiation attenuation in the soil and ash. Therefore, it required no further confirmation. The approximation simplifies calculations and eliminates the dependence of external exposure on ash thickness and location.

Applicability-This approximation is applied to the external exposure submodel for the volcanic ash scenario in Sections 6.3.2.6 and 6.5.5.1.

\subsubsection{Submodels for the Volcanic Ash Scenario}

To understand radionuclide transport among biosphere components (Figure 6.3-4), the biosphere conceptual model is divided into the six parts consistent with the components in the interaction matrix (Table 6.3-4). Considering the human receptor component to be composed of three parts, there are eight parts considered. Relationships among these parts or submodels (Figure 6.3-4) show important mechanisms of radionuclide migration from the source through the media to the human receptor. The BDCF box (Figure 6.3-4) is not considered a submodel; rather, it represents the results of the ERMYN.

\subsubsection{Description of Conceptual Model for the Volcanic Ash Scenario}

The biosphere conceptual model for the volcanic ash scenario is based on the radionuclide transfer interaction matrix (Table 6.3-4). Because many radionuclide transfer mechanisms are the same in the groundwater and volcanic ash scenarios, the submodels are similar. The following description of the volcanic ash conceptual model focuses on the differences between the two scenarios.

Volcanic Ash Source-Volcanic ash deposited on the ground is the source of radionuclides for the volcanic ash scenario. In the biosphere model, activity concentrations of radionuclides in the deposited ash are defined as a constant in units of $\mathrm{Bq} / \mathrm{m}^{2}$. The source, ash, is not considered a submodel because it is an input to the biosphere model.

Surface Soil Submodel-The surface soil submodel converts the volcanic ash source from areal radionuclide concentrations $\left(\mathrm{Bq} / \mathrm{m}^{2}\right)$ to radionuclide concentrations in a mass of ash (or the ash-soil mixture; $\mathrm{Bq} / \mathrm{kg}$ ) on the ground. Because of different soil-mixing mechanisms on cultivated and noncultivated lands, and because of the different contributions to human radiation exposure, the consequences of volcanic ash deposition are calculated differently for these two areas. When deposited on cultivated land (Table 6.3-4, Element [1,2]), volcanic ash would be mixed with surface soil due to plowing and irrigation (Element [2,1]). When deposited on noncultivated land, ash would be mixed with native soil and windblown dust if the layer is thin, or it would remain undiluted if the layer is thick (Approximation 12). 


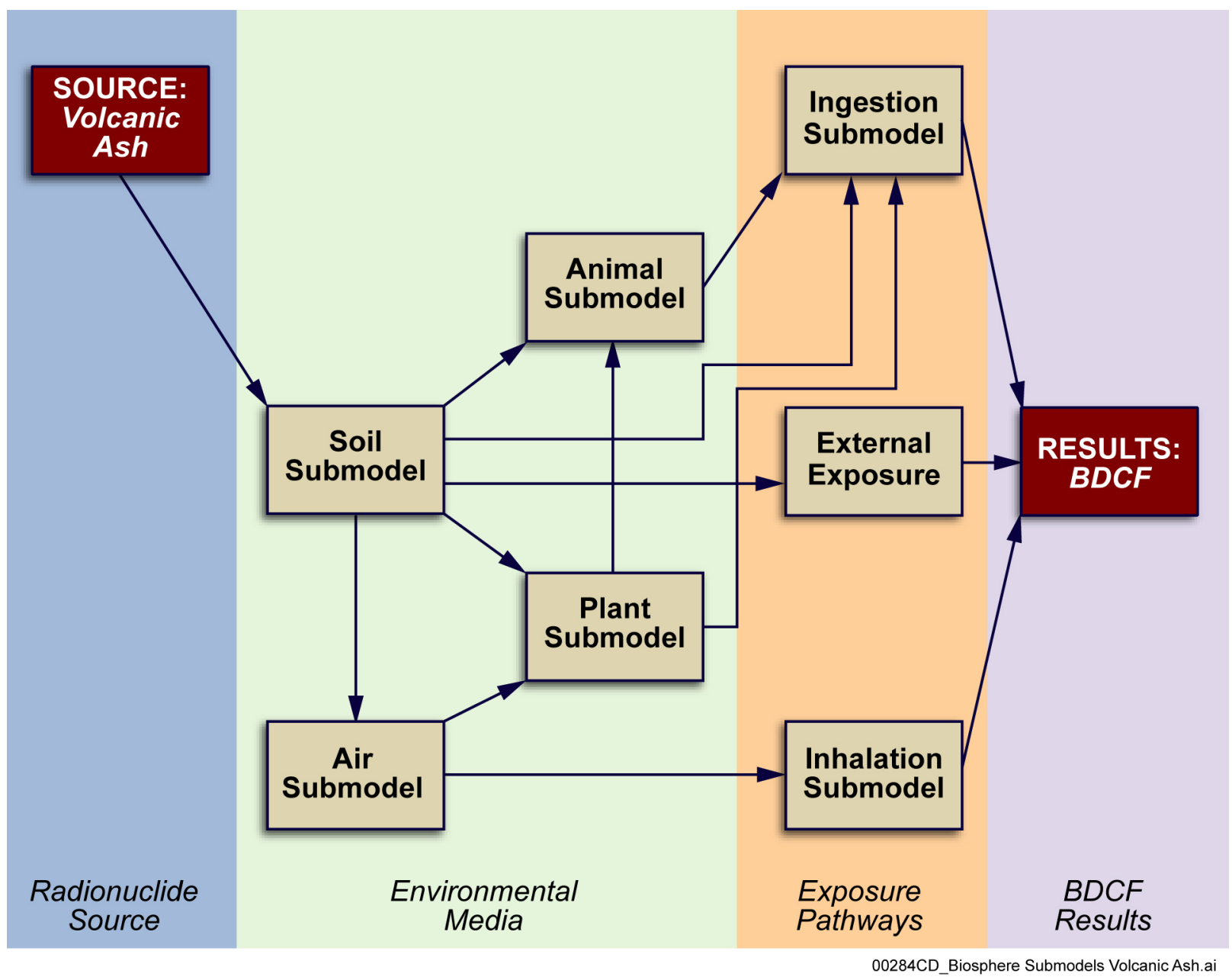

Figure 6.3-4. Relationship Between the Biosphere Submodels for the Volcanic Ash Scenario

On cultivated lands, ash would mix with surface soils and lose the characteristics of the original ash (e.g., ash density) because there would be a small amount of ash compared with a large amount of surface soil. In the submodel, the ash-soil mixture is treated as regular soil except that it is contaminated. The contaminated soil is the source of contamination for crops and animal products, and it is the source for inadvertent soil ingestion because soil ingestion by humans likely would be from consuming crops, particularly those that would be unwashed.

On noncultivated lands, ash would not readily mix with surface soil because the land would not be tilled and irrigated, and because the low amount of precipitation would limit downward migration of radionuclide through the soil profile. If the ash layer is thin, resuspension and subsequent redeposition could mix the volcanic ash and surface soil particles. To characterize this type of mixing, a critical thickness (a hypothetical layer of soil from which soil particles would be more readily resuspended) must be considered. If the deposited ash thickness is thinner than the critical thickness, the ash would mix with the top layer of host soil, but the ash characteristics are assumed to remain unchanged even when mixing with the host soil particles occurs (Approximation 12). If the deposited layer of ash is greater than the critical thickness, this type of mixing will not occur, as all resuspended particles would originate, at least initially, 
from the top layer of original ash. Therefore, to convert the volcanic ash source term from areal radionuclide concentration $\left(\mathrm{Bq} / \mathrm{m}^{2}\right)$ to mass radionuclide concentration in ash (or its soil mixture; $\mathrm{Bq} / \mathrm{kg}$ ), the thickness of the deposited ash is required. The converted radionuclide concentrations of ash (or the ash-soil mixture) are used to calculate radionuclide concentrations in the air. This is the source of radionuclide resuspension used for human inhalation.

As discussed for the groundwater scenario, some radionuclide transfer mechanisms, including harvest removal, fertilization, and crop weathering, are implicitly considered in the biosphere surface soil submodel. Other removal mechanisms (including radionuclide decay, and wind and water erosion) are not considered in the biosphere model but are incorporated in the TSPA-LA model, where changes to the source term are tracked. Unlike the groundwater scenario, where soil contamination could be deep, volcanic ash deposition would be relatively shallow. Thus, the source for any external exposure is considered to be contained in a thin layer on the top of the ground (Approximation 16).

There are several outputs from this submodel. The activity concentration of radionuclides on cultivated lands is used to calculate the contamination of crops and animal products, and it is used to calculate the activity intake from inadvertent soil ingestion. The activity concentrations on noncultivated lands are used to calculate the contamination in the air, which contributes to human inhalation. The surface concentration of radionuclides is used to calculate external exposure. The relationships among these submodels are shown in Figure 6.3-4.

Air Submodel-The purpose of the air submodel is to calculate radionuclide concentrations in the air from the resuspension of contaminated ash-soil particles and from the gaseous release of ${ }^{222} \mathrm{Rn}$ from deposited volcanic ash (Table 6.3-4, Element [2,3]). Because there would be relatively little cultivated land compared with the total amount of the ash-contaminated land, it is assumed that human inhalation dose comes only from noncultivated land (Approximation 13). Soil resuspended from cultivated land is the source of contaminated soil particles for deposition on plants.

An elevated concentration of airborne particulates (mass loading) is expected after a volcanic eruption (BSC 2004 [DIRS 169458], Sections 6.2 and 6.3), but mass loading would decrease with time (Approximation 14) because the ash eventually would settle onto the ground and mix with surface soil. Some human activities cause elevated mass loading relative to average levels (BSC 2004 [DIRS 169458], Sections 6.1.1 and 6.2.1). Therefore, mass loading is related to human activity, similar to that for the groundwater scenario. Radon gas released from deposited ash is considered, but the evaluation of ${ }^{222} \mathrm{Rn}$ concentrations in the air is simplified by using a radon release factor from ${ }^{226} \mathrm{Ra}$ contaminated ground (Approximation 15).

The output of the submodel, the activity concentrations of radionuclides in the air, is used for calculating the radionuclide intake by inhalation of contaminated air and the direct deposition of ash on plant surfaces (Figure 6.3-4).

Plant Submodel-The purpose of the plant submodel is to calculate radionuclide concentrations in various crops that would be consumed by humans and farm animals. Crop contamination is assumed to come from cultivated land where deposited volcanic ash and surface soil are uniformly mixed (Approximation 12). Two mechanisms cause the contamination of crops: plant 
root uptake due to contaminated surface soil (Table 6.3-4, Element $[2,4]$ ) and deposition of resuspended particulates on plants (Element $[3,4]$ ).

Except for the absence of direct deposition of irrigation water on crop leaves, radionuclide transfer mechanisms from soil to plants and from particulates to plants are the same as those used in the groundwater scenario. The crop types also are the same in both scenarios.

Output from the submodel, activity concentrations of radionuclides in crops, is used as input to calculate radionuclide intake by ingestion of contaminated crops and to calculate the contamination of animal products (Figure 6.3-4 and Table 6.3-4).

Animal Submodel-The purpose of the animal submodel is to calculate radionuclide concentrations in animal products using two radionuclide transfer mechanisms for animal products: ingestion of contaminated soil (Table 6.3-4, Element [2,5]) and feed (Element [4,5]).

Except for removing the transfer mechanism for ingesting contaminated water, other submodel treatments (including animal product types and equilibrium conditions) are the same as those in the groundwater scenario. The output of the submodel, the activity concentration of radionuclides in animal products, is used to calculate radionuclide intake by ingestion of contaminated animal products (Figure 6.3-4).

External Exposure Submodel-The purpose of the external exposure submodel is to calculate human radiation exposure from contaminated volcanic ash deposited on the surface of the ground (Table 6.3-4, Element [2,7]). The annual EDE is calculated for this pathway. As discussed for the surface soil submodel, the ash deposit is anticipated to be relatively shallow. It is assumed that all radionuclides will stay on the ground surface (Approximation 16), and external exposure does not depend on the thickness of the ash. As in the groundwater scenario, air submersion and other external exposures from contaminated media are not considered in the submodel because they contribute relatively little to the overall external dose to the human receptor, as shown in Section 7.4.8.

The evaluation method for doses from external exposure to radionuclides on the surface of the ground are similar to the external exposure submodel used in the groundwater scenario, except that the surface source and the dose coefficients are different. The output of the submodel, annual doses from external exposure, contribute to the all-pathway dose, which is then used to calculate BDCFs.

Inhalation Submodel-The purpose of the inhalation submodel is to calculate human radiation doses due to the inhalation of radionuclides. The model includes inhalation of resuspended volcanic ash and short-lived decay products of ${ }^{222} \mathrm{Rn}$. The 50-year CEDE resulting from annual intake of radionuclides by inhalation is calculated for this pathway. Two types of contaminated sources are considered to cause human inhalation dose: contaminated resuspended particulates and radon gas (Table 6.3-4, Element [3,7]).

To account for variation in ash thickness (discussed in the soil submodel) and changes in mass loading over time (discussed for the air submodel), the inhalation dose depends on the thickness of the ash and changes over time as dust levels decrease. These functions of time and ash thickness is carried into the TSPA-LA model. Similar to the inhalation submodel for the 
groundwater scenario, the inhalation dose depends on airborne concentrations, breathing rates, and exposure time. Breathing rates and exposure times are related to human activities, and exposure time varies among population groups (Section 6.4.7.1). These parameters are linked to human activities and population groups to incorporate uncertainty from those parameters into the submodel.

The output of the inhalation submodel, annual inhalation dose, contributes to the all-pathway dose, which is used to calculate BDCFs (Figure 6.3-4).

Ingestion Submodel-The purpose of the ingestion submodel is to calculate the radiation dose from ingestion of radionuclides. The 50-year CEDE resulting from annual intake of radionuclides by ingestion is calculated for this pathway. The ingestion submodel includes eight contaminated foodstuffs: four types of plants (leafy vegetables, other vegetables, fruit, and grain) (Table 6.3-4, Element [4,7]) and four types of animal products (meat, poultry, milk, and eggs) (Element [5,7]), plus inadvertent soil ingestion (Element [2,7]). Inputs to this submodel are the radionuclide concentrations in foodstuffs and the soil.

These media concentrations, when combined with the corresponding consumption rates and dose conversion factors, are used to produce ingestion doses. The output of the ingestion submodel, annual ingestion dose, contributes to the all-pathway dose, which is used to calculate BDCFs (Figure 6.3-4).

BDCFs and ERMYN Results - The all-pathway dose is the sum of the radionuclide-specific annual doses from the external, inhalation, and ingestion exposure pathways. The all-pathway dose is expressed in terms of TEDE. The BDCFs (Sv/year) $/\left(\mathrm{Bq} / \mathrm{m}^{2}\right)$ are numerically equal to the all-pathway dose from a unit activity concentration in the deposited volcanic ash. The calculation of radionuclide concentrations in volcanic ash is carried out in the TSPA-LA model. The total dose calculated in the TSPA-LA model is the sum of the products of the radionuclide-specific BDCFs, considering changes in mass loading with time and the deposited ash thickness, and the time-dependent activity concentrations of radionuclides in the volcanic ash for all of the radionuclides in the TSPA-LA model.

\subsubsection{Alternative Conceptual Models}

Performance assessment for a repository at Yucca Mountain must consider ACMs of features and processes that are consistent with available data and current scientific understanding, and they must evaluate the effects that ACMs have on the performance of the geologic repository (10 CFR 63.114(c) [DIRS 156605]). From the guidelines for the treatment of ACMs in the TSPA-LA (BSC 2002 [DIRS 158794], Section 2), a conceptual model can only be alternative if it:

- Differs in important ways from the selected conceptual model

- Is consistent with available data and current scientific understanding

- Is reasonable, which has been interpreted as implying that there is some precedent for the alternative, such as prior use by other analysts, and that there is a physical basis for the alternative. 
Based on this definition of an ACM, there are no alternative groundwater or volcanic ash conceptual models applicable to the entire reference biosphere. However, there are ACMs for submodels and components. This section identifies ACMs from published biosphere models that differ from the corresponding submodel or parts of submodel in the ERMYN (Table 6.3-5). These ACMs are screened and evaluated qualitatively and quantitatively, and mathematical representations of these ACMs are presented and compared with the calculations used in the validation of ERMYN (Section 7.3). Finally, numerical comparisons between the ACMs and the ERMYN are performed, and justifications are provided for why ACMs are not selected (Section 7.4).

The ACMs come from five published biosphere models with corresponding submodels or parts of submodels that are compared with the ERMYN (Section 7.1.2). These published biosphere models are GENII/GENII-S (Napier et al. 1988 [DIRS 157927]; Leigh et al. 1993 [DIRS 100464]), BIOMASS ERB2A (BIOMASS 2000 [DIRS 154522]), RESRAD (Yu et al. 2001 [DIRS 159465]), EPRI-YM (Smith et al. 1996 [DIRS 101085]), and NCRP-129 (NCRP 1999 [DIRS 155894]). To be considered as an ACM, submodels or parts of submodels must be conceptually or mathematically different from those in the ERMYN. Mathematical simplifications or different treatments, such as numerical or analytical methods, are not considered to be ACMs. Brief descriptions of the seven identified ACMs are presented below and summarized in Table 6.3-5.

ACM 1, Radon Release from Soil (Air Submodel)-The selected conceptual model for radon is based on a radon release factor from radium contaminated soil. An ACM relies on modeling radon transport in the soil and the atmosphere (Yu et al. 2001 [DIRS 159465]). A numerical comparison between the selected model and the ACM shows that the ${ }^{222} \mathrm{Rn}$ concentrations are comparable (Section 7.4.3.1). Because the ACM required more input data, it is not selected.

ACM 2, Evaporative Cooler (Air Submodel)-The selected conceptual model for evaporative coolers is based on evenly evaporating contaminated water into the airflow of an evaporative cooler. An ACM calculates radionuclide concentrations based on evaporation and differences in humidity. An evaluation of these two methods shows that they produce equivalent results (Section 7.4.3.2).

ACM 3, Direct Deposition of Irrigated Water (Plant Submodel)-The ERMYN considers radionuclides in irrigation water to be directly translocated into edible plant parts with accumulation and weathering occurring during the growing period. This conceptual model is also used in published biosphere models (e.g., GENII-S and RESRAD). An ACM presented in BIOMASS ERB2A considers two processes, movement of deposited radionuclides from external plant surfaces into the plant tissues, and movement of radionuclides from plant tissues into edible parts of the crop. This ACM applies weathering to contaminants that remain on external plant surfaces, and food-processing losses are considered. This ACM may be conceptually more realistic than simulating radionuclide transfers directly from water to edible portions of the plants, but it is not commonly used because the input data typically are not available. The two models are evaluated using the same input values, or using default data used in BIOMASS ERB2A (Sections 7.4.4.1). This ACM and the approach used in the ERMYN produce comparable results for reasonable input values. 
ACM 4, Direct Deposition of Airborne Particulates (Plant Submodel)-In the ERMYN, resuspended soil deposited on crop leaves is treated in the same manner as intercepted irrigation water. Other published biosphere models take a different approach. An ACM is based on a contamination factor for the external contamination of crops, which is similar to a soil-to-plant transfer factor. Differences between the ERMYN and the ACM are evaluated (Section 7.4.4.3) using the same input values when the parameters are comparable or using default data from the published ACM. The evaluation shows that the ERMYN and the ACM approaches produce comparable results for reasonable input values.

ACM 5, Animal Product Contamination (Animal Submodel)—The ERMYN considers animal contamination resulting from the consumption of contaminated water, soil, and feed. The GENII-S model only includes the consumption of water and feed, and the BIOMASS ERB2A model includes an additional pathway: inhalation of contaminated air by animals. These animal transport pathways are compared to determine their relative importance (Section 7.4.5). Soil ingestion is an important contributor to the total activity concentration in meat and, therefore, is included in the ERMYN. The inhalation of contaminated dust contributes little to concentrations in meat, and, therefore, it is not included.

ACM 6, ${ }^{14} \mathrm{C}$ Special Submodel $\left({ }^{14} \mathrm{C}\right.$ Submodel)-The ERMYN ${ }^{14} \mathrm{C}$ conceptual submodel is based on experimental results (Yu et al. 2001 [DIRS 159465], Section L) and concerns the release of ${ }^{14} \mathrm{CO}_{2}$ gas from soil. The ERMYN considers external exposure, inhalation of ${ }^{14} \mathrm{CO}_{2}$ gas, and soil ingestion pathways, and includes the uptake of ${ }^{14} \mathrm{CO}_{2}$ gas into plants during photosynthesis. An ACM in GENII-S only considers the uptake of ${ }^{14} \mathrm{C}$ by roots. The two submodels are compared to evaluate any differences (Section 7.4.7). BIOMASS ERB2A presents another ${ }^{14} \mathrm{C}$ ACM, but it requires input parameters that are not available. The mathematical representations are compared (Section 7.3.6), but numerical comparisons are not conducted because of the lack of appropriate input values. The GENII-S ACM is not used because the ERMYN ${ }^{14} \mathrm{C}$ special submodel is more realistic and results in higher ${ }^{14} \mathrm{C}$ concentrations in plants (Section 7.4.7).

ACM 7, Environment-Specific Inhalation Submodel (Inhalation Submodel)-In the ERMYN, inhalation exposure is considered to be a function of environment and human activity because many model parameters (e.g., mass loading, breathing rate, and exposure time) are related to human activities. Similar models, called microenvironmental models, have been used to assess exposure to particulate matter and other contaminants (Duan 1982 [DIRS 162466]; Klepeis 1999 [DIRS 160094]; Mage 1985 [DIRS 162465]). The previous biosphere model and all of the compared biosphere models use average values for these input parameters (Section 7.3.8). An evaluation (Section 7.4.9) numerically compares the ERMYN method with those in the other models. The approaches produce comparable results for reasonable model input values. The environment-specific approach is used in the ERMYN because uncertainty associated with the input parameters can be considered. 
Table 6.3-5. Alternative Conceptual Models Considered

\begin{tabular}{|c|c|c|}
\hline $\begin{array}{c}\text { Alternative } \\
\text { Conceptual Model }\end{array}$ & Overview & Screening Assessment and Basis \\
\hline $\begin{array}{l}\text { Radon release from } \\
\text { soil (Air Submodel) }\end{array}$ & $\begin{array}{l}\text { This ACM considers radon transport in the soil and } \\
\text { the atmosphere, which requires more input data. } \\
\text { The ERMYN does not include these processes and } \\
\text { uses a simple release factor. }\end{array}$ & $\begin{array}{l}\text { This ACM is screened from the } \\
\text { biosphere model based on an } \\
\text { analysis (Section 7.4.3.1) showing } \\
\text { that the ACM and the ERMYN } \\
\text { produce comparable results. }\end{array}$ \\
\hline $\begin{array}{l}\text { Evaporative cooler } \\
\text { (Air Submodel) }\end{array}$ & $\begin{array}{l}\text { This ACM considers an inhalation dose from } \\
\text { aerosols generated from evaporative coolers and is } \\
\text { based on calculating radionuclide concentrations in } \\
\text { the air due to an increase in humidity. The ERMYN } \\
\text { uses a submodel based on the amount of water } \\
\text { evaporated rather than an increase in humidity. }\end{array}$ & $\begin{array}{l}\text { This ACM is screened from the } \\
\text { biosphere model based on an } \\
\text { analysis (Section 7.4.3.2) showing } \\
\text { that this ACM and the ERMYN } \\
\text { produce comparable results. }\end{array}$ \\
\hline $\begin{array}{l}\text { Direct deposition of } \\
\text { irrigation water } \\
\text { (Plant Submodel) }\end{array}$ & $\begin{array}{l}\text { This ACM considers two processes, one where the } \\
\text { deposited radionuclides move from external plant } \\
\text { surfaces into the plant tissues, and then from plant } \\
\text { tissues into the edible portion of the crop. } \\
\text { Weathering is applied only to contaminants that } \\
\text { remain on external plant surfaces. Food } \\
\text { processing loss is also considered in the ACM. } \\
\text { The ERMYN conceptual model considers the } \\
\text { radionuclides in irrigation water to be directly } \\
\text { translocated to the edible parts of plants with } \\
\text { weathering and accumulation during the growing } \\
\text { period, but without food preparation loss. }\end{array}$ & $\begin{array}{l}\text { This ACM is screened from the } \\
\text { biosphere model based on an } \\
\text { analysis (Section 7.4.4.1) showing } \\
\text { that this ACM and the ERMYN } \\
\text { produce comparable results. }\end{array}$ \\
\hline $\begin{array}{l}\text { Direct deposition of } \\
\text { airborne particulates } \\
\text { (Plant Submodel) }\end{array}$ & $\begin{array}{l}\text { This ACM is based on the crop external } \\
\text { contamination. This contamination factor is very } \\
\text { similar to a soil-to-plant transfer factor. The } \\
\text { ERMYN conceptual model considers the deposited } \\
\text { airborne particles on crop leaves acting the same } \\
\text { way as the intercepted irrigation water. }\end{array}$ & $\begin{array}{l}\text { This ACM is screened from the } \\
\text { biosphere model based on an } \\
\text { analysis (Section 7.4.4.3) showing } \\
\text { that this ACM and the ERMYN } \\
\text { produce comparable results for } \\
\text { reasonable input values. }\end{array}$ \\
\hline $\begin{array}{l}\text { Animal product } \\
\text { contamination } \\
\text { (Animal Submodel) }\end{array}$ & $\begin{array}{l}\text { Two pathways are considered in this ACM: animal } \\
\text { inhalation of contaminated air and animal soil } \\
\text { ingestion. The ERMYN conceptual model excludes } \\
\text { the inhalation of contaminated air, but it includes } \\
\text { animal soil ingestion. }\end{array}$ & $\begin{array}{l}\text { This ACM is screened from the } \\
\text { biosphere model based on an } \\
\text { analysis (Section 7.4.5) showing that } \\
\text { soil ingestion is important but that } \\
\text { inhalation of contaminated air is not. }\end{array}$ \\
\hline $\begin{array}{l}{ }^{14} \mathrm{C} \text { special } \\
\text { submodel } \\
\left({ }^{14} \mathrm{C} \text { Special }\right. \\
\text { Submodel })\end{array}$ & $\begin{array}{l}\text { This ACM considered root uptake only. The } \\
\text { ERMYN includes external exposure, inhalation of } \\
{ }^{14} \mathrm{C} \text { gas, soil ingestion pathways, and }{ }^{14} \mathrm{C} \text { transfer } \\
\text { into plants through photosynthesis. }\end{array}$ & $\begin{array}{l}\text { This } A C M \text { is screened from the } \\
\text { biosphere model based on an } \\
\text { analysis (Section } 7.4 .7 \text { ) showing that } \\
\text { the selected }{ }^{14} \mathrm{C} \text { special submodel } \\
\text { considers more processes of }{ }^{14} \mathrm{C} \\
\text { contamination in plants than this } \\
\text { ACM, which results in a higher }{ }^{14} \mathrm{C} \\
\text { concentration in plants. }\end{array}$ \\
\hline $\begin{array}{l}\text { Environment-specific } \\
\text { inhalation submodel } \\
\text { (Inhalation } \\
\text { Submodel) }\end{array}$ & $\begin{array}{l}\text { This ACM uses average values of input parameters } \\
\text { for inhalation exposure. ERMYN considers } \\
\text { inhalation exposure as a function of the } \\
\text { environment because many model parameters, } \\
\text { such as mass loading, breathing rate, and } \\
\text { exposure time, differ among environments and } \\
\text { activities. }\end{array}$ & $\begin{array}{l}\text { This ACM is screened from the } \\
\text { biosphere model based on an } \\
\text { analysis (Section 7.4.9) showing that } \\
\text { the ACM and the ERMYN produce } \\
\text { comparable results. In addition, it is } \\
\text { easier to address uncertainty in the } \\
\text { input parameters using environment- } \\
\text { specific values. }\end{array}$ \\
\hline
\end{tabular}

$\mathrm{ACM}=$ alternative conceptual model; ERMYN=Environmental Radiation Model for Yucca Mountain, Nevada 


\subsubsection{FEPs Considered in the Biosphere Conceptual Model}

The radionuclide transfer interaction matrixes, Table 6.3-2 for the groundwater scenario and Table 6.3-4 for the volcanic ash scenario, also serve as a tool to map the included FEPs into the biosphere submodels. To document that the conceptual models address all included FEPs, Table 6.3-6 provides a list of the included FEPs, shows where each FEP is mapped in the interaction matrixes (Tables 6.3-2 and 6.3-4), and lists the submodels where each FEP is addressed. The disposition of these FEPs in the biosphere mathematical model, submodels, and associated equations and parameters is discussed in Section 6.7.1.

Table 6.3-6. Mapping of FEPs to Interaction Matrices and Relevant Submodel

\begin{tabular}{|c|c|c|c|c|}
\hline $\begin{array}{l}\text { LA FEP } \\
\text { Number }\end{array}$ & FEP Name & $\begin{array}{c}\text { Matrix for } \\
\text { Groundwater }^{\text {a }}\end{array}$ & $\begin{array}{c}\text { Matrix for } \\
\text { Volcanic Ash }\end{array}$ & Biosphere Submodels $^{c}$ \\
\hline 1.2.04.07.0A & Ashfall & - & $(1,1)(1,2)$ & Soil, Plant, Air \\
\hline 1.3.01.00.0A & Climate change & $\begin{array}{l}(2,2)(4,4)(6,6) \\
(3,7)\end{array}$ & $(4,4)$ & $\begin{array}{l}\text { Soil, Plant, Fish, }{ }^{14} \mathrm{C} \text {, } \\
\text { Inhalation }\end{array}$ \\
\hline 1.3.07.02.0A & Water table rise affects $S Z$ & $(1,1)$ & - & $\begin{array}{l}\text { Soil, Air, Plant, }{ }^{14} \mathrm{C} \text {, Animal, } \\
\text { Fish, Ingestion }\end{array}$ \\
\hline 1.4.07.01.0A & Water management activities & $(1,4)(1,6)$ & - & Plant, Fish \\
\hline 1.4.07.02.0A & Wells & $(1,1)$ & - & $\begin{array}{l}\text { Soil, Air, Plant, }{ }^{14} \mathrm{C} \text {, Animal, } \\
\text { Fish, Ingestion }\end{array}$ \\
\hline 2.2.08.01.0A & $\begin{array}{l}\text { Chemical characteristics of } \\
\text { groundwater in the SZ }\end{array}$ & $(1,1)$ & - & $\begin{array}{l}\text { Soil, Plant, Animal, Fish, } \\
\text { Ingestion, Inhalation }\end{array}$ \\
\hline 2.3.02.01.0A & Soil type & $(2,2)(4,4)$ & $(2,2)(4,4)$ & Soil, Plant, ${ }^{14} \mathrm{C}$ \\
\hline 2.3.02.02.0A & $\begin{array}{l}\text { Radionuclide accumulation } \\
\text { in soils }\end{array}$ & $\begin{array}{l}(1,2)(2,1)(2,2) \\
(3,2)(4,2)(5,2)\end{array}$ & $(4,2)(5,2)$ & Soil \\
\hline 2.3.02.03.0A & $\begin{array}{l}\text { Soil and sediment transport } \\
\text { in the biosphere }\end{array}$ & $(2,3)(3,2)$ & $(2,3)(3,2)$ & Soil, Air \\
\hline 2.3.04.01.0A & $\begin{array}{l}\text { Surface water transport and } \\
\text { mixing }\end{array}$ & $(1,1)$ & - & $\begin{array}{l}\text { Soil, Air, Plant, }{ }^{14} \mathrm{C}, \\
\text { Animal, Fish, Ingestion }\end{array}$ \\
\hline 2.3.11.01.0A & Precipitation & $(2,2)(4,4)$ & - & Soil, Plant, ${ }^{14} \mathrm{C}$ \\
\hline 2.3.13.01.0A & Biosphere characteristics & $\begin{array}{l}(2,2)(3,3)(4,4) \\
(6,6)(3,7)\end{array}$ & $(2,2)(3,3)(4,4)$ & $\begin{array}{l}\text { Soil, Air, Plant, Fish, }{ }^{14} \mathrm{C} \text {, } \\
\text { Inhalation }\end{array}$ \\
\hline 2.3.13.02.0A & $\begin{array}{l}\text { Radionuclide alteration } \\
\text { during biosphere transport }\end{array}$ & $\begin{array}{l}(2,2)(2,1)(2,3) \\
(1,4)(2,4)(1,6) \\
(4,2)\end{array}$ & $(1,4)(2,4)(4,2)$ & $\begin{array}{l}\text { Soil, Plant, Animal, Fish, } \\
\text { Inhalation, Ingestion, } \\
\text { Evaporative cooler. }\end{array}$ \\
\hline 2.4.01.00.0A & $\begin{array}{l}\text { Human characteristics } \\
\text { (physiology, metabolism) }\end{array}$ & $(7,7)$ & $(7,7)$ & $\begin{array}{l}\text { External exposure, } \\
\text { Inhalation, Ingestion }\end{array}$ \\
\hline 2.4.04.01.0A & Human lifestyle & $(7,7)$ & $(7,7)$ & $\begin{array}{l}\text { Air, External exposure, } \\
\text { Inhalation, Ingestion }\end{array}$ \\
\hline 2.4.07.00.0A & Dwellings & $(1,3)(2,7)(3,7)$ & $(2,7)(3,7)$ & $\begin{array}{l}\text { Air, External exposure, } \\
\text { Inhalation }\end{array}$ \\
\hline 2.4.08.00.0A & $\begin{array}{l}\text { Wild and natural land and } \\
\text { water use }\end{array}$ & $(5,5)(2,7)(5,7)$ & - & $\begin{array}{l}\text { Air, Animal , External } \\
\text { exposure, Ingestion }\end{array}$ \\
\hline 2.4.09.01.0B & $\begin{array}{l}\text { Agricultural land use and } \\
\text { irrigation }\end{array}$ & $\begin{array}{l}(2,2)(3,3)(4,4) \\
(5,5)(6,6)\end{array}$ & $\begin{array}{l}(2,2)(3,3)(4,4) \\
(5,5)\end{array}$ & $\begin{array}{l}\text { Soil, Air, Plant, External } \\
\text { exposure, Inhalation, } \\
\text { Animal, }{ }^{14} \mathrm{C} \text {, Fish }\end{array}$ \\
\hline 2.4.09.02.0A & Animal farms and fisheries & $(5,5)(6,6)$ & $(5,5)$ & Animal, Fish \\
\hline 2.4.10.00.0A & $\begin{array}{l}\text { Urban and industrial land } \\
\text { and water use }\end{array}$ & $(2,7)$ & $(2,7)$ & $\begin{array}{l}\text { Soil, Air, }{ }^{14} \mathrm{C} \text {, External } \\
\text { exposure, Inhalation }\end{array}$ \\
\hline
\end{tabular}


Table 6.3-6. Mapping of FEPs to Interaction Matrices and Relevant Submodel (Continued)

\begin{tabular}{|c|c|c|c|c|}
\hline $\begin{array}{l}\text { LA FEP } \\
\text { Number }\end{array}$ & FEP Name & $\begin{array}{c}\text { Matrix for } \\
\text { Groundwater }\end{array}$ & $\begin{array}{c}\text { Matrix for } \\
\text { Volcanic Ash }\end{array}$ & Biosphere Submodels ${ }^{c}$ \\
\hline 3.1.01.01.0A & $\begin{array}{l}\text { Radioactive decay and } \\
\text { ingrowth }\end{array}$ & All $^{d}$ & All ${ }^{d}$ & $\begin{array}{l}\text { Soil, Air, Plant, Animal, Fish, } \\
\text { External exposure, Inhalation, } \\
\text { Ingestion }\end{array}$ \\
\hline 3.2.10.00.0A & $\begin{array}{l}\text { Atmospheric transport of } \\
\text { contaminants }\end{array}$ & $(1,3)(2,3)$ & $(2,3)$ & Air, ${ }^{14} \mathrm{C}$ \\
\hline 3.3.01.00.0A & $\begin{array}{l}\text { Contaminated drinking water, } \\
\text { foodstuffs and drugs }\end{array}$ & $(4,4)(5,5)(6,6)$ & $(4,4)(5,5)$ & Plant, Animal, Fish, Ingestion \\
\hline 3.3.02.01.0A & Plant uptake & $(1,4)(2,4)(3,4)$ & $(2,4)(3,4)$ & Plant, ${ }^{14} \mathrm{C}$ \\
\hline 3.3.02.02.0A & Animal uptake & $(1,5)(2,5)(4,5)$ & $(2,5)(4,5)$ & Animal, ${ }^{14} \mathrm{C}$ \\
\hline 3.3.02.03.0A & Fish uptake & $(1,6)$ & - & Fish \\
\hline 3.3.03.01.0A & $\begin{array}{l}\text { Contaminated non-food } \\
\text { products and exposure }\end{array}$ & $(2,7)$ & $(2,7)$ & External Exposure \\
\hline 3.3.04.01.0A & Ingestion & $\begin{array}{l}(1,7)(2,7)(4,7) \\
(5,7)(6,7)\end{array}$ & $(2,7)(4,7)(5,7)$ & Ingestion \\
\hline 3.3.04.02.0A & Inhalation & $(3,7)$ & $(3,7)$ & Inhalation \\
\hline 3.3.04.03.0A & External exposure & $(2,7)$ & $(2,7)$ & External exposure \\
\hline 3.3.05.01.0A & Radiation doses & $\begin{array}{l}(1,7)(2,7)(3,7) \\
(4,7)(5,7)(6,7)\end{array}$ & $\begin{array}{l}(2,7)(3,7)(4,7) \\
(5,7)\end{array}$ & $\begin{array}{l}\text { External exposure, Inhalation, } \\
\text { Ingestion }\end{array}$ \\
\hline 3.3.08.00.0A & $\begin{array}{l}\text { Radon and radon daughter } \\
\text { exposure }\end{array}$ & $(2,3)(3,7)$ & $(2,3)(3,7)$ & Air, Inhalation \\
\hline
\end{tabular}

Source: The LA FEP list (DTN: MO0407SEPFEPLA.000. [DIRS 170760]) is the source of FEPs for the biosphere model.

${ }^{a}$ Elements of the radionuclide transfer interaction matrix for the groundwater scenario shown in Table 6.3-2. Index "i" in (i,j) indicates the row in the matrix, while index " $\mathrm{j}$ " in (i,j) indicates the column in the matrix. The linkage between the elements in the matrix and FEPs is based on the biosphere model and the FEP description.

${ }^{\mathrm{b}}$ Elements of the radionuclide transfer interaction matrix for the volcanic ash scenario shown in Table 6.3-4. Index "i" in (i,j) indicates the row in the matrix, while index "j" in (i,j) indicates the column in the matrix.

${ }^{c}$ Relationships among the submodels are shown in Figures 6.3-2 and 6.3-4. Note that ${ }^{14} \mathrm{C}$ has its own special submodel for the groundwater scenario.

d "All" means that radionuclide decay is considered in all radionuclide transport in environment media and all human exposure pathways.

FEP=feature, event, or process; LA=license application; $S Z=$ saturated zone

\subsubsection{Radionuclide Decay and Ingrowth}

The TSPA-LA is conducted for the suite of radionuclides identified as important contributors to the total dose from radionuclides released from the repository at Yucca Mountain (BSC 2002 [DIRS 160059], Section 7). Consistent with the TSPA-LA, the ERMYN considers the same suite of primary radionuclides (Section 6.1.3). The primary radionuclides are long-lived and are accompanied by short-lived decay products, which are included in the ERMYN to correctly account for the radiological consequences of the decay chains.

Radionuclides with high atomic numbers (greater than or equal to 82) typically have long chains of radioactive decay products. The consideration of radionuclide decay and ingrowth as a function of time for all members of a decay chain, although conceptually simple, can be computationally intensive and frequently adds little value to analyses that only consider 
long-lived radionuclides. Therefore, in the ERMYN, radionuclides with half-lives less than 180 days are treated as if they were always in secular equilibrium with the parent radionuclides (Approximation 2). The secular equilibrium of the parent and progeny applies to the entire biosphere system.

This approximation eliminates the need to consider long decay chains from radionuclides with high atomic numbers. This approximation is reasonable, considering the long time frame of the TSPA modeling. This approach also does not underestimate the concentrations of decay products because the activity concentration of short-lived radionuclides is highest when in secular equilibrium with the long-lived parents (the activity concentrations are equal). The dose contributions of the primary radionuclide and the short-lived decay products can be determined using the activity concentration of the primary radionuclide and the effective dose conversion factors or effective dose coefficients for the chain considered. The latter are produced by adding the dose conversion factors or dose coefficients for the parent radionuclide and those for the short-lived decay products as modified by the branching fraction of the decay products.

The radionuclides of interest for TSPA-LA (Table 6.1-1), with their short-lived (half-life, $\mathrm{T}_{1 / 2}$ less than $180 \mathrm{~d}$ ) decay products, associated branching ratios, and half-lives, are shown in Table 6.3-7. The table is divided into five sections, including:

- Relatively light radionuclides that decay to a stable nuclide or have only one or two radioactive decay products

- Uranium series radionuclides $(4 n+2)$

- Thorium series radionuclides (4n)

- Actinium series radionuclides $(4 n+3)$

- Neptunium series radionuclides $(4 n+1)$.

The method of combining dose contributions of decay products with those of their parents is discussed in the sections about the external exposure (Sections 6.4.7 and 6.5.5), inhalation (Section 6.4.8), and ingestion submodels (Section 6.4.9).

Table 6.3-7. Radionuclides of Interest and Their Decay Products

\begin{tabular}{|c|c|c|c|c|}
\hline \multicolumn{2}{|c|}{ Primary Radionuclide } & \multirow{2}{*}{$\begin{array}{c}\text { Decay Product }{ }^{\mathrm{c}} \\
\text { (branching fraction if not 100\%, } \\
\text { half-life) }\end{array}$} & \multicolumn{2}{|c|}{ Terminal Nuclide } \\
\hline Radionuclide & Half-life $(y r)^{c}$ & & Nuclide & Half-life (yr) ${ }^{d}$ \\
\hline C-14 & 5730 & - & $\mathrm{N}-14$ & * \\
\hline $\mathrm{Cl}-36$ & $3.01 \mathrm{E}+05$ & - & $\begin{array}{l}\text { Ar-36 (1.9\%) } \\
\text { S-36 (98.1\%) }\end{array}$ & * \\
\hline Se-79 & $6.5 \mathrm{E}+04$ & - & $\mathrm{Br}-79$ & * \\
\hline Sr-90D ${ }^{a}$ & 29.12 & Y-90 (64.0 hr) & Zr-90 & * \\
\hline Tc-99 & $2.13 \mathrm{E}+05$ & - & Ru-99 & * \\
\hline Sn-126D & $1.0 \mathrm{E}+05$ & $\begin{array}{l}\text { Sb-126m (19.0 min) } \\
\text { Sb-126 (14\%, } 12.4 \mathrm{~d})\end{array}$ & Te-126 & * \\
\hline I-129 & $1.57 \mathrm{E}+07$ & - & Xe-129 & * \\
\hline Cs-135 & $2.3 \mathrm{E}+06$ & - & Ba-135 & * \\
\hline Cs-137D & 30.0 & Ba-137m (94.6\%, $2.552 \mathrm{~min})$ & Ba-137 & * \\
\hline Pu-242 & $3.763 \mathrm{E}+05$ & - & $\mathrm{U}-238$ & $4.468 \mathrm{E}+09$ \\
\hline
\end{tabular}


Table 6.3-7. Radionuclides of Interest and Their Decay Products (Continued)

\begin{tabular}{|c|c|c|c|c|}
\hline \multicolumn{2}{|c|}{ Primary Radionuclide } & \multirow{2}{*}{$\begin{array}{l}\text { Decay Product }{ }^{\mathrm{c}} \\
\text { (branching fraction if not } 100 \% \text {, } \\
\text { half-life) }\end{array}$} & \multicolumn{2}{|c|}{ Terminal Nuclide } \\
\hline Radionuclide & Half-life $(y r)^{c}$ & & Nuclide & Half-life $(y r)^{d}$ \\
\hline U-238D & $4.468 \mathrm{E}+09$ & $\begin{array}{l}\text { Th-234 (24.10 d) } \\
\text { Pa-234m }(99.80 \%, 1.17 \mathrm{~min}) \\
\text { Pa-234 (0.33\%, 6.7 hr) }\end{array}$ & U-234 & $2.445 \mathrm{E}+05$ \\
\hline Pu-238 & 87.74 & - & $\mathrm{U}-234$ & $2.445 \mathrm{E}+05$ \\
\hline $\mathrm{U}-234$ & $2.445 \mathrm{E}+05$ & - & Th-230 & $7.7 \mathrm{E}+04$ \\
\hline Th-230 & $7.7 \mathrm{E}+04$ & - & Ra-226 & $1.60 \mathrm{E}+03$ \\
\hline Ra-226D & $1.60 \mathrm{E}+03$ & $\begin{array}{l}\text { Rn-222 (3.8235 d) } \\
\text { Po-218 (3.05 min) } \\
\text { Pb-214 (99.98\%, 26.8 min) } \\
\text { At-218 (0.02\%, 2 sec) } \\
\text { Bi-214 (19.9 min) } \\
\left.\text { Po-214 (99.98\%, } 1.643 \times 10^{-4} \mathrm{sec}\right) \\
\text { Tl-210 (0.02\%, } 1.3 \mathrm{~min})\end{array}$ & $\mathrm{Pb}-210$ & $2.23 \mathrm{E}+01$ \\
\hline $\mathrm{Pb}-210 \mathrm{D}$ & 22.3 & $\begin{array}{l}\operatorname{Bi}-210(5.012 \mathrm{~d}) \\
\text { Po-210 }(138.38 \mathrm{~d})\end{array}$ & $\mathrm{Pb}-206$ & * \\
\hline Pu-240 & $6.537 \mathrm{E}+03$ & - & $\mathrm{U}-236$ & $2.3415 \mathrm{E}+07$ \\
\hline $\mathrm{U}-236$ & $2.3415 \mathrm{E}+07$ & - & Th-232 & $1.405 \mathrm{E}+10$ \\
\hline Th-232 & $1.405 \mathrm{E}+10$ & - & Ra-228 & $5.75 \mathrm{E}+00$ \\
\hline $\mathrm{Ra}-228 \mathrm{D}^{\mathrm{b}}$ & 5.75 & Ac-228 (6.13 hr) & Th-228 & $1.9131 \mathrm{E}+00$ \\
\hline $\mathrm{U}-232$ & 72 & - & Th-228 & $1.9131 \mathrm{E}+00$ \\
\hline Th-228D & 1.9131 & $\begin{array}{l}\text { Ra-224 (3.66 d) } \\
\text { Rn-220 (55.6 sec) } \\
\text { Po-216 (0.15 sec) } \\
\text { Pb-212 (10.64 hr) } \\
\text { Bi-212 (60.55 min) } \\
\text { Po-212 (64.07\%, } 0.305 \mu-\mathrm{sec}) \\
\text { Tl-208 (35.93\%, } 3.07 \mathrm{~min})\end{array}$ & $\mathrm{Pb}-208$ & 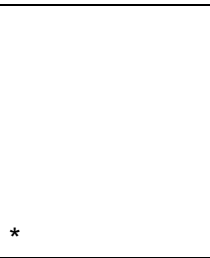 \\
\hline Am-243D & $7.38 \mathrm{E}+03$ & $\mathrm{~Np}-239(2.355 \mathrm{~d})$ & Pu-239 & $2.406 \mathrm{E}+04$ \\
\hline Pu-239 & $2.4065 \mathrm{E}+04$ & - & U-235 & $7.038 \mathrm{E}+08$ \\
\hline U-235D & $7.038 \mathrm{E}+08$ & Th-231 (25.52 hr) & $\mathrm{Pa}-231$ & $3.276 \mathrm{E}+04$ \\
\hline $\mathrm{Pa}-231$ & $3.276 \mathrm{E}+04$ & - & Ac-227 & $2.1773 \mathrm{E}+01$ \\
\hline$A c-227 D$ & 21.773 & $\begin{array}{l}\text { Th-227 (98.62\%, } 18.718 \mathrm{~d}) \\
\text { Fr-223 (1.38\%, } 21.8 \mathrm{~min}) \\
\text { Ra-223 (11.434 d) } \\
\text { Rn-219 (3.96 sec) } \\
\text { Po-215 (1.78 millisec) } \\
\text { Pb-211 (36.1 min) } \\
\text { Bi-211 (2.14 min) } \\
\text { Tl-207 (99.72\%, } 4.77 \mathrm{~min}) \\
\text { Po-211 (0.28\%, } 0.516 \mathrm{sec})\end{array}$ & $\mathrm{Pb}-207$ & 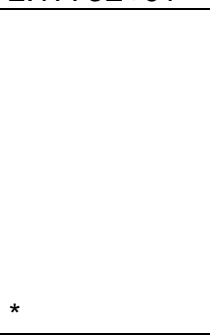 \\
\hline $\mathrm{Am}-241$ & 432.2 & - & $\mathrm{Np}-237$ & $2.14 \mathrm{E}+06$ \\
\hline Np-237D & $2.14 \mathrm{E}+06$ & Pa-233 (27.0 d) & $U-233$ & $1.585 \mathrm{E}+05$ \\
\hline $\mathrm{U}-233$ & $1.585 \mathrm{E}+05$ & - & Th-229 & $7.34 \mathrm{E}+03$ \\
\hline Th-229D & $7.34 \mathrm{E}+03$ & $\begin{array}{l}\text { Ra-225 (14.8 d) } \\
\text { Ac-225 (10.0 d) } \\
\text { Fr-221 (4.8 min) } \\
\text { At-217 (32.3 millisec) } \\
\text { Bi-213 (45.65 min) } \\
\text { Po-213 (97.84\%, } 4.2 \mu-s e c) \\
\text { Tl-209 (2.16\%, } 2.2 \mathrm{~min}) \\
\text { Pb-209 (3.253 hr })\end{array}$ & $\mathrm{Bi}-209$ & * \\
\hline
\end{tabular}

a " $D$ " indicates that the radionuclide is treated with the short-lived ( $T_{1 / 2}$ less than $180 \mathrm{~d}$ ) decay products.

${ }^{\mathrm{b}}$ Indented radionuclides are long-lived decay products considered separately from the parents.

c Source: BSC (2004 [DIRS 169671] Table 6-23).

"A "*" denotes a stable nuclide. 
If the half-life of a decay product is longer than $180 \mathrm{~d}$, but not long enough to be considered a primary radionuclide for the TSPA-LA, the decay product is treated in the same way as the long-lived parent. That is, the radionuclide is tracked individually in the ERMYN, including the calculation of the BDCF. The BDCF for an individually tracked decay product is added to the BDCF for the parent (Table 6.3-7), assuming the secular equilibrium of the parent and decay products in the source (i.e., groundwater or volcanic ash). However, following release, the decay product is transported in the environment independently of the parent. For example, ${ }^{232} \mathrm{U}$ decays to ${ }^{228} \mathrm{Th}$. ${ }^{228} \mathrm{Th}$ is not a primary radionuclide for the TSPA-LA, but the half-life is sufficiently long that it exhibits different environmental transport behaviors, especially as related to leaching, soil-to-plant transfer, and transfer from animal feed to animal products.

If a decay product of a primary radionuclide also is a primary radionuclide, the activity of this radionuclide arising from the decay of the parent radionuclide and the activity that is originally present in the source as a primary radionuclide are tracked independently in the biosphere model. For example, ${ }^{243} \mathrm{Am}$ and ${ }^{239} \mathrm{Pu}$ are primary radionuclides, but ${ }^{243} \mathrm{Am}$ decays to ${ }^{239} \mathrm{Pu}$, so there are two sources of ${ }^{239} \mathrm{Pu}$. If groundwater containing both of these primary radionuclides is used for irrigation, the total activity concentration of ${ }^{239} \mathrm{Pu}$ in the soil results from ${ }^{239} \mathrm{Pu}$ in the groundwater and from ${ }^{239} \mathrm{Pu}$ produced in the soil by the decay of ${ }^{243} \mathrm{Am}$. These two fractions of ${ }^{239} \mathrm{Pu}$ are independently accounted for in the biosphere model, and the activity concentrations in the soil depend on the concentrations of the primary radionuclides (i.e., ${ }^{239} \mathrm{Pu}$ and ${ }^{243} \mathrm{Am}$, respectively) in the groundwater source. Ingrowth of decay products in the soil is discussed in Section 6.4.1.2.

\subsection{MATHEMATICAL MODEL FOR THE GROUNDWATER SCENARIO}

The objective of the groundwater biosphere model (Section 6.1) is to calculate BDCFs $(\mathrm{Sv} /$ year $) /\left(\mathrm{Bq} / \mathrm{m}^{3}\right)$ for the TSPA-LA model. The source term for the groundwater scenario is the activity concentration of a radionuclide in groundwater $\left(\mathrm{Bq} / \mathrm{m}^{3}\right)$. This is a time-dependent quantity, calculated outside the biosphere model by the TSPA-LA model, and is unknown until the TSPA-LA model is run. It is assumed in the biosphere model that the radionuclide concentration in groundwater does not change over time (Approximation 1). The radiation dose can be calculated as a product of the source term (activity concentration in groundwater) and the BDCF, which is source-term independent. A BDCF for the groundwater scenario is numerically equal to the all-pathway dose that the RMEI receives under specific biosphere conditions (Section 6.3.1) when the RMEI is exposed to radionuclide contamination in environmental media arising from continuous use of groundwater containing a unit concentration of the radionuclide.

The mathematical model for the groundwater scenario, similar to the biosphere conceptual model for the groundwater scenario (Section 6.3.1), is presented as a series of submodels. The relationship among the submodels (Figure 6.3-2) is described in Section 6.3.1. The same notation is used for the same parameter in all submodels so that the linkage between submodels can be traced. Because the ERMYN is largely derived from the GENII-S biosphere model, the two models share most mathematical equations, which were originally described in Napier et al. (1988 [DIRS 157927]). For the equations in Sections 6.4 and 6.5, taken from the GENII/GENII-S model, the source is not specifically referenced; but if the equation is from another source, the source is cited. In Section 7.3, each submodel described in this section is 
compared with other published biosphere models for model validation, and the sources of equations in the ERMYN are described in detail.

In the following sections, representative data for many parameters are introduced from the five supporting analyses shown in Figure 1-1. These data are summarized in Table 6.6-3. They are introduced here to provide insight into the various submodel developed in ERMYN, and are used in Section 7 for validation. From the perspective of this report, none of these data are direct inputs.

\subsubsection{Surface Soil Submodel}

The surface soil submodel is designed to evaluate radionuclide accumulation in, and removal from, the upper layer of the soil (down to the tilling depth) where all plant roots are assumed to be located (Approximation 7). For the groundwater scenario, surface soil is contaminated as a result of using contaminated groundwater for irrigation. The surface soil submodel is based on the BIOMASS ERB2A model (BIOMASS 2000 [DIRS 154522], Section 7).

If contaminated groundwater is used to irrigate cultivated soils, radionuclide concentrations in the soil will build up at a rate determined by the physical and chemical properties of the soil and the radionuclides. On land irrigated for a long time, radionuclide concentrations depend on the rate of accumulation and removal, and they will reach equilibrium concentrations when the rates of addition and removal are equal. Long-lived isotopes of elements that bind readily to soil particles will not reach equilibrium concentrations for thousands of years, whereas relatively short-lived or mobile radioisotopes will approach equilibrium concentrations after only a few years (Section 7.4.2).

\subsubsection{Primary Radionuclides in the Surface Soil}

Radionuclides can be removed from the surface soil by leaching into the deep soil, surface soil erosion, crop harvest removal, and radioactive decay. Although crop harvesting may be an important mechanism for radionuclide removal on cultivated lands, this mechanism is not considered in the biosphere model because it is considered to compensate for the reintroduction of radionuclides into the soil when contaminated cow manure is used as fertilizer (Approximation 4).

Because irrigation rates differ among crop types, radionuclide concentrations in the surface soil depend on the specific use of cultivated land. However, crop rotation over a longer period of time would average out the differing radionuclide concentrations caused by different crop irrigation levels (Approximation 3). Therefore, it is reasonable to use an average annual irrigation rate to estimate long-term radionuclide concentrations in surface soil. The long-term concentration is used to evaluate radionuclide uptake from soil by plants and to evaluate inhalation, inadvertent soil ingestion, and external exposures. Using this simplification, the average activity concentration of a radionuclide in the surface soil does not depend on the crop type.

The mathematical representation of the primary radionuclide addition and removal processes in the surface soil is expressed by the following differential equation and the initial condition. Decay product ingrowth in the surface soil is discussed in the next subsection. 


$$
\left\{\begin{array}{l}
\frac{d C s_{i}(t)}{d t}=C w_{i}(t) I R(t)-\left(\lambda_{d, i}+\lambda_{l, i}+\lambda_{e}\right) C s_{i}(t) \\
C s_{i}(t)=0, \text { when } t=0
\end{array}\right.
$$

where

$$
\begin{aligned}
C s_{i}(t)= & \text { activity concentration of radionuclide } i \text { in surface soil per unit area at time } \mathrm{t} \\
& \left(\mathrm{Bq} / \mathrm{m}^{2}\right) \\
i & \text { primary radionuclide index, used for entire biosphere model } \\
t & \text { time variable (year) } \\
C w_{i}(t)= & \text { activity concentration of radionuclide } i \text { in the groundwater at time } t\left(\mathrm{~Bq} / \mathrm{m}^{3}\right) \\
I R(t)= & \text { annual average irrigation rate on land (annual irrigation rate) }(\mathrm{m} / \mathrm{year}) \\
\lambda_{d, i}= & \text { radioactive decay constant for radionuclide } i \text { (/year); this can be calculated } \\
& \text { from radionuclide half-life (Table } 6.3-7) \text { using the conversion } \ln (2) / T_{d, i}, \text { where } \\
& T_{d, i} \text { is half-life of radionuclide } i \text { (year) } \\
\lambda_{l, i}= & \text { average annual leaching removal constant for radionuclide } i(/ \text { year) } \\
\lambda_{e}= & \text { average annual surface soil erosion removal constant (/year) }
\end{aligned}
$$

When radionuclide concentrations in the surface soil reach equilibrium, they do not change with time, $\frac{d C s_{i}(t)}{d t}=0$, and they are not time dependent $C s_{i}(t)=C s_{i}$. As noted previously, it is assumed in the biosphere model that radionuclide concentrations in groundwater do not change over the time of modeling interest: $C w_{i}(t)=C w_{i}($ Approximation 1).

The average crop irrigation rate in Equation 6.4.1-1, $I R(t)$, is an annual average irrigation rate deposited on the soil, which is developed based on the irrigation rates for individual crop types. The typical range of irrigation rates is from 0.5 to $1.0 \mathrm{~m} /$ year (BSC 2004 [DIRS 169673], Section 6.5). The average irrigation rate is time-dependent because of climate changes predicted for the Yucca Mountain region. However, for a defined climate, the average irrigation is not a function of time: $I R(t)=I R$. As discussed in Section 6.2, climate change does not change the overall biosphere conceptual model as long as the general characteristics of the biosphere are consistent with the arid and semi-arid climate. The model can be adopted for different climates within these boundaries through selection of climate-dependent parameter values, such that they match the desired climate.

The leaching removal constant, $\lambda_{l, i}$, is an element-specific input parameter. This important parameter is discussed further in Section 6.4.1.3. The surface soil erosion removal constant, $\lambda_{e}$, represents the rate of radionuclide loss from the surface soil due to wind erosion. The value of this radionuclide-independent parameter is strongly site-specific and depends on environmental characteristics and land use. This parameter is further discussed in Section 6.4.1.4.

When the radionuclide concentration in the groundwater and the crop irrigation rate are not time-dependent, the analytical solution of Equation 6.4.1-1 is expressed as 


$$
C s_{i}(t)=\frac{C w_{i} I R}{\lambda_{d, i}+\lambda_{l, i}+\lambda_{e}}\left[1-e^{-\left(\lambda_{d, i}+\lambda_{l, i}+\lambda_{e}\right) t}\right]
$$

All parameters in Equation 6.4.1-2 are defined in Equation 6.4.1-1. The term $\lambda_{d, i}+\lambda_{l, i}+\lambda_{e}$ can be replaced with one parameter, the effective removal constant, $\lambda_{\text {eff,i }}$. Thus, Equation 6.4.1-2 can be expressed as

$$
C s_{i}(t)=\frac{C w_{i} I R}{\lambda_{e f f, i}}\left(1-e^{-\lambda_{e f f, i} t}\right)
$$

The effective removal constant is an important parameter that determines the rate at which radionuclides approach equilibrium concentrations in the surface soil. For example, the time $(t)$ required for soil concentrations to reach 95 percent of the equilibrium value can be derived from Equation 6.4.1-3, and is equal to $\ln (20) / \lambda_{\text {eff }, i}$. Although it theoretically takes an infinite amount of time to reach equilibrium, the time to reach a fixed percentage of the equilibrium value would be finite, and 95 percent is a close approximation to equilibrium. It takes about 10 to 2,500 years for the radionuclides considered in the model to reach the 95 percent equilibrium concentration in surface soils (Section 7.4.2).

With time, radionuclide concentrations eventually would reach the equilibrium concentration (Approximation 5). The equilibrium concentration in the soil, $\mathrm{Cs}_{i}$, is expressed as the ratio of the radionuclide addition rate to the removal rate

$$
C s_{i}=\frac{C w_{i} I R}{\lambda_{e f f, i}}
$$

The activity concentration of a radionuclide in surface soil calculated from Equation 6.4.1-4 is given in units of activity per unit area $\left(\mathrm{Bq} / \mathrm{m}^{2}\right)$. It can be converted to activity concentration in Bq per unit mass of surface soil using

$$
C s_{m, i}=\frac{C s_{i}}{\rho_{s}}
$$

where

$$
\begin{aligned}
C s_{m, i}= & \text { equilibrium activity concentration of radionuclide } i \text { in surface soil per unit mass } \\
& (\mathrm{Bq} / \mathrm{kg}) \\
C s_{i}= & \begin{array}{l}
\text { equilibrium activity concentration of radionuclide } i \text { in surface soil per unit area } \\
\left(\mathrm{Bq} / \mathrm{m}^{2}\right)(\text { defined in Equation } 6.4 .1-4)
\end{array} \\
\rho_{s}= & \text { areal density of surface soil }\left(\mathrm{kg} / \mathrm{m}^{2}\right) .
\end{aligned}
$$

Surface soil density is calculated in the submodel using

$$
\rho_{s}=\rho \times d
$$


where

$$
\begin{array}{ll}
\rho & =\text { bulk density of surface soil }\left(\mathrm{kg} / \mathrm{m}^{3}\right) \\
d & =\text { depth of surface soil }(\mathrm{m})
\end{array}
$$

The soil bulk density ranges from about 1,300 to $1,700 \mathrm{~kg} / \mathrm{m}^{3}$ (Table $6.6-3$ ). The depth of the surface soil is based on tillage depth and ranges from 5 to about $30 \mathrm{~cm}$ (Table 6.6-3).

In the biosphere model, soil is divided into two compartments: the surface soil layer encompassing the crop root zone, and the deep soil where radionuclides are assumed inaccessible to plants (Approximation 7). The depth of the surface soil controls the partition between these two compartments, and, therefore, controls the effective "capacity" of the compartment for the amount of radionuclides available for further mobilization in the biosphere (e.g., by crop uptake).

This method is considered valid for long-lived radionuclides and for the conditions of sustained irrigation with water containing constant concentrations of given radionuclides (Approximation 1). This approach does not underestimate radionuclide concentrations in surface soil because it is assumed that equilibrium conditions exist in the soil.

\subsubsection{Radionuclide Decay and Ingrowth in the Surface Soil}

Radionuclide decay and ingrowth in surface soil due to introduction of primary radionuclides in irrigation water are considered in this section. As described in Sections 6.3.1.4 and 6.3.5, short-lived decay products (half-lives less than 180 days) are treated as if they were in secular equilibrium with the parent radionuclide (Approximation 2). The buildup of long-lived decay products (which may also be primary radionuclides) in the surface soil is considered separately from the parent primary radionuclides (Section 6.4.1.1) because decay product concentrations in the soil are calculated differently. The radiation dose contribution from the decay products is included with the primary radionuclide.

For decay chains in the surface soil, decay products are produced at the rate that the parent radionuclides decay. Removal mechanisms include decay of the decay product, leaching from the surface soil, and removal by soil erosion. The general differential equation (Lamarsh 1983 [DIRS 149069], Section 2.9) describing the rate of change in the number of atoms of the decay product is given by

$$
\left\{\begin{array}{l}
\frac{d N_{l}(t)}{d t}=\lambda_{d, l-1} N_{l-1}(t)-\left(\lambda_{d, l}+\lambda_{l, l}+\lambda_{e}\right) N_{l}(t) \\
N_{l}(t)=N_{l-1}(t)=0 \text {, when } t=0
\end{array}\right.
$$

where

$$
\begin{aligned}
& N_{l}(t)=\text { number of atoms for the } \mathrm{l}^{\text {th }} \text { decay product in a decay chain in the surface soil } \\
& N_{l-1}(t)=\text { number of atoms for the }(l-1)^{\text {th }} \text { decay product in a decay chain in the surface soil } \\
& l
\end{aligned}
$$


$\lambda_{d, l-1}=$ radioactive decay constant for the $(l-1)^{\text {th }}$ decay product (/year)

$\lambda_{d, l}=$ radioactive decay constant for the $l^{\text {th }}$ decay product (/year)

$\lambda_{l, l}=$ average annual leaching removal constant $\left(\lambda_{l}\right)$ for the $l^{\text {th }}$ decay product (/year)

$\lambda_{e} \quad=$ average annual surface soil erosion removal constant (/year)

If the number of atoms in Equation 6.4.1-7 is multiplied by the decay constant, $\lambda_{d, l}$, and divided by the soil surface area, $A$, the resulting expression can be used to calculate the rate of change of activity concentration of a radionuclide in the surface soil equation (Lamarsh 1983 [DIRS 149069], Section 2.9), such that Equation 6.4.1-7 becomes

$$
\left\{\begin{array}{l}
\frac{d C s_{l}(t)}{d t}=\lambda_{d, l} C s_{l-1}(t)-\left(\lambda_{d, l}+\lambda_{l, l}+\lambda_{e}\right) C s_{l}(t) \\
C s_{l}(t)=C s_{l-1}(t)=0 \text {, when } t=0
\end{array}\right.
$$

where

$$
\begin{array}{ll}
C s_{l}(t)=\frac{\lambda_{d, l} N_{l}(t)}{A}=\begin{array}{l}
\text { activity concentration of a decay product } l \text { in surface soil } \\
\left(\mathrm{Bq} / \mathrm{m}^{2}\right)
\end{array} \\
C s_{l-1}(t)=\frac{\lambda_{d, l-1} N_{l-1}(t)=}{A}=\begin{array}{l}
\text { activity concentration of a primary radionuclide if }(l=1) \text { or a } \\
\text { decay product }(l-1) \text { in surface soil }\left(\mathrm{Bq} / \mathrm{m}^{2}\right)
\end{array}=\begin{array}{l}
\text { index of radionuclide decay chain } l=0 \text { for a primary } \\
\text { radionuclide and Equation } 6.4 .1-1 \text { is used; } l=1 \text { for the first } \\
\text { decay product }
\end{array}
\end{array}
$$

and the other parameters are defined in Equation 6.4.1-7.

When the activity concentration in surface soil, $\operatorname{Cs}_{l}(t)$, is a function of time, the analytical solution of Equation 6.4.1-8 for each decay product of the primary radionuclide can be complicated. However, the solutions for all radionuclides in a decay chain can be obtained analytically when the activity concentration in the soil is evaluated at equilibrium for the primary radionuclides and the decay products (Approximation 5); that is, $\frac{d C s_{l}(t)}{d t}=0$. The solution can be expressed as

$$
C s_{l}=\frac{\lambda_{d, l}}{\lambda_{d, l}+\lambda_{l, l}+\lambda_{e}} C s_{l-1}=\frac{\lambda_{d, l}}{\lambda_{\text {eff }, l}} C s_{l-1}
$$

where

$$
\begin{aligned}
C s_{l}= & \text { equilibrium activity concentration of a decay product } l \text { in surface soil }\left(\mathrm{Bq} / \mathrm{m}^{2}\right) \\
C s_{l-1}= & \text { equilibrium activity concentration of a primary radionuclide if } l=1 \text {, or a decay } \\
& \text { product }(l-1) \text { in surface soil }\left(\mathrm{Bq} / \mathrm{m}^{2}\right)
\end{aligned}
$$


$\lambda_{\text {eff }, l}=$ effective removal constant for the $t^{\text {th }}$ decay product (/year)

and the other parameters are defined in Equation 6.4.1-7.

For surface soil, Equation 6.4.1-9 describes relationships among activity concentrations for consecutive members of decay chains produced by the decay of primary radionuclides. For short-lived decay products, the decay constant $\left(\lambda_{d}\right)$ is approximately equal to the effective removal constant $\left(\lambda_{\text {eff }}\right)$, and the activity concentration in the soil is the same as that for the immediate predecessor, thereby, demonstrating that for short-lived radionuclides, the secular equilibrium is not perturbed by the other physical removal processes.

The activity concentrations of decay products in the soil are expressed as a constant fraction of the parent concentrations (Equation 6.4.1-9), which simplifies the calculation of activity concentrations for decay products in the surface soil. In addition, by combining short-lived radionuclides with their longer-lived parents (Section 6.3.5), the number of radionuclides in a decay chain can be reduced. However, even if reduced, the number of primary radionuclides in a decay chain could be as high as six (see ${ }^{242} \mathrm{Pu}$, Table 6.3-7). The concentrations of decay products in the soil depended on the decay constants and the effective removal constants (Equation 6.4.1-9). Typical effective removal constants for the radionuclides of interest to biosphere modeling range from $1 \times 10^{-4} /$ year to $1 \times 10^{-1} /$ year (Section 7.4.2). If radionuclides have a long half-life (on the order of $1 \times 10^{5}$ year), which corresponds to a small decay constant $\left(<1 \times 10^{-5} /\right.$ year $)$, it is not necessary to include them as a decay product of the parent primary radionuclide. Thus, these radionuclides can be considered decay chain "stoppers" for a decay chain originating with a primary radionuclide. The radionuclides that meet this condition are ${ }^{238} \mathrm{U},{ }^{234} \mathrm{U},{ }^{236} \mathrm{U},{ }^{232} \mathrm{Th},{ }^{235} \mathrm{U},{ }^{237} \mathrm{~Np}$, and ${ }^{233} \mathrm{U}$, which terminate the decay chains of ${ }^{242} \mathrm{Pu}$, ${ }^{238} \mathrm{U} /{ }^{238} \mathrm{Pu},{ }^{240} \mathrm{Pu},{ }^{236} \mathrm{U},{ }^{239} \mathrm{Pu},{ }^{241} \mathrm{Am}$, and ${ }^{237} \mathrm{~Np}$, respectively (Table 6.3-7).

By combining short-lived decay products with their longer-lived predecessors, and neglecting the contribution from very long-lived decay products and their progeny, the number of decay chain members that are explicitly considered in the ERMYN is reduced. The abridged chains are shown in Table 6.4-1.

Only the decay chain for ${ }^{234} \mathrm{U}$ includes a third long-lived decay product, (Pb-210D; Table 6.4-1). The contribution to the ${ }^{234} \mathrm{U}$ dose from ${ }^{226} \mathrm{Ra}$, with additional consideration of ${ }^{222} \mathrm{Rn}$ and progeny, is expected to be higher than the dose contribution from $\mathrm{Pb}-210 \mathrm{D}$ and progeny because of the inhalation dose contribution from radon progeny. Therefore, the third decay product, $\mathrm{Pb}-210 \mathrm{D}$, of the abbreviated ${ }^{234} \mathrm{U}$ decay chain is not considered (see Table 6.10-3). This reduces the number of decay products from primary radionuclides are explicitly modeled in the ERMYN to, at most, two. In Section 7.4.2.2, the radionuclide decay chains used in the GENII-S and ERMYNs are compared to verify that all of the decay products are properly considered. 
Table 6.4-1. The Primary Radionuclides and Their Decay Chains for Soil

\begin{tabular}{|l|l|l|l|}
\hline \multicolumn{1}{|c|}{ Primary Radionuclide } & \multicolumn{1}{|c|}{$\begin{array}{c}\text { 1st Long-Lived Decay } \\
\text { Product }\end{array}$} & $\begin{array}{c}\text { 2nd Long-Lived Decay } \\
\text { Product }\end{array}$ & \multicolumn{1}{|c|}{$\begin{array}{c}\text { 3rd Long-Lived Decay } \\
\text { Product }\end{array}$} \\
\hline U-234 & Th-230 & Ra-226D & Pb-210D \\
\hline Th-230 & Ra-226D & Pb-210D & - \\
\hline Ra-226D & ab-210D & - & - \\
\hline Th-232 & Ra-228D & Th-228D & - \\
\hline Ra-228D & Th-228 & -- & - \\
\hline U-232 & Th-228 & - & - \\
\hline Am-243D & Pu-239 & - & - \\
\hline U-235D & Pa-231 & Ac-227D & - \\
\hline Pa-231 & Ac-227D & - & - \\
\hline U-233 & Th-229D & - & - \\
\hline
\end{tabular}

NOTE: Table entries were derived from Table 6.3-7 using the method discussed in this section.

a $A$ "D" after a radionuclide symbol denotes that the radionuclide is treated together with its short-lived (less than $180 \mathrm{~d}$ ) decay product.

${ }^{\text {b }{ }^{228} \mathrm{Ra} \text { and }}{ }^{235} \mathrm{U}$ are not primary radionuclides (Table 6.1-1), but their half-lives are long enough to consider them separately from the parent radionuclides, and they are treated as a primary radionuclide in the decay chain (Section 6.3.5).

\subsubsection{Radionuclide Leaching from the Surface Soil}

The residence time of radionuclides in the soil can influence the contribution to the total exposure of the receptor. Therefore, the biosphere assessment must account for the removal of radionuclides by leaching from the surface soil to the deep soil. Radionuclides removed from the surface soil by leaching would no longer be available to many of the environmental transport and receptor exposure pathways. In an arid climate, leaching may be enhanced by overwatering, which is a common practice to prevent the buildup of salts deposited in soil from irrigation water (BSC 2004 [DIRS 169673], Section 6.9). Under wetter conditions, such as those predicted to occur in the future at Yucca Mountain, leaching also would occur when excess precipitation flows through the surface soil, primarily during the winter.

The process of leaching contaminants from the surface soil is evaluated using element-specific leaching coefficients. Leaching coefficients are calculated using a relationship developed by Baes and Sharp (1983 [DIRS 109606]). This equation is used in other biosphere models as discussed in the model comparison section (Section 7.3.1.1). The equation for the leaching removal constant, $\lambda_{l, i}$, is expressed as

$$
\lambda_{l, i}=\frac{O W}{d \times \theta\left(1+\frac{\rho}{\theta} K d_{i}\right)}
$$

where

$$
\begin{array}{lll}
O W & =\text { crop overwatering rate }(\mathrm{m} / \text { year }) \\
\theta & =\text { volumetric water content of soil (dimensionless) }
\end{array}
$$


$K_{i}=$ solid-liquid partition coefficient for radionuclide $i$ in surface soil

$$
\left(\mathrm{Bq} / \mathrm{kg}_{\text {solid }}\right) /\left(\mathrm{Bq} / \mathrm{m}^{3} \text { liquid }\right)=\left(\mathrm{m}^{3} \text { liquid } / \mathrm{kg}_{\text {solid }}\right)
$$

and the other parameters are defined in Equations 6.4.1-1 and 6.4.1-6.

In arid regions, the overwatering rate usually is determined by calculating the amount of water required to flush accumulated salts out of the surface soil to maintain productivity. The value of this parameter is on the order of $10 \mathrm{~cm} /$ year (BSC 2004 [DIRS 169673]). The volumetric water content of soil is defined as the fraction of the soil volume representing water-filled porosity. The value of this parameter depends on soil texture and ranges from less than 0.1 (dry soils) to 0.4 to 0.5 (water-saturated soils), with typical values of about 0.2 to 0.3 . The partition coefficients depend on soil characteristics, with values ranging over several orders of magnitude (i.e., $1 \times 10^{-3}$ to $1 \times 10^{0} \mathrm{~m}^{3} / \mathrm{kg}$; BSC 2004 [DIRS 169459]).

\subsubsection{Surface Soil Erosion}

Under natural conditions, the rate of soil removal by erosion, generally, is in approximate equilibrium with the rate of soil development from soil forming processes, and under these conditions, soil depth is relatively constant (Troeh et al. 1980 [DIRS 110012], p. 4). Human activities tend to accelerate the rate of soil removal. The removal of surface soil by erosion would result in the loss of radionuclides attached to the soil particles. The rate of radionuclide removal from surface soils is quantified in the ERMYN using a surface soil erosion removal constant $\left(\lambda_{e}\right)$, as introduced in Equation 6.4.1-1. The erosion rate is developed in BSC (2004 [DIRS 169459]), and the surface soil erosion removal constant is evaluated as

$$
\lambda_{e}=\frac{E R}{d \times \rho}
$$

where

$E R=$ annual average erosion rate for the surface soil $\left(\mathrm{kg} /\left(\mathrm{m}^{2}\right.\right.$ year $\left.)\right)$ and the other parameters are defined in Equations 6.4.1-1 and 6.4.1-6.

\subsubsection{Air Submodel}

The air submodel addresses the transport of radionuclides from contaminated water and soil to the air. Particle transport from soil to air is considered to occur primarily via resuspension. The release of radioactive gasses from the soil for species such as radon or carbon dioxide results predominantly from diffusion caused by concentration gradients at the soil-air interface. The transport of radionuclides from water to air also may result from the use of evaporative coolers (for a discussion of the rationale for including this pathway see Appendix D).

\subsubsection{Resuspended Particles from Surface Soil}

Resuspension is the process by which material deposited from the atmosphere onto the ground is subsequently returned to the atmosphere. In the ERMYN, radionuclide concentrations in the air are used to estimate the inhalation dose (Section 6.4.8) and crop contamination by the deposition of resuspended particles onto plant surfaces (Section 6.4.3). Radionuclide concentrations in the 
air are estimated differently for the inhalation exposure and for particulate deposition on crops, primarily because of the differences in mass loading among environments.

For direct deposition on crops, the activity concentration of resuspended particles is linked to the activity concentration in the local surface soil. The relationship between these quantities is expressed as

$$
C a_{p, i}=S \times C s_{m, i}=\frac{\mathrm{S}}{\rho_{s}} C s_{i}
$$

where

$$
\begin{aligned}
C a_{p, i}= & \text { activity concentration of radionuclide } i \text { in the air from soil resuspension }\left(\mathrm{Bq} / \mathrm{m}^{3}\right) \\
C s_{m, i}= & \text { equilibrium activity concentration of radionuclide } i \text { in the surface soil per unit } \\
& \text { mass }(\mathrm{Bq} / \mathrm{kg})(\text { Equation } 6.4 .1-5) \\
S & =\text { mass concentration of resuspended particles; mass loading }\left(\mathrm{kg} / \mathrm{m}^{3}\right) \\
C s_{i}= & \text { equilibrium activity concentration of radionuclide } i \text { in the surface soil per unit } \\
& \text { area }\left(\mathrm{Bq} / \mathrm{m}^{2}\right) \\
\rho_{s}= & \text { areal density of surface soil }\left(\mathrm{kg} / \mathrm{m}^{2}\right)(\text { Equation } 6.4 .1-6)
\end{aligned}
$$

The mass loading of resuspended particulates, $S$, (Equation 6.4.2-1), is characteristic of cultivated fields, and is developed based on annual average values (BSC 2004 [DIRS 169458]).

The activity concentration of resuspended particles, used for the assessment of inhalation doses, is calculated for the five environments associated with the human activities considered. In addition, the enhancement factor for the activity concentration of resuspended particulates, $f_{\text {enhance}}$, is defined as the ratio of activity concentration of resuspended particles $(\mathrm{Bq} / \mathrm{kg})$ to total surface soil activity concentration for a given radionuclide $(\mathrm{Bq} / \mathrm{kg})$. The enhancement factor accounts for the fact that the activity concentration of resuspended particulates may be different from that of the soil where they originated. For example, for soil particles contaminated by irrigation water, the contaminant would be adsorbed onto particles in the form of a thin film on the particle surface. The surface coating would result in an increased activity concentration for smaller particles compared to that of larger particles because surface area per unit mass is greater for smaller particles. The enhancement factor is discussed in BSC (2004 [DIRS 169459], Section 6.5).

To account for variation and uncertainty in the characteristics of the RMEI and concentrations of radionuclides in the biosphere, the ERMYN uses a micro-environmental modeling approach to calculate inhalation dose and external exposure. For micro-environmental models, the total exposure environment (i.e., the biosphere) is divided into segments, or environments, with different concentrations of contaminants. The contaminant concentration, time spent exposed to the contaminant, and intake rate or exposure factor (e.g., breathing rate and shielding factor) is determined for each environment, and the total dose is calculated as the sum of the dose from all environments (Mage 1985 [DIRS 162465], pp. 409 and 410). Micro-environmental models are commonly used to evaluate exposure to particulate matter and other contaminants (Duan 1982 [DIRS 162466]; Mage 1985 [DIRS 162465]; Klepeis 1999 [DIRS 160094]). Radionuclide concentrations in the air are calculated as 


$$
C a_{h, i, n}=f_{\text {enhance }, n} C s_{m, i} S_{n}=f_{\text {enhance }, n} \frac{S_{n}}{\rho_{s}} C s_{i}
$$

where

$$
\begin{aligned}
& C a_{h, i, n}=\begin{array}{l}
\text { activity concentration of radionuclide } i \text { in the air from soil resuspension for } \\
\text { the assessment of human inhalation exposure in environment } n\left(\mathrm{~Bq} / \mathrm{m}^{3}\right)
\end{array} \\
& \begin{array}{ll} 
& \text { enhancement factor for the activity concentration of resuspended particulates } \\
f_{\text {enhance, } n} & \text { in environments } n \text { (dimensionless) }
\end{array} \\
& \begin{aligned}
S_{n} & \text { concentration of total resuspended particulates (mass loading) for evaluation } \\
& \text { of inhalation exposure for environment } n\left(\mathrm{~kg} / \mathrm{m}^{3}\right)
\end{aligned} \\
& \quad=\quad \text { index of the environments (see below) } \\
& \text { and the other parameters are defined in Equation } 6.4 .2-1 .
\end{aligned}
$$

Five environments associated with different human activities are considered in the ERMYN, four in the contaminated area: active outdoors $(n=1)$, inactive outdoors $(n=2)$, active indoors $(n=3)$, asleep indoors $(n=4)$, and one outside of the contaminated area $(n=5)$. The activity concentration in the air outside the contaminated area is zero. These mutually exclusive environments represent behavioral and environmental combinations for which the receptor would receive a substantially different rate of exposure via inhalation or external exposure.

Active Outdoors - This category includes time spent outdoors in contaminated areas conducting activities that would resuspend soil, including dust-generating activities while working (e.g., plowing, excavating, and livestock operations) and recreating outdoors (e.g., gardening, landscaping, and riding horses or motorbikes). Because dust concentrations decrease rapidly after the dust-disturbing activities cease (e.g., Pinnick et al. 1985 [DIRS 159577], pp. 103 and 104), this category is limited to the time when the activities are occurring.

Inactive Outdoors - This category includes time spent outdoors in contaminated areas engaged in activities that do not resuspend soil (e.g., sitting, swimming, walking on turf or paved surfaces, barbecuing, and equipment maintenance). This category also includes time spent commuting within the contaminated area because the major roads in the Amargosa Valley are paved.

Active Indoors - This category includes time spent indoors in contaminated areas, awake, and including work time. This value is calculated as the amount of time not spent in the other environments.

Asleep Indoors - This category includes time spent indoors in contaminated areas sleeping.

Away from Potentially Contaminated Area-This category includes time spent away from areas potentially contaminated by groundwater or ash, including time spent commuting to work and working outside the contaminated areas.

The mass concentration of resuspended particulates in the air, $S_{n}$, depends on the environment and the type of activities conducted there. Values of mass loading range over several orders of 
magnitude, from $6 \times 10^{-8} \mathrm{~kg} / \mathrm{m}^{3}$ for indoor environments to $1 \times 10^{-5} \mathrm{~kg} / \mathrm{m}^{3}$ for dusty outdoor environments (BSC 2004 [DIRS 169458], Section 7).

\subsubsection{Aerosols from Evaporative Cooler Operation}

About 73 percent of the residents in Amargosa Valley used evaporative coolers during 1997 (DOE 1997 [DIRS 100332], p. 20), and these coolers might transfer water-borne contaminants to the indoor air. Thus, the ERMYN must include an estimate of the radionuclide concentrations in indoor air when evaporative coolers are in operation so that the radiation dose to the human receptor inhaling the contaminated air can be evaluated. A search of the scientific literature did not find environmental assessments that considered this transport process from water to indoor air. Rather than ignore the process, as had been done in previous assessments, a model was developed to incorporate the process into ERMYN. Based on how evaporative coolers operate and the conservation of radioactivity (i.e., activity transferred to air is equal to the loss of activity from water), the equation $\left(C a_{e, i}\right)\left(F_{\text {air }}\right)=\left(f_{\text {evap }}\right)\left(M_{\text {water }}\right)\left(C w_{i}\right)$ is rearranged, and radionuclide concentrations in indoor air are estimated as

$$
C a_{e, i}=f_{\text {evap }} \frac{M_{\text {water }}}{F_{\text {air }}} C w_{i}
$$

where

$$
\begin{array}{ll}
C a_{e, i} & =\text { activity concentration of radionuclide } i \text { in the air resulting from the operation } \\
& \text { of an evaporative cooler }\left(\mathrm{Bq} / \mathrm{m}^{3}\right) \\
f_{\text {evap }} & =\text { fraction of radionuclides in water transferred to indoor air (dimensionless) } \\
M_{\text {water }} & =\text { water evaporation rate (water use) for an evaporative cooler }\left(\mathrm{m}^{3} / \mathrm{hr}\right) \\
F_{\text {air }} & =\text { air flow rate for an evaporative cooler }\left(\mathrm{m}^{3} / \mathrm{hr}\right) \\
C w_{i} & =\text { activity concentration of radionuclide } i \text { in the groundwater }\left(\mathrm{Bq} / \mathrm{m}^{3}\right)
\end{array}
$$

The fraction of radionuclides that remain in the reservoir, bleed-off water, or in the pads of evaporative cooler are not further modeled because the associated exposure pathways do not contribute significantly to the RMEI's dose, as shown in Appendix D. Evaporation and air flow rates are estimated based on specifications of residential evaporative cooling units. The typical evaporation rate is about $20 \mathrm{~L} / \mathrm{hr}$, and typical air flow rates range from 2,000 to $10,000 \mathrm{~m}^{3} / \mathrm{hr}$ (BSC 2004 [DIRS 169672]). The fraction of radionuclides transferred from the water to the indoor air is an important parameter that is not available in the literature, but the theoretical range is from 0 to 1 (BSC 2004 [DIRS 169672]).

Radon, primarily ${ }^{222} \mathrm{Rn}$, released from evaporative coolers is not considered in this submodel, and justification is provided in Section 7.4.3.1. The calculation of activity concentrations in the air resulting from evaporative coolers does not include consideration of radionuclide buildup in the indoor air. This is because the air flow associated with the use of these coolers would result in dilution and elimination of the airborne radionuclides (Approximation 11). 


\subsubsection{Radon Exhalation from Surface Soil}

The inhalation of radon decay products is a major, and in many cases, the dominant dose contributor of internal radiation when radium isotopes are present in the soil (Yu et al. 2001 [DIRS 159465], p. C-3). Radon-222, a decay product of ${ }^{226} \mathrm{Ra}$ (a primary radionuclide; Table 6.3-7), is the most common radon isotope. Other radon isotopes, such as ${ }^{220} \mathrm{Rn}$ and ${ }^{219} \mathrm{Rn}$, are less important because they are short-lived and their parent radium isotopes, ${ }^{224} \mathrm{Ra}$, and ${ }^{223} \mathrm{Ra}$, respectively, are short-lived and are not designated as radionuclides of interest in the TSPA-LA. Thus, ${ }^{222} \mathrm{Rn}$ is the only radon isotope considered in the ERMYN.

Outdoor Radon Concentration-The concentration of radon outdoors is estimated from the amount of radon exhaled from the soil. A screening calculation, based on the concentration ratio (i.e., the release factor of ${ }^{222} \mathrm{Rn}$ ) of ${ }^{222} \mathrm{Rn}$ activity in the air to ${ }^{226} \mathrm{Ra}$ activity in the soil (NCRP 1999 [DIRS 155894], Section 4.3.6) is used to estimate the concentration of radon in outdoor air as

$$
C a_{g, R n-222, n=1 \& 2}=f_{m, R n-222} C s_{m, R a-226}
$$

where

$$
\begin{array}{lll}
C a_{g, R n-222, n=1 \& 2}= & \text { activity concentration of }{ }^{222} \mathrm{Rn} \text { in outdoor air }(\mathrm{n}=1 \text { and } 2 \text { for active } \\
& \text { outdoor and inactive outdoor; defined in Equation } 6.4 .2-2)\left(\mathrm{Bq} / \mathrm{m}^{3}\right) \\
= & \text { concentration ratio of }{ }^{222} \mathrm{Rn} \text { activity in the air to }{ }^{226} \mathrm{Ra} \text { activity in soil } \\
f_{m, R n-222} & \text { (radon release factor) }\left(\mathrm{kg} / \mathrm{m}^{3}\right) \\
C s_{m, R a-226} & \text { activity concentration of }{ }^{226} \mathrm{Ra} \text { in surface soil }(\mathrm{Bq} / \mathrm{kg})
\end{array}
$$

Using an average outdoor ${ }^{222} \mathrm{Rn}$ concentration value of $10 \mathrm{~Bq} / \mathrm{m}^{3}$ and an average ${ }^{226} \mathrm{Ra}$ concentration in the soil of $40 \mathrm{~Bq} / \mathrm{kg}$ (NCRP 1999 [DIRS 155894], Section 4.3.6), the release factor for ${ }^{222} \mathrm{Rn}$ would be $0.25\left(\mathrm{~Bq} / \mathrm{m}^{3}\right) /(\mathrm{Bq} / \mathrm{kg})$. This value is large compared to the resuspension contribution for ${ }^{226} \mathrm{Ra}$. For example, using a typical mass loading of about $1 \times 10^{-8}$ to $1 \times 10^{-6} \mathrm{~kg} / \mathrm{m}^{3}$ (Table 6.6-3) and a ${ }^{226} \mathrm{Ra}$ concentration in the soil of $1 \mathrm{~Bq} / \mathrm{kg}$, the ${ }^{226} \mathrm{Ra}$ concentration in the air due to resuspension would be $1 \times 10^{-8}$ to $1 \times 10^{-6} \mathrm{~Bq} / \mathrm{m}^{3}$, whereas the ${ }^{222} \mathrm{Rn}$ concentration in the air would be $0.25 \mathrm{~Bq} / \mathrm{m}^{3}$.

Indoor Radon Concentration-The method for calculating the indoor concentration of radon, taken from RESRAD (Yu et al. 2001 [DIRS 159465], Equation C.12), was developed for a single-story house built on contaminated soil, assuming steady-state conditions between the rate of radon entry into the house and the rate of removal. The main sources of indoor radon are the soil beneath the house and the entry of outdoor air. The surface soil beneath the house contains the equilibrium concentration of ${ }^{226} \mathrm{Ra}$, consistent with the approach of modeling radionuclide concentrations in the soil used in the ERMYN and to eliminate speculation on previous land use. This approach provides upper bound for the indoor radon concentration because it is unlikely that all houses would be built on soil that was continuously irrigated over a period of time sufficiently long to reach the equilibrium concentration of ${ }^{226} \mathrm{Ra}$. Indoor radon concentration is expressed as 


$$
C a_{g, R n-222, n=3 \& 4}=\frac{J_{\text {indoor }}}{v H}+C a_{g, R n-222, n=1 \& 2}
$$

where

$$
\begin{aligned}
\mathrm{Ca}_{g, R n-222, n=384}= & \text { activity concentration of }{ }^{222} \mathrm{Rn} \text { in indoor air }(\mathrm{n}=3 \text { and } 4 \text { for active } \\
& \text { indoor and asleep indoor; defined in Equation } 6.4 .2-2)\left(\mathrm{Bq} / \mathrm{m}^{3}\right) \\
= & \text { radon flux density from the house floor }\left(\mathrm{Bq} /\left(\mathrm{m}^{2} \mathrm{sec}\right)\right) \\
= & \text { interior wall height of the house }(\mathrm{m}) \\
= & \text { house ventilation rate, or air exchange rate }(/ \mathrm{sec}) . \text { This parameter had } \\
& \text { two values: a normal rate }\left(v_{n}\right) \text { and a higher rate used when evaporative } \\
& \text { coolers are in operation }\left(v_{e}\right)
\end{aligned}
$$

and the other parameters are defined in Equation 6.4.2-4.

The radon flux density from the floor of the house can be expressed as a proportion of the total radon flux density from contaminated outdoor soil as

$$
J_{\text {indoor }}=f_{\text {house }} \times J_{\text {outdoor }}
$$

where

$$
\begin{array}{ll}
J_{\text {outdoor }}= & \text { radon flux density from outdoor contaminated soil }\left(\mathrm{Bq} /\left(\mathrm{m}^{2} \mathrm{sec}\right)\right) \\
f_{\text {house }} & \text { fraction of radon released into a house from soil beneath the house } \\
& \text { (dimensionless) }
\end{array}
$$

By combining the Equations 6.4.2-5 and 6.4.2-6, the indoor radon concentration can be calculated as

$$
\begin{aligned}
C a_{g, R n-222, n=3 \& 4} & =C a_{g, R n-222, n=1 \& 2}\left(\frac{f_{\text {house }}}{v_{n} H} \frac{J_{\text {outdoor }}}{C a_{g, R n-222, n=1 \& 2}}+1\right) \\
& =C a_{g, R n-222, n=1 \& 2}\left(\frac{f_{\text {house }}}{C F_{R n-222} v_{n} H}+1\right) \\
& =C a_{g, R n-222, n=1 \& 2} I F_{n, R n-222}
\end{aligned}
$$

where

$\mathrm{CF}_{\mathrm{Rn-222}}=$ ratio of ${ }^{222} \mathrm{Rn}$ concentration in outdoor air to ${ }^{222} \mathrm{Rn}$ flux density from outdoor soil ( $\mathrm{sec} / \mathrm{m})$

$I F_{n, R n-222}=$ indoor ${ }^{222} \mathrm{Rn}$ increase factor for normal ventilation rate (dimensionless) and the other parameters are defined in Equations 6.4.2-5, and 6.4.2-6.

During operation of an evaporative cooler, the increase in indoor radon concentration relative to the outdoor concentration would be relatively low because of the high ventilation rate. The indoor radon concentration during that period can be estimated by 


$$
\begin{gathered}
C a_{g, R n-222, e}=C a_{g, R n-222, n=1 \& 2}\left(\frac{f_{\text {house }}}{C F_{R n-222} v_{e} H}+1\right) \\
=C a_{g, R n-222, n=1 \& 2} I F_{e, R n-222}
\end{gathered}
$$

where

$$
\begin{aligned}
& C a_{g, R n-222, e}= \begin{array}{l}
\text { activity concentration of }{ }^{222} \mathrm{Rn} \text { in indoor air during evaporative cooler } \\
\text { operation }\left(\mathrm{Bq} / \mathrm{m}^{3}\right)
\end{array} \\
& I F_{e, R n-222}= \begin{array}{l}
\text { indoor }{ }^{222} \mathrm{Rn} \text { increase factor for a high ventilation rate during } \\
\text { evaporative cooler operation (dimensionless) }
\end{array} \\
& \text { and the other parameters are defined in Equation 6.4.2-5 and 6.4.2-6. }
\end{aligned}
$$

Radon enters the indoor space through cracks and other openings in the floor and foundations. The fraction of radon released into a house from soil would depend on the type and number of such openings in the floor in the house (typical range of 0.10 to 0.25 ; Table 6.6-3). The fraction retained in the house would primarily depend on the ventilation rate. Ventilation rates would depend on ambient temperatures because during cold weather, residents would be expected to close up their houses to retain heat, but during warmer weather, they would be expected to open their houses to allow ventilation. Ventilation rates range from about 0.3 to 2.9 air exchanges per hour; however, when evaporative coolers are in operation, house air exchange rates range from 1 to 30 exchanges per hour (BSC 2004 [DIRS 169672], Section 6.6.2). Similar to the radon release factor, $f_{m, R n-222}$, the ratio of the concentration of ${ }^{222} \mathrm{Rn}$ in outdoor air to ${ }^{222} \mathrm{Rn}$ flux density from radium contaminated outdoor soil is based on a global average value, about $300 \mathrm{sec} / \mathrm{m}$ $\left(10 \mathrm{~Bq} / \mathrm{m}^{3}\right.$ to $0.033 \mathrm{~Bq} /\left(\mathrm{m}^{2} \mathrm{sec}\right)$ ) (BSC 2004 [DIRS 169672], Section 6.6). Because of evaporative coolers, indoor radon concentrations must be considered separately when the cooler is turned on or off, as discussed in Section 6.4.8.4.

The radon contribution from evaporative coolers and household water use is excluded from the ERMYN, as justified in Section 7.4.3.1.

\subsubsection{Plant Submodel}

If groundwater is contaminated, irrigated crops become contaminated by the deposition of radionuclides onto the above-ground plant parts and through root uptake of radionuclides from the soil. The plant submodel includes both processes.

Radionuclides would be deposited on plant surfaces from contaminated irrigation water and from resuspension of contaminated surface soil. Some of the radionuclides intercepted by crops would be retained on, or in, the plant and some would be removed by weathering. Contamination of plants by direct deposition would be important for elements that have low rates of root uptake (e.g., actinides: plutonium, neptunium, and americium). For environmentally mobile elements (e.g., technetium, iodine, and chlorine), root uptake usually is more important than direct deposition (see values of transfer factors; Table 6.6-3). Radionuclide concentrations in the surface soil are considered in the surface soil submodel (Section 6.4.1), and radionuclide concentrations in the air are considered in the air submodel (Section 6.4.2). 
Soil splash due to irrigation or rain is an additional mechanism used in some biosphere models (e.g., BIOMASS 2000 [DIRS 154522], Section 7). However, the process of soil splash is considered equivalent to direct deposition of resuspended particles on plant surfaces (Section 6.4.3.3). Therefore, soil splash is not considered separately in the ERMYN. Furthermore, none of the published biosphere models considers dust deposition combined with irrigation or rain splash. For model validation, this ACM 4 (direct deposition of airborne particulates) is compared with the methods used in the plant submodel (Section 7.4.4.3).

Based on the typical approach used in assessments of environmental radionuclide releases, the ERMYN includes four types of crops consumed by humans: leafy vegetables, other vegetables, fruit, and grain. Leafy vegetables include plants such as lettuce, spinach, and cabbage, the edible portions of which (i.e., the leaves) grow aboveground, are exposed, and can be eaten directly with little processing (Napier et al. 1988 [DIRS 157927], Section 4.7.4). Other vegetables include root crops (e.g., carrots and potatoes) and crops where the edible parts are unlikely to be exposed directly (e.g., peas and beans that grow inside pods). Fruits include a variety of products such as berries, grapes, currants, melons, pomes (e.g., apples), and drupes (e.g., peaches). Grains include seed-producing crops such as wheat, corn, and barley.

In addition to crops consumed by humans, crops consumed by animals are considered in the plant submodel. Fresh forage (e.g., alfalfa) consumed by beef cattle and dairy cows, and grain fed to poultry and egg-laying hens are considered (see Approximation 8 about animal diets). The mathematical submodel presented in this section applies to all types of crops, but some input parameters are crop-type specific.

The plant submodel does not include radionuclide decay following harvest because the radionuclides of interest are long-lived and decay little during short-term storage. The activity concentration in crops is calculated for the wet-weight of the edible plant portions. The three environmental transport processes (roots, water, and dust) are considered independent, and the total activity concentration in crops is the sum of the three contributions, estimated as

$$
C p_{i, j}=C p_{\text {root }, i, j}+C p_{\text {water }, i, j}+C p_{\text {dust }, i, j}
$$

where

$$
\begin{aligned}
& C p_{i, j} \quad=\text { activity concentration of radionuclide } i \text { in crop type } \mathrm{j}(\mathrm{Bq} / \mathrm{kg} \text { wet }) \\
& j \quad=\text { crop-type index; } j=1 \text { for leafy vegetables, } 2 \text { for other vegetables, } 3 \text { for fruit, } \\
& 4 \text { for grain (consumed by humans and poultry), and } 5 \text { for fresh forage feed } \\
& \text { (used for beef cattle and dairy cows) } \\
& C p_{\text {root }, i, j}=\text { activity concentration of radionuclide } i \text { in crop type } j \text { contributed from plant } \\
& \text { root uptake }(\mathrm{Bq} / \mathrm{kg} \text { wet }) \\
& C p_{\text {water }, i, j}=\text { activity concentration of radionuclide } i \text { in crop type } j \text { contributed from direct } \\
& \text { deposition on crop leaves due to interception of contaminated irrigation } \\
& \text { water }\left(\mathrm{Bq} / \mathrm{kg}_{\text {wet }}\right) \\
& C p_{\text {dust }, i, j}=\text { activity concentration of radionuclide } i \text { in crop type } j \text { contributed from the } \\
& \text { direct deposition on crop leaves due to interception of resuspended particles } \\
& \text { from contaminated soil }\left(\mathrm{Bq} / \mathrm{kg}_{\text {wet }}\right)
\end{aligned}
$$




\subsubsection{Root Uptake}

The radionuclides in the contaminated irrigation water would be transferred to the crops through root uptake. The extent to which plant roots absorb radionuclides from the soil depends on the physiology of the plant, the properties of the soil, and the characteristics of the radionuclide. The uptake of radionuclides by plants usually is considered proportional to radionuclide concentrations in the soil.

The surface soil submodel is used to calculate radionuclide concentrations in the surface layer of the soil. It is assumed that all roots remain in the surface layer, which maximizes the uptake of radionuclides in the submodel (Approximation 7). This approach eliminates the need to determine the fraction of roots in the surface soil. If radionuclides are taken up by the plant roots, the contamination will be internal to the plants and not subject to removal by weathering or food processing. The activity concentration of radionuclides in crops from root uptake is estimated as

$$
C p_{\text {root }, i, j}=C s_{m, i} F_{s \rightarrow p, i, j} D W_{j}
$$

where

$$
\begin{aligned}
C s_{m, i}= & \text { activity concentration of radionuclide } i \text { in surface soil }(\mathrm{Bq} / \mathrm{kg} \text { dry soil }) \\
F_{s \rightarrow p, i, j}= & \text { soil-to-plant transfer factor for radionuclide } i \text { and crop type } j(\mathrm{~Bq} / \mathrm{kg} \text { dry plant } \\
& \text { per } \left.\mathrm{Bq} / \mathrm{kg}_{\text {dry soil }}\right) \\
D W_{j}= & \text { dry-to-wet weight ratio for edible parts of crop type } j\left(\mathrm{~kg} \text { dry-plant } / \mathrm{kg}_{\text {wet-plant }}\right)
\end{aligned}
$$

The soil-to-plant transfer factor is defined as the ratio of activity concentration of a given radionuclide in dry soil to the activity concentration in dry plants. Observed values of transfer factors vary widely, mainly because of different soils, vegetation types, and environmental conditions (UNSCEAR 2000 [DIRS 158644], p. 39). Because the values of transfer factors represent crop types, rather than the individual crop species, there is uncertainty associated with this parameter. The values of radionuclide-specific and crop-type-specific soil-to-plant transfer factors are developed in BSC (2004 [DIRS 169672]), which also includes a more detailed discussion of the transfer factors. The dry-to-wet weight ratio is represented by the fraction of dry weight of foodstuff in the total (wet) weight of the foodstuff. Typical values range from a few percent for leafy vegetables to about 90 percent for grain (BSC 2004 [DIRS 169673]).

\subsubsection{Uptake Following Foliar Interception of Irrigation Water}

Radionuclide transfer to plants through foliar uptake involves three processes: interception, translocation, and retention (IAEA 1994 [DIRS 100458], Section 2). Interception is the process by which radionuclide contaminants in the atmosphere are deposited on plant surfaces in wet (irrigation water) or dry (resuspended soil) forms. The plant submodel includes two mechanisms of radionuclide uptake from the deposition of contaminants on plants: leaf uptake following interception of irrigation water (this section) and leaf uptake following interception of resuspended particles (Section 6.4.3.3). 
Translocation is the process by which chemical elements, initially deposited on the leaf surface, move from the site of deposition to the edible parts of the plant, including parts not directly affected by deposition. The fraction of radionuclides translocated depends on the plant species, the chemical and physical forms of the radionuclides, the stage of plant development, and weathering conditions. In this submodel, translocation refers to that portion of activity initially deposited on a plant surface that contributes to activity in edible parts of the plant, regardless of the external or internal nature of contamination.

Retention is the result of weathering and other field losses, in which a fraction of the radionuclides initially intercepted by foliage is detached from plant surfaces and deposited on the ground. In the ERMYN, the calculation of activity deposited on leaves is based on daily average irrigation rates per crop type, and is carried out within the plant submodel. The activity deposited on the ground is calculated based on the average annual irrigation rate for all crops, which incorporates crop rotation and land use changes, and is carried out within the soil submodel. These two irrigation rates are linked, as the activity deposited on the ground is not depleted by foliar deposition (Approximation 6).

In the arid to semi-arid region at Yucca Mountain, crops must be irrigated frequently. Therefore, deposition of radionuclides on plant surfaces by irrigation is modeled as a quasi-continuous process throughout the plant growing season. Radionuclide concentrations in crops due to leaf uptake from contaminated irrigation water sprayed on plants is expressed as

$$
C p_{\text {water }, i, j}=\frac{D w_{i, j} f_{o, j} R w_{j} T_{j}}{\lambda_{w} Y_{j}}\left(1-e^{-\lambda_{w} t_{g, j}}\right)
$$

where

$$
\begin{aligned}
& D w_{i, j}=\text { deposition rate of radionuclide } i \text { due to application of irrigation water on crop } \\
& \text { type } j\left(\mathrm{~Bq} /\left(\mathrm{m}^{2} \mathrm{~d}\right)\right) \\
& f_{o, j}=\text { fraction of irrigation applied using overhead methods for plant type } j \\
& \text { (dimensionless) } \\
& R w_{j} \quad=\quad \text { interception fraction of irrigation water for crop type } j \text { (dimensionless) } \\
& T_{j} \quad=\text { translocation factor for crop type } j \text { (dimensionless) } \\
& \lambda_{w}=\text { weathering constant (per d), which can be calculated from weathering half-life } \\
& \text { ( } T_{w} \text { in units of day) by } \lambda_{w}=\ln (2) / T_{w} \\
& t_{g, j}=\text { crop growing time for crop type } j(\mathrm{~d}) \\
& Y_{j}=\text { crop yield or wet biomass for crop type } j\left(\mathrm{~kg}_{\text {wet weight }} / \mathrm{m}^{2}\right)
\end{aligned}
$$

For overhead irrigation (i.e., sprinkler or spray), the rate of radionuclide deposition onto the crops, $D w_{i, j}$, is the product of the irrigation rate and the radionuclide concentration in the water. In this submodel, the radionuclide deposition rate from irrigation water is estimated as

$$
D w_{i, j}=C w_{i} I R D_{j}
$$


where

$I R D_{j}=$ daily average irrigation rate for crop type $j$ during the growing season $(\mathrm{m} / \mathrm{d})$ and the other parameter is defined in Equation 6.4.1-1.

The daily irrigation rates for crops vary during the growing season; therefore, a daily average rate over the entire growing season is used in the submodel. As noted previously, the daily average irrigation rate for a crop (Equation 6.4.3-4) and the annual average irrigation rate on land (Equation 6.4.1-1) serve different purposes. The daily average irrigation rate for a crop type is used to calculate activity deposited on the crop leaves in the plant submodel. The annual average irrigation rate is used to calculate the activity deposited on surface soil. The two irrigation rates are determined based on overwatering requirements, precipitation, and evapotranspiration in BSC (2004 [DIRS 169673]).

The fraction of water applied using overhead irrigation methods, $f_{o, j}$, is not considered in other biosphere models. However, the rate of contaminant deposition on leaves depends on how irrigation water is applied, and some irrigation methods (e.g., flood and drip irrigation) that cause little foliar deposition are used in the Amargosa Valley (BSC 2004 [DIRS 169673], Section 6.3). Thus, this parameter, which is crop-type specific with values ranging from about 0.25 to one, is used to incorporate variation related to site-specific irrigation methods.

The interception fraction for irrigation water, $R w_{j}$, quantifies the initial fraction of radionuclides deposited on plant surfaces following irrigation with contaminated water. The possible range for this parameter is 0 to 1 . Values for the irrigation interception fraction can be determined using empirical equations or values from the literature. In this submodel, the interception fraction is estimated using an empirical equation (Hoffman et al. 1989 [DIRS 124110]) based on crop biomass and the amount and intensity of precipitation and irrigation. The empirical equation was developed using multiple regression analysis on data from experiments where simulated rain was applied to three types of plants: clover, fescue, and mixed grasses. The simulated rain contained five radionuclides: ${ }^{131} \mathrm{I},{ }^{7} \mathrm{Be},{ }^{141} \mathrm{Ce},{ }^{95} \mathrm{Nb}$, and ${ }^{85} \mathrm{Sr} .{ }^{131} \mathrm{I}$ and ${ }^{7} \mathrm{Be}$ were applied in the form of dissolved species in irrigation water, but the remaining radionuclides were used as tracers in the form of insoluble polystyrene microspheres (Hoffman et al. 1992 [DIRS 124114]). Because the majority of radioactive contaminants in the Yucca Mountain groundwater would be soluble, the results of the ${ }^{131} \mathrm{I}$ and ${ }^{7} \mathrm{Be}$ experiments are of interest for the groundwater scenario.

The experiments indicate that anionic ${ }^{131} \mathrm{I}$, present as periodate $\left(\mathrm{IO}_{4}{ }^{-}\right)$, is essentially removed with water after the vegetation surface became saturated, and that cationic ${ }^{7} \mathrm{Be}^{2+}$ is adsorbed to, or settles out on, the plant surfaces. The discrepancy between the behavior of the anionic and cationic species is consistent with a negative charge on the plant surface. Hoffman et al. (1995 [DIRS 124120]) show similar experimental results involving six soluble radionuclides, including ${ }^{51} \mathrm{Cr}$ (as $\mathrm{CrO}_{4}{ }^{2-}$ ), ${ }^{85} \mathrm{Sr}\left(\right.$ as $\mathrm{Sr}^{2+}$ ), ${ }^{109} \mathrm{Cd}\left(\right.$ as $\mathrm{Cd}^{2+}$ ), ${ }^{7} \mathrm{Be}$ (as $\mathrm{Be}^{2+}$ ), ${ }^{131} \mathrm{I}$ (as $\mathrm{I}^{-}$), and ${ }^{35} \mathrm{~S}$ (as $\mathrm{SO}_{4}{ }^{2-}$ ). The results indicate that ${ }^{7} \mathrm{Be}$ has the highest interception fraction, while ${ }^{131} \mathrm{I}$ has the lowest.

The empirical equation for the interception fraction, $R w$, from Hoffman et al. (1989 [DIRS 124110]), which is based on the results of experiments with ${ }^{7} \mathrm{Be}$ and ${ }^{131} \mathrm{I}$, is expressed as

$$
R w_{j}=K_{1} D B_{j}^{K_{2}} I A_{j}^{K_{3}} I^{K_{4}}
$$


where

$$
\begin{aligned}
& R w_{j} \quad=\quad \text { interception fraction of irrigation water for crop type } j \\
& \text { (dimensionless) } \\
& K_{1}, K_{2}, K_{3} \text {, and } K_{4}=\text { empirical constants }\left(K_{1} \text { is in units of }\left(\mathrm{kg} / \mathrm{m}^{2}\right)^{-K 2}(\mathrm{~mm})^{-K 3}(\mathrm{~cm} / \mathrm{hr})^{-K 4}\right. \text {, } \\
& \text { and } K_{2}, K_{3} \text { and } K_{4} \text { are dimensionless) } \\
& D B_{j} \quad=\text { standing biomass of crop type } j \text { (in units of } \mathrm{kg} \text { dry weight } / \mathrm{m}^{2} \text { ) } \\
& I A_{j} \quad=\text { amount of irrigation per application event for crop type } j \text { (value in } \\
& \text { units of } \mathrm{mm} \text { ) } \\
& \text { I } \quad=\text { irrigation intensity (value in units of } \mathrm{cm} / \mathrm{hr} \text { ) }
\end{aligned}
$$

Because this is a regression equation from experimental data, values for the input parameters must be used in the units specified above. The empirical constants in Equation 6.4.3-5, developed based on given parameter units for standing biomass, irrigation amount, and irrigation intensity, depend on the plant type and contaminant form. The recommended values (Hoffman et al. (1989 [DIRS 124110]) are:

$$
\begin{array}{llll}
K_{1}=2.29 & \text { for beryllium }\left(\mathrm{Be}^{+}\right) ; & K_{1}=1.54 & \text { for iodine }\left(\mathrm{I}^{-}\right) \\
K_{2}=0.695 & \text { for beryllium }\left(\mathrm{Be}^{+}\right) ; & K_{2}=0.697 & \text { for iodine }\left(\mathrm{I}^{-}\right) \\
K_{3}=-0.29 & \text { for beryllium }\left(\mathrm{Be}^{+}\right) ; & K_{3}=-0.909 & \text { for iodine }\left(\mathrm{I}^{-}\right) \\
K_{4}=-0.341 & \text { for beryllium }\left(\mathrm{Be}^{+}\right) ; & K_{4}=-0.049 & \text { for iodine }\left(\mathrm{I}^{-}\right)
\end{array}
$$

Because radionuclides in the groundwater may be present as different species, the empirical constants cannot be determined unequivocally. Therefore, the ERMYN uses a simplified approach based on the empirical constants for beryllium. This approach results in the highest values of the irrigation interception fraction because the experimental results indicate that beryllium cations, $\mathrm{Be}^{2+}$, in the simulated rain water have the highest interception fraction among the species tested (Hoffman et al. (1989 [DIRS 124110]; Hoffman et al. (1995 [DIRS 124120]).

The standing biomass of the growing crop, $D B$, is a crop-type-specific parameter that represents the capacity of the plants to intercept irrigation water. A typical range for dry biomass is 0.1 to $1.0 \mathrm{~kg} / \mathrm{m}^{2}$ (BSC 2004 [DIRS 169673], Section 6.1).

The amount of irrigation per irrigation event, $I A$, in the experiment ranged from 1 to $30 \mathrm{~mm}$, while the experimental irrigation (or rain) intensity, $I$, ranged from 1.4 to $12.2 \mathrm{~cm} / \mathrm{hr}$ (Hoffman et al. 1995 [DIRS 124120]). Some parameter values representative of crop irrigation in arid and semi-arid environments exceed the ranges used in these experiments (BSC 2004 [DIRS 169673]). Therefore, a numerical evaluation is used to ensure that the use of this equation is valid (Section 7.4.4.2).

The translocation factor, $T_{j}$ (Equation 6.4.3-3), is the ratio of activity on $1 \mathrm{~m}^{2}$ of edible plant parts at harvest $\left(\mathrm{Bq} / \mathrm{m}^{2}\right)$ to the activity retained on $1 \mathrm{~m}^{2}$ of foliage at the time of deposition $\left(\mathrm{Bq} / \mathrm{m}^{2}\right)$ (IAEA 1994 [DIRS 100458], p. 12). This factor is equal to the fraction of a chemical element initially deposited on the leaf surface that is retained and translocated to the edible plant parts. The possible range for this parameter is zero to one (BSC 2004 [DIRS 169672]).

Radionuclide concentrations on vegetation may be reduced by a variety of processes (e.g., the actions of wind, washout, surface abrasion, volatilization, and growth of new tissue) other than 
by radioactive decay. These processes can be described by a first order removal submodel with an aggregated weathering constant or weathering rate (IAEA 2001 [DIRS 158519], Section 5.1.1.2). Similar to radionuclide half-life and decay constants, the relationship between the weathering half-life, $T_{w}$, and the weathering constant, $\lambda_{w}$, is $T_{w}=\ln 2 / \lambda_{w}$. The weathering half-life describes the time that it would take for the amount of contaminant deposited on a plant to be reduced to one-half of the initial value. The range of values for the weathering half-life reported in the literature is 6 to $56 \mathrm{~d}$ (Till and Meyer 1983 [DIRS 101895], p. 5-36). A typically used value for the weathering half-life is $14 \mathrm{~d}$ (BSC 2004 [DIRS 169672], Section 6.2.2.3). There is evidence that the weathering half-life may depend on the plant type and the radionuclide (Smith et al. 1996 [DIRS 101085], p. 5-30); however, this dependence usually is not included in biosphere models.

The crop growing time, $t_{g, j}$ depends on the crop type and climatic conditions (BSC 2004 [DIRS 169673], Section 6.4). The crop growing time and weathering rate are used in the exponential term in Equation 6.4.3-3. Because the typical value of the weathering half-life is $14 \mathrm{~d}$, the system is considered to reach equilibrium after about three weathering half-lives, a period typically shorter than the crop growing time. Therefore, calculations of activity concentration in crops resulting from the foliar interception of contaminant usually are insensitive to the value of the crop growing time.

The wet yield of crops, $Y_{j}$, is used to describe the mass of edible plant parts grown per unit area of farmland. This is a crop-type-specific parameter. The range of crop yields for agricultural and garden crops typically grown in southern Nevada is about $0.6 \mathrm{~kg} / \mathrm{m}^{2}$ for grain to about $4 \mathrm{~kg} / \mathrm{m}^{2}$ for other vegetables (BSC 2004 [DIRS 169673], Section 6.11).

\subsubsection{Uptake by Foliar Interception of Airborne Particulates}

The other environmental transport pathway leading to radionuclide contamination of plant surfaces is deposition of resuspended soil, particularly on foliar surfaces. The mathematical representation of this process is similar to that used to represent plant uptake by foliar interception of irrigation water (Equation 6.4.3-3). The radionuclide concentration in crops contributed from uptake by foliar interception of airborne particulates is expressed as

$$
C p_{d u s t, i, j}=\frac{D a_{i} R a_{j} T_{j}}{\lambda_{w} Y_{j}}\left(1-e^{-\lambda_{w} t_{g, j}}\right)
$$

where

$\begin{aligned} D a_{i}= & \text { deposition rate of radionuclide } i \text { with resuspended particulates }\left(\mathrm{Bq} /\left(\mathrm{m}^{2} \mathrm{~d}\right)\right) \\ R a_{j} & = \\ & \text { interception fraction for airborne particulates for crop type } j \\ & \text { (dimensionless); see Equation 6.4.3-8 }\end{aligned}$

and the other parameters are defined in Equations 6.4.3-1 and 6.4.3-3.

The deposition rate of contaminated airborne particles, $D a_{i}$, quantifies the combined effect of contaminant removal from the atmosphere by several processes, such as gravitational settling, diffusion, and turbulent transport. The deposition rate, which can be derived by letting a uniform 
volumetric activity fall with an average velocity representative of the assembly of particulates for a defined period of time, is mathematically represented as

$$
D a_{i}=8.64 \times 10^{4} C a_{p, i} V_{d}
$$

where

$$
\begin{aligned}
C a_{p, i}= & \text { activity concentration of radionuclide } i \text { in the air used for evaluation of } \\
& \text { activity deposition on crops }\left(\mathrm{Bq} / \mathrm{m}^{3}\right) \text {, see Equation } 6.4 .2-1 \\
V_{d} & \text { dry deposition velocity for airborne particulates }(\mathrm{m} / \mathrm{sec}) \\
8.64 \times 10^{4}= & \text { unit conversion factor }(\mathrm{sec} / \mathrm{d})
\end{aligned}
$$

The dry deposition velocity for airborne particulates, $V_{d}$, is a function of particle size. For climatic conditions in the Amargosa Valley, the appropriate velocity ranges from $5 \times 10^{-4}$ to $3 \times 10^{-2} \mathrm{~m} / \mathrm{s}$ (BSC 2004 [DIRS 169672], Section 6.2.2.1).

The interception fraction for airborne particulates, $R a_{j}$, quantifies the initial fractional deposition of radionuclides on plant surfaces from dry deposition. This parameter is crop-type dependent with a range from zero to one. The value of this parameter can be selected from the literature or calculated using an empirical formula. An empirical formula, selected to estimate the value of the interception fraction for airborne particulates, is

$$
R a_{j}=1.0-e^{-a_{j} D B_{j}}
$$

where

$a_{j}=$ an empirical factor for crop type $j\left(\mathrm{~m}^{2} / \mathrm{kg}\right.$ dry biomass)

and the other parameters are defined in Equations 6.4.3-5 and 6.4.3-6.

This empirical equation, including the values of the empirical factor is adopted from the GENII-S model. The recommended values for this factor are 2.9 for leafy vegetables, fresh forage feed, and grain; and 3.6 for root, other vegetables, and fruit (Napier et al. 1988 [DIRS 157927], Section 4.7.4). This empirical formula is modified to use dry biomass (BSC 2004 [DIRS 169673], Section 6.1) rather than wet standing biomass of growing vegetation times the dry-to-wet biomass ratio (Section 7.3.3.3).

\subsubsection{Animal Submodel}

The animal food chain involves the transfer of radionuclides from animal feed, water, and soil to animal products consumed by the receptor. In this submodel, humans consume meat, poultry, milk, and eggs, four food categories commonly used in radiological assessment models. The regional food consumption survey (DOE 1997 [DIRS 100332]), the basis for calculating consumption rates of locally produced animal products in the Amargosa Valley (BSC 2004 [DIRS 169671]), also includes these four categories. This submodel does not consider the inhalation of contaminated air by animals (Section 6.3.1). 
Beef is the representative animal product for all meat (including beef, pork, wild game, and other meat; Approximation 9). To incorporate the use of wild and natural land and water (FEP 2.4.08.00.0A), consumption rates for local game were obtained from the Amargosa Valley food consumption survey (DOE 1997 [DIRS 100332]). The consumption rate for meat is then calculated by combining the consumption rates for wild game and all other meats (BSC 2004 [DIRS 169671], Section 6.4.2). This is done because game could eat crops from irrigated farmlands and drink from irrigation canals and fish ponds. Milk from cows is the representative milk product, chickens are the representative poultry, and chicken eggs are the representative type of eggs (Approximation 9).

Beef cattle and dairy cows are raised using locally grown fresh forage, and poultry and laying hens are fed locally grown grain (Approximation 8). Animals drink contaminated groundwater. Radionuclide concentrations in the soil consumed by animals is based on the long-term concentration obtained using the annual average irrigation rate (Section 6.4.1). Radionuclide decay is not considered in the animal submodel because of the long half-life of the primary radionuclides (Table 6.3-7).

Radionuclide concentrations in animal products are calculated based on a media equilibrium model that uses transfer coefficients to relate the daily radionuclide intake by animals to radionuclide concentrations in animal products. The transfer coefficient represents the fraction of daily radionuclide intake $(\mathrm{Bq} / \mathrm{d}$ ) that transfers into the animal product (based on mass, $\mathrm{Bq} / \mathrm{kg}$, or volume, $\mathrm{Bq} / \mathrm{L}$ ). The daily radionuclide intake includes contributions from feed, water, and ingested soil. Radionuclide concentrations in animal products $\left(C d_{i, k}\right)$ are estimated as

$$
C d_{i, k}=C d_{\text {feed } i, k}+C d_{\text {water } i, k}+C d_{\text {soil } i, k}
$$

where

$$
\begin{aligned}
& C d_{i, k}=\text { activity concentration of radionuclide } i \text { in animal product } k(\mathrm{~Bq} / \mathrm{kg} \text { fresh weight } \\
& \text { or } \mathrm{Bq} / \mathrm{L} \text { for milk) } \\
& k=\text { animal product index; } k=1 \text { for beef, } 2 \text { for milk, } 3 \text { for poultry, } 4 \text { for eggs } \\
& C d_{\text {feed } i, k}=\text { activity concentration of radionuclide } i \text { in animal product } k \text { due to ingestion } \\
& \text { of contaminated animal feed (Bq/kg or Bq/L for milk) } \\
& C d_{\text {water } i, k}=\text { activity concentration of radionuclide } i \text { in animal product } k \text { due to ingestion } \\
& \text { of contaminated water }(\mathrm{Bq} / \mathrm{kg} \text { or } \mathrm{Bq} / \mathrm{L} \text { for milk) } \\
& C d_{\text {soil } i, k}=\text { activity concentration of radionuclide } i \text { in animal product } k \text { due to ingestion } \\
& \text { of contaminated soil (Bq/kg or Bq/L for milk) }
\end{aligned}
$$

\subsubsection{Animal Feed}

The radionuclide concentrations in specific animal products from the ingestion of contaminated feed is given by

$$
C d_{\text {feed } i, k}=F m_{i, k} C p_{i, j} Q f_{k}
$$


where

$$
\begin{array}{ll}
F m_{i, k}= & \text { animal intake-to-animal product transfer coefficient for radionuclide } i \text { and } \\
& \text { animal product } k(\mathrm{~d} / \mathrm{kg} \text { fresh weight } \text { or } \mathrm{d} / \mathrm{L} \text { for milk) } \\
C p_{i, j}= & \text { activity concentration of radionuclide } i \text { in animal feed } j(\mathrm{~Bq} / \mathrm{kg} \text { fresh weight }) \\
j & =\begin{array}{l}
\text { animal feed index; } j=5 \text { fresh forage for beef }(k=1) \text { and milk }(k=3), \text { while } \\
j=4 \text { grain for poultry }(k=2) \text { and egg hens }(k=4)
\end{array} \\
k & =\begin{array}{l}
\text { animal product index; see above } \\
Q f_{k}
\end{array} \quad \text { animal consumption rate of feed }(\mathrm{kg} / \mathrm{d})
\end{array}
$$

The radionuclide concentrations in animal feed are taken from the plant submodel. The transfer coefficients for individual animal products depend on the element, the chemical form, and the type of animal product. Direct measurements of transfer coefficients are scarce (IAEA 1994 [DIRS 100458], p. 38). Many published values are derived from sources other than explicit experimental data, such as stable element concentrations in feed and animal tissues, extrapolation from single dose tracer experiments, and the approximation of analogous behavior by chemically similar elements. Therefore, uncertainty in the transfer coefficient values is considerable for most elements. The range of values for transfer coefficients span orders of magnitude (e.g., $1 \times 10^{-5}$ to $1 \times 10^{-1} \mathrm{~d} / \mathrm{kg}$ or $\mathrm{d} / \mathrm{L}$ ) and are dependent on the element and product type (BSC 2004 [DIRS 169672], Section 6.3).

The animal feed consumption rates depend on the species, mass, age, growth rate, and other variables. The values used in biosphere models are for mature animals. Variation in consumption rates reported in the literature is relatively low. Typical values for animal feed are 30 to $70 \mathrm{~kg} / \mathrm{d}$ for beef cattle and dairy cows, and about 0.12 to $0.4 \mathrm{~kg} / \mathrm{d}$ for poultry and laying hens (BSC 2004 [DIRS 169672], Section 6.3).

\subsubsection{Animal Drinking Water}

Radionuclide concentrations in animal products contributed from ingestion of contaminated drinking water is estimated as

$$
C d_{\text {water } i, k}=F m_{i, k} C w_{i} Q w_{k}
$$

where

$C w_{i}=$ activity concentration of radionuclide $i$ in the groundwater $(\mathrm{Bq} / \mathrm{L})$

$Q w_{k}=$ animal consumption rate of drinking water $(\mathrm{L} / \mathrm{d})$

and the other parameters are defined in Equation 6.4.4-2

As with animal feed, consumption rates for drinking water are based on mature animals, they are animal-type specific, and the data in the literature show little variation. Typical consumption rates from the literature are 50 to $160 \mathrm{~L} / \mathrm{d}$ for beef cattle and dairy cows and about $0.3 \mathrm{~L} / \mathrm{d}$ for poultry and laying hens (BSC 2004 [DIRS 169672], Section 6.3). 


\subsubsection{Animal Soil Ingestion}

Ingestion of contaminated soil is another source of radionuclide intake for animals. This pathway is important because of the high soil equilibrium concentrations for some radionuclides. The radionuclide concentration in animal products contributed from the ingestion of contaminated soil is estimated as

$$
C d_{\text {soil } i, k}=F m_{i, k} C s_{m, i} Q s_{k}
$$

where

$C s_{m, i}=$ equilibrium activity concentration of radionuclide $i$ in the surface soil per unit mass $(\mathrm{Bq} / \mathrm{kg})$

$Q s_{k} \quad=$ animal consumption rate of soil $(\mathrm{kg} / \mathrm{d})$

and the other parameters are defined in Equation 6.4.4-2.

As in the other cases, inadvertent soil ingestion is estimated for mature animals, and the values are animal-type specific. Typical consumption rates for beef cattle and dairy cows are $1 \mathrm{~kg} / \mathrm{d}$ or less (BSC 2004 [DIRS 169672], Section 6.3.2). The equilibrium activity concentration of radionuclides in the surface soil is taken from the surface soil submodel.

\subsubsection{Fish Submodel}

The ERMYN includes radionuclide transport through an aquatic food chain because there is a fish farm in Amargosa Valley (YMP 1999 [DIRS 158212], Tables 8 and 9), which was fully operational from 1988 until at least 1999 (Roe 2002 [DIRS 160674]). The primary customer for the catfish was the Nevada Division of Wildlife, which used the fish to stock ponds and lakes in southern Nevada. The farm owner also allowed individuals, including residents of Amargosa Valley, to fish the ponds.

Most models for assessing radionuclide transport in aquatic systems are based on the observation that aquatic organisms assimilate radionuclides proportional to radionuclide concentrations in the water (Napier et al. 1998 [DIRS 157927] and Yu et al. [DIRS 159465]). These models are based on equilibrium systems and use equilibrium concentration ratios (also called bioaccumulation factors) to quantify the uptake of radionuclides by aquatic organisms. The bioaccumulation factor is the ratio of the activity concentration in edible portions of animal tissue to that in the water $(\mathrm{Bq} / \mathrm{kg}$ wet to $\mathrm{Bq} / \mathrm{L})$. In natural aquatic systems, fish take in radionuclides directly from water and feed. In the Amargosa Valley fish farm, the fish consume commercial feed, which is produced outside the Amargosa Valley and, presumably, is uncontaminated (Roe 2002 [DIRS 160674]. Therefore, models based on equilibrium concentrations in various components of the aquatic system provide an upper bound for the activity concentration in Amargosa Valley fish. Radionuclide concentrations in fish are expressed as

$$
C f_{i}=C w_{f, i} B F_{i}
$$


where

$\begin{array}{ll}C f_{i} & =\text { activity concentration of radionuclide } i \text { in fish }(\mathrm{Bq} / \mathrm{kg} \text { wet }) \\ C w_{f, i} & =\begin{array}{l}\text { activity concentration of radionuclide } i \text { in fishpond water, at the time of } \\ \text { harvest }(\mathrm{Bq} / \mathrm{L})\end{array} \\ B F_{i} & =\text { bioaccumulation factor for radionuclide } i \text { in freshwater fish }(\mathrm{L} / \mathrm{kg})\end{array}$

The bioaccumulation factors are element- and species-specific, and for a given element and species, bioaccumulation factors range over several orders of magnitude (BSC 2004 [DIRS 169672], Section 6.4; IAEA 2001 [DIRS 158519], p. 72).

The calculation of radionuclide bioaccumulation in farm-raised fish uses the activity concentration in pond water at the time of harvest. Due to the need to replace water lost by evaporation and the relatively long fish-breeding cycle, the activity concentration in pond water may be higher than that in the groundwater used to fill the ponds. Therefore, a water concentration-modifying factor is used to account for the increase in activity concentration due to evaporation. The fish submodel parameters are developed in BSC (2004 [DIRS 169672], Section 6.4). The activity concentration in the fish is calculated as

$$
C f_{i}=C w_{i} \quad M F_{i} \quad B F_{i}
$$

where

$C w_{i}=$ activity concentration of radionuclide $i$ in groundwater $(\mathrm{Bq} / \mathrm{L})$

$M F_{i} \quad=\quad$ water concentration modifying factor for radionuclide $i$ (dimensionless)

and the other parameters are defined in Equation 6.4.5-1.

Radionuclides also might enter the fish ponds by the deposition of resuspended soil particles. The total amount of deposited material is estimated using a submodel similar to that for direct deposition on crops (plant submodel; Equation 6.4.3-7). Equation 6.4.3-7 uses equations from the air (Equation 6.4.2-1) and surface soil submodels (Equations 6.4.1-4 and 6.4.1-5). Using typical values, $C w=1 \mathrm{~Bq} / \mathrm{m}^{3}, I R=1 \mathrm{~m} /$ year, $\lambda_{\text {eff }}=0.001 /$ year, $\rho_{s}=250 \mathrm{~kg} / \mathrm{m}^{2}, V_{d}=0.008 \mathrm{~m} / \mathrm{sec}$, and $S=1 \times 10^{-7} \mathrm{~kg} / \mathrm{m}^{3}$ (Table 6.6-3, and Table 7.4-4), the annual deposition rate for contaminated airborne particles is $D a=0.1 \mathrm{~Bq} /\left(\mathrm{m}^{2}\right.$ year $)$. This deposition rate is lower than the activity added annually to the fishponds per unit surface area to replace the water lost by evaporation, which is about $2 \mathrm{~Bq} /\left(\mathrm{m}^{2}\right.$ year $)$. This value was calculated based on a unit concentration in water $\left(C w=1 \mathrm{~Bq} / \mathrm{m}^{3}\right)$ and an annual evaporation rate of $2 \mathrm{~m}$ /year (BSC 2004 [DIRS 169672], Section 6.4). Water evaporation from the fishponds is incorporated into the water concentration-modifying factor $(M F)$.

\subsection{6 $\quad{ }^{14} \mathrm{C}$ Special Submodel}

${ }^{14} \mathrm{C}$ is a primary radionuclide for the groundwater scenario (Section 6.1.3). Because carbon is so common in the environment and because the ${ }^{14} \mathrm{C}$ transport pathways differ from those of other radionuclides, ${ }^{14} \mathrm{C}$ transport in the biosphere is considered differently than other radionuclides. Possible ${ }^{14} \mathrm{C}$ transport pathways include emission from the soil, uptake by crops through roots, 
uptake by crops through leaves (via photosynthesis), and accumulation in animal products. The ERMYN includes ${ }^{14} \mathrm{C}$ in the ${ }^{14} \mathrm{C}$ special submodel for the groundwater scenario.

In the groundwater scenario, ${ }^{14} \mathrm{C}$ initially is introduced into the soil from contaminated irrigation water. Subsequently, a fraction of the ${ }^{14} \mathrm{C}$ is released by gaseous emission into the atmosphere as ${ }^{14} \mathrm{CO}_{2}$. In the atmosphere, ${ }^{14} \mathrm{CO}_{2}$ becomes incorporated into crops via photosynthesis, resulting in increased levels of ${ }^{14} \mathrm{C}$ in the crops. The uptake of ${ }^{14} \mathrm{C}$ in crops may also occur via the root system; however, root uptake is less important than foliar uptake (BIOMASS 2001 [DIRS 159468], T3FM/WD01, p. 47).

The RESRAD (Yu et al. 2001 [DIRS 159465], Appendix L) and BIOMASS ERB2A (BIOMASS 2000 [DIRS 154522], Appendix A) models include ${ }^{14} \mathrm{C}$ special submodels that address the transport of gaseous carbon species from soil through the atmosphere and into plants. These ${ }^{14} \mathrm{C}$ special submodels are based on experimental results, which indicated that ${ }^{14} \mathrm{C}$ is quickly released from the soil as ${ }^{14} \mathrm{CO}_{2}$. The BIOMASS model also considers the direct absorption of ${ }^{14} \mathrm{C}$ by plant leaves from intercepted irrigation water.

The ERMYN ${ }^{14} \mathrm{C}$ special submodel is based on RESRAD (Yu et al. 2001 [DIRS 159465], Appendix L). This submodel comparison is discussed in Section 7.3.6. The following are considered for developing the ${ }^{14} \mathrm{C}$ special submodel:

- The ${ }^{14} \mathrm{C}$ special submodel should include all important exposure pathways for the other radionuclides.

- For inhalation exposure, the airborne sources of ${ }^{14} \mathrm{C}$ are gaseous species of carbon released from the soil following irrigation with ${ }^{14} \mathrm{C}$-contaminated groundwater. This approach differs from the other radionuclides, where resuspended contaminated soil is the source for inhalation exposure.

- To assess the activity concentration of ${ }^{14} \mathrm{C}$ gas in the air, a finite area is irrigated at the average annual irrigation rate. The resulting activity concentration in the air is required to evaluate the inhalation dose and plant leaf uptake.

- Long-term accumulation of ${ }^{14} \mathrm{C}$ in the soil is not considered because of the rapid loss of ${ }^{14} \mathrm{C}$ from the soil primarily by emission of ${ }^{14} \mathrm{CO}_{2}$.

\subsubsection{1 $\quad{ }^{14} \mathrm{C}$ in Soil}

Mechanisms of ${ }^{14} \mathrm{C}$ loss from soil include leaching, soil erosion, and radioactive decay, similar to the other radionuclides. However, ${ }^{14} \mathrm{C}$ transport in the environment is controlled by an additional loss mechanism, emission loss, which is not applicable to other primary radionuclides. Due to the volatility of ${ }^{14} \mathrm{C}$ in soil, it is quickly released via gaseous emission as ${ }^{14} \mathrm{CO}_{2}$. Sheppard et al. (1991 [DIRS 159545]) measured the rate of ${ }^{14} \mathrm{C}$ loss from soil in outdoor lysimeter experiments. Carbon loss from the soil, measured by the emission rate (the evasion rate in RESRAD), is 12/year for clay and loamy soils, and 22/year for sandy and organic soils (Yu et al. 2001 [DIRS 159465] p. L-16). Thus, ${ }^{14} \mathrm{C}$ concentrations in surface soil reach equilibrium within 1 
to 2 months. Emission is the dominant mechanism for removing ${ }^{14} \mathrm{C}$ from the soil. In comparison, losses due to leaching, radioactive decay, and soil erosion are slight (Table 7.4-4).

The calculation of ${ }^{14} \mathrm{C}$ concentration in the soil is based on equilibrium conditions between ${ }^{14} \mathrm{C}$ gains and losses. Using a differential equation similar to Equation 6.4.1-1 and the solution similar to Equation 6.4.1-4, the concentration of ${ }^{14} \mathrm{C}$ in the soil is expressed as

$$
C s_{C-14, j}=\frac{C w_{C-14} I R_{j}}{\lambda_{d, C-14}+\lambda_{l, C-14}+\lambda_{e}+\lambda_{a, C-14}}
$$

where

$$
\begin{array}{ll}
C s_{\mathrm{C}-14, j}= & \begin{array}{l}
\text { activity concentration of }{ }^{14} \mathrm{C} \text { in surface soil for the crop type or exposure } \\
\text { pathway } j\left(\mathrm{~Bq} / \mathrm{m}^{2}\right)
\end{array} \\
j & \begin{array}{l}
\text { crop-type or pathway index; } j=1 \text { for leafy vegetables, } 2 \text { for other } \\
\text { vegetables, } 3 \text { for fruit, } 4 \text { for grain, and } 5 \text { for fresh forage; } j=0 \text { for the }
\end{array} \\
& \text { pathways including inhalation, soil ingestion, and external exposure } \\
C W_{C-14}= & \text { activity concentration of }{ }^{14} \mathrm{C} \text { in irrigation water }\left(\mathrm{Bq} / \mathrm{m}^{3}\right) \\
I R_{j} & =\begin{array}{l}
\text { crop irrigation rate; } j=1 \text { to } 5 \text { for individual crop types }\left(I R D_{j}\right) \text { and } j=0 \text { for } \\
\\
\quad \text { the average annual irrigation rate }(\mathrm{m} / \text { year) }
\end{array} \\
\lambda_{d, C-14}= & \text { radioactive decay constant for }{ }^{14} \mathrm{C} \text { (per year) } \\
\lambda_{1, C-14}= & \text { leaching removal constant for }{ }^{14} \mathrm{C} \text { (per year) } \\
\lambda_{e}= & \text { the surface soil erosion removal constant (per year) } \\
\lambda_{a, C-14}= & \text { emission rate constant of }{ }^{14} \mathrm{C} \text { from the soil to the air (per year) }
\end{array}
$$

Because crops take up ${ }^{14} \mathrm{C}$ from the local soil and air, and because ${ }^{14} \mathrm{C}$ is released rapidly from the soil, irrigation in the ${ }^{14} \mathrm{C}$ submodel is considered locally and only during the crop growing season. Therefore, the daily average irrigation rate for crop type $j, I R D_{j}$, introduced in the plant submodel, is considered appropriate. This input parameter is used in the ${ }^{14} \mathrm{C}$ submodel with a simple conversion of units from $\mathrm{m} / \mathrm{d}$ to $\mathrm{m} /$ year. This conversion is needed because all removal rates are in units of per year. Modifying this equation to convert all removal rates to units of per day gives the same results. The average annual irrigation rate for farmland, $I R$, in the surface soil submodel is used for calculating the dose from inhalation, soil ingestion, and external exposure.

Equation 6.4.6-1 is analogous to Equation 6.4.1-4, except that the effective removal constant,

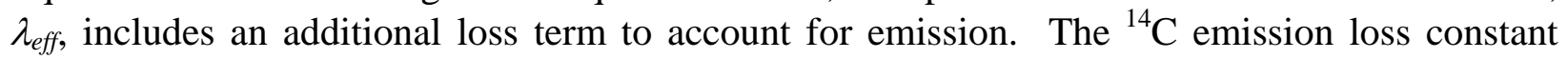
depends on the soil type, but not strongly (Yu et al. 2001 [DIRS 159465] p. L-16).

\subsubsection{2 $\quad{ }^{14} \mathrm{C}$ in the Air}

Due to the volatility of ${ }^{14} \mathrm{C}$, the activity concentration of this radionuclide in the air cannot be modeled using the soil resuspension submodel. A separate submodel for the gaseous release of ${ }^{14} \mathrm{C}$ from the soil $\left(\mathrm{as} \mathrm{CO}_{2}\right)$ is used to predict ${ }^{14} \mathrm{C}$ concentrations in the air. The flux density of gaseous ${ }^{14} \mathrm{C}$ from soil to air is estimated as

$$
\operatorname{EVSN}_{j}=\operatorname{Cs}_{C-14, j} \lambda_{a, C-14}
$$


where

EVSN $_{j}=$ average flux density of gaseous ${ }^{14} \mathrm{C}$ from contaminated soil for the crop exposure pathway $j\left(\mathrm{~Bq} /\left(\mathrm{m}^{2}\right.\right.$ year $\left.)\right)$

and the other parameters are defined in Equation 6.4.6-1.

If $C s_{C-14, j}$ in Equation 6.4.6-2 is substituted using Equation 6.4.6-1, the flux density of gaseous ${ }^{14} \mathrm{C}$ from the soil is almost equal to the total deposition rate of ${ }^{14} \mathrm{C}\left(C w_{C-14, j} \times I R_{j}\right)$ because the emission loss constant $\left(\lambda_{a, C-14}=22\right.$ year; Table 6.6-3) is much larger than the remaining components of the effective removal constant $\left(\lambda_{d, C-14}+\lambda_{l, C-14}+\lambda_{e}=0.01 /\right.$ year; Table 7.4-4). This indicates that the atmosphere, rather than the soil, is the main source of ${ }^{14} \mathrm{C}$ available for further dispersion in the environment. It must be noted that the gaseous ${ }^{14} \mathrm{C}$ flux of concern to biosphere modeling originates from irrigated land only. After it is released into the air, ${ }^{14} \mathrm{C}$ is diluted by mixing with uncontaminated air. The ${ }^{14} \mathrm{C}$ concentration in the air above cropland irrigated by contaminated groundwater is estimated using the total ${ }^{14} \mathrm{C}$ release rate and the potential mixing volume of air as

$$
C a_{g, C-14, j}=\frac{E V S N_{j} \times \mathrm{A}}{3.16 \times 10^{7} H_{\text {mix }} U \sqrt{A}}=\frac{E V S N_{j} \times \sqrt{\mathrm{A}}}{3.16 \times 10^{7} H_{\text {mix }} U}
$$

where

$$
\begin{aligned}
& C a_{g, C-14, j} \quad=\text { activity concentration of }{ }^{14} \mathrm{C} \text { in the air for the crop type or exposure } \\
& \text { pathway } j\left(\mathrm{~Bq} / \mathrm{m}^{3}\right) \\
& \text { A } \quad=\text { surface area of irrigated land }\left(\mathrm{m}^{2}\right) \text {, which is calculated by } W / I R \text {, where } \\
& W \text { is annual total groundwater usage }\left(\mathrm{m}^{3} / \text { year) and } I R\right. \text { is annual average } \\
& \text { irrigation rate (m/year) defined in Section 6.4.1.1 } \\
& \mathrm{H}_{\text {mix }} \quad=\text { mixing height of }{ }^{14} \mathrm{CO}_{2}(\mathrm{~m}) \\
& U=\text { annual average wind speed }(\mathrm{m} / \mathrm{sec}) \\
& 3.16 \times 10^{7}=\text { unit conversion factor from year to seconds based on } 1 \text { year }=365.25 \mathrm{~d} \\
& \text { (sec/year) }
\end{aligned}
$$

and the other parameters are defined in Equation 6.4.6-2.

The airborne concentration of ${ }^{14} \mathrm{C}$ depends on crop types or exposure pathways. The surface area of irrigated land, $A$, is estimated using an annual water demand of 3,000 acre-feet (10 CFR 63.312 [DIRS 156605]) and the average annual irrigation rate. The square root of irrigated area, $A$, is the estimated length of one side of the square with surface area $A$. The mixing height of gaseous ${ }^{14} \mathrm{C}, H_{\text {mix }}$, depends on the specific pathway and is less for crop uptake than for human uptake. The same is true for the annual average wind speed, $U$, which is slower close to the ground in the plant growing zone than it is in the human breathing zone. Values for the parameters in Equation 6.4.6-3 are developed in BSC (2004 [DIRS 169672], Section 6.7).

The RESRAD model (Yu et al. 2001 [DIRS 159465], p. L-15) uses an additional parameter to account for the fraction of time when wind is blowing over the contaminated area and towards the receptor. In the RESRAD model, the value of this parameter is 0.5 because the receptor is located at the edge of a relatively small contaminated area. In the ERMYN, the receptor is 
located within the contaminated area, where wind blowing from any direction exposes the receptor. Therefore, wind direction is not considered in the ERMYN.

\subsubsection{3 $\quad{ }^{14} \mathrm{C}$ in Crops}

In the environment, the transport of ${ }^{14} \mathrm{C}$ follows that of stable carbon (Yu et al. 2001 [DIRS 159465], p. L-15). Two transport pathways are considered for the uptake of ${ }^{14} \mathrm{C}$ by plants: direct root uptake of ${ }^{14} \mathrm{C}$ and leaf uptake of ${ }^{14} \mathrm{CO}_{2}$ released from soil to the atmosphere by gaseous emission. The latter mechanism is dominant because plants acquire most carbon from the atmosphere during photosynthesis (Yu et al. 2001 [DIRS 159465], p. L-18). In the biosphere model, the activity concentration of ${ }^{14} \mathrm{C}$ in crops resulting from root uptake is calculated as

$$
C p_{\text {root } \mathrm{C}-14, j}=\frac{C s_{C-14, j}}{\rho_{s}} \frac{F S \times f C_{\text {plant }, j}}{f C_{\text {soil }}}
$$

where

$$
\begin{aligned}
& C p_{\text {root } C-14, j}=\text { activity concentration of }{ }^{14} \mathrm{C} \text { in the edible parts of crop type } j \text { resulting } \\
& \text { from root uptake }(\mathrm{Bq} / \mathrm{kg} \text { wet weight }) \\
& j=\text { crop-type index; which is the same as that defined in Equation 6.4.6-1, } \\
& \text { except it does not include } j=0 \text {. } \\
& \mathrm{Cs}_{\mathrm{C}-14, j}=\text { activity concentration of }{ }^{14} \mathrm{C} \text { in surface soil for crop type } j\left(\mathrm{~Bq} / \mathrm{m}^{2}\right) \\
& \rho_{s} \quad=\text { areal density of surface soil }\left(\mathrm{kg} / \mathrm{m}^{2}\right) \\
& f c_{\text {plant }, j}=\text { fraction of stable carbon in crop type } j \text { (dimensionless, based on } \\
& \mathrm{kg} \text { carbon } / \mathrm{kg} \text { wet crop) } \\
& \text { Fs } \quad=\text { fraction of soil-derived carbon in plants (dimensionless) } \\
& f c_{\text {soil }}=\text { fraction of stable carbon in soil (dimensionless, based on } \mathrm{kg} \text { carbon } / \mathrm{kg} \text { soil) }
\end{aligned}
$$

The fraction of stable carbon in the soil, $f c_{\text {soil }}$, is defined as the mass of carbon per unit mass of soil. The fraction varies depending on soil type, with a typical value on the order of a few percent (BSC 2004 [DIRS 169672]). A value of 0.03 is recommended for the RESRAD model (Yu et al. 2001 [DIRS 159465], p. L-17).

The activity concentration of ${ }^{14} \mathrm{C}$ in crops resulting from the uptake through the leaves via photosynthesis is estimated as

$$
C p_{\text {leaf } \mathrm{C}-14, j}=C a_{C-14, j} \frac{F a \times f c_{\text {plant }, j}}{f c_{a i r}}
$$

where

$$
\begin{array}{ll}
C p_{\text {leaf } C-14, j}= & \text { activity concentration of }{ }^{14} \mathrm{C} \text { in edible parts of crop type } j \text { resulting from } \\
& \text { leaf uptake }\left(\mathrm{Bq} / \mathrm{kg}_{\text {wet weight }}\right) \\
C a_{C-14, j} & \text { activity concentration of }{ }^{14} \mathrm{C} \text { in the air for the crop type } j\left(\mathrm{~Bq} / \mathrm{m}^{3}\right) \\
\mathrm{Fa} & =\text { fraction of air-derived carbon in plants }(\text { dimensionless }) \\
f C_{\text {air }} & \text { concentration of stable carbon in the air }\left(\mathrm{kg} \text { carbon } / \mathrm{m}^{3}\right)
\end{array}
$$


The concentration of stable carbon in the air, $f c_{a i r}$, can be derived from the average global value, about $1.8 \times 10^{-4} \mathrm{~kg} / \mathrm{m}^{3}$ (BSC 2004 [DIRS 169672], Section 6.7). By combining Equation 6.4.6-4 and Equation 6.4.6-5, Equation 6.4.6-6, the total concentration of ${ }^{14} \mathrm{C}$ in plants, can be obtained as

$$
C p_{C-14, j}=f C_{\text {plant }, j}\left[\left(F a \frac{C a_{g, C-14, j}}{f C_{\text {air }}}\right)+\left(F s \frac{C s_{C-14, j}}{\rho_{s} f C_{\text {soil }}}\right)\right]
$$

where

$C p_{C-14, j}=$ activity concentration of ${ }^{14} \mathrm{C}$ in edible parts of crop type $j(\mathrm{~Bq} / \mathrm{kg}$ wet weight $)$ and the other parameters are defined in Equations 6.4.6-4 and 6.4.6-5.

The fraction of carbon in plants derived from air, $F a$, ranges from 0 to 1 , and experiments indicate that the fraction of carbon in plants derived from air is close to 1 (Yu et al. 2001 [DIRS 159465], p. L-17). A value of $F a=0.98$ is recommended for the RESRAD model (Yu et al. 2001 [DIRS 159465], p. L-20). The fraction of carbon in plants derived from soil, Fs, is complementary to the fraction of carbon in plants derived from air (i.e., $F s=1-F a$ ), with a value of about 0.02 (Yu et al. 2001 [DIRS 159465], p. L-17).

The fraction of stable carbon in plants, $f c_{\text {plant }, \text {, is }}$ is crop-type specific, and defined as the mass of carbon per unit wet mass of a plant. Default values for this parameter in the RESRAD and GENII-S models are 0.09 for fruits, vegetables, and fresh forage; and 0.40 for grain (BSC 2004 [DIRS 169672], Section 6.7).

\subsubsection{4 $\quad{ }^{14} \mathrm{C}$ in Animal Products}

The activity concentration of ${ }^{14} \mathrm{C}$ in animal products is derived from the animal feed, soil, and drinking water. The transfer of ${ }^{14} \mathrm{C}$ from feed to animal products is modeled using the same route as that of stable carbon. The ${ }^{14} \mathrm{C}$ activity concentration in animal products is calculated as

$$
C d_{C-14, k}=\frac{\left(C p_{C-14, j} \times Q f_{k}\right)+\left(C w_{C-14} \times Q w_{k}\right)+\left(C s_{C-14} \times Q s_{k}\right)}{\left(f c_{\text {plant }, j} \times Q f_{k}\right)+\left(f c_{\text {water }} \times Q w_{k}\right)+\left(f c_{\text {soil }} \times Q s_{k}\right)} \times f c_{\text {anim }, k}
$$

where

$C d_{C-14, k}=$ activity concentration of ${ }^{14} \mathrm{C}$ in animal product $k(\mathrm{~Bq} / \mathrm{kg} ; \mathrm{Bq} / \mathrm{L}$ for milk)

$\mathrm{Cw}_{\mathrm{C}-14}=$ activity concentration of ${ }^{14} \mathrm{C}$ in groundwater $(\mathrm{Bq} / \mathrm{L})$

$f c_{\text {water }}=$ concentration of stable carbon in water $(\mathrm{kg} / \mathrm{L})$

$f c_{\text {anim, } k}=$ fraction of stable carbon in animal product $k$ (dimensionless, based on $\mathrm{kg}$ carbon $/ \mathrm{kg}$ animal product)

and the other parameters are defined in Equations 6.4.4-1 to 6.4.4-4, 6.4.6-1, and 6.4.6-6.

The concentration of stable carbon in water, $f c_{\text {water }}$, is on the order of $1 \times 10^{-5} \mathrm{~kg} / \mathrm{L}$ (BSC 2004

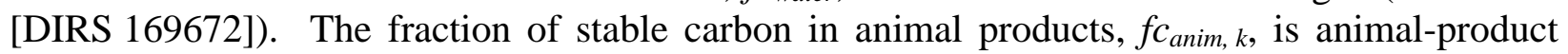


dependent. The GENII and RESRAD models use 0.24 for beef, 0.2 for poultry, 0.07 for milk, and 0.15 for eggs (BSC 2004 [DIRS 169672]).

By comparing the three ${ }^{14} \mathrm{C}$ sources (feed, water, and soil), the main source for animal ${ }^{14} \mathrm{C}$ intake is from feed, as there is only a small amount of carbon in water and soil (Napier et al. 1988 [DIRS 157927] p. 4.89).

\subsubsection{5 $\quad{ }^{14} \mathrm{C}$ in Fish}

The activity concentration of ${ }^{14} \mathrm{C}$ in fish is calculated using the method developed for other radionuclides, as discussed in Section 6.4.5.

\subsubsection{External Exposure Submodel}

The dose received from external sources of radiation is attributed to high energy beta- and gamma-emitting radionuclides in contaminated media such as soil, air, and water. For external exposure, radiation emitters are external to the human body, and, therefore, exposure occurs only when a person is near or in contact with the contaminated media. The primary external exposure pathways include exposure to contamination on or in the ground (ground exposure), air submersion, and water immersion.

External exposure of the human receptor can be evaluated using radionuclide media concentrations, exposure times, and dose coefficients. Dose coefficients, tabulated in FGR-12 (Eckerman and Ryman 1993 [DIRS 107684]), convert media concentrations into EDEs per unit exposure time. In this section, the EDE from external exposure is referred to as dose from external exposure.

\subsubsection{Exposure to Contaminated Soil}

The dose coefficients for external exposure to contaminated soils given in the FGR-12 are derived for a source, which when seen from the location of an exposed individual, is uniform and effectively semi-infinite in extent (Eckerman and Ryman 1993 [DIRS 107684], p. 2). For consistency, it is assumed that the same exposure geometry applies to the human receptor in the ERMYN (Approximation 10). For the groundwater scenario, the irrigated area is limited in extent, and, although noncultivated areas could become contaminated by surface soil transport, the contamination levels would be lower than those on the irrigated land. However, due to crop rotation and changes in land use over the long period considered for evaluation of repository performance, areas not farmed at any given time may have been irrigated previously and, thus, are considered to remain contaminated. Therefore, it is conservative to assume that the surface soil is contaminated at equilibrium radionuclide concentrations (see the surface soil submodel, Section 6.4.1).

The annual dose to a receptor from external exposure to primary radionuclide $i$ in contaminated soil may include contributions from other primary radionuclides formed in the soil as a result of radioactive decay of radionuclide $i$, as explained in Section 6.3.5. The combined dose is estimated as 


$$
D_{\text {ext, }, i}=\sum_{l} D_{\text {ext,l}}=\sum_{l} E D C i_{\text {soil }, l} \frac{C s_{l}}{d}\left[\sum_{n} f_{e x t, l, n}\left(\sum_{m} P P_{m}\left(3600 \times t_{n, m}\right)\right)\right]
$$

where

$$
\begin{aligned}
& D_{\text {ext,i }}=\text { annual dose from external exposure to primary radionuclide } i \text { in soil } \\
& \text { (Sv/year) } \\
& D_{\text {ext, },}=\text { dose from external exposure to long-lived radionuclide } l \text { (Table 6.4-1) in a } \\
& \text { decay chain of a primary radionuclide } i \text { (Sv/year) } \\
& l \quad=\text { index of long-lived radionuclide in a decay chain; } l=0 \text { for primary } \\
& \text { radionuclide, } \\
& E D C i_{\text {soil }, l}=\text { effective dose coefficient for exposure to soil contaminated to an infinite } \\
& \text { depth for a long-lived radionuclide } l \text { in a decay chain of a primary } \\
& \text { radionuclide } i\left(\mathrm{~Sv} / \mathrm{sec} \text { per } \mathrm{Bq} / \mathrm{m}^{3}\right) \text {. Calculation of effective dose coefficients } \\
& \text { is discussed in Section 6.4.7.2. } \\
& \text { Cs } I=\text { equilibrium activity concentration in surface soil for a long-lived } \\
& \text { radionuclide } l \text { in a decay chain of a primary radionuclide } i\left(\mathrm{~Bq} / \mathrm{m}^{2}\right) \\
& d \quad=\text { depth of surface soil (m) } \\
& f_{\text {ext }, l, n}=\text { external shielding factor for exposure to radionuclide } l \text { in the soil at } \\
& \text { environment } n \text { (dimensionless) } \\
& n=\text { environment index; } n=1 \text { for active outdoors, } 2 \text { for inactive outdoors, } 3 \text { for } \\
& \text { active indoors, } 4 \text { for asleep indoors, and } 5 \text { for away from the contaminated } \\
& \text { area } \\
& m=\text { population group index; } m=1 \text { for local outdoor workers, } 2 \text { for local indoor } \\
& \text { workers, } 3 \text { for commuters, and } 4 \text { for nonworkers } \\
& P P_{m} \quad=\quad \text { fraction of total population in population group } m \text { (population proportion) } \\
& \text { (dimensionless) } \\
& t_{n, m} \quad=\quad \text { exposure times of population group } m \text { spent in environment } n \text { (exposure } \\
& \text { time) (hr/year) } \\
& 3600=\text { unit conversion of hours to seconds; 3,600 (s/hr) }
\end{aligned}
$$

To account for variation and uncertainty in the amount of time the receptor would spend in environment $n$ (Section 6.4.2.1), four mutually exclusive population groups, $m$, are considered. These groups represent the range of behaviors that would most influence the amount of time that people are exposed to radionuclides via external exposure and inhalation. Variation among individuals in these exposure pathways is influenced primarily by the amount of time they spent indoors and outdoors within contaminated areas (and the amount of time they spent away from contaminated areas). For adults, variation among these time factors primarily is a function of occupational characteristics, as people working outside the contaminated area, generally, would experience less exposure than people who remain within the area, and people who work outdoors would be exposed at a different level than those who remained indoors. Therefore, the categories are based on work location and type of occupation. Estimates of the proportion of the adult population of Amargosa Valley in each group are developed in BSC (2004 [DIRS 169671] Section 6.3.1). 
Local Outdoor Workers-This group includes residents who work outdoors and disturb (and therefore resuspend) contaminated soil.

Local Indoor Workers-Local indoor workers are residents who work indoors (or outdoors in enclosed vehicles) in areas contaminated by groundwater or ash. The proportion of the population in this group is calculated as the proportion not in the other groups.

Commuters - This group includes residents who work outside the contaminated area.

Nonworkers - Nonworkers are residents who are unemployed or otherwise not in the labor force, including retired persons.

The effective dose coefficient for exposure to soil contaminated to an infinite depth, $E D C i_{\text {soil, }}$, is a radionuclide-specific parameter. Unlike the submodels for crop contamination, the external exposure submodel uses an infinite depth of contaminated soil, rather than the depth of the surface soil. This difference accounts for emissions from radionuclides that leach out of the surface soil into the deep soil, but that still could contribute to the radiation field above the air-ground interface. Radionuclide concentrations in the surface soil are used to calculate the external exposure, even though radionuclide concentrations in the deep soil might not be contaminated at the same high level. As discussed in Section 6.3.5, the short-lived decay products are assumed to be in equilibrium with the long-lived parent radionuclides, and their dose coefficients are combined with the parent dose coefficient to produce an effective dose coefficient. The development of effective dose coefficients, which includes contributions from the short-lived decay products, is discussed in Section 6.4.7.2.

The equilibrium activity concentration of radionuclide $i$ and its decay product $l$ in soil, $C s_{i}$ and $\mathrm{Cs}_{l}$, respectively, are calculated using Equation 6.4.1-4 and Equation 6.4.1-9, which are discussed with respect to the surface soil submodel (Section 6.4.1).

In the submodel, an external shielding factor, $f_{\text {ext,l,n, }}$, is used to account for the reduction in external exposure offered by dwellings. For the outdoors $(n=1,2$, and 5$)$, where normally there would be no shielding, the value for $f_{e x t, l, n}$ is equal to one. For indoor environments ( $n=3$ and 4), shielding is radionuclide dependent because of radiation characteristics. Shielding factor values range from 0 to 1; however, the typical values, even for the most penetrating radiation emissions, do not exceed 0.4 (BSC 2004 [DIRS 169671], Section 6.6). Radionuclides with penetrating (high energy) gamma rays have a higher shielding factor than radionuclides with low energy gamma or beta rays.

The external exposure time, $t_{n, m}$, is the annual duration that population group $m$ spend in environment $n$ (BSC 2004 [DIRS 169671]). The fraction of the total population in population group $m, P P_{m}$, is based on current Amargosa Valley census data (BSC 2004 [DIRS 169671]). The fractional exposure time for the active-indoor category and the proportion of indoor-workers are calculated as one minus the sum of the other fractional exposure times and population proportions, respectively. The method is provided in BSC (BSC 2004 [DIRS 169671], Section 6.3), and calculations are carried in the ERMYN to incorporate the uncertainty of these parameters. 
The external exposure submodel does not include air submersion exposure because air contamination is a secondary process following the resuspension of contaminated surface soil. Because most of the radionuclides of interest are long-lived alpha emitters, the inhalation dose is higher than any subsequent air submersion dose. The external exposure submodel does not include water immersion exposure because of the emission characteristics of the radionuclides involved (primarily long-lived alpha emitters) and the relatively short exposure times expected in water. Justification for excluding these two pathways is presented in the validation section (Section 7.4.8). In addition, the external exposure arising from accumulation of activity in evaporative coolers is not considered as justified in Appendix D.

\subsubsection{Effective Dose Coefficients for Exposure to Contaminated Soil}

Dose coefficients for exposure to soil contaminated to an infinite depth are evaluated in BSC (2004 [DIRS 169671], Section 6.5). As discussed in Section 6.3.5, the dose contributions from short-lived decay products are combined with those of their long-lived parent radionuclides. The development of the combined, or effective, dose coefficients is shown in Table 6.4-2. The first column in the table contains the names of the primary radionuclides, followed by a " $\mathrm{D}$ " if short-lived decay products are included. The second column lists the short-lived decay products, the dose contributions of which are combined with those of the parent radionuclide. Dose coefficients for the individual radionuclides are shown in the third column. The effective dose coefficients, calculated by summing the dose coefficients for all short-lived decay products under a primary radionuclide, with consideration of the branching fraction, are given in the last column of the table. The calculation method can be expressed as

$$
E D C i_{\text {soil }, i}=\sum_{s} D C i_{\text {soil, }, s} \times B N_{s}
$$

where

$$
\begin{aligned}
& D C i_{\text {soil,s }}= \text { dose coefficient for exposure to soil contaminated to an infinite depth for } \\
& \text { short-lived radionuclide } s \text { in the decay chain of primary radionuclide } i \\
&\left(\text { Sv/sec per } \mathrm{Bq} / \mathrm{m}^{3}\right) \\
& s \quad= \text { index of short-lived radionuclide decay chain under a primary radionuclide } i \\
& B N_{s}=\begin{array}{l}
\text { branching fraction for short-lived radionuclide } s \text { in the decay chain of } \\
\text { primary radionuclide } i \text { (dimensionless) }
\end{array}
\end{aligned}
$$

and the other parameter is defined in Equation 6.4.7-1

The values shown in Table 6.4-2 are for demonstration purposes only. The ERMYN uses branching fractions and dose coefficients as inputs to calculate effective dose coefficients in GoldSim (Section 6.8). 
Table 6.4-2. Effective Dose Coefficients for Exposure to Soil Contaminated to an Infinite Depth

\begin{tabular}{|c|c|c|c|}
\hline $\begin{array}{c}\text { Primary } \\
\text { Radionuclide }\end{array}$ & $\begin{array}{c}\text { Decay Product }{ }^{\mathrm{C}} \text { (branching fraction if } \\
\text { not } 100 \% \text {, half-life) }\end{array}$ & $\begin{array}{l}\text { Dose coefficient } \\
\left(D C i_{\text {soil }}\right)^{d} \\
(\mathrm{~Sv} / \mathrm{sec}) /\left(\mathrm{Bq} / \mathrm{m}^{3}\right)\end{array}$ & $\begin{array}{c}\text { Effective dose } \\
\text { coefficient }\left(E D C i_{\text {soil }}\right) \\
(\text { Sv/sec }) /\left(\mathrm{Bq} / \mathrm{m}^{3}\right) \\
\end{array}$ \\
\hline C-14 & - & $7.20 \mathrm{E}-23$ & $7.20 \mathrm{E}-23$ \\
\hline $\mathrm{Cl}-36$ & - & $1.28 \mathrm{E}-20$ & $1.28 \mathrm{E}-20$ \\
\hline Se-79 & - & 9.96E-23 & 9.96E-23 \\
\hline Sr-90D ${ }^{a}$ & Y-90 (64.0 hr) & $\begin{array}{l}3.77 \mathrm{E}-21 \\
1.28 \mathrm{E}-19 \\
\end{array}$ & $1.32 \mathrm{E}-19$ \\
\hline Tc-99 & - & $6.72 \mathrm{E}-22$ & $6.72 \mathrm{E}-22$ \\
\hline Sn-126D & $\begin{array}{l}\text { Sb-126m (19.0 min }) \\
\text { Sb-126 (14\%, } 12.4 \mathrm{~d})\end{array}$ & $\begin{array}{l}7.89 \mathrm{E}-19 \\
4.98 \mathrm{E}-17 \\
9.16 \mathrm{E}-17\end{array}$ & $6.34 \mathrm{E}-17$ \\
\hline $\mathrm{I}-129$ & - & $6.93 \mathrm{E}-20$ & $6.93 \mathrm{E}-20$ \\
\hline Cs-135 & - & $2.05 E-22$ & $2.05 \mathrm{E}-22$ \\
\hline Cs-137D & Ba-137m (94.6\%, $2.552 \mathrm{~min})$ & $\begin{array}{l}4.02 \mathrm{E}-21 \\
1.93 \mathrm{E}-17 \\
\end{array}$ & $1.83 \mathrm{E}-17$ \\
\hline Pu-242 & - & $6.85 E-22$ & $6.85 \mathrm{E}-22$ \\
\hline U-238D & $\begin{array}{l}\text { Th-234 (24.10 d) } \\
\text { Pa-234m (99.80\%, } 1.17 \mathrm{~min}) \\
\text { Pa-234 (0.33\%, 6.7 hr) }\end{array}$ & $\begin{array}{l}5.52 \mathrm{E}-22 \\
1.29 \mathrm{E}-19 \\
4.80 \mathrm{E}-19 \\
6.18 \mathrm{E}-17\end{array}$ & 8.13E-19 \\
\hline $\mathrm{Pu}-238$ & - & 8.10E-22 & $8.10 \mathrm{E}-22$ \\
\hline U-234 & - & $2.15 E-21$ & $2.15 E-21$ \\
\hline Th-230 & - & $6.47 E-21$ & $6.47 E-21$ \\
\hline Ra-226D & $\begin{array}{l}\text { Rn-222 (3.8235 d) } \\
\text { Po-218 (3.05 min) } \\
\text { Pb-214 (99.98\%, 26.8 min }) \\
\text { At-218 }(0.02 \%, 2 \mathrm{sec}) \\
\text { Bi-214 }(19.9 \mathrm{~min}) \\
\text { Po-214 }\left(99.98 \%, 1.64 \times 10^{-4} \mathrm{sec}\right) \\
\text { Tl-210 }(0.02 \%, 1.3 \mathrm{~min})^{\mathrm{e}}\end{array}$ & $\begin{array}{l}1.70 \mathrm{E}-19 \\
1.26 \mathrm{E}-20 \\
3.02 \mathrm{E}-22 \\
7.18 \mathrm{E}-18 \\
3.13 \mathrm{E}-20 \\
5.25 \mathrm{E}-17 \\
2.75 \mathrm{E}-21 \\
0.00 \mathrm{E}+00 \\
\end{array}$ & $5.99 \mathrm{E}-17$ \\
\hline $\mathrm{Pb}-210 \mathrm{D}$ & $\begin{array}{l}\operatorname{Bi}-210(5.012 d) \\
\text { Po-210 (138.38 d) }\end{array}$ & $\begin{array}{l}1.31 \mathrm{E}-20 \\
1.93 \mathrm{E}-20 \\
2.80 \mathrm{E}-22 \\
\end{array}$ & 3.27E-20 \\
\hline $\mathrm{Pu}-240$ & - & $7.85 E-22$ & $7.85 \mathrm{E}-22$ \\
\hline $\mathrm{U}-236$ & - & 1.15E-21 & 1.15E-21 \\
\hline Th-232 & - & $2.79 \mathrm{E}-21$ & $2.79 \mathrm{E}-21$ \\
\hline $\mathrm{Ra}-228 \mathrm{D}^{\mathrm{b}}$ & Ac-228 (6.13 hr) & $\begin{array}{l}0.00 \mathrm{E}+00 \\
3.20 \mathrm{E}-17 \\
\end{array}$ & $3.20 \mathrm{E}-17$ \\
\hline $\mathrm{U}-232$ & - & $4.83 \mathrm{E}-21$ & 4.83E-21 \\
\hline
\end{tabular}


Table 6.4-2. Effective Dose Coefficients for Exposure to Soil Contaminated to an Infinite Depth (Continued)

\begin{tabular}{|c|c|c|c|}
\hline $\begin{array}{c}\text { Primary } \\
\text { Radionuclide }\end{array}$ & $\begin{array}{c}\text { Decay Product }{ }^{\mathrm{C}} \text { (branching fraction if } \\
\text { not } 100 \% \text {, half-life) }\end{array}$ & $\begin{array}{l}\text { Dose coefficient } \\
\left(D C i_{\text {soil }}\right)^{d} \\
(\mathrm{~Sv} / \mathrm{sec}) /\left(\mathrm{Bq} / \mathrm{m}^{3}\right)\end{array}$ & $\begin{array}{c}\text { Effective dose } \\
\text { coefficient }\left(E D C i_{\text {soil }}\right) \\
(\mathrm{Sv} / \mathrm{sec}) /\left(\mathrm{Bq} / \mathrm{m}^{3}\right)\end{array}$ \\
\hline Th-228D & $\begin{array}{l}\text { Ra-224 (3.66 d) } \\
\text { Rn-220 (55.6 sec) } \\
\text { Po-216 (0.15 sec) } \\
\text { Pb-212 (10.64 hr) } \\
\text { Bi-212 (60.55 min) } \\
\text { Po-212 (64.07\%, } 0.305 \mu-s e c) \\
\text { Tl-208 (35.93\%, 3.07 min) }\end{array}$ & $\begin{array}{l}4.25 \mathrm{E}-20 \\
2.74 \mathrm{E}-19 \\
1.23 \mathrm{E}-20 \\
5.58 \mathrm{E}-22 \\
3.77 \mathrm{E}-18 \\
6.27 \mathrm{E}-18 \\
0.00 \mathrm{E}+00 \\
1.23 \mathrm{E}-16\end{array}$ & $5.46 \mathrm{E}-17$ \\
\hline Am-243D & Np-239 (2.355 d) & $\begin{array}{l}7.60 \mathrm{E}-19 \\
4.03 \mathrm{E}-18\end{array}$ & 4.79E-18 \\
\hline Pu-239 & - & $1.58 \mathrm{E}-21$ & $1.58 \mathrm{E}-21$ \\
\hline$U-235 D$ & Th-231 (25.52 hr) & $\begin{array}{l}3.86 \mathrm{E}-18 \\
1.95 \mathrm{E}-19\end{array}$ & $4.06 \mathrm{E}-18$ \\
\hline Pa-231 & - & $1.02 \mathrm{E}-18$ & $1.02 \mathrm{E}-18$ \\
\hline$A c-227 D$ & $\begin{array}{l}\text { Th-227 (98.62\%, } 18.718 \mathrm{~d}) \\
\text { Fr-223 (1.38\%, } 21.8 \mathrm{~min}) \\
\text { Ra-223 (11.434 d) } \\
\text { Rn-219 (3.96 sec) } \\
\text { Po-215 (1.78 millisec) } \\
\text { Pb-211 (36.1 min) } \\
\text { Bi-211 (2.14 min) } \\
\text { Tl-207 (99.72\%, } 4.77 \text { min) } \\
\text { Po-211 (0.28\%, } 0.516 \mathrm{sec})\end{array}$ & $\begin{array}{l}2.65 \mathrm{E}-21 \\
2.79 \mathrm{E}-18 \\
1.06 \mathrm{E}-18 \\
3.23 \mathrm{E}-18 \\
1.65 \mathrm{E}-18 \\
5.44 \mathrm{E}-21 \\
1.64 \mathrm{E}-18 \\
1.37 \mathrm{E}-18 \\
1.06 \mathrm{E}-19 \\
2.55 \mathrm{E}-19\end{array}$ & $1.08 \mathrm{E}-17$ \\
\hline Am-241 & - & $2.34 \mathrm{E}-19$ & $2.34 \mathrm{E}-19$ \\
\hline Np-237D & $\mathrm{Pa}-233(27.0 \mathrm{~d})$ & $\begin{array}{l}4.17 \mathrm{E}-19 \\
5.46 \mathrm{E}-18\end{array}$ & $5.88 \mathrm{E}-18$ \\
\hline U-233 & - & $7.48 \mathrm{E}-21$ & $7.48 \mathrm{E}-21$ \\
\hline Th-229D & $\begin{array}{l}\text { Ra-225 (14.8 d) } \\
\text { Ac-225 (10.0 d) } \\
\text { Fr-221 (4.8 min) } \\
\text { At-217 (32.3 millisec) } \\
\text { Bi-213 (45.65 min) } \\
\text { Po-213 (97.84\%, } 4.2 \mu-s e c) \\
\text { Tl-209 (2.16\%, } 2.2 \text { min) } \\
\text { Pb-209 (3.253 hr) }\end{array}$ & $\begin{array}{l}1.72 \mathrm{E}-18 \\
5.90 \mathrm{E}-20 \\
3.41 \mathrm{E}-19 \\
8.22 \mathrm{E}-19 \\
9.49 \mathrm{E}-21 \\
4.10 \mathrm{E}-18 \\
0.00 \mathrm{E}+00 \\
6.92 \mathrm{E}-17 \\
4.14 \mathrm{E}-21\end{array}$ & 8.55E-18 \\
\hline
\end{tabular}

a A "D" indicates that the radionuclide is treated with its short-lived (less than $180 \mathrm{~d}$ ) decay products.

b Indented radionuclides are long-lived decay products considered separately from the parents.

c Branching fractions and half-lives are from Eckerman and Ryman (1993 [DIRS 107684], Table A.1).

d Dose coefficient source (BSC 2004 [DIRS 169671], Section 6.5).

e Half-life of TI-210 is taken from Lide and Frederikse (1997 [DIRS 103178], p.11-125).

$\mathrm{DC}=$ dose coefficients 


\subsubsection{Inhalation Exposure Submodel}

The inhalation submodel is used to calculate radiation doses caused by the inhalation of contaminated air. The inhalation dose is estimated using radionuclide concentrations in the air, parameters describing conditions of human exposure, and dose conversion factors for inhalation exposure that convert radionuclide intake to CEDE. Activity concentrations in the air are discussed in Section 6.4.2 (air submodel). In contrast to external exposure, where emissions arise from outside the human body, inhalation and ingestion exposures arise from radiation emitted inside the body, and the exposure continues for as long as the radionuclides are in the body. Therefore, inhalation and ingestion doses are presented in terms of the CEDE, which represents the EDE integrated over the 50-year commitment period. The CEDE, although delivered over the commitment period, is assigned to the period of intake (1 year). Analogous to the external exposure submodel, the CEDE is referred to as the dose or the annual dose. This submodel includes three types of air contamination: resuspension of contaminated soil (Section 6.4.2.1), aerosols from evaporative coolers (Section 6.4.2.2), and gaseous emissions from soil, which includes exhalation of ${ }^{222} \mathrm{Rn}$ (Section 6.4.2.3) and gaseous emissions of ${ }^{14} \mathrm{C}$ (Section 6.4.6.2). The total inhalation dose is the sum of the doses resulting from these three inhalation exposure pathways, expressed as

$$
D_{i n h, i}=D_{i n h, p, i}+D_{i n h, e, i}+D_{i n h, g, i}
$$

where

$$
\begin{aligned}
& D_{i n h, i}= \text { annual dose from inhalation exposure to radionuclide } i \text { (Sv/year) } \\
& D_{\text {inh, }, i}= \begin{array}{l}
\text { annual dose from inhalation exposure to radionuclide } i \text { in resuspended } \\
\text { particles (Sv/year) }
\end{array} \\
& D_{\text {inh,e,i }}=\begin{array}{l}
\text { annual dose from inhalation exposure to radionuclide } i \text { resulting from the } \\
\text { operation of an evaporative cooler (Sv/year) }
\end{array} \\
& D_{\text {inh,g,i }}=\begin{array}{l}
\text { annual dose from inhalation exposure to radionuclide } i \text { resulting from } \\
\text { gaseous emission from the soil (Sv/year). This term applies only to the } \\
\text { inhalation of }{ }^{222} \mathrm{Rn} \text { gas and the inhalation of }{ }^{14} \mathrm{CO}_{2}
\end{array}
\end{aligned}
$$

\subsubsection{Inhalation of Resuspended Particles}

Exposure from the inhalation of radionuclides in resuspended particles depends on a number of factors, including activity concentrations in air, indoor and outdoor exposure times, the particle size distribution, the radionuclide, the chemical form of the radionuclide, and the breathing rate of the receptor. Although the resuspension pathway usually is not an important contributor to long-term exposure for most locations, it is potentially important in the dusty environments associated with some human activities (NCRP 1999 [DIRS 155894], p. 59).

As discussed for the air submodel (Section 6.4.2), radionuclide concentrations in the air, $C a_{h, i, n}$, are calculated for five environments associated with human activities and characterized by different concentrations of resuspended particles. The receptor used for the performance assessment of the repository is the RMEI, the characteristics of which are based on the lifestyles and behaviors of people residing in the Amargosa Valley. Evaluation of the inhalation exposure 
to the RMEI involves considering various population groups within the Amargosa Valley population. Therefore, parameter values for this pathway depend on the environment, the population group, or both. The inhalation submodel includes five environments, four in the contaminated area (active outdoors, inactive outdoors, active indoors, and asleep indoors) and one away from the contaminated area. The population groups include commuters, local outdoor workers, local indoor workers, and nonworkers (Section 6.4.7).

Annual doses resulting from the inhalation of primary radionuclides should also include exposure to radionuclides in the decay chain (as discussed for the surface soil submodel; Section 6.4.1.2) because the resuspended particles would come from the surface soil where radionuclides would build up during long-term irrigation. The combined dose is estimated as

$$
D_{\text {inh,p,i }}=\sum_{l} D_{i n h, p, l}=\sum_{l} E D C F_{i n h, l}\left[\sum_{n} C a_{h, l, n} B R_{n} \sum_{m}\left(P P_{m} t_{n, m}\right)\right]
$$

where

$$
\begin{aligned}
& D_{\text {inh, }, i} \quad=\quad \text { annual dose from inhalation exposure to primary radionuclide } i \text { in } \\
& \text { resuspended particles (Sv/year) } \\
& D_{\text {inh, }, l}=\text { annual dose from inhalation exposure to long-lived radionuclide } l \text { in a } \\
& \text { decay chain of primary radionuclide } i \text { in resuspended particles (Sv/year) } \\
& l \quad=\text { radionuclide index for a decay chain, } l=0 \text { for primary radionuclide, } 1 \text { for } \\
& \text { the first long-lived decay product, } 2 \text { for the second long-lived decay } \\
& \text { product } \\
& E D C F_{\text {inh, } l}=\text { effective dose conversion factor for inhalation of long-lived radionuclide } l \\
& \text { in a decay chain of primary radionuclide } i(\mathrm{~Sv} / \mathrm{Bq}) \text {. Calculation of } \\
& \text { effective dose conversion factors is discussed in Section 6.4.8.5. } \\
& n=\text { environment index; } n=1 \text { for active outdoors, } 2 \text { for inactive outdoors, } 3 \text { for } \\
& \text { active indoors, } 4 \text { for asleep indoors, and } 5 \text { for away from the contaminated } \\
& \text { area } \\
& C a_{h, l, n}=\text { activity concentration of radionuclide } l \text { in a decay chain of primary } \\
& \text { radionuclide } i \text { in air for environment } n\left(\mathrm{~Bq} / \mathrm{m}^{3}\right) \\
& B R_{n} \quad=\text { breathing rate for environment } n\left(\mathrm{~m}^{3} / \mathrm{hr}\right) \\
& m \quad=\text { population group index; } m=1 \text { for local outdoor workers, } 2 \text { for local indoor } \\
& \text { workers, } 3 \text { for commuters, and } 4 \text { for nonworkers } \\
& P P_{m} \quad=\text { fraction of the total population in population group } m \text { (population } \\
& \text { proportion) (dimensionless) } \\
& t_{n, m} \quad=\text { annual amount of time that population group } m \text { spends in environment } n \\
& \text { (exposure time) (hr/year) }
\end{aligned}
$$

The effective dose conversion factor for inhalation of radionuclide $l, E D C F_{\text {inh,l }}$ includes contributions from the short-lived decay products. The development of this parameter is described in Section 6.4.8.5. The inhalation exposure time, $t_{n, m}$, is the annual amount of time that population $m$ spends in environment $n$, depending upon the characteristics of the receptor (BSC 2004 [DIRS 169671]). The breathing rate, $B R_{n}$ varies among environments. 


\subsubsection{Inhalation of Aerosols from Evaporative Coolers}

In 1997, about 73 percent of surveyed Amargosa Valley residents used evaporative coolers (DOE 1997 [DIRS 100332], p. 20). Therefore, this submodel includes the inhalation of radionuclides introduced into indoor air by coolers as an exposure pathway. During the operation of evaporative coolers, the indoor air exchange rate is high, the residence time for the air inside the dwelling is short, and only the primary radionuclides and decay products in secular equilibrium in the groundwater are considered in this portion of the submodel.

Evaporative coolers are not expected to result in a large transport of outdoor particulates into the indoor space because the cooler pads filter out the particulates. The potential contribution of contaminated indoor air to the outdoors and to outdoor inhalation exposure is not incorporated in the submodel because the dilution of that air would be large.

Evaporative coolers are not usually operated year-round; most people use them only during the hotter months (BSC 2004 [DIRS 169671] Section 6.3.4.2). Therefore, the indoor inhalation exposure time is modified by an evaporative cooler use factor that represents the fraction of time when evaporative coolers are used. Even during the use period, evaporative coolers are not operated continuously. However, it is assumed that indoor radionuclide concentrations do not decrease when an evaporative cooler is temporarily turned off (Approximation 11).

The inhalation dose attributable to evaporative cooler operation is estimated using a method similar to Equation 6.4.8-2, as

$$
D_{\text {inh }, e, i}=E D C F_{\text {inh }, i} C a_{e, i} f_{\text {cooler }} f_{\text {use }} \sum_{n=3}^{4} B R_{n}\left(\sum_{m} P P_{m} t_{n, m}\right)
$$

where

$$
\begin{aligned}
& D_{\text {inh,e,i }}=\text { annual dose from inhalation of primary radionuclide } i \text { from evaporative } \\
& \text { cooler operation (Sv/year) } \\
& E D C F_{i n h, i}=\text { effective dose conversion factor for inhalation of radionuclide } i(\mathrm{~Sv} / \mathrm{Bq}) \\
& \mathrm{Ca}_{e, i}=\text { activity concentration of radionuclide } i \text { in indoor air attributable to the } \\
& \text { evaporative cooler operation }\left(\mathrm{Bq} / \mathrm{m}^{3}\right) \\
& n=\text { environment index ( } n=3 \text { or } n=4 \text { denotes an indoor environment) } \\
& \text { fooler } \quad=\text { fraction of houses with evaporative coolers (dimensionless) } \\
& \text { fuse } \quad=\text { annual evaporative cooler use factor (dimensionless) } \\
& \text { and the other parameters are defined in Equation 6.4.8-2. }
\end{aligned}
$$

The activity concentration of radionuclides in indoor air attributable to the operation of evaporative coolers is discussed in the air submodel (Section 6.4.2.2). The annual evaporative cooler use factor and the fraction of houses with evaporative coolers are developed based on a site-specific survey in Amargosa Valley (DOE 1997 [DIRS 100332]) and temperatures representative of present-day and future predicted climatic conditions there, as documented in BSC (2004 [DIRS 169671], Section 6.3.4). 


\subsubsection{Inhalation of ${ }^{14} \mathrm{C}$}

This submodel includes another potential inhalation exposure pathway: the inhalation of gaseous ${ }^{14} \mathrm{C}$ released from irrigated soil. After ${ }^{14} \mathrm{C}$ is released from soil as ${ }^{14} \mathrm{CO}_{2}$, it is dispersed in the outdoor and indoor environments. There are no mechanisms that would greatly change the indoor concentration of ${ }^{14} \mathrm{C}$ relative to the outdoor concentration, so both are considered the same. The inhalation dose from ${ }^{14} \mathrm{C}$ is calculated using a method similar to that used to calculate the inhalation dose from resuspended particulates (Section 6.4.8.1)

$$
\begin{aligned}
D_{i n h, g, C-14} & =\sum_{n} D_{i n h, g, C-14, n} \\
& =D C F_{i n h, C-14} C a_{g, C-14} \sum_{n} B R_{n}\left(\sum_{m} P P_{m} t_{n, m}\right)
\end{aligned}
$$

where

$$
\begin{array}{ll}
D_{i n h, g, C-14} & \text { annual dose from inhalation of gaseous }{ }^{14} \mathrm{C}(\mathrm{Sv} / \text { year }) \\
D_{i n h, g, C-14, n} & =\text { annual dose from inhalation of gaseous }{ }^{14} \mathrm{C} \text { for environment } n \text { (Sv/year) } \\
C a_{g, C-14} & \text { activity concentration of }{ }^{14} \mathrm{C} \text { in air }\left(\mathrm{Bq} / \mathrm{m}^{3}\right) \\
D C F_{\text {inh,C-14 }} & =\text { dose conversion factor for inhalation of }{ }^{14} \mathrm{C}(\mathrm{Sv} / \mathrm{Bq}) \\
\text { and the other parameters are defined in Equations } 6.4 .6-3,6.4 .8-1, \text { and } 6.4 .8-2 .
\end{array}
$$

\subsubsection{Inhalation of Radon Decay Products}

The release of ${ }^{222} \mathrm{Rn}$ from the soil, and its subsequent decay in the air through a series of short-lived decay products, causes an inhalation dose. In the soil, ${ }^{226} \mathrm{Ra}$ is considered to have originated from irrigation water or from radioactive decay of other radionuclides initially present in irrigation water (Section 6.4.1.2).

The dose due to inhalation of radon decay products is evaluated separately for indoor environments when evaporative coolers are off and when they are in operation because of the increase in ventilation caused by the operation of coolers. The total radon dose from ${ }^{222} \mathrm{Rn}$ decay products is evaluated as

$$
\begin{aligned}
D_{\text {inh }, g, R n-222}= & \sum_{n} D_{i n h, g, R n-222, n} \\
= & \sum_{n=1}^{5} C a_{g, R n-222, n} F_{n} D C F_{i n h, R n-222, n} B R_{n}\left(\sum_{m} P P_{m} t_{n, m}\right)+ \\
& \sum_{n=3}^{4} C a_{g, R n-222, e} f_{\text {cooler }} f_{\text {use }} D C F_{i n h, R n-222, n} B R_{n}\left(\sum_{m} P P_{m} t_{n, m}\right)
\end{aligned}
$$


where

$$
\begin{aligned}
& D_{i n h, g, R n-222}=\text { annual dose from inhalation of }{ }^{222} \mathrm{Rn} \text { decay products (Sv/year) } \\
& D_{i n h, g, R n-222 n}=\text { annual dose from inhalation of }{ }^{222} \mathrm{Rn} \text { decay products for } \\
& \text { environment } n \text { (Sv/year) } \\
& C a_{g, R n-222, n}=\text { activity concentration of }{ }^{222} \mathrm{Rn} \text { in environment } n\left(\mathrm{~Bq} / \mathrm{m}^{3}\right) \\
& F_{n} \quad=\text { correction factor to account for the use of evaporative coolers in } \\
& \text { indoor environment } n \text { (dimensionless), } 1 \text { for } n=1 \& 2 \text {, and } \\
& \left(1-f_{\text {cooler }} \times f_{\text {use }}\right) \text { for } n=3 \& 4 \\
& C a_{g, R n-222, e} \quad=\text { activity concentration of }{ }^{222} \mathrm{Rn} \text { in indoor air at a high ventilation rate } \\
& \text { during evaporative cooler in operation }\left(\mathrm{Bq} / \mathrm{m}^{3}\right) \\
& D C F_{i n h, R n-222, n}=\text { dose conversion factor for inhalation of }{ }^{222} \mathrm{Rn} \text { decay products for } \\
& \text { environment } n(\mathrm{~Sv} / \mathrm{Bq})
\end{aligned}
$$

The dose conversion factor for the inhalation of ${ }^{222} \mathrm{Rn}$ decay products for environment $n$ can be further expressed as

$$
D C F_{i n h, R n-222, n}=D C F_{i n h, R n-222} E F_{R n-222, n}
$$

where

$$
\begin{array}{ll}
E F_{R n-22, n}= & \begin{array}{l}
\text { equilibrium factor for } \\
\text { (dimensionless) }
\end{array} \\
D C F_{\text {inh, } R n-222} \mathrm{Rn} \text { decay products for environment } n & \begin{array}{l}
\text { dose conversion factor for inhalation of } \\
\text { equilibrium }
\end{array}
\end{array}
$$

The equilibrium factor permits estimating the potential alpha energy concentration from the measurement of radon gas (here ${ }^{222} \mathrm{Rn}$ ), and is defined as the ratio of the actual potential alpha energy concentration to that prevailing if all decay products in the ${ }^{222} \mathrm{Rn}$ series are in equilibrium with the parent radon. The equilibrium factor depends on the environment, such as indoors and outdoors (BSC 2004 [DIRS 169672], Section 6.6). By combining Equations 6.4.8-5 and 6.4.8-6, the inhalation dose from the ${ }^{222} \mathrm{Rn}$ decay products is evaluated as

$$
\begin{aligned}
D_{i n h, g, R n-222}= & \sum_{n} D_{i n h, g, R n-222, n} \\
= & D C F_{i n h, R n-222} \sum_{n=1}^{5} C a_{g, R n-222, n} F_{n} E F_{R n-222, n} B R_{n}\left(\sum_{m} P P_{m} t_{n, m}\right)+ \\
& D C F_{i n h, R n-222} \sum_{n=3}^{4} C a_{g, R n-222, e} f_{\text {cooler }} f_{\text {use }} E F_{R n-222, n} B R_{n}\left(\sum_{m} P P_{m} t_{n, m}\right)
\end{aligned}
$$

where the parameters are defined in Equations 6.4.8-3, 6.4.8-5, and 6.4.8-6. 
For the TSPA-LA, ${ }^{222} \mathrm{Rn}$ is a decay product of ${ }^{226} \mathrm{Ra}$, not a primary radionuclide. This radon inhalation dose contribution is added into the BDCF for ${ }^{226} \mathrm{Ra}$ or to radionuclides that have ${ }^{226} \mathrm{Ra}$ as a decay product.

The calculation of dose from radon decay products is based on the indoor and outdoor concentrations of radon gas. The indoor radon concentration is equal to outdoor radon concentration plus a contribution of radon from soil beneath the house, which depends on the house ventilation rate. Two ventilation rates are considered in the submodel: a high rate when coolers are in use, and a low rate when coolers are not in use. When evaporative coolers are in use, the contribution of radon from soil beneath the house would be limited because the high ventilation rate would prevent radon buildup. However, when evaporative coolers are not in operation, or for houses that had no evaporative cooler, a higher radon contribution from soil beneath the house would be expected. Radon from household water use (e.g., showers and evaporative coolers), typically, is of minor importance (Section 7.4.3.1). Therefore, the submodel does not include this pathway.

\subsubsection{Effective Dose Conversion Factor for Inhalation}

The effective inhalation dose conversion factors used in the ERMYN are based on dose conversion factors obtained from FGR-11 (Eckerman et al. 1988 [DIRS 101069], Table 2.1). These factors represent the radionuclide-specific CEDE for the 50-year commitment period per unit activity intake. As discussed in Section 6.3.5, the dose contributions from short-lived decay products are combined with those of the long-lived parent radionuclide to produce effective dose conversion factors. The development of the effective inhalation dose conversion factors is shown in Table 6.4-3. The calculation method can be expressed as

$$
E D C F_{i n h, i}=\sum_{s} D C F_{i n h, s} \times B N_{s}
$$

where

$$
\begin{aligned}
& D C F_{i n h, s}=\text { dose conversion factor for inhalation for short-lived radionuclide } s \text { in the } \\
& \text { decay chain of primary radionuclide } i(\mathrm{~Sv} / \mathrm{Bq}) \\
& S \quad=\quad \text { index of short-lived radionuclide decay chain under a primary radionuclide } i \\
& B N_{s} \quad=\text { branching fraction for short-lived radionuclide } s \text { in the decay chain of } \\
& \text { primary radionuclide } i \text { (dimensionless) }
\end{aligned}
$$

and the other parameter is defined in Equation 6.4.8-2.

Similar to Table 6.4-2, the values in Table 6.4-3 are for demonstration purposes only. The ERMYN uses branching fractions and dose conversion factors as inputs to calculate the effective dose conversion factors in GoldSim (Section 6.8).

The inhalation dose conversion factor for some radionuclides have different values depending on the chemical compound of the radionuclide and the lung clearance class. Because the chemical form of radionuclides in the biosphere is not known, the highest values of dose conversion factors from among those available, generally, are chosen. However, the highest inhalation dose conversion factor value for ${ }^{90} \mathrm{Sr}$ is for the chemical compound $\mathrm{SrTiO}_{3}$, which is not a common 
chemical compound expected in the biosphere (Rittmann 1993 [DIRS 107744], Section 2), so it is not used. The justification for selecting inhalation dose conversion factors is provided in BSC (2004 [DIRS 169671], Section 6.5).

Table 6.4-3. Effective Dose Conversion Factors for Inhalation

\begin{tabular}{|c|c|c|c|c|}
\hline $\begin{array}{c}\text { Primary } \\
\text { Radionuclide }\end{array}$ & $\begin{array}{l}\text { Lung Clearance } \\
\text { Class }\end{array}$ & $\begin{array}{l}\text { Decay Product }{ }^{\mathrm{c}} \text { (branching } \\
\text { fraction if not } 100 \% \text {, half-life) }\end{array}$ & $\begin{array}{c}\text { Dose } \\
\text { Conversion } \\
\text { Factor }\left(D C F_{\text {inh }}\right) \\
{ }_{\mathrm{d}}(\mathrm{Sv} / \mathrm{Bq})\end{array}$ & $\begin{array}{c}\text { Effective Dose } \\
\text { Conversion } \\
\text { Factor } \\
\text { (EDCF inh) } \\
\text { (Sv/Bq) }\end{array}$ \\
\hline C-14 & $\mathrm{CO}_{2}$ & - & $6.36 \mathrm{E}-12$ & $6.36 \mathrm{E}-12$ \\
\hline $\mathrm{Cl}-36$ & W & - & 5.93E-09 & 5.93E-09 \\
\hline Se-79 & W & - & 2.66E-09 & $2.66 \mathrm{E}-09$ \\
\hline Sr-90D ${ }^{a}$ & $\begin{array}{l}\mathrm{D} \\
\mathrm{Y}\end{array}$ & Y-90 (64.0 hr) & $\begin{array}{l}6.47 \mathrm{E}-08 \\
2.28 \mathrm{E}-09 \\
\end{array}$ & $6.70 \mathrm{E}-08$ \\
\hline Tc-99 & W & - & $2.25 \mathrm{E}-09$ & $2.25 \mathrm{E}-09$ \\
\hline Sn-126D & $\begin{array}{l}\text { W } \\
D \\
W\end{array}$ & $\begin{array}{l}\text { Sb-126m (19.0 min) } \\
\text { Sb-126 (14\%, } 12.4 \text { d) }\end{array}$ & $\begin{array}{l}2.69 \mathrm{E}-08 \\
9.17 \mathrm{E}-12 \\
3.17 \mathrm{E}-09\end{array}$ & $2.74 \mathrm{E}-08$ \\
\hline $\mathrm{I}-129$ & $\mathrm{D}$ & - & 4.69E-08 & 4.69E-08 \\
\hline Cs-135 & $\mathrm{D}$ & - & 1.23E-09 & $1.23 \mathrm{E}-09$ \\
\hline Cs-137D & $\begin{array}{l}\mathrm{D} \\
-\end{array}$ & Ba-137m (94.6\%, $2.552 \mathrm{~min})$ & $\begin{array}{l}8.63 \mathrm{E}-09 \\
0.00 \mathrm{E}+00\end{array}$ & 8.63E-09 \\
\hline Pu-242 & W & - & $1.11 \mathrm{E}-04$ & $1.11 \mathrm{E}-04$ \\
\hline $\mathrm{U}-238 \mathrm{D}$ & $\begin{array}{l}\mathrm{Y} \\
\mathrm{Y} \\
\mathrm{Y}\end{array}$ & $\begin{array}{l}\text { Th-234 (24.10 d) } \\
\text { Pa-234m (99.80\%, } 1.17 \mathrm{~min}) \\
\text { Pa-234 (0.20\%, 6.7 hr) }\end{array}$ & $\begin{array}{l}3.20 \mathrm{E}-05 \\
9.47 \mathrm{E}-09 \\
0.00 \mathrm{E}+00 \\
2.20 \mathrm{E}-10\end{array}$ & $3.20 \mathrm{E}-05$ \\
\hline $\mathrm{Pu}-238$ & $\mathrm{~W}$ & - & 1.06E-04 & 1.06E-04 \\
\hline U-234 & $\mathrm{Y}$ & - & 3.58E-05 & 3.58E-05 \\
\hline Th-230 & $\mathrm{W}$ & - & 8.80E-05 & 8.80E-05 \\
\hline Ra-226D & $\begin{array}{l}\text { W } \\
- \\
\bar{D} \\
\bar{D} \\
- \\
-\end{array}$ & $\begin{array}{l}\text { Rn-222 (3.8235 d) } \\
\text { Po-218 (3.05 min) } \\
\text { Pb-214 (99.98\%, } 26.8 \mathrm{~min}) \\
\text { At-218 }(0.02 \%, 2 \mathrm{sec}) \\
\text { Bi-214 }(19.9 \mathrm{~min}) \\
\text { Po-214 }\left(99.98 \%, 1.64 \times 10^{-4} \mathrm{sec}\right) \\
\text { Tl-210 }(0.02 \%, 1.3 \mathrm{~min})^{\mathrm{e}}\end{array}$ & $\begin{array}{c}2.32 \mathrm{E}-06 \\
0.00 \mathrm{E}+00^{f} \\
0.00 \mathrm{E}+00 \\
2.11 \mathrm{E}-09 \\
0.00 \mathrm{E}+00 \\
1.78 \mathrm{E}-09 \\
0.00 \mathrm{E}+00 \\
0.00 \mathrm{E}+00\end{array}$ & $2.32 \mathrm{E}-06$ \\
\hline $\mathrm{Pb}-210 \mathrm{D}$ & $\begin{array}{l}D \\
W \\
D\end{array}$ & $\begin{array}{l}\operatorname{Bi}-210(5.012 \mathrm{~d}) \\
\text { Po-210 (138.38 d) }\end{array}$ & $\begin{array}{l}3.67 \mathrm{E}-06 \\
5.29 \mathrm{E}-08 \\
2.54 \mathrm{E}-06 \\
\end{array}$ & $6.26 \mathrm{E}-06$ \\
\hline Pu-240 & $\mathrm{W}$ & - & 1.16E-04 & 1.16E-04 \\
\hline U-236 & $\mathrm{Y}$ & - & 3.39E-05 & 3.39E-05 \\
\hline Th-232 & $\mathrm{W}$ & - & 4.43E-04 & 4.43E-04 \\
\hline
\end{tabular}


Table 6.4-3. Effective Dose Conversion Factors for Inhalation (Continued)

\begin{tabular}{|c|c|c|c|c|}
\hline $\begin{array}{c}\text { Primary } \\
\text { Radionuclide }\end{array}$ & $\begin{array}{l}\text { Lung Clearance } \\
\text { Class }\end{array}$ & $\begin{array}{l}\text { Decay Product }{ }^{\mathrm{C}} \text { (branching } \\
\text { fraction if not } 100 \% \text {, half-life) }\end{array}$ & $\begin{array}{c}\text { Dose } \\
\text { Conversion } \\
\text { Factor }\left(D C F_{i n h}\right) \\
{ }_{d}(\mathrm{~Sv} / \mathrm{Bq})\end{array}$ & $\begin{array}{l}\text { Effective Dose } \\
\text { Conversion } \\
\text { Factor } \\
\left(E D C F_{i n h}\right) \\
\text { (Sv/Bq) }\end{array}$ \\
\hline$R a-228 D^{b}$ & $\begin{array}{l}\mathrm{W} \\
\mathrm{D}\end{array}$ & Ac-228 (6.13 hr) & $\begin{array}{l}1.29 \mathrm{E}-06 \\
8.33 \mathrm{E}-08\end{array}$ & $1.37 \mathrm{E}-06$ \\
\hline $\mathrm{U}-232$ & $\bar{Y}$ & - & 1.78E-04 & $1.78 \mathrm{E}-04$ \\
\hline Th-228D & $\begin{array}{l}\text { Y } \\
W \\
- \\
- \\
D \\
D \\
- \\
-\end{array}$ & $\begin{array}{l}\text { Ra-224 (3.66 d) } \\
\text { Rn-220 (55.6 sec) } \\
\text { Po-216 (0.15 sec) } \\
\text { Pb-212 (10.64 hr) } \\
\text { Bi-212 (60.55 min) } \\
\text { Po-212 (64.07\%, } 0.305 \mu-\mathrm{sec}) \\
\text { TI-208 (35.93\%, } 3.07 \mathrm{~min})\end{array}$ & $\begin{array}{l}9.23 \mathrm{E}-05 \\
8.53 \mathrm{E}-07 \\
0.00 \mathrm{E}+00 \\
0.00 \mathrm{E}+00 \\
4.56 \mathrm{E}-08 \\
5.83 \mathrm{E}-09 \\
0.00 \mathrm{E}+00 \\
0.00 \mathrm{E}+00\end{array}$ & 9.32E-05 \\
\hline Am-243D & $\begin{array}{l}\mathrm{W} \\
\mathrm{W}\end{array}$ & $\mathrm{Np}-239(2.355 \mathrm{~d})$ & $\begin{array}{l}1.19 \mathrm{E}-04 \\
6.78 \mathrm{E}-10 \\
\end{array}$ & $1.19 \mathrm{E}-04$ \\
\hline Pu-239 & W & - & 1.16E-04 & 1.16E-04 \\
\hline U-235D & $\begin{array}{l}Y \\
Y\end{array}$ & Th-231 (25.52 hr) & $\begin{array}{l}3.32 \mathrm{E}-05 \\
2.37 \mathrm{E}-10 \\
\end{array}$ & 3.32E-05 \\
\hline $\mathrm{Pa}-231$ & W & - & 3.47E-04 & $3.47 \mathrm{E}-04$ \\
\hline Ac-227D & $\begin{array}{l}\mathrm{D} \\
\mathrm{Y} \\
\mathrm{D} \\
\mathrm{W} \\
- \\
- \\
\mathrm{D} \\
- \\
- \\
-\end{array}$ & $\begin{array}{l}\text { Th-227 (98.62\%, } 18.718 \mathrm{~d}) \\
\text { Fr-223 (1.38\%, 21.8 min) } \\
\text { Ra-223 (11.434 d) } \\
\text { Rn-219 (3.96 sec) } \\
\text { Po-215 (1.78 millisec) } \\
\text { Pb-211 (36.1 min) } \\
\text { Bi-211 (2.15 min) } \\
\text { Tl-207 (99.72\%, } 4.77 \mathrm{~min}) \\
\text { Po-211 (0.28\%, 0.516 sec) }\end{array}$ & $\begin{array}{l}1.81 \mathrm{E}-03 \\
4.37 \mathrm{E}-06 \\
1.68 \mathrm{E}-09 \\
2.12 \mathrm{E}-06 \\
0.00 \mathrm{E}+00 \\
0.00 \mathrm{E}+00 \\
2.35 \mathrm{E}-09 \\
0.00 \mathrm{E}+00 \\
0.00 \mathrm{E}+00 \\
0.00 \mathrm{E}+00\end{array}$ & 1.82E-03 \\
\hline Am-241 & $\mathrm{W}$ & - & $1.20 \mathrm{E}-04$ & $1.20 \mathrm{E}-04$ \\
\hline$N p-237 D$ & $\begin{array}{l}W \\
Y\end{array}$ & $\mathrm{~Pa}-233(27.0 \mathrm{~d})$ & $\begin{array}{l}1.46 \mathrm{E}-04 \\
2.58 \mathrm{E}-09 \\
\end{array}$ & 1.46E-04 \\
\hline $\mathrm{U}-233$ & $\mathrm{Y}$ & - & 3.66E-05 & $3.66 \mathrm{E}-05$ \\
\hline
\end{tabular}


Table 6.4-3. Effective Dose Conversion Factors for Inhalation (Continued)

\begin{tabular}{|c|c|c|c|c|}
\hline $\begin{array}{c}\text { Primary } \\
\text { Radionuclide }\end{array}$ & $\begin{array}{c}\text { Lung Clearance } \\
\text { Class }\end{array}$ & $\begin{array}{l}\text { Decay Product }{ }^{\mathrm{c}} \text { (branching } \\
\text { fraction if not } 100 \% \text {, half-life) }\end{array}$ & $\begin{array}{c}\text { Dose } \\
\text { Conversion } \\
\text { Factor }\left(D C F_{\text {inh }}\right) \\
{ }^{\mathrm{d}}(\mathrm{Sv} / \mathrm{Bq}) \\
\end{array}$ & $\begin{array}{c}\text { Effective Dose } \\
\text { Conversion } \\
\text { Factor } \\
\text { (EDCF } \text { Finh }) \\
\text { (Sv/Bq) } \\
\end{array}$ \\
\hline Th-229D & $\begin{array}{l}\text { W } \\
W \\
D \\
- \\
- \\
D \\
- \\
-\end{array}$ & $\begin{array}{l}\text { Ra-225 (14.8 d) } \\
\text { Ac-225 (10.0 d) } \\
\text { Fr-221 (4.8 min) } \\
\text { At-217 (32.3 millisec) } \\
\text { Bi-213 (45.65 min) } \\
\text { Po-213 (97.84\%, } 4.2 \mu-s e c) \\
\text { Tl-209 (2.16\%, } 2.2 \mathrm{~min}) \\
\text { Pb-209 (3.253 hr) }\end{array}$ & $\begin{array}{l}5.80 \mathrm{E}-04 \\
2.10 \mathrm{E}-06 \\
2.92 \mathrm{E}-06 \\
0.00 \mathrm{E}+00 \\
0.00 \mathrm{E}+00 \\
4.63 \mathrm{E}-09 \\
0.00 \mathrm{E}+00 \\
0.00 \mathrm{E}+00 \\
2.56 \mathrm{E}-11\end{array}$ & 5.85E-04 \\
\hline
\end{tabular}

a $A$ " $D$ " indicates that the radionuclide is treated with its short-lived (less than $180 \mathrm{~d}$ ) decay products.

${ }^{b}$ Indented radionuclides are long-lived decay products considered separately from the parents.

c Branching fractions and half-lives are from Eckerman and Ryman (1993 [DIRS 107684], Table A.1).

d Dose conversion factor source (BSC 2004 [DIRS 169671], Section 6.5).

e Half-life of TI-210 is taken from Lide and Frederikse (1997 [DIRS 103178] p.11-125).

${ }^{f}$ Dose conversion factor for inhalation of short-lived decay products of ${ }^{222} \mathrm{Rn}$ (alpha emitters) is calculated separately and is equal to $1.33 \times 10^{-8} \mathrm{~Sv} / \mathrm{Bq}$ (BSC 2004 [DIRS 169671], Section 6.5.4).

$\mathrm{DCF}=$ dose conversion factor

\subsubsection{Ingestion Exposure Submodel}

When contaminated groundwater is used to produce food for humans or farm animals, the ingestion of that food should result in a radiation dose. Inadvertent ingestion of contaminated soil also results in a radiation dose. The ingestion submodel addresses human doses from ingesting contaminated drinking water, four types of crops (leafy vegetables, other vegetables, fruits, and grain), four types of animal products (meat in the form of beef, poultry, milk, and eggs), freshwater fish, and soil. The ingestion dose, analogous to the inhalation dose, is calculated as the CEDE for the 50-year committed period resulting from 1 year of intake.

The methods for calculating activity concentrations in contaminated crops, animal products, and fish are discussed with respect to the plant (Section 6.4.3), animal (Section 6.4.4), and fish submodels (Section 6.4.5), respectively. The source of water for human consumption, animal consumption, irrigation, and fish farming is untreated groundwater. The rate of inadvertent soil ingestion by adults is used in the submodel, but purposeful soil ingestion is excluded. The total ingestion dose for a radionuclide includes contributions from all of these sources, and is expressed as

$$
D_{i n g, i}=D_{i n g, w, i}+D_{i n g, p, i}+D_{i n g, d, i}+D_{i n g, f, i}+D_{i n g, s, i}
$$

where

$$
\begin{aligned}
& D_{\text {ing } i}=\text { annual dose from ingestion of radionuclide } i \text { (Sv/year) } \\
& D_{\text {ing,w,i }}=\text { annual dose from ingestion of radionuclide } i \text { in drinking water (Sv/year) } \\
& D_{\text {ing, }, i}=\text { annual dose from ingestion of radionuclide } i \text { in crops (Sv/year) }
\end{aligned}
$$


$D_{\text {ing,d,i }}=$ annual dose from ingestion of radionuclide $i$ in animal products (Sv/year)

$D_{\text {ing,f,i }}=$ annual dose from ingestion of radionuclide $i$ in fish (Sv/year)

$D_{\text {ing,s,i }}=$ annual dose from inadvertent ingestion of radionuclide $i$ in surface soil (Sv/year)

These ingestion pathways are discussed in detail in the following subsections.

All short-lived radionuclides (half-life less than $180 \mathrm{~d}$ ) are assumed to be in equilibrium with the long-lived primary radionuclides, and the effective dose conversion factors for the long-lived primary radionuclides includes their dose contribution (Approximation 2). In addition, for pathways such as ingestion of crops, animal products, and soil, the contribution due to radionuclide decay and ingrowth in surface soil is added into the primary radionuclides. The method is discussed in Section 6.4.1.2.

\subsubsection{Ingestion of Drinking Water}

The drinking water pathway, generally, is an important ingestion pathway for the groundwater scenario (BSC 2004 [DIRS 169674], Table 6.2-10). For this pathway, the primary radionuclides are assumed to be in the groundwater and accompanied by their short-lived decay products. The annual dose from ingestion of radionuclides in drinking water is expressed as

$$
D_{i n g, w, i}=E D C F_{i n g, i} C w_{i} U w
$$

where

$$
\begin{aligned}
E D C F_{\text {ing }, i}= & \text { effective dose conversion factor for ingestion of radionuclide } i(\mathrm{~Sv} / \mathrm{Bq}) ; \\
& \text { calculation of effective dose conversion factors for ingestion is discussed in } \\
& \text { Section } 6.4 .9 .6 \\
= & \text { activity concentration of radionuclide } i \text { in groundwater }(\mathrm{Bq} / \mathrm{L}) \\
C w_{i}=\begin{array}{l}
\text { annual consumption rate of contaminated drinking water by the receptor } \\
\text { (L/year) }
\end{array} &
\end{aligned}
$$

The development of effective ingestion dose conversion factors is discussed in Section 6.4.9.6. The annual water consumption rate for the receptor is specified in 10 CFR 63.312(d) ([DIRS 156605]) as $2 \mathrm{~L} / \mathrm{d}$ (730 L/year).

\subsubsection{Ingestion of Crop Foodstuffs}

Ingestion of crops also is an important pathway for the groundwater scenario (CRWMS M\&O 2001 [DIRS 152539], p. 78). Radionuclide decay during storage (between harvest and consumption) is not considered because only long-lived primary radionuclides are of concern for the biosphere model. The annual dose to a receptor from ingestion of primary radionuclide $i$ in foodstuffs should include all radionuclides ( $l$ ) in a decay chain (if one exists), as discussed in the surface soil submodel (Section 6.4.1.2). The annual dose from ingestion of contaminated crops is expressed as 


$$
D_{\text {ing, } p, i}=\sum_{l} D_{\text {ing }, p, l}=\sum_{l}\left[E D C F_{\text {ing,l }} \sum_{j}\left(C p_{l, j} U p_{j}\right)\right]
$$

where

$$
\begin{aligned}
& D_{\text {ing,p,i }}=\text { annual dose from ingestion of primary radionuclide } i \text { in crops (Sv/year) } \\
& D_{\text {ing, }, l}=\quad=\text { annual dose from ingestion of long-lived radionuclide } l \text { in decay chain of } \\
& \text { primary radionuclide } i \text { in crops (Sv/year) } \\
& l \quad=\quad \text { index of radionuclide decay chain } l=0 \text { for primary radionuclide } \\
& E D C F_{\text {ing, } l}=\text { effective dose conversion factor for ingestion of radionuclide } l \text { in decay } \\
& \text { chain of primary radionuclide } i(\mathrm{~Sv} / \mathrm{Bq}) \\
& C p_{l, j} \quad=\quad \text { activity concentration of primary radionuclide } l \text { in the crop type } j(\mathrm{~Bq} / \mathrm{kg}) \\
& j \quad=\quad \text { index of crop type, } j=1 \text { for leafy vegetables, } 2 \text { for other vegetables, } 3 \text { for } \\
& \text { fruit, and } 4 \text { for grain } \\
& U p_{j} \quad=\text { annual consumption rate of locally produced crop type } j(\mathrm{~kg} / \text { year) }
\end{aligned}
$$

The activity concentrations of radionuclides in crops is discussed in the plant submodel (Section 6.4.3). The consumption rates used in Equation 6.4.9-3 apply only to locally produced crops (BSC 2004 [DIRS 169671]); imported crops are considered to be uncontaminated.

\subsubsection{Ingestion of Animal Products}

Animal product foodstuffs may become contaminated if animals are raised using contaminated feed and water. This submodel does not include radionuclide decay during storage of feed, but it does include contributions from radionuclide decay chains. For animals, all feed and water is contaminated. The annual dose from ingestion of contaminated animal products is expressed as

$$
D_{\text {ing }, d, i}=\sum_{l} D_{i n g, d, l}=\sum_{l}\left[E D C F_{i n g, l} \sum_{k}\left(C d_{l, k} U d_{k}\right)\right]
$$

where

$$
\begin{aligned}
& D_{\text {ing,d,i }}=\text { annual dose from ingestion of primary radionuclide } i \text { (Sv/year) } \\
& D_{\text {ing,d,l }}=\text { annual dose from ingestion of radionuclide } l \text { in decay chain of primary } \\
& \text { radionuclide } i \text { in animal products (Sv/year) } \\
& C d_{l, k}=\text { activity concentration of primary radionuclide } l \text { in the animal product } \\
& \text { type } k(\mathrm{~Bq} / \mathrm{kg}) \\
& k=\quad \text { index of animal products, } k=1 \text { for meat, } 2 \text { for poultry, } 3 \text { for milk, } \\
& \text { and } 4 \text { for eggs } \\
& U d_{k}=\text { annual consumption rate of locally produced animal product type } k \\
& \text { ( } \mathrm{kg} / \text { year) }
\end{aligned}
$$

and the other parameter is defined in Equation 6.4.9-3.

The activity concentrations of radionuclides in animal products are discussed in the animal submodel (Section 6.4.4). The consumption rates in Equation 6.4.9-4 apply only to locally 
produced animal products (BSC 2004 [DIRS 169671]); imported animal products are considered to be uncontaminated.

\subsubsection{Ingestion of Fish}

The ingestion of locally produced fish is another potential exposure pathway in the groundwater scenario. As discussed for the fish submodel (Section 6.4.5), groundwater is used as a water source for fishponds. Radionuclide decay and ingrowth in the ponds is not considered because fishpond water is not expected to be used for very long (Roe 2002 [DIRS 160674]). All short-lived decay products are considered to be in equilibrium with the long-lived parent radionuclide (Section 6.4.5). Contaminated groundwater is the only water source for fish. The annual dose from ingestion of contaminated fish is calculated as

$$
D_{\text {ing, }, \text { i }}=E D C F_{\text {ing, } i} C f_{i} U f
$$

where

$$
\begin{array}{lll}
D_{\text {ing,f,i }} & =\text { annual dose from ingestion of primary radionuclide } i \text { in fish (Sv/year) } \\
C f_{i} & =\text { activity concentration of primary radionuclide } i \text { in fish }(\mathrm{Bq} / \mathrm{kg}) \\
U f & =\text { annual consumption rate of locally produced fish (kg/year) }
\end{array}
$$

The parameter, $E D C F_{\text {ing,i, }}$, is defined in Equation 6.4.9-2. Activity concentrations in fish are discussed in the fish submodel (Section 6.4.5). The consumption rates used in Equation 6.4.9-5 apply only to locally produced fish (BSC 2004 [DIRS 169671], Section 6.4); imported fish is considered to be uncontaminated.

\subsubsection{Inadvertent Soil Ingestion}

In the groundwater scenario, soils are contaminated during irrigation, and the duration of irrigation is taken to be sufficiently long that radionuclides and decay products accumulate in the soil and reach equilibrium (Approximation 5). As a result, soils contain more radioactivity than any other biosphere component. To account for radionuclides consumed with contaminated soil, the ERMYN includes inadvertent soil consumption as an ingestion pathway. Modeling activity concentrations in the soil involves tracking the accumulation and loss of all decay products of a primary radionuclide (Section 6.4.1.2). The annual dose from inadvertent ingestion of contaminated soil is calculated as

$$
D_{\text {ing,s,i }}=\sum_{l} D_{i n g, s, l}=\sum_{l}\left(E D C F_{i n g, l} C s_{m, l} U s\right)
$$

where

$$
\begin{aligned}
& D_{\text {ing,s,i }}= \begin{array}{l}
\text { annual dose from ingestion of primary radionuclide } i \text { in the surface soil } \\
\text { (Sv/year) }
\end{array} \\
& D_{\text {ing,s,l }=}=\begin{array}{l}
\text { annual dose from ingestion of long-lived radionuclide } l \text { in the decay chain of } \\
\text { a primary radionuclide } i \text { in the surface soil (Sv/year) }
\end{array} \\
& C s_{m, l}=\begin{array}{l}
\text { mass-based activity concentration of primary radionuclide } l \text { in the surface } \\
\text { soil }(\mathrm{Bq} / \mathrm{kg})
\end{array}
\end{aligned}
$$


Us $\quad=$ annual consumption rate of contaminated soil $(\mathrm{kg} / \mathrm{year})$

and the other parameter is defined in Equation 6.4.9-3.

Activity concentrations of radionuclides in the surface soil are discussed in the surface soil submodel (Section 6.4.1). Estimates of soil ingestion rates usually have wide uncertainty distributions, and typical values for adults are on the order of several tens to a few hundred milligrams per day (BSC 2004 [DIRS 169671], Section 6.4).

\subsubsection{Effective Dose Conversion Factor for Ingestion}

The effective ingestion dose conversion factors used in the ERMYN are based on dose conversion factors from FGR-11 (Eckerman et al. 1988 [DIRS 101069], Table 2.2), which represent the CEDE for the 50-year commitment period per unit of activity intake of a radionuclide. As discussed in Section 6.3.5, dose contributions from short-lived decay products are combined with those of the long-lived parent radionuclide to produce effective dose conversion factors. The development of effective ingestion dose conversion factors is shown in Table 6.4-4. The calculation method can be expressed as

$$
E D C F_{i n g, i}=\sum_{s} D C F_{i n g, s} \times B N_{s}
$$

where

$$
\begin{aligned}
& D C F_{\text {ing }, s}=\text { dose conversion factor for ingestion for short-lived radionuclide } s \text { in the } \\
& s=\text { index of short-lived radionuclide decay chain under a primary radionuclide } i \\
& B N_{s} \quad=\text { branching fraction for short-lived radionuclide } s \text { in the decay chain of } \\
& \text { primary radionuclide } i \text { (dimensionless) }
\end{aligned}
$$

and the other parameter is defined in Equation 6.4.9.2.

Similar to Table 6.4-2, the values in Table 6.4-4 are for demonstration purposes only. The ERMYN uses branching fractions and dose conversion factors as inputs to calculate the effective dose conversion factors in GoldSim (Section 6.8).

The ingestion dose conversion factors for some radionuclides have several values, depending on the chemical form of the radionuclides. Because the chemical form of radionuclides may be different in different environmental media and is not exactly known, the highest values of dose conversion factors from among the available choices are selected. The justification for selecting ingestion dose conversion factors is provided in BSC (2004 [DIRS 169671], Section 6.5). 
Table 6.4-4. Effective Dose Conversion Factors for Ingestion

\begin{tabular}{|c|c|c|c|c|}
\hline $\begin{array}{c}\text { Primary } \\
\text { Radionuclide }\end{array}$ & $\begin{array}{l}\text { Fractional } \\
\text { Uptake }\end{array}$ & $\begin{array}{l}\text { Decay Product }{ }^{\mathrm{c}} \text { (branching } \\
\text { fraction if not } 100 \% \text {, half-life) }\end{array}$ & $\begin{array}{c}\text { Dose Conversion } \\
\text { Factor }\left(D C F_{\text {ing }}\right)^{d} \\
(\mathrm{~Sv} / \mathrm{Bq})\end{array}$ & $\begin{array}{l}\text { Effective Dose } \\
\text { Conversion } \\
\text { Factor }\left(E D C F_{\text {ing }}\right) \\
\text { (Sv/Bq) }\end{array}$ \\
\hline C-14 & 1.0 & 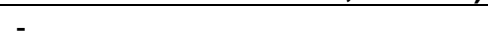 & $5.64 \mathrm{E}-10$ & $5.64 \mathrm{E}-10$ \\
\hline $\mathrm{Cl}-36$ & 1.0 & - & $8.18 \mathrm{E}-10$ & 8.18E-10 \\
\hline Se-79 & $8 \mathrm{E}-1$ & - & $2.35 \mathrm{E}-09$ & $2.35 \mathrm{E}-09$ \\
\hline Sr-90D ${ }^{a}$ & $\begin{array}{l}3 \mathrm{E}-1 \\
1 \mathrm{E}-4\end{array}$ & Y-90 (64.0 hr) & $\begin{array}{l}3.85 \mathrm{E}-08 \\
2.91 \mathrm{E}-09\end{array}$ & 4.14E-08 \\
\hline Tc-99 & $8 \mathrm{E}-1$ & - & $3.95 \mathrm{E}-10$ & $3.95 \mathrm{E}-10$ \\
\hline Sn-126D & $\begin{array}{l}2 \mathrm{E}-2 \\
1 \mathrm{E}-2 \\
1 \mathrm{E}-2\end{array}$ & $\begin{array}{l}\text { Sb-126m (19.0 min) } \\
\text { Sb-126 (14\%, } 12.4 \mathrm{~d})\end{array}$ & $\begin{array}{l}5.27 \mathrm{E}-09 \\
2.54 \mathrm{E}-11 \\
2.89 \mathrm{E}-09\end{array}$ & 5.70E-09 \\
\hline $1-129$ & 1.0 & - & $7.46 \mathrm{E}-08$ & $7.46 \mathrm{E}-08$ \\
\hline Cs-135 & 1.0 & - & 1.91E-09 & 1.91E-09 \\
\hline Cs-137D & $\begin{array}{c}1.0 \\
-\end{array}$ & Ba-137m (94.6\%, $2.552 \mathrm{~min})$ & $\begin{array}{l}1.35 \mathrm{E}-08 \\
0.00 \mathrm{E}+00\end{array}$ & 1.35E-08 \\
\hline Pu-242 & $1 \mathrm{E}-3$ & - & $9.08 \mathrm{E}-07$ & $9.08 \mathrm{E}-07$ \\
\hline U-238D & $\begin{array}{c}5 \mathrm{E}-2 \\
2 \mathrm{E}-4 \\
- \\
1 \mathrm{E}-3 \\
\end{array}$ & $\begin{array}{l}\text { Th-234 (24.10 d) } \\
\text { Pa-234m }(99.80 \%, 1.17 \mathrm{~min}) \\
\mathrm{Pa}-234(0.33 \%, 6.7 \mathrm{hr})\end{array}$ & $\begin{array}{l}6.88 \mathrm{E}-08 \\
3.69 \mathrm{E}-09 \\
0.00 \mathrm{E}+00 \\
5.84 \mathrm{E}-10 \\
\end{array}$ & $7.25 \mathrm{E}-08$ \\
\hline Pu-238 & $1 \mathrm{E}-3$ & - & $8.65 \mathrm{E}-07$ & 8.65E-07 \\
\hline $\mathrm{U}-234$ & $5 \mathrm{E}-2$ & - & 7.66E-08 & $7.66 \mathrm{E}-08$ \\
\hline Th-230 & $2 \mathrm{E}-4$ & - & $1.48 \mathrm{E}-07$ & $1.48 \mathrm{E}-07$ \\
\hline Ra-226D & $\begin{array}{c}2 \mathrm{E}-1 \\
- \\
- \\
2 \mathrm{E}-1 \\
- \\
5 \mathrm{E}-2 \\
- \\
- \\
\end{array}$ & $\begin{array}{l}\text { Rn-222 }(3.8235 \mathrm{~d}) \\
\text { Po-218 }(3.05 \mathrm{~min}) \\
\text { Pb-214 }(99.98 \%, 26.8 \mathrm{~min}) \\
\text { At-218 }(0.02 \%, 2 \mathrm{sec}) \\
\text { Bi-214 }(19.9 \mathrm{~min}) \\
\text { Po-214 }\left(99.98 \%, 1.64 \times 10^{-4} \mathrm{sec}\right) \\
\text { Tl-210 }(0.02 \%, 1.3 \mathrm{~min})^{\text {e }}\end{array}$ & $\begin{array}{c}3.58 \mathrm{E}-07 \\
0.00 \mathrm{E}+00 \\
0.00 \mathrm{E}+00 \\
1.69 \mathrm{E}-10 \\
0.00 \mathrm{E}+00 \\
7.64 \mathrm{E}-11 \\
0.00 \mathrm{E}+00 \\
0.00 \mathrm{E}+00\end{array}$ & $3.58 \mathrm{E}-07$ \\
\hline $\mathrm{Pb}-210 \mathrm{D}$ & $\begin{array}{l}2 \mathrm{E}-1 \\
5 \mathrm{E}-2 \\
1 \mathrm{E}-1\end{array}$ & $\begin{array}{l}\text { Bi-210 (5.012 d) } \\
\text { Po-210 (138.38 d) }\end{array}$ & $\begin{array}{l}1.45 \mathrm{E}-06 \\
1.73 \mathrm{E}-09 \\
5.14 \mathrm{E}-07 \\
\end{array}$ & 1.97E-06 \\
\hline Pu-240 & $1 \mathrm{E}-3$ & - & $9.56 \mathrm{E}-07$ & $9.56 \mathrm{E}-07$ \\
\hline $\mathrm{U}-236$ & $5 \mathrm{E}-2$ & - & $7.26 \mathrm{E}-08$ & $7.26 \mathrm{E}-08$ \\
\hline Th-232 & $2 \mathrm{E}-4$ & - & 7.38E-07 & 7.38E-07 \\
\hline Ra-228D ${ }^{b}$ & $\begin{array}{l}2 \mathrm{E}-1 \\
1 \mathrm{E}-3 \\
\end{array}$ & Ac-228 (6.13 hr) & $\begin{array}{l}3.88 \mathrm{E}-07 \\
5.85 \mathrm{E}-10 \\
\end{array}$ & 3.89E-07 \\
\hline TC-99 & $8 \mathrm{E}-1$ & - & $3.95 \mathrm{E}-10$ & $3.95 \mathrm{E}-10$ \\
\hline U-232 & $5 \mathrm{E}-2$ & - & 3.54E-07 & 3.54E-07 \\
\hline
\end{tabular}


Table 6.4-4. Effective Dose Conversion Factors for Ingestion (Continued)

\begin{tabular}{|c|c|c|c|c|}
\hline $\begin{array}{c}\text { Primary } \\
\text { Radionuclide }\end{array}$ & $\begin{array}{l}\text { Fractional } \\
\text { Uptake }\end{array}$ & $\begin{array}{l}\text { Decay Product }{ }^{\mathrm{c}} \text { (branching } \\
\text { fraction if not } 100 \% \text {, half-life) }\end{array}$ & $\begin{array}{l}\text { Dose Conversion } \\
\text { Factor }\left(D C F_{i n g}\right)^{d} \\
(\text { Sv/Bq) }\end{array}$ & $\begin{array}{c}\text { Effective Dose } \\
\text { Conversion } \\
\text { Factor }\left(E D C F_{\text {ing }}\right) \\
\text { (Sv/Bq) }\end{array}$ \\
\hline Th-228D & $\begin{array}{c}2 \mathrm{E}-4 \\
2 \mathrm{E}-1 \\
- \\
- \\
2 \mathrm{E}-1 \\
5 \mathrm{E}-2 \\
- \\
-\end{array}$ & $\begin{array}{l}\text { Ra-224 (3.66 d) } \\
\text { Rn-220 (55.6 sec) } \\
\text { Po-216 (0.15 sec) } \\
\text { Pb-212 (10.64 hr) } \\
\text { Bi-212 (60.55 min) } \\
\text { Po-212 (64.07\%, } 0.305 \mu-\mathrm{sec}) \\
\text { Tl-208 (35.93\%, } 3.07 \mathrm{~min})\end{array}$ & $\begin{array}{l}1.07 \mathrm{E}-07 \\
9.89 \mathrm{E}-08 \\
0.00 \mathrm{E}+00 \\
0.00 \mathrm{E}+00 \\
1.23 \mathrm{E}-08 \\
2.87 \mathrm{E}-10 \\
0.00 \mathrm{E}+00 \\
0.00 \mathrm{E}+00\end{array}$ & $2.18 \mathrm{E}-07$ \\
\hline Am-243D & $\begin{array}{l}1 \mathrm{E}-3 \\
1 \mathrm{E}-3 \\
\end{array}$ & Np-239 (2.355 d) & $\begin{array}{l}9.79 \mathrm{E}-07 \\
8.82 \mathrm{E}-10 \\
\end{array}$ & 9.80E-07 \\
\hline Pu-239 & $1 \mathrm{E}-3$ & - & $9.56 \mathrm{E}-07$ & $9.56 \mathrm{E}-07$ \\
\hline U-235D & $\begin{array}{l}5 \mathrm{E}-2 \\
2 \mathrm{E}-4\end{array}$ & Th-231 (25.52 hr) & $\begin{array}{l}7.19 \mathrm{E}-08 \\
3.65 \mathrm{E}-10\end{array}$ & 7.23E-08 \\
\hline Pa-231 & $1 \mathrm{E}-3$ & - & 2.86E-06 & 2.86E-06 \\
\hline Ac-227D & $\begin{array}{c}1 \mathrm{E}-3 \\
2 \mathrm{E}-4 \\
1.0 \\
2 \mathrm{E}-1 \\
- \\
- \\
2 \mathrm{E}-1 \\
- \\
- \\
- \\
\end{array}$ & $\begin{array}{l}\text { Th-227 (98.62\%, } 18.718 \mathrm{~d}) \\
\text { Fr-223 (1.38\%, } 21.8 \mathrm{~min}) \\
\text { Ra-223 (11.434 d) } \\
\text { Rn-219 (3.96 sec) } \\
\text { Po-215 (1.78 millisec) } \\
\text { Pb-211 (36.1 min) } \\
\text { Bi-211 (2.14 min) } \\
\text { Tl-207 (99.72\%, } 4.77 \mathrm{~min}) \\
\text { Po-211 (0.28\%, } 0.516 \mathrm{sec})\end{array}$ & $\begin{array}{l}3.80 \mathrm{E}-06 \\
1.03 \mathrm{E}-08 \\
2.33 \mathrm{E}-09 \\
1.78 \mathrm{E}-07 \\
0.00 \mathrm{E}+00 \\
0.00 \mathrm{E}+00 \\
1.42 \mathrm{E}-10 \\
0.00 \mathrm{E}+00 \\
0.00 \mathrm{E}+00 \\
0.00 \mathrm{E}+00\end{array}$ & 3.99E-06 \\
\hline Am-241 & $1 E-3$ & - & $9.84 \mathrm{E}-07$ & 9.84E-07 \\
\hline Np-237D & $\begin{array}{l}1 \mathrm{E}-3 \\
1 \mathrm{E}-3 \\
\end{array}$ & $\mathrm{~Pa}-233(27.0 \mathrm{~d})$ & $\begin{array}{l}1.20 \mathrm{E}-06 \\
9.81 \mathrm{E}-10 \\
\end{array}$ & 1.20E-06 \\
\hline $\mathrm{U}-233$ & $5 \mathrm{E}-2$ & - & $7.81 \mathrm{E}-08$ & $7.81 \mathrm{E}-08$ \\
\hline Th-229D & $\begin{array}{c}2 \mathrm{E}-4 \\
2 \mathrm{E}-1 \\
1 \mathrm{E}-3 \\
- \\
- \\
5 \mathrm{E}-2 \\
- \\
- \\
2 \mathrm{E}-1\end{array}$ & $\begin{array}{l}\text { Ra-225 (14.8 d) } \\
\text { Ac-225 (10.0 d) } \\
\text { Fr-221 (4.8 min) } \\
\text { At-217 (32.3 millisec) } \\
\text { Bi-213 (45.65 min) } \\
\text { Po-213 (97.84\%, } 4.2 \mu-\mathrm{sec}) \\
\text { Tl-209 (2.16\%, 2.2 min) } \\
\text { Pb-209 (3.253 hr) }\end{array}$ & $\begin{array}{l}9.54 \mathrm{E}-07 \\
1.04 \mathrm{E}-07 \\
3.00 \mathrm{E}-08 \\
0.00 \mathrm{E}+00 \\
0.00 \mathrm{E}+00 \\
1.95 \mathrm{E}-10 \\
0.00 \mathrm{E}+00 \\
0.00 \mathrm{E}+00 \\
5.75 \mathrm{E}-11\end{array}$ & 1.09E-06 \\
\hline
\end{tabular}

${ }^{a} \mathrm{~A}$ " $\mathrm{D}$ " after a radionuclide symbol denotes that the radionuclide is treated together with the short-lived (less than 180 d) decay products.

${ }^{\mathrm{b}}$ Indented radionuclides are long-lived decay products considered separately from the parents.

${ }^{c}$ Branching fractions and half-lives are from Eckerman and Ryman (1993 [DIRS 107684], Table A.1).

d Dose conversion factor source (BSC 2004 [DIRS 169671], Section 6.5).

${ }^{\mathrm{e}}$ Half-life of Tl-210 is taken from Lide and Frederikse (1997 [DIRS 103178] p.11-125).

DCF=dose conversion factor 


\subsubsection{All-Pathway Dose and Biosphere Dose Conversion Factor Calculations}

Input to the TSPA from the biosphere model consists of radionuclide-specific BDCFs from all exposure pathways. BDCFs for the groundwater scenario are numerically equal to annual doses to the receptor for a unit activity concentration of a specific radionuclide in groundwater. This section explains how the BDCFs are calculated.

\subsubsection{All-Pathway Doses}

The all-pathway annual dose for an individual primary radionuclide, expressed in terms of the TEDE, is the sum of the annual effective dose equivalent from external exposure and the CEDE from the annual radionuclide intake into the body by ingestion and inhalation. The annual dose for a radionuclide $i$ includes any contributions from the decay products, and is calculated as

$$
D_{\text {all }, i}=D_{\text {ext }, i}+D_{\text {inh }, i}+D_{\text {ing }, i}
$$

where

$$
\begin{aligned}
& D_{a l l, i}=\text { all-pathway annual dose from internal and external exposure to primary } \\
& \text { radionuclide } i \text { (Sv/year) } \\
& D_{\text {ext }, i}=\text { annual dose from external exposure to primary radionuclide } i \text { (Sv/year) } \\
& D_{\text {inh, } i}=\text { annual dose from inhalation exposure to radionuclide } i \text { (Sv/year) } \\
& D_{\text {ing, } i}=\text { annual dose from ingestion exposure to radionuclide } i \text { (Sv/year) }
\end{aligned}
$$

\subsubsection{Biosphere Dose Conversion Factors for the Groundwater Scenario}

Contaminated groundwater is the only source of radionuclides in the biosphere under the groundwater scenario. The dose from all exposure pathways discussed in previous sections is linearly proportional to this source, as summarized in Table 6.4-5. As shown in Table 6.4-5, all quantities calculated in the submodels, including radionuclide concentrations in the environmental media and the dose from various exposure pathways, are proportional to the radionuclide concentration in the groundwater. Thus, the biosphere model contribution to the dose assessment is separated from the source (i.e., radionuclide concentration in the groundwater). The biosphere contributions are the BDCFs. In the ERMYN, all-pathway doses could be calculated for any concentration of radionuclides in the water. To obtain the BDCFs, the all-pathway doses are divided by their respective radionuclide concentrations in the water. Thus, the BDCF for a radionuclide is numerically equal to the dose for a unit activity concentration of this radionuclide in the water. For the groundwater scenario, the BDCFs are calculated as

$$
B D C F_{i}=\frac{D_{a l l, i}}{C w_{i}}
$$

where

$B D C F_{i}=$ BDCF for radionuclide $i$ in the groundwater scenario (Sv/year per $\left.\mathrm{Bq} / \mathrm{m}^{3}\right)$

$D_{\text {all }, i}=$ all-pathway annual dose for radionuclide $i$ (Sv/year) 
$C w_{i}=$ activity concentration of radionuclide $i$ in groundwater $\left(\mathrm{Bq} / \mathrm{m}^{3}\right)$

To support the dose calculations, different sets of BDCFs are generated for present-day and future climate states.

Table 6.4-5. Summary of the Biosphere Submodels for the Groundwater Scenario

\begin{tabular}{|c|c|c|c|c|}
\hline Submodel & Quantity Calculated in Submodel & Section & Simplified Equation $^{\text {a }}$ & $\begin{array}{l}\text { Equation } \\
\text { Number }\end{array}$ \\
\hline \multirow[t]{2}{*}{ Soil } & $\begin{array}{l}\text { Activity concentration of a primary } \\
\text { radionuclide in surface soil }\end{array}$ & 6.4.1.1 & $C s_{i}=K 1 C w_{i}$ & $6.4 .1-4$ \\
\hline & $\begin{array}{l}\text { Activity concentration of a decay } \\
\text { product in surface soil }\end{array}$ & 6.4 .1 .2 & $C s_{i}=K 2 C s_{i}=K 2 K 1 C w_{i}$ & $6.4 .1-9$ \\
\hline \multirow[t]{3}{*}{ Air } & $\begin{array}{l}\text { Activity concentration of a } \\
\text { radionuclide in air from soil } \\
\text { resuspension }\end{array}$ & 6.4.2.1 & $C a_{i}=K 3 C s_{i}=K 3 K 1 C w_{i}$ & $\begin{array}{l}\text { 6.4.2-1, } \\
6.4 .2-2\end{array}$ \\
\hline & $\begin{array}{l}\text { Activity concentration of a } \\
\text { radionuclide in air from operation of } \\
\text { evaporative cooler }\end{array}$ & 6.4 .2 .2 & $C a_{i}=K 4 C w_{i}$ & $6.4 .2-3$ \\
\hline & $\begin{array}{l}\text { Activity concentration of radon gas } \\
\text { in air }\end{array}$ & 6.4 .2 .3 & $\begin{array}{l}C a_{R n-222}=K 5 C s_{R a-226}=K 5 \\
K 1 C W_{R a-226}\end{array}$ & $6.4 .2-4$ \\
\hline \multirow[t]{3}{*}{ Plant } & $\begin{array}{l}\text { Activity concentration of a } \\
\text { radionuclide in crops from root } \\
\text { uptake }\end{array}$ & 6.4 .3 .1 & $C p_{i}=K 6 C s_{i}=K 6 K 1 C w_{i}$ & $6.4 .3-2$ \\
\hline & $\begin{array}{l}\text { Activity concentration of a } \\
\text { radionuclide in crops from foliar } \\
\text { interception of irrigation water }\end{array}$ & 6.4.3.2 & $C p_{i}=K 7 C w_{i}$ & $\begin{array}{l}\text { 6.4.3-3 } \\
6.4 .3-4\end{array}$ \\
\hline & $\begin{array}{l}\text { Activity concentration of a } \\
\text { radionuclide in crops from foliar } \\
\text { interception of resuspended soil }\end{array}$ & 6.4.3.3 & $C p_{i}=K 8 C a_{i}=K 8 K 3 K 1 C w_{i}$ & $\begin{array}{l}\text { 6.4.3-6, } \\
6.4 .3-7\end{array}$ \\
\hline \multirow[t]{3}{*}{ Animal } & $\begin{array}{l}\text { Activity concentration of a } \\
\text { radionuclide in animal product from } \\
\text { animal feed }\end{array}$ & 6.4 .4 .1 & $C d_{i}=K 9 C p_{i}=K 9 K 10 C w_{i}$ & $6.4 .4-2$ \\
\hline & $\begin{array}{l}\text { Activity concentration of a } \\
\text { radionuclide in animal product from } \\
\text { drinking water }\end{array}$ & 6.4 .4 .2 & $C d_{i}=K 11 C w_{i}$ & $6.4 .4-3$ \\
\hline & $\begin{array}{l}\text { Activity concentration of a } \\
\text { radionuclide in animal product from } \\
\text { soil ingestion }\end{array}$ & 6.4 .4 .3 & $C d_{i}=K 12 C s_{i}=K 12 K 1 C w_{i}$ & $6.4 .4-4$ \\
\hline Fish & $\begin{array}{l}\text { Activity concentration of a } \\
\text { radionuclide in fish }\end{array}$ & 6.4 .5 & $C f_{i}=K 13 C w_{i}$ & $6.4 .5-2$ \\
\hline \multirow[t]{4}{*}{${ }^{14} \mathrm{C}$} & Activity concentration of ${ }^{14} \mathrm{C}$ in soil & 6.4.6.1 & $C s_{C-14}=K 14 C w_{C-14}$ & $6.4 .6-1$ \\
\hline & Activity concentration of ${ }^{14} \mathrm{C}$ in air & 6.4 .6 .2 & $C a_{C-14}=K 15 C s_{C-14}=K 15 K 1 C W_{C-14}$ & $\begin{array}{l}6.4 .6-2 \\
6.4 .6-3\end{array}$ \\
\hline & Activity concentration of ${ }^{14} \mathrm{C}$ in crops & 6.4.6.3 & $\begin{array}{l}C p_{C-14}=K 16 C s_{C-14}+K 17 \\
C a_{C-14}=K 18 C W_{C-14}\end{array}$ & $6.4 .6-6$ \\
\hline & $\begin{array}{l}\text { Activity concentration of }{ }^{14} \mathrm{C} \text { in } \\
\text { animal products }\end{array}$ & 6.4.6.4 & $\begin{array}{l}C d_{C-14}=K 19 C p_{C-14}+K 20 C W_{C-14}+ \\
K 21 C s_{C-14}=K 22 C W_{C-14}\end{array}$ & $6.4 .6-7$ \\
\hline $\begin{array}{l}\text { External } \\
\text { Exposure }\end{array}$ & External exposure dose & 6.4 .7 .1 & $D_{\text {ext }, i}=K 23 C s_{i}=K 23 K 1 C w_{i}$ & $6.4 .7-1$ \\
\hline \multirow[t]{3}{*}{ Inhalation } & $\begin{array}{l}\text { Inhalation dose from airborne } \\
\text { particulates }\end{array}$ & 6.4.8.1 & $D_{i n h, i}=K 24 C a_{i}=K 24 K 3 K 1 C w_{i}$ & $6.4 .8-2$ \\
\hline & $\begin{array}{l}\text { Inhalation dose from evaporative } \\
\text { cooler operation }\end{array}$ & 6.4 .8 .2 & $D_{i n h, i}=K 25 C a_{i}=K 25 K 4 C w_{i}$ & $6.4 .8-3$ \\
\hline & Inhalation dose from ${ }^{14} \mathrm{C}$ & 6.4.8.3 & $D_{\text {inh, } C-14}=K 26 C a_{C-14}=K 27 C W_{C-14}$ & $6.4 .8-4$ \\
\hline
\end{tabular}


Table 6.4-5. Summary of the Biosphere Submodels for the Groundwater Scenario (Continued)

\begin{tabular}{|l|l|l|l|l|}
\hline Submodel & Quantity Calculated in Submodel & Section & \multicolumn{1}{|c|}{ Simplified Equation ${ }^{\text {a }}$} & \multicolumn{1}{|c|}{$\begin{array}{c}\text { Equation } \\
\text { Number }\end{array}$} \\
\hline $\begin{array}{l}\text { Inhalation } \\
\text { (continued) }\end{array}$ & $\begin{array}{l}\text { Inhalation dose from radon decay } \\
\text { products }\end{array}$ & 6.4 .8 .4 & $\begin{array}{l}D_{\text {inh, } R n-222}=K 28 \\
C a_{R n-222}=K 29 C W_{\text {Ra-226 }}\end{array}$ & $6.4 .8-7$ \\
\hline \multirow{5}{*}{ Ingestion } & Ingestion dose from water & 6.4 .9 .1 & $D_{\text {ing, } i}=K 30 C W_{i}$ & $6.4 .9-2$ \\
\cline { 2 - 5 } & Ingestion dose from crops & 6.4 .9 .2 & $D_{\text {ing, } i}=K 31 C p_{i}=K 31 K 32 C W_{i}$ & $6.4 .9-3$ \\
\cline { 2 - 5 } & Ingestion dose from animal products & 6.4 .9 .3 & $D_{\text {ing, } i}=K 33 C d_{i}=K 34 C W_{i}$ & $6.4 .9-4$ \\
\cline { 2 - 5 } & Ingestion dose from fish & 6.4 .9 .4 & $D_{\text {ing, } i}=K 35 C f_{i}=K 35 K 13 C W_{i}$ & $6.4 .9-5$ \\
\cline { 2 - 5 } & Ingestion dose from soil & 6.4 .9 .5 & $D_{\text {ing, } i}=K 36 C s_{i}=K 36 K 1 C W_{i}$ & $6.4 .9-6$ \\
\hline
\end{tabular}

${ }^{a}$ The proportionality constants, $\mathrm{K} 1, \mathrm{~K} 2, \ldots$ in this table can be derived from the equation identified in the last column. These constants are used to show that the dose is proportional to the source, $\mathrm{Cw}_{i}$, the groundwater concentration.

\subsubsection{Pathway Contribution to Dose}

The all-pathway dose is the sum of doses from all exposure pathways in the ERMYN, which are addressed in Sections 6.4.7, 6.4.8, and 6.4.9. Therefore, the ERMYN can be used to determine the importance of individual exposure pathways. The annual all-pathway dose is the sum of the component pathway doses, expressed as

$$
\begin{aligned}
D_{\text {all }, i}= & \sum_{p} D_{p, i}=D_{e x t, i}+D_{i n h, i}+D_{i n g, i} \\
= & D_{e x t, i}+D_{i n h, p, i}+D_{i n h, \mathrm{e}, i}+D_{i n h, \mathrm{~g}, i}+D_{i n g, w, i}+D_{i n g, p, i, 1}+D_{i n g, p, i, 2}+D_{i n g, p, i, 3} \\
& \quad+D_{i n g, p, i, 4}+D_{i n g, d, i, 1}+D_{i n g, d, i, 2}+D_{i n g, d, i, 3}+D_{i n g, d, i, 4}+D_{i n g, f, i}+D_{i n g, s, i}
\end{aligned}
$$

where

$$
\begin{aligned}
& D_{\text {all,i }} \quad=\text { annual all-pathway dose for primary radionuclide } i \text { (Sv/year) } \\
& D_{p, i} \quad=\text { annual dose from exposure pathway } p \text { for primary radionuclide } i \text { (Sv/year) } \\
& \text { and the other parameters are defined in Equations 6.4.7-1, 6.4.8-1, and 6.4.9-2 to 6.4.9-6. }
\end{aligned}
$$

By analogy, the all-pathway BDCF for individual radionuclides are calculated as the sum of pathway BDCFs, which can be expressed, using Equation 6.4.10-2, as

$$
B D C F_{i}=\sum_{p} B D C F_{p, i}
$$

where

$B D C F_{p, i}=$ BDCF of individual pathway $p$ for radionuclide $i\left(\mathrm{~Sv} /\right.$ year per $\left.\mathrm{Bq} / \mathrm{m}^{3}\right)$ and the other parameters are defined in Equation 6.4.10-2 and 6.4.10-3.

The ERMYN can be used to calculate doses and BDCFs for individual long-lived radionuclides in the decay chain of a primary radionuclide, which can provide insight into the importance of individual members of the decay chain following long-term irrigation and radionuclide buildup in surface soil. 


\subsubsection{Use of BDCFs in the Total System Performance Assessment Model}

The assessment of annual doses is carried out in the TSPA model using the BDCFs as input parameters. The TSPA model is used to calculate groundwater concentrations at the source of the groundwater in the biosphere (the well or spring) for each radionuclide. Annual doses from individual radionuclides can be estimated as

$$
D_{\text {all }, i}(t)=B D C F_{i} \times C w_{i}(t)
$$

where

$D_{a l l, i}(t)=$ time dependent all-pathway annual dose for radionuclide $i$ (Sv/year)

$C w_{i}(t)=$ time dependent activity concentration of radionuclide $i$ in groundwater $\left(\mathrm{Bq} / \mathrm{m}^{3}\right)$

and the other parameter is defined in Equation 6.4.10-2.

The total annual dose is the sum of annual doses from the individual radionuclides tracked by the TSPA model. These individual radionuclides, referred to as primary radionuclides, include the contribution from the short-lived decay products (half-life less than $180 \mathrm{~d}$ ). The annual dose, the final output from the TSPA-LA model, is used to determine compliance with individual protection standard. The total annual dose is calculated in the TSPA-LA as

$$
D_{\text {total }}(t)=\sum_{i} D_{a l l, i}(t)
$$

where

$D_{\text {total }}(t)=$ time-dependent total annual dose to a defined receptor resulting from radionuclides released from the repository, including contributions from all radionuclides considered in the TSPA (Sv/year)

and the other parameter is defined in Equation 6.4.10-5.

Equation 6.4.10-6 is based on a linear relationship between radionuclide concentrations in the groundwater and the resulting dose. BDCFs are calculated based on a constant activity concentration of radionuclides in groundwater (Approximation 1) and equilibrium radionuclide concentrations in surface soil (Approximation 5). Equilibrium conditions will be reached if irrigation is sustained for a sufficiently long period of time, which would differ among radionuclides (ranging from years to a few thousand years; Sections 6.4.1 and 7.4.2) and would depend on radionuclide addition and removal rates from the soil. After equilibrium concentrations are reached, those concentrations would remain constant if the irrigation continues and the agricultural practices, climate, and soil characteristics remain unchanged (Approximation 3).

In the soil, radionuclides such as the isotopes of thorium, plutonium, and americium, have low removal rates, but they also have low rates of buildup, taking on the order of a few thousand years to approach equilibrium. The approximation of a constant radionuclide concentrations in the groundwater may not be valid for long time frames. The annual dose for a point in time, $t$, is 
calculated using activity concentrations in the groundwater at time $t, C w(t)$. Therefore, the product of the groundwater concentration and the BDCF represents the dose that would result if the same radionuclide concentration in the water persisted before time $t$ long enough for the radionuclide to reach equilibrium. This calculation is conservative if radionuclide concentrations in the groundwater increase, which would be expected for the 10,000-year compliance period. If radionuclide concentrations in the groundwater decrease quickly with time, the decrease in the true radionuclide buildup in the soil would be slower (i.e., the activity concentration in the soil would be higher) than predicted by this submodel. However, in the biosphere model, it is assumed that irrigation on the same farmland would continue for long periods of time (Approximation 3). If irrigation is not sustained, this may compensate for a long-term decrease in activity levels in the groundwater. In addition, during the period of time when the activity concentration in the groundwater is expected to decrease, the time step in the TSPA model will be long, and the concentrations will be constant during the time step. Therefore, the ERMYN is unlikely to underestimate the dose to the receptor, regardless of the point in time.

\subsection{MATHEMATICAL MODEL FOR THE VOLCANIC ASH SCENARIO}

The mathematical model described in this section is based on the conceptual model for the volcanic ash scenario (Section 6.3.2). Similar to the groundwater scenario, the goal of this mathematical model is to calculate BDCFs for the TSPA-LA model. Because variation in radionuclide concentrations in deposited volcanic ash is not part of the biosphere model, BDCFs are calculated based on a unit source in volcanic ash deposited on the ground $\left(1 \mathrm{~Bq} / \mathrm{m}^{2}\right)$. The TSPA-LA model calculates radiation dose as a product of the time-dependent source term and the source-independent BDCF. The time-dependent source term is subject to radioactive decay, volcanic ash redistribution, surface soil erosion, and other removal mechanisms.

The mathematical model consists of individual submodels. The relationship among the submodels is shown in Figure 6.3-4 and described in Section 6.3.2. In the mathematical model, linkages among the submodels can be traced by the consistent use of notation among submodels. As described for the conceptual model (Section 6.3.2.6), there are seven submodels, two fewer than in the groundwater scenario (the fish and ${ }^{14} \mathrm{C}$ submodels are not relevant). The main difference between the two scenarios is the release source. However, the radionuclide transfers among submodels are similar, and, therefore, many of the approximations and simplifications for the groundwater scenario apply to the volcanic ash scenario. The final dose calculations, the BDCFs, and their use in the TSPA-LA model, is discussed in Section 6.5.8.

\subsubsection{Surface Soil Submodel}

The surface soil submodel for the volcanic ash scenario differs from the groundwater scenario (Section 6.4.1) primarily because a volcanic eruption would spread ash over a large area (i.e., the entire Yucca Mountain region), while irrigating would contaminate the relatively small farming area. The scenarios also differ because radionuclides would not accumulate in the surface soil because they are not continuously added to the environment, as is the case for contaminated irrigation water.

The radionuclide source for this scenario is volcanic ash deposited on the surface of the ground $\left(\mathrm{Bq} / \mathrm{m}^{2}\right)$. The source, $C s_{i}(t)$, is time dependent because of radionuclide decay, volcanic ash 
redistribution, surface erosion, and other removal mechanisms. This time-dependent source, calculated using the disruptive events submodel in the TSPA-LA model, is not directly used in the ERMYN. To calculate the biosphere contribution for the dose assessment, this source term is separated into two parts, such that

$$
C s_{i}(t)=C s_{i} \times S T_{i}(t)
$$

where

$$
\begin{array}{ll}
C s_{i}(t)= & \text { time dependent activity concentration of radionuclide } i \text { in ash deposited on the } \\
& \text { ground surface }\left(\mathrm{Bq} / \mathrm{m}^{2}\right) \\
i & \text { index of primary radionuclide } \\
C s_{i}= & \text { activity concentration of radionuclide } i \text { in ash deposited on the ground surface } \\
& \left(\mathrm{Bq} / \mathrm{m}^{2}\right)
\end{array}
$$

Equation 6.5.1-1 is a mathematical treatment of the volcanic ash source, such that the time dependent source, $\mathrm{Cs}_{i}(t)$, could be considered a constant, unit source of radionuclide $i$ in ash deposited on the ground $\left(\mathrm{Bq} / \mathrm{m}^{2}\right)$. Because a unit source is considered in the ERMYN, the biosphere contribution to the TSPA-LA is the BDCFs, and the time function of the volcanic ash source term, $S T_{i}(t)$, is numerically equal to $C s_{i}(t)$.

Two important factors, land use and ash thickness, are considered in this submodel because the concentration of resuspended radionuclides would differ on cultivated and uncultivated lands. On agricultural and other cultivated land (e.g., gardens), the ash deposit would be uniformly mixed with surface soil during tilling, such that a mix of soil and ash would be resuspended. On uncultivated land, undiluted ash would be resuspended from thick deposits, and a mix of ash and soil would be resuspended from thin deposits.

Radionuclide decay and ingrowth in the source are a function of time. Short-lived decay products (half-life less than $180 \mathrm{~d}$ ) are in equilibrium with parent radionuclides (Approximation 2), similar to the groundwater scenario (Section 6.3.5). Dose contributions from short-lived decay products are included with the long-lived primary radionuclides. For a long-lived decay product that also is a primary radionuclide, the ERMYN does not consider ingrowth; rather, ingrowth is considered in the time dependent source $\left(S T_{i}(t)\right)$. For a relatively

long-lived decay product that is not a primary radionuclide (e.g., ${ }^{228} \mathrm{Th}$ from ${ }^{232} \mathrm{U}$ ), the BDCF of the decay product is initially developed based on secular equilibrium with the long-lived primary radionuclide in volcanic ash source, and then it is added to the BDCF for the primary radionuclide.

\subsubsection{Cultivated Land}

On cultivated lands, irrigation and tilling uniformly mix the volcanic ash with uncontaminated surface soil to the depth of the root zone (Approximation 12). However, the ash could be thicker than the tilling depth if a large quantity of ash is deposited at the location of the receptor or if a large quantity of ash is transported to the farm fields by fluvial processes. If that happens, radionuclide concentrations in the surface soil would be the same as those in the volcanic ash 
because the volcanic ash would not be mixed with uncontaminated surface soil and the ash below the root zone would not contribute to crop contamination. However, thick deposits are unlikely on cultivated fields in the Amargosa Valley. Therefore, to simplify the computations, regardless of the ash thickness, radionuclide concentrations in the surface soil of cultivated lands are calculated based on the total amount of radionuclides in the ash deposited on the ground $\left(\mathrm{Bq} / \mathrm{m}^{2}\right)$.

Areal radionuclide concentrations in the surface soil are converted to radionuclide mass concentrations in the surface soil, similar to the groundwater soil submodel. Because only a thin layer of ash is expected as discussed in the rationale for Approximation 12 (Section 6.3.2.4), the soil bulk density is assumed to remain unchanged after it is mixed with volcanic ash (Approximation 12). This approximation allows surface soil density to be considered as an independent input parameter that is not a function of thickness of ash deposited on the top of the surface soil. The activity concentration in surface soil mass can then be converted by

$$
C s_{m, i}=\frac{C s_{i}}{\rho \times d}=\frac{C s_{i}}{\rho_{s}}
$$

where

$$
\begin{array}{lll}
C s_{m, i} & = & \text { activity concentration of radionuclide } i \text { in a mixture of soil and ash on } \\
& \text { cultivated land }(\mathrm{Bq} / \mathrm{kg}) \\
\rho & = & \text { bulk density of the surface soil }\left(\mathrm{kg} / \mathrm{m}^{3}\right) \\
d & = & \text { depth of the surface soil in the cultivated land }(\mathrm{m}) \\
\rho_{s} & =\text { areal density of surface soil }\left(\mathrm{kg} / \mathrm{m}^{2}\right)
\end{array}
$$

and the other parameters are defined in Equation 6.5.1-1.

These soil-related parameters are expected to be the same as those used for the groundwater scenario (BSC 2004 [DIRS 169459]). The radionuclide concentration in a mixture of soil and ash on cultivated land is used to estimate radionuclide transfers to plant foodstuffs and animal products, and transfers directly to the receptor by inadvertent soil ingestion. These processes are modeled in the plant, animal, and ingestion submodels, respectively. Radionuclide concentrations in the surface soil would not change with the thickness of deposited volcanic ash because the contaminated ash would be uniformly diluted in the surface soil to the tilling depth.

\subsubsection{Noncultivated Land}

On noncultivated lands, volcanic ash would not quickly mix with surface soil, and the proportion of resuspended particles comprised of contaminated ash (versus clean soil) depends on the thickness of the ash deposit. To account for this process, a critical thickness is considered in the submodel. The critical thickness is the layer from which particles are resuspended, which is, at most, a few millimeters thick (BSC 2004 [DIRS 169672]). If the ash thickness is equal to or greater than the critical thickness, only ash is resuspended because clean soil is covered by too much ash to be resuspended, and only a portion of the ash would be available for resuspension. If the deposit is thinner than the critical thickness, the resuspended material would be a mix of ash particles and clean soil. 
The calculated areal activity concentration in the deposited ash $\left(\mathrm{Bq} / \mathrm{m}^{2}\right)$ is the same regardless of the ash thickness relative to the critical thickness. If the ash thickness is less than the critical thickness, the entire volume of ash (and all of the activity it contains) and uncontaminated soil, which together are within the critical thickness, is resuspended. If the ash thickness is greater than the critical thickness, the volume of ash (and the activity it contains within the critical thickness) is resuspended. Under these conditions, the volume of resuspended ash does not contain all of the activity that is initially deposited because the entire volume of ash (and all of the activity) will not be available for resuspension. Thus, the activity concentration per unit mass without dilution can be expressed as a fraction of the activity concentration per unit mass when there is dilution. The ratio of critical thickness to ash thickness is the fraction used for the calculation.

Radionuclide concentrations in the resuspended material (i.e., the mass of mixed ash and soil or the undiluted original ash) would depend on the ash thickness $\left(d_{a}\right)$ and the critical thickness $\left(d_{c}\right)$. Ash thickness is calculated in the TSPA-LA model. Similar to Equation 6.5.1-2, the mass concentration is calculated as

$$
C s_{m c, i}\left(d_{a}\right)=\left\{\begin{array}{lr}
\frac{C s_{i}}{\rho_{a} \times d_{c}}=C s_{m c, i} & \text { when } d_{a}<d_{c} \\
\frac{C s_{i}}{\rho_{a} \times d_{a}}=\frac{C s_{i}}{\rho_{a} \times d_{c}} \frac{d_{c}}{d_{a}}=C s_{m c, i} \frac{d_{c}}{d_{a}} & \text { when } d_{a} \geq d_{c}
\end{array}\right.
$$

where

$$
\begin{aligned}
C s_{m c, i}\left(d_{a}\right)= & \text { activity concentration of radionuclide } i \text { in volcanic ash or in the mix of ash } \\
& \text { and dust of noncultivated land }(\mathrm{Bq} / \mathrm{kg}) \\
\rho_{a} & \text { bulk density of volcanic ash }\left(\mathrm{kg} / \mathrm{m}^{3}\right) \\
d_{c} & \text { critical thickness for resuspension on noncultivated lands }(\mathrm{m}) \\
d_{a} & =\text { thickness of ash deposited on the ground }(\mathrm{m}) \\
C s_{m c, i}= & \text { activity concentration of radionuclide } i \text { in the mass of resuspendable ash or } \\
& \text { in the mix of ash and dust }(\mathrm{Bq} / \mathrm{kg})
\end{aligned}
$$

and the other parameters are defined in Equation 6.5.1-1.

The bulk density of volcanic ash, $\rho_{a}$, is lower than the soil bulk density (BSC 2004 [DIRS 169459]).

Equation 6.5.1-3 is rewritten as

$$
C s_{m c, i}\left(d_{a}\right)=C s_{m c, i} \times g\left(d_{a}\right)
$$

where $g\left(d_{a}\right)$, a function of volcanic ash thickness (dimensionless), can be expressed as

$$
g\left(d_{a}\right)= \begin{cases}1 & \text { when } d_{a}<d_{c} \\ \frac{d_{c}}{d_{a}} & \text { when } d_{a} \geq d_{c}\end{cases}
$$

and the other parameters are defined in Equation 6.5.1-3. 
The radionuclide concentration of volcanic ash or mixed ash and soil for noncultivated land, as defined in Equation 6.5.1-4 and Equation 6.5.1-5, is used to estimate the radionuclide transfer from volcanic ash to resuspended ash in the air (Approximation 13), and then to estimate the human inhalation dose. The radionuclide concentration in the critical thickness for surface particle resuspension is a reference concentration is used for developing the inhalation pathway contribution to BDCFs. For noncultivated lands, radionuclide leaching is likely to be low because of the limited precipitation and lack of irrigation. Consequently, using the depth of ash on noncultivated land for determining inhalation dose is a conservative approach.

\subsubsection{Air Submodel}

The air submodel for the volcanic ash scenario has two components: the resuspension of particles from deposited volcanic ash or from a mixture of ash and soil, and the exhalation of radon gas from radium-contaminated volcanic ash. Because groundwater is uncontaminated in the volcanic ash scenario, radionuclide transfer into indoor air from evaporative coolers is not considered. This submodel also does not consider ${ }^{14} \mathrm{C}$ in the air because it is not a radionuclide considered important to the TSPA-LA in the volcanic ash scenario (Section 6.1.3).

\subsubsection{Resuspension of Volcanic Ash or a Mixture of Soil and Ash}

As discussed in Section 6.5.1, volcanic ash would settle on cultivated and noncultivated lands. On cultivated lands, ash would be well mixed with uncontaminated soil to the depth of the surface soil. On noncultivated lands, volcanic ash would not be mixed with surface soil unless the amount of ash is less than the critical thickness (Section 6.5.1.2). Radionuclide concentrations in the air are estimated separately for the two cases. The mixture of soil and ash on cultivated lands is used for the deposition of resuspended particles onto plant surface (Section 6.5.3), while the volcanic ash or mixture of soil and ash from noncultivated land is used for the inhalation dose (Section 6.5.6).

For resuspended particles deposited on crops, only particles from the local farm fields or gardens would be deposited on the plants (Approximation 13). Therefore, the submodel is based on the same mass concentration of radionuclides in the surface soil and in the airborne particles

$$
C a_{p, i}=C s_{m, i} S
$$

where

$C a_{p, i}=$ activity concentration of radionuclide $i$ in the air for crop deposition $\left(\mathrm{Bq} / \mathrm{m}^{3}\right)$

$S=$ concentration of total resuspended particulates (mass loading) for direct deposition on crops $\left(\mathrm{kg} / \mathrm{m}^{3}\right)$

and the other parameters are defined in Equation 6.5.1-2.

The mass loading term, $S$, is characteristic of cultivated areas in a dry climate after a volcanic eruption. The value for mass loading likely would be different from the value used in the groundwater scenario (BSC 2004 [DIRS 169458]). 
Similar to the groundwater scenario, the enhancement factor, defined as the ratio of airborne particle concentration $(\mathrm{Bq} / \mathrm{kg})$ to average surface soil concentration $(\mathrm{Bq} / \mathrm{kg})$, is used in the calculation of airborne activity concentrations used to determine the inhalation dose. Similar to Equation 6.4.2-2, radionuclide concentrations in the air inhaled by the receptor are calculated as

$$
C a_{h, i, n}\left(d_{a}, t\right)=f_{\text {enhance, } n} C s_{m c, i}\left(d_{a}\right) S_{n}(t)
$$

where

$$
\begin{aligned}
& C a_{h, i, n}\left(d_{a}, t\right)=\text { activity concentration of radionuclide } i \text { in the air for environment } n \text { at time } \\
& t \text { and ash thickness } d_{a}\left(\mathrm{~Bq} / \mathrm{m}^{3}\right) \\
& n=\text { index of the environments, } n=1 \text { for active outdoors; } 2 \text { for inactive } \\
& \text { outdoors; } 3 \text { for active indoors; } 4 \text { for asleep indoors; and } 5 \text { for away from } \\
& \text { the contaminated area } \\
& f_{\text {enhance } n}=\text { enhancement factor for the activity concentration of resuspended particles } \\
& \text { (dimensionless) for environment } n \\
& S_{n}(t) \quad=\text { total average annual mass loading (the concentration of total resuspended } \\
& \text { particulates in the air) in environment } n \text { at time } t \text { following a volcanic } \\
& \text { eruption }\left(\mathrm{kg} / \mathrm{m}^{3}\right) \\
& \text { and the other parameters are defined in Equation 6.5.1-3. }
\end{aligned}
$$

Mass loading would be higher for some time after a volcanic eruption because there would be more unconsolidated, fine particles on the soil surface that would be readily resuspended by wind, human activity, or other disturbances. Over time, the ash would be consolidated into the soil or removed by erosion, and mass loading would return to levels experienced before the eruption (i.e., nominal mass loading; Approximation 14). This approximation is based on measurements of mass loading after the eruption of Mount St. Helens and other volcanoes (BSC 2004 [DIRS 169458], Section 6.2 and 6.3). To account for this effect, radionuclide concentrations in the air used for the calculation of inhalation dose are separated into two parts. The first part is for the period immediately after the eruption and before ash stabilization. The second part is for the time when mass loading has returned to preeruption levels. This second part is ash-thickness dependent, but not time dependent. By separating the time and ash thickness components, radionuclide concentrations immediately after the eruption can be evaluated.

The dependence of mass loading on time is expressed as

$$
S_{n}(t)=S_{n}+S_{v, n} f(t)
$$

where

$$
\begin{aligned}
S_{n}= & \text { nominal annual average mass loading (the concentration of total resuspended } \\
& \text { particulates) in environment } n\left(\mathrm{~kg} / \mathrm{m}^{3}\right) \\
S_{v, n}= & \text { elevated, post-volcanic }(v) \text { annual average mass loading (the concentration of } \\
& \text { total resuspended particulates) in environment } n \text {, in addition to } S_{n}, \text { during the } \\
& \text { first year following a volcanic eruption }\left(\mathrm{kg} / \mathrm{m}^{3}\right)
\end{aligned}
$$


$f(t)=$ mass loading time function, which described the rate of change in mass loading after a volcanic eruption

and the other parameters are defined in Equation 6.5.2-2.

Mass loading is an important parameter for the inhalation pathway because the radionuclide concentration in air is proportional to mass loading. Mass loading depends on the environment and human activities, and values range from about $1 \times 10^{-8}$ to $1 \times 10^{-5} \mathrm{~kg} / \mathrm{m}^{3}$ (BSC 2004 [DIRS 169458], Section 6.2). Mass loading in indoor environments is considerably lower than in some outdoor environments. Mass loading distributions for nominal conditions, $S_{n}$, are the same as in the groundwater scenario (Equation 6.4.2-2); under post-volcanic conditions, $S_{v, n}$, mass loading would be elevated due to the volcanic eruption. The parameter $S_{v, n}$ represents the initial increased mass loading and differs among environments (BSC 2004 [DIRS 169458]). The mass loading time function, $f(t)$, which is expected to rapidly decrease with time (BSC 2004 [DIRS 169458], Section 6.3), is carried into the TSPA-LA model to evaluate the final dose (Section 6.5.8).

Combining Equations 6.5.1-4, 6.5.2-2, and 6.5.2-3, radionuclide concentrations in the air used for the assessment of inhalation dose are calculated in two parts, one steady state and one time-dependent. The ash thickness dependent function, $g\left(d_{a}\right)$ defined in Equation 6.5.1-5, is applied to both parts, as

$$
\begin{aligned}
C a_{h, i, n}\left(d_{a}, t\right) & =f_{\text {enhance, } n} C s_{m c, i} g\left(d_{a}\right)\left[S_{n}+S_{v, n} f(t)\right] \\
& =f_{\text {enhance }, n} C s_{m c, i} g\left(d_{a}\right) S_{n}+f_{\text {enhance }, n} C s_{m c, i} g\left(d_{a}\right) S_{v, n} f(t) \\
& =C a_{h, i, n} g\left(d_{a}\right)+C a_{v, i, n} g\left(d_{a}\right) f(t)
\end{aligned}
$$

where

$$
\begin{gathered}
C a_{h, i, n}=\begin{array}{l}
\text { activity concentration of radionuclide } i \text { in the air in environment } n \text {, calculated } \\
\text { from the nominal mass loading levels }\left(\mathrm{Bq} / \mathrm{m}^{3}\right)
\end{array} \\
C a_{v, i, n}=\begin{array}{l}
\text { activity concentration of radionuclide } i \text { in the air in environment } n \text {, calculated } \\
\text { from the post-volcanic mass loading levels }\left(\mathrm{Bq} / \mathrm{m}^{3}\right)
\end{array} \\
\text { and the other parameters are defined in Equations } 6.5 .1-3,6.5 .1-5,6.5 .2-2 \text {, and } 6.5 .2-3 .
\end{gathered}
$$

\subsubsection{Exhalation of Radon from the Ground Surface}

The volcanic ash scenario considers the release of radon from volcanic ash deposited on the ground surface. Because the layer of contaminated volcanic ash is expected to be relatively thin (less than $10 \mathrm{~cm}$, Approximation 12), the use of a radon release factor developed for the groundwater scenario (Section 6.4.2.3), $f_{m, R n-222}=0.25\left(\mathrm{~Bq} / \mathrm{m}^{3}\right) /(\mathrm{Bq} / \mathrm{kg})$, is not valid because it is based on a thickness of ${ }^{226} \mathrm{Ra}$-contaminated soil that is infinite with respect to radon transport. However, in the case of volcanic ash and soil mixing, the distribution of radium concentration in the soil would not be immediately known, and, in addition, the assessment of radon release from a limited contaminated source would require a radon diffusion submodel, for which more input data would be needed. Therefore, a simplified method for estimating the radon concentration in the air, using the relationship between the concentration of ${ }^{226} \mathrm{Ra}$ in the surface soil $\left(\mathrm{Bq} / \mathrm{m}^{2}\right)$, 
${ }^{222} \mathrm{Rn}$ flux density from soil $\left(\mathrm{Bq} /\left(\mathrm{m}^{2} \mathrm{~s}\right)\right)$, and ${ }^{222} \mathrm{Rn}$ in the air $\left(\mathrm{Bq} / \mathrm{m}^{3}\right)$ is used. This method is based on a thin layer of ${ }^{226} \mathrm{Ra}$ on the ground surface and the approximation that all of the ${ }^{222} \mathrm{Rn}$ is released into the air (Approximation 15).

Based on this approximation, if the ${ }^{226} \mathrm{Ra}$ concentration on the top of the ground is $1 \mathrm{~Bq} / \mathrm{m}^{2}$, then one atom of ${ }^{226} \mathrm{Ra} / \mathrm{m}^{2} / \mathrm{s}$ would decay to ${ }^{222} \mathrm{Rn}$. Because all of the ${ }^{222} \mathrm{Rn}$ would be released into the air, the ${ }^{222} \mathrm{Rn}$ flux density from soil would be one atom per $\mathrm{m}^{2} / \mathrm{sec}$. To convert the number of ${ }^{222} \mathrm{Rn}$ atoms $\left(N_{R n-222}\right)$ into activity of ${ }^{222} \mathrm{Rn}\left(A_{R n-222}\right)$, a half-life $\left(T_{1 / 2, R n-222}\right)$ of $3.8235 \mathrm{~d}$ $\left(3.3 \times 10^{5} \mathrm{~s}\right.$, Table 6.3-7) is used as

$$
A_{R n-222}=\frac{\ln (2)}{T_{1 / 2, R n-222}} N_{R n-222}=\frac{\ln (2)}{3.3 \times 10^{5}(\mathrm{sec} .)} \times 1(\text { atom })=2 \times 10^{-6} \text { (Bq) (Eq. 6.5.2-5) }
$$

This indicates that one atom of ${ }^{222} \mathrm{Rn}$ has an activity of about $2 \times 10^{-6} \mathrm{~Bq}$. Then, ${ }^{222} \mathrm{Rn}$ flux density per $1 \mathrm{~Bq} / \mathrm{m}^{2}$ of ${ }^{226} \mathrm{Ra}$ from the ground surface is expressed as

$$
F D_{R n-222}=\frac{1\left(\text { atom } /\left(\mathrm{m}^{2} \mathrm{~s}\right)\right)_{\mathrm{Rn}-222}}{1\left(\mathrm{~Bq} / \mathrm{m}^{2}\right)_{\mathrm{Ra}-226}}=2 \times 10^{-6} \frac{\mathrm{Bq} /\left(\mathrm{m}^{2} \mathrm{~s}\right)}{\left(\mathrm{Bq} / \mathrm{m}^{2}\right)}
$$

where

$$
\begin{aligned}
F D_{R n-222}= & \text { radon release factor for the volcanic ash scenario, defined as flux density of } \\
& { }^{222} \mathrm{Rn} \text { per unit }{ }^{226} \mathrm{Ra} \text {-activity on the ground surface }\left(\left(\mathrm{Bq} /\left(\mathrm{m}^{2} \mathrm{~s}\right)\right) /\left(\mathrm{Bq} / \mathrm{m}^{2}\right)=/ \mathrm{s}\right)
\end{aligned}
$$

Using the ratio of ${ }^{222} \mathrm{Rn}$ activity concentration in the air to the flux density, $C F_{R n-222}$, from a large contaminated area outdoors (which is introduced in the air submodel for the groundwater scenario for calculating the indoor radon concentration; Section 6.4.2.3), the release factor of ${ }^{222} \mathrm{Rn}$ from ${ }^{226} \mathrm{Ra}$ surface concentration is estimated as

$$
f_{s, R n-222}=F D_{R n-222} \times C F_{R n-222}
$$

where

$$
\begin{aligned}
& f_{s, R n-222}=\text { ratio of }{ }^{222} \mathrm{Rn} \text { activity concentration in the air to the }{ }^{226} \mathrm{Ra} \text { activity } \\
& \text { concentration on the ground surface }\left(\left(\mathrm{Bq} / \mathrm{m}^{3}\right) /\left(\mathrm{Bq} / \mathrm{m}^{2}\right)=/ \mathrm{m}\right) \\
& C F_{R n-222}=\text { ratio of }{ }^{222} \mathrm{Rn} \text { concentration in air to flux density from soil for outdoors } \\
& \left(\left(\mathrm{Bq} / \mathrm{m}^{3}\right) /\left(\mathrm{Bq} /\left(\mathrm{m}^{2} \mathrm{~s}\right)\right)=(\mathrm{sec} / \mathrm{m})\right. \\
& \text { and the other parameters are defined in Equation 6.5.2-6. }
\end{aligned}
$$


Using the release factor for ${ }^{222} \mathrm{Rn}\left(f_{s, R n-222}\right)$ and an equation similar to Equation 6.4.2-5, the activity concentration of radon in air is estimated as

$$
\begin{aligned}
C a_{g, R n-222} & =f_{s, R n-222} C s_{R a-226} \\
& =F D_{R n-222} C F_{R n-222} C s_{R a-226}
\end{aligned}
$$

where

$\mathrm{Ca}_{g, \mathrm{Rn}-222}=$ activity concentration of ${ }^{222} \mathrm{Rn}$ in air $\left(\mathrm{Bq} / \mathrm{m}^{3}\right)$

$\mathrm{Cs}_{\text {Ra-226 }}=$ activity concentration of ${ }^{226} \mathrm{Ra}$ in surface soil $\left(\mathrm{Bq} / \mathrm{m}^{2}\right)$

and the other parameters are defined in Equation 6.5.2-7.

Using a typical value of $C F_{R n-222}=300 \mathrm{~s} / \mathrm{m}$ (BSC 2004 [DIRS 169672], Section 6.6.1), the radon release factor is about $0.0006\left(\mathrm{~Bq} / \mathrm{m}^{3}\right) /\left(\mathrm{Bq} / \mathrm{m}^{2}\right)$. This estimate is an upper bound because all of the radon in the ash is released into the air.

Unlike the groundwater scenario, indoor radon concentrations for the volcanic ash scenario are considered to be the same as the outdoor radon concentration because the initial amount of indoor volcanic ash would be limited. Even if new houses are built on contaminated land, the release of ${ }^{222} \mathrm{Rn}$ indoors is limited because the thin layer of ash would be removed or mixed with the surface soil during home construction. Because an upper bound value for outdoor concentrations is used, it is not necessary to consider an additional indoor radon source.

\subsubsection{Plant Submodel}

Under the volcanic ash scenario, ash contaminates surface soil and surface water. The biosphere model does not include permanent surface waters (e.g., rivers, lakes, or reservoirs; Section 6.3.1.1), and it does not consider surface water contamination. Groundwater could become contaminated if radionuclides from volcanic ash leached into the deep soil, but this process is not considered in the biosphere model. Therefore, the plant submodel for this scenario only considers contaminated soil and air, which contaminate crops by root uptake and dust deposition.

The plant submodel for the volcanic ash scenario is similar to the groundwater scenario. The discussions in Section 6.4.3 on crop type, mechanisms of crop contamination, and radionuclide decay are valid for the volcanic ash scenario. Equations presented in this section are the same as those in Section 6.4.3, except that contaminated water is not considered. The radionuclide concentrations in specific plant foodstuffs are estimated as

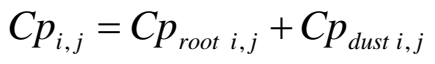

where

$$
\begin{aligned}
C p_{i, j}= & \text { activity concentration of radionuclide } i \text { in crop type } j(\mathrm{~Bq} / \mathrm{kg}) \\
j= & \text { crop-type index; } \mathrm{j}=1 \text { for leafy vegetables, } 2 \text { for other vegetables, } 3 \text { for fruit, } \\
& 4 \text { for grain (used for both human and animals), and } 5 \text { for fresh forage feed } \\
& \text { (used for beef cattle and dairy cows) }
\end{aligned}
$$




$$
\begin{aligned}
C p_{\text {root } i, j=}= & \text { activity concentration of radionuclide } i \text { in crop type } j \text { contributed from plant } \\
& \text { root uptake }(\mathrm{Bq} / \mathrm{kg}) \\
C p_{\text {dust } i, j=}= & \text { activity concentration of radionuclide } i \text { in crop type } j \text { contributed from plant } \\
& \text { leaf uptake due to the deposition of resuspended particulates on crop } \\
& \text { surfaces }(\mathrm{Bq} / \mathrm{kg})
\end{aligned}
$$

\subsubsection{Root Uptake}

Root uptake is estimated using the same methods as those for the groundwater scenario (Section 6.4.3). It is assumed that all plant roots are in the surface soil (to the tilling depth) to maximize radionuclide uptake (Approximation 7). This approach eliminates the need for determining the fraction of roots in a defined thickness of surface soil. Radionuclides taken up by plant roots are internal to the plants and not subject to removal by weathering or food processing. The activity concentration of radionuclides in crops contributed from root uptake is expressed as

$$
C p_{\text {root }, i, j}=C s_{m, i} F_{s \rightarrow p, i, j} D W_{j}
$$

where

$$
\begin{aligned}
& C p_{\text {root }, i, j}=\text { activity concentration of radionuclide } i \text { in crop type } j \text { contributed from root } \\
& \text { uptake }(\mathrm{Bq} / \mathrm{kg} \text { wet weight of edible portions of the plant) } \\
& C s_{m, i}=\text { activity concentration of radionuclide } i \text { in surface of cultivated soil } \\
& \left(\mathrm{Bq} / \mathrm{kg}_{\text {dry soil }}\right) \\
& F_{s \rightarrow p, i, j}=\text { soil-to-plant transfer factor for radionuclide } i \text { and crop type } j(\mathrm{~Bq} / \mathrm{kg} \text { dry-plant } \\
& \text { per } \mathrm{Bq} / \mathrm{kg} \text { dry-soil }) \\
& D W_{j} \quad=\text { dry-to-wet weight ratio for edible parts of crop type } j\left(\mathrm{~kg}_{\text {dry-plant }} / \mathrm{kg}_{\text {wet-plant }}\right)
\end{aligned}
$$

The activity concentration of radionuclides in surface soil, $C s_{m, i}$, is calculated using Equation 6.5.1-2. The soil-to-plant transfer factor and the dry-to-wet ratio (Section 6.4.3.1) are expected to be the same for the groundwater and volcanic ash scenarios (BSC 2004 [DIRS 169672], Section 7; BSC 2004 [DIRS 169673], Section 7).

\subsubsection{Uptake from Resuspended Soil}

The second mechanism of crop contamination is the deposition of resuspended soil on leaf surfaces. Similar to the groundwater scenario (Section 6.4.3.3), radionuclide concentrations in plant foodstuffs from uptake by foliar interception of airborne particulates are expressed as

$$
C p_{\text {dust }, i, j}=\frac{D a_{i} R a_{j} T_{j}}{\lambda_{w} Y_{j}}\left(1-e^{-\lambda_{w} t_{g, j}}\right)
$$

where

$$
\begin{aligned}
& D a_{i}=\text { deposition rate of radionuclide } i \text { in resuspended soil }\left(\mathrm{Bq} /\left(\mathrm{m}^{2} \mathrm{~d}\right)\right. \text { ). } \\
& R a_{j}=\text { interception fraction for resuspended soil (dimensionless) }
\end{aligned}
$$




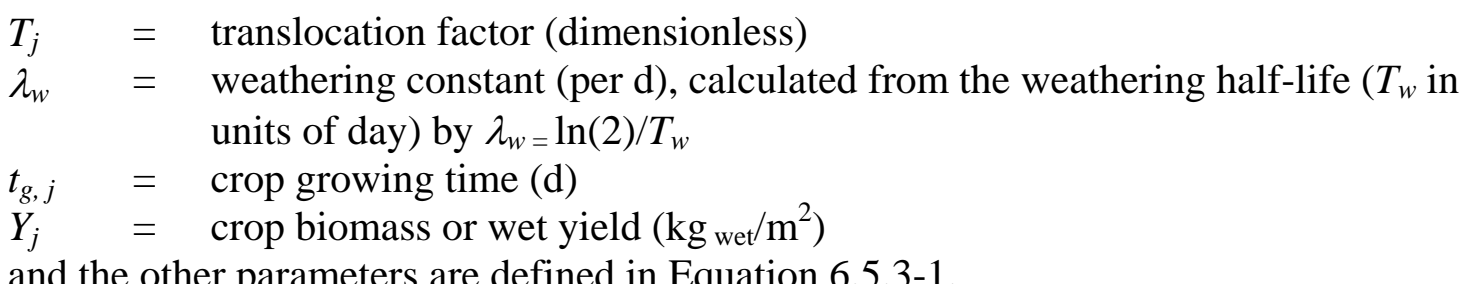

The translocation factor, weathering constant, crop growing time, and crop biomass are expected to be the same in the groundwater and volcanic ash scenarios (BSC 2004 [DIRS 169672], Section 7; BSC 2004 [DIRS 169673] Section 7). The deposition rate of contaminated resuspended soil, $D a_{i}$, quantifies the combined effect of contaminant removal from the atmosphere by several processes, such as gravitational settling, diffusion, and turbulent transport. The deposition rate is expressed as

$$
D a_{i}=8.64 \times 10^{4} C a_{p, i} V_{d}
$$

where

$$
\begin{aligned}
C a_{p, i}= & \text { activity concentration of radionuclide } i \text { in the air used for evaluation of } \\
& \text { activity deposition on the crops }\left(\mathrm{Bq} / \mathrm{m}^{3}\right) . \\
V_{d} & \text { deposition velocity for resuspended soil }(\mathrm{m} / \mathrm{sec}) \\
8.64 \times 10^{4}= & \text { unit conversion factor }(\mathrm{sec} / \mathrm{d})
\end{aligned}
$$

Activity concentrations of radionuclides in the air are calculated using Equation 6.5.2-1. The deposition velocity for resuspended soil, $V_{d}$, is a function of particle size. The value of this parameter is developed considering site-specific conditions in the Yucca Mountain region (BSC 2004 [DIRS 169672] Section 6.2.2).

The interception fraction for resuspended soil, $R a_{j}$, quantifies the initial fractional deposition of radionuclides on plant surfaces from dry deposition. This parameter is crop-type dependent and ranges in value from zero to one. Values for this parameter are estimated using an empirical formula as

$$
R a_{j}=1.0-e^{-a_{j} D B_{j}}
$$

where

$$
\begin{array}{ll}
a_{j} & =\text { an empirical factor }\left(\mathrm{m}^{2} / \mathrm{kg} \text { dry biomass }\right) \\
D B_{j} & =\text { the dry standing biomass of crop type } j \text { (value in units of } \mathrm{kg} \text { dry weigh } / \mathrm{m}^{2} \text { ) } \\
\text { and the other parameters are defined in Equation 6.5.3-3. }
\end{array}
$$

This empirical equation is adopted from the GENII-S model, including the values of the empirical factor. The recommended values of the empirical factor are 2.9 for leafy vegetables, fresh forage feed, and grain, and 3.6 for root and other vegetables plus fruit (Napier et al. 1988 [DIRS 157927], p. 4.69). The empirical formula is modified for this submodel to use dry biomass rather than wet standing biomass of growing vegetation multiplied by the dry-to-wet weight ratio (Sections 6.4.3.3 and 7.3.3.3). 


\subsubsection{Animal Submodel}

The animal product submodel is used to evaluate the accumulation of radionuclides in animal products that later would be consumed by humans. Two pathways are considered for the contamination of animal products: ingestion of contaminated feed and soil. Inhalation of contaminated air is not considered in the submodel because it is much less important than ingestion (Section 7.4.5).

Discussions presented for the groundwater scenario (Section 6.4.4) are valid for the volcanic ash scenario. In addition, as mentioned in the plant submodel, only fresh forage is considered as feed for beef cattle and dairy cows, and only stored grain is considered as feed for poultry and laying hens.

Radionuclide decay is not considered in this submodel because the growing time and storage time (time between harvest and consumption) for animal feed are short compared with the half life of the radionuclides of interest. The radionuclide concentration in animal products $\left(C d_{i, k}\right)$ is evaluated as

$$
C d_{i, k}=C d_{f e e d, i, k}+C d_{\text {soil }, i, k}
$$

where

$$
\begin{aligned}
& C d_{i, k}=\text { activity concentration of radionuclide } i \text { in animal product } k(\mathrm{~Bq} / \mathrm{kg} \text { fresh weight } \\
& \text { or } \mathrm{Bq} / \mathrm{L} \text { milk) } \\
& k=\text { animal product index; } k=1 \text { for beef, } 2 \text { for poultry, } 3 \text { for milk, and } 4 \text { for eggs } \\
& C d_{\text {feed,i,k }}=\text { activity concentration of radionuclide } i \text { in animal product } k \text { due to ingestion } \\
& \text { of contaminated animal feed }(\mathrm{Bq} / \mathrm{kg} \text { or } \mathrm{Bq} / \mathrm{L}) \\
& C d_{\text {soil,i,k }}=\text { activity concentration of radionuclide } i \text { in animal product } k \text { due to ingestion } \\
& \text { of contaminated soil }(\mathrm{Bq} / \mathrm{kg} \text { or } \mathrm{Bq} / \mathrm{L})
\end{aligned}
$$

\subsubsection{Animal Feed}

The radionuclide concentrations in animal products, resulting from the ingestion of contaminated animal feed, is evaluated as

$$
C d_{\text {feed }, i, k}=F m_{i, k} C p_{i, j} Q f_{k}
$$

where

$$
\begin{array}{ll}
F m_{i, k}= & \text { animal intake-to-animal product transfer coefficient for radionuclide } i \text { and } \\
& \text { animal product } k(\mathrm{~d} / \mathrm{kg} \text { fresh weight } \text { or } \mathrm{d} / \mathrm{L}) \\
C p_{i, j}= & \text { activity concentration of radionuclide } i \text { in animal feed } j\left(\mathrm{~Bq} / \mathrm{kg}_{\text {fresh weight }}\right) \\
j & =\text { animal feed index; } j=5 \text { for fresh forage, } j=4 \text { for grain } \\
k & =\text { animal product index; } k=1 \text { for beef, } 2 \text { for milk, } 3 \text { for poultry, and } 4 \text { for eggs } \\
Q f_{k}= & \text { animal consumption rate of feed }(\mathrm{kg} \text { fresh weight } / \mathrm{d})
\end{array}
$$


Radionuclide concentrations in animal feed are calculated in the plant submodel (Section 6.5.3). The transfer coefficients for the animal products and animal consumption rates are considered to be the same in the volcanic ash and groundwater scenarios (BSC 2004 [DIRS 169672], Section 7).

\subsubsection{Animal Soil Ingestion}

Animal soil ingestion is an important pathway (Section 7.4.5) because soil is the initial source of contamination in the volcanic ash scenario. To be consistent with the methods used for the contamination of foodstuffs, radionuclide concentrations in the contaminated soil that an animal ingests are calculated for cultivated lands (Equation 6.5.1-2). Radionuclide concentrations in animal products from the ingestion of contaminated soil are evaluated as

$$
C d_{\text {soil } i, k}=F m_{i, k} C s_{m, i} Q s_{k}
$$

where

$$
\begin{aligned}
C s_{m, i}= & \text { activity concentration of radionuclide } i \text { in contaminated soil under } \\
& \text { equilibrium conditions }(\mathrm{Bq} / \mathrm{kg}) \\
Q s_{k} & =\text { animal consumption rate of soil }(\mathrm{kg} / \mathrm{d})
\end{aligned}
$$

and the other parameters are defined in Equation 6.5.4-2.

Inadvertent soil ingestion by animals is estimated for mature animals, and the values are animal-type specific. Typical literature values for beef cattle and dairy cows are about $1 \mathrm{~kg} / \mathrm{d}$ (BSC 2004 [DIRS 169672], Section 6.3).

\subsubsection{External Exposure Submodel}

As in the groundwater scenario, the external exposure submodel for the volcanic ash scenario considers a human receptor exposed only to contaminated soil. Other external exposure pathways, air submersion and water immersion, are excluded because of the low contribution likely from air submersion (Section 7.4.8) and the lack of a contaminated source for water immersion. Air submersion could be important during a volcanic eruption, but the ERMYN does not consider any scenarios during a volcanic eruption. Dose from external exposure is calculated as the annual EDE.

\subsubsection{External Exposure to Contaminated Ground Surface}

Under the volcanic ash scenario, a thin layer of contaminated volcanic ash would be deposited on the surface of the ground. It would be improper to use a fixed volumetric source for the volcanic ash because the thickness of the source (i.e., the layer of ash) would be unknown, and it may change through time. To simplify the calculations, it is assumed (Approximation 16) that, regardless of the actual thickness of the ash, all radionuclides are only in a thin layer on the ground, which gives a higher external dose than if radionuclides mix with the soil. The annual external exposure, based on a modification of Equation 6.4.7-1 for the groundwater scenario, is evaluated as 


$$
D_{e x t, i}=E D C s_{\text {soil }, i} C s_{i} \sum_{n} f_{e x t, i, n}\left(\sum_{m} P P_{m}\left(3600 \times t_{n, m}\right)\right)
$$

where

$$
\begin{aligned}
& D_{\text {ext, } i}=\text { dose from external exposure to radionuclide } i \text { in deposited volcanic ash } \\
& \mathrm{Cs}_{i} \quad=\text { activity concentration of radionuclide } i \text { in deposited volcanic ash }\left(\mathrm{Bq} / \mathrm{m}^{2}\right) \\
& f_{\text {ext,inn }}=\text { external shielding factor for exposure to radionuclide } i \text { in the ground at } \\
& n=\text { environment index; } n=1 \text { for active outdoors, } 2 \text { for inactive outdoors, } 3 \text { for } \\
& \text { active indoors, } 4 \text { for asleep indoors, and } 5 \text { for away from the contaminated } \\
& \text { area } \\
& m=\text { population group index; } m=1 \text { for local outdoor workers, } 2 \text { for local indoor } \\
& \text { workers, } 3 \text { for commuters, and } 4 \text { for nonworkers } \\
& P P_{m} \quad=\text { fraction of total population in population group } m \text { (population proportion) } \\
& \text { (dimensionless) } \\
& t_{n, m} \quad=\text { time spent by population group } m \text { in environment } n \text { (exposure time) } \\
& \text { (hr/year). } \\
& 3600=\text { unit conversion of hours to seconds; 3,600 (sec/hr) }
\end{aligned}
$$

Similar to the volumetric source discussed in Section 6.4.7, the short-lived decay products in equilibrium with their long-lived primary radionuclides are considered in the effective dose coefficients for contaminated ground surface. Development of the effective dose coefficients is discussed below. The radionuclide concentration in the surface soil is the source term for this scenario.

The external shielding factor, $f_{e x t, i, n}$, accounts for the reduction in external exposure offered by dwellings. Outdoors ( $n=1,2$, and 5), there is no shielding reduction and the value of $f_{e x t, i, n}$ is considered to be one. For indoor environments ( $n=3$ and 4 ), the shielding factor is radionuclide dependent (e.g., strong gamma-ray emitters would be more penetrating and have a higher factor than would weak gamma emitters). Consequently, values for the shielding factor range from zero to one; however, typical values, even for the most penetrating radiation emissions, do not exceed 0.4. Values for this parameter should be the same as those used in the groundwater scenario, as developed by BSC (2004 [DIRS 169671]).

The external exposure time, $t_{n, m}$, the amount of time spent annually in environment $n$ by population $m$, depends on the life style of the receptor. The fraction of the total population in population group $m, P P_{m}$, is based on Amargosa valley census data (BSC 2004 [DIRS 169671], Section 6.3). The spatial distribution of contamination differs between the two exposure scenarios. Therefore, the fraction of the population that works outside the contaminated area 
(i.e., commuters) and the time it would take them to leave the area differs, and different values for exposure times and population proportions must be used for the two scenarios.

\subsubsection{Effective Dose Coefficients for Contaminated Ground Surface}

Dose coefficients for exposure to a contaminated ground surface are evaluated in BSC (2004 [DIRS 169671], Section 6.5) and are based on the values from FGR-12 (Eckerman and Ryman 1993 [DIRS 107684], Table III.3). Dose contributions from short-lived decay products are combined with those of the long-lived parent radionuclides (Section 6.3.5). Effective dose coefficients (Table 6.5-1) are calculated as the sum of the dose coefficients for a long-lived radionuclide and the short-lived decay products, with consideration of the branching ratios. The calculation is expressed as

$$
E D C s_{\text {soil }, i}=\sum_{s} D C s_{\text {soil }, s} \times B N_{s}
$$

where

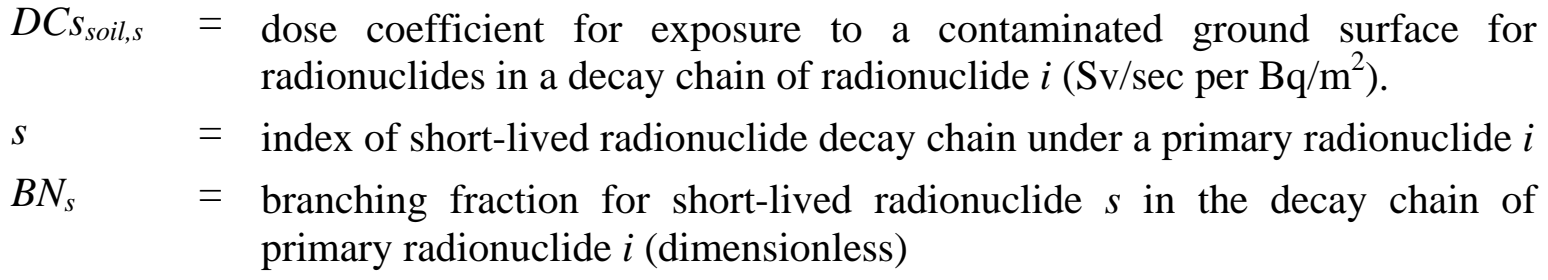

\begin{tabular}{|c|c|c|c|}
\hline $\begin{array}{c}\text { Primary } \\
\text { Radionuclide }\end{array}$ & $\begin{array}{c}\text { Decay Product }{ }^{c} \\
\text { (branching fraction if not } 100 \% \text {, } \\
\text { half-life) }\end{array}$ & $\begin{array}{l}\text { Dose Coefficient } \\
\text { Value }^{\mathrm{d}}\left(D C s_{\text {soil }}\right) \\
(\mathrm{Sv} / \mathrm{sec}) /\left(\mathrm{Bq} / \mathrm{m}^{2}\right)\end{array}$ & $\begin{array}{c}\text { Effective Dose } \\
\text { Coefficient }(E D C \text { s soil }) \\
(\text { Sv/sec }) /\left(\mathrm{Bq} / \mathrm{m}^{2}\right)\end{array}$ \\
\hline Sr-90D ${ }^{a}$ & Y-90 (64.0 hr) & $\begin{array}{l}2.84 \mathrm{E}-19 \\
5.32 \mathrm{E}-18 \\
\end{array}$ & $5.60 \mathrm{E}-18$ \\
\hline TC-99 & - & 7.80E-20 & 7.80E-20 \\
\hline Sn-126D & $\begin{array}{l}\text { Sb-126m (19.0 min) } \\
\text { Sb-126 (14\%, } 12.4 \mathrm{~d})\end{array}$ & $\begin{array}{l}5.47 \mathrm{E}-17 \\
1.52 \mathrm{E}-15 \\
2.78 \mathrm{E}-15 \\
\end{array}$ & 1.96E-15 \\
\hline Cs-137D & Ba-137m (94.6\%, $2.552 \mathrm{~min})$ & $\begin{array}{l}2.85 \mathrm{E}-19 \\
5.86 \mathrm{E}-16\end{array}$ & $5.55 \mathrm{E}-16$ \\
\hline
\end{tabular}

the other parameter is defined in Equation 6.5.5-1.

Similar to Table 6.4-2, the values in Table 6.5-1 are for demonstration purposes only. The ERMYN uses branching fractions and dose coefficients as inputs to calculate the effective dose conversion factors in GoldSim (Section 6.9).

Table 6.5-1. Effective Dose Coefficients for Exposure to Contaminated Ground Surface 
Table 6.5-1. Effective Dose Coefficients for Exposure to Contaminated Ground Surface (Continued)

\begin{tabular}{|c|c|c|c|}
\hline $\begin{array}{c}\text { Primary } \\
\text { Radionuclide } \\
\end{array}$ & $\begin{array}{c}\text { Decay Product }{ }^{\mathrm{c}} \\
\text { (branching fraction if not } 100 \% \text {, } \\
\text { half-life) }\end{array}$ & $\begin{array}{l}\text { Dose Coefficient } \\
\text { Value }^{\mathrm{d}}\left(D C s_{\text {soii }}\right) \\
(\mathrm{Sv} / \mathrm{sec}) /\left(\mathrm{Bq} / \mathrm{m}^{2}\right)\end{array}$ & $\begin{array}{c}\text { Effective Dose } \\
\text { Coefficient }\left(E D C s_{\text {soil }}\right) \\
(\text { Sv/sec }) /\left(\mathrm{Bq} / \mathrm{m}^{2}\right) \\
\end{array}$ \\
\hline Pu-242 & - & 6.67E-19 & 6.67E-19 \\
\hline U-238D & $\begin{array}{l}\text { Th-234 (24.10 d) } \\
\text { Pa-234m (99.80\%, } 1.17 \mathrm{~min}) \\
\text { Pa-234 (0.33\%, } 6.7 \mathrm{hr})\end{array}$ & $\begin{array}{l}5.51 \mathrm{E}-19 \\
8.32 \mathrm{E}-18 \\
1.53 \mathrm{E}-17 \\
1.84 \mathrm{E}-15 \\
\end{array}$ & $3.02 \mathrm{E}-17$ \\
\hline $\mathrm{Pu}-238$ & - & $8.38 \mathrm{E}-19$ & 8.38E-19 \\
\hline U-234 & - & $7.48 \mathrm{E}-19$ & $7.48 \mathrm{E}-19$ \\
\hline Th-230 & - & 7.50E-19 & $7.50 \mathrm{E}-19$ \\
\hline Ra-226D & $\begin{array}{l}\text { Rn-222 (3.8235 d) } \\
\text { Po-218 (3.05 min) } \\
\text { Pb-214 (99.98\%, } 26.8 \mathrm{~min}) \\
\text { At-218 }(0.02 \%, 2 \mathrm{sec}) \\
\text { Bi-214 }(19.9 \mathrm{~min}) \\
\text { Po-214 }\left(99.98 \%, 1.64 \times 10^{-4} \mathrm{sec}\right) \\
\text { Tl-210 }(0.02 \%, 1.3 \mathrm{~min})\end{array}$ & $\begin{array}{l}6.44 \mathrm{E}-18 \\
3.95 \mathrm{E}-19 \\
8.88 \mathrm{E}-21 \\
2.44 \mathrm{E}-16 \\
4.18 \mathrm{E}-18 \\
1.41 \mathrm{E}-15 \\
8.13 \mathrm{E}-20 \\
0.00 \mathrm{E}+00 \\
\end{array}$ & $1.66 \mathrm{E}-15$ \\
\hline $\mathrm{Pb}-210 \mathrm{D}$ & $\begin{array}{l}\text { Bi-210 (5.012 d) } \\
\text { Po-210 (138.38 d) }\end{array}$ & $\begin{array}{l}2.48 \mathrm{E}-18 \\
1.05 \mathrm{E}-18 \\
8.29 \mathrm{E}-21 \\
\end{array}$ & $3.54 \mathrm{E}-18$ \\
\hline Pu-240 & - & 8.03E-19 & 8.03E-19 \\
\hline U-236 & - & $6.50 \mathrm{E}-19$ & $6.50 \mathrm{E}-19$ \\
\hline Th-232 & - & $5.51 \mathrm{E}-19$ & $5.51 \mathrm{E}-19$ \\
\hline $\mathrm{Ra}-228 \mathrm{D}^{\mathrm{b}}$ & Ac-228 (6.13 hr) & $\begin{array}{l}0.00 \mathrm{E}+00 \\
9.28 \mathrm{E}-16\end{array}$ & $9.28 \mathrm{E}-16$ \\
\hline $\mathrm{U}-232$ & - & $1.01 \mathrm{E}-18$ & $1.01 \mathrm{E}-18$ \\
\hline Th-228D & $\begin{array}{l}\text { Ra-224 (3.66 d) } \\
\text { Rn-220 (55.6 sec) } \\
\text { Po-216 (0.15 sec) } \\
\text { Pb-212 (10.64 hr) } \\
\text { Bi-212 (60.55 min) } \\
\text { Po-212 (64.07\%, } 0.305 \mu-\mathrm{sec}) \\
\text { Tl-208 (35.93\%, 3.07 min })^{\text {e }}\end{array}$ & $\begin{array}{l}2.35 \mathrm{E}-18 \\
9.57 \mathrm{E}-18 \\
3.81 \mathrm{E}-19 \\
1.65 \mathrm{E}-20 \\
1.43 \mathrm{E}-16 \\
1.79 \mathrm{E}-16 \\
0.00 \mathrm{E}+00 \\
2.98 \mathrm{E}-15 \\
\end{array}$ & $1.41 \mathrm{E}-15$ \\
\hline Am-243D & Np-239 (2.355 d) & $\begin{array}{l}5.35 \mathrm{E}-17 \\
1.63 \mathrm{E}-16\end{array}$ & 2.17E-16 \\
\hline Pu-239 & - & 3.67E-19 & 3.67E-19 \\
\hline $\mathrm{U}-235 \mathrm{D}$ & Th-231 (25.52 hr) & $\begin{array}{l}1.48 \mathrm{E}-16 \\
1.85 \mathrm{E}-17 \\
\end{array}$ & $1.67 \mathrm{E}-16$ \\
\hline $\mathrm{Pa}-231$ & - & 4.07E-17 & 4.07E-17 \\
\hline
\end{tabular}


Table 6.5-1. Effective Dose Coefficients for Exposure to Contaminated Ground Surface (Continued)

\begin{tabular}{|c|c|c|c|}
\hline $\begin{array}{c}\text { Primary } \\
\text { Radionuclide }\end{array}$ & $\begin{array}{c}\text { Decay Product }{ }^{\mathrm{c}} \\
\text { (branching fraction if not } 100 \% \text {, } \\
\text { half-life) }\end{array}$ & $\begin{array}{l}\text { Dose Coefficient } \\
\text { Value }^{\mathrm{d}}\left(\mathrm{DCs_{ \text {soil } } )}\right. \\
(\mathrm{Sv} / \mathrm{sec}) /\left(\mathrm{Bq} / \mathrm{m}^{2}\right)\end{array}$ & $\begin{array}{c}\text { Effective Dose } \\
\text { Coefficient }\left(E D C s_{\text {soil }}\right) \\
(\text { Sv/sec }) /\left(\mathrm{Bq} / \mathrm{m}^{2}\right) \\
\end{array}$ \\
\hline Ac-227D & $\begin{array}{l}\text { Th-227 (98.62\%, } 18.718 \mathrm{~d}) \\
\text { Fr-223 (1.38\%, 21.8 min) } \\
\text { Ra-223 (11.434 d) } \\
\text { Rn-219 (3.96 sec) } \\
\text { Po-215 (1.78 millisec) } \\
\text { Pb-211 (36.1 min) } \\
\text { Bi-211 (2.14 min) } \\
\text { Tl-207 (99.72\%, } 4.77 \mathrm{~min}) \\
\text { Po-211 (0.28\%, } 0.516 \mathrm{sec})\end{array}$ & $\begin{array}{l}1.57 \mathrm{E}-19 \\
1.04 \mathrm{E}-16 \\
5.65 \mathrm{E}-17 \\
1.28 \mathrm{E}-16 \\
5.49 \mathrm{E}-17 \\
1.74 \mathrm{E}-19 \\
5.08 \mathrm{E}-17 \\
4.58 \mathrm{E}-17 \\
3.76 \mathrm{E}-18 \\
7.61 \mathrm{E}-18\end{array}$ & 3.87E-16 \\
\hline Am-241 & - & 2.75E-17 & $2.75 \mathrm{E}-17$ \\
\hline Np-237D & $\mathrm{Pa}-233(27.0 \mathrm{~d})$ & $\begin{array}{l}2.87 \mathrm{E}-17 \\
1.95 \mathrm{E}-16 \\
\end{array}$ & $2.24 \mathrm{E}-16$ \\
\hline U-233 & - & 7.16E-19 & 7.16E-19 \\
\hline Th-229D & $\begin{array}{l}\text { Ra-225 (14.8 d) } \\
\text { Ac-225 (10.0 d) } \\
\text { Fr-221 (4.8 min) } \\
\text { At-217 (32.3 millisec) } \\
\text { Bi-213 (45.65 min) } \\
\text { Po-213 (97.84\%, } 4.2 \mu \text {-sec) } \\
\text { Tl-209 (2.16\%, } 2.2 \mathrm{~min}) \\
\text { Pb-209 (3.253 hr) }\end{array}$ & $\begin{array}{l}8.54 \mathrm{E}-17 \\
1.33 \mathrm{E}-17 \\
1.58 \mathrm{E}-17 \\
2.98 \mathrm{E}-17 \\
3.03 \mathrm{E}-19 \\
1.32 \mathrm{E}-16 \\
0.00 \mathrm{E}+00 \\
1.90 \mathrm{E}-15 \\
3.01 \mathrm{E}-19\end{array}$ & $3.18 \mathrm{E}-16$ \\
\hline
\end{tabular}

${ }^{a} \mathrm{~A}$ "D" after a radionuclide symbol denotes that the radionuclide is treated together with the short-lived (less than $180 \mathrm{~d}$ ) decay product.

${ }^{\mathrm{b}}$ Indented radionuclides are long-lived decay products considered separately from the parents.

c Branching fractions and half-lives are from Eckerman and Ryman (1993 [DIRS 107684], Table A.1).

${ }^{d}$ Dose coefficient source (BSC 2004 [DIRS 169671], Section 6.5).

${ }^{\mathrm{e}}$ Half-life of TI-210 is taken from Lide and Frederikse (1997 [DIRS 103178], p.11-125).

$\mathrm{DC}=$ dose coefficient

\subsubsection{Inhalation Submodel}

The inhalation submodel for the volcanic ash scenario is more complicated than that for the groundwater scenario because the input parameters, radionuclide concentrations in air and ground, vary with time and thickness of the ash (Equation 6.5.2-4). The inhalation dose is calculated as the CEDE for the 50-year committed period resulting from annual intake of radionuclides by inhalation. Two sources of contamination in air are considered: resuspended particles (Section 6.5.2.1) and radon gas (Section 6.5.2.2). The total inhalation dose is the sum of the dose from both sources, which is evaluated as

$$
D_{i n h, i}\left(d_{a}, t\right)=D_{i n h, p, i}\left(d_{a}, t\right)+D_{i n h, g, i}
$$


where

$$
\begin{array}{ll}
D_{\text {inh,i }}\left(d_{a}, t\right)= & \begin{array}{l}
\text { annual dose from inhalation of radionuclide } i \text { for ash thickness } d_{a} \text { at } \\
\text { time } t(\mathrm{~Sv} / \text { year })
\end{array} \\
D_{\text {inh,p,i }}\left(d_{a}, t\right)=\begin{array}{l}
\text { annual dose from inhalation of radionuclide } i \text { resulting from exposure to } \\
\text { resuspended particles }(p) \text { for ash thickness } d_{a} \text { at time } t(\mathrm{~Sv} / \mathrm{year})
\end{array} \\
\begin{array}{l}
\text { annual dose from inhalation of radionuclides resulting from gaseous } \\
\text { emission }(g) \text { of radionuclide } i \text { in the ash (Sv/year) }
\end{array}
\end{array}
$$

\subsubsection{Inhalation of Resuspended Particles}

The inhalation dose is calculated considering specific environments associated with human activities and population groups. For this scenario, there are two components to the radionuclide concentrations in the air, one related to nominal mass loading and one related to post-volcanic, time-dependent, mass loading, both of which depend on ash thickness (Section 6.5.2.1). Therefore, the inhalation dose calculation can be separated into two items and expressed as

$$
\begin{aligned}
& D_{i n h, p, i}\left(d_{a}, t\right)= E D C F_{i n h, i} \sum_{n} C a_{h, i, n}\left(d_{a}, t\right) B R_{n}\left(\sum_{m} P P_{m} t_{n, m}\right) \\
&=E D C F_{i n h, i} \sum_{n} C a_{h, i, n} g\left(d_{a}\right) B R_{n}\left(\sum_{m} P P_{m} t_{n, m}\right) \\
&+E D C F_{i n h, i} \sum_{n} C a_{v, i, n} g\left(d_{a}\right) f(t) B R_{n}\left(\sum_{m} P P_{m} t_{n, m}\right) \\
&=D_{i n h, p, i} g\left(d_{a}\right)+D_{i n h, v, i} g\left(d_{a}\right) f(t)
\end{aligned}
$$

where

$$
\begin{aligned}
& D_{\text {inh,p,i }}\left(d_{a}, t\right)=\text { annual dose from inhalation of radionuclide } i \text { resulting from exposure to } \\
& \text { resuspended particles for an ash thickness of } d_{a} \text { at time } t \text { (Sv/year) } \\
& E D C F_{\text {inh }, i}=\text { effective dose conversion factor for inhalation of primary radionuclide } i \\
& (\mathrm{~Sv} / \mathrm{Bq}) \\
& n=\text { environment index; } n=1 \text { for active outdoors, } 2 \text { for inactive outdoors, } \\
& 3 \text { for active indoors, } 4 \text { for asleep indoors, and } 5 \text { for away from the } \\
& \text { contaminated area } \\
& B R_{n} \quad=\text { breathing rate for environment } n\left(\mathrm{~m}^{3} / \mathrm{hr}\right) \\
& m \quad=\text { population group index; } m=1 \text { for local outdoor workers, } 2 \text { for local } \\
& \text { indoor workers, } 3 \text { for commuters, and } 4 \text { for nonworkers } \\
& P P_{m} \quad=\text { proportion of population in group } m \text { (dimensionless) } \\
& t_{n, m} \quad=\text { time spent by population group } m \text { in environment } n \text { (exposure time) } \\
& \text { (hr/year) } \\
& D_{\text {inh, }, i} \quad=\text { annual dose from inhalation of radionuclide } i \text { resulting from exposure to } \\
& \text { nominal mass loading }(p) \text { after a volcanic eruption (Sv/year) }
\end{aligned}
$$


$D_{i n h, v, i}=$ annual dose from inhalation of radionuclide $i$ resulting from exposure to post-volcanic mass loading $(v)$ in addition to nominal mass loading following a volcanic eruption (Sv/year)

and the other parameters are defined in Equations 6.5.1-3, 6.5.1.4, 6.5.2-2, 6.5.2-3, and 6.5.2-4 .

The effective dose conversion factors for inhalation are discussed in Section 6.4.8.5 and shown in Table 6.4-3. The inhalation exposure time, $t_{n, m}$, is the annual amount of time that population $m$ spends in environment $n$, and depends on the life style of the receptor (BSC 2004 [DIRS 169671], Section 6.3). The breathing rate, $B R_{n}$, varies with the environment. The fraction of the total population in population group $m, P P_{m}$, is based on Amargosa Valley census data (BSC 2004 [DIRS 169671]).

\subsubsection{Inhalation of Radon Decay Products}

The only gaseous radionuclide considered in the submodel is ${ }^{222} \mathrm{Rn}$, which would be released from ${ }^{226} \mathrm{Ra}$ in volcanic ash. Considering an infinitely large contaminated area (Section 6.5.2.2), the radon concentration in the air is estimated using a conversion factor between ${ }^{226} \mathrm{Ra}$ activity concentration on the top of the ground and ${ }^{222} \mathrm{Rn}$ activity concentration in air. The indoor ${ }^{222} \mathrm{Rn}$ concentration is the same as the outdoor ${ }^{222} \mathrm{Rn}$ concentration (Section 6.5.2.2). Under the volcanic ash scenario, the ${ }^{222} \mathrm{Rn}$ inhalation dose calculation does not require consideration of elevated indoor concentration of ${ }^{222} \mathrm{Rn}$ produced as a result of operating an evaporative cooler. Using the radon inhalation dose conversion factor derived in Section 6.4.8.5 (Equation 6.4.8-8), the radon inhalation dose is evaluated as

$$
\begin{aligned}
D_{\text {inh, }, \text {, Rn-222 }} & =C a_{g, R n-222} \sum_{n} D C F_{i n h, R n-222, n} B R_{n}\left(\sum_{m} P P_{m} t_{n, m}\right) \\
& =C a_{g, R n-222} D C F_{i n h, R n-222} \sum_{n} E F_{R n-222, n} B R_{n}\left(\sum_{m} P P_{m} t_{n, m}\right)
\end{aligned}
$$

where

$$
\begin{aligned}
& D_{\text {inh,g,Rn-222 }}=\text { annual dose from inhalation of }{ }^{222} \mathrm{Rn} \text { decay products (Sv/year) } \\
& D C F_{i n h, R n-222, n}=\text { dose conversion factor for inhalation of }{ }^{222} \mathrm{Rn} \text { decay products in } \\
& \text { environment } n(\mathrm{~Sv} / \mathrm{Bq}) \\
& D C F_{\text {inh,Rn-222 }}=\text { dose conversion factor for inhalation of }{ }^{222} \mathrm{Rn} \text { decay products at } \\
& 100 \text { percent equilibrium } \\
& E F_{R n-222, n}=\text { equilibrium factor for }{ }^{222} \mathrm{Rn} \text { decay products in environment } n \\
& \text { (dimensionless) }
\end{aligned}
$$

and the other parameters are defined in Equations 6.5.2-8 and 6.5.6-2. 
The radon inhalation dose contribution is to be added into the BDCF for ${ }^{226} \mathrm{Ra}$. By combining Equations 6.5.6-2 and 6.5.6-3, Equation 6.5.6-1 can be rewritten as

$$
\begin{aligned}
D_{i n h, i}\left(d_{a}, t\right) & =D_{i n h, p, i}\left(d_{a}, t\right)+D_{i n h, g, i} \\
& =D_{i n h, v i} g\left(d_{a}\right) f(t)+D_{i n h, p, i} g\left(d_{a}\right)+D_{i n h, g i}
\end{aligned}
$$

where all parameters are defined in Equations 6.5.6-1, 6.5.6-2, and 6.5.6-3.

\subsubsection{Ingestion Submodel}

Because groundwater is uncontaminated in the volcanic ash scenario, the ingestion submodel only considers contaminated crops (leafy vegetables, other vegetables, fruits, and grain), contaminated animal products (meat, poultry, milk, and eggs), and inadvertent ingestion of contaminated soil. The ingestion dose, analogous to the inhalation dose, is calculated as the CEDE for the 50-year committed period resulting from the annual intake of radionuclides by ingestion. The contaminated foodstuffs are discussed in the plant (Section 6.5.3) and animal submodels (Section 6.5.4). The total ingestion dose includes contributions from all these sources, and for an individual radionuclide is expressed as

$$
D_{\text {ing }, i}=D_{\text {ing }, p, i}+D_{i n g, d, i}+D_{i n g, s, i}
$$

where

$$
\begin{aligned}
D_{\text {ing }, i}= & \text { annual dose from ingestion of radionuclide } i \text { (Sv/year) } \\
D_{\text {ing, }, \mathrm{i}, i}= & \text { annual dose from ingestion of radionuclide } i \text { in crops (Sv/year) } \\
D_{\text {ing,d,i }}= & \text { annual dose from ingestion of radionuclide } i \text { in animal products (Sv/year) } \\
D_{\text {ing, }, i}= & \text { annual dose from inadvertent ingestion of radionuclide } i \text { in surface soil } \\
& \text { (Sv/year) }
\end{aligned}
$$

These ingestion pathways are discussed in detail in the following subsections.

\subsubsection{Ingestion of Crop Foodstuffs}

The ingestion of contaminated crops is an important pathway for the volcanic scenario, similar to the groundwater scenario, and includes four types of crop foodstuffs. The storage time from harvest to consumption is not considered because only long-lived radionuclides are of concern in the biosphere model. Annual doses from the ingestion of contaminated crops are evaluated as

$$
D_{\text {ing }, p, i}=E D C F_{\text {ing, } i} \sum_{j}\left(C p_{i, j} U p_{j}\right)
$$

where

$$
\begin{array}{ll}
D_{\text {ing, }, i}= & \text { annual dose from ingestion of primary radionuclide } i \text { in crops }(\mathrm{Sv} / \mathrm{year}) \\
E D C F_{\text {ing, } i}= & \text { effective dose conversion factor for ingestion of primary radionuclide } i \\
& (\mathrm{~Sv} / \mathrm{Bq}) \\
C p_{i, j} & \text { activity concentration of primary radionuclide } i \text { in crop type } j(\mathrm{~Bq} / \mathrm{kg}) \\
j & \text { index of crop type, } j=1 \text { for leafy vegetables, } 2 \text { for other vegetables, } 3 \text { for }
\end{array}
$$




$$
\begin{aligned}
& \text { Up } \text { fruit, and } 4 \text { for grain } \\
& \text { annual consumption rate of locally produced contaminated crop type } \\
& j(\mathrm{~kg} / \text { year) }
\end{aligned}
$$

The effective dose conversion factors for ingestion are discussed in Section 6.4.9.6 and shown in Table 6.4-4. The activity concentrations of radionuclides in crops are discussed in the plant submodel (Section 6.5.3). The consumption rates used in Equation 6.5.7-2 apply only to locally produced crops (BSC 2004 [DIRS 169671]).

\subsubsection{Ingestion of Animal Products}

The ingestion of animal products includes the four animal product pathways used for the groundwater scenario. Similar to crops, the storage time from harvest to consumption is not considered. Annual doses from the ingestion of contaminated animal products are evaluated as

$$
D_{\text {ing, }, i, i}=E D C F_{\text {ing, } i} \sum_{k}\left(C d_{i, k} U d_{k}\right)
$$

where

$$
\begin{aligned}
& D_{\text {ing,d, } i}=\text { annual dose from ingestion of primary radionuclide } i \text { in animal products } \\
& \text { (Sv/year) } \\
& C d_{i, k}=\text { activity concentration of primary radionuclide } i \text { in animal product } k(\mathrm{~Bq} / \mathrm{kg}) \\
& k=\text { index of animal products, } k=1 \text { for meat, } 2 \text { for poultry, } 3 \text { for milk, and } 4 \text { for } \\
& \text { eggs } \\
& U d_{k}=\text { annual consumption rate of locally produced animal product } k \text { (kg/year) } \\
& \text { and the other parameter is defined in Equation 6.5.7-2. }
\end{aligned}
$$

The activity concentrations of radionuclides in animal products are discussed in the animal submodel (Section 6.5.4). The consumption rates used in Equation 6.5.7-3 apply only to locally produced animal products; imported animal products are considered to be uncontaminated (BSC 2004 [DIRS 169671], Section 6.4).

\subsubsection{Inadvertent Soil Ingestion}

Inadvertent soil ingestion may be an important pathway for the volcanic ash scenario because contaminated ash deposited on the soil surface is the source of contamination. Annual doses from inadvertent ingestion of radionuclides in contaminated soil is evaluated as

$$
D_{i n g, s, i}=E D C F_{i n g, i} C s_{m, i} U s
$$

where

$$
\begin{aligned}
D_{\text {ing, }, \mathrm{i}, \mathrm{i}}= & \text { annual dose from inadvertent ingestion of primary radionuclide } i \text { in the surface } \\
& \text { soil }(\mathrm{Sv} / \mathrm{year}) \\
C s_{m, i}= & \begin{array}{l}
\text { activity concentration of a primary radionuclide } i \text { in the surface soil of } \\
\text { cultivated land }(\mathrm{Bq} / \mathrm{kg})
\end{array}
\end{aligned}
$$


Us $\quad=$ annual consumption rate of contaminated soil $(\mathrm{kg} /$ year $)$

and the other parameter is defined in Equation 6.5.7-2.

The activity concentration of radionuclides in the surface soil is discussed in the surface soil submodel (Section 6.5.1). Estimates of soil ingestion rates have wide uncertainty distributions, with typical values for adults on the order of several tens to a few hundred milligrams per day (BSC 2004 [DIRS 169671], Section 6.4).

\subsubsection{All-Pathway Dose and Biosphere Dose Conversion Factor Calculation}

Under the volcanic ash scenario, radionuclides in the ash are the source for all radiation pathways, and all depend linearly on this source. The biosphere contribution to the TSPA-LA for dose assessments is separated from the source. The biosphere contribution has three terms, one that is constant, one that depends on the thickness of the ash, and one that depends on time and the thickness of the ash (Equation 6.5.6-4). The time function is due to the expected decline in mass loading after a volcanic eruption. Because the radionuclide source term is time dependent (due to ash redistribution and radionuclide decay), time-dependent dose calculations are carried out in the TSPA-LA model rather than in the ERMYN. In addition, two of the BDCF components depend on ash thickness. Because ash thickness is not an input parameter to the ERMYN, this dose calculation also is carried out in the TSPA-LA model. This section provides the method to calculate the final dose based on the BDCFs discussed in the previous sections.

\subsubsection{All-Pathway Dose}

The all-pathway annual dose for an individual primary radionuclide, expressed in terms of the TEDE, is the sum of the annual EDE from external exposure and the annual CEDE from the radionuclide intake into the body by ingestion and inhalation, and is evaluated as

$$
\begin{aligned}
D_{a l l, i}\left(d_{a}, t\right) & =D_{e x t, i}+D_{i n h, i}\left(d_{a}, t\right)+D_{i n g, i} \\
& =D_{e x t, i}+D_{i n g, i}+D_{i n h, g, i}+D_{i n h, v, i} g\left(d_{a}\right) f(t)+D_{i n h, p, i} g\left(d_{a}\right) \\
& =D_{e x t, i n g, R n, i}+D_{i n h, v, i} g\left(d_{a}\right) f(t)+D_{i n h, p, i} g\left(d_{a}\right)
\end{aligned}
$$

where

$$
\begin{aligned}
& D_{\text {all,i }}\left(d_{a}, t\right)= \begin{array}{l}
\text { all-pathway annual dose from internal and external exposure to } \\
\text { radionuclide } i \text { for ash deposited to a depth of } d_{a} \text { at time } t(\mathrm{~Sv} / \text { year) }
\end{array} \\
& D_{\text {ext ing,Rn,i }}=\begin{array}{l}
\text { annual dose from external exposure, ingestion, and inhalation of radon } \\
\text { decay products for radionuclide } i \text { (Sv/year) }
\end{array}
\end{aligned}
$$

and the other parameters are defined in Equations 6.5.5-1, 6.5.6-1, 6.5.6-2, 6.5.6-4, and 6.5.7-1. 


\subsubsection{Biosphere Dose Conversion Factors for the Volcanic Ash Scenario}

The radionuclide concentration in the volcanic ash deposited on the surface of the ground is the only source of radionuclide contamination for the volcanic ash scenario. All radiation pathways discussed in the previous sections depend linearly on this source, which is summarized in Table 6.5-2.

Table 6.5-2. Summary of the Biosphere Submodels for the Volcanic Ash Scenario

\begin{tabular}{|c|c|c|c|c|}
\hline Submodel & Quantity Calculated in Submodel & Section & Simplified Equation & $\begin{array}{c}\text { Equation } \\
\text { Number }\end{array}$ \\
\hline \multirow[t]{2}{*}{ Soil } & $\begin{array}{l}\text { Activity concentration of a primary } \\
\text { radionuclide on cultivated lands }\end{array}$ & 6.5 .1 .1 & $C s_{m, i}=K 1 C s_{i}$ & $6.5 .1-2$ \\
\hline & $\begin{array}{l}\text { Activity concentration of a primary } \\
\text { radionuclide on noncultivated lands }\end{array}$ & 6.5 .1 .2 & $C s_{m c, i}=K 2 C s_{i}$ & $6.5 .1-3$ \\
\hline \multirow[t]{2}{*}{ Air } & $\begin{array}{l}\text { Activity concentration of a radionuclide in air } \\
\text { from soil resuspension }\end{array}$ & 6.5 .2 .1 & $C a_{i}=K 3 C s_{i}$ & $6.5 .2-2$ \\
\hline & Activity concentration of radon gas in air & 6.5.2.2 & $C a_{R n-222}=K 4 C s_{R a-226}$ & $6.5 .2-8$ \\
\hline \multirow[t]{2}{*}{ Plant } & $\begin{array}{l}\text { Activity concentration of a radionuclide in } \\
\text { crops from root uptake }\end{array}$ & 6.5 .3 .1 & $C p_{i}=K 5 C s_{i}$ & $6.5 .3-2$ \\
\hline & $\begin{array}{l}\text { Activity concentration of a radionuclide in } \\
\text { crops from foliar interception of resuspended } \\
\text { soil }\end{array}$ & 6.5 .3 .2 & $C p_{i}=K 6 C a_{i}=K 6 K 3 C s_{i}$ & $\begin{array}{l}6.5 .3-3 \\
6.5 .3-4\end{array}$ \\
\hline \multirow[t]{2}{*}{ Animal } & $\begin{array}{l}\text { Activity concentration of a radionuclide in } \\
\text { animal product from animal feed }\end{array}$ & 6.5 .4 .1 & $C d_{i}=K 7 C p_{l}=K 8 C s_{i}$ & $6.5 .4-2$ \\
\hline & $\begin{array}{l}\text { Activity concentration of a radionuclide in } \\
\text { animal product from soil ingestion }\end{array}$ & 6.5 .4 .2 & $C d_{i}=K 9 C s_{i}$ & $6.5 .4-3$ \\
\hline $\begin{array}{l}\text { External } \\
\text { Exposure }\end{array}$ & External exposure dose & 6.5 .5 .1 & $D_{\text {ext }, i}=K 10 C s_{i}$ & $6.5 .5-1$ \\
\hline \multirow[t]{2}{*}{ Inhalation } & Inhalation dose from airborne particulates & 6.5.6.1 & $D_{i n h, i}=K 11 C a_{i}=K 11 K 3 C s_{i}$ & $6.5 .6-2$ \\
\hline & Inhalation dose from radon decay products & 6.5 .6 .2 & $\begin{array}{l}D_{\text {inh,Rn-222 }}=K 12 C a_{R n-222} \\
=K 12 \mathrm{~K} 4 \mathrm{Cs}_{\mathrm{Ra}-226}\end{array}$ & $6.5 .6-3$ \\
\hline \multirow[t]{3}{*}{ Soil } & Ingestion dose from crops & 6.5.7.1 & $D_{\text {ing }, i}=K 13 C p_{i}=K 14 C s_{i}$ & $6.5 .7-2$ \\
\hline & Ingestion dose from animal products & 6.5.7.2 & $D_{\text {ing }, i}=K 15 C d_{i}=K 16 C s_{i}$ & $6.5 .7-3$ \\
\hline & Ingestion dose from soil & 6.5.7.3 & $D_{\text {ing }, i}=K 17 C s_{i}$ & $6.5 .7-4$ \\
\hline
\end{tabular}

NOTE: The constants in this table are specific to the volcanic ash scenario and are not the same as the constants in Table 6.4-5.

In the simplified equations shown in Table 6.5-2, the proportionality constants (e.g., $\mathrm{K} 1, \mathrm{~K} 2, \mathrm{~K}_{\mathrm{i}}$ ) can be derived from the referenced equations. The dose is proportional to the activity concentration of radionuclide $i$ in surface soil per unit area, $C s_{i}\left(\mathrm{~Bq} / \mathrm{m}^{2}\right)$.

Because of the need to consider ash thickness and time in the biosphere model, the posteruption BDCFs for the volcanic ash scenario are calculated as a function of ash thickness and time. In the TSPA-SR, the time dependence was eliminated using conservative transition phase BDCFs (CRWMS M\&O 2000 [DIRS 153246]). Alternatively, modeling the BDCFs as a function of ash thickness allows for applying the BDCFs to any ash thickness. Also, the new format for BDCFs allows for incorporating source variation, including ash redistribution, radionuclide decay and ingrowth, and surface erosion in the TSPA-LA model. 
All of the pathways are linearly proportional to the areal radionuclide concentration in the volcanic ash on the ground (Table 6.5-2). Thus, the biosphere contribution for the dose assessment can be separated from the source. The radionuclide-specific BDCFs are the final products from the ERMYN, and these are used in the TSPA-LA model. For the volcanic ash scenario, the radionuclide-specific BDCFs are calculated as

$$
\begin{aligned}
\operatorname{BDCF}_{i}\left(d_{a}, t\right) & =\frac{D_{a l l, i}\left(d_{a}, t\right)}{C s_{i}} \\
& =\frac{D_{e x t, i n g, R n, i}}{C s_{i}}+\frac{D_{i n h, v, i}}{C s_{i}} g\left(d_{a}\right) f(t)+\frac{D_{i n h, p, i}}{C s_{i}} g\left(d_{a}\right) \\
& =B D C F_{\text {ext }, i n g, R n, i}+\left(B D C F_{i n h, v, i} f(t)+B D C F_{i n h, p, i}\right) g\left(d_{a}\right)
\end{aligned}
$$

where

$$
\begin{aligned}
& B D C F_{i}\left(d_{a}, t\right)=\text { BDCF component for radionuclide } i \text { for an ash deposition depth of } d_{a} \\
& \text { at time } t \text { following a volcanic eruption (Sv/year per } \mathrm{Bq} / \mathrm{m}^{2} \text { ) } \\
& B D C F_{\text {ext,ing,Rn, } i}=\text { BDCF component for radionuclide } i \text { for external exposure, ingestion, } \\
& \text { and inhalation of radon decay products (Sv/year per } \mathrm{Bq} / \mathrm{m}^{2} \text { ) } \\
& B D C F_{\text {inh, }, i}=\text { BDCF component for radionuclide } i \text { for inhalation of post-volcanic } \\
& \text { mass loading in addition to nominal mass loading following a } \\
& \text { volcanic eruption (Sv/year per } \mathrm{Bq} / \mathrm{m}^{2} \text { ) } \\
& B D C F_{\text {inh,p,i }}=\text { BDCF component for radionuclide } i \text { for inhalation of nominal mass } \\
& \text { loading following a volcanic eruption (Sv/year per } \mathrm{Bq} / \mathrm{m}^{2} \text { ) } \\
& \text { and the other parameters are defined in Equations 6.5.1-1 and 6.5.8-1. }
\end{aligned}
$$

For the volcanic ash scenario, three BDCF components are provided to the TSPA-LA model. The first one is for the time-independent component, $B D C F_{i}$, which includes external exposure, radon inhalation, and ingestion. The second one is for the ash thickness dependent component, $B D C F_{\text {inh,p, }}$, which includes inhalation of resuspension particles at normal condition. The last one is for the ash thickness and time dependent component, $B D C F_{\text {inh, }, i}$, which includes inhalation of resuspended particles under post-volcanic conditions.

\subsubsection{Use of BDCFs in the Total System Performance Assessment Model}

Similar to the groundwater scenario, the expected annual dose calculation for the volcanic scenario is calculated in the TSPA-LA model, where input parameters include the radionuclide-specific BDCFs, the mass loading decrease function, and the thickness of the volcanic ash. The TSPA-LA code is used to calculate the time-dependent amount of volcanic ash deposited on the ground. The total annual dose is the sum of the individual annual doses from radionuclides tracked by the TSPA-LA, including the doses from any short-lived decay products incorporated into the BDCFs (half-life less than $180 \mathrm{~d}$ ). The expected annual dose is the final output of the TSPA-LA model. This quantity is used to determine compliance with the individual protection standard, which is one of the performance measures for the repository. The total annual dose, as a function of time and conditional on the occurrence of an eruption, is calculated using the TSPA-LA model as 


$$
\begin{array}{rl}
D_{\text {total }}\left(d_{a}, t\right)= & \sum_{i} B D C F_{i}\left(d_{a}, t\right) \times C s_{i}(t) \\
=\sum_{i} & B D C F_{\text {ext,ing, Rn,i }} \times C s_{i}(t) \\
& +\sum_{i}\left(B D C F_{i n h, v, i} f(t)+B D C F_{i n h, p, i}\right) g\left(d_{a}\right) \times C s_{i}(t)
\end{array}
$$

where

$$
\begin{aligned}
D_{\text {total }}\left(d_{a}, t\right)= & \text { time-dependent total annual dose to a defined receptor resulting from the } \\
& \text { release of radionuclides from the repository including contributions from } \\
& \text { all radionuclides considered in the TSPA-LA (Sv/year) } \\
C s_{i}(t)= & \text { time dependent activity concentration of radionuclide } i \text { in volcanic ash } \\
& \text { deposited on the ground }\left(\mathrm{Bq} / \mathrm{m}^{2}\right)
\end{aligned}
$$

and the other parameters are defined in Equation 6.5.8.2.

\subsection{CONSIDERATION OF MODEL UNCERTAINTY}

The ERMYN is based on the included FEPs, but it includes many approximations, simplifications, and idealizations, and, therefore, uncertainties must be considered. Uncertainty in the results of models comes from the conceptual model (e.g., from decisions concerning the inclusion or exclusion of pathways), the mathematical model (e.g., from the use of simplified analytical methods), and the input parameters (e.g., when represented by distributions of parameter values). When models are executed, the conceptual and mathematical model uncertainties are fixed, but if input distributions are used and multiple model simulations are realized, the output will be a range of values that can be used to characterize uncertainty distributions for the output values. The ERMYN has capabilities for simulating biosphere processes using variable inputs. In this section, uncertainty in the ERMYN from the conceptual model, mathematical model, and input parameters are discussed.

\subsubsection{Conceptual Model Uncertainty}

Uncertainty generated by the conceptual model comes from decisions regarding FEPs screening, approximations, and the selection of ACMs, but this uncertainty cannot be quantitatively evaluated. However, if a model is constructed carefully from individual submodels, the conceptual model uncertainty usually will be unimportant to overall model uncertainty. In the biosphere model, many submodels have the simple form of radionuclide concentration in media $X=$ constant of proportionality $\times$ radionuclide concentration in media $Y$, where the constant of proportionality is empirically determined. These constants are generally represented by distributions, thereby incorporating model uncertainty into the data uncertainty.

FEPs Screening-The biosphere model is based on a comprehensive list of the included FEPs; however, screening decisions to include or exclude FEPs could contribute to model uncertainty. Although this uncertainty is not evaluated quantitatively, it is likely that these decisions add little additional uncertainty because all applicable, important FEPs are represented in the conceptual model (BSC 2004 [DIRS 169826]). 
Human Receptor-The characteristics of the RMEI are defined by regulation (10 CFR 63.312 [DIRS 156605]). These regulations substantially reduce uncertainty about the dietary and lifestyle characteristics of the receptor and the selection of environmental transport and exposure pathways that are applicable to the receptor. Development of the attributes and behavioral characteristics of the RMEI involves uncertainties related to site-specific information. These uncertainties are considered in input parameter uncertainty (discussed below), rather than conceptual model uncertainty.

Consideration of Human Exposure Pathways-All applicable pathways are considered during development of the conceptual model (Sections 6.3.1 and 6.3.2), and only applicable pathways shown to have little influence on the results are excluded. For example, air submersion and water immersion pathways are not included because numerical comparisons made between dose coefficients and typical exposure times indicate that they are not important when compared with the included pathways (Section 7.4.8). The increase in overall uncertainty in the ERMYN results from not considering pathways with demonstrable inconsequential contributions to the BDCFs is small.

Environmental Transport of Radionuclides - Interaction matrices (Table 6.3-2, groundwater scenario; Table 6.3-4, volcanic ash scenario; Table 6.3-6, verification) are used to summarize radionuclide transfers between biosphere model components (environmental media). Because applicable, important FEPs are considered in the interaction matrices, it is expected that the important radionuclide transfer mechanisms are considered during development of the ERMYN. Of the transfer mechanisms considered, only those shown to have little influence on model results (e.g., inhalation of resuspended particles by livestock; see Section 7.4.5) are excluded from the model. Therefore, uncertainty due to omission or exclusion of radionuclide transfer mechanisms is low.

Alternative Conceptual Model-ACMs are discussed in Section 6.3.3. Based on evaluations (Section 7.4), the selected submodels and components are considered more reasonable than the excluded ACMs, and the main uncertainties associated with the ACMs are captured in the ERMYN. Therefore, screening of ACMs added little uncertainty to the final results.

Future Conditions-Regulation 10 CFR 63.305 ([DIRS 156605]) states that the DOE should not project changes in society, the biosphere (other than climate), human biology, and increases or decreases in human knowledge and technology. Therefore, uncertainty due to changes in the lifestyle and biology of the receptor, or conditions in the biosphere other than climate, are not considered in the ERMYN.

\subsubsection{Mathematical Model Uncertainty}

Uncertainty associated with mathematical models comes from how accurately a conceptual model is represented by the mathematical equations. Selection of the mathematical model used to express a conceptual model is mainly based on the appropriateness of the model representation and on data availability. When data are not available, approximations and simplifications are used to develop a reasonable mathematical model so that the processes can be quantitatively evaluated. Uncertainty associated with the approximations and simplifications becomes part of mathematical model uncertainty. Mathematical model uncertainty usually cannot be 
quantitatively evaluated unless it is included in the input parameters (see discussion on mathematical representation in Section 6.6.1).

Uncertainty for all of the modeling approximations discussed in Sections 6.3.1.4 and 6.3.2.4 are summarized in Table 6.6-1. The approximations are constructed to be conservative, but not overly conservative, with respect to the implications of compounding conservatism in the ERMYN results. When an approximation is reasonable, uncertainty about how the approximation represents reality is expected to be relatively small. However, approximations are necessary to reduce the numerical requirements of the mathematical model and the details required in the associated input data. The tradeoff between model uncertainty and simplified methods is considered acceptable if the dose estimates are not substantially underestimated. This section does not quantitatively evaluate uncertainty associated with the approximations; rather, it discusses the qualitative uncertainty due to using the approximations.

The mathematical representations of transport and exposure pathways used in the ERMYN were developed from a review of applicable methods in numerous biosphere and radiological assessment models. Appropriate methods were chosen from among those reviewed and, if necessary, adapted to match site-specific conditions, the requirements of 10 CFR Part 63, and the needs of the TSPA (Sections 6.4, 6.5, 7.1, and 7.3). For the few processes for which no appropriate method had been previously used (e.g., increase in radionuclide concentrations in fish ponds from water evaporation, transfer of radionuclides from water to air during the operation of evaporative coolers), approximations or new methods were developed generally based on mass conservation arguments (Sections 5, 6.4, 6.5, and 7.3.3).

The mathematical model was validated by comparing the computational methods used in the ERMYN to the methods of five published biosphere and radiological assessment models (see Section 7). To validate the model, the methods and calculations used in each submodel were compared to the analogous methods and calculations in the validation models. In almost all cases, the methods used by those models produced the same or very similar results to those from the ERMYN. Therefore, it is concluded that the methods used in the ERMYN are well documented and accepted by the scientific community and that the results are consistent with output from other process-level models. Based on the comparisons conducted for model validation, it is concluded that the uncertainty associated with the mathematical methods used to calculate BDCFs is similar to that in other biosphere models. 
Table 6.6-1. Uncertainty Considerations for All Approximations Used in the Model

\begin{tabular}{|c|c|c|c|}
\hline $\begin{array}{l}\text { Approx. } \\
\text { No. }\end{array}$ & $\begin{array}{c}\text { Approximation } \\
\text { Description }\end{array}$ & Uncertainty Consideration & Conclusion \\
\hline 1 & $\begin{array}{l}\text { Radionuclide } \\
\text { concentrations in the } \\
\text { groundwater are } \\
\text { constant }\end{array}$ & $\begin{array}{l}\text { Radionuclide buildup in the soil would eventually } \\
\text { reach equilibrium conditions; however, the time to } \\
\text { reach these conditions would depend on the } \\
\text { individual radionuclide. See also Approximation } 5 .\end{array}$ & $\begin{array}{l}\text { Low uncertainty for } \\
\text { most radionuclides, } \\
\text { higher uncertainty } \\
\text { for radionuclides that } \\
\text { take a long time to } \\
\text { reach equilibrium. }\end{array}$ \\
\hline 2 & $\begin{array}{l}\text { Short-lived (less than } \\
180 \mathrm{~d} \text { ) decay products } \\
\text { are in equilibrium with } \\
\text { the long-lived primary } \\
\text { radionuclide }\end{array}$ & $\begin{array}{l}\text { For dose assessment of long-lived radionuclides, this } \\
\text { approximation has low uncertainty because even if } \\
\text { short-lived decay products are not in secular } \\
\text { equilibrium, they should be close. }\end{array}$ & Low uncertainty \\
\hline 3 & $\begin{array}{l}\text { Radionuclide } \\
\text { concentrations in the } \\
\text { air are calculated using } \\
\text { an annual average } \\
\text { irrigation rate }\end{array}$ & $\begin{array}{l}\text { It is more reasonable to assume crop rotation than to } \\
\text { assume single crops are grown forever on a single } \\
\text { farm field. By using a distribution for the long-term } \\
\text { irrigation input parameter, model uncertainty is } \\
\text { considered in input parameter uncertainty. }\end{array}$ & $\begin{array}{l}\text { Input parameters } \\
\text { include uncertainty }\end{array}$ \\
\hline 4 & $\begin{array}{l}\text { Harvest removal is } \\
\text { compensated by the } \\
\text { use of cow manure for } \\
\text { fertilizer }\end{array}$ & $\begin{array}{l}\text { These mechanisms are not modeled because of a } \\
\text { lack of input data. This approximation is considered } \\
\text { conservative. Uncertainty introduced by this } \\
\text { approximation is not evaluated quantitatively. }\end{array}$ & $\begin{array}{l}\text { Uncertainty not } \\
\text { evaluated } \\
\text { mathematically }\end{array}$ \\
\hline 5 & $\begin{array}{l}\text { Radionuclides in } \\
\text { surface soil are at } \\
\text { equilibrium } \\
\text { concentrations, i.e., } \\
\text { the rate of addition of } \\
\text { activity in surface soil } \\
\text { from irrigation is } \\
\text { balanced by removal } \\
\text { mechanisms }\end{array}$ & $\begin{array}{l}\text { For most radionuclides of interest, it takes less than a } \\
\text { few hundred years to reach equilibrium in irrigated } \\
\text { soil. For these radionuclides, uncertainty in } \\
\text { accumulation is low because the performance } \\
\text { assessment time is longer than the time required to } \\
\text { reach equilibrium. However, a few radionuclides take } \\
\text { thousands of years to reach equilibrium, and, } \\
\text { although uncertainty can be estimated for these } \\
\text { radionuclides, the ERMYN results do not include this } \\
\text { uncertainty. }\end{array}$ & $\begin{array}{l}\text { Low uncertainty for } \\
\text { most radionuclides, } \\
\text { higher uncertainty } \\
\text { for radionuclides that } \\
\text { take a long time to } \\
\text { reach equilibrium } \\
\text { throughout the root } \\
\text { depth. However, } \\
\text { uncertainty would be } \\
\text { reduced because } \\
\text { buildup in thin top } \\
\text { layer of soil (e.g., } \\
3 \text { mm for } \\
\text { resuspension) could } \\
\text { be more rapid. }\end{array}$ \\
\hline 6 & $\begin{array}{l}\text { Irrigation water initially } \\
\text { deposited on the crop } \\
\text { leaf surface is subject } \\
\text { to weathering } \\
\text { deposition }\end{array}$ & $\begin{array}{l}\text { Mathematical evaluation of weathering deposition is } \\
\text { not conducted because crops only retain a fraction of } \\
\text { the contaminants from irrigation water (Section } 7.4 .4 \text { ). } \\
\text { This approximation contributes little uncertainty to the } \\
\text { results. }\end{array}$ & Low uncertainty \\
\hline 7 & $\begin{array}{l}\text { Crop roots grow in the } \\
\text { upper layer of the } \\
\text { surface soil (tilling } \\
\text { depth), regardless of } \\
\text { the actual surface soil } \\
\text { depth }\end{array}$ & $\begin{array}{l}\text { When radionuclides reach steady state, the } \\
\text { radionuclide concentration is not dependent on } \\
\text { surface soil depth. This approximation contributes } \\
\text { little uncertainty for equilibrium conditions. }\end{array}$ & Low uncertainty \\
\hline 8 & $\begin{array}{l}\text { Cows are fed with } \\
\text { forage; chickens are } \\
\text { fed with grain }\end{array}$ & $\begin{array}{l}\text { Feeds are based on site-specific information. The } \\
\text { plant submodel includes variation in radionuclide } \\
\text { concentrations in feed, which partially accounts for } \\
\text { uncertainty in the types of animal feed. }\end{array}$ & $\begin{array}{l}\text { Input parameters } \\
\text { partially include } \\
\text { uncertainty }\end{array}$ \\
\hline
\end{tabular}


Table 6.6-1. Uncertainty Considerations for All Approximations Used in the Model (Continued)

\begin{tabular}{|c|c|c|c|}
\hline $\begin{array}{l}\text { Approx. } \\
\text { No. }\end{array}$ & $\begin{array}{l}\text { Approximation } \\
\text { Description }\end{array}$ & Uncertainty Consideration & Conclusion \\
\hline 9 & $\begin{array}{l}\text { Animal product types } \\
\text { (meat, milk, poultry } \\
\text { and eggs) are } \\
\text { representative }\end{array}$ & $\begin{array}{l}\text { Each animal product type in the ERMYN may include } \\
\text { more than one product. The most common animal } \\
\text { products are selected to represent the groups, and } \\
\text { variation is incorporated into input parameter } \\
\text { uncertainty. }\end{array}$ & $\begin{array}{l}\text { Input parameters } \\
\text { include uncertainty }\end{array}$ \\
\hline 10 & $\begin{array}{l}\text { Dose coefficients for } \\
\text { exposure to soil that is } \\
\text { contaminated to an } \\
\text { infinite depth }\end{array}$ & $\begin{array}{l}\text { Differences between dose coefficients for infinite and } \\
\text { limited soil depths are compared for two sets of dose } \\
\text { coefficients (Eckerman and Ryman } 1993 \\
\text { [DIRS 107684], Tables III-6 and III-7). The } \\
\text { differences are small ( } 10 \text { percent), and uncertainty } \\
\text { due to this approximation is low. }\end{array}$ & Low uncertainty \\
\hline 11 & $\begin{array}{l}\text { Radionuclide } \\
\text { concentrations in } \\
\text { indoor air are due only } \\
\text { to radionuclides } \\
\text { introduced from the } \\
\text { use of contaminated } \\
\text { water in evaporative } \\
\text { coolers }\end{array}$ & $\begin{array}{l}\text { Radionuclide accumulation in indoor air is unlikely } \\
\text { because a large volume of air would be circulated. } \\
\text { Radionuclide decay is possible, but unlikely because } \\
\text { the residence time of the air would be short, even if } \\
\text { the evaporative coolers are temporarily turned off. } \\
\text { Therefore, uncertainty due to this approximation is } \\
\text { low. }\end{array}$ & Low uncertainty \\
\hline 12 & $\begin{array}{l}\text { The mixing of volcanic } \\
\text { ash and surface soil } \\
\text { depends on land use }\end{array}$ & $\begin{array}{l}\text { This approximation allows realistic considerations of } \\
\text { volcanic ash mixing with surface soil. Thus, it } \\
\text { contributes little uncertainty to the results. }\end{array}$ & Low uncertainty \\
\hline 13 & $\begin{array}{l}\text { Resuspended ash } \\
\text { deposited on plants } \\
\text { comes from cultivated } \\
\text { lands, while ash for } \\
\text { human inhalation } \\
\text { comes from } \\
\text { uncultivated lands }\end{array}$ & $\begin{array}{l}\text { This approximation eliminates considering the mixing } \\
\text { of resuspended particles from different lands, as } \\
\text { there is no information to evaluate how the mixing } \\
\text { occurs. Because inhalation is the predominant } \\
\text { pathway, it is conservative to assume that ash is } \\
\text { undiluted or only slightly diluted. However, this } \\
\text { uncertainty could not be evaluated quantitatively. }\end{array}$ & $\begin{array}{l}\text { Uncertainty not } \\
\text { evaluated } \\
\text { mathematically }\end{array}$ \\
\hline 14 & $\begin{array}{l}\text { Time dependent mass } \\
\text { loading }\end{array}$ & $\begin{array}{l}\text { Mass loading used for calculation of inhalation dose } \\
\text { decreases with time after a volcanic eruption, but the } \\
\text { specific decrease rate depends on many factors. } \\
\text { Uncertainty due to this approximation is accounted } \\
\text { for using a distribution function to describe the } \\
\text { decrease rate. Mass loading used for calculation of } \\
\text { radionuclide deposition on crops from soil } \\
\text { resuspension is not time dependent. Because } \\
\text { inhalation is the predominant pathway, this } \\
\text { conservatism is not likely to affect the model result or } \\
\text { its uncertainty. }\end{array}$ & $\begin{array}{l}\text { Input parameter } \\
\text { includes uncertainty }\end{array}$ \\
\hline 15 & $\begin{array}{l}\text { Radon gas is released } \\
\text { from volcanic ash on } \\
\text { the ground surface }\end{array}$ & $\begin{array}{l}\text { It is conservative to assume that all }{ }^{222} \mathrm{Rn} \text { from }{ }^{226} \mathrm{Ra} \\
\text { is released into the air. However, uncertainty is not } \\
\text { evaluated quantitatively because of dynamic changes } \\
\text { in the volcanic ash thickness. }\end{array}$ & $\begin{array}{l}\text { Uncertainty not } \\
\text { evaluated } \\
\text { mathematically }\end{array}$ \\
\hline 16 & $\begin{array}{l}\text { External exposure } \\
\text { from volcanic ash on } \\
\text { the ground surface }\end{array}$ & $\begin{array}{l}\text { It is conservative to assume that radionuclides in } \\
\text { volcanic ash are concentrated on the surface of the } \\
\text { ground. However, uncertainty is not evaluated } \\
\text { quantitatively because of dynamic changes in the } \\
\text { volcanic ash thickness. }\end{array}$ & $\begin{array}{l}\text { Uncertainty not } \\
\text { evaluated } \\
\text { mathematically }\end{array}$ \\
\hline
\end{tabular}




\subsubsection{Input Parameter Uncertainty}

The mathematical models for the groundwater (Section 6.4) and volcanic ash scenarios (Section 6.5) require many input parameters. Typically, parameter uncertainty is represented by probability distribution functions. General considerations of uncertainty in the input parameters is discussed below, followed by a list of the input parameters and further discussions of the general parameter uncertainty.

Site-Specific Data-Site-specific and regional information was used to develop parameter distributions in the supporting data documents shown in Figure 1-1. Where necessary, gaps in that information were filled by data published in scientific literature for location with similar environmental conditions to those in the Amargosa Valley. The resulting parameter distributions, developed to describe the FEPs in the reference biosphere, were ensured to be consistent with the current conditions in the Yucca Mountain region. The consistency was achieved by defining parametric uncertainties to include the range of compatibility with conditions in regions comparable to that surrounding Yucca Mountain.

Parameter Distributions-All ERMYN input parameters can be represented by probability distributions, but fixed values are used for a few parameters. Discussion of parameter uncertainty represented by distribution functions is presented in Section 6.6.3.2.

Fixed Parameter Values-Justification is provided if a fixed value is used for a parameter. In general, fixed values are used for parameters that have little influence on the modeling results or are associated with limited uncertainty and variation. Therefore, parameters with fixed values have relatively minor contributions, and likely add little uncertainty to the final BDCF results. An exception are dose coefficients for intakes of radionuclides. There is a considerable uncertainty associated with their values; however, it is not customary to include uncertainty in dose coefficients in dose assessments conducted for the purpose of demonstrating compliance with regulatory requirements.

Parameter Correlation-For parameters that use distributions, the probability density functions are considered to vary independently unless information indicates that pairs of parameters are correlated. When parameters are correlated, correlation coefficients are developed, although information on parameter correlation is limited. In general, the effect of neglecting covariance is to imprecisely estimate variability in the results. For positively correlated parameters, neglecting correlation typically results in underestimating variation in the ERMYN results, and for negatively correlated parameters, neglecting correlation typically results in overestimating variation in the results.

\subsubsection{List of Input Parameters}

Parameter definitions are given in the sections of this report where parameters are introduced in the submodels. A complete list of input parameters, including their use in the groundwater and volcanic ash scenarios, is provided in Table 6.6-2. The table indicates whether the same or different values of a parameter should be used for present-day or future climate conditions and to which scenario the parameter applies. These parameters are grouped based on the submodels for the two scenarios. Because of simplifications in the table, the parameter names may not be 
exactly the same as those used when the parameter is first described. The parameter notations, shown in the third column, are exactly the same as those used in the appropriate mathematical equation identified in the column labeled "Eqn.”. The characterization of uncertainty is shown, and either a fixed value or a distribution is given. Further discussion of parameter uncertainty is provided in the following sections. The number of values used in each parameter, array, or matrix (e.g., five types of crops, climate change impacts for many agricultural related parameters, and scenario change impacts for some particle resuspension related parameters) is presented, as is a DIRS reference to the report where parameter values are determined and discussed. To be consistent with the requirements of 10 CFR 63.305(b), and (c) only those parameters that would be affected by a future change in climate have different values for future conditions.

Table 6.6-2. Summary of Parameters Used in the ERMYN for the Two Scenarios

\begin{tabular}{|c|c|c|c|c|c|c|c|c|}
\hline Submodel & Parameter Name & Symbol & Eqn. $^{a}$ & Dist. & $\begin{array}{l}\text { Array } \\
\text { No. }\end{array}$ & $\begin{array}{l}\text { Climate } \\
\text { Change }^{c}\end{array}$ & $\begin{array}{l}\text { Scenario } \\
\text { Change }^{\mathrm{d}}\end{array}$ & Ref. ${ }^{e}$ \\
\hline \multirow[t]{13}{*}{$\begin{array}{l}\text { Surface } \\
\text { Soil }\end{array}$} & $\begin{array}{l}\text { Radionuclide concentration } \\
\text { in groundwater }\end{array}$ & $C w_{i}$ & 6.4.1-1 & Fixed & 28 & Same & Water & $\begin{array}{l}\text { Unit } \\
\text { source }\end{array}$ \\
\hline & Annual irrigation rate & IR & 6.4.1-1 & Dist. & $1 \times 2$ & Different & Water & $\begin{array}{l}\text { [DIRS } \\
\text { 169673] }\end{array}$ \\
\hline & Radionuclide half-life & $T_{d, i}$ & 6.4.1-1 & Fixed & 31 & Same & Same & $\begin{array}{l}\text { [DIRS } \\
169671]\end{array}$ \\
\hline & Surface soil erosion rate & $E R$ & 6.4.1-11 & Dist. & 1 & Same & Water & $\begin{array}{l}\text { [DIRS } \\
169459]\end{array}$ \\
\hline & Soil bulk density & $\rho$ & 6.4.1-6 & Dist. & 1 & Same & Same & $\begin{array}{l}\text { [DIRS } \\
169459]\end{array}$ \\
\hline & Surface soil depth & $d$ & 6.4.1-6 & Dist. & 1 & Same & Same & $\begin{array}{l}\text { [DIRS } \\
\text { 169673] }\end{array}$ \\
\hline & $\begin{array}{l}\text { Soil solid-liquid partition } \\
\text { coefficient }\end{array}$ & $K d_{i}$ & 6.4.1-10 & Dist. & 17 & Same & Water & $\begin{array}{l}\text { [DIRS } \\
\text { 169459] }\end{array}$ \\
\hline & Overwatering rate & OW & 6.4.1-10 & Dist. & $1 \times 2$ & Different & Water & $\begin{array}{l}\text { [DIRS } \\
169673]\end{array}$ \\
\hline & Volumetric water content & $\theta$ & 6.4.1-10 & Dist. & 1 & Same & Water & $\begin{array}{l}\text { [DIRS } \\
169459]\end{array}$ \\
\hline & $\begin{array}{l}\text { Radionuclide concentration } \\
\text { in ash deposited on the } \\
\text { ground }\end{array}$ & $C s_{i}$ & 6.5.1-3 & Fixed & 23 & Same & Ash & $\begin{array}{l}\text { Unit } \\
\text { source }\end{array}$ \\
\hline & Ash bulk density & $\rho_{a}$ & $6.5 .1-3$ & Dist. & 1 & Same & Ash & $\begin{array}{l}\text { [DIRS } \\
169459]\end{array}$ \\
\hline & $\begin{array}{l}\text { Thickness of ash deposited } \\
\text { on the ground }\end{array}$ & $d_{a}$ & 6.5.1-3 & Dist. & 1 & Same & Ash & $\begin{array}{l}\text { TSPA-LA } \\
\text { model }\end{array}$ \\
\hline & $\begin{array}{l}\text { Critical thickness for } \\
\text { resuspension }\end{array}$ & $d_{c}$ & 6.5.1-3 & Dist & 1 & Same & Ash & $\begin{array}{l}\text { [DIRS } \\
\text { 169672] }\end{array}$ \\
\hline \multirow[t]{3}{*}{ Air } & Mass loading for crops & $s$ & $\begin{array}{l}6.4 .2-1 \\
6.5 .2-1 \\
\end{array}$ & Dist. & $1 \times 2$ & Same & Different & $\begin{array}{l}\text { [DIRS } \\
169458]\end{array}$ \\
\hline & $\begin{array}{l}\text { Mass loading for receptor } \\
\text { environments at nominal } \\
\text { condition }\end{array}$ & $S_{n}$ & $6.4 .2-2$ & Dist. & 5 & Same & Same & $\begin{array}{l}\text { [DIRS } \\
169458]\end{array}$ \\
\hline & $\begin{array}{l}\text { Mass loading for receptor } \\
\text { environments at post- } \\
\text { volcanic condition }\end{array}$ & $S_{v, n}$ & $6.5 .2-3$ & Dist. & 5 & Same & Ash & $\begin{array}{l}\text { [DIRS } \\
169458]\end{array}$ \\
\hline
\end{tabular}


Table 6.6-2. Summary of Parameters Used in the ERMYN for the Two Scenarios (Continued)

\begin{tabular}{|c|c|c|c|c|c|c|c|c|}
\hline Submodel & Parameter Name & Symbol & Eqn. $^{a}$ & Dist. & $\begin{array}{l}\text { Array } \\
\text { No. }\end{array}$ & $\begin{array}{l}\text { Climate } \\
\text { Change }^{c}\end{array}$ & $\begin{array}{l}\text { Scenario } \\
\text { Change }^{\mathrm{d}}\end{array}$ & Ref. ${ }^{e}$ \\
\hline \multirow[t]{9}{*}{$\begin{array}{l}\text { Air } \\
\text { (Continued) }\end{array}$} & $\begin{array}{l}\text { Resuspension } \\
\text { enhancement factor }\end{array}$ & $f_{\text {enhance, } n}$ & $\begin{array}{l}6.4 .2-2 \\
6.5 .2-2\end{array}$ & Dist. & $5 \times 2$ & Same & Different & $\begin{array}{l}\text { [DIRS } \\
169459]\end{array}$ \\
\hline & $\begin{array}{l}\text { Fraction of radionuclide } \\
\text { from water to air }\end{array}$ & $f_{\text {evap }}$ & $6.4 .2-3$ & Dist. & 1 & Same & Water & $\begin{array}{l}\text { [DIRS } \\
169672]\end{array}$ \\
\hline & Water evaporation rate & $M_{\text {water }}$ & $6.4 .2-3$ & Dist. & 1 & Same & Water & $\begin{array}{l}\text { [DIRS } \\
169672]\end{array}$ \\
\hline & $\begin{array}{l}\text { Evaporative cooler air flow } \\
\text { rate }\end{array}$ & $F_{\text {air }}$ & $6.4 .2-3$ & Dist. & 1 & Same & Water & $\begin{array}{l}\text { [DIRS } \\
169672]\end{array}$ \\
\hline & Radon release factor & $f_{m, R n-222}$ & $6.4 .2-4$ & Dist. & 1 & Same & Water & $\begin{array}{l}\text { [DIRS } \\
169672]\end{array}$ \\
\hline & Interior wall height & $H$ & $6.4 .2-5$ & Dist. & 1 & Same & Water & $\begin{array}{l}\text { [DIRS } \\
\text { 169672] }\end{array}$ \\
\hline & $\begin{array}{l}\text { House ventilation rate (for } \\
\text { normal or evaporative } \\
\text { condition) }\end{array}$ & $v$ & $6.4 .2-5$ & Dist & $1 \times 2$ & Same & Water & $\begin{array}{l}\text { [DIRS } \\
\text { 169672] }\end{array}$ \\
\hline & $\begin{array}{l}\text { Fraction of radon from soil } \\
\text { entering into the house }\end{array}$ & $f_{\text {house }}$ & $6.4 .2-6$ & Dist. & 1 & Same & Water & $\begin{array}{l}\text { [DIRS } \\
169672]\end{array}$ \\
\hline & $\begin{array}{l}\text { Ratio of }{ }^{222} \mathrm{Rn} \\
\text { concentration in air to flux } \\
\text { density from soil }\end{array}$ & $C F_{R n-222}$ & $6.4 .2-7$ & Dist. & 1 & Same & Same & $\begin{array}{l}\text { [DIRS } \\
\text { 169672] }\end{array}$ \\
\hline \multirow[t]{12}{*}{ Plant } & Soil-to-plant transfer factor & $F_{s \rightarrow p, i, j}$ & $6.4 .3-2$ & Dist. & $16 \times 5$ & Same & Same & $\begin{array}{l}\text { [DIRS } \\
\text { 169672] }\end{array}$ \\
\hline & Dry-to-wet weight ratio & $D W_{j}$ & 6.4.3-2 & Dist. & 5 & Same & Same & $\begin{array}{l}\text { [DIRS } \\
\text { 169673] }\end{array}$ \\
\hline & Translocation factor & $T_{j}$ & 6.4.3-3 & $\begin{array}{l}\text { Dist./ } \\
\text { Fixed }\end{array}$ & 5 & Same & Same & $\begin{array}{l}\text { [DIRS } \\
169672]\end{array}$ \\
\hline & $\begin{array}{l}\text { Fraction of overhead } \\
\text { irrigation }\end{array}$ & $f_{o, j}$ & 6.4.3-3 & Dist. & 5 & Same & Water & $\begin{array}{l}\text { [DIRS } \\
169673]\end{array}$ \\
\hline & Weathering half-life & $T_{w}$ & 6.4.3-3 & Dist. & 1 & Same & Same & $\begin{array}{l}\text { [DIRS } \\
169672]\end{array}$ \\
\hline & Crop growing time & $t_{g, j}$ & 6.4.3-3 & Fixed & $5 \times 2$ & Different & Same & $\begin{array}{l}\text { [DIRS } \\
169673]\end{array}$ \\
\hline & Crop wet yield & $Y_{j}$ & 6.4.3-3 & Dist. & 5 & Same & Same & $\begin{array}{l}\text { [DIRS } \\
\text { 169673] }\end{array}$ \\
\hline & Daily irrigation rate & $I R D_{j}$ & $6.4 .3-4$ & Dist. & $5 \times 2$ & Different & Water & $\begin{array}{l}\text { [DIRS } \\
\text { 169673] }\end{array}$ \\
\hline & Crop dry biomass & $D B_{j}$ & $6.4 .3-5$ & Dist. & 5 & Same & Same & $\begin{array}{l}\text { [DIRS } \\
169673]\end{array}$ \\
\hline & $\begin{array}{l}\text { Irrigation amount per } \\
\text { application }\end{array}$ & $I A_{j}$ & $6.4 .3-5$ & Dist. & $5 \times 2$ & Different & Water & $\begin{array}{l}\text { [DIRS } \\
\text { 169673] }\end{array}$ \\
\hline & Irrigation intensity & I & 6.4.3-5 & Dist. & 1 & Same & Water & $\begin{array}{l}\text { [DIRS } \\
169673]\end{array}$ \\
\hline & Dry deposition velocity & $V_{d}$ & 6.4.3-7 & Dist. & 1 & Same & Same & $\begin{array}{l}\text { [DIRS } \\
169672]\end{array}$ \\
\hline
\end{tabular}


Table 6.6-2. Summary of Parameters Used in the ERMYN for the Two Scenarios (Continued)

\begin{tabular}{|c|c|c|c|c|c|c|c|c|}
\hline Submodel & Parameter Name & Symbol & Eqn. $^{a}$ & Dist. & $\begin{array}{l}\text { Array } \\
\text { No. }\end{array}$ & $\begin{array}{c}\text { Climate } \\
\text { Change }^{c}\end{array}$ & $\begin{array}{l}\text { Scenario } \\
\text { Change }\end{array}$ & Ref. $^{\mathrm{e}}$ \\
\hline \multirow[t]{4}{*}{ Animal } & $\begin{array}{l}\text { Animal product transfer } \\
\text { coefficient }\end{array}$ & $F m_{i, k}$ & $6.4 .4-2$ & Dist. & $16 \times 4$ & Same & Same & $\begin{array}{l}\text { [DIRS } \\
\text { 169672] }\end{array}$ \\
\hline & $\begin{array}{l}\text { Animal consumption rate of } \\
\text { feed }\end{array}$ & $Q f_{k}$ & $6.4 .4-2$ & Dist. & 4 & Same & Same & $\begin{array}{l}\text { [DIRS } \\
\text { 169672] }\end{array}$ \\
\hline & $\begin{array}{l}\text { Animal consumption rate of } \\
\text { water }\end{array}$ & $Q w_{k}$ & 6.4.4-3 & $\begin{array}{l}\text { Dist./ } \\
\text { Fixed }\end{array}$ & 4 & Same & Water & $\begin{array}{l}\text { [DIRS } \\
\text { 169672] }\end{array}$ \\
\hline & $\begin{array}{l}\text { Animal consumption rate of } \\
\text { soil }\end{array}$ & $Q s_{k}$ & $6.4 .4-4$ & Dist. & 4 & Same & Same & $\begin{array}{l}\text { [DIRS } \\
169672]\end{array}$ \\
\hline \multirow[t]{2}{*}{ Fish } & Bioaccumulation factor & $B F_{i}$ & $6.4 .5-1$ & Dist. & 17 & Same & Water & $\begin{array}{l}\text { [DIRS } \\
\text { 169672] }\end{array}$ \\
\hline & $\begin{array}{l}\text { Water concentration } \\
\text { modifying factor }\end{array}$ & $M F_{i}$ & $6.4 .5-2$ & Dist. & $17 \times 2$ & Different & Water & $\begin{array}{l}\text { [DIRS } \\
\text { 169672] }\end{array}$ \\
\hline \multirow[t]{11}{*}{${ }^{14} \mathrm{C}$} & ${ }^{14} \mathrm{C}$ emission rate & $\lambda_{a, C-14}$ & $6.4 .6-1$ & Fixed & 1 & Same & Water & $\begin{array}{l}\text { [DIRS } \\
\text { 169672] }\end{array}$ \\
\hline & $\begin{array}{l}\text { Annual total groundwater } \\
\text { usage }\end{array}$ & $w$ & $6.4 .6-3$ & Fixed & 1 & Same & Water & $\begin{array}{l}\text { [DIRS } \\
\text { 169672] }\end{array}$ \\
\hline & $\begin{array}{l}\text { Annual average wind } \\
\text { speed (for crops or } \\
\text { inhalation) }\end{array}$ & $U$ & $6.4 .6-3$ & Dist. & $1 \times 2$ & Same & Water & $\begin{array}{l}\text { [DIRS } \\
\text { 169672] }\end{array}$ \\
\hline & $\begin{array}{l}{ }^{14} \mathrm{C} \text { mixing height (for crops } \\
\text { or inhalation) }\end{array}$ & $H_{\text {mix }}$ & $6.4 .6-3$ & Fixed & $1 \times 2$ & Same & Water & $\begin{array}{l}\text { [DIRS } \\
\text { 169672] }\end{array}$ \\
\hline & $\begin{array}{l}\text { Fraction of soil-derived } \\
\text { carbon in plants }\end{array}$ & Fs & $6.4 .6-4$ & Fixed & 1 & Same & Water & $\begin{array}{l}\text { [DIRS } \\
\text { 169672] }\end{array}$ \\
\hline & $\begin{array}{l}\text { Fraction of stable carbon in } \\
\text { plant }\end{array}$ & $F C_{\text {plant }, j}$ & $6.4 .6-4$ & Fixed & 5 & Same & Water & $\begin{array}{l}\text { [DIRS } \\
169672]\end{array}$ \\
\hline & $\begin{array}{l}\text { Fraction of air-derived } \\
\text { carbon in plants }\end{array}$ & $\mathrm{Fa}$ & $6.4 .6-5$ & Fixed & 1 & Same & Water & $\begin{array}{l}\text { [DIRS } \\
\text { 169672] }\end{array}$ \\
\hline & $\begin{array}{l}\text { Fraction of stable carbon in } \\
\text { soil }\end{array}$ & $f C_{\text {soil }}$ & $6.4 .6-4$ & Fixed & 1 & Same & Water & $\begin{array}{l}\text { [DIRS } \\
\text { 169672] }\end{array}$ \\
\hline & $\begin{array}{l}\text { Concentration of stable } \\
\text { carbon in air }\end{array}$ & $f C_{\text {air }}$ & $6.4 .6-5$ & Fixed & 1 & Same & Water & $\begin{array}{l}\text { [DIRS } \\
\text { 169672] }\end{array}$ \\
\hline & $\begin{array}{l}\text { Concentration of stable } \\
\text { carbon in water }\end{array}$ & $F C_{\text {water }}$ & $6.4 .6-7$ & Fixed & 1 & Same & Water & $\begin{array}{l}\text { [DIRS } \\
169672]\end{array}$ \\
\hline & $\begin{array}{l}\text { Fraction of stable carbon in } \\
\text { animal product }\end{array}$ & $F C_{\text {anim, } k}$ & 6.4.6-7 & Fixed & 4 & Same & Water & $\begin{array}{l}\text { [DIRS } \\
\text { 169672] }\end{array}$ \\
\hline \multirow[t]{5}{*}{$\begin{array}{l}\text { External } \\
\text { Exposure }\end{array}$} & Population proportion & $P P_{m}$ & $6.4 .7-1$ & Dist. & 4 & Same & Different & $\begin{array}{l}\text { [DIRS } \\
169671]\end{array}$ \\
\hline & $\begin{array}{l}\text { Exposure time by } \\
\text { population group and } \\
\text { environment }\end{array}$ & $t_{n, m}$ & $6.4 .7-1$ & Dist. & $5 \times 4$ & Same & Different & $\begin{array}{l}\text { [DIRS } \\
\text { 169671] }\end{array}$ \\
\hline & Building shielding factor & $f_{\text {ext } i, n}$ & $6.4 .7-1$ & Fixed & 31 & Same & Same & $\begin{array}{l}\text { [DIRS } \\
\text { 169671] }\end{array}$ \\
\hline & Branching fraction & $B N_{s}$ & $6.4 .7-2$ & Fixed & 75 & Same & Same & $\begin{array}{l}\text { [DIRS } \\
\text { 169671] }\end{array}$ \\
\hline & $\begin{array}{l}\text { Dose coefficient for } \\
\text { exposure contaminated to } \\
\text { an infinite depth }\end{array}$ & $D C i_{\text {soil, }, i}$ & $6.4 .7-2$ & Fixed & 75 & Same & Water & $\begin{array}{l}\text { [DIRS } \\
\text { 169671] }\end{array}$ \\
\hline
\end{tabular}


Table 6.6-2. Summary of Parameters Used in the ERMYN for the Two Scenarios (Continued)

\begin{tabular}{|c|c|c|c|c|c|c|c|c|}
\hline Submodel & Parameter Name & Symbol & Eqn. $^{a}$ & Dist. & $\begin{array}{l}\text { Array } \\
\text { No. }\end{array}$ & $\begin{array}{l}\text { Climate } \\
\text { Change }^{c}\end{array}$ & $\begin{array}{l}\text { Scenario } \\
\text { Change }^{d}\end{array}$ & Ref. $^{e}$ \\
\hline $\begin{array}{l}\text { External } \\
\text { Exposure } \\
\text { (Continued) }\end{array}$ & $\begin{array}{l}\text { Dose coefficient for } \\
\text { exposure to contaminated } \\
\text { ground surface }\end{array}$ & $D C s_{\text {soil, } i}$ & $6.5 .5-2$ & Fixed & 75 & Same & Ash & $\begin{array}{l}\text { [DIRS } \\
169671]\end{array}$ \\
\hline \multirow[t]{6}{*}{ Inhalation } & Breathing rate & $B R_{n}$ & 6.4.8-2 & Fixed & 5 & Same & Same & $\begin{array}{l}\text { [DIRS } \\
169671]\end{array}$ \\
\hline & $\begin{array}{l}\text { Fraction of houses with } \\
\text { evaporative coolers }\end{array}$ & $f_{\text {cooler }}$ & $6.4 .8-3$ & Dist. & 1 & Same & Water & $\begin{array}{l}\text { [DIRS } \\
169671]\end{array}$ \\
\hline & $\begin{array}{l}\text { Evaporative cooler use } \\
\text { factor }\end{array}$ & $f_{\text {use }}$ & 6.4.8-3 & Dist. & 1 & Different & Water & $\begin{array}{l}\text { [DIRS } \\
169671]\end{array}$ \\
\hline & $\begin{array}{l}\text { Equilibrium factor for }{ }^{222} \mathrm{Rn} \\
\text { decay products }\end{array}$ & $E F_{R n-222, n}$ & 6.4.8-6 & Dist. & 5 & Same & Same & $\begin{array}{l}\text { [DIRS } \\
169672]\end{array}$ \\
\hline & $\begin{array}{l}\text { Dose conversion factor for } \\
\text { inhalation of }{ }^{222} \mathrm{Rn} \text { decay } \\
\text { products at } 100 \% \\
\text { equilibrium }\end{array}$ & $\begin{array}{l}\text { DCF inh, } \\
\text { Rn-222 }\end{array}$ & 6.4.8-6 & Fixed & 1 & Same & Same & $\begin{array}{l}\text { [DIRS } \\
169671]\end{array}$ \\
\hline & $\begin{array}{l}\text { Dose conversion factor for } \\
\text { inhalation }\end{array}$ & $D C F_{i n h, s}$ & 6.4.8-8 & Fixed & 75 & Same & Same & $\begin{array}{l}\text { [DIRS } \\
169671]\end{array}$ \\
\hline \multirow[t]{6}{*}{ Ingestion } & Consumption rate of water & Uw & 6.4.9-2 & Fixed & 1 & Same & Water & $\begin{array}{l}\text { [DIRS } \\
169671]\end{array}$ \\
\hline & $\begin{array}{l}\text { Consumption rate of locally } \\
\text { produced crop foodstuffs }\end{array}$ & $U p_{j}$ & 6.4.9-3 & Dist. & 4 & Same & Same & $\begin{array}{l}\text { [DIRS } \\
\text { 169671] }\end{array}$ \\
\hline & $\begin{array}{l}\text { Consumption rate of locally } \\
\text { produced animal products }\end{array}$ & $U d_{k}$ & 6.4.9-4 & Dist. & 4 & Same & Same & $\begin{array}{l}\text { [DIRS } \\
\text { 169671] }\end{array}$ \\
\hline & $\begin{array}{l}\text { Consumption rate of locally } \\
\text { produced fish }\end{array}$ & Uf & 6.4.9-5 & Dist. & 1 & Same & Water & $\begin{array}{l}\text { [DIRS } \\
169671]\end{array}$ \\
\hline & $\begin{array}{l}\text { Inadvertent soil ingestion } \\
\text { rate }\end{array}$ & Us & 6.4.9-6 & Dist. & 1 & Same & Same & $\begin{array}{l}\text { [DIRS } \\
169671]\end{array}$ \\
\hline & $\begin{array}{l}\text { Dose conversion factor for } \\
\text { ingestion }\end{array}$ & $D C F_{i n g, s}$ & 6.4.9-7 & Fixed & 75 & Same & Same & $\begin{array}{l}\text { [DIRS } \\
169671]\end{array}$ \\
\hline
\end{tabular}

${ }^{a}$ Equation number where the parameter is introduced. The equation for the groundwater scenario is listed unless the parameter is only for the volcanic ash scenario.

$\mathrm{b}$ The number of values for the parameter, which depends on the radionuclides and elements $(i=31$ primary radionuclides included in the ERMYN for the groundwater scenario (fewer for the volcanic scenario); these radionuclides result from total of 75 radionuclides including short-lived radionuclides, 17 elements corresponding to primary radionuclides, 16 elements for transfer factors and coefficients because carbon is a special element), crop types ( $j=5$ including forage; 4 used for plant ingestion pathways), animal products $(k=4)$, population groups $(m=4)$, environments $(n=5)$, climate conditions $(n=2)$, and exposure scenarios $(n=2)$.

c Indicates if the parameter values change due to climate conditions, "Same" means the same value is used for all climate states, "Different" means different values are used in different climate states.

d Indicates where the parameter is used. "Same" means it is used in both scenarios with the same value, "Different" means it is used in both scenarios with different values, "Water" means it is used only in the groundwater scenario, and "Ash" means it is used only in the volcanic ash scenario.

e Reference number for the reports where the parameter values are developed: BSC (2004 [DIRS 169673]), BSC (2004 [DIRS 169459]), BSC (2004 [DIRS 169458]), BSC (2004 [DIRS 169671]), and BSC (2004 [DIRS 169672]). 


\subsubsection{Consideration of Parameter Uncertainty}

As discussed in the previous section, sources of data for parameter development vary with the input parameter types and the available information. Section 63.114(b) requires that the performance assessment must "account for uncertainties and variabilities in parameter values and provide the technical basis for parameter ranges, probability distributions, or bounding values used in the performance assessment." This section briefly describes how uncertainties and variabilities in parameter values are accounted for in the parameter distributions and provides the technical bases for the ranges, distributions, or bounding parameter values.

Two types of probability distribution functions are considered. The first type is a population distribution, which describes the probability of any reasonable event occurring within the population. If the distribution is statistically normal, it is defined using the mean and standard deviation of a sample from the population. The second type of probability distribution is a distribution of the population mean, which describes the probability of occurrence of any reasonable mean value for the population. For statistically normal distributions, this second type of distribution is defined using the mean and standard error of the mean. Other distributions are considered (e.g., lognormal, uniform, and triangular) if the data are not statistically normal. Because some distributions range from negative infinity to positive infinity, the distributions are truncated to eliminate physically impossible input values and to make the distribution meaningful.

When a parameter is developed based on the mean characteristics of a group, the distribution of the parameter represents variation and uncertainty in the mean value. For normal distributions, such parameters are characterized using the mean as the reasonable estimate and the standard error as a measure of variance or parameter range (the second type described above). Based on the requirements in 10 CFR 63.305 [DIRS 156605], parameters in this category include the diet and lifestyle of the RMEI (e.g., consumption rates and time spent in various environments), which primarily are used in the external exposure, inhalation, and ingestion submodels.

When parameters describe processes or properties of a group, even if the processes or properties are constant over time or space, the distribution is selected to represent the entire range of variation among individuals within the group. For normal distributions, the distributions are characterized using the mean as a reasonable estimate and the standard deviation as a measure of variance (the first type described above). For many parameters, lognormal distributions better describe the variation, and a geometric mean is used for a reasonable estimate and the geometric standard deviation is used for the variance. Parameters in this category include many environmental transport and agricultural parameters (e.g., transfer factors, irrigation rates, biomass, and dry-wet ratio) that are used in the plant, animal, and fish submodels.

If evidence indicates that a parameter contributes little to overall dose, or if it has little impact on model uncertainty, then a fixed value may be used for the input parameter. Parameters in this category include the transfer of ${ }^{14} \mathrm{C}$ among soil, air, crop, and animal product components, which are evaluated in the ${ }^{14} \mathrm{C}$ special submodel.

Cumulative distributions, or piece-wise linear distributions, are used for some model input parameters. A cumulative distribution is defined as a set of input values and corresponding 
cumulative probabilities developed based on empirical data points. The probability density function for this type of distribution may contain distinct, irregular peaks.

This section provides a brief summary of parameter uncertainty consideration because every parameter is developed separately based on available information. Details concerning development of the input parameters are presented in the five reports (BSC 2004 [DIRS 169673]; BSC 2004 [DIRS 169459]; BSC 2004 [DIRS 169458]; BSC 2004 [DIRS 169671]; BSC 2004 [DIRS 169672]), as discussed in Section 1 and Figure 1-1.

\subsubsection{Summary of Parameter Uncertainty}

A summary of representative parameter values and probability distribution functions are presented in Table 6.6-3. The table includes parameter values and uncertainty information taken from the five parameter reports (Figure 1-1), including the distribution type, the mean or mode, standard deviation or standard error, range (minimum and maximum), and brief notes describing uncertainty considerations. The parameter values in Table 6.6-3 are shown in accordance with the ERMYN parameters shown in Table 6.6-2. To show parameter values and distributions, parameters with subscript indices in the mathematical model are shown for each individual value in Table 6.6-3. The purpose of the table is to summarize possible model input values that are developed under the current TWP (BSC 2004 [DIRS 169573]). Other parameter distributions may be used with this model if they are within the limits of the model described in Section 8.2.

The parameter values listed in Table 6.6-3 use the same units as the data reports (Figure 1-1). The units may not be the same as those in the equations shown in Sections 6.4 and 6.5. This is not a discrepancy between the model and inputs, as the GoldSim software automatically converts to the proper units.

\subsubsection{Uncertainty of Model Results}

When models that use parameters with distribution functions are realized, parameter values are sampled from the range of possible values. For each realization, the values are fixed for the run to produce one deterministic calculation for the entire model. However, the realized parameter values change for each realization, and, therefore, the model results differ for each realization. After many realizations, the results yield a distribution of possible outcomes (i.e., a distribution function), which reflect the possibility of the result in the uncertainty. This simulation method, built into the GoldSim software, includes two sampling methods: Monte Carlo or Latin Hypercube. A detailed description of the software is presented below.

Uncertainty in the ERMYN results is considered for all of the input parameters that are represented by uncertainty distributions. Uncertainty from conservative approximations and simplifications cannot be evaluated quantitatively. 


\begin{tabular}{|c|c|c|c|c|c|c|c|c|c|}
\hline $\begin{array}{l}\text { Sub- } \\
\text { model }\end{array}$ & \multicolumn{2}{|c|}{ Parameter Name } & $\begin{array}{c}\text { Distribution } \\
\text { Type }\end{array}$ & Units & $\begin{array}{l}\text { Mean, } \\
\text { Mode, } \\
\text { Average }^{a}\end{array}$ & SDISE $^{a}$ & Min. ${ }^{b}$ & Max. ${ }^{b}$ & Notes $^{c}$ \\
\hline \multirow[t]{23}{*}{$\begin{array}{l}\text { Surface } \\
\text { Soil }\end{array}$} & \multicolumn{2}{|c|}{$\begin{array}{l}\text { Radionuclide concentration in } \\
\text { groundwater }\end{array}$} & Fixed & $\mathrm{Bq} / \mathrm{m}^{3}$ & 1 & - & - & - & $\begin{array}{l}\text { Unit activity concentration }-\mathrm{a} \\
\text { source term for the } \\
\text { groundwater scenario. }\end{array}$ \\
\hline & \multirow[t]{4}{*}{$\begin{array}{l}\text { Annual average } \\
\text { irrigation rate }\end{array}$} & $\begin{array}{l}\text { Present-day } \\
\text { climate/ Lower } \\
\text { bound monsoon } \\
\text { climate }\end{array}$ & \multirow[t]{4}{*}{ Normal } & \multirow[t]{4}{*}{$\mathrm{m} / \mathrm{yr}$} & 0.94 & 0.08 & 0.73 & 1.15 & \multirow{4}{*}{$\begin{array}{l}\text { (BSC } 2003 \text { [DIRS 160976]) } \\
\text { Normal distribution used for } \\
\text { long-term annual irrigation. } \\
\text { The term "present-day climate" } \\
\text { is used for modern interglacial } \\
\text { climate (Section 6.1.1.2). The } \\
\text { term "future climate" is used for } \\
\text { the upper bound of the glacial } \\
\text { transition climate } \\
\text { (Section 6.1.1.2). }\end{array}$} \\
\hline & & $\begin{array}{l}\text { Upper bound } \\
\text { monsoon climate }\end{array}$ & & & 0.52 & - & - & - & \\
\hline & & $\begin{array}{l}\text { Lower bound } \\
\text { future climate }\end{array}$ & & & 0.88 & - & - & - & \\
\hline & & $\begin{array}{l}\text { Upper bound } \\
\text { future climate }\end{array}$ & & & 0.50 & 0.04 & 0.40 & 0.60 & \\
\hline & \multicolumn{2}{|c|}{ Radionuclide half-life } & Fixed & $\mathrm{yr}$ & See Notes & - & - & - & $\begin{array}{l}\text { Values presented in Table 6.3- } \\
7 \text { are from BSC } 2003 \\
\text { [DIRS 161241] }\end{array}$ \\
\hline & \multicolumn{2}{|c|}{ Surface soil erosion rate } & Triangular & $\mathrm{kg} /\left(\mathrm{m}^{2} \mathrm{yr}\right)$ & $\begin{array}{l}0.49 \text { (mean) } \\
0.19 \text { (mode) }\end{array}$ & - & 0.19 & 1.1 & $\begin{array}{l}\text { Mean given in the source report } \\
\text { (BSC } 2003 \text { [DIRS 161239]) for } \\
\text { deterministic calculation. }\end{array}$ \\
\hline & \multicolumn{2}{|l|}{ Soil bulk density } & Triangular & $\mathrm{kg} / \mathrm{m}^{3}$ & $\begin{array}{l}1500 \text { (mean } \\
\text { and mode) }\end{array}$ & - & 1300 & 1700 & (BSC 2003 [DIRS 161239]) \\
\hline & \multicolumn{2}{|c|}{ Surface soil depth } & Uniform & $\mathrm{m}$ & 0.25 & - & 0.05 & 0.30 & $\begin{array}{l}\text { Tilling depth. Mean given in } \\
\text { the data source report. } \\
\text { (BSC } 2003 \text { [DIRS 160976]) }\end{array}$ \\
\hline & \multirow{14}{*}{$\begin{array}{l}\text { Soil solid-liquid } \\
\text { partition } \\
\text { coefficient }\end{array}$} & Carbon & \multirow[t]{14}{*}{ Lognormal } & \multirow[t]{14}{*}{$\mathrm{L} / \mathrm{kg}$} & $1.8 \mathrm{E}+01$ & $6.0 \mathrm{E}+00$ & - & - & \multirow{14}{*}{$\begin{array}{l}\text { Partition coefficients are } \\
\text { correlated with the soil-to-plant } \\
\text { transfer factors. The } \\
\text { correlation coefficient is }-0.8 \text {. } \\
\text { (BSC } 2003 \text { [DIRS 161239]) }\end{array}$} \\
\hline & & Chlorine & & & $1.4 \mathrm{E}-01$ & $6.0 \mathrm{E}+00$ & - & - & \\
\hline & & Selenium & & & $1.5 \mathrm{E}+02$ & $6.0 \mathrm{E}+00$ & - & - & \\
\hline & & Strontium & & & $2.0 \mathrm{E}+01$ & $5.5 E+00$ & - & - & \\
\hline & & Technetium & & & 1.4E-01 & $6.0 \mathrm{E}+00$ & - & - & \\
\hline & & Tin & & & $4.5 E+02$ & $6.0 \mathrm{E}+00$ & - & - & \\
\hline & & Iodine & & & $4.5 \mathrm{E}+00$ & $7.4 \mathrm{E}+00$ & - & - & \\
\hline & & Cesium & & & $4.4 \mathrm{E}+03$ & $3.7 \mathrm{E}+00$ & - & - & \\
\hline & & Lead & & & $1.6 \mathrm{E}+04$ & $4.1 \mathrm{E}+00$ & - & - & \\
\hline & & Radium & & & $3.6 \mathrm{E}+04$ & $2.2 \mathrm{E}+01$ & - & - & \\
\hline & & Actinium & & & $1.5 \mathrm{E}+03$ & $6.0 \mathrm{E}+00$ & - & - & \\
\hline & & Thorium & & & $3.0 \mathrm{E}+03$ & $8.2 \mathrm{E}+00$ & - & - & \\
\hline & & Protactinium & & & $1.8 \mathrm{E}+03$ & $6.0 \mathrm{E}+00$ & - & - & \\
\hline & & Uranium & & & $3.3 E+01$ & $2.5 \mathrm{E}+01$ & - & - & \\
\hline
\end{tabular}


Table 6.6-3. Summary of Representative Input Parameter Values and Their Distributions (Continued)

\begin{tabular}{|c|c|c|c|c|c|c|c|c|c|}
\hline $\begin{array}{l}\text { Sub- } \\
\text { model }\end{array}$ & \multicolumn{2}{|c|}{ Parameter Name } & $\begin{array}{c}\text { Distribution } \\
\text { Type }\end{array}$ & Units & $\begin{array}{c}\text { Mean, } \\
\text { Mode, } \\
\text { Average }^{\mathrm{a}} \\
\end{array}$ & SDISE $^{a}$ & Min. ${ }^{b}$ & Max. ${ }^{b}$ & Notes $^{c}$ \\
\hline \multirow{10}{*}{$\begin{array}{l}\text { Surface } \\
\text { Soil } \\
\text { (Continued) }\end{array}$} & & Neptunium & & & $2.5 \mathrm{E}+01$ & $3.3 \mathrm{E}+00$ & - & - & \\
\hline & & Plutonium & & & $1.2 \mathrm{E}+03$ & $3.3 \mathrm{E}+00$ & - & - & \\
\hline & & Americium & & & $2.0 \mathrm{E}+03$ & 1.3E+01 & - & - & \\
\hline & \multirow[t]{2}{*}{$\begin{array}{l}\text { Overwatering } \\
\text { rate }\end{array}$} & $\begin{array}{l}\text { Present-day } \\
\text { climate }\end{array}$ & Cumulative & $\mathrm{m} / \mathrm{yr}$ & 0.079 & - & $\begin{array}{l}0.009 \\
0.030 \\
0.045 \\
0.076 \\
0.128 \\
0.233 \\
0.275 \\
\end{array}$ & $\begin{array}{r}0 \% \\
19 \% \\
38 \% \\
57 \% \\
76 \% \\
95 \% \\
100 \% \\
\end{array}$ & \multirow[t]{2}{*}{$\begin{array}{l}\text { Irrigation water passing through } \\
\text { crop root zone. } \\
\text { (BSC 2003 [DIRS 160976]) }\end{array}$} \\
\hline & & Future climate & Cumulative & $\mathrm{m} / \mathrm{yr}$ & 0.067 & - & $\begin{array}{l}0.004 \\
0.02 \\
0.047 \\
0.072 \\
0.104 \\
0.15 \\
0.177 \\
\end{array}$ & $\begin{array}{r}0 \% \\
19 \% \\
38 \% \\
57 \% \\
76 \% \\
95 \% \\
100 \% \\
\end{array}$ & \\
\hline & \multicolumn{2}{|c|}{ Volumetric water content } & Uniform & - & 0.23 & - & 0.18 & 0.28 & (BSC 2003 [DIRS 161239]) \\
\hline & \multicolumn{2}{|c|}{$\begin{array}{l}\text { Radionuclide concentration in ash } \\
\text { deposited on ground surface }\end{array}$} & Fixed & $\mathrm{Bq} / \mathrm{m}^{2}$ & 1 & - & - & - & $\begin{array}{l}\text { Unit areal activity concentration } \\
- \text { a source term for the } \\
\text { volcanic ash scenario. }\end{array}$ \\
\hline & \multicolumn{2}{|c|}{ Ash bulk density } & Fixed & $\mathrm{kg} / \mathrm{m}^{3}$ & 1000 & & & & (BSC 2003 [DIRS 161239]) \\
\hline & \multicolumn{2}{|c|}{$\begin{array}{l}\text { Thickness of ash deposited on the } \\
\text { ground }\end{array}$} & - & - & - & - & - & - & $\begin{array}{l}\text { Ash thickness is calculated in } \\
\text { the TSPA-LA model. }\end{array}$ \\
\hline & \multicolumn{2}{|c|}{$\begin{array}{l}\text { Critical thickness for the } \\
\text { resuspension }\end{array}$} & Uniform & $\mathrm{mm}$ & 2 & - & 1 & 3 & $\begin{array}{l}\text { Mean calculated from uniform } \\
\text { distribution. BSC } 2003 \\
\text { [DIRS 160964] }\end{array}$ \\
\hline \multirow[t]{10}{*}{ Air } & \multirow{2}{*}{$\begin{array}{l}\text { Mass loading for } \\
\text { crops }\end{array}$} & Nominal & \multirow[t]{2}{*}{ Triangular } & \multirow[t]{2}{*}{$\mathrm{mg} / \mathrm{m}^{3}$} & 0.12 & - & 0.025 & 0.200 & \multirow[t]{2}{*}{ (BSC 2003 [DIRS 160965]) } \\
\hline & & Post-volcanic & & & 0.24 & - & 0.050 & 0.600 & \\
\hline & \multirow{4}{*}{$\begin{array}{l}\text { Mass loading for } \\
\text { receptor } \\
\text { environments at } \\
\text { nominal } \\
\text { condition }\end{array}$} & Active outdoors & \multirow[t]{4}{*}{ Triangular } & \multirow[t]{4}{*}{$\mathrm{mg} / \mathrm{m}^{3}$} & 5.00 & - & 1.000 & 10.000 & \multirow{4}{*}{$\begin{array}{l}\text { Used for the groundwater } \\
\text { scenario and for the long-term } \\
\text { inhalation component for the } \\
\text { volcanic ash scenario. (BSC } \\
2003 \text { [DIRS 160965]) }\end{array}$} \\
\hline & & \begin{tabular}{|l|} 
Inactive \\
outdoors
\end{tabular} & & & 0.06 & - & 0.025 & 0.100 & \\
\hline & & Active indoors & & & 0.10 & - & 0.060 & 0.175 & \\
\hline & & Asleep indoors & & & 0.03 & - & 0.010 & 0.050 & \\
\hline & \multirow{4}{*}{$\begin{array}{l}\text { Mass loading for } \\
\text { receptor } \\
\text { environments at } \\
\text { post-volcanic } \\
\text { condition }\end{array}$} & Active outdoors & \multirow[t]{4}{*}{ Triangular } & \multirow[t]{4}{*}{$\mathrm{mg} / \mathrm{m}^{3}$} & 2.50 & - & 0.000 & 5.000 & \multirow{4}{*}{$\begin{array}{l}\text { Used for short term inhalation } \\
\text { component for the volcanic ash } \\
\text { scenario. (BSC } 2003 \\
\text { [DIRS 160965]) }\end{array}$} \\
\hline & & \begin{tabular}{|l|} 
Inactive \\
outdoors \\
\end{tabular} & & & 0.06 & - & 0.025 & 0.200 & \\
\hline & & Active indoors & & & 0.10 & - & 0.060 & 0.175 & \\
\hline & & Asleep indoors & & & 0.03 & - & 0.010 & 0.060 & \\
\hline
\end{tabular}


Table 6.6-3. Summary of Representative Input Parameter Values and Their Distributions (Continued)

\begin{tabular}{|c|c|c|c|c|c|c|c|c|c|}
\hline $\begin{array}{l}\text { Sub- } \\
\text { model }\end{array}$ & \multicolumn{2}{|c|}{ Parameter Name } & $\begin{array}{l}\text { Distribution } \\
\text { Type }\end{array}$ & Units & $\begin{array}{c}\text { Mean, } \\
\text { Mode, } \\
\text { Average }^{\mathrm{a}} \\
\end{array}$ & SDISE $^{a}$ & Min. ${ }^{b}$ & Max. ${ }^{b}$ & Notes $^{c}$ \\
\hline \multirow[t]{16}{*}{$\begin{array}{l}\text { Air } \\
\text { (Continued) }\end{array}$} & \multirow{2}{*}{$\begin{array}{l}\text { Mass loading } \\
\text { function } \\
\mathrm{f}(t)=S_{0} e^{-\lambda t} \text {, with } \\
\text { constant } \lambda \text { value }\end{array}$} & \begin{tabular}{|l|} 
For initial ash \\
depth $<10 \mathrm{~mm}$
\end{tabular} & \multirow[t]{2}{*}{ Triangular } & \multirow[t]{2}{*}{ /yr } & 0.33 & - & 0.2 & 2.0 & \multirow{2}{*}{$\begin{array}{l}\text { This decay function is used in } \\
\text { the TSPA-LA model. (BSC } \\
2003 \text { [DIRS 160965]) }\end{array}$} \\
\hline & & $\begin{array}{l}\text { For initial ash } \\
\text { depth } \geq 10 \mathrm{~mm}\end{array}$ & & & 0.20 & - & 0.125 & 1.0 & \\
\hline & \multirow[t]{3}{*}{$\begin{array}{l}\text { Enhancement } \\
\text { factor at nominal } \\
\text { condition }\end{array}$} & Active outdoors & Cumulative & - & 4.0 & - & $\begin{array}{l}2.2 \\
4.0 \\
6.5 \\
\end{array}$ & $\begin{array}{r}0 \% \\
50 \% \\
100 \% \\
\end{array}$ & \multirow{7}{*}{$\begin{array}{l}\text { Active outdoors is an } \\
\text { environment where soil } \\
\text { disturbing activities are } \\
\text { conducted; there are no soil } \\
\text { disturbing activities in the other } \\
\text { environments. Mean taken } \\
\text { from } 50 \text { percent value. (BSC } \\
2003 \text { [DIRS 161239]) }\end{array}$} \\
\hline & & \begin{tabular}{|l} 
Inactive \\
outdoors \\
\end{tabular} & Cumulative & & 0.7 & - & $\begin{array}{l}0.21 \\
0.70\end{array}$ & $\begin{array}{r}0 \% \\
50 \%\end{array}$ & \\
\hline & & \begin{tabular}{|l|} 
Active indoors \\
Asleep indoors \\
\end{tabular} & & & & & 1.04 & $100 \%$ & \\
\hline & \multirow{4}{*}{$\begin{array}{l}\text { Enhancement } \\
\text { factor at post- } \\
\text { volcanic } \\
\text { condition }\end{array}$} & Active outdoors & Cumulative & \multirow[t]{4}{*}{-} & 4.4 & - & $\begin{array}{l}2.8 \\
4.4 \\
8.4 \\
\end{array}$ & $\begin{array}{r}0 \% \\
50 \% \\
100 \% \\
\end{array}$ & \\
\hline & & $\begin{array}{l}\text { Inactive } \\
\text { outdoors }\end{array}$ & Cumulative & & \multirow[t]{3}{*}{0.7} & \multirow[t]{3}{*}{-} & $\begin{array}{l}0.21 \\
0.70\end{array}$ & $\begin{array}{r}0 \% \\
50 \%\end{array}$ & \\
\hline & & Active indoors & & & & & 1.04 & $100 \%$ & \\
\hline & & Asleep indoors & & & & & & & \\
\hline & \multicolumn{2}{|c|}{$\begin{array}{l}\text { Fraction of radionuclides from } \\
\text { water into air }\end{array}$} & Uniform & - & 0.5 & - & 0 & 1 & $\begin{array}{l}\text { Mean calculated from uniform } \\
\text { distribution. (BSC } 2003 \\
\text { [DIRS 160964]) }\end{array}$ \\
\hline & \multicolumn{2}{|c|}{$\begin{array}{l}\text { Water evaporation rate of } \\
\text { evaporative cooler }\end{array}$} & lognormal & $\mathrm{L} / \mathrm{hr}$ & 17 & 1.7 & - & - & \multirow{2}{*}{$\begin{array}{l}\text { Water evaporation rate is given } \\
\text { as geometric mean and } \\
\text { standard deviation. Airflow rate } \\
\text { is correlated with water } \\
\text { evaporative rate (correlation } \\
\text { coefficient }=0.8 \text { ); mean is } 50 \\
\text { percent value. (BSC } 2003 \\
\text { [DIRS 160964]) }\end{array}$} \\
\hline & \multicolumn{2}{|c|}{ Air flow rate of evaporative cooler } & Cumulative & $\mathrm{m}^{3} / \mathrm{hr}$ & 8300 & - & $\begin{array}{r}1700 \\
8300 \\
10200\end{array}$ & $\begin{array}{r}0 \% \\
50 \% \\
100 \%\end{array}$ & \\
\hline & \multicolumn{2}{|c|}{ Radon release factor } & Fixed & $\begin{array}{l}\left(\mathrm{Bq} / \mathrm{m}^{3}\right) / \\
(\mathrm{Bq} / \mathrm{kg})\end{array}$ & 0.25 & - & - & - & $\begin{array}{l}\text { Global average value. (BSC } \\
2003 \text { [DIRS 160964]) }\end{array}$ \\
\hline & \multicolumn{2}{|l|}{ Interior wall height } & Cumulative & $\mathrm{m}$ & 2.3 & - & $\begin{array}{l}2.1 \\
2.3 \\
2.7 \\
\end{array}$ & $\begin{array}{r}0 \% \\
50 \% \\
100 \% \\
\end{array}$ & $\begin{array}{l}\text { Mean taken from } 50 \text { percent } \\
\text { value. (BSC } 2003 \text { [DIRS } \\
\text { 160964]) }\end{array}$ \\
\hline & \multirow{2}{*}{$\begin{array}{l}\text { House ventilation } \\
\text { rate }\end{array}$} & For regular time & Lognormal & \multirow[t]{2}{*}{$/ \mathrm{hr}$} & 1 & 1.1 & 0.35 & 2.9 & \\
\hline & & $\begin{array}{l}\text { For evaporative } \\
\text { cooler on }\end{array}$ & Uniform & & 15.5 & - & 1 & 30 & $\begin{array}{l}\text { Mean calculated from uniform } \\
\text { distribution. (BSC } 2003 \\
\text { [DIRS 160964]) }\end{array}$ \\
\hline
\end{tabular}


Table 6.6-3. Summary of Representative Input Parameter Values and Their Distributions (Continued)

\begin{tabular}{|c|c|c|c|c|c|c|c|c|c|}
\hline $\begin{array}{l}\text { Sub- } \\
\text { model }\end{array}$ & \multicolumn{2}{|c|}{ Parameter Name } & $\begin{array}{l}\text { Distribution } \\
\text { Type }\end{array}$ & Units & $\begin{array}{c}\text { Mean, } \\
\text { Mode, } \\
\text { Average }^{\mathrm{a}}\end{array}$ & SDISE $^{a}$ & Min. ${ }^{b}$ & Max. ${ }^{b}$ & Notes $^{c}$ \\
\hline \multirow[t]{2}{*}{$\begin{array}{l}\text { Air } \\
\text { (Continued) }\end{array}$} & \multicolumn{2}{|c|}{$\begin{array}{l}\text { Fraction of radon from soil entering } \\
\text { into the house }\end{array}$} & Uniform & - & 0.175 & - & 0.10 & 0.25 & $\begin{array}{l}\text { Mean calculated from uniform } \\
\text { distribution. (BSC } 2003 \\
\text { [DIRS 160964]) }\end{array}$ \\
\hline & \multicolumn{2}{|c|}{$\begin{array}{l}\text { Ratio of }{ }^{222} \mathrm{Rn} \text { concentration in air } \\
\text { to flux density from soil }\end{array}$} & Fixed & $\begin{array}{l}\left.\mathrm{Bq} \mathrm{m} \mathrm{m}^{-3}\right) /(\mathrm{Bq} \\
\left.\mathrm{n}^{-2} \mathrm{sec}^{-1}\right)\end{array}$ & 300 & - & - & - & $\begin{array}{l}\text { Global average value. (BSC } \\
2003 \text { [DIRS 160964]) }\end{array}$ \\
\hline \multirow[t]{32}{*}{ Plant } & \multirow{16}{*}{$\begin{array}{l}\text { Soil-to-plant } \\
\text { transfer factor for } \\
\text { leafy vegetables }\end{array}$} & Chlorine & \multirow[t]{16}{*}{ Lognormal } & \multirow{16}{*}{$\begin{array}{l}\left(\mathrm{Bq} / \mathrm{kg}_{\text {plant }}\right) / \\
\left(\mathrm{Bq} / \mathrm{kg}_{\text {soil }}\right)\end{array}$} & $6.4 \mathrm{E}+01$ & 2.0 & $1.1 \mathrm{E}+01$ & $3.8 \mathrm{E}+02$ & \multirow{16}{*}{$\begin{array}{l}\text { Transfer factors are given in } \\
\text { geometric mean and geometric } \\
\text { standard deviation. The lower } \\
\text { and upper bounds are } 0.01 \text { and } \\
0.99 \text { percentile values. (BSC } \\
2003 \text { [DIRS 160964]) }\end{array}$} \\
\hline & & Selenium & & & $4.6 \mathrm{E}-02$ & 3.8 & 1.4E-03 & $1.4 \mathrm{E}+00$ & \\
\hline & & Strontium & & & $1.7 E+00$ & 2.0 & 2.9E-01 & $1.0 \mathrm{E}+01$ & \\
\hline & & Technetium & & & $4.6 \mathrm{E}+01$ & 2.6 & $3.8 \mathrm{E}+00$ & $5.5 \mathrm{E}+02$ & \\
\hline & & Tin & & & $3.8 \mathrm{E}-02$ & 2.0 & $6.4 \mathrm{E}-03$ & $2.3 \mathrm{E}-01$ & \\
\hline & & Iodine & & & $2.6 \mathrm{E}-02$ & 9.9 & 7.2E-05 & $9.7 \mathrm{E}+00$ & \\
\hline & & Cesium & & & $1.2 \mathrm{E}-01$ & 2.5 & $1.2 \mathrm{E}-02$ & $1.2 \mathrm{E}+00$ & \\
\hline & & Lead & & & 1.5E-02 & 4.6 & 3.0E-04 & 7.7E-01 & \\
\hline & & Radium & & & $6.8 \mathrm{E}-02$ & 2.7 & 5.1E-03 & 9.2E-01 & \\
\hline & & Actinium & & & 4.3E-03 & 2.0 & $7.2 \mathrm{E}-04$ & $2.6 \mathrm{E}-02$ & \\
\hline & & Thorium & & & 4.3E-03 & 2.8 & $3.2 \mathrm{E}-04$ & 5.9E-02 & \\
\hline & & Protactinium & & & 4.6E-03 & 3.8 & 1.4E-04 & 1.4E-01 & \\
\hline & & Uranium & & & $1.1 \mathrm{E}-02$ & 2.0 & $1.8 \mathrm{E}-03$ & $6.6 \mathrm{E}-02$ & \\
\hline & & Neptunium & & & $5.9 \mathrm{E}-02$ & 4.4 & 1.3E-03 & $2.6 \mathrm{E}+00$ & \\
\hline & & Plutonium & & & $2.9 \mathrm{E}-04$ & 2.0 & 4.9E-05 & 1.7E-03 & \\
\hline & & Americium & & & 1.2E-03 & 2.5 & 1.2E-04 & 1.3E-02 & \\
\hline & \multirow{16}{*}{$\begin{array}{l}\text { Soil-to-plant } \\
\text { transfer factor for } \\
\text { other vegetables }\end{array}$} & Chlorine & \multirow[t]{16}{*}{ Lognormal } & \multirow{16}{*}{$\begin{array}{l}\left(\mathrm{Bq} / \mathrm{kg}_{\text {plant }}\right) / \\
\left(\mathrm{Bq} / \mathrm{kg}_{\text {soil }}\right)\end{array}$} & $6.4 \mathrm{E}+01$ & 2.0 & $1.1 \mathrm{E}+01$ & $3.8 \mathrm{E}+02$ & \multirow{16}{*}{$\begin{array}{l}\text { Transfer factors are given in } \\
\text { geometric mean and geometric } \\
\text { standard deviation. The lower } \\
\text { and upper bounds are } 0.01 \text { and } \\
0.99 \text { percentile values. (BSC } \\
2003 \text { [DIRS 160964]) }\end{array}$} \\
\hline & & Selenium & & & 4.6E-02 & 3.8 & 1.4E-03 & $1.4 \mathrm{E}+00$ & \\
\hline & & Strontium & & & 7.9E-01 & 2.0 & 1.4E-01 & $4.5 E+00$ & \\
\hline & & Technetium & & & $4.4 \mathrm{E}+00$ & 3.7 & 1.5E-01 & $1.2 \mathrm{E}+02$ & \\
\hline & & Tin & & & 1.5E-02 & 3.6 & 5.3E-04 & 4.0E-01 & \\
\hline & & Iodine & & & $3.2 \mathrm{E}-02$ & 4.4 & $7.0 \mathrm{E}-04$ & $1.5 \mathrm{E}+00$ & \\
\hline & & Cesium & & & $5.0 \mathrm{E}-02$ & 2.0 & 8.4E-03 & $3.0 \mathrm{E}-01$ & \\
\hline & & Lead & & & 9.0E-03 & 3.1 & 5.0E-04 & 1.6E-01 & \\
\hline & & Radium & & & $1.2 \mathrm{E}-02$ & 5.3 & 1.6E-04 & 8.6E-01 & \\
\hline & & Actinium & & & 1.1E-03 & 4.9 & 1.8E-05 & $6.6 \mathrm{E}-02$ & \\
\hline & & Thorium & & & 4.4E-04 & 5.6 & 5.3E-06 & 3.6E-02 & \\
\hline & & Protactinium & & & 1.1E-03 & 10.0 & 3.0E-06 & 4.3E-01 & \\
\hline & & Uranium & & & 6.0E-03 & 2.8 & $4.2 \mathrm{E}-04$ & 8.5E-02 & \\
\hline & & Neptunium & & & 3.1E-02 & 4.9 & 5.0E-04 & $1.9 E+00$ & \\
\hline & & Plutonium & & & 1.9E-04 & 2.0 & 3.3E-05 & 1.1E-03 & \\
\hline & & Americium & & & 4.0E-04 & 2.6 & 3.5E-05 & 4.6E-03 & \\
\hline
\end{tabular}


Table 6.6-3. Summary of Representative Input Parameter Values and Their Distributions (Continued)

\begin{tabular}{|c|c|c|c|c|c|c|c|c|c|}
\hline $\begin{array}{l}\text { Sub- } \\
\text { model }\end{array}$ & \multicolumn{2}{|c|}{ Parameter Name } & $\begin{array}{c}\text { Distribution } \\
\text { Type }\end{array}$ & Units & $\begin{array}{c}\text { Mean, } \\
\text { Mode, } \\
\text { Average }^{a}\end{array}$ & SDISE $^{a}$ & Min. ${ }^{b}$ & Max. $^{\mathbf{b}}$ & Notes $^{c}$ \\
\hline \multirow{32}{*}{$\begin{array}{l}\text { Plant } \\
\text { (Continued) }\end{array}$} & \multirow{16}{*}{$\begin{array}{l}\text { Soil-to-plant } \\
\text { transfer factor for } \\
\text { fruit }\end{array}$} & Chlorine & \multirow[t]{16}{*}{ Lognormal } & \multirow{16}{*}{$\begin{array}{l}\text { (Bq/kg plant)/ } \\
\text { (Bq/kg soil })\end{array}$} & $6.4 \mathrm{E}+01$ & 2.0 & $1.1 \mathrm{E}+01$ & $3.8 \mathrm{E}+02$ & \multirow{16}{*}{$\begin{array}{l}\text { Transfer factors are given in } \\
\text { geometric mean and geometric } \\
\text { standard deviation. The lower } \\
\text { and upper bounds are } 0.01 \text { and } \\
0.99 \text { percentile values. (BSC } \\
2003 \text { [DIRS 160964]) }\end{array}$} \\
\hline & & Selenium & & & $4.6 \mathrm{E}-02$ & 3.8 & $1.4 \mathrm{E}-03$ & $1.4 \mathrm{E}+00$ & \\
\hline & & Strontium & & & $2.9 \mathrm{E}-01$ & 2.3 & $3.6 \mathrm{E}-02$ & $2.4 \mathrm{E}+00$ & \\
\hline & & Technetium & & & $4.3 \mathrm{E}+00$ & 4.6 & 8.7E-02 & $2.1 \mathrm{E}+02$ & \\
\hline & & Tin & & & 1.5E-02 & 3.6 & 5.3E-04 & 4.0E-01 & \\
\hline & & Iodine & & & 5.7E-02 & 2.8 & 4.1E-03 & 7.9E-01 & \\
\hline & & Cesium & & & $5.6 \mathrm{E}-02$ & 2.8 & $3.8 \mathrm{E}-03$ & $8.1 \mathrm{E}-01$ & \\
\hline & & Lead & & & $1.2 \mathrm{E}-02$ & 3.3 & $5.8 \mathrm{E}-04$ & 2.6E-01 & \\
\hline & & Radium & & & 7.3E-03 & 4.3 & $1.6 \mathrm{E}-04$ & $3.2 \mathrm{E}-01$ & \\
\hline & & Actinium & & & $8.5 E-04$ & 3.4 & 3.7E-05 & $2.0 \mathrm{E}-02$ & \\
\hline & & Thorium & & & 2.9E-04 & 4.9 & $4.8 \mathrm{E}-06$ & 1.7E-02 & \\
\hline & & Protactinium & & & 1.1E-03 & 10.0 & 3.0E-06 & 4.3E-01 & \\
\hline & & Uranium & & & $6.3 \mathrm{E}-03$ & 2.9 & $3.9 \mathrm{E}-04$ & 1.0E-01 & \\
\hline & & Neptunium & & & 3.4E-02 & 6.9 & 2.3E-04 & $5.0 E+00$ & \\
\hline & & Plutonium & & & 1.8E-04 & 3.4 & 7.8E-06 & $4.2 \mathrm{E}-03$ & \\
\hline & & Americium & & & $5.4 \mathrm{E}-04$ & 2.3 & $6.5 \mathrm{E}-05$ & $4.5 \mathrm{E}-03$ & \\
\hline & \multirow{16}{*}{$\begin{array}{l}\text { Soil-to-plant } \\
\text { transfer factor for } \\
\text { grain }\end{array}$} & Chlorine & \multirow[t]{16}{*}{ Lognormal } & \multirow{16}{*}{\begin{tabular}{|l}
$\left(\mathrm{Bq} / \mathrm{kg}_{\text {plant }}\right) /$ \\
$\left(\mathrm{Bq} / \mathrm{kg}_{\text {soil }}\right)$
\end{tabular}} & $2.4 \mathrm{E}+01$ & 8.4 & 1.0E-01 & $5.8 \mathrm{E}+03$ & \multirow{16}{*}{$\begin{array}{l}\text { Transfer factors are given in } \\
\text { geometric mean and geometric } \\
\text { standard deviation. The lower } \\
\text { and upper bounds are } 0.01 \text { and } \\
0.99 \text { percentile values. (BSC } \\
2003 \text { [DIRS 160964]) }\end{array}$} \\
\hline & & Selenium & & & $2.9 \mathrm{E}-02$ & 2.0 & $4.8 \mathrm{E}-03$ & 1.7E-01 & \\
\hline & & Strontium & & & 1.7E-01 & 2.0 & $2.8 \mathrm{E}-02$ & $1.0 \mathrm{E}+00$ & \\
\hline & & Technetium & & & $1.6 \mathrm{E}+00$ & 4.3 & $3.8 \mathrm{E}-02$ & $6.8 \mathrm{E}+01$ & \\
\hline & & Tin & & & $9.2 \mathrm{E}-03$ & 2.0 & 1.5E-03 & $5.5 \mathrm{E}-02$ & \\
\hline & & Iodine & & & $2.5 \mathrm{E}-02$ & 10.0 & $6.6 \mathrm{E}-05$ & $9.4 \mathrm{E}+00$ & \\
\hline & & Cesium & & & $2.0 \mathrm{E}-02$ & 2.2 & $2.7 \mathrm{E}-03$ & $1.6 \mathrm{E}-01$ & \\
\hline & & Lead & & & 5.5E-03 & 2.1 & 8.2E-04 & $3.8 \mathrm{E}-02$ & \\
\hline & & Radium & & & $3.1 \mathrm{E}-03$ & 4.0 & 8.8E-05 & $1.1 \mathrm{E}-01$ & \\
\hline & & Actinium & & & 5.4E-04 & 2.9 & $3.6 \mathrm{E}-05$ & 8.0E-03 & \\
\hline & & Thorium & & & 1.7E-04 & 5.2 & $2.4 \mathrm{E}-06$ & $1.2 \mathrm{E}-02$ & \\
\hline & & Protactinium & & & 9.5E-04 & 7.2 & 5.9E-06 & 1.5E-01 & \\
\hline & & Uranium & & & 1.1E-03 & 3.6 & 4.1E-05 & $3.1 \mathrm{E}-02$ & \\
\hline & & Neptunium & & & $4.4 \mathrm{E}-03$ & 6.9 & 3.1E-05 & 6.3E-01 & \\
\hline & & Plutonium & & & 1.9E-05 & 4.2 & $4.8 \mathrm{E}-07$ & $7.8 \mathrm{E}-04$ & \\
\hline & & Americium & & & 7.5E-05 & 3.2 & 3.8E-06 & 1.5E-03 & \\
\hline
\end{tabular}


Table 6.6-3. Summary of Representative Input Parameter Values and Their Distributions (Continued)

\begin{tabular}{|c|c|c|c|c|c|c|c|c|c|}
\hline $\begin{array}{c}\text { Sub- } \\
\text { model }\end{array}$ & \multicolumn{2}{|c|}{ Parameter Name } & $\begin{array}{c}\text { Distribution } \\
\text { Type }\end{array}$ & Units & $\begin{array}{c}\text { Mean, } \\
\text { Mode, } \\
\text { Average a }\end{array}$ & SDISE $^{a}$ & Min. ${ }^{b}$ & Max. ${ }^{b}$ & Notes $^{c}$ \\
\hline \multirow{19}{*}{$\begin{array}{l}\text { Plant } \\
\text { (Continued) }\end{array}$} & \multirow{16}{*}{$\begin{array}{l}\text { Soil-to-plant } \\
\text { transfer factor for } \\
\text { forage }\end{array}$} & Chlorine & \multirow[t]{16}{*}{ Lognormal } & \multirow{16}{*}{$\begin{array}{l}\text { (Bq/kg plant) } / \\
\left(\mathrm{Bq} / \mathrm{kg}_{\text {soil }}\right)\end{array}$} & $7.5 \mathrm{E}+01$ & 2.0 & $1.3 \mathrm{E}+01$ & $4.5 \mathrm{E}+02$ & \multirow{16}{*}{$\begin{array}{l}\text { Transfer factors are given in } \\
\text { geometric mean and geometric } \\
\text { standard deviation. The lower } \\
\text { and upper bounds are } 0.01 \text { and } \\
0.99 \text { percentile values. (BSC } \\
2003 \text { [DIRS 160964]) }\end{array}$} \\
\hline & & Selenium & & & 1.5E-01 & 5.5 & 1.9E-03 & $1.3 \mathrm{E}+01$ & \\
\hline & & Strontium & & & $2.1 \mathrm{E}+00$ & 2.1 & 3.2E-01 & $1.3 \mathrm{E}+01$ & \\
\hline & & Technetium & & & $2.7 \mathrm{E}+01$ & 2.7 & $2.1 \mathrm{E}+00$ & $3.5 \mathrm{E}+02$ & \\
\hline & & Tin & & & 1.6E-01 & 5.8 & 1.7E-03 & $1.5 \mathrm{E}+01$ & \\
\hline & & Iodine & & & 4.0E-02 & 10.0 & 1.1E-04 & $1.5 \mathrm{E}+01$ & \\
\hline & & Cesium & & & 1.3E-01 & 3.3 & 6.3E-03 & $2.8 \mathrm{E}+00$ & \\
\hline & & Lead & & & $1.8 \mathrm{E}-02$ & 7.0 & $1.2 \mathrm{E}-04$ & $2.8 \mathrm{E}+00$ & \\
\hline & & Radium & & & $8.2 \mathrm{E}-02$ & 3.0 & $4.9 \mathrm{E}-03$ & $1.4 \mathrm{E}+00$ & \\
\hline & & Actinium & & & 1.7E-02 & 5.4 & $2.2 \mathrm{E}-04$ & $1.3 \mathrm{E}+00$ & \\
\hline & & Thorium & & & 1.0E-02 & 4.2 & 2.5E-04 & 3.9E-01 & \\
\hline & & Protactinium & & & 1.9E-02 & 6.7 & $1.4 \mathrm{E}-04$ & $2.5 E+00$ & \\
\hline & & Uranium & & & 1.7E-02 & 6.1 & 1.6E-04 & $1.9 \mathrm{E}+00$ & \\
\hline & & Neptunium & & & $5.8 \mathrm{E}-02$ & 5.6 & $6.8 \mathrm{E}-04$ & $4.9 \mathrm{E}+00$ & \\
\hline & & Plutonium & & & $1.0 \mathrm{E}-03$ & 10.0 & 2.7E-06 & $3.9 \mathrm{E}-01$ & \\
\hline & & Americium & & & $2.1 \mathrm{E}-03$ & 10.0 & 5.5E-06 & 7.9E-01 & \\
\hline & \multirow[t]{3}{*}{$\begin{array}{l}\text { Dry-to-wet weight } \\
\text { ratio }\end{array}$} & $\begin{array}{l}\text { Leafy } \\
\text { vegetables }\end{array}$ & \multirow[t]{3}{*}{ Cumulative } & \multirow[t]{3}{*}{$\mathrm{kg}$ dry $/ \mathrm{kg}_{\text {wet }}$} & 0.070 & - & $\begin{array}{l}0.041 \\
0.054 \\
0.06 \\
0.078 \\
0.081 \\
0.084 \\
0.093 \\
\end{array}$ & $\begin{array}{r}0 \% \\
17 \% \\
33 \% \\
50 \% \\
67 \% \\
83 \% \\
100 \% \\
\end{array}$ & \multirow[t]{3}{*}{$\begin{array}{l}\text { Means given in the source } \\
\text { reports. (BSC } 2003 \\
\text { [DIRS 160976]) }\end{array}$} \\
\hline & & $\begin{array}{l}\text { Other } \\
\text { vegetables }\end{array}$ & & & 0.103 & - & $\begin{array}{l}0.035 \\
0.063 \\
0.078 \\
0.08 \\
0.103 \\
0.122 \\
0.24 \\
\end{array}$ & $\begin{array}{r}0 \% \\
17 \% \\
33 \% \\
50 \% \\
67 \% \\
83 \% \\
100 \% \\
\end{array}$ & \\
\hline & & Fruit & & & 0.120 & - & $\begin{array}{l}0.062 \\
0.084 \\
0.102 \\
0.155 \\
0.194 \\
\end{array}$ & $\begin{array}{r}0 \% \\
25 \% \\
50 \% \\
75 \% \\
100 \% \\
\end{array}$ & \\
\hline
\end{tabular}


Table 6.6-3. Summary of Representative Input Parameter Values and Their Distributions (Continued)

\begin{tabular}{|c|c|c|c|c|c|c|c|c|c|c|}
\hline $\begin{array}{l}\text { Sub- } \\
\text { model }\end{array}$ & \multicolumn{3}{|c|}{ Parameter Name } & $\begin{array}{c}\text { Distribution } \\
\text { Type }\end{array}$ & Units & $\begin{array}{c}\text { Mean, } \\
\text { Mode, } \\
\text { Average }^{\text {a }}\end{array}$ & SDISE $^{a}$ & Min. ${ }^{b}$ & Max. ${ }^{b}$ & Notes $^{c}$ \\
\hline \multirow[t]{23}{*}{$\begin{array}{l}\text { Plant } \\
\text { (Continued) }\end{array}$} & \multirow{2}{*}{\multicolumn{2}{|c|}{$\begin{array}{l}\text { Dry-to-wet weight } \\
\text { ratio (cont.) }\end{array}$}} & Grain & & & 0.903 & - & $\begin{array}{l}0.891 \\
0.896 \\
0.906 \\
0.918\end{array}$ & $\begin{array}{r}0 \% \\
33 \% \\
67 \% \\
100 \%\end{array}$ & \\
\hline & & & Forage & & & 0.220 & - & $\begin{array}{l}0.182 \\
0.227 \\
0.238 \\
\end{array}$ & $\begin{array}{r}0 \% \\
75 \% \\
100 \% \\
\end{array}$ & \\
\hline & \multirow{5}{*}{\multicolumn{2}{|c|}{$\begin{array}{l}\text { Translocation } \\
\text { factor }\end{array}$}} & \begin{tabular}{|l} 
Leafy \\
vegetation
\end{tabular} & Fixed & - & 1.0 & - & - & - & BSC 2003 [DIRS 160964] \\
\hline & & & \begin{tabular}{|l|} 
Other \\
vegetation
\end{tabular} & Cumulative & - & 0.1 & - & $\begin{array}{l}0.05 \\
0.10\end{array}$ & $\begin{array}{r}0 \% \\
50 \%\end{array}$ & $\begin{array}{l}\text { Mean taken from } 50 \text { percent } \\
\text { value. }\end{array}$ \\
\hline & & & Fruit & & & & & 0.30 & $100 \%$ & \\
\hline & & & Grain & & & & & & & \\
\hline & & & Forage & Fixed & - & 1.0 & - & - & - & \\
\hline & \multirow{5}{*}{\multicolumn{2}{|c|}{$\begin{array}{l}\text { Fraction of } \\
\text { overhead } \\
\text { irrigation }\end{array}$}} & \begin{tabular}{|l} 
Leafy \\
vegetation
\end{tabular} & Normal & - & 0.75 & 0.10 & 0.49 & 1.0 & (BSC 2003 [DIRS 160976]) \\
\hline & & & \begin{tabular}{|l|} 
Other \\
vegetation
\end{tabular} & & & 0.75 & 0.10 & 0.49 & 1.0 & \\
\hline & & & Fruit & & & 0.50 & 0.10 & 0.24 & 1.0 & \\
\hline & & & Grain & & & 0.90 & 0.05 & 0.77 & 1.0 & \\
\hline & & & Forage & & & 0.90 & 0.05 & 0.77 & 1.0 & \\
\hline & \multicolumn{3}{|c|}{ Weathering half-life } & Cumulative & $d$ & 14 & - & $\begin{array}{r}5 \\
14 \\
30 \\
\end{array}$ & $\begin{array}{r}0 \% \\
50 \% \\
100 \% \\
\end{array}$ & $\begin{array}{l}\text { Mean taken from } 50 \text { percent } \\
\text { value. (BSC } 2003 \text { [DIRS } \\
160964] \text { ) }\end{array}$ \\
\hline & \multirow{10}{*}{$\begin{array}{l}\text { Crop } \\
\text { grow- } \\
\text { ing } \\
\text { time }\end{array}$} & \multirow{5}{*}{$\begin{array}{l}\text { Present } \\
\text {-day } \\
\text { climate }\end{array}$} & $\begin{array}{l}\text { Leafy } \\
\text { vegetation }\end{array}$ & \multirow[t]{5}{*}{ Fixed } & \multirow[t]{5}{*}{$d$} & 75 & - & - & - & \multirow[t]{5}{*}{ (BSC 2003 [DIRS 160976]) } \\
\hline & & & $\begin{array}{l}\text { Other } \\
\text { vegetation }\end{array}$ & & & 80 & - & - & - & \\
\hline & & & Fruit & & & 160 & - & - & - & \\
\hline & & & Grain & & & 200 & - & - & - & \\
\hline & & & Forage & & & 75 & - & - & - & \\
\hline & & \multirow[t]{5}{*}{\begin{tabular}{|l|} 
Future \\
climate
\end{tabular}} & $\begin{array}{l}\text { Leafy } \\
\text { vegetation }\end{array}$ & \multirow[t]{5}{*}{ Fixed } & \multirow[t]{5}{*}{$d$} & 75 & - & - & - & \multirow[t]{5}{*}{ (BSC 2003 [DIRS 160976]) } \\
\hline & & & $\begin{array}{l}\text { Other } \\
\text { vegetation }\end{array}$ & & & 100 & - & - & - & \\
\hline & & & Fruit & & & 105 & - & - & - & \\
\hline & & & Grain & & & 185 & - & - & - & \\
\hline & & & Forage & & & 90 & - & - & - & \\
\hline
\end{tabular}


Table 6.6-3. Summary of Representative Input Parameter Values and Their Distributions (Continued)

\begin{tabular}{|c|c|c|c|c|c|c|c|c|c|}
\hline $\begin{array}{l}\text { Sub- } \\
\text { model }\end{array}$ & \multicolumn{2}{|c|}{ Parameter Name } & $\begin{array}{c}\text { Distribution } \\
\text { Type }\end{array}$ & Units & $\begin{array}{l}\text { Mean, } \\
\text { Mode, } \\
\text { Averaqe }^{\mathrm{a}}\end{array}$ & $\mathrm{SD} / \mathrm{SE}^{\mathrm{a}}$ & Min. ${ }^{b}$ & Max. ${ }^{b}$ & Notes $^{c}$ \\
\hline \multirow[t]{5}{*}{$\begin{array}{l}\text { Plant } \\
\text { (Continued) }\end{array}$} & \multirow[t]{5}{*}{ Crop wet yield } & \begin{tabular}{|l|} 
Leafy \\
vegetation
\end{tabular} & \multirow[t]{5}{*}{ Cumulative } & \multirow[t]{5}{*}{$\mathrm{kg} / \mathrm{m}^{2}$} & 3.30 & - & $\begin{array}{l}1.08 \\
1.46 \\
1.78 \\
2.01 \\
2.98 \\
3.25 \\
3.83 \\
7.79 \\
7.85 \\
\end{array}$ & $\begin{array}{r}0 \% \\
5 \% \\
20 \% \\
35 \% \\
50 \% \\
65 \% \\
80 \% \\
95 \% \\
100 \% \\
\end{array}$ & \multirow[t]{5}{*}{$\begin{array}{l}\text { Means given in the source } \\
\text { reports. (BSC 2003 } \\
\text { [DIRS 160976]) }\end{array}$} \\
\hline & & $\begin{array}{l}\text { Other } \\
\text { vegetation }\end{array}$ & & & 4.13 & - & $\begin{array}{l}2.8 \\
3.37 \\
3.56 \\
3.64 \\
4.92 \\
5.15 \\
6.61\end{array}$ & $\begin{array}{r}0 \% \\
5 \% \\
28 \% \\
51 \% \\
72 \% \\
95 \% \\
100 \%\end{array}$ & \\
\hline & & Fruit & & & 2.75 & - & $\begin{array}{l}0.73 \\
1.51 \\
2.67 \\
2.92 \\
3.00 \\
3.63 \\
6.89\end{array}$ & $\begin{array}{r}0 \% \\
5 \% \\
28 \% \\
51 \% \\
72 \% \\
95 \% \\
100 \%\end{array}$ & \\
\hline & & Grain & & & 0.59 & - & $\begin{array}{l}0.27 \\
0.28 \\
0.44 \\
0.54 \\
1.1 \\
1.22\end{array}$ & $\begin{array}{r}0 \% \\
5 \% \\
35 \% \\
65 \% \\
95 \% \\
100 \%\end{array}$ & \\
\hline & & \begin{tabular}{|l|} 
Forage \\
\end{tabular} & & & 2.14 & - & $\begin{array}{l}0.69 \\
1.02 \\
1.87 \\
5.78 \\
6.28\end{array}$ & $\begin{array}{r}0 \% \\
5 \% \\
73 \% \\
95 \% \\
100 \%\end{array}$ & \\
\hline
\end{tabular}


Table 6.6-3. Summary of Representative Input Parameter Values and Their Distributions (Continued)

\begin{tabular}{|c|c|c|c|c|c|c|c|c|c|}
\hline $\begin{array}{c}\text { Sub- } \\
\text { model }\end{array}$ & \multicolumn{2}{|c|}{ Parameter Name } & $\begin{array}{c}\text { Distribution } \\
\text { Type }\end{array}$ & Units & $\begin{array}{c}\text { Mean, } \\
\text { Mode, } \\
\text { Average }^{\text {a }}\end{array}$ & $\mathrm{SD} / \mathrm{SE}^{\mathrm{a}}$ & Min. ${ }^{b}$ & Max. ${ }^{b}$ & Notes $^{c}$ \\
\hline \multirow[t]{5}{*}{$\begin{array}{l}\text { Plant } \\
\text { (Continued) }\end{array}$} & \multirow[t]{5}{*}{$\begin{array}{l}\text { Crop dry } \\
\text { biomass }\end{array}$} & \begin{tabular}{|l|} 
Leafy \\
vegetation
\end{tabular} & \multirow[t]{5}{*}{ Cumulative } & \multirow[t]{5}{*}{$\mathrm{kg} / \mathrm{m}^{2}$} & 0.21 & - & $\begin{array}{l}0.10 \\
0.13 \\
0.14 \\
0.15 \\
0.16 \\
0.18 \\
0.30 \\
0.42 \\
0.50 \\
\end{array}$ & $\begin{array}{r}0 \% \\
5 \% \\
20 \% \\
35 \% \\
50 \% \\
65 \% \\
80 \% \\
95 \% \\
100 \% \\
\end{array}$ & \multirow[t]{5}{*}{$\begin{array}{l}\text { Means given in the source } \\
\text { reports. (BSC 2003 } \\
\text { [DIRS 160976]) }\end{array}$} \\
\hline & & \begin{tabular}{|l|} 
Other \\
vegetation
\end{tabular} & & & 0.43 & - & $\begin{array}{l}0.30 \\
0.40 \\
0.41 \\
0.43 \\
0.44 \\
0.46 \\
0.60\end{array}$ & $\begin{array}{r}0 \% \\
5 \% \\
28 \% \\
51 \% \\
73 \% \\
95 \% \\
100 \%\end{array}$ & \\
\hline & & Fruit & & & 0.62 & - & $\begin{array}{l}0.10 \\
0.56 \\
0.60 \\
0.65 \\
0.68 \\
1.30\end{array}$ & $\begin{array}{r}0 \% \\
5 \% \\
35 \% \\
65 \% \\
95 \% \\
100 \%\end{array}$ & \\
\hline & & Grain & & & 1.13 & - & $\begin{array}{l}0.50 \\
0.61 \\
0.74 \\
1.20 \\
1.97 \\
2.20\end{array}$ & $\begin{array}{r}0 \% \\
5 \% \\
35 \% \\
65 \% \\
95 \% \\
100 \% \\
\end{array}$ & \\
\hline & & Forage & & & 0.48 & - & $\begin{array}{l}0.10 \\
0.23 \\
0.34 \\
1.38 \\
1.50\end{array}$ & $\begin{array}{r}0 \% \\
5 \% \\
73 \% \\
95 \% \\
100 \%\end{array}$ & \\
\hline
\end{tabular}


Table 6.6-3. Summary of Representative Input Parameter Values and Their Distributions (Continued)

\begin{tabular}{|c|c|c|c|c|c|c|c|c|c|c|}
\hline $\begin{array}{l}\text { Sub- } \\
\text { model }\end{array}$ & \multicolumn{3}{|c|}{ Parameter Name } & $\begin{array}{c}\text { Distribution } \\
\text { Type }\end{array}$ & Units & $\begin{array}{c}\text { Mean, } \\
\text { Mode, } \\
\text { Average a }\end{array}$ & $\mathrm{SD} / \mathrm{SE}^{\mathrm{a}}$ & Min. ${ }^{b}$ & Max. & Notes $^{c}$ \\
\hline \multirow[t]{5}{*}{$\begin{array}{l}\text { Plant } \\
\text { (Continued) }\end{array}$} & \multirow[t]{5}{*}{\begin{tabular}{|l|} 
Daily \\
irriga- \\
tion \\
rate
\end{tabular}} & \multirow[t]{5}{*}{$\begin{array}{l}\text { Present } \\
\text {-day } \\
\text { climate }\end{array}$} & \begin{tabular}{|l} 
Leafy \\
vegetation
\end{tabular} & \multirow[t]{5}{*}{ Cumulative } & \multirow[t]{5}{*}{$\mathrm{mm} / \mathrm{d}$} & 5.40 & - & $\begin{array}{l}4.00 \\
5.11 \\
5.19 \\
5.21 \\
5.38 \\
5.46 \\
5.98 \\
7.06\end{array}$ & $\begin{array}{r}0 \% \\
5 \% \\
20 \% \\
35 \% \\
50 \% \\
80 \% \\
95 \% \\
100 \%\end{array}$ & \multirow[t]{5}{*}{$\begin{array}{l}\text { Means given in the source } \\
\text { reports. (BSC 2003 } \\
\text { [DIRS 160976]) }\end{array}$} \\
\hline & & & \begin{tabular}{|l|} 
Other \\
vegetation
\end{tabular} & & & 7.55 & - & $\begin{array}{r}5.00 \\
6.05 \\
6.65 \\
6.85 \\
7.62 \\
8.19 \\
8.32 \\
9.18 \\
10.83\end{array}$ & $\begin{array}{r}0 \% \\
5 \% \\
20 \% \\
35 \% \\
50 \% \\
65 \% \\
80 \% \\
95 \% \\
100 \%\end{array}$ & \\
\hline & & & Fruit & & & 7.38 & - & $\begin{array}{r}4.00 \\
5.38 \\
7.00 \\
7.56 \\
8.35 \\
8.60 \\
10.15\end{array}$ & $\begin{array}{r}0 \% \\
5 \% \\
28 \% \\
51 \% \\
72 \% \\
95 \% \\
100 \%\end{array}$ & \\
\hline & & & Grain & & & 4.64 & - & $\begin{array}{l}3.00 \\
3.44 \\
3.58 \\
3.86 \\
7.67 \\
9.05\end{array}$ & $\begin{array}{r}0 \% \\
5 \% \\
35 \% \\
65 \% \\
95 \% \\
100 \%\end{array}$ & \\
\hline & & & Forage & & & 6.54 & - & $\begin{array}{r}5.00 \\
5.84 \\
6.18 \\
9.00 \\
10.62\end{array}$ & $\begin{array}{r}0 \% \\
5 \% \\
73 \% \\
95 \% \\
100 \%\end{array}$ & \\
\hline
\end{tabular}


Table 6.6-3. Summary of Representative Input Parameter Values and Their Distributions (Continued)

\begin{tabular}{|c|c|c|c|c|c|c|c|c|c|c|}
\hline $\begin{array}{l}\text { Sub- } \\
\text { model }\end{array}$ & \multicolumn{3}{|c|}{ Parameter Name } & $\begin{array}{c}\text { Distribution } \\
\text { Type }\end{array}$ & Units & $\begin{array}{c}\text { Mean, } \\
\text { Mode, } \\
\text { Average a }\end{array}$ & $\mathrm{SD} / \mathrm{SE}^{\mathrm{a}}$ & Min. ${ }^{b}$ & Max. & Notes $^{c}$ \\
\hline \multirow[t]{5}{*}{$\begin{array}{l}\text { Plant } \\
\text { (Continued) }\end{array}$} & \multirow[t]{5}{*}{\begin{tabular}{|l|} 
Daily \\
irriga- \\
tion \\
rate \\
(cont.)
\end{tabular}} & \multirow[t]{5}{*}{\begin{tabular}{|l|} 
Future \\
climate
\end{tabular}} & \begin{tabular}{|l|} 
Leafy \\
vegetation
\end{tabular} & \multirow[t]{5}{*}{ Cumulative } & \multirow[t]{5}{*}{$\mathrm{mm} / \mathrm{d}$} & 3.81 & - & $\begin{array}{l}3.00 \\
3.34 \\
3.51 \\
3.86 \\
3.92 \\
4.02 \\
4.18 \\
4.93\end{array}$ & $\begin{array}{r}0 \% \\
5 \% \\
20 \% \\
50 \% \\
65 \% \\
80 \% \\
95 \% \\
100 \%\end{array}$ & \multirow[t]{5}{*}{$\begin{array}{l}\text { Means given in the source } \\
\text { reports. (BSC 2003 } \\
\text { [DIRS 160976]) }\end{array}$} \\
\hline & & & \begin{tabular}{|l|} 
Other \\
vegetation
\end{tabular} & & & 3.84 & - & $\begin{array}{l}2.00 \\
2.73 \\
3.08 \\
3.48 \\
4.08 \\
4.16 \\
4.43 \\
4.95 \\
5.84\end{array}$ & $\begin{array}{r}0 \% \\
5 \% \\
20 \% \\
35 \% \\
50 \% \\
65 \% \\
80 \% \\
95 \% \\
100 \%\end{array}$ & \\
\hline & & & Fruit & & & 3.90 & - & $\begin{array}{l}2.00 \\
2.51 \\
3.48 \\
4.33 \\
4.38 \\
4.79 \\
5.65\end{array}$ & $\begin{array}{r}0 \% \\
5 \% \\
28 \% \\
51 \% \\
72 \% \\
95 \% \\
100 \%\end{array}$ & \\
\hline & & & Grain & & & 3.36 & - & $\begin{array}{l}1.00 \\
1.99 \\
3.42 \\
3.93 \\
4.11 \\
4.85\end{array}$ & $\begin{array}{r}0 \% \\
5 \% \\
35 \% \\
65 \% \\
95 \% \\
100 \%\end{array}$ & \\
\hline & & & Forage & & & 4.14 & - & $\begin{array}{l}3.00 \\
3.64 \\
4.01 \\
5.03 \\
5.94\end{array}$ & $\begin{array}{r}0 \% \\
5 \% \\
73 \% \\
95 \% \\
100 \%\end{array}$ & \\
\hline
\end{tabular}


Table 6.6-3. Summary of Representative Input Parameter Values and Their Distributions (Continued)

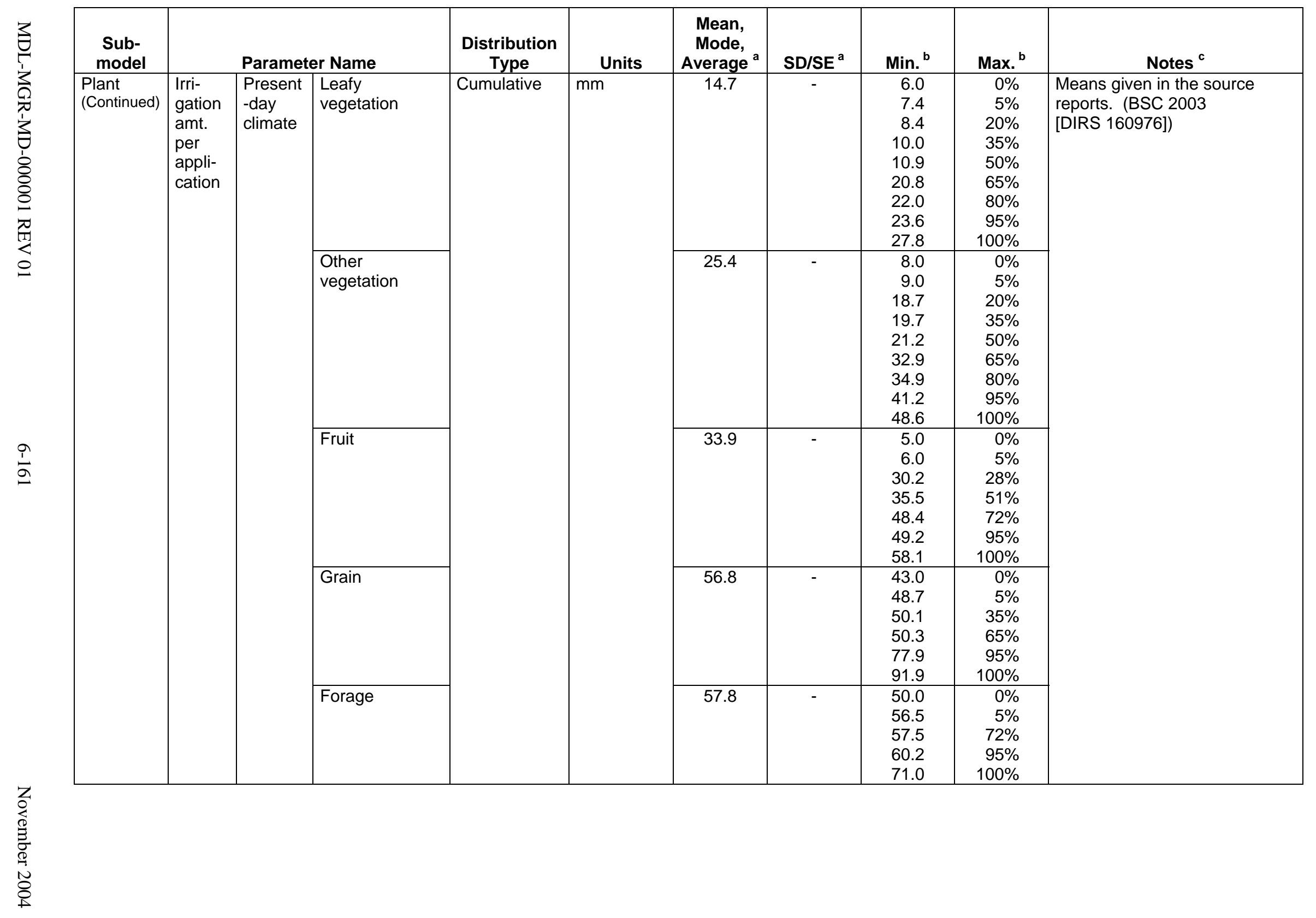


Table 6.6-3. Summary of Representative Input Parameter Values and Their Distributions (Continued)

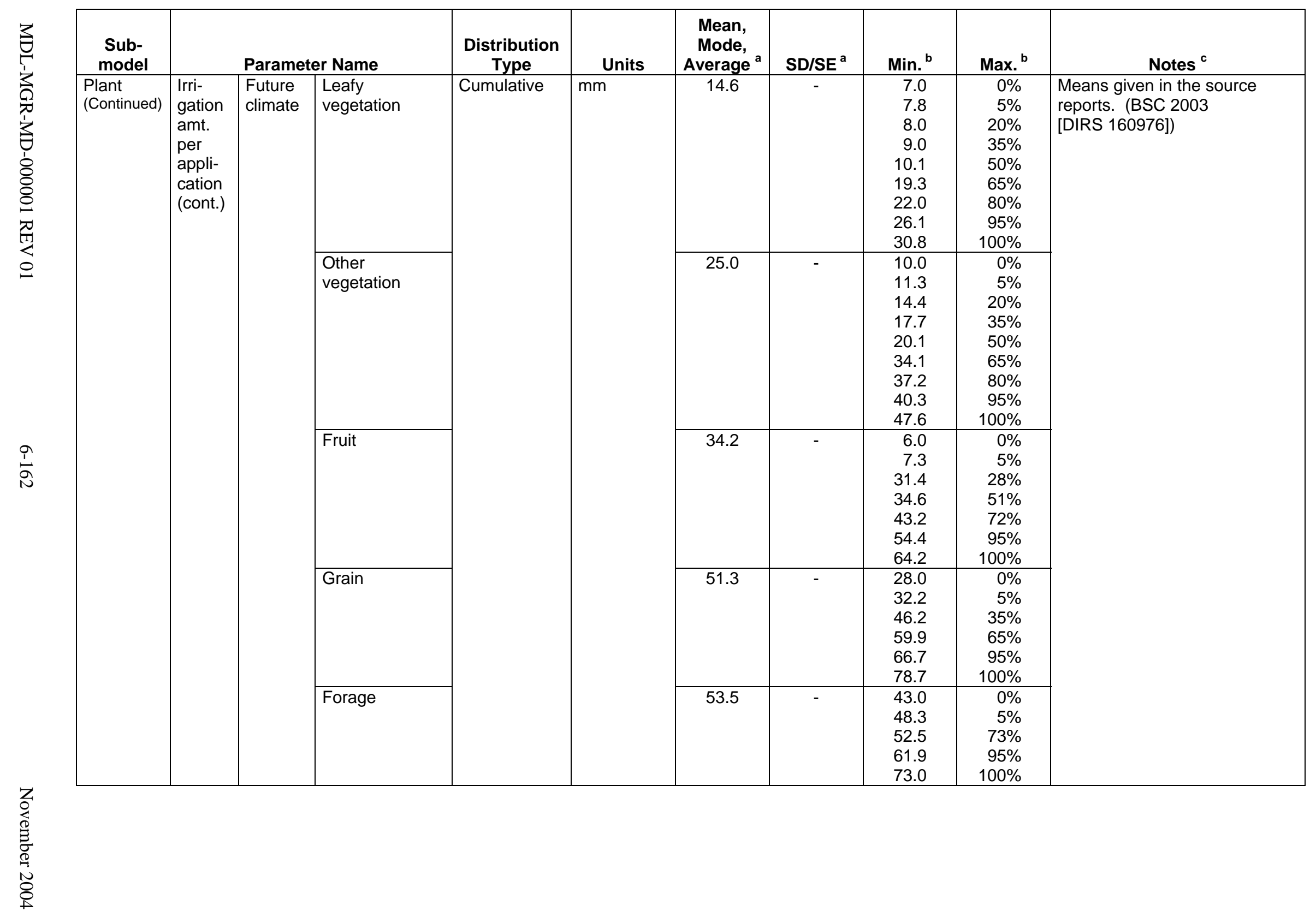


Table 6.6-3. Summary of Representative Input Parameter Values and Their Distributions (Continued)

\begin{tabular}{|c|c|c|c|c|c|c|c|c|c|}
\hline $\begin{array}{l}\text { Sub- } \\
\text { model }\end{array}$ & \multicolumn{2}{|c|}{ Parameter Name } & $\begin{array}{c}\text { Distribution } \\
\text { Type }\end{array}$ & Units & $\begin{array}{c}\text { Mean, } \\
\text { Mode, } \\
\text { Average }^{\text {a }}\end{array}$ & SDISE $^{a}$ & Min. ${ }^{b}$ & Max. ${ }^{b}$ & Notes $^{c}$ \\
\hline \multirow{2}{*}{$\begin{array}{l}\text { Plant } \\
\text { (Continued) }\end{array}$} & \multicolumn{2}{|c|}{ Irrigation intensity } & Uniform & $\mathrm{cm} / \mathrm{hr}$ & 4.25 & - & 1.0 & 7.5 & (BSC 2003 [DIRS 160976]) \\
\hline & \multicolumn{2}{|c|}{ Dry deposition velocity } & Cumulative & $\mathrm{m} / \mathrm{sec}$ & $8 E-3$ & - & $\begin{array}{l}3 E-4 \\
1 E-3 \\
8 E-3 \\
3 E-2 \\
3 E-1 \\
\end{array}$ & $\begin{array}{r}0 \% \\
16 \% \\
50 \% \\
84 \% \\
100 \% \\
\end{array}$ & $\begin{array}{l}\text { Mean taken from } 50 \text { percent } \\
\text { value. } \\
\text { (BSC } 2003 \text { [DIRS 160964]) }\end{array}$ \\
\hline \multirow[t]{32}{*}{ Animal } & \multirow{16}{*}{$\begin{array}{l}\text { Animal product } \\
\text { transfer } \\
\text { coefficients for } \\
\text { meat }\end{array}$} & Chlorine & \multirow[t]{16}{*}{ Lognormal } & \multirow[t]{16}{*}{$\mathrm{d} / \mathrm{kg}$} & 4.6E-02 & 2.0 & 7.7E-03 & 2.7E-01 & \multirow{16}{*}{$\begin{array}{l}\text { Transfer coefficients are given } \\
\text { in geometric mean and } \\
\text { geometric standard deviation. } \\
\text { The lower and upper bounds } \\
\text { are } 0.01 \text { and } 0.99 \text { percentile } \\
\text { values. (BSC } 2003 \\
\text { [DIRS 160964]) }\end{array}$} \\
\hline & & Selenium & & & 8.8E-02 & 5.8 & 9.6E-04 & $8.0 \mathrm{E}+00$ & \\
\hline & & Strontium & & & $1.4 \mathrm{E}-03$ & 4.4 & 3.1E-05 & $6.2 \mathrm{E}-02$ & \\
\hline & & Technetium & & & 1.1E-03 & 7.2 & $6.9 \mathrm{E}-06$ & $1.8 \mathrm{E}-01$ & \\
\hline & & Tin & & & 1.9E-02 & 4.6 & 3.8E-04 & 9.9E-01 & \\
\hline & & Iodine & & & 1.0E-02 & 2.8 & $6.8 \mathrm{E}-04$ & 1.5E-01 & \\
\hline & & Cesium & & & $2.4 \mathrm{E}-02$ & 2.6 & $2.1 \mathrm{E}-03$ & 2.7E-01 & \\
\hline & & Lead & & & $6.3 \mathrm{E}-04$ & 2.6 & 5.4E-05 & 7.5E-03 & \\
\hline & & Radium & & & 8.1E-04 & 2.1 & 1.1E-04 & 5.7E-03 & \\
\hline & & Actinium & & & 7.9E-05 & 8.2 & 3.5E-07 & 1.8E-02 & \\
\hline & & \begin{tabular}{|l|} 
Thorium \\
\end{tabular} & & & 1.1E-04 & 10.0 & 2.8E-07 & 4.0E-02 & \\
\hline & & Protactinium & & & 6.6E-05 & 10.0 & $1.8 \mathrm{E}-07$ & $2.5 \mathrm{E}-02$ & \\
\hline & & \begin{tabular}{|l|} 
Uranium \\
\end{tabular} & & & $4.8 \mathrm{E}-04$ & 3.0 & $2.9 \mathrm{E}-05$ & $7.8 \mathrm{E}-03$ & \\
\hline & & Neptunium & & & $3.4 \mathrm{E}-04$ & 8.8 & 1.3E-06 & 9.0E-02 & \\
\hline & & Plutonium & & & 1.3E-05 & 10.0 & 3.3E-08 & 4.7E-03 & \\
\hline & & Americium & & & 3.4E-05 & 9.0 & $1.2 \mathrm{E}-07$ & 9.9E-03 & \\
\hline & \multirow{16}{*}{$\begin{array}{l}\text { Animal product } \\
\text { transfer } \\
\text { coefficients for } \\
\text { milk }\end{array}$} & Chlorine & \multirow[t]{16}{*}{ Lognormal } & \multirow[t]{16}{*}{$d / L$} & $1.8 \mathrm{E}-02$ & 2.0 & $2.9 \mathrm{E}-03$ & 1.0E-01 & \multirow{16}{*}{$\begin{array}{l}\text { Transfer coefficients are given } \\
\text { in geometric mean and } \\
\text { geometric standard deviation. } \\
\text { The lower and upper bounds } \\
\text { are } 0.01 \text { and } 0.99 \text { percentile } \\
\text { values. (BSC } 2003 \\
\text { [DIRS 160964]) }\end{array}$} \\
\hline & & Selenium & & & 5.7E-03 & 2.5 & 5.5E-04 & 6.0E-02 & \\
\hline & & Strontium & & & 1.7E-03 & 2.0 & $2.8 \mathrm{E}-04$ & 1.0E-02 & \\
\hline & & Technetium & & & 2.1E-03 & 6.0 & 2.0E-05 & $2.1 \mathrm{E}-01$ & \\
\hline & & Tin & & & 1.1E-03 & 2.0 & $1.8 \mathrm{E}-04$ & $6.3 \mathrm{E}-03$ & \\
\hline & & \begin{tabular}{|l|} 
Iodine \\
\end{tabular} & & & $9.1 \mathrm{E}-03$ & 2.0 & $1.5 \mathrm{E}-03$ & $5.4 \mathrm{E}-02$ & \\
\hline & & Cesium & & & 7.7E-03 & 2.0 & $1.3 \mathrm{E}-03$ & $4.6 \mathrm{E}-02$ & \\
\hline & & Lead & & & 1.7E-04 & 3.0 & 1.0E-05 & $2.9 \mathrm{E}-03$ & \\
\hline & & Radium & & & $5.8 \mathrm{E}-04$ & 2.0 & 1.0E-04 & 3.4E-03 & \\
\hline & & Actinium & & & 7.6E-06 & 4.1 & 2.0E-07 & $2.9 \mathrm{E}-04$ & \\
\hline & & \begin{tabular}{|l|} 
Thorium \\
\end{tabular} & & & 4.4E-06 & 2.0 & 7.4E-07 & 2.6E-05 & \\
\hline & & Protactinium & & & 4.4E-06 & 2.0 & 7.4E-07 & $2.6 \mathrm{E}-05$ & \\
\hline & & \begin{tabular}{|l|} 
Uranium \\
\end{tabular} & & & 4.9E-04 & 2.0 & 8.1E-05 & $2.9 \mathrm{E}-03$ & \\
\hline & & Neptunium & & & $6.3 E-06$ & 2.0 & 1.0E-06 & 3.9E-05 & \\
\hline & & \begin{tabular}{|l|} 
Plutonium \\
\end{tabular} & & & $2.3 \mathrm{E}-07$ & 7.7 & $1.2 \mathrm{E}-09$ & 4.4E-05 & \\
\hline & & Americium & & & 1.6E-06 & 4.2 & 3.9E-08 & 6.3E-05 & \\
\hline
\end{tabular}


Table 6.6-3. Summary of Representative Input Parameter Values and Their Distributions (Continued)

\begin{tabular}{|c|c|c|c|c|c|c|c|c|c|}
\hline $\begin{array}{l}\text { Sub- } \\
\text { model }\end{array}$ & \multicolumn{2}{|c|}{ Parameter Name } & $\begin{array}{c}\text { Distribution } \\
\text { Type }\end{array}$ & Units & $\begin{array}{c}\text { Mean, } \\
\text { Mode, } \\
\text { Average }^{\mathrm{a}}\end{array}$ & SDISE $^{a}$ & Min. ${ }^{b}$ & Max. $^{b}$ & Notes $^{c}$ \\
\hline \multirow{36}{*}{$\begin{array}{l}\text { Animal } \\
\text { (Continued) }\end{array}$} & \multirow{16}{*}{$\begin{array}{l}\text { Animal product } \\
\text { transfer } \\
\text { coefficients for } \\
\text { poultry }\end{array}$} & Chlorine & \multirow[t]{16}{*}{ Lognormal } & \multirow[t]{16}{*}{$\mathrm{d} / \mathrm{kg}$} & 3.0E-02 & 2.0 & 5.0E-03 & 1.8E-01 & \multirow{16}{*}{$\begin{array}{l}\text { Transfer coefficients are given } \\
\text { in geometric mean and } \\
\text { geometric standard deviation. } \\
\text { The lower and upper bounds } \\
\text { are } 0.01 \text { and } 0.99 \text { percentile } \\
\text { values. (BSC } 2003 \\
\text { [DIRS 160964]) }\end{array}$} \\
\hline & & Selenium & & & $5.1 \mathrm{E}+00$ & 3.6 & $1.9 \mathrm{E}-01$ & $1.4 \mathrm{E}+02$ & \\
\hline & & Strontium & & & 3.1E-02 & 5.8 & 3.4E-04 & $2.9 \mathrm{E}+00$ & \\
\hline & & Technetium & & & $6.3 E-02$ & 10.0 & 1.7E-04 & $2.4 \mathrm{E}+01$ & \\
\hline & & Tin & & & $3.5 \mathrm{E}-02$ & 10.0 & 9.4E-05 & $1.3 \mathrm{E}+01$ & \\
\hline & & Iodine & & & 5.5E-02 & 9.7 & 1.6E-04 & $1.9 \mathrm{E}+01$ & \\
\hline & & Cesium & & & $2.6 \mathrm{E}+00$ & 9.8 & $7.2 \mathrm{E}-03$ & $9.3 \mathrm{E}+02$ & \\
\hline & & Lead & & & $2.5 \mathrm{E}-02$ & 10.0 & 6.6E-05 & $9.3 \mathrm{E}+00$ & \\
\hline & & Radium & & & 1.7E-02 & 10.0 & 4.4E-05 & $6.3 \mathrm{E}+00$ & \\
\hline & & Actinium & & & 4.0E-03 & 2.0 & 6.7E-04 & $2.4 \mathrm{E}-02$ & \\
\hline & & Thorium & & & 5.9E-03 & 8.0 & 2.7E-05 & $1.3 \mathrm{E}+00$ & \\
\hline & & Protactinium & & & 3.0E-03 & 2.0 & 5.1E-04 & $1.8 \mathrm{E}-02$ & \\
\hline & & Uranium & & & 2.4E-01 & 10.0 & $6.5 \mathrm{E}-04$ & $9.2 \mathrm{E}+01$ & \\
\hline & & Neptunium & & & 3.6E-03 & 2.0 & 6.0E-04 & 2.1E-02 & \\
\hline & & Plutonium & & & $1.2 \mathrm{E}-03$ & 10.0 & $3.2 \mathrm{E}-06$ & 4.6E-01 & \\
\hline & & Americium & & & $1.8 \mathrm{E}-03$ & 10.0 & $4.8 \mathrm{E}-06$ & $6.7 \mathrm{E}-01$ & \\
\hline & \multirow{16}{*}{$\begin{array}{l}\text { Animal product } \\
\text { transfer } \\
\text { coefficients for } \\
\text { eggs }\end{array}$} & Chlorine & \multirow[t]{16}{*}{ Lognormal } & \multirow[t]{16}{*}{$\mathrm{d} / \mathrm{kg}$} & $4.4 \mathrm{E}-02$ & 10.0 & 1.2E-04 & $1.7 \mathrm{E}+01$ & \multirow{16}{*}{$\begin{array}{l}\text { Transfer coefficients are given } \\
\text { in geometric mean and } \\
\text { geometric standard deviation. } \\
\text { The lower and upper bounds } \\
\text { are } 0.01 \text { and } 0.99 \text { percentile } \\
\text { values. (BSC } 2003 \\
\text { [DIRS 160964]) }\end{array}$} \\
\hline & & Selenium & & & $7.3 \mathrm{E}+00$ & 2.0 & $1.2 \mathrm{E}+00$ & $4.4 \mathrm{E}+01$ & \\
\hline & & Strontium & & & 2.7E-01 & 2.0 & 4.5E-02 & $1.6 \mathrm{E}+00$ & \\
\hline & & Technetium & & & $2.4 \mathrm{E}+00$ & 2.0 & 4.0E-01 & $1.4 \mathrm{E}+01$ & \\
\hline & & Tin & & & 8.7E-02 & 10.0 & $2.3 \mathrm{E}-04$ & $3.3 \mathrm{E}+01$ & \\
\hline & & Iodine & & & $2.6 \mathrm{E}+00$ & 2.0 & $4.4 \mathrm{E}-01$ & $1.6 \mathrm{E}+01$ & \\
\hline & & Cesium & & & 5.9E-01 & 2.3 & $7.2 \mathrm{E}-02$ & $4.8 \mathrm{E}+00$ & \\
\hline & & Lead & & & 5.6E-02 & 10.0 & 1.5E-04 & $2.1 \mathrm{E}+01$ & \\
\hline & & Radium & & & $3.9 \mathrm{E}-04$ & 10.0 & $1.0 \mathrm{E}-06$ & $1.5 \mathrm{E}-01$ & \\
\hline & & Actinium & & & 2.9E-03 & 2.3 & 3.4E-04 & $2.5 \mathrm{E}-02$ & \\
\hline & & Thorium & & & 3.5E-03 & 7.3 & 2.0E-05 & 5.9E-01 & \\
\hline & & Protactinium & & & 2.0E-03 & 2.0 & 3.4E-04 & $1.2 \mathrm{E}-02$ & \\
\hline & & Uranium & & & $6.3 \mathrm{E}-01$ & 2.5 & $6.0 \mathrm{E}-02$ & $6.7 \mathrm{E}+00$ & \\
\hline & & Neptunium & & & 3.4E-03 & 2.4 & 3.4E-04 & 3.3E-02 & \\
\hline & & Plutonium & & & 1.7E-03 & 7.4 & 9.7E-06 & 2.9E-01 & \\
\hline & & Americium & & & 4.9E-03 & 2.0 & $8.2 \mathrm{E}-04$ & 2.9E-02 & \\
\hline & \multirow{4}{*}{$\begin{array}{l}\text { Animal } \\
\text { consumption rate } \\
\text { of feed }\end{array}$} & Meat & \multirow[t]{4}{*}{ Uniform } & \multirow[t]{4}{*}{$\mathrm{kg} / \mathrm{d}$} & 48.5 & - & 29 & 68 & \multirow{4}{*}{$\begin{array}{l}\text { Mean calculated from uniform } \\
\text { distribution. (BSC } 2003 \\
\text { [DIRS 160964]) }\end{array}$} \\
\hline & & Milk & & & 61.5 & - & 50 & 73 & \\
\hline & & Poultry & & & 0.26 & - & 0.12 & 0.40 & \\
\hline & & Eggs & & & 0.26 & - & 0.12 & 0.40 & \\
\hline
\end{tabular}


Table 6.6-3. Summary of Representative Input Parameter Values and Their Distributions (Continued)

\begin{tabular}{|c|c|c|c|c|c|c|c|c|c|}
\hline $\begin{array}{l}\text { Sub- } \\
\text { model }\end{array}$ & \multicolumn{2}{|c|}{ Parameter Name } & $\begin{array}{l}\text { Distribution } \\
\text { Type }\end{array}$ & Units & $\begin{array}{c}\text { Mean, } \\
\text { Mode, } \\
\text { Average }^{\text {a }}\end{array}$ & SDISE $^{a}$ & Min. ${ }^{b}$ & Max. $^{b}$ & Notes $^{c}$ \\
\hline \multirow{8}{*}{$\begin{array}{l}\text { Animal } \\
\text { (Continued) }\end{array}$} & \multirow{4}{*}{$\begin{array}{l}\text { Animal } \\
\text { consumption rate } \\
\text { of water }\end{array}$} & Meat & Fixed & \multirow[t]{4}{*}{$\mathrm{L} / \mathrm{d}$} & 60 & - & - & - & \multirow{4}{*}{$\begin{array}{l}\text { Mean calculated from uniform } \\
\text { distribution. (BSC } 2003 \\
\text { [DIRS 160964]) }\end{array}$} \\
\hline & & Milk & Uniform & & 80 & - & 60 & 100 & \\
\hline & & Poultry & Fixed & & 0.5 & - & - & - & \\
\hline & & Eggs & Fixed & & 0.5 & - & - & - & \\
\hline & \multirow{4}{*}{$\begin{array}{l}\text { Animal } \\
\text { consumption rate } \\
\text { of soil }\end{array}$} & Meat & \multirow[t]{4}{*}{ Uniform } & \multirow[t]{4}{*}{$\mathrm{kg} / \mathrm{d}$} & 0.7 & - & 0.4 & 1.0 & \multirow{4}{*}{$\begin{array}{l}\text { Mean calculated from uniform } \\
\text { distribution. (BSC } 2003 \\
\text { [DIRS 160964]) }\end{array}$} \\
\hline & & Milk & & & 0.95 & - & 0.8 & 1.1 & \\
\hline & & Poultry & & & 0.02 & - & 0.01 & 0.03 & \\
\hline & & Eggs & & & 0.02 & - & 0.01 & 0.03 & \\
\hline \multirow[t]{21}{*}{ Fish } & \multirow{17}{*}{$\begin{array}{l}\text { Bioaccumulation } \\
\text { factor }\end{array}$} & Carbon & \multirow[t]{17}{*}{ Lognormal } & \multirow[t]{17}{*}{$\mathrm{L} / \mathrm{kg}$} & $4.6 \mathrm{E}+03$ & 3.2 & $2.3 \mathrm{E}+02$ & $9.2 \mathrm{E}+04$ & \multirow{17}{*}{$\begin{array}{l}\text { Bioaccumulation factors are } \\
\text { given in geometric mean and } \\
\text { geometric standard deviation. } \\
\text { The lower and upper bounds } \\
\text { are } 0.01 \text { and } 0.99 \text { percentile } \\
\text { values. (BSC } 2003 \\
\text { [DIRS 160964]) }\end{array}$} \\
\hline & & Chlorine & & & $2.2 \mathrm{E}+02$ & 5.6 & $2.6 \mathrm{E}+00$ & $1.9 \mathrm{E}+04$ & \\
\hline & & Selenium & & & $2.3 E+02$ & 2.0 & $3.9 \mathrm{E}+01$ & $1.2 \mathrm{E}+03$ & \\
\hline & & Strontium & & & $4.6 \mathrm{E}+01$ & 2.0 & $7.8 \mathrm{E}+00$ & $2.8 \mathrm{E}+02$ & \\
\hline & & Technetium & & & $2.0 \mathrm{E}+01$ & 2.0 & $3.3 \mathrm{E}+00$ & $1.2 \mathrm{E}+02$ & \\
\hline & & Tin & & & $2.5 \mathrm{E}+03$ & 2.0 & $4.2 \mathrm{E}+02$ & $1.5 \mathrm{E}+04$ & \\
\hline & & Iodine & & & $4.5 \mathrm{E}+01$ & 2.6 & $3.8 \mathrm{E}+00$ & $5.3 E+02$ & \\
\hline & & Cesium & & & $3.5 \mathrm{E}+03$ & 2.2 & $4.7 \mathrm{E}+02$ & $2.5 E+04$ & \\
\hline & & Lead & & & $2.9 \mathrm{E}+02$ & 2.5 & $2.7 \mathrm{E}+01$ & $3.1 \mathrm{E}+03$ & \\
\hline & & Radium & & & $6.7 \mathrm{E}+01$ & 2.2 & $9.2 E+00$ & $5.0 \mathrm{E}+02$ & \\
\hline & & Actinium & & & $2.9 \mathrm{E}+01$ & 3.0 & $1.7 \mathrm{E}+00$ & $5.0 \mathrm{E}+02$ & \\
\hline & & Thorium & & & $1.1 \mathrm{E}+02$ & 2.5 & $1.0 \mathrm{E}+01$ & $1.2 \mathrm{E}+03$ & \\
\hline & & Protactinium & & & $1.2 \mathrm{E}+01$ & 2.0 & $2.0 \mathrm{E}+00$ & $7.1 \mathrm{E}+01$ & \\
\hline & & Uranium & & & $1.4 \mathrm{E}+01$ & 3.0 & $8.4 \mathrm{E}-01$ & $2.3 E+02$ & \\
\hline & & Neptunium & & & $3.0 \mathrm{E}+01$ & 2.9 & $1.9 \mathrm{E}+00$ & $4.7 E+02$ & \\
\hline & & Plutonium & & & $4.1 \mathrm{E}+01$ & 4.7 & 7.9E-01 & $2.2 \mathrm{E}+03$ & \\
\hline & & Americium & & & $5.2 \mathrm{E}+01$ & 2.3 & $5.8 \mathrm{E}+00$ & $4.6 \mathrm{E}+02$ & \\
\hline & \multirow{2}{*}{$\begin{array}{l}\text { Modifying factor } \\
\text { for present-day } \\
\text { climate }\end{array}$} & Carbon & Fixed & - & 1 & - & - & - & \multirow{4}{*}{$\begin{array}{l}\text { Developed based on estimates } \\
\text { of water evaporation in } \\
\text { fishpond. Mean calculated } \\
\text { from uniform distribution. } \\
\text { (BSC } 2003 \text { [DIRS 160964]) }\end{array}$} \\
\hline & & Other elements & Uniform & - & 4.15 & - & 2.2 & 6.1 & \\
\hline & \multirow{2}{*}{$\begin{array}{l}\text { Modifying factor } \\
\text { for future climate }\end{array}$} & Carbon & Fixed & - & 1 & - & - & - & \\
\hline & & Other elements & Uniform & - & 2.4 & - & 1.5 & 3.3 & \\
\hline
\end{tabular}


Table 6.6-3. Summary of Representative Input Parameter Values and Their Distributions (Continued)

\begin{tabular}{|c|c|c|c|c|c|c|c|c|c|}
\hline $\begin{array}{c}\text { Sub- } \\
\text { model }\end{array}$ & \multicolumn{2}{|c|}{ Parameter Name } & $\begin{array}{c}\text { Distribution } \\
\text { Type }\end{array}$ & Units & $\begin{array}{l}\text { Mean, } \\
\text { Mode, } \\
\text { Average }^{\text {a }}\end{array}$ & SDISE $^{a}$ & Min. ${ }^{b}$ & Max. ${ }^{b}$ & Notes $^{c}$ \\
\hline \multirow[t]{20}{*}{${ }^{14} \mathrm{C}$} & \multicolumn{2}{|c|}{${ }^{14} \mathrm{C}$ emission rate } & Fixed & /yr & 22 & - & - & - & $\begin{array}{l}\text { Fixed value for sandy soil. } \\
\text { (BSC } 2003 \text { [DIRS 160964]) }\end{array}$ \\
\hline & \multicolumn{2}{|c|}{ Annual total groundwater usage } & Fixed & $\mathrm{m}^{3} / \mathrm{yr}$ & 3714450 & - & - & - & $\begin{array}{l}\text { From } 10 \text { CFR } 63 \text {, contaminated } \\
\text { groundwater volume. }\end{array}$ \\
\hline & \multirow{2}{*}{$\begin{array}{l}\text { Annual average } \\
\text { wind speed }\end{array}$} & For inhalation & \multirow[t]{2}{*}{ Uniform } & \multirow[t]{2}{*}{$\mathrm{m} / \mathrm{sec}$} & 2.45 & - & 2.1 & 2.8 & \multirow{2}{*}{$\begin{array}{l}\text { Wind speed at } 1 \mathrm{~m} \text { for crops } \\
\text { and } 2 \mathrm{~m} \text { for inhalation. (BSC } \\
2003 \text { [DIRS 160964]) }\end{array}$} \\
\hline & & For crops & & & 1.9 & - & 1.5 & 2.3 & \\
\hline & \multirow[t]{2}{*}{${ }^{14} \mathrm{C}$ mixing height } & For inhalation & \multirow[t]{2}{*}{ Fixed } & \multirow[t]{2}{*}{$\mathrm{m}$} & 2 & - & - & - & \multirow{4}{*}{$\begin{array}{l}\text { Fixed values are from RESRAD } \\
\text { code. } \\
\text { (BSC } 2003 \text { [DIRS 160964]) }\end{array}$} \\
\hline & & For crops & & & 1 & - & - & - & \\
\hline & \multicolumn{2}{|c|}{$\begin{array}{l}\text { Fraction of air-derived carbon in } \\
\text { plants }\end{array}$} & Fixed & - & 0.98 & - & - & - & \\
\hline & \multicolumn{2}{|c|}{$\begin{array}{l}\text { Fraction of soil-derived carbon in } \\
\text { plants }\end{array}$} & Fixed & - & 0.02 & - & - & - & \\
\hline & \multirow[t]{5}{*}{$\begin{array}{l}\text { Fraction of stable } \\
\text { carbon in plant }\end{array}$} & \begin{tabular}{|l} 
Leafy \\
vegetation
\end{tabular} & \multirow[t]{5}{*}{ Fixed } & \multirow[t]{5}{*}{-} & 0.09 & - & - & - & \multirow{12}{*}{$\begin{array}{l}\text { Fixed values are from RESRAD } \\
\text { and GENII/GENII-S codes. } \\
\text { (BSC } 2003 \text { [DIRS 160964]) }\end{array}$} \\
\hline & & $\begin{array}{l}\text { Other } \\
\text { vegetation }\end{array}$ & & & 0.09 & - & - & - & \\
\hline & & Fruit & & & 0.09 & - & - & - & \\
\hline & & Grain & & & 0.40 & - & - & - & \\
\hline & & Forage & & & 0.09 & - & - & - & \\
\hline & \multicolumn{2}{|c|}{ Fraction of stable carbon in soil } & Fixed & - & 0.03 & - & - & - & \\
\hline & \multicolumn{2}{|c|}{$\begin{array}{l}\text { Concentration of stable carbon in } \\
\text { air }\end{array}$} & Fixed & $\mathrm{kg} / \mathrm{m}^{3}$ & $1.8 \mathrm{E}-4$ & - & - & - & \\
\hline & \multicolumn{2}{|c|}{$\begin{array}{l}\text { Concentration of stable carbon in } \\
\text { water }\end{array}$} & Fixed & $\mathrm{kg} / \mathrm{L}$ & $2.0 \mathrm{E}-5$ & - & - & - & \\
\hline & \multirow{4}{*}{$\begin{array}{l}\text { Fraction of stable } \\
\text { carbon in animal } \\
\text { product }\end{array}$} & Meat & \multirow[t]{4}{*}{ Fixed } & \multirow[t]{4}{*}{-} & 0.24 & - & - & - & \\
\hline & & Milk & & & 0.07 & - & - & - & \\
\hline & & Poultry & & & 0.20 & - & - & - & \\
\hline & & Eggs & & & 0.15 & - & - & - & \\
\hline \multirow[t]{8}{*}{$\begin{array}{l}\text { External } \\
\text { Exposure }\end{array}$} & \multirow{4}{*}{$\begin{array}{l}\text { Population } \\
\text { proportion for } \\
\text { groundwater } \\
\text { scenario }\end{array}$} & $\begin{array}{l}\text { Outdoor } \\
\text { workers }\end{array}$ & \multirow[t]{4}{*}{ Uniform } & \multirow[t]{4}{*}{$\%$} & 5.5 & 2.6 & 2.9 & 8.1 & \multirow{8}{*}{$\begin{array}{l}\text { Based on census data. Indoor } \\
\text { worker data calculated in the } \\
\text { submodel as one minus the } \\
\text { sum of the other three } \\
\text { percentages. (BSC } 2003 \\
\text { [DIRS 161241]) }\end{array}$} \\
\hline & & Indoor workers $^{\text {d }}$ & & & $16.1^{\mathrm{d}}$ & d & - & - & \\
\hline & & Commuters & & & 39.2 & 5.3 & 33.9 & 44.5 & \\
\hline & & Nonworkers & & & 39.2 & 4.8 & 34.4 & 44.0 & \\
\hline & \multirow{4}{*}{$\begin{array}{l}\text { Population } \\
\text { proportion for } \\
\text { volcanic ash } \\
\text { scenario }\end{array}$} & $\begin{array}{l}\text { Outdoor } \\
\text { workers }\end{array}$ & Uniform & $\%$ & 5.5 & 2.6 & 2.9 & 10.7 & \\
\hline & & Indoor workers $^{\mathrm{d}}$ & & & $42.8^{\mathrm{d}}$ & d & - & - & \\
\hline & & Commuters & & & 12.5 & 3.8 & 4.9 & 16.3 & \\
\hline & & Nonworkers & & & 39.2 & 4.8 & 34.4 & 44.0 & \\
\hline
\end{tabular}


Table 6.6-3. Summary of Representative Input Parameter Values and Their Distributions (Continued)

\begin{tabular}{|c|c|c|c|c|c|c|c|c|c|c|}
\hline $\begin{array}{l}\text { Sub- } \\
\text { model }\end{array}$ & & Paramet & er Name & $\begin{array}{c}\text { Distribution } \\
\text { Type }\end{array}$ & Units & $\begin{array}{c}\text { Mean, } \\
\text { Mode, } \\
\text { Average }^{\text {a }}\end{array}$ & SDISE $^{a}$ & Min. ${ }^{b}$ & Max. ${ }^{b}$ & Notes $^{c}$ \\
\hline \multirow{30}{*}{$\begin{array}{l}\text { External } \\
\text { Exposure } \\
\text { (Continued) }\end{array}$} & \multirow{20}{*}{$\begin{array}{l}\text { For } \\
\text { GW }\end{array}$} & \multirow{5}{*}{$\begin{array}{l}\text { Exposure } \\
\text { time for } \\
\text { outdoor } \\
\text { workers }\end{array}$} & Active outdoors & \multirow[t]{5}{*}{ Lognormal } & \multirow[t]{5}{*}{$\mathrm{hr} / \mathrm{d}$} & 3.1 & 0.2 & 2.6 & 3.7 & \multirow{20}{*}{$\begin{array}{l}\text { Activity time budget, time for } \\
\text { active indoors is calculated in } \\
\text { the submodel as } 24 \mathrm{hr} / \mathrm{d} \text { minus } \\
\text { the sum of times in the other } \\
\text { four environments. (BSC } 2003 \\
\text { [DIRS 161241]) }\end{array}$} \\
\hline & & & \begin{tabular}{|l|} 
Inactive \\
outdoors
\end{tabular} & & & 4.0 & 0.3 & 3.3 & 4.8 & \\
\hline & & & Active indoors $^{\mathrm{d}}$ & & & $6.6^{\mathrm{d}}$ & d & - & - & \\
\hline & & & Asleep indoors & & & 8.3 & 0.1 & 8.0 & 8.6 & \\
\hline & & & Away from area & & & 2.0 & 0.4 & 1.2 & 3.3 & \\
\hline & & \multirow{5}{*}{$\begin{array}{l}\text { Exposure } \\
\text { time for } \\
\text { indoor } \\
\text { workers }\end{array}$} & Active outdoors & \multirow[t]{5}{*}{ Lognormal } & \multirow[t]{5}{*}{$\mathrm{hr} / \mathrm{d}$} & 0.3 & 0.1 & 0.1 & 0.7 & \\
\hline & & & \begin{tabular}{|l|} 
Inactive \\
outdoors \\
\end{tabular} & & & 1.3 & 0.2 & 0.9 & 1.9 & \\
\hline & & & Active indoors $^{\mathrm{d}}$ & & & $12.1^{d}$ & d & - & - & \\
\hline & & & Asleep indoors & & & 8.3 & 0.1 & 8.0 & 8.6 & \\
\hline & & & Away from area & & & 2.0 & 0.4 & 1.2 & 3.3 & \\
\hline & & \multirow{5}{*}{$\begin{array}{l}\text { Exposure } \\
\text { time for } \\
\text { com- } \\
\text { muters }\end{array}$} & Active outdoors & \multirow[t]{5}{*}{ Lognormal } & \multirow[t]{5}{*}{$\mathrm{hr} / \mathrm{d}$} & 0.3 & 0.1 & 0.1 & 0.7 & \\
\hline & & & \begin{tabular}{|l|} 
Inactive \\
outdoors \\
\end{tabular} & & & 1.4 & 0.2 & 1.0 & 2.0 & \\
\hline & & & Active indoors $^{d}$ & & & $6.0^{\mathrm{d}}$ & ${ }^{d}$ & - & - & \\
\hline & & & Asleep indoors & & & 8.3 & 0.1 & 8.0 & 8.6 & \\
\hline & & & Away from area & & & 8.0 & 0.5 & 6.8 & 9.4 & \\
\hline & & \multirow{5}{*}{\begin{tabular}{|l|} 
Exposure \\
time for \\
non \\
workers
\end{tabular}} & Active outdoors & \multirow[t]{5}{*}{ Lognormal } & \multirow[t]{5}{*}{$\mathrm{hr} / \mathrm{d}$} & 0.3 & 0.1 & 0.1 & 0.7 & \\
\hline & & & $\begin{array}{l}\text { Inactive } \\
\text { outdoors }\end{array}$ & & & 1.2 & 0.2 & 0.8 & 1.8 & \\
\hline & & & Active indoors $^{\mathrm{d}}$ & & & $12.2^{\mathrm{d}}$ & $\mathrm{d}$ & - & - & \\
\hline & & & Asleep indoors & & & 8.3 & 0.1 & 8.0 & 8.6 & \\
\hline & & & Away from area & & & 2.0 & 0.4 & 1.2 & 3.3 & \\
\hline & \multirow{10}{*}{\begin{tabular}{|l} 
For \\
VA
\end{tabular}} & \multirow{5}{*}{$\begin{array}{l}\text { Exposure } \\
\text { time for } \\
\text { outdoor } \\
\text { workers }\end{array}$} & Active outdoors & \multirow[t]{5}{*}{ Lognormal } & \multirow[t]{5}{*}{$\mathrm{hr} / \mathrm{d}$} & 3.1 & 0.2 & 2.6 & 3.7 & \multirow{10}{*}{$\begin{array}{l}\text { Activity time budget, time for } \\
\text { active indoors is calculated in } \\
\text { the submodel as } 24 \mathrm{hr} / \mathrm{d} \text { minus } \\
\text { the sum of times in the other } \\
\text { four environments. (BSC } 2003 \\
\text { [DIRS 161241]) }\end{array}$} \\
\hline & & & \begin{tabular}{|l} 
Inactive \\
outdoors
\end{tabular} & & & 4.2 & 0.3 & 3.5 & 5.0 & \\
\hline & & & Active indoors $^{\mathrm{d}}$ & & & $6.4^{\mathrm{d}}$ & d & - & - & \\
\hline & & & Asleep indoors & & & 8.3 & 0.1 & 8.0 & 8.6 & \\
\hline & & & Away from area & & & 2.0 & 0.4 & 1.2 & 3.3 & \\
\hline & & \multirow{5}{*}{$\begin{array}{l}\text { Exposure } \\
\text { time for } \\
\text { indoor } \\
\text { workers }\end{array}$} & Active outdoors & \multirow[t]{5}{*}{ Lognormal } & \multirow[t]{5}{*}{$\mathrm{hr} / \mathrm{d}$} & 0.3 & 0.1 & 0.1 & 0.7 & \\
\hline & & & \begin{tabular}{|l|} 
Inactive \\
outdoors
\end{tabular} & & & 1.5 & 0.2 & 1.1 & 2.1 & \\
\hline & & & Active indoors $^{\mathrm{d}}$ & & & $11.9^{d}$ & d & - & - & \\
\hline & & & Asleep indoors & & & 8.3 & 0.1 & 8.0 & 8.6 & \\
\hline & & & Away from area & & & 2.0 & 0.4 & 1.2 & 3.3 & \\
\hline
\end{tabular}


Table 6.6-3. Summary of Representative Input Parameter Values and Their Distributions (Continued)

\begin{tabular}{|c|c|c|c|c|c|c|c|c|c|c|}
\hline $\begin{array}{l}\text { Sub- } \\
\text { model }\end{array}$ & \multicolumn{3}{|c|}{ Parameter Name } & $\begin{array}{c}\text { Distribution } \\
\text { Type }\end{array}$ & Units & $\begin{array}{c}\text { Mean, } \\
\text { Mode, } \\
\text { Average a }\end{array}$ & SDISE $^{a}$ & Min. ${ }^{b}$ & Max. ${ }^{b}$ & Notes $^{c}$ \\
\hline \multirow{34}{*}{$\begin{array}{l}\text { External } \\
\text { Exposure } \\
\text { (Continued) }\end{array}$} & \multirow{10}{*}{$\begin{array}{l}\text { For } \\
\text { VA }\end{array}$} & \multirow{5}{*}{$\begin{array}{l}\text { Exposure } \\
\text { time for } \\
\text { com- } \\
\text { muters }\end{array}$} & Active outdoors & \multirow[t]{5}{*}{ Lognormal } & \multirow[t]{5}{*}{$\mathrm{hr} / \mathrm{d}$} & 0.3 & 0.1 & 0.1 & 0.7 & \\
\hline & & & \begin{tabular}{|l|} 
Inactive \\
outdoors \\
\end{tabular} & & & 2.0 & 0.2 & 1.5 & 2.6 & \\
\hline & & & Active indoors $^{\mathrm{d}}$ & & & $5.1^{\mathrm{d}}$ & d & - & - & \\
\hline & & & Asleep indoors & & & 8.3 & 0.1 & 8.0 & 8.6 & \\
\hline & & & Away from area & & & 8.3 & 0.6 & 6.9 & 10.0 & \\
\hline & & \multirow{5}{*}{\begin{tabular}{|l} 
Exposure \\
time for \\
non \\
workers
\end{tabular}} & Active outdoors & \multirow[t]{5}{*}{ Lognormal } & \multirow[t]{5}{*}{$\mathrm{hr} / \mathrm{d}$} & 0.3 & 0.1 & 0.1 & 0.7 & \\
\hline & & & \begin{tabular}{|l|} 
Inactive \\
outdoors
\end{tabular} & & & 1.2 & 0.2 & 0.8 & 1.8 & \\
\hline & & & Active indoors $^{d}$ & & & $12.2^{\mathrm{d}}$ & d & - & - & \\
\hline & & & Asleep indoors & & & 8.3 & 0.1 & 8.0 & 8.6 & \\
\hline & & & Away from area & & & 2.0 & 0.4 & 1.2 & 3.3 & \\
\hline & \multirow{24}{*}{\multicolumn{2}{|c|}{$\begin{array}{l}\text { Building } \\
\text { shielding factor }\end{array}$}} & C-14 & \multirow[t]{24}{*}{ Fixed } & \multirow[t]{24}{*}{-} & 0.2 & - & - & - & \multirow{24}{*}{$\begin{array}{l}\text { External shielding factors } \\
\text { developed from literature data. } \\
\text { (BSC } 2003 \text { [DIRS 161241]) }\end{array}$} \\
\hline & & & $\mathrm{Cl}-36$ & & & 0.4 & - & - & - & \\
\hline & & & Se-79 & & & 0.1 & - & - & - & \\
\hline & & & Sr-90D & & & 0.4 & - & - & - & \\
\hline & & & Tc-99 & & & 0.2 & - & - & - & \\
\hline & & & Sn-126D & & & 0.4 & - & - & - & \\
\hline & & & $\mathrm{I}-129$ & & & 0.1 & - & - & - & \\
\hline & & & Cs-135 & & & 0.1 & - & - & - & \\
\hline & & & Cs-137D & & & 0.4 & - & - & - & \\
\hline & & & Pu-242 & & & 0.1 & - & - & - & \\
\hline & & & U-238D & & & 0.4 & - & - & - & \\
\hline & & & Pu-238 & & & 0.1 & - & - & - & \\
\hline & & & U-234 & & & 0.2 & - & - & - & \\
\hline & & & Th-230 & & & 0.3 & - & - & - & \\
\hline & & & Ra-226D & & & 0.4 & - & - & - & \\
\hline & & & Pb-210D & & & 0.4 & - & - & - & \\
\hline & & & Pu-240 & & & 0.1 & - & - & - & \\
\hline & & & U-236 & & & 0.1 & - & - & - & \\
\hline & & & Th-232 & & & 0.2 & - & - & - & \\
\hline & & & Ra-228D & & & 0.4 & - & - & - & \\
\hline & & & U-232 & & & 0.3 & - & - & - & \\
\hline & & & Th-228D & & & 0.4 & - & - & - & \\
\hline & & & Am-243D & & & 0.4 & - & - & - & \\
\hline & & & Pu-239 & & & 0.3 & - & - & - & \\
\hline
\end{tabular}


Table 6.6-3. Summary of Representative Input Parameter Values and Their Distributions (Continued)

\begin{tabular}{|c|c|c|c|c|c|c|c|c|c|}
\hline $\begin{array}{l}\text { Sub- } \\
\text { model }\end{array}$ & \multicolumn{2}{|c|}{ Parameter Name } & $\begin{array}{l}\text { Distribution } \\
\text { Type }\end{array}$ & Units & $\begin{array}{l}\text { Mean, } \\
\text { Mode, } \\
\text { Average }^{\text {a }}\end{array}$ & SD/SE ${ }^{a}$ & Min. ${ }^{b}$ & Max. ${ }^{b}$ & Notes ${ }^{c}$ \\
\hline \multirow{9}{*}{$\begin{array}{l}\text { External } \\
\text { Exposure } \\
\text { (Continued) }\end{array}$} & \multirow{7}{*}{$\begin{array}{l}\text { Building } \\
\text { shielding factor }\end{array}$} & U-235D & \multirow{7}{*}{ Fixed } & \multirow[t]{7}{*}{-} & 0.4 & - & - & - & \\
\hline & & $\mathrm{Pa}-231$ & & & 0.4 & - & - & - & \\
\hline & & Ac-227D & & & 0.4 & - & - & - & \\
\hline & & Am-241 & & & 0.2 & - & - & - & \\
\hline & & $\mathrm{Np}-237 \mathrm{D}$ & & & 0.4 & - & - & - & \\
\hline & & $\mathrm{U}-233$ & & & 0.4 & - & - & - & \\
\hline & & Th-229D & & & 0.4 & - & - & - & \\
\hline & \multicolumn{2}{|c|}{$\begin{array}{l}\text { Dose coefficient for exposure } \\
\text { contaminated to an infinite depth }\end{array}$} & Fixed & $\begin{array}{l}(\mathrm{Sv} / \mathrm{yr}) / \\
\left(\mathrm{Bq} / \mathrm{m}^{3}\right) \\
\end{array}$ & See Notes & - & - & - & (BSC 2003 [DIRS 161241]) \\
\hline & \multicolumn{2}{|c|}{$\begin{array}{l}\text { Dose coefficient for exposure to } \\
\text { contaminated ground surface }\end{array}$} & Fixed & $\begin{array}{l}(\mathrm{Sv} / \mathrm{yr}) / \\
\left(\mathrm{Bq} / \mathrm{m}^{2}\right)\end{array}$ & See Notes & - & - & - & (BSC 2003 [DIRS 161241]) \\
\hline & \multicolumn{2}{|c|}{ Branching fraction } & Fixed & - & See Notes & - & - & - & (BSC 2003 [DIRS 161241]) \\
\hline \multirow[t]{12}{*}{ Inhalation } & \multirow[t]{5}{*}{ Breathing rate } & Active outdoors & \multirow[t]{5}{*}{ Fixed } & \multirow[t]{5}{*}{$\mathrm{m}^{3} / \mathrm{hr}$} & 1.57 & - & - & - & \multirow[t]{5}{*}{ (BSC 2003 [DIRS 161241]). } \\
\hline & & $\begin{array}{l}\text { Inactive } \\
\text { outdoors }\end{array}$ & & & 1.08 & - & - & - & \\
\hline & & Active indoors & & & 1.08 & - & - & - & \\
\hline & & Asleep indoors & & & 0.39 & - & - & - & \\
\hline & & Away from area & & & 1.08 & - & - & - & \\
\hline & \multicolumn{2}{|c|}{$\begin{array}{l}\text { Dose conversion factor for } \\
\text { inhalation }\end{array}$} & Fixed & $\mathrm{Sv} / \mathrm{Bq}$ & See Notes & - & - & - & (BSC 2003 [DIRS 161241]). \\
\hline & \multicolumn{2}{|c|}{$\begin{array}{l}\text { Fraction of houses with } \\
\text { evaporative coolers }\end{array}$} & Binomial & - & 0.738 & $\begin{array}{l}\text { Sample } \\
\text { size }=187\end{array}$ & - & - & $\begin{array}{l}\text { Developed from site-specific } \\
\text { survey done in Amargosa }\end{array}$ \\
\hline & \multirow[t]{2}{*}{$\begin{array}{l}\text { Evaporative } \\
\text { cooler use factor }\end{array}$} & $\begin{array}{l}\text { Present-day } \\
\text { climate }\end{array}$ & \multirow[t]{2}{*}{ Uniform } & \multirow[t]{2}{*}{-} & 0.39 & - & 0.32 & 0.46 & \multirow{2}{*}{$\begin{array}{l}\text { Valley. Mean calculated from } \\
\text { uniform distribution. (BSC } 2003 \\
\text { [DIRS 161241]) }\end{array}$} \\
\hline & & Future climate & & & 0.085 & - & 0.03 & 0.14 & \\
\hline & \multirow{2}{*}{$\begin{array}{l}\text { Equilibrium } \\
\text { factor for }{ }^{222} \mathrm{Rn} \\
\text { decay products }\end{array}$} & Outdoors & Uniform & - & 0.6 & - & 0.5 & 0.7 & \multirow{2}{*}{$\begin{array}{l}\text { Mean calculated from uniform } \\
\text { distribution. (BSC } 2003 \\
\text { [DIRS 161241]) }\end{array}$} \\
\hline & & Indoors & Uniform & - & 0.4 & - & 0.3 & 0.5 & \\
\hline & \multicolumn{2}{|c|}{$\begin{array}{l}\text { Dose conversion factor for } \\
\text { inhalation of }{ }^{222} \mathrm{Rn} \text { decay products }\end{array}$} & Fixed & $\mathrm{Sv} / \mathrm{Bq}$ & $1.33 \mathrm{E}-8$ & - & - & - & $\begin{array}{l}\text { (BSC } 2003 \text { [DIRS 161241]), } \\
\text { see Equation 6.4.8-6. }\end{array}$ \\
\hline
\end{tabular}


Table 6.6-3. Summary of Representative Input Parameter Values and Their Distributions (Continued)

\begin{tabular}{|c|c|c|c|c|c|c|c|c|c|}
\hline $\begin{array}{l}\text { Sub- } \\
\text { model }\end{array}$ & \multicolumn{2}{|c|}{ Parameter Name } & $\begin{array}{l}\text { Distribution } \\
\text { Type }\end{array}$ & Units & $\begin{array}{c}\text { Mean, } \\
\text { Mode, } \\
\text { Average }^{\mathrm{a}}\end{array}$ & SDISE $^{a}$ & Min. $^{b}$ & Max. $^{b}$ & Notes $^{c}$ \\
\hline \multirow[t]{12}{*}{ Ingestion } & \multicolumn{2}{|c|}{ Consumption rate of water } & Fixed & $\mathrm{L} / \mathrm{d}$ & 2 & - & - & - & Required by 10 CFR 63.312 \\
\hline & \multirow{4}{*}{$\begin{array}{l}\text { Consumption } \\
\text { rate of locally } \\
\text { produced crop } \\
\text { foodstuffs }\end{array}$} & $\begin{array}{l}\text { Leafy } \\
\text { vegetables }\end{array}$ & \multirow[t]{4}{*}{ Lognormal } & \multirow[t]{4}{*}{$\mathrm{kg} / \mathrm{yr}$} & 3.78 & 0.88 & - & - & \multirow{9}{*}{$\begin{array}{l}\text { Developed based on site- } \\
\text { specific survey. Mean/Mode } \\
\text { column represents the mean } \\
\text { and SD/SE column represents } \\
\text { the standard deviation for } \\
\text { lognormal distribution. }\end{array}$} \\
\hline & & $\begin{array}{l}\text { Other } \\
\text { vegetables }\end{array}$ & & & 4.73 & 0.67 & - & - & \\
\hline & & Fruit & & & 12.68 & 1.36 & - & - & \\
\hline & & Grain & & & 0.23 & 0.11 & - & - & \\
\hline & \multirow{4}{*}{$\begin{array}{l}\text { Consumption } \\
\text { rate of locally } \\
\text { produced animal } \\
\text { products }\end{array}$} & Meat & \multirow[t]{4}{*}{ Lognormal } & \multirow[t]{4}{*}{$\mathrm{kg} / \mathrm{yr}$} & 2.85 & 0.65 & - & - & \\
\hline & & Milk & & & 4.66 & 1.68 & - & - & \\
\hline & & Poultry & & & 0.42 & 0.13 & - & - & \\
\hline & & Eggs & & & 5.30 & 0.83 & - & - & \\
\hline & \multicolumn{2}{|c|}{$\begin{array}{l}\text { Consumption rate of locally } \\
\text { produced fish }\end{array}$} & Lognormal & $\mathrm{kg} / \mathrm{yr}$ & 0.23 & 0.10 & - & - & \\
\hline & \multicolumn{2}{|c|}{ Inadvertent soil ingestion rate } & Cumulative & $\mathrm{mg} / \mathrm{d}$ & 100 & - & $\begin{array}{r}50 \\
100 \\
200 \\
\end{array}$ & $\begin{array}{r}0 \% \\
50 \% \\
100 \% \\
\end{array}$ & $\begin{array}{l}\text { Recommended by the U.S. } \\
\text { Environmental Protection } \\
\text { Agency. }\end{array}$ \\
\hline & \multicolumn{2}{|c|}{$\begin{array}{l}\text { Dose conversion factor for } \\
\text { ingestion }\end{array}$} & Fixed & Sv/Bq & See notes & - & - & - & $\begin{array}{l}\text { Based on FGR-11. Values } \\
\text { presented in Table 6.4-4. }\end{array}$ \\
\hline
\end{tabular}

a a The "Mean, Mode, Average" column represents the value for a fixed distribution, the mean for a normal or lognormal distribution, the mode for a triangular distribution, or the average value for distributions that do not require a mean, mode, or average, such as uniform distribution or cumulative distribution, in which values in this column are taken from the sources listed below or calculated as the 50th-percentile value. Data in this column are used in model verification (Section 6.10) and model validation (Sections 7.3 and 7.4). "SD/SE" represents the standard deviation or standard error for the described input distribution, such as normal distribution and lognormal distribution. However, for the transfer factors, transfer coefficients, and partition coefficients the "Mean, Mode, Average" represents the geometric mean and SD/SE represents geometric standard deviation for their lognormal distributions.

b "Min." represents the lower bounding value, and "Max." represents the upper bounding value for most distribution types, except for cumulative distributions, in which the "Min." column is the value, and the "Max." column is the corresponding accumulative percentage.

c Source: The data presented in this Table were taken from the original analyses documents, viz BSC 2003 [DIRS 160964]; BSC 2003 [DIRS 161239]; BSC 2003 [DIRS 160976]; BSC 2003 [DIRS 160965]; and BSC 2003 [DIRS 161241]. These documents have been revised to BSC 2004 [DIRS 169672]; BSC 2004 [DIRS 169459]; BSC 2004 [DIRS 169673]; BSC 2004 [DIRS 169458]; and BSC 2004 [DIRS 169671], respectively. As a result of the revision, there were a few minor changes to the input data. These changes are identified, discussed, and evaluated in BSC 2004 [DIRS 169674] for the nominal release scenario and BSC 2004 [DIRS 167287] for the volcanic scenario.

d Within the block of parameters, this parameter is the dependent variable whose expected value and distribution is determined by the defined distribution of the remaining parameters in the block and the necessary boundary conditions (such as 24 hours per day, percentage total having to be 100 percent).

$\mathrm{GW}=$ groundwater scenario, LA=license application; SD=standard deviation, SE=standard error; TSPA=total system performance assessment; VA=volcanic ash scenario 


\subsection{BIOSPHERE MODEL ENHANCEMENT}

The ERMYN, which is based on the previously used GENII-S model, incorporates several enhancements relative to the older model. The enhancements are discussed in this section. The disposition of included FEPs (Table 6.2-1) within the biosphere model is discussed in Section 6.7.1. The mathematical model (Sections 6.4 and 6.5) could not be implemented using the GENII-S code, used for the TSPA-SR, due to the incorporation of new exposure pathways, and a different model implementation tool is selected to accommodate the changes. In this section, the ERMYN and the previous model performed with GENII-S (Section 6.7.2) are compared. Justification for building the ERMYN in GoldSim is presented (Section 6.7.3).

\subsubsection{Disposition of FEPs within Biosphere Mathematical Model}

The FEPs considered in the biosphere conceptual model are discussed in Section 6.3.4. Table 6.7-1 describes how those FEPs are dispositioned in the mathematical model. Many of the FEPs (primarily features) are represented in the input parameters. For these FEPs, the related input parameters are identified and the disposition of the FEP through development and use of the input parameters is described (Table 6.7-1). Other FEPs (primarily events and processes) are incorporated into the equations described in Sections 6.4 and 6.5. For these FEPs, the submodels and equations related to the FEPs are identified, and the associated model parameters are listed. Some parameters address several FEPs, and one FEP may be linked to several parameters. Based on the evaluation, all FEPs considered in the conceptual model (Section 6.3.4) are adequately dispositioned in the ERMYN mathematical model.

Table 6.7-1. Disposition of the Included FEPs within the Biosphere Mathematical Model

\begin{tabular}{|c|c|c|c|c|}
\hline $\begin{array}{l}\text { LA FEP } \\
\text { Number }\end{array}$ & FEP Name & $\begin{array}{l}\text { Biosphere } \\
\text { Submodel }^{a}\end{array}$ & $\begin{array}{l}\text { Model Parameters that } \\
\text { Address the FEP }\end{array}$ & Disposition within ERMYN \\
\hline \multirow[t]{2}{*}{ 1.2.04.07.0A } & \multirow[t]{2}{*}{ Ashfall } & $\begin{array}{l}\text { Soil } \\
\text { Plant }\end{array}$ & $\begin{array}{l}\text { Radionuclide concentration in ash } \\
\text { deposited on ground surface } \\
\text { Ash bulk density } \\
\text { Soil to plant transfer factor }\end{array}$ & $\begin{array}{l}\text { Volcanic ash is the initial source } \\
\text { of contamination for the volcanic } \\
\text { ash exposure scenario } \\
\text { (Section 6.3.2). }\end{array}$ \\
\hline & & Air & $\begin{array}{l}\text { Mass loading for crops } \\
\text { Mass loading for receptor } \\
\text { environments } \\
\text { Mass loading time function }\end{array}$ & $\begin{array}{l}\text { Initial ashfall depth is considered } \\
\text { in development of the mass } \\
\text { loading parameters (BSC } 2004 \\
\text { [DIRS 169458], Section } 6.2 \text { and } \\
6.3 \text { ). }\end{array}$ \\
\hline \multirow[t]{5}{*}{ 1.3.01.00.0A } & \multirow[t]{5}{*}{$\begin{array}{l}\text { Climate } \\
\text { change }\end{array}$} & Soil & $\begin{array}{l}\text { Annual irrigation rate } \\
\text { Overwatering rate }\end{array}$ & \multirow{5}{*}{$\begin{array}{l}\text { Separate distributions developed } \\
\text { for listed parameters based on } \\
\text { present-day and predicted future } \\
\text { climatic conditions (BSC } 2004 \\
\text { [DIRS 169673], Sections } 6.4 \text {, } \\
6.5,6.7,6.8 \text { and } 6.9 \text {; BSC } 2004 \\
\text { [DIRS 169672], Sections } 6.4 \\
\text { and 6.7; BSC 2004 } \\
\text { [DIRS 169671], } \\
\text { Sections 6.3.4.2). Separate sets } \\
\text { of BDCFs are developed for the } \\
\text { present-day and future climates. }\end{array}$} \\
\hline & & Plant & $\begin{array}{l}\text { Growing time } \\
\text { Irrigation amount per application } \\
\text { Daily irrigation rate }\end{array}$ & \\
\hline & & Fish & $\begin{array}{l}\text { Water concentration modifying } \\
\text { factor }\end{array}$ & \\
\hline & & ${ }^{14} \mathrm{C}$ & $\begin{array}{l}\text { Annual irrigation rate } \\
\text { Daily irrigation rate } \\
\text { Surface area of irrigated land }\end{array}$ & \\
\hline & & Inhalation & Evaporative cooler use factor & \\
\hline
\end{tabular}


Table 6.7-1. Disposition of the Included FEPs within the Biosphere Mathematical Model (Continued)

\begin{tabular}{|c|c|c|c|c|}
\hline $\begin{array}{l}\text { LA FEP } \\
\text { Number }\end{array}$ & FEP Name & $\begin{array}{l}\text { Biosphere } \\
\text { Submodel }^{a}\end{array}$ & $\begin{array}{l}\text { Model Parameters that } \\
\text { Address the FEP }{ }^{b}\end{array}$ & Disposition within ERMYN \\
\hline 1.3.07.02.0A & $\begin{array}{l}\text { Water table } \\
\text { rise affects SZ }\end{array}$ & $\begin{array}{l}\text { Soil, Air, } \\
\text { Plant, }{ }^{14} \mathrm{C}, \\
\text { Animal, } \\
\text { Fish, } \\
\text { Ingestion }\end{array}$ & $\begin{array}{l}\text { Radionuclide concentration in } \\
\text { groundwater }\end{array}$ & $\begin{array}{l}\text { Conceptual and mathematical } \\
\text { models for the groundwater } \\
\text { scenario are applicable to } \\
\text { surface water flowing from a } \\
\text { spring or other discharge point } \\
\text { as the source of contaminants } \\
\text { (Section 6.3.1). }\end{array}$ \\
\hline \multirow[t]{2}{*}{ 1.4.07.01.0A } & \multirow{2}{*}{$\begin{array}{l}\text { Water } \\
\text { management } \\
\text { activities }\end{array}$} & Plant & $\begin{array}{l}\text { Fraction of overhead irrigation } \\
\text { Irrigation intensity }\end{array}$ & \multirow{2}{*}{$\begin{array}{l}\text { Distributions for the values of the } \\
\text { listed parameters are developed } \\
\text { based in part on the types of } \\
\text { water distribution and storage } \\
\text { systems in Amargosa Valley for } \\
\text { crop irrigation (BSC } 2004 \\
\text { [DIRS 169673], Sections } 6.3 \text { and } \\
\text { 6.6) and fish farming (BSC } 2004 \\
\text { [DIRS 169672], Section 6.4). }\end{array}$} \\
\hline & & Fish & $\begin{array}{l}\text { Water concentration modifying } \\
\text { factor }\end{array}$ & \\
\hline 1.4.07.02.0A & Wells & $\begin{array}{l}\text { Soil, Air, } \\
\text { Plant, }{ }^{14} \mathrm{C}, \\
\text { Animal, } \\
\text { Fish, } \\
\text { Ingestion }\end{array}$ & $\begin{array}{l}\text { Radionuclide concentration in } \\
\text { groundwater }\end{array}$ & $\begin{array}{l}\text { A well is initial source of } \\
\text { contaminated groundwater for } \\
\text { the groundwater scenario } \\
\text { (Section 6.3.1). }\end{array}$ \\
\hline \multirow[t]{5}{*}{ 2.2.08.01.0A } & \multirow{5}{*}{$\begin{array}{l}\text { Chemical } \\
\text { characteristics } \\
\text { of groundwater } \\
\text { in the SZ }\end{array}$} & Soil & Partition coefficient & \multirow{3}{*}{$\begin{array}{l}\text { Parameter distributions } \\
\text { developed to reflect possible } \\
\text { variations due to chemical } \\
\text { characteristics of groundwater in } \\
\text { SZ ( Sections } 6.4 .1,6.4 .3,6.4 .4 \text { ). }\end{array}$} \\
\hline & & Plant & Soil-to-plant transfer factor & \\
\hline & & $\begin{array}{l}\text { Animal } \\
\text { Fish }\end{array}$ & Animal product transfer coefficient & \\
\hline & & Ingestion & Dose conversion factors & \multirow{2}{*}{$\begin{array}{l}\text { Where multiple dose conversion } \\
\text { factors are defined, the largest } \\
\text { value is used to eliminate the } \\
\text { possibility of underestimating } \\
\text { dose (Sections } 6.4 .58 \text { and } \\
6.4 .9 .6 \text { ). }\end{array}$} \\
\hline & & Inhalation & Dose conversion factors & \\
\hline \multirow[t]{3}{*}{ 2.3.02.01.0A } & \multirow[t]{3}{*}{ Soil type } & Soil & $\begin{array}{l}\text { Surface soil depth (tillage depth) } \\
\text { Soil partition coefficient } \\
\text { Soil and ash bulk density } \\
\text { Surface soil erosion rate } \\
\text { Volumetric water content }\end{array}$ & \multirow{3}{*}{$\begin{array}{l}\text { Distributions for listed } \\
\text { parameters developed based in } \\
\text { part on characteristics of the soil } \\
\text { types in northern Amargosa } \\
\text { Valley (BSC 2004 } \\
\text { [DIRS 169673], Sections 6.6, } \\
6.10 \text { and 6.12; BSC } 2004 \\
\text { [DIRS 169459], Sections } 6.2, \\
6.4,6.6 \text {, and 6.7; and (BSC 2004 } \\
\text { [DIRS 169672], Section 6.7.1). }\end{array}$} \\
\hline & & Plant & Irrigation Intensity & \\
\hline & & ${ }^{14} \mathrm{C}$ & ${ }^{14} \mathrm{C}$ emission rate from soil & \\
\hline 2.3.02.02.0A & $\begin{array}{l}\text { Radionuclide } \\
\text { accumulation } \\
\text { in soils }\end{array}$ & Soil & $\begin{array}{l}\text { Annual irrigation rate } \\
\text { Overwatering rate } \\
\text { Surface soil depth (tillage depth) } \\
\text { Soil solid-liquid partition } \\
\text { coefficient } \\
\text { Soil bulk density } \\
\text { Volumetric water content } \\
\text { Surface soil erosion rate } \\
\text { Critical thickness for the } \\
\text { resuspension }\end{array}$ & $\begin{array}{l}\text { The soil submodel includes the } \\
\text { accumulation of radionuclides in } \\
\text { the soil from irrigation water } \\
\text { (Eq. } 6.4 .1-2 \text { and associated } \\
\text { equations). }\end{array}$ \\
\hline
\end{tabular}


Table 6.7-1. Disposition of the Included FEPs within the Biosphere Mathematical Model (Continued)

\begin{tabular}{|c|c|c|c|c|}
\hline $\begin{array}{l}\text { LA FEP } \\
\text { Number }\end{array}$ & FEP Name & $\begin{array}{l}\text { Biosphere } \\
\text { Submodel }^{a}\end{array}$ & $\begin{array}{l}\text { Model Parameters that } \\
\text { Address the FEP }{ }^{b}\end{array}$ & Disposition within ERMYN \\
\hline \multirow[t]{2}{*}{ 2.3.02.03.0A } & \multirow[t]{2}{*}{$\begin{array}{l}\text { Soil and } \\
\text { sediment } \\
\text { transport in the } \\
\text { biosphere }\end{array}$} & Soil & $\begin{array}{l}\text { Surface soil erosion rate } \\
\text { Soil bulk density } \\
\text { Dry deposition velocity } \\
\text { Critical thickness for resuspension }\end{array}$ & $\begin{array}{l}\text { The soil submodel includes soil } \\
\text { loss and gain on farm fields } \\
\text { (groundwater scenario, } \\
\text { Eq. 6.4.1-11; volcanic ash } \\
\text { scenario, Eq. 6.5.1-4). The } \\
\text { ERMYN does not develop } \\
\text { thickness of ash deposited on } \\
\text { the ground (Eq. 6.5.1-4). }\end{array}$ \\
\hline & & Air & $\begin{array}{l}\text { Mass loading decrease constant } \\
\text { in mass loading time function }\end{array}$ & $\begin{array}{l}\text { Selected distribution based in } \\
\text { part on the influence of ash } \\
\text { redistribution on changes in } \\
\text { mass loading through time (BSC } \\
2004 \text { [DIRS 169458], } \\
\text { Section 6.3). }\end{array}$ \\
\hline 2.3.04.01.0A & $\begin{array}{l}\text { Surface water } \\
\text { transport and } \\
\text { mixing }\end{array}$ & $\begin{array}{l}\text { Soil, Air, } \\
\text { Plant, }{ }^{14} \mathrm{C} \\
\text { Animal, Fish } \\
\text { Ingestion }\end{array}$ & $\begin{array}{l}\text { Radionuclide concentration in } \\
\text { groundwater }\end{array}$ & $\begin{array}{l}\text { The conceptual and } \\
\text { mathematical models for the } \\
\text { groundwater scenario are } \\
\text { applicable to water discharged to } \\
\text { the surface, and the subsequent } \\
\text { transport of radionuclides in } \\
\text { surface water. Mixing is not } \\
\text { considered because there are no } \\
\text { sources of uncontaminated } \\
\text { water in the reference biosphere } \\
\text { (Section 6.3.1). }\end{array}$ \\
\hline \multirow[t]{3}{*}{ 2.3.11.01.0A } & \multirow[t]{3}{*}{ Precipitation } & Soil & $\begin{array}{l}\text { Annual irrigation rate } \\
\text { Overwatering rate }\end{array}$ & \multirow{3}{*}{$\begin{array}{l}\text { Distributions of parameters } \\
\text { developed based in part on } \\
\text { variation and uncertainty in } \\
\text { precipitation for the present-day } \\
\text { and predicted future climate } \\
\text { (BSC } 2004 \text { [DIRS 169673], } \\
\text { Sections } 6.5,6.7,6.8 \text {, and 6.9). }\end{array}$} \\
\hline & & Plant & $\begin{array}{l}\text { Irrigation amount per application } \\
\text { Daily irrigation rate }\end{array}$ & \\
\hline & & ${ }^{14} \mathrm{C}$ & $\begin{array}{l}\text { Daily irrigation rate } \\
\text { Annual irrigation rate }\end{array}$ & \\
\hline \multirow[t]{6}{*}{ 2.3.13.01.0A } & \multirow[t]{6}{*}{$\begin{array}{l}\text { Biosphere } \\
\text { characteristics }\end{array}$} & Soil & $\begin{array}{l}\text { Annual irrigation rate } \\
\text { Overwatering rate }\end{array}$ & \multirow{6}{*}{$\begin{array}{l}\text { Distributions of parameters } \\
\text { developed based in part on } \\
\text { variation and uncertainty in site- } \\
\text { specific characteristics of the } \\
\text { reference biosphere, such as } \\
\text { temperature, wind speed, and } \\
\text { evaporation rate (BSC } 2004 \\
\text { [DIRS 169673], Sections } 6.4 \text {, } \\
6.5,6.6,6.7,6.8 \text {, and } 6.9) ; \text { BSC } \\
2004 \text { [DIRS 169671], } \\
\text { Sections } 6.3 .4 .2 \text {; BSC } 2004 \\
\text { [DIRS 169672], Sections } 6.2 .2 .1 \text {, } \\
6.4 .3,6.5 .2, \text { and } 6.7 .2) \text {. Some } \\
\text { other biosphere characteristics } \\
\text { covered by other FEPs, such as } \\
\text { soil type (2.3.02.01.0A) and } \\
\text { precipitation (2.3.11.01.0A). }\end{array}$} \\
\hline & & Air & Water evaporation rate & \\
\hline & & Plant & $\begin{array}{l}\text { Dry deposition velocity } \\
\text { Daily irrigation rate } \\
\text { Irrigation application } \\
\text { Irrigation intensity } \\
\text { Growing time }\end{array}$ & \\
\hline & & Fish & $\begin{array}{l}\text { Water concentration modifying } \\
\text { factor }\end{array}$ & \\
\hline & & ${ }^{14} \mathrm{C}$ & Annual average wind speed & \\
\hline & & Inhalation & Evaporative cooler use factor & \\
\hline
\end{tabular}


Table 6.7-1. Disposition of the Included FEPs within the Biosphere Mathematical Model (Continued)

\begin{tabular}{|c|c|c|c|c|}
\hline $\begin{array}{l}\text { LA FEP } \\
\text { Number }\end{array}$ & FEP Name & $\begin{array}{l}\text { Biosphere } \\
\text { Submodel }^{a}\end{array}$ & $\begin{array}{l}\text { Model Parameters that } \\
\text { Address the FEP }\end{array}$ & Disposition within ERMYN \\
\hline \multirow[t]{5}{*}{ 2.3.13.02.0A } & \multirow{5}{*}{$\begin{array}{l}\text { Radionuclide } \\
\text { alteration } \\
\text { during } \\
\text { biosphere } \\
\text { transport }\end{array}$} & Soil & Partition coefficient & \multirow{3}{*}{$\begin{array}{l}\text { Parameter distributions } \\
\text { developed to reflect possible } \\
\text { variations due to chemical } \\
\text { characteristics of groundwater in } \\
\text { SZ (Sections } 6.4 .1,6.4 .3,6.4 .4 \text { ). }\end{array}$} \\
\hline & & Plant & Soil-to-plant transfer factor & \\
\hline & & $\begin{array}{l}\text { Animal } \\
\text { Fish }\end{array}$ & Animal product transfer coefficient & \\
\hline & & Ingestion & Dose conversion factors & \multirow{2}{*}{$\begin{array}{l}\text { Where multiple dose conversion } \\
\text { factors are defined, the largest } \\
\text { value is used to eliminate the } \\
\text { possibility of underestimating } \\
\text { dose (Sections } 6.4 .58 \text { and } \\
6.4 .9 .6) \text {. }\end{array}$} \\
\hline & & Inhalation & Dose conversion factors & \\
\hline \multirow[t]{3}{*}{ 2.4.01.00.0A } & \multirow[t]{3}{*}{$\begin{array}{l}\text { Human } \\
\text { characteristics } \\
\text { (physiology, } \\
\text { metabolism) }\end{array}$} & $\begin{array}{l}\text { External } \\
\text { exposure }\end{array}$ & $\begin{array}{l}\text { Dose coefficient for exposure to } \\
\text { soil contaminated to an infinite } \\
\text { depth } \\
\text { Dose coefficient for exposure to } \\
\text { contaminated ground surface }\end{array}$ & \multirow{3}{*}{$\begin{array}{l}\text { Physiology and metabolism of } \\
\text { the human receptor are } \\
\text { considered in developing the } \\
\text { listed parameters (BSC } 2004 \\
\text { [DIRS 169671], Sections } 6.3 .3 \text {, } \\
6.5 .3 .1,6.5 .3 .2,6.5 .4 \text {, and } \\
6.5 .5 \text { ). }\end{array}$} \\
\hline & & Inhalation & $\begin{array}{l}\text { Breathing rate } \\
\text { Dose conversion factor for } \\
\text { inhalation } \\
\text { Dose conversion factor for } \\
\text { inhalation of radon decay } \\
\text { products }\end{array}$ & \\
\hline & & Ingestion & $\begin{array}{l}\text { Dose conversion factor for } \\
\text { ingestion }\end{array}$ & \\
\hline \multirow[t]{4}{*}{ 2.4.04.01.0A } & \multirow[t]{4}{*}{$\begin{array}{l}\text { Human } \\
\text { lifestyle }\end{array}$} & Air & $\begin{array}{l}\text { Mass loading for receptor } \\
\text { environments }\end{array}$ & \multirow{4}{*}{$\begin{array}{l}\text { Distributions based, in part, on } \\
\text { variation and uncertainty of the } \\
\text { lifestyles and characteristics of } \\
\text { people living in Amargosa Valley } \\
\text { (BSC } 2004 \text { [DIRS 169458], } \\
\text { Sections } 6.1 \text { and 6.2; BSC } 2004 \\
\text { [DIRS } 169671 \text { ], Sections } 6.3 \text { and } \\
6.4 \text { ). Influence of human lifestyle } \\
\text { on external exposure are } \\
\text { considered (Eq. } 6.4 .7-1 \text { for the } \\
\text { groundwater scenario; } \\
\text { Eq. 6.5.5-1 for the volcanic ash } \\
\text { scenario). Influences on } \\
\text { inhalation pathway are } \\
\text { considered (Eqs. 6.4.8-2 to } \\
6.4 .8-7 \text { for the groundwater } \\
\text { scenario; Eqs. 6.5.6-2 and } \\
6.5 .6-3 \text { for the volcanic ash } \\
\text { scenario). Influences on the } \\
\text { ingestion pathway are } \\
\text { considered (Eqs. 6.4.9-2 to } \\
6.4 .9-6 \text { for the groundwater } \\
\text { scenario; Eqs. 6.5.7-2 to } 6.5 .7-4 \\
\text { for the volcanic ash scenario). }\end{array}$} \\
\hline & & $\begin{array}{l}\text { External } \\
\text { exposure }\end{array}$ & $\begin{array}{l}\text { Population proportion } \\
\text { Exposure time }\end{array}$ & \\
\hline & & Inhalation & $\begin{array}{l}\text { Population proportion } \\
\text { Exposure time } \\
\text { Fraction of house with } \\
\text { evaporative cooler } \\
\text { Evaporative cooler usage factor } \\
\text { by climate }\end{array}$ & \\
\hline & & Ingestion & $\begin{array}{l}\text { Consumption rate of water } \\
\text { Consumption rate of locally } \\
\text { produced crop foodstuffs } \\
\text { Consumption rate of locally } \\
\text { produced animal products } \\
\text { Consumption rate of locally } \\
\text { produced fish } \\
\text { Inadvertent soil ingestion rate }\end{array}$ & \\
\hline
\end{tabular}


Table 6.7-1. Disposition of the Included FEPs within the Biosphere Mathematical Model (Continued)

\begin{tabular}{|c|c|c|c|c|}
\hline $\begin{array}{l}\text { LA FEP } \\
\text { Number }\end{array}$ & FEP Name & $\begin{array}{l}\text { Biosphere } \\
\text { Submodel }^{a}\end{array}$ & $\begin{array}{l}\text { Model Parameters that } \\
\text { Address the FEP }{ }^{b}\end{array}$ & Disposition within ERMYN \\
\hline \multirow[t]{3}{*}{ 2.4.07.00.0A } & \multirow[t]{3}{*}{ Dwellings } & Air & $\begin{array}{l}\text { Water evaporation rate } \\
\text { Evaporative cooler air flow rate } \\
\text { Interior wall height } \\
\text { House ventilation rate }\end{array}$ & \multirow{3}{*}{$\begin{array}{l}\text { Distributions based in part on } \\
\text { uncertainty and variation in the } \\
\text { characteristics of types of } \\
\text { dwellings in northern Amargosa } \\
\text { Valley (BSC } 2004 \\
\text { [DIRS 169671], Sections } 6.3 .4 .1 \text {, } \\
\text { 6.3.4.2, and 6.6; BSC } 2004 \\
\text { [DIRS 169672], Sections } 6.5 \text { and } \\
\text { 6.6.2). }\end{array}$} \\
\hline & & $\begin{array}{l}\text { External } \\
\text { exposure }\end{array}$ & Building shielding factor & \\
\hline & & Inhalation & $\begin{array}{l}\text { Fraction of houses with } \\
\text { evaporative coolers } \\
\text { Evaporative cooler use factor }\end{array}$ & \\
\hline \multirow[t]{4}{*}{ 2.4.08.00.0A } & \multirow{4}{*}{$\begin{array}{l}\text { Wild and } \\
\text { natural land } \\
\text { and water use }\end{array}$} & Air & $\begin{array}{l}\text { Mass loading for receptor } \\
\text { environments }\end{array}$ & \multirow{4}{*}{$\begin{array}{l}\text { Distributions based in part on } \\
\text { uncertainty and variation in the } \\
\text { use of wild and natural lands and } \\
\text { the rate of consumption of wild } \\
\text { game by the receptor (BSC } 2004 \\
\text { [DIRS 169458], Sections } 6.1 \text { and } \\
\text { 6.2; BSC } 2004 \text { [DIRS 169671], } \\
\text { Sections } 6.3 .2 \text { and 6.4.2). }\end{array}$} \\
\hline & & $\begin{array}{l}\text { External } \\
\text { exposure }\end{array}$ & Exposure time & \\
\hline & & Inhalation & Exposure time & \\
\hline & & Ingestion & $\begin{array}{l}\text { Annual consumption rate of } \\
\text { locally produced animal products }\end{array}$ & \\
\hline \multirow[t]{8}{*}{ 2.4.09.01.0B } & \multirow{8}{*}{$\begin{array}{l}\text { Agricultural } \\
\text { land use and } \\
\text { irrigation }\end{array}$} & Soil & $\begin{array}{l}\text { Annual irrigation rate } \\
\text { Overwatering rate }\end{array}$ & \multirow{8}{*}{ 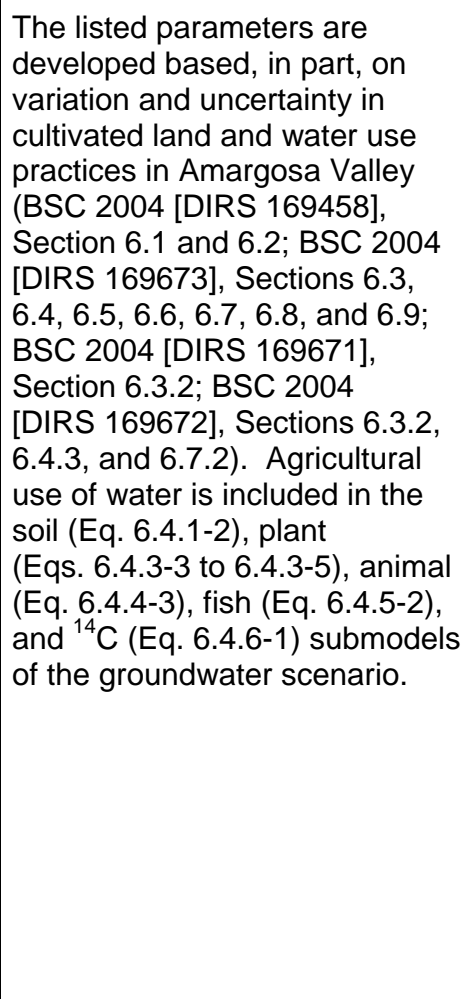 } \\
\hline & & Air & $\begin{array}{l}\text { Mass loading for receptor } \\
\text { environments } \\
\text { Mass loading for crops }\end{array}$ & \\
\hline & & Plant & $\begin{array}{l}\text { Fraction of overhead irrigation } \\
\text { Crop growing time } \\
\text { Irrigation intensity } \\
\text { Tillage depth } \\
\text { Irrigation amount per application } \\
\text { Daily irrigation rate }\end{array}$ & \\
\hline & & $\begin{array}{l}\text { External } \\
\text { exposure }\end{array}$ & Exposure time & \\
\hline & & $\begin{array}{l}\text { Inhalation } \\
\text { exposure }\end{array}$ & $\begin{array}{l}\text { Exposure time } \\
\text { Enhancement factor }\end{array}$ & \\
\hline & & Animal & Animal consumption rate of water & \\
\hline & & ${ }^{14} \mathrm{C}$ & $\begin{array}{l}\text { Annual irrigation rate } \\
\text { Daily irrigation rate } \\
\text { Overwatering rate } \\
\text { Surface area of irrigated land } \\
\end{array}$ & \\
\hline & & Fish & $\begin{array}{l}\text { Water concentration modifying } \\
\text { factor }\end{array}$ & \\
\hline \multirow[t]{2}{*}{ 2.4.09.02.0A } & \multirow[t]{2}{*}{$\begin{array}{l}\text { Animal farms } \\
\text { and fisheries }\end{array}$} & Animal & $\begin{array}{l}\text { Animal consumption rate of feed } \\
\text { Animal consumption rate of water } \\
\text { Animal consumption rate of soil }\end{array}$ & \multirow{2}{*}{$\begin{array}{l}\text { Parameters developed based, in } \\
\text { part, on variation and uncertainty } \\
\text { in animal and fish farming } \\
\text { practices (BSC } 2004 \\
\text { [DIRS 169672], Sections } 6.3 .2 \text {, } \\
6.4 .3 \text {, and 6.4.5) }\end{array}$} \\
\hline & & Fish & $\begin{array}{l}\text { Water concentration modifying } \\
\text { factor }\end{array}$ & \\
\hline
\end{tabular}


Table 6.7-1. Disposition of the Included FEPs within the Biosphere Mathematical Model (Continued)

\begin{tabular}{|c|c|c|c|c|}
\hline $\begin{array}{l}\text { LA FEP } \\
\text { Number }\end{array}$ & FEP Name & $\begin{array}{l}\text { Biosphere } \\
\text { Submodel }^{a}\end{array}$ & $\begin{array}{l}\text { Model Parameters that } \\
\text { Address the FEP }\end{array}$ & Disposition within ERMYN \\
\hline \multirow[t]{5}{*}{ 2.4.10.00.0A } & \multirow{5}{*}{$\begin{array}{l}\text { Urban and } \\
\text { Industrial land } \\
\text { and water use }\end{array}$} & Soil & Annual Irrigation rate & \multirow{5}{*}{$\begin{array}{l}\text { Distributions developed based in } \\
\text { part on uncertainty and variation } \\
\text { in land and water use practices } \\
\text { in residential and industrial } \\
\text { settings in Amargosa Valley } \\
\text { (BSC } 2004 \text { [DIRS 169458], } \\
\text { Section } 6.1 \text { and 6.2; BSC 2004 } \\
\text { [DIRS } 169671 \text { ], Section 6.3.2; } \\
\text { BSC } 2004 \text { [DIRS 169672], } \\
\text { Section 6.5). Use of } \\
\text { contaminated water in residential } \\
\text { and urban environments } \\
\text { included in soil (Eq. 6.4.1-2) and } \\
\text { air (Eq. 6.4.2-3) submodels of } \\
\text { the groundwater scenario. }\end{array}$} \\
\hline & & Air & $\begin{array}{l}\text { Mass loading for receptor } \\
\text { environments } \\
\text { Water evaporation rate }\end{array}$ & \\
\hline & & ${ }^{14} \mathrm{C}$ & Annual Irrigation rate & \\
\hline & & $\begin{array}{l}\text { External } \\
\text { exposure }\end{array}$ & Exposure time & \\
\hline & & Inhalation & Exposure time & \\
\hline \multirow[t]{4}{*}{ 3.1.01.01.0A } & \multirow[t]{4}{*}{$\begin{array}{l}\text { Radioactive } \\
\text { decay and } \\
\text { ingrowth }\end{array}$} & $\begin{array}{l}\text { Soil, Air, } \\
\text { Plant, } \\
\text { Animal, Fish }\end{array}$ & $\begin{array}{l}\text { Activity concentration of a decay } \\
\text { product in soil, air, plants, and } \\
\text { animal products }\end{array}$ & \multirow{4}{*}{$\begin{array}{l}\text { Radionuclide decay and } \\
\text { ingrowth in surface soils is } \\
\text { included in the soil (Eq. 6.4.1-9), } \\
\text { external exposure (Eq. 6.4.7-1), } \\
\text { inhalation (Eqs. 6.4.8-2 to } \\
6.4 .8-7 \text { ), and ingestion } \\
\text { (Eq. 6.4.9-3 to 6.4.9-6) } \\
\text { submodels of the groundwater } \\
\text { scenario. Also included in the } \\
\text { external exposure (Eq. 6.5.5-1), } \\
\text { inhalation (Eq. 6.5.6-2 to } \\
\text { 6.5.6-4), and ingestion } \\
\text { (Eq. 6.5.7-2 to 6.5.7-4) } \\
\text { submodels of the volcanic ash } \\
\text { scenario. Also included in } \\
\text { associated dose conversion } \\
\text { factors and dose coefficients } \\
\text { (BSC } 2004 \text { [DIRS 169671], } \\
\text { Section } 6.5 \text {. }\end{array}$} \\
\hline & & $\begin{array}{l}\text { External } \\
\text { exposure }\end{array}$ & $\begin{array}{l}\text { Dose coefficient for exposure to } \\
\text { soil contaminated to an infinite } \\
\text { depth } \\
\text { Dose coefficient for exposure to } \\
\text { contaminated ground surface }\end{array}$ & \\
\hline & & Inhalation & $\begin{array}{l}\text { Dose conversion factor for } \\
\text { inhalation } \\
\text { Dose conversion factor for } \\
\text { inhalation of radon decay } \\
\text { products }\end{array}$ & \\
\hline & & Ingestion & $\begin{array}{l}\text { Dose conversion factor for } \\
\text { ingestion }\end{array}$ & \\
\hline
\end{tabular}


Table 6.7-1. Disposition of the Included FEPs within the Biosphere Mathematical Model (Continued)

\begin{tabular}{|c|c|c|c|c|}
\hline $\begin{array}{l}\text { LA FEP } \\
\text { Number }\end{array}$ & FEP Name & $\begin{array}{l}\text { Biosphere } \\
\text { Submodel }^{a}\end{array}$ & $\begin{array}{l}\text { Model Parameters that } \\
\text { Address the FEP }{ }^{b}\end{array}$ & Disposition within ERMYN \\
\hline \multirow[t]{2}{*}{ 3.2.10.00.0A } & \multirow[t]{2}{*}{$\begin{array}{l}\text { Atmospheric } \\
\text { transport of } \\
\text { contaminants }\end{array}$} & Air & $\begin{array}{l}\text { Mass loading for crops } \\
\text { Mass loading for receptor } \\
\text { environments } \\
\text { Soil bulk density } \\
\text { Tillage depth } \\
\text { Resuspension enhancement } \\
\text { factor } \\
\text { Fraction of radionuclide transfer } \\
\text { from water to air } \\
\text { Water evaporation rate } \\
\text { Evaporative cooler air flow rate } \\
\text { Radon release factor } \\
\text { Interior wall height } \\
\text { House ventilation rate } \\
\text { Fraction of }{ }^{222} \text { Rn from soil } \\
\text { entering the house } \\
\text { Ratio of }{ }^{222} \text { Rn concentration in air } \\
\text { to flux density from soil }\end{array}$ & \multirow[t]{2}{*}{$\begin{array}{l}\text { The process of atmospheric } \\
\text { transport is included in the air } \\
\text { submodel for the groundwater } \\
\text { scenario (Eqs. } 6.4 .2-1 \text { to } \\
6.4 .2-8 \text { ), the air submodel for the } \\
\text { volcanic ash scenario } \\
\text { (Eqs. } 6.5 .2-1 \text { to } 6.5 .2-8 \text { ), and the } \\
{ }^{14} \text { C special submodel for the } \\
\text { groundwater scenario } \\
\text { (Eq. 6.4.6-2 and } 6.4 .6-3 \text { ). }\end{array}$} \\
\hline & & ${ }^{14} \mathrm{C}$ & $\begin{array}{l}{ }^{14} \mathrm{C} \text { emission rate } \\
\text { Surface area of irrigated land } \\
\text { Annual average wind speed } \\
{ }^{14} \mathrm{C} \text { mixing height } \\
\text { Concentration of stable carbon in } \\
\text { air }\end{array}$ & \\
\hline 3.3.01.00.0A & $\begin{array}{l}\text { Contaminated } \\
\text { drinking water, } \\
\text { foodstuffs and } \\
\text { drugs }\end{array}$ & $\begin{array}{l}\text { Plant, } \\
\text { Animal, } \\
\text { Fish, } \\
\text { Ingestion }\end{array}$ & $\begin{array}{l}\text { Consumption rates of locally } \\
\text { produced crop foodstuffs } \\
\text { Consumption rates of locally } \\
\text { produced animal products } \\
\text { Consumption rates of locally } \\
\text { produced fish } \\
\text { Consumption rate of water } \\
\text { Inadvertent soil ingestion rate }\end{array}$ & $\begin{array}{l}\text { The listed parameters quantify } \\
\text { intake of locally produced food } \\
\text { and locally obtained water. } \\
\text { Distributions of intake of locally } \\
\text { produced food are based on a } \\
\text { survey of the people of } \\
\text { Amargosa Valley (BSC } 2004 \\
\text { [DIRS } 169671 \text { ], Section } 6.4 \text { ). } \\
\text { The ingestion submodel includes } \\
\text { the intake of food, water, and soil } \\
\text { (groundwater scenario, } \\
\text { Eqs. 6.4.9-2 to 6.4.9-6; volcanic } \\
\text { ash scenario, Eqs. 6.5.7-2 to } \\
6.5 .7-4 \text { ). Calculated radionuclide } \\
\text { concentrations in foodstuffs } \\
\text { (Sections } 6.4 .3,6.4 .4,6.4 .5 \text {, } \\
6.4 .6,6.5 .3,6.5 .4 \text { ) also address } \\
\text { this FEP }\end{array}$ \\
\hline
\end{tabular}


Table 6.7-1. Disposition of the Included FEPs within the Biosphere Mathematical Model (Continued)

\begin{tabular}{|c|c|c|c|c|}
\hline $\begin{array}{l}\text { LA FEP } \\
\text { Number }\end{array}$ & FEP Name & $\begin{array}{l}\text { Biosphere } \\
\text { Submodel }^{a}\end{array}$ & $\begin{array}{l}\text { Model Parameters that } \\
\text { Address the FEP }{ }^{b}\end{array}$ & Disposition within ERMYN \\
\hline \multirow[t]{2}{*}{ 3.3.02.01.0A } & \multirow[t]{2}{*}{ Plant uptake } & Plant & $\begin{array}{l}\text { Soil-to-plant transfer factor } \\
\text { Dry-to-wet ratio } \\
\text { Fraction of overhead irrigation } \\
\text { Translocation factor } \\
\text { Weathering half-life } \\
\text { Crop growing time } \\
\text { Crop wet yield } \\
\text { Daily irrigation rate } \\
\text { Crop dry biomass } \\
\text { Irrigation amount per application } \\
\text { Irrigation intensity } \\
\text { Dry deposition velocity }\end{array}$ & \multirow[t]{2}{*}{$\begin{array}{l}\text { The process of plant uptake of } \\
\text { radionuclides is included in the } \\
\text { plant submodel for the } \\
\text { groundwater (Eqs. } 6.4 .3-1 \text { to } \\
6.4 .3-8 \text { ) and volcanic ash } \\
\text { scenarios (Eqs. } 6.5 .3-1 \text { to } \\
6.5 .3-5 \text { ), and in the }{ }^{14} \mathrm{C} \text { special } \\
\text { submodel for the groundwater } \\
\text { scenario (Eqs. } 6.4 .6-4 \text { and } \\
6.4 .6-6 \text { ). }\end{array}$} \\
\hline & & ${ }^{14} \mathrm{C}$ & $\begin{array}{l}\text { Soil bulk density } \\
\text { Fraction of air-derived carbon in } \\
\text { plants } \\
\text { Fraction of soil-derived carbon in } \\
\text { plants } \\
\text { Fraction of stable carbon in crops } \\
\text { Fraction of stable carbon in soil } \\
\text { Concentration of stable carbon in } \\
\text { air }\end{array}$ & \\
\hline \multirow[t]{2}{*}{ 3.3.02.02.0A } & \multirow[t]{2}{*}{ Animal uptake } & Animal & $\begin{array}{l}\text { Animal product transfer coefficient } \\
\text { Animal consumption rate of feed } \\
\text { Animal consumption rate of water } \\
\text { Animal consumption rate of soil }\end{array}$ & \multirow{2}{*}{$\begin{array}{l}\text { The animal submodel includes } \\
\text { the processes of radionuclide } \\
\text { uptake by farm animals } \\
\text { (groundwater scenario, } \\
\text { Eqs. } 6.4 .4-1 \text { to } 6.4 .4-4 \text {; volcanic } \\
\text { ash scenario, Eqs. } 6.5 .4-1 \text { to } \\
6.5 .4-3 \text { ), and in the }{ }^{14} \mathrm{C} \text { special } \\
\text { submodel for the groundwater } \\
\text { scenario (Eq. } 6.4 .6-7 \text { ). }\end{array}$} \\
\hline & & ${ }^{14} \mathrm{C}$ & $\begin{array}{l}\text { Fraction of stable carbon in } \\
\text { animal product } \\
\text { Animal consumption rate of feed } \\
\text { Animal consumption rate of water } \\
\text { Animal consumption rate of soil } \\
\text { Fraction of stable carbon in crops } \\
\text { Concentration of stable carbon in } \\
\text { water }\end{array}$ & \\
\hline 3.3.02.03.0A & Fish uptake & Fish & $\begin{array}{l}\text { Bioaccumulation factor } \\
\text { Water concentration modifying } \\
\text { factor }\end{array}$ & $\begin{array}{l}\text { The fish submodel includes the } \\
\text { bioaccumulation of radionuclides } \\
\text { in fish (groundwater scenario, } \\
\text { Eqs. } 6.4 .5-1 \text { and } 6.4 .5-2 \text { ). The } \\
\text { accumulation of radionuclides in } \\
\text { farm animals is considered in the } \\
\text { animal uptake FEP } \\
(3.3 .02 .02 .0 \mathrm{~A}) \text {. }\end{array}$ \\
\hline 3.3.03.01.0A & $\begin{array}{l}\text { Contaminated } \\
\text { non-food } \\
\text { products and } \\
\text { exposure }\end{array}$ & $\begin{array}{l}\text { External } \\
\text { exposure }\end{array}$ & $\begin{array}{l}\text { See parameter list under FEP } \\
\text { 3.3.04.03.0A }\end{array}$ & $\begin{array}{l}\text { The external exposure submodel } \\
\text { implicitly considers the FEP } \\
\text { because these contaminated } \\
\text { products cause external } \\
\text { exposure that is no worse than } \\
\text { exposure of contaminated soil. }\end{array}$ \\
\hline
\end{tabular}


Table 6.7-1. Disposition of the Included FEPs within the Biosphere Mathematical Model (Continued)

\begin{tabular}{|c|c|c|c|c|}
\hline $\begin{array}{l}\text { LA FEP } \\
\text { Number }\end{array}$ & FEP Name & $\begin{array}{l}\text { Biosphere } \\
\text { Submodel }^{a}\end{array}$ & $\begin{array}{l}\text { Model Parameters that } \\
\text { Address the FEP }\end{array}$ & Disposition within ERMYN \\
\hline 3.3.04.01.0A & Ingestion & Ingestion & $\begin{array}{l}\text { Dose conversion factor for } \\
\text { ingestion } \\
\text { Consumption rate of water } \\
\text { Consumption rate of locally } \\
\text { produced crop foodstuffs } \\
\text { Consumption rate of locally } \\
\text { produced animal products } \\
\text { Consumption rate of locally } \\
\text { produced fish } \\
\text { Inadvertent soil ingestion rate }\end{array}$ & $\begin{array}{l}\text { The ingestion submodels include } \\
\text { ingestion of contaminated food, } \\
\text { drinking water, and } \\
\text { contaminated soil (groundwater } \\
\text { scenario, Eqs. } 6.4 .9-1 \text { to } 6.4 .9-6 \text {; } \\
\text { volcanic ash scenario, } \\
\text { Eqs. 6.5.7-1 to 6.5.7-4). }\end{array}$ \\
\hline 3.3.04.02.0A & Inhalation & Inhalation & $\begin{array}{l}\text { Dose conversion factor for } \\
\text { inhalation } \\
\text { Breathing rate } \\
\text { Exposure time } \\
\text { Population proportion } \\
\text { Equilibrium factor for }{ }^{222} \mathrm{Rn} \text { decay } \\
\text { products } \\
\text { Dose conversion factor for } \\
\text { inhalation of }{ }^{222} \mathrm{Rn} \text { decay products } \\
\text { Critical thickness for resuspension } \\
\text { Fraction of dwellings with } \\
\text { evaporative coolering systems } \\
\text { Evaporator cooler usage factor }\end{array}$ & $\begin{array}{l}\text { The inhalation submodel } \\
\text { includes inhalation of } \\
\text { contaminated resuspended } \\
\text { particles, aerosols from } \\
\text { evaporative coolers, }{ }^{14} \mathrm{C} \text {, and } \\
\text { radon decay products } \\
\text { (groundwater scenario, } \\
\text { Eqs. } 6.4 .8-1 \text { to } 6.4 .8-7 \text {; volcanic } \\
\text { ash scenario, Eqs. } 6.5 .6-1 \text { to } \\
6.5 .6-4 \text { ). }\end{array}$ \\
\hline 3.3.04.03.0A & $\begin{array}{l}\text { External } \\
\text { exposure }\end{array}$ & $\begin{array}{l}\text { External } \\
\text { exposure }\end{array}$ & $\begin{array}{l}\text { Dose coefficient for exposure to } \\
\text { soil contaminated to an infinite } \\
\text { depth } \\
\text { Dose coefficient for exposure to } \\
\text { contaminated ground surface } \\
\text { Exposure time } \\
\text { Population proportions } \\
\text { Building shielding factor }\end{array}$ & $\begin{array}{l}\text { The external exposure submodel } \\
\text { includes external exposure to } \\
\text { contaminated materials } \\
\text { (groundwater scenario, } \\
\text { Eq. 6.4.7-1; volcanic ash } \\
\text { scenario, Eq. 6.5.5-1). }\end{array}$ \\
\hline 3.3.05.01.0A & $\begin{array}{l}\text { Radiation } \\
\text { doses }\end{array}$ & $\begin{array}{l}\text { External } \\
\text { exposure, } \\
\text { Inhalation, } \\
\text { Ingestion }\end{array}$ & $\begin{array}{l}\text { Dose coefficient for exposure to } \\
\text { soil contaminated to an infinite } \\
\text { depth } \\
\text { Dose coefficient for exposure to } \\
\text { contaminated ground surface } \\
\text { Dose conversion factor for } \\
\text { inhalation } \\
\text { Dose conversion factor for } \\
\text { ingestion } \\
\text { BDCFs }\end{array}$ & $\begin{array}{l}\text { Calculation of the predicted } \\
\text { annual dose to the receptor for a } \\
\text { unit activity concentration of a } \\
\text { radionuclide (i.e., BDCF) is } \\
\text { described in Eq. } 6.4 .10-1 \text { and } \\
6.4 .10-2 \text { for the groundwater } \\
\text { scenario and } 6.5 .8-1 \text { and } 6.5 .8-2 \\
\text { for the volcanic ash scenario. }\end{array}$ \\
\hline
\end{tabular}


Table 6.7-1. Disposition of the Included FEPs within the Biosphere Mathematical Model (Continued)

\begin{tabular}{|c|c|c|c|c|}
\hline $\begin{array}{l}\text { LA FEP } \\
\text { Number }\end{array}$ & FEP Name & $\begin{array}{l}\text { Biosphere } \\
\text { Submodel }^{\mathrm{a}}\end{array}$ & $\begin{array}{l}\text { Model Parameters that } \\
\text { Address the FEP }\end{array}$ & Disposition within ERMYN \\
\hline 3.3.08.00.0A & $\begin{array}{l}\text { Radon and } \\
\text { radon } \\
\text { daughter } \\
\text { exposure }\end{array}$ & $\begin{array}{l}\text { Air, } \\
\text { Inhalation }\end{array}$ & $\begin{array}{l}\text { Radon release factor } \\
\text { Interior wall height } \\
\text { House ventilation rate } \\
\text { Fraction of }{ }^{222} \mathrm{Rn} \text { from soil } \\
\text { entering the house } \\
\text { Ratio of }{ }^{222} \mathrm{Rn} \text { concentration to } \\
\text { flux density for outdoors } \\
\text { Equilibrium factor for }{ }^{222} \mathrm{Rn} \text { decay } \\
\text { products } \\
\text { Fraction of radionuclide transfer } \\
\text { from water to air } \\
\text { Dose conversion factor for radon } \\
\text { decay products }\end{array}$ & $\begin{array}{l}\text { The air submodels include radon } \\
\text { concentrations (groundwater } \\
\text { scenario, Eqs. } 6.4 .2-4 \text { to } 6.4 .2-8 \text {; } \\
\text { volcanic ash scenario, } \\
\text { Eqs. } 6.5 .2-5 \text { to } 6.5 .2-8 \text { ). The } \\
\text { inhalation submodel includes the } \\
\text { consequences of inhaling radon } \\
\text { and the decay products } \\
\text { (groundwater scenario, } \\
\text { Eqs. } 6.4 .8-5 \text { to } 6.4 .8-7 ; \text { volcanic } \\
\text { ash scenario, Eqs. } 6.5 .6-3 \text { and } \\
6.5 .6-4 \text { ). }\end{array}$ \\
\hline
\end{tabular}

${ }^{a}$ Relationships among submodels shown in Figures 6.3-2 and 6.3-4. Mathematical representations described in Sections 6.4 and 6.5 .

${ }^{b}$ Model parameters for each submodel presented in Sections 6.4 and 6.5; also summarized in Section 6.6. ERMYN=Environmental Radiation Model for Yucca Mountain, Nevada; FEP=feature, event, or process; LA=license application; SZ=saturated zone

\subsubsection{Comparison of Biosphere Models}

Improvements in the current biosphere model are identified by comparing the ERMYN with the GENII-S model previously used for the TSPA-SR. Forty items or issues are compared (Table 6.7-2), many of which are new exposure pathways that were not considered in the TSPA-SR (e.g., a radon exposure pathway, inhalation of aerosols from evaporative cooling, and soil ingestion by domestic farm animals). The overall effect of these changes cannot be evaluated until the model has been used to generate BDCFs.

Table 6.7-2. Comparison of Biosphere Models for the TSPA-LA and TSPA-SR

\begin{tabular}{|c|c|c|c|c|}
\hline No. & $\begin{array}{c}\text { Item } \\
\text { Compared }\end{array}$ & GENII-S (TSPA-SR) $^{a}$ & ERMYN (TSPA-LA) ${ }^{b}$ & $\begin{array}{l}\text { Used in } \\
\text { TSPA-LA } \\
\text { Scenario }\end{array}$ \\
\hline 1 & $\begin{array}{l}\text { Parameter } \\
\text { uncertainty }\end{array}$ & $\begin{array}{l}\text { Limited number of probability } \\
\text { distribution parameters. }\end{array}$ & $\begin{array}{l}\text { Every parameter can be represented } \\
\text { by probability distribution function. }\end{array}$ & Both \\
\hline 2 & $\begin{array}{l}\text { Number of } \\
\text { realizations per } \\
\text { simulation }\end{array}$ & $\begin{array}{l}\text { Scenario dependent, but typically } \\
\text { less than } 200 \text { realizations. }\end{array}$ & $\begin{array}{l}\text { High limit on number of realizations } \\
\text { (e.g. } 5,000 \text { realizations). }\end{array}$ & Both \\
\hline 3 & $\begin{array}{l}\text { Cumulative } \\
\text { probability } \\
\text { results }\end{array}$ & $\begin{array}{l}\text { Some cumulative probability results } \\
\text { calculated incorrectly. }\end{array}$ & Not considered. & Both \\
\hline 4 & $\begin{array}{l}\text { Long-term } \\
\text { irrigation rate } \\
\text { for cultivated } \\
\text { lands }\end{array}$ & Not considered. & $\begin{array}{l}\text { Average irrigation rate includes } \\
\text { consideration of crop rotation and } \\
\text { changes in the use of cultivated land. }\end{array}$ & Groundwater \\
\hline 5 & $\begin{array}{l}\text { Crop rotation } \\
\text { and land use }\end{array}$ & $\begin{array}{l}\text { Not considered; assumed one crop } \\
\text { grown indefinitely on each plot of } \\
\text { land. }\end{array}$ & $\begin{array}{l}\text { Incorporated using an average } \\
\text { irrigation rate. }\end{array}$ & Groundwater \\
\hline
\end{tabular}


Table 6.7-2. Comparison of Biosphere Models for the TSPA-LA and TSPA-SR (Continued)

\begin{tabular}{|c|c|c|c|c|}
\hline No. & $\begin{array}{c}\text { Item } \\
\text { Compared }\end{array}$ & GENII-S (TSPA-SR) ${ }^{a}$ & ERMYN (TSPA-LA) ${ }^{b}$ & $\begin{array}{l}\text { Used in } \\
\text { TSPA-LA } \\
\text { Scenario }\end{array}$ \\
\hline 6 & $\begin{array}{l}\text { Average } \\
\text { radionuclide } \\
\text { concentration in } \\
\text { soil }\end{array}$ & $\begin{array}{l}\text { Home lawn irrigation rate used for } \\
\text { evaluation of external exposure, } \\
\text { inhalation, and soil ingestion. }\end{array}$ & $\begin{array}{l}\text { Calculated using the annual average } \\
\text { irrigation rate and the annual average } \\
\text { of activity loss mechanisms from soil; } \\
\text { used for plant submodel, external } \\
\text { exposure, inhalation, and soil } \\
\text { ingestion. }\end{array}$ & Groundwater \\
\hline 7 & $\begin{array}{l}\text { Leaching } \\
\text { removal into } \\
\text { deep soil }\end{array}$ & Only represented by a fixed value. & $\begin{array}{l}\text { Represented by a probability } \\
\text { distribution. }\end{array}$ & Groundwater \\
\hline 8 & $\begin{array}{l}\text { Surface soil } \\
\text { erosion }\end{array}$ & $\begin{array}{l}\text { Not considered in GENII-S code, but } \\
\text { considered externally. }\end{array}$ & $\begin{array}{l}\text { One of removal mechanisms in } \\
\text { surface soil. }\end{array}$ & Groundwater \\
\hline 9 & $\begin{array}{l}\text { Radionuclide } \\
\text { accumulation in } \\
\text { soil }\end{array}$ & $\begin{array}{l}\text { Fixed leaching rate only and } \\
\text { calculated based on multiple } \\
\text { irrigation periods. }\end{array}$ & $\begin{array}{l}\text { Equilibrium activity concentration of } \\
\text { radionuclides in surface soil. }\end{array}$ & Groundwater \\
\hline 10 & $\begin{array}{l}\text { Harvest } \\
\text { removal and } \\
\text { use of cow } \\
\text { manure as } \\
\text { fertilizer }\end{array}$ & $\begin{array}{l}\text { Theoretically includes harvest } \\
\text { removal, but the code does not } \\
\text { execute this part of the submodel. }\end{array}$ & $\begin{array}{l}\text { The ERMYN does not include these } \\
\text { two mechanisms because they } \\
\text { balance out. }\end{array}$ & Both \\
\hline 11 & $\begin{array}{l}\text { Volcanic ash } \\
\text { deposited on } \\
\text { cultivated land }\end{array}$ & $\begin{array}{l}\text { Uniformly mixed in soil up to a } \\
\text { defined depth. }\end{array}$ & $\begin{array}{l}\text { Ash and surface soil mixed uniformly } \\
\text { to tilling depth. Mixture is soil-like. } \\
\text { Used for plant submodel and soil } \\
\text { ingestion. }\end{array}$ & Volcanic Ash \\
\hline 12 & $\begin{array}{l}\text { Volcanic ash } \\
\text { deposited on } \\
\text { noncultivated } \\
\text { land }\end{array}$ & Not considered. & $\begin{array}{l}\text { Ash mixes with surface soil if ash } \\
\text { thickness is less than critical } \\
\text { thickness. Mixture is ash-like. Used } \\
\text { for inhalation of resuspended soil and } \\
\text { source term for external exposure. }\end{array}$ & Volcanic Ash \\
\hline 13 & $\begin{array}{l}\text { Enhancement } \\
\text { factor }\end{array}$ & Not considered. & $\begin{array}{l}\text { Correction for enhancement of } \\
\text { airborne activity concentration } \\
\text { relative to surface soil. }\end{array}$ & Both \\
\hline 14 & $\begin{array}{l}\text { Mass loading } \\
\text { for crops }\end{array}$ & $\begin{array}{l}\text { Not directly used; used a related } \\
\text { parameter of resuspension factor. }\end{array}$ & $\begin{array}{l}\text { Parameter, based on farm field } \\
\text { conditions, used to calculate } \\
\text { deposition of particles on crop } \\
\text { surface. }\end{array}$ & Both \\
\hline 15 & $\begin{array}{l}\text { Mass loading } \\
\text { for inhalation }\end{array}$ & Used average value. & $\begin{array}{l}\text { Environment related, based on } \\
\text { human activity, including active } \\
\text { outdoors, inactive outdoors, active } \\
\text { indoors, asleep indoors, and outside } \\
\text { of contaminated area. }\end{array}$ & Both \\
\hline 16 & $\begin{array}{l}\text { Time- } \\
\text { dependent } \\
\text { mass loading }\end{array}$ & Not considered. & $\begin{array}{l}\text { Includes time function for decreasing } \\
\text { mass loading after a volcanic } \\
\text { eruption. }\end{array}$ & Volcanic Ash \\
\hline 17 & $\begin{array}{l}\text { Radionuclide } \\
\text { release from } \\
\text { evaporative } \\
\text { cooler }\end{array}$ & Not considered. & $\begin{array}{l}\text { Includes submodel to calculate } \\
\text { radionuclide concentration in indoor } \\
\text { air from operation of evaporative } \\
\text { coolers. }\end{array}$ & Groundwater \\
\hline
\end{tabular}


Table 6.7-2. Comparison of Biosphere Models for the TSPA-LA and TSPA-SR (Continued)

\begin{tabular}{|c|c|c|c|c|}
\hline No. & $\begin{array}{c}\text { Item } \\
\text { Compared }\end{array}$ & GENII-S (TSPA-SR) $^{a}$ & ERMYN (TSPA-LA) b & $\begin{array}{l}\text { Used in } \\
\text { TSPA-LA } \\
\text { Scenario }\end{array}$ \\
\hline 18 & $\begin{array}{l}\text { Radon release } \\
\text { from soil into air }\end{array}$ & Not considered. & $\begin{array}{l}\text { Considered based on parameter of } \\
\text { radon release factor. }\end{array}$ & Both \\
\hline 19 & $\begin{array}{l}\text { Soil-to-plant } \\
\text { transfer factor } \\
\text { for fresh forage }\end{array}$ & $\begin{array}{l}\text { Used one transfer factor for leafy } \\
\text { vegetables. }\end{array}$ & $\begin{array}{l}\text { Includes parameter value based on } \\
\text { literature. }\end{array}$ & Both \\
\hline 20 & $\begin{array}{l}\text { Fraction of } \\
\text { overhead } \\
\text { irrigation } \\
\text { method }\end{array}$ & Not considered. & $\begin{array}{l}\text { Includes parameter to better } \\
\text { represent site-specific agricultural } \\
\text { practice. }\end{array}$ & Groundwater \\
\hline 21 & $\begin{array}{l}\text { Crop daily } \\
\text { irrigation rate }\end{array}$ & $\begin{array}{l}\text { Correlation between annual } \\
\text { irrigation rate and duration not } \\
\text { addressed. }\end{array}$ & $\begin{array}{l}\text { Includes parameter to eliminate } \\
\text { irrigation duration and its correlation } \\
\text { with annual irrigation rate. }\end{array}$ & Groundwater \\
\hline 22 & $\begin{array}{l}\text { Irrigation } \\
\text { interception } \\
\text { fraction }\end{array}$ & A single value for all crop types. & $\begin{array}{l}\text { Includes parameter based on site- } \\
\text { specific irrigation practices, including } \\
\text { irrigation methods, irrigation } \\
\text { frequency, and crop type; crop-type } \\
\text { dependent. }\end{array}$ & Groundwater \\
\hline 23 & $\begin{array}{l}\text { Translocation } \\
\text { factor and } \\
\text { weathering rate }\end{array}$ & Represented by fixed values. & $\begin{array}{l}\text { Represented by probability } \\
\text { distribution functions. }\end{array}$ & Both \\
\hline 24 & $\begin{array}{l}\text { Crop yield and } \\
\text { biomass }\end{array}$ & $\begin{array}{l}\text { Not used correctly (Sections 6.4.3.3 } \\
\text { and 7.3.3.3). }\end{array}$ & $\begin{array}{l}\text { Crop yield represents crop foodstuffs } \\
\text { and crop biomass represents the } \\
\text { total standing biomass for the } \\
\text { interception of irrigation water and } \\
\text { particle deposition. }\end{array}$ & Both \\
\hline 25 & $\begin{array}{l}\text { Animal soil } \\
\text { ingestion }\end{array}$ & Not considered. & Included. & Both \\
\hline 26 & $\begin{array}{l}{ }^{14} \mathrm{C} \text { release } \\
\text { from soil in form } \\
\text { of gaseous } \\
\text { species }\end{array}$ & Not considered. & $\begin{array}{l}\text { Considers release of gaseous } \\
\text { species of carbon from soil and } \\
\text { subsequent uptake by plant and } \\
\text { inhalation by humans. }\end{array}$ & Groundwater \\
\hline 27 & $\begin{array}{l}{ }^{14} \mathrm{C} \text { leaching } \\
\text { removal } \\
\text { constant }\end{array}$ & $\begin{array}{l}\text { Value is fixed in the code; user input } \\
\text { not used. }\end{array}$ & User defined. & Groundwater \\
\hline 28 & $\begin{array}{l}{ }^{14} \mathrm{C} \text { pathways of } \\
\text { external } \\
\text { exposure, } \\
\text { inhalation, and } \\
\text { soil ingestion }\end{array}$ & Not considered. & Included. & Groundwater \\
\hline 29 & Crop ${ }^{14} \mathrm{C}$ uptake & Includes root uptake only. & $\begin{array}{l}\text { Submodel differentiated between }{ }^{14} \mathrm{C} \\
\text { fractions derived from air and soil. }\end{array}$ & Groundwater \\
\hline 30 & $\begin{array}{l}\text { Dose } \\
\text { coefficients for } \\
\text { exposure to } \\
\text { contaminated } \\
\text { soil }\end{array}$ & $\begin{array}{l}\text { Dose coefficients for } 15-\mathrm{cm} \\
\text { contaminated soil are used. }\end{array}$ & $\begin{array}{l}\text { Dose coefficients for infinite depth } \\
\text { account for radionuclides removed } \\
\text { from surface soil by leaching. }\end{array}$ & Groundwater \\
\hline 31 & $\begin{array}{l}\text { Dose } \\
\text { coefficients for } \\
\text { exposure to } \\
\text { contaminated } \\
\text { ground surface }\end{array}$ & $\begin{array}{l}\text { Radionuclides mixed in surface soil; } \\
\text { used same method for groundwater } \\
\text { scenario. }\end{array}$ & $\begin{array}{l}\text { Dose coefficients for the ground } \\
\text { surface are used for thin layer of } \\
\text { volcanic ash deposited on the } \\
\text { ground. }\end{array}$ & Volcanic Ash \\
\hline
\end{tabular}


Table 6.7-2. Comparison of Biosphere Models for the TSPA-LA and TSPA-SR (Continued)

\begin{tabular}{|c|c|c|c|c|}
\hline No. & $\begin{array}{c}\text { Item } \\
\text { Compared }\end{array}$ & GENII-S (TSPA-SR) $^{a}$ & ERMYN (TSPA-LA) ${ }^{b}$ & $\begin{array}{l}\text { Used in } \\
\text { TSPA-LA } \\
\text { Scenario } \\
\end{array}$ \\
\hline 32 & $\begin{array}{l}\text { Indoor external } \\
\text { exposure }\end{array}$ & Not considered. & Considers building shielding factor. & Both \\
\hline 33 & $\begin{array}{l}\text { External and } \\
\text { inhalation } \\
\text { exposure time }\end{array}$ & A single effective value is used. & $\begin{array}{l}\text { Environment specific; based on site- } \\
\text { specific information. }\end{array}$ & Both \\
\hline 34 & $\begin{array}{l}\text { Human activity } \\
\text { budget }\end{array}$ & Not considered. & $\begin{array}{l}\text { Determines external and inhalation } \\
\text { exposure times for receptor. }\end{array}$ & Both \\
\hline 35 & $\begin{array}{l}\text { Dose } \\
\text { conversion } \\
\text { factors for } \\
\text { inhalation } \\
\end{array}$ & $\begin{array}{l}\text { Not consistent with FGR-11 } \\
\text { (Eckerman } 1988 \text { [DIRS 101069]) }\end{array}$ & $\begin{array}{l}\text { Taken from FGR-11 (Eckerman } 1988 \\
\text { [DIRS 101069]) }\end{array}$ & Both \\
\hline 36 & Breathing rate & A fixed average value. & $\begin{array}{l}\text { Environment related, based on } \\
\text { human activity. }\end{array}$ & Both \\
\hline 37 & $\begin{array}{l}\text { Inhalation dose } \\
\text { from aerosols } \\
\text { released from } \\
\text { evaporative } \\
\text { cooler }\end{array}$ & Not considered. & Considered. & Groundwater \\
\hline 38 & $\begin{array}{l}\text { Inhalation dose } \\
\text { from radon } \\
\text { decay products }\end{array}$ & Not considered. & Considered. & Both \\
\hline 39 & $\begin{array}{l}\text { Dose } \\
\text { conversion } \\
\text { factors for } \\
\text { ingestion } \\
\end{array}$ & $\begin{array}{l}\text { Not consistent with FGR-11 } \\
\text { (Eckerman } 1988 \text { [DIRS 101069]) }\end{array}$ & $\begin{array}{l}\text { Taken from FGR-11 (Eckerman } 1988 \\
\text { [DIRS 101069]) }\end{array}$ & Both \\
\hline 40 & $\begin{array}{l}\text { BDCFs for } \\
\text { volcanic ash } \\
\text { scenario }\end{array}$ & Several sets of BDCFs calculated. & $\begin{array}{l}\text { Three components of BDCF: } \\
\text { external and ingestion, long-term } \\
\text { inhalation, and short-term inhalation. }\end{array}$ & Volcanic Ash \\
\hline \multicolumn{5}{|c|}{$\begin{array}{l}\text { Source: GENII manual (Napier et al. } 1988 \text { [DIRS 157927], GENII-S user guide (Leigh et al. } 1993 \text { [DIRS 100464]), } \\
\text { and BDCFs for TSPA-SR (CRWMS M\&O } 2001 \text { [DIRS 152536]; CRWMS M\&O } 2001 \text { [DIRS 152539]). } \\
\text { bource: Sections } 6.1 \text { to 6.6. }\end{array}$} \\
\hline & & & n, NV; LA=license application; SR=site & \\
\hline
\end{tabular}

\subsubsection{Software Selection}

Based on biosphere model comparisons (Table 6.7-2), the new biosphere conceptual model (ERMYN) can not be implemented using the old (GENII-S) code. Existing codes that could be used for the Yucca Mountain biosphere model were examined, but no off-the-shelf computer codes are capable of implementing the ERMYN (Sections 6.4 and 6.5) or of implementing the uncertainty treatment (Section 6.6). The GoldSim software (GoldSim Technology Group 2002 [DIRS 160643]) can perform probabilistic analyses and can simulate processes to address uncertainty. Therefore, the ERMYN is built using GoldSim.

The GoldSim software supports 13 types of stochastic distributions: uniform, normal, lognormal, triangular, cumulative, discrete, Poisson, beta, gamma, Weibull, binomial, Student's $t$, and boolean distributions. The most frequently used distributions in the ERMYN are lognormal, normal, uniform, and cumulative distributions. 
Only the eight basic elements in the GoldSim environment are used in the ERMYN (Figure 6.7-1). The Data element is used for the input of fixed data or to combine several stochastic inputs into a data array. Sometimes, a simple calculation is done in the Data element. The Stochastic element is used to input distribution data. The one-dimensional Table element is used to store all radionuclide-related input parameters so that they can be searched later. The Expression element, the most frequently used element, is used for all calculations. The Sum element is used for some simple additions. The Data and Expression elements accept data arrays, which are used to simplify calculation expressions. The Selector element is used to select parameter values from a database. The Result element is used to present the final distribution results. The Container box is used to separate submodels and calculation tasks.

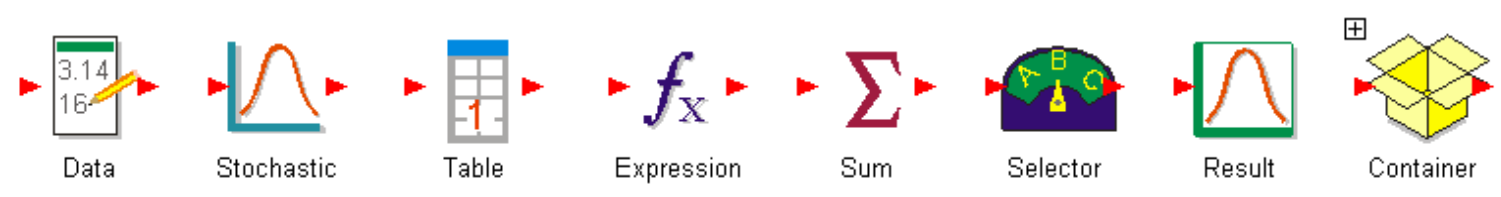

Figure 6.7-1. Basic Elements in the GoldSim Environment

\subsection{GOLDSIM IMPLEMENTATION OF THE BIOSPHERE MODEL FOR THE GROUNDWATER SCENARIO}

As discussed previously, the ERMYN is implemented using GoldSim stochastic simulation software. For transparency, the biosphere models for the two exposure scenarios are built separately. This section describes the implementation of the ERMYN model for the groundwater scenario in GoldSim and shows the overall model algorithm and submodel structures. The GoldSim file (ERMYN_GW.gsm) is part of the model output, which is listed in Appendix A. As discussed in Section 6.4, ERMYN_GW is structured as a series of submodels. Under each submodel, the linkage of GoldSim elements to the submodel input parameters in an equation is tabulated in this section. A color code is used in GoldSim to aid in distinguishing among items displayed on the computer screen. Text descriptions are shown in green. Element names are shown in black for calculated quantities and in blue for input parameters. If the name of a container box is shown in blue, it contains at least one input parameter. The cover page for the ERMYN_GW simulation system for biosphere modeling (Figure 6.8-1) shows the title, the version and other text (green), and one container box, Biosphere_Model, which is shown in blue to indicate that it contains at least one input parameter.

The Biosphere_Model container holds the submodel containers and the radionuclide data (Figure 6.8-2). GoldSim is an object-oriented graphical program, and the overall model structure looks much like the block diagram of the conceptual model for the groundwater scenario shown in Figure 6.3-2. Each container in GoldSim corresponds to a submodel or to a component model if a container is at a lower level. 


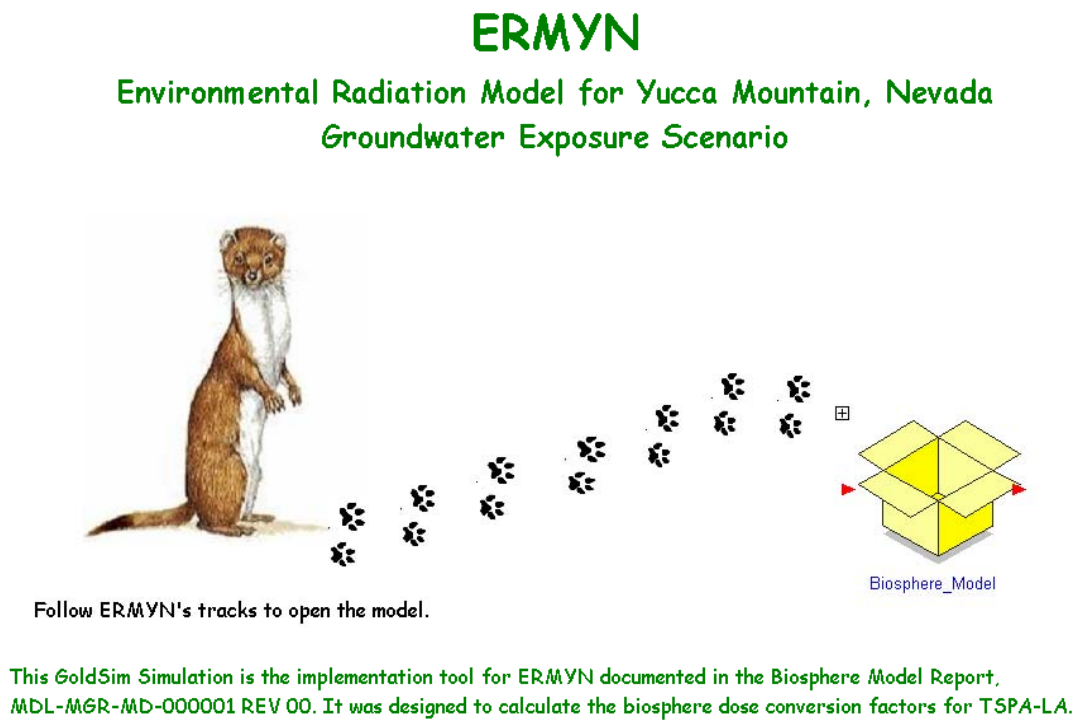

Figure 6.8-1. Cover Page for the ERMYN_GW Model in GoldSim

Ten containers, including eight submodels $\left({ }^{14} \mathrm{C}\right.$ does not have a specific box), one results box, and one radionuclide database box are shown in Figure 6.8-2. Each box is discussed in detail in the following subsections. Only two input parameters can be changed at this level: Radionuclide and Water_Source. Radionuclide can be selected only from the data element of Radionuclide_List (index $i$ in the equations) that is built in the Nuclide_Database container. Water_Source is the radionuclide concentration in the groundwater ( $C w_{i}$ in the equation), which has a default value of $1 \mathrm{~Bq} / \mathrm{m}^{3}$. GoldSim can run in deterministic or stochastic modes by adjusting settings in the MasterClock. If the stochastic mode is chosen, the number of realizations, the sampling method (Monte Carlo or Latin Hypercube), and a random seed number is set. Because the BDCFs are not a function of time, the time option is disabled for the ERMYN.

As discussed in Section 6.4, the biosphere model involves radionuclides linearly transported from one medium to another. The model algorithm is based on the submodels in the order discussed in Section 6.4. The calculated results in one submodel are then used in the next applicable submodel. The arrows in Figure 6.8-2 indicate the relationships and calculation logic flow among submodels.

Many calculations are performed using data vectors to reduce the number of elements. Twelve data array labels are used: primary radionuclides $(i=31)$, total number of radionuclides $(i=75)$, pathways $(n=15)$, plant types $(j=5)$, crop food types $(j=4)$, animal product types $(k=4)$, number of long-lived decay products $(l=3)$, population groups $(m=4)$, environments $(n=5)$, air submodel pathway $(n=4)$, crop uptake pathway $(n=4)$, and animal uptake pathway $(n=4)$. These data sets and submodel pathways are discussed in the mathematical model (Section 6.4). 


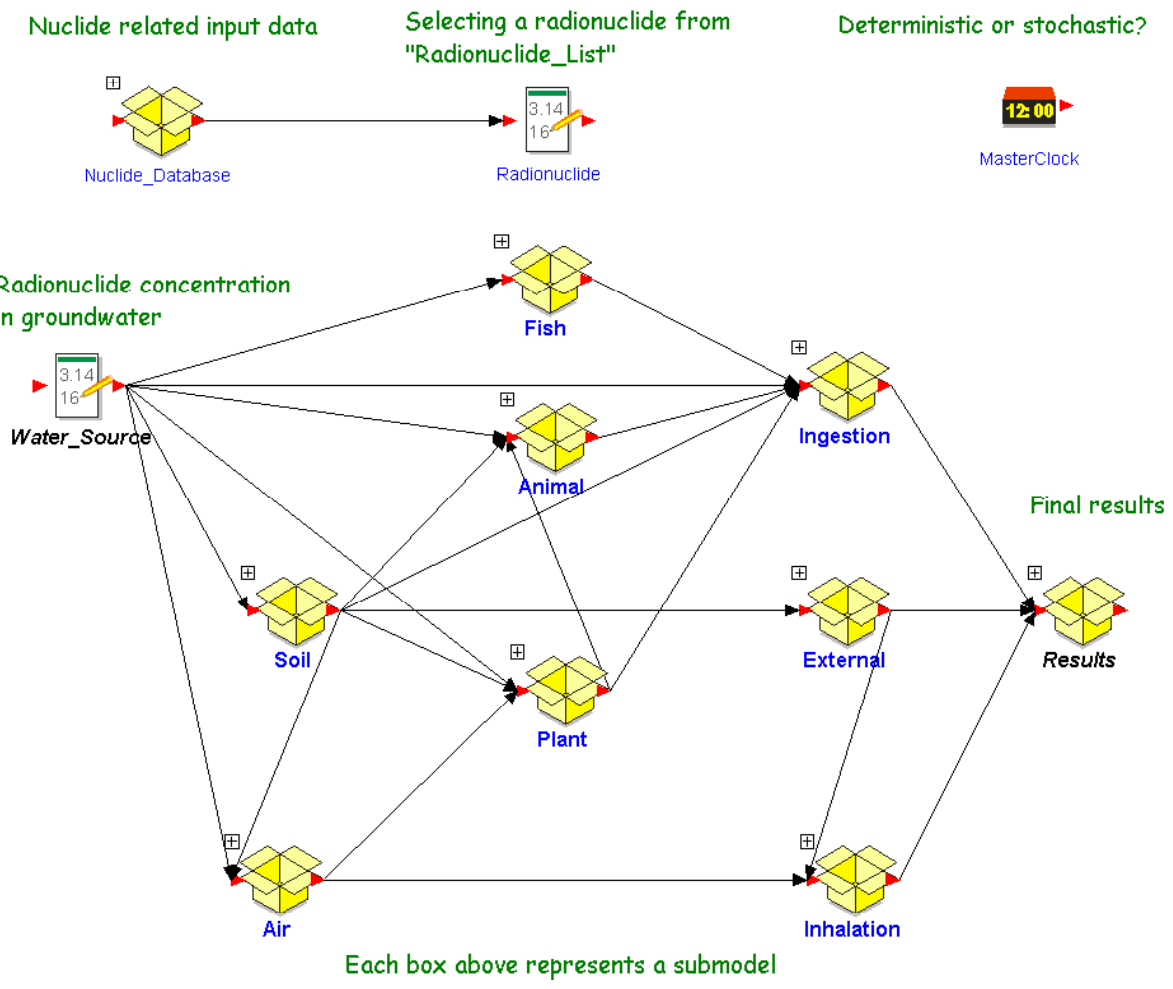

Figure 6.8-2. Graphical Representation of the Groundwater Scenario in GoldSim

\subsubsection{Nuclide Database}

The Nuclide_Database container (Figure 6.8-3) includes all radionuclide-related input parameters (under the Nuclide_Data container) and their selection in the model (under the Data_Selection container). Besides the two containers, there is one data element, Radionuclide_List, which includes 31 radionuclides, three more than the number of radionuclides of interest for the TSPA-LA (Table 6.1-1). The three extra radionuclides are ${ }^{228} \mathrm{Ra}$, ${ }^{228} \mathrm{Th}$, and ${ }^{235} \mathrm{U}$. The ERMYN includes these radionuclides because they are part of decay chains (Table 6.3-7).

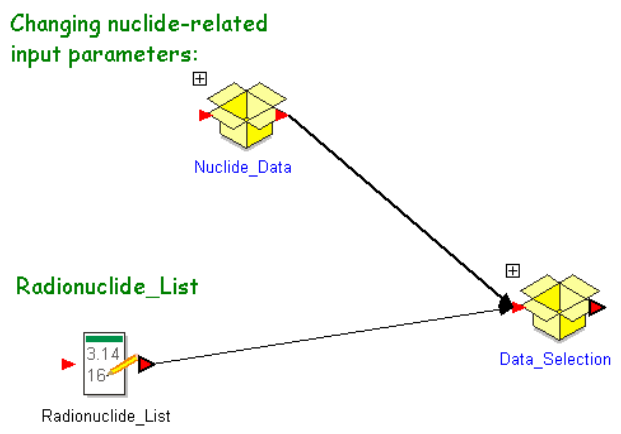

Figure 6.8-3. Nuclide Database Container 
The Nuclide_Data container includes five subcontainers (element-specific partition coefficients, crop transfer factors, animal product transfer coefficients, fish bioaccumulation factors, and nuclear data). For example, the Crop_Transfer container (Figure 6.8-4) includes 80 distribution parameters for 16 elements and 5 plant types. The form of the other three containers (Animal_Transfer, Fish_Transfer, Kd_Coefficients) is similar. The Nuclear_Data container includes radionuclide half-lives, branching fractions, external dose coefficients for contaminated soil, and dose conversion factors for inhalation and ingestion. All nuclear input data have fixed values and are in array form.
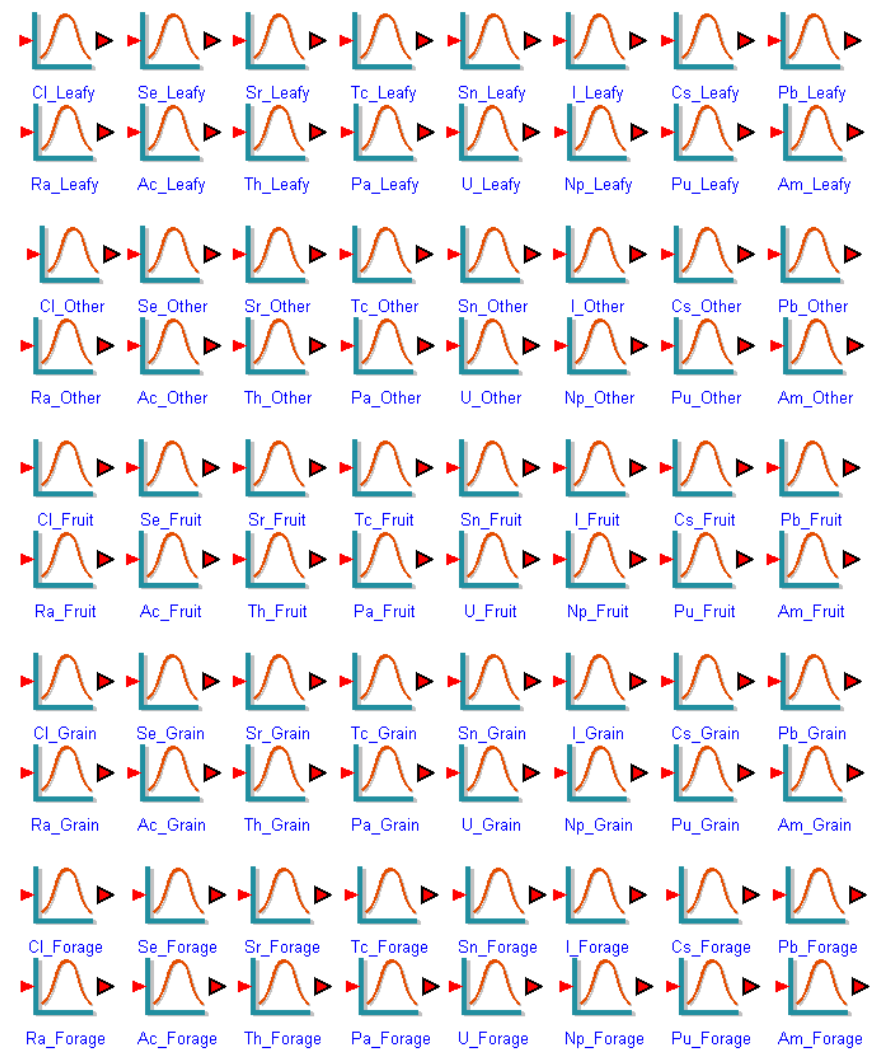

Figure 6.8-4. Input Parameters for Crop Transfer Factors

Within the Data_Selection container (Figure 6.8-5), dose coefficients and dose conversion factors of short-lived radionuclides are added to the corresponding primary radionuclides, which are calculated under the Effective_DCF (Figure 6.8-6). Three containers determine 15 radionuclide-specific input parameters for the primary radionuclides, the first decay products, and the second decay products (if the primary radionuclide has decay products) (Figure 6.8-5). After a radionuclide is selected for processing through the model, the radionuclide specific input parameters are determined using Selector elements (Figure 6.8-7). Parameter names, GoldSim element types, data sources, data types, related mathematical equation numbers, and notations are shown in Table 6.8-1. Information for the decay product containers (Decay1_Rn and Decay2_Rn) is not tabulated, but it is similar to that shown for the primary radionuclides (Table 6.8-1). The selection of decay products is discussed in Section 6.4.1.2 and Table 6.4-1. 
After the radionuclide-related input parameters are selected, the values are passed to the appropriate submodels.

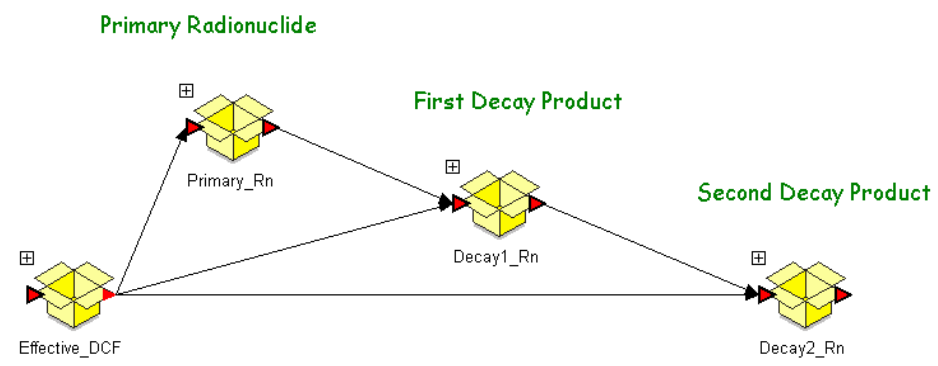

Figure 6.8-5. Radionuclide-Specific Input Parameter Selection

Calculate decay constants

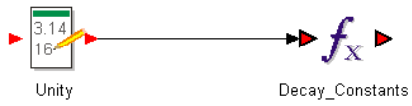

Calculate efective dose coefficients and conversion factors
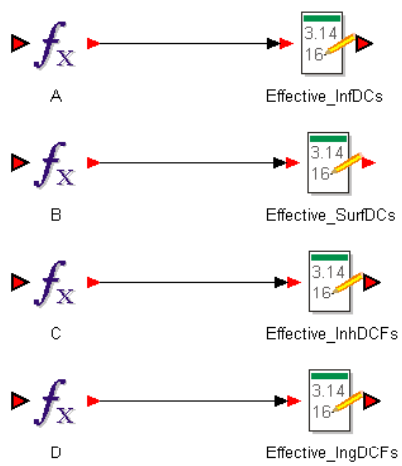

Figure 6.8-6. Calculation of Effective Dose Coefficients and Dose Conversion Factors 


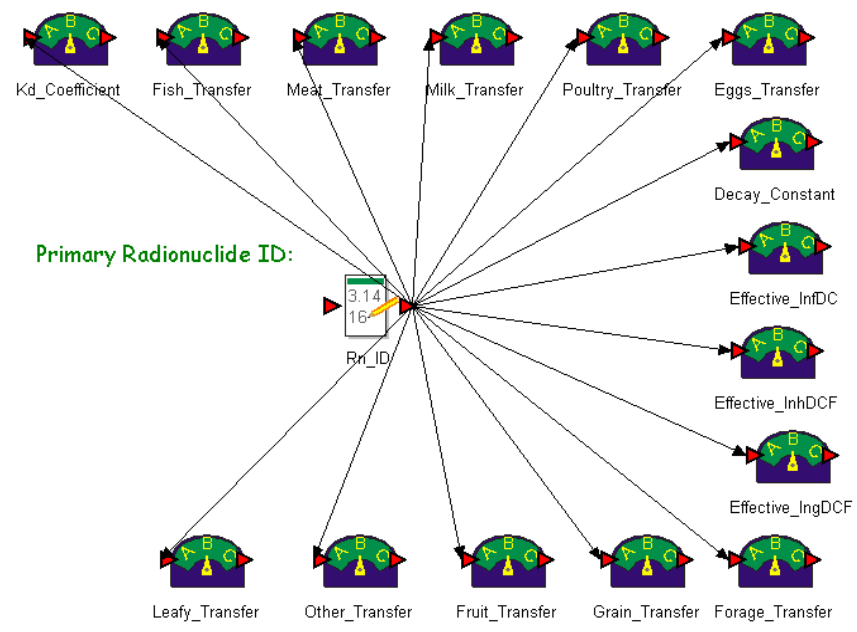

Figure 6.8-7. Primary_Rn Container and Selection of the Second Decay Product

Table 6.8-1. Radionuclide-Related Input Parameters in the Nuclide_Database Container

\begin{tabular}{|c|c|c|c|c|c|c|c|}
\hline $\begin{array}{l}\text { First Level } \\
\text { Box Name }\end{array}$ & $\begin{array}{l}\text { Second } \\
\text { Level } \\
\text { Box } \\
\text { Name }\end{array}$ & $\begin{array}{c}\text { Parameter or } \\
\text { Container Name }\end{array}$ & $\begin{array}{c}\text { Element } \\
\text { Type }^{\text {a }}\end{array}$ & $\begin{array}{c}\text { Data } \\
\text { Source }\end{array}$ & Data Type ${ }^{c}$ & Equation & Notation \\
\hline \multicolumn{3}{|c|}{ Radionuclide_List } & Data & Input & Vector(31) & $6.4 .1-1$ & $i$ \\
\hline \multirow{5}{*}{$\begin{array}{l}\text { Nuclide__ } \\
\text { Data }\end{array}$} & \multicolumn{2}{|c|}{ Kd_Coefficients } & Container & \multirow{5}{*}{\multicolumn{4}{|c|}{ Not shown in detail }} \\
\hline & \multirow{4}{*}{\multicolumn{2}{|c|}{$\begin{array}{l}\text { Crop_Transfers } \\
\text { Animal_Transfers } \\
\text { Fish_Transfers } \\
\text { Nuclear_Data }\end{array}$}} & & & & & \\
\hline & & & & & & & \\
\hline & & & & & & & \\
\hline & & & & & & & \\
\hline \multirow{10}{*}{$\begin{array}{l}\text { Data } \\
\text { Selection }\end{array}$} & \multirow{10}{*}{$\begin{array}{l}\text { Effective } \\
\text { _DCF }\end{array}$} & Unity & Data & - & Vector(31) & - & - \\
\hline & & Decay_Constants & Expression & Cal'ed & Vector(31) & $6.4 .1-1$ & $\lambda_{d i}$ \\
\hline & & A & Expression & Cal'ed & Vector(31) & $6.4 .7-2$ & $D C F_{\text {soil }, i}$ \\
\hline & & $\mathrm{B}$ & Expression & Cal'ed & Vector(31) & Not used & - \\
\hline & & $\mathrm{C}$ & Expression & Cal'ed & Vector(31) & $6.4 .8-8$ & $D C i_{i n h, i}$ \\
\hline & & $\mathrm{D}$ & Expression & Cal'ed & Vector(31) & 6.4.9-7 & $D F_{\text {ing }, i}$ \\
\hline & & Effective_InfDCs & Data & Cal'ed & Vector(31) & $6.4 .7-2$ & $E D C i_{\text {soil }, i}$ \\
\hline & & Effective_SurDCs & Data & Cal'ed & Vector(31) & Not used & - \\
\hline & & Effective_InhDCFs & Data & Cal'ed & Vector(31) & $6.4 .8-8$ & $E D C F_{i n h, i}$ \\
\hline & & Effective_IngDCFs & Data & Cal'ed & Vector(31) & 6.4.9-6 & $E D C F_{\text {ing }, i}$ \\
\hline
\end{tabular}


Table 6.8-1. Radionuclide-Related Input Parameters in the Nuclide_Database Container (Continued)

\begin{tabular}{|c|c|c|c|c|c|c|c|}
\hline $\begin{array}{l}\text { First Level } \\
\text { Box Name }\end{array}$ & $\begin{array}{c}\text { Second } \\
\text { Level } \\
\text { Box } \\
\text { Name }\end{array}$ & $\begin{array}{c}\text { Parameter or } \\
\text { Container Name }\end{array}$ & $\begin{array}{c}\text { Element } \\
\text { Type }^{\text {a }}\end{array}$ & $\begin{array}{c}\text { Data } \\
\text { Source }^{b}\end{array}$ & Data Type $^{c}$ & Equation & Notation \\
\hline \multirow{18}{*}{$\begin{array}{l}\text { Data_- } \\
\text { Selection } \\
\text { (Cont.) }\end{array}$} & \multirow{11}{*}{$\begin{array}{l}\text { Primary_ } \\
\text { Rn }\end{array}$} & Rn_ID & Data & Dbase & Scalar & $6.4 .1-1$ & 1 \\
\hline & & Decay_Constant & Selector & Dbase & Scalar & 6.4.1-1 & $\lambda_{d I}$ \\
\hline & & Effective_InfDC & Selector & Dbase & Scalar & 6.4.7-1 & $E D C i_{\text {soil }, i}$ \\
\hline & & Effective_InhDCF & Selector & \begin{tabular}{|l|} 
Dbase \\
\end{tabular} & Scalar & $6.4 .8-2$ & $E D C F_{i n h, i}$ \\
\hline & & Effective_IngDCF & Selector & Dbase & Scalar & $6.4 .9-2$ & $E D C F_{i n g, i}$ \\
\hline & & Kd_Coefficient & Selector & Dbase & Scalar & $6.4 .1-10$ & $K d_{i}$ \\
\hline & & Fish_Transfer & Selector & Dbase & Scalar & $6.4 .5-1$ & $B F_{i}$ \\
\hline & & Beef_Transfer & Selector & Dbase & Scalar & 6.4.4-2 & $F m_{i, 1}$ \\
\hline & & Poultry_Transfer & Selector & \begin{tabular}{|l} 
Dbase \\
\end{tabular} & Scalar & $6.4 .4-2$ & $F m_{i, 2}$ \\
\hline & & Milk_Transfer & Selector & Dbase & Scalar & 6.4.4-2 & $F m_{i, 3}$ \\
\hline & & Eggs_Transfer & Selector & \begin{tabular}{|l|} 
Dbase \\
\end{tabular} & Scalar & $6.4 .4-2$ & $F m_{i, 4}$ \\
\hline & & Leafy_Transfer & Selector & Dbase & Scalar & 6.4.3-2 & $F_{S \rightarrow p, i, 1}$ \\
\hline & & Other_Transfer & Selector & Dbase & Scalar & $6.4 .3-2$ & $F_{S \rightarrow p, i, 2}$ \\
\hline & & Fruit_Transfer & Selector & Dbase & Scalar & $6.4 .3-2$ & $F_{S \rightarrow p, i, 3}$ \\
\hline & & Grain_Transfer & Selector & Dbase & Scalar & 6.4.3-2 & $F_{S \rightarrow p, i, 4}$ \\
\hline & & Forage_Transfer & Selector & Dbase & Scalar & $6.4 .3-2$ & $F_{S \rightarrow p, i, 5}$ \\
\hline & \multicolumn{2}{|c|}{ Decay1_Rn } & Container & \multicolumn{4}{|c|}{ Not shown in detail } \\
\hline & \multicolumn{2}{|c|}{ Decay2 Rn } & Container & \multicolumn{4}{|c|}{ Not shown in detail } \\
\hline
\end{tabular}

NOTE: The following notes apply to all GoldSim tables in Section 6.8.

${ }^{a}$ Element type is the GoldSim element type used for inputs, calculations, and other manipulations (Section 6.7.3).

b If the data source is "Input," the parameter values are entered in the GoldSim element. If the source is "Dbase," values are taken from a database, or calculated values. If the data source is "Cal'ed," it is a calculated quantity. If the source and the corresponding equation notation are dashes (-), the element is added for GoldSim array calculations.

${ }^{c}$ Data types are scalar (a single value) or array (a set of values). A one-dimensional array is called a vector, and a two-dimensional array is called a matrix. The number of values in the array is given in parenthesis.

\subsubsection{Surface Soil Submodel}

The mathematical equations for the surface soil submodel are discussed in Section 6.4.1. The contents of the submodel container are shown in Figure 6.8-8. All GoldSim elements in the surface soil submodel are listed in Table 6.8-2. The submodel includes five lower-level containers. The SoilModel_Input container includes all input parameters in the submodel (Figure 6.8-9), which are all distribution parameters. The Soil_Conc container includes calculations of the leaching factor (Equation 6.4.1-10) and the equilibrium concentration for primary radionuclides (Equation 6.4.1-4). The Soil_Conc_1 container includes calculations of the soil buildup of the first decay product, while the Soil_Conc_2 container includes calculations of the buildup of the second decay product. The calculation of decay-product buildup is similar to the calculations for primary radionuclides in the Soil_Conc container, but uses Equation 6.4.1-9 and radionuclide-specific input parameters. Although the special submodel for ${ }^{14} \mathrm{C}$ in the soil is discussed separately (Section 6.4.6), the soil concentration of ${ }^{14} \mathrm{C}$ is included in the C14_Soil container in the surface soil submodel. 
This submodel calculates the saturation concentration of radionuclides in surface soil

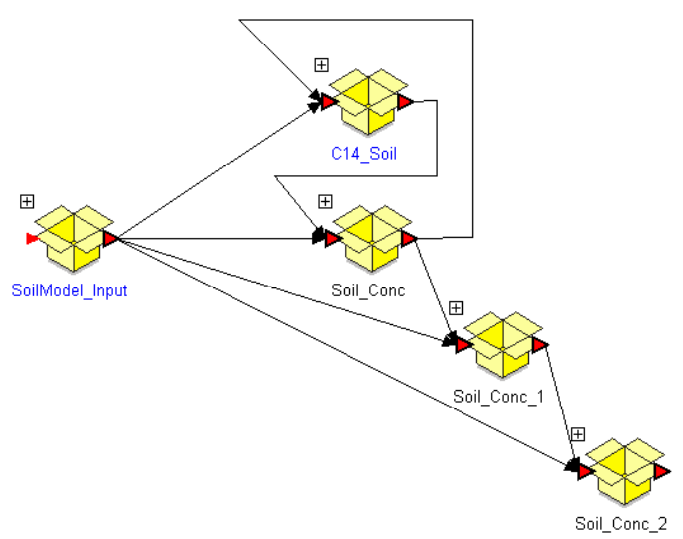

Figure 6.8-8. Soil Submodel Container

Parameters for calculating radionuclide concentration in surface soil (decay constant in Nuclide Database)
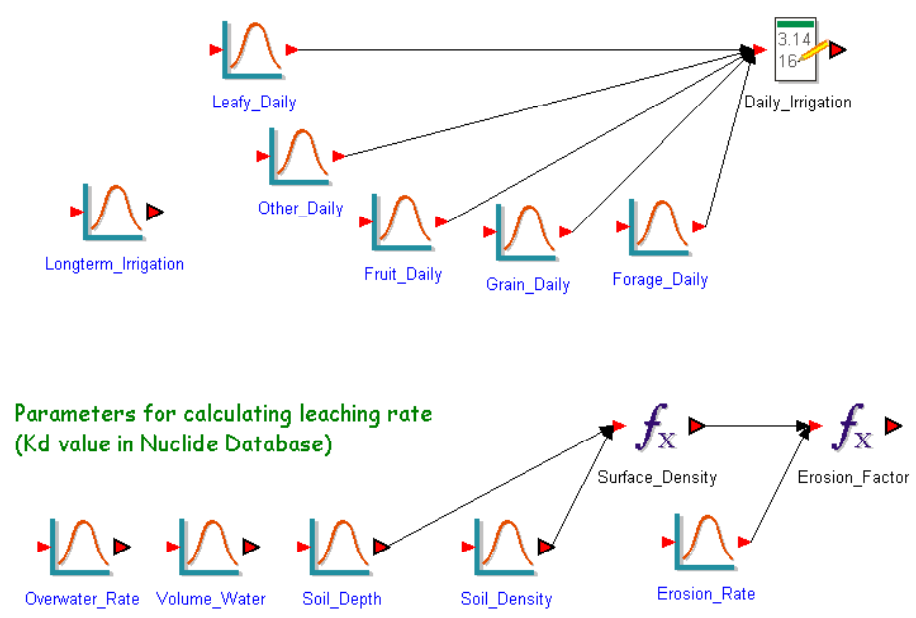

Figure 6.8-9. Input Parameter Container (SoilModel_Input) for the Soil Submodel 
Table 6.8-2. Parameters in the Surface Soil Submodel

\begin{tabular}{|c|c|c|c|c|c|c|}
\hline $\begin{array}{c}\text { Low Level Box } \\
\text { Name }\end{array}$ & Parameter Name & $\begin{array}{c}\text { Element } \\
\text { Type }\end{array}$ & $\begin{array}{c}\text { Data } \\
\text { Source }\end{array}$ & Data Type & Equation & Notation \\
\hline \multirow[t]{14}{*}{ SoilModel_Input } & Leafy_Daily & Stochastic & Input & Scalar & 6.4.3-4 & $I R D_{1}$ \\
\hline & Other_Daily & Stochastic & Input & Scalar & 6.4.3-4 & $I R D_{2}$ \\
\hline & Fruit_Daily & Stochastic & Input & Scalar & $6.4 .3-4$ & $I R D_{3}$ \\
\hline & Grain_Daily & Stochastic & Input & Scalar & 6.4.3-4 & $I R D_{4}$ \\
\hline & Forage_Daily & Stochastic & Input & Scalar & 6.4.3-4 & $I R D_{5}$ \\
\hline & Daily_Irrigation & Data & Cal'ed & Vector(5) & $6.4 .3-4$ & $I R D_{j}$ \\
\hline & Longterm Irrigation & Stochastic & Input & Scalar & 6.4.1-1 & IR \\
\hline & Overwater_Rate & Stochastic & Input & Scalar & $6.4 .1-10$ & OW \\
\hline & Volume_Water & Stochastic & Input & Scalar & $6.4 .1-10$ & $\theta$ \\
\hline & Erosion_Rate & Stochastic & Input & Scalar & 6.4.1-11 & $E R$ \\
\hline & Erosion_Factor & Expression & Cal'ed & Scalar & 6.4.1-11 & $\lambda_{e}$ \\
\hline & Soil_Depth & Stochastic & Input & Scalar & $6.4 .1-6$ & $d$ \\
\hline & Soil_Density & Stochastic & Input & Scalar & 6.4.1-6 & $\rho$ \\
\hline & Surface_Density & Expression & Cal'ed & Scalar & 6.4.1-5 & $\rho_{\mathrm{S}}$ \\
\hline \multirow[t]{4}{*}{ C14_Soil } & Emission_Factor & Data & Input & Scalar & 6.4.6-1 & $\lambda_{a, C-14}$ \\
\hline & C14_Zero & Data & - & Vector(5) & - & - \\
\hline & C14Conc_CropSoil & Expression & Cal'ed & Vector(5) & 6.4.6-1 & $\mathrm{CS}_{\mathrm{C}-14, j}$ \\
\hline & C14Conc InhSoil & Expression & Cal'ed & Scalar & $6.4 .6-1$ & $C S_{C-14,0}$ \\
\hline \multirow[t]{6}{*}{ Soil_Conc } & Partition_Coefficient & Data & Dbase & Scalar & $6.4 .1-10$ & $K d_{i}$ \\
\hline & Leaching_Factor & Expression & Cal'ed & Scalar & $6.4 .1-10$ & $\lambda_{l, i}$ \\
\hline & Decay_Factor & Data & Dbase & Scalar & $6.4 .1-1$ & $\lambda_{d, i}$ \\
\hline & Effective_Removal & Expression & Cal'ed & Scalar & 6.4.1-3 & $\lambda_{\text {eff }, i}$ \\
\hline & Saturated_Conc & Expression & Cal'ed & Scalar & 6.4.1-4 & $C s_{i}$ \\
\hline & SatMass_Conc & Expression & Cal'ed & Scalar & $6.4 .1-5$ & $C s_{m, i}$ \\
\hline \multirow[t]{6}{*}{ Soil_Conc_1 } & Partition_Coefficient_1 & Data & Dbase & Scalar & $6.4 .1-10$ & $K d_{1}$ \\
\hline & Leaching_Factor_1 & Expression & Cal'ed & Scalar & $6.4 .1-10$ & $\lambda_{1,1}$ \\
\hline & Decay_Factor_1 & Data & Dbase & Scalar & $6.4 .1-1$ & $\lambda_{d, 1}$ \\
\hline & Effective_Removal_1 & Expression & Cal'ed & Scalar & 6.4.1-3 & $\lambda_{\text {eff,1 }}$ \\
\hline & Saturated_Conc_1 & Expression & Cal'ed & Scalar & 6.4.1-9 & $\mathrm{Cs}_{1}$ \\
\hline & SatMass_Conc_1 $\overline{1}$ & Expression & Cal'ed & Scalar & $6.4 .1-5$ & $C s_{m, 1}$ \\
\hline \multirow[t]{6}{*}{ Soil_Conc_2 } & Partition_Coefficient_2 & Data & Dbase & Scalar & $6.4 .1-10$ & $K d_{2}$ \\
\hline & Leaching_Factor_2 & Expression & Cal'ed & Scalar & $6.4 .1-10$ & $\lambda_{1,2}$ \\
\hline & Decay_Factor_2 & \begin{tabular}{|l|} 
Data \\
\end{tabular} & Dbase & Scalar & $6.4 .1-1$ & $\lambda_{d, 2}$ \\
\hline & Effective_Removal_2 & Expression & Cal'ed & Scalar & 6.4.1-3 & $\lambda_{\text {eff }, 2}$ \\
\hline & Saturated_Conc_2 & Expression & Cal'ed & Scalar & 6.4.1-9 & $\mathrm{Cs}_{2}$ \\
\hline & SatMass_Conc_2 & Expression & Cal'ed & Scalar & $6.4 .1-5$ & $\mathrm{Cs}_{m, 2}$ \\
\hline
\end{tabular}

NOTE: See notes for Table 6.8-1.

\subsubsection{Air Submodel}

The mathematical equations used for the air submodel are discussed in Section 6.4.2. All parameters in the air submodel are summarized in Table 6.8-3. This submodel includes five lower-level containers (Figure 6.8-10). The AirModel_Input container includes two input parameters: mass loading and the enhancement factor (Figure 6.8-11). The Dust_Air container calculates radionuclide concentrations in the air due to resuspended particles for direct deposition on crops (Equation 6.4.2-1) and human inhalation (Equation 6.4.2-2). Because particle resuspension is linked to the surface soil, decay product accumulation in surface soil is considered in the soil container. The Radon_Air container includes calculations related to the release of radon gas from ${ }^{226} \mathrm{Ra}$-contaminated soil. Radon-222 is a decay product of the primary radionuclide ${ }^{226} \mathrm{Ra}$, and a decay product of ${ }^{226} \mathrm{Ra}$, which is a decay product of ${ }^{230} \mathrm{Th}$. The special 
submodel for ${ }^{14} \mathrm{C}$ in the air (Section 6.4.6.2) is included in the C14_Air container of the air submodel. Radionuclide concentrations in the air due to aerosols from evaporative coolers are calculated in the Evaporative_Air container. Because aerosols are released directly from contaminated groundwater, a decay chain due to radionuclide buildup in the soil is not considered.

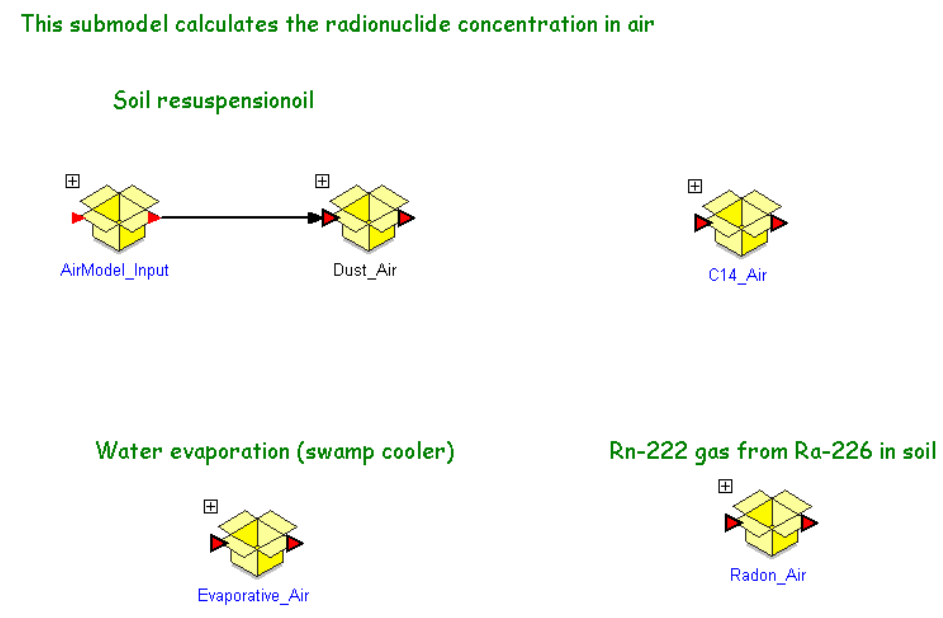

Figure 6.8-10. Air Submodel Container

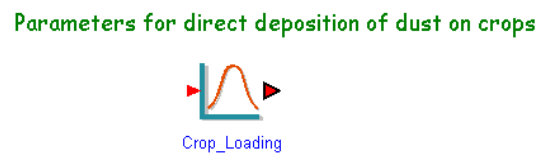

Parameter for dust Inhalation
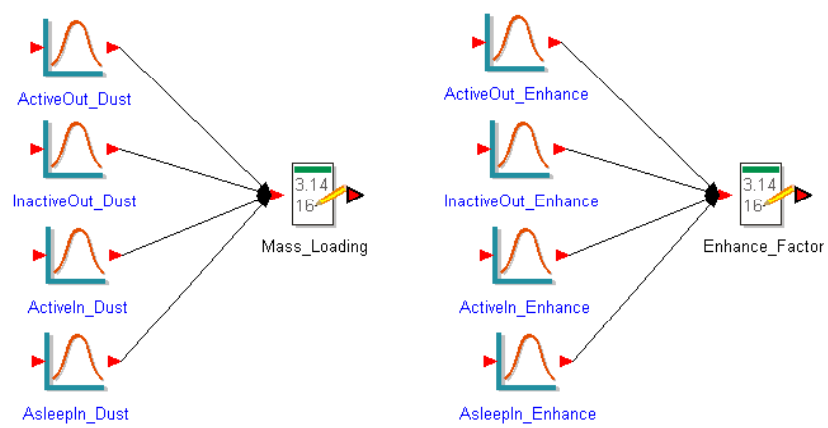

Figure 6.8-11. Input Parameter Container (AirModel_Input) for the Air Submodel 
Table 6.8-3. Parameters in the Air Submodel

\begin{tabular}{|c|c|c|c|c|c|c|}
\hline $\begin{array}{c}\text { Low Level Box } \\
\text { Name }\end{array}$ & Parameter Name & $\begin{array}{c}\text { Element } \\
\text { Type }\end{array}$ & $\begin{array}{c}\text { Data } \\
\text { Source }\end{array}$ & $\begin{array}{l}\text { Data } \\
\text { Type }\end{array}$ & Equation & Notation \\
\hline \multirow{11}{*}{ AirModel_Input } & Crop_Loading & Stochastic & Input & Scalar & $6.4 .2-1$ & $S$ \\
\hline & ActiveOut_Dust & Stochastic & Input & Scalar & 6.4.2-2 & $S_{1}$ \\
\hline & InactiveOut_Dust & Stochastic & Input & Scalar & $6.4 .2-2$ & $S_{2}$ \\
\hline & Activeln_Dust & Stochastic & Input & Scalar & 6.4.2-2 & $S_{3}$ \\
\hline & Asleepln_Dust & Stochastic & Input & Scalar & $6.4 .2-2$ & $\mathrm{~S}_{4}$ \\
\hline & Mass_Loading & Data & Cal'ed & Vector(5) & $6.4 .2-2$ & $S_{n}$ \\
\hline & ActiveOut_Enhance & Stochastic & Input & Scalar & $6.4 .2-2$ & $f_{\text {enhance, } 1}$ \\
\hline & InactiveOut_Enhance & Stochastic & Input & Scalar & 6.4.2-2 & $f_{\text {enhance, } 2}$ \\
\hline & Activeln_Enhance & Stochastic & Input & Scalar & $6.4 .2-2$ & $f_{\text {enhance, } 3}$ \\
\hline & Asleepln_Enhance & Stochastic & Input & Scalar & $6.4 .2-2$ & $f_{\text {enhance, } 4}$ \\
\hline & Enhance_Factor & Data & Cal'ed & Vector(5) & 6.4.2-2 & $f_{\text {enhance }, n}$ \\
\hline \multirow[t]{6}{*}{ Dust_Air } & AirConc_Crop & Expression & Cal'ed & Scalar & $6.4 .2-1$ & $C a_{p, i}$ \\
\hline & AirConc_Crop_1 & Expression & Cal'ed & Scalar & 6.4.2-1 & $C a_{p, 1}$ \\
\hline & AirConc_Crop_2 & Expression & Cal'ed & Scalar & 6.4.2-1 & $C a_{p, 2}$ \\
\hline & AirConc_Inh & Expression & Cal'ed & Vector(5) & $6.4 .2-2$ & $C a_{h, i, n}$ \\
\hline & AirConc_Inh_1 & Expression & Cal'ed & Vector(5) & $6.4 .2-2$ & $C a_{h, 1, n}$ \\
\hline & AirConc_Inh_2 & Expression & Cal'ed & Vector(5) & $6.4 .2-2$ & $C a_{h, 2, n}$ \\
\hline \multirow[t]{9}{*}{ C14_Air } & Mixing_Height_Crop & Data & Input & Scalar & 6.4.6-3 & $H_{\text {mix }}$ \\
\hline & Wind_Speed_Crop & Stochastic & Input & Scalar & 6.4.6-3 & $U$ \\
\hline & Irrigation_Area & Data & Input & Scalar & 6.4.6-3 & $A$ \\
\hline & Wind_Speed_Inh & Stochastic & Input & Scalar & 6.4.6-3 & $U$ \\
\hline & Mixing_Height_Inh & Data & Input & Scalar & 6.4.6-3 & $H_{\text {mix }}$ \\
\hline & C14Conc_Air & Expression & Cal'ed & Scalar & 6.4.6-3 & $C a_{g, C-14}$ \\
\hline & C14_Flux & Expression & Cal'ed & Scalar & 6.4.6-2 & $E V S N_{j}$ \\
\hline & C14Conc_Inh & Expression & Cal'ed & Scalar & 6.4.6-3 & $C a_{\text {g.C-14,0 }}$ \\
\hline & C14Air_Inh & Data & Cal'ed & Vector(5) & 6.4.6-3 & $C a_{g, C-14, j}$ \\
\hline \multirow[t]{4}{*}{ Evaporative_Air } & Evap_Fraction & Stochastic & Input & Scalar & 6.4.2-3 & $f_{\text {evap }}$ \\
\hline & Water_Usage & Stochastic & Input & Scalar & 6.4.2-3 & $M_{\text {water }}$ \\
\hline & Airflow_Rate & Stochastic & Input & Scalar & 6.4.2-3 & $F_{\text {air }}$ \\
\hline & AirConc_Evap & Expression & Cal'ed & Scalar & $6.4 .2-3$ & $C a_{e, i}$ \\
\hline \multirow[t]{11}{*}{ Radon_Air } & Radon_ConcRatio & Stochastic & \begin{tabular}{|l|} 
Input \\
\end{tabular} & Scalar & $6.4 .2-4$ & $f_{m, R n-222}$ \\
\hline & AirConc_Radon & Expression & Cal'ed & Scalar & $6.4 .2-4$ & $C a_{g, R n-222}$ \\
\hline & AirConc_Radon_1 & Expression & Cal'ed & Scalar & $6.4 .2-4$ & $C a_{g, R n-222}$ \\
\hline & AirConc_Radon_2 & Expression & Cal'ed & Scalar & 6.4.2-4 & $C a_{g, R n-222}$ \\
\hline & Evap_Ventilation & Stochastic & \begin{tabular}{|l|} 
Input \\
\end{tabular} & Scalar & $6.4 .2-5$ & $v_{e}$ \\
\hline & RnFraction_Indoor & Stochastic & Input & Scalar & 6.4.2-6 & $f_{\text {house }}$ \\
\hline & House_Height & Stochastic & Input & Scalar & $6.4 .2-5$ & $\mathrm{H}$ \\
\hline & Radon_FluxRatio & Data & Input & Scalar & $6.4 .2-7$ & $C_{\text {Rn-222 }}$ \\
\hline & Normal_Ventilation & Stochastic & Input & Scalar & $6.4 .2-5$ & $V_{n}$ \\
\hline & Indoor_RnEvap & Expression & Cal'ed & Scalar & $6.4 .2-8$ & $I F_{e, R n-222}$ \\
\hline & Indoor_RnNormal & Expression & Cal'ed & Scalar & 6.4.2-7 & $I F_{n, R n-222}$ \\
\hline
\end{tabular}

NOTE: See notes for Table 6.8-1.

\subsubsection{Plant Submodel}

Mathematical equations for the plant submodel are discussed in Section 6.4.3. All parameters in the submodel are listed in Table 6.8-4. The contents of the submodel container (Figure 6.8-12) include five lower-level containers. The PlantModel_Input container includes many input parameters related to agriculture (Figure 6.8-13). There is one container under the PlantModel_Input container, which contains input parameters related to irrigation. Each mechanism of radionuclide transfer into crops is considered separately. The Water_Uptake container includes calculations of radionuclide concentrations in crops (Equations 6.4.3-3, 
6.4.3-4, and 6.4.3-5) due to foliar interception of contaminated groundwater. One element, the Intercept_Factor, combines the interception fractions for the various crops into a vector and checks to determine if the sum exceeds 1.0 (this parameter can not exceed 1.0; Section 6.4.3.2). The Root_Uptake container includes calculations of the radionuclide concentrations in crops (Equation 6.4.3-2) due to root uptake of radionuclides. The Dust_Uptake container includes calculations of the radionuclide concentrations in crops (Equations 6.4.3-6, 6.4.3-7, and 6.4.3-8) due to the deposition of resuspended contaminated soil. The C14_Crop container includes the calculations of transfer of ${ }^{14} \mathrm{C}$ into plants (Section 6.4.6.3).

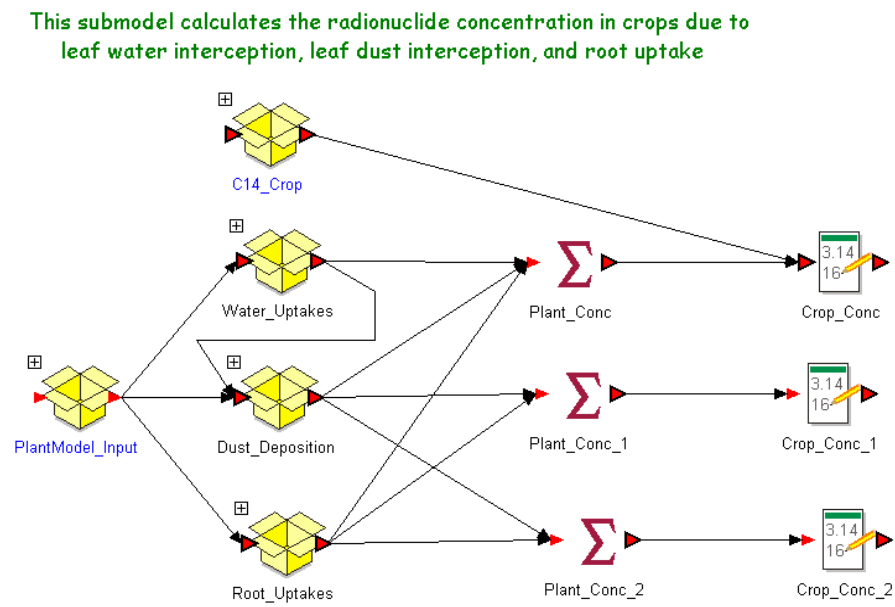

Figure 6.8-12. Plant Submodel Container
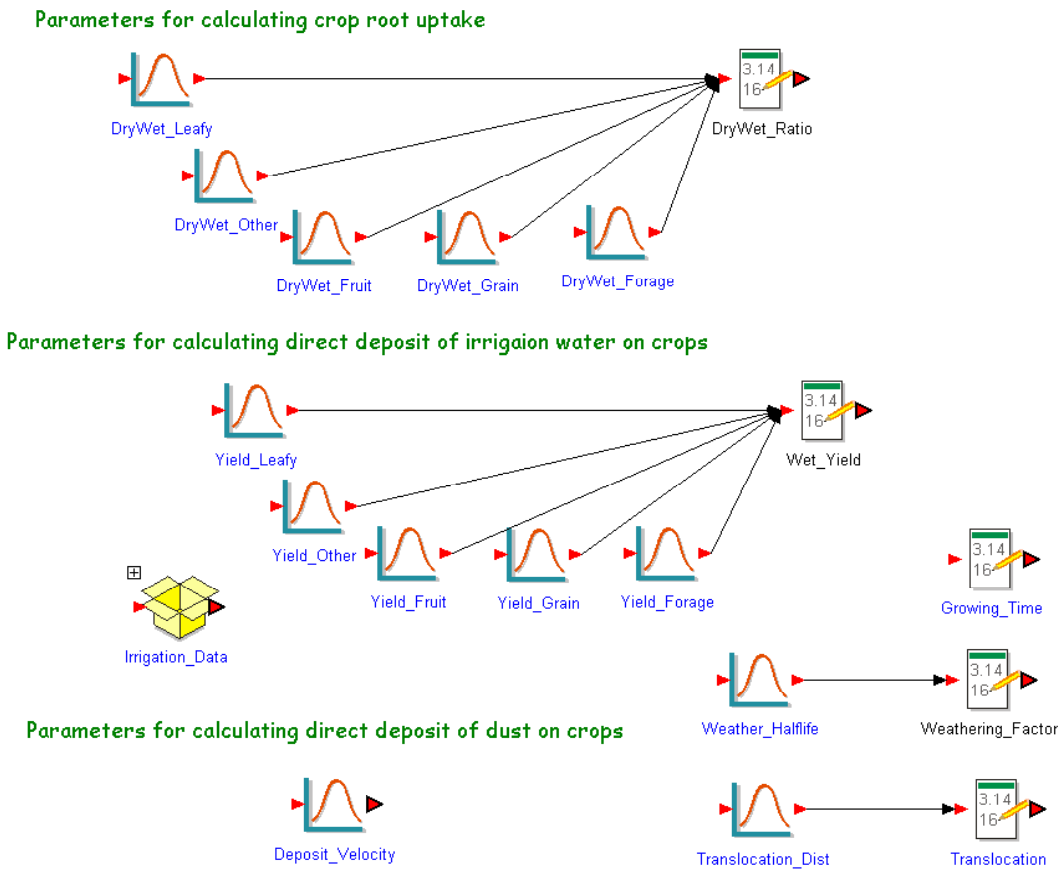

Figure 6.8-13. Input Parameter Container (PlantModel_Input) for the Plant Submodel 
Table 6.8-4. Parameters in the Plant Submodel

\begin{tabular}{|c|c|c|c|c|c|c|c|}
\hline $\begin{array}{c}\text { First Level } \\
\text { Name }\end{array}$ & $\begin{array}{r}\text { Secor } \\
\text { Level } N\end{array}$ & Parameter Name & $\begin{array}{l}\text { Element } \\
\text { Type }\end{array}$ & $\begin{array}{c}\text { Data } \\
\text { Source }\end{array}$ & $\begin{array}{l}\text { Data } \\
\text { Type }\end{array}$ & Equation & Notation \\
\hline \multirow{35}{*}{$\begin{array}{l}\text { Plant } \\
\text { Model__ } \\
\text { Input }\end{array}$} & \multicolumn{2}{|c|}{ DryWet_Leafy } & Stochastic & Input & Scalar & $6.4 .3-2$ & $D W_{1}$ \\
\hline & \multicolumn{2}{|c|}{ DryWet_Other } & Stochastic & Input & Scalar & $6.4 .3-2$ & $D W_{2}$ \\
\hline & \multicolumn{2}{|c|}{ DryWet_Fruit } & Stochastic & Input & Scalar & $6.4 .3-2$ & $D W_{3}$ \\
\hline & \multicolumn{2}{|c|}{ DryWet_Grain } & Stochastic & Input & Scalar & $6.4 .3-2$ & $D W_{4}$ \\
\hline & \multicolumn{2}{|c|}{ DryWet Forage } & Stochastic & Input & Scalar & $6.4 .3-2$ & $D W_{5}$ \\
\hline & \multicolumn{2}{|c|}{ DryWet_Ratio } & Data & Cal'ed & Vector(5) & $6.4 .3-2$ & $D W_{j}$ \\
\hline & \multicolumn{2}{|c|}{ Yield_Leafy } & Stochastic & Input & Scalar & $6.4 .3-3$ & $Y_{1}$ \\
\hline & \multicolumn{2}{|c|}{ Yield Other } & Stochastic & Input & Scalar & $6.4 .3-3$ & $Y_{2}$ \\
\hline & \multicolumn{2}{|c|}{ Yield_Fruit } & Stochastic & Input & Scalar & $6.4 .3-3$ & $Y_{3}$ \\
\hline & \multicolumn{2}{|c|}{ Yield_Grain } & Stochastic & Input & Scalar & $6.4 .3-3$ & $Y_{4}$ \\
\hline & \multicolumn{2}{|c|}{ Yield_Forage } & Stochastic & Input & Scalar & $6.4 .3-3$ & $Y_{5}$ \\
\hline & \multicolumn{2}{|c|}{ Wet_Yield } & Data & Cal'ed & Vector(5) & $6.4 .3-3$ & $Y_{j}$ \\
\hline & \multicolumn{2}{|c|}{ Growing_Time } & Data & Input & Vector(5) & 6.4.3-3 & $t_{g, j}$ \\
\hline & \multicolumn{2}{|c|}{ Weather_Halflife } & Stochastic & input & Scalar & $6.4 .3-3$ & $T_{w}$ \\
\hline & \multicolumn{2}{|c|}{ Weathering_Factor } & Data & Cal'ed & Scalar & $6.4 .3-3$ & $\lambda_{w}$ \\
\hline & \multicolumn{2}{|c|}{ Translocation_Dist } & Stochastic & Input & Scalar & $6.4 .3-3$ & $T_{j}$ \\
\hline & \multicolumn{2}{|c|}{ Translocation } & Data & Input & Vector(5) & $6.4 .3-3$ & $T_{j}$ \\
\hline & \multicolumn{2}{|c|}{ Deposit_Velocity } & Stochastic & Input & Scalar & 6.4.3-7 & $V_{d}$ \\
\hline & \multirow{17}{*}{$\begin{array}{l}\text { Irrigation } \\
\text { _Data }\end{array}$} & DryBiom Leafy & Stochastic & Input & Scalar & $6.4 .3-5$ & $D B_{1}$ \\
\hline & & DryBiom_Other & Stochastic & Input & Scalar & 6.4.3-5 & $D B_{2}$ \\
\hline & & DryBiom_Fruit & Stochastic & Input & Scalar & $6.4 .3-5$ & $D B_{3}$ \\
\hline & & DryBiom_Grain & Stochastic & Input & Scalar & $6.4 .3-5$ & $D B_{4}$ \\
\hline & & DryBiom_Forage & Stochastic & Input & Scalar & 6.4.3-5 & $D B_{5}$ \\
\hline & & IrriAmt_Leafy & Stochastic & Input & Scalar & $6.4 .3-5$ & $I A_{1}$ \\
\hline & & IrriAmt_Other & Stochastic & Input & Scalar & $6.4 .3-5$ & $I A_{2}$ \\
\hline & & IrriAmt Fruit & Stochastic & Input & Scalar & $6.4 .3-5$ & $I A_{3}$ \\
\hline & & IrriAmt_Grain & Stochastic & Input & Scalar & $6.4 .3-5$ & $I A_{4}$ \\
\hline & & IrriAmt_Forage & Stochastic & Input & Scalar & $6.4 .3-5$ & $I A_{5}$ \\
\hline & & Irrigation_Intensity & Stochastic & Input & Scalar & $6.4 .3-5$ & 1 \\
\hline & & Overhead_Leafy & Stochastic & Input & Scalar & $6.4 .3-3$ & $f_{0,1}$ \\
\hline & & Overhead_Other & Stochastic & Input & Scalar & $6.4 .3-3$ & $f_{0,2}$ \\
\hline & & Overhead_Fruit & Stochastic & Input & Scalar & $6.4 .3-3$ & $\frac{f_{0,3}}{f_{0,3}}$ \\
\hline & & Overhead_Grain & Stochastic & Input & Scalar & $6.4 .3-3$ & $f_{0,4}$ \\
\hline & & Overhead_Forage & Stochastic & Input & Scalar & $6.4 .3-3$ & $f_{0,5}$ \\
\hline & & Overhead_Factor & Data & Cal'ed & Vector(5) & $6.4 .3-3$ & $f_{o, j}$ \\
\hline & ExpGron & & Expression & Cal'ed & Scalar & $6.4 .3-3$ & $e^{-\lambda \omega t g, 1}$ \\
\hline Uptakes & ExpGron & & Expression & Cal'ed & Scalar & $6.4 .3-3$ & $e^{-\lambda w t g, 2}$ \\
\hline & ExpGron & & Expression & Cal'ed & Scalar & $6.4 .3-3$ & $e^{-\lambda \omega t g, 3}$ \\
\hline & ExpGron & & Expression & Cal'ed & Scalar & $6.4 .3-3$ & $e^{-\lambda W t g, 4}$ \\
\hline & ExpGron & & Expression & Cal'ed & Scalar & $6.4 .3-3$ & $e^{-\lambda \omega t g, 5}$ \\
\hline & Growing & & Data & Cal'ed & Vector(5) & $6.4 .3-3$ & $1-e^{-\lambda \omega t g, j}$ \\
\hline & Other_Fa & & Expression & Cal'ed & Vector(5) & $6.4 .3-3$ & $\begin{array}{l}T_{j} /\left(Y_{j} \lambda_{w}\right) \\
\left(1-e^{-\lambda w ~ t g, j}\right)\end{array}$ \\
\hline & IntFrac_L & & Expression & Cal'ed & Scalar & 6.4.3-3 & $R w_{1}$ \\
\hline & IntFrac__ & & Expression & Cal'ed & Scalar & $6.4 .3-3$ & $R W_{2}$ \\
\hline & IntFrac_F & & Expression & Cal'ed & Scalar & $6.4 .3-3$ & $R W_{3}$ \\
\hline & IntFrac__ & & Expression & Cal'ed & Scalar & $6.4 .3-3$ & $R w_{4}$ \\
\hline & IntFrac_F & & Expression & Cal'ed & Scalar & $6.4 .3-3$ & $R w_{5}$ \\
\hline & Intercept & & Data & Cal'ed & Vector(5) & $6.4 .3-3$ & $R w_{j}$ \\
\hline & Irrigation & cept & Expression & Cal'ed & Vector(5) & $6.4 .3-3$ & $D w_{i, j} R w$ \\
\hline & Water_U & & Expression & Cal'ed & Vector(5) & $6.4 .3-3$ & $C p_{\text {water } i, j}$ \\
\hline
\end{tabular}


Table 6.8-4. Parameters in the Plant Submodel (Continued)

\begin{tabular}{|c|c|c|c|c|c|c|}
\hline $\begin{array}{l}\text { First Level } \\
\text { Name }\end{array}$ & \begin{tabular}{c|c}
$\begin{array}{c}\text { Second } \\
\text { Level Name }\end{array}$ & Parameter Name \\
\end{tabular} & $\begin{array}{c}\text { Element } \\
\text { Type }\end{array}$ & $\begin{array}{c}\text { Data } \\
\text { Source }\end{array}$ & $\begin{array}{l}\text { Data } \\
\text { Type }\end{array}$ & Equation & Notation \\
\hline \multirow{6}{*}{$\begin{array}{l}\text { Root } \\
\text { Uptakes }\end{array}$} & Transfer_Factor & Data & Dbase & Vector(5) & $6.4 .3-2$ & $F_{S \rightarrow p, i, j}$ \\
\hline & Transfer_Factor_1 & Data & Dbase & Vector(5) & 6.4.3-2 & $F_{S \rightarrow p, 1, j}$ \\
\hline & Transfer_Factor_2 & Data & Dbase & Vector(5) & $6.4 .3-2$ & $F_{S \rightarrow 0,2 j}$ \\
\hline & Root_Uptake & Expression & Cal'ed & Vector(5) & $6.4 .3-2$ & $C p_{\text {root }, i, j}$ \\
\hline & Root_Uptake_1 & Expression & Cal'ed & Vector(5) & $6.4 .3-2$ & $C p_{\text {root }, 1, j}$ \\
\hline & Root_Uptake_2 & Expression & Cal'ed & Vector(5) & $6.4 .3-2$ & $C p_{\text {root }, 2, j}$ \\
\hline \multirow{13}{*}{$\begin{array}{l}\text { Dust } \\
\text { Uptakes }\end{array}$} & \multirow{2}{*}{\begin{tabular}{|l|} 
Dust_Factor \\
ExpDust Leafy
\end{tabular}} & Data & Input & Vector(5) & 6.4.3-8 & $a_{j}$ \\
\hline & & Expression & Cal'ed & Scalar & 6.4.3-8 & $e^{-a_{1}{ }_{1} B_{1}}$ \\
\hline & ExpDust_Other & Expression & Cal'ed & Scalar & $6.4 .3-8$ & $e^{-a_{2}}{ }^{D B}{ }_{2}$ \\
\hline & ExpDust Fruit & Expression & Cal'ed & Scalar & $6.4 .3-8$ & $e^{-a_{3} D B_{3}}$ \\
\hline & ExpDust_Grain & Expression & Cal'ed & Scalar & 6.4.3-8 & $e^{-a}{ }_{4}^{D B}{ }_{4}$ \\
\hline & ExpDust_Forage & \begin{tabular}{|l|} 
Expression \\
\end{tabular} & Cal'ed & Scalar & 6.4.3-8 & $e^{-a_{5} D B_{5}}$ \\
\hline & Dust_Intercept & Data & Cal'ed & Vector(5) & $6.4 .3-8$ & $R a_{j}$ \\
\hline & Air_Interception & Expression & Cal'ed & Vector(5) & $6.4 .3-6$ & $D a_{i, j} R a_{j}$ \\
\hline & Air_Interception_1 & Expression & Cal'ed & Vector(5) & $6.4 .3-6$ & $D a_{1, j} R a_{j}$ \\
\hline & Air_Interception_2 & Expression & Cal'ed & Vector(5) & 6.4.3-6 & $D a_{2, j} R a_{j}$ \\
\hline & Dust Uptake & Expression & Cal'ed & Vector(5) & $6.4 .3-6$ & $C p_{\text {dust }, i, j}$ \\
\hline & Dust_Uptake_1 & Expression & Cal'ed & Vector(5) & $6.4 .3-6$ & $C p_{\text {dust }, 1, j}$ \\
\hline & Dust_Uptake_2 & Expression & Cal'ed & Vector(5) & 6.4.3-6 & $C p_{\text {dust }, 2, j}$ \\
\hline \multirow[t]{8}{*}{ C14_Crop } & & \begin{tabular}{|l|} 
Data \\
\end{tabular} & Input & Vector(5) & 6.4.6-6 & $f C_{\text {plant }, j}$ \\
\hline & $\begin{array}{l}\text { Crop_Carbon } \\
\text { Air Carbon }\end{array}$ & Data & Input & Scalar & $6.4 .6-5$ & $f C_{\text {air }}$ \\
\hline & Soil_Carbon & Data & Input & Scalar & 6.4.6-4 & $f C_{\text {soil }}$ \\
\hline & Cair_Uptake & Data & Input & Scalar & $6.4 .6-6$ & $\mathrm{Fa}$ \\
\hline & Csoil_Uptake & Data & Input & Scalar & $6.4 .6-6$ & $F S$ \\
\hline & C14Crop_Air & Expression & Cal'ed & Vector(5) & $6.4 .6-6$ & $\begin{array}{l}F a C a_{C-14} / \\
f_{\text {air }}\end{array}$ \\
\hline & C14Crop_Soil & Expression & Cal'ed & Vector(5) & 6.4.6-6 & $\begin{array}{l}F S C S_{C-14} / \\
\left(f C_{\text {soil }} \rho_{S}\right)\end{array}$ \\
\hline & C14Conc_Crop & Expression & Cal'ed & Vector(5) & $6.4 .6-6$ & $C p_{C-14, j}$ \\
\hline \multicolumn{2}{|c|}{ Plant_Conc } & \begin{tabular}{|l|} 
Sum \\
\end{tabular} & Cal'ed & $\begin{array}{l}\text { Vector(5) } \\
\end{array}$ & 6.4.3-1 & $C p_{i, j}$ \\
\hline Plant_Conc & & Sum & Cal'ed & Vector(5) & 6.4.3-1 & $C p_{1, j}$ \\
\hline Plant_Conc & & Sum & Cal'ed & Vector(5) & 6.4.3-1 & $C p_{2, j}$ \\
\hline Crop_Conc & & Data & Cal'ed & Vector(4) & 6.4.3-1 & $C p_{i, j}$ \\
\hline Crop_Conc & & Data & Cal'ed & Vector(4) & 6.4.3-1 & $C p_{1, j}$ \\
\hline Crop_Conc & & Data & Cal'ed & Vector(4) & 6.4.3-1 & $C p_{2, j}$ \\
\hline
\end{tabular}

NOTE: See notes for Table 6.8-1.

\subsubsection{Animal Submodel}

The mathematical equations for the animal submodel are discussed in Section 6.4.4. All parameters in the submodel are listed in Table 6.8-5. The contents of the animal submodel container (Figure 6.8-14) include three lower level containers. The AnimalModel_Input container includes all animal consumption rates for feed, water, and soil (Figure 6.8-15). The Animal_Ingestions container includes three containers for calculating animal uptake from the consumption of contaminated animal feed (Equation 6.4.4-2), contaminated water (Equation 6.4.4-3), and contaminated soil (Equation 6.4.4-4) for four types of animal products. The C14_Animal container includes the calculation of ${ }^{14} \mathrm{C}$ transfer to animal products (Section 6.4.6.4). 


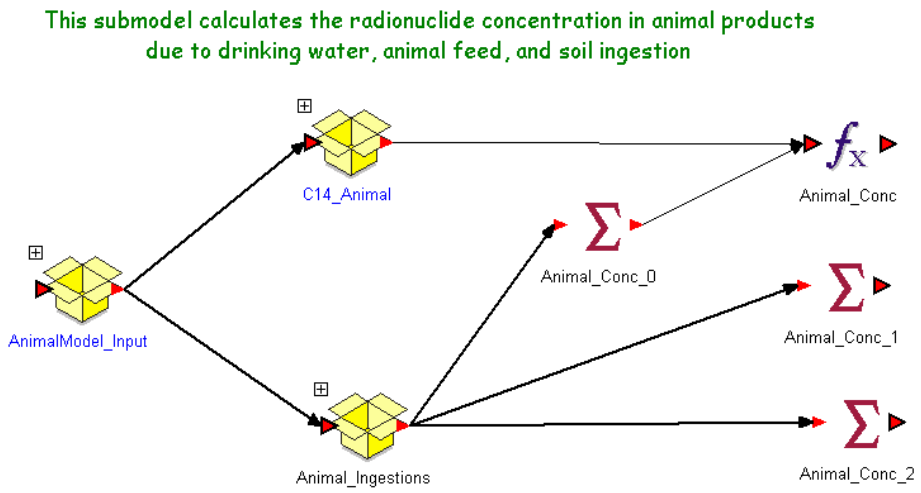

Figure 6.8-14. Animal Submodel Container

Parameters for calculating radionuclide concentration in animal products
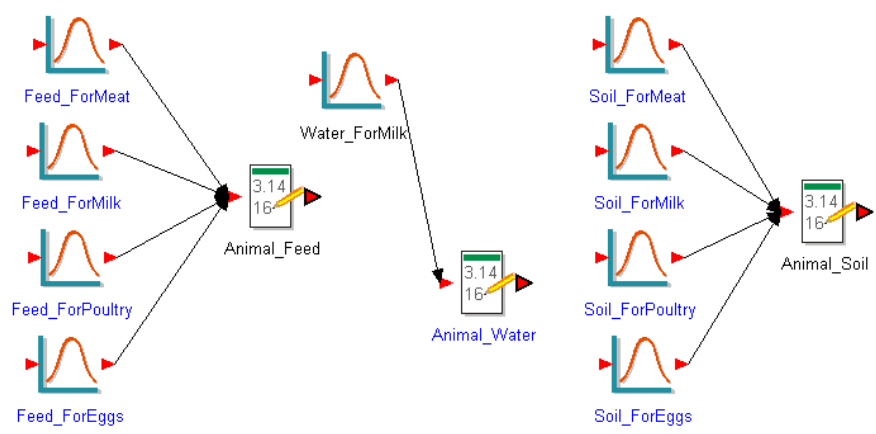

Transfer Coefficeints for radionuclides (taken from Nuclide Database)
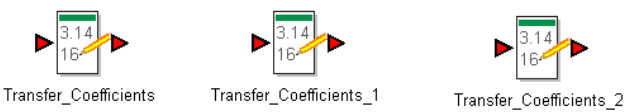

Figure 6.8-15. Input Parameter Container (AnimalModel_Input) for the Animal Submodel 
Table 6.8-5. Parameters in the Animal Submodel

\begin{tabular}{|c|c|c|c|c|c|c|}
\hline $\begin{array}{l}\text { Low Level } \\
\text { Name }\end{array}$ & Parameter Name & Element Type & $\begin{array}{c}\text { Data } \\
\text { Source }\end{array}$ & Data Type & Equation & Notation \\
\hline \multirow{15}{*}{$\begin{array}{l}\text { Animal Model_ } \\
\text { Input }\end{array}$} & Water_ForMilk & Stochastic & Input & Scalar & $6.4 .4-3$ & $Q w_{2}$ \\
\hline & Animal_Water & Data & Input & Vector(4) & 6.4.4-3 & $Q w_{k}$ \\
\hline & Feed_ForMeat & Stochastic & Input & Scalar & $6.4 .4-2$ & $Q f_{1}$ \\
\hline & Feed_ForMilk & Stochastic & Input & Scalar & 6.4.4-2 & $Q f_{2}$ \\
\hline & Feed_ForPoultry & Stochastic & Input & Scalar & 6.4.4-2 & $Q f_{3}$ \\
\hline & Feed_ForEggs & Stochastic & Input & Scalar & $6.4 .4-2$ & $Q f_{4}$ \\
\hline & Animal_Feed & Data & Cal'ed & Vector(4) & 6.4.4-2 & $Q f_{k}$ \\
\hline & Soil_ForMeat & Stochastic & Input & Scalar & 6.4.4-4 & $Q s_{1}$ \\
\hline & Soil_ForMilk & Stochastic & Input & Scalar & 6.4.4-4 & $Q s_{2}$ \\
\hline & Soil_ForPoultry & Stochastic & Input & Scalar & 6.4.4-4 & $Q s_{3}$ \\
\hline & Soil_ForEggs & Stochastic & Input & Scalar & 6.4.4-4 & $Q s_{4}$ \\
\hline & Animal_Soil & Data & Cal'ed & Vector(4) & 6.4.4-4 & $Q s_{k}$ \\
\hline & Transfer_Coefficients & Data & Dbase & Vector(4) & $6.4 .4-2$ & $F m_{i, k}$ \\
\hline & Transfer_Coefficients_1 & Data & Dbase & Vector(4) & 6.4.4-2 & $F m_{1, k}$ \\
\hline & Transfer_Coefficients_2 & Data & Dbase & Vector(4) & 6.4.4-2 & $F m_{2, k}$ \\
\hline \multirow{10}{*}{$\begin{array}{l}\text { Animal_- } \\
\text { Ingestion }\end{array}$} & Water_Contribution & Expression & Cal'ed & Vector(4) & 6.4.4-3 & $C d_{\text {water } i, k}$ \\
\hline & Feed_Conc & Data & Cal'ed & Vector(4) & 6.4.3-1 & $C p_{i, j}$ \\
\hline & Feed_Conc_1 & Data & Cal'ed & Vector(4) & 6.4.3-1 & $C p_{1, j}$ \\
\hline & Feed_Conc_2 & Data & Cal'ed & Vector(4) & 6.4.3-1 & $C p_{2, j}$ \\
\hline & Feed_Contribution & Expression & Cal'ed & Vector(4) & 6.4.4-2 & $C d_{\text {feed } i, k}$ \\
\hline & Feed_Contribution_1 & Expression & Cal'ed & Vector(4) & 6.4.4-2 & $C d_{\text {feed } 1, k}$ \\
\hline & Feed_Contribution_2 & Expression & Cal'ed & Vector(4) & 6.4.4-2 & $C d_{\text {feed } 2, k}$ \\
\hline & Soil_Contribution & Expression & Cal'ed & Vector(4) & 6.4.4-4 & $C d_{\text {soil } l, k}$ \\
\hline & Soil_Contribution_1 & Expression & Cal'ed & Vector(4) & 6.4.4-4 & $C d_{\text {soil } 1, k}$ \\
\hline & Soil_Contribution_2 & Expression & Cal'ed & Vector(4) & 6.4.4-4 & $\mathrm{Cd}_{\text {soil } 2, \mathrm{k}}$ \\
\hline \multirow[t]{11}{*}{ C14_Animal } & Animal_Carbon & Data & Input & Vector(4) & 6.4.6-7 & $f c_{\text {anim }, k}$ \\
\hline & C14Conc_Feed & Data & Cal'ed & Vector(4) & 6.4.6-6 & $C p_{C-14, j}$ \\
\hline & C14From_Feed & Expression & Cal'ed & Vector(4) & 6.4.6-7 & $C p_{C-14, j} Q f_{k}$ \\
\hline & C14From_Water & Expression & Cal'ed & Vector(4) & 6.4.6-7 & $C w_{C-14} Q w_{k}$ \\
\hline & C14From_Soil & Expression & Cal'ed & Vector(4) & 6.4.6-7 & $C s_{C-14} Q s_{k}$ \\
\hline & Feed_Carbon & Data & Input & Vector(4) & 6.4.6-6 & $f c_{\text {plant }, j}$ \\
\hline & CFrom_Feed & Expression & Cal'ed & Vector(4) & 6.4.6-7 & $f c_{\text {plant }, j} Q f_{k}$ \\
\hline & Water_Carbon & Data & Input & Scalar & 6.4.6-7 & $f C_{\text {water }}$ \\
\hline & Cfrom_Water & Expression & Cal'ed & Vector(4) & 6.4.6-7 & $f c_{\text {water }} Q w_{k}$ \\
\hline & Cfrom_Soil & Expression & Cal'ed & Vector(4) & 6.4.6-7 & $f c_{\text {soil }} Q s_{k}$ \\
\hline & C14Conc_Animal & Expression & Cal'ed & Vector(4) & 6.4.6-7 & $C d_{C-14, k}$ \\
\hline \multicolumn{2}{|l|}{ Animal_Conc } & Expression & Cal'ed & Vector(4) & 6.4.4-1 & $C d_{i, k}$ \\
\hline \multicolumn{2}{|l|}{ Animal_Conc_0 } & Sum & Cal'ed & Vector(4) & 6.4.4-1 & $C d_{i, k}$ \\
\hline \multicolumn{2}{|l|}{ Animal_Conc_1 } & Sum & Cal'ed & Vector(4) & 6.4.4-1 & $C d_{1, k}$ \\
\hline \multicolumn{2}{|l|}{ Animal_Conc_2 } & Sum & Cal'ed & Vector(4) & 6.4.4-1 & $\mathrm{Cd}_{2, k}$ \\
\hline
\end{tabular}

NOTE: See notes for Table 6.8-1.

\subsubsection{Fish Submodel}

The fish submodel (Figure 6.8-16) is described in Section 6.4.5. The fish submodel container includes calculations of activity concentration in the fish. Because ${ }^{14} \mathrm{C}$ transport to fish is the same as that for other radionuclides, the ${ }^{14} \mathrm{C}$ special submodel is not considered separately. All parameters in the submodel are listed in Table 6.8-6. 


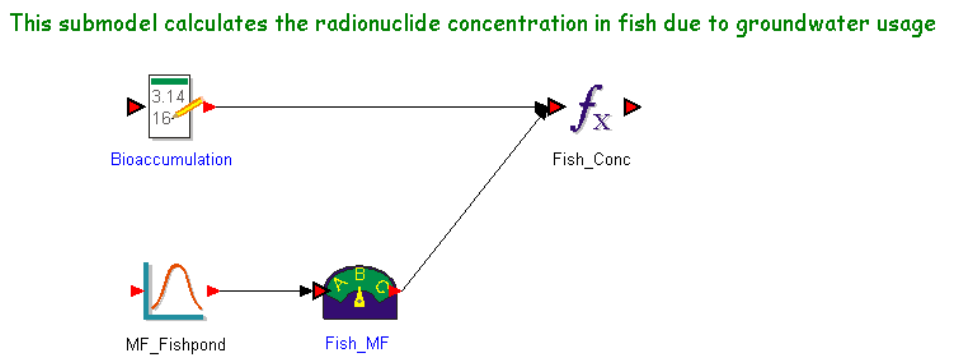

Figure 6.8-16. Fish Submodel Container

Table 6.8-6. Parameters in the Fish Submodel

\begin{tabular}{|l|l|l|l|l|l|l|}
\hline $\begin{array}{l}\text { Submodel } \\
\text { Box Name }\end{array}$ & Parameter Name & Element Type & \multicolumn{1}{|c|}{ Data Source } & \multicolumn{1}{|c|}{ Data Type } & Equation & Notation \\
\hline Fish & Bioaccumulation & Data & Dbase & Scalar & $6.4 .5-1$ & $B F_{i}$ \\
\cline { 2 - 7 } & MF_Fishpond & Stochastic & Input & Scalar & $6.4 .5-2$ & $M F_{i}$ \\
\cline { 2 - 7 } & Fish_MF & Selector & Input & Scalar & $6.4 .5-2$ & $M F_{i}$ \\
\cline { 2 - 7 } & Fish_Conc & Expression & Cal'ed & Scalar & $6.4 .5-2$ & $C_{i}$ \\
\hline
\end{tabular}

NOTE: See notes for Table 6.8-1.

\subsubsection{External Exposure Submodel}

The external exposure submodel is discussed in Section 6.4.7. All parameters in the submodel are listed in Table 6.8-7. This submodel includes one lower level container (Figure 6.8-17). The External_Input container (Figure 6.8-18) contains all of the external exposure related input parameters for this submodel plus the input data for the population groups and associated time budgets. Model calculation is included in the External_Model container. Decay products that build up in the soil as a result of the decay of primary radionuclides are considered in the calculation of external exposure to soil as a result of radionuclide buildup in the soil.

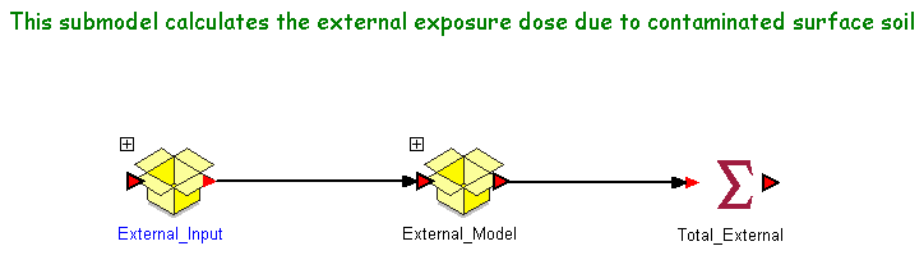

Figure 6.8-17. External Exposure Submodel Container 


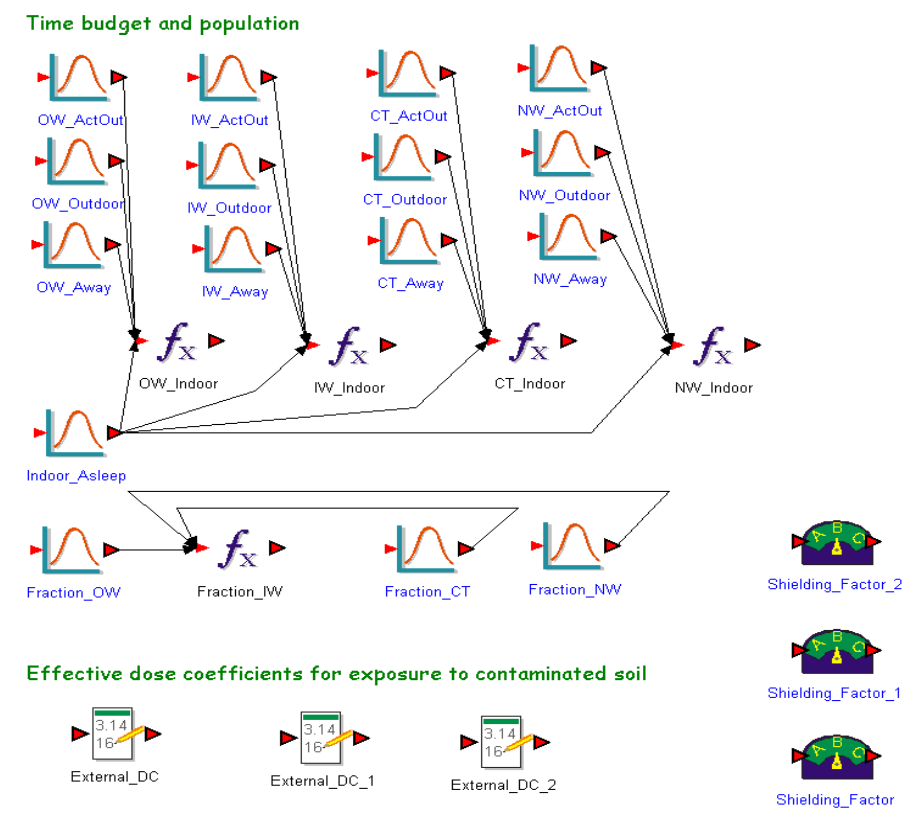

Figure 6.8-18. Input Parameter Container (External_Input) for the External Exposure Submodel

Table 6.8-7. Parameters in the External Exposure Submodel

\begin{tabular}{|c|c|c|c|c|c|c|}
\hline $\begin{array}{c}\text { Low Level } \\
\text { Name }\end{array}$ & Parameter Name & Element Type & $\begin{array}{c}\text { Data } \\
\text { Source }\end{array}$ & Data Type & Equation & Notation \\
\hline \multirow{21}{*}{$\begin{array}{l}\text { External__ } \\
\text { Input }\end{array}$} & OW_ActOut & Stochastic & Input & Scalar & $6.4 .7-1$ & $t_{1,1}$ \\
\hline & IW_ActOut & Stochastic & Input & Scalar & 6.4.7-1 & $t_{1,2}$ \\
\hline & CT_ActOut & Stochastic & Input & Scalar & 6.4.7-1 & $t_{1,3}$ \\
\hline & NW_ActOut & Stochastic & Input & Scalar & 6.4.7-1 & $t_{1,4}$ \\
\hline & OW_Outdoor & Stochastic & Input & Scalar & 6.4.7-1 & $t_{2,1}$ \\
\hline & IW_Outdoor & Stochastic & Input & Scalar & 6.4.7-1 & $t_{2,2}$ \\
\hline & CT_Outdoor & Stochastic & Input & Scalar & 6.4.7-1 & $t_{2,3}$ \\
\hline & NW_Outdoor & Stochastic & Input & Scalar & 6.4.7-1 & $t_{2,4}$ \\
\hline & OW_Away & Stochastic & Input & Scalar & 6.4.7-1 & $t_{5,1}$ \\
\hline & IW_Away & Stochastic & Input & Scalar & $6.4 .7-1$ & $t_{5,2}$ \\
\hline & CT_Away & Stochastic & Input & Scalar & 6.4.7-1 & $t_{5,3}$ \\
\hline & NW_Away & Stochastic & Input & Scalar & 6.4.7-1 & $t_{5,4}$ \\
\hline & Indoor_Asleep & Stochastic & Input & Scalar & 6.4.7-1 & $t_{4, m}$ \\
\hline & OW_Indoor & Expression & Cal'ed & Scalar & 6.4.7-1 & $t_{3,1}$ \\
\hline & IW_Indoor & Expression & Cal'ed & Scalar & 6.4.7-1 & $t_{3,2}$ \\
\hline & CT_Indoor & Expression & Cal'ed & Scalar & $6.4 .7-1$ & $t_{3,3}$ \\
\hline & NW_Indoor & Expression & Cal'ed & Scalar & 6.4.7-1 & $t_{3,4}$ \\
\hline & Fraction_OW & Stochastic & Input & Scalar & 6.4.7-1 & $P P_{1}$ \\
\hline & Fraction_IW & Expression & Cal'ed & Scalar & 6.4.7-1 & $P P_{2}$ \\
\hline & Fraction_CT & Stochastic & Input & Scalar & 6.4.7-1 & $P P_{3}$ \\
\hline & Fraction_NW & Stochastic & Input & Scalar & 6.4.7-1 & $P P_{4}$ \\
\hline
\end{tabular}


Table 6.8-7. Parameters in the External Exposure Submodel (Continued)

\begin{tabular}{|c|c|c|c|c|c|c|}
\hline $\begin{array}{c}\text { Low Level } \\
\text { Name }\end{array}$ & Parameter Name & Element Type & $\begin{array}{c}\text { Data } \\
\text { Source }\end{array}$ & Data Type & Equation & Notation \\
\hline \multirow{6}{*}{$\begin{array}{l}\text { External__ } \\
\text { Input } \\
\text { (Continued) }\end{array}$} & Shielding_Factor & Data & Input & Scalar & 6.4.7-1 & $f_{\text {ext }, i, 3 / 4}$ \\
\hline & Shielding_Factor_1 & Data & Input & Scalar & 6.4.7-1 & $f_{\text {ext, } 1,3 / 4}$ \\
\hline & Shielding_Factor_2 & Data & Input & Scalar & 6.4.7-1 & $f_{\text {ext }, 2,3 / 4}$ \\
\hline & External_DC & Data & Dbase & Scalar & 6.4.7-1 & $E D C i_{\text {soil, }, 0}$ \\
\hline & External_DC_1 & Data & Dbase & Scalar & 6.4.7-1 & $E D C i_{\text {soil, } 1}$ \\
\hline & External_DC_2 & Data & Dbase & Scalar & 6.4.7-1 & $E D C i_{\text {soil }, 2}$ \\
\hline \multirow{11}{*}{$\begin{array}{l}\text { External_- } \\
\text { Model }\end{array}$} & Exposure_times & Data & Cal'ed & Matrix $(5,4)$ & 6.4.7-1 & $t_{n, m}$ \\
\hline & Population & Data & Cal'ed & Vector(4) & 6.4.7-1 & $P P_{m}$ \\
\hline & Weighted_Time & Expression & Cal'ed & Vector(5) & 6.4.7-1 & $P P_{m} t_{n, m}$ \\
\hline & Weighted_Outdoor & Expression & Cal'ed & Scalar & 6.4.7-1 & $P P_{m} t_{1, m}+P P_{m} t_{2, m}$ \\
\hline & Weighted_Indoor & Expression & Cal'ed & Scalar & 6.4.7-1 & $P P_{m} t_{3, m}+P P_{m} t_{4, m}$ \\
\hline & External_Time & Expression & Cal'ed & Scalar & 6.4.7-1 & $\sum f_{e x t, i, n}\left(\sum P P_{m} t_{n, m}\right)$ \\
\hline & External_Time_1 & Expression & Cal'ed & Scalar & 6.4.7-1 & $\sum f_{e x t, 1, n}\left(\sum P P_{m} t_{n, m}\right)$ \\
\hline & External_Time_2 & Expression & Cal'ed & Scalar & 6.4.7-1 & $\sum f_{e x t, 2, n}\left(\sum P P_{m} t_{n, m}\right)$ \\
\hline & External_Dose & Expression & Cal'ed & Scalar & 6.4.7-1 & $D_{\text {ext } 0}$ \\
\hline & External_Dose_1 & Expression & Cal'ed & Scalar & 6.4.7-1 & $D_{\text {ext } 1}$ \\
\hline & External_Dose_2 & Expression & Cal'ed & Scalar & 6.4.7-1 & $D_{\text {ext } 2}$ \\
\hline \multicolumn{2}{|c|}{ Total_External } & Expression & Cal'ed & Scalar & 6.4.7-1 & $D_{\text {ext } i}$ \\
\hline
\end{tabular}

NOTE: See notes for Table 6.8-1.

\subsubsection{Inhalation Submodel}

The mathematical equations for the inhalation submodel are discussed in Section 6.4.8. All parameters in the submodel are listed in Table 6.8-8. The contents of this submodel container include three lower level containers (Figure 6.8-19). The Inhalation_Input container includes all inhalation related input parameters used in this submodel (Figure 6.8-20). The time budget for the receptor is calculated in the external submodel container. The Dust_Inhalation container includes the calculations of human inhalation of contaminated resuspended particles. Decay products are considered for resuspended particles in the air as the impact of radionuclide buildup in soil. This container also includes calculations for ${ }^{14} \mathrm{C}$ gas inhalation. The Radon_Inhalation container includes calculations of the radon inhalation dose due to exhalation of radon gas from ${ }^{226} \mathrm{Ra}$-contaminated soil. The dose from inhalation of contaminated aerosols generated from evaporative coolers is calculated in the expression element Cooler_Inhalation. 
This submodel calculates the inhalation dose due to contaminated dust in air

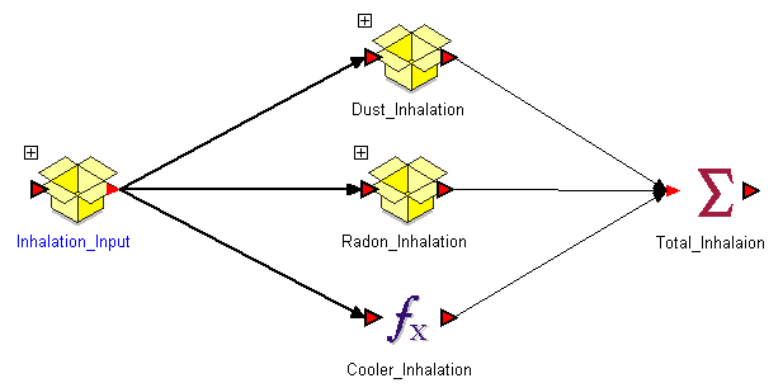

Figure 6.8-19. Inhalation Submodel Container

Input parameters for breathing rates

Radon dose conversion factor
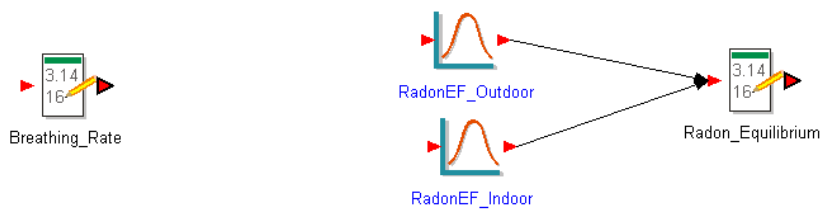

Input Parameters for evaporative cooler exposure

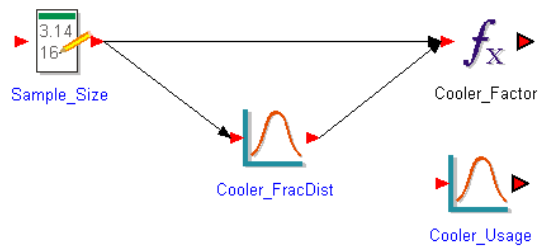

Effective dose conversion factors for inhalation
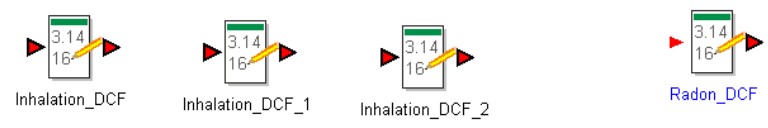

Figure 6.8-20. Input Parameter Container (Inhalation_Input) for the Inhalation Submodel 
Table 6.8-8. Parameters in the Inhalation Submodel

\begin{tabular}{|c|c|c|c|c|c|c|}
\hline $\begin{array}{l}\text { Low Level } \\
\text { Box Name }\end{array}$ & Parameter Name & $\begin{array}{l}\text { Element } \\
\text { Type }\end{array}$ & $\begin{array}{c}\text { Data } \\
\text { Source }\end{array}$ & Data Type & Equation & Notation \\
\hline \multirow{12}{*}{$\begin{array}{l}\text { Inhalation_- } \\
\text { Input }\end{array}$} & Breathing_Rate & Data & Input & Vector(5) & $6.4 .8-2$ & $B R_{n}$ \\
\hline & Sample_Size & Data & Input & Scalar & 6.4.8-3 & $f_{\text {cooler }}$ \\
\hline & Cooler_FacDist & Stochastic & Input & Scalar & 6.4.8-3 & $f_{\text {cooler }}$ \\
\hline & Cooler_Factor & Expression & Cal'ed & Scalar & 6.4.8-3 & $f_{\text {cooler }}$ \\
\hline & Cooler_Usage & Stochastic & Input & Scalar & 6.4.8-3 & $f_{\text {use }}$ \\
\hline & RadonEF_Outdoor & Stochastic & Input & Scalar & 6.4.8-6 & $E F_{R n-222,1 \& 2}$ \\
\hline & RadonEF_Indoor & Stochastic & Input & Scalar & 6.4.8-6 & $E F_{R n-222,3 \& 4}$ \\
\hline & Radon_Equilibrium & Data & Cal'ed & Vector(5) & 6.4.8-6 & $E F_{R n-222, n}$ \\
\hline & Radon_DCF & Data & Input & Scalar & 6.4.8-6 & $D_{C} F_{i n h, R n-222}$ \\
\hline & Inhalation_DCF & Data & Dbase & Scalar & 6.4.8-2 & $E D C F_{i n h, I}$ \\
\hline & Inhalation_DCF_1 & Data & Dbase & Scalar & 6.4.8-2 & $E D C F_{i n h, 1}$ \\
\hline & Inhalation_DCF_2 & Data & Dbase & Scalar & 6.4.8-2 & $E D C F_{i n h, 2}$ \\
\hline \multirow{10}{*}{$\begin{array}{l}\text { Dust } \\
\text { Inhalation }\end{array}$} & C14Gas_Inhalation & Expression & Cal'ed & Vector(5) & 6.4.8-4 & $D_{\text {inh, }, \text {, C-14, }}$ \\
\hline & Total_C14 & Sum & Cal'ed & Scalar & 6.4.8-4 & $D_{\text {inh, }, \text {, C-14 }}$ \\
\hline & Activity_Inhalation & Expression & Cal'ed & Vector(5) & 6.4.8-2 & $D_{\text {inh }, p, 0, n}$ \\
\hline & Activity_Inhalation_1 & Expression & Cal'ed & Vector(5) & 6.4.8-2 & $D_{\text {inh }, p 1, n}$ \\
\hline & Activity_Inhalation_2 & Expression & Cal'ed & Vector(5) & 6.4.8-2 & $D_{\text {inh }, p 2, n}$ \\
\hline & Inhalation_Dose_0 & Sum & Cal'ed & Scalar & 6.4.8-2 & $D_{\text {inh }, p, 0}$ \\
\hline & Inhalation_Dose & Expression & Cal'ed & Scalar & 6.4.8-2 & $D_{\text {inh }, p, 0}$ \\
\hline & Inhalation_Dose_1 & Sum & Cal'ed & Scalar & 6.4.8-2 & $D_{\text {inh }, p, 1}$ \\
\hline & Inhalation_Dose_2 & Sum & Cal'ed & Scalar & 6.4.8-2 & $D_{\text {inh }, p, 2}$ \\
\hline & Total_Dust & Sum & Cal'ed & Scalar & 6.4.8-2 & $D_{\text {inh }, p, i}$ \\
\hline \multirow[t]{8}{*}{$\begin{array}{l}\text { Radon_- } \\
\text { Inhalation }\end{array}$} & Radon_Correction & Data & Cal'ed & Vector(5) & $\begin{array}{l}6.4 .8-7 \\
6.4 .2-7 \\
6.4 .2-8\end{array}$ & $\begin{array}{l}1 @ n=1 \& 2 \\
\left(1-f_{\text {cooler }} f_{\text {use }}\right) I F_{n}+ \\
f_{\text {cooler }} f_{\text {use }} I F_{e} @ \\
n=3 \& 4 \\
0 @ n=5 \\
\end{array}$ \\
\hline & Rn_Inhalation & Expression & Cal'ed & Vector(5) & 6.4.8-7 & $D_{i n h, g, R n-222, n}$ \\
\hline & Rn_Inhalation_1 & Expression & Cal'ed & Vector(5) & 6.4.8-7 & $D_{\text {inh }, g, R n-222, n}$ \\
\hline & Rn_Inhalation_2 & Expression & Cal'ed & Vector(5) & 6.4.8-7 & $D_{\text {inh }, g, R n-222, n}$ \\
\hline & Radon_Dose & Sum & Cal'ed & Scalar & 6.4.8-7 & $D_{\text {inh }, g, R n-222}$ \\
\hline & Radon_Dose_1 & Sum & Cal'ed & Scalar & 6.4.8-7 & $D_{\text {inh, }, \text { Rn-222 }}$ \\
\hline & Radon_Dose_2 & Sum & Cal'ed & Scalar & 6.4.8-7 & $D_{\text {inh, }, \text { Rn-222 }}$ \\
\hline & Total_Radon & Sum & Cal'ed & Scalar & 6.4.8-7 & $D_{\text {inh, } g, R n-222}$ \\
\hline \multicolumn{2}{|c|}{ Cooler_Inhalation } & Expression & Cal'ed & Scalar & 6.4.8-3 & $D_{\text {inh }, e, i}$ \\
\hline \multicolumn{2}{|c|}{ Total_Inhalation } & Sum & Cal'ed & Scalar & 6.4.8-1 & $D_{\text {inh }, i}$ \\
\hline
\end{tabular}

NOTE: See notes for Table 6.8-1. 


\subsubsection{Ingestion Submodel}

The human ingestion pathways include 11 individual pathways (Section 6.4.9). All parameters in the submodel are listed in Table 6.8-9. This submodel includes two lower level containers (Figure 6.8-21). The Ingestion_Input container includes various human foodstuff consumption rates (Figure 6.8-22). The Ingestion_Model container includes calculations of the ingestion dose from each foodstuff for primary radionuclides. Contributions from long-lived decay products that accumulate in the soil are calculated if applicable. To provide the results of exposure pathway analysis, ingestion dose is presented for individual pathways and radionuclides.

This submodel calculates the ingestion dose due to consumption of various foodstuffs

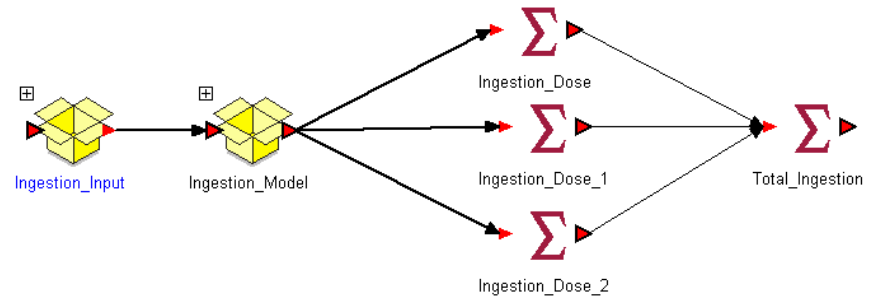

Figure 6.8-21. Ingestion Submodel Container

Human consumption rates of foodstuffs
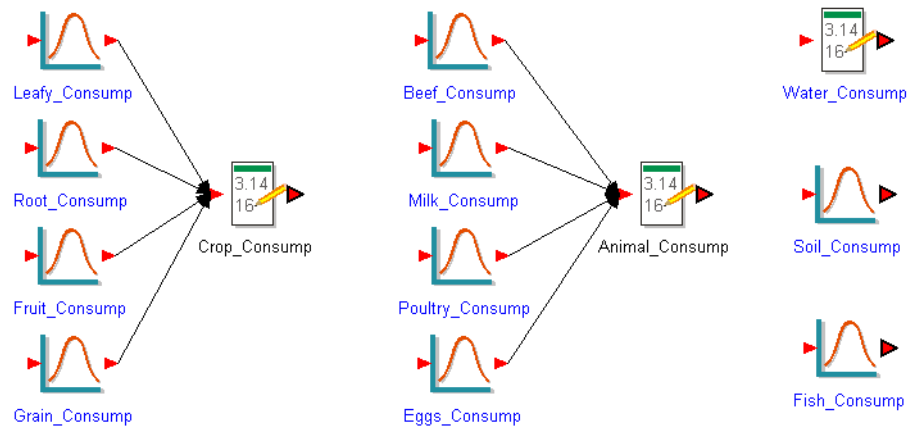

Effective dose conversion factor for ingestion
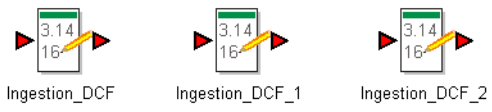

Figure 6.8-22. Input Parameter Container (Ingestion_Input) for the Ingestion Submodel 
Table 6.8-9. Parameters in the Ingestion Submodel

\begin{tabular}{|c|c|c|c|c|c|c|}
\hline $\begin{array}{l}\text { Low Level } \\
\text { Box Name }\end{array}$ & Parameter Name & $\begin{array}{l}\text { Element } \\
\text { Type }\end{array}$ & $\begin{array}{c}\text { Data } \\
\text { Source }\end{array}$ & Data Type & Equation & Notation \\
\hline \multirow{16}{*}{$\begin{array}{l}\text { Ingestion_- } \\
\text { Input }\end{array}$} & Leafy_Consump & Stochastic & Input & Scalar & $6.4 .9-3$ & $U p_{1}$ \\
\hline & Other_Consump & Stochastic & Input & Scalar & $6.4 .9-3$ & $U p_{2}$ \\
\hline & Fruit_Consump & Stochastic & Input & Scalar & $6.4 .9-3$ & $U p_{3}$ \\
\hline & Grain_Consump & Stochastic & Input & Scalar & $6.4 .9-3$ & $U p_{4}$ \\
\hline & Crop_Consump & Data & Cal'ed & Vector(4) & $6.4 .9-3$ & $U p_{j}$ \\
\hline & Beef_Consump & Stochastic & Input & Scalar & $6.4 .9-4$ & $U d_{1}$ \\
\hline & Poultry_Consump & Stochastic & Input & Scalar & $6.4 .9-4$ & $U d_{2}$ \\
\hline & Milk_Consump & Stochastic & Input & Scalar & $6.4 .9-4$ & $U d_{3}$ \\
\hline & Eggs_Consump & Stochastic & Input & Scalar & $6.4 .9-4$ & $U d_{4}$ \\
\hline & Animal_Consump & Data & Cal'ed & Vector(4) & $6.4 .9-4$ & $U d_{k}$ \\
\hline & Water_Consump & Data & Input & Scalar & $6.4 .9-2$ & Uw \\
\hline & Fish_Consump & Stochastic & Input & Scalar & $6.4 .9-5$ & Uf \\
\hline & Soil_Consump & Stochastic & Input & Scalar & $6.4 .9-6$ & Us \\
\hline & Ingestion_DCF & Data & Dbase & Scalar & $6.4 .9-2$ & $E D C F_{\text {ing, } 0}$ \\
\hline & Ingestion_DCF_1 & Data & Dbase & Scalar & $6.4 .9-2$ & $E D C F_{\text {ing, } 1}$ \\
\hline & Ingestion_DCF_2 & Data & Dbase & Scalar & $6.4 .9-2$ & $E D C F_{\text {ing, } 2}$ \\
\hline \multirow{14}{*}{$\begin{array}{l}\text { Ingestion_ } \\
\text { Model }\end{array}$} & Water_Ingestion & Expression & Cal'ed & Scalar & $6.4 .9-2$ & $D_{\text {ing,w,i }}$ \\
\hline & Fish_Ingestion & Expression & Cal'ed & Scalar & $6.4 .9-5$ & $D_{\text {ing }, f, i}$ \\
\hline & Crop_Ingestion & Expression & Cal'ed & Vector(4) & $6.4 .9-3$ & $D_{\text {ing, } p, 0}$ \\
\hline & Crop_Ingestion_1 & Expression & Cal'ed & Vector(4) & $6.4 .9-3$ & $D_{\text {ing,p,1 }}$ \\
\hline & Crop_Ingestion_2 & Expression & Cal'ed & Vector(4) & $6.4 .9-3$ & $D_{\text {ing }, p, 2}$ \\
\hline & Crop_Pathway & Sum & Cal'ed & Vector(4) & $6.4 .9-3$ & $D_{\text {ing, } p, i}$ \\
\hline & Animal_Ingestion & Expression & Cal'ed & Vector(4) & $6.4 .9-4$ & $D_{\text {ing, } d, 0}$ \\
\hline & Animal_Ingestion_1 & Expression & Cal'ed & Vector(4) & $6.4 .9-4$ & $D_{\text {ing, } d, 1}$ \\
\hline & Animal_Ingestion_2 & Expression & Cal'ed & Vector(4) & 6.4.9-4 & $D_{\text {ing,d,2 }}$ \\
\hline & Animal_Pathway & Sum & Cal'ed & Vector(4) & 6.4.9-4 & $D_{\text {ing, }, d, i}$ \\
\hline & Soil_Ingestion & Expression & Cal'ed & Scalar & $6.4 .9-6$ & $D_{\text {ing }, \mathrm{s}, 0}$ \\
\hline & Soil_Ingestion_1 & Expression & Cal'ed & Scalar & $6.4 .9-6$ & $D_{\text {ing, }, 1,1}$ \\
\hline & Soil_Ingestion_2 & Expression & Cal'ed & Scalar & 6.4.9-6 & $D_{\text {ing, }, \mathrm{s}, 2}$ \\
\hline & Soil_Pathway & Sum & Cal'ed & Scalar & $6.4 .9-6$ & $D_{\text {ing }, \mathrm{s}, i}$ \\
\hline \multicolumn{2}{|c|}{ Ingestion_Dose } & Sum & Cal'ed & Scalar & 6.4.9-1 & $D_{\text {ing }, 0}$ \\
\hline \multicolumn{2}{|c|}{ Ingestion_Dose_1 } & Sum & Cal'ed & Scalar & 6.4.9-1 & $D_{\text {ing, } 1}$ \\
\hline \multicolumn{2}{|c|}{ Ingestion_Dose_2 } & Sum & Cal'ed & Scalar & $6.4 .9-1$ & $D_{\text {ing,2 }}$ \\
\hline \multicolumn{2}{|c|}{ Total_ingestion } & Sum & Cal'ed & Scalar & $6.4 .9-1$ & $D_{\text {ing,i }}$ \\
\hline
\end{tabular}

NOTE: See notes for Table 6.8-1.

\subsubsection{BDCF Results}

The TEDEs and BDCFs for individual radionuclides are discussed in Section 6.4.10. All parameters in the submodel are listed in Table 6.8-10. The ERMYN GoldSim model calculates the total dose from a radionuclide, the dose from each exposure pathway, the dose from primary radionuclide and the decay products, and the activity concentration of the radionuclide in the environmental media (Figure 6.8-23). 


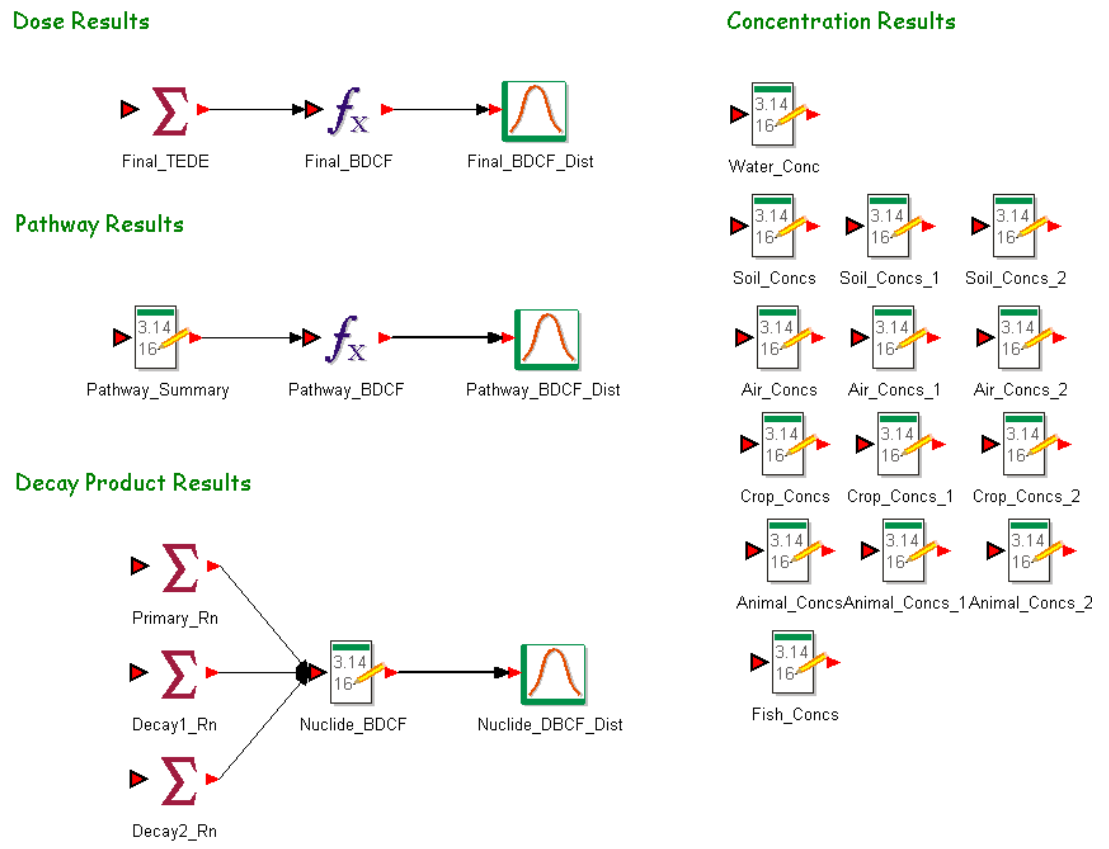

Figure 6.8-23. Final BDCF Result Container

Table 6.8-10. Parameters in the Final BDCF Results

\begin{tabular}{|l|l|l|l|l|l|}
\hline \multicolumn{1}{|c|}{ Parameter Name } & \multicolumn{1}{c|}{ Element Type } & Data Source & \multicolumn{1}{c|}{ Data Type } & \multicolumn{1}{c|}{ Equation } & \multicolumn{1}{c|}{ Notation } \\
\hline Final_TEDE & Sum & Cal'ed & Scalar & $6.4 .10-1$ & $D_{\text {all,i }}$ \\
\hline Final_BDCF & Expression & Cal'ed & Scalar & $6.4 .10-2$ & $B D C F_{i}$ \\
\hline Pathway_Summary & Data & Cal'ed & Vector(15) & $6.4 .10-3$ & $D_{p, i}$ \\
\hline Pathway_BDCF & Expression & Cal'ed & Vector(15) & $6.4 .10-4$ & $B D C F_{p, i}$ \\
\hline Primary_Rn & Sum & Cal'ed & Scalar & - & - \\
\hline Decay1_Rn & Sum & Cal'ed & Scalar & - & - \\
\hline Decay2_Rn & Sum & Cal'ed & Scalar & - & - \\
\hline Nuclide_BDCF & Data & Cal'ed & Vector(3) & - & - \\
\hline Final_BDCF_Dist & Result & Cal'ed & Scalar & - & - \\
\hline Pathway_BDCF_Dist & Result & Cal'ed & Vector(15) & - & - \\
\hline Nuclide_BDCF_Dist & Result & Cal'ed & Vector(3) & - & - \\
\hline Water_Conc & Data & Cal'ed & Scalar & $6.4 .1-1$ & $C w$ \\
\hline Soil_Concs & Data & Cal'ed & Scalar & $6.4 .1-5$ & $C s_{m, i}$ \\
\hline Soil_Concs_1 & Data & Cal'ed & Scalar & $6.4 .1-9$ & $C s_{m, 1}$ \\
\hline Soil_Concs_2 & Data & Cal'ed & Scalar & $6.4 .1-9$ & $C s_{m, 2}$ \\
\hline Air_Concs & Data & Cal'ed & Matrix(4,5) & $6.4 .2-1$ & $C a_{i}$ \\
\hline Air_Concs_1 & Data & Cal'ed & Matrix(4,5) & $6.4 .2-1$ & $C a_{1}$ \\
\hline Air_Concs_2 & Data & Cal'ed & Matrix(4,5) & $6.4 .2-1$ & $C a_{2}$ \\
\hline Crop_Concs & Data & Cal'ed & Matrix(5,4) & $6.4 .3-1$ & $C p_{i}$ \\
\hline
\end{tabular}


Table 6.8-10. Parameters in the Final BDCF Results (Continued)

\begin{tabular}{|l|l|l|l|l|l|}
\hline \multicolumn{1}{|c|}{ Parameter Name } & \multicolumn{1}{c|}{ Element Type } & Data Source & \multicolumn{1}{c|}{ Data Type } & \multicolumn{1}{c|}{ Equation } & \multicolumn{1}{c|}{ Notation } \\
\hline Crop_Concs_1 & Data & Cal'ed & Matrix(5,4) & $6.4 .3-1$ & $C p_{1}$ \\
\hline Crop_Concs_2 & Data & Cal'ed & Matrix(5,4) & $6.4 .3-1$ & $C p_{2}$ \\
\hline Animal_Concs & Data & Cal'ed & Matrix $(4,4)$ & $6.4 .4-1$ & $C d_{i}$ \\
\hline Animal_Concs_1 & Data & Cal'ed & Matrix(4,4) & $6.4 .4-1$ & $C d_{1}$ \\
\hline Animal_Concs_2 & Data & Cal'ed & Matrix(4,4) & $6.4 .4-1$ & $C d_{2}$ \\
\hline Fish_Concs & Data & Cal'ed & Scalar & $6.4 .5-1$ & $C f$ \\
\hline
\end{tabular}

NOTE: See notes for Table 6.8-1.

\subsection{GOLDSIM IMPLEMENTATION OF THE BIOSPHERE MODEL FOR THE VOLCANIC ASH SCENARIO}

This section describes the ERMYN model for the volcanic ash scenario (ERMYN_VA) and shows the overall model algorithm, submodel structures, input parameters, and calculated results. Many parts of the GoldSim volcanic ash model are similar to those used in the groundwater model (ERMYN_GW; Section 6.8). The GoldSim file, ERMYN_VA.gsm, is part of the output, which is listed in Appendix A. Similar to the mathematical model (Section 6.5), the ERMYN_VA is structured as a series of submodels. In each submodel, the linkage of the GoldSim elements to input parameters is tabulated. The description of the ERMYN_VA design is simplified because many parts are the same as those used in the groundwater model, ERMYN_GW. As with the ERMYN_GW model, there is only one container box (Biosphere_Model) on the cover page of the ERMYN_VA model (Figure 6.9-1).

\section{ERMYN}

Environmental Radiation Model for Yucca Mountain, Nevada Volcanic Ash Exposure Scenario

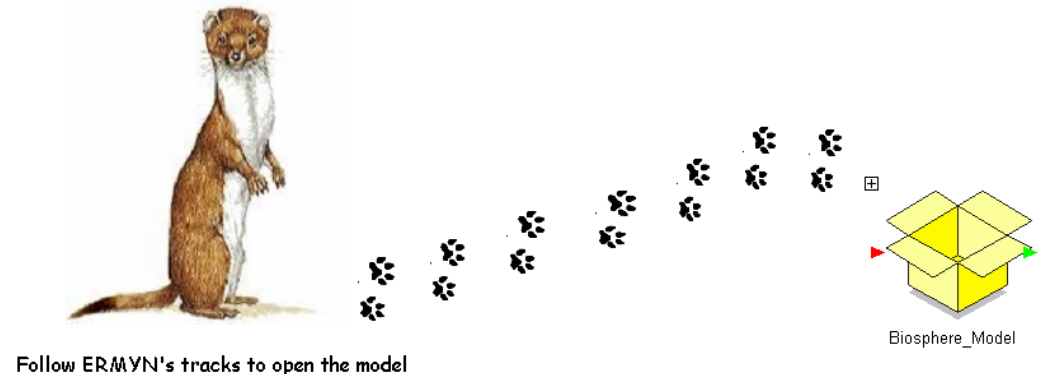

This GoldSim Simulation is the implementation tool for ERMYN documented in the Biosphere Model Report, MDL-MGR-MD-000001 REV 00. It was designed to calculate the biosphere dose conversion factors for TSPA-LA.

Figure 6.9-1. Cover Page for the ERMYN_VA Model in GoldSim 
The Biosphere_Model container holds the submodel containers and the radionuclide data (Figure 6.9-2). The overall model structure looks much like the block diagram of the conceptual model for the volcanic ash scenario shown in Figure 6.3-4, and each container in the GoldSim simulation corresponds to a submodel.

Nine containers, including seven submodels, one results box, and one radionuclide database box, are shown in Figure 6.9-2. The fish and ${ }^{14} \mathrm{C}$ special submodels are excluded from the volcanic ash scenario. Each box is discussed in detail in the following sections. Only two input parameters can be changed at this level: Radionuclide and Ash_Source. The Radionuclide parameter can only be selected from the data element of Radionuclide_List that is built in the Nuclide_Database container. Ash_Source represents the radionuclide concentration of radionuclides deposited on the ground ( $\mathrm{Cs}_{i}$ in the equation), which has a default value of $1 \mathrm{~Bq} / \mathrm{m}^{2}$. GoldSim can be run in deterministic and stochastic modes by adjusting settings in the MasterClock. If the stochastic mode is chosen, the number of realizations, the sampling method (Monte Carlo or Latin Hypercube), and the random seed number need to be selected. Because the BDCF is not a function of time, the time option is disabled.

Similar to the ERMYN_GW model, many calculations are performed using the data array to reduce the number of GoldSim elements used in the ERMYN. Thirteen data arrays are used with the following labels: primary radionuclides $(i=31)$, total number of radionuclides $(i=75)$, pathways $(n=15)$, plant types $(j=5)$, crop food types $(j=4)$, animal product types $(k=4)$, number of decay products $(l=3)$, population groups $(m=4)$, environments $(n=5)$, soil conditions $(n=2)$, air submodel pathway $(n=4)$, crop uptake pathway $(n=4)$, and animal uptake pathway $(n=4)$. These data sets and submodel pathways are discussed in Section 6.5.
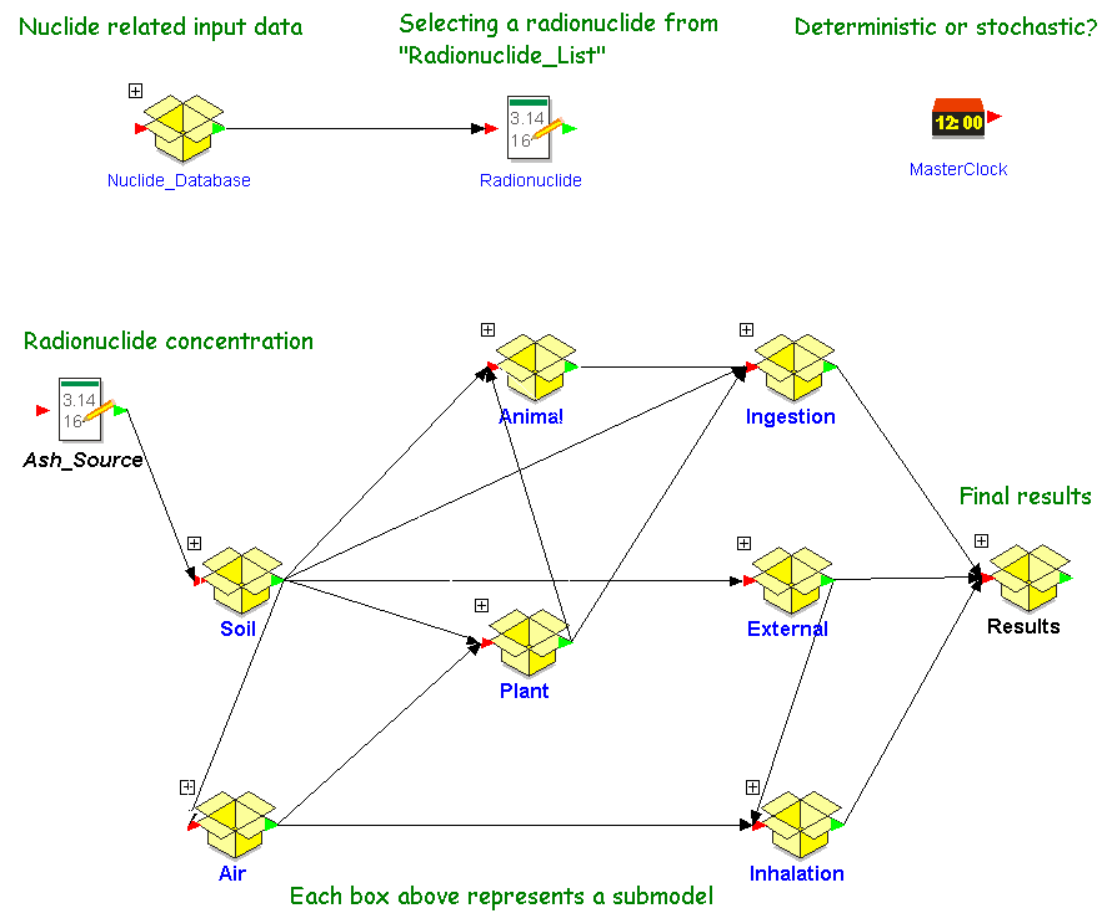

Figure 6.9-2. Biosphere Model for the Volcanic Ash Scenario 


\subsubsection{Nuclide Database}

The nuclide database for the volcanic ash scenario is similar to the one used for the groundwater scenario, except that there are no radioactive decay products accumulating in the soil. Thirty-one primary radionuclides are included in the ERMYN_VA (see Section 6.8.1). The Nuclide_Database container is the same as that used for the ERMYN_GW (Figure 6.8-3) (even though fewer radionuclides are considered in this scenario). The Nuclide_Data container does not include the Fish_Transfer container that is used in the groundwater scenario. Only two lower-level containers are in the Data_Selection container. Thirteen radionuclide-specific input parameters are selected using the Selector elements (Figure 6.9-3).

All GoldSim elements in this database are presented in Table 6.9-1. This table lists input parameters for each lower level container and their characteristics, including parameter name, GoldSim element type, data source, data types, equation number where the equation is originally defined, and symbol notation.

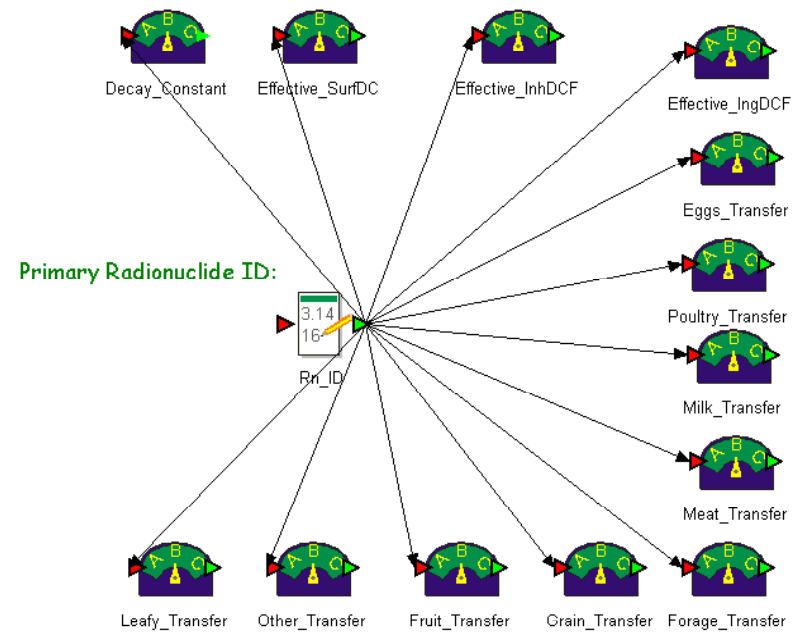

Figure 6.9-3. Nuclide Database Container and Radionuclide List

Table 6.9-1. Radionuclide Related Input Parameters

\begin{tabular}{|c|c|c|c|c|c|c|c|}
\hline $\begin{array}{l}\text { First Level } \\
\text { Box Name }\end{array}$ & $\begin{array}{c}\text { Second } \\
\text { Level } \\
\text { Box } \\
\text { Name }\end{array}$ & $\begin{array}{c}\text { Parameter or } \\
\text { Container Name }\end{array}$ & $\begin{array}{c}\text { Element } \\
\text { Type }^{\mathrm{a}}\end{array}$ & $\begin{array}{c}\text { Data } \\
\text { Source }\end{array}$ & Data Type ${ }^{c}$ & Equation & Notation \\
\hline \multicolumn{3}{|c|}{ Radionuclide_List } & Data & Input & Vector(31) & 6.5.1-1 & $i$ \\
\hline \multirow{4}{*}{$\begin{array}{l}\text { Nuclide__ } \\
\text { Data }\end{array}$} & \multicolumn{2}{|c|}{ Kd_Coefficients } & Container & \multirow{4}{*}{\multicolumn{4}{|c|}{ Not shown in detail }} \\
\hline & \multicolumn{2}{|c|}{ Crop_Transfers } & & & & & \\
\hline & \multicolumn{2}{|c|}{ Animal_Transfers } & & & & & \\
\hline & \multicolumn{2}{|c|}{ Nuclear_Data } & & & & & \\
\hline
\end{tabular}


Table 6.9-1. Radionuclide Related Input Parameters (Continued)

\begin{tabular}{|c|c|c|c|c|c|c|c|}
\hline $\begin{array}{l}\text { First Level } \\
\text { Box Name }\end{array}$ & $\begin{array}{l}\text { Second } \\
\text { Level } \\
\text { Box } \\
\text { Name } \\
\end{array}$ & $\begin{array}{c}\text { Parameter or } \\
\text { Container Name }\end{array}$ & $\begin{array}{c}\text { Element } \\
\text { Type }^{\mathrm{a}}\end{array}$ & $\begin{array}{c}\text { Data } \\
\text { Source }\end{array}$ & Data Type ${ }^{c}$ & Equation & Notation \\
\hline \multirow{24}{*}{$\begin{array}{l}\text { Data } \\
\text { Selection }\end{array}$} & \multirow{10}{*}{$\begin{array}{l}\text { Effective } \\
\text { DCF }\end{array}$} & Unity & Data & - & Vector(31) & - & - \\
\hline & & Decay_Constants & Expression & Cal'ed & Vector(31) & 6.4.1-1 & $\lambda_{d i}$ \\
\hline & & A & Expression & Cal'ed & Vector(31) & Not used & - \\
\hline & & $\mathrm{B}$ & Expression & Cal'ed & Vector(31) & $6.5 .5-2$ & $E D C s_{\text {soil }, i}$ \\
\hline & & $\mathrm{C}$ & Expression & Cal'ed & Vector(31) & $6.4 .8-8$ & $E D C F_{i n h, i}$ \\
\hline & & $\mathrm{D}$ & Expression & Cal'ed & Vector(31) & 6.4.9-7 & $E D C F_{i n q, i}$ \\
\hline & & Effective_InfDCs & Data & Cal'ed & Vector(31) & Not used & - \\
\hline & & Effective_SurDCs & Data & Cal'ed & Vector(31) & $6.5 .5-2$ & $E D C s_{\text {soil, }, i}$ \\
\hline & & Effective_InhDCFs & Data & Cal'ed & Vector(31) & $6.4 .8-8$ & $E D C F_{i n h, i}$ \\
\hline & & Effective_IngDCFs & Data & Cal'ed & Vector(31) & $6.4 .9-7$ & $E D C F_{i n g, i}$ \\
\hline & \multirow{14}{*}{$\begin{array}{l}\text { Primary_ } \\
\text { Rn }\end{array}$} & Rn_ID & Data & Dbase & Scalar & 6.4.1-1 & $i$ \\
\hline & & Decay_Constant & Selector & Dbase & Scalar & 6.4.1-1 & $\lambda_{d i}$ \\
\hline & & Effective_SurDC & Selector & Dbase & Scalar & $6.5 .5-1$ & $E D C s_{\text {soil, }, i}$ \\
\hline & & Effective_InhDCF & Selector & Dbase & Scalar & $6.5 .6-2$ & $E D C F_{i n h, i}$ \\
\hline & & Effective_IngDCF & Selector & Dbase & Scalar & $6.5 .7-2$ & $E D C F_{\text {ing }, i}$ \\
\hline & & Beef_Transfer & Selector & Dbase & Scalar & $6.5 .4-2$ & $F m_{i, 1}$ \\
\hline & & Poultry_Transfer & Selector & Dbase & Scalar & $6.5 .4-2$ & $F m_{i, 2}$ \\
\hline & & Milk_Transfer & Selector & Dbase & Scalar & $6.5 .4-2$ & $F m_{i, 3}$ \\
\hline & & Eggs_Transfer & Selector & Dbase & Scalar & $6.5 .4-2$ & $F m_{i, 4}$ \\
\hline & & Leafy_Transfer & Selector & Dbase & Scalar & $6.5 .3-2$ & $F_{S \rightarrow p, i, 1}$ \\
\hline & & Other_Transfer & Selector & Dbase & Scalar & $6.5 .3-2$ & $F_{s \rightarrow p, i, 2}$ \\
\hline & & Fruit_Transfer & Selector & Dbase & Scalar & 6.5.3-2 & $\mathrm{Fs} \rightarrow \mathrm{p}, \mathrm{i}, 3$ \\
\hline & & Grain_Transfer & Selector & Dbase & Scalar & $6.5 .3-2$ & $\mathrm{Fs} \rightarrow \mathrm{p}, \mathrm{i}, 4$ \\
\hline & & Forage_Transfer & Selector & Dbase & Scalar & 6.5.3-2 & $\mathrm{Fs} \rightarrow \mathrm{p}, \mathrm{i}, 5$ \\
\hline
\end{tabular}

NOTE: The following notes apply to all GoldSim tables in Section 6.9.

${ }^{a}$ Element type is the GoldSim element type used for inputs, calculations, and other manipulations (Section 6.7.3).

b If the data source is "Input," the parameter values are entered in the GoldSim element. If the source is "Dbase," values are taken from a database, or calculated values. If the data source is "Cal'ed," it is a calculated quantity. If the source and the corresponding equation notation are dashes (-), the element is added for GoldSim array calculations.

${ }^{c}$ Data types are scalar (a single value) or array (a set of values). A one-dimensional array is called a vector, and a two-dimensional array is called a matrix. The number of values in the array is given in parenthesis.

\subsubsection{Surface Soil Submodel}

The mathematical equations for the surface soil submodel are discussed in Section 6.5.1. All parameters used in this submodel are listed in Table 6.9-2. The contents of the submodel container are shown in Figure 6.9-4. Only a few GoldSim elements are used for calculating the radionuclide concentrations on cultivated (Equation 6.5.1-2) and noncultivated lands (Equation 6.5.1-3). 
This submodel calculates the radionuclide concentration in surface soil and ash/dust mixture

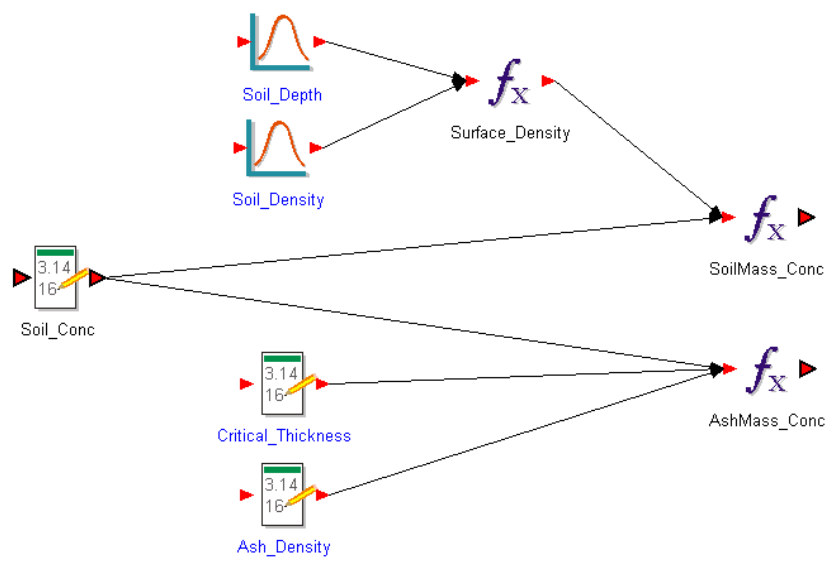

Figure 6.9-4. Surface Soil Submodel Container

Table 6.9-2. Parameters in the Surface Soil Submodel

\begin{tabular}{|l|l|l|l|l|l|}
\hline \multicolumn{1}{|c|}{ Parameter Name } & Element Type & \multicolumn{1}{c|}{ Data Source } & \multicolumn{1}{c|}{ Data Type } & \multicolumn{1}{c|}{ Equation } & \multicolumn{1}{c|}{ Notation } \\
\hline Soil_Conc & Data & Input & Scalar & $6.5 .1-1$ & $C s_{i}$ \\
\hline Soil_Depth & Stochastic & Input & Scalar & $6.5 .1-2$ & $d$ \\
\hline Soil_Density & Stochastic & Input & Scalar & $6.5 .1-2$ & $\rho$ \\
\hline Surface_Density & Expression & Cal'ed & Scalar & $6.5 .1-2$ & $\rho_{s}$ \\
\hline SoilMass_Conc & Expression & Cal'ed & Scalar & $6.5 .1-2$ & $C s_{m, i}$ \\
\hline Critical_Thickness & Data & Input & Scalar & $6.5 .1-3$ & $d_{c}$ \\
\hline Ash_Density & Data & Input & Scalar & $6.5 .1-3$ & $\rho_{a}$ \\
\hline AshMass_Conc & Expression & input & Scalar & $6.5 .1-3$ & $C s_{m c, i}$ \\
\hline
\end{tabular}

NOTE: See notes for Table 6.9-1.

\subsubsection{Air Submodel}

The mathematical equations used for the air submodel are discussed in Section 6.5.2. All parameters used in this submodel are listed in Table 6.9-3. The contents of the submodel container are shown in Figure 6.9-5. One lower level container is included in the submodel. The AirModel_Input container calculates the mass loading and enhancement factors that are used for calculating the radionuclide concentration in the air due to resuspended particles. This submodel calculates radionuclide concentrations in the air for crop deposition (Equation 6.5.2-1) and human inhalation (Equation 6.5.2-2). Airborne concentrations of radon gas released from ${ }^{226} \mathrm{Ra}$-contaminated soils are calculated using Equation 6.5.2-8. 
This submodel calculates the radionuclide concentration in air

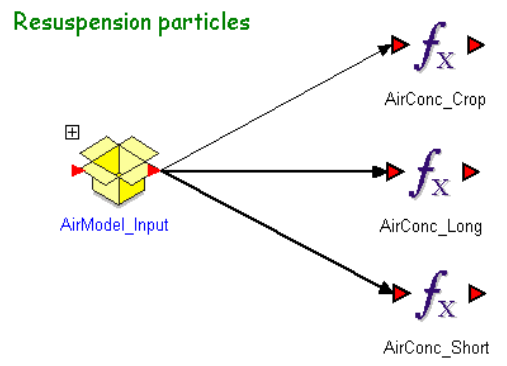

$\mathrm{Rn}-222$ gas from $\mathrm{Ra}-226$ in ash

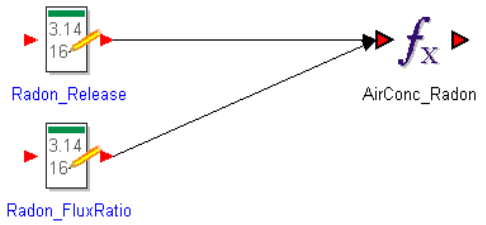

Figure 6.9-5. Air Submodel Container

Table 6.9-3. Parameters in the Air Submodel

\begin{tabular}{|c|c|c|c|c|c|c|}
\hline $\begin{array}{c}\text { Low Layer Box } \\
\text { Name }\end{array}$ & Parameter Name & $\begin{array}{l}\text { Element } \\
\text { Type }\end{array}$ & $\begin{array}{c}\text { Data } \\
\text { Source }\end{array}$ & Data Type & Equation & Notation \\
\hline \multirow[t]{16}{*}{ AirModel_Input } & Crop_Loading & Stochastic & Input & Scalar & $6.5 .2-1$ & $S$ \\
\hline & ActiveOut_Dust & Stochastic & Input & Scalar & $6.5 .2-3$ & $S_{1}$ \\
\hline & InactiveOut_Dust & Stochastic & Input & Scalar & 6.5.2-3 & $S_{2}$ \\
\hline & Activeln_Dust & Stochastic & Input & Scalar & 6.5.2-3 & $S_{3}$ \\
\hline & Asleepln_Dust & Stochastic & Input & Scalar & 6.5.2-3 & $S_{4}$ \\
\hline & Mass_Loading & Data & Cal'ed & Vector(5) & $6.5 .2-3$ & $S_{n}$ \\
\hline & ActiveOut_Ash & Stochastic & Input & Scalar & 6.5.2-3 & $S_{V, 1}$ \\
\hline & InactiveOut_Ash & Stochastic & Input & Scalar & $6.5 .2-3$ & $S_{V, 2}$ \\
\hline & Activeln_Ash & Stochastic & Input & Scalar & 6.5.2-3 & $S_{V, 3}$ \\
\hline & Asleepln_Ash & Stochastic & Input & Scalar & 6.5.2-3 & $S_{V, 4}$ \\
\hline & Ash_Loading & Data & Cal'ed & Vector(5) & $6.5 .2-3$ & $S_{V, n}$ \\
\hline & ActiveOut_Enhance & Stochastic & Input & Scalar & $6.5 .2-2$ & $f_{\text {enhance, } 1}$ \\
\hline & InactiveOut_Enhance & Stochastic & Input & Scalar & $6.5 .2-2$ & $f_{\text {enhance, } 2}$ \\
\hline & Activeln_Enhance & Stochastic & Input & Scalar & $6.5 .2-2$ & $f_{\text {enhance, } 3}$ \\
\hline & Asleepln_Enhance & Stochastic & Input & Scalar & $6.5 .2-2$ & $f_{\text {enhance }, 4}$ \\
\hline & Enhance_Factor & Data & Cal'ed & Vector(5) & $6.5 .2-2$ & $f_{\text {enhance, } n}$ \\
\hline \multicolumn{2}{|l|}{ AirConc_Crop } & Expression & Cal'ed & Scalar & 6.5.2-1 & $C a_{p, i}$ \\
\hline \multicolumn{2}{|l|}{ AirConc_Short } & Expression & Cal'ed & Vector(5) & $6.5 .2-4$ & $C a_{h, i, n}$ \\
\hline \multicolumn{2}{|l|}{ AirConc_Long } & Expression & Cal'ed & Vector(5) & $6.5 .2-4$ & $C a_{v, i, n}$ \\
\hline \multicolumn{2}{|l|}{ Radon_Release } & Data & Input & Scalar & $6.5 .2-6$ & $F D_{R n-222}$ \\
\hline \multicolumn{2}{|l|}{ RnFlux_Ratio } & Data & Input & Scalar & 6.5.2-7 & $C F_{R n-222}$ \\
\hline \multicolumn{2}{|l|}{ AirConc_Radon } & Expression & Cal'ed & Scalar & $6.5 .2-8$ & $C a_{g, R n-222}$ \\
\hline
\end{tabular}

NOTE: See notes for Table 6.9-1. 


\subsubsection{Plant Submodel}

Mathematical equations for the plant submodel are discussed in Section 6.5.3. All parameters used in this submodel are listed in Table 6.9-4. The contents of the submodel container are shown in Figure 6.9-6. This submodel includes two lower level containers. The Root_Uptake container (Equation 6.5.3-2) calculates radionuclide concentrations in crops due to contaminated soil. The Dust_Uptake container (Equation 6.5.3-3) calculates radionuclide concentrations in crops due to direct deposition of resuspended soil from cultivated lands.

This submodel calculates the radionuclide concentration in crops

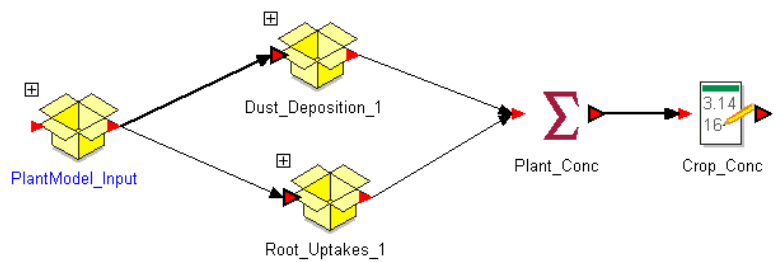

Figure 6.9-6. Plant Submodel Container

Table 6.9-4. Parameters in the Plant Submodel

\begin{tabular}{|c|c|c|c|c|c|c|}
\hline $\begin{array}{l}\text { Low Level } \\
\text { Box Name }\end{array}$ & Parameter Name & $\begin{array}{c}\text { Element } \\
\text { Type }\end{array}$ & $\begin{array}{c}\text { Data } \\
\text { Source }\end{array}$ & Data Type & Equation & Notation \\
\hline \multirow{23}{*}{$\begin{array}{l}\text { PlantModel__ } \\
\text { Input }\end{array}$} & DryWet_Leafy & Stochastic & Input & Scalar & $6.5 .3-2$ & $D W_{1}$ \\
\hline & DryWet_Other & Stochastic & Input & Scalar & 6.5.3-2 & $D W_{2}$ \\
\hline & DryWet_Fruit & Stochastic & Input & Scalar & $6.5 .3-2$ & $D W_{3}$ \\
\hline & DryWet_Grain & Stochastic & Input & Scalar & 6.5.3-2 & $D W_{4}$ \\
\hline & DryWet_Forage & Stochastic & Input & Scalar & 6.5.3-2 & $D W_{5}$ \\
\hline & DryWet_Ratio & Data & Cal'ed & Vector(5) & $6.5 .3-2$ & $D W_{j}$ \\
\hline & Yield_Leafy & Stochastic & Input & Scalar & $6.5 .3-3$ & $Y_{1}$ \\
\hline & Yield_Other & Stochastic & Input & Scalar & 6.5.3-3 & $Y_{2}$ \\
\hline & Yield_Fruit & Stochastic & Input & Scalar & 6.5.3-3 & $Y_{3}$ \\
\hline & Yield_Grain & Stochastic & Input & Scalar & 6.5.3-3 & $Y_{4}$ \\
\hline & Yield_Forage & Stochastic & Input & Scalar & 6.5.3-3 & $Y_{5}$ \\
\hline & Wet_Yield & Data & Cal'ed & Vector(5) & 6.5.3-3 & $Y_{j}$ \\
\hline & DryBiom_Leafy & Stochastic & Input & Scalar & 6.5.3-5 & $D B_{1}$ \\
\hline & DryBiom_Other & Stochastic & Input & Scalar & $6.5 .3-5$ & $D B_{2}$ \\
\hline & DryBiom_Fruit & Stochastic & Input & Scalar & 6.5.3-5 & $D B_{3}$ \\
\hline & DryBiom_Grain & Stochastic & Input & Scalar & 6.5.3-5 & $D B_{4}$ \\
\hline & DryBiom_Forage & Stochastic & Input & Scalar & 6.5.3-5 & $D B_{5}$ \\
\hline & Deposity_Velocity & Stochastic & Input & Scalar & 6.5.3-4 & $V_{d}$ \\
\hline & Weather_Halflife & Stochastic & input & Scalar & 6.5.3-3 & $T_{w}$ \\
\hline & Weathering_Factor & Data & Cal'ed & Scalar & 6.5.3-3 & $\lambda_{w}$ \\
\hline & Translocation_Dist & Stochastic & Input & Scalar & 6.5.3-3 & $T_{j}$ \\
\hline & Translocation & Data & Input & Vector(5) & $6.5 .3-3$ & $T_{j}$ \\
\hline & Growing_Time & Data & Input & Vector(5) & 6.5.3-3 & $t_{g, j}$ \\
\hline
\end{tabular}


Table 6.9-4. Parameters in the Plant Submodel (Continued)

\begin{tabular}{|c|c|c|c|c|c|c|}
\hline $\begin{array}{l}\text { Low Level } \\
\text { Box Name }\end{array}$ & Parameter Name & $\begin{array}{l}\text { Element } \\
\text { Type }\end{array}$ & $\begin{array}{c}\text { Data } \\
\text { Source }\end{array}$ & Data Type & Equation & Notation \\
\hline \multirow{16}{*}{$\begin{array}{l}\text { Dust__ } \\
\text { Deposition }\end{array}$} & Dust_Factor & Data & Input & Vector(5) & 6.5.3-5 & $a_{j}$ \\
\hline & ExpDust_Leafy & Expression & Cal'ed & Scalar & $6.5 .3-5$ & $e^{-a_{1} D B}{ }_{1}$ \\
\hline & ExpDust_Other & Expression & Cal'ed & Scalar & $6.5 .3-5$ & $e^{-a_{2} D B_{2}}$ \\
\hline & ExpDust_Fruit & Expression & Cal'ed & Scalar & $6.5 .3-5$ & $e^{-a_{3} D B_{3}}$ \\
\hline & ExpDust_Grain & Expression & Cal'ed & Scalar & $6.5 .3-5$ & $e^{-a_{4} D B}{ }_{4}$ \\
\hline & ExpDust_Forage & Expression & Cal'ed & Scalar & $6.5 .3-5$ & $e^{-a_{5} D B_{5}}$ \\
\hline & Dust_Intercept & Data & Cal'ed & Vector(5) & $6.5 .3-5$ & $R a_{j}$ \\
\hline & ExpGrow_Leafy & Expression & Cal'ed & Scalar & 6.5.3-3 & $e^{-\lambda w t g, 1}$ \\
\hline & ExpGrow_Other & Expression & Cal'ed & Scalar & 6.5.3-3 & $e^{-\lambda w t g, 2}$ \\
\hline & ExpGrow_Fruit & Expression & Cal'ed & Scalar & 6.5.3-3 & $e^{-\lambda w \operatorname{tg}, 3}$ \\
\hline & ExpGrow_Grain & Expression & Cal'ed & Scalar & 6.5.3-3 & $e^{-\lambda w t g, 4}$ \\
\hline & ExpGrow_Forage & Expression & Cal'ed & Scalar & 6.5.3-3 & $e^{-\lambda w \operatorname{tg}, 5}$ \\
\hline & Growing_Factors & Data & Cal'ed & Vector(5) & $6.5 .3-3$ & $1-e^{-\lambda w \operatorname{tg}, j}$ \\
\hline & Other_Factor & Expression & Cal'ed & Vector(5) & $6.5 .3-3$ & $T_{j} /\left(Y_{j} \lambda_{w}\right)\left(1-e^{-\lambda w t g, j}\right)$ \\
\hline & Air_Interception & Expression & Cal'ed & Vector(5) & 6.5.3-3 & $R a_{j}$ \\
\hline & Dust_Uptake & Expression & Cal'ed & Vector(5) & 6.5.3-3 & $C p_{\text {dust } i, j}$ \\
\hline \multirow[t]{2}{*}{ Root_Uptakes } & Transfer_Factor & Data & Dbase & Vector(5) & $6.5 .3-2$ & $F_{S \rightarrow p i, j}$ \\
\hline & Root_Uptake & Expression & Cal'ed & Vector(5) & $6.5 .3-2$ & $C p_{\text {root } i, j}$ \\
\hline \multicolumn{2}{|l|}{ Plant Conc } & Sum & Cal'ed & Vector(5) & 6.5.3-1 & $C p_{i, j}$ \\
\hline \multicolumn{2}{|l|}{ Crop Conc } & Data & Cal'ed & Vector(4) & $6.5 .3-1$ & $C p_{i, j}$ \\
\hline
\end{tabular}

NOTE: See notes for Table 6.9-1.

\subsubsection{Animal Submodel}

Mathematical equations for the animal submodel are discussed in Section 6.5.4. All parameters used in this submodel are listed in Table 6.9-5. The animal submodel container includes two lower level containers (Figure 6.9-7). The AnimalModel_Input container (Equation 6.5.4-1) includes input parameters for the animal submodel. The Animal_Ingestion container calculates radionuclide concentrations in animal products due to the ingestion of contaminated feed (Equation 6.5.4-2) and contaminated soil (Equation 6.5.4-3).

This submodel calculates the radionuclide concentration in animal products

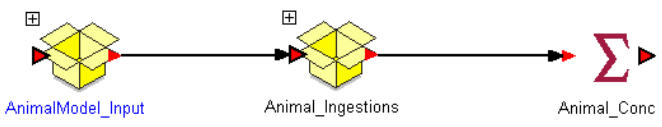

Figure 6.9-7. Animal Submodel Container 
Table 6.9-5. Parameters in the Animal Submodel

\begin{tabular}{|c|c|c|c|c|c|c|}
\hline $\begin{array}{l}\text { Low Level } \\
\text { Box Name }\end{array}$ & Parameter Name & $\begin{array}{l}\text { Element } \\
\text { Type }\end{array}$ & Data Source & Data Type & Equation & Notation \\
\hline \multirow{11}{*}{$\begin{array}{l}\text { Animal } \\
\text { Model_Input }\end{array}$} & Feed_ForMeat & Stochastic & Input & Scalar & $6.5 .4-2$ & $Q f_{1}$ \\
\hline & Feed_ForMilk & Stochastic & Input & Scalar & $6.5 .4-2$ & $Q f_{2}$ \\
\hline & Feed_ForPoultry & Stochastic & Input & Scalar & 6.5.4-2 & $Q f_{3}$ \\
\hline & Feed_ForEggs & Stochastic & Input & Scalar & 6.5.4-2 & $Q f_{4}$ \\
\hline & Animal_Feed & Data & Cal'ed & Vector(4) & 6.5.4-2 & $Q f_{k}$ \\
\hline & Soil_ForMeat & Stochastic & Input & Scalar & 6.5.4-3 & $Q s_{1}$ \\
\hline & Soil_ForMilk & Stochastic & Input & Scalar & 6.5.4-3 & $Q s_{2}$ \\
\hline & Soil_ForPoultry & Stochastic & Input & Scalar & 6.5.4-3 & $Q s_{3}$ \\
\hline & Soil_ForEggs & Stochastic & Input & Scalar & 6.5.4-3 & $Q s_{4}$ \\
\hline & Animal_Soil & Data & Cal'ed & Vector(4) & 6.5.4-3 & $Q s_{k}$ \\
\hline & Transfer_Coefficients & Data & Dbase & Vector(4) & 6.5.4-2 & $F m_{i, k}$ \\
\hline \multirow{3}{*}{$\begin{array}{l}\text { Animal__} \\
\text { Ingestions }\end{array}$} & Feed_Conc & Data & Cal'ed & Vector(4) & 6.5.3-1 & $C p_{i, j}$ \\
\hline & Feed_Contribution & Expression & Cal'ed & Vector(4) & 6.5.4-2 & $C d_{\text {feed,i,k }}$ \\
\hline & Soil_Contribution & Expression & Cal'ed & Vector(4) & 6.5.4-3 & $C d_{\text {soil, }, i, k}$ \\
\hline \multicolumn{2}{|l|}{ Animal Conc } & Sum & Cal'ed & Vector(4) & 6.5.4-1 & $C d_{i, k}$ \\
\hline
\end{tabular}

NOTE: See notes for Table 6.9-1.

\subsubsection{External Exposure Submodel}

Mathematical equations for the external exposure submodel are discussed in Section 6.5.5. All parameters used in this submodel are listed in Table 6.9-6. The external exposure submodel container includes two lower level containers (Figure 6.9-8). The External_Input container is used for input data for the population groups and the associated time budgets, and the External_Model container calculates the effective external exposure time and the human external radiation dose (Equation 6.5.5-1).

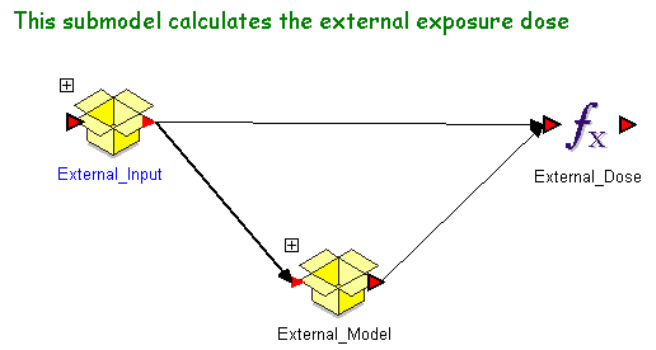

Figure 6.9-8. External Exposure Submodel Container 
Table 6.9-6. Parameters in the External Exposure Submodel

\begin{tabular}{|c|c|c|c|c|c|c|}
\hline $\begin{array}{c}\text { Low Level Box } \\
\text { Name }\end{array}$ & Parameter Name & $\begin{array}{c}\text { Element } \\
\text { Type }\end{array}$ & $\begin{array}{c}\text { Data } \\
\text { Source }\end{array}$ & Data Type & Equation & Notation \\
\hline \multirow[t]{23}{*}{ External_Input } & OW_ActOut & Stochastic & Input & Scalar & 6.5.5-1 & $t_{1,1}$ \\
\hline & IW_ActOut & Stochastic & Input & Scalar & 6.5.5-1 & $t_{1,2}$ \\
\hline & CT_ActOut & Stochastic & Input & Scalar & 6.5.5-1 & $t_{1,3}$ \\
\hline & NW_ActOut & Stochastic & Input & Scalar & 6.5.5-1 & $t_{1,4}$ \\
\hline & OW_Outdoor & Stochastic & Input & Scalar & 6.5.5-1 & $t_{2,1}$ \\
\hline & IW_Outdoor & Stochastic & Input & Scalar & 6.5.5-1 & $t_{2,2}$ \\
\hline & CT_Outdoor & Stochastic & Input & Scalar & 6.5.5-1 & $t_{2,3}$ \\
\hline & NW_Outdoor & Stochastic & Input & Scalar & 6.5.5-1 & $t_{2,4}$ \\
\hline & OW_Away & Stochastic & Input & Scalar & 6.5.5-1 & $t_{5,1}$ \\
\hline & IW_Away & Stochastic & Input & Scalar & $6.5 .5-1$ & $t_{5,2}$ \\
\hline & CT_Away & Stochastic & Input & Scalar & 6.5.5-1 & $t_{5,3}$ \\
\hline & NW_Away & Stochastic & Input & Scalar & 6.5.5-1 & $t_{5,4}$ \\
\hline & Indoor_Asleep & Stochastic & Input & Scalar & 6.5.5-1 & $t_{4, m}$ \\
\hline & OW_Indoor & Expression & Cal'ed & Scalar & 6.5.5-1 & $t_{3,1}$ \\
\hline & IW_Indoor & Expression & Cal'ed & Scalar & 6.5.5-1 & $t_{3,2}$ \\
\hline & CT_Indoor & Expression & Cal'ed & Scalar & 6.5.5-1 & $t_{3,3}$ \\
\hline & NW_Indoor & Expression & Cal'ed & Scalar & 6.5.5-1 & $t_{3,4}$ \\
\hline & Fraction_OW & Stochastic & Input & Scalar & 6.5.5-1 & $P P_{1}$ \\
\hline & Fraction_IW & Expression & Cal'ed & Scalar & $6.5 .5-1$ & $P P_{2}$ \\
\hline & Fraction_CT & Stochastic & Input & Scalar & 6.5.5-1 & $P P_{3}$ \\
\hline & Fraction_NW & Stochastic & Input & Scalar & $6.5 .5-1$ & $\mathrm{PP}_{4}$ \\
\hline & Shielding_Factor & Data & Input & Scalar & 6.5.5-1 & $f_{\text {ext }, i, 3 / 4}$ \\
\hline & External_DC & Data & Dbase & Scalar & 6.5.5-1 & $E D C s_{\text {soil, } i}$ \\
\hline \multirow{6}{*}{$\begin{array}{l}\text { External_ } \\
\text { Model }\end{array}$} & Exposure_times & Data & Cal'ed & Matrix $(5,4)$ & 6.5.5-1 & $t_{n, m}$ \\
\hline & Population & Data & Cal'ed & Vector(4) & 6.5.5-1 & $P P_{m}$ \\
\hline & Weighted_Time & Expression & Cal'ed & Vector(5) & $6.5 .5-1$ & $P P_{m} t_{n, m}$ \\
\hline & Weighted_Outdoor & Expression & Cal'ed & Scalar & $6.5 .5-1$ & $P P_{m} t_{1, m}+P P_{m} t_{2, m}$ \\
\hline & Weighted_Indoor & Expression & Cal'ed & Scalar & $6.5 .5-1$ & $P P_{m} t_{3, m}+P P_{m} t_{4, m}$ \\
\hline & External_Time & Expression & Cal'ed & Scalar & 6.5.5-1 & $\sum f_{e x t, i, n}\left(\sum P P_{m} t_{n, m}\right)$ \\
\hline \multicolumn{2}{|l|}{ External_Dose } & Expression & Cal'ed & Scalar & 6.5.5-1 & $D_{e x t, i}$ \\
\hline
\end{tabular}

NOTE: See notes for Table 6.9-1.

\subsubsection{Inhalation Submodel}

Mathematical equations for the inhalation submodel are discussed in Section 6.5.6. All parameters used in this submodel are listed in Table 6.9-7. The contents of the inhalation submodel container include one lower level container (Figure 6.9-9). The Inhalation_Input container includes inhalation-related input parameters (e.g., breathing rates and radon data). The time budgets for the receptor are calculated in the External Model container. Inhalation doses are calculated under normal and post-volcanic conditions (Equation 6.5.6-1). The radon inhalation dose due to exhalation of radon gas from ${ }^{226} \mathrm{Ra}$ in volcanic ash on the ground is also included in this submodel (Equation 6.5.6-4). 
This submodel calculates the inhalation dose

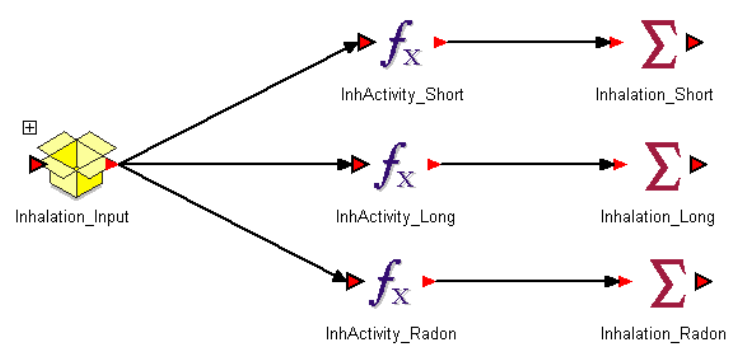

Figure 6.9-9. Inhalation Submodel Container

Table 6.9-7. Parameters in the Inhalation Submodel

\begin{tabular}{|c|c|c|c|c|c|c|}
\hline $\begin{array}{c}\text { Low Level Box } \\
\text { Name }\end{array}$ & Parameter Name & $\begin{array}{l}\text { Element } \\
\text { Type }\end{array}$ & $\begin{array}{l}\text { Data } \\
\text { Source }\end{array}$ & Data Type & Equation & Notation \\
\hline \multirow[t]{6}{*}{ Inhalation_Input } & Breathing_Rate & Data & Input & Vector(5) & $6.5 .6-2$ & $B R_{n}$ \\
\hline & RadonEF_Outdoor & Stochastic & Input & Scalar & $6.5 .6-3$ & $E F_{R n-222,1 \& 2}$ \\
\hline & RadonET_Indoor & Stochastic & Input & Scalar & $6.5 .6-3$ & $E F_{R n-222,3 \& 4}$ \\
\hline & Radon_Equilibrium & Data & Cal'ed & Vector(5) & $6.5 .6-3$ & $E F_{R n-222, n}$ \\
\hline & Radon_DCF & Data & Input & Scalar & $6.5 .6-3$ & $D_{\text {inh,Rn-222 }}$ \\
\hline & Inhalation_DCF & Data & Dbase & Scalar & $6.5 .6-2$ & $D C F_{i n h, i}$ \\
\hline \multicolumn{2}{|l|}{ InhActivity_Short } & Expression & Cal'ed & Vector(5) & $6.5 .6-2$ & $D_{\text {inh }, v, i}$ \\
\hline \multicolumn{2}{|l|}{ InhActivity_Long } & Expression & Cal'ed & Vector(5) & $6.5 .6-2$ & $D_{\text {inh }, p, i}$ \\
\hline \multicolumn{2}{|l|}{ InhActivity_Radon } & Expression & Cal'ed & Vector(5) & $6.5 .6-3$ & $D_{\text {inh }, g, R n-222, n}$ \\
\hline \multicolumn{2}{|l|}{ Inhalation_Short } & Sum & Cal'ed & Scalar & $6.5 .6-4$ & $D_{\text {inh }, v, i}$ \\
\hline \multicolumn{2}{|l|}{ Inhalation_Long } & Sum & Cal'ed & Scalar & $6.5 .6-4$ & $D_{\text {inh }, p, i}$ \\
\hline \multicolumn{2}{|l|}{ Inhalation_Radon } & Sum & Cal'ed & Scalar & $6.5 .6-3$ & $D_{\text {inh, }, \text { Rn-222 }}$ \\
\hline
\end{tabular}

NOTE: See notes for Table 6.9-1.

\subsubsection{Ingestion Submodel}

Mathematical equations for the human ingestion submodel, which include nine pathways, are discussed in Section 6.5.7. All parameters used in this submodel are listed in Table 6.9-8. The contents of the GoldSim submodel container include one lower level container (Figure 6.9-10). The Ingestion_Input container includes human foodstuff consumption rates. To provide the results of pathway analysis, the ingestion dose is presented for individual pathways and for total ingestion. 


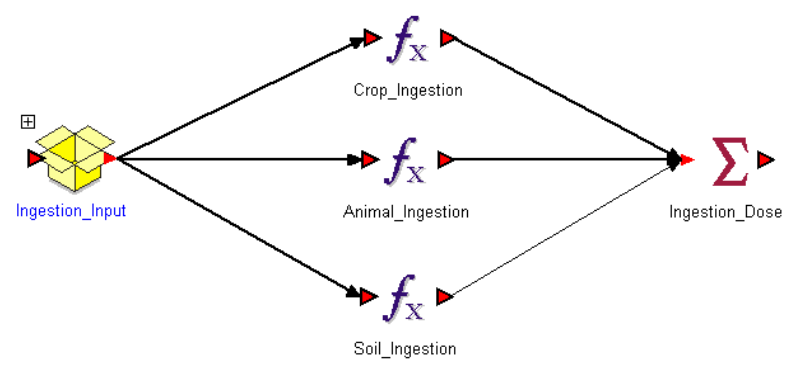

Figure 6.9-10. Ingestion Submodel Container

Table 6.9-8. Parameters in the Ingestion Submodel

\begin{tabular}{|c|c|c|c|c|c|c|}
\hline $\begin{array}{c}\text { Low Level Box } \\
\text { Name }\end{array}$ & Parameter Name & $\begin{array}{c}\text { Element } \\
\text { Type }\end{array}$ & $\begin{array}{c}\text { Data } \\
\text { Source }\end{array}$ & Data Type & Equation & Notation \\
\hline \multirow[t]{12}{*}{ Ingestion_Input } & Leafy_Consump & Stochastic & Input & Scalar & $6.5 .7-2$ & $U p_{1}$ \\
\hline & Other_Consump & Stochastic & Input & Scalar & $6.5 .7-2$ & $U p_{2}$ \\
\hline & Fruit_Consump & Stochastic & Input & Scalar & $6.5 .7-2$ & $U p_{3}$ \\
\hline & Grain_Consump & Stochastic & Input & Scalar & $6.5 .7-2$ & $U p_{4}$ \\
\hline & Crop_Consump & Data & Cal'ed & Vector(4) & $6.5 .7-2$ & $U p_{j}$ \\
\hline & Beef_Consump & Stochastic & Input & Scalar & $6.5 .7-3$ & $U d_{1}$ \\
\hline & Poultry_Consump & Stochastic & Input & Scalar & $6.5 .7-3$ & $U d_{2}$ \\
\hline & Milk_Consump & Stochastic & Input & Scalar & $6.5 .7-3$ & $U d_{3}$ \\
\hline & Eggs_Consump & Stochastic & Input & Scalar & $6.5 .7-3$ & $U d_{4}$ \\
\hline & Animal_Consump & Data & Cal'ed & Vector(4) & $6.5 .7-3$ & $U d_{k}$ \\
\hline & Soil_Consump & Stochastic & Input & Scalar & $6.5 .7-4$ & Us \\
\hline & Ingestion_DCF & Data & Dbase & Scalar & $6.5 .7-2$ & $E D C F_{\text {ing, } i}$ \\
\hline \multicolumn{2}{|l|}{ Crop_Ingestion } & Expression & Cal'ed & Vector(4) & $6.5 .7-2$ & $D_{\text {ing,p,j }}$ \\
\hline \multicolumn{2}{|l|}{ Animal_Ingestion } & Expression & Cal'ed & Vector(4) & $6.5 .7-3$ & $D_{\text {ing, } d, k}$ \\
\hline \multicolumn{2}{|l|}{ Soil_Ingestion } & Expression & Cal'ed & Scalar & 6.5.7-4 & $D_{\text {ing,s }}$ \\
\hline \multicolumn{2}{|l|}{ Ingestion_Dose } & Sum & Cal'ed & Scalar & $6.5 .7-1$ & $D_{\text {ing }, 0}$ \\
\hline
\end{tabular}

NOTE: See notes for Table 6.9-1.

\subsubsection{BDCF Results}

The TEDE and BDCF for each radionuclide are discussed in Section 6.5.8. All parameters used in this submodel are listed in Table 6.9-9. The BDCF for volcanic ash deposition has three parts. The first part includes external exposure, radon inhalation, and ingestion pathways; the second part includes inhalation of resuspended particles at post-volcanic levels, and the third part includes inhalation of resuspended particles at normal levels. In GoldSim, the pathway results are also calculated (Figure 6.9-11). 
External, radon inhalation \& ingestion $B D C F$

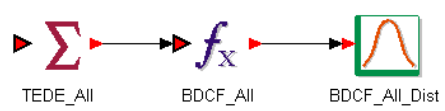

Short term inhalation BDCF

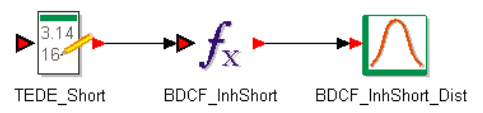

Long term inhalation BDCF

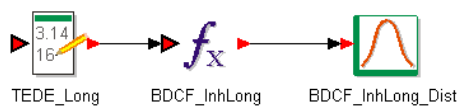

Pathway results

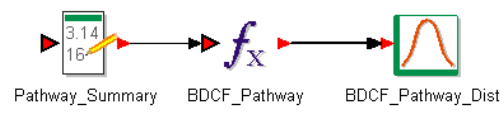

Concentration Results

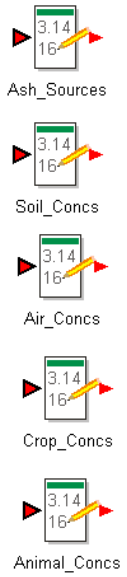

Figure 6.9-11. Final BDCF Result Container

Table 6.9-9. Parameters in the Final BDCF Results

\begin{tabular}{|l|l|l|l|l|l|}
\hline \multicolumn{1}{|c|}{ Parameter Name } & Element Type & Data Source & \multicolumn{1}{c|}{ Data Type } & \multicolumn{1}{c|}{ Equation } & \multicolumn{1}{c|}{ Notation } \\
\hline TEDE_All & Sum & Cal'ed & Scalar & $6.5 .8-1$ & $D_{\text {all,i }}$ \\
\hline BDCF_All & Expression & Cal'ed & Scalar & $6.5 .8-2$ & $B D C F_{i}$ \\
\hline TEDE_Short & Data & Cal'ed & Scalar & $6.5 .8-1$ & $D_{\text {inh, }, i}$ \\
\hline BDCF_InhShort & Expression & Cal'ed & Scalar & $6.5 .8-2$ & BDCF $F_{\text {inh, }, i}$ \\
\hline TEDE_Long & Data & Cal'ed & Scalar & $6.5 .8-1$ & $D_{\text {inh, }, i}$ \\
\hline BDCF_InhLong & Expression & Cal'ed & Scalar & $6.5 .8-2$ & BDCF inh,,$i$ \\
\hline Pathway_Summary & Data & Cal'ed & Vector(12) & $6.4 .10-3$ & $D_{p, i}$ \\
\hline Pathway_BDCF & Expression & Cal'ed & Vector(12) & $6.4 .10-4$ & $B D C F_{p, i}$ \\
\hline BDCF_All_Dist & Result & Cal'ed & Scalar & - & - \\
\hline BDCF_InhShort_Dist & Result & Cal'ed & Scalar & - & - \\
\hline BDCF_InhLong_Dist & Result & Cal'ed & Scalar & - & - \\
\hline BDCF_Pathway_Dist & Result & Cal'ed & Vector(12) & - & - \\
\hline
\end{tabular}

NOTE: See notes for Table 6.9-1.

\subsection{VERIFICATION OF THE ERMYN IMPLEMENTATION IN GOLDSIM}

Verification of the ERMYN in GoldSim is carried out by executing the model and comparing the results with results of hand calculations based on the equations described in Sections 6.4 and 6.5. Several test cases are performed to ensure that the ERMYN_GW and ERMYN_VA model is correctly implemented. The test cases are carried out as deterministic runs because stochastic runs are simply sets of many deterministic runs, and the correctness of the stochastic simulation is a property of the GoldSim software. 
In addition to verifying the deterministic realization with hand calculations, tests of the stochastic calculations are carried out in which the results of stochastic realizations are compared with the results of the deterministic realization. This was done to gain confidence that the stochastic results include the deterministic result, which have been verified with hand calculations. Because the sample means of input parameter distributions from some recommended distributions do not match the verification values of the input parameters, the mean of the output from the stochastic realizations is not expected to exactly match the results of the deterministic run. However, the deterministic results are expected to be within the range of the output distributions from the stochastic results.

As implemented in GoldSim, the ERMYN calculates BDCFs for individual radionuclides one at a time. Correlations between BDCFs for primary radionuclides may exist. BDCF correlations are part of the biosphere model results, which are documented and discussed in the two BDCF reports shown in Figure 1-1.

\subsubsection{Verification of the Model Implementation for the Groundwater Scenario}

\subsubsection{Verification of Deterministic Calculations}

Input parameters for verifying the model implementation for the groundwater scenario are taken from the Mean-Mode-Average column in Table 6.6-3. Three radionuclides, ${ }^{239} \mathrm{Pu},{ }^{14} \mathrm{C}$, and ${ }^{226} \mathrm{Ra}$, are tested under the groundwater scenario for the present-day climate conditions. For the base case, ${ }^{239} \mathrm{Pu}$ is selected (Table $6.10-1$ ), ${ }^{14} \mathrm{C}$ is selected for the ${ }^{14} \mathrm{C}$ special submodel (Table 6.10-2), and ${ }^{226} \mathrm{Ra}$ and decay products are selected for the radon dose calculations (Table 6.10-3). In the tables, the hand calculation equations are listed so that the calculations can be reproduced using inputs from Table 6.6-3. In addition, the results from GoldSim are presented with the corresponding GoldSim element names. The results from both methods are the same, indicating that the GoldSim implementation of the ERMYN (groundwater scenario; Section 6.4) is correct. The GoldSim files described in this section are listed in Appendix A.

The verification results for ${ }^{239} \mathrm{Pu}$ are presented in Table 6.10-1, in which the verification is presented in the order of each submodel discussed in Section 6.4. The hand-calculated results from the important equations in the biosphere model are presented in the table for comparison with the GoldSim results.

Verification results for ${ }^{14} \mathrm{C}$ are presented in Table 6.10 -2. Because ${ }^{14} \mathrm{C}$ is a special radionuclide, the model for ${ }^{14} \mathrm{C}$ (Section 6.6) in the environmental media differs from the model for the other radionuclides. Therefore, the content of this table is different from Table 6.10-1.

Another special radionuclide is ${ }^{226} \mathrm{Ra}$. This radionuclide produces radon gas, which eventually decays to a long-lived decay product $\left({ }^{210} \mathrm{~Pb}\right)$ that would accumulate in irrigated soils. Furthermore, consideration of different radon concentrations indoors and outdoors, the circumstances involving the operation of evaporative coolers, and normal ventilation conditions, made the calculation more complicated. For exposures related to the accumulation of radionuclides in surface soils, the contribution from the ${ }^{210} \mathrm{~Pb}$ decay product is considered (Table 6.10-3). 


\begin{tabular}{|c|c|c|c|c|c|c|c|c|c|}
\hline \multirow[b]{2}{*}{ Submodel } & \multicolumn{6}{|c|}{ Parameter in Mathematical Model } & \multicolumn{2}{|c|}{ Parameter in GoldSim } & \multirow[b]{2}{*}{ Notes $^{c}$} \\
\hline & \multicolumn{2}{|c|}{ Parameter Name } & $\begin{array}{l}\text { Notation in } \\
\text { Equation }\end{array}$ & $\begin{array}{l}\text { Equation } \\
\text { Number }\end{array}$ & Units & $\begin{array}{l}\text { Calculated } \\
\text { Value }^{\mathrm{a}}\end{array}$ & Element Name & $\begin{array}{l}\text { ERMYN_GW } \\
\text { Result }^{\text {b }}\end{array}$ & \\
\hline \multirow[t]{5}{*}{ Soil } & \multicolumn{2}{|c|}{ Leaching removal constant } & $\lambda_{1, \text { Pu-239 }}$ & $6.4 .1-10$ & /yr & 1.76E-04 & Leaching_Factor & $1.76 \mathrm{E}-04$ & Same \\
\hline & \multicolumn{2}{|c|}{ Erosion removal constant } & $\lambda_{e}$ & 6.4.1-11 & /yr & 1.31E-03 & Erosion_Factor & 1.31E-03 & Same \\
\hline & \multicolumn{2}{|c|}{ Effective removal constant } & $\lambda_{\text {eff,Pu-239 }}$ & $6.4 .1-3$ & /yr & 1.51E-03 & Effective_Removal & 1.51E-03 & Same \\
\hline & \multicolumn{2}{|c|}{ Concentration in surface soil } & $\mathrm{CS}_{\mathrm{Pu}-239}$ & $6.4 .1-4$ & $\mathrm{~Bq} / \mathrm{m}^{2}$ & 622 & Saturated_Conc & 622 & Same \\
\hline & \multicolumn{2}{|c|}{ Concentration in soil mass } & $C s_{m, P u-239}$ & $6.4 .1-5$ & $\mathrm{~Bq} / \mathrm{kg}$ & 1.66 & SatMass_Conc & 1.66 & Same \\
\hline \multirow[t]{7}{*}{ Air } & \multicolumn{2}{|c|}{ Concentration in air for crop } & $C a_{p, P u-239}$ & $6.4 .2-1$ & $\mathrm{~Bq} / \mathrm{m}^{3}$ & 1.99E-07 & AirConc_Crop & 1.99E-07 & Same \\
\hline & \multirow{5}{*}{$\begin{array}{l}\text { Concentration in } \\
\text { air for inhalation }\end{array}$} & Active outdoors & $C a_{h, P u-239,1}$ & \multirow[t]{5}{*}{$6.4 .2-2$} & \multirow[t]{5}{*}{$\mathrm{Bq} / \mathrm{m}^{3}$} & $3.32 \mathrm{E}-05$ & \multirow[t]{5}{*}{ AirConc_Inh } & $3.32 \mathrm{E}-05$ & Same \\
\hline & & Inactive outdoors & $C a_{h, P u-239,2}$ & & & $6.97 \mathrm{E}-08$ & & $6.97 \mathrm{E}-08$ & Same \\
\hline & & Active indoors & $C a_{h, P u-239,3}$ & & & $1.16 \mathrm{E}-07$ & & 1.16E-07 & Same \\
\hline & & Asleep indoors & $C a_{h, P u-239,4}$ & & & $3.48 \mathrm{E}-08$ & & 3.48E-08 & Same \\
\hline & & Away from area & $C a_{h, P u-239,5}$ & & & $0.00 \mathrm{E}+00$ & & $0.00 \mathrm{E}+00$ & Same \\
\hline & \multicolumn{2}{|c|}{$\begin{array}{l}\text { Concentration in air from evaporative } \\
\text { cooler }\end{array}$} & $C a_{e, P u-239}$ & $6.4 .2-3$ & $\mathrm{~Bq} / \mathrm{m}^{3}$ & $1.02 \mathrm{E}-06$ & AirConc_Evap & $1.02 \mathrm{E}-06$ & Same \\
\hline \multirow[t]{20}{*}{ Plant } & \multirow{5}{*}{$\begin{array}{l}\text { Concentration due } \\
\text { to root uptake }\end{array}$} & Leafy vegetation & $C p_{\text {root }, \text { Pu-239, } 1}$ & \multirow[t]{5}{*}{$6.4 .3-2$} & \multirow[t]{5}{*}{$\mathrm{Bq} / \mathrm{kg}$} & 3.37E-05 & \multirow[t]{5}{*}{ Root_Uptake } & 3.37E-05 & Same \\
\hline & & Other vegetation & $C p_{\text {root, } P u-239,2}$ & & & $3.25 E-05$ & & $3.25 \mathrm{E}-05$ & Same \\
\hline & & Fruit & Cp root, Pu-239,3 & & & 3.58E-05 & & 3.58E-05 & Same \\
\hline & & Grain & $C p_{\text {root }, \text { Pu-239,4 }}$ & & & $2.85 E-05$ & & $2.85 \mathrm{E}-05$ & Same \\
\hline & & Forage & $C p_{\text {root,Pu-239,5 }}$ & & & 3.65E-04 & & 3.65E-04 & Same \\
\hline & \multirow{5}{*}{$\begin{array}{l}\text { Irrigation } \\
\text { interception } \\
\text { fraction }\end{array}$} & Leafy vegetation & $R W_{1}$ & \multirow[t]{5}{*}{$6.4 .3-5$} & \multirow[t]{5}{*}{-} & 0.217 & \multirow[t]{5}{*}{ Intercept_Fractor } & 0.217 & Same \\
\hline & & Other vegetation & $R W_{2}$ & & & 0.304 & & 0.304 & Same \\
\hline & & Fruit & $R w_{3}$ & & & 0.361 & & 0.361 & Same \\
\hline & & Grain & $R W_{4}$ & & & 0.472 & & 0.472 & Same \\
\hline & & Forage & $R W_{5}$ & & & 0.259 & & 0.259 & Same \\
\hline & & Leafy vegetation & $C p_{\text {water,Pu-239,1 }}$ & $6.4 .3-3$ & $\mathrm{~Bq} / \mathrm{kg}$ & $5.24 \mathrm{E}-03$ & Water_Uptake & $5.24 \mathrm{E}-03$ & Same \\
\hline & to water & Other vegetation & $C p_{\text {water }, \text { Pu-239,2 }}$ & & & 8.27E-04 & & 8.27E-04 & Same \\
\hline & interception & Fruit & $C p_{\text {water,Pu-239,3 }}$ & & & $9.78 \mathrm{E}-04$ & & $9.78 \mathrm{E}-04$ & Same \\
\hline & & Grain & $C p_{\text {water,Pu-239,4 }}$ & & & $6.74 \mathrm{E}-03$ & & $6.74 \mathrm{E}-03$ & Same \\
\hline & & Forage & $C p_{\text {water }, \text { Pu-239,5 }}$ & & & 1.40E-02 & & 1.40E-02 & Same \\
\hline & Dust interception & Leafy vegetation & $R a_{1}$ & $6.4 .3-8$ & - & 0.456 & Dust_Intercept & 0.456 & Same \\
\hline & fraction & Other vegetation & $\mathrm{Ra}_{2}$ & & & 0.787 & & 0.787 & Same \\
\hline & & Fruit & $R a_{3}$ & & & 0.893 & & 0.893 & Same \\
\hline & & Grain & $\mathrm{Ra}_{4}$ & & & 0.962 & & 0.962 & Same \\
\hline & & Forage & $R a_{5}$ & & & 0.751 & & 0.751 & Same \\
\hline
\end{tabular}




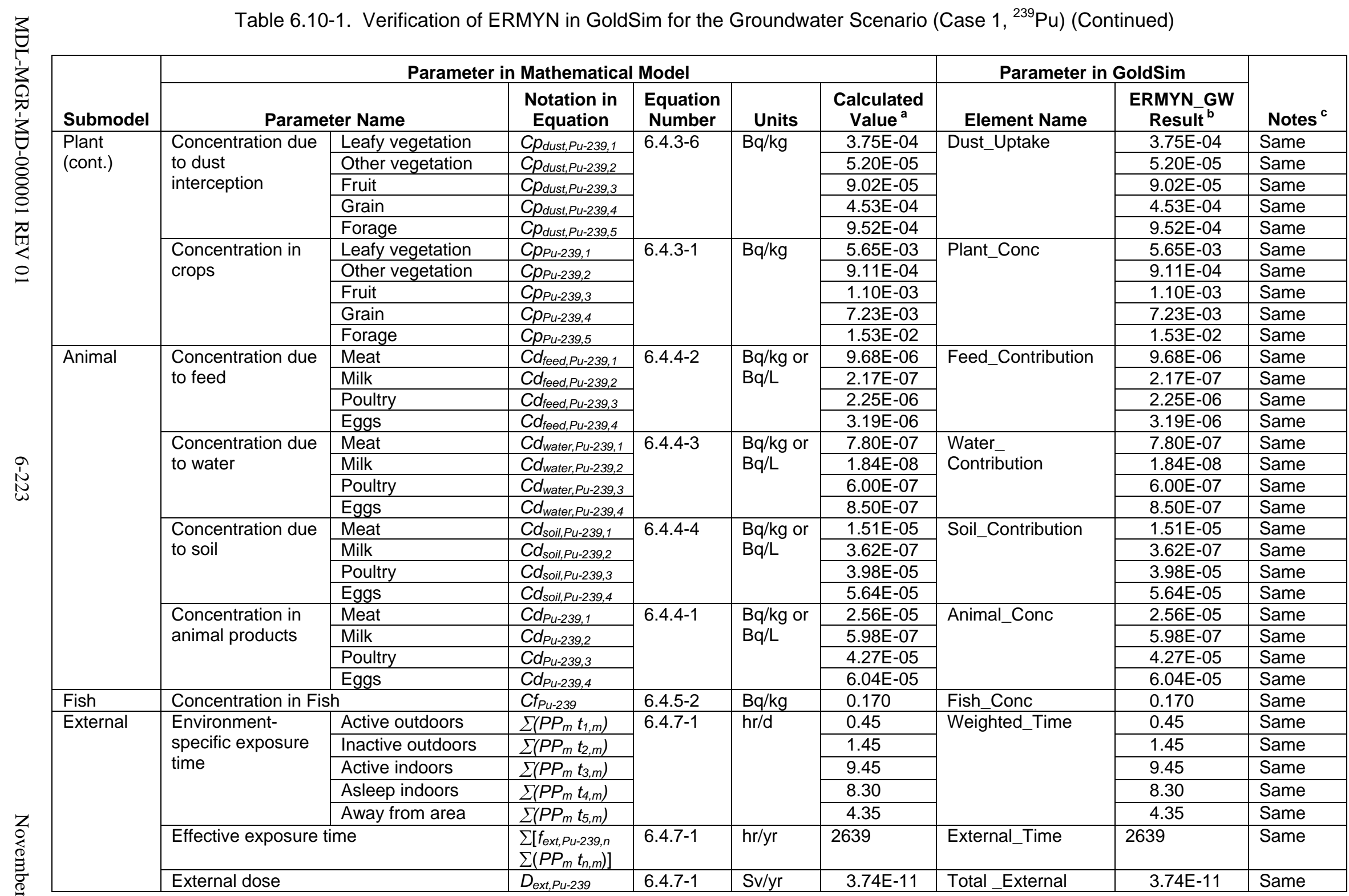




\begin{tabular}{|c|c|c|c|c|c|c|c|c|c|}
\hline \multirow[b]{2}{*}{ Submodel } & \multicolumn{6}{|c|}{ Parameter in Mathematical Model } & \multicolumn{2}{|c|}{ Parameter in GoldSim } & \multirow[b]{2}{*}{ Notes ${ }^{\circ}$} \\
\hline & \multicolumn{2}{|c|}{ Parameter Name } & $\begin{array}{l}\text { Notation in } \\
\text { Equation }\end{array}$ & $\begin{array}{l}\text { Equation } \\
\text { Number }\end{array}$ & Units & $\begin{array}{l}\text { Calculated } \\
\text { Value }^{a}\end{array}$ & Element Name & $\begin{array}{l}\text { ERMYN_GW } \\
\text { Result }^{\mathbf{b}}\end{array}$ & \\
\hline \multirow[t]{3}{*}{ Inhalation } & \multicolumn{2}{|c|}{ Inhalation dose for particles } & $D_{\text {inh, }, \text { Pu-239 }}$ & $6.4 .8-2$ & Sv/yr & $1.06 \mathrm{E}-06$ & Total Dust & $1.06 \mathrm{E}-06$ & Same \\
\hline & \multirow{2}{*}{\multicolumn{2}{|c|}{$\begin{array}{l}\text { Inhalation dose from evaporative cooler } \\
\text { Inhalation dose }\end{array}$}} & $D_{\text {inh }, e, \text { Pu-239 }}$ & $6.4 .8-3$ & Sv/yr & $1.68 \mathrm{E}-07$ & Cooler_Inhalation & $1.68 \mathrm{E}-07$ & Same \\
\hline & & & $D_{\text {inh, }, \text { Pu-239 }}$ & $6.4 .8-1$ & Sv/yr & $1.23 \mathrm{E}-06$ & Total_Inhalation & $1.23 \mathrm{E}-06$ & Same \\
\hline \multirow[t]{12}{*}{ Ingestion } & \multicolumn{2}{|c|}{ Ingestion dose for water } & $D_{\text {ing,w,Pu-239 }}$ & $6.4 .9-2$ & Sv/yr & $6.98 \mathrm{E}-07$ & Water_Ingestion & $6.98 \mathrm{E}-07$ & Same \\
\hline & \multirow{4}{*}{$\begin{array}{l}\text { Ingestion dose for } \\
\text { crops }\end{array}$} & Leafy vegetation & $D_{\text {ing, }, p, \text { Pu-239, } 1}$ & \multirow[t]{4}{*}{$6.4 .9-3$} & Sv/yr & $2.04 \mathrm{E}-08$ & \multirow[t]{4}{*}{ Crop_Ingestion } & $2.04 \mathrm{E}-08$ & Same \\
\hline & & Other vegetation & $D_{\text {ing,p,Pu-239, } 2}$ & & Sv/yr & 4.12E-09 & & $4.12 \mathrm{E}-09$ & Same \\
\hline & & Fruit & $D_{\text {ing, }, p, P u-239,3}$ & & Sv/yr & $1.34 \mathrm{E}-08$ & & $1.34 \mathrm{E}-08$ & Same \\
\hline & & Grain & $D_{\text {ing, }, p, P u-239,4}$ & & Sv/yr & $1.59 \mathrm{E}-09$ & & $1.59 \mathrm{E}-09$ & Same \\
\hline & \multirow{4}{*}{$\begin{array}{l}\text { Ingestion dose for } \\
\text { animal products }\end{array}$} & Meat & 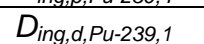 & \multirow[t]{4}{*}{$6.4 .9-4$} & Sv/yr & $6.96 \mathrm{E}-11$ & \multirow[t]{4}{*}{ Animal_Ingestion } & $6.96 \mathrm{E}-11$ & Same \\
\hline & & Milk & $D_{\text {ing, } d, P u-239,2}$ & & Sv/yr & $2.66 \mathrm{E}-12$ & & $2.66 \mathrm{E}-12$ & Same \\
\hline & & Poultry & $D_{\text {ing, } d, P u-239,3}$ & & Sv/yr & $1.71 \mathrm{E}-11$ & & $1.71 \mathrm{E}-11$ & Same \\
\hline & & Eggs & $D_{\text {ing, } d, P u-239,4}$ & & Sv/yr & $3.06 \mathrm{E}-10$ & & $3.06 \mathrm{E}-10$ & Same \\
\hline & \multicolumn{2}{|c|}{ Ingestion dose for fish } & $D_{\text {ing, } f \text { Pu-239 }}$ & $6.4 .9-5$ & Sv/yr & $3.74 \mathrm{E}-08$ & Fish_Ingestion & $3.74 \mathrm{E}-08$ & Same \\
\hline & \multirow{2}{*}{\multicolumn{2}{|c|}{$\begin{array}{l}\text { Ingestion dose for soil } \\
\text { Ingestion dose }\end{array}$}} & $D_{\text {ing, }, \text { Pu-239 }}$ & $6.4 .9-6$ & Sv/yr & $5.79 \mathrm{E}-08$ & Soil_Ingestion & $5.79 \mathrm{E}-08$ & Same \\
\hline & & & $D_{\text {ing, Pu-239 }}$ & $6.4 .9-1$ & Sv/yr & $8.34 \mathrm{E}-07$ & Total_Ingestion & $8.34 \mathrm{E}-07$ & Same \\
\hline \multirow{2}{*}{$\begin{array}{l}\text { All } \\
\text { Pathway }\end{array}$} & \multicolumn{2}{|c|}{$\begin{array}{l}\text { Ingestion dose } \\
\text { All-pathway dose }\end{array}$} & $D_{\text {all, Pu-239 }}$ & $6.4 .10-1$ & Sv/yr & $2.06 \mathrm{E}-06$ & Final_TEDE & $2.06 \mathrm{E}-06$ & Same \\
\hline & \multicolumn{2}{|l|}{ BDCF } & $B D C F_{P u-239}$ & $6.4 .10-2$ & $\begin{array}{l}(\mathrm{rem} / \mathrm{yr}) / \\
(\mathrm{pCi} / \mathrm{L})\end{array}$ & $7.63 E-03$ & Final_BDCF & $7.63 \mathrm{E}-03$ & Same \\
\hline \multicolumn{10}{|c|}{$\begin{array}{l}\text { a Input data for this verification taken from the "mean, mode, or average" column in Table 6.6-3. Calculations performed in Microsoft Excel } 97 \text { SR-2. The } \\
\text { electronic file, ERMYN verification.xIs, which contains this calculation in worksheet GWPu239, is listed in Appendix A. } \\
\text { "The ERMYN is realized in GoldSim using the deterministic mode and data from the "mean, mode, or average" column in Table 6.6-3. The electronic file, } \\
\text { ERMYN_GW_PU239verf.gsm, for this run is listed in Appendix A. } \\
\text { c Comparison of results from Calculated Value (Column 6) and ERMYN_GW Result (Column 8). }\end{array}$} \\
\hline
\end{tabular}


Table 6.10-2. Verification of ERMYN in GoldSim for the Groundwater Scenario (Case $2,{ }^{14} \mathrm{C}$ )

\begin{tabular}{|c|c|c|c|c|c|c|c|c|c|}
\hline \multirow[b]{2}{*}{ Submodel } & \multicolumn{6}{|c|}{ Parameter in Mathematical Model } & \multicolumn{2}{|c|}{ Parameter in GoldSim } & \multirow[b]{2}{*}{ Notes ${ }^{c}$} \\
\hline & \multicolumn{2}{|c|}{ Parameter Name } & $\begin{array}{c}\text { Notation in } \\
\text { Equation }\end{array}$ & $\begin{array}{c}\text { Equation } \\
\text { Number }\end{array}$ & Units & $\begin{array}{c}\text { Calculated } \\
\text { Value }^{\mathrm{a}}\end{array}$ & Element Name & $\begin{array}{l}\text { ERMYN_GW } \\
\text { Result }{ }^{\text {b }}\end{array}$ & \\
\hline \multirow[t]{9}{*}{${ }^{14} \mathrm{C} /$ Soil } & \multicolumn{2}{|c|}{ Leaching removal constant } & $\lambda_{1, C-14}$ & 6.4.1-10 & /yr & 1.16E-02 & Leaching_Factor & 1.16E-02 & Same \\
\hline & \multicolumn{2}{|c|}{ Erosion removal constant } & $\lambda_{e}$ & 6.4.1-11 & /yr & $1.31 \mathrm{E}-03$ & Erosion_Factor & $1.31 \mathrm{E}-03$ & Same \\
\hline & \multicolumn{2}{|c|}{ Effective removal constant } & $\lambda_{\text {eff,C-14 }}$ & 6.4.1-3 & /yr & 1.30E-02 & Effective_Removal & 1.30E-02 & Same \\
\hline & \multicolumn{2}{|c|}{ Concentration in surface soil } & $C S_{0, C-14}$ & 6.4.6-1 & $\mathrm{Bq} / \mathrm{m}^{2}$ & 4.27E-02 & C14Conc_InhSoil & 4.27E-02 & Same \\
\hline & \multirow{5}{*}{$\begin{array}{l}\text { Concentration in } \\
\text { crop land }\end{array}$} & Leafy vegetation & $C S_{1, C-14}$ & \multirow[t]{5}{*}{ 6.4.6-1 } & \multirow[t]{5}{*}{$\mathrm{Bq} / \mathrm{m}^{2}$} & $8.96 \mathrm{E}-02$ & \multirow[t]{5}{*}{ C14Conc_CropSoil } & 8.96E-02 & \multirow[t]{5}{*}{ Same } \\
\hline & & Other vegetation & $\mathrm{CS}_{2, \mathrm{C}-14}$ & & & $1.25 \mathrm{E}-01$ & & $1.25 \mathrm{E}-01$ & \\
\hline & & Fruit & $C s_{3, C-14}$ & & & $1.22 \mathrm{E}-01$ & & $1.22 \mathrm{E}-01$ & \\
\hline & & Grain & $\mathrm{CS}_{4, \mathrm{C}-14}$ & & & 7.70E-02 & & 7.70E-02 & \\
\hline & & Forage & $C S_{5, C-14}$ & & & 1.09E-01 & & $1.09 \mathrm{E}-01$ & \\
\hline \multirow[t]{6}{*}{${ }^{14} \mathrm{C} /$ Air } & \multicolumn{2}{|c|}{ Concentration in air for inhalation } & $C a_{h, C-14}$ & 6.4.6-3 & $\mathrm{Bq} / \mathrm{m}^{3}$ & 1.21E-05 & C14Conc_Inh & $1.21 \mathrm{E}-05$ & Same \\
\hline & \multirow{5}{*}{$\begin{array}{l}\text { Concentration in } \\
\text { air for crops }\end{array}$} & Leafy vegetation & $C a_{h, C-14,1}$ & \multirow[t]{5}{*}{$6.4 .6-3$} & \multirow[t]{5}{*}{$\mathrm{Bq} / \mathrm{m}^{3}$} & $6.54 \mathrm{E}-05$ & \multirow[t]{5}{*}{ C14Conc_Air } & $6.54 \mathrm{E}-05$ & Same \\
\hline & & Other vegetation & $C a_{h, c-14,2}$ & & & 9.14E-05 & & 9.14E-05 & Same \\
\hline & & Fruit & $C a_{h, C-14,3}$ & & & 8.93E-05 & & 8.93E-05 & Same \\
\hline & & Grain & $C a_{h, c-14,4}$ & & & $5.62 \mathrm{E}-05$ & & $5.62 \mathrm{E}-05$ & Same \\
\hline & & Forage & $C a_{h, C-14,5}$ & & & 7.91E-05 & & 7.91E-05 & Same \\
\hline \multirow[t]{5}{*}{${ }^{14}$ C/Plant } & \multirow{5}{*}{$\begin{array}{l}\text { Concentration in } \\
\text { crops }\end{array}$} & Leafy vegetation & $C p_{C-14,1}$ & \multirow[t]{5}{*}{ 6.4.6-6 } & \multirow[t]{5}{*}{$\mathrm{Bq} / \mathrm{kg}$} & 3.20E-02 & \multirow[t]{5}{*}{ C14Conc_Crop } & 3.20E-02 & Same \\
\hline & & Other vegetation & $C p_{C-14,2}$ & & & $4.48 \mathrm{E}-02$ & & 4.48E-02 & Same \\
\hline & & Fruit & $C p_{C-14,3}$ & & & $4.38 \mathrm{E}-02$ & & 4.38E-02 & Same \\
\hline & & Grain & $C p_{C-14,4}$ & & & $1.22 \mathrm{E}-01$ & & $1.22 \mathrm{E}-01$ & Same \\
\hline & & Forage & $C p_{C-14,5}$ & & & $3.88 \mathrm{E}-02$ & & $3.88 \mathrm{E}-02$ & Same \\
\hline${ }^{14} \mathrm{C} /$ Animal & Concentration in & Meat & $C d_{C-14,1}$ & 6.4.6-7 & $\mathrm{Bq} / \mathrm{kg}$ & $1.06 \mathrm{E}-01$ & C14Conc_Animal & $1.06 \mathrm{E}-01$ & Same \\
\hline & animal product & Milk & $C d_{C-14,2}$ & & & $3.10 \mathrm{E}-02$ & & 3.10E-02 & Same \\
\hline & & Poultry & $C d_{C-14,3}$ & & & $6.18 \mathrm{E}-02$ & & $6.18 \mathrm{E}-02$ & Same \\
\hline & & Eggs & $C d_{C-14,4}$ & & & 4.63E-02 & & 4.63E-02 & Same \\
\hline Fish & Concentration in $\mathrm{F}$ & & $C f_{C-14}$ & $6.4 .5-2$ & $\mathrm{~Bq} / \mathrm{kg}$ & 4.6 & Fish_Conc & 4.6 & Same \\
\hline External & Environment- & Active outdoors & $\Sigma\left(P P_{m} t_{1, m}\right)$ & 6.4.7-1 & $\mathrm{hr} / \mathrm{d}$ & 0.45 & Weighted_Time & 0.45 & Same \\
\hline & specific exposure & Inactive outdoors & $\Sigma\left(P P_{m} t_{2, m}\right)$ & & & 1.45 & & 1.45 & Same \\
\hline & time & Active indoors & $\Sigma\left(P P_{m} t_{3, m}\right)$ & & & 9.45 & & 9.45 & Same \\
\hline & & Asleep indoors & $\Sigma\left(P P_{m} t_{4, m}\right)$ & & & 8.30 & & 8.30 & Same \\
\hline & & Away from area & $\sum\left(P P_{m} t_{5, m}\right)$ & & & 4.35 & & 4.35 & Same \\
\hline & Effective exposure & ime & $\begin{array}{l}\sum\left[f_{\text {ext }, C-14, n}\right. \\
\left.\sum\left(P P_{m} t_{n, m}\right)\right]\end{array}$ & 6.4.7-1 & $\mathrm{hr} / \mathrm{yr}$ & 1991 & External_Time & 1991 & Same \\
\hline & External dose & & $D_{\text {ext, } C-14}$ & 6.4.7-1 & Sv/yr & $8.82 \mathrm{E}-17$ & Total_External & $8.82 \mathrm{E}-17$ & Same \\
\hline Inhalation & Inhalation dose for & ${ }^{4} \mathrm{C}$ gas & $D_{\text {inh }, g, C-14}$ & 6.4.8-4 & Sv/yr & 4.41E-13 & Total_C14 & $4.41 \mathrm{E}-13$ & Same \\
\hline & Inhalation dose fro & evaporative cooler & $D_{\text {inh }, e, C-14}$ & \begin{tabular}{|l|}
$6.4 .8-3$ \\
\end{tabular} & Sv/yr & 9.20E-15 & Cooler_Inhalation & 9.20E-15 & Same \\
\hline & Inhalation dose & & $D_{\text {inh, } C-14}$ & 6.4.8-1 & Sv/yr & $4.50 \mathrm{E}-13$ & Total_Inhalation & $4.50 \mathrm{E}-13$ & Same \\
\hline
\end{tabular}


Table 6.10-2. Verification of ERMYN in GoldSim for the Groundwater Scenario (Case $2,{ }^{14} \mathrm{C}$ ) (Continued)

\begin{tabular}{|c|c|c|c|c|c|c|c|c|c|}
\hline \multirow[b]{2}{*}{ Submodel } & \multicolumn{6}{|c|}{ Parameter in Mathematical Model } & \multicolumn{2}{|c|}{ Parameter in GoldSim } & \multirow[b]{2}{*}{ Notes $^{c}$} \\
\hline & \multicolumn{2}{|c|}{ Parameter Name } & $\begin{array}{c}\text { Notation in } \\
\text { Equation }\end{array}$ & $\begin{array}{l}\text { Equation } \\
\text { Number }\end{array}$ & Units & $\begin{array}{c}\text { Calculated } \\
\text { Value }^{\text {a }}\end{array}$ & Element Name & $\begin{array}{c}\text { ERMYN_GW } \\
\text { Result }{ }^{b}\end{array}$ & \\
\hline \multirow[t]{12}{*}{ Ingestion } & \multicolumn{2}{|c|}{ Ingestion dose for water } & $D_{\text {ing }, w, C-14}$ & $6.4 .9-2$ & Sv/yr & $4.12 \mathrm{E}-10$ & Water_Ingestion & $4.12 \mathrm{E}-10$ & Same \\
\hline & \multirow{4}{*}{$\begin{array}{l}\text { Ingestion dose for } \\
\text { crops }\end{array}$} & Leafy vegetation & $D_{\text {ing, },, C-14,1}$ & \multirow[t]{4}{*}{ 6.4.9-3 } & Sv/yr & $6.83 \mathrm{E}-11$ & \multirow[t]{4}{*}{ Crop_Ingestion } & $6.83 \mathrm{E}-11$ & Same \\
\hline & & Other vegetation & $D_{\text {ing, } p, C-14,2}$ & & Sv/yr & $1.19 \mathrm{E}-10$ & & 1.19E-10 & Same \\
\hline & & Fruit & $D_{\text {ing, } p, C-14,3}$ & & Sv/yr & $3.13 E-10$ & & $3.13 E-10$ & Same \\
\hline & & Grain & $D_{\text {ing }, p, C-14,4}$ & & Sv/yr & $1.59 \mathrm{E}-11$ & & $1.59 \mathrm{E}-11$ & Same \\
\hline & \multirow{4}{*}{$\begin{array}{l}\text { Ingestion dose for } \\
\text { animal products }\end{array}$} & Meat & $D_{\text {ing }, d, C-14,1}$ & \multirow[t]{4}{*}{$6.4 .9-4$} & Sv/yr & $1.71 \mathrm{E}-10$ & \multirow[t]{4}{*}{ Animal_Ingestion } & $1.71 \mathrm{E}-10$ & Same \\
\hline & & Milk & $D_{\text {ing }, d, C-14,2}$ & & Sv/yr & $8.15 \mathrm{E}-11$ & & $8.15 \mathrm{E}-11$ & Same \\
\hline & & Poultry & $D_{\text {ing }, d, C-14,3}$ & & Sv/yr & $1.46 \mathrm{E}-11$ & & $1.46 \mathrm{E}-11$ & Same \\
\hline & & Eggs & $D_{\text {ing }, d, C-14,4}$ & & Sv/yr & $1.38 \mathrm{E}-10$ & & $1.39 \mathrm{E}-10$ & Rounding \\
\hline & \multicolumn{2}{|c|}{ Ingestion dose for fish } & $D_{\text {ing, } f, C-14}$ & $6.4 .9-5$ & Sv/yr & $5.97 \mathrm{E}-10$ & Fish_Ingestion & 5.97E-10 & Same \\
\hline & \multicolumn{2}{|c|}{ Ingestion dose for soil } & $D_{\text {ing,s,C-14 }}$ & $6.4 .9-6$ & Sv/yr & $2.35 E-15$ & Soil_Ingestion & $2.35 E-15$ & Same \\
\hline & \multicolumn{2}{|c|}{ Ingestion dose } & $D_{\text {ing, C-14 }}$ & $6.4 .9-1$ & Sv/yr & $1.93 \mathrm{E}-09$ & Total_Ingestion & $1.93 \mathrm{E}-09$ & Same \\
\hline \multirow[t]{2}{*}{ All Pathway } & \multicolumn{2}{|l|}{ All-pathway dose } & $D_{\text {all, }, C-14}$ & $6.4 .10-1$ & Sv/yr & 1.93E-09 & Final_TEDE & 1.93E-09 & Same \\
\hline & \multicolumn{2}{|l|}{ BDCF } & $B D C F_{C-14}$ & $6.4 .10-2$ & $\begin{array}{l}(\mathrm{rem} / \mathrm{yr}) / \\
(\mathrm{pCi} / \mathrm{L})\end{array}$ & $7.15 \mathrm{E}-06$ & Final_BDCF & $7.15 \mathrm{E}-06$ & Same \\
\hline
\end{tabular}

a Input data for this verification taken from the "mean, mode, or average" column in Table 6.6-3. Calculations performed in Microsoft Excel 97 SR-2. The electronic file, ERMYN verification.xls, which contains this calculation in worksheet GWC14, is listed in Appendix A.

b The ERMYN is realized in GoldSim using the deterministic mode and data from the "mean, mode, or average" column in Table 6.6-3. The electronic file, ERMYN_GW_C14verf.gsm, for this run is listed in Appendix A.

${ }^{c}$ Comparison of results from Calculated Value (Column 6) and ERMYN_GW Result (Column 8).

BDCF=biosphere dose conversion factor; ERMYN=Environmental Radiation Model for Yucca Mountain, Nevada; GW=groundwater 


\begin{tabular}{|c|c|c|c|c|c|c|c|c|c|}
\hline \multirow[b]{2}{*}{ Submodel } & \multicolumn{6}{|c|}{ Parameter in Mathematical Model } & \multicolumn{2}{|c|}{ Parameter in GoldSim } & \multirow[b]{2}{*}{ Notes $^{c}$} \\
\hline & \multicolumn{2}{|c|}{ Parameter Name } & $\begin{array}{c}\text { Notation in } \\
\text { Equation }\end{array}$ & $\begin{array}{l}\text { Equation } \\
\text { Number }\end{array}$ & Units & $\begin{array}{c}\text { Calculated } \\
\text { Value }^{\mathrm{a}}\end{array}$ & Element Name & $\begin{array}{l}\text { ERMYN_GW } \\
\text { Result }{ }^{\text {R }}\end{array}$ & \\
\hline \multirow[t]{9}{*}{ Soil } & \multicolumn{2}{|c|}{ Leaching removal constant } & $\lambda_{1, R a-226}$ & $6.4 .1-10$ & /yr & 5.85E-06 & Leaching_Factor & 5.85E-06 & Same \\
\hline & \multicolumn{2}{|c|}{ Erosion removal constant } & $\lambda_{e}$ & 6.4.1-11 & /yr & 1.31E-03 & Erosion_Factor & 1.31E-03 & Same \\
\hline & \multicolumn{2}{|c|}{ Effective removal constant } & $\lambda_{\text {eff,Ra-226 }}$ & $6.4 .1-3$ & /yr & \begin{tabular}{|l|}
$1.75 E-03$ \\
\end{tabular} & Effective_Removal & 1.75E-03 & Same \\
\hline & \multicolumn{2}{|c|}{ Concentration in surface soil } & $C S_{R a-226}$ & 6.4.1-4 & $\mathrm{Bq} / \mathrm{m}^{2}$ & 538 & Saturated_Conc & 538 & Same \\
\hline & \multicolumn{2}{|c|}{ Concentration in soil mass } & $C S_{m, R a-226}$ & $6.4 .1-5$ & $\mathrm{~Bq} / \mathrm{kg}$ & \begin{tabular}{|l|}
1.44 \\
\end{tabular} & SatMass_Conc & 1.44 & Same \\
\hline & \multicolumn{2}{|c|}{ Leaching removal constant of $\mathrm{Pb}-210$} & $\lambda_{1, P b-210}$ & 6.4.1-10 & $/ \mathrm{yr}$ & $1.32 \mathrm{E}-05$ & Leaching_Factor_1 & $1.32 \mathrm{E}-05$ & Same \\
\hline & \multicolumn{2}{|c|}{ Effective removal constant of $\mathrm{Pb}-210$} & $\lambda_{\text {eff,Pb-210 }}$ & $6.4 .1-3$ & /yr & $3.24 \mathrm{E}-02$ & $\begin{array}{l}\text { Effective_Removal_ } \\
1\end{array}$ & $3.24 \mathrm{E}-02$ & Same \\
\hline & \multicolumn{2}{|c|}{ Concentration in surface soil of $\mathrm{Pb}-210$} & $\mathrm{CS}_{P b-210}$ & 6.4.1-9 & $\mathrm{Bq} / \mathrm{m}^{2}$ & $5.17 \mathrm{E}+02$ & Saturated_Conc_1 & $5.17 \mathrm{E}+02$ & Same \\
\hline & \multicolumn{2}{|c|}{ Concentration in soil mass of $\mathrm{Pb}-210$} & $C s_{m, P b-210}$ & 6.4.1-5 & $\mathrm{Bq} / \mathrm{kg}$ & 1.38 & SatMass_Conc_1 & 1.38 & Same \\
\hline \multirow[t]{16}{*}{ Air } & \multicolumn{2}{|c|}{ Concentration in air for crop } & $C a_{p, R a-226}$ & $6.4 .2-1$ & $\mathrm{~Bq} / \mathrm{m}^{3}$ & $1.72 \mathrm{E}-07$ & AirConc_Crop & $1.72 \mathrm{E}-07$ & Same \\
\hline & \multirow{5}{*}{$\begin{array}{l}\text { Concentration in } \\
\text { air for inhalation }\end{array}$} & Active outdoors & $C a_{h, R a-226,1}$ & \multirow[t]{5}{*}{$6.4 .2-2$} & \multirow[t]{5}{*}{$\mathrm{Bq} / \mathrm{m}^{3}$} & 2.87E-05 & \multirow[t]{5}{*}{ AirConc_Inh } & 2.87E-05 & Same \\
\hline & & Inactive outdoors & $C a_{h, R a-226,2}$ & & & $6.03 E-08$ & & $6.03 E-08$ & Same \\
\hline & & Active indoors & $C a_{h, R a-226,3}$ & & & $1.01 \mathrm{E}-07$ & & 1.01E-07 & Same \\
\hline & & Asleep indoors & $C a_{h, R a-226,4}$ & & & 3.02E-08 & & 3.02E-08 & Same \\
\hline & & Away from area & $C a_{h, R a-226,5}$ & & & $0.00 \mathrm{E}+00$ & & $0.00 \mathrm{E}+00$ & Same \\
\hline & \multicolumn{2}{|c|}{$\begin{array}{l}\text { Concentration in air from evaporative } \\
\text { cooler }\end{array}$} & $C a_{e, R a-226}$ & $6.4 .2-3$ & $\mathrm{~Bq} / \mathrm{m}^{3}$ & $1.02 \mathrm{E}-06$ & AirConc_Evap & $1.02 \mathrm{E}-06$ & Same \\
\hline & \multicolumn{2}{|c|}{ Radon concentration in outdoor air } & $C a_{g, R n-222}$ & $6.4 .2-4$ & $\mathrm{~Bq} / \mathrm{m}^{3}$ & 0.359 & AirConc_Radon & 0.359 & Same \\
\hline & \multicolumn{2}{|c|}{ Radon indoor factor for normal condition } & $I F_{n, R n-222}$ & 6.4.2-7 & - & 1.913 & Indoor_RnNormal & 1.913 & Same \\
\hline & \multicolumn{2}{|c|}{$\begin{array}{l}\text { Radon indoor factor for evaporative } \\
\text { condition }\end{array}$} & $I F_{e, R n-222}$ & $6.4 .2-7$ & - & 1.059 & Indoor_RnEvap & 1.059 & Same \\
\hline & \multicolumn{2}{|c|}{ Concentration in air of $\mathrm{Pb}-210$ for crop } & $C a_{p, P b-210}$ & $6.4 .2-1$ & $\mathrm{~Bq} / \mathrm{m}^{3}$ & $1.65 \mathrm{E}-07$ & AirConc_Crop_1 & $1.65 \mathrm{E}-07$ & Same \\
\hline & \multirow{5}{*}{$\begin{array}{l}\text { Concentration in } \\
\text { air for inhalation of } \\
\mathrm{Pb}-210\end{array}$} & Active outdoors & $C a_{h, P b-210,1}$ & \multirow[t]{5}{*}{$6.4 .2-2$} & $\mathrm{~Bq} / \mathrm{m}^{3}$ & 2.75E-05 & AirConc_Inh_1 & 2.75E-05 & Same \\
\hline & & Inactive outdoors & $C a_{h, P b-210,2}$ & & & 5.79E-08 & & $5.79 \mathrm{E}-08$ & Same \\
\hline & & Active indoors & $C a_{h, P b-210,3}$ & & & 9.64E-08 & & 9.64E-08 & Same \\
\hline & & \begin{tabular}{|l|} 
Asleep indoors \\
\end{tabular} & $C a_{h, P b-210,4}$ & & & $2.89 \mathrm{E}-08$ & & $2.89 \mathrm{E}-08$ & Same \\
\hline & & Away from area & $C a_{h, P b-210,5}$ & & & $0.00 \mathrm{E}+00$ & & $0.00 \mathrm{E}+00$ & Same \\
\hline Plant & Concentration due & Leafy vegetation & $C p_{\text {root,Ra-2261 }}$ & 6.4.3-2 & $\mathrm{Bq} / \mathrm{kg}$ & $6.83 \mathrm{E}-03$ & Root_Uptake & $6.83 \mathrm{E}-03$ & Same \\
\hline & to root uptake & Other vegetation & Cp root,Ra-226,2 & & & 1.77E-03 & & 1.77E-03 & Same \\
\hline & & Fruit & $C p_{\text {root }, R a-226,3}$ & & & 1.26E-03 & & 1.26E-03 & Same \\
\hline & & Grain & $C p_{\text {root,Ra-226,4 }}$ & & & 4.02E-03 & & 4.02E-03 & Same \\
\hline & & Forage & $C p_{\text {root }, R a-226,5}$ & & & $2.59 \mathrm{E}-02$ & & $2.59 \mathrm{E}-02$ & Same \\
\hline
\end{tabular}


Table 6.10-3. Verification of ERMYN in GoldSim for the Groundwater Scenario (Case 3, ${ }^{226} \mathrm{Ra}$ ) (Continued)

\begin{tabular}{|c|c|c|c|c|c|c|c|c|c|}
\hline \multirow[b]{2}{*}{ Submodel } & \multicolumn{6}{|c|}{ Parameter in Mathematical Model } & \multicolumn{2}{|c|}{ Parameter in GoldSim } & \multirow[b]{2}{*}{ Notes $^{c}$} \\
\hline & \multicolumn{2}{|c|}{ Parameter Name } & $\begin{array}{c}\text { Notation in } \\
\text { Equation }\end{array}$ & $\begin{array}{c}\text { Equation } \\
\text { Number }\end{array}$ & Units & $\begin{array}{c}\text { Calculated } \\
\text { Value }^{\text {a }}\end{array}$ & Element Name & $\begin{array}{c}\text { ERMYN_GW } \\
\text { Result }^{b}\end{array}$ & \\
\hline \multirow{35}{*}{$\begin{array}{l}\text { Plant } \\
\text { (cont.) }\end{array}$} & \multirow{5}{*}{$\begin{array}{l}\text { Irrigation } \\
\text { interception } \\
\text { fraction }\end{array}$} & Leafy vegetation & $R W_{1}$ & \multirow[t]{5}{*}{$6.4 .3-5$} & \multirow[t]{5}{*}{-} & 0.217 & \multirow[t]{5}{*}{ Intercept_Fractor } & 0.217 & Same \\
\hline & & Other vegetation & $R W_{2}$ & & & 0.304 & & 0.304 & Same \\
\hline & & Fruit & $R W_{3}$ & & & 0.361 & & 0.361 & Same \\
\hline & & Grain & $R W_{4}$ & & & 0.472 & & 0.472 & Same \\
\hline & & Forage & $R W_{5}$ & & & 0.259 & & 0.259 & Same \\
\hline & \multirow{5}{*}{$\begin{array}{l}\text { Concentration due } \\
\text { to water } \\
\text { interception }\end{array}$} & Leafy vegetation & $C p_{\text {water,Ra-226,1 }}$ & \multirow[t]{5}{*}{$6.4 .3-3$} & \multirow[t]{5}{*}{$\mathrm{Bq} / \mathrm{kg}$} & $5.24 \mathrm{E}-03$ & \multirow[t]{5}{*}{ Water_Uptake } & $5.24 \mathrm{E}-03$ & Same \\
\hline & & Other vegetation & $C p_{\text {water,Ra-226,2 }}$ & & & 8.27E-04 & & 8.27E-04 & Same \\
\hline & & Fruit & $C p_{\text {water,Ra-226,3 }}$ & & & 9.78E-04 & & $9.78 \mathrm{E}-04$ & Same \\
\hline & & Grain & $C p_{\text {water,Ra-226,4 }}$ & & & $6.74 \mathrm{E}-03$ & & $6.74 \mathrm{E}-03$ & Same \\
\hline & & Forage & $C p_{\text {water,Ra-226,5 }}$ & & & $1.40 \mathrm{E}-02$ & & $1.40 \mathrm{E}-02$ & Same \\
\hline & \multirow{5}{*}{$\begin{array}{l}\text { Dust interception } \\
\text { fraction }\end{array}$} & Leafy vegetation & $R a_{1}$ & \multirow[t]{5}{*}{ 6.4.3-8 } & \multirow[t]{5}{*}{-} & 0.456 & \multirow[t]{5}{*}{ Dust_Intercept } & 0.456 & Same \\
\hline & & Other vegetation & $\mathrm{Ra}_{2}$ & & & 0.787 & & 0.787 & Same \\
\hline & & \begin{tabular}{|l|} 
Fruit \\
\end{tabular} & $R a_{3}$ & & & 0.893 & & 0.893 & Same \\
\hline & & Grain & $\mathrm{Ra}_{4}$ & & & 0.962 & & 0.962 & Same \\
\hline & & Forage & $R a_{5}$ & & & 0.751 & & 0.751 & Same \\
\hline & \multirow{5}{*}{$\begin{array}{l}\text { Concentration due } \\
\text { to dust interception }\end{array}$} & Leafy vegetation & $C p_{\text {dust }, R a-226,1}$ & \multirow[t]{5}{*}{ 6.4.3-6 } & \multirow[t]{5}{*}{$\mathrm{Bq} / \mathrm{kg}$} & $3.24 \mathrm{E}-04$ & \multirow[t]{5}{*}{ Dust_Uptake } & $3.24 \mathrm{E}-04$ & Same \\
\hline & & Other vegetation & $C p_{\text {dust }, R a-226,2}$ & & & 4.50E-05 & & 4.50E-05 & Same \\
\hline & & \begin{tabular}{|l|} 
Fruit \\
\end{tabular} & $C p_{\text {dust,Ra-226,3 }}$ & & & 7.81E-05 & & 7.81E-05 & Same \\
\hline & & Grain & $C p_{\text {dust,Ra-226,4 }}$ & & & 3.92E-04 & & 3.92E-04 & Same \\
\hline & & Forage & $C p_{\text {dust,Ra-226,5 }}$ & & & 8.24E-04 & & 8.24E-04 & Same \\
\hline & \multirow{5}{*}{$\begin{array}{l}\text { Concentration in } \\
\text { crops }\end{array}$} & Leafy vegetation & $C p_{R a-226,1}$ & \multirow[t]{5}{*}{ 6.4.3-1 } & \multirow[t]{5}{*}{$\mathrm{Bq} / \mathrm{kg}$} & $1.24 \mathrm{E}-02$ & \multirow[t]{5}{*}{ Plant_Conc } & 1.24E-02 & Same \\
\hline & & Other vegetation & $C p_{R a-226,2}$ & & & $2.65 \mathrm{E}-03$ & & $2.65 \mathrm{E}-03$ & Same \\
\hline & & Fruit & $C p_{R a-226,3}$ & & & 2.31E-03 & & 2.31E-03 & Same \\
\hline & & Grain & $C p_{R a-226,4}$ & & & $1.12 \mathrm{E}-02$ & & $1.12 \mathrm{E}-02$ & Same \\
\hline & & Forage & $C p_{R a-226,5}$ & & & 4.08E-02 & & 4.08E-02 & Same \\
\hline & Concentration of & Leafy vegetation & $C p_{\text {root }, P b-210,1}$ & 6.4.3-2 & $\mathrm{Bq} / \mathrm{kg}$ & 1.45E-03 & Root_Uptake_1 & $1.45 \mathrm{E}-03$ & Same \\
\hline & $\mathrm{Pb}-210$ due to root & Other vegetation & $C p_{\text {root }, P b-210,2}$ & & & $1.28 \mathrm{E}-03$ & & $1.28 \mathrm{E}-03$ & Same \\
\hline & uptake & Fruit & $C p_{\text {root }, P b-210,3}$ & & & $1.98 \mathrm{E}-03$ & & $1.98 \mathrm{E}-03$ & Same \\
\hline & & Grain & $C p_{\text {root }, P b-210,4}$ & & & $6.84 \mathrm{E}-03$ & & 6.84E-03 & Same \\
\hline & & Forage & $C p_{\text {root }, P b-210,5}$ & & & $5.45 \mathrm{E}-03$ & & $5.45 \mathrm{E}-03$ & Same \\
\hline & Concentration of & Leafy vegetation & $C p_{\text {dust }, P b-210,1}$ & 6.4.3-6 & $\mathrm{Bq} / \mathrm{kg}$ & 3.11E-04 & Dust_Uptake_1 & 3.11E-04 & Same \\
\hline & $\mathrm{Pb}-210$ due to dust & Other vegetation & $C p_{\text {dust }, P b-210,2}$ & & & 4.32E-05 & & 4.32E-05 & Same \\
\hline & interception & \begin{tabular}{|l|} 
Fruit \\
\end{tabular} & $C p_{\text {dust }, P b-210,3}$ & & & 7.49E-05 & & 7.49E-05 & Same \\
\hline & & Grain & $C p_{\text {dust }, P b-210,4}$ & & & 3.76E-04 & & 3.76E-04 & Same \\
\hline & & Forage & $C p_{\text {dust }, P b-210,5}$ & & & 7.90E-04 & & 7.90E-04 & Same \\
\hline
\end{tabular}




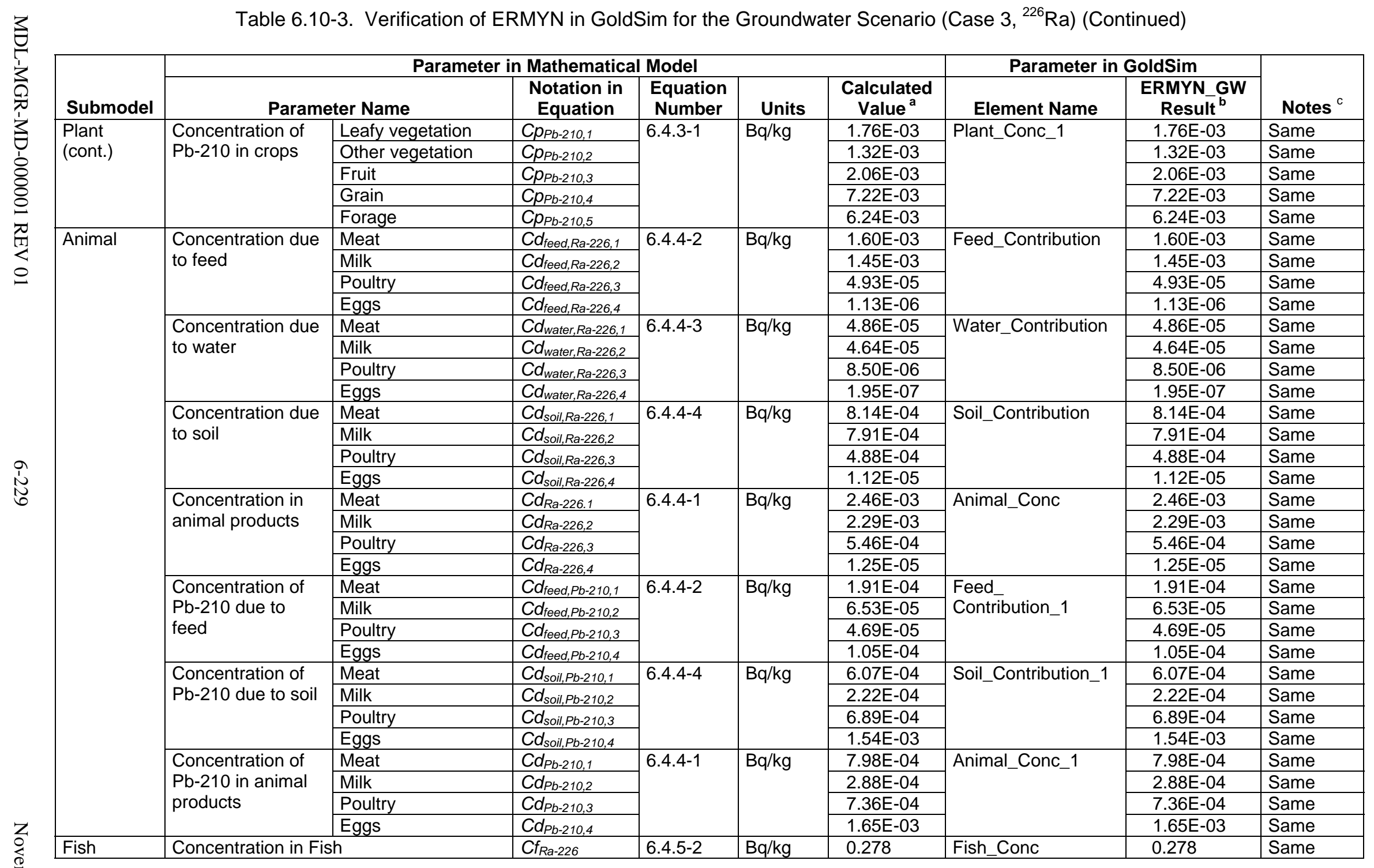


Table 6.10-3. Verification of ERMYN in GoldSim for the Groundwater Scenario (Case $3,{ }^{226} \mathrm{Ra}$ ) (Continued)

\begin{tabular}{|c|c|c|c|c|c|c|c|c|c|}
\hline \multirow[b]{2}{*}{ Submodel } & \multicolumn{6}{|c|}{ Parameter in Mathematical Model } & \multicolumn{2}{|c|}{ Parameter in GoldSim } & \multirow[b]{2}{*}{ Notes $^{\mathrm{c}}$} \\
\hline & \multicolumn{2}{|c|}{ Parameter Name } & $\begin{array}{c}\text { Notation in } \\
\text { Equation }\end{array}$ & $\begin{array}{c}\text { Equation } \\
\text { Number }\end{array}$ & Units & $\begin{array}{c}\text { Calculated } \\
\text { Value }^{\mathrm{a}}\end{array}$ & Element Name & $\begin{array}{c}\text { ERMYN_GW } \\
\text { Result }\end{array}$ & \\
\hline \multirow[t]{10}{*}{ External } & \multirow{5}{*}{$\begin{array}{l}\text { Environment- } \\
\text { specific exposure } \\
\text { time }\end{array}$} & Active outdoors & $\Sigma\left(P P_{m} t_{1, m}\right)$ & \multirow[t]{5}{*}{$6.4 .7-1$} & \multirow[t]{5}{*}{$\mathrm{hr} / \mathrm{d}$} & 0.45 & \multirow[t]{5}{*}{ Weighted_Time } & 0.45 & Same \\
\hline & & Inactive outdoors & $\sum\left(P P_{m} t_{2, m}\right)$ & & & 1.45 & & 1.45 & Same \\
\hline & & Active indoors & $\Sigma\left(P P_{m} t_{3, m}\right)$ & & & 9.45 & & 9.45 & Same \\
\hline & & Asleep indoors & $\sum\left(P P_{m} t_{4, m}\right)$ & & & 8.30 & & 8.30 & Same \\
\hline & & Away from area & $\sum\left(P P_{m} t_{5, m}\right)$ & & & 4.35 & & 4.35 & Same \\
\hline & \multicolumn{2}{|c|}{ Effective exposure time } & $\begin{array}{l}\sum\left[f_{\text {ext,Ra-226,n }}\right. \\
\left.\sum\left(P P_{m} t_{n, m}\right)\right]\end{array}$ & $6.4 .7-1$ & $\mathrm{hr} / \mathrm{yr}$ & 3288 & External_Time & 3288 & Same \\
\hline & \multicolumn{2}{|c|}{ External dose from Ra-226D } & - & 6.4.7-1 & Sv/yr & \begin{tabular}{|l|}
$1.53 E-06$ \\
\end{tabular} & External_Dose & 1.53E-06 & Same \\
\hline & \multicolumn{2}{|c|}{ Effective exposure time } & $\begin{array}{l}\sum\left[f_{e x t, P b-210, n}\right. \\
\left.\sum\left(P P_{m} t_{n, m}\right)\right]\end{array}$ & $6.4 .7-1$ & $\mathrm{hr} / \mathrm{yr}$ & 3288 & External_Time_1 & 3288 & Same \\
\hline & \multicolumn{2}{|c|}{ External dose from $\mathrm{Pb}-210 \mathrm{D}$} & - & 6.4.7-1 & Sv/yr & 7.99E-10 & External_Dose_1 & $7.99 \mathrm{E}-10$ & Same \\
\hline & \multicolumn{2}{|c|}{ Total external dose } & $D_{\text {ext,Ra-226 }}$ & 6.4.7-1 & Sv/yr & 1.53E-06 & Total_External & 1.53E-06 & Same \\
\hline \multirow[t]{5}{*}{ Inhalation } & \multicolumn{2}{|c|}{ Inhalation dose for particles } & $D_{\text {inh, }, \text { Ra-226 }}$ & $6.4 .8-2$ & Sv/yr & 1.84E-08 & Inhalation_Dose & 1.84E-08 & Same \\
\hline & \multicolumn{2}{|c|}{ Inhalation dose from evaporative cooler } & $D_{\text {inh,e, Ra-226 }}$ & $6.4 .8-3$ & Sv/yr & 3.36E-09 & Cooler_Inhalation & 3.36E-09 & Same \\
\hline & \multicolumn{2}{|c|}{ Inhalation dose from radon } & $D_{\text {inh, }, R n-222}$ & 6.4.8-7 & Sv/yr & 1.80E-05 & Total_Radon & $1.80 \mathrm{E}-05$ & Same \\
\hline & \multicolumn{2}{|c|}{$\begin{array}{l}\text { Inhalation dose for particles from } \mathrm{Pb}- \\
210 \mathrm{D}\end{array}$} & - & $6.4 .8-2$ & Sv/yr & 4.76E-08 & Inhalation_Dose_1 & 4.76E-08 & Same \\
\hline & \multicolumn{2}{|c|}{ Total Inhalation dose } & $D_{\text {inh, Ra-226 }}$ & 6.4.8-1 & Sv/yr & 1.81E-05 & Total_Inhalation & 1.81E-05 & Same \\
\hline \multirow[t]{16}{*}{ Ingestion } & \multicolumn{2}{|c|}{ Ingestion dose for water } & $D_{\text {ing }, w, R a-226}$ & $6.4 .9-2$ & Sv/yr & $2.62 \mathrm{E}-07$ & Water_Ingestion & $2.62 \mathrm{E}-07$ & Same \\
\hline & \multirow{4}{*}{$\begin{array}{l}\text { Ingestion dose for } \\
\text { crops }\end{array}$} & Leafy vegetation & $D_{\text {ing, } p, R a-226,1}$ & 6.4.9-3 & Sv/yr & 1.68E-08 & Crop_Ingestion & 1.68E-08 & Same \\
\hline & & Other vegetation & $D_{\text {ing, } p, R a-226,2}$ & & & 4.48E-09 & & 4.48E-09 & Same \\
\hline & & Fruit & $D_{\text {ing, } p, R a-226,3}$ & & & 1.05E-08 & & 1.05E-08 & Same \\
\hline & & Grain & $D_{\text {ing, } p, R a-226,4}$ & & & 9.19E-10 & & 9.19E-10 & Same \\
\hline & \multirow{4}{*}{$\begin{array}{l}\text { Ingestion dose for } \\
\text { animal products }\end{array}$} & Meat & $D_{\text {ing, }, \text { Ra-226,1 }}$ & \multirow[t]{4}{*}{$6.4 .9-4$} & \multirow[t]{4}{*}{ Sv/yr } & $2.52 \mathrm{E}-09$ & \multirow[t]{4}{*}{ Animal_Ingestion } & $2.52 \mathrm{E}-09$ & Same \\
\hline & & Milk & $D_{\text {ing, }, \text {,Ra-226,2 }}$ & & & 3.83E-09 & & 3.83E-09 & Same \\
\hline & & Poultry & $D_{\text {ing, } d, R a-226,3}$ & & & $8.22 \mathrm{E}-11$ & & $8.22 \mathrm{E}-11$ & Same \\
\hline & & Eggs & $D_{\text {ing, }, \text { Ra-226,4 }}$ & & & $2.38 \mathrm{E}-11$ & & $2.38 \mathrm{E}-11$ & Same \\
\hline & Ingestion dose for & & $D_{\text {ing }, f, R a-226}$ & $6.4 .9-5$ & Sv/yr & $2.29 \mathrm{E}-08$ & Fish_Ingestion & 2.29E-08 & Same \\
\hline & Ingestion dose for & & $D_{\text {ing }, \mathrm{s}, \mathrm{Ra}-226}$ & 6.4.9-6 & Sv/yr & 1.88E-08 & Soil_Ingestion & 1.88E-08 & Same \\
\hline & Ingestion dose fron & Ra-226 & - & 6.4.9-1 & Sv/yr & $3.43 E-07$ & Ingestion_Dose & $3.43 E-07$ & Same \\
\hline & Ingestion dose for & Leafy vegetation & $D_{\text {ing }, p, P b-210,1}$ & $6.4 .9-3$ & Sv/yr & 1.31E-08 & Crop_Ingestion_1 & $1.31 \mathrm{E}-08$ & Same \\
\hline & crops from $\mathrm{Pb}-210$ & Other vegetation & $D_{\text {ing, } p, P b-210,2}$ & & & 1.23E-08 & & 1.23E-08 & Same \\
\hline & & Fruit & $D_{\text {ing, } p, P b-210,3}$ & & & $5.13 \mathrm{E}-08$ & & $5.13 \mathrm{E}-08$ & Same \\
\hline & & Grain & $D_{\text {ing, } p, P b-210,4}$ & & & 3.26E-09 & & 3.26E-09 & Same \\
\hline
\end{tabular}


Table 6.10-3. Verification of ERMYN in GoldSim for the Groundwater Scenario (Case $3,{ }^{226} \mathrm{Ra}$ ) (Continued)

\begin{tabular}{|c|c|c|c|c|c|c|c|c|c|}
\hline \multirow[b]{2}{*}{ Submodel } & \multicolumn{6}{|c|}{ Parameter in Mathematical Model } & \multicolumn{2}{|c|}{ Parameter in GoldSim } & \multirow[b]{2}{*}{ Notes } \\
\hline & \multicolumn{2}{|c|}{ Parameter Name } & $\begin{array}{c}\text { Notation in } \\
\text { Equation }\end{array}$ & $\begin{array}{c}\text { Equation } \\
\text { Number }\end{array}$ & Units & $\begin{array}{c}\text { Calculated } \\
\text { Value }^{\mathrm{a}}\end{array}$ & Element Name & $\begin{array}{c}\text { ERMYN_GW } \\
\text { Result }^{b}\end{array}$ & \\
\hline \multirow{7}{*}{$\begin{array}{l}\text { Ingestion } \\
\text { (cont.) }\end{array}$} & \multirow{4}{*}{$\begin{array}{l}\text { Ingestion dose for } \\
\text { animal products } \\
\text { from } \mathrm{Pb}-210\end{array}$} & Meat & $D_{\text {ing, } d, P b-210,1}$ & \multirow[t]{4}{*}{$6.4 .9-4$} & \multirow[t]{4}{*}{ Sv/yr } & 4.47E-09 & \multirow[t]{4}{*}{ Animal_Ingestion_1 } & 4.47E-09 & Same \\
\hline & & Milk & $D_{\text {ing }, d, P b-210,2}$ & & & 2.64E-09 & & 2.64E-09 & Same \\
\hline & & Poultry & $D_{\text {ing, }, \mathrm{d}, \mathrm{Pb}-210,3}$ & & & $6.07 \mathrm{E}-10$ & & $6.07 \mathrm{E}-10$ & Same \\
\hline & & Eggs & $D_{\text {ing, } d, P b-210,4}$ & & & 1.72E-08 & & $1.72 \mathrm{E}-08$ & Same \\
\hline & \multicolumn{2}{|c|}{ Ingestion dose for soil from $\mathrm{Pb}-210$} & $D_{\text {ing }, s, P b-210}$ & 6.4.9-6 & Sv/yr & 9.89E-08 & Soil_Ingestion 1 & 9.89E-08 & Same \\
\hline & \multicolumn{2}{|c|}{ Ingestion dose from $\mathrm{Pb}-210$} & - & 6.4.9-1 & Sv/yr & $2.04 \mathrm{E}-07$ & Ingestion_Dose_1 & $2.04 \mathrm{E}-07$ & Same \\
\hline & \multicolumn{2}{|c|}{ Total ingestion dose } & $D_{\text {ing,Ra-226 }}$ & 6.4.9-1 & Sv/yr & $5.46 \mathrm{E}-07$ & Total_Ingestion & $5.46 \mathrm{E}-07$ & Same \\
\hline \multirow[t]{2}{*}{ All Pathway } & \multicolumn{2}{|l|}{ All-pathway dose } & $D_{\text {all,Ra-226 }}$ & \begin{tabular}{|l|}
$6.4 .10-1$ \\
\end{tabular} & Sv/yr & 2.02E-05 & Final_TEDE & 2.02E-05 & Same \\
\hline & \multicolumn{2}{|l|}{$\mathrm{BDCF}$} & $B D C F_{R a-226}$ & $6.4 .10-2$ & $\begin{array}{l}(\mathrm{rem} / \mathrm{yr}) / \\
(\mathrm{pCi} / \mathrm{L})\end{array}$ & 7.46E-02 & Final_BDCF & 7.46E-02 & Same \\
\hline
\end{tabular}

a Input data for this verification taken from the "mean, mode, or average" column in Table 6.6-3. Calculations performed in Microsoft Excel 97 SR-2. The electronic file, ERMYN verification.xIs, which contains this calculation in worksheet GWRa226, is listed in Appendix A.

b The ERMYN is realized in GoldSim using the deterministic mode and data from the "mean, mode, or average" column in Table 6.6-3. The electronic file, ERMYN_GW_Ra226verf.gsm, for this run is listed in Appendix A.

c Comparison of results from Calculated Value (Column 6) and ERMYN_GW Result (Column 8).

o BDCF=biosphere dose conversion factor; ERMYN=Environmental Radiation Model for Yucca Mountain, Nevada; GW=groundwater 


\subsubsection{Verification of Stochastic Calculations}

To verify the stochastic calculations implemented in the ERMYN, results from deterministic and stochastic runs are compared for ${ }^{239} \mathrm{Pu}$ (Table 6.10-4). Differences between the deterministic and stochastic runs are within 30 percent which is relatively small when compared to the standard deviation of the stochastic runs (i.e., about one standard deviation or less). This is because the deterministic inputs ("Mean, Mode, or Average" column in Table 6.6-3) were selected as representative of the parameter distribution used in the stochastic calculations. Because they propagate the expected parametric uncertainties to the results, the stochastic results are considered more realistic than the deterministic ones.

Table 6.10-4. Results from Deterministic and Stochastic Runs for the Groundwater Scenario

\begin{tabular}{|l|c|c|}
\hline \multicolumn{1}{|c|}{ Parameter } & Deterministic Results & $\begin{array}{c}\text { Stochastic Results } \\
\text { (mean and standard deviation) }\end{array}$ \\
\hline External exposure dose (Sv/yr) & $3.74 \mathrm{E}-11$ & $(4.13 \pm 2.02) \mathrm{E}-11$ \\
\hline Inhalation dose (Sv/yr) & $1.23 \mathrm{E}-06$ & $(1.52 \pm 0.91) \mathrm{E}-06$ \\
\hline Ingestion dose (Sv/yr) & $8.34 \mathrm{E}-07$ & $(9.57 \pm 2.29) \mathrm{E}-07$ \\
\hline BDCF (rem/yr)/(pCi/L) & $7.63 \mathrm{E}-03$ & $(9.16 \pm 3.59) \mathrm{E}-03$ \\
\hline
\end{tabular}

NOTE: Deterministic calculations done using ERMYN_GW_Pu239verf.gsm; stochastic calculations done using ERMYN_GW_Pu239stoc.gsm; both listed in Appendix A.

\subsubsection{Verification of the Model Implementation for the Volcanic Ash Scenario}

\subsubsection{Verification of Deterministic Calculations}

Similar to the groundwater scenario, verification of the implementation of the model for the volcanic ash scenario in GoldSim is performed using reasonable estimates of input values (Table 6.6-3). Two radionuclides, ${ }^{239} \mathrm{Pu}$ and ${ }^{226} \mathrm{Ra}$, are tested for the present-day climate. For the base case, ${ }^{239} \mathrm{Pu}$ is selected, and for the radon dose calculations, ${ }^{226} \mathrm{Ra}$ is selected. The GoldSim files described in this section are listed in Appendix A.

The verification results for ${ }^{239} \mathrm{Pu}$ are presented in Table 6.10-5, in which the comparison is presented in the order of each submodel discussed in Section 6.5. The hand-calculated results from the important equations in the biosphere model are presented in the table for comparison with the GoldSim results.

Verification results for ${ }^{226} \mathrm{Ra}$ are presented in Table 6.10-6. The only difference between ${ }^{226} \mathrm{Ra}$ and ${ }^{239} \mathrm{Pu}$ is the radon inhalation calculation for ${ }^{226} \mathrm{Ra}$. The long-lived decay product, ${ }^{210} \mathrm{~Pb}$, considered in the groundwater scenario because it accumulates in irrigated soils, is not included in the ${ }^{226} \mathrm{Ra}$ BDCF for the volcanic ash scenario because it is considered to be a primary radionuclide in the volcanic ash. The results from both methods are the same, indicating that the ERMYN (volcanic ash scenario; Section 6.5) implementation is correct. 


\begin{tabular}{|c|c|c|c|c|c|c|c|c|c|}
\hline \multirow[b]{2}{*}{ Submodel } & \multicolumn{6}{|c|}{ Parameter in Mathematical Model } & \multicolumn{2}{|c|}{ Parameter in GoldSim } & \multirow[b]{2}{*}{ Notes $^{c}$} \\
\hline & \multicolumn{2}{|c|}{ Parameter Name } & $\begin{array}{c}\text { Notation in } \\
\text { Equation }\end{array}$ & $\begin{array}{l}\text { Equation } \\
\text { Number }\end{array}$ & Units & $\begin{array}{c}\text { Calculated } \\
\text { Value }^{\mathrm{a}}\end{array}$ & Element Name & $\begin{array}{c}\text { ERMYN_VA } \\
\text { Result }{ }^{\text {b }}\end{array}$ & \\
\hline \multirow[t]{2}{*}{ Soil } & \multicolumn{2}{|c|}{ Concentration in agricultural land } & $C s_{m, P u-239}$ & $6.5 .1-2$ & $\mathrm{~Bq} / \mathrm{kg}$ & 2.67E-03 & SoilMass_Conc & 2.67E-03 & Same \\
\hline & \multicolumn{2}{|c|}{ Concentration in nonagricultural land } & $C s_{m c, P u-239}$ & \begin{tabular}{|l|}
$6.5 .1-3$ \\
\end{tabular} & $\mathrm{~Bq} / \mathrm{kg}$ & 5.00E-01 & AshMass_Conc & 5.00E-01 & Same \\
\hline \multirow[t]{11}{*}{ Air } & \multicolumn{2}{|c|}{ Concentration in air for crop } & $C a_{p, P u-239}$ & $6.5 .2-1$ & $\mathrm{~Bq} / \mathrm{m}^{3}$ & $6.40 \mathrm{E}-10$ & AirConc_Crop & $6.40 \mathrm{E}-10$ & Same \\
\hline & \multirow{5}{*}{$\begin{array}{l}\text { Concentration in } \\
\text { air at nominal } \\
\text { condition }\end{array}$} & Active outdoors & $C a_{h, P u-239,1}$ & \multirow[t]{5}{*}{$6.5 .2-4$} & \multirow[t]{5}{*}{$\mathrm{Bq} / \mathrm{m}^{3}$} & 1.10E-05 & \multirow[t]{5}{*}{ AirConc_Long } & 1.10E-05 & Same \\
\hline & & Inactive outdoors & $C a_{h, P u-239,2}$ & & & $2.10 \mathrm{E}-08$ & & $2.10 \mathrm{E}-08$ & Same \\
\hline & & Active indoors & $C a_{h, P u-239,3}$ & & & $3.50 \mathrm{E}-08$ & & $3.50 \mathrm{E}-08$ & Same \\
\hline & & Asleep indoors & $C a_{h, P u-239,4}$ & & & 1.05E-08 & & $1.05 \mathrm{E}-08$ & Same \\
\hline & & Away from area & $C a_{h, P u-239,5}$ & & & $0.00 \mathrm{E}+00$ & & $0.00 \mathrm{E}+00$ & Same \\
\hline & \multirow{5}{*}{$\begin{array}{l}\text { Concentration in } \\
\text { air at post volcanic } \\
\text { condition }\end{array}$} & Active outdoors & $C a_{V, P u-239,1}$ & \multirow[t]{5}{*}{$6.5 .2-4$} & \multirow[t]{5}{*}{$\mathrm{Bq} / \mathrm{m}^{3}$} & 5.50E-06 & \multirow[t]{5}{*}{ AirConc_Short } & 5.50E-06 & Same \\
\hline & & Inactive outdoors & $C a_{v, P u-239,2}$ & & & 2.10E-08 & & $2.10 \mathrm{E}-08$ & Same \\
\hline & & Active indoors & $C a_{V, P u-239,3}$ & & & 3.50E-08 & & 3.50E-08 & Same \\
\hline & & Asleep indoors & $C a_{V, P u-239,4}$ & & & $1.05 \mathrm{E}-08$ & & $1.05 \mathrm{E}-08$ & Same \\
\hline & & Away from area & $C a_{V, P u-239,5}$ & & & $0.00 \mathrm{E}+00$ & & $0.00 \mathrm{E}+00$ & Same \\
\hline \multirow[t]{20}{*}{ Plant } & \multirow{5}{*}{$\begin{array}{l}\text { Concentration due } \\
\text { to root uptake }\end{array}$} & Leafy vegetation & $C p_{\text {root }, \text { Pu-239, } 1}$ & \multirow[t]{5}{*}{$6.5 .3-2$} & \multirow[t]{5}{*}{$\mathrm{Bq} / \mathrm{kg}$} & 5.41E-08 & \multirow[t]{5}{*}{ Root_Uptake } & 5.41E-08 & Same \\
\hline & & Other vegetation & Cp $p_{\text {root }, \text { Pu-239, } 2}$ & & & $5.22 \mathrm{E}-08$ & & $5.22 \mathrm{E}-08$ & Same \\
\hline & & Fruit & $C p_{\text {root,Pu-239,3 }}$ & & & 5.76E-08 & & 5.76E-08 & Same \\
\hline & & Grain & Cp $p_{\text {root }, \text { Pu-239, } 4}$ & & & 4.58E-08 & & 4.58E-08 & Same \\
\hline & & Forage & $C p_{\text {root }, \text { Pu-239, } 5}$ & & & 5.87E-07 & & 5.87E-07 & Same \\
\hline & \multirow{5}{*}{$\begin{array}{l}\text { Dust interception } \\
\text { fraction }\end{array}$} & Leafy vegetation & $R a_{1}$ & \multirow[t]{5}{*}{$6.5 .3-5$} & \multirow[t]{5}{*}{-} & 0.456 & \multirow[t]{5}{*}{ Dust_Intercept } & 0.456 & Same \\
\hline & & Other vegetation & $\mathrm{Ra}_{2}$ & & & 0.787 & & 0.787 & Same \\
\hline & & Fruit & $R a_{3}$ & & & 0.893 & & 0.893 & Same \\
\hline & & Grain & $\mathrm{Ra}_{4}$ & & & 0.962 & & 0.962 & Same \\
\hline & & Forage & $R a_{5}$ & & & 0.751 & & 0.751 & Same \\
\hline & Concentration due & Leafy vegetation & $C p_{\text {dust }, \text { Pu-239,1 }}$ & $6.5 .3-3$ & $\mathrm{~Bq} / \mathrm{kg}$ & 1.20E-06 & Dust_Uptake & 1.20E-06 & Same \\
\hline & to dust interception & Other vegetation & $C p_{\text {dust,Pu-239,2 }}$ & & & 1.67E-07 & & 1.67E-07 & Same \\
\hline & & Fruit & $C p_{\text {dust }, P u-239,3}$ & & & 2.90E-07 & & $2.90 \mathrm{E}-07$ & Same \\
\hline & & Grain & $C p_{\text {dust }, P u-239,4}$ & & & $1.46 \mathrm{E}-06$ & & $1.46 \mathrm{E}-06$ & Same \\
\hline & & Forage & $C p_{\text {dust }, \text { Pu-239, } 5}$ & & & 3.06E-06 & & 3.06E-06 & Same \\
\hline & Concentration in & Leafy vegetation & $C p_{P u-239,1}$ & 6.5.3-1 & $\mathrm{Bq} / \mathrm{kg}$ & 1.26E-06 & Plant_Conc & 1.26E-06 & Same \\
\hline & crops & Other vegetation & $C p_{P u-239,2}$ & & & $2.19 \mathrm{E}-07$ & & $2.19 \mathrm{E}-07$ & Same \\
\hline & & Fruit & $C p_{P u-239,3}$ & & & $3.48 \mathrm{E}-07$ & & $3.48 \mathrm{E}-07$ & Same \\
\hline & & Grain & $C p_{P u-239,4}$ & & & $1.50 \mathrm{E}-06$ & & $1.50 \mathrm{E}-06$ & Same \\
\hline & & Forage & $C p_{P u-239,5}$ & & & 3.65E-06 & & 3.65E-06 & Same \\
\hline
\end{tabular}


Table 6.10-5. Verification of ERMYN in GoldSim for the Volcanic Ash Scenario (Case 1, ${ }^{239} \mathrm{Pu}$ ) (Continued)

\begin{tabular}{|c|c|c|c|c|c|c|c|c|c|}
\hline \multirow[b]{2}{*}{ Submodel } & \multicolumn{6}{|c|}{ Parameter in Mathematical Model } & \multicolumn{2}{|c|}{ Parameter in GoldSim } & \multirow[b]{2}{*}{ Notes ${ }^{c}$} \\
\hline & \multicolumn{2}{|c|}{ Parameter Name } & $\begin{array}{l}\text { Notation in } \\
\text { Equation }\end{array}$ & $\begin{array}{l}\text { Equation } \\
\text { Number }\end{array}$ & Units & $\begin{array}{c}\text { Calculated } \\
\text { Value }^{\text {a }}\end{array}$ & Element Name & $\begin{array}{l}\text { ERMYN_VA } \\
\text { Result }^{\text {b }}\end{array}$ & \\
\hline \multirow[t]{12}{*}{ Animal } & \multirow{4}{*}{$\begin{array}{l}\text { Concentration due } \\
\text { to feed }\end{array}$} & Meat & $C d_{f e e d, P u-239,1}$ & \multirow[t]{4}{*}{$6.5 .4-2$} & \multirow[t]{4}{*}{$\mathrm{Bq} / \mathrm{kg}$} & $2.30 \mathrm{E}-09$ & \multirow[t]{4}{*}{ Feed_Contribution } & 2.30E-09 & Same \\
\hline & & Milk & $C d_{\text {feed,Pu-239,2 }}$ & & & $5.16 \mathrm{E}-11$ & & $5.16 \mathrm{E}-11$ & Same \\
\hline & & Poultry & $\mathrm{Cd}_{\text {feed,Pu-239,3 }}$ & & & 4.69E-10 & & $4.69 \mathrm{E}-10$ & Same \\
\hline & & Eggs & $C d_{\text {feed,Pu-239,4 }}$ & & & $6.64 \mathrm{E}-10$ & & 6.64E-10 & Same \\
\hline & \multirow{4}{*}{$\begin{array}{l}\text { Concentration due } \\
\text { to soil }\end{array}$} & Meat & $C d_{\text {soil,Pu-239,1 }}$ & \multirow[t]{4}{*}{$6.5 .4-3$} & \multirow[t]{4}{*}{$\mathrm{Bq} / \mathrm{kg}$} & $2.43 \mathrm{E}-08$ & \multirow[t]{4}{*}{ Soil_Contribution } & $2.43 \mathrm{E}-08$ & Same \\
\hline & & Milk & $C d_{\text {soil, }, \text { Pu-239,2 }}$ & & & $5.83 \mathrm{E}-10$ & & $5.83 \mathrm{E}-10$ & Same \\
\hline & & Poultry & $C d_{\text {soil,Pu-239,3 }}$ & & & $6.40 \mathrm{E}-08$ & & $6.40 \mathrm{E}-08$ & Same \\
\hline & & Eggs & $C d_{\text {soil,Pu-239,4 }}$ & & & 9.07E-08 & & 9.07E-08 & Same \\
\hline & \multirow{4}{*}{$\begin{array}{l}\text { Concentration in } \\
\text { animal products }\end{array}$} & Meat & $C d_{P u-239,1}$ & \multirow[t]{4}{*}{$6.5 .4-1$} & \multirow[t]{4}{*}{$\mathrm{Bq} / \mathrm{kg}$} & $2.66 \mathrm{E}-08$ & \multirow[t]{4}{*}{ Animal_Conc } & $2.66 \mathrm{E}-08$ & Same \\
\hline & & Milk & $C d_{P u-239,2}$ & & & $6.34 \mathrm{E}-10$ & & $6.34 \mathrm{E}-10$ & Same \\
\hline & & Poultry & $C d_{P u-239,3}$ & & & $6.45 \mathrm{E}-08$ & & $6.45 \mathrm{E}-08$ & Same \\
\hline & & Eggs & $C d_{P u-239,4}$ & & & 9.13E-08 & & 9.13E-08 & Same \\
\hline \multirow[t]{7}{*}{ External } & \multirow{5}{*}{$\begin{array}{l}\text { Environment- } \\
\text { specific exposure } \\
\text { time }\end{array}$} & Active outdoors & $\sum\left(P P_{m} t_{1, m}\right)$ & \multirow[t]{5}{*}{$6.5 .5-1$} & \multirow[t]{5}{*}{$\mathrm{hr} / \mathrm{d}$} & 0.45 & \multirow[t]{5}{*}{ Weighted_Time } & 0.45 & Same \\
\hline & & Inactive outdoors & $\sum\left(P P_{m} t_{2, m}\right)$ & & & 1.59 & & 1.59 & Same \\
\hline & & Active indoors & $\Sigma\left(P P_{m} t_{3, m}\right)$ & & & 10.87 & & 10.87 & Same \\
\hline & & Asleep indoors & $\sum\left(P P_{m} t_{4, m}\right)$ & & & 8.30 & & 8.30 & Same \\
\hline & & Away from area & $\sum\left(P P_{m} t_{5, m}\right)$ & & & 2.79 & & 2.79 & Same \\
\hline & \multicolumn{2}{|c|}{ Effective exposure time } & $\begin{array}{l}\sum\left[f_{\text {ext,Pu-239,n }}\right. \\
\left.\sum\left(P P_{m} t_{n, m}\right)\right]\end{array}$ & $6.5 .5-1$ & $\mathrm{hr} / \mathrm{yr}$ & 2848 & External_Time & 2848 & Same \\
\hline & \multicolumn{2}{|l|}{ External dose } & $D_{\text {ext,Pu-239 }}$ & $6.5 .5-1$ & Sv/yr & $3.76 \mathrm{E}-12$ & Total_External & $3.76 \mathrm{E}-12$ & Same \\
\hline \multirow[t]{2}{*}{ Inhalation } & \multicolumn{2}{|c|}{$\begin{array}{l}\text { Long-term inhalation dose (at nominal } \\
\text { condition) }\end{array}$} & $D_{\text {inh,p,Pu-239 }}$ & $6.5 .6-2$ & Sv/yr & $3.53 \mathrm{E}-07$ & Inhalation_Long & 3.53E-07 & Same \\
\hline & \multicolumn{2}{|c|}{$\begin{array}{l}\text { Short-term inhalation dose (at post- } \\
\text { volcanic condition) }\end{array}$} & $D_{\text {inh }, v, P u-239}$ & $6.5 .6-2$ & Sv/yr & $1.86 \mathrm{E}-07$ & Inhalation_Short & $1.86 \mathrm{E}-07$ & Same \\
\hline Ingestion & Ingestion dose for & Leafy vegetation & $D_{\text {ing, } p, P u-239,1}$ & $6.5 .7-2$ & Sv/yr & $4.55 \mathrm{E}-12$ & Crop_Ingestion & $4.55 \mathrm{E}-12$ & Same \\
\hline & crops & Other vegetation & $D_{\text {ing,p,Pu-239,2 }}$ & & & 9.92E-13 & & 9.92E-13 & Same \\
\hline & & Fruit & $D_{\text {ing, }, \text { Pu- } 239,3}$ & & & $4.21 \mathrm{E}-12$ & & $4.21 \mathrm{E}-12$ & Same \\
\hline & & Grain & $D_{\text {ing, } p, P u-239,4}$ & & & 3.30E-13 & & 3.30E-13 & Same \\
\hline & Ingestion dose for & Meat & $D_{\text {ing, } d, P u-239,1}$ & $6.5 .7-3$ & Sv/yr & $7.24 \mathrm{E}-14$ & Animal_Ingestion & $7.24 \mathrm{E}-14$ & Same \\
\hline & animal products & Milk & $D_{\text {ing }, d, P u-239,2}$ & & & $2.83 \mathrm{E}-15$ & & $2.83 \mathrm{E}-15$ & Same \\
\hline & & Poultry & $D_{\text {ing }, d, P u-239,3}$ & & & $2.59 \mathrm{E}-14$ & & $2.59 \mathrm{E}-14$ & Same \\
\hline & & Eggs & $D_{\text {ing, } d, P u-239,4}$ & & & $4.63 \mathrm{E}-13$ & & $4.63 \mathrm{E}-13$ & Same \\
\hline & Ingestion dose for $\mathrm{s}$ & & $D_{\text {ing }, \mathrm{S}, \mathrm{Pu}-239}$ & $6.5 .7-4$ & Sv/yr & 9.31E-11 & Soil_Ingestion & 9.31E-11 & Same \\
\hline & Ingestion dose & & $D_{\text {ing,Pu-239 }}$ & 6.5.7-1 & Sv/yr & $1.04 \mathrm{E}-10$ & Total_Ingestion & $1.04 \mathrm{E}-10$ & Same \\
\hline
\end{tabular}




\begin{tabular}{|c|c|c|c|c|c|c|c|c|}
\hline \multirow[b]{2}{*}{ Submodel } & \multicolumn{5}{|c|}{ Parameter in Mathematical Model } & \multicolumn{2}{|c|}{ Parameter in GoldSim } & \multirow[b]{2}{*}{ Notes $^{\mathrm{c}}$} \\
\hline & Parameter Name & $\begin{array}{l}\text { Notation in } \\
\text { Equation }\end{array}$ & $\begin{array}{l}\text { Equation } \\
\text { Number }\end{array}$ & Units & $\begin{array}{c}\text { Calculated } \\
\text { Value }^{\mathrm{a}}\end{array}$ & Element Name & $\begin{array}{c}\text { ERMYN_VA } \\
\text { Result }\end{array}$ & \\
\hline \multirow{6}{*}{$\begin{array}{l}\text { All } \\
\text { Pathway }\end{array}$} & Dose for external, radon, and ingestion & $D_{\text {all, Pu-239 }}$ & $6.5 .8-1$ & Sv/yr & $1.08 \mathrm{E}-10$ & TEDE_All & $1.08 \mathrm{E}-10$ & Same \\
\hline & Inhalation dose at post-volcanic condition & $D_{\text {inh }, v, \text { Pu-239 }}$ & $6.5 .8-1$ & Sv/yr & 1.86E-07 & TEDE_Short & $1.86 \mathrm{E}-07$ & Same \\
\hline & Inhalation dose at normal condition & $D_{\text {inh, }, \text {, Pu-239 }}$ & $6.5 .8-1$ & Sv/yr & 3.53E-07 & TEDE_Long & 3.53E-07 & Same \\
\hline & BDCF for external, radon, and ingestion & $B D C F_{P u-239}$ & $6.5 .8-2$ & $\begin{array}{l}(\mathrm{rem} / \mathrm{yr}) / \\
\left(\mathrm{pCi} / \mathrm{m}^{2}\right)\end{array}$ & $3.98 \mathrm{E}-10$ & BDCF_All & $3.98 \mathrm{E}-10$ & Same \\
\hline & $\begin{array}{l}\text { BDCF for inhalation at post-volcanic } \\
\text { condition }\end{array}$ & $\begin{array}{l}\text { BDCF } F_{\text {inh }, v, P u-} \\
239\end{array}$ & $6.5 .8-2$ & $\begin{array}{l}(\mathrm{rem} / \mathrm{yr}) / \\
\left(\mathrm{pCi} / \mathrm{m}^{2}\right)\end{array}$ & $6.90 \mathrm{E}-07$ & BDCF_InhShort & $6.90 \mathrm{E}-07$ & Same \\
\hline & BDCF for inhalation at normal condition & $\begin{array}{l}\text { BDCF } F_{\text {inh }, p, P u-} \\
239\end{array}$ & $6.5 .8-2$ & $\begin{array}{l}(\mathrm{rem} / \mathrm{yr}) / \\
\left(\mathrm{pCi} / \mathrm{m}^{2}\right)\end{array}$ & $1.30 \mathrm{E}-06$ & BDCF_InhLong & $1.30 \mathrm{E}-06$ & Same \\
\hline
\end{tabular}

a Input data for this verification taken from the "mean, mode, or average" column in Table 6.6-3. Calculations performed in Microsoft Excel 97 SR-2. The electronic file, ERMYN verification.xIs, which contains this calculation in worksheet VAPu239, is listed in Appendix A.

b The ERMYN is realized in GoldSim using the deterministic mode and data from the "mean, mode, or average" column in Table 6.6-3. The electronic file,

ERMYN_VA_PU239verf.gsm, for this run is listed in Appendix A.

${ }^{c}$ Comparison of results from Calculated Value (Column 6) and ERMYN_GW Result (Column 8).

$\mathrm{BDCF}=$ biosphere dose conversion factor; ERMYN=Environmental Radiation Model for Yucca Mountain, Nevada; VA=volcanic ash

Table 6.10-6. Verification of ERMYN in GoldSim for the Volcanic Ash Scenario (Case 2, ${ }^{226} \mathrm{Ra}$ )

\begin{tabular}{|c|c|c|c|c|c|c|c|c|c|}
\hline \multirow[b]{2}{*}{ Submodel } & \multicolumn{6}{|c|}{ Parameter in Mathematical Model } & \multicolumn{2}{|c|}{ Parameter in GoldSim } & \multirow[b]{2}{*}{ Notes ${ }^{c}$} \\
\hline & \multicolumn{2}{|c|}{ Parameter Name } & $\begin{array}{l}\text { Notation in } \\
\text { Equation }\end{array}$ & $\begin{array}{l}\text { Equation } \\
\text { Number }\end{array}$ & Units & $\begin{array}{c}\text { Calculated } \\
\text { Value }^{\mathrm{a}}\end{array}$ & Element Name & $\begin{array}{c}\text { ERMYN_VA } \\
\text { Result }^{b}\end{array}$ & \\
\hline \multirow[t]{2}{*}{ Soil } & \multicolumn{2}{|c|}{ Concentration in agricultural land } & $C S_{m, R a-226}$ & $6.5 .1-2$ & $\mathrm{~Bq} / \mathrm{kg}$ & 2.67E-03 & SoilMass_Conc & $2.67 \mathrm{E}-03$ & Same \\
\hline & \multicolumn{2}{|c|}{ Concentration in nonagricultural land } & $C s_{m c, R a-226}$ & $6.5 .1-3$ & $\mathrm{~Bq} / \mathrm{kg}$ & $5.00 \mathrm{E}-01$ & AshMass Conc & $5.00 \mathrm{E}-01$ & Same \\
\hline \multirow[t]{12}{*}{ Air } & \multicolumn{2}{|c|}{ Concentration in air for crop } & $C a_{p, R a-226}$ & $6.5 .2-1$ & $\mathrm{~Bq} / \mathrm{m}^{3}$ & $6.40 \mathrm{E}-10$ & AirConc_Crop & $6.40 \mathrm{E}-10$ & Same \\
\hline & \multirow{5}{*}{$\begin{array}{l}\text { Concentration in } \\
\text { air at nominal } \\
\text { condition }\end{array}$} & Active outdoors & $C a_{h, R a-226,1}$ & \multirow[t]{5}{*}{$6.5 .2-4$} & \multirow[t]{5}{*}{$\mathrm{Bq} / \mathrm{m}^{3}$} & $1.10 \mathrm{E}-05$ & \multirow[t]{5}{*}{ AirConc_Long } & $1.10 \mathrm{E}-05$ & Same \\
\hline & & Inactive outdoors & $C a_{h, R a-226,2}$ & & & $2.10 \mathrm{E}-08$ & & $2.10 \mathrm{E}-08$ & Same \\
\hline & & Active indoors & $C a_{h, R a-226,3}$ & & & 3.50E-08 & & 3.50E-08 & Same \\
\hline & & Asleep indoors & $C a_{h, R a-226,4}$ & & & $1.05 \mathrm{E}-08$ & & $1.05 \mathrm{E}-08$ & Same \\
\hline & & Away from area & $C a_{h, R a-226,5}$ & & & $0.00 \mathrm{E}+00$ & & $0.00 \mathrm{E}+00$ & Same \\
\hline & \multirow{5}{*}{$\begin{array}{l}\text { Concentration in } \\
\text { air at post volcanic } \\
\text { condition }\end{array}$} & Active outdoors & $C a_{V, R a-226,1}$ & \multirow[t]{5}{*}{$6.5 .2-4$} & \multirow[t]{5}{*}{$\mathrm{Bq} / \mathrm{m}^{3}$} & $5.50 \mathrm{E}-06$ & \multirow[t]{5}{*}{ AirConc_Short } & $5.50 \mathrm{E}-06$ & Same \\
\hline & & Inactive outdoors & $C a_{V, R a-226,2}$ & & & $2.10 \mathrm{E}-08$ & & $2.10 \mathrm{E}-08$ & Same \\
\hline & & Active indoors & $C a_{v, R a-226,3}$ & & & $3.50 \mathrm{E}-08$ & & $3.50 \mathrm{E}-08$ & Same \\
\hline & & Asleep indoors & $C a_{v, R a-226,4}$ & & & 1.05E-08 & & $1.05 E-08$ & Same \\
\hline & & Away from area & $C a_{V, R a-226,5}$ & & & $0.00 \mathrm{E}+00$ & & $0.00 \mathrm{E}+00$ & Same \\
\hline & \multicolumn{2}{|c|}{ Radon concentration in air } & $C a_{g, R n-222}$ & $6.5 .2-8$ & $\mathrm{~Bq} / \mathrm{m}^{3}$ & $6.00 \mathrm{E}-04$ & AirConc_Radon & $6.00 \mathrm{E}-04$ & Same \\
\hline
\end{tabular}


Table 6.10-6. Verification of ERMYN in GoldSim for the Volcanic Ash Scenario (Case 2, ${ }^{226} \mathrm{Ra}$ ) (Continued)

\begin{tabular}{|c|c|c|c|c|c|c|c|c|c|}
\hline \multirow[b]{2}{*}{ Submodel } & \multicolumn{6}{|c|}{ Parameter in Mathematical Model } & \multicolumn{2}{|c|}{ Parameter in GoldSim } & \multirow[b]{2}{*}{ Notes ${ }^{c}$} \\
\hline & \multicolumn{2}{|c|}{ Parameter Name } & \begin{tabular}{|c|c|}
$\begin{array}{c}\text { Notation in } \\
\text { Equation }\end{array}$ \\
\end{tabular} & $\begin{array}{c}\begin{array}{c}\text { Equation } \\
\text { Number }\end{array} \\
\end{array}$ & Units & $\begin{array}{c}\text { Calculated } \\
\text { Value }^{\mathrm{a}}\end{array}$ & Element Name & $\begin{array}{c}\text { ERMYN_VA } \\
\text { Result }\end{array}$ & \\
\hline \multirow[t]{20}{*}{ Plant } & \multirow{5}{*}{$\begin{array}{l}\text { Concentration due } \\
\text { to root uptake }\end{array}$} & Leafy vegetation & $C p_{\text {root }, R a-226,2}$ & \multirow[t]{5}{*}{ 6.5.3-2 } & \multirow[t]{5}{*}{$\mathrm{Bq} / \mathrm{kg}$} & 1.27E-05 & \multirow[t]{5}{*}{ Root_Uptake } & 1.27E-05 & Same \\
\hline & & Other vegetation & $C p_{\text {root }, R a-226,2}$ & & & 3.30E-06 & & 3.30E-06 & Same \\
\hline & & Fruit & $C p_{\text {root,Ra-226,3 }}$ & & & $2.34 \mathrm{E}-06$ & & 2.34E-06 & Same \\
\hline & & Grain & $C p_{\text {root,Ra-226,4 }}$ & & & $7.46 \mathrm{E}-06$ & & $7.46 \mathrm{E}-06$ & Same \\
\hline & & Forage & $C p_{\text {root }, R a-226,5}$ & & & 4.81E-05 & & 4.81E-05 & Same \\
\hline & \multirow{5}{*}{$\begin{array}{l}\text { Dust interception } \\
\text { fraction }\end{array}$} & Leafy vegetation & $R a_{1}$ & \multirow[t]{5}{*}{$6.5 .3-5$} & \multirow[t]{5}{*}{-} & 0.456 & \multirow[t]{5}{*}{ Dust_Intercept } & 0.456 & Same \\
\hline & & Other vegetation & $\mathrm{Ra}_{2}$ & & & 0.787 & & 0.787 & Same \\
\hline & & Fruit & $R a_{3}$ & & & 0.893 & & 0.893 & Same \\
\hline & & Grain & $\mathrm{Ra}_{4}$ & & & 0.962 & & 0.962 & Same \\
\hline & & Forage & $R a_{5}$ & & & 0.751 & & 0.751 & Same \\
\hline & \multirow{5}{*}{$\begin{array}{l}\text { Concentration due } \\
\text { to dust interception }\end{array}$} & Leafy vegetation & $C p_{\text {dust }, R a-226,1}$ & \multirow[t]{5}{*}{ 6.5.3-3 } & \multirow[t]{5}{*}{$\mathrm{Bq} / \mathrm{kg}$} & 1.20E-06 & \multirow[t]{5}{*}{ Dust_Uptake } & 1.20E-06 & Same \\
\hline & & Other vegetation & $C p_{\text {dust,Ra-226,2 }}$ & & & 1.67E-07 & & $1.67 \mathrm{E}-07$ & Same \\
\hline & & Fruit & $C p_{\text {dust }, R a-226,3}$ & & & $2.90 \mathrm{E}-07$ & & $2.90 \mathrm{E}-07$ & Same \\
\hline & & Grain & $C p_{\text {dust }, R a-226,4}$ & & & 1.46E-06 & & 1.46E-06 & Same \\
\hline & & Forage & $C p_{\text {dust,Ra-226,5 }}$ & & & $3.06 \mathrm{E}-06$ & & $3.06 \mathrm{E}-06$ & Same \\
\hline & \multirow{5}{*}{$\begin{array}{l}\text { Concentration in } \\
\text { crops }\end{array}$} & Leafy vegetation & $C p_{R a-226,1}$ & \multirow[t]{5}{*}{ 6.5.3-1 } & \multirow[t]{5}{*}{$\mathrm{Bq} / \mathrm{kg}$} & 1.39E-05 & \multirow[t]{5}{*}{ Plant_Conc } & 1.39E-05 & Same \\
\hline & & Other vegetation & $C p_{R a-226,2}$ & & & $3.46 \mathrm{E}-06$ & & 3.46E-06 & Same \\
\hline & & Fruit & $C p_{R a-226,3}$ & & & $2.63 \mathrm{E}-06$ & & 2.63E-06 & Same \\
\hline & & Grain & $C p_{R a-226,4}$ & & & 8.92E-06 & & 8.92E-06 & Same \\
\hline & & Forage & $C p_{R a-226,5}$ & & & 5.12E-05 & & 5.12E-05 & Same \\
\hline \multirow[t]{12}{*}{ Animal } & \multirow{4}{*}{$\begin{array}{l}\text { Concentration due } \\
\text { to feed }\end{array}$} & Meat & $C d_{\text {feed,Ra-226,1 }}$ & \multirow[t]{4}{*}{$6.5 .4-2$} & \multirow[t]{4}{*}{$\mathrm{Bq} / \mathrm{kg}$} & 2.01E-06 & \multirow[t]{4}{*}{ Feed_Contribution } & 2.01E-06 & Same \\
\hline & & \begin{tabular}{|l|} 
Milk \\
\end{tabular} & $C d_{\text {feed,Ra-226,2 }}$ & & & 1.83E-06 & & $1.83 \mathrm{E}-06$ & Same \\
\hline & & Poultry & $C d_{\text {feed,Ra-226,3 }}$ & & & 3.94E-08 & & 3.94E-08 & Same \\
\hline & & \begin{tabular}{|l|} 
Eggs \\
\end{tabular} & $C d_{\text {feed,Ra-226,4 }}$ & & & $9.05 \mathrm{E}-10$ & & 9.05E-10 & Same \\
\hline & Concentration due & Meat & $C d_{\text {soil,Ra-226,1 }}$ & 6.5.4-3 & $\mathrm{Bq} / \mathrm{kg}$ & 1.51E-06 & Soil_Contribution & 1.51E-06 & Same \\
\hline & to soil & \begin{tabular}{|l|} 
Milk \\
\end{tabular} & $C d_{\text {soil, Ra-226,2 }}$ & & & 1.47E-06 & & 1.47E-06 & Same \\
\hline & & \begin{tabular}{|l|} 
Poultry \\
\end{tabular} & $\mathrm{Cd}_{\text {soil, Ra-226,3 }}$ & & & 9.07E-07 & & 9.07E-07 & Same \\
\hline & & Eggs & $C d_{\text {soil,Ra-226,4 }}$ & & & $2.08 \mathrm{E}-08$ & & $2.08 \mathrm{E}-08$ & Same \\
\hline & Concentration in & Meat & $C d_{R a-226,1}$ & 6.5.4-1 & $\mathrm{Bq} / \mathrm{kg}$ & 3.52E-06 & Animal_Conc & 3.52E-06 & Same \\
\hline & animal products & \begin{tabular}{|l|} 
Milk \\
\end{tabular} & $\mathrm{Cd}_{\mathrm{Ra-226,2}}$ & & & 3.29E-06 & & 3.29E-06 & Same \\
\hline & & \begin{tabular}{|l|} 
Poultry \\
\end{tabular} & $\mathrm{Cd}_{\mathrm{Ra-226,3}}$ & & & $9.46 \mathrm{E}-07$ & & 9.46E-07 & Same \\
\hline & & Eggs & $\mathrm{Cd}_{\mathrm{Ra}-226,4}$ & & & 2.17E-08 & & 2.17E-08 & Same \\
\hline
\end{tabular}


Table 6.10-6. Verification of ERMYN in GoldSim for the Volcanic Ash Scenario (Case 2, ${ }^{226} \mathrm{Ra}$ ) (Continued)

\begin{tabular}{|c|c|c|c|c|c|c|c|c|c|}
\hline \multirow[b]{2}{*}{ Submodel } & \multicolumn{6}{|c|}{ Parameter in Mathematical Model } & \multicolumn{2}{|c|}{ Parameter in GoldSim } & \multirow[b]{2}{*}{ Notes $^{\mathrm{c}}$} \\
\hline & \multicolumn{2}{|c|}{ Parameter Name } & $\begin{array}{l}\text { Notation in } \\
\text { Equation }\end{array}$ & $\begin{array}{l}\text { Equation } \\
\text { Number }\end{array}$ & Units & $\begin{array}{l}\text { Calculated } \\
\text { Value }^{\mathrm{a}}\end{array}$ & Element Name & $\begin{array}{l}\text { ERMYN_VA } \\
\text { Result }\end{array}$ & \\
\hline \multirow[t]{7}{*}{ External } & \multirow{5}{*}{$\begin{array}{l}\text { Environment- } \\
\text { specific exposure } \\
\text { time }\end{array}$} & Active outdoors & $\sum\left(P P_{m} t_{1, m}\right)$ & \multirow[t]{5}{*}{$6.5 .5-1$} & \multirow[t]{5}{*}{$\mathrm{hr} / \mathrm{d}$} & 0.45 & \multirow[t]{5}{*}{ Weighted_Time } & 0.45 & Same \\
\hline & & Inactive outdoors & $\sum\left(P P_{m} t_{2, m}\right)$ & & & 1.59 & & 1.59 & Same \\
\hline & & Active indoors & $\sum\left(P P_{m} t_{3, m}\right)$ & & & 10.87 & & 10.87 & Same \\
\hline & & Asleep indoors & $\sum\left(P P_{m} t_{4, m}\right)$ & & & 8.30 & & 8.30 & Same \\
\hline & & Away from area & $\sum\left(P P_{m} t_{5, m}\right)$ & & & 2.79 & & 2.79 & Same \\
\hline & \multicolumn{2}{|c|}{ Effective exposure time } & $\begin{array}{l}\sum\left[f_{\text {ext,Ra-226,n }}\right. \\
\left.\sum\left(P P_{m} t_{n, m}\right)\right]\end{array}$ & $6.5 .5-1$ & $\mathrm{hr} / \mathrm{yr}$ & 3548 & External_Time & 3548 & Same \\
\hline & \multicolumn{2}{|l|}{ External dose } & $D_{\text {ext }, R a-226}$ & $6.5 .5-1$ & Sv/yr & $2.12 \mathrm{E}-08$ & Total_External & $2.12 \mathrm{E}-08$ & Same \\
\hline \multirow[t]{3}{*}{ Inhalation } & \multicolumn{2}{|c|}{$\begin{array}{l}\text { Long-term inhalation dose (at nominal } \\
\text { condition) }\end{array}$} & $D_{\text {inh,p,Ra-226 }}$ & $6.5 .6-2$ & Sv/yr & 7.06E-09 & Inhalation_Long & 7.06E-09 & Same \\
\hline & \multicolumn{2}{|c|}{$\begin{array}{l}\text { Short-term inhalation dose (at post- } \\
\text { volcanic condition) }\end{array}$} & $D_{\text {inh, }, \text { }, R a-226}$ & $6.5 .6-2$ & Sv/yr & 3.74E-09 & Inhalation_Short & 3.74E-09 & Same \\
\hline & \multicolumn{2}{|c|}{ Inhalation dose from radon } & $D_{\text {inh }, R n-222}$ & $6.5 .6-3$ & Sv/yr & $2.17 E-08$ & Inhalation_Radon & $2.17 \mathrm{E}-08$ & \\
\hline \multirow[t]{10}{*}{ Ingestion } & \multirow{4}{*}{$\begin{array}{l}\text { Ingestion dose for } \\
\text { crops }\end{array}$} & Leafy vegetation & $D_{\text {ing, } p, R a-226,1}$ & \multirow[t]{4}{*}{$6.5 .7-2$} & \multirow[t]{4}{*}{ Sv/yr } & $1.88 \mathrm{E}-11$ & \multirow[t]{4}{*}{ Crop_Ingestion } & $1.88 \mathrm{E}-11$ & Same \\
\hline & & Other vegetation & $D_{\text {ing, } p, R a-226,2}$ & & & $5.87 \mathrm{E}-12$ & & $5.87 \mathrm{E}-12$ & Same \\
\hline & & Fruit & $D_{\text {ing, }, \text { Ra-226,3 }}$ & & & 1.19E-11 & & $1.19 \mathrm{E}-11$ & Same \\
\hline & & Grain & $D_{\text {ing, } p, R a-226,4}$ & & & $7.35 \mathrm{E}-13$ & & $7.35 \mathrm{E}-13$ & Same \\
\hline & \multirow{4}{*}{$\begin{array}{l}\text { Ingestion dose for } \\
\text { animal products }\end{array}$} & Meat & $D_{\text {ing,d,Ra-226,1 }}$ & \multirow[t]{4}{*}{$6.5 .7-3$} & \multirow[t]{4}{*}{ Sv/yr } & $3.60 \mathrm{E}-12$ & \multirow[t]{4}{*}{ Animal_Ingestion } & $3.60 \mathrm{E}-12$ & Same \\
\hline & & Milk & $D_{\text {ing, }, \text { Ra-226,2 }}$ & & & $5.50 \mathrm{E}-12$ & & $5.50 \mathrm{E}-12$ & Same \\
\hline & & Poultry & $D_{\text {ing, } d, R a-226,3}$ & & & $1.42 \mathrm{E}-13$ & & $1.42 \mathrm{E}-13$ & Same \\
\hline & & Eggs & $D_{\text {ing, } d, R a-226,4}$ & & & 4.12E-14 & & $4.12 \mathrm{E}-14$ & Same \\
\hline & \multicolumn{2}{|c|}{ Ingestion dose for soil } & $D_{\text {ing }, \mathrm{s}, \mathrm{Ra}-226}$ & $6.5 .7-4$ & Sv/yr & 3.49E-11 & Soil_Ingestion & $3.49 \mathrm{E}-11$ & Same \\
\hline & \multicolumn{2}{|c|}{ Ingestion dose } & $D_{\text {ing,Ra-226 }}$ & $6.5 .7-1$ & Sv/yr & 8.15E-11 & Total_Ingestion & $8.15 \mathrm{E}-11$ & Same \\
\hline $\begin{array}{l}\text { All } \\
\text { Pathway }\end{array}$ & \multicolumn{2}{|c|}{ Dose for external, radon, and ingestion } & $D_{\text {all,Ra-226 }}$ & $6.5 .8-1$ & Sv/yr & 4.30E-08 & TEDE_All & 4.30E-08 & Same \\
\hline
\end{tabular}


Table 6.10-6. Verification of ERMYN in GoldSim for the Volcanic Ash Scenario (Case 2, ${ }^{226} \mathrm{Ra}$ ) (Continued)

.

\begin{tabular}{|c|c|c|c|c|c|c|c|c|c|}
\hline \multirow{2}{*}{ Submodel } & \multicolumn{6}{|c|}{ Parameter in Mathematical Model } & \multicolumn{2}{|c|}{ Parameter in GoldSim } & \multirow[b]{2}{*}{ Notes $^{c}$} \\
\hline & \multicolumn{2}{|c|}{ Parameter Name } & $\begin{array}{l}\text { Notation in } \\
\text { Equation }\end{array}$ & $\begin{array}{l}\text { Equation } \\
\text { Number }\end{array}$ & Units & $\begin{array}{l}\text { Calculated } \\
\text { Value }^{a}\end{array}$ & Element Name & $\begin{array}{l}\text { ERMYN_VA } \\
\text { Result }^{b}\end{array}$ & \\
\hline \multirow[t]{6}{*}{ External } & $\begin{array}{l}\text { Environment- } \\
\text { specific exposure } \\
\text { time }\end{array}$ & Active outdoors & $\sum\left(P P_{m} t_{1, m}\right)$ & $6.5 .5-1$ & $\mathrm{hr} / \mathrm{d}$ & 0.45 & Weighted_Time & 0.45 & Same \\
\hline & \multicolumn{2}{|c|}{ Inhalation dose at post-volcanic condition } & $D_{\text {inh }, v, R a-226}$ & 6.5.8-1 & Sv/yr & 3.74E-09 & TEDE_Short & 3.74E-09 & Same \\
\hline & \multicolumn{2}{|c|}{ Inhalation dose at normal condition } & $D_{\text {inh }, p, R a-226}$ & 6.5.8-1 & Sv/yr & 7.06E-09 & TEDE_Long & 7.06E-09 & Same \\
\hline & \multicolumn{2}{|c|}{ BDCF for external, radon, and ingestion } & $B D C F_{R a-226}$ & $6.5 .8-2$ & $\begin{array}{l}(\mathrm{rem} / \mathrm{yr}) / \\
\left(\mathrm{pCi} / \mathrm{m}^{2}\right)\end{array}$ & 1.59E-07 & BDCF_All & 1.59E-07 & Same \\
\hline & \multicolumn{2}{|c|}{$\begin{array}{l}\text { BDCF for inhalation at post-volcanic } \\
\text { condition }\end{array}$} & $\begin{array}{l}\text { BDCF inh,v,Ra- } \\
226\end{array}$ & $6.5 .8-2$ & $\begin{array}{l}(\mathrm{rem} / \mathrm{yr}) / \\
\left(\mathrm{pCi} / \mathrm{m}^{2}\right)\end{array}$ & 1.38E-08 & BDCF_InhShort & 1.38E-08 & Same \\
\hline & \multicolumn{2}{|c|}{ BDCF for inhalation at normal condition } & $\begin{array}{l}\text { BDCF } \text { inh,p,Ra- } \\
226\end{array}$ & $6.5 .8-2$ & $\begin{array}{l}(\mathrm{rem} / \mathrm{yr}) / \\
\left(\mathrm{pCi} / \mathrm{m}^{2}\right)\end{array}$ & $2.61 \mathrm{E}-08$ & BDCF_InhLong & $2.61 \mathrm{E}-08$ & Same \\
\hline
\end{tabular}

${ }^{a}$ Input data for this verification taken from the "mean, mode, or average" column in Table 6.6-3. Calculations performed in Microsoft Excel 97 SR-2. The electronic file, ERMYN verification.xIs, which contains this calculation in worksheet VARa226, is listed in Appendix A.

b The ERMYN is realized in GoldSim using the deterministic mode and data from the "mean, mode, or average" column in Table 6.6-3. The electronic file, ERMYN_VA_RA226verf.gsm, for this run is listed in Appendix A.

${ }^{c}$ Comparison of results from Calculated Value (Column 6) and ERMYN_GW Result (Column 8). 


\subsubsection{Verification of Stochastic Calculations}

Similar to the groundwater scenario, deterministic and stochastic runs for ${ }^{239} \mathrm{Pu}$ are performed using input parameters from Table 6.6-3. The results (Table 6.10-7) reveal small differences between the deterministic and stochastic runs, except for the ingestion dose and BDCF for combined external and ingestion pathways. In the groundwater scenario, radionuclide concentrations in surface soil depend on several stochastic parameters, such as the partition coefficient, and are thus calculated as stochastic variables. Unlike the groundwater scenario, radionuclide concentrations in ash deposited in the ground, which is the source of radionuclides for the volcanic ash scenario, are equal to the unit areal activity concentration. This results in a relatively small difference between the deterministic and stochastic calculations. For the ingestion pathway, sample means and reasonable estimates for some parameters, such as soil depth and transfer coefficients, are different. As before, differences between the BDCFs calculated using the two methods are not significant because the deterministic results are within the one standard deviation of the stochastic mean values. The stochastic results are considered more realistic.

Table 6.10-7. Results from the Deterministic and Stochastic Runs for the Volcanic Ash Scenario

\begin{tabular}{|l|c|c|}
\hline \multicolumn{1}{|c|}{ Parameter } & $\begin{array}{c}\text { Deterministic } \\
\text { Results }\end{array}$ & $\begin{array}{c}\text { Stochastic Results } \\
\text { (mean and standard deviation) }\end{array}$ \\
\hline External dose (Sv/yr) & $3.76 \mathrm{E}-12$ & $(3.80 \pm 0.07) \mathrm{E}-12$ \\
\hline Inhalation dose; post-volcanic conditions (Sv/yr) & $1.86 \mathrm{E}-07$ & $(2.47 \pm 1.49) \mathrm{E}-07$ \\
\hline Inhalation dose; normal conditions (Sv/yr) & $3.53 \mathrm{E}-07$ & $(4.97 \pm 3.05) \mathrm{E}-07$ \\
\hline Ingestion dose (Sv/yr) & $1.04 \mathrm{E}-10$ & $(3.30 \pm 4.27) \mathrm{E}-10$ \\
\hline BDCF for external exposure and ingestion $(\mathrm{rem} / \mathrm{yr}) /\left(\mathrm{pCi} / \mathrm{m}^{2}\right)$ & $3.98 \mathrm{E}-10$ & $(1.24 \pm 1.58) \mathrm{E}-09$ \\
\hline BDCF for inhalation; post-volcanic conditions $(\mathrm{rem} / \mathrm{yr}) /\left(\mathrm{pCi} / \mathrm{m}^{2}\right)$ & $6.90 \mathrm{E}-07$ & $(9.12 \pm 5.52) \mathrm{E}-07$ \\
\hline BDCF for inhalation; normal conditions $(\mathrm{rem} / \mathrm{yr}) /\left(\mathrm{pCi} / \mathrm{m}^{2}\right)$ & $1.30 \mathrm{E}-06$ & $(1.84 \pm 1.13) \mathrm{E}-06$ \\
\hline
\end{tabular}

NOTE: Deterministic calculations done using ERMYN_GW_Pu239verf.gsm; stochastic calculations done using ERMYN_GW_Pu239stoc.gsm; both are listed in Appendix A. 


\section{INTENTIONALLY LEFT BLANK}




\section{VALIDATION}

As presented in Sections 1 and 6, this report was revised in accordance with Revision 3 of the TWP (BSC 2004 [DIRS 169573]). This TWP (BSC 2004 [DIRS 169573], Section 1) explicitly excludes any work scope related to model development and/or validation because the biosphere model development and validation was completed during development of Revision 0 of this report (BSC 2003 [DIRS 166507]) in accordance with Revision 1 of the TWP (BSC 2003 [DIRS 163602]) and the procedures and guidelines effective at the time the work was performed. Consistent with those procedures, the validation methodology and criteria were consistent with the Scientific Processes Guidelines Manual (SPGM) (BSC 2002 [DIRS 160313], which was subsequently cancelled. The description of the model validation methodology from the SPGM was incorporated into the planning (AP-2.27Q) and modeling (AP-SIII.10Q) procedures followed for this revision. Therefore, the biosphere model validation remains appropriate, as described in this section. This revision was developed to correct typographical errors, update references, enhance transparency and traceability of the report, and to bring it up to date with project procedures.

As required by AP-SIII.10Q, developed models had to be validated to ensure that they are suitable for the intended purpose. The approach to validating the biosphere model is specified in Revision 1 of the biosphere TWP (BSC 2003 [DIRS 163602], Section 2). The SPGM (BSC 2002 [DIRS 160313], Appendix B) specified the levels of model importance and validation, and it described three levels of validation and associated requirements to ensure an appropriate level of confidence in the model. Although the SPGM stated that the biosphere model only requires Level I validation for the groundwater scenario (BSC 2002 [DIRS 160313], p. B-9), the initial issue of this report validated the ERMYN to Level II (which includes Level I requirements). There were two reasons for doing this. First, the ERMYN, which supports the TSPA-LA, is considerably changed from the older biosphere model. Second, Level II validation requirements are more stringent and provide a greater level of confidence in the model. The SPGM also states that Level III validation is required for biosphere submodels associated with igneous activity, such as soil thickness, removal, and aeolian and fluvial redistribution (BSC 2002 [DIRS 160313], Appendix B). However, these items are part of the radionuclide source term for the volcanic ash scenario, which is not part of the biosphere model.

The ERMYN incorporates the requirements of 10 CFR 63 [DIRS 156605], which established the characteristics of the reference biosphere (10 CFR 63.305) and the RMEI (10 CFR 63.312). The results of the ERMYN are used to demonstrate compliance with the postclosure individual protection standard (10 CFR 63.311 [DIRS 156605]).

The output of the biosphere model has a direct impact on the results of the TSPA-LA dose calculations because the total (all radionuclides) dose is calculated as the sum of the products of radionuclide-specific BDCFs and the concentration of individual radionuclides in the source. Variation due to uncertainty in the BDCFs had a small impact on previous dose calculations for the groundwater scenario (CRWMS M\&O 2000 [DIRS 153246], p. 5-20), at least in part because BDCF distributions were narrow relative to the distribution of radionuclide concentrations in the groundwater. 
Based on Revision 1 of the TWP (BSC 2003 [DIRS 163602], Section 2), the biosphere model was validated to a level of confidence commensurate with Level II validation (BSC 2002 [DIRS 160313], p. B-9). This conclusion is based on the facts below.

The ERMYN deals with a specific environment, is not extrapolated over large distances, and generally does not depend on time. For some radionuclides, however, the surface soil submodel includes long periods of time by assuming constant land use (with irrigation and attendant leaching). This exception only applies to radionuclides with high solid-to-liquid partition coefficients, which would inhibit radionuclide accumulation in soil. The biosphere model employs a steady-state (long time) solution for the concentration of radionuclides in soil, that allows consideration of radionuclide build-up in soil with additional loss mechanisms. Parameters developed for this submodel incorporate long time frames using parameter value distributions for the rates of accumulation and depletion, which address uncertainty in the parameter values.

Overall uncertainty in the ERMYN is due to uncertainties in the conceptual representation, mathematical representation, and parameter values in each component submodel. Uncertainty associated with each submodel, which is quantified and propagated through the biosphere model, is due to uncertainty in the input parameters. A typical range of BDCF values, due to uncertainty in the input parameter values, is about one order of magnitude (BSC 2004 [DIRS 169674], Section 6.2.3; BSC 2004 [DIRS 167287], Section 6.2.3). This range is relatively low compared with other TSPA components, and it indicates that variation in the overall dose, which is many orders of magnitude, dwarfs variability in the BDCF values (CRWMS M\&O 2000 [DIRS 153246], Section 5). Uncertainty in the ERMYN is evaluated through submodel comparisons.

The level of confidence necessary for the ERMYN is based on guidance in Revision 1 of the TWP for Level II validation (BSC 2002 [DIRS 163602], Section 2), which states that a submodel is considered valid if it is consistent with submodels used and documented in other national or international dose assessment programs, and not using an alternative submodel can be justified based on an explanation of the differences between it and the selected submodel. The biosphere model is valid if the model includes all of the applicable radionuclide transport processes and radiation dose pathways, and if each of the corresponding submodels is validated using the methods and criteria listed above.

The ERMYN was validated to establish confidence that the conceptual (Section 6.3) and mathematical models (Sections 6.4 and 6.5) adequately represent the biosphere systems, processes, and phenomena in the reference biosphere. The model validation approach, including the selection of published biosphere models for the comparison and the validation criteria are discussed in Section 7.1. The conceptual models (Section 7.2) and mathematical models (Section 7.3) were compared to identify similarities and differences. For submodels that differ, numerical comparisons were made (Section 7.4), and the ERMYN submodels were justified. Finally, validation of the ERMYN implementation in GoldSim was performed by comparing results from the GoldSim implementation with those generated by the GENII-S implementation for the TSPA-SR (Section 7.5). The range of validation for the input parameters is presented in Section 7.6. The conclusions of an external review are presented in Section 7.7. 


\subsection{VALIDATION APPROACH}

In this section, the biosphere model validation approach, the selection of published biosphere models and supporting information used for corroboration, and the criteria and level of confidence for the ACMs are discussed. AP-SIII.10Q at 5.3.2(b) places three requirements on the validation of the mathematical model and its underlying conceptual model. The first concerns the selection of input parameter and/or input data (in this case, as discussed in Section 4.1, these data are equations). As discussed in the following subsections, this was achieved by a review of other biosphere models developed by reputable groups and used to evaluate the consequences of radionuclide transport and exposure. In addition, as discussed in the parameter development reports (Figure 1-1), data used to develop the model input parameters were selected based on site-specific information, including reasonable uncertainty and variation, and adequately described diet and lifestyle of the RMEI and the conditions in the Yucca Mountain region. The second requirement involves calibration of the model. Comparisons were made using ERMYN and the other biosphere models to demonstrate that there is little if any significant difference among predictions. These issues are discussed in Section 7.2 and 7.3. The final requirement is to discuss the impacts of uncertainties to model results. By virtue of the close predictive agreement between ERMYN and other biosphere model, this does not raise a concern on the results. The uncertainties in the predictions are primarily governed by the uncertainties in the multitude of input parameters. By being built on the GoldSim platform supporting stochastic evaluations, the ERMYN has the required capability to propagate and appropriately incorporate uncertainty in all parameters, as defined by the source documentation (BSC 2004 [DIRS 169672]; BSC 2004 [DIRS 169459]; BSC 2004 [DIRS 169673]; BSC 2004 [DIRS 169458]; BSC 2004 [DIRS 169671]). Thus the impacts of uncertainties are reflected in the BDCFs developed by use of ERMYN (BSC 2004 [DIRS 167287] and BSC 2004 [DIRS 169674].

The ERMYN (Section 6) is based on the previous biosphere model, implemented in GENII-S, for the TSPA-SR radiation dose assessments. The current model includes many improvements and modifications to represent site-specific conditions (Section 6.7.2). As stated in Section 6.1, the purpose and objective of the ERMYN is to provide the capabilities for TSPA-LA to calculate annual dose to the receptor that would result if radionuclides from the geologic repository were released into the environment with groundwater or ash at unit concentrations. Limitations in the model are specified in Section 8.2. 


\subsubsection{Confidence Building During Model Development to Establish the Scientific Basis and Accuracy for Intended Use}

Confidence building during model development was achieved by satisfying six SPGM requirements (BSC 2002 [DIRS 160313], p. B-1), each of which is discussed below to document the decisions and activities that were taken to generate confidence in the model during development of the conceptual and mathematical models. Further, the development of the model was documented in accordance with the requirements of Section 5.3.2(b) of AP-SIII.10Q as follows:

a) Selection of the input parameters and/or input data and a discussion of how the selection builds confidence in the model. [AP-SIII.10Q 5.3.2(b) (1) and AP-2.27Q Attachment 3 Level I(a)] — Input parameters were defined when they first appeared in the mathematical model description. The physical meaning and typical ranges were discussed, but the input parameter values were evaluated and developed in other reports (Figure 1-1 and brief discussion in Section 4).

b) Formulate defensible approximations and simplifications [AP-2.27Q Attachment 3 Level I (b)] —All modeling approximations and simplifications are discussed in Sections 6.3.1.4 and 6.3.2.4. Rationales for the approximations, and their use in the biosphere model, are given in Sections 6.3.1.4 and 6.3.2.4.

c) Ensure consistency with physical principles, such as conservation of mass, energy, and momentum [AP-2.27Q Attachment 3 Level I (c)] - All equations are consistent with basic physical principles. Conservation of mass and radionuclides was preserved in most of the developed equations, except when "double counting" was used to ensure that the risk to the receptor is not underestimated, such as in Approximation 6. Units for the parameters are checked to ensure consistency. See the summary discussion on equations and their sources in Section 4.1 and Table 4.1.

d) Discussion of the impacts of uncertainties to model results, including how the model represents important future state (aleatoric), parameters, and alternative model uncertainties [AP-SIII.10Q 5.3.2(b)(3) and AP-2.27Q Level I (d)] Present-day and future climate states are considered in the ERMYN, and different data sets will be developed for the two climate states. Uncertainties associated with the conceptual and mathematical models are discussed (Section 6.6). The source of parameter uncertainty is discussed, and a summary of parameter uncertainty distributions is presented (Table 6.6-3).

e) Description of the calibration activities, and/or initial boundary condition runs, and/or run convergences, simulation conditions have been set up to span the range of intended use and avoid inconsistent output, and a discussion of how the activity or activities builds confidence in the environment. [AP-SIII.10Q 5.3.2(b)(2) and AP-2.27Q Attachment 3 Level I (e)]—The ERMYN is implemented using GoldSim software, which provides the necessary simulation environment. Most input parameters are entered as distributions representing reasonable ranges. These parameter distributions are incorporated in the ERMYN using GoldSim. Outputs from 
model realizations are obtained while sampling over the full range of input parameters. Therefore, the simulated results are consistent with the input ranges and distributions. The model implementation is conducted in other analyses; therefore, there are no instances of run convergences or noncovergences. However, the linear nature of the model (i.e., the sum of multiple products) as implemented with the simplifying approximations indicates that convergence cannot be a concern. Calibration was performed by comparison of the ERYMN model to other biosphere models to demonstrate that there is little if any significant difference among predictions. The calibrations by comparison to other models are discussed in Sections 7.2 through 7.4.

f) Ensure that model predications (performance parameters) adequately represent the range of possible outcomes, consistent with important uncertainties-Most of the parameters are represented by distributions, and the number of realizations is large enough (e.g., 1,000) to ensure that the input parameters are sampled over the full range of values and that the model outcomes encompass the full range of possible values.

\subsubsection{Post-Development Model Validation to Support the Scientific Basis of the Model}

Level II validation requires confidence building activities as described in Section 7.1.1, plus one additional postdevelopment model validation method (BSC 2002 [DIRS 160313], p. B-2) selected from a list of seven methods (AP-SIII.10Q):

1) Corroboration of model results with data acquired from the laboratory, field experiments, analogue studies, or other relevant observations, not previously used to develop or calibrate the model

2) Corroboration of results with alternative mathematical models

3) Corroboration with data published in refereed journals or literature

4) Peer Review per AP-2.12Q, Peer Review

5) Technical review, planned in the applicable TWP, by reviewers independent of the development, checking, and interdisciplinary review of the model documentation (the Responsible Manager/Lead may not participate in this technical review of products for which they are directly responsible)

6) Corroboration of abstraction model results to the results of the validated process model(s) from which the abstraction was derived

7) Corroboration of pretest model predictions to data collected during the associated testing.

An additional method can be used to gain confidence per AP-SIII.10Q, Section 5.3.2(d): Technical review through publication in a refereed professional journal or review by an external agency, documented by the external agency, may be used to demonstrate additional confidence in the model, if publication or review is used in conjunction with one or more of the postdevelopment validation techniques described in Section 5.3.2(c) of AP-SIII.10Q. 
Because the ERMYN is complex and requires hundreds of input values (Section 6.6), it would be difficult to collect all input data through field experiments, laboratory experiments, or other testing on site within a limited time frame. Therefore, comparing model results with data from experimental or other testing is not realistic, and validation methods 1 and 7 cannot be used. Accordingly, corroboration of ERMYN results with alternative mathematical models (validation method 2) is the principal method for validating the model. Alternative mathematical models result from ACMs or from different mathematical representations of the same conceptual model. Validation methods 3 and 6 also are used for comparing results from the ERMYN with results from the selected published models and with data from the published literature.

In addition, AP-SIII.10Q allows the use of technical reviews by external agencies to demonstrate additional confidence in models. Technical review activities for the ERMYN include an international peer review of the previous biosphere model by an IAEA International Review Team (IAEA 2001 [DIRS 155188]), a model validation status review by experts at Lawrence Livermore National Laboratory (LLNL) (BSC 2003 [DIRS 170030]), and a technical review of the ERMYN performed separately and independently by another expert at LLNL (Daniels 2003 [DIRS 163016]). Recommendations from the first two reviews were incorporated in the ERMYN. The third review, conducted after the ERMYN was developed, evaluated functions and improvements in the ERMYN (Section 7.7).

The ERMYN was validated through corroboration of the conceptual approach, the mathematical representations, and comparison of the results of individual submodels with the results of published biosphere models that have been used elsewhere for dose assessments. This validation approach was stated in Revision 1 of the TWP (BSC 2003 [DIRS 163602], p. 20) as follows:

The biosphere model will be validated through corroboration of the conceptual approach, mathematical representation, and the modeling results for each submodel with those of other published biosphere models. If the mathematical representations from the published models are mathematically equivalent to, and result in, approximately the same numerical values as the one used in the Yucca Mountain biosphere model, the submodel is considered validated. If the mathematical representations from the published models are not equivalent to the one used in the Yucca Mountain model, a numerical comparison will be conducted by using the appropriate input parameter values to exercise the submodels. Corroboration (i.e. numerical similarity) of the submodels will be considered demonstrated if the results of the numerical comparison are within a factor of 2 over the applicable range of input parameters. This factor of 2 was defined by BSC (2002 [DIRS 160313], p. B-9). If the numerical results differ by more than a factor of 2 from the expected range of input parameters, corroboration will not be assumed and the modeling methods will be evaluated, and the selection of a specific submodel will be justified. The justification of the selected submodel will be documented in the report.

The main step in validation was corroborating each ERMYN submodel with commonly used published models to ensure that the ERMYN submodels are appropriate. Validation includes the review of the radiation dose assessment context, evaluation of the biosphere conceptual model and ACMs, consideration of scenarios and radiation pathways, comparison of mathematical 
submodels with published biosphere models, and documentation of ACMs. The selection of applicable, state-of-the-art published biosphere models (i.e., the validation models) for use in validation is described in Section 7.1.2. The following criteria were used to establish the adequacy of the scientific basis for the model and to determine if the needed level of confidence for the model is met.

If the mathematical representations in the ERMYN or validation models include a parameter that is not used in the other, and if that parameter has no influence on the numerical results for the Yucca Mountain scenarios, the mathematical representations are judged to be equivalent, and no further justification or comparison was required. For example, if a validation model has a parameter that is not used in the ERMYN, but the parameter is multiplicative and equal to 1 for the Yucca Mountain scenarios, the two representations are mathematically equivalent. Similarly, if a parameter used in the ERMYN is not used in the validation models because of approximations or site-specific conditions, the equations are mathematically equivalent.

If the validation and ERMYN submodels are mathematically different, they were compared numerically using input parameter values from Table 6.6-3 and, if necessary, default or reasonable values for the validation model. Simple comparisons are presented in Section 7.3. Complex comparisons requiring a series of calculations or the use of a spreadsheet are presented in Section 7.4. The data from Table 6.6-3 used for model validation were not used to develop the model.

If the validation and ERMYN submodels produced different results, but the difference is less than or equal to a factor of two, the numerical results are similar and no further justification is necessary. Therefore, demonstrating numerical similarity validates the item in the ERMYN. The comparisons were made by evaluating results at the level of submodels or equations in a submodel, which ensures that differences that could substantially influence dose calculations were identified. A factor of two was chosen for validation of a submodel because such a difference within a submodel is small relative to the approximately order-of-magnitude range of variation in BDCFs. In addition, it is small relative to the order-of-magnitude (or greater) range in variation in some parameters that are likely to have an important influence on the results of the model, such as mass loading, partition coefficients, and transfer factors. Thus, this criterion is sufficiently accurate for its intended use and consistent with parameter uncertainty.

If the validation and ERMYN submodels produced different results, and the difference was more than a factor of two, the difference is evaluated to ensure that it is reasonable. Justification for the selected approach is provided, which usually is based on site-specific or realistically predictable conditions, better incorporating uncertainty, or avoiding over- or underestimating dose calculations.

The overall approach for validating the ERMYN provides an appropriate level of confidence that the ERMYN methods are appropriate and sufficiently accurate for their intended use because they are similar to, or produce results that are similar to, published state-of-the-art environmental radiation models. In cases where the validation and ERMYN submodels produce different results, this approach ensures that the differences are consistent with available data, incorporated for valid reasons, and that they improve the model. 


\subsubsection{Selection of Supporting Information}

The primary information used to validate the ERMYN were the descriptions of published biosphere models that have been used nationally and internationally for environmental radiation dose assessments. Eleven models were reviewed, and although none use exactly the same pathways as the ERMYN, they all have comparable submodels (Sections 6.4 and 6.5). Five models were selected for validation because they are representative, in common use, and available. The five selected models, referred to as validation models, were:

- GENII/GENII-S (Napier et al. 1988 [DIRS 157927]; Leigh et al. 1993 [DIRS 100464]) -This generic computer model for assessing radiation doses was developed by the Pacific Northwest and Sandia National Laboratories. The model supports various sources, including contaminated groundwater, contaminated soil, and air dispersion. This model can be used for evaluating individual and population doses, and for chronic and acute releases. The GENII-S model was used to calculate BDCFs for the TSPA-SR, and limitations in GENII-S (Table 6.7-2) are the basis for developing the ERMYN.

- BIOMASS ERB2A (BIOMASS 2000 [DIRS 154522])—This model, designed for groundwater contamination scenarios using various generic exposure pathways, provides systematic methods for performing postclosure radiation dose assessments for geologic repositories. It was developed by the IAEA Division of Radiation and Waste Safety. This report is one of a series that provides an example reference biosphere with an agricultural well. Useful information includes the assessment context, biosphere identification, and some input parameter values. BIOMASS ERB2A is not Yucca Mountain specific, but details of the mathematical model were useful.

- EPRI-YM (Smith et al. 1996 [DIRS 101085]) — This model was developed in 1996 by the Electric Power Research Institute to model a groundwater release at Yucca Mountain. It is one of the few published biosphere models for Yucca Mountain. The report provides a method for identifying biosphere FEPs, identifying other dose assessment requirements for a groundwater scenario at Yucca Mountain, and it presents the mathematical model, the selection of input data, and useful site-specific information. A new revision of the EPRI-YM model uses the BIOMASS ERB2A model (EPRI 2002 [DIRS 158069], Section 8).

- RESRAD (Yu et al. 2001 [DIRS 159465])—This generic, but comprehensive tool for estimating radiation doses and risks from radioactive materials in the environment was developed by the Argonne National Laboratory. It is designed for soil contamination scenarios and is useful for comparison with the volcanic ash scenario. The methods for evaluating the movement of radionuclides are considered to be among the best, they are widely accepted in the scientific community, and they are widely used by government agencies and institutions (Yu et al. 2001 [DIRS 159465], p. xvii). Although this model uses radionuclide concentrations in the soil as the primary source, it allows modeling of radionuclide leaching from soil to groundwater and subsequent use of contaminated groundwater for irrigation. Therefore, this model was useful for comparison with the Yucca Mountain scenarios. 
- NCRP-129 (NCRP 1999 [DIRS 155894])-This document, developed by the National Council on Radiation Protection and Measurements, provides screening limits for contaminated surface soil and reviews factors relevant to site-specific studies. Designed for soil contamination scenarios, this model was useful for comparison with the volcanic ash scenario. The screening limits are calculated conservatively so that no further action is needed if radionuclide concentrations are below the limits. Although the methods are simple, they provide bounding limits for estimations.

Six other biosphere models were reviewed, but they were not used for direct comparison with the ERMYN because they are similar to the validation models, they do not apply to the Yucca Mountain scenarios, or the methods are not commonly used. These documents are:

- CNWRA 97-009 (LaPlante and Poor 1997 [DIRS 101079])—This document, produced by the Center for Nuclear Waste Regulatory Analyses, provides information and analyses to support the selection of critical groups and reference biospheres for the Yucca Mountain scenarios. Because this model is based on GENII-S, it was not compared with the ERMYN.

- NUREG/CR-5512 (Kennedy and Strenge 1992 [DIRS 103776])—This document, produced by the Pacific Northwest Laboratory, provides generic and site-specific estimates of radiation doses for exposure to residual radioactive contamination after the decommissioning of NRC-licensed facilities. Although the document does not directly mention using the GENII model, the methods, input parameters, and default values are similar to those in the GENII manual (Napier et al. 1988 [DIRS 157927]). In addition, the scenario in this model is similar to that used in RESRAD.

- NUREG/CR-3332 (Till and Meyer 1983 [DIRS 101895])—This NRC document provides systematic methods for performing generic radiological assessments. This document is cited in newer models, including GENII/GENII-S and RESRAD. This model was not compared because it is similar to newer models.

- NCRP-76 (NCRP 1984 [DIRS 103784]) - This document, produced by the National Council on Radiation Protection and Measurements, provides systematic methods for performing generic radiological assessments. This document is cited in newer models, including GENII/GENII-S and RESRAD, and was not compared because it is similar to newer models.

- BIOTRAC (Zach et al. 1996 [DIRS 103831])—This document, produced by Atomic Energy of Canada Limited, Whiteshell Laboratories, describes the Canadian biosphere model for assessing the radiological consequences of radioactive waste disposal. This model was developed in association with an environmental impact statement for a postclosure assessment case. The Canadian biosphere is considerably different from Yucca Mountain, so this model was not used in the comparisons.

- Swedish Biosphere Model (Karlsson et al. 2001 [DIRS 159470])—This document, produced by the Swedish Nuclear Fuel and Waste Management Company, describes a site-specific biosphere model for a Swedish geological repository. Several release 
scenarios are considered, including a groundwater release, but not a volcanic release. Because this model is similar to BIOMASS ERB2A (BIOMASS 2000 [DIRS 154522]), it was not used in the comparisons.

\subsection{COMPARISON OF BIOSPHERE CONCEPTUAL MODELS}

The biosphere conceptual models for the groundwater (Section 6.3.1) and volcanic ash scenarios (Section 6.3.2) are based on site-specific biosphere FEPs summarized in Section 6.2 and further discussed in Sections 6.3.4 and 6.7.1. To validate the ERMYN, the biosphere conceptual model, assessment context, scenarios, submodels, and pathways were examined and compared with the validation models. This Section documents the results of the comparisons.

\subsubsection{Review of Biosphere Assessment Context}

The assessment context is the general overview of the framework and the objective of the modeling problem, including justification of why a particular assessment approach is suitable for the intended purpose. Among the five validation models (Section 7.1.3), only two are specific for a groundwater scenario and a geological repository: BIOMASS ERB2A (BIOMASS 2000 [DIRS 154522]) and EPRI-YM (Smith et al. 1996 [DIRS 101085]). The authors of these models provide details of the conceptual models, including the biosphere system identification, justification, and description. GENII-S, RESRAD, and NCRP-129 are generic models that do not have a specific assessment context and, therefore, were not compared in this section.

Nine aspects of the assessment context for the ERMYN (groundwater scenario) were compared with those from the BIOMASS ERB2A and EPRI-YM models (Table 7.2-1). The comparison indicated that, while the purpose of the models differ, they have many identical or equivalent aspects, and the only major difference is the lower amount of locally produced foodstuffs in the ERMYN (societal assumption). In the BIOMASS ERB2A and EPRI-YM models, most foodstuffs are locally produced. However, consumption rates for locally produced food in the ERMYN are based on a site-specific survey, which shows that only a small percentage of the Amargosa population are farmers, that the population imports most of their food, and that agricultural production is limited to a few crops.

The assessment endpoint for the ERMYN is a TEDE based on the ICRP-26/30 dosimetric methodology (10 CFR 63.2 [DIRS 156605]). Therefore, the human dosimetric model and the corresponding dose conversion factors and dose coefficients should be compatible with this methodology (e.g., Eckerman et al. 1988 [DIRS 101069]; Eckerman and Ryman 1993 [DIRS 107684]), even though this is an older method than is used in the BIOMASS and EPRI-YM models. 
Table 7.2-1. Assessment Context for the ERMYN, BIOMASS ERB2A, and EPRI-YM Biosphere Models for Groundwater Contamination

\begin{tabular}{|c|c|c|c|c|}
\hline Issue & ERMYN $^{\mathrm{a}}$ & BIOMASS ERB2A $^{b}$ & EPRI-YM $^{\mathrm{c}}$ & Comparison \\
\hline $\begin{array}{l}\text { Assessment } \\
\text { Purpose }\end{array}$ & $\begin{array}{l}\text { Develop dose } \\
\text { assessment capability } \\
\text { for the TSPA-LA } \\
\text { model. }\end{array}$ & $\begin{array}{l}\text { Develop reference } \\
\text { biosphere modeling } \\
\text { capabilities for an } \\
\text { agricultural well } \\
\text { scenario. }\end{array}$ & $\begin{array}{l}\text { Develop biosphere } \\
\text { model to facilitate } \\
\text { independent dose } \\
\text { assessment for the } \\
\text { Yucca Mountain } \\
\text { repository. }\end{array}$ & $\begin{array}{l}\text { ERMYN and EPRI are } \\
\text { equivalent; BIOMASS } \\
\text { is different. }\end{array}$ \\
\hline $\begin{array}{l}\text { Assessment } \\
\text { Endpoint }\end{array}$ & $\begin{array}{l}\text { BDCF based on TEDE } \\
\text { for RMEI. }\end{array}$ & $\begin{array}{l}\text { Annual individual } \\
\text { effective dose for } \\
\text { critical groups. }\end{array}$ & $\begin{array}{l}\text { Annual individual } \\
\text { effective dose to } \\
\text { members of } \\
\text { hypothetical critical } \\
\text { groups. }\end{array}$ & $\begin{array}{l}\text { All three are } \\
\text { equivalent: ERMYN } \\
\text { uses TEDE from } \\
\text { ICRP } 30 \text { (ICRP } 1979 \\
\text { [DIRS 110386]; } \\
\text { ICRP 1980 } \\
\text { [DIRS 110351]; } \\
\text { ICRP 1981 } \\
\text { [DIRS 110352]) for the } \\
\text { RMEI; BIOMASS and } \\
\text { EPRI use effective } \\
\text { dose from ICRP } 72 \\
\text { (ICRP 1996 } \\
\text { [DIRS 152446]) for } \\
\text { critical groups. }\end{array}$ \\
\hline $\begin{array}{l}\text { Assessment } \\
\text { Philosophy }\end{array}$ & $\begin{array}{l}\text { All parameters are } \\
\text { developed based on a } \\
\text { reasonable, but } \\
\text { conservative } \\
\text { approach, "equitable" } \\
\text { if possible, and } \\
\text { "cautious" when } \\
\text { unsure. }\end{array}$ & $\begin{array}{l}\text { "Equitable" except for } \\
\text { definition of the critical } \\
\text { group, which should } \\
\text { invoke a "cautious" } \\
\text { approach. }\end{array}$ & $\begin{array}{l}\text { "Cautious" for critical } \\
\text { groups, "equitable" for } \\
\text { other aspects. }\end{array}$ & $\begin{array}{l}\text { All three are } \\
\text { equivalent: BIOMASS } \\
\text { and EPRI are cautious } \\
\text { for human receptor, } \\
\text { while ERMYN is } \\
\text { equitable for human } \\
\text { receptor. }\end{array}$ \\
\hline Repository Type & $\begin{array}{l}\text { Deep repository for } \\
\text { long-lived solid } \\
\text { radioactive waste. }\end{array}$ & $\begin{array}{l}\text { Deep repository for } \\
\text { long-lived solid } \\
\text { radioactive waste. }\end{array}$ & $\begin{array}{l}\text { Deep repository for } \\
\text { long-lived solid } \\
\text { radioactive waste. }\end{array}$ & All three are the same. \\
\hline Site Context & $\begin{array}{l}\text { Specific to the } \\
\text { Amargosa Valley; } \\
\text { groundwater use, } \\
\text { limited climate change. }\end{array}$ & $\begin{array}{l}\text { Generic inland } \\
\text { repository; aquifer at } \\
\text { accessible depth, no } \\
\text { biosphere change. }\end{array}$ & $\begin{array}{l}\text { Vicinity of Yucca } \\
\text { Mountain; } \\
\text { groundwater use, } \\
\text { present climate. }\end{array}$ & $\begin{array}{l}\text { All three are } \\
\text { equivalent: ERMYN } \\
\text { and EPRI are site- } \\
\text { specific; BIOMASS is } \\
\text { generic. }\end{array}$ \\
\hline $\begin{array}{l}\text { Geosphere- } \\
\text { Biosphere } \\
\text { Interface }\end{array}$ & $\begin{array}{l}\text { A well from which } \\
\text { contaminated } \\
\text { groundwater is } \\
\text { pumped; used for } \\
\text { drinking, agriculture, } \\
\text { and domestic } \\
\text { purposes. }\end{array}$ & $\begin{array}{l}\text { A well intruding into } \\
\text { aquifer plume with } \\
\text { pumping at a rate } \\
\text { consistent with } \\
\text { domestic and } \\
\text { agricultural use. }\end{array}$ & $\begin{array}{l}\text { A deep well sunk into } \\
\text { aquifer adjacent to the } \\
\text { repository footprint. }\end{array}$ & All three are the same. \\
\hline Source Term & $\begin{array}{l}\text { Constant unit } \\
\text { concentration for each } \\
\text { radionuclide }\left(\mathrm{Bq} / \mathrm{m}^{3} \text { or }\right. \\
\mathrm{pCi} / \mathrm{L}) \text {. }\end{array}$ & $\begin{array}{l}\text { Constant unit } \\
\text { concentration for each } \\
\text { radionuclide }\left(\mathrm{Bq} / \mathrm{m}^{3}\right) \text {. }\end{array}$ & $\begin{array}{l}\text { Constant radionuclide } \\
\text { flux from the well } \\
\text { (mol/year), which can } \\
\text { be converted to } \\
\left(\mathrm{Bq} / \mathrm{m}^{3}\right) .\end{array}$ & All three are the same. \\
\hline
\end{tabular}


Table 7.2-1. Assessment Context for the ERMYN, BIOMASS ERB2A, and EPRI-YM Biosphere Models for Groundwater Contamination (Continued)

\begin{tabular}{|c|c|c|c|c|}
\hline Issue & ERMYN $^{a}$ & BIOMASS ERB2A $^{b}$ & EPRI-YM $^{c}$ & Comparison \\
\hline $\begin{array}{l}\text { Societal } \\
\text { Assumptions }\end{array}$ & $\begin{array}{l}\text { Current lifestyle of } \\
\text { Amargosa Valley } \\
\text { residents; rural } \\
\text { community, vegetable } \\
\text { garden, farm animals, } \\
\text { and fishponds. Small } \\
\text { fraction of foodstuffs } \\
\text { locally produced. }\end{array}$ & $\begin{array}{l}\text { Agricultural } \\
\text { community, using } \\
\text { modern cultivation and } \\
\text { animal husbandry } \\
\text { practices. Community } \\
\text { capable of producing a } \\
\text { high proportion of the } \\
\text { total diet of most } \\
\text { foodstuffs. }\end{array}$ & $\begin{array}{l}\text { All foods assumed to } \\
\text { be produced locally } \\
\text { using modern farming } \\
\text { practices, consistent } \\
\text { with the modern well } \\
\text { abstraction } \\
\text { technology. }\end{array}$ & $\begin{array}{l}\text { The three differ: } \\
\text { ERMYN uses site- } \\
\text { specific data; } \\
\text { BIOMASS and EPRI } \\
\text { assume all foodstuffs } \\
\text { locally produced. }\end{array}$ \\
\hline Time Frame & $\begin{array}{l}\text { Up to } 10,000 \text { years, up } \\
\text { to } 1 \text { million years. }\end{array}$ & Up to 1 million years. & Up to 1 million years. & $\begin{array}{l}\text { All three are the same } \\
\text { within the model } \\
\text { applicability and } \\
\text { limitations. }\end{array}$ \\
\hline
\end{tabular}

a This report.

b BIOMASS (2000 [DIRS 154522]).

c Smith et al. (1996 [DIRS 101085]).

d Dependent upon applicability of the reference biosphere, as defined for this model, to represent the future biospheres.

BDCF=biosphere dose conversion factor; ERMYN=Environmental Radiation Model for Yucca Mountain, Nevada; $\mathrm{LA}=$ =license application; RMEI=reasonably maximally exposed individual; TEDE=total effective dose equivalent; TSPA=total system performance assessment

The human receptor for the ERMYN is the RMEI (10 CFR 63.312 [DIRS 156605]), although the model can be used for other receptors. In the BIOMASS and EPRI-YM models, the human receptor is the critical group. In BIOMASS ERB2A, several groups are considered in finding the most highly exposed critical group.

Two assessment philosophies, cautious and equitable, are used in the biosphere models. The cautious assessment philosophy is based on the assumption that the disposal of radioactive waste represents an involuntary risk from a man-made source from which future generations will derive no benefit (Smith et al. 1996 [DIRS 101085], Section 3.2.1). This assessment philosophy is applied to the critical groups in the BIOMASS and EPRI-YM models. In the ERMYN, parameters for which there is a lack of information (e.g., translocation factor) are developed based on the cautious assessment philosophy. The equitable assessment philosophy is based on the assumption that radioactive waste disposal constitutes a health risk to present and future generations, and that this health risk is similar to other risks that society chooses to tolerate (Smith et al. 1996 [DIRS 101085], Section 3.2.2). In the ERMYN, the equitable assessment philosophy is applied to developing input parameters for which there is sufficient information.

The three biosphere models are all specific to a deep geologic repository with a possible radionuclide release through groundwater contamination. Although groundwater transport is not the focus of these studies, an agricultural well pumping contaminated groundwater is the interface between the geosphere and biosphere. A source term of unit concentration of a radionuclide in the groundwater is used to evaluate the biosphere contribution. This indicates that the approach of separating the source term and biosphere contribution is reasonable and acceptable. 


\subsubsection{Consideration of Scenarios, Submodels, and Pathways}

The exposure scenarios in the ERMYN arise from radionuclide releases in groundwater and volcanic ash. For the groundwater exposure scenario, groundwater is the only source of water for people living in the Amargosa Valley. Other dose assessment models (BIOMASS ERB2A and EPRI-YM) consider this scenario (Section 7.2.1). The validation models do not include a volcanic ash exposure scenario. The RESRAD and NCRP-129 models include onsite soil contamination, which is similar to the volcanic ash deposited scenario, and these two models are compared with the ERMYN for that scenario.

The biosphere conceptual model is based on selected biosphere components (i.e., FEPs), which are the fundamental elements of the submodels. Radionuclide transfer interaction matrices are used in the specific biosphere models (BIOMASS ERB2A and EPRI-YM), and are all similar. The generic biosphere models (GENII-S, RESRAD, and NCRP-129) do not use an interaction matrix, but present their conceptual models. However, their mathematical models are similar to those that use the interaction matrix. Typical biosphere components include groundwater, surface soil, air, plants, animals, fish, and human receptors. Some models, developed for wetter environments, include perennial surface water, sediments, and source terms in deep soil. These components are not used in the ERMYN because they are not present in the arid Yucca Mountain region.

The human exposure pathways and the associated submodels for the five validation models and the two Yucca Mountain models (TSPA-SR and TSPA-LA) were compared (Table 7.2-2). The results of this comparison indicate that the ERMYN includes all but three of the pathways in the other models. Justification for excluding air submersion and water immersion is given in Section 7.4.8. The ingestion of animal offal is excluded because there is no indication of animal offal in the diet of Amargosa residents. All seven models include most of the exposure pathways, although some pathways are only used in a few models. Detailed comparisons of the ERMYN submodels with the submodels in the validation models are described in the next section.

Table 7.2-2. Pathways and Submodels in Seven Biosphere Models

\begin{tabular}{|c|c|c|c|c|c|c|c|c|c|}
\hline \multicolumn{2}{|r|}{ Pathway } & Submodel & ERMYN & $\begin{array}{l}\text { YMP. } \\
\text { SR }\end{array}$ & $\begin{array}{l}\text { GENII- } \\
\text { S }\end{array}$ & $\begin{array}{l}\text { BIO- } \\
\text { MASS }\end{array}$ & $\begin{array}{l}\text { EPRI- } \\
\text { YM }\end{array}$ & $\begin{array}{l}\text { RES- } \\
\text { RAD }\end{array}$ & $\begin{array}{l}\text { NCRP. } \\
129\end{array}$ \\
\hline \multirow{3}{*}{$\begin{array}{l}\text { External } \\
\text { exposure }\end{array}$} & Contaminated soil & \multirow{3}{*}{ Soil, External } & $x$ & $x$ & $x$ & $X$ & $x$ & $X$ & $X$ \\
\hline & Air submersion & & - & - & $\mathrm{X}$ & - & - & - & - \\
\hline & Water immersion & & - & - & $X$ & $X$ & - & - & - \\
\hline \multirow[t]{3}{*}{$\begin{array}{l}\text { Inhalation } \\
\text { dose }\end{array}$} & $\begin{array}{l}\text { Resuspended soil (air } \\
\text { dust) }\end{array}$ & \multirow[t]{3}{*}{ Air, Inhalation } & $x$ & $x$ & $x$ & $\mathrm{X}$ & $\mathrm{X}$ & $x$ & $\mathrm{X}$ \\
\hline & $\begin{array}{l}\text { Radioactive gas } \\
\left({ }^{14} \mathrm{C},{ }^{222} \mathrm{Rn}\right)\end{array}$ & & $\mathrm{X}$ & - & - & - & - & $\mathrm{X}$ & $\mathrm{X}$ \\
\hline & Water evaporation & & $X$ & - & - & $\mathrm{X}$ & - & - & - \\
\hline \multirow{8}{*}{$\begin{array}{l}\text { Ingestion } \\
\text { dose }\end{array}$} & Soil & Soil, Ingestion & $\mathrm{X}$ & $\mathrm{X}$ & $\mathrm{X}$ & $\mathrm{X}$ & $\mathrm{X}$ & $\mathrm{X}$ & $\mathrm{X}$ \\
\hline & Drinking water & Ingestion & $X$ & $\mathrm{X}$ & $X$ & $X$ & $\mathrm{X}$ & $X$ & - \\
\hline & Leafy vegetables & \multirow[t]{4}{*}{ Plant, Ingestion } & $\mathrm{X}$ & $X$ & $X$ & $\mathrm{X}$ & $\mathrm{X}$ & $\mathrm{X}$ & $\mathrm{X}$ \\
\hline & Root vegetables & & $\mathrm{X}$ & $\mathrm{X}$ & $\mathrm{X}$ & $\mathrm{X}$ & $\mathrm{X}$ & $\mathrm{X}$ & $\mathrm{X}$ \\
\hline & Fruit & & $\mathrm{X}$ & $X$ & $\mathrm{X}$ & - & $\mathrm{X}$ & - & $\mathrm{X}$ \\
\hline & Grain & & $\mathrm{X}$ & $X$ & $\mathrm{X}$ & $\mathrm{X}$ & $\mathrm{X}$ & - & $\mathrm{X}$ \\
\hline & Fresh feed for cows & \multirow[t]{2}{*}{ Plant } & $\mathrm{X}$ & $\mathrm{X}$ & $\mathrm{X}$ & $\mathrm{X}$ & $\mathrm{X}$ & $\mathrm{X}$ & $\mathrm{X}$ \\
\hline & Stored feed for birds & & $\mathrm{X}$ & $\mathrm{X}$ & $\mathrm{X}$ & - & $\mathrm{X}$ & - & - \\
\hline
\end{tabular}


Table 7.2-2. Pathways and Submodels in Seven Biosphere Models (Continued)

\begin{tabular}{|c|c|c|c|c|c|c|c|c|c|}
\hline \multicolumn{2}{|r|}{ Pathway } & \multirow{2}{*}{$\begin{array}{c}\text { Submodel } \\
\text { Animal, Ingestion }\end{array}$} & \multirow{2}{*}{$\begin{array}{l}\text { ERMYN } \\
X\end{array}$} & \multirow{2}{*}{$\begin{array}{c}\text { YMP. } \\
\text { SR } \\
X\end{array}$} & \multirow{2}{*}{\begin{tabular}{|c} 
GENII- \\
$\mathbf{S}$ \\
$\mathrm{X}$
\end{tabular}} & \multirow{2}{*}{$\begin{array}{l}\text { BIO- } \\
\text { MASS } \\
X\end{array}$} & \multirow{2}{*}{$\begin{array}{c}\text { EPRI- } \\
\text { YM } \\
X\end{array}$} & \multirow{2}{*}{$\begin{array}{l}\text { RES- } \\
\text { RAD } \\
X\end{array}$} & \multirow{2}{*}{$\begin{array}{c}\text { NCRP. } \\
129 \\
\end{array}$} \\
\hline Ingestion & Meat & & & & & & & & \\
\hline dose & \begin{tabular}{|l|} 
Milk \\
\end{tabular} & & $\mathrm{X}$ & $\mathrm{X}$ & $\mathrm{X}$ & $\mathrm{X}$ & $\mathrm{X}$ & $\mathrm{X}$ & $\mathrm{X}$ \\
\hline & Offal & & - & - & - & $\mathrm{X}$ & $\mathrm{X}$ & - & - \\
\hline & Poultry & & $\mathrm{X}$ & $X$ & $X$ & - & $X$ & - & - \\
\hline & Eggs & & $\mathrm{X}$ & $\mathrm{X}$ & $\mathrm{X}$ & - & $\mathrm{X}$ & - & - \\
\hline & Fish & Fish, Ingestion & $\mathrm{X}$ & $\mathrm{X}$ & $\mathrm{X}$ & $\mathrm{X}$ & $X$ & $\mathrm{X}$ & - \\
\hline Total Dose & All & All & $x$ & $x$ & $x$ & $x$ & $X$ & $x$ & $X$ \\
\hline
\end{tabular}

Source: This table is from Section 6 of this report, CRWMS M\&O (2001 [DIRS 152539]), Napier et al. (1988 [DIRS 157927]), BIOMASS (2000 [DIRS 154522]), Smith et al. (1996 [DIRS 101085]), Yu et al. (2001 [DIRS 159465]), and NCRP (1999 [DIRS 155894]).

ERMYN=Environmental Radiation Model for Yucca Mountain, Nevada

\subsection{COMPARISON OF BIOSPHERE MATHEMATICAL SUBMODELS}

To validate the ERMYN, details of the mathematical representations of the biosphere processes in the ERMYN are compared with similar representations in the validation models (Section 7.1.2). Detailed comparisons, given in the following sections, begin with a summary table (Table 7.3-1) that lists all items compared. For each submodel, the comparison focuses on the core part of the submodels (Sections 6.4 and 6.5). Equation derivations and simple calculations (e.g., unit conversion and summations) are excluded.

Table 7.3-1. Summary of Mathematical Model Comparisons

\begin{tabular}{|c|c|c|c|c|}
\hline Submodel & Table & Item Compared & Equation & Comparison Result \\
\hline \multirow[t]{2}{*}{ Soil } & $7.3-2$ & $\begin{array}{l}\text { Radionuclide concentration in } \\
\text { surface soil }\end{array}$ & $\begin{array}{l}6.4 .1-2 \\
6.4 .1-4 \\
6.4 .1-9 \\
\end{array}$ & $\begin{array}{l}\text { All models use a method equivalent to the } \\
\text { ERMYN. }\end{array}$ \\
\hline & 7.3-3 & $\begin{array}{l}\text { Removal coefficients for } \\
\text { surface soil }\end{array}$ & $\begin{array}{l}\text { 6.4.1-10, } \\
6.4 \cdot 1-11\end{array}$ & Same as above. \\
\hline \multirow[t]{3}{*}{ Air } & $7.3-4$ & $\begin{array}{l}\text { Soil resuspension in air } \\
\text { submodel }\end{array}$ & $\begin{array}{l}6.4 .2-1 \\
6.4 .2-2 \\
6.5 .2-1 \\
6.5 .2-2 \\
\end{array}$ & Same as above. \\
\hline & $7.3-5$ & $\begin{array}{l}\text { Radon release from radium } \\
\text { contaminated soil }\end{array}$ & $\begin{array}{l}6.4 .2-4 \\
6.4 .2-7 \\
6.5 .2-8\end{array}$ & $\begin{array}{l}\text { Only RESRAD and NCRP-129 include the } \\
\text { radon pathway. The ERMYN uses the } \\
\text { NCRP-129 method, which differs from the } \\
\text { RESRAD method. }\end{array}$ \\
\hline & 7.3-6 & $\begin{array}{l}\text { Radionuclide concentration } \\
\text { indoors from the operation of } \\
\text { evaporative coolers }\end{array}$ & $6.4 .2-3$ & No validation models include this pathway. \\
\hline \multirow[t]{3}{*}{ Plant } & 7.3-7 & $\begin{array}{l}\text { Crop contamination due to root } \\
\text { uptake }\end{array}$ & $\begin{array}{l}\text { 6.4.3-2, } \\
6.5 .3-2 \\
\end{array}$ & $\begin{array}{l}\text { All models use the same method as the } \\
\text { ERMYN. }\end{array}$ \\
\hline & 7.3-8 & $\begin{array}{l}\text { Direct deposition on crop leaf } \\
\text { surfaces due to interception of } \\
\text { irrigation water }\end{array}$ & 6.4.3-3 & $\begin{array}{l}\text { All models except BIOMASS ERB2A use the } \\
\text { same method as the ERMYN. }\end{array}$ \\
\hline & 7.3-9 & Irrigation deposition rate & 6.4.3-4 & Same as above. \\
\hline
\end{tabular}


Table 7.3-1. Summary of Mathematical Model Comparisons (Continued)

\begin{tabular}{|c|c|c|c|c|}
\hline Submodel & Table & Item Compared & Equation & Comparison Result \\
\hline \multirow[t]{4}{*}{$\begin{array}{l}\text { Plant } \\
\text { (Cont'd) }\end{array}$} & $7.3-10$ & Interception fraction of irrigation & $6.4 .3-5$ & $\begin{array}{l}\text { All models use a fixed value independent of } \\
\text { irrigation practices. }\end{array}$ \\
\hline & 7.3-11 & $\begin{array}{l}\text { Direct deposition on leaf } \\
\text { surfaces due to interception of } \\
\text { resuspended soil }\end{array}$ & $\begin{array}{l}\text { 6.4.3-6, } \\
6.5 .3-3\end{array}$ & $\begin{array}{l}\text { The ERMYN uses the same method as } \\
\text { GENII-S and RESRAD. EPRI-YM, BIOMASS } \\
\text { ERB2A, and NCRP-129 use a different } \\
\text { method. }\end{array}$ \\
\hline & 7.3-12 & Dust deposition rate & $\begin{array}{l}\text { 6.4.3-7, } \\
6.5 .3-4\end{array}$ & Same as above. \\
\hline & 7.3-13 & $\begin{array}{l}\text { Interception fraction for } \\
\text { resuspended soil }\end{array}$ & $\begin{array}{l}\text { 6.4.3-8, } \\
6.5 .3-5\end{array}$ & Same as above. \\
\hline \multirow[t]{4}{*}{ Animal } & 7.3-14 & $\begin{array}{l}\text { Animal product contamination } \\
\text { due to animal feed }\end{array}$ & $\begin{array}{l}6.4 .4-2 \\
6.5 .4-2\end{array}$ & Same as above. \\
\hline & 7.3-15 & $\begin{array}{l}\text { Animal product contamination } \\
\text { due to drinking water }\end{array}$ & $6.4 .4-3$ & Same as above. \\
\hline & 7.3-16 & $\begin{array}{l}\text { Animal product contamination } \\
\text { due to soil ingestion }\end{array}$ & $\begin{array}{l}\text { 6.4.4-4, } \\
6.5 .4-3\end{array}$ & All models except GENII-S use this process. \\
\hline & 7.3-17 & $\begin{array}{l}\text { Animal product contamination } \\
\text { due to dust inhalation }\end{array}$ & - & $\begin{array}{l}\text { This process is only included in the BIOMASS } \\
\text { ERB2A model. }\end{array}$ \\
\hline Fish & 7.3-18 & $\begin{array}{l}\text { Fish contamination due to } \\
\text { fishpond water }\end{array}$ & $\begin{array}{l}6.4 .5-1 \\
6.4 .5-2\end{array}$ & $\begin{array}{l}\text { All models use the same method as the } \\
\text { ERMYN, except for not including evaporation } \\
\text { of fishpond water. }\end{array}$ \\
\hline \multirow[t]{5}{*}{${ }^{14} \mathrm{C}$} & 7.3-19 & $\begin{array}{l}{ }^{14} \mathrm{C} \text { special submodel for soil } \\
\text { contamination }\end{array}$ & 6.4.6-1 & $\begin{array}{l}\text { ERMYN and RESRAD use the same method; } \\
\text { GENII-S and BIOMASS ERB2A use different } \\
\text { methods; EPRI-YM and NCRP-129 did not } \\
\text { include }{ }^{14} \mathrm{C} \text {. }\end{array}$ \\
\hline & $7.3-20$ & $\begin{array}{l}{ }^{14} \mathrm{C} \text { special submodel for air } \\
\text { contamination }\end{array}$ & 6.4.6-3 & Same as above. \\
\hline & $7.3-21$ & $\begin{array}{l}{ }^{14} \mathrm{C} \text { special submodel for plant } \\
\text { contamination }\end{array}$ & 6.4.6-6 & Same as above. \\
\hline & 7.3-22 & $\begin{array}{l}{ }^{14} \mathrm{C} \text { special submodel for animal } \\
\text { product contamination }\end{array}$ & 6.4.6-7 & Same as above. \\
\hline & $7.3-22$ & $\begin{array}{l}{ }^{14} \mathrm{C} \text { special submodel for animal } \\
\text { product contamination }\end{array}$ & 6.4.6-7 & Same as above. \\
\hline External & 7.3-23 & $\begin{array}{l}\text { External exposure to } \\
\text { contaminated soil }\end{array}$ & $\begin{array}{l}\text { 6.4.7-1, } \\
6.5 .5-1\end{array}$ & $\begin{array}{l}\text { All models use the same method as the } \\
\text { ERMYN, except for not using exposure time } \\
\text { budgets. Few models consider air } \\
\text { submersion and water immersion. }\end{array}$ \\
\hline Inhalation & $7.3-24$ & Inhalation dose & $\begin{array}{l}\text { 6.4.8-2, } \\
6.4 .8-3 \\
6.4 .8-4 \\
6.4 .8-7 \\
6.5 .6-2 \\
6.5 .6-3 \\
\end{array}$ & $\begin{array}{l}\text { All models use the same method as the } \\
\text { ERMYN for air particle inhalation, except that } \\
\text { exposure time budgets are not included. Few } \\
\text { validation models use radon and }{ }^{14} \mathrm{C} \text { gas } \\
\text { inhalation pathways, and none include } \\
\text { evaporative coolers. }\end{array}$ \\
\hline \multirow[t]{3}{*}{ Ingestion } & $7.3-25$ & Water ingestion & $6.4 .9-2$ & $\begin{array}{l}\text { All models use the same method as the } \\
\text { ERMYN, but the number of ingestion } \\
\text { pathways differs among models. }\end{array}$ \\
\hline & $7.3-26$ & Crop ingestion & $\begin{array}{l}\text { 6.4.9-3, } \\
6.5 .7-2 \\
\end{array}$ & Same as above. \\
\hline & $7.3-27$ & Animal product ingestion & $\begin{array}{l}\text { 6.4.9-4, } \\
6.5 .7-3\end{array}$ & Same as above. \\
\hline
\end{tabular}


Table 7.3-1. Summary of Mathematical Model Comparisons (Continued)

\begin{tabular}{|c|c|c|c|c|}
\hline Submodel & Table & Item Compared & Equation & Comparison Result \\
\hline \multirow{2}{*}{$\begin{array}{l}\text { Ingestion } \\
\text { (Cont'd) }\end{array}$} & $7.3-28$ & Fish ingestion & $6.4 .9-5$ & Same as above. \\
\hline & $7.3-29$ & Soil ingestion & $\begin{array}{l}\text { 6.4.9-6, } \\
\text { 6.5.7-4 }\end{array}$ & Same as above. \\
\hline
\end{tabular}

ERMYN=Environmental Radiation Model for Yucca Mountain, Nevada

\subsubsection{Comparison of Surface Soil Submodels}

The surface soil submodels for the groundwater (Section 6.4.1) and volcanic ash (Section 6.5.1) scenarios are different because they have different radionuclide source terms. Under the groundwater scenario, long-term irrigation causes radionuclide buildup in the soil. In contrast, ash deposited on the ground contaminates the surface soil during a single volcanic eruption, but radionuclides do not build up in the surface soil. Therefore, separate comparisons are presented for each scenario.

\subsubsection{Comparison of Surface Soil Submodels for the Groundwater Scenario}

For the groundwater scenario, two items in the surface soil submodel are compared: calculations of radionuclide concentrations in the soil (Table 7.3-2) and removal coefficients (Table 7.3-3). The conversion of radionuclide concentrations in the surface soil to concentrations in the soil mass (Equations 6.4.1-5 and 6.4.1-6) are based on the fundamental relationship between mass and volume, which does not require further comparison and validation.

GENII-S, BIOMASS ERB2A, and EPRI-YM address groundwater contamination and include radionuclide buildup in the soil, although equilibrium conditions are not used directly in the dose calculations of some models. The EPRI-YM model does not present mathematical equations or solutions for long-term radionuclide buildup in the surface soil, so it is excluded from the comparison. For the RESRAD model, soil contamination is the primary source term, and although it includes irrigation, this is a secondary source calculated from radionuclide removal in the surface soil. Contaminated irrigation water is not a radionuclide source in the NCRP-129 model.

Calculations of soil concentration of radionuclides in the ERMYN (Equation 6.4.1-1) and BIOMASS models (BIOMASS 2000 [DIRS 154522], Equation 8) are based on similar differential equations and similar initial conditions for the long-term irrigation source. The analytical solution of the differential equation (Equation 6.4.1-2) gives radionuclide concentrations in the surface soil at any time. The GENII-S model does not use the same analytical solution; it uses a numerical method that gives results similar to the analytical solution (Table 7.3-2). A comparison of the treatment of decay products in the ERMYN and GENII-S models is presented in Section 7.4.2.2. The BIOMASS model documentation (BIOMASS 2000 [DIRS 154522]) does not describe the treatment of long-lived decay products in surface soils. Results of the comparison indicate that the calculations of radionuclide concentrations in the soil in the ERMYN, GENII-S, and BIOMASS models are equivalent. The only other differences in the ERMYN, GENII-S, and BIOMASS models for calculating soil concentrations involve calculating removal coefficients (Tables 7.3-2 and 7.3-3). 
The methods for calculating removal coefficients are the same in the ERMYN, GENII-S, BIOMASS, and EPRI-YM models, with two exceptions (Table 7.3-3). First, the GENII-S model does not directly include removal by erosion, but because this process can be included in the leaching removal constant by replacing it with an effective removal coefficient, the mathematical approaches are equivalent. Second, the GENII-S and BIOMASS models include harvest removal in calculating radionuclide concentrations in the soil. The ERMYN does not use a harvest removal factor (Section 6.4.1.1) because fertilization with animal manure compensates for harvest removal (Approximation 4). Based on this approximation, the mathematical expressions are equivalent.

Because the mathematical representations are the same or give equivalent results, the soil submodel for the groundwater scenario is considered validated.

\subsubsection{Comparison of Surface Soil Submodels for the Volcanic Ash Scenario}

GENII-S, RESRAD, and NCRP-129 include soil contamination in a manner applicable to the volcanic ash scenario. The radionuclide source term for these models is in units of $\mathrm{Bq} / \mathrm{m}^{3}$ or $\mathrm{Bq} / \mathrm{kg}$ in soil. Because the source term in the ERMYN is in units of $\mathrm{Bq} / \mathrm{m}^{2}$, it is converted to radionuclide concentration per mass of soil $(\mathrm{Bq} / \mathrm{kg})$ based on the depth of the surface soil on cultivated lands (Equation 6.5.1-2) or the critical thickness for ash resuspension on noncultivated lands (Equation 6.5.1-3). The conversion is based on the fundamental relationship between mass and volume, it is the same in all three models, and it does not require further comparison.

Environmental radiation dose assessments for scenarios similar to the volcanic ash scenario have not been documented in the published literature. Therefore, the approach described in Approximation 12 (the mixing of ash and soil on noncultivated lands, and the dependence of resuspended soil radionuclide concentrations on ash depth) is unique to the ERMYN. This approach is expressed in Equations 6.5.1-3 through 6.5.1-5 (Section 6.5.1). The influence of ash depth on activity concentrations in soil is expressed as the ratio of the critical thickness to the actual thickness for ash depths greater than the critical thickness. Because the critical thickness is about 1 to $3 \mathrm{~mm}$ (BSC 2004 [DIRS 169672]), it is possible that the depth of ash initially deposited at the location of the receptor would be at least twice that of the critical thickness; this method could result in a difference of more than a factor of two. Additional justification for selecting this method is required for validation.

The justification for this approach is provided in Approximation 12 (Section 6.3.2.4). If the volcanic ash deposit is thin (less than the critical thickness), all volcanic ash is readily resuspended. If volcanic ash deposit is thick (more than critical thickness), only a fraction of the volcanic ash is readily resuspended. This method is reasonable because it uses the same radionuclide concentration in volcanic ash (in volume or mass) as that in resuspended ash particles, and it eliminates overestimating airborne concentrations, which occurs if all deposited radionuclides $\left(\mathrm{Bq} / \mathrm{m}^{2}\right)$ are considered to be within the critical thickness. Based on the above comparison and this justification, the soil submodel for the volcanic scenario is validated. 


\begin{tabular}{|c|c|c|c|}
\hline Document & Mathematical Model & $\begin{array}{c}\text { Comparison with the ERMYN (Equations 6.4.1-1, } \\
6.4 .1-2,6.4 .1-4 \text {, and 6.4.1-9) }\end{array}$ & Reference \\
\hline GENII-S & 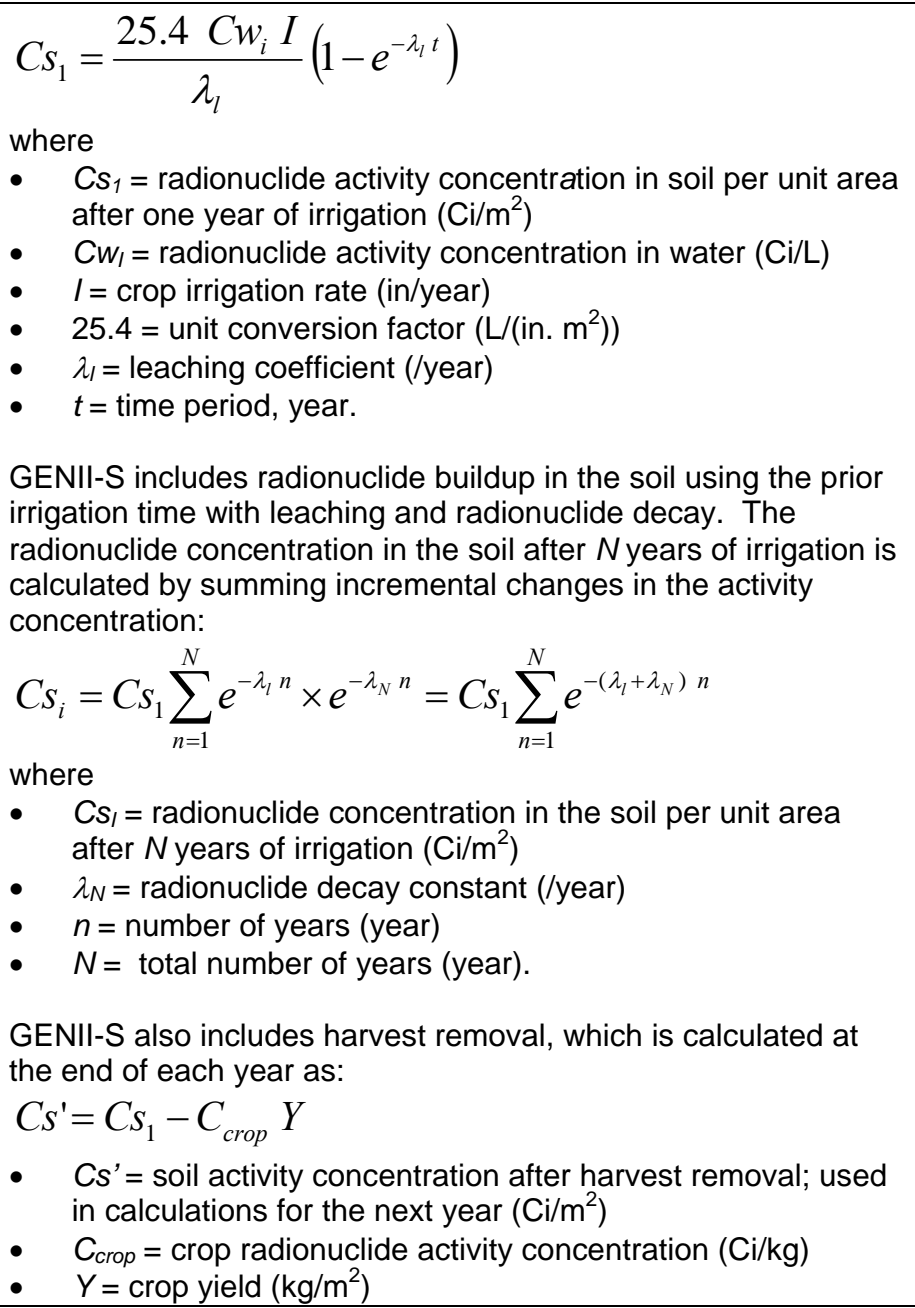 & $\begin{array}{l}\text { GENII-S includes removal by leaching and decay, } \\
\text { but not erosion, although erosion can be combined } \\
\text { with the leaching removal constant. } \\
\text { GENII-S calculates long-term radionuclide buildup } \\
\text { in the soil by combining single-year net } \\
\text { contributions with the number of prior irrigation } \\
\text { years. This method is equivalent to the analytical } \\
\text { solution (Eq. } 6.4 .1-1) \text { because the sum of the } \\
\text { exponential terms, } \sum[\exp (-\lambda)] \text { can be represented } \\
\text { by the expression [1-exp(- } \lambda \text { N)]/[1-exp(- }-\lambda)] \text {. } \\
\text { Although the GENII-S code shows that harvest } \\
\text { removal is included, this part of the model is not } \\
\text { actually executed. } \\
\text { The two approaches are different, but the result is the } \\
\text { same. In the GENII-S model for the TSPA-SR, BDCFs } \\
\text { are calculated at several irrigation time periods to find } \\
\text { the equilibrium condition (CRWMS M\&O 2001 } \\
\text { [DIRS } 153207] \text {. The ERMYN calculates only the } \\
\text { equilibrium condition. The ERMYN also calculates the } \\
\text { equilibrium concentration for long-lived decay products } \\
\text { that are also primary radionuclides (Equation } 6.4 .1-9 \text { ). } \\
\text { The GENII/GENII-S manual does not provide the } \\
\text { analytical equations for its calculation, but it calculates } \\
\text { activity concentration in the soil for the long-lived decay } \\
\text { products at a specific time. A comparison is performed } \\
\text { (Section } 7.4 .2 \text { for the equilibrium status of the long- } \\
\text { lived decay products between GENII-S and ERMYNs. }\end{array}$ & $\begin{array}{l}\text { Napier et al. 1988 } \\
\text { [DIRS 157927], } \\
\text { p. 4.57, Eq. 4.6.2. } \\
\text { Calculation } \\
\text { methods for } \\
\text { radionuclide soil } \\
\text { buildup, harvest } \\
\text { removal, } \\
\text { radionuclide decay, } \\
\text { and ingrowth are } \\
\text { from the GENII-S } \\
\text { source code, which } \\
\text { is part of the } \\
\text { GENII-S software } \\
\text { package } \\
\text { (SNL 1998 } \\
\text { [DIRS 117076]). }\end{array}$ \\
\hline
\end{tabular}




\begin{tabular}{|c|c|c|c|}
\hline Document & Mathematical Model & $\begin{array}{c}\text { Comparison with the ERMYN (Equations 6.4.1-1, } \\
6.4 .1-2,6.4 .1-4, \text { and } 6.4 .1-9)\end{array}$ & Reference \\
\hline $\begin{array}{l}\text { BIOMASS } \\
\text { ERB2A }\end{array}$ & 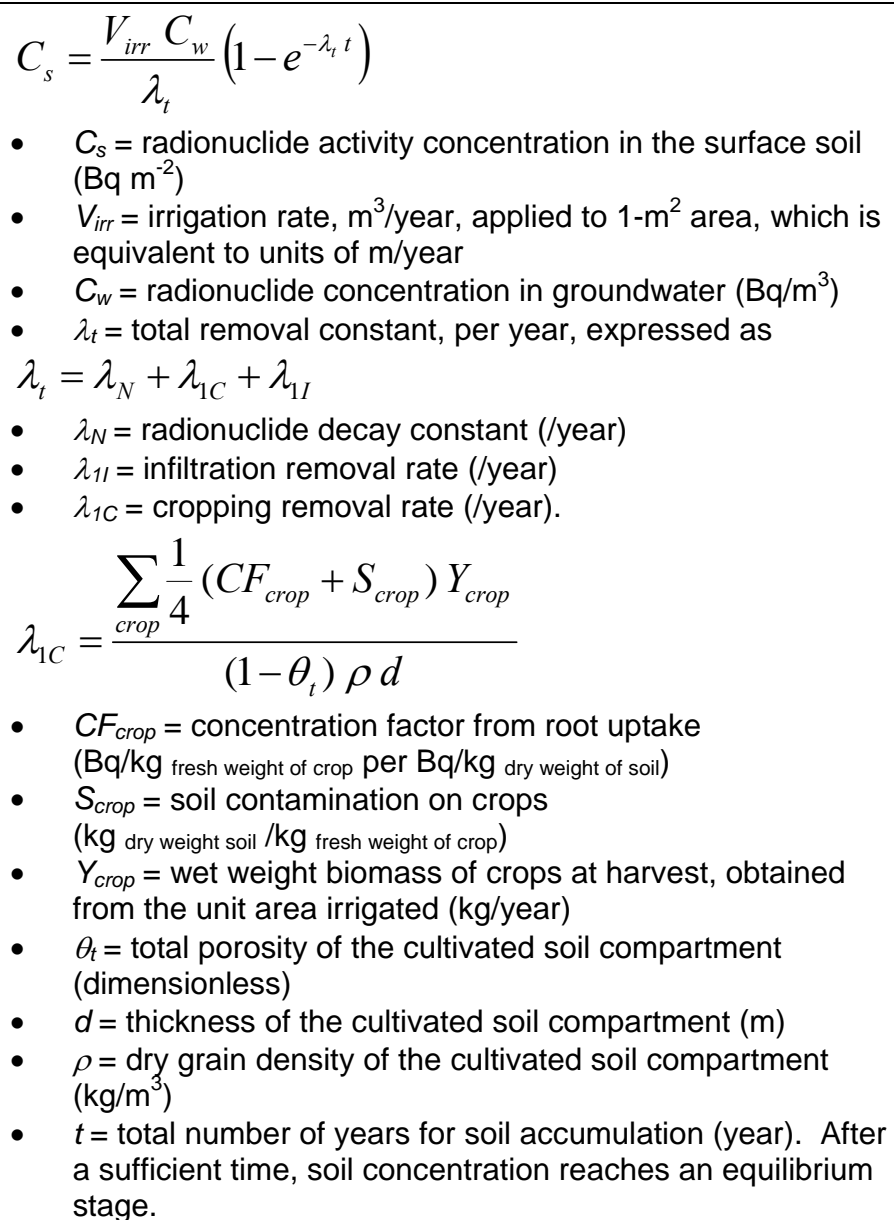 & $\begin{array}{l}\text { BIOMASS ERB2A includes leaching, harvest removal } \\
\text { (cropping), and decay removal. It also gives the } \\
\text { method for erosion removal. Harvest removal } \\
\text { calculations are based on root uptake and external } \\
\text { contamination, but not leaf uptake due to interception of } \\
\text { irrigation, which may be the major contributor for most } \\
\text { radionuclides. } \\
\text { BIOMASS ERB2A uses equilibrium conditions for dose } \\
\text { calculations (BIOMASS } 2000 \text { [DIRS } 154522] \text {, } \\
\text { pp. } 60 \text { and } 61 \text {, Tables } 22 \text { to } 25 \text { ); therefore, it is the } \\
\text { same as the ERMYN. } \\
\text { BIOMASS ERB2A is the same as the ERMYN except } \\
\text { that it includes harvest removal and excludes the } \\
\text { buildup of long-lived decay products in surface soils. } \\
\text { BIOMASS ERB2A does not describe the treatment of } \\
\text { long-lived decay products accumulation in surface soils. }\end{array}$ & $\begin{array}{l}\text { BIOMASS } 2000 \\
\text { [DIRS } 154522] \text {, } \\
\text { p. 33, Eq. } 7 \text { and } 8 . \\
\text { The differential } \\
\text { equation in Eq. } 8 \\
\text { had inconsistent } \\
\text { units for the source } \\
\text { term. It is modified } \\
\text { using irrigation rate } \\
\text { as } \mathrm{m} / \text { year and } \\
\text { surface soil } \\
\text { concentration as } \\
\text { Bq/ } \mathrm{m}^{2} \text {. Assumes a } \\
\text { constant } \\
\text { radionuclide } \\
\text { concentration in } \\
\text { groundwater over } \\
\text { the time of interest. }\end{array}$ \\
\hline EPRI-YM & $\begin{array}{l}\text { Equation for calculating radionuclide concentrations in the } \\
\text { surface soil is not given in the document. }\end{array}$ & N/A & $\begin{array}{l}\text { Smith et al. } 1996 \\
\text { [DIRS 101085], } \\
\text { Section 5. }\end{array}$ \\
\hline
\end{tabular}




\begin{tabular}{|c|c|c|c|}
\hline Document & Mathematical Model & $\begin{array}{c}\text { Comparison with the ERMYN (Equations 6.4.1-1, } \\
6.4 .1-2,6.4 .1-4 \text {, and 6.4.1-9) }\end{array}$ & Reference \\
\hline RESRAD & Soil contamination, irrigation is a secondary source. & N/A & $\begin{array}{l}\text { Yu et al. } 2001 \\
\text { [DIRS 159465]. }\end{array}$ \\
\hline NCRP-129 & Not included in the model. & N/A & $\begin{array}{l}\text { NCRP } 1999 \\
\text { [DIRS 155894]. }\end{array}$ \\
\hline
\end{tabular}

ERMYN=Environmental Radiation Model for Yucca Mountain, Nevada

Table 7.3-3. Comparison of Removal Coefficients for Surface Soil

\begin{tabular}{|c|c|c|c|}
\hline Document & Mathematical Model & $\begin{array}{l}\text { Comparison with the ERMYN } \\
\text { (Equation 6.4.1-10, and 6.4.1-11) }\end{array}$ & Reference \\
\hline GENII-S & 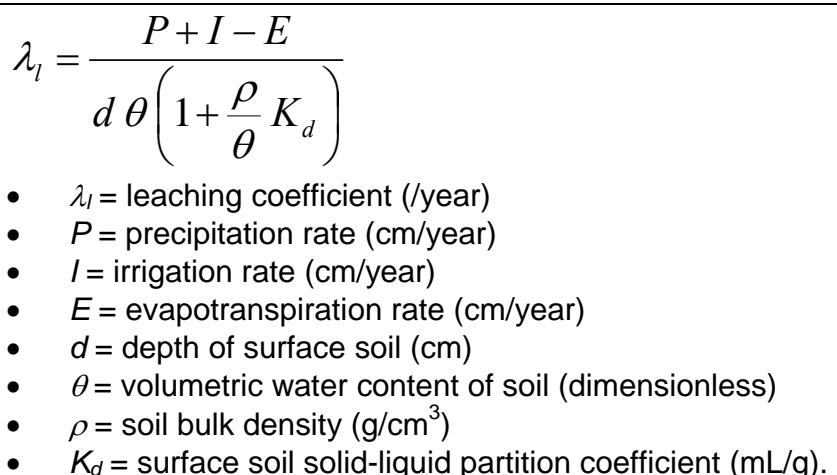 & $\begin{array}{l}\text { The formula shown in the GENII manual } \\
\text { (Eq. 4.6.3) is incorrect (CRWMS M\&O } 2001 \\
\text { [DIRS 152517]). The correct leaching } \\
\text { coefficient equation was used in the } \\
\text { TSPA-SR. The formula presented here is the } \\
\text { correct one, which is also the equation used } \\
\text { in the ERMYN, with the input parameter of } \\
\text { overwatering rate }(O W) \text { replacing the } \\
\text { equivalent }(P+I-E) \text { term. }\end{array}$ & $\begin{array}{l}\text { Napier et al. 1988 } \\
\text { [DIRS 157927]. The } \\
\text { leaching coefficient is a } \\
\text { GENII-S input parameter. It } \\
\text { is calculated using the } \\
\text { equation documented in } \\
\text { CRWMS M\&O (2001 } \\
\text { [DIRS 152517], p. 25) for the } \\
\text { TSPA-SR. The equation in } \\
\text { GENII (Napier et al. 1988 } \\
\text { [DIRS 157927], p. } 4.58 \text {, } \\
\text { Eq. 4.6.3) is not used. }\end{array}$ \\
\hline
\end{tabular}


Table 7.3-3. Comparison of Removal Coefficients for Surface Soil (Continued)

\begin{tabular}{|c|c|c|c|}
\hline Document & Mathematical Model & $\begin{array}{l}\text { Comparison with the ERMYN } \\
\text { (Equation } 6.4 .1-10 \text {, and 6.4.1-11) }\end{array}$ & Reference \\
\hline $\begin{array}{l}\text { BIOMASS } \\
\text { ERB2A }\end{array}$ & 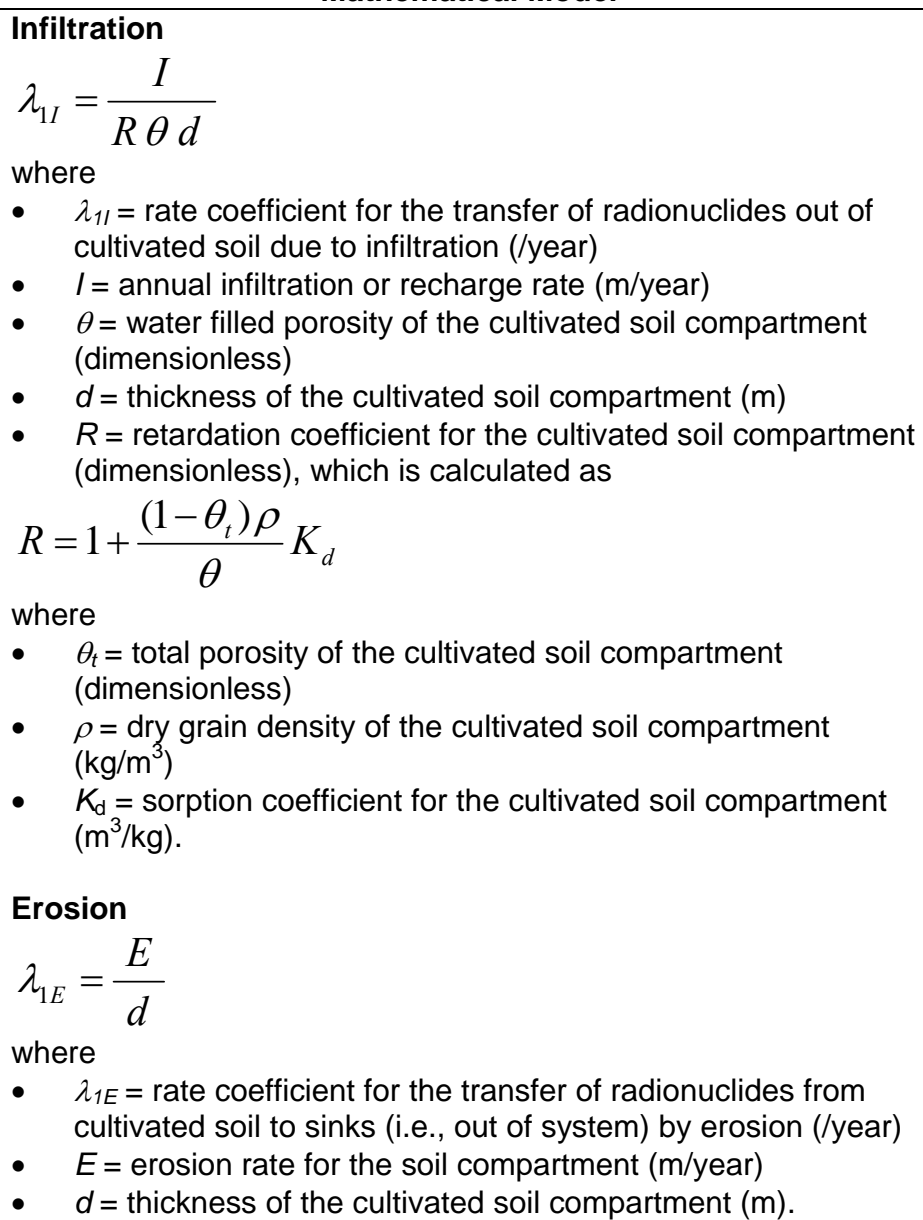 & $\begin{array}{l}\text { The leaching rate equation is the same as the } \\
\text { ERMYN with the following equivalent } \\
\text { parameters: } \\
\text { The overwatering rate (OW) in the } \\
\text { ERMYN is equivalent to annual } \\
\text { infiltration }(I) \text { in BIOMASS ERB2A } \\
\text { The soil bulk density }(\rho) \text { in the ERMYN is } \\
\text { equivalent to the cultivated soil density }(1 \\
\left.-\theta_{t}\right)(\rho) \text { in BIOMASS ERB2A }\end{array}$ & $\begin{array}{l}\text { BIOMASS 2000 } \\
\text { [DIRS } 154522], \text { pp. } 32 \text { and } \\
33 \text {, Eqs. } 4 \text { to } 6 .\end{array}$ \\
\hline
\end{tabular}




\begin{tabular}{|c|c|c|c|}
\hline Document & Mathematical Model & $\begin{array}{c}\text { Comparison with the ERMYN } \\
\text { (Equation 6.4.1-10, and 6.4.1-11) }\end{array}$ & Reference \\
\hline EPRI-YM & $\begin{array}{l}\text { EPRI-YM uses the same method as BIOMASS ERB2A. } \\
\text { Equations are the same, but parameter names may differ from } \\
\text { those used in BIOMASS. }\end{array}$ & EPRI-YM is the same as BIOMASS. & $\begin{array}{l}\text { Smith et al. } 1996 \\
\text { [DIRS 101085], Eqs. 5.2, } \\
\text { 5.3, and 5.5. }\end{array}$ \\
\hline RESRAD & $\begin{array}{l}\text { RESRAD uses contaminated soil as initial source, which can be } \\
\text { removed from surface soils to deep soil, and eventually to the } \\
\text { groundwater. }\end{array}$ & N/A & $\begin{array}{l}\text { Yu et al. } 2001 \\
\text { [DIRS 159465]. }\end{array}$ \\
\hline NCRP-129 & Not included in the model. & N/A & NCRP 1999 [DIRS 155894]. \\
\hline
\end{tabular}

ERMYN=Environmental Radiation Model for Yucca Mountain, Nevada; SR=site recommendation; TSPA=total system performance assessment 


\subsubsection{Validation of the Air Submodel}

Particle resuspension is included in the air submodel for both scenarios (Sections 6.4.2.1 and 6.5.2.1), and both scenarios include exhalation of radon from radium-contaminated soil (Sections 6.4.2.3 and 6.5.2.2). The groundwater scenario also includes generation of contaminated aerosols by evaporative coolers (Section 6.4.2.2) and the release of ${ }^{14} \mathrm{C}$ gas into the air. Validation of the modeling of the processes of particle resuspension from surface soils, radon exhalation from radium-contaminated soil, and generation of contaminated aerosols by evaporative coolers is described in the following sections, and the model for ${ }^{14} \mathrm{C}$ gas in the air is validated in Section 7.3.6.

\subsubsection{Particle Resuspension from Surface Soil}

Particle resuspension from surface soils is treated similarly for both scenarios, as both use an environment-specific mass loading and enhancement factor. The volcanic ash scenario also includes modeling of mass loading decrease following a volcanic eruption. Radionuclide concentrations in the air are calculated differently for direct deposition on crops (Equations 6.4.2-1 and 6.5.2-1) and human inhalation (Equations 6.4.2-2 and 6.5.2-2). Mass loading for calculating human inhalation exposure depends on specific environments with different levels of activity.

All five validation models include particle resuspension from surface soils, and the core parts of the submodels are the same (Table 7.3-4) because each is based on the concentration of particulate matter in air (mass loading) and each assumes that the resuspended particles and the surface soil have the same radionuclide concentration per unit mass. The differences between the validation models and the ERMYN (Table 7.3-4) are:

- BIOMASS includes a retardation coefficient, which in a system in equilibrium is the ratio of the total mass of a contaminant in a unit volume of the soil to the mass in solution in that volume of soil. (Retardation coefficient expresses how much slower a contaminant present in the water moves in the soil than does the water itself.) Typical values for the retardation coefficient are much larger than 1 for most radionuclides (based on the retardation coefficient calculation in Table 7.3-3 for BIOMASS ERB2A and input parameters in Table 6.6-3), so the part of the equation in Table 7.3-4 with the retardation term, (R-1)/R, is about equal to one. However, ${ }^{99} \mathrm{Tc}$ and ${ }^{36} \mathrm{Cl}$ have low partition coefficient $\left(K_{d}\right)$ values $(0.14 \mathrm{~L} / \mathrm{kg}$; Table 6.6-3), which results in a lower retardation coefficient $(R=1.5)$ and causes the retardation term to be about 0.33 . Thus, the radionuclide concentrations in air calculated using the two methods differs by more than a factor of two. Excluding the retardation factor from the ERMYN is justified because radionuclides initially present in soil water could attach to soil particles upon water evaporation from this layer and become resuspended. Therefore, using this factor could underestimate radionuclide concentrations in the air, and the retardation coefficient is excluded to avoid underestimating doses for low partition coefficient radionuclides (i.e., those radionuclides that are not highly sorbing and thus their concentrations in the soil water are relatively high compared to their concentrations in the soil.) However, because these radionuclides do not accumulate in the soil to a large degree, their contribution to the inhalation dose is relatively low. 
- RESRAD includes an area factor to account for the portion of contaminated land. For the groundwater scenario, the total area contaminated by long-term irrigation would be larger because of land use rotation. In the volcanic ash scenario, contaminated ash would be deposited over the entire Amargosa Valley. Thus, the area factor would be about one for both cases and the two methods are mathematically equivalent.

- RESRAD includes a cover-and-depth factor to account for the effects of burying radioactive waste. For the Yucca Mountain scenarios, all radionuclides are in the surface soil, and this factor would be set at one, so the methods are mathematically equivalent.

- None of the validation models use environment-specific mass loading. The ERMYN uses this method to account for differences in mass loading among, and uncertainty within, various environments. A numerical comparison of the ERMYN and validation model methods is presented in Section 7.4.9. The difference in total activity inhaled per day calculated by the two methods is less than a factor of two (Table 7.4-19), and, therefore, the two methods are numerically similar.

- The enhancement factor in the ERMYN (Equations 6.4.2-2 and 6.5.2-2) is taken from NCRP-129. This factor is included in the calculations of activity concentrations in air inhaled by the receptor to account for differences in activity concentrations between surface soil and resuspended soil particles. This is an environment-specific parameter with an average value of about four for the active outdoor environment, and an average of less than one for all other environments used in the ERMYN (Table 6.6-3). Including the enhancement factor causes a difference in the calculated airborne activity concentrations by a factor of less than two, and, therefore, the two methods are numerically similar.

- None of the validation models address mass loading as a function of time. The ERMYN includes a time function to account for the decrease in mass loading after a volcanic eruption (Equation 6.5.2-2), which avoids overestimating the inhalation dose. Concentrations of resuspended particles during the first year after a volcanic eruption that deposits a thin layer of ash at the location of the receptor likely would not be more than twice as high as those prior to the eruption (BSC 2004 [DIRS 169458], Section 6.2). For example, average annual concentrations of total suspended particles one year after the eruption of Mount St. Helens at six sites with less than $1 \mathrm{~mm}$ to about $10 \mathrm{~mm}$ of deposited ash were from about 0 to 90 percent higher than the year prior to the eruption (BSC 2004 [DIRS 169458], Table 6.2.2-1). However, if a large quantity of ash is deposited at the location of the receptor, concentrations in some environments could more than double the mass loading and change airborne concentrations by more than a factor of two. If the submodel does not include the mass loading time function, airborne concentrations remain at high levels throughout the period of calculation. Therefore, the addition of the time function could result in a difference of two or more for some conditions, and an evaluation and justification are needed to validate this portion of the submodel. Concentrations of airborne particles after volcanic eruptions decrease toward preeruption levels within a relatively short time. For example, concentrations of total suspended particles at six sites in Washington returned to preeruption levels within three 
to eight months after the eruption of Mount St. Helens, and concentrations decreased after other eruptions at similar rates (BSC 2004 [DIRS 169458], Section 6.3). The decrease results from consolidation of ash particles, incorporation of ash into the soil, and removal of ash by residents and natural processes. These processes also would occur in the Yucca Mountain region, although possibly at a different rate (BSC 2004 [DIRS 169458], Section 6.3). Omitting the mass loading time function results in overestimating airborne activity concentrations during the long period following a volcanic eruption for which doses will be calculated. Because the time function is based on measurement data, because the processes that cause the changes also would occur in the Yucca Mountain region, and because omission of the function results in an invalid overestimation of concentrations, this portion of the air submodel is valid for the volcanic ash scenario.

Based on above discussions, it is concluded that the calculations of particle resuspension from surface soil in the air submodel are mathematically equivalent, numerically similar, or the approach is justified because it includes site-specific or realistically predictable conditions, and, therefore, this portion of the ERMYN air submodel is validated. 


\begin{tabular}{|c|c|c|c|}
\hline Document & Mathematical Model & $\begin{array}{l}\text { Comparison with the ERMYN } \\
\text { (Equations 6.4.2-1, 6.4.2-2, 6.5.2-1, and 6.5.2-2) }\end{array}$ & Reference \\
\hline GENII-S & $\begin{array}{l}C a_{i}=C s_{i} M \\
-\quad C a_{i}=\text { radionuclide concentration in the air }\left(\mathrm{Ci} / \mathrm{m}^{3}\right) \\
\text { - } \quad C s_{I}=\text { radionuclide activity concentration in soil }\left(\mathrm{Ci} / \mathrm{m}^{2}\right) \\
\text { - } M=\text { resuspension factor }(/ \mathrm{m}), \text { calculated as } \\
M=\frac{S}{\rho_{s}} \\
\text { - } \quad S=\text { mass loading, or concentration of total resuspended } \\
\text { particles }\left(\mathrm{kg} / \mathrm{m}^{3}\right) \\
\text { - } \quad \rho_{\mathrm{s}}=\text { surface soil density }\left(\mathrm{kg} / \mathrm{m}^{2}\right) .\end{array}$ & $\begin{array}{l}\text { The GENII-S model uses average mass loading, } \\
\text { which is the same as mass loading for crops in the } \\
\text { ERMYN (Equations 6.4.2-1 and 6.5.2-1). } \\
\text { Particle resuspension for inhalation in the ERMYN } \\
\text { uses an enhancement factor and environment- } \\
\text { specific mass loading (Eqs. } 6.4 .2-2 \text { and } 6.5 .2-2) \text {. It } \\
\text { also uses time dependent mass loading during the } \\
\text { transition period after a volcanic eruption } \\
\text { (Eq. 6.5.2-3). }\end{array}$ & $\begin{array}{l}\text { Napier et al. } 1988 \\
\text { [DIRS 157927], } \\
\text { p. 4.63, Eq. 4.7.2, } \\
\text { and p. 4.64, } \\
\text { Eq. 4.7.3b. }\end{array}$ \\
\hline $\begin{array}{l}\text { BIOMASS } \\
\text { ERB2A }\end{array}$ & 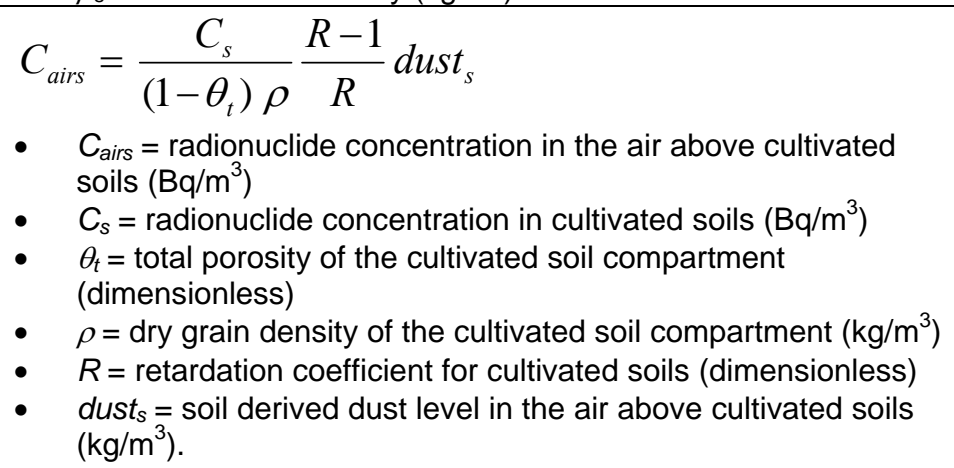 & $\begin{array}{l}\text { BIOMASS is equivalent to the GENII-S based on: } \\
\text { - The } S \text { term in GENII-S is equivalent to the dust } \\
\text { - } \quad \text { The } C_{s} / \rho_{s} \text { term in GENII-S is equivalent to the } \\
C_{s} /\left[\left(1-\theta_{t}\right) \rho\right] \text { term in BIOMASS } \\
\text { BIOMASS uses a retardation coefficient }(R) \\
\text { (defined in Table } 7.3-3) \text { which causes the } \\
\text { activity concentrations in the air to be element } \\
\text { specific. Because } R \text { is much larger than } 1 \text { for } \\
\text { most radionuclides, }(R-1) / R \text { is close to } 1 \text { for } \\
\text { most radionuclides (see the retardation } \\
\text { coefficient calculations in Table } 7.3-3) \text {. }\end{array}$ & $\begin{array}{l}\text { BIOMASS } 2000 \\
\text { [DIRS 154522], } \\
\text { p. 36, Eq. } 14 .\end{array}$ \\
\hline EPRI-YM & $\begin{array}{ll}\text { - } & C_{\text {airs }}=\text { radionuclide concentration in the air }\left(\mathrm{Bq} / \mathrm{m}^{3}\right) \\
\text { - } & C_{\text {sed }}=\text { radionuclide concentration in sediments or soils }\left(\mathrm{Bq} / \mathrm{m}^{3}\right) \\
\text { - } & \theta_{\text {tus }}=\text { total porosity of the sediment or soil compartment } \\
& (\text { dimensionless }) \\
\text { - } & \rho_{\text {gsed }}=\text { grain density of the sediment or soil compartment } \\
& \left(\mathrm{kg} / \mathrm{m}^{3}\right) \\
\text { - } & d u s t_{\text {sed }}=\text { dust level associated with sediments or soils }\left(\mathrm{kg} / \mathrm{m}^{3}\right) .\end{array}$ & EPRI-YM is similar to ERMYN. & $\begin{array}{l}\text { Smith et al. } 1996 \\
\text { [DIRS 101085], } \\
\text { p. 5-14 Eq. 5.16. }\end{array}$ \\
\hline
\end{tabular}




\begin{tabular}{|c|c|c|c|}
\hline Document & Mathematical Model & $\begin{array}{l}\text { Comparison with the ERMYN } \\
\text { (Equations 6.4.2-1, 6.4.2-2, 6.5.2-1, and 6.5.2-2) }\end{array}$ & Reference \\
\hline RESRAD & 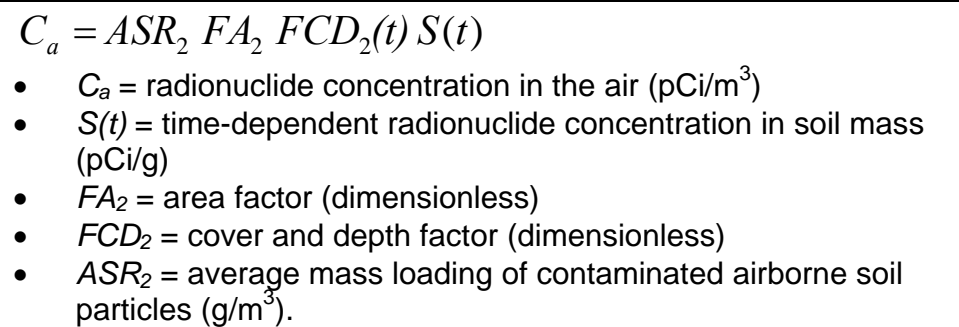 & $\begin{array}{l}\text { RESRAD uses an area factor and a cover-and- } \\
\text { depth factor. These two factors are used for } \\
\text { underground contaminants with a limited area and } \\
\text { depth of contamination, which is not used in the } \\
\text { ERMYN. Without these two factors, RESRAD is the } \\
\text { same as GENII-S. }\end{array}$ & $\begin{array}{l}\text { Yu et al. } 2001 \\
\text { [DIRS 159465], } \\
\text { p. B-6, Eq. B.1. } \\
\text { Only the parts of } \\
\text { the equation } \\
\text { applicable to } \\
\text { activity } \\
\text { concentrations in } \\
\text { the air are shown. }\end{array}$ \\
\hline NCRP-129 & 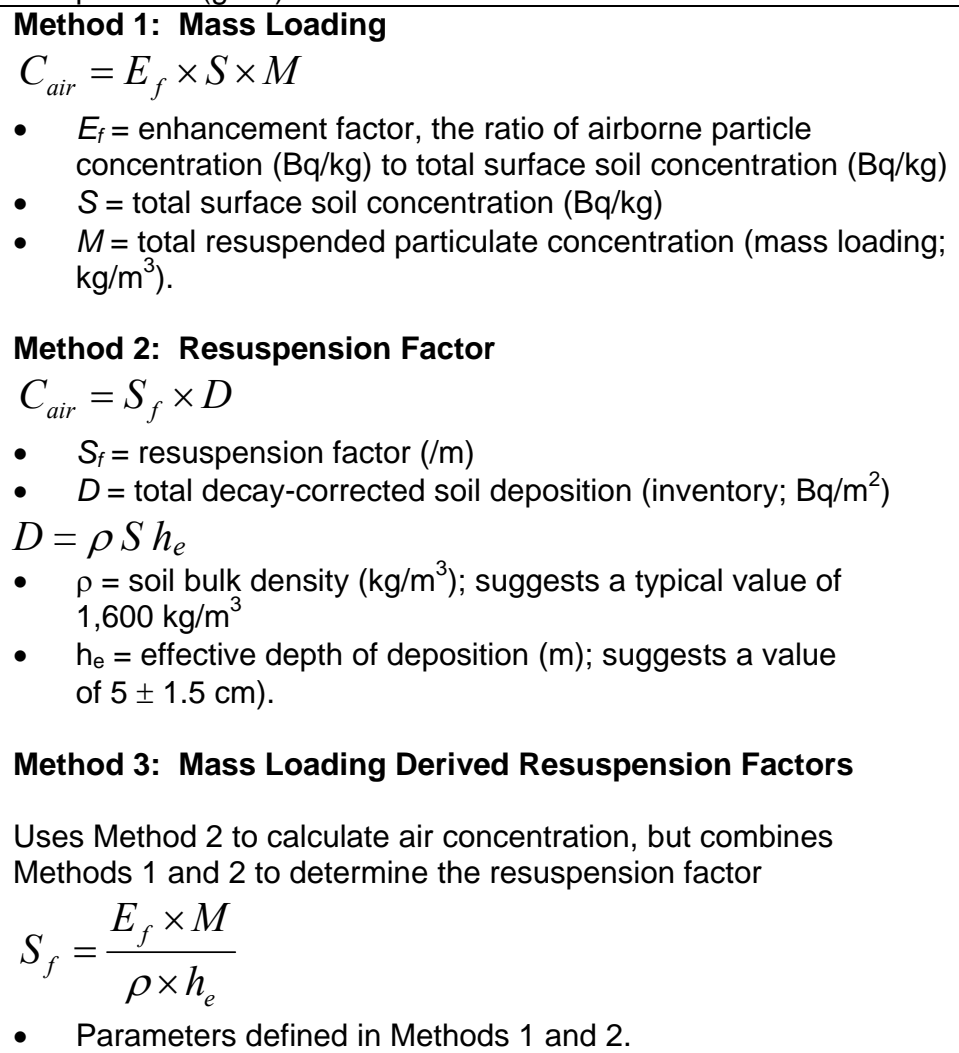 & $\begin{array}{l}\text { Method } 1 \text { is the same as the ERMYN. Methods } 2 \\
\text { and } 3 \text {, suggested in NCRP-129, are not often used } \\
\text { due to lack of input data, such as the resuspension } \\
\text { factor and the effective depth of deposition. }\end{array}$ & $\begin{array}{l}\text { NCRP } 1999 \\
\text { [DIRS } 155894] \\
\text { pp. } 64 \text { to } 71 \\
\text { Eqs. } 4.3 \text { to } 4.13 .\end{array}$ \\
\hline
\end{tabular}

ERMYN=Environmental Radiation Model for Yucca Mountain, Nevada 


\subsubsection{Radon from Radium-Contaminated Soil}

The ERMYN air submodel includes the release of radon from radium-contaminated soil for both exposure scenarios. Under the groundwater scenario, radon concentrations are calculated separately for outdoor air (radon released from surface soil) and indoor air (based on the outdoor radon and radon released from the soil under the foundation of a house; Section 6.4.2.3). For the volcanic ash scenario, the same calculation is used for indoor and outdoor air because there would be no contaminated ash under existing buildings or it would be removed during construction of new buildings. Among the five validation models, RESRAD includes indoor and outdoor radon, NCRP-129 only includes outdoor radon, and the other three validation models do not include radon. RESRAD uses relatively sophisticated radon methods that calculate radon emanation from the soil and diffusion in the air.

The indoor air radon calculation for the groundwater scenario (Section 6.4.2.3) is similar to that used in the RESRAD model (Table 7.3-5) with the following exceptions:

- RESRAD uses a radon decay constant that is ignored in the ERMYN because the typical house ventilation rate $(0.5 / \mathrm{hr})$ is larger than the radon decay constant $(0.0076 / \mathrm{hr})$.

- RESRAD uses an indoor area factor to account for partially contaminated house foundations, which does not apply to the Yucca Mountain scenarios.

- The indoor radon flux parameter in the RESRAD model is not defined as proportional to the outdoor radon flux.

Therefore, the indoor air radon calculations in the RESRAD model are mathematically equivalent to those in the ERMYN.

The NCRP-129 model uses a simple radon release factor, which the ERMYN uses to determine outdoor radon concentrations for the groundwater scenario (Equation 6.4.2-4). The ERMYN uses a similar method to determine indoor and outdoor radon concentrations for the volcanic ash scenario (Equation 6.5.2-8) using a release factor calculated by Equation 6.5.2-7 (Section 6.5.2). This is different from RESRAD, and, therefore, the methods are compared numerically (Section 7.4.3.1 and Appendix B). The general differential equation and the boundary conditions given in RESRAD are solved using the ERMYN parameter values or RESRAD default parameter values if the parameters are not used in the ERMYN. Using RESRAD, the ${ }^{222} \mathrm{Rn}$ release factor for ${ }^{226} \mathrm{Ra}$ in the soil is $0.19\left(\mathrm{~Bq} / \mathrm{m}^{3}\right) /(\mathrm{Bq} / \mathrm{kg}$ ) (Section 7.4.3.1 and Table II-1), which is similar to the value of $0.25\left(\mathrm{~Bq} / \mathrm{m}^{3}\right) /(\mathrm{Bq} / \mathrm{kg})$ from the ERMYN groundwater scenario (Section 6.4.2.3). For the volcanic scenario, RESRAD produces a ${ }^{222} \mathrm{Rn}$ release factor of $0.0005\left(\mathrm{~Bq} / \mathrm{m}^{3}\right) /\left(\mathrm{Bq} / \mathrm{m}^{2}\right)$ using a radon emanation coefficient of 1 (Section 7.4.3.1). This is similar to the value of $0.0006\left(\mathrm{~Bq} / \mathrm{m}^{3}\right) /\left(\mathrm{Bq} / \mathrm{m}^{2}\right)$ from the ERMYN volcanic scenario (Section 6.5.2.2). Because the ${ }^{222} \mathrm{Rn}$ release factors calculated using the RESRAD and ERMYN methods differ by less than a factor of two, the two methods are numerically similar.

Based on these considerations, the radon calculations are mathematically equivalent or numerically similar, and, therefore, the ERMYN methods are validated. 
Table 7.3-5. Comparison of Radon Release from Radium Contaminated Soil

\begin{tabular}{|c|c|c|c|}
\hline Document & Mathematical Model & $\begin{array}{l}\text { Comparison with the ERMYN } \\
\text { (Equations 6.4.2-4, and 6.4.2-7) }\end{array}$ & Reference \\
\hline GENII-S & Not included. & N/A & $\begin{array}{l}\text { Napier et al. } 1988 \\
\text { [DIRS 157927]. }\end{array}$ \\
\hline $\begin{array}{l}\text { BIOMASS } \\
\text { ERB2A }\end{array}$ & Not included. & $\mathrm{N} / \mathrm{A}$ & $\begin{array}{l}\text { BIOMASS 2000 } \\
\text { [DIRS 154522], Section } 7 .\end{array}$ \\
\hline EPRI-YM & Not included. & $\mathrm{N} / \mathrm{A}$ & $\begin{array}{l}\text { Smith et al. 1996 } \\
\text { [DIRS 101085], Section 5.2. }\end{array}$ \\
\hline RESRAD & 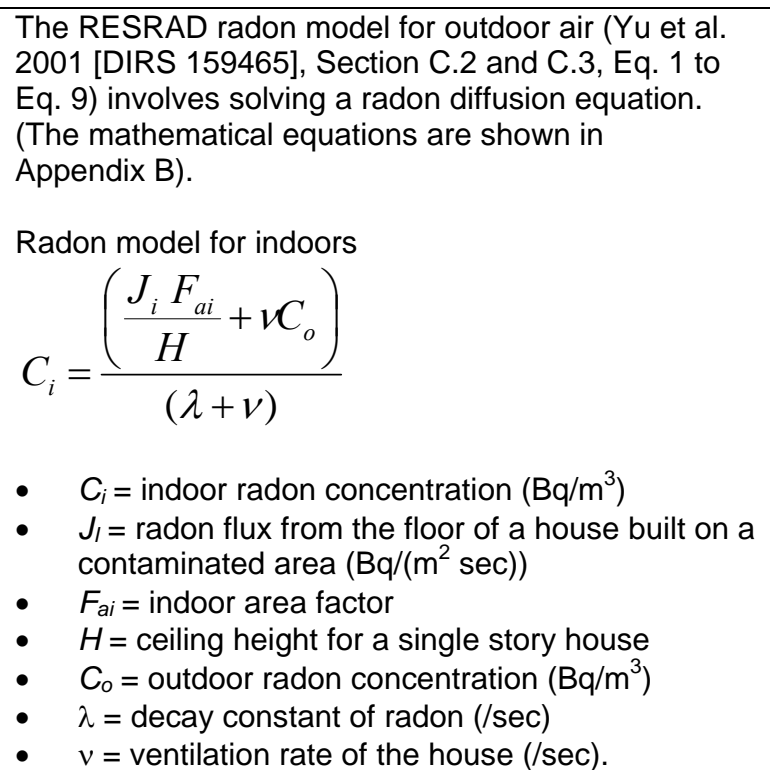 & $\begin{array}{l}\text { The RESRAD submodel for radon concentrations in } \\
\text { outdoor air requires site-specific information, such as the } \\
\text { diffusion coefficient for radon in the soil and the radon } \\
\text { emanation coefficient, which are not available. } \\
\text { For radon concentrations in indoor air, RESRAD is the } \\
\text { same as ERMYN (Eq. } 6.4 .2-7) \text {, with the following } \\
\text { approximation and equivalent quantities: } \\
\text { The value of } v \text { is much larger than } \lambda \text {; typical house } \\
\text { ventilation rates are about } 0.5 / h r \text {, while the radon } \\
\text { decay constant is } 0.0076 / h r . \\
\text { The indoor radon flux is a fraction of the outdoor flux, } \\
J_{l}=f_{\text {house }} J_{o} \text {, and the ratio of radon concentration to } \\
\text { flux }\left(C_{o} / J_{o}\right) \text { could be observed in the natural } \\
\text { environment. } \\
\text { The indoor area factor, the fraction of the foundation } \\
\text { area that is built on contaminated soil, is } 100 \text { percent. }\end{array}$ & $\begin{array}{l}\text { Yu et al. 2001 } \\
\text { [DIRS 159465], Appendix C. }\end{array}$ \\
\hline NCRP-129 & $\begin{array}{l}f_{m, R n-222}=\frac{C a_{g R n-222}}{C s_{m, R a-226}} \\
\text { Based on the average }{ }^{226} \text { Ra concentration measured } \\
\text { in soil of } 40 \mathrm{~Bq} / \mathrm{kg} \text {, and an average outdoor air radon } \\
\text { gas concentration of } 10 \mathrm{~Bq} / \mathrm{m}^{3}, \\
f_{m, R n-222}=0.25 \mathrm{~Bq} / \mathrm{m}^{3} / \mathrm{Bq} / \mathrm{kg}\end{array}$ & $\begin{array}{l}\text { For the groundwater scenario, the radon release factor (or } \\
\text { the ratio of average outdoor radon in the air to }{ }^{226} \mathrm{Ra} \text { in the } \\
\text { soil) used in NCRP- } 129 \text { is used in the ERMYN for } \\
\text { calculating outdoor radon concentration (Eq 6.4.2-4). } \\
\text { For the volcanic ash scenario, the radon release factor is } \\
\text { defined as the ratio of the average airborne radon } \\
\text { concentration to the areal concentration of }{ }^{226} \mathrm{Ra} \text { in the } \\
\text { soil. This method is linked to the ratio of average outdoor } \\
\text { airborne radon to the average outdoor radon flux density } \\
\text { from radium contaminated soil. }\end{array}$ & $\begin{array}{l}\text { NCRP 1999 [DIRS 155894], } \\
\text { Section 4.3.6. }\end{array}$ \\
\hline
\end{tabular}

ERMYN=Environmental Radiation Model for Yucca Mountain, Nevada 


\subsubsection{Contaminated Aerosols from the Operation of Evaporative Cooler}

Evaporative coolers operated using contaminated water would generate contaminated aerosols, and air submodel for the groundwater scenario includes this pathway (Section 6.4.2.2). Calculations of radionuclide concentrations in the air are based on the operating characteristics of evaporative coolers (typical rates for water use and airflow) and the conservation of mass (water and radionuclides).

None of the validation models includes the evaporative cooler exposure pathway. Therefore, the ERMYN method is validated by comparing it with a different mathematical model. Radionuclide concentrations in indoor air can be estimated by accounting for evaporation in terms of the difference in absolute humidity between indoor and outdoor air. Based on physical principles, a fraction of the radionuclides in the contaminated water would be released into the indoor air with the water vapor from the evaporative cooler. The evaporation process would create a difference between the absolute humidity in the indoor and outdoor air. This alternative method is presented in Table 7.3-6 based on known absolute humidity values for indoor and outdoor air. Relative humidity can also be used for this because absolute humidity can be determined if the temperature and relative humidity are known. A numerical comparison of the results from the two approaches is documented in Section 7.4.3.2, and the activity concentrations differ by a factor of two (Table 7.4-8). Therefore, the two methods of calculating aerosol concentrations are numerically similar, and this portion of the submodel is validated.

\subsubsection{Validation of the Plant Submodel}

The plant submodel is used in the groundwater (Section 6.4.3) and volcanic ash scenarios (Section 6.5.3). All five validation models use plant submodels to calculate radionuclide concentrations in crops or other human foodstuffs. The ERMYN includes all radionuclide transfer mechanisms considered in the validation models, including absorption through roots (both scenarios), direct deposition of contaminated water (groundwater scenario only), and soil or ash (both scenarios) on above-ground plant parts. These three aspects of the plant submodel are validated separately in the following sections. Because each aspect of plant submodel is mathematically equivalent, numerically similar, or the approach is justified because it includes site-specific or realistically predictable conditions, the ERMYN plant submodel is validated.

\subsubsection{Root Uptake}

All validation models use similar methods for crop root uptake (Table 7.3-7), with the following differences:

- GENII-S model divides the root zone into two compartments to separately account for deep soil contamination as an initial source of contaminants. However, the contamination in both Yucca Mountain scenarios is on the soil surface, and the ERMYN assumes that all roots are in the surface soil (Approximation 7). Thus, if used, the GENII-S parameters “deep soil concentration" and "root penetration factor" would be zero in the ERMYN, making the two methods mathematically equivalent. 
- GENII-S includes a parameter for residual soil concentration from deposition in previous years. This parameter accounts for radionuclide accumulation in the soil, which approaches zero as radionuclides reach equilibrium conditions, which the ERMYN assumes (Approximation 5). Thus, the methods are mathematically equivalent.

- ERMYN includes the parameter dry-to-wet ratio because the transfer factors used in Equations 6.4.3-2 and 6.5.3-2 are based on dry plant weight. None of the validation models directly use a dry-to-wet ratio because their transfer factors are in units of wet plant weight. Thus, the methods are mathematically equivalent.

- BIOMASS includes a parameter for the fraction of internal contamination retained after food processing. The default value for this parameter is one (i.e., no loss during processing), and processing losses are not included in the ERMYN; therefore, the methods are mathematically equivalent.

- RESRAD uses an area factor to account for the portion of contaminated land. Under the groundwater scenario, the total area contaminated by long-term irrigation would be large because of land use rotation. Under the volcanic ash scenario, contaminated ash would be deposited over the entire Amargosa Valley. Thus, the area factor would be one for both scenarios, and this calculation is mathematically equivalent to the ERMYN.

- RESRAD includes a cover-and-depth factor to account for the effects of burying radioactive waste. Because all radionuclides are in the surface soil for the ERMYN scenarios, this factor would be set at one. Therefore, this calculation is mathematically equivalent to the ERMYN.

Thus, the crop root uptake portion of the ERMYN plant submodel is mathematically equivalent to the five validation models, and, therefore, it is validated.

Table 7.3-6. Comparison of Radionuclide Concentration in Indoor Air from the Operation of an Evaporative Cooler

\begin{tabular}{|c|c|c|c|}
\hline Document & Mathematical Model & Comparison with the ERMYN (Equations 6.4.2-3) & Reference \\
\hline & 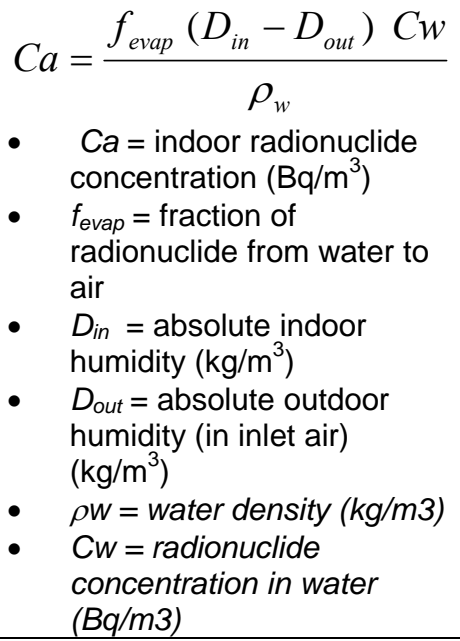 & $\begin{array}{l}\text { The fundamentals of the two methods are the same, } \\
\text { but the ERMYN includes the amount of water used } \\
\text { by the evaporative cooler as the amount of water } \\
\text { vapor in the air. This ACM considers differences } \\
\text { between absolute indoor and outdoor humidity, which } \\
\text { is from water used by evaporative coolers. }\end{array}$ & $\begin{array}{l}\text { This } \\
\text { method is } \\
\text { based on } \\
\text { physical } \\
\text { principles. }\end{array}$ \\
\hline
\end{tabular}

$\mathrm{ACM}=$ alternative conceptual model; ERMYN=Environmental Radiation Model for Yucca Mountain, Nevada 


\begin{tabular}{|c|c|c|c|}
\hline Document & Mathematical Model & $\begin{array}{l}\text { Comparison with the ERMYN } \\
\text { (Equations 6.4.3-2 and 6.5.3-2) }\end{array}$ & Reference \\
\hline GENII-S & $\begin{array}{ll}C r_{i p}=\left[C d_{i} R_{p 2}+\left(\frac{C s_{i}}{P}+\mathrm{Cb}_{\mathrm{i}}\right) R_{p 1}\right] B v_{i p} \\
\text { - } \quad C r_{i p}=\text { plant concentration from root uptake for } \\
\text { radionuclide } i \text { and plant type } p(\mathrm{Ci} / \mathrm{kg}) \\
B v_{i p}=\text { soil-to-plant transfer factor for radionuclide } i \text { and } \\
\text { plant type } p \text { (dimensionless) } \\
\quad C b_{i}=\text { residual soil concentration from previous years } \\
\text { deposition of radionuclide } i(\mathrm{pCi} / \mathrm{kg}) \\
\text { - } \quad C s i=\text { area soil concentration for radionuclide } i\left(\mathrm{Ci} / \mathrm{m}^{2}\right) \\
-\quad P d_{i}=\text { deep soil concentration for radionuclide } i(\mathrm{Ci} / \mathrm{kg}) \\
-\quad R_{p 1}=\text { root penetration factor for surface soil } \\
\text { (dimensionless) } \\
R_{p 2}=\text { root penetration factor for deep soil } \\
\text { (dimensionless). }\end{array}$ & $\begin{array}{l}\text { GENII-S is similar to the ERMYN with following } \\
\text { approximations and simplifications: } \\
\text { Deep soil is not contaminated }\left(C d_{i}=0\right) \text {, and all crop } \\
\text { roots are in the surface soil }\left(R_{p 1}=1, \text { and } R_{p s}=0\right) \\
\text { (Approximation } 7) \\
\text { Residual soil concentrations from previous years }\left(C b_{i}\right) \\
\text { are at the equilibrium condition in surface soils } \\
\text { (Approximation } 5) \\
\text { The GENII-S equation does not include the dry-to-wet } \\
\text { ratio, but it could be considered as the soil-to-plant } \\
\text { transfer factor }\left(B v_{i p}\right) \text { based on the wet weight, or } \\
B v_{i p}=F_{S \rightarrow p} D W \text {, in the ERMYN. }\end{array}$ & $\begin{array}{l}\text { Napier et al. } 1988 \\
\text { [DIRS 157927], } \\
\text { p. 4.67, Eq. 4.7.7. }\end{array}$ \\
\hline $\begin{array}{l}\text { BIOMASS } \\
\text { ERB2A }\end{array}$ & $\begin{array}{l}C_{\text {crop }, \text { root }}=\frac{F_{p 2} C F_{\text {crop }} C_{s}}{\left(1-\theta_{t}\right) \rho} \\
\text { - } \quad C_{\text {crop,root }}=\text { plant root uptake }(\mathrm{Bq} / \mathrm{kg} \text { wet plant }) \\
\text { - } \quad C_{s}=\text { radionuclide concentration in soil }\left(\mathrm{Bq} / \mathrm{m}^{3}\right) \\
\theta_{t}=\text { total soil porosity (dimensionless) } \\
\text { - } \quad \rho=\text { dry grain density of soil }\left(\mathrm{kg} / \mathrm{m}^{3}\right) \\
\quad C F_{c r o p}=\text { concentration factor from root uptake for crops } \\
(\mathrm{Bq} / \mathrm{kg} \text { wet plant per } \mathrm{pCi} / \mathrm{kg} \text { dry soil }) \\
F_{p 2}=\text { fraction of internal contamination associated with } \\
\text { edible parts of the plant at harvest that is retained after } \\
\text { food processing (dimensionless). }\end{array}$ & $\begin{array}{l}\text { BIOMASS ERB2A is the same as the ERMYN, except for } \\
\text { internal contamination losses due to food processing. } \\
\text { Because the default value is } 1 \text {, the two submodels are the } \\
\text { same with the following equivalent parameters: } \\
\text { - } \quad C_{s} /\left[\left(1-\theta_{t}\right) \rho\right] \text { in BIOMASS ERB2A is equivalent to } \\
C_{s} / \rho_{S} \text { in ERMYN } \\
\quad C F_{c r o p} \text { in BIOMASS ERB2A is equivalent to } F_{S \rightarrow p} D W \text { in } \\
\text { ERMYN. }\end{array}$ & $\begin{array}{l}\text { BIOMASS } 2000 \\
\text { [DIRS 154522], } \\
\text { p. 35, Eq. } 11 .\end{array}$ \\
\hline RESRAD & $\begin{array}{ll}E_{p 1}=S(0) F A_{p} F C D_{p 1} B_{j v} \\
\text { - } & E_{p 1}=\text { plant root uptake }\left(\mathrm{Bq} / \mathrm{g}_{\text {wet plant }}\right) \\
\text { - } & S(0)=\text { initial radionuclide concentration in soil }\left(\mathrm{Bq} / \mathrm{g}_{\mathrm{dry}} \text { soil) }\right. \\
\text { - } & F A_{p}=\text { area factor (dimensionless) } \\
\text { - } & F C D_{p 1}=\text { cover-and-depth factor (dimensionless) } \\
& B_{j v}=\text { vegetable-soil transfer factor for root uptake } \\
& \left(\mathrm{Bq} / \mathrm{g}_{\text {wet plant }} \text { per } \mathrm{Bq} / \mathrm{g} \text { dry soil). }\right.\end{array}$ & $\begin{array}{l}\text { RESRAD is designed for soil contamination. The equation } \\
\text { shown at left is modified to eliminate radionuclide and time } \\
\text { dependence, and other subscripts are simplified to reflect } \\
\text { root uptake. Two parameters, the area factor and the } \\
\text { cover-and-depth factor, are used for underground } \\
\text { contaminants with limited area and depth, which is not } \\
\text { used in the ERMYN. } \\
\text { RESRAD uses the same equation as the ERMYN with the } \\
\text { following equivalent parameter: } \\
\text { - } \quad B_{i, j} \text { in RESRAD is equivalent to } F_{S \rightarrow p} D W \text { in ERMYN. }\end{array}$ & $\begin{array}{l}\text { Yu et al. } 2001 \\
\text { [DIRS 159465], } \\
\text { Eq. 3.11, Eqs. D.1 } \\
\text { and D.8. }\end{array}$ \\
\hline
\end{tabular}




\begin{tabular}{|c|c|c|c|}
\hline Document & Mathematical Model & $\begin{array}{l}\text { Comparison with the ERMYN } \\
\text { (Equations } 6.4 .3-2 \text { and } 6.5 .3-2 \text { ) }\end{array}$ & Reference \\
\hline EPRI-YM & 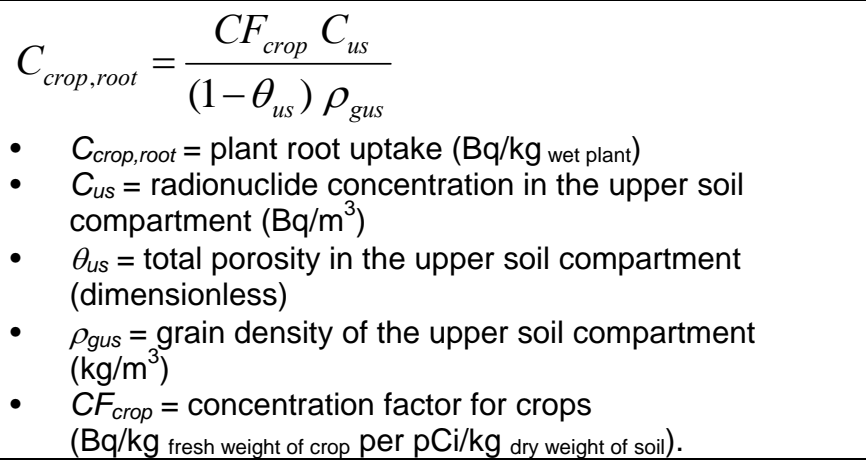 & $\begin{array}{l}\text { EPRI-YM uses the same equation as BIOMASS ERB2A, } \\
\text { except for the fraction of contaminant retained after food } \\
\text { processing }\left(F_{p 2}\right) \text {. Thus, it is the same as the ERMYN. }\end{array}$ & $\begin{array}{l}\text { Smith et al. } 1996 \\
\text { [DIRS 101085], } \\
\text { p. 5-8, Eq. 5.9. }\end{array}$ \\
\hline NCRP-129 & $\begin{array}{l}C_{i, \text { root }}=S \times B_{v} \\
\text { - } \quad C_{i, \text { root }}=\text { concentration in a given type of vegetation due to } \\
\text { root uptake }\left(\mathrm{Bq} / \mathrm{kg}_{\text {wet plant }}\right) \\
\text { - } \quad S=\text { radionuclide concentration in the surface soil } \\
(\mathrm{Bq} / \mathrm{kg} \text { dry soil }) \\
B_{v}=\text { an empirically determined soil-to-vegetation transfer } \\
\text { factor for roots, usually expressed as } \mathrm{Bq} / \mathrm{kg} \text { wet vegetation). }\end{array}$ & $\begin{array}{l}\text { NCRP-129 uses the same equation as RESRAD, except } \\
\text { for no area factor, and no cover-and-depth factor. Thus, it } \\
\text { is the same as the ERMYN. }\end{array}$ & $\begin{array}{l}\text { NCRP } 1999 \\
\text { [DIRS 155894], } \\
\text { Section } 5.1 \\
\text { Eq. } 5.2, \text { p. } 92\end{array}$ \\
\hline
\end{tabular}

ERMYN=Environmental Radiation Model for Yucca Mountain, Nevada 


\subsubsection{Uptake from Irrigation Water}

Four of the five validation models, GENII-S, BIOMASS ERB2A, EPRI-YM, and RESRAD, include crop uptake from contaminated irrigation water as a result of direct deposition on leaf surfaces. NCRP-129, which applies only to a soil contamination scenario, does not address this process. The BIOMASS ERB2A and EPRI-YM methods for calculating concentrations in fresh forage (animal feed) are different from those used to calculate concentrations in other crops. These methods are not shown in the comparison (Table 7.3-8) because they are based on cattle fed in pastures, which differs from the farming methods in the Amargosa Valley. The methods these two models use for calculating concentrations in human foodstuffs are compared in Table 7.3-8. The two components for calculating radionuclide uptake, the irrigation deposition rate (Table 7.3-9) and the interception fraction due to irrigation water intercepted by leaf surfaces (Table 7.3-10), are compared separately. Based on the comparisons, the calculations are mathematically equivalent, numerically similar, or have differences that result from model improvements and site-specific conditions, and this portion of the ERMYN plant submodel is validated.

Radionuclides in Crops-Among these four validation models, GENII-S and RESRAD use methods similar to the ERMYN. The BIOMASS ERB2A method includes more transfer processes, is conceptually different from the ERMYN method, and is an ACM (Section 6.3.3). The numerical comparison is described below and in Section 7.4.4.1. The EPRI-YM equation has an error that allows the total activity in the crops to exceed activity in the originally intercepted irrigation water if the fraction of external contamination $\left(F_{\text {crop }}\right)$ is zero. Therefore, the EPRI-YM approach is not valid and is not directly considered here. Differences between the ERMYN and the validation models are:

- None of the validation models use a parameter for the fraction of overhead irrigation (Equation 6.4.3-3). Values of this parameter, ranging from 0 to 1, can be lower than 0.5 for crops such as fruits that normally are drip irrigated (BSC 2004 [DIRS 169673], Section 6.3). Values of 0.5 or lower result in activity concentrations due to irrigation deposition that differ by a factor of two or more, and this portion of the submodel must be further justified. There are three basic methods for irrigating field crops, orchards, and gardens: surface (i.e., flood), drip, and sprinkler irrigation. Of these, only sprinkling deposits radionuclides directly on plant surfaces. Irrigation methods differ among crop types. Drip irrigation often is used on orchard and gardens, and overhead sprinklers and surface irrigation often are used on fields (BSC 2004 [DIRS 169673], Section 6.3). In the Amargosa Valley in 1997, about 85 percent of field crops were irrigated with overhead sprinklers and all of the fruit and nut crops were irrigated with drip systems (BSC 2004 [DIRS 169673], Table 6.3-1). There is little information about the preferred methods of irrigating gardens in the Amargosa Valley; therefore, there is uncertainty in the proportion of crops that would be contaminated by overhead irrigation. The fraction of overhead irrigation is a justifiable improvement to the ERMYN because it allows for considering site-specific differences in irrigation methods among crop types, accounts for uncertainty in irrigation methods used, and prevents overestimating contamination via this pathway by avoiding the approximation that all crops are irrigated with sprinkler systems. Therefore, using this parameter in the ERMYN plant submodel is justified. 
- GENII-S includes parameters that account for radioactive decay during the time from harvest to consumption (holdup time and decay constant). The holdup time, generally, is days to weeks for fresh produce, which is short relative to radioactive decay rates for the long-lived radionuclides in the ERMYN (Table 6.3-7). Thus, the exponential factor in GENII-S, which includes the holdup time and decay constant, approaches one, and the methods are mathematically equivalent.

The BIOMASS ERB2A method includes two translocation processes: translocation from external plant surfaces into the plant tissues $\left(F_{a b s}\right)$ and translocation from plant tissues into the edible portion of the crop $\left(F_{\text {trans }}\right)$. It also includes internal $\left(F_{p 2}\right)$ and external $\left(F_{p 3}\right)$ losses due to food processing. Furthermore, it considers that weathering losses occur only during the interval between the last irrigation and harvest $(T)$ rather than over the entire growing period. Because frequent irrigation is required during the entire growing season in southern Nevada (BSC 2004 [DIRS 169673], Section 6.5), this consideration is invalid for the ERMYN. In addition, several parameters used in the BIOMASS ERB2A model, such as the interval time $(T)$ and the absorption fraction $\left(F_{a b s}\right)$, are not commonly used in environmental radiation models and, therefore, are hard to quantify. The BIOMASS and ERMYN methods are numerically compared using input values from Table 6.6-3 and, where necessary, default values from the BIOMASS model (Section 7.4.4.1), and the results differ by a factor of two (Table 7.4-9). Thus, the methods are numerically similar.

Irrigation Deposition Rate-The irrigation deposition rate (Equation 6.4.3-4) is used to calculate the direct deposition rate of radionuclides due to application of irrigation water for crops. The ERMYN and the applicable validation models calculate the deposition rate by multiplying the water concentration by an irrigation rate. The structure of the equations and the input parameters differ in the following ways:

- GENII-S uses an annual irrigation rate divided by the number of months crops are irrigated. The ERMYN, instead, uses a daily irrigation rate to eliminate the correlation between irrigation rates and growing season lengths. These terms are mathematically equivalent because they both represent the rate of irrigation application per unit time during the growing season.

- RESRAD uses a factor $(F 1)$ for the proportion of irrigation water that is contaminated. This value equals one in the ERMYN because contaminated groundwater is the only source of irrigation water. Thus, the portion of the RESRAD equation with this factor and the associated ratio of surface water concentration to soil concentration (WSR $)$ becomes one, making the methods mathematically equivalent.

- BIOMASS ERB2A, EPRI-YM, and RESRAD use an annual irrigation rate, without considering the length of the growing season, to determine the average deposition rate. When converted to an annualized rate per day (as is done when divided by the weathering factor in Equation 6.4.3-4), rates based on irrigating over an entire year result in lower deposition rates than rates based on the growing season. This difference is greater than a factor of two for all crops with a growing season of less than six months, and the following evaluation and justification are provided to validate this portion of the submodel. Many of the crops commonly grown in farms and gardens in 
the Amargosa Valley have growing seasons of less than 4 months, and no crops types are irrigated all year (BSC 2004 [DIRS 169673], Section 6.4). Using an annual irrigation rate for these crops results in underestimating radionuclide deposition because a rate divided over an entire year would be lower than a rate divided over the growing season. The method in the ERMYN is used to match the site-specific gardening and agricultural practices in the Amargosa Valley, and to avoid underestimating the irrigation deposition rate. Thus, this portion of the submodel is justified.

Interception Fraction-The ERMYN method for calculating the proportion of radionuclides in irrigation water intercepted by crops differs from the method in the five validation models, which all include the interception fraction as a single parameter. Default values for the fraction range from 0.05 to 0.3 (Table 7.3-10). In contrast, the ERMYN uses an empirical equation from Hoffman et al. (1989 [DIRS 124110]) for calculating the interception faction. This equation is based on the interception of ${ }^{7}$ Be (i.e., beryllium-7), which has a high interception fraction (Section 6.4.3.2). Inputs to this equation are crop biomass, irrigation amount applied per application, and irrigation intensity. The first two inputs differ among crops, so different distributions are calculated for each crop type.

To compare these methods numerically, interception fractions are calculated using average values for each crop type, and the interception fraction values range from 0.24 for leafy vegetables to 0.51 for grains (Table 7.4-10). Some of the values differ by more than a factor of two from the default values used in the validation models, so an evaluation and justification for the ERMYN method is provided. The primary reason the empirical equation is used in the ERMYN is to incorporate variation and uncertainty in irrigation rates and the types of crops grown in the Amargosa Valley. Hoffman et al. (1989 [DIRS 124110]) show that the proportion of radionuclides intercepted differs depending on the size of plants (i.e., aboveground biomass), the rate at which water is applied, the amount of water applied, and the charge carried by the chemical element. Therefore, a single value per crop type is not adequate because there are a substantial number of crops per crop type grown in the Amargosa Valley (BSC 2004 [DIRS 169673], Section 7 and Appendix A). The ERMYN method accounts for differences in irrigation requirements and growth forms of the crops. It also accounts for differences resulting from climate change.

Although experiments indicated that the interception fraction depends on the charge carried by the chemical element (Hoffman et al. 1989 [DIRS 124110]), there is not enough information to calculate radionuclide-specific interception fraction values. Therefore, conservative empirical constants based on the ${ }^{7}$ Be results are used in the ERMYN (Section 6.4.3.2).

The simulated irrigation conditions used by Hoffman et al. (amount of rain $=1$ to $30 \mathrm{~mm}$; rain intensity $=2$ to $12 \mathrm{~cm} / \mathrm{hr}$ ), generally, are comparable with irrigation practices in the Amargosa Valley (Table 6.6-3), except that the amount of irrigation per application for grain and forage (about $55 \mathrm{~mm}$ ) is higher than the simulated amount of rain. However, the equation is relatively insensitive to the irrigation amount. For example, changing the irrigation application from 15 to $65 \mathrm{~mm}$ (and holding the other factors constant at average values) changes the interception fraction from 0.34 to 0.23 (BSC 2004 [DIRS 169673], Section 6.7). The dry biomass of crops in the Amargosa Valley (Table 6.6-3) is generally higher than the experimental conditions used to develop the equation (Hoffman et al. 1989 [DIRS 124110]). However, the interception fraction 
asymptotes to one at relatively low values of dry biomass (BSC 2004 [DIRS 169673], Figure 6.1-1), so the equation is insensitive to larger biomass values. Thus, the method is applicable to the site-specific input values in the ERMYN. The full range of parameter values used to calculate the interception fraction were stochastically sampled as part of the verification of the model (Section 6.10.1.2). The resulting range of interception fractions was from 0.10 to 0.69 for leafy vegetables with a mean value of $0.23,0.20$ to 0.69 for other vegetables with a mean value of $0.33,0.09$ to 1.0 for fruits with a mean value of $0.40,0.23$ to 1.0 for grain with a mean value of 0.49 , and 0.08 to 0.81 for forage with a mean value of 0.25 (Appendix A, ERMYN_GW_Pu239stoc.gsm).

Anspaugh (1987 [DIRS 123696]) reviewed the literature on the retention of radionuclides deposited on crop surfaces. In general, the interception fractions in that report are within the range of average values calculated using the ERMYN method (Table 7.4-10). A few of the reviewed studies reported interception fractions higher than the mean values shown in Table 7.4-10 (e.g., greater than 0.7) but within the range of results when the full range of input parameters are stochastically sampled. The amount of rainfall or irrigation applied in these studies was only a fraction of a millimeter per wetting event. Because the irrigation applications in Table 7.4-10 are higher (mean of 15 to $58 \mathrm{~mm}$; Table 6.6-3), the calculated interception fractions (Equation 6.4.3-5) are expected to be lower than the levels obtained experimentally using low application rates.

In summary, the empirical method in the ERMYN for calculating the irrigation interception fraction incorporates variation and uncertainty in the fraction resulting from differences among crops and in irrigation practices in the Amargosa Valley. The ERMYN method is applicable to the site-specific conditions and is relatively insensitive to input values outside the range of experimental values. The average values calculated using site-specific inputs are similar to values reported in the literature. This method, therefore, is reasonable, and this portion of the submodel is justified. 


\begin{tabular}{|c|c|c|c|}
\hline Document & Mathematical Model & Comparison with the ERMYN (Equation 6.4.3-3) & Reference \\
\hline GENII-S & 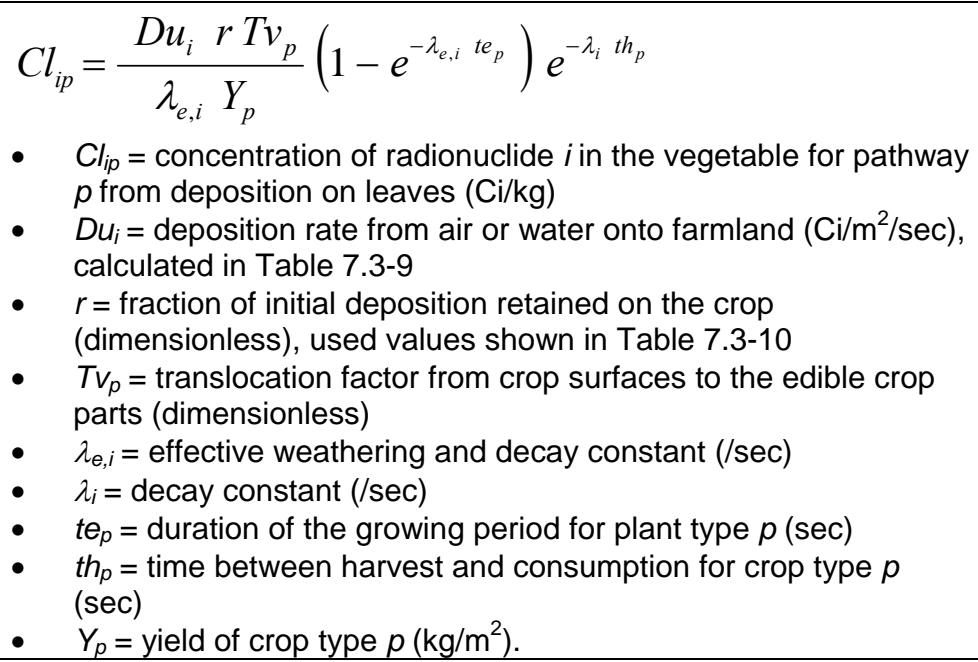 & $\begin{array}{l}\text { Holdup time is not used in ERMYN, as it only uses } \\
\text { radioactive decay, which is not important for } \\
\text { long-lived radionuclides (Section 6.3.1.6). The } \\
\text { fraction of overhead irrigation is used in the ERMYN } \\
\text { to consider other irrigation methods used in } \\
\text { Amargosa Valley. }\end{array}$ & $\begin{array}{l}\text { Napier et al. } \\
1988 \\
\text { [DIRS 157927], } \\
\text { p. } 4.67, \\
\text { Eq. } 4.7 .6 .\end{array}$ \\
\hline RESRAD & $\begin{array}{l}F W R_{j 34 k}=\frac{I_{r r} f_{r}^{\prime} T_{j v k}}{\lambda_{w} Y_{v k}}\left(1-e^{-\lambda_{w} t_{e k}}\right) \\
\text { - } \quad F W R_{j 34 k}=\text { plant-food/water concentration ratio }\left(\mathrm{m}^{3} / \mathrm{kg}\right) \\
\text { - } \quad I_{r r}=\text { deposition rate from air or water onto farmland (m/year) } \\
\text { - } \quad f_{r}^{\prime}=\text { fraction of deposited radionuclide retained on vegetation } \\
\text { - } \quad T_{j v k}=\text { foliage-to-food radionuclide transfer coefficient for the } j \text { th } \\
\text { principal radionuclide and } k \text { th food class (dimensionless) } \\
\text { - } \quad \lambda_{w i}=\text { weathering removal constant for vegetation (/year) } \\
\text { - } \quad t_{e k}=\text { time of exposure of the } k \text { th food class to contamination } \\
\text { - } \quad Y_{v k}=\text { wet-weight crop yield for the } k \text { th food class }\left(\mathrm{kg} / \mathrm{m}^{2}\right) .\end{array}$ & $\begin{array}{l}\text { RESRAD calculates the plant-food/water } \\
\text { concentration ratio }\left(\mathrm{m}^{3} / \mathrm{kg}\right) \text {. The radionuclide } \\
\text { concentrations in plants are calculated by using this } \\
\text { value multiplying the water concentration } \\
\left(\mathrm{Cw}-\mathrm{Bq} / \mathrm{m}^{3}\right) \text { in the model. } \\
\text { RESRAD is equivalent to ERMYN, except for the } \\
\text { addition of the parameter "fraction of overhead } \\
\text { irrigation" to the ERMYN. }\end{array}$ & $\begin{array}{l}\text { Yu et al. } 2001 \\
\text { [DIRS 159465], } \\
\text { p. D-14, } \\
\text { Eq. D.14. }\end{array}$ \\
\hline
\end{tabular}


Table 7.3-8. Comparison of Direct Deposition on Leaf Surfaces Due to the Interception of Irrigation Water (Continued)

\begin{tabular}{|c|c|c|c|}
\hline Document & Mathematical Model & Comparison with the ERMYN (Equation 6.4.3-3) & Reference \\
\hline $\begin{array}{l}\text { BIOMASS } \\
\text { ERB2A }\end{array}$ & 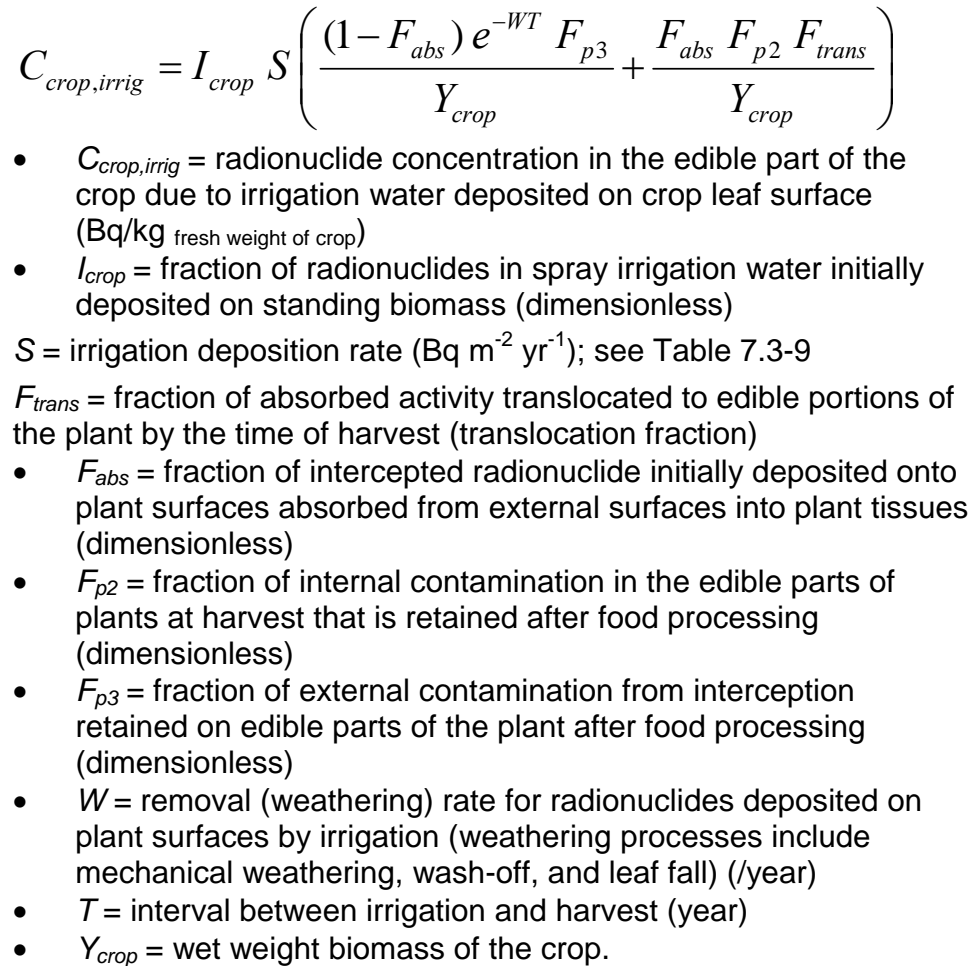 & 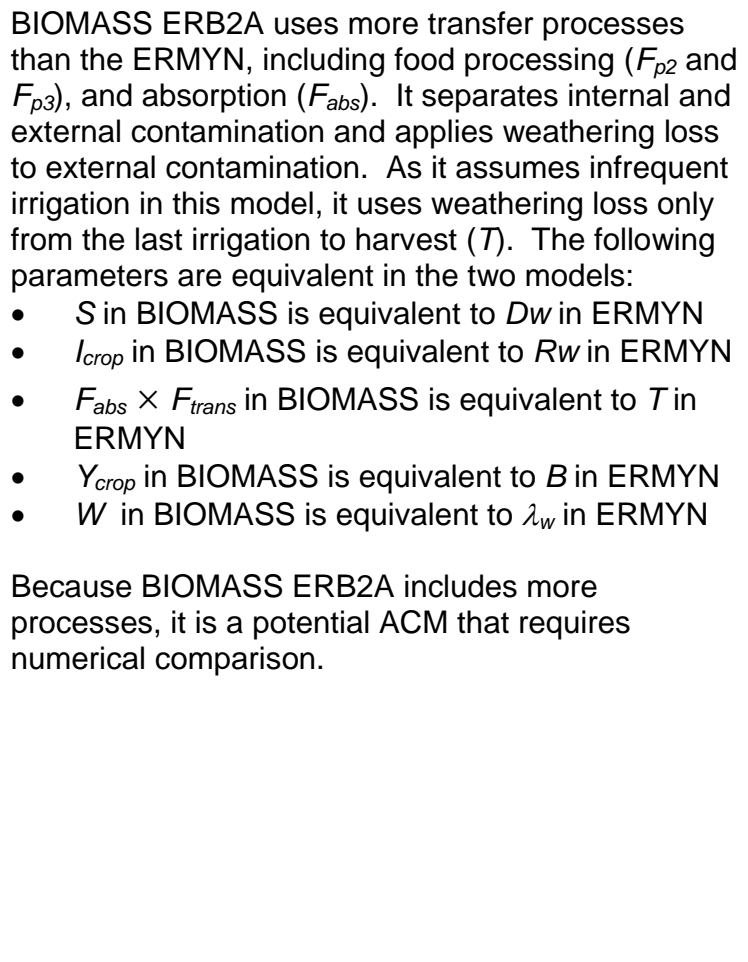 & $\begin{array}{l}\text { BIOMASS } 2000 \\
\text { [DIRS 154522], } \\
\text { p. 35, Eq. } 11 .\end{array}$ \\
\hline
\end{tabular}




\begin{tabular}{|c|c|c|c|}
\hline Document & Mathematical Model & Comparison with the ERMYN (Equation 6.4.3-3) & Reference \\
\hline EPRI-YM & 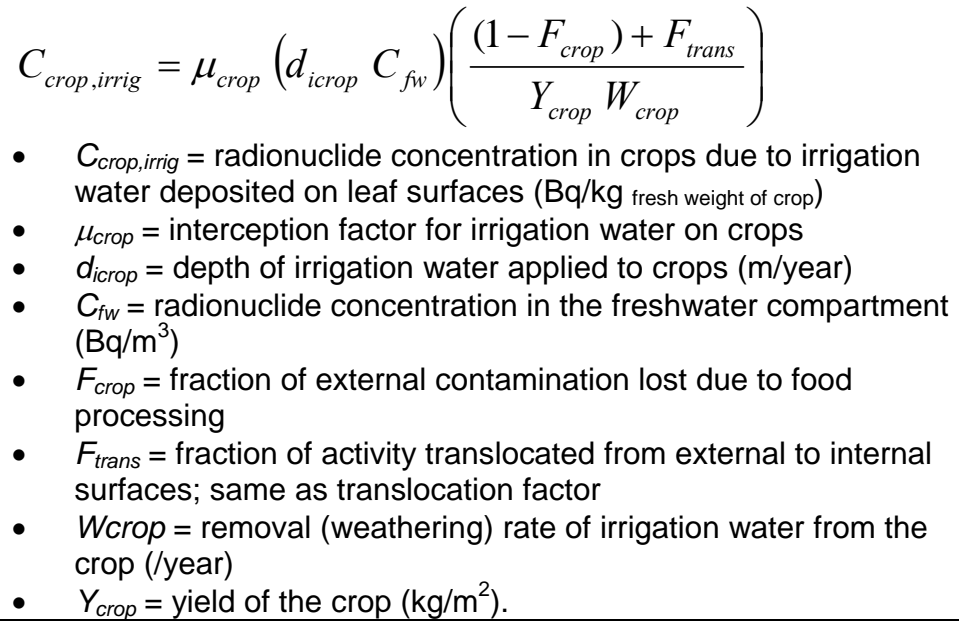 & $\begin{array}{l}\text { The EPRI-YM equation has an error that adds the } \\
\text { translocation }\left(F_{\text {trans }}\right) \text { to the external contamination } \\
\left(F_{\text {crop }}\right) \text { factor. For } F_{\text {crop }}=0 \text {, the total activity in the } \\
\text { crop could exceed that originally in intercepted } \\
\text { irrigation water. }\end{array}$ & $\begin{array}{l}\text { Smith et al. } 1996 \\
\text { [DIRS 101085], } \\
\text { p. 5-8, Eq. 5.9. }\end{array}$ \\
\hline NCRP-129 & Not included in the model. & Not applicable. & $\begin{array}{l}\text { NCRP } 1999 \\
\text { [DIRS 155894]. }\end{array}$ \\
\hline
\end{tabular}

ERMYN=Environmental Radiation Model for Yucca Mountain, Nevada

Table 7.3-9. Comparison of Irrigation Deposition Rate

\begin{tabular}{|c|c|c|c|}
\hline Document & Mathematical Model & Comparison with the ERMYN (Equation 6.4.3-4) & Reference \\
\hline GENII-S & 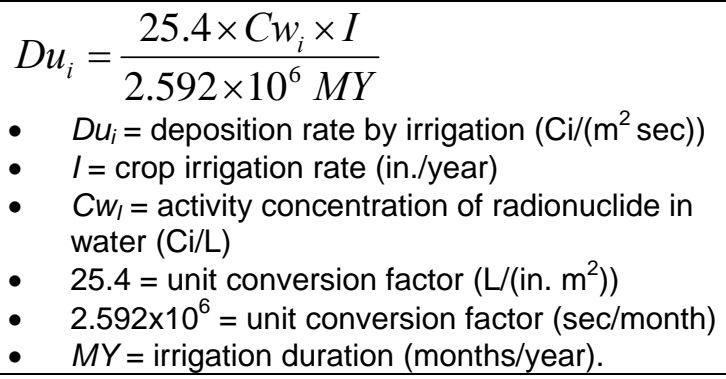 & $\begin{array}{l}\text { GENII-S uses the duration of irrigation (MY) during the crop } \\
\text { growing season. In the ERMYN, this parameter is used in the } \\
\text { daily irrigation rate }(I R D) \text { for crops and is equivalent to }(I \div \mathrm{MY}) \text { in } \\
\text { GENII-S. The two models are the same, except for different } \\
\text { units and notations. }\end{array}$ & $\begin{array}{l}\text { Napier et al. } 1988 \\
\text { [DIRS 157927], } \\
\text { p. 4.57, Eq. 4.6.2. }\end{array}$ \\
\hline
\end{tabular}


Table 7.3-9. Comparison of Irrigation Deposition Rate (Continued)

\begin{tabular}{|c|c|c|c|}
\hline Document & Mathematical Model & Comparison with the ERMYN (Equation 6.4.3-4) & Reference \\
\hline $\begin{array}{l}\text { BIOMASS } \\
\text { ERB2A }\end{array}$ & $\begin{array}{ll}S= & V_{\text {irr }} C_{w} \\
\text { - } & S=\text { activity deposition by irrigation }(\mathrm{Bq} / \text { year or } \\
& \mathrm{Bq} \mathrm{m}^{-2} \mathrm{yr}^{-1} \text { depending on the irrigation rate term) } \\
& V_{\text {irr }}=\text { irrigation rate }\left(\mathrm{m}^{3} / \text { year }\right) ; \text { this quantity is } \\
& \text { equivalent to the amount of irrigation water }(\mathrm{m}) \\
& \text { applied to a unit area }\left(\mathrm{m}^{2}\right) \text { per unit time (year) } \\
& (\mathrm{m} / \text { year) } \\
& C_{w}=\text { radionuclide concentration in groundwater } \\
& \left(\mathrm{Bq} / \mathrm{m}^{3}\right) .\end{array}$ & $\begin{array}{l}\text { The irrigation deposition rate in BIOMASS ERB2A is uniformly } \\
\text { distributed throughout the year, not just during the growing } \\
\text { season, as is used in ERMYN and GENII-S. In addition, a single } \\
\text { irrigation rate is applied to all crop types. Most crops do not } \\
\text { grow throughout the year, and irrigation should occur only during } \\
\text { crop growing season. The difference between annual irrigation } \\
\text { rates and daily irrigation rates is not a simple conversion of units } \\
\text { because the annual irrigation rate considers the total amount of } \\
\text { irrigation in a year and disregards when and how the irrigation } \\
\text { occurs. }\end{array}$ & $\begin{array}{l}\text { BIOMASS } 2000 \\
\text { [DIRS 154522], } \\
\text { p. 32, Eq. } 3 .\end{array}$ \\
\hline RESRAD & $\begin{array}{l}D=I_{r r}\left[W S R_{1} F 1+W S R_{2}(1-F 1)\right] S(0) \\
\text { - } \quad D=\text { radionuclide deposition rate (this parameter is } \\
\text { added for comparison) }\left(\mathrm{Bq}^{-2} \mathrm{yr}^{-1}\right) \\
\text { - } \quad I_{r r}=\text { irrigation rate }(\mathrm{m} / \mathrm{year}) \\
\quad W S R_{1}=\text { ratio of groundwater concentration to the } \\
\text { soil concentration of radionuclide }(\mathrm{g} / \mathrm{L}) ; \mathrm{g} / \mathrm{m}^{3} \text { is } \\
\text { required for canceling units } \\
\text { - } \quad F 1=\text { groundwater use as a fraction of water from } \\
\text { a contaminated source (dimensionless) } \\
\text { - } W S R_{2}=\text { ratio of surface water concentration to the } \\
\text { soil concentration of radionuclide }(\mathrm{g} / \mathrm{L}) ; \mathrm{g} / \mathrm{m}^{3} \text { is } \\
\text { required for canceling units } \\
S(0)=\text { initial radionuclide concentration in soil } \\
(\mathrm{Bq} / \mathrm{g} \text { dry soil). }\end{array}$ & $\begin{array}{l}\text { In the RESRAD document (Yu et al. } 2001 \text { [DIRS 159465]), the } \\
\text { mathematical representation is based on initial soil } \\
\text { contamination, and the source term is discussed in Section } 3 \text { of } \\
\text { that document, not Appendix D. The equation shown at left is } \\
\text { modified to eliminate radionuclide and time dependence. Other } \\
\text { subscripts are simplified to reflect irrigation deposition. The } \\
\text { terms WSR } 1 \text { S }(0) \text { is equivalent to the calculation of } \\
\text { concentrations in groundwater in the ERMYN. RESRAD is the } \\
\text { same as the ERMYN when there is no surface water }\left(W S R_{2}=0\right) \\
\text { and all groundwater is contaminated }(F 1=1) \text {. The unit } \\
\text { conversion at left may be different from that shown in the manual } \\
\text { because the item compared is not presented directly. } \\
\text { Similar to BIOMASS ERB2A, RESRAD does not use irrigation } \\
\text { duration; rather, it uses an annual rate for the entire year. In } \\
\text { addition, a single irrigation rate is used for all crop types. }\end{array}$ & $\begin{array}{l}\text { Yu et al. } 2001 \\
\text { [DIRS 159465], } \\
\text { Eq. 3.11, Eq. D.14 } \\
\text { and D.2. }\end{array}$ \\
\hline EPRI-YM & $\begin{array}{ll}S= & d_{\text {icorp }} C_{f w} \\
\text { - } & S=\text { radionuclide deposition rate for irrigation }^{-1} \\
& \left(\mathrm{~Bq} \mathrm{~m}^{-2} \mathrm{yr}^{-1}\right) \\
\text { - } & d_{\text {iicorp }}=\text { depth of irrigation water applied to crops } \\
& (\mathrm{m} / \mathrm{year}) \\
\text { - } \quad & C_{f w}=\text { radionuclide concentration in the freshwater } \\
& \text { compartment }\left(\mathrm{Bq} / \mathrm{m}^{3}\right) .\end{array}$ & EPRI-YM is the same as BIOMASS ERB2A. & $\begin{array}{l}\text { Smith et al. } 1996 \\
\text { [DIRS 101085], } \\
\text { p. 5-8. }\end{array}$ \\
\hline NCRP-129 & Not included. & Not applicable. & $\begin{array}{l}\text { NCRP } 1999 \\
\text { [DIRS 155894]. }\end{array}$ \\
\hline
\end{tabular}




\begin{tabular}{|c|c|c|c|}
\hline Document & Mathematical Model & Comparison with the ERMYN (Equation 6.4.3-5) & Reference \\
\hline GENII-S & $\begin{array}{l}\text { No mathematical representation for the interception } \\
\text { fraction of irrigation in GENII-S. A default value of } \\
0.25 \text { is used for all crop types. }\end{array}$ & \multirow{4}{*}{$\begin{array}{l}\text { The intercept fraction for irrigation is an important parameter to } \\
\text { estimate direct deposition on leaf surfaces due to the } \\
\text { interception of irrigation water. A single value is used in most } \\
\text { biosphere models. An empirical equation based on experiments } \\
\text { conducted by Hoffman et al. (1989 [DIRS 124110]) is used in the } \\
\text { ERMYN because the experiments are applicable to irrigation } \\
\text { practices in the Yucca Mountain area, and because this equation } \\
\text { can be used to develop interception fractions for each crop type. }\end{array}$} & $\begin{array}{l}\text { Napier et al. } 1988 \\
\text { [DIRS 157927], } \\
\text { p. } 4.69 .\end{array}$ \\
\hline $\begin{array}{l}\text { BIOMASS } \\
\text { ERB2A }\end{array}$ & $\begin{array}{l}\text { No mathematical representation for the interception } \\
\text { fraction of irrigation in BIOMASS ERB2A. A default } \\
\text { value of } 0.3 \text { is used for all crop types. }\end{array}$ & & $\begin{array}{l}\text { BIOMASS 2000 } \\
\text { [DIRS 154522], } \\
\text { p. 35, Eq. } 11 .\end{array}$ \\
\hline RESRAD & $\begin{array}{l}\text { No mathematical representation for the interception } \\
\text { fraction of irrigation in RESRAD. A default value of } \\
0.25 \text { is used for all crop types. }\end{array}$ & & $\begin{array}{l}\text { Yu et al. } 2001 \\
\text { [DIRS 159465], } \\
\text { Section D.2, } \\
\text { Eq. D.9. }\end{array}$ \\
\hline EPRI-YM & $\begin{array}{l}\text { No mathematical representation for the interception } \\
\text { fraction of irrigation in EPRI-YM. A value of } 0.05 \text { for } \\
\text { grain and } 0.3 \text { for other crop types is used. }\end{array}$ & & $\begin{array}{l}\text { Smith et al. } 1996 \\
\text { [DIRS 101085], } \\
\text { p. 5-25. }\end{array}$ \\
\hline NCRP-129 & Not included. & Not applicable. & $\begin{array}{l}\text { NCRP } 1999 \\
\text { [DIRS 155894]. }\end{array}$ \\
\hline
\end{tabular}

ERMYN=Environmental Radiation Model for Yucca Mountain, Nevada 


\subsubsection{Uptake from Resuspended Soil}

Direct deposition of radionuclides on leaf surfaces due to the interception of resuspended soil is another mechanism by which crops could become contaminated. The ERMYN, GENII-S, and RESRAD models address dust deposition and the subsequent transfer of radionuclides to crops in a manner similar to the interception of irrigation water. The BIOMASS ERB2A, EPRI-YM, and NCRP-129 models address crop surface contamination using a ratio factor (similar to the soil-to-plant transfer factor in the root uptake process). These two approaches are compared mathematically (Table 7-3.11). Two components, the dust deposition rate (Table 7.3-12) and the interception fraction (Table 7.3-13), are compared separately. The results show that the methods are mathematically equivalent, numerically similar, or have differences resulting from model improvements and site-specific conditions, which validates this portion of the ERMYN plant submodel.

Radionuclides in Crops-The ERMYN calculates radionuclide concentrations in plants due to foliar interception of airborne particles (Equations 6.4.3-6 and 6.5.3-3) using a method similar to that used by GENII-S and RESRAD (Table 7.3-11). This method considers the transfer of radionuclides into crops through dust deposition on leaf surfaces, a mechanism similar to the deposition of irrigation water on leaf surfaces. The only difference among these models is that GENII-S includes radioactive decay during the time between harvest and consumption (i.e., holdup time). As explained for water interception, the holdup time is short relative to the decay time of the long-lived radionuclides, and, therefore, the methods are mathematically equivalent.

BIOMASS ERB2A, EPRI-YM, and NCRP-129 address crop surface contamination using a contamination factor, a method that differs from the ERMYN method and is identified as an ACM (Section 6.3.3). A numerical comparison (Section 7.4.4.3) shows that the activity concentration calculated using the ERMYN method for other vegetables $\left(3.1 \times 10^{-5} \mathrm{~Bq} / \mathrm{kg}\right.$; Table 7.4-11) differs by less than a factor of two from the concentration for vegetables and grains using the alternative method $\left(2.0 \times 10^{-5} \mathrm{~Bq} / \mathrm{kg}\right)$. In contrast, the ERMYN result for leafy vegetables $\left(2.2 \times 10^{-4} \mathrm{~Bq} / \mathrm{kg}\right)$ is an order of magnitude higher than that for the alternative method for vegetables and grains. It is, however, similar to the concentration for forage $\left(2.0 \times 10^{-4} \mathrm{~Bq} / \mathrm{kg}\right)$ calculated using the alternative method in BIOMASS, EPRI-YM, and NCRP-129. This is because the ERMYN uses a high translocation factor for leafy vegetables and forage, whereas the analogous factor in the alternative method (the soil contamination factor) is high only for forage (Section 7.4.4). Because leafy vegetables and forage have similar growth forms (i.e., the consumed portion of the plant, the leaves, are aboveground and directly exposed), the same, high translocation factor should be used for both. Therefore, the alternative method underestimates the radionuclide concentration in leafy vegetables, but the models are numerically similar for the applicable comparison of leafy vegetables to forage. Thus, this portion of the submodel is justified.

Dust Deposition Rate-The ERMYN method for calculating the dust deposition rate (Equations 6.4.3-7 and 6.5.3-4) is the same as that in GENII-S and RESRAD, except for the units and associated unit conversion factors (Table 7.3-12). The alternative approach used in the BIOMASS ERB2A, EPRI-YM, and NCRP-129 models does not require calculating the dust deposition rate. The differences between the ERMYN method and the alternative approach to 
calculate radionuclide concentrations in plants due to foliar interception of airborne particles are compared above.

Interception Fraction-The ERMYN method for calculating the initial fractional deposition of radionuclides on plant surfaces from dry deposition (Equations 6.4.3-8 and 6.5.3-5) is similar to the GENII-S method (Table 7.3-13), with one exception described below. RESRAD uses a fixed value for the interception fraction of resuspended soil, with a default value of 0.25 (Table 7.3-13). Justification is provided below based on a numerical comparison between the RESRAD default value and the calculated values in the ERMYN. The alternative approach used in the BIOMASS ERB2A, EPRI-YM, and NCRP-129 models does not require a dust interception fraction. Differences between the ERMYN method and the alternative approach for calculating radionuclide concentrations in plants due to foliar interception of airborne particles are already compared.

- The ERMYN and GENII-S models use different measurements of dry biomass for calculating the dust interception fraction. The ERMYN includes a parameter (dry biomass; Equations 6.4.3-8 and 6.5.3-5) that is defined as the dry weight of aboveground standing biomass. GENII-S calculates dry biomass as the product of crop yield (wet weight) and a dry-to-wet ratio. To evaluate possible differences between these methods, values of the dry biomass parameter are compared to the product of the yield and the dry-to-wet ratio using average parameter values (Table 6.6-3). Dry biomass differed by less than a factor of two for all crop types except grains, which differed by a factor of 2.1 (average dry biomass $=1.13 \mathrm{~kg} / \mathrm{m}^{2}$; yield $\times$ dry-to-wet ratio $\left.=0.59 \mathrm{~kg} / \mathrm{m}^{2} \times 0.903=0.53 \mathrm{~kg} / \mathrm{m}^{2}\right)$. Because these two approaches may result in estimates of dry biomass that differ by more than a factor of two, a justification for the ERMYN method is provided. The equation in the ERMYN and GENII-S models was originally developed by Pinder, Ciravolo, and Bowling, as reported by Napier et al. (1988 [DIRS 157927], p. 4.69). This empirical equation requires measurements of dry biomass. Although the GENII manual correctly describes dry biomass as a product of the dry-to-wet biomass ratio and wet standing biomass (Table 7.3-13), the GENII-S code does not require the dry-to-wet biomass ratio as a model input; rather, it uses the dry-to-wet ratio for crop products. This is an incorrect method of calculating dry biomass because the dry-to-wet ratios for aboveground biomass and crop products may differ substantially, especially for root crops and crops for which only a small portion of the aboveground biomass is harvested (e.g., grains). In contrast, the ERMYN parameter for dry biomass represents total aboveground, standing-crop biomass. Estimates of the dry biomass parameter in the ERMYN are based on measurements of crop aboveground dry biomass or on measured ratios of aboveground dry biomass to crop yield (the harvest index; BSC 2004 [DIRS 169673], Section 6.1). Therefore, the ERMYN method is justified because it correctly requires estimates of aboveground dry biomass.

- RESRAD does not calculate the fraction of resuspended particles intercepted by plants; rather, it uses a default value of 0.25 for all crop types. Dust interception fractions calculated using the ERMYN for average values (Table 6.6-3) range from 0.456 for leafy vegetables to 0.959 for grains (Table 6.10-1). These two approaches produce interception fractions that differ by more than a factor of two, so a justification for the ERMYN method is provided. Field experiments with dry-deposited particles indicate 
that the interception fraction depends on the deposited materials, particle sizes, and crop types (IAEA 1996 [DIRS 160402], Table I). One experimental result shows that about 96 percent of the deposited ${ }^{212} \mathrm{~Pb}$ is intercepted by leaves (IAEA 1996 [DIRS 160402], p. 13). Based on the experimental results, a single value of interception fraction for all crop types does not reflect differences among crops, and the default value of 0.25 in the RESRAD model is too low for some crops. Therefore, the method used in the ERMYN is justified.

\subsubsection{Validation of the Animal Submodel}

The animal submodel is used in the groundwater (Section 6.4.4) and volcanic ash (Section 6.5.4) scenarios, except that the drinking water pathway is not included in the volcanic ash scenario. All five validation models use an animal submodel, although some of the models include more contamination processes than others. Each part of the ERMYN animal submodel is validated, as discussed in detail in the following four sections. Because the ERMYN animal submodel and the animal submodels in the validation models are mathematically equivalent, numerically similar, or have differences that result from model improvements, the ERMYN animal submodel is validated.

\subsubsection{Animal Feed}

All five validation models use the same method as the ERMYN for calculating radionuclide concentrations in animal products due to contaminated animal feed (Equations 6.4.4-2 and 6.5.4-2), with two exceptions (Table 7.3-14). First, the GENII-S and NCRP-129 models calculate radionuclide decay during the holdup time between harvest and consumption. The holdup time is short (generally days to weeks for fresh forage; weeks to months for grain) relative to the rate of radioactive decay for long-lived radionuclides (Table 6.3-7). Thus, the exponential factor in the GENII-S and NCRP-129 models, which includes the holdup time and decay constant, approaches one, and the methods are mathematically equivalent. Second, the GENII-S and NCRP-129 models include a parameter for the proportion of contaminated animal feed. In the ERMYN, this parameter value is assumed to be 100 percent (Approximation 8), and, therefore, the methods are mathematically equivalent. 


\begin{tabular}{|c|c|c|c|}
\hline Document & Mathematical Model & $\begin{array}{l}\text { Comparison with the ERMYN } \\
\text { (Equation } 6.4 .3-6 \text { and } 6.5 .3-3 \text { ) }\end{array}$ & Reference \\
\hline GENII-S & $\begin{array}{l}\text { GENII-S uses the same equation as is used for the irrigation } \\
\text { interception, except for a different deposition rate, } D u_{i} \text {, and } \\
\text { interception fraction, } r \text { (Table } 7.3-8) \text {. }\end{array}$ & $\begin{array}{l}\text { GENII-S is similar to the ERMYN. Holdup time is not } \\
\text { used in the ERMYN because the radionuclides of } \\
\text { interest are long-lived. }\end{array}$ & $\begin{array}{l}\text { Napier et al. } 1988 \\
\text { [DIRS 157927], } \\
\text { p. 4.67, Eq. 4.7.6. }\end{array}$ \\
\hline RESRAD & $\begin{array}{l}\text { RESRAD is similar to the equation for irrigation interception, } \\
\text { except that irrigation rate, } I_{r r} \text {, replaces particle deposition } \\
\text { velocity, } V_{d} \text {, with unit conversions (Table } 7.3-8 \text { ). }\end{array}$ & $\begin{array}{l}\text { RESRAD is the same as the ERMYN. RESRAD } \\
\text { calculates a plant-food to air concentration ratio }\left(\mathrm{m}^{3} / \mathrm{g}\right) \text {. } \\
\text { Radionuclide concentrations in plants are calculated } \\
\text { using this ratio multiplied by the air concentration (Ca- } \\
\left.\mathrm{Bq} / \mathrm{m}^{3}\right) \text {. }\end{array}$ & $\begin{array}{l}\text { Yu et al. 2001 } \\
\text { [DIRS 159465], } \\
\text { Eq. D.9 and D.10. }\end{array}$ \\
\hline $\begin{array}{l}\text { BIOMASS } \\
\text { ERB2A }\end{array}$ & 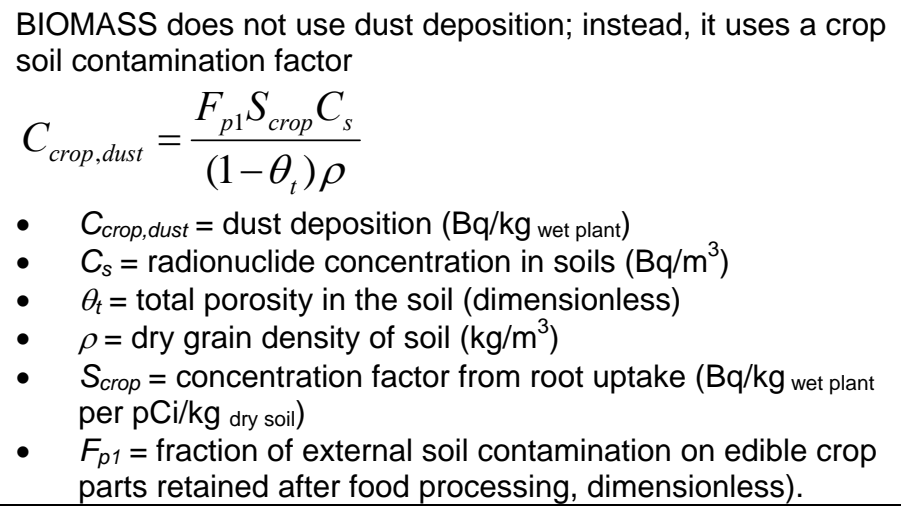 & \multirow[t]{2}{*}{$\begin{array}{l}\text { These three dose assessments use a different method } \\
\text { to calculate dust deposition on crops. A crop soil } \\
\text { contamination factor, in units of } \mathrm{kg} \text { dry soil per } \mathrm{kg} \text { wet plant or } \\
\mathrm{kg} \text { dry plant, is used. } \\
\text { BIOMASS uses an equation similar to EPRI-YM, except } \\
F_{p 1} \text { in BIOMASS is equivalent to }\left(1-F_{\text {crop }}\right) \text {. NCRP-129 } \\
\text { uses a mass soil concentration with no food process } \\
\text { losses. These three models use the same method. }\end{array}$} & $\begin{array}{l}\text { BIOMASS 2000 } \\
\text { [DIRS 154522], } \\
\text { p. 35, Eq. } 11 .\end{array}$ \\
\hline EPRI-YM & $\begin{array}{l}\text { EPRI-YM does not use dust deposition; instead, it uses a crop } \\
\text { soil contamination factor } \\
C_{\text {crop }, \text { dust }}=\frac{\left(1-F_{c r o p}\right) S_{c r o p} C_{u s}}{\left(1-\theta_{u s}\right) \rho_{g u s}} \\
\text { - } \quad C_{\text {crop,dust }}=\text { dust deposition }\left(\mathrm{Bq} / \mathrm{kg}_{\text {wet plant }}\right) \\
\text { - } \quad F_{\text {crop }}=\text { fraction of external contamination on the crop lost to } \\
\text { food processing } \\
\text { - } \quad C_{u s}=\text { radionuclide concentration in the upper soil } \\
\text { compartment }\left(\mathrm{Bq} / \mathrm{m}^{3}\right) \\
\text { - } \quad \theta_{u s}=\text { total porosity in the upper soil compartment } \\
\text { (dimensionless) } \\
\text { - } \rho_{\text {gus }}=\text { grain density of the upper soil compartment }\left(\mathrm{kg} / \mathrm{m}^{3}\right) \\
\text { - } S_{c r o p}=\text { soil contamination on crops }(\mathrm{kg} \text { dry weight soil } \mathrm{per} \\
\mathrm{kg} \text { fresh weight of crop). }\end{array}$ & & $\begin{array}{l}\text { Smith et al. } 1996 \\
\text { [DIRS 101085], } \\
\text { p. 5-8, Eq. 5.9. }\end{array}$ \\
\hline
\end{tabular}




\begin{tabular}{|c|c|c|c|}
\hline Document & Mathematical Model & $\begin{array}{l}\text { Comparison with the ERMYN } \\
\text { (Equation } 6.4 .3-6 \text { and } 6.5 .3-3 \text { ) }\end{array}$ & Reference \\
\hline NCRP-129 & $\begin{array}{l}\text { NCRP-129 does not use dust deposition; instead, is uses } \\
\text { transfer factors from soil to vegetation due to soil adhesion for } \\
\text { resuspension processes } \\
C_{i, \text { dust }}=S \times B_{v^{\prime}} \\
\text { - } \quad C_{i, \text { dust }}=\text { concentration in a crop type due to dust deposition } \\
\quad(\mathrm{Bq} / \mathrm{kg} \text { wet plant) } \\
\text { - } \quad S=\text { radionuclide concentration in surface soil (Bq } / \mathrm{kg} \text { dry soil) } \\
\text { - } \quad B_{v^{\prime}}=\text { transfer factor for the net effect of all resuspension } \\
\text { processes. }\end{array}$ & & $\begin{array}{l}\text { NCRP } 1999 \\
\text { [DIRS 155894], } \\
\text { p. 92, Eq. 5.2. }\end{array}$ \\
\hline
\end{tabular}

ERMYN=Environmental Radiation Model for Yucca Mountain, Nevada

Table 7.3-12. Comparison of the Dust Deposition Rate

\begin{tabular}{|c|c|c|c|}
\hline Document & Mathematical Model & $\begin{array}{l}\text { Comparison with the ERMYN } \\
\text { (Equations 6.4.3-7 and 6.5.3-4) }\end{array}$ & Reference \\
\hline RESRAD & $\begin{array}{l}D=3.16 \times 10^{7} \times V_{d} \times A S R_{3} \times S(0) \\
\text { - } \quad D=\text { deposition rate for resuspended soil (this parameter is } \\
\text { added for the comparison) }\left(\mathrm{Bq} /\left(\mathrm{m}^{2} \text { year }\right)\right) \\
\text { - } \quad 3.16 \times 10^{7}=\text { unit conversion }(\mathrm{sec} / \mathrm{year}) \\
\text { - } \quad V_{d}=\text { deposition velocity for radionuclide }(\mathrm{m} / \mathrm{sec}) \\
\quad A S R_{3}=\text { air/soil concentration ratio, specified as the } \\
\text { average mass loading of airborne contaminated soil } \\
\text { particles in a garden during the growing season }\left(\mathrm{g} / \mathrm{m}^{3}\right) \\
\text { - } \mathrm{S}(0)=\text { initial radionuclide concentration in soil }(\mathrm{Bq} / \mathrm{g} \mathrm{dry} \text { soil). }\end{array}$ & $\begin{array}{l}\text { RESRAD and ERMYN are the same with the following } \\
\text { equivalent quantities: } A S R_{3} \times S(0) \text { in RESRAD is } \\
\text { equivalent to } C_{a} \text {, in ERMYN. The unit conversion (to } \\
\text { the left) is different from that shown in the manual } \\
\text { because the item compared is not presented directly. }\end{array}$ & $\begin{array}{l}\text { Yu et al. } 2001 \\
\text { [DIRS 159465], } \\
\text { Eq. 3.11, Eq. D.9 } \\
\text { and D.10. }\end{array}$ \\
\hline $\begin{array}{l}\text { BIOMASS } \\
\text { ERB2A }\end{array}$ & $\begin{array}{l}\text { Dust deposition is not included; instead, it uses a crop soil } \\
\text { contamination factor. }\end{array}$ & Not applicable. & $\begin{array}{l}\text { BIOMASS } 2000 \\
\text { [DIRS 154522]. }\end{array}$ \\
\hline
\end{tabular}


Table 7.3-12. Comparison of the Dust Deposition Rate (Continued)

\begin{tabular}{|l|l|l|l|}
\hline Document & \multicolumn{1}{|c|}{ Mathematical Model } & \multicolumn{1}{|c|}{$\begin{array}{c}\text { Comparison with the ERMYN } \\
\text { (Equations 6.4.3-7 and 6.5.3-4) }\end{array}$} & \multicolumn{1}{c|}{ Reference } \\
\hline EPRI-YM & $\begin{array}{l}\text { Dust deposition is not included; instead, it uses a crop soil } \\
\text { contamination factor. See the interception fraction for } \\
\text { resuspended soil. }\end{array}$ & $\begin{array}{l}\text { Smith et al. 1996 } \\
\text { [DIRS 101085]. }\end{array}$ \\
\cline { 1 - 2 } NCRP-129 & $\begin{array}{l}\text { Dust deposition is not included; instead, it uses transfer factors } \\
\text { from soil to vegetation due to soil adhesion for resuspension } \\
\text { processes. }\end{array}$ & $\begin{array}{l}\text { NCRP 1999 1999 } \\
\text { [DIRS 155894]. }\end{array}$ \\
\hline
\end{tabular}

ERMYN=Environmental Radiation Model for Yucca Mountain, Nevada

Table 7.3-13. Comparison of Interception Fraction for Resuspended Soil

\begin{tabular}{|c|c|c|c|}
\hline Document & Mathematical Model & $\begin{array}{l}\text { Comparison with the ERMYN (Equations 6.4.3-8 } \\
\text { and 6.5.3-5) }\end{array}$ & Reference \\
\hline RESRAD & $\begin{array}{l}\text { No mathematical representation for the interception fraction of } \\
\text { resuspended soil. A default value of } 0.25 \text { is used for all plant } \\
\text { types. }\end{array}$ & $\begin{array}{l}\text { The default value is comparable to the results of } \\
\text { empirical equation. }\end{array}$ & $\begin{array}{l}\text { Yu et al. } 2001 \\
\text { [DIRS 159465], } \\
\text { Section D.2, p. D-12. }\end{array}$ \\
\hline $\begin{array}{l}\text { BIOMASS } \\
\text { ERB2A }\end{array}$ & $\begin{array}{l}\text { Dust deposition is not included; instead, a crop soil } \\
\text { contamination factor is used. A default value of } \\
2 \times 10^{-4} \mathrm{~kg} \text { dry soil per } \mathrm{kg} \text { wet plant is used for leafy vegetables, root } \\
\text { vegetables, and grain, and } 2 \times 10^{-3} \text { is used for fresh forage } \\
\text { feed. }\end{array}$ & $\begin{array}{l}\text { Due to differences between the models, it was } \\
\text { necessary to conduct the numerical comparison } \\
\text { described in Section } 7.4 .4 \text {. The results showed } \\
\text { that the contribution of direct deposition on crop } \\
\text { leaves due to the interception of resuspended soil } \\
\text { were numerically similar. }\end{array}$ & $\begin{array}{l}\text { BIOMASS 2000 } \\
\text { [DIRS 154522], p. 41, } \\
\text { Table 13. }\end{array}$ \\
\hline
\end{tabular}


Table 7.3-13. Comparison of Interception Fraction for Resuspended Soil (Continued)

\begin{tabular}{|c|c|c|c|}
\hline Document & Mathematical Model & $\begin{array}{c}\text { Comparison with the ERMYN (Equations 6.4.3-8 } \\
\text { and 6.5.3-5) }\end{array}$ & Reference \\
\hline EPRI-YM & $\begin{array}{l}\text { Dust deposition is not included; instead, a crop soil } \\
\text { contamination factor is used. Selected values are } \\
1.3 \times 10^{-4} \mathrm{~kg} \text { dry soil per kg wet plant for leafy vegetables and grain, } \\
2.0 \times 10^{-4} \text { for root vegetables and fruit, and } 8.0 \times 10^{-3} \text { for fresh } \\
\text { forage feed. }\end{array}$ & & $\begin{array}{l}\text { Smith et al. } 1996 \\
\text { [DIRS 101085, p. 5-25. }\end{array}$ \\
\hline NCRP-129 & $\begin{array}{l}\text { Dust deposition is not included; instead, transfer factors from } \\
\text { soil to vegetation due to soil adhesion for resuspension } \\
\text { processes are used. A value of } 1 \times 10^{-4} \mathrm{~kg}_{\text {dry soil }} \text { per } \mathrm{kg} \text { wet plant } \\
\text { is suggested for cultivated land use. The range is also } \\
\text { suggested to be } 10 \text { times lower or higher than the } \\
\text { recommended value. }\end{array}$ & & $\begin{array}{l}\text { NCRP } 1999 \\
\text { [DIRS 155894], p. 104, } \\
\text { Table 5.7. }\end{array}$ \\
\hline
\end{tabular}

ERMYN=Environmental Radiation Model for Yucca Mountain, Nevada; IAEA=International Atomic Energy Agency 


\subsubsection{Drinking Water}

The NCRP-129 model does not use contaminated water, but the other four validation models use the same method as the ERMYN (Equation 6.4.4-3), with two exceptions (Table 7.3-15). First, the GENII-S model includes radionuclide decay during the holdup time between pumping of groundwater and consumption by animals, which is short relative to the rate of radioactive decay for long-lived radionuclides (Table 6.3-7). Thus, the methods are mathematically equivalent. Second, the GENII-S model uses a parameter for the proportion of contaminated water. Because all water is contaminated in the ERMYN, the value of this parameter is one, and the methods are mathematically equivalent.

\subsubsection{Soil Ingestion}

Three of the validation models (BIOMASS ERB2A, RESRAD, and EPRI-YM) include soil ingestion, and they all use the same method as the ERMYN (Equations 6.4.4-4 and 6.5.4-3; Table 7.3-16). Therefore, they are mathematically equivalent.

The other two models do not include this process. The omission of this contamination mechanism is an ACM (Section 6.3.3), and a numerical evaluation is conducted to evaluate the importance of soil ingestion to animal product contamination (Section 7.4.5). Omitting soil ingestion by animals results in a difference in radionuclide concentrations in meat by more than a factor of two because this process may account for about 75 percent of the total concentration (Table 7.4-12). The ERMYN includes soil ingestion by animals to avoid underestimating the dose from this pathway. Therefore, this addition to the ERMYN animal submodel is justified.

\subsubsection{Dust Inhalation}

The ERMYN does not include dust inhalation as a mechanism for animal product contamination. Only two of the validation models (BIOMASS ERB2A and EPRI-YM) include this process (Table 7.3-17). Because of a lack of animal data on transfer coefficients for dust inhalation, the two models use human data. Dust inhalation is an ACM (Section 6.3.3, the same ACM as for animal soil ingestion), and the ERMYN and BIOMASS animal submodels are compared to evaluate the importance of this pathway (Section 7.4.5). The two models (which are similar except for this pathway) produce similar estimates of meat concentrations (ERMYN: $2.64 \times 10^{-5} \mathrm{~Bq} / \mathrm{kg}$; BIOMASS: $2.62 \times 10^{-5} \mathrm{~Bq} / \mathrm{kg}$ ), but the contribution from dust inhalation $\left(3.4 \times 10^{-10} \mathrm{~Bq} / \mathrm{kg}\right)$ to the total concentration in meat in the BIOMASS model is negligible. Therefore, the methods are numerically similar, and including dust inhalation by animals is unnecessary. 


\begin{tabular}{|c|c|c|c|}
\hline Document & Mathematical Model & $\begin{array}{l}\text { Comparison with the ERMYN } \\
\text { (Equations 6.4.4-2 and 6.5.4-2) }\end{array}$ & Reference \\
\hline GENII-S & 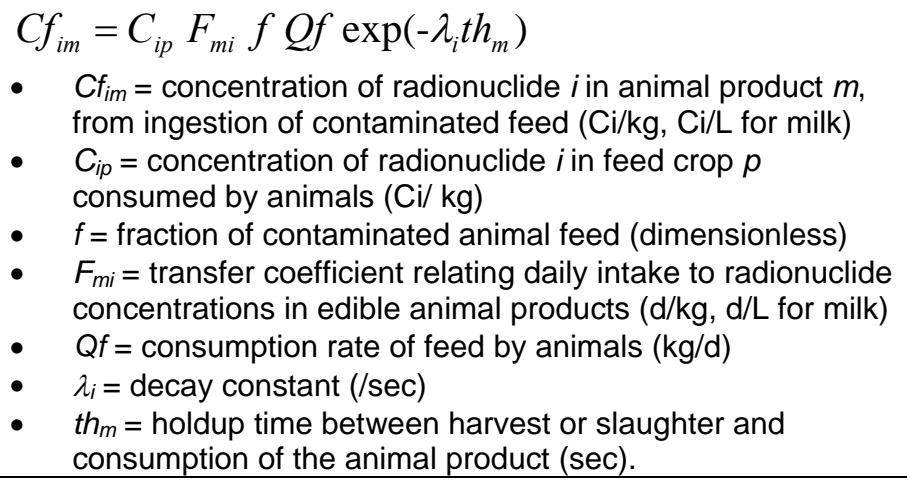 & $\begin{array}{l}\text { GENII-S uses a holdup time for animal products to } \\
\text { account for radionuclide decay, which can be ignored } \\
\text { for long-lived radionuclides. GENII-S also uses a } \\
\text { parameter for the fraction of contaminated animal feed, } \\
\text { which would be } 1.0 \text { in the ERMYN. Except for these } \\
\text { differences, the GENII-S and ERMYNs are the same. }\end{array}$ & $\begin{array}{l}\text { Napier et al. } 1988 \\
\text { [DIRS 157927], } \\
\text { p. 4.70, Eq. 4.7.11. }\end{array}$ \\
\hline $\begin{array}{l}\text { BIOMASS } \\
\text { ERB2A }\end{array}$ & $\begin{array}{l}C_{\text {prod,fodd }}=T F_{\text {proding }} C_{\text {fodd }} I N G_{\text {fodd }} \\
\text { - } \quad C_{\text {prod, fodd }}=\text { radionuclide concentration in an animal product } \\
\text { due to ingestion of animal fodder }(\mathrm{Bq} / \mathrm{kg}) \\
\text { - } \quad C_{\text {fodd }}=\text { radionuclide concentration in animal fodder } \\
(\mathrm{Bq} / \mathrm{kg} \text { fresh weight of fodder }) \\
\text { - } \quad T F_{\text {proding }}=\text { transfer factor for ingestion for animal products } \\
\left(\mathrm{d} / \mathrm{kg}_{\text {fresh weight of product })}\right. \\
\text { - } \quad \mathrm{IG}_{\text {fodd }}=\text { consumption rate of fodder by animals } \\
\left(\mathrm{kg}_{\text {fresh weight }} / \mathrm{d}\right) .\end{array}$ & BIOMASS ERB2A and ERMYN use the same method. & $\begin{array}{l}\text { BIOMASS } 2000 \\
\text { [DIRS 154522], } \\
\text { p. 36, Eq. } 13 .\end{array}$ \\
\hline EPRI-YM & $\begin{array}{l}\text { EPRI-YM uses the same equation as BIOMASS ERB2A, except } \\
\text { for a few notations. }\end{array}$ & EPRI-YM and ERMYN use the same method. & $\begin{array}{l}\text { Smith et al. } 1996 \\
\text { [DIRS 101085], } \\
\text { p. 5-10, Eq. 5.11. }\end{array}$ \\
\hline
\end{tabular}




\begin{tabular}{|c|c|c|c|}
\hline Document & Mathematical Model & $\begin{array}{l}\text { Comparison with the ERMYN } \\
\text { (Equations 6.4.4-2, and 6.5.4-2) }\end{array}$ & Reference \\
\hline RESRAD & 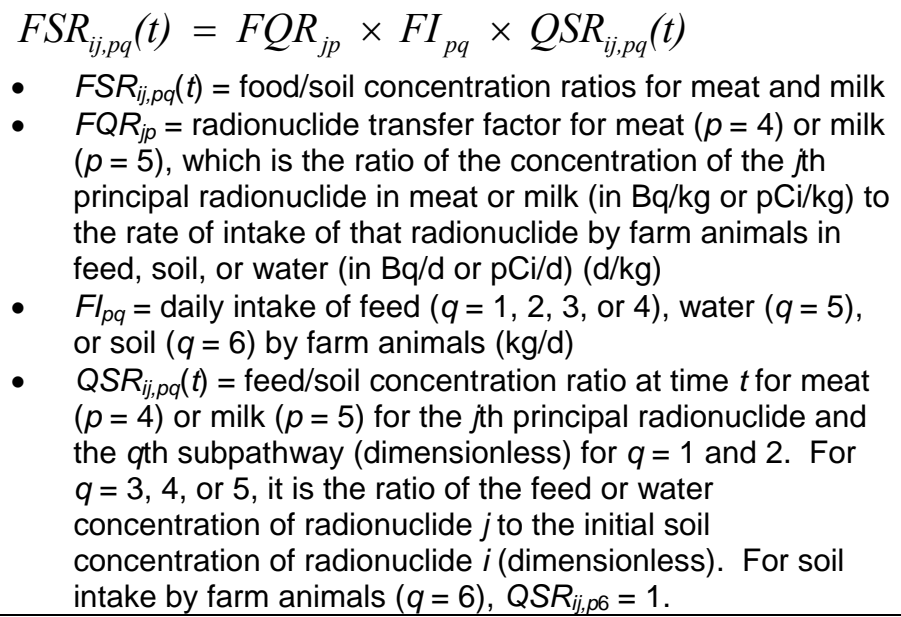 & $\begin{array}{l}\text { This equation is for the contamination of all animal } \\
\text { products, including feed, soil, and water, which are the } \\
\text { same animal pathways used in the ERMYN. RESRAD } \\
\text { and ERMYN are the same, except that the RESRAD } \\
\text { equation is based on food/soil concentration ratios. }\end{array}$ & $\begin{array}{l}\text { Yu et al. } 2001 \\
\text { [DIRS 159465], } \\
\text { p. D-15, Eq. D.15. }\end{array}$ \\
\hline NCRP-129 & 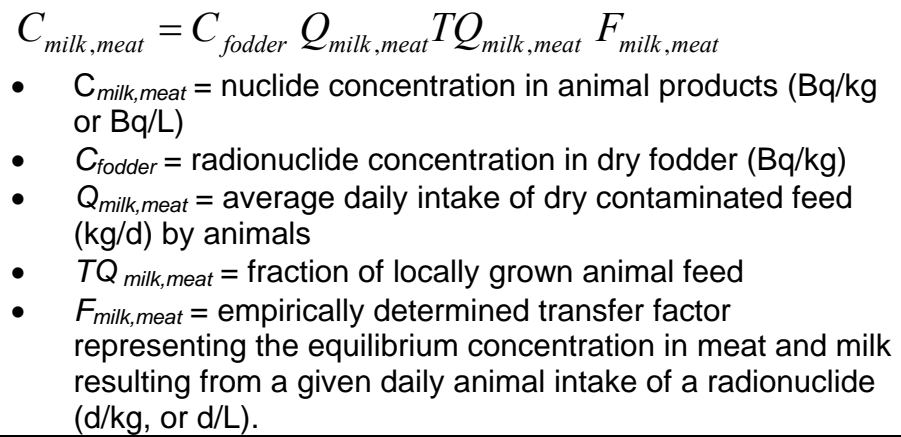 & $\begin{array}{l}\text { NCRP-129 is the same as GENII-S, except that the } \\
\text { holdup decay term is not shown here, although it is } \\
\text { used in the ingestion submodel (NCRP } 1999 \\
\text { [DIRS 155894], p. 91, Eq. 5.1). }\end{array}$ & $\begin{array}{l}\text { NCRP } 1999 \\
\text { [DIRS 155894], } \\
\text { p. 93, Eq. 5.3. }\end{array}$ \\
\hline
\end{tabular}

ERMYN=Environmental Radiation Model for Yucca Mountain, Nevada 


\begin{tabular}{|c|c|c|c|}
\hline Document & Mathematical Model & $\begin{array}{l}\text { Comparison with the ERMYN } \\
\text { (Equation 6.4.4-3) }\end{array}$ & Reference \\
\hline GENII-S & 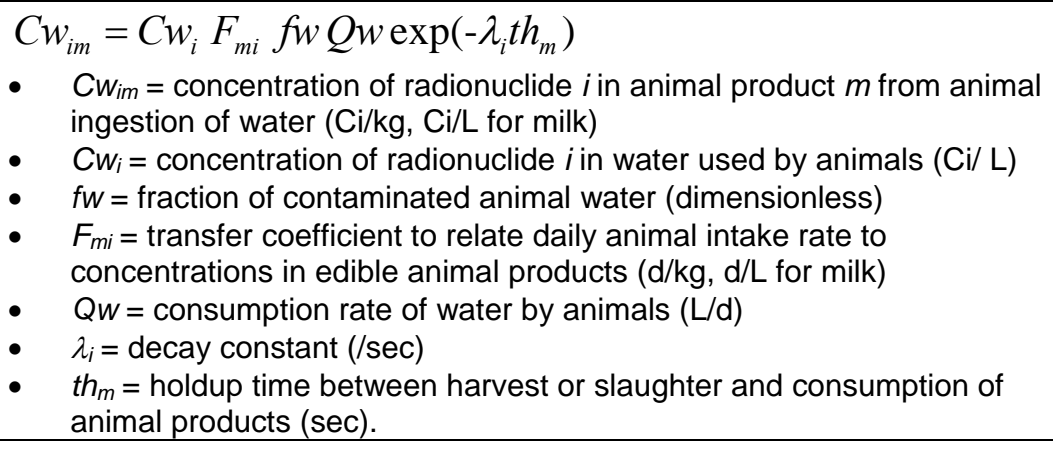 & $\begin{array}{l}\text { GENII-S uses animal product holdup } \\
\text { time for radionuclide decay, which can } \\
\text { be ignored for long-lived radionuclides. } \\
\text { It also uses the fraction of animal water } \\
\text { contaminated. Except for these } \\
\text { two differences, GENII-S and ERMYN } \\
\text { are the same. }\end{array}$ & $\begin{array}{l}\text { Napier et al. } 1988 \\
\text { [DIRS 157927], p. } 4.71 \text {, } \\
\text { Eq. 4.7.12. }\end{array}$ \\
\hline $\begin{array}{l}\text { BIOMASS } \\
\text { ERB2A }\end{array}$ & $\begin{array}{l}C_{\text {prod,fodd }}=T F_{\text {proding }} C_{w} I N G_{w a} \\
\text { - } \quad C_{\text {prod, fodd }}=\text { radionuclide concentration in animal products due to } \\
\text { ingestion of animal fodder }(\mathrm{Bg} / \mathrm{kg}) \\
\text { - } C_{w}=\text { radionuclide concentrations in groundwater } \\
(\mathrm{Bq} / \mathrm{kg} \text { fresh weight of fodder }) \\
\text { - } \quad T F_{\text {proding }} \text { transfer factor for ingestion for animal products } \\
\text { (d/kg fresh weight of product) } \\
\left.\text { - } \quad I N G_{w a}=\text { consumption rate of water by animals, } \mathrm{kg} \text { fresh weight } / \mathrm{d}\right) .\end{array}$ & $\begin{array}{l}\text { BIOMASS ERB2A and ERMYN use the } \\
\text { same method. }\end{array}$ & $\begin{array}{l}\text { BIOMASS 2000 } \\
\text { [DIRS 154522], p. 36, } \\
\text { Eq. } 13 .\end{array}$ \\
\hline EPRI-YM & $\begin{array}{l}\text { EPRI-YM uses the same equation as BIOMASS ERB2A, except for a few } \\
\text { notations. }\end{array}$ & $\begin{array}{l}\text { EPRI-YM and ERMYN use the same } \\
\text { method. }\end{array}$ & $\begin{array}{l}\text { Smith et al. } 1996 \\
\text { [DIRS 101085], p. 5-10, } \\
\text { Eq. 5.11. }\end{array}$ \\
\hline RESRAD & Mathematical equation shown in Table 7.3-14. & $\begin{array}{l}\text { RESRAD is the same as ERMYN, } \\
\text { except that the equation is based on } \\
\text { food/soil concentration ratios. }\end{array}$ & $\begin{array}{l}\text { Yu et al. } 2001 \\
\text { [DIRS 159465], p. D-15, } \\
\text { Eq. D.15. }\end{array}$ \\
\hline NCRP-129 & Not included. & Not applicable. & $\begin{array}{l}\text { NCRP } 1999 \\
\text { [DIRS 155894]. }\end{array}$ \\
\hline
\end{tabular}

ERMYN=Environmental Radiation Model for Yucca Mountain, Nevada 


\begin{tabular}{|c|c|c|c|}
\hline Document & Mathematical Model & $\begin{array}{l}\text { Comparison with ERMYN } \\
\text { (Equation 6.4.4-4 and 6.5.4-3) }\end{array}$ & Reference \\
\hline GENII-S & Not included. & Not applicable. & $\begin{array}{l}\text { Napier et al. } 1988 \\
\text { [DIRS 157927], } \\
\text { Section 4.7.5. }\end{array}$ \\
\hline $\begin{array}{l}\text { BIOMASS } \\
\text { ERB2A }\end{array}$ & $\begin{array}{l}C_{\text {prod,water }}=T F_{\text {proding }} \frac{C_{s} I N G_{s a}}{\left(1-\theta_{t}\right) \rho+\theta \rho_{w}} \\
\text { - } \quad C_{\text {prod,water }} \text { radionuclide concentrations in animal products due to the } \\
\text { ingestion of drinking water }(\mathrm{Bq} / \mathrm{kg}) \\
\text { - } \quad T F_{\text {proding }}=\text { transfer factor for ingestion for animal products } \\
(\mathrm{d} / \mathrm{kg} \text { fresh weight of product }) \\
\text { - } \quad C_{s}=\text { radionuclide concentrations in cultivated soil }\left(\mathrm{Bq} / \mathrm{m}^{3}\right) \\
\text { - } \quad I N G_{\text {sa }}=\text { consumption rate of cultivated soil by animals } \\
\quad(\mathrm{kg} \text { wet weight of soil } / \mathrm{d}) \\
\text { - } \quad \theta_{t}=\text { total porosity of the cultivated soil compartment (dimensionless) } \\
\text { - } \quad \rho=\text { dry grain density of the cultivated soil compartment }\left(\mathrm{kg} / \mathrm{m}^{3}\right) \\
\text { - } \quad \theta=\text { water filled porosity of the cultivated soil compartment } \\
\text { - (dimensionless) } \\
\end{array}$ & $\begin{array}{l}\text { BIOMASS ERB2A and ERMYN use the } \\
\text { same method, except soil concentration } \\
\text { requires a unit conversion. }\end{array}$ & $\begin{array}{l}\text { BIOMASS 2000 } \\
\text { [DIRS 154522], p. 36, } \\
\text { Eq. } 13 .\end{array}$ \\
\hline EPRI-YM & $\begin{array}{l}\text { EPRI-YM and BIOMASS ERB2A use the same equation, except for a few } \\
\text { notations. }\end{array}$ & $\begin{array}{l}\text { EPRI-YM and ERMYN use the same } \\
\text { method. }\end{array}$ & $\begin{array}{l}\text { Smith et al. } 1996 \\
\text { [DIRS 101085], p. 5-10, } \\
\text { Eq. 5.11. }\end{array}$ \\
\hline RESRAD & Mathematical equation shown in Table 7.3-14. & $\begin{array}{l}\text { RESRAD and ERMYN use the same } \\
\text { method, except the equation is derived } \\
\text { based on the food/soil concentration } \\
\text { ratios. }\end{array}$ & $\begin{array}{l}\text { Yu et al. } 2001 \\
\text { [DIRS 159465], p. D-15 } \\
\text { Eq. D.15. }\end{array}$ \\
\hline NCRP-129 & Not included. & Not applicable. & $\begin{array}{l}\text { NCRP 1999, } \\
\text { [DIRS 155894]. }\end{array}$ \\
\hline
\end{tabular}

ERMYN=Environmental Radiation Model for Yucca Mountain, Nevada 
Table 7.3-17. Comparison of Animal Product Contamination Due to Dust Inhalation

\begin{tabular}{|c|c|c|c|}
\hline Document & Mathematical Model & $\begin{array}{l}\text { Comparison with the ERMYN } \\
\text { (Not considered in ERMYN) }\end{array}$ & Reference \\
\hline GENII-S & Not considered. & Not applicable. & $\begin{array}{l}\text { Napier et al. } 1988 \\
\text { [DIRS 157927], } \\
\text { Section 4.7.5. }\end{array}$ \\
\hline $\begin{array}{l}\text { BIOMASS } \\
\text { ERB2A }\end{array}$ & 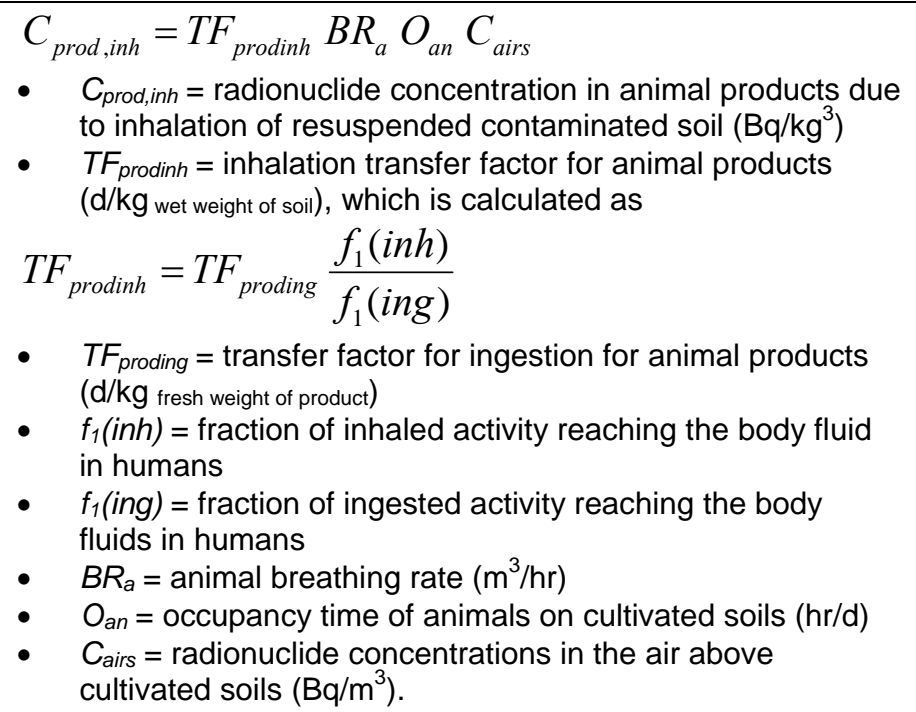 & $\begin{array}{l}\text { BIOMASS ERB2A includes animal inhalation } \\
\text { of contaminated dust using a transfer factor for } \\
\text { inhalation based on human data (BIOMASS } \\
2000 \text { [DIRS 154522], p. 36, Eq. 13). ERMYN } \\
\text { does not include this pathway. A numerical } \\
\text { comparison shows that the contribution is } \\
\text { small (Section } 7.4 .5 \text { ). }\end{array}$ & $\begin{array}{l}\text { BIOMASS 2000 } \\
\text { [DIRS 154522], p. 36, } \\
\text { Eq. } 13 .\end{array}$ \\
\hline EPRI-YM & $\begin{array}{l}\text { EPRI-YM and BIOMASS ERB2A use similar equations and use } \\
\text { the same conversion factor for ingestion and inhalation } \\
\left(\text { CF }_{\text {prod }}=T F_{\text {proding }}=T F_{\text {prodinh }}\right) \text {. }\end{array}$ & EPRI-YM and ERMYN are similar. & $\begin{array}{l}\text { Smith et al. } 1996 \\
\text { [DIRS 101085], p. 5-10, } \\
\text { Eq. 5.11. }\end{array}$ \\
\hline RESRAD & Not included. & Not applicable. & $\begin{array}{l}\text { Yu et al. } 2001 \\
\text { [DIRS 159465], Appendix D. }\end{array}$ \\
\hline NCRP-129 & Not included. & Not applicable. & NCRP 1999 [DIRS 155894]. \\
\hline
\end{tabular}

ERMYN=Environmental Radiation Model for Yucca Mountain, Nevada 


\subsubsection{Validation of the Fish Submodel}

Two of the five validation models (GENII-S and RESRAD) use a fish submodel, and both use methods similar to the ERMYN (Equation 6.4.5-2) with three exceptions (Table 7.3-18):

- GENII-S includes radionuclide decay during the holdup period between harvest and consumption. Holdup time is short (generally days to weeks) relative to the rate of decay for the long-lived radionuclides in the ERMYN (Table 6.3-7). Thus, the exponential factor in GENII-S that includes the holdup time and decay constant approaches one, making the methods mathematically equivalent.

- RESRAD uses a dietary fraction and a contamination factor in calculating fish contamination. These parameters are incorporated into the fish consumption rate in the ERMYN ingestion submodel; therefore, the methods are mathematically equivalent.

- GENII-S and RESRAD do not include a water concentration modifying factor. Values of that factor depend on the climate and differ among radionuclides. Typical values are 1 for ${ }^{14} \mathrm{C}$ and 4.15 for other radionuclides under present-day climate conditions (Table 6.6-3). Omission of the water concentration modifying factor could result in a difference by more than a factor of two in estimates of radionuclide concentrations in fish. The ERMYN includes this factor to account for increases in activity concentrations due to evaporation. This addition is necessary because the fish ponds in Amargosa Valley are small, the evaporation rate is high, and the activity concentration likely would increase when additional groundwater is added to compensate for evaporation (Section 6.4.5). This addition is justified because it is based on site-specific conditions in the Amargosa Valley and because it avoids underestimating radionuclide concentrations in fish.

Thus, the fish submodels are mathematically equivalent or the differences are due to model improvements or site-specific conditions, and, therefore, the ERMYN submodel is validated. 


\begin{tabular}{|c|c|c|c|}
\hline Document & Mathematical Model & $\begin{array}{l}\text { Comparison with the ERMYN } \\
\text { (Equation 6.4.5-2) }\end{array}$ & Reference \\
\hline GENII-S & 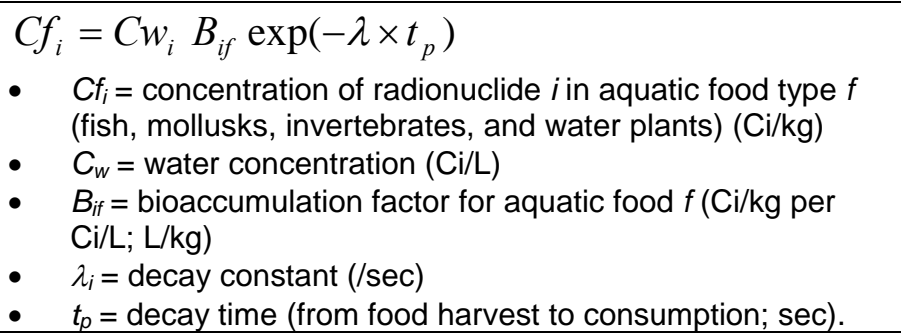 & $\begin{array}{l}\text { The GENII-S model is capable of including } \\
\text { more aquatic foods in fresh and salt water, but } \\
\text { only freshwater fish are included in the Yucca } \\
\text { Mountain biosphere. The GENII-S fish } \\
\text { submodel is the same as the ERMYN, except } \\
\text { that the ERMYN includes a modifying factor for } \\
\text { pond water evaporation. }\end{array}$ & $\begin{array}{l}\text { Napier et al. } 1988 \\
\text { [DIRS 157927], p. 4.66, } \\
\text { Eq. 4.7.5a. }\end{array}$ \\
\hline $\begin{array}{l}\text { BIOMASS } \\
\text { ERB2A }\end{array}$ & Not included. & Not applicable. & $\begin{array}{l}\text { BIOMASS } 2000 \\
\text { [DIRS 154522], Section } 7 .\end{array}$ \\
\hline EPRI-YM & Not included. & Not applicable. & $\begin{array}{l}\text { Smith et al. } 1996 \\
\text { [DIRS 101085]. }\end{array}$ \\
\hline RESRAD & 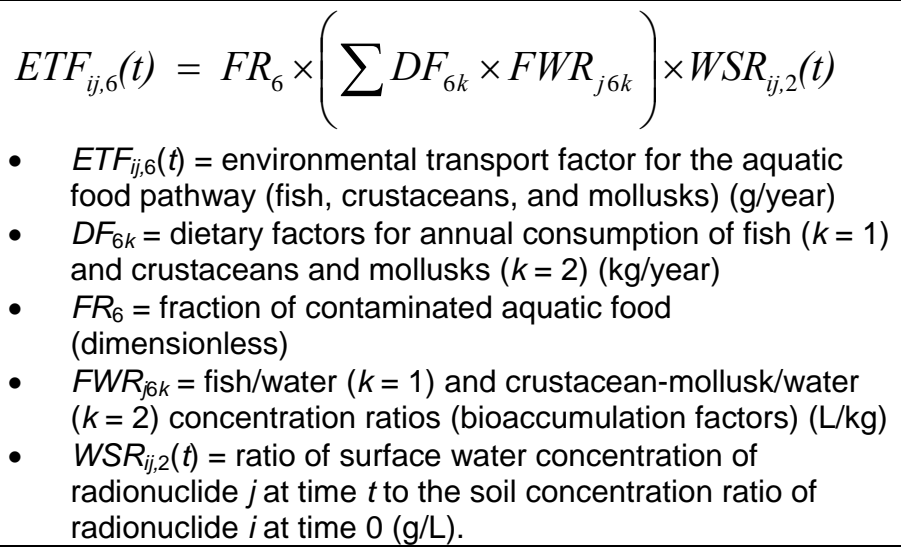 & $\begin{array}{l}\text { RESRAD includes several aquatic food } \\
\text { pathways, and it uses a dietary factor and a } \\
\text { contamination factor, which are included in the } \\
\text { ERMYN consumption rates (ingestion } \\
\text { submodel). For the fish pathway, RESRAD } \\
\text { and GENII-S are equivalent, except the } \\
\text { RESRAD equation is based on a food/soil } \\
\text { concentration ratio. }\end{array}$ & $\begin{array}{l}\text { Yu et al. } 2001 \\
\text { [DIRS 159465], p. D-18, } \\
\text { Eq. D.21. }\end{array}$ \\
\hline NCRP-129 & Not included. & Not applicable. & NCRP 1999 [DIRS 155894]. \\
\hline
\end{tabular}

ERMYN=Environmental Radiation Model for Yucca Mountain, Nevada 


\subsubsection{Validation of the ${ }^{14} \mathrm{C}$ Special Submodel}

The ERMYN includes a ${ }^{14} \mathrm{C}$ special submodel. Among the five validation models, three (GENII-S, RESRAD, and BIOMASS ERB2A) include ${ }^{14} \mathrm{C}$ special submodels. The GENII-S submodel only includes crops, animal products, and fish contamination. The ERMYN, RESRAD, and BIOMASS ERB2A models include these three pathways plus soil and air contamination (which can cause external exposure to contaminated soil, inhalation of contaminated air, and inadvertent soil ingestion). Including soil and air contamination in the ERMYN ${ }^{14} \mathrm{C}$ special model is an improvement over the GENII-S model. Comparisons of the methods for calculating ${ }^{14} \mathrm{C}$ concentrations in soil, air, plants, and animals are presented below. Concentrations of ${ }^{14} \mathrm{C}$ in fish are calculated in the fish submodel (Section 6.4.5).

Soil Contamination-In the ERMYN, the calculation of ${ }^{14} \mathrm{C}$ soil contamination resulting from irrigation (Equation 6.4.6-1) is similar to the method used in the soil submodel for other radionuclides (Equation 6.4.1-2). BIOMASS ERB2A is the only validation model that directly calculates ${ }^{14} \mathrm{C}$ soil contamination from irrigation water; RESRAD includes soil contamination as the initial source term. The ERMYN and BIOMASS ERB2A models use the same methods, with the following exceptions (Table 7.3-19):

- BIOMASS ERB2A includes the weathering of ${ }^{14} \mathrm{C}$ from plants as a soil contamination source. The ERMYN assumes that all radionuclides in irrigation water eventually are deposited in the soil (Approximation 6). Therefore, the parameters in the BIOMASS ERB2A model for calculating weathering are unnecessary in the ERMYN, and the methods are mathematically equivalent.

- BIOMASS ERB2A does not include losses of ${ }^{14} \mathrm{C}$ due to erosion or radionuclide decay. These loss mechanisms are used in the ERMYN ${ }^{14} \mathrm{C}$ submodel to maintain consistency with the soil submodel. The rate of loss from erosion and radionuclide decay is inconsequential compared to the ${ }^{14} \mathrm{C}$ emission rate (about 22/year; Table 6.6-3). Therefore, including losses due to erosion and radionuclide decay do not affect the results of the equation, and the methods are mathematically equivalent.

Air Contamination-In the air, ${ }^{14} \mathrm{C}$ contamination is caused by the release of ${ }^{14} \mathrm{C}$ from groundwater-contaminated soil (due to the volatility of ${ }^{14} \mathrm{C}$ ), as almost all ${ }^{14} \mathrm{C}$ in the soil is quickly released into the air. The BIOMASS ERB2A and RESRAD models include ${ }^{14} \mathrm{C}$ in the air, using methods that are the same as the ERMYN, with the following exceptions (Table 7.3-20):

- BIOMASS ERB2A and RESRAD include a factor for the proportion of time that the wind blows from the contaminated source to the receptor. In the ERMYN, the area contaminated by long-term irrigation surrounds the receptor, so this factor would be 1.0. Therefore, these methods are mathematically equivalent.

- BIOMASS ERB2A uses radionuclide concentrations per unit volume $\left(\mathrm{Bq} / \mathrm{m}^{3}\right)$ and includes parameters for the width and volume of the plant canopy. The ERMYN uses concentrations per unit area and replaces the width and volume parameters with a 
parameter for the size of the irrigated area. Thus, the differences are unit conversions, and the methods are mathematically equivalent.

- The calculation of ${ }^{14} \mathrm{C}$ flux density from contaminated soil (evasion rate) differs in the RESRAD and ERMYNs because of differences in the source term. The differences are unit conversions (Table 7.3-20), and the methods are mathematically equivalent.

Plant Contamination-Plant contamination by ${ }^{14} \mathrm{C}$ is included in the GENII-S, RESRAD, and BIOMASS ERB2A models. The ERMYN method (Equation 6.4.6-6) is the same as that used in RESRAD (Table 7.3-21). BIOMASS ERB2A uses a different method that requires additional input parameters, the measurements of which are not available, and the model documentation (BIOMASS 2000 [DIRS 154522]) does not include recommended values. Therefore, a numerical comparison is not possible, and this portion of BIOMASS ERB2A is not used to validate the ERMYN.

The GENII-S and ERMYNs use substantially different methods for calculating ${ }^{14} \mathrm{C}$ plant contamination, so the models are compared numerically (Section 7.4.7). The ${ }^{14} \mathrm{C}$ concentration in leafy vegetables calculated using the ERMYN method $\left(3.2 \times 10^{-2} \mathrm{~Bq} / \mathrm{kg}\right.$; Table $\left.7.4-13\right)$ is about six times higher than the value calculated using the GENII-S method $\left(5.8 \times 10^{-3} \mathrm{~Bq} / \mathrm{kg}\right)$. However, using a more appropriate removal rate, the results of the ERMYN and GENII-S models are almost the same (Section 7.4.7). Using the default removal rate, the GENII-S method underestimates ${ }^{14} \mathrm{C}$ contamination in plants because it only includes contamination from root uptake, whereas the ERMYN, BIOMASS ERB2A, and RESRAD models include the uptake of ${ }^{14} \mathrm{CO}_{2}$ during photosynthesis. Because of the high emission rate of ${ }^{14} \mathrm{C}$ from the soil (Table 6.6-3), uptake of ${ }^{14} \mathrm{C}$ via photosynthesis is an important transfer process. Therefore, the ERMYN method is justified because it includes this important transfer process to avoid underestimating dose.

Animal Contamination-The GENII-S, RESRAD, and BIOMASS ERB2A models include animal product contamination by ${ }^{14} \mathrm{C}$. The ERMYN method (Equation 6.4.6-7) is the same as the RESRAD method (Table 7.3-22). BIOMASS ERB2A uses a different method that requires additional input parameters, the measurements of which are not available, and the model documentation (BIOMASS 2000 [DIRS 154522]) does not include recommended values. Therefore, a numerical comparison is not possible. The GENII-S model is similar to the ERMYN, except that soil ingestion is not included in GENII-S. As described in Sections 7.3.4 and 7.4.5, ingestion of soil is an important contamination mechanism for animals. Therefore, the method used in the ERMYN is justified because it avoids underestimating ${ }^{14} \mathrm{C}$ concentrations in animals. 


\begin{tabular}{|c|c|c|c|}
\hline Document & Mathematical Model & $\begin{array}{c}\text { Comparison with the ERMYN } \\
\text { (Equation 6.4.6-1) }\end{array}$ & Reference \\
\hline GENII-S & Not included. & $\begin{array}{l}\text { GENII-S does not include }{ }^{14} \mathrm{C} \\
\text { contamination in the soil. }\end{array}$ & $\begin{array}{l}\text { Napier et al. } 1988 \\
\text { [DIRS 157927]. }\end{array}$ \\
\hline $\begin{array}{l}\text { BIOMASS } \\
\text { ERB2A }\end{array}$ & 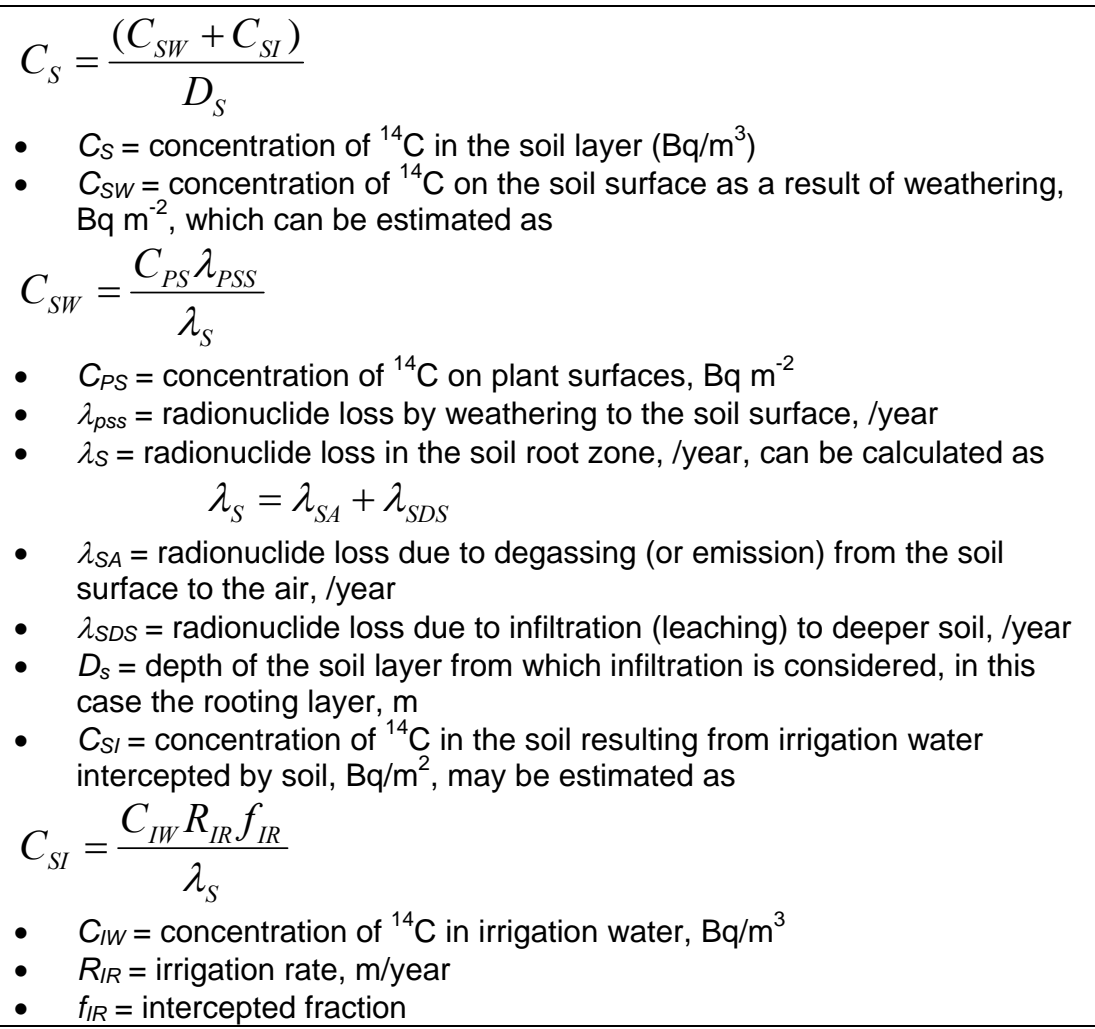 & $\begin{array}{l}\text { BIOMASS ERB2A includes }{ }^{14} \mathrm{C} \text { soil } \\
\text { contamination from irrigation and } \\
\text { weathering of plant surfaces. This differs } \\
\text { from the BIOMASS ERB2A model for all } \\
\text { other radionuclides, in which all irrigation } \\
\text { water is deposited in the soil (same as } \\
\text { Approximation 6). BIOMASS ERB2A } \\
\text { uses two loss mechanisms (leaching and } \\
\text { emission), but not erosion (which is } \\
\text { inconsistent with the regular model in } \\
\text { which erosion is limited). BIOMASS } \\
\text { ERB2A and ERMYN are similar if it is } \\
\text { assumed that the intercepted irrigation } \\
\text { water is eventually deposited on the soil. }\end{array}$ & $\begin{array}{l}\text { BIOMASS } 2000 \\
\text { [DIRS 154522], } \\
\text { Appendix A. }\end{array}$ \\
\hline EPRI-YM & Not included. & Not applicable. & $\begin{array}{l}\text { Smith et al. } 1996 \\
\text { [DIRS 101085]. }\end{array}$ \\
\hline RESRAD & $\begin{array}{l}\text { The source term is the concentration of }{ }^{14} \mathrm{C} \text { in contaminated soil }(\mathrm{Sc}-14) \text {, and, } \\
\text { therefore, it is not a calculated value. }\end{array}$ & Source term in RESRAD. & $\begin{array}{l}\text { Yu et al. } 2001 \\
\text { [DIRS 159465], } \\
\text { Appendix L. }\end{array}$ \\
\hline NCRP-129 & Not included. & Not applicable. & $\begin{array}{l}\text { NCRP } 1999 \\
\text { [DIRS 155894]. }\end{array}$ \\
\hline
\end{tabular}


Table 7.3-20. Comparison of ${ }^{14} \mathrm{C}$ Special Model for Air Contamination

\begin{tabular}{|c|c|c|c|}
\hline Document & Mathematical Model & $\begin{array}{l}\text { Comparison with the ERMYN } \\
\text { (Equation 6.4.6-2 and 6.4.6-3) }\end{array}$ & Reference \\
\hline GENII-S & Not included. & $\begin{array}{l}\text { GENII-S does not include }{ }^{14} \mathrm{C} \\
\text { contamination in the air. }\end{array}$ & $\begin{array}{l}\text { Napier et al. } 1988 \\
\text { [DIRS 157927]). }\end{array}$ \\
\hline $\begin{array}{l}\text { BIOMASS } \\
\text { ERB2A }\end{array}$ & $\begin{array}{l}C_{S A}=\frac{C_{S} \lambda_{S A}}{\lambda_{A}} \\
\text { - } C_{S A}=\text { concentration of }{ }^{14} \mathrm{C} \text { in the canopy due to degassing }\left(\mathrm{Bq} / \mathrm{m}^{3}\right) \\
\text { - } \quad C_{S}=\text { concentration of }{ }^{14} \mathrm{C} \text { on the soil surface }\left(\mathrm{Bq} / \mathrm{m}^{3}\right) \\
\lambda_{S A}=\text { radionuclide loss due to degassing (or emission) from the soil } \\
\text { surface to the air (/year) } \\
\lambda_{A}=\text { radionuclide loss in the canopy (/year), calculated as } \\
\lambda_{A}=\frac{f \times U \times w_{C} \times h_{c}}{v_{C}} \\
\text { - } \quad f=\text { factor to account for the geometry of the contaminated area, varying } \\
\text { wind directions } \\
\text { - } \quad \begin{array}{l}\quad=\text { average wind speed (m/year) } \\
\text { direction (m) }\end{array} \\
\text { - } \quad h_{c}=\text { assumed height of the canopy layer }(\mathrm{m}) \\
\text { - } \quad v_{c}=\text { volume of the contaminated plant canopy }\left(\mathrm{m}^{3}\right)\end{array}$ & $\begin{array}{l}\text { BIOMASS ERB2A and ERMYN are the } \\
\text { same, except for the geometry of the } \\
\text { contaminated area and the varying wind } \\
\text { direction. Because most or all of the } \\
\text { area would be contaminated, this } \\
\text { parameter is unnecessary in the } \\
\text { ERMYN. The BIOMASS model uses } \\
\text { radionuclide concentration in units of } \\
\text { Bq } / \mathrm{m}^{3} \text {, as shown in the equation where } \\
v_{c} \text { is the volume of contaminated plant } \\
\text { canopy. In contrast, the ERMYN uses } \\
\text { units of Bq } / \mathrm{m}^{2} \text { and an area (A) parameter } \\
\text { (Equation } 6.4 .6-3) \text {. }\end{array}$ & $\begin{array}{l}\text { BIOMASS 2000 } \\
\text { [DIRS 154522], } \\
\text { pp. } 69 \text { and 70, } \\
\text { Appendix A. }\end{array}$ \\
\hline EPRI-YM & Not included. & Not applicable. & $\begin{array}{l}\text { Smith et al. } 1996 \\
\text { [DIRS 101085]. }\end{array}$ \\
\hline
\end{tabular}




\begin{tabular}{|c|c|c|c|}
\hline Document & Mathematical Model & $\begin{array}{l}\text { Comparison with the ERMYN } \\
\text { (Equation } 6.4 .6-2 \text { and } 6.4 .6-3 \text { ) }\end{array}$ & Reference \\
\hline RESRAD & 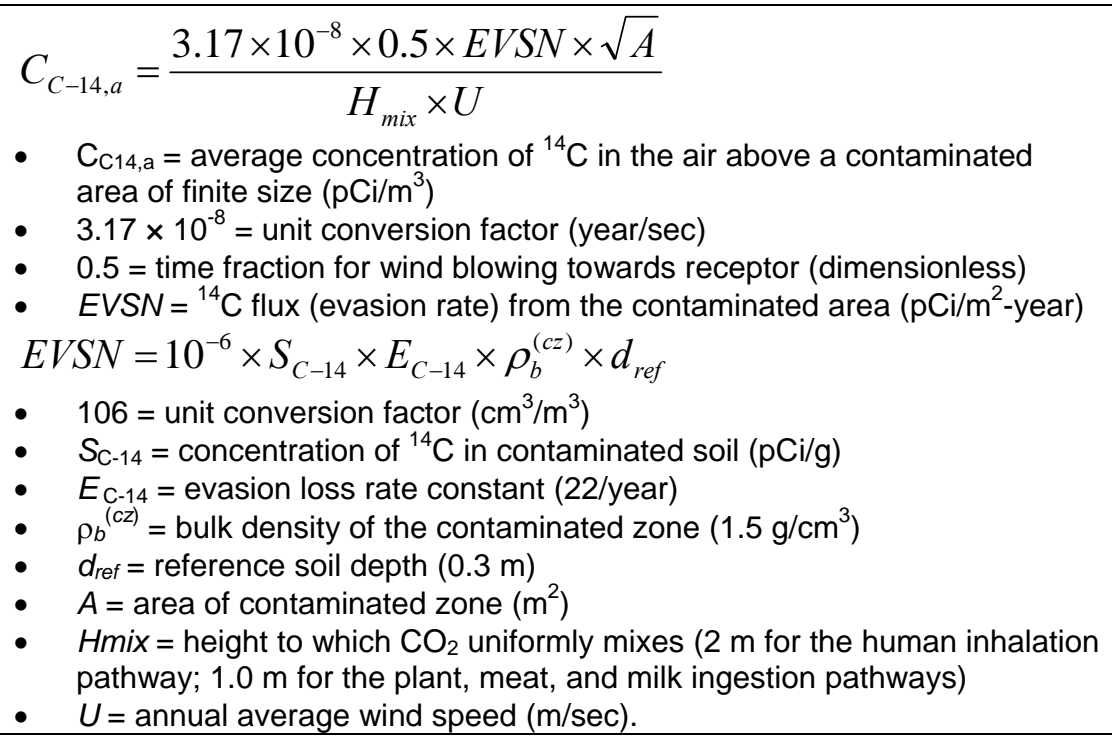 & $\begin{array}{l}\text { The ERMYN uses the RESRAD } \\
\text { equation, except that the time fraction for } \\
\text { wind blowing toward the receptor is set } \\
\text { to } 1.0 \text { (Section } 6.4 .6 .2) \text { because the } \\
\text { ERMYN does not need this parameter. } \\
\text { Because of different source terms, the } \\
{ }^{14} \mathrm{C} \text { flux density, EVSN, calculations are } \\
\text { different in the RESRAD and ERMYNs: } \\
C S_{C-14}\left(B q / m^{2}\right) \text { in ERMYN is equivalent to } \\
10^{6} \times S_{c-14} \times \rho_{b}{ }^{(c z)} \times d_{r e f} \text { in RESRAD. }\end{array}$ & $\begin{array}{l}\text { Yu et al. } 2001 \\
\text { [DIRS 159465], } \\
\text { p. L-15, Eq. L.25. }\end{array}$ \\
\hline NCRP-129 & Not included. & Not applicable. & $\begin{array}{l}\text { NCRP } 1999 \\
\text { [DIRS 155894]. }\end{array}$ \\
\hline
\end{tabular}

ERMYN=Environmental Radiation Model for Yucca Mountain, Nevada 


\begin{tabular}{|c|c|c|c|}
\hline Document & Mathematical Model & $\begin{array}{c}\text { Comparison with the ERMYN } \\
\text { (Equation 6.4.6-6) }\end{array}$ & Reference \\
\hline GENII-S & 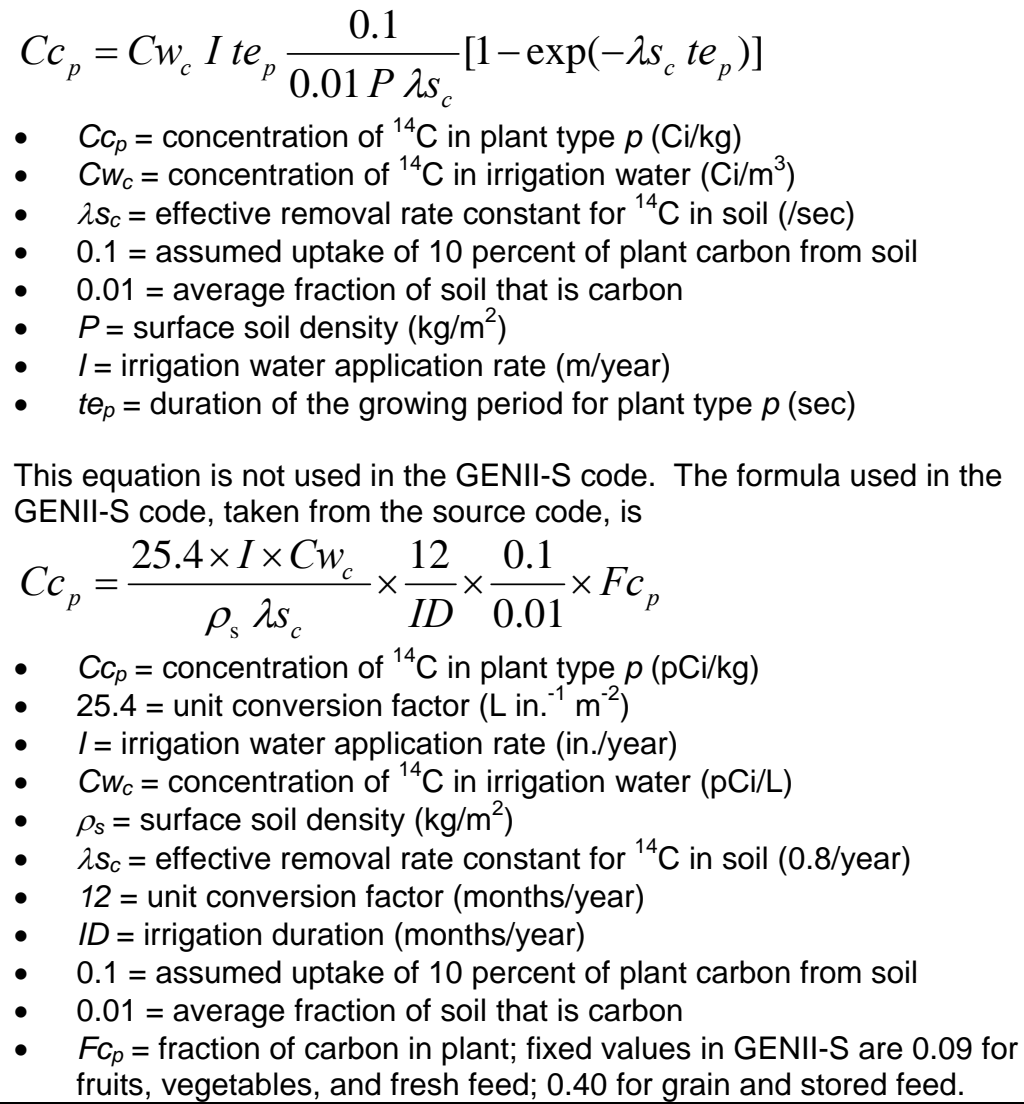 & $\begin{array}{l}\text { This equation has errors: } \\
\text { 1) the units conversion is incorrect } \\
\text { 2) the duration of the growing period, te } \\
\text { should not be proportional to the } \\
\text { concentration of }{ }^{14} \mathrm{C} \text { in the plants } \\
\text { 3) the fraction of carbon in plants, } F c_{p} \text {, } \\
\text { which is proportional to the }{ }^{14} \mathrm{C} \\
\text { concentration, is missing } \\
\text { GENII-S considers that }{ }^{14} \mathrm{C} \\
\text { contamination in plants occurs only } \\
\text { through root uptake. Because the }{ }^{14} \mathrm{C} \\
\text { special model for plant contamination in } \\
\text { the GENII-S and ERMYNs are different, } \\
\text { a numerical comparison between the } \\
\text { GENII-S and ERMYNs is made in } \\
\text { Section 7.4.7. }\end{array}$ & $\begin{array}{l}\text { Napier et al. } 1988 \\
\text { [DIRS 157927], } \\
\text { p. 4.89, Eq. 4.7.32. } \\
\text { The }{ }^{14} \mathrm{C} \text { source } \\
\text { code is in the } \\
\text { GENII-S code } \\
\text { (SNL 1998 } \\
\text { [DIRS 117076], } \\
\text { CANDH } \\
\text { subroutine), which } \\
\text { is listed in } \\
\text { Appendix A, } \\
\text { Table A-6. }\end{array}$ \\
\hline
\end{tabular}


Table 7.3-21. Comparison of ${ }^{14} \mathrm{C}$ Special Model for Plant Contamination (Continued)

\begin{tabular}{|c|c|c|c|}
\hline Document & Mathematical Model & $\begin{array}{l}\text { Comparison with the ERMYN } \\
\text { (Equation 6.4.6-6) }\end{array}$ & Reference \\
\hline $\begin{array}{l}\text { BIOMASS } \\
\text { ERB2A }\end{array}$ & 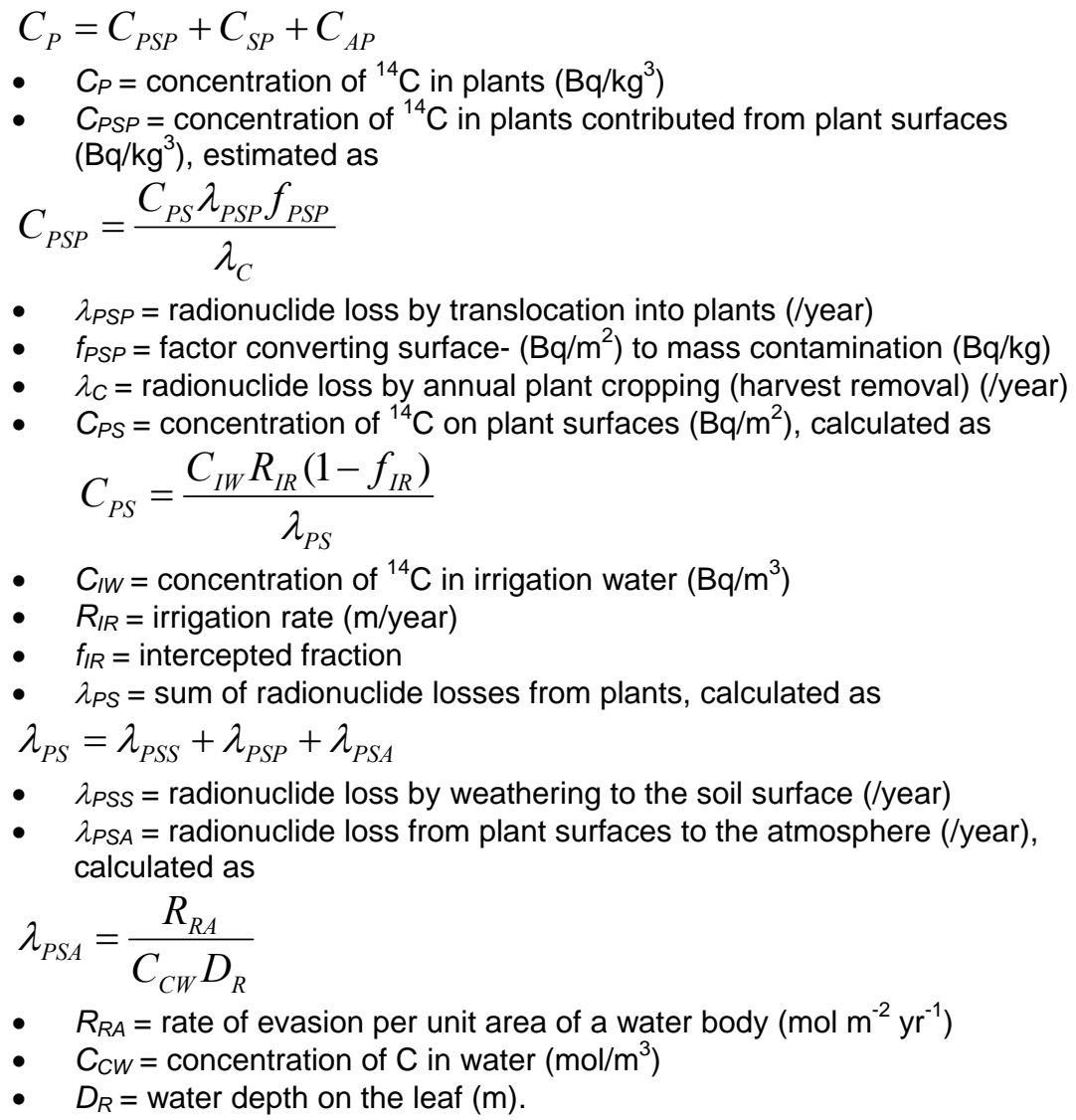 & $\begin{array}{l}\text { The BIOMASS ERB2A model includes } \\
\text { plant contamination from plant surfaces, } \\
\text { soil, and air. This method requires many } \\
\text { input parameters that are not given in the } \\
\text { document, which makes numerical } \\
\text { comparisons impossible. }\end{array}$ & $\begin{array}{l}\text { BIOMASS 2000 } \\
\text { [DIRS 154522], } \\
\text { Appendix A. }\end{array}$ \\
\hline
\end{tabular}

客 


\begin{tabular}{|c|c|c|c|}
\hline Document & Mathematical Model & $\begin{array}{c}\text { Comparison with the ERMYN } \\
\text { (Equation 6.4.6-6) }\end{array}$ & Reference \\
\hline $\begin{array}{l}\text { BIOMASS } \\
\text { ERB2A } \\
\text { (cont.) }\end{array}$ & 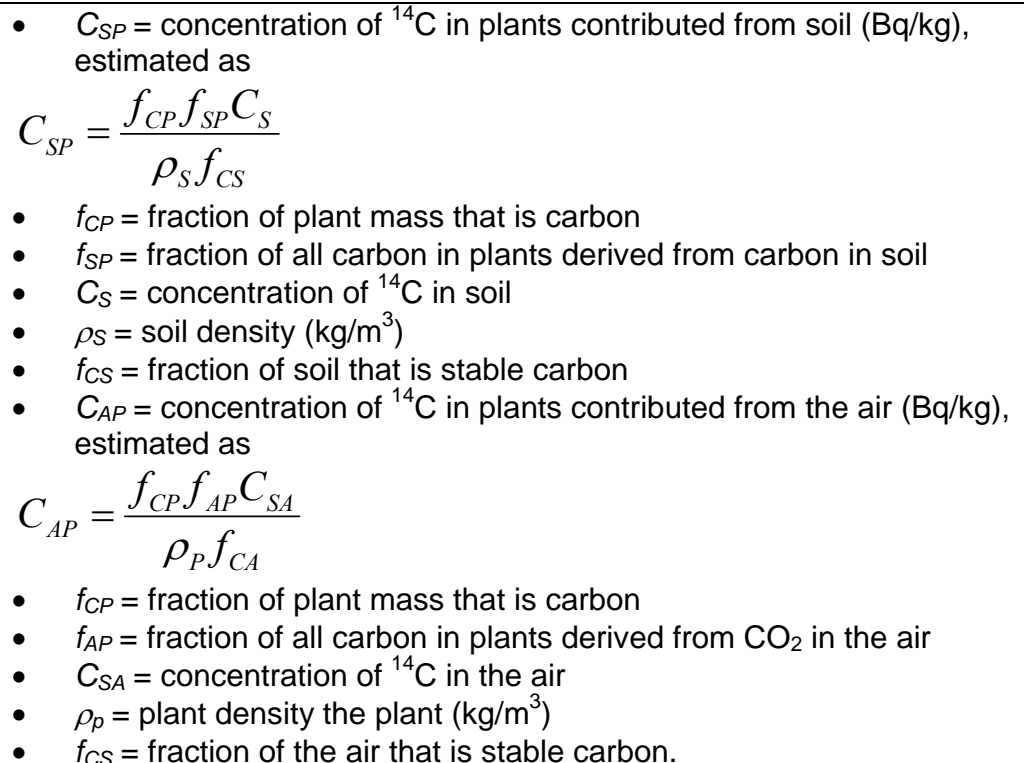 & & \\
\hline EPRI-YM & Not included. & Not applicable. & $\begin{array}{l}\text { Smith et al. } 1996 \\
\text { [DIRS 101085]. }\end{array}$ \\
\hline
\end{tabular}




\begin{tabular}{|c|c|c|c|}
\hline Document & Mathematical Model & $\begin{array}{l}\text { Comparison with the ERMYN } \\
\text { (Equation 6.4.6-6) }\end{array}$ & Reference \\
\hline RESRAD & 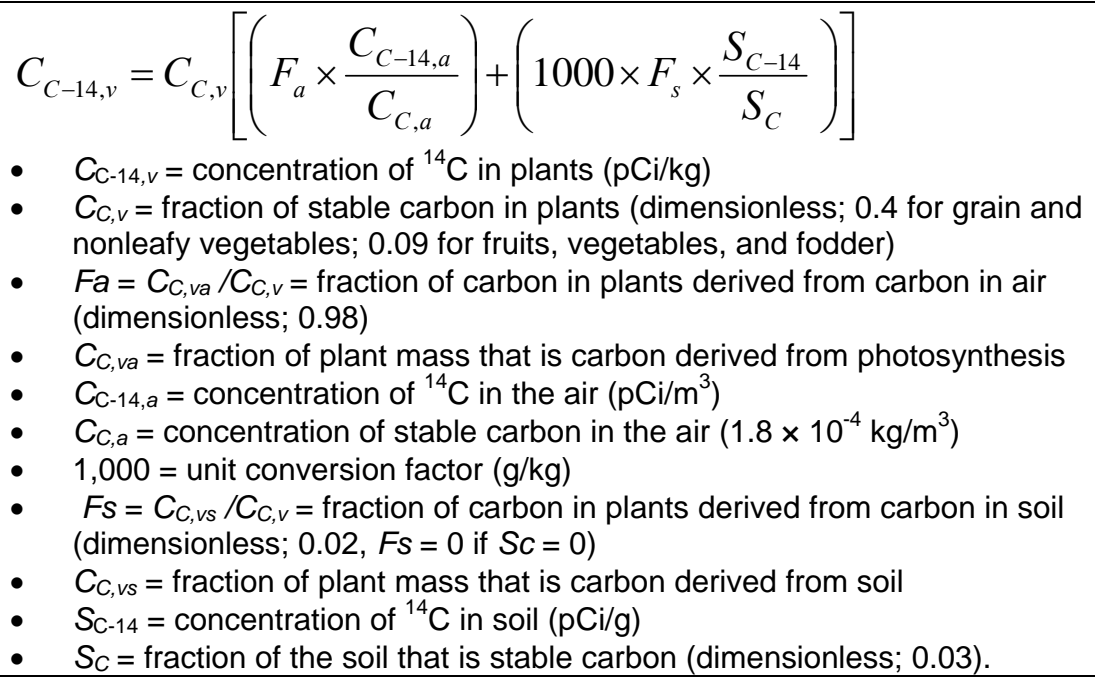 & $\begin{array}{l}\text { RESRAD and ERMYN are the same, } \\
\text { except for the notations and units for } \\
\text { some parameters. }\end{array}$ & $\begin{array}{l}\text { Yu et al. } 2001 \\
\text { [DIRS 159465], } \\
\text { p. L-20, Eq. L.31. }\end{array}$ \\
\hline NCRP-129 & Not included. & Not applicable. & $\begin{array}{l}\text { NCRP } 1999 \\
\text { [DIRS 155894]. }\end{array}$ \\
\hline
\end{tabular}

ERMYN=Environmental Radiation Model for Yucca Mountain, Nevada 


\begin{tabular}{|c|c|c|c|}
\hline Document & Mathematical Model & $\begin{array}{c}\text { Comparison with the ERMYN } \\
\text { (Equation 6.4.6-7) }\end{array}$ & Reference \\
\hline GENII-S & 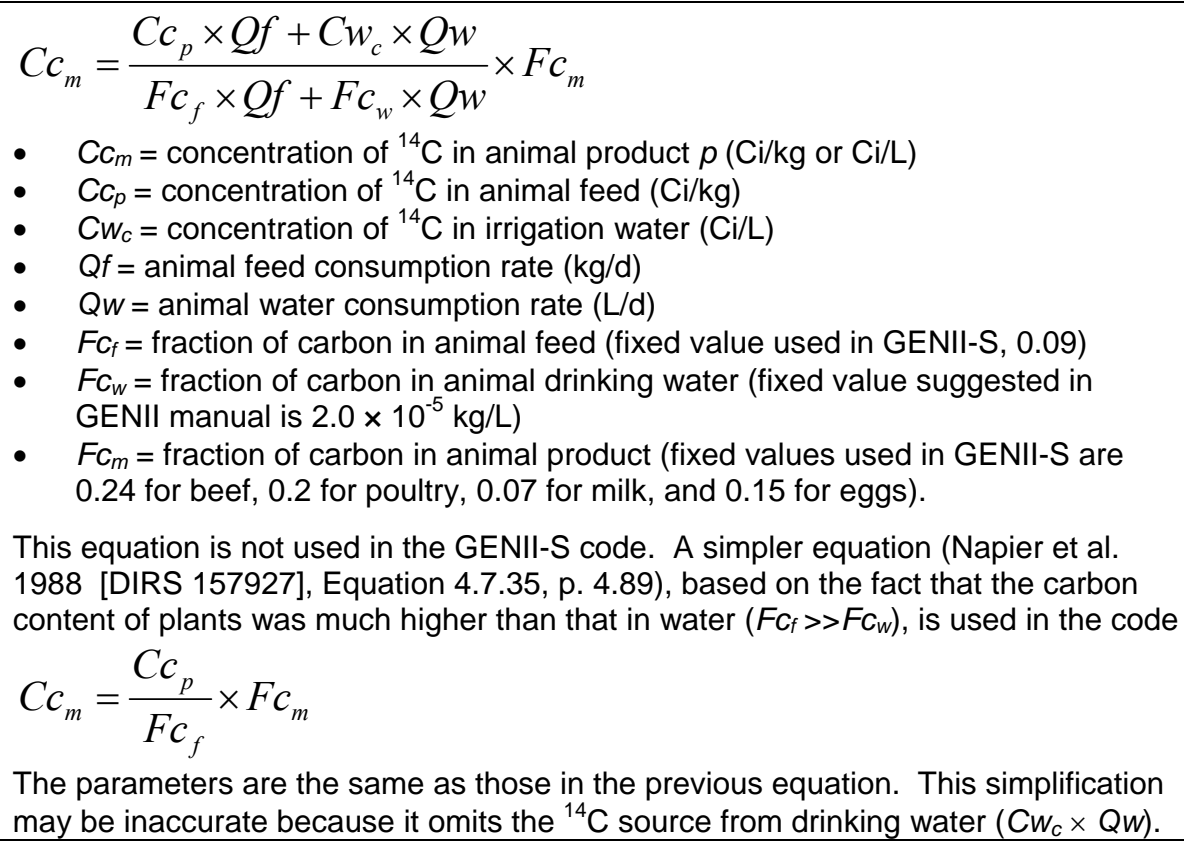 & $\begin{array}{l}\text { GENII-S and ERMYN are } \\
\text { equivalent, except that GENII-S } \\
\text { does not include soil ingestion by } \\
\text { animals, which is similar to the way } \\
\text { other radionuclides are treated. }\end{array}$ & $\begin{array}{l}\text { Napier et al. } 1988 \\
\text { [DIRS 157927], } \\
\text { p. 4.89, Eq. 4.7.34 } \\
\text { and Eq. 4.7.35. }\end{array}$ \\
\hline $\begin{array}{l}\text { BIOMASS } \\
\text { ERB2A }\end{array}$ & $\begin{array}{l}C_{A, C 14}=C_{A, C 12}\left(f_{A, W} \frac{C_{W, C 14}}{C_{W, C 12}}+f_{A, S} \frac{C_{S, C 14}}{C_{S, C 12}}+f_{A, P} \frac{C_{P, C 14}}{C_{P, C 12}}\right) \\
-C_{x, C 12}=\text { fraction of the mass of material that is stable carbon, where } \mathrm{X}=A \text { for } \\
\text { animal products, } W \text { is water, } S \text { is soil, and } P \text { is plants }(\mathrm{Bq} / \mathrm{kg}) \\
\text { - } \quad f_{A, X}=\text { fraction of carbon derived from medium } X(\text { water, soil, or plants) } \\
\quad C_{X, C 14}=\text { activity concentration of }{ }^{14} \mathrm{C} \text { on the material, where } \mathrm{X}=\mathrm{A} \text { is animal } \\
\text { product, } W \text { is water, } S \text { is soil, and } P \text { is plants }(\mathrm{Bq} / \mathrm{kg}) .\end{array}$ & $\begin{array}{l}\text { BIOMASS ERB2A includes }{ }^{14} \mathrm{C} \\
\text { contamination in animal products } \\
\text { through water, soil, and plants, } \\
\text { which is the same as the ERMYN, } \\
\text { but it uses a different method for } \\
\text { calculating radionuclide } \\
\text { concentrations in animal products. } \\
\text { The values are not given in the } \\
\text { document, which makes numerical } \\
\text { comparison impossible. }\end{array}$ & $\begin{array}{l}\text { BIOMASS } 2000 \\
\text { [DIRS 154522], } \\
\text { pp. } 70 \text { and } 71, \\
\text { Appendix A. }\end{array}$ \\
\hline EPRI-YM & Not included. & Not applicable. & $\begin{array}{l}\text { Smith et al. } 1996 \\
\text { [DIRS 101085]. }\end{array}$ \\
\hline
\end{tabular}


Table 7.3-22. Comparison of ${ }^{14} \mathrm{C}$ Special Model for Animal Product Contamination (Continued)

\begin{tabular}{|c|c|c|c|}
\hline Document & Mathematical Model & $\begin{array}{c}\text { Comparison with the ERMYN } \\
\text { (Equation 6.4.6-7) }\end{array}$ & Reference \\
\hline RESRAD & 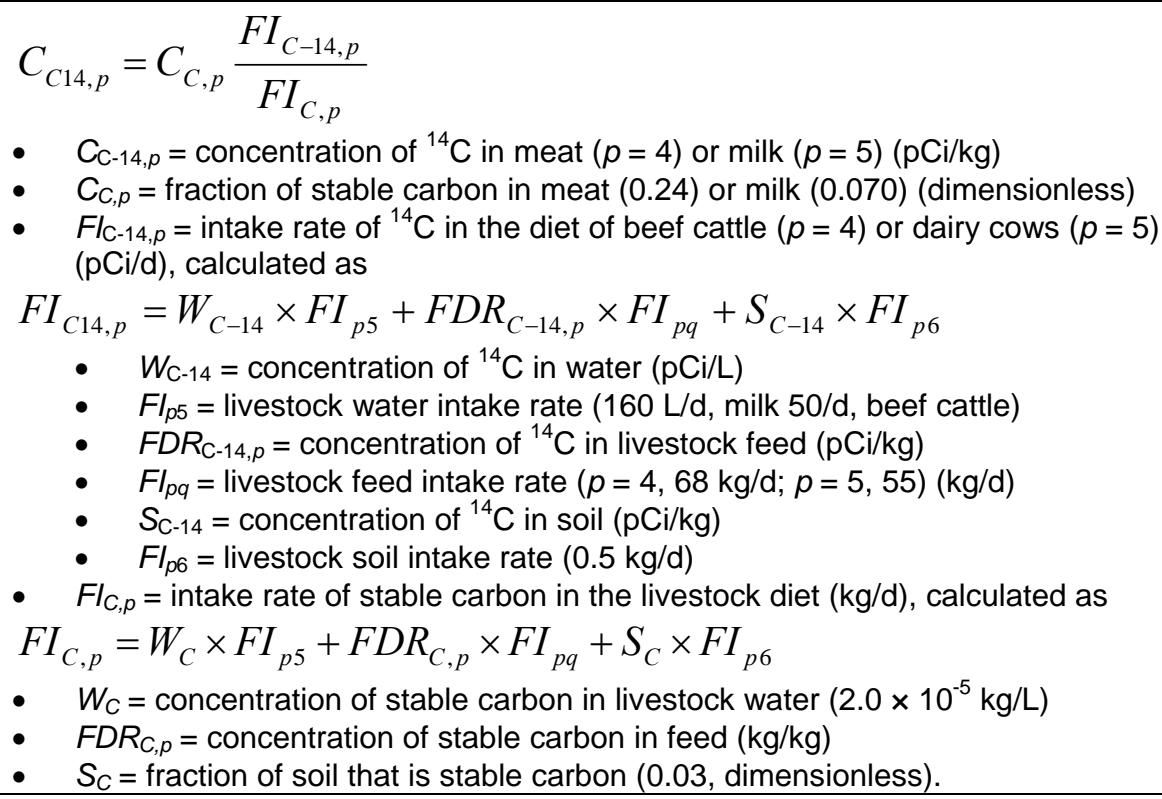 & $\begin{array}{l}\text { RESRAD and ERMYN are the } \\
\text { same, except for notations and } \\
\text { units for some parameters. }\end{array}$ & $\begin{array}{l}\text { Yu et al. } 2001 \\
\text { [DIRS 159465], } \\
\text { p. L-21, Eq. L.33, } \\
\text { L.34, and L.36. }\end{array}$ \\
\hline NCRP-129 & Not included. & Not applicable. & $\begin{array}{l}\text { NCRP } 1999 \\
\text { [DIRS 155894]. }\end{array}$ \\
\hline
\end{tabular}

ERMYN=Environmental Radiation Model for Yucca Mountain, Nevada 


\subsubsection{Validation of the External Exposure Submodel}

The method used to calculate the external exposure dose in the ERMYN is similar to that used in the validation models (Table 7.3-23). All of the models calculate dose as a product of a radionuclide-specific dose coefficient, radionuclide concentrations in the surface soil, and exposure time. All of the validation models include external exposure to contaminated soil. In addition, the GENII-S model includes air submersion and water immersion, and the BIOMASS ERB2A model includes water immersion (Table 7.2-2). A comparison of dose coefficients for external exposure to contaminated soil, air submersion, and water immersion evaluates the importance of these pathways (Section 7.4.8). The potential doses from air submersion and water immersion are inconsequential compared to soil exposure, and they are excluded from the ERMYN dose assessments.

There are several differences between the ERMYN methods (Equations 6.4.7-1 and 6.5.5-1) and the validation model methods (Table 7.2-23):

- The ERMYN uses environment-specific exposure times and shielding factors for five environments and four population groups. The RESRAD and NCRP-129 models use two environments (indoors and outdoors), but the other validation models do not consider different environments. None of the validation models includes differences among population groups. The ERMYN approach accounts for variation in shielding factors among the various environments where a receptor lives, and variation and uncertainty in exposure times among segments of the receptor population. A numerical comparison of inhalation exposure to particulate matter using the ERMYN micro-environmental approach and the single-environment approach used in other models demonstrates that the calculation of exposure rates differ by less than a factor of two (Table 7.4-19). This comparison also is valid for the external exposure submodel because the same values for exposure times and population groups are used for both submodels. Based on this numerical comparison, the ERMYN methods are numerically similar to the validation models.

- The dose coefficients and their units differ among models and scenarios because the type of dose coefficient depends on the type and distribution of contaminants. For example, the dose coefficients for exposure to soil contaminated to an infinite depth $\left(\mathrm{Bq} / \mathrm{m}^{3}\right)$ in the ERMYN groundwater scenario (Approximation 10) are similar to those in the BIOMASS ERB2A model. In contrast, dose coefficients for contaminated soil surface $\left(\mathrm{Bq} / \mathrm{m}^{2}\right)$ in the ERMYN volcanic scenario (Approximation 16) are similar to GENII-S. Differences in notations for dose coefficients and associated factors in the ERMYN and validation model equations (e.g., density correction $W_{S}$ in NCRP-129) are required for unit conversion; therefore, the methods are mathematically equivalent.

- The ERMYN, NCRP-129, and RESRAD models include a building shielding factor associated with time spent indoors. In the ERMYN, this radionuclide-specific parameter accounts for the reduction in external exposure caused by dwellings. The GENII-S, BIOMASS ERB2A, and EPRI-YM models do not differentiate between time spent indoors and outdoors and do not include a shielding factor. However, this factor can be incorporated into exposure time by reducing or excluding time spent indoors, and 
therefore, the methods are mathematically equivalent. This factor, not used in the previous biosphere model, is included based on a suggestion made during a model validation status review (BSC 2003 [DIRS 170030], Section 6.17.5). Inclusion of this factor is a biosphere model improvement.

- The RESRAD model includes factors for the size and shape of the contaminated area. Under the groundwater scenario, the total area contaminated is large because of long-term irrigation land-use rotation. Under the volcanic ash scenario, contaminated ash would be deposited over the entire Amargosa Valley. Thus, these factors would be about 1.0 for both scenarios and this method is mathematically equivalent to the ERMYN.

- RESRAD includes a cover-and-depth factor for the effects of burying radioactive waste. For both scenarios in the ERMYN, all radionuclides are in the surface soil, so this factor would be set at 1.0. Therefore, this method is mathematically equivalent to the ERMYN.

- The NCRP-129 model includes different exposure rates for children and adults. However, 10 CFR 63.312(e) requires basing the characteristics of the RMEI on an adult, so this factor would equal 1.0, making the methods mathematically equivalent.

Because the mathematical representations are mathematically equivalent or the results are numerically similar, the external exposure submodel is validated. 


\begin{tabular}{|c|c|c|c|}
\hline Document & Mathematical Model & $\begin{array}{l}\text { Comparison with the ERMYN } \\
\text { (Equations 6.4.7-1 and 6.5.5-1) }\end{array}$ & Reference \\
\hline GENII-S & $\begin{array}{l}D x_{i}=t s C s_{i} D s_{i} \\
\text { - } \quad D x_{i}=\text { dose to an individual from exposure to contaminated ground for } \\
\text { radionuclide } i(\text { rem }) \\
\text { - } D s_{i}=\text { external dose conversion factor for exposure to a planar surface } \\
\text { contaminated by radionuclide } i\left(\text { rem } / \mathrm{hr} \text { per } \mathrm{Ci} / \mathrm{m}^{2}\right) \\
\text { - } \quad C s_{i}=\text { soil concentration of radionuclide } i\left(\mathrm{Ci} / \mathrm{m}^{2}\right) \\
\text { - } s=\text { time of exposure to contaminated ground }(\mathrm{hr}) .\end{array}$ & $\begin{array}{l}\text { In the GENII-S manual, surface } \\
\text { contamination is similar to } \\
\text { Equation } 6.4 .7-1 \text { in ERMYN. The } \\
\text { GENII-S code can also use volumetric } \\
\text { dose coefficients, which are similar to } \\
\text { Equation } 6.4 .7-1 \text { in ERMYN. The } \\
\text { ERMYN also includes a shielding factor } \\
\text { and an environment-specific time budget, } \\
\text { but GENII-S does not. }\end{array}$ & $\begin{array}{l}\text { Napier et al. } 1988 \\
\text { [DIRS 157927], } \\
\text { p. 4.84, Eq. 4.7.24. }\end{array}$ \\
\hline $\begin{array}{l}\text { BIOMASS } \\
\text { ERB2A }\end{array}$ & $\begin{array}{l}D_{\text {exsoil }}=O_{s} D C_{\text {exts }} C_{s} \\
\text { - } \quad D_{\text {exsoil }}=\text { individual dose from external irradiation from the soil (Sv/year) } \\
\text { - } \quad D C_{\text {exts }}=\text { dose factor for external irradiation from the soil }\left(\mathrm{Sv} / \mathrm{hr} \text { per } \mathrm{Bq} / \mathrm{m}^{3}\right) \\
-\quad C_{s}=\text { radionuclide activity concentration in cultivated soil }\left(\mathrm{Bq} / \mathrm{m}^{3}\right) \\
O_{s}=\text { individual occupancy in the soil compartment (hr/year). }\end{array}$ & $\begin{array}{l}\text { BIOMASS ERB2A uses a method } \\
\text { equivalent to that in the ERMYN } \\
\text { (Equation 7.1), except for the lack of a } \\
\text { shielding factor and environment-specific } \\
\text { time budgets. }\end{array}$ & $\begin{array}{l}\text { BIOMASS } 2000 \\
\text { [DIRS 154522], } \\
\text { p. } 37, \text { Eq. } 17 .\end{array}$ \\
\hline EPRI-YM & 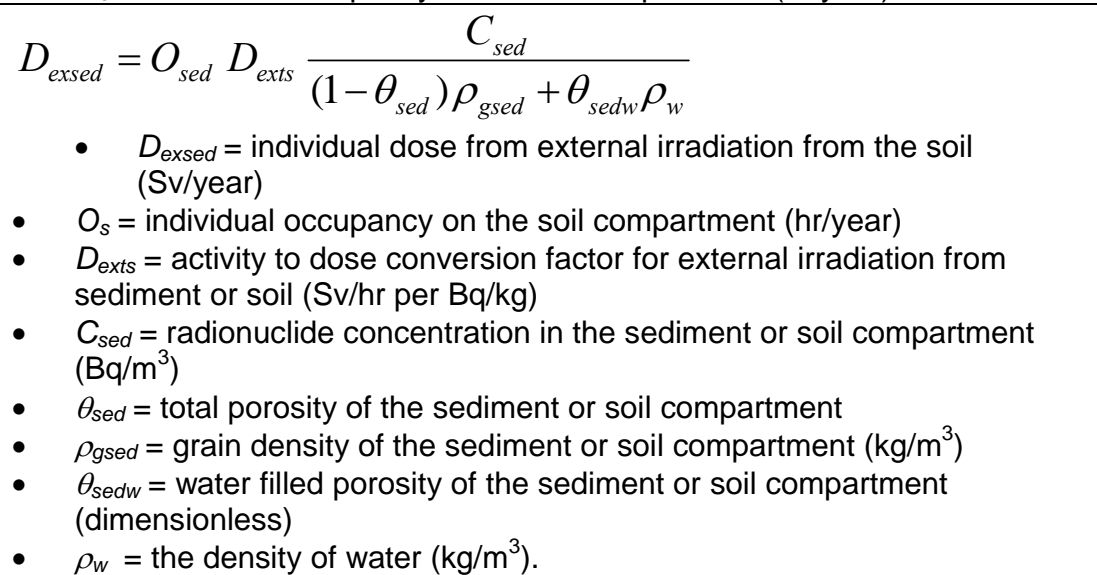 & $\begin{array}{l}\text { EPRI-YM uses a method equivalent to } \\
\text { BIOMASS ERB2A. The units for dose } \\
\text { conversion factors are different } \\
(\text { Sv } / \mathrm{hr} \text { per Bq } / \mathrm{kg} \text { in EPRI-YM and Sv/hr } \\
\text { per Bq/m } \mathrm{m}^{3} \text { in BIOMASS ERB2A), which } \\
\text { changes the form of the equation. }\end{array}$ & $\begin{array}{l}\text { Smith et al. } 1996 \\
\text { [DIRS 101085], } \\
\text { p. 5-12, Eq. 5.14. }\end{array}$ \\
\hline
\end{tabular}




\begin{tabular}{|c|c|c|c|}
\hline Document & Mathematical Model & $\begin{array}{l}\text { Comparison with the ERMYN } \\
\text { (Equations 6.4.7-1 and 6.5.5-1) }\end{array}$ & Reference \\
\hline RESRAD & 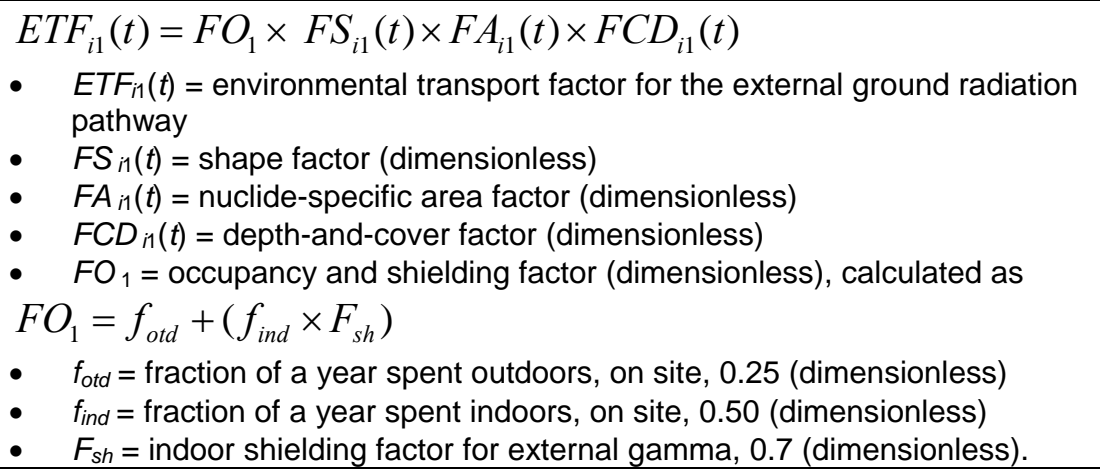 & $\begin{array}{l}\text { The RESRAD model includes several } \\
\text { factors, including shape, area, depth, } \\
\text { and cover factors that are not in ERMYN. } \\
\text { An indoor shielding factor is also used. } \\
\text { The equation calculates the } \\
\text { environmental transport factor. To } \\
\text { convert } E T F \text { to dose, multiply ETF by the } \\
\text { effective dose equivalent conversion } \\
\text { factor }(D C F i 1-\text { mrem/year per pCi/g) and } \\
\text { the source term }(S(t)-p C i / g) \text {. }\end{array}$ & $\begin{array}{l}\text { Yu et al. } 2001 \\
\text { [DIRS 159465], } \\
\text { Appendix A, p. A-7, } \\
\text { Eq. A-7. }\end{array}$ \\
\hline NCRP-129 & $\begin{array}{l}E_{\text {ext }}=D f_{\text {ext }} \times\left[T_{\text {out }}+\left(T_{\text {in }} \times S F\right)\right] \times(C / A) \times W_{s} \times S(z, t) \\
\text { - } \quad E_{\text {extl }}=\text { average annual exposure to an exposed individual per unit } \\
\text { radionuclide concentration from external radiation }(\mathrm{Sv} /(\mathrm{Bq} / \mathrm{kg})) \\
\text { - } \quad D f_{\text {ext }}=\text { dose factor for a particular radionuclide }(\mathrm{Sv} / \mathrm{year} \text { per } \mathrm{Bq} / \mathrm{kg}) \\
\mathrm{S}(\mathrm{z}, \mathrm{t})=\text { radionuclide concentration in the soil, averaged over the } 1 \text { year } \\
\text { interval }(t) \text { for which the dose is calculated }(\mathrm{Bq} / \mathrm{kg} \text { dry soil); varies with depth } \\
\text { ( } z) \text { beneath the ground surface } \\
\text { - } \quad T_{\text {out }}=\text { the mean fraction of time spent outdoors on a contaminated site } \\
T_{\text {in }}=\text { the mean fraction of time spent indoors in a dwelling on a } \\
\text { contaminated site } \\
\text { - } S F=\text { shielding factor or ratio of the dose indoors to the unshielded } \\
\text { outdoor dose } \\
\text { Ws = density correction due to soil moisture (i.e., the ratio of dry soil } \\
\text { density to the actual in situ bulk density) } \\
\text { C/A = ratio of the external dose to children to that for adults when children } \\
\text { are present. }\end{array}$ & $\begin{array}{l}\text { NCRP-129 uses soil density and child- } \\
\text { exposure correction factors that are not } \\
\text { in ERMYN. E Extl should be in units of Sv } \\
\text { in the equation when } S(z, t) \text { is the } \\
\text { radionuclide concentration in the soil (in } \\
\text { units of Bq/kg). NCRP-129 is equivalent } \\
\text { to ERMYN, except that the soil density } \\
\text { correction factor and exposure for } \\
\text { children are not used in the ERMYN. }\end{array}$ & $\begin{array}{l}\text { NCRP } 1999 \\
\text { [DIRS 155894], } \\
\text { p. 34, Eq. 3.1. }\end{array}$ \\
\hline
\end{tabular}

ERMYN=Environmental Radiation Model for Yucca Mountain, Nevada 


\subsubsection{Validation of the Inhalation Submodel}

The ERMYN (Equation 6.4.8-2 and 6.5.6-2) and all of the validation models (Table 7.3-24) use the same general approach for calculating the dose from inhalation of particulate matter as the product of radionuclide-specific dose conversion factors, breathing rates, airborne particle concentrations, and exposure times. The ERMYN also uses this approach for calculating exposure to aerosols generated from operating evaporative coolers (Equation 6.4.8-3), inhalation of ${ }^{14} \mathrm{C}$ (Equation 6.4.8-4), and inhalation of radon decay products (Equation 6.4.8-5 and 6.5.6-3). Differences between the ERMYN methods for calculating the inhalation dose for all airborne contaminants and the methods used in the validation models are:

- Four of the five validation models calculate average inhalation exposure using average breathing rates, air concentrations, and inhalation times (Table 7.3-24). Only the NCRP-129 model considers two environments (indoors and outdoors) for these parameters, which is similar to the microenvironment concept in the ERMYN. The ERMYN includes five environments and four population groups to incorporate variation and uncertainty in concentrations of radionuclides within the receptor environment and exposure times among segments of the receptor population. A numerical comparison of the ERMYN micro-environment method and the single-environment method evaluates the effects of these differences (Section 7.4.9). Using average values for the single-environment method, the two approaches produce similar results $\left(1.5 \times 10^{-5}\right.$ versus $1.25 \times 10^{-5} \mathrm{~Bq} / \mathrm{day}$; Table 7.4-19), and additional validation of this portion of the submodel is not required.

- None of the validation models considers the inhalation dose as a function of time. The inhalation dose for the ERMYN volcanic scenario is treated as a function of time (Equation 6.5.6-2) to account for decreases in mass loading following a volcanic eruption. This approach is evaluated and justified in the validation of the air submodel (Section 7.3.2).

- None of the validation models considers the inhalation dose as a function of ash depth. This is done in the ERMYN volcanic ash scenario (Equation 6.5.6-2) to account for differences in activity concentrations in the air that result from a thin layer of ash or as the ash layer is depleted by erosion (Approximation 12). This approach is evaluated and justified in the validation of the soil submodel (Section 7.3.1).

- None of the validation models include inhalation of aerosols from evaporative coolers. The ERMYN method for calculating this inhalation dose (Equation 6.4.8-3) is similar to the methods in the validation models for particulate matter, except that the ERMYN calculation includes parameters to quantify the proportion of houses with evaporative coolers and the proportion of the year that coolers are used. The calculation of the inhalation dose from radon decay products (Equation 6.4.8-7) includes these factors because of different radon accumulation levels in indoor air when evaporative coolers are tuned on and off. The average cooler use factor may be as low as 0.08 for predicted future climatic conditions (Table 6.6-3); therefore, these factors could result in a difference of more than a factor of two. The ERMYN includes these parameters because some people in the Amargosa Valley do not use evaporative coolers, and coolers are not 
operated year-round (DOE 1997 [DIRS 100332]). Excluding these parameters results in overestimating the inhalation dose. These parameters are justified because they account for site-specific conditions and prevent overestimating dose.

- BIOMASS ERB2A includes the inhalation of aerosols from water sprays, but it does not provide the method for calculating aerosol concentrations, although it does give a default value of $1.0 \times 10^{-11} \mathrm{~m}^{3}$ water $/ \mathrm{m}^{3}$ air (BIOMASS 2000 [DIRS 154522], Table 13), which corresponds to $1.0 \times 10^{-11} \mathrm{~Bq} / \mathrm{m}^{3}$ for a unit concentration of contaminated groundwater $\left(1 \mathrm{~Bq} / \mathrm{m}^{3}\right)$. This airborne concentration is lower than the typical concentration for resuspended particles $\left(10^{-5}\right.$ to $10^{-8} \mathrm{~Bq} / \mathrm{m}^{3}$, Table $\left.6.10-1\right)$. In addition, the BIOMASS ERB2A default value for exposure time to aerosols is $36.5 \mathrm{~h} /$ year (BIOMASS 2000 [DIRS 154522], Table 13), which is lower than exposure time to resuspended particles (10.9 hr/day, Table 7.4-19) in the ERMYN. Therefore, excluding this pathway from the ERMYN would change the estimated inhalation dose by less than a factor of two, and, therefore, it does not require further justification.

- RESRAD includes a factor for the size of the contaminated area. Under the groundwater scenario, the total area contaminated is large due to long-term irrigation and land-use rotation. Under the volcanic ash scenario, contaminated ash is deposited over the entire Amargosa Valley. Thus, this factor would be about 1.0 for both scenarios, and the RESRAD and ERMYN methods are mathematically equivalent.

- RESRAD includes a cover-and-depth factor for the effect of burying radioactive waste. Under both ERMYN scenarios, all radionuclides are in the surface soil, so this factor would be 1.0. Therefore, the RESRAD and ERMYN methods are mathematically equivalent.

Because the models are mathematically equivalent or produce similar results, the inhalation submodel is validated. 


\begin{tabular}{|c|c|c|c|}
\hline Document & Mathematical Model & $\begin{array}{l}\text { Comparison with the ERMYN } \\
\text { (Equations 6.4.8-2 and 6.5.6-2) }\end{array}$ & Reference \\
\hline GENII-S & $\begin{array}{ll} & D n_{i}=3.15 \times 10^{7} B \times C a_{i} \times f \times D h_{i} \\
\text { - } & D n_{i}=\text { individual dose from inhalation (rem/year) } \\
\text { - } & 3.15 \times 10^{7}=\text { the number of seconds per year }(\mathrm{sec} / \mathrm{year}) \\
\text { - } & B=\text { breathing rate for exposed individuals }\left(\mathrm{m}^{3} / \mathrm{sec}\right) \\
& C a_{i}=\text { average air concentration of radionuclide } i\left(\mathrm{Ci} / \mathrm{m}^{3}\right) \\
\text { - } & f=\text { fraction of year exposed to inhalation (dimensionless) } \\
\text { - } & D h_{i}=\text { inhalation dose conversion factor for radionuclide } i(\mathrm{rem} / \mathrm{Ci}) .\end{array}$ & $\begin{array}{l}\text { The GENII-S inhalation submodel uses an } \\
\text { average breathing rate, air concentration, } \\
\text { and annual exposure time. It is simpler } \\
\text { than the ERMYN, which uses } \\
\text { environment-specific breathing rates and } \\
\text { air concentrations, and time budgets for } \\
\text { exposure time. }\end{array}$ & $\begin{array}{l}\text { Napier et al. } \\
1988 \\
\text { [DIRS 157927], } \\
\text { p. 4.63, } \\
\text { Eq. 4.7.1a. }\end{array}$ \\
\hline $\begin{array}{l}\text { BIOMASS } \\
\text { ERB2A }\end{array}$ & $\begin{array}{l}D_{\text {dust }}=D C_{\text {inh }} C_{\text {airs }} B R O_{s} \\
\text { - } \quad D_{\text {dust }}=\text { individual dose from dust inhalation (Sv/year) } \\
\text { - } \quad D C_{\text {inh }}=\text { dose coefficient for inhalation }(\mathrm{Sv} / \mathrm{Bq}) \\
\text { - } C_{\text {airs }}=\text { radionuclide concentration in the air above the cultivated soil } \\
\text { compartment }\left(\mathrm{Bq} / \mathrm{m}^{3}\right) \\
\text { - } \quad B R=\text { breathing rate of humans in the soil compartment }\left(\mathrm{m}^{3} / \mathrm{hr}\right) \\
\quad O_{s}=\text { individual occupancy in the soil compartment (hr/year) } \\
D_{\text {aero }}=D C_{\text {inh }} A I R_{\text {aero }} B R O_{\text {aero }} C_{w} \\
\text { - } \quad D_{\text {aero }}=\text { individual dose from the inhalation of aerosols (Sv/year) } \\
\text { - } \quad A I R_{\text {aero }}=\text { aerosol level in the air in the area affected by aerosol/spray } \\
\left(\mathrm{m}^{3} / \mathrm{m}^{3}\right) \\
\text { - } \quad O_{\text {aero }}=\text { individual occupancy in the area affected by aerosols (hr/year). }\end{array}$ & $\begin{array}{l}\text { In BIOMASS, the inhalation of dust is the } \\
\text { same as in the GENII-S submodel. } \\
\text { However, BIOMASS includes the } \\
\text { inhalation of aerosols and sprays, which is } \\
\text { not important, as discussed in the text. }\end{array}$ & $\begin{array}{l}\text { BIOMASS } 2000 \\
\text { [DIRS 154522], } \\
\text { p. } 38, \text { Eq. } 19 .\end{array}$ \\
\hline EPRI-YM & $\begin{array}{ll}D_{\text {dust }}=D_{\text {inh }} B R_{\text {sed }} O_{\text {sed }} C_{\text {airs }} \\
\text { - } \quad D_{\text {dust }}=\text { individual dose from dust inhalation (Sv/year) } \\
\text { - } \quad D_{\text {inh }}=\text { conversion factor for activity to dose for inhalation }(\mathrm{Sv} / \mathrm{Bq}) \\
-\quad B R_{\text {sed }}=\text { human breathing rate in the sediment or soil compartment }\left(\mathrm{m}^{3} / \mathrm{hr}\right) \\
\text { - } \quad O_{\text {sed }}=\text { individual occupancy in the sediment or soil compartment }(\mathrm{hr} / \mathrm{year}) \\
\text { - } \quad \mathrm{C}_{\text {airs }}=\text { radionuclide concentration in the air }\left(\mathrm{Bq} / \mathrm{m}^{3}\right)(\text { Table } 7.3-4) .\end{array}$ & $\begin{array}{l}\text { The EPRI-YM inhalation submodel is the } \\
\text { same as that in the BIOMASS submodel. }\end{array}$ & $\begin{array}{l}\text { Smith et al. } 1996 \\
\text { [DIRS 101085], } \\
\text { p. 5-14, Eq. 5.16. }\end{array}$ \\
\hline
\end{tabular}




\begin{tabular}{|c|c|c|c|}
\hline Document & Mathematical Model & $\begin{array}{l}\text { Comparison with the ERMYN } \\
\text { (Equations 6.4.8-2 and 6.5.6-2) }\end{array}$ & Reference \\
\hline RESRAD & 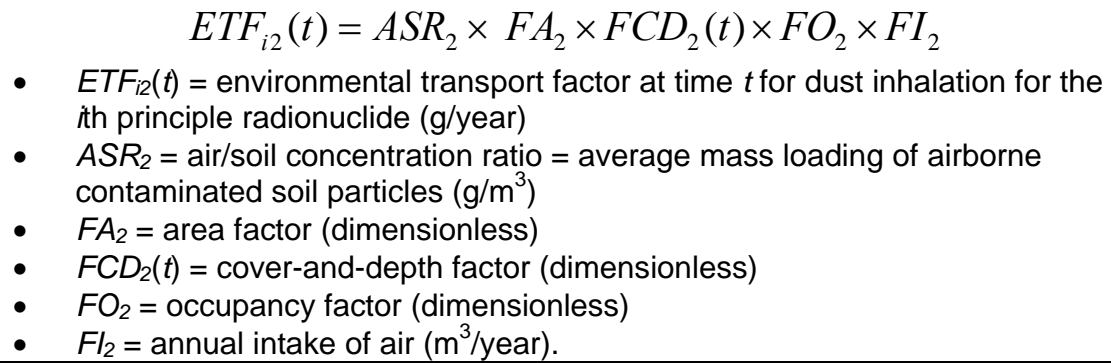 & $\begin{array}{l}\text { RESRAD includes several factors, } \\
\text { including contaminated soil depth, } \\
\text { uncontaminated soil cover, and area } \\
\text { contaminated. To convert ETF into dose, } \\
\text { ETF is multiplied by the CEDE conversion } \\
\left.\text { factor for inhalation ( } D C F_{i 2}-\mathrm{mrem} / \mathrm{pCi}\right) \\
\text { and the soil source }(\mathrm{S}(\mathrm{t})-\mathrm{pCi} / \mathrm{g}) \text {. Without } \\
\text { these factors, the RESRAD inhalation } \\
\text { submodel is similar to GENII-S. }\end{array}$ & $\begin{array}{l}\text { Yu et al. } 2001 \\
\text { [DIRS 159465], } \\
\text { p. B-6, Eq. 8.1. }\end{array}$ \\
\hline NCRP-129 & 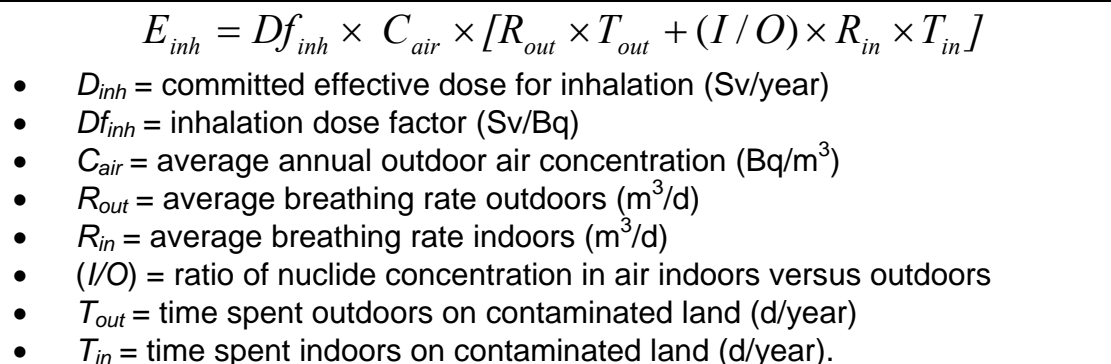 & $\begin{array}{l}\text { The NCRP-129 inhalation submodel uses } \\
\text { breathing rates and radionuclide } \\
\text { concentrations in the air for indoor and } \\
\text { outdoor environments, which is similar to } \\
\text { the concept used in the ERMYN for } \\
\text { environment-related inhalation. } \\
\text { NCRP-129 is similar to ERMYN, except } \\
\text { for using fewer human activity and } \\
\text { environment categories. }\end{array}$ & $\begin{array}{l}\text { NCRP } 1999 \\
\text { [DIRS 155894], } \\
\text { p. 64, Eq. 4.2. }\end{array}$ \\
\hline
\end{tabular}

$\mathrm{CEDE}=$ committed effective dose equivalent; ERMYN=Environmental Radiation Model for Yucca Mountain, Nevada 


\subsubsection{Validation of the Ingestion Submodel}

The ERMYN and the five validation models use the same methods for calculating ingestion doses, although the number of ingestion pathways differs among the models. All of the models include soil ingestion by humans and use similar methods (Table 7.3-29). All five validation models also use crop (Table 7.3-26) and animal (Table 7.3-27) ingestion pathways. Vegetables, beef, and milk are the most common types of food in the submodels. Water ingestion is included in the ERMYN groundwater water scenario (Equation 6.4.9-2). Among the five validation models, only NCRP-129 excludes the ingestion of drinking water (Table 7.3-25). The ingestion of aquatic foods (e.g., fish) is included in the GENII-S and RESRAD models (Table 7.3-28). Using the same general methods, the ERMYN and the five validation models calculate ingestion doses as the product of radionuclide-specific dose conversion factors, radionuclide concentrations in the ingested media, and ingestion rates. The validation models differ from the ERMYN submodel in the following ways:

- GENII-S and NCRP-129 include radionuclide decay during the holdup time before consumption. The time between harvest or groundwater pumping and consumption is small relative to the half-life of long-lived radionuclides (Table 6.3-7). Thus, the terms in the GENII-S and NCRP-129 models that include the holdup time and decay constant approach 1.0, making the methods mathematically equivalent.

- GENII-S and EPRI-YM include factors for the portion of groundwater that is treated or uncontaminated (Table 7.3-25). Because Amargosa Valley groundwater is not treated and all water is contaminated, the percentage of uncontaminated water would be 0.0 , and, therefore, the methods are mathematically equivalent.

- NCRP-129 includes a parameter for the fraction of crop consumption derived from the contaminated site (Table 7.3-26). Because all crops in the Amargosa Valley are contaminated in the ERMYN, this parameter would be 1.0, and the methods are mathematically equivalent.

- BIOMASS ERB2A includes a water content factor in the estimate of soil density in the soil ingestion dose calculation (Table 7.3-29). This factor is not used in the ERMYN because ingested soil on hands and crops most likely would be dry. The ERMYN uses the dry bulk density of surface soil (Equation 6.4.1-6), which is about $1,500 \mathrm{~kg} / \mathrm{m}^{3}$ for soils in the Amargosa Valley (Table 6.6-3). The additional factor in the BIOMASS equation is calculated as the product of the soil volumetric water content (about 0.23 ; Table 6.6-3) and water density $\left(1,000 \mathrm{~kg} / \mathrm{m}^{3}\right)$, and is equal to about $230 \mathrm{~kg} / \mathrm{m}^{3}$. Including the water content factor would change the estimate of soil density by a factor of less than 1.2. This small difference requires no further justification.

- NCRP-129 uses an occupational exposure modification factor to account for differences in soil ingestion rates among people working in different occupations (Table 7.3-29). Soil ingestion rates in the ERMYN are based on the occupations and lifestyles of Amargosa Valley residents (BSC 2004 [DIRS 169671], Section 6.4.3). Thus, this parameter is not required in the ERMYN submodel and the methods are mathematically equivalent.

Based on these comparisons, the ERMYN ingestion submodel is supported by the validation models and, therefore, is validated. 


\begin{tabular}{|c|c|c|c|}
\hline Document & Mathematical Model & $\begin{array}{c}\text { Comparison with the ERMYN } \\
\text { (Equation 6.4.9-2) }\end{array}$ & Reference \\
\hline GENII-S & $\begin{array}{ll} & D w_{i}=U_{w} C w_{i} T f_{i} \exp \left(-\lambda_{i} \times t p\right) D g_{i} \\
\text { - } & D w_{i}=\text { individual dose from water ingestion for radionuclide } i \\
& \text { (rem/year) } \\
\text { - } & D g_{i}=\text { ingestion dose conversion factor for radionuclide } i(\text { rem/Ci) } \\
- & U_{w}=\text { individual water intake rate }(\mathrm{L} / \text { year) } \\
\text { - } & C w_{i}=\text { water concentration of radionuclide } i(\mathrm{Ci} / \mathrm{L}) \\
- & T f_{i}=\text { water treatment purification factor (dimensionless) } \\
- & \lambda_{i}=\text { radiological decay constant for radionuclide } i(/ \mathrm{sec}) \\
\text { - } & t p=\text { transit time through the water distribution system }(\mathrm{sec}) . \\
\end{array}$ & $\begin{array}{l}\text { The GENII-S submodel uses decay time, } \\
\text { which is negligible for long-lived } \\
\text { radionuclides. It also uses a water } \\
\text { treatment factor, which is not in the } \\
\text { ERMYN because groundwater in the } \\
\text { Amargosa Valley is not treated. With these } \\
\text { two exceptions, the models are the same. }\end{array}$ & $\begin{array}{l}\text { Napier et al. } 1988 \\
\text { [DIRS 157927], p. } 4.65 \text {, } \\
\text { Eq. 4.7.4a. }\end{array}$ \\
\hline $\begin{array}{l}\text { BIOMASS } \\
\text { ERB2A }\end{array}$ & $\begin{array}{ll}D_{w}=I N G_{w} D_{\text {ing }} \mathrm{C}_{w} \\
\text { - } & D_{w}=\text { individual dose from consumption of groundwater ( } \mathrm{Sv} / \text { year) } \\
\text { - } & D_{\text {ing }}=\text { dose coefficient for ingestion (Sv/Bq) } \\
\text { - } & I N G_{w}=\text { individual consumption rate for groundwater }\left(\mathrm{m}^{3} / \mathrm{year}\right) \\
\text { - } & C_{w}=\text { radionuclide activity concentration in groundwater }\left(\mathrm{Bq} \mathrm{m}^{3}\right) .\end{array}$ & $\begin{array}{l}\text { The BIOMASS ERB2A water ingestion } \\
\text { dose calculations are the same as those in } \\
\text { the ERMYN. }\end{array}$ & $\begin{array}{l}\text { BIOMASS } 2000 \\
\text { [DIRS 154522], p. 34, } \\
\text { Eq. } 9 .\end{array}$ \\
\hline EPRI-YM & $\begin{array}{l}D_{f w}=I N G_{f w} D_{i n g} F F_{f w} \mathrm{C}_{f w} \\
\text { - } \quad D_{f w}=\text { individual dose from consuming drinking water (Sv/year) } \\
\text { - } \quad D_{i n g}=\text { activity to dose conversion factor for ingestion }(\mathrm{Sv} / \mathrm{Bq}) \\
\text { - } \quad I N G_{f w}=\text { individual ingestion rate of freshwater }\left(\mathrm{m}^{3} / \text { year) }\right. \\
\text { - } \quad F F_{f w}=\text { fraction of activity in the filtered freshwater } \\
\text { - } \quad C_{f w}=\text { radionuclide concentration in the freshwater compartment } \\
\left(\mathrm{Bq} / \mathrm{m}^{3}\right) .\end{array}$ & $\begin{array}{l}\text { The EPRI-YM and ERMYN submodels are } \\
\text { the same, except that water treatment is } \\
\text { included in EPRI-YM, but not in ERMYN. }\end{array}$ & $\begin{array}{l}\text { Smith et al. } 1996 \\
\text { [DIRS 101085], p. 5-7, } \\
\text { Eq. 5.6. }\end{array}$ \\
\hline RESRAD & $\begin{array}{l}H_{E, i p}(t)=D S R_{i j}(t) \times S_{i}(0) \\
\text { - } \quad H_{E, i p}(t)=\text { average annual TEDE received at time } t \text { by a member of } \\
\text { the critical population group from the ith principal radionuclide } \\
\text { transported through the pth environmental pathway together with } \\
\text { its associated decay products (mSv/year or mrem/year) } \\
D S R_{i p}(t)=\text { dose to soil-concentration ratio for the } i \text { th principal } \\
\text { radionuclide and } p \text { th environmental pathway }((\mathrm{mSv} / \mathrm{year}) /(\mathrm{Bq} / \mathrm{g}) \text { or } \\
(\mathrm{mrem} / \mathrm{year}) /(\mathrm{pCi} / \mathrm{g})) \\
\text { - } \\
S_{i}(0)=\text { initial concentration of the } i \text { th principal radionuclide in a } \\
\text { uniformly contaminated zone }(\mathrm{Bq} / \mathrm{g} \text { or } \mathrm{pCi} / \mathrm{g}) .\end{array}$ & $\begin{array}{l}\text { RESRAD calculates the environmental } \\
\text { transport factor for various pathways, then } \\
\text { calculates the dose to soil-concentration } \\
\text { ratios }(D S R) \text {, then calculates the dose }\left(H_{E}\right) \text {. } \\
\text { Although the equation has a different } \\
\text { layout, the method is the same as the } \\
\text { ERMYN. }\end{array}$ & $\begin{array}{l}\text { Yu et al. } 2001 \\
\text { [DIRS 159465], p. 3-10 } \\
\text { and 3-11, Eq. 3.6 \& 3.9. }\end{array}$ \\
\hline NCRP-129 & Not included. & Not applicable. & $\begin{array}{l}\text { NCRP } 1999 \\
\text { [DIRS 155894]. }\end{array}$ \\
\hline
\end{tabular}

ERMYN=Environmental Radiation Model for Yucca Mountain, Nevada; TEDE=total effective dose equivalent 


\begin{tabular}{|c|c|c|c|}
\hline Document & Mathematical Model & $\begin{array}{l}\text { Comparison with the ERMYN } \\
\text { (Equations 6.4.9-3 and 6.5.7-2) }\end{array}$ & Reference \\
\hline GENII-S & 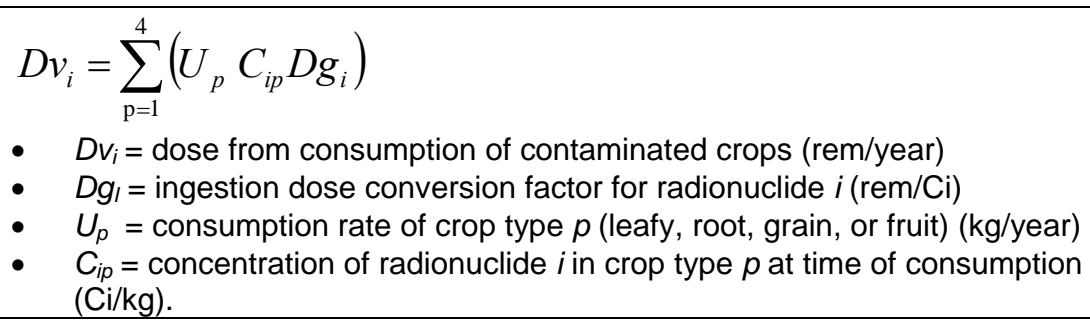 & $\begin{array}{l}\text { GENII-S and ERMYN use the } \\
\text { same method to calculate the crop } \\
\text { ingestion dose. }\end{array}$ & $\begin{array}{l}\text { Napier et al. } 1988 \\
\text { [DIRS 157927], } \\
\text { p. 4.69, Eq. 4.7.9. }\end{array}$ \\
\hline $\begin{array}{l}\text { BIOMASS } \\
\text { ERB2A }\end{array}$ & $\begin{array}{ll}D_{\text {crop }}=I N G_{\text {crop }} D_{\text {ing }} \mathrm{C}_{\text {crop }} \\
\text { - } \quad D_{\text {crop }}=\text { individual dose from consumption of crops (Sv/year) } \\
\text { - } \quad I N G_{\text {crop }}=\text { individual consumption rate for crops (kg/year) } \\
\text { - } \quad D_{\text {ing }}=\text { dose coefficient for ingestion }(\mathrm{Sv} / \mathrm{Bq}) \\
\text { crop }=\text { radionuclide activity concentration in edible crop parts }(\mathrm{Bq} / \mathrm{kg}) .\end{array}$ & $\begin{array}{l}\text { BIOMASS ERB2A and ERMYN } \\
\text { use the same method to calculate } \\
\text { the crop ingestion dose. }\end{array}$ & $\begin{array}{l}\text { BIOMASS } 2000 \\
\text { [DIRS 154522], p. 34, } \\
\text { Eq. } 10 .\end{array}$ \\
\hline EPRI-YM & $\begin{array}{l}D_{\text {crop }}=I N G_{\text {crop }} D_{\text {ing }} \mathrm{C}_{\text {crop }} \\
\text { - } \quad D_{\text {crop }}=\text { individual dose from consumption of crops (Sv/year) } \\
\text { - } \quad I N G_{\text {crop }}=\text { individual ingestion rate of crops (kg/year) } \\
\text { - } \quad D_{\text {ing }}=\text { activity to dose conversion factor for ingestion }(\mathrm{Sv} / \mathrm{Bq}) \\
\quad C_{\text {crop }}=\text { radionuclide concentration in crops }(\mathrm{Bq} / \mathrm{kg}) .\end{array}$ & $\begin{array}{l}\text { EPRI-YM and ERMYN are the } \\
\text { same. }\end{array}$ & $\begin{array}{l}\text { Smith et al. } 1996 \\
\text { [DIRS 101085], p. 5-8, } \\
\text { Eq. 5.8. }\end{array}$ \\
\hline RESRAD & The same equation for water ingestion dose (see Table 7.3-25). & $\begin{array}{l}\text { RESRAD calculates the } \\
\text { environmental transport factor for } \\
\text { various pathways, then calculates } \\
\text { the dose to soil-concentration } \\
\text { ratios }(D S R) \text {, then calculates the } \\
\text { dose }\left(H_{E}\right) \text {. Although the equation } \\
\text { has a different layout, the method } \\
\text { is the same as the other models. }\end{array}$ & $\begin{array}{l}\text { Yu et al. 2001 } \\
\text { [DIRS 159465], p. 3-10 } \\
\text { and 3-11, Eq. 3.6 and } \\
3.9 \text {. }\end{array}$ \\
\hline
\end{tabular}




\begin{tabular}{|c|c|c|c|}
\hline Document & Mathematical Model & $\begin{array}{l}\text { Comparison with the ERMYN } \\
\text { (Equations 6.4.9-3 and 6.5.7-2) }\end{array}$ & Reference \\
\hline NCRP-129 & 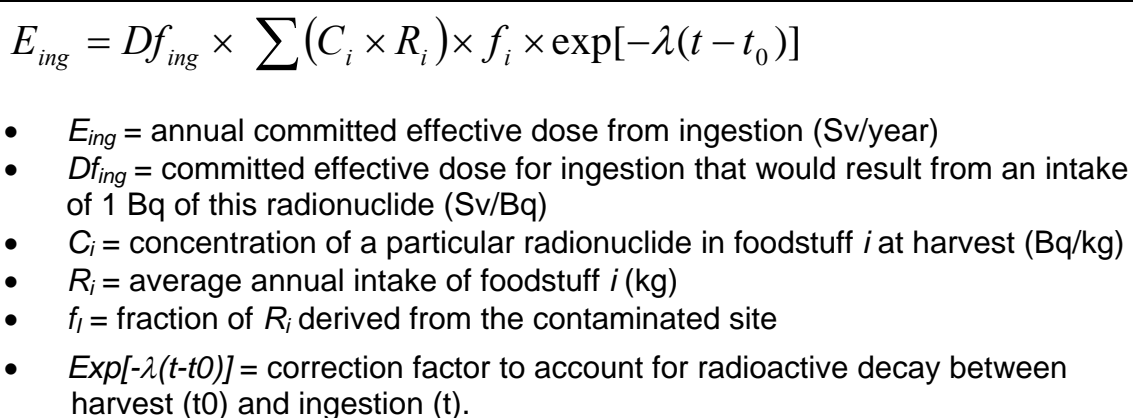 & $\begin{array}{l}\text { NCRP-129 uses holding times, } \\
\text { which are negligible for long-lived } \\
\text { radionuclides. It also uses the } \\
\text { fraction of contaminated food } \\
\text { consumed, which is incorporated } \\
\text { into the consumption rate in the } \\
\text { ERMYN. }\end{array}$ & $\begin{array}{l}\text { NCRP-129 uses } \\
\text { holding times, which } \\
\text { are negligible for long- } \\
\text { lived radionuclides. It } \\
\text { also uses the fraction } \\
\text { of contaminated food } \\
\text { consumed, which is } \\
\text { incorporated into the } \\
\text { consumption rate in } \\
\text { the ERMYN. }\end{array}$ \\
\hline
\end{tabular}

ERMYN=Environmental Radiation Model for Yucca Mountain, Nevada

Table 7.3-27. Comparison of Animal Product Ingestion Pathway

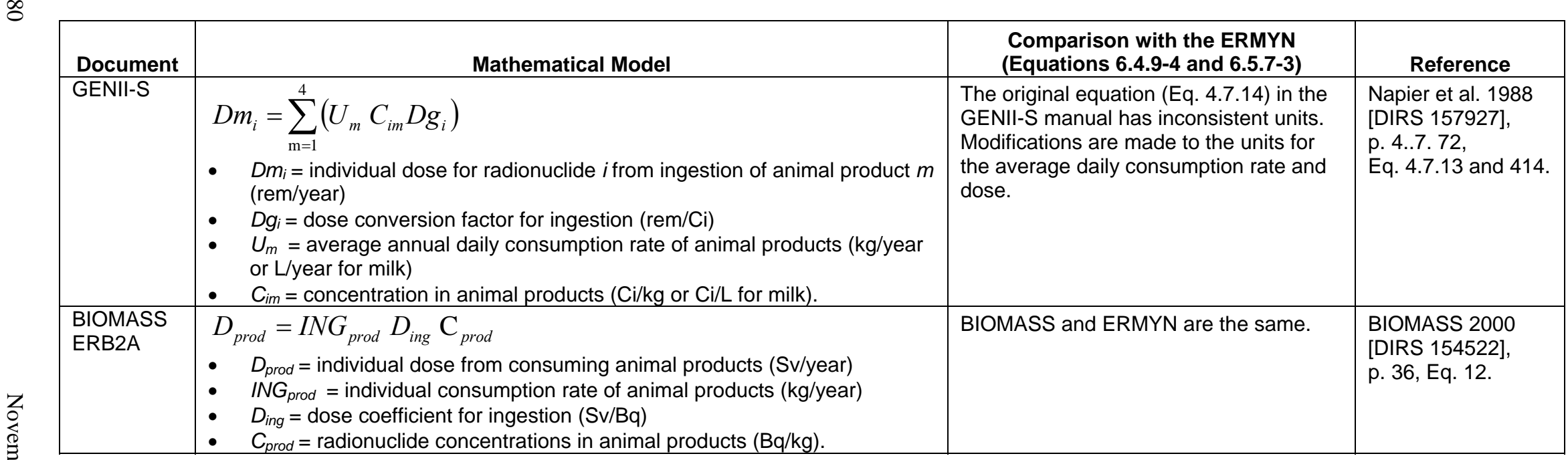




\begin{tabular}{|c|c|c|c|}
\hline Document & Mathematical Model & $\begin{array}{l}\text { Comparison with the ERMYN } \\
\text { (Equations 6.4.9-4 and 6.5.7-3) }\end{array}$ & Reference \\
\hline EPRI-YM & $\begin{array}{l}D_{\text {prod }}=I N G_{\text {prod }} D_{\text {ing }} C_{\text {prod }} \\
\text { - } \quad D_{\text {prod }}=\text { individual dose from consuming animal products (Sv/year) } \\
\text { - } \quad I N G_{\text {prod }}=\text { individual consumption rate of animal products }(\mathrm{kg} / \mathrm{year}) \\
\text { - } \quad D_{\text {ing }}=\text { activity to dose conversion factor for ingestion }(\mathrm{Sv} / \mathrm{Bq}) \\
\quad C_{\text {prod }}=\text { radionuclide concentration in animal products }(\mathrm{Bq} / \mathrm{kg}) .\end{array}$ & $\begin{array}{l}\text { EPRI-YM and ERMYN use the same } \\
\text { method. }\end{array}$ & $\begin{array}{l}\text { Smith et al. } 1996 \\
\text { [DIRS 101085], } \\
\text { p. 5-9, Eq. 5.10. }\end{array}$ \\
\hline RESRAD & The same equation for water ingestion dose (see Table 7.3-25). & $\begin{array}{l}\text { RESRAD calculates the environmental } \\
\text { transport factor for various pathways, } \\
\text { then calculates the dose to soil- } \\
\text { concentration ratios }(D S R) \text {, then } \\
\text { calculates the dose }\left(H_{E}\right) \text {. Although the } \\
\text { equation has a different layout, the } \\
\text { method is the same as the other models. }\end{array}$ & $\begin{array}{l}\text { Yu et al. } 2001 \\
\text { [DIRS 159465], } \\
\text { p. 3-10, and 3-11, } \\
\text { Eq. } 3.6 \& 3.9 \text {. }\end{array}$ \\
\hline NCRP-129 & The same equation for crop ingestion (see Table 7.4-25). & $\begin{array}{l}\text { NCRP- } 129 \text { model includes holding time, } \\
\text { which is negligible for long-lived } \\
\text { radionuclides. }\end{array}$ & $\begin{array}{l}\text { NCRP } 1999 \\
\text { [DIRS 155894], } \\
\text { p. } 91, \text { Eq. } 5.1 .\end{array}$ \\
\hline
\end{tabular}

ERMYN=Environmental Radiation Model for Yucca Mountain, Nevada

Table 7.3-28. Comparison of Fish Ingestion Pathway

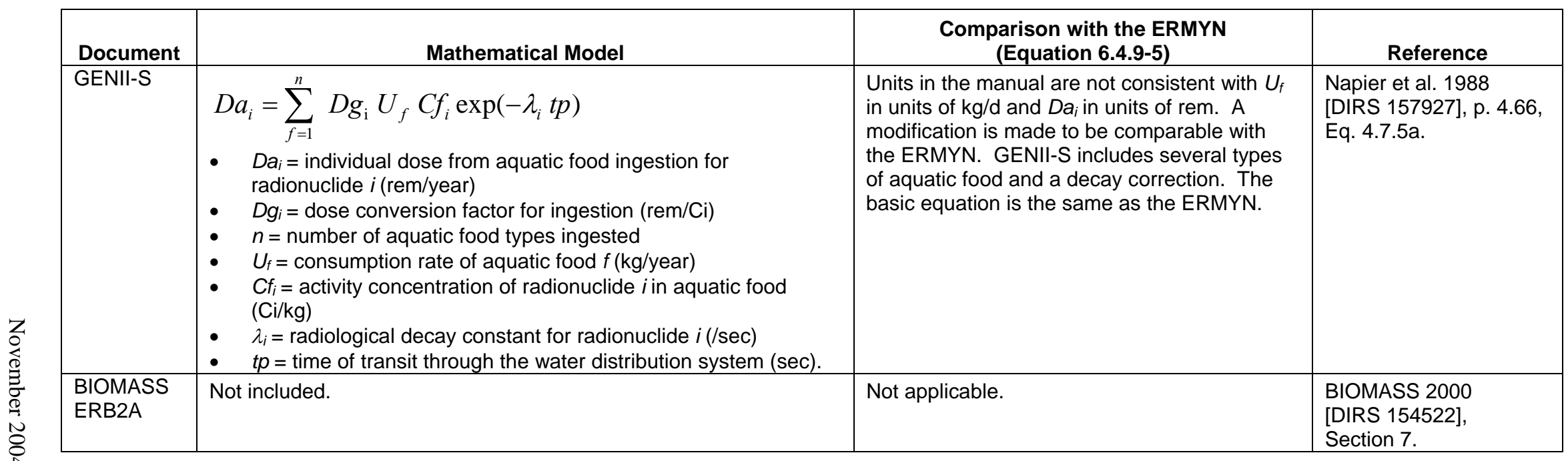


Table 7.3-28. Comparison of Fish Ingestion Pathway (Continued)

\begin{tabular}{|c|c|c|c|}
\hline Document & Mathematical Model & $\begin{array}{c}\text { Comparison with the ERMYN } \\
\text { (Equation 6.4.9-5) }\end{array}$ & Reference \\
\hline EPRI-YM & Not included. & Not applicable. & $\begin{array}{l}\text { Smith et al. } 1996 \\
\text { [DIRS 101085]. }\end{array}$ \\
\hline RESRAD & The same equation for water ingestion dose (see Table 7.3-25). & $\begin{array}{l}\text { RESRAD calculates the environmental } \\
\text { transport factor for various pathways, } \\
\text { calculates the dose to soil-concentration ratios } \\
(D S R) \text {, and then calculates the dose }\left(H_{E}\right) \text {. } \\
\text { Although the equation has a different layout, } \\
\text { the method is the same as the other models. }\end{array}$ & $\begin{array}{l}\text { Yu et al. } 2001 \\
\text { [DIRS 159465], pp. 3-10 } \\
\text { and 3-11, Eq. 3.6 and } \\
\text { 3.9. }\end{array}$ \\
\hline NCRP-129 & Not included. & Not applicable. & $\begin{array}{l}\text { NCRP } 1999 \\
\text { [DIRS 155894]. }\end{array}$ \\
\hline
\end{tabular}

ERMYN=Environmental Radiation Model for Yucca Mountain, Nevada

Table 7.3-29. Comparison of Soil Ingestion Pathway

\begin{tabular}{|c|c|c|c|}
\hline Document & Mathematical Model & $\begin{array}{l}\text { Comparison with the ERMYN } \\
\text { (Equations 6.4.9-6 and 6.5.7-4) }\end{array}$ & Reference \\
\hline GENII-S & $\begin{array}{ll}D d_{i}=D g_{i} t d U_{\text {soil }} \frac{C s_{i}}{\rho_{s}} \\
\text { - } \quad D d_{i}=\text { dose to an individual from inadvertent soil ingestion (rem/year) } \\
\text { - } \quad D g_{i}=\text { dose conversion factor for ingestion }(\mathrm{rem} / \mathrm{Ci}) \\
\text { - } \quad U_{s}=\text { daily average soil ingestion rate }(\mathrm{mg} / \mathrm{d}) \\
\text { - } \quad t d=\text { period of exposure to ingestion of soil }(\mathrm{d} / \mathrm{year}) \\
\text { - } \quad C_{s}=\text { radionuclide activity concentration in the soil per unit area }\left(\mathrm{Ci} / \mathrm{m}^{2}\right) \\
\quad \rho_{s}=\text { surface soil density }\left(\mathrm{mg} / \mathrm{m}^{2}\right) .\end{array}$ & $\begin{array}{l}\text { GENII-S does not calculate the annual } \\
\text { dose. A modification is made to be } \\
\text { consistent with other components of the } \\
\text { dose calculation. GENII-S and ERMYN } \\
\text { use the same submodel. }\end{array}$ & $\begin{array}{l}\text { Napier et al. } 1988 \\
\text { [DIRS 157927], } \\
\text { p. 4.85, Eq. 4.7.25. }\end{array}$ \\
\hline $\begin{array}{l}\text { BIOMASS } \\
\text { ERB2A }\end{array}$ & $\begin{array}{ll} & C_{s o i l}=D_{\text {ing }} I N G_{\text {soil }} \frac{C_{s}}{\left(1-\theta_{t}\right) \rho+\theta \rho_{w}} \\
\text { - } & D_{\text {soil }}=\text { individual dose from soil consumption (Sv/year) } \\
\text { - } & D_{\text {ing }}=\text { dose coefficient for ingestion (Sv/Bq) } \\
\text { - } & I N G_{\text {soil }}=\text { individual consumption rate of soil }(\mathrm{kg} / \mathrm{year} \text { wet weight }) \\
- & C_{s}=\text { radionuclide activity concentration in cultivated soil }\left(\mathrm{Bq} / \mathrm{m}^{3}\right) \\
\text { - } & \theta_{t}=\text { total porosity of the cultivated soil compartment } \\
\text { - } & \rho=\text { dry grain density of the cultivated soil compartment }\left(\mathrm{kg} / \mathrm{m}^{3}\right) \\
- & \theta=\text { water filled porosity of the cultivated soil compartment }(\mathrm{dimensionless}) \\
\text { - } & \rho_{w}=\text { density of water }\left(\mathrm{kg} / \mathrm{m}^{3}\right) .\end{array}$ & $\begin{array}{l}\text { BIOMASS and ERMYN are equivalent. } \\
\text { BIOMASS includes water content in the } \\
\text { soil concentration. Because inadvertent } \\
\text { soil ingestion by humans is from hands } \\
\text { and food, not cultivated soil, the water } \\
\text { content should be lower than that for } \\
\text { cultivated soil. The method used in the } \\
\text { ERMYN is a more conservative because } \\
\text { including water content in the equation } \\
\text { reduces radionuclide concentrations in } \\
\text { dry soil. }\end{array}$ & $\begin{array}{l}\text { BIOMASS } 2000 \\
\text { [DIRS 154522], } \\
\text { p. } 37 \text {, Eq. } 16 .\end{array}$ \\
\hline
\end{tabular}




\begin{tabular}{|c|c|c|c|}
\hline Document & Mathematical Model & $\begin{array}{l}\text { Comparison with the ERMYN } \\
\text { (Equations 6.4.9-6 and 6.5.7-4) }\end{array}$ & Reference \\
\hline EPRI-YM & 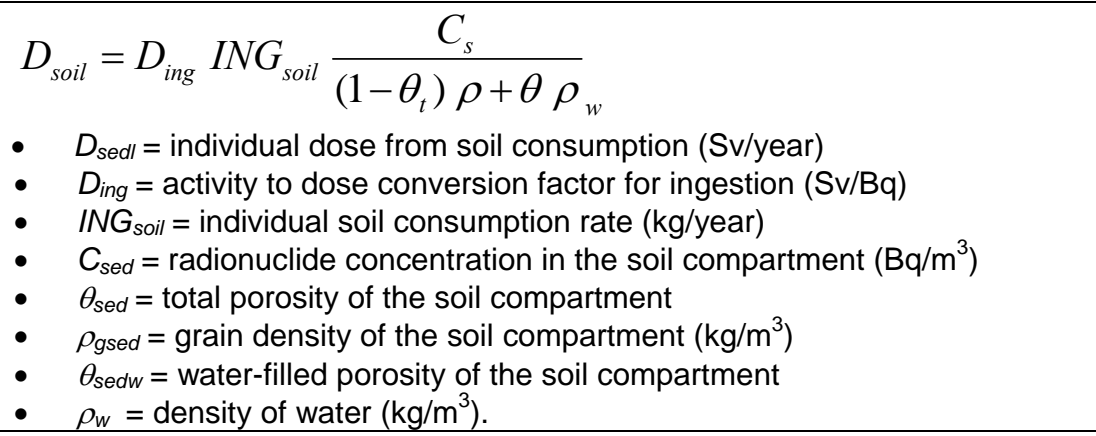 & $\begin{array}{l}\text { EPRI-YM and ERMYN use the same } \\
\text { method. }\end{array}$ & $\begin{array}{l}\text { Smith et al. } 1996 \\
\text { [DIRS 101085], } \\
\text { p. 5-12, Eq. 5.13. }\end{array}$ \\
\hline RESRAD & $\begin{array}{l}\qquad E T F_{j 8}(t)=F S I \times F A_{8} \times F C D_{8}(t) \times F O_{8} \\
\text { - } \quad E T F_{j 8}(t)=\text { environmental transport factor at time } t \text { for soil ingestion for the } \\
\text { ith principle radionuclide (g/year) } \\
-\quad F S I=\text { annual intake of soil (g/year) } \\
-\quad F A_{8}=\text { area factor (dimensionless) } \\
-F C D_{8}(t)=\text { cover-and-depth factor (dimensionless) } \\
-F O_{8}=\text { occupancy factor (dimensionless). }\end{array}$ & $\begin{array}{l}\text { RESRAD uses several factors, including } \\
\text { depth of the contaminated soil, } \\
\text { uncontaminated soil cover, and } \\
\text { contaminated area, which are not used in } \\
\text { ERMYN. To convert ETF to dose, ETF } \\
\text { is multiplied by the dose coefficients } \\
(D C F-\mathrm{mrem} / \mathrm{pCi}) \text { and the source terms } \\
(\mathrm{S}(t)-\mathrm{pCi} / \mathrm{g})(\mathrm{Yu} \text { et al. } 2001 \\
\text { [DIRS 159465], Eq. } 3.6 \text { and } 3.9) \text {. }\end{array}$ & $\begin{array}{l}\text { Yu et al. 2001 } \\
\text { [DIRS 159465], } \\
\text { p. F-3, Eq. F.2. }\end{array}$ \\
\hline NCRP-129 & $\begin{array}{cl} & E_{\text {soil }}=D f_{\text {ing }} \times C_{\text {soil }} \times I_{\text {soil }} \times T \times O F \\
\text { - } & E_{\text {soil }}=\text { committed effective dose for soil ingestion }(\mathrm{Sv} / \mathrm{year}) \\
\text { - } & D f_{\text {ing }}=\text { ingestion dose factor }(\mathrm{Sv} / \mathrm{Bq}) \\
\text { - } & C_{\text {soil }}=\text { average concentration in top } 5 \mathrm{~cm} \text { of soil }(\mathrm{Bq} / \mathrm{kg}) \\
\text { - } & I_{\text {soil }}=\text { average soil ingestion rate during the exposure period }(\mathrm{kg} / \mathrm{d}) \\
& \quad \mathrm{I}=\text { exposure duration }(\mathrm{d} / \mathrm{year}) \\
& \mathrm{OF}=\text { occupational exposure modification factor. }\end{array}$ & $\begin{array}{l}\text { NCRP-129 uses an occupational } \\
\text { exposure modification factor, which is } \\
\text { used for workers. This factor is included } \\
\text { in the ERMYN soil consumption rate. } \\
\text { Therefore, NCRP-129 and ERMYN use } \\
\text { equivalent methods. }\end{array}$ & $\begin{array}{l}\text { NCRP } 1999 \\
\text { [DIRS 155894], } \\
\text { p. 133, Eq. 5.4. }\end{array}$ \\
\hline
\end{tabular}

ERMYN=Environmental Radiation Model for Yucca Mountain, Nevada 


\subsection{NUMERICAL COMPARISON OF MODEL RESULTS}

This section describes some of the numerical comparisons conducted to evaluate the approximations (Sections 6.3.1.4 and 6.3.2.4) and ACMs (Section 6.3.3), to validate the ERMYN (Section 7.3), and to determine if the effective dose coefficients and the effective dose conversion factors (Section 6.4) used to consider short-lived decay products are valid.

The model validation criteria for numerical comparisons are specified in Revision 1 of the TWP (BSC 2003 [DIRS 163602], p. 20), and implementation of the criteria is described in Section 7.1. In all cases where the ERMYN and the five validation models are not mathematically equivalent, a numerical comparison is required. Simple comparisons are presented in Section 7.3, and complex numerical comparisons are presented in this section. If the difference in the results between the ERMYN and the validation model are within a factor of 2, numerical similarity between the models is demonstrated. If the results differ by more than a factor of 2, further evaluation and justification of the selected method is included in Section 7.3.

To make the model comparisons more realistic, input parameters are selected mainly from the ERMYN input values (Section 6.6.3), and, if possible, the same parameter values are used in all of the comparisons. When parameters are specific to a particular validation model, default values for that model are used.

\subsubsection{Radionuclide Decay and Ingrowth}

This Section presents a validation of the methods used for the effective dose coefficients (Section 6.4.7.2), the effective dose conversion factor for inhalation (Section 6.4.8.5), and the effective dose conversion factor for ingestion (Section 6.4.9.6) by comparing the ERMYN results with those from the RESRAD model (Yu et al. 2001 [DIRS 159465]). This comparison does not involve an ACM (Sections 6.3.3 and 7.3).

As discussed in Section 6.3.5, the ERMYN assumes that a radionuclide with a half-life of less than $180 \mathrm{~d}$ is always in secular equilibrium with the long-lived parent radionuclide (Approximation 2). The half-life cutoff is based on the intended use of the model. From a data file, RMDLIB.DAT (Appendix A) in the GENII-S model (SNL 1998 [DIRS 117076]), it can be determined that GENII-S uses a one-hour cutoff for the half-life, as that model is suitable for acute radionuclide releases. Using a high value for the half-life cutoff simplifies the ERMYN by eliminating many short-lived radionuclide decay chains, while still maintaining the accuracy of the model for a long-term repository dose assessment.

This simplification is used in the RESRAD code, a code widely used by the DOE and DOE contractors, the NRC, U.S. Environmental Protection Agency, and many other organizations (Yu et al. 2001 [DIRS 159465], p. xi). The effective dose coefficients and conversion factors calculated in the ERMYN (Sections 6.4.7.2, 6.4.8.5 and 6.4.9.6) are compared with the RESRAD values (Tables 7.4-1, 7.4-2 and 7.4-3, respectively). These comparisons indicate that the values derived from the two models are the same, except for small differences due to rounding. The effective dose coefficients for exposure to contaminated ground (Section 6.5.5.2) are not compared because RESRAD does not use this set of dosimetric data, but the method to derive these values is the same as those used for calculating the values compared in this section. 
Table 7.4-1. Effective Dose Coefficients for Exposure to Soil Contaminated to an Infinite Depth

\begin{tabular}{|c|c|c|c|c|}
\hline $\begin{array}{c}\text { Primary } \\
\text { Radionuclide }\end{array}$ & $\begin{array}{c}\text { Effective DC }^{\mathrm{a}} \\
\text { (Sv/sec) } / \\
(\mathrm{Bq} / \mathrm{m} 3)\end{array}$ & $\begin{array}{c}\text { Effective DC } \\
\text { (mrem/yr) } \\
(\mathrm{pCi} / \mathrm{g})\end{array}$ & $\begin{array}{c}\text { RESRAD DC }{ }^{c} \\
(\mathrm{mrem} / \mathrm{yr}) l \\
(\mathrm{pCi} / \mathrm{g})\end{array}$ & Notes $^{d}$ \\
\hline C-14 & 7.20E-23 & 1.35E-05 & 1.34E-05 & Rounding differences \\
\hline $\mathrm{Cl}-36$ & $1.28 \mathrm{E}-20$ & $2.39 \mathrm{E}-03$ & $2.39 \mathrm{E}-03$ & Same \\
\hline Se-79 & 9.96E-23 & 1.86E-05 & 1.86E-05 & Same \\
\hline Sr-90D & $1.32 \mathrm{E}-19$ & $2.47 \mathrm{E}-02$ & $2.46 \mathrm{E}-02$ & Rounding differences \\
\hline Tc-99 & $6.72 E-22$ & 1.26E-04 & 1.26E-04 & Same \\
\hline Sn-126D & $6.34 \mathrm{E}-17$ & $1.19 \mathrm{E}+01$ & n.a. & Not available in RESRAD \\
\hline I-129 & 6.93E-20 & 1.30E-02 & $1.29 \mathrm{E}-02$ & Rounding differences \\
\hline Cs-135 & 2.05E-22 & 3.83E-05 & 3.83E-05 & Same \\
\hline Cs-137D & $1.83 \mathrm{E}-17$ & $3.42 \mathrm{E}+00$ & $3.41 \mathrm{E}+00$ & Rounding differences \\
\hline Pu-242 & $6.85 E-22$ & $1.28 \mathrm{E}-04$ & $1.28 \mathrm{E}-04$ & Same \\
\hline U-238D & 8.13E-19 & $1.52 \mathrm{E}-01$ & 1.37E-01 & $\begin{array}{l}\text { Correction made in RESRAD code } 6.1 \\
\text { to be } 1.52 \mathrm{E}-01\end{array}$ \\
\hline Pu-238 & $8.10 \mathrm{E}-22$ & 1.51E-04 & 1.51E-04 & Same \\
\hline U-234 & $2.15 E-21$ & 4.02E-04 & 4.02E-04 & Same \\
\hline Th-230 & $6.47 \mathrm{E}-21$ & $1.21 \mathrm{E}-03$ & $1.21 \mathrm{E}-03$ & Same \\
\hline Ra-226D & 5.99E-17 & $1.12 \mathrm{E}+01$ & $1.12 \mathrm{E}+01$ & Same \\
\hline $\mathrm{Pb}-210 \mathrm{D}$ & 3.27E-20 & $6.11 \mathrm{E}-03$ & $6.10 \mathrm{E}-03$ & $\begin{array}{l}\text { Rounding differences (Pb-210D + } \\
\text { Po-210) }\end{array}$ \\
\hline Pu-240 & 7.85E-22 & 1.47E-04 & 1.47E-04 & Same \\
\hline U-236 & 1.15E-21 & $2.15 \mathrm{E}-04$ & $2.15 \mathrm{E}-04$ & Same \\
\hline Th-232 & $2.79 E-21$ & 5.21E-04 & $5.21 \mathrm{E}-04$ & Same \\
\hline Ra-228D & 3.20E-17 & $5.98 \mathrm{E}+00$ & $5.98 \mathrm{E}+00$ & Same \\
\hline $\mathrm{U}-232$ & 4.83E-21 & 9.03E-04 & 9.02E-04 & Rounding differences \\
\hline Th-228D & $5.46 \mathrm{E}-17$ & $1.02 \mathrm{E}+01$ & $1.02 \mathrm{E}+01$ & Same \\
\hline Am-243D & $4.79 \mathrm{E}-18$ & 8.96E-01 & 8.95E-01 & Rounding differences \\
\hline Pu-239 & $1.58 \mathrm{E}-21$ & 2.95E-04 & 2.95E-04 & Same \\
\hline U-235D & 4.06E-18 & 7.59E-01 & 7.57E-01 & Rounding differences \\
\hline Pa-231 & $1.02 \mathrm{E}-18$ & 1.91E-01 & $1.91 \mathrm{E}-01$ & Same \\
\hline Ac-227D & 1.08E-17 & $2.02 \mathrm{E}+00$ & $2.01 \mathrm{E}+00$ & Rounding differences \\
\hline Am-241 & 2.34E-19 & 4.38E-02 & 4.37E-02 & Rounding differences \\
\hline Np-237D & $5.88 \mathrm{E}-18$ & $1.10 \mathrm{E}+00$ & $1.10 E+00$ & Same \\
\hline U-233 & 7.48E-21 & $1.40 \mathrm{E}-03$ & 1.40E-03 & Same \\
\hline Th-229D & 8.55E-18 & $1.60 \mathrm{E}+00$ & $1.60 \mathrm{E}+00$ & Same \\
\hline
\end{tabular}

${ }^{\mathrm{a}}$ From Table 6.4-2.

b Unit conversion from Column 2 using soil density $=1.6 \mathrm{~g} / \mathrm{cm}^{3}$, in order to compare the values with RESRAD values in Column 4.

c Source: RESRAD (Yu et al. 2001 [DIRS 159465], Table A.1).

${ }^{d}$ Using a conversion factor of $1.87 \times 10^{17}$, ten values differ slightly due to rounding differences.

$\mathrm{DC}=$ dose coefficient 
Table 7.4-2. Effective Equivalent Conversion Factors for Inhalation

\begin{tabular}{|c|c|c|c|c|c|}
\hline $\begin{array}{c}\text { Primary } \\
\text { Radionuclide }\end{array}$ & $\begin{array}{c}\text { Inhalation } \\
\text { Class }^{c}\end{array}$ & $\begin{array}{c}\text { Effective DCF }{ }^{\text {a }} \\
\text { (Sv/Bq) }\end{array}$ & $\begin{array}{c}\text { Effective DCF }{ }^{b} \\
(\mathrm{mrem} / \mathrm{pCi})\end{array}$ & $\begin{array}{c}\text { RESRAD DCF }^{c} \\
(\mathrm{mrem} / \mathrm{pCi})\end{array}$ & Notes \\
\hline C-14 & $\mathrm{CO}_{2}$ & $6.36 \mathrm{E}-12$ & 2.35E-08 & $2.35 \mathrm{E}-08$ & Same \\
\hline $\mathrm{Cl}-36$ & W & 5.93E-09 & $2.19 \mathrm{E}-05$ & $2.19 \mathrm{E}-05$ & Same \\
\hline Se-79 & W & 2.66E-09 & 9.84E-06 & 9.84E-06 & Same \\
\hline Sr-90D & $\mathrm{D}$ & $6.68 \mathrm{E}-08$ & $2.47 \mathrm{E}-04$ & $2.47 \mathrm{E}-04$ & Same \\
\hline Tc-99 & W & $2.25 \mathrm{E}-09$ & $8.33 \mathrm{E}-06$ & 8.33E-06 & Same \\
\hline Sn-126D & W & $2.74 \mathrm{E}-08$ & 1.53E-03 & n.a. & Not available in RESRAD \\
\hline $\mathrm{I}-129$ & $\mathrm{D}$ & $4.69 \mathrm{E}-08$ & 1.74E-04 & 1.74E-04 & Same \\
\hline Cs-135 & $\mathrm{D}$ & 1.23E-09 & $4.55 \mathrm{E}-06$ & 4.55E-06 & Same \\
\hline Cs-137D & $\mathrm{D}$ & 8.63E-09 & 3.19E-05 & 3.19E-05 & Same \\
\hline $\mathrm{Pu}-242$ & W & $1.11 \mathrm{E}-04$ & 4.11E-01 & 4.11E-01 & Same \\
\hline U-238D & $\mathrm{Y}$ & $3.20 \mathrm{E}-05$ & $1.18 \mathrm{E}-01$ & $1.18 \mathrm{E}-01$ & Same \\
\hline Pu-238 & W & 1.06E-04 & 3.92E-01 & 3.92E-01 & Same \\
\hline U-234 & $\mathrm{Y}$ & $3.58 \mathrm{E}-05$ & $1.32 \mathrm{E}-01$ & $1.32 \mathrm{E}-01$ & Same \\
\hline Th-230 & W & $8.80 \mathrm{E}-05$ & 3.26E-01 & 3.26E-01 & Same \\
\hline Ra-226D & W & $2.32 \mathrm{E}-06$ & 8.60E-03 & 8.60E-03 & Same \\
\hline $\mathrm{Pb}-210 \mathrm{D}$ & $\mathrm{D}$ & $6.26 \mathrm{E}-06$ & $2.32 \mathrm{E}-02$ & $2.32 \mathrm{E}-02$ & Same (Pb-210D + Po-210) \\
\hline Pu-240 & W & 1.16E-04 & $4.29 \mathrm{E}-01$ & 4.29E-01 & Same \\
\hline U-236 & $\mathrm{Y}$ & 3.39E-05 & 1.25E-01 & 1.25E-01 & Same \\
\hline Th-232 & W & 4.43E-04 & $1.64 \mathrm{E}+00$ & $1.64 \mathrm{E}+00$ & Same \\
\hline $\mathrm{Ra}-228 \mathrm{D}$ & W & $1.37 \mathrm{E}-06$ & $5.08 \mathrm{E}-03$ & $5.08 \mathrm{E}-03$ & Same \\
\hline U-232 & $\mathrm{Y}$ & 1.78E-04 & 6.59E-01 & $6.59 \mathrm{E}-01$ & Same \\
\hline Th-228D & $\mathrm{Y}$ & 9.32E-05 & 3.45E-01 & $3.45 \mathrm{E}-01$ & Same \\
\hline Am-243D & W & 1.19E-04 & 4.40E-01 & 4.40E-01 & Same \\
\hline Pu-239 & W & 1.16E-04 & $4.29 \mathrm{E}-01$ & 4.29E-01 & Same \\
\hline U-235D & $\mathrm{Y}$ & 3.32E-05 & 1.23E-01 & 1.23E-01 & Same \\
\hline $\mathrm{Pa}-231$ & W & 3.47E-04 & $1.28 \mathrm{E}+00$ & $1.28 \mathrm{E}+00$ & Same \\
\hline Ac-227D & $\mathrm{D}$ & $1.82 \mathrm{E}-03$ & $6.72 \mathrm{E}+00$ & $6.72 \mathrm{E}+00$ & Same \\
\hline Am-241 & W & 1.20E-04 & 4.44E-01 & 4.44E-01 & Same \\
\hline Np-237D & W & $1.46 \mathrm{E}-04$ & $5.40 \mathrm{E}-01$ & 5.40E-01 & Same \\
\hline U-233 & $\mathrm{Y}$ & 3.66E-05 & 1.35E-01 & 1.35E-01 & Same \\
\hline Th-229D & W & 5.85E-04 & $2.16 \mathrm{E}+00$ & $2.16 \mathrm{E}+00$ & Same \\
\hline
\end{tabular}

${ }^{\mathrm{a}}$ From Table 6.4-3.

${ }^{b}$ Unit conversion from Column 3.

c Source: RESRAD (Yu et al. 2001 [DIRS 159465], Table B.1).

$\mathrm{DCF}=$ dose conversion factor 
Table 7.4-3. Effective Dose Conversion Factors for Ingestion

\begin{tabular}{|c|c|c|c|c|c|}
\hline $\begin{array}{c}\text { Primary } \\
\text { Radionuclide }\end{array}$ & $f_{1}{ }^{c, d}$ & $\begin{array}{c}\text { Effective DCF }{ }^{\text {a }} \\
\text { (Sv/Bq) }\end{array}$ & $\begin{array}{c}\text { Effective DCF }{ }^{b} \\
(\mathrm{mrem} / \mathrm{pCi})\end{array}$ & $\begin{array}{l}\text { RESRAD }^{\mathrm{c}} \\
(\mathrm{mrem} / \mathrm{pCi})\end{array}$ & Notes \\
\hline C-14 & 1.0 & $5.64 \mathrm{E}-10$ & 2.09E-06 & 2.09E-06 & Same \\
\hline $\mathrm{Cl}-36$ & 1.0 & $8.18 \mathrm{E}-10$ & 3.03E-06 & 3.03E-06 & Same \\
\hline Se-79 & $8 \mathrm{E}-1$ & 2.35E-09 & 8.70E-06 & 8.70E-06 & Same \\
\hline Sr-90D & $3 \mathrm{E}-1$ & 4.14E-08 & 1.53E-04 & 1.53E-04 & Same \\
\hline Tc-99 & $8 \mathrm{E}-1$ & $3.95 \mathrm{E}-10$ & $1.46 \mathrm{E}-06$ & 1.46E-06 & Same \\
\hline Sn-126D & $2 \mathrm{E}-2$ & 5.70E-09 & 2.11E-05 & n.a. & Not available in RESRAD \\
\hline $\mathrm{I}-129$ & 1.0 & 7.46E-08 & $2.76 \mathrm{E}-04$ & $2.76 \mathrm{E}-04$ & Same \\
\hline Cs-135 & 1.0 & 1.91E-09 & 7.07E-06 & 7.07E-06 & Same \\
\hline Cs-137D & 1.0 & 1.35E-08 & 5.00E-05 & 5.00E-05 & Same \\
\hline Pu-242 & $1 \mathrm{E}-3$ & $9.08 \mathrm{E}-07$ & 3.36E-03 & 3.36E-03 & Same \\
\hline U-238D & $5 E-2$ & $7.25 \mathrm{E}-08$ & $2.68 \mathrm{E}-04$ & $2.69 \mathrm{E}-04$ & Rounding difference \\
\hline Pu-238 & $1 \mathrm{E}-3$ & 8.65E-07 & $3.20 \mathrm{E}-03$ & $3.20 \mathrm{E}-03$ & Same \\
\hline U-234 & $5 \mathrm{E}-2$ & 7.66E-08 & 2.83E-04 & 2.83E-04 & Same \\
\hline Th-230 & $2 \mathrm{E}-4$ & $1.48 \mathrm{E}-07$ & $5.48 \mathrm{E}-04$ & $5.48 \mathrm{E}-04$ & Same \\
\hline Ra-226D & $2 \mathrm{E}-1$ & $3.58 \mathrm{E}-07$ & 1.33E-03 & 1.33E-03 & Same \\
\hline $\mathrm{Pb}-210 \mathrm{D}$ & $2 \mathrm{E}-1$ & 1.97E-06 & 7.27E-03 & 7.27E-03 & Same (Pb-210D + Po-210) \\
\hline Pu-240 & $1 \mathrm{E}-3$ & 9.56E-07 & 3.54E-03 & 3.54E-03 & Same \\
\hline $\mathrm{U}-236$ & $5 E-2$ & $7.26 \mathrm{E}-08$ & $2.69 \mathrm{E}-04$ & 2.69E-04 & Same \\
\hline Th-232 & $2 \mathrm{E}-4$ & 7.38E-07 & 2.73E-03 & 2.73E-03 & Same \\
\hline $\mathrm{Ra}-228 \mathrm{D}$ & $2 \mathrm{E}-1$ & 3.89E-07 & $1.44 \mathrm{E}-03$ & 1.44E-03 & Same \\
\hline U-232 & $5 E-2$ & 3.54E-07 & 1.31E-03 & $1.31 \mathrm{E}-03$ & Same \\
\hline Th-228D & $2 \mathrm{E}-4$ & $2.18 \mathrm{E}-07$ & 8.08E-04 & 8.08E-04 & Same \\
\hline Am-243D & $1 \mathrm{E}-3$ & 9.80E-07 & 3.63E-03 & 3.63E-03 & Same \\
\hline Pu-239 & $1 \mathrm{E}-3$ & 9.56E-07 & 3.54E-03 & 3.54E-03 & Same \\
\hline U-235D & $5 \mathrm{E}-2$ & 7.23E-08 & 2.67E-04 & 2.67E-04 & Same \\
\hline Pa-231 & $1 \mathrm{E}-3$ & 2.86E-06 & 1.06E-02 & 1.06E-02 & Same \\
\hline Ac-227D & $1 \mathrm{E}-3$ & 3.99E-06 & $1.48 \mathrm{E}-02$ & 1.48E-02 & Same \\
\hline Am-241 & $1 \mathrm{E}-3$ & 9.84E-07 & 3.64E-03 & 3.64E-03 & Same \\
\hline Np-237D & $1 \mathrm{E}-3$ & $1.20 \mathrm{E}-06$ & 4.44E-03 & 4.44E-03 & Same \\
\hline U-233 & $5 E-2$ & 7.81E-08 & $2.89 \mathrm{E}-04$ & 2.89E-04 & Same \\
\hline Th-229D & $2 \mathrm{E}-4$ & 1.09E-06 & 4.03E-03 & 4.03E-03 & Same \\
\hline
\end{tabular}

${ }^{\mathrm{a}}$ From Table 6.4-4.

${ }^{\mathrm{b}}$ Unit conversion from Column 3.

c Source: RESRAD (Yu et al. 2001 [DIRS 159465], Table D.1).

${ }^{d} f_{1}=$ fractional uptake from the small intestine to blood for common chemical forms of the radionuclide.

$\mathrm{DCF}=$ dose conversion factor 


\subsubsection{Surface Soil Submodel}

The surface soil submodel for the groundwater scenario is used to calculate the accumulation of radionuclides in cultivated soils after long-term irrigation. It is assumed that equilibrium conditions have been reached, and radionuclide concentrations in the soil are calculated only under equilibrium conditions (Approximation 5). An evaluation is conducted (Section 7.4.2.1) to verify that the radionuclides considered would reach equilibrium conditions by 10,000 years. Furthermore, in the ERMYN, decay-chain radionuclides are considered to be in equilibrium (Section 6.4.1.2). Therefore, the radionuclide decay chains in the ERMYN are compared with those in the GENII-S model to evaluate whether the results are comparable, and to ensure that the ERMYN includes all of the important radionuclides (Section 7.4.2.2).

\subsubsection{Time Required to Establish Equilibrium Condition}

As discussed in Section 6.4.1, the time to reach 95-percent equilibrium (i.e., the 95-percent equilibrium time) can be calculated using the effective removal constant. The effective removal factor is controlled mainly by a leaching constant (Equation 6.4.1-10), unless the leaching constant is less than the erosion constant $\left(1.3 \times 10^{-4}\right)$, which corresponds to a partition coefficient value of about $140 \mathrm{~L} / \mathrm{kg}$ (Table 7.4-4). The erosion constant, leaching removal constant, effective removal constant, and 95-percent equilibrium time are calculated (Table 7.4-4) using input parameter values for the expected future climate (Table 6.6-3). Values for future climate conditions are used because of the lower leaching rate and longer time to reach equilibrium for that climate. Using mean partition coefficients and erosion rates, all radionuclides reach the 95-percent conditions in less than 2,300 years. If uncertainties in the partition coefficients and erosion rates are considered, variation in the leaching constants could be large. However, when the leaching rate is low, erosion is important. Therefore, using the 95-percent equilibrium condition for radionuclide accumulation in the soil is a valid approach that does not underestimate the radiation dose.

Table 7.4-4. Time to Reach 95-Percent Equilibrium

\begin{tabular}{|l|c|c|c|c|c|c|}
\hline $\begin{array}{c}\text { Primary } \\
\text { Radionuclide }\end{array}$ & $\begin{array}{c}\text { Decay } \\
\text { Constant } \\
\text { (lyr) }\end{array}$ & $\begin{array}{c}\text { Erosion } \\
\text { Constant } \\
(\text { /yr) }\end{array}$ & $\begin{array}{c}\text { Partition } \\
\text { Coefficient } \\
\text { (L/kg) }\end{array}$ & $\begin{array}{c}\text { Calculated } \\
\text { Leaching } \\
\text { Constant } \\
\text { (lyr) }\end{array}$ & $\begin{array}{c}\text { Effective } \\
\text { Removal } \\
\text { Constant } \\
(\text { /yr) }\end{array}$ & $\begin{array}{c}\text { Equilibrium } \\
\text { time @ 95\% } \\
\text { (yr) }\end{array}$ \\
\hline C-14 & $1.21 \mathrm{E}-04$ & $1.31 \mathrm{E}-03$ & $1.8 \mathrm{E}+01$ & $9.8 \mathrm{E}-03$ & $22^{\mathrm{a}}$ & 1 \\
\hline Cl-36 & $2.30 \mathrm{E}-06$ & $1.31 \mathrm{E}-03$ & $1.4 \mathrm{E}-01$ & $6.1 \mathrm{E}-01$ & $6.10 \mathrm{E}-01$ & 5 \\
\hline Se-79 & $1.07 \mathrm{E}-05$ & $1.31 \mathrm{E}-03$ & $1.5 \mathrm{E}+02$ & $1.2 \mathrm{E}-03$ & $2.51 \mathrm{E}-03$ & 1195 \\
\hline Sr-90D & $2.38 \mathrm{E}-02$ & $1.31 \mathrm{E}-03$ & $2.0 \mathrm{E}+01$ & $8.9 \mathrm{E}-03$ & $3.40 \mathrm{E}-02$ & 88 \\
\hline Tc-99 & $3.25 \mathrm{E}-06$ & $1.31 \mathrm{E}-03$ & $1.4 \mathrm{E}-01$ & $6.1 \mathrm{E}-01$ & $6.10 \mathrm{E}-01$ & 5 \\
\hline Sn-126 & $6.93 \mathrm{E}-06$ & $1.31 \mathrm{E}-03$ & $4.5 \mathrm{E}+02$ & $4.0 \mathrm{E}-04$ & $1.71 \mathrm{E}-03$ & 1751 \\
\hline I-129 & $4.41 \mathrm{E}-08$ & $1.31 \mathrm{E}-03$ & $4.5 \mathrm{E}+00$ & $3.8 \mathrm{E}-02$ & $3.97 \mathrm{E}-02$ & 75 \\
\hline Cs-135 & $3.01 \mathrm{E}-07$ & $1.31 \mathrm{E}-03$ & $4.4 \mathrm{E}+03$ & $4.1 \mathrm{E}-05$ & $1.35 \mathrm{E}-03$ & 2223 \\
\hline Cs-137D & $2.31 \mathrm{E}-02$ & $1.31 \mathrm{E}-03$ & $4.4 \mathrm{E}+03$ & $4.1 \mathrm{E}-05$ & $2.45 \mathrm{E}-02$ & 123 \\
\hline Pu-242 & $1.84 \mathrm{E}-06$ & $1.31 \mathrm{E}-03$ & $1.2 \mathrm{E}+03$ & $1.5 \mathrm{E}-04$ & $1.46 \mathrm{E}-03$ & 2056 \\
\hline
\end{tabular}


Table 7.4-4. Time to Reach 95-Percent Equilibrium (Continued)

\begin{tabular}{|l|c|c|c|c|c|c|}
\hline $\begin{array}{c}\text { Primary } \\
\text { Radionuclide }\end{array}$ & $\begin{array}{c}\text { Decay } \\
\text { Constant } \\
\text { (lyr) }\end{array}$ & $\begin{array}{c}\text { Erosion } \\
\text { Constant } \\
\text { (lyr) }\end{array}$ & $\begin{array}{c}\text { Partition } \\
\text { Coefficient } \\
\text { (L/kg) }\end{array}$ & $\begin{array}{c}\text { Calculated } \\
\text { Leaching } \\
\text { Constant } \\
\text { (lyr) }\end{array}$ & $\begin{array}{c}\text { Effective } \\
\text { Removal } \\
\text { Constant } \\
\text { (lyr) }\end{array}$ & $\begin{array}{c}\text { Equilibrium } \\
\text { time @ 95\% } \\
\text { (yr) }\end{array}$ \\
\hline U-238D & $1.55 \mathrm{E}-10$ & $1.31 \mathrm{E}-03$ & $3.3 \mathrm{E}+01$ & $5.4 \mathrm{E}-03$ & $6.70 \mathrm{E}-03$ & 447 \\
\hline Pu-238 & $7.90 \mathrm{E}-03$ & $1.31 \mathrm{E}-03$ & $1.2 \mathrm{E}+03$ & $1.5 \mathrm{E}-04$ & $9.36 \mathrm{E}-03$ & 320 \\
\hline U-234 & $2.83 \mathrm{E}-06$ & $1.31 \mathrm{E}-03$ & $3.3 \mathrm{E}+01$ & $5.4 \mathrm{E}-03$ & $6.70 \mathrm{E}-03$ & 447 \\
\hline Th-230 & $9.00 \mathrm{E}-06$ & $1.31 \mathrm{E}-03$ & $3.0 \mathrm{E}+03$ & $6.0 \mathrm{E}-05$ & $1.38 \mathrm{E}-03$ & 2178 \\
\hline Ra-226D & $4.33 \mathrm{E}-04$ & $1.31 \mathrm{E}-03$ & $3.6 \mathrm{E}+04$ & $5.0 \mathrm{E}-06$ & $1.74 \mathrm{E}-03$ & 1717 \\
\hline Pb-210D & $3.11 \mathrm{E}-02$ & $1.31 \mathrm{E}-03$ & $1.6 \mathrm{E}+04$ & $1.1 \mathrm{E}-05$ & $3.24 \mathrm{E}-02$ & 92 \\
\hline Pu-240 & $1.06 \mathrm{E}-04$ & $1.31 \mathrm{E}-03$ & $1.2 \mathrm{E}+03$ & $1.5 \mathrm{E}-04$ & $1.56 \mathrm{E}-03$ & 1918 \\
\hline U-236 & $2.96 \mathrm{E}-08$ & $1.31 \mathrm{E}-03$ & $3.3 \mathrm{E}+01$ & $5.4 \mathrm{E}-03$ & $6.70 \mathrm{E}-03$ & 447 \\
\hline Th-232 & $4.93 \mathrm{E}-11$ & $1.31 \mathrm{E}-03$ & $3.0 \mathrm{E}+03$ & $6.0 \mathrm{E}-05$ & $1.37 \mathrm{E}-03$ & 2193 \\
\hline \multicolumn{1}{|c|}{ Ra-228D } & $1.21 \mathrm{E}-01$ & $1.31 \mathrm{E}-03$ & $3.6 \mathrm{E}+04$ & $5.0 \mathrm{E}-06$ & $1.22 \mathrm{E}-01$ & 25 \\
\hline U-232 & $9.63 \mathrm{E}-03$ & $1.31 \mathrm{E}-03$ & $3.3 \mathrm{E}+01$ & $5.4 \mathrm{E}-03$ & $1.63 \mathrm{E}-02$ & 184 \\
\hline \multicolumn{1}{|c|}{ Th-228D } & $3.62 \mathrm{E}-01$ & $1.31 \mathrm{E}-03$ & $3.0 \mathrm{E}+03$ & $6.0 \mathrm{E}-05$ & $3.64 \mathrm{E}-01$ & 8 \\
\hline Am-243D & $9.39 \mathrm{E}-05$ & $1.31 \mathrm{E}-03$ & $2.0 \mathrm{E}+03$ & $8.9 \mathrm{E}-05$ & $1.49 \mathrm{E}-03$ & 2011 \\
\hline Pu-239 & $2.88 \mathrm{E}-05$ & $1.31 \mathrm{E}-03$ & $1.2 \mathrm{E}+03$ & $1.5 \mathrm{E}-04$ & $1.48 \mathrm{E}-03$ & 2018 \\
\hline U-235D & $9.85 \mathrm{E}-10$ & $1.31 \mathrm{E}-03$ & $3.3 \mathrm{E}+01$ & $5.4 \mathrm{E}-03$ & $6.70 \mathrm{E}-03$ & 447 \\
\hline Pa-231 & $2.12 \mathrm{E}-05$ & $1.31 \mathrm{E}-03$ & $1.8 \mathrm{E}+03$ & $9.9 \mathrm{E}-05$ & $1.43 \mathrm{E}-03$ & 2099 \\
\hline Ac-227D & $3.18 \mathrm{E}-02$ & $1.31 \mathrm{E}-03$ & $1.5 \mathrm{E}+03$ & $1.2 \mathrm{E}-04$ & $3.33 \mathrm{E}-02$ & 90 \\
\hline Am-241 & $1.60 \mathrm{E}-03$ & $1.31 \mathrm{E}-03$ & $2.0 \mathrm{E}+03$ & $8.9 \mathrm{E}-05$ & $3.00 \mathrm{E}-03$ & 999 \\
\hline Np-237D & $3.24 \mathrm{E}-07$ & $1.31 \mathrm{E}-03$ & $2.5 \mathrm{E}+01$ & $7.1 \mathrm{E}-03$ & $8.41 \mathrm{E}-03$ & 356 \\
\hline U-233 & $4.37 \mathrm{E}-06$ & $1.31 \mathrm{E}-03$ & $3.3 \mathrm{E}+01$ & $5.4 \mathrm{E}-03$ & $6.70 \mathrm{E}-03$ & 447 \\
\hline Th-229D & $9.44 \mathrm{E}-05$ & $1.31 \mathrm{E}-03$ & $3.0 \mathrm{E}+03$ & $6.0 \mathrm{E}-05$ & $1.46 \mathrm{E}-03$ & 2051 \\
\hline NOTE: Can & & & & & \\
\hline
\end{tabular}

NOTE: Calculations performed using Microsoft Excel 97 SR-2, and the electronic file, ERMYN validation.xIs, which is listed in Appendix A. Columns 2 and 4 are from Tables 6.3-7 and 6.6-3, respectively. Column 3 is calculated using Equation 6.4.1-11. Column 5 is calculated using Equation 6.4.1-10. The last two columns are calculated using the method provided in Section 6.4.1.1. All input data are taken from Table 6.6-3.

a This value is the ${ }^{14} \mathrm{C}$ emission rate (Table 6.6-3).

\subsubsection{Comparison of Radionuclide Decay Chains in the GENII-S Model and ERMYN}

When radionuclide decay and ingrowth are modeled, decay chains often are evaluated to determine where the chains approximately stop, permitting the calculations to be terminated and the computational problem to be simplified. This is especially true for the transuranic radionuclides with long-decay chains. The only decay and ingrowth included in the ERMYN is related to radionuclide buildup in the soil, where radionuclide decay chains are included in the surface soil submodel (Section 6.4.1.2). In this section, radionuclide decay chains in the GENII-S and ERMYNs are compared to determine if all of the decay products are properly considered.

A data file, RMDLIB.DAT (Appendix A), is used in the GENII-S model (SNL 1998 [DIRS 117076]) to control radionuclide decay chains. Part of this data file is shown in the 
GENII-S manual (Leigh et al. 1993 [DIRS 100464], p. 5-69). Only the high atomic number ( $Z$ greater than or equal to 82) radionuclides are compared. Comparisons are made for each high-Z primary radionuclide and associated decay chain (Table 7.4-5). The comparison indicates that the two models use similar methods to control the decay chains, and, therefore, the radionuclide decay chains are properly considered in the ERMYN.

Table 7.4-5. Radionuclide Decay Chains Included in the GENII-S Model and ERMYN

\begin{tabular}{|c|c|c|c|}
\hline $\begin{array}{c}\text { Primary } \\
\text { Radionuclide }\end{array}$ & GENII-S $^{a}$ & ERMYN $^{\text {b }}$ & Notes \\
\hline Pu-242 & No decay chain & No decay chain & Same \\
\hline $\mathrm{U}-238$ & Decay chain: Th-234 (Pa-234m) / Pa234 & U-238D & Same \\
\hline Pu-238 & Decay chain: U-234 & No decay chain & $\begin{array}{l}\text { Low contribution from } \\
\text { ingrowth of U-234 } \\
\text { due to long half-life }\end{array}$ \\
\hline U-234 & No decay chain & $\begin{array}{l}\text { Decay chain: Th-230 / } \\
\text { Ra-226D / Pb-210D }\end{array}$ & $\begin{array}{l}\text { ERMYN includes the } \\
\text { decay chain, which } \\
\text { adds a small } \\
\text { contribution from the } \\
\text { decay products }\end{array}$ \\
\hline Th-230 & $\begin{array}{l}\text { Decay chain: Ra-226 / Rn-222 (Po-218, } \\
\text { Pb-214, Bi-214, Po-214) / Pb-210 / Bi-210 / } \\
\text { Po-210 }\end{array}$ & $\begin{array}{l}\text { Decay chain: Ra-226D / } \\
\text { Pb-210D }\end{array}$ & Equivalent \\
\hline Ra-226 & $\begin{array}{l}\text { Decay chain: Rn-222 (Po-218, Pb-214, } \\
\text { Bi-214, Po-214) / Pb-210 / Bi-210 / Po-210 }\end{array}$ & $\begin{array}{l}\text { Decay chain: Ra-226D / } \\
\text { Pb-210D }\end{array}$ & Equivalent \\
\hline $\mathrm{Pb}-210$ & Decay chain: Pb-210 / Bi-210 / Po-210 & $\mathrm{Pb}-210 \mathrm{D}$ & Equivalent \\
\hline $\mathrm{Pu}-240$ & Decay chain: U-236 & No decay chain & Equivalent \\
\hline $\mathrm{U}-236$ & No decay chain & No decay chain & Same \\
\hline Th-232 & $\begin{array}{l}\text { Decay chain: Ra-228 / Ac-228 / Th-228 / } \\
\text { Ra-224 (Rn-220, Po-216) / Pb-212 / Bi-212 }\end{array}$ & $\begin{array}{l}\text { Decay chain: Ra-228D / } \\
\text { Th-228D }\end{array}$ & Equivalent \\
\hline Ra-228 & $\begin{array}{l}\text { Decay chain: Ac-228 / Th-228 / Ra-224 } \\
\text { (Rn-220, Po-216) / Pb-212 / Bi-212 }\end{array}$ & $\begin{array}{l}\text { Decay chain: Ra-228D / } \\
\text { Th-228D }\end{array}$ & Equivalent \\
\hline $\mathrm{U}-232$ & $\begin{array}{l}\text { Decay chain: Th-228 / Ra-224 (Rn-220, } \\
\text { Po-216) / Pb-212 / Bi-212 }\end{array}$ & Decay chain: Th-228D & Equivalent \\
\hline Th-228 & $\begin{array}{l}\text { Decay chain: Ra-224 (Rn-220, Po-216) / } \\
\text { Pb-212 / Bi-212 }\end{array}$ & Th-228D & Equivalent \\
\hline Am-243 & Decay chain: Np-239 / Pu-239 & $\begin{array}{l}\text { Decay chain: Am-243D / } \\
\text { Pu-239 }\end{array}$ & Same \\
\hline Pu-239 & No decay chain & No decay chain & Same \\
\hline U-235 & $\begin{array}{l}\text { Decay chain: Th-231 / Pa-231 / Ac-227 / } \\
\text { Th-227 / Fr-227 / Ra-223 (Rn-129, Po-215, } \\
\text { Pb-211, Bi-211, TL-207) }\end{array}$ & $\begin{array}{l}\text { Decay chain: U-235D / } \\
\text { Pa-231 / Ac-227D }\end{array}$ & Equivalent \\
\hline Pa-231 & $\begin{array}{l}\text { Decay chain: Ac-227 / Th-227 / Fr-227 / } \\
\text { Ra-223 (Rn-129, Po-215, Pb-211, Bi-211, } \\
\text { TL-207) }\end{array}$ & Decay chain: Ac-227D & Equivalent \\
\hline Ac-227 & $\begin{array}{l}\text { Decay chain: Th-227 / Fr-227 / Ra-223 } \\
\text { (Rn-129, Po-215, Pb-211, Bi-211, TL-207) }\end{array}$ & Ac-227D & Equivalent \\
\hline Am-241 & Decay chain: Np-237 / Pa-233 & No decay chain & $\begin{array}{l}\text { Low contribution from } \\
\text { Np-237, due to long } \\
\text { half-life }\end{array}$ \\
\hline Np-237 & Decay chain: Pa-233 & Np-237D & Same \\
\hline
\end{tabular}


Table 7.4-5. Radionuclide Decay Chains Included in the GENII-S Model and ERMYN (Continued)

\begin{tabular}{|l|l|l|l|}
\hline $\begin{array}{c}\text { Primary } \\
\text { Radionuclide }\end{array}$ & \multicolumn{1}{|c|}{ GENII-S $^{\text {a }}$} & \multicolumn{1}{|c|}{ ERMYN $^{\text {b }}$} & \multicolumn{1}{c|}{ Notes $^{-1}$} \\
\hline U-233 & $\begin{array}{l}\text { Decay chain: Th-229 / Ra-225 / Ac-225 } \\
\text { (Fr-221, At-217, Bi-213, Po-213, TI-209, } \\
\text { Pb-209) }\end{array}$ & Decay chain: Th-229D & Equivalent \\
\hline Th-229 & $\begin{array}{l}\text { Decay chain: Ra-225 / Ac-225 (Fr-221, } \\
\text { At-217, Bi-213, Po-213, Tl-209, Pb-209) }\end{array}$ & Th-229D & Equivalent \\
\hline
\end{tabular}

a Decay products in parenthesis are in a secular equilibrium with the parent radionuclides, but the decay chain is considered for those separated by a slash (/). From file RMDLIB.DAT in Appendix A.

b " $D$ " indicates that a short half-life (less than $180 \mathrm{~d}$ ) decay product is considered with the primary radionuclide (Section 6.3.5 and Table 6.4-1).

ERMYN=Environmental Radiation Model for Yucca Mountain, Nevada

\subsubsection{Air Submodel}

The GENII-S model does not include airborne dose contributions from radon and evaporative coolers. Therefore, the importance of these pathways is evaluated to determine if they warrant inclusion in the ERMYN. In addition, a numerical comparison of an ACM for calculating the dose contribution from ${ }^{222} \mathrm{Rn}$ inhalation (ACM 1, Sections 6.3.3 and 7.3.2.2) is documented in this section.

\subsubsection{Radon Pathway}

An evaluation (Table 7.4-6) estimates the contribution of ${ }^{222} \mathrm{Rn}$ to the total dose from ${ }^{226} \mathrm{Ra}$. The information for this evaluation is from the verification calculations (Section 6.10, Tables 6.10-3 and 6.10-6). For the groundwater and volcanic ash scenarios, 89 percent and 50 percent, respectively, of the ${ }^{226} \mathrm{Ra}$ dose is from ${ }^{222} \mathrm{Rn}$ inhalation (Table 7.4-6). The ERMYN includes this pathway because the dose contribution from ${ }^{222} \mathrm{Rn}$ is high.

Table 7.4-6. Radon Contribution to the Final ${ }^{226}$ Ra Dose

\begin{tabular}{|l|c|c|c|}
\hline \multicolumn{1}{|c|}{ Scenario } & $\begin{array}{c}\text { Total }^{226} \text { Ra Including } \\
\text { (Sv/yr) }\end{array}$ & $\begin{array}{c}{ }^{222} \mathbf{R n} \\
{ }^{222} \mathbf{R n} \text { Contribution } \\
\text { (Sv/yr) }\end{array}$ & Ratio (Rn/Ra) \\
\hline Groundwater $^{\text {a }}$ & $2.02 \mathrm{E}-05$ & $1.80 \mathrm{E}-05$ & $89.1 \%$ \\
\hline${\text { Volcanic } \text { Ash }^{\mathrm{b}}}^{\mathrm{b}}$ & $4.30 \mathrm{E}-08^{\mathrm{c}}$ & $2.17 \mathrm{E}-08$ & $50.5 \%$ \\
\hline
\end{tabular}

${ }^{a}$ Data from Table 6.10-3.

b Data from Table 6.10-6.

c This value is for all pathways except inhalation, which has two components $\left(7.06 \times 10^{-9}\right.$ Sv/year for the nominal condition, and $3.74 \times 10^{-9} \mathrm{~Sv} /$ year for the post-volcanic condition) under the volcanic ash scenario (Table 6.10-6).

The methods for assessing dose from ${ }^{222} \mathrm{Rn}$ inhalation are based on a concentration ratio, or release factor, of the average concentration of ${ }^{222} \mathrm{Rn}$ in the air $\left(\mathrm{Bq} / \mathrm{m}^{3}\right)$ and the average concentration of ${ }^{226} \mathrm{Ra}$ in the soil $\left(\mathrm{Bq} / \mathrm{kg}\right.$ for groundwater scenario, Section $6.4 .2 .3 ; \mathrm{Bq} / \mathrm{m}^{2}$ for volcanic ash scenario, Section 6.5.2.2). The ERMYN method is different from the RESRAD radon diffusion method ( $\mathrm{Yu}$ et al. 2001 [DIRS 159465], Appendix C; see also Section 7.3.2.2). The RESRAD method is considered an ACM (Section 6.3.3). According to the model validation approach, a numerical comparison between these two methods is required. The detailed analytical solution for radon diffusion is discussed in Appendix B. The general differential equation and the boundary conditions given in RESRAD are solved using the ERMYN input 
parameter values or RESRAD default parameter values if the parameters are not included in the ERMYN. The ${ }^{222} \mathrm{Rn}$ release factor for volumetric ${ }^{226} \mathrm{Ra}$ in the soil is $0.19\left(\mathrm{~Bq} / \mathrm{m}^{3}\right) /(\mathrm{Bq} / \mathrm{kg})$ (Table B-1). This value is similar to the ERMYN value, which is $0.25\left(\mathrm{~Bq} / \mathrm{m}^{3}\right) /(\mathrm{Bq} / \mathrm{kg})$ for the groundwater scenario (Section 6.4.2.3).

To compare ${ }^{222} \mathrm{Rn}$ release factors for the volcanic scenario, the amount of ${ }^{222} \mathrm{Rn}$ released into air from a thin contaminated layer on the ground surface must be calculated. This calculation is documented in Appendix B, and the results (Table 7.4-7) are taken from Table B-2.

Compared with the selected radon release factor for the volcanic ash scenario, $0.0006\left(\mathrm{~Bq} / \mathrm{m}^{3}\right) /\left(\mathrm{Bq} / \mathrm{m}^{2}\right)$ (Section 6.5.2.2), the calculated result for a thin layer of contaminated soil, $0.00013\left(\mathrm{~Bq} / \mathrm{m}^{3}\right) /\left(\mathrm{Bq} / \mathrm{m}^{2}\right)$, is 5 times lower than the selected value. The difference is due to the approximation that all radon released from the soil goes into the air (Approximation 15), which considers the radon emanation coefficient $(\varepsilon)$ to equal 1 . The default coefficient used in the RESRAD calculation is 0.25 (Table B-1). If a coefficient of 1 is used, the calculated radon release factor is $0.0005\left(\mathrm{~Bq} / \mathrm{m}^{3}\right) /\left(\mathrm{Bq} / \mathrm{m}^{2}\right)$, which is similar to the value used in the ERMYN.

In conclusion, the selected radon release factors for the groundwater and volcanic ash scenarios, $0.25\left(\mathrm{~Bq} / \mathrm{m}^{3}\right) /(\mathrm{Bq} / \mathrm{kg})$ and $0.0006\left(\mathrm{~Bq} / \mathrm{m}^{3}\right) /\left(\mathrm{Bq} / \mathrm{m}^{2}\right)$, respectively, are within a factor of 2 of the RESRAD method for the two scenarios, $0.19\left(\mathrm{~Bq} / \mathrm{m}^{3}\right) /(\mathrm{Bq} / \mathrm{kg})$ and $0.0005\left(\mathrm{~Bq} / \mathrm{m}^{3}\right) /\left(\mathrm{Bq} / \mathrm{m}^{2}\right)$, respectively. Thus, the radon pathway is important and must be included in the ERMYN. The selected radon model is simple and valid, and ACM 1 (Section 6.3.3) does not need further consideration for comparable input values.

Table 7.4-7. Radon Release Factors Due to Radium Contaminated Soil

\begin{tabular}{|c|c|c|c|c|}
\hline $\begin{array}{c}\text { Depth of } \\
\text { Contaminant }(\mathbf{m})\end{array}$ & $\begin{array}{c}\text { Source } \\
\text { Exponential Term }\end{array}$ & $\begin{array}{c}\text { Rn-222 Release } \\
\text { Factor } \\
\left(\mathrm{Bq} / \mathbf{m}^{\mathbf{3}}\right) /(\mathrm{Bq} / \mathbf{k g})\end{array}$ & $\begin{array}{c}\text { Surface Soil } \\
\text { Density } \\
\left(\mathbf{k g} / \mathbf{m}^{\mathbf{2}}\right)\end{array}$ & $\begin{array}{c}\text { Rn-222 Release } \\
\text { Factor } \\
\left(\mathbf{B q} / \mathbf{m}^{\mathbf{3}}\right) /\left(\mathbf{B q} / \mathbf{m}^{\mathbf{2}}\right)\end{array}$ \\
\hline 0.003 & 0.0031 & $5.86 \mathrm{E}-04$ & 4.5 & 0.00013 \\
\hline 0.01 & 0.0102 & $1.95 \mathrm{E}-03$ & 15 & 0.00013 \\
\hline 0.02 & 0.0205 & $3.91 \mathrm{E}-03$ & 30 & 0.00013 \\
\hline 0.05 & 0.0512 & $9.76 \mathrm{E}-03$ & 75 & 0.00013 \\
\hline 0.1 & 0.1021 & $1.95 \mathrm{E}-02$ & 300 & 0.00013 \\
\hline 0.2 & 0.2020 & $3.85 \mathrm{E}-02$ & 750 & 0.00013 \\
\hline 0.5 & 0.4716 & $9.00 \mathrm{E}-02$ & 1500 & 0.00012 \\
\hline 1 & 0.7716 & $1.47 \mathrm{E}-01$ & 3000 & 0.00010 \\
\hline 2 & 0.9673 & $1.85 \mathrm{E}-01$ & 7500 & 0.00006 \\
\hline 5 & 0.9999 & $1.91 \mathrm{E}-01$ & 15000 & 0.00003 \\
\hline 10 & 1.0000 & $1.91 \mathrm{E}-01$ & & 0.00001 \\
\hline
\end{tabular}

NOTE: This table is taken from Appendix B, Table B-2.

As mentioned in Section 6.4.2.2, ${ }^{222} \mathrm{Rn}$ released from evaporative coolers is not considered in the air submodel because the amount of radon released from the water would be relatively low compared to that released from radium at equilibrium concentrations in soils. The ${ }^{222} \mathrm{Rn}$ in the groundwater used in evaporative coolers should be in equilibrium with ${ }^{226} \mathrm{Ra}$ from the repository. Using typical values given above, the ratio of the ${ }^{222} \mathrm{Rn}$ concentration in the air to the ${ }^{226} \mathrm{Ra}$ concentration in the water, which would be the same as the ${ }^{222} \mathrm{Rn}$ concentration in water at secular equilibrium, is estimated at a level of $10^{-6}$ (for the evaporation rate of $20 \mathrm{~L} / \mathrm{hr}$ and the air 
flow rate of $8,000 \mathrm{~m}^{3} / \mathrm{hr}$ ). This ratio is lower than a typical value of $10^{-4}$ for radon dissolved in water entering indoor air through de-emanation (UNSCEAR 2000 [DIRS 158644], p. 102).

As mentioned in Section 6.4.2.3, the justification for excluding the radon contributions from evaporative coolers and household water use from the ERMYN are provided here. Radon concentrations in indoor air resulting from water use can be estimated based on the rates of water use and house ventilation. As mentioned in Section 6.4.2.2, a typical radon release ratio between the concentration of ${ }^{222} \mathrm{Rn}$ in the air and the concentration of ${ }^{226} \mathrm{Ra}$ in the water is about $1 \times 10^{-4}$ (Yu et al. 2001 [DIRS 159465], p. C-13). This value can be verified using

$$
\frac{C a_{g, R n-222}}{C w_{R a-226}}=\frac{f_{w a} U_{w}}{\left(\lambda_{R n-222}+v\right) V}
$$

where

$$
\begin{array}{ll}
f_{w a} & =\text { transfer efficiency of radon from water to air (dimensionless) } \\
U_{w} & =\text { household water use rate }(\mathrm{L} / \mathrm{hr}) \\
\lambda_{R n-222} & =\text { decay constant of }{ }^{222} \mathrm{Rn}(0.0076 / \mathrm{hr}) \\
v & =\text { house ventilation rate }(/ \mathrm{hr}) \\
V & =\text { volume of the house }(\mathrm{L})
\end{array}
$$

Using typical values suggested in RESRAD, $f_{w a}=0.55, U_{w}=9.5 \mathrm{~L} / \mathrm{hr}$ for each individual (assuming 4 people in a household), $V=75,000 \mathrm{~L}, v=1 / \mathrm{hr}$ (when evaporative coolers are not in operation), and $\lambda_{R n-222}=0.0076 / \mathrm{hr}$ (Yu et al. 2001 [DIRS 159465], p. C-15), the concentration ratio of ${ }^{222} \mathrm{Rn}$ in the air to ${ }^{226} \mathrm{Ra}$ in the water can be calculated as $2.8 \times 10^{-4}$. Equation 7.4.3-1 can also apply to a room, such as a bathroom, where water use would be high. Using values for a shower, $f_{w a}=0.7, U_{w}=300 \mathrm{~L} / \mathrm{hr}, v \times V=0.13 \mathrm{~m}^{3} / \mathrm{min}=7,800 \mathrm{~L} / \mathrm{hr}$ (McKone and Bogen 1992 [DIRS 160440], p. 93; McKone and Daniels 1991 [DIRS 160441], p. 50), the ratio in Equation 7.4.3-1 for a shower would be 0.027, which is two orders of magnitude higher than that for the house. However, a typical shower lasts for only about $10 \mathrm{~min}$, while a person typically spends more than 8 hrs per day in the house. Therefore, a 10-min shower may cause only twice as much radon exposure as would $8 \mathrm{hrs}$ of home occupancy due to radon released from domestic use of water.

A typical outdoor radon concentration over soil contaminated by the long-term use of irrigation water can be estimated using equilibrium concentrations of ${ }^{226} \mathrm{Ra}$ in the soil (Equations 6.4.1-4 and 6.4.1-5) and a ${ }^{222} \mathrm{Rn}$ release factor (Equation 6.4.2-4). Using typical values (IR=1 m/year, $\lambda_{\text {eff, } R a-226}=0.001 /$ year, $\rho_{s}=250 \mathrm{~kg} / \mathrm{m}^{2}$, and $\left.f_{m, R n-222}=0.25\left(\mathrm{~Bq} / \mathrm{m}^{3}\right) /(\mathrm{Bq} / \mathrm{kg})\right)$, the concentration ratio of ${ }^{222} \mathrm{Rn}$ in the air to ${ }^{226} \mathrm{Ra}$ in the contaminated irrigation water would be about 1 . This ratio is higher than the ratios for radon dissolved in water entering indoor air $\left(1 \times 10^{-4}\right.$ to $\left.1 \times 10^{-3}\right)$ and for radon released by evaporative coolers $\left(10^{-6}\right)$, which are discussed in Section 6.4.2.2. Therefore, it is reasonable to ignore the indoor radon contributions from the use of contaminated household water and evaporative coolers because the radon exposure from contaminated soil is several orders of magnitude higher. 


\subsubsection{Evaporative Cooler}

The ERMYN includes radionuclide concentrations in aerosols from evaporative coolers using a submodel based on the mechanical operation of an evaporative cooler (Section 6.4.2.2). To validate this submodel, it is compared with an alternative method based on the difference between absolute humidity values in indoor and outdoor air caused by evaporative coolers (Table 7.3-6).

The results from the two methods differ by a factor of 2 (Table 7.4-8), which meets the validation criteria for numerical similarity, and the selected method is valid. There is less uncertainty in defining the operating parameters of evaporative coolers, such as air flow rate and water evaporation rate, than required to quantify temporal variation in absolute humidity values. This evaluation indicates that ACM 2 (Section 6.3.3) does not need further consideration, and the chosen method is valid.

Table 7.4-8. Comparison of Evaporative Cooler Model

\begin{tabular}{|c|c|c|c|c|c|c|}
\hline \multirow{3}{*}{$\begin{array}{l}\text { Equation } \\
\text { Parameter }\end{array}$} & \multicolumn{3}{|c|}{ ERMYN } & \multicolumn{3}{|c|}{ Alternative } \\
\hline & \multicolumn{3}{|c|}{$C a_{e}=f_{\text {evap }} \frac{M_{\text {water }}}{F_{\text {air }}} C w$} & \multicolumn{3}{|c|}{$C a=\frac{f_{\text {evap }}\left(D_{\text {in }}-D_{\text {out }}\right) C w}{\rho_{w}}$} \\
\hline & Notation & Value & Units & Notation & Value & Units \\
\hline Release fraction & $f_{\text {evap }}$ & 0.5 & - & $f_{\text {evap }}$ & 0.5 & - \\
\hline Concentration in water & $\mathrm{Cw}$ & 1 & $\mathrm{~Bq} / \mathrm{m}^{3}$ & Cw & 1 & $\mathrm{~Bq} / \mathrm{m}^{3}$ \\
\hline Water evaporation rate & $M_{\text {water }}$ & 17 & $\mathrm{~L} / \mathrm{hr}$ & - & - & - \\
\hline Air flow rate & $F_{\text {air }}$ & 8,300 & $\mathrm{~m}^{3} / \mathrm{hr}$ & - & - & - \\
\hline Absolute humidity (outdoors) & - & - & - & $D_{\text {out }}$ & 4.8 & $\mathrm{~g} / \mathrm{m}^{3}$ \\
\hline Absolute humidity (indoors) & - & - & - & $D_{\text {in }}$ & 8.7 & $\mathrm{~g} / \mathrm{m}^{3}$ \\
\hline Water density & - & - & - & $\rho_{w}$ & 1,000 & $\mathrm{~kg} / \mathrm{m}^{3}$ \\
\hline Concentration in air & $C a_{e}$ & $1.0 \mathrm{E}-6$ & $\mathrm{~Bq} / \mathrm{m}^{3}$ & $C a_{e}$ & $2.0 \mathrm{E}-6$ & $\mathrm{~Bq} / \mathrm{m}^{3}$ \\
\hline
\end{tabular}

NOTE: Outdoor absolute humidity is representative of conditions at Yucca Mountain Weather Station \#9 (Gate-510; CRWMS M\&O 1997 [DIRS 100117] p. A-11), and indoor absolute humidity is based on $24^{\circ} \mathrm{C}$ and 40 percent relative humidity. The calculation of absolute humidity from relative humidity and temperature is documented in Appendix C. All other values from the "mean, mode, average" column in Table 6.6-3.

ERMYN=Environmental Radiation Model for Yucca Mountain, Nevada

\subsubsection{Plant Uptake Submodel}

Three numerical comparisons (direct deposition of irrigation water on crop surfaces, crop interception fraction for irrigation water, and direct deposition of resuspended soil on crop surfaces) are required to validate the plant submodel (Section 7.3.3), which are described in this section.

\subsubsection{Direct Deposition of Irrigation Water on Crop Surfaces}

The methods in the ERMYN (Section 6.4.3.2) and BIOMASS ERB2A (Section 7.3.3.2) models for calculating concentrations from the direct deposition of irrigation water on crop surfaces are compared (Table 7.4-9). The results indicate that the two methods could differ by a factor of two. Some input parameters in the BIOMASS ERB2A model (e.g., absorption fraction and 
interval time) are not commonly used in the validation models, but they have a large influence on the results of the model. For example, the absorption fraction, which is 0.5 in BIOMASS ERB2A, is a high value for leaf water absorption. If a lower, perhaps more realistic, value is used (e.g., 0.3), the differences between the two models would be even smaller. The fraction of radionuclides that transfer from irrigation water to crops can be estimated using data in Table 7.4-9. The amount of radioactive material in crops per unit area is small, about $0.03 \mathrm{~Bq} / \mathrm{m}^{2}\left(0.015 \mathrm{~Bq} / \mathrm{kg} \times 2 \mathrm{~kg} / \mathrm{m}^{2}\right)$, while the total amount of radioactive material in irrigation water is larger, $0.45 \mathrm{~Bq} / \mathrm{m}^{2}\left(1 \mathrm{~Bq} / \mathrm{m}^{3} \times 0.006 \mathrm{~m} / \mathrm{d} \times 75 \mathrm{~d}\right)$.

In conclusion, the two methods are numerically similar. This evaluation shows that ACM 3 (Section 6.3.3) does not need further consideration, and the chosen method is justified.

Table 7.4-9. Comparison of Direct Deposition of Irrigation Water on Crop Surfaces

\begin{tabular}{|c|c|c|c|c|c|c|}
\hline Model & \multicolumn{3}{|c|}{ ERMYN $^{a}$} & \multicolumn{3}{|c|}{ BIOMASS ERB2A ${ }^{b}$} \\
\hline Equation & $C p_{w}=\frac{C v}{n}$ & $\frac{f_{o} R w}{Y}$ & $\left.-e^{-\lambda_{w} t_{g}}\right)$ & $\begin{array}{l}C_{c r o p, w}= \\
{\left[\left(1-F_{a b s}\right)\right.}\end{array}$ & $\begin{array}{l}\frac{V_{i r r} C_{w}}{Y_{c r o p}} \times \\
{ }^{W T} F_{p 3}+F\end{array}$ & $F_{p 2} F_{t r a n s}$ \\
\hline Parameter & Notation & Value & Units & Notation & Value & Units \\
\hline Yearly Irrigation Rate & - & - & - & $V_{\text {irr }}$ & 0.45 & $\mathrm{~m} / \mathrm{yr}$ \\
\hline Daily irrigation rate & $I R D$ & 0.006 & $\mathrm{~m} / \mathrm{d}$ & - & - & - \\
\hline Concentration & $C_{w}$ & 1 & $\mathrm{~Bq} / \mathrm{m}^{3}$ & $\mathrm{C}_{w}$ & 1 & $\mathrm{~Bq} / \mathrm{m}^{3}$ \\
\hline Absorption fraction & - & - & - & $F_{a b s}$ & 0.5 & - \\
\hline Internal wash left & - & - & - & $F_{p 2}$ & 1 & - \\
\hline External wash left & - & - & - & $F_{p 3}$ & 0.1 & - \\
\hline Translocation & $T$ & 1 & - & $F_{\text {trans }}$ & 1 & - \\
\hline Overhead fraction & $f_{o}$ & 1 & - & - & - & - \\
\hline Interception & $R_{w}$ & 0.25 & - & $I_{\text {crop }}$ & 0.25 & - \\
\hline Weathering & $\lambda_{w}$ & 0.05 & $/ d$ & W & 18 & /yr \\
\hline Wet yield & $Y$ & 2 & $\mathrm{~kg} / \mathrm{m}^{2}$ & $Y_{\text {crop }}$ & 2 & $\mathrm{~kg} / \mathrm{m}^{2} / \mathrm{yr}$ \\
\hline Growing time & $\operatorname{tg}$ & 75 & $\mathrm{~d}$ & - & - & - \\
\hline Interval time & - & - & - & $T$ & 0.02 & $\mathrm{yr}$ \\
\hline Fraction - weather & $f_{w}$ & 0.976 & - & - & - & - \\
\hline Fraction - external & - & - & - & $F_{\text {ext }}$ & 0.035 & - \\
\hline Fraction - internal & - & - & - & $F_{\text {int }}$ & 0.5 & - \\
\hline Leaf water deposition & $C p_{w}$ & $1.5 \mathrm{E}-2$ & $\mathrm{~Bq} / \mathrm{kg}$ & $C_{\text {crop,w }}$ & $3.0 \mathrm{E}-2$ & $\mathrm{~Bq} / \mathrm{kg}$ \\
\hline
\end{tabular}

NOTE: To simplify the equations, the radionuclide and crop-type indices (subscripts) are not shown.

${ }^{a}$ Input values from Table 6.6-3 if available; reasonable values used when there are multiple values per crop type.

b Input values are the same as those for the ERMYN, or default values from BIOMASS ERB2A (BIOMASS 2000, [DIRS 154522], Section 7.4).

ERMYN=Environmental Radiation Model for Yucca Mountain, Nevada

\subsubsection{Crop Interception Fraction for Irrigation Water}

The crop interception fraction for irrigation is calculated in the ERMYN using an empirical equation (Equation 6.4.3-5), while all five validation models use a fixed value. Therefore, the models are compared to determine if the results are similar. The calculated results (mean values) for five crop types (Table 7.4-10) range from 0.24 to 0.51 , with higher values for crops with more leaf surface (i.e., larger surface area). The results for some of the values differ by more than a factor of two, and an evaluation of the differences and justification for selecting the 
ERMYN method is presented in Section 7.3.3.2. It is concluded that the selected method is justified, and this part of the ERMYN plant submodel is validated.

Table 7.4-10. Calculated Interception Fraction for Irrigation Water

\begin{tabular}{|l|c|c|}
\hline \multicolumn{1}{|c|}{ Interception Fraction } & Notation & Calculated Mean Value \\
\hline Leafy vegetables & $R w_{1}$ & 0.236 \\
\hline Other vegetables & $R w_{2}$ & 0.332 \\
\hline Fruit & $R W_{3}$ & 0.394 \\
\hline Grain & $R w_{4}$ & 0.514 \\
\hline Forage & $R w_{5}$ & 0.282 \\
\hline
\end{tabular}

NOTE: Calculated mean values from Table 6.10-1.

\subsubsection{Direct Deposition of Resuspended Soil on Crop Surfaces}

The ERMYN (Section 6.4.3.3) and BIOMASS ERB2A (Section 7.3.3.3) models use different methods for calculating crop contamination by direct deposition of resuspended soil on crop surfaces. The BIOMASS ERB2A method is an ACM (Section 6.3.3), and, therefore, the two methods are compared. The equations presented here are simplified by eliminating radionuclide and crop-type indexes. Input parameter values are taken from Table 6.6-3, except that some default values for unique parameters are taken from the BIOMASS ERB2A model (BIOMASS 2000 [DIRS 154522], Section 7.4). Because the method used in the ERMYN is sensitive to crop type due to translocation factor values, leafy vegetables and other vegetables are used to calculate radionuclide concentrations in crops from dust deposition (Table 7.4-11). The results of this comparison are evaluated in Section 7.3.3.3. The ERMYN overestimates radionuclide concentrations in leafy vegetables relative to the BIOMASS ERB2A method, but the models are numerically similar for the applicable comparison of leafy vegetables to forage, and, therefore, the method selected for the ERMYN plant submodel is justified. This evaluation shows that ACM 4 (Section 6.3.3) does not need further consideration.

Table 7.4-11. Comparison of Direct Deposition of Resuspended Soil on Crop Surfaces

\begin{tabular}{|c|c|c|c|c|c|c|c|}
\hline Model & \multicolumn{4}{|c|}{ ERMYN $^{a}$} & \multicolumn{3}{|c|}{ BIOMASS ERB2A $^{b}$} \\
\hline Equation & $C p_{d}=-$ & $\frac{s_{m} S V_{d} R a}{\lambda_{w} Y}$ & $-\left(1-e^{-\lambda_{w}}\right.$ & & $C_{c r o p, d}=$ & $\frac{F_{p 1} S_{\text {crop }} C_{s}}{\left(1-\theta_{t}\right) \rho}$ & \\
\hline Parameter & Notation & $\begin{array}{l}\text { Value for } \\
\text { leafy veg. }\end{array}$ & $\begin{array}{l}\text { Value for } \\
\text { other veg }\end{array}$ & Units & Notation & Value & Units \\
\hline $\begin{array}{l}\text { Soil volume } \\
\text { concentration }\end{array}$ & - & - & - & - & Cs & 1,500 & $\mathrm{~Bq} / \mathrm{m}^{3}$ \\
\hline Soil grain density & - & - & - & - & $\rho$ & 2,650 & $\mathrm{~kg} / \mathrm{m}^{3}$ \\
\hline Total porosity & - & - & - & - & $\theta_{\mathrm{t}}$ & $0.434^{c}$ & - \\
\hline $\begin{array}{l}\text { Soil mass } \\
\text { concentration }\end{array}$ & $C s_{m}$ & 1 & 1 & $\mathrm{~Bq} / \mathrm{kg}$ & $C s_{m}$ & 1 & $\mathrm{~Bq} / \mathrm{kg}$ \\
\hline Mass loading & $S$ & $1.2 \mathrm{E}-7$ & $1.2 \mathrm{E}-7$ & $\mathrm{~kg} / \mathrm{m}^{3}$ & - & - & - \\
\hline Deposit velocity & $V_{d}$ & 0.008 & 0.008 & $\mathrm{~m} / \mathrm{sec}$ & - & - & - \\
\hline External soil left & - & - & - & - & $F_{p 1}$ & 0.1 & - \\
\hline Soil contamination & - & - & - & - & $S_{\text {crop }}$ & $\begin{array}{ll}2.0 \mathrm{E}-4 & \text { (all others) } \\
2.0 \mathrm{E}-3 & \text { (forage) }\end{array}$ & $\mathrm{kg} / \mathrm{kg}$ \\
\hline
\end{tabular}


Table 7.4-11. Comparison of Direct Deposition of Resuspended Soil on Crop Surfaces (Continued)

\begin{tabular}{|c|c|c|c|c|c|c|c|}
\hline Model & \multicolumn{4}{|c|}{ ERMYN $^{a}$} & \multicolumn{3}{|c|}{ BIOMASS ERB2A ${ }^{b}$} \\
\hline Equation & $C p_{d}=\frac{C}{C}$ & $\frac{{ }_{m} S V_{d} R a}{\lambda_{w} Y}$ & $\left(1-e^{-\lambda_{w} t_{g}}\right.$ & & $C_{c r o p, d}=$ & $\frac{{ }_{p 1} S_{c r o p} C_{s}}{\left(1-\theta_{t}\right) \rho}$ & \\
\hline Parameter & Notation & $\begin{array}{l}\text { Value for } \\
\text { leafy veg. }\end{array}$ & $\begin{array}{l}\text { Value for } \\
\text { other veg. }\end{array}$ & Units & Notation & Value & Units \\
\hline Translocation & $T$ & 1 & 0.1 & - & - & - & - \\
\hline Weathering & $\lambda_{w}$ & 0.05 & 0.05 & $/ \mathrm{d}$ & - & - & - \\
\hline Wet yield & $Y$ & 3.3 & 4.13 & $\mathrm{~kg} / \mathrm{m}^{2}$ & - & - & - \\
\hline Growing time & $t_{g}$ & 75 & 80 & $d$ & - & - & - \\
\hline Air interception ${ }^{\mathrm{d}}$ & $R a$ & 0.456 & 0.787 & - & - & - & - \\
\hline Dust deposition & $C p_{d}$ & $2.2 \mathrm{E}-4$ & $3.1 \mathrm{E}-5$ & $\mathrm{~Bq} / \mathrm{kg}$ & $C_{\text {crop }, d}$ & $\begin{array}{l}2.0 \mathrm{E}-5 \text { (all others) } \\
2.0 \mathrm{E}-4 \text { (forage) }\end{array}$ & $\mathrm{Bq} / \mathrm{kg}$ \\
\hline
\end{tabular}

a Input values from the "mean, mode, average" column in Table 6.6-3 if available.

${ }^{b}$ Input values are the same as those for the ERMYN or default values from the BIOMASS ERB2A report (BIOMASS 2000, [DIRS 154522], Section 7.4).

c Value from Table 6.6-3 as the ERMYN has this parameter, although it is not used in this calculation.

'Values from Table 6.10-1, "Dust interception fraction."

ERMYN=Environmental Radiation Model for Yucca Mountain, Nevada

\subsubsection{Animal Submodel}

Three mechanisms for the contamination of animal products are included in the ERMYN: animal consumption of contaminated feed, water, and soil (Sections 6.4.4 and 6.5.4). Only two mechanisms, feed and drinking water, are included in the GENII-S model. The BIOMASS ERB2A model includes animal dust inhalation (Section 7.3.4) as an additional mechanism. Animal soil ingestion and animal dust inhalation are identified as ACMs (Section 6.3.3), and the importance of these alternative pathways is evaluated in this section.

This evaluation uses "meat" as an example animal product and ${ }^{239} \mathrm{Pu}$ as a test radionuclide. The equations are shown in Sections 6.4.3 and 6.4.4, and results from the ERMYN are taken from Table 6.10-1. The BIOMASS ERB2A model is described in Section 7.3.4, and the equations for calculating radionuclide concentrations in feed and animal products are shown in Table 7.4-12. Because BIOMASS ERB2A uses one irrigation rate for all crops and for soil accumulation, a direct comparison is not meaningful. Thus, radionuclide concentrations in the soil are from the results of the ERMYN for surface soil submodel, soil grain density is from the default values in the BIOMASS model, and total porosity is calculated to match the soil bulk density in the ERMYN. The retardation coefficient is calculated from the partition coefficient and other parameters in the ERMYN. All other parameter values are from the "mean, mode, average" column in Table 6.6-3, if possible, or default values from BIOMASS ERB2A (BIOMASS 2000 [DIRS 154522], Section 7.4). The calculations (Table 7.4-12) are performed in an Excel spreadsheet, ERMYN validation.xls, which is listed in Appendix A.

The two models are equivalent, as the calculated concentrations in meat differ by only a few percent (Table 7.4-12). The ingestion of soil accounts for about 52 to 60 percent of the total concentration in meat for both models (Table 7.4-12), and, therefore, animal soil ingestion is an important mechanism that must be included in the animal submodel. The inclusion of animal soil ingestion is an improvement in the ERMYN compared with the previous biosphere model. In contrast, animal inhalation of resuspended soil contributes little to dose (about five orders of 
magnitude less than the total concentration in meat calculated with the BIOMASS ERB2A model; Table 7.4-12) and is not necessary. This evaluation shows that ACM 5 (Section 6.3.3) does not need further consideration.

Table 7.4-12. Numerical Calculation of Animal Uptake Submodel

\begin{tabular}{|c|c|c|c|c|c|c|}
\hline \multirow{2}{*}{$\begin{array}{c}\text { Model } \\
\text { Equation }\end{array}$} & \multicolumn{3}{|c|}{ ERMYN $^{a}$} & \multicolumn{3}{|c|}{ BIOMASS ERB2A ${ }^{b}$} \\
\hline & \multicolumn{3}{|c|}{$\begin{array}{l}\text { Equations shown in } \\
\text { Sections } 6.4 .3 \text { and } 6.4 .4 \text {, } \\
\text { calculations shown in } \\
\text { Table } 6.10-1 .\end{array}$} & \multicolumn{3}{|c|}{$\begin{aligned} & C_{\text {fodd }}= \frac{\left(C F_{\text {past }}+S_{\text {past }}\right) C_{s}}{\left(1-\theta_{t}\right) \rho} \\
&+\frac{I_{\text {past }} V_{\text {irr }} C_{w}}{S B_{\text {past }} W_{\text {past }}+365 I N G_{\text {fodd }} S D} \\
& C_{\text {prod }}= T F_{\text {proding }}\left(C_{\text {fodd }} I N G_{\text {fodd }}+C_{w} I N G_{w a}\right)+ \\
& \frac{C_{s} I N G_{s a} T F_{\text {proding }}}{\left(1-\theta_{t}\right) \rho+\theta \rho_{w}}+\left(B R_{a} O_{a n} C_{\text {air }}\right) T F_{\text {prodin }}\end{aligned}$} \\
\hline Parameter & Notation & Value & Units & Notation & Value & Units \\
\hline Concentration in soil mass & $C s_{m}$ & 1.66 & $\mathrm{~Bq} / \mathrm{kg}$ & $C s_{m}$ & 1.66 & $\mathrm{~Bq} / \mathrm{kg}$ \\
\hline Concentration in soil volume & - & - & - & Cs & 2489 & $\mathrm{pCi} / \mathrm{m}^{3}$ \\
\hline Retardation coefficient & - & - & - & $R$ & 7827 & - \\
\hline Grain density & - & - & - & $\rho$ & 2.650 & $\mathrm{~kg} / \mathrm{m}^{3}$ \\
\hline Total porosity & - & - & - & $\theta_{t}$ & 0.434 & - \\
\hline Mass loading for crops & $S$ & $1.20 \mathrm{E}-07$ & $\mathrm{~kg} / \mathrm{m}^{3}$ & Dusts $_{s}$ & 1.20E-07 & $\mathrm{kg} / \mathrm{m}^{3}$ \\
\hline Concentration in air for crop & $\mathrm{Ca}$ & $1.99 \mathrm{E}-07$ & $\mathrm{~Bq} / \mathrm{m}^{3}$ & $C_{\text {air }}$ & $1.99 \mathrm{E}-07$ & $\mathrm{~Bq} / \mathrm{m}^{3}$ \\
\hline Soil-to-plant transfer factor & $F_{S \rightarrow 05}$ & $1.0 \mathrm{E}-03$ & (dry) & $C F_{\text {past }}$ & $2.2 \mathrm{E}-04^{\mathrm{c}}$ & (wet) \\
\hline Soil contamination on pasture & - & - & - & $S_{\text {past }}$ & $2.0 \mathrm{E}-03$ & $\mathrm{~kg} / \mathrm{kg}$ \\
\hline Daily irrigation rate & $I R D_{5}$ & 6.54 & $\mathrm{~mm} / \mathrm{d}$ & $V_{\text {irr }}$ & 2.39 & $\mathrm{~m} / \mathrm{yr}$ \\
\hline Interception fraction for irrigation & $R W_{5}$ & 0.259 & - & $I_{\text {past }}$ & 0.259 & - \\
\hline Crop wet yield & $Y_{5}$ & 2.14 & $\mathrm{~kg} / \mathrm{m}^{2}$ & $S B_{\text {past }}$ & 2.14 & $\mathrm{~kg} / \mathrm{m}^{2}$ \\
\hline Weathering half-live or constant & $L w$ & 14 & $\mathrm{~d}$ & W & 18.1 & $/ \mathrm{yr}$ \\
\hline Animal consumption rate of feed & $Q f, 1$ & 75 & $\mathrm{~kg} / \mathrm{d}$ & $I N G_{\text {fodd }}$ & 48.5 & $\mathrm{~kg} / \mathrm{d}$ \\
\hline Number of animals in the area & - & - & - & $S D$ & $2.0 \mathrm{E}-04$ & - \\
\hline Concentration due to root uptake & $C p, r, 5$ & 3.65E-04 & $\mathrm{Bq} / \mathrm{kg}$ & Codd,root & 3.65E-04 & $\mathrm{Bq} / \mathrm{kg}$ \\
\hline $\begin{array}{l}\text { Concentration due to water } \\
\text { deposition }\end{array}$ & $C p, w, 5$ & $1.40 \mathrm{E}-02$ & $\mathrm{~Bq} / \mathrm{kg}$ & $C_{\text {fodd, iri }}$ & $1.59 \mathrm{E}-02$ & $\mathrm{~Bq} / \mathrm{kg}$ \\
\hline $\begin{array}{l}\text { Concentration due to dust } \\
\text { deposition }\end{array}$ & $C p, d, 5$ & $9.52 \mathrm{E}-04$ & $\mathrm{~Bq} / \mathrm{kg}$ & $C_{\text {fodd, dust }}$ & $3.32 \mathrm{E}-03$ & $\mathrm{~Bq} / \mathrm{kg}$ \\
\hline Concentration in crops & $C p_{5}$ & $1.53 \mathrm{E}-02$ & $\mathrm{~Bq} / \mathrm{kg}$ & $C_{\text {fodd }}$ & $1.96 \mathrm{E}-02$ & $\mathrm{~Bq} / \mathrm{kg}$ \\
\hline Animal consumption rate of feed & $Q f, 1$ & 48.5 & $\mathrm{~kg} / \mathrm{d}$ & $I N G_{\text {fodd }}$ & 48.5 & $\mathrm{~kg} / \mathrm{d}$ \\
\hline Animal consumption rate of water & $Q W, 1$ & 60 & L/d & $I N G_{w a}$ & 0.06 & $\mathrm{~m}^{3} / \mathrm{d}$ \\
\hline Animal consumption rate of soil & $Q s, 1$ & 0.7 & $\mathrm{~kg} / \mathrm{d}$ & $I N G_{\text {soil }}$ & 0.7 & $\mathrm{~kg} / \mathrm{d}$ \\
\hline $\begin{array}{l}\text { Animal product transfer } \\
\text { coefficient }\end{array}$ & $F m_{1}$ & $1.30 \mathrm{E}-05$ & $\mathrm{~d} / \mathrm{kg}$ & $T F_{\text {proding }}$ & $1.30 \mathrm{E}-05$ & $\mathrm{~d} / \mathrm{kg}$ \\
\hline Water density & - & - & - & $\rho_{w}$ & 1000 & $\mathrm{~kg} / \mathrm{m}^{3}$ \\
\hline Volumetric water content & - & - & - & $\theta$ & 0.23 & - \\
\hline Animal breathing rate & - & - & - & $B R_{a}$ & 5.4 & $\mathrm{~m}^{3} / \mathrm{hr}$ \\
\hline Animal occupancy time & - & - & - & $O_{a n}$ & 24 & $\mathrm{hr} / \mathrm{d}$ \\
\hline $\begin{array}{l}\text { Transfer coefficient for animal } \\
\text { inhalation }\end{array}$ & - & - & - & $T F_{\text {prodinh }}$ & $1.3 \mathrm{E}-05^{\mathrm{d}}$ & $\mathrm{d} / \mathrm{kg}$ \\
\hline From animal feed $(\mathrm{Bg} / \mathrm{kg})$ & & $9.68 \mathrm{E}-06$ & & & & \\
\hline
\end{tabular}


Table 7.4-12. Numerical Calculation of Animal Uptake Submodel (Continued)

\begin{tabular}{|c|c|c|c|c|c|c|}
\hline Model & \multicolumn{3}{|c|}{ ERMYN $^{\mathrm{a}}$} & \multicolumn{3}{|c|}{ BIOMASS ERB2A $^{\text {b }}$} \\
\hline Equation & \multicolumn{3}{|c|}{$\begin{array}{l}\text { Equations shown in } \\
\text { Sections } 6.4 .3 \text { and } 6.4 .4 \text {, } \\
\text { calculations shown in } \\
\text { Table } 6.10-1 .\end{array}$} & \multicolumn{3}{|c|}{$\begin{aligned} & C_{\text {fodd }}= \frac{\left(C F_{\text {past }}+S_{\text {past }}\right) C_{s}}{\left(1-\theta_{t}\right) \rho} \\
&+\frac{I_{\text {past }} V_{\text {irr }} C_{w}}{S B_{\text {past }} W_{\text {past }}+365 I N G_{\text {fodd }} S D} \\
& C_{\text {prod }}= T F_{\text {proding }}\left(C_{\text {fodd }} I N G_{\text {fodd }}+C_{w} I N G_{w a}\right)+ \\
& \frac{C_{s} I N G_{s a} T F_{p r o d i n g}}{\left(1-\theta_{t}\right) \rho+\theta \rho_{w}}+\left(B R_{a} O_{a n} C_{a i r}\right) T F_{p r o d i n}\end{aligned}$} \\
\hline Parameter & Notation & Value & Units & Notation & Value & Units \\
\hline From animal water $(\mathrm{Bq} / \mathrm{kg})$ & $C d, w, 1$ & $7.80 \mathrm{E}-07$ & $\mathrm{~Bq} / \mathrm{kg}$ & $\mathrm{C}_{\text {prod,wa }}$ & 7.80E-07 & $\mathrm{Bq} / \mathrm{kg}$ \\
\hline From soil $(\mathrm{Bq} / \mathrm{kg})$ & $C d_{s, 1}$ & $1.51 \mathrm{E}-05$ & $\mathrm{~Bq} / \mathrm{kg}$ & $\mathrm{C}_{\text {prod,soil }}$ & $1.31 \mathrm{E}-05$ & $\mathrm{~Bq} / \mathrm{kg}$ \\
\hline From dust inhalation $(\mathrm{Bq} / \mathrm{kg})$ & & & & $\mathrm{C}_{\text {prod,inh }}$ & $3.4 \mathrm{E}-10$ & $\mathrm{~Bq} / \mathrm{kg}$ \\
\hline Meat concentration $(\mathrm{Bg} / \mathrm{kg})$ & & $256 E-05$ & & & & \\
\hline
\end{tabular}

${ }^{a}$ Results from Table 6.10-1. Some input values shown for comparison.

b BIOMASS ERB2A calculations are based on the equations in this table and input parameter values from the ERMYN (Table 6.6-3), if possible, or default values suggested in BIOMASS ERB2A (BIOMASS 2000 [DIRS 154522], Section 7.4).

${ }^{c}$ Value from soil-to-plant transfer factor (dry) $\times$ dry-to-wet ratio (forage) $=1 \mathrm{E}-3 \times 0.22=2.2 \mathrm{E}-4$; Table 6.6-3.

${ }^{d}$ Value selected to be the same as the transfer coefficient (ingestion), as suggested by BIOMASS (2000 [DIRS 154522], Table 14 to 17). Therefore, the animal product transfer coefficient for meat, $1.3 \mathrm{E}-5 \mathrm{~d} / \mathrm{kg}$, is selected for Pu-239 (Table 6.6-3).

ERMYN=Environmental Radiation Model for Yucca Mountain, Nevada

\subsubsection{Fish Submodel}

There are no ACMs for the fish submodel (Section 6.3.3), and no complex numerical comparisons are required to validate the submodel.

\subsection{7 $\quad{ }^{14} \mathrm{C}$ Special Submodel}

The ${ }^{14} \mathrm{C}$ special submodel in the ERMYN is different from the GENII-S submodel. The major difference is the release of ${ }^{14} \mathrm{C}$ from the soil into the air as radioactive carbon dioxide gas $\left({ }^{14} \mathrm{CO}_{2}\right)$. Furthermore, the ERMYN plant uptake submodel includes photosynthesis using ${ }^{14} \mathrm{CO}_{2}$ gas from the air. These differences are an ACM. The ERMYN and GENII-S methods for calculating concentrations of ${ }^{14} \mathrm{C}$ in plants are compared to validate the ${ }^{14} \mathrm{C}$ special submodel (Section 7.3.6).

The ERMYN and GENII-S ${ }^{14} \mathrm{C}$ special submodels are described in Sections 6.4.6 and 7.3.6, respectively. The ${ }^{14} \mathrm{C}$ concentrations in crops are calculated in Table 6.10-2. For example, the ${ }^{14} \mathrm{C}$ concentration in leafy vegetables is $0.032 \mathrm{~Bq} / \mathrm{kg}$. Using the same input parameter values as the ERMYN (Table 6.10-2), if possible, the corresponding value, $0.0058 \mathrm{~Bq} / \mathrm{kg}$, is calculated using the GENII-S method (Table 7.3-21). The comparisons, including the input parameters, are shown in Table 7.4-13. Because the effective removal rate for ${ }^{14} \mathrm{C}$ is hard coded in GENII-S, a value of $0.8 /$ year is used. For leafy vegetables, a daily irrigation rate of $5.40 \mathrm{~mm} / \mathrm{d}$ and a growing time of $75 \mathrm{~d}$ are used in the ERMYN. Thus, the corresponding annual irrigation rate of 
$15.9 \mathrm{in} /$ year and irrigation duration of 2.5 month/year are used for GENII-S. The calculations (Table 7.4-13) are performed in an Excel spreadsheet, ERMYN validation.xls, which is listed in Appendix A.

The ERMYN submodel gives a ${ }^{14} \mathrm{C}$ concentration for leafy vegetables that is about six times higher that the GENII-S value (Table 7.4-13). However, the removal rate ( $\lambda s_{c}=0.8 /$ year) in the GENII-S model, which is written into the computer code, is inappropriate. A more appropriate removal rate, 0.132/year, was developed in the previous biosphere model (CRWMS M\&O 2001 [DIRS 152539], Table 3). If this value is used in the GENII-S model, the crop ${ }^{14} \mathrm{C}$ concentration would be six times higher than the value shown in Table 7.4-13, and the ERMYN and GENII-S results would be almost the same. In addition, the ERMYN submodel is conceptually correct in that plants take carbon dioxide from air, instead of directly from the soil. Therefore, the ${ }^{14} \mathrm{C}$ submodel in the ERMYN is valid because it includes photosynthesis. The RESRAD and BIOMASS ERB2A models also include photosynthesis. An evaluation of these methods, and a justification for selecting the method used in the ERMYN ${ }^{14} \mathrm{C}$ special submodel, is presented in Section 7.3.6. This evaluation shows that ACM 6 (Section 6.3.3) does not need further consideration.

Table 7.4-13. Evaluation of Plant Uptake in ${ }^{14} \mathrm{C}$ Special Submodel

\begin{tabular}{|c|c|c|c|c|c|c|}
\hline & \multirow{2}{*}{\multicolumn{3}{|c|}{$\begin{array}{l}\text { ERMYN }^{\text {a }} \\
\text { Equation shown in Section 6.4.6, } \\
\text { calculations shown in Table 6.10-2 }\end{array}$}} & \multicolumn{3}{|c|}{ GENII-S $^{b}$} \\
\hline \multirow{2}{*}{$\begin{array}{l}\text { Equation } \\
\text { Parameter }\end{array}$} & & & & $C c_{p}=\frac{25.4}{}$ & $\frac{C w_{c}}{s_{c}} \times \frac{12}{I D}$ & $\frac{0.1}{0.01} \times F c_{p}$ \\
\hline & Notation & Value & Units & Notation & Value & Units \\
\hline Removal rate & & & & $\lambda s_{c}$ & 0.8 & /yr \\
\hline Surface density & $\rho_{\mathrm{S}}$ & 375 & $\mathrm{~kg} / \mathrm{m}^{2}$ & $\rho_{S}$ & 375 & $\mathrm{~kg} / \mathrm{m}^{2}$ \\
\hline Concentration in water & Cw & 1 & $\mathrm{~Bq} / \mathrm{m}^{3}$ & Cw & 0.027 & $\mathrm{pCi} / \mathrm{L}$ \\
\hline Fraction of carbon & $f c_{\text {plant }}$ & 0.09 & - & $F C_{p}$ & 0.09 & - \\
\hline Irrigation rate & IRD & 5.40 & $\mathrm{~mm} / \mathrm{d}$ & I & 15.9 & in./yr \\
\hline Irrigation duration & $\mathrm{Tg}$ & 75 & $\mathrm{~d}$ & ID & 2.5 & month/yr \\
\hline C-14 concentration & & & & $C c_{p}$ & 1.57E-1 & $\mathrm{pCi} / \mathrm{kg}$ \\
\hline C-14 concentration & $C p$ & $3.2 E-02$ & $\mathrm{~Bq} / \mathrm{kg}$ & $C c_{p}$ & $5.8 E-03$ & $\mathrm{~Bq} / \mathrm{kg}$ \\
\hline
\end{tabular}

${ }^{a}$ Results from Table 6.10-2; some input values shown for comparison.

b Calculation described in Table 7.3-21; input values from Table 6.6-3.

ERMYN=Environmental Radiation Model for Yucca Mountain, Nevada

\subsubsection{External Exposure Submodel}

For the ERMYN, the external exposure submodel only includes surface soil contamination, although external exposure to air and water contamination is possible (Sections 6.4.7 and 6.5.5). In this section, calculations are presented to evaluate the importance of air submersion and water immersion relative to exposure to contaminated soil.

\subsubsection{Air Submersion}

Air submersion is applicable to both exposure scenarios. To evaluate the importance of air submersion, dose coefficients for air submersion (including short-lived decay products) are 
developed (Table 7.4-14) and compared with dose coefficients for soil contamination (Table 6.4-2).

The dose coefficients for air submersion (Table 7.4-14) are in the same units as those for exposure to soil contaminated to an infinite depth (Table 6.4-2). However, the source of the radionuclides for the two dose coefficients differs: air versus soil. The ratio of the dose coefficients for air submersion to those for soil exposure (air/soil; Table 7.4-15) range from 1,471 to 6,051 (i.e., on the order of $10^{3}$ ).

To compare the relative importance of air submersion and soil exposure, activity concentrations in the air and soil are estimated. A soil contamination level of $1 \mathrm{~Bq} / \mathrm{m}^{3}$ corresponds to an activity concentration (in mass units) of $1 \times 10^{-3} \mathrm{~Bq} / \mathrm{kg}$, based on a soil density of $1 \times 10^{3} \mathrm{~kg} / \mathrm{m}^{3}$. If air contamination is only from contaminated resuspended soil, activity concentrations in the air can be calculated using the air submodel (Section 6.4.2, Equation 6.4.2-1). Using typical values of air mass loading (ranging from $10^{-6}$ to $10^{-7} \mathrm{~kg} / \mathrm{m}^{3}$; BSC 2004 [DIRS 169458], Section 6.1), the air concentration is about $10^{-9}$ to $10^{-10} \mathrm{~Bq} / \mathrm{m}^{3}$. Therefore, the activity concentration ratio between air submersion and soil exposure is on the order of $10^{-9}\left(10^{-9} \mathrm{~Bq} / \mathrm{m}^{3} / 1 \mathrm{~Bq} / \mathrm{m}^{3}\right)$. Considering the ratio of dose coefficients at the level of $10^{3}$, the air submersion dose is about 1 million times lower than soil exposure if the exposure times are the same, which is true because both exposures occur simultaneously. Because air submersion is much less important than soil exposure, there is no impact when this pathway is excluded from the ERMYN.

Table 7.4-14. Effective Dose Coefficients for Air Submersion

\begin{tabular}{|c|c|c|c|}
\hline $\begin{array}{c}\text { Primary } \\
\text { Radionuclide }\end{array}$ & Short-Lived Decay Product ${ }^{c}$ & $\begin{array}{c}\text { DC Value }{ }^{d} \\
(\mathrm{~Sv} / \mathrm{sec}) /(\mathrm{Bq} / \mathrm{m} 3)\end{array}$ & $\begin{array}{c}\text { Effective DC } \\
(\text { (Sv/sec)/(Bq/m3) }\end{array}$ \\
\hline C-14 & 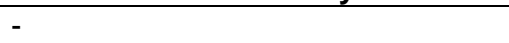 & $2.24 \mathrm{E}-19$ & $2.24 \mathrm{E}-19$ \\
\hline $\mathrm{Cl}-36$ & - & $2.23 \mathrm{E}-17$ & $2.23 \mathrm{E}-17$ \\
\hline Se-79 & - & $3.03 \mathrm{E}-19$ & $3.03 E-19$ \\
\hline Sr-90D ${ }^{a}$ & Y-90 (64.0 hr) & $\begin{array}{l}7.53 \mathrm{E}-18 \\
1.90 \mathrm{E}-16\end{array}$ & $1.98 \mathrm{E}-16$ \\
\hline Tc-99 & - & $1.62 \mathrm{E}-18$ & $1.62 \mathrm{E}-18$ \\
\hline Sn-126 & $\begin{array}{l}\text { Sb-126m (19.0 min) } \\
\text { Sb-126 (14\%, } 12.4 \text { day) }\end{array}$ & $\begin{array}{l}2.11 \mathrm{E}-15 \\
7.50 \mathrm{E}-14 \\
1.37 \mathrm{E}-13\end{array}$ & $9.63 E-14$ \\
\hline $1-129$ & - & $3.80 \mathrm{E}-16$ & $3.80 \mathrm{E}-16$ \\
\hline Cs-135 & - & $5.65 \mathrm{E}-19$ & $5.65 \mathrm{E}-19$ \\
\hline Cs-137D & Ba-137m (94.6\%, $2.552 \mathrm{~min})$ & $\begin{array}{l}7.74 \mathrm{E}-18 \\
2.88 \mathrm{E}-14 \\
\end{array}$ & $2.73 \mathrm{E}-14$ \\
\hline Pu-242 & - & $4.01 \mathrm{E}-18$ & $4.01 \mathrm{E}-18$ \\
\hline U-238D & $\begin{array}{l}\text { Th-234 (24.10 d) } \\
\text { Pa-234m }(99.80 \%, 1.17 \mathrm{~min}) \\
\mathrm{Pa}-234(0.33 \%, 6.7 \mathrm{hr})\end{array}$ & $\begin{array}{l}3.41 \mathrm{E}-18 \\
3.38 \mathrm{E}-16 \\
7.19 \mathrm{E}-16 \\
9.34 \mathrm{E}-14\end{array}$ & $1.37 \mathrm{E}-15$ \\
\hline Pu-238 & - & $4.88 \mathrm{E}-18$ & $4.88 \mathrm{E}-18$ \\
\hline $\mathrm{U}-234$ & - & $7.63 \mathrm{E}-18$ & $7.63 \mathrm{E}-18$ \\
\hline Th-230 & - & $1.74 \mathrm{E}-17$ & $1.74 \mathrm{E}-17$ \\
\hline
\end{tabular}


Table 7.4-14. Effective Dose Coefficients for Air Submersion (Continued)

\begin{tabular}{|c|c|c|c|}
\hline $\begin{array}{c}\text { Primary } \\
\text { Radionuclide }\end{array}$ & Short-Lived Decay Product ${ }^{c}$ & $\begin{array}{c}\text { DC Value }{ }^{d} \\
(\mathrm{~Sv} / \mathrm{sec}) /(\mathrm{Bq} / \mathrm{m} 3)\end{array}$ & $\begin{array}{c}\text { Effective DC } \\
(\mathrm{Sv} / \mathrm{sec}) /(\mathrm{Bq} / \mathrm{m} 3)\end{array}$ \\
\hline Ra-226D & $\begin{array}{l}\text { Rn-222 (3.8235 d) } \\
\text { Po-218 (3.05 min) } \\
\text { Pb-214 (99.98\%, 26.8 min) } \\
\text { At-218 (0.02\%, 2 sec) } \\
\text { Bi-214 (19.9 min) } \\
\text { Po-214 (99.98\%, } 1.64 \times 10-4 \mathrm{sec}) \\
\text { Tl-210 (0.02\%, } 1.3 \mathrm{~min})\end{array}$ & $\begin{array}{l}3.15 \mathrm{E}-16 \\
1.91 \mathrm{E}-17 \\
4.48 \mathrm{E}-19 \\
1.18 \mathrm{E}-14 \\
1.19 \mathrm{E}-16 \\
7.65 \mathrm{E}-14 \\
4.08 \mathrm{E}-18 \\
0.00 \mathrm{E}+00\end{array}$ & $8.86 \mathrm{E}-14$ \\
\hline $\mathrm{Pb}-210 \mathrm{D}$ & $\begin{array}{l}\text { Bi-210 (5.012 d) } \\
\text { Po-210 (138.38 d) }\end{array}$ & $\begin{array}{l}5.64 \mathrm{E}-17 \\
3.29 \mathrm{E}-17 \\
4.16 \mathrm{E}-19\end{array}$ & 8.97E-17 \\
\hline $\mathrm{Pu}-240$ & - & $4.75 \mathrm{E}-18$ & $4.75 \mathrm{E}-18$ \\
\hline U-236 & - & $5.01 \mathrm{E}-18$ & $5.01 \mathrm{E}-18$ \\
\hline Th-232 & - & $8.72 \mathrm{E}-18$ & $8.72 \mathrm{E}-18$ \\
\hline $\mathrm{Ra}-228 \mathrm{D}^{b}$ & Ac-228 (6.13 hr) & $\begin{array}{l}0.00 \mathrm{E}+00 \\
4.78 \mathrm{E}-14 \\
\end{array}$ & $4.78 \mathrm{E}-14$ \\
\hline $\mathrm{U}-232$ & - & $1.42 \mathrm{E}-17$ & $1.42 \mathrm{E}-17$ \\
\hline Th-228D & $\begin{array}{l}\text { Ra-224 (3.66 d) } \\
\text { Rn-220 (55.6 sec) } \\
\text { Po-216 (0.15 sec) } \\
\text { Pb-212 (10.64 hr) } \\
\text { Bi-212 (60.55 min) } \\
\text { Po-212 (64.07\%, } 0.305 \mu-s e c) \\
\text { Tl-208 (35.93\%, } 3.07 \mathrm{~min})\end{array}$ & $\begin{array}{l}9.20 \mathrm{E}-17 \\
4.71 \mathrm{E}-16 \\
1.85 \mathrm{E}-17 \\
8.29 \mathrm{E}-19 \\
6.87 \mathrm{E}-15 \\
9.24 \mathrm{E}-15 \\
0.00 \mathrm{E}+00 \\
1.77 \mathrm{E}-13\end{array}$ & 8.03E-14 \\
\hline$A m-243 D$ & Np-239 (2.355 d) & $\begin{array}{l}2.18 \mathrm{E}-15 \\
7.69 \mathrm{E}-15\end{array}$ & 9.87E-15 \\
\hline $\mathrm{Pu}-239$ & - & $4.24 \mathrm{E}-18$ & $4.24 \mathrm{E}-18$ \\
\hline U-235D & Th-231 (25.52 hr) & $\begin{array}{l}7.20 \mathrm{E}-15 \\
5.22 \mathrm{E}-16 \\
\end{array}$ & $7.72 \mathrm{E}-15$ \\
\hline $\mathrm{Pa}-231$ & - & $1.72 \mathrm{E}-15$ & $1.72 \mathrm{E}-15$ \\
\hline AC-227D & $\begin{array}{l}\text { Th-227 (98.62\%, } 18.718 \mathrm{~d}) \\
\text { Fr-223 (1.38\%, 21.8 min) } \\
\text { Ra-223 (11.434 d) } \\
\text { Rn-219 (3.96 sec) } \\
\text { Po-215 (1.78 millisec) } \\
\text { Pb-211 (36.1 min) } \\
\text { Bi-211 (2.15 min) } \\
\text { Tl-207 (99.72\%, } 4.77 \mathrm{~min}) \\
\text { Po-211 (0.28\%, } 0.516 \mathrm{sec})\end{array}$ & $\begin{array}{l}5.82 \mathrm{E}-18 \\
4.88 \mathrm{E}-15 \\
2.29 \mathrm{E}-15 \\
6.09 \mathrm{E}-15 \\
2.68 \mathrm{E}-15 \\
8.43 \mathrm{E}-18 \\
2.49 \mathrm{E}-15 \\
2.22 \mathrm{E}-15 \\
1.62 \mathrm{E}-16 \\
3.81 \mathrm{E}-16\end{array}$ & $1.85 \mathrm{E}-14$ \\
\hline Am-241 & - & $8.18 \mathrm{E}-16$ & $8.18 \mathrm{E}-16$ \\
\hline Np-237D & $\mathrm{Pa}-233(27.0 \mathrm{~d})$ & $\begin{array}{l}1.03 \mathrm{E}-15 \\
9.35 \mathrm{E}-15\end{array}$ & $1.04 \mathrm{E}-14$ \\
\hline
\end{tabular}


Table 7.4-14. Effective Dose Coefficients for Air Submersion (Continued)

\begin{tabular}{|c|c|c|c|}
\hline $\begin{array}{c}\text { Primary } \\
\text { Radionuclide }\end{array}$ & Short-Lived Decay Product ${ }^{c}$ & $\begin{array}{c}\text { DC Value }{ }^{d} \\
(\mathrm{~Sv} / \mathrm{sec}) /(\mathrm{Bq} / \mathrm{m} 3)\end{array}$ & $\begin{array}{c}\text { Effective DC } \\
(\text { Sv/sec)/(Bq/m3) }\end{array}$ \\
\hline U-233 & 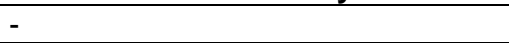 & $1.63 \mathrm{E}-17$ & 1.63E-17 \\
\hline Th-229D & $\begin{array}{l}\text { Ra-225 (14.8 d) } \\
\text { Ac-225 (10.0 d) } \\
\text { Fr-221 (4.8 min) } \\
\text { At-217 (32.3 millisec) } \\
\text { Bi-213 (45.65 min) } \\
\text { Po-213 (97.84\%, } 4.2 \mu-\mathrm{sec}) \\
\text { Tl-209 (2.16\%, } 2.2 \mathrm{~min}) \\
\text { Pb-209 (3.253 hr) }\end{array}$ & $\begin{array}{l}3.83 \mathrm{E}-15 \\
2.79 \mathrm{E}-16 \\
7.21 \mathrm{E}-16 \\
1.46 \mathrm{E}-15 \\
1.48 \mathrm{E}-17 \\
6.39 \mathrm{E}-15 \\
0.00 \mathrm{E}+00 \\
1.02 \mathrm{E}-13 \\
8.12 \mathrm{E}-18\end{array}$ & $1.49 \mathrm{E}-14$ \\
\hline
\end{tabular}

a " $\mathrm{D}$ " after a radionuclide symbol denotes that the radionuclide is treated together with the short half-life (less than 180 d) decay product.

b Indented radionuclides are long-lived decay products considered separately from the parents.

${ }^{c}$ Branching fraction and half-life from FGR-12 (Eckerman and Ryman 1993 [DIRS 107684], Table A.1).

d Values from FGR-12 (Eckerman and Ryman 1993 [DIRS 107684], Table III.1).

$\mathrm{DC}=$ dose coefficient

Table 7.4-15. Comparison of Dose Coefficients for Infinite Depth Soil and Air Submersion

\begin{tabular}{|c|c|c|c|}
\hline $\begin{array}{c}\text { Primary } \\
\text { Radionuclide }\end{array}$ & $\begin{array}{l}\text { Infinite Depth Soil Effective DC }{ }^{\mathrm{c}} \\
(\mathrm{Sv} / \mathrm{sec}) /(\mathrm{Bq} / \mathrm{m} 3)\end{array}$ & $\begin{array}{c}\text { Air Submersion Effective DC }{ }^{d} \\
(\text { Sv } / \mathrm{sec}) /(\mathrm{Bq} / \mathrm{m} 3)\end{array}$ & $\begin{array}{l}\text { Ratio }^{\mathrm{e}} \\
\text { (Air/Soil) }\end{array}$ \\
\hline C-14 & $7.20 \mathrm{E}-23$ & $2.24 \mathrm{E}-19$ & 3111 \\
\hline $\mathrm{Cl}-36$ & $1.28 \mathrm{E}-20$ & $2.23 \mathrm{E}-17$ & 1742 \\
\hline Se-79 & $9.96 \mathrm{E}-23$ & 3.03E-19 & 3042 \\
\hline Sr-90D ${ }^{a}$ & $1.32 \mathrm{E}-19$ & 1.98E-16 & 1500 \\
\hline Tc-99 & $6.72 \mathrm{E}-22$ & $1.62 \mathrm{E}-18$ & 2411 \\
\hline Sn-126 & $6.34 \mathrm{E}-17$ & 9.63E-14 & 1519 \\
\hline $\mathrm{I}-129$ & 6.93E-20 & $3.80 \mathrm{E}-16$ & 5483 \\
\hline Cs-135 & $2.05 \mathrm{E}-22$ & 5.65E-19 & 2756 \\
\hline Cs-137D & 1.83E-17 & $2.73 \mathrm{E}-14$ & 1492 \\
\hline Pu-242 & $6.85 E-22$ & 4.01E-18 & 5854 \\
\hline U-238D & 8.13E-19 & $1.37 \mathrm{E}-15$ & 1685 \\
\hline Pu-238 & 8.10E-22 & 4.88E-18 & 6025 \\
\hline U-234 & $2.15 E-21$ & 7.63E-18 & 3549 \\
\hline Th-230 & $6.47 \mathrm{E}-21$ & $1.74 \mathrm{E}-17$ & 2689 \\
\hline Ra-226D & 5.99E-17 & 8.86E-14 & 1479 \\
\hline $\mathrm{Pb}-210 \mathrm{D}$ & $3.27 \mathrm{E}-20$ & 8.97E-17 & 2743 \\
\hline Pu-240 & 7.85E-22 & 4.75E-18 & 6051 \\
\hline U-236 & 1.15E-21 & $5.01 \mathrm{E}-18$ & 4357 \\
\hline Th-232 & $2.79 E-21$ & $8.72 \mathrm{E}-18$ & 3125 \\
\hline $\mathrm{Ra}-228 \mathrm{D}^{\mathrm{b}}$ & $3.20 \mathrm{E}-17$ & 4.78E-14 & 1494 \\
\hline $\mathrm{U}-232$ & 4.83E-21 & $1.42 \mathrm{E}-17$ & 2940 \\
\hline Th-228D & 5.46E-17 & 8.03E-14 & 1471 \\
\hline
\end{tabular}


Table 7.4-15. Comparison of Dose Coefficients for Infinite Depth Soil and Air Submersion (Continued)

\begin{tabular}{|l|c|c|c|}
\hline $\begin{array}{c}\text { Primary } \\
\text { Radionuclide }\end{array}$ & $\begin{array}{c}\text { Infinite Depth Soil Effective DC } \\
\text { (Sv/sec)/(Bq/m3) }\end{array}$ & $\begin{array}{c}\text { Air Submersion Effective DC }{ }^{\mathbf{d}} \\
\text { (Sv/sec)/(Bq/m3) }\end{array}$ & $\begin{array}{c}\text { Ratio }^{{ }^{\mathbf{e}}} \\
\text { (Air/Soil) }\end{array}$ \\
\hline Am-243D & $4.79 \mathrm{E}-18$ & $9.87 \mathrm{E}-15$ & 2061 \\
\hline Pu-239 & $1.58 \mathrm{E}-21$ & $4.24 \mathrm{E}-18$ & 2684 \\
\hline U-235D & $4.06 \mathrm{E}-18$ & $7.72 \mathrm{E}-15$ & 1901 \\
\hline Pa-231 & $1.02 \mathrm{E}-18$ & $1.72 \mathrm{E}-15$ & 1686 \\
\hline Ac-227D & $1.08 \mathrm{E}-17$ & $1.85 \mathrm{E}-14$ & 1713 \\
\hline Am-241 & $2.34 \mathrm{E}-19$ & $8.18 \mathrm{E}-16$ & 3496 \\
\hline Np-237D & $5.88 \mathrm{E}-18$ & $1.04 \mathrm{E}-14$ & 1769 \\
\hline U-233 & $7.48 \mathrm{E}-21$ & $1.63 \mathrm{E}-17$ & 2179 \\
\hline Th-229D & $8.55 \mathrm{E}-18$ & $1.49 \mathrm{E}-14$ & 1743 \\
\hline
\end{tabular}

a " $D$ " after a radionuclide symbol denotes that the radionuclide is treated together with the short half-life (less than $180 \mathrm{~d}$ ) decay product.

${ }^{b}$ Indented radionuclides are long-lived decay products considered separately from the parents.

c Data from Table 6.4-2.

'Data from Table 7.4-14.

e Calculated from Column 3 divided by Column 2.

$\mathrm{DC}=$ dose coefficient

\subsubsection{Water Immersion}

Immersion in contaminated water only applies to the groundwater scenario, where water immersion involves activities such as swimming or bathing in contaminated water. To evaluate the importance of the water immersion exposure pathway, dose coefficients (including short-lived decay products) are developed for water immersion (Table 7.4-16), similar to the dose coefficients for soil contamination (Table 6.4-2).

The dose coefficients for water immersion (Table 7.4-16) are in the same units (Sv/sec) $/\left(\mathrm{Bq} / \mathrm{m}^{3}\right)$ as those for exposure to soil contaminated to an infinite depth. However, the source of radionuclides differ: water versus soil. The ratio of dose coefficient for water immersion (Table 7.4-16) to soil exposure (Table 6.4-2) range from 2.9 to 14.1 (Table 7.4-17), with iodine and plutonium having the largest ratios (greater than 10).

To compare the relative importance of water immersion and soil exposure, exposure times and media concentrations are evaluated. Typically, daily baths last about $20 \mathrm{~min}$, while showers last about 10 min (EPA 1997 [DIRS 116135], pp. 15 and 16). Typical rates for swimming are one swim per month that lasts for about 60 min (EPA 1997 [DIRS 116135], pp. 15 to 17), which gives an average swimming time of about $2 \mathrm{~min} / \mathrm{d}$. Therefore, the average daily water immersion time is about $17 \mathrm{~min} / \mathrm{d}$, based on equal frequency of bathing and showering. The effective soil exposure time is radionuclide dependent because of the external shielding factor. Based on the calculated environment-specific outdoor time of $1.90 \mathrm{hr} / \mathrm{d}$, an indoor time of $17.75 \mathrm{hr} / \mathrm{d}$ (Table 6.10-1), and the shielding factors, the average effective exposure times and the ratios of soil exposure time to water immersion time are calculated (Table 7.4-18).

In addition, radionuclide concentrations in the water and soil are estimated. If the water contamination is $1 \mathrm{~Bq} / \mathrm{m}^{3}$, equilibrium soil concentrations after long-term irrigation are estimated using Equations 6.4.1-4 and 6.4.1-5. Using an annual irrigation rate of $0.5 \mathrm{~m} /$ year for the 
expected future climate, a surface soil depth of $0.25 \mathrm{~m}$ (Table 6.6-3), and an effective removal constant (Table 7.4-4), the average radionuclide concentration in the soil is calculated (Table 7.4-18). Using a unit concentration in the water, the ratio of radionuclide concentrations in the water and soil is numerically equal to the values of radionuclide concentration in soil.

The soil exposure and water immersion doses depend on exposure time, media concentrations, and dose coefficients, and a comparison of soil exposure to water immersion can be calculated as the soil/water exposure time ratio times the soil/water radionuclide concentration ratio, and then divided by the water/soil dose coefficient ratio. Soil exposure doses range from 13 (for ${ }^{99} \mathrm{Tc}$ ) to more than $12,000\left({ }^{231} \mathrm{~Pa}\right)$ times higher than that for water immersion (Table 7.4-18). Because the external exposure pathway is not important compared with all pathways (e.g., about 7.6 percent of the total dose for ${ }^{226} \mathrm{Ra}$ and less than 0.01 percent for ${ }^{239} \mathrm{Pu}$ and ${ }^{14} \mathrm{C}$; Tables $6.10-1$ to $6.10-3$ ), eliminating the water immersion pathway is reasonable and does not underestimate the dose. Because water immersion is much less important than soil exposure, there is no impact when this pathway is excluded from the ERMYN.

Table 7.4-16. Effective Dose Coefficients for Water Immersion

\begin{tabular}{|c|c|c|c|}
\hline $\begin{array}{c}\text { Primary } \\
\text { Radionuclide }\end{array}$ & Short-Lived Decay Product ${ }^{c}$ & $\begin{array}{c}\text { DC Value }{ }^{\mathrm{d}} \\
(\mathrm{Sv} / \mathrm{sec}) /(\mathrm{Bq} / \mathrm{m} 3)\end{array}$ & $\begin{array}{c}\text { Effective DC } \\
(\mathrm{Sv} / \mathrm{sec}) /(\mathrm{Bq} / \mathrm{m} 3)\end{array}$ \\
\hline C-14 & 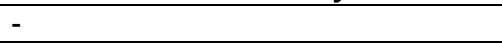 & $4.39 \mathrm{E}-22$ & $4.39 \mathrm{E}-22$ \\
\hline $\mathrm{Cl}-36$ & - & $4.48 \mathrm{E}-20$ & $4.48 \mathrm{E}-20$ \\
\hline Se-79 & - & $5.93 \mathrm{E}-22$ & $5.93 \mathrm{E}-22$ \\
\hline Sr-90D ${ }^{a}$ & Y-90 (64.0 hr) & $\begin{array}{l}1.46 \mathrm{E}-20 \\
3.63 \mathrm{E}-19 \\
\end{array}$ & $3.78 \mathrm{E}-19$ \\
\hline Tc-99 & - & $3.14 \mathrm{E}-21$ & $3.14 \mathrm{E}-21$ \\
\hline Sn-126 & $\begin{array}{l}\text { Sb-126m (19.0 min) } \\
\text { Sb-126 (14\%, } 12.4 \text { day) }\end{array}$ & $\begin{array}{l}4.76 \mathrm{E}-18 \\
1.63 \mathrm{E}-16 \\
2.99 \mathrm{E}-16 \\
\end{array}$ & $2.10 \mathrm{E}-16$ \\
\hline I-129 & - & $8.91 \mathrm{E}-19$ & $8.91 \mathrm{E}-19$ \\
\hline Cs-135 & - & $1.10 \mathrm{E}-21$ & $1.10 \mathrm{E}-21$ \\
\hline Cs-137D & $\mathrm{Ba}-137 \mathrm{~m}(94.6 \%, 2.552 \mathrm{~min})$ & $\begin{array}{l}1.49 \mathrm{E}-20 \\
6.26 \mathrm{E}-17\end{array}$ & $5.92 \mathrm{E}-17$ \\
\hline Pu-242 & - & $9.35 \mathrm{E}-21$ & $9.35 \mathrm{E}-21$ \\
\hline U-238D & $\begin{array}{l}\text { Th-234 (24.10 d) } \\
\text { Pa-234m }(99.80 \%, 1.17 \mathrm{~min}) \\
\mathrm{Pa}-234(0.33 \%, 6.7 \mathrm{hr})\end{array}$ & $\begin{array}{l}7.95 \mathrm{E}-21 \\
7.64 \mathrm{E}-19 \\
1.52 \mathrm{E}-18 \\
2.03 \mathrm{E}-16\end{array}$ & $2.96 \mathrm{E}-18$ \\
\hline $\mathrm{Pu}-238$ & - & $1.14 \mathrm{E}-20$ & $1.14 \mathrm{E}-20$ \\
\hline $\mathrm{U}-234$ & - & $1.75 \mathrm{E}-20$ & $1.75 \mathrm{E}-20$ \\
\hline Th-230 & - & $3.94 \mathrm{E}-20$ & $3.94 \mathrm{E}-20$ \\
\hline Ra-226D & $\begin{array}{l}\text { Rn-222 (3.8235 d) } \\
\text { Po-218 (3.05 min) } \\
\text { Pb-214 (99.98\%, 26.8 min) } \\
\text { At-218 (0.02\%, 2 sec) } \\
\text { Bi-214 (19.9 min) } \\
\text { Po-214 (99.98\%, } 1.64 \times 10-4 \mathrm{sec}) \\
\text { Tl-210 }(0.02 \%, 1.3 \mathrm{~min}) \\
\end{array}$ & $\begin{array}{l}6.95 \mathrm{E}-19 \\
4.16 \mathrm{E}-20 \\
9.71 \mathrm{E}-22 \\
2.59 \mathrm{E}-17 \\
2.75 \mathrm{E}-19 \\
1.66 \mathrm{E}-16 \\
8.85 \mathrm{E}-21 \\
0.00 \mathrm{E}+00 \\
\end{array}$ & 1.93E-16 \\
\hline $\mathrm{Pb}-210 \mathrm{D}$ & $\begin{array}{l}\text { Bi-210 }(5.012 \mathrm{~d}) \\
\text { Po-210 (138.38 d) }\end{array}$ & $\begin{array}{l}1.31 \mathrm{E}-19 \\
6.33 \mathrm{E}-20 \\
9.03 \mathrm{E}-22 \\
\end{array}$ & $1.95 \mathrm{E}-19$ \\
\hline Pu-240 & - & $1.11 \mathrm{E}-20$ & $1.11 \mathrm{E}-20$ \\
\hline U-236 & - & $1.16 \mathrm{E}-20$ & $1.16 \mathrm{E}-20$ \\
\hline
\end{tabular}


Table 7.4-16. Effective Dose Coefficients for Water Immersion (Continued)

\begin{tabular}{|c|c|c|c|}
\hline $\begin{array}{c}\text { Primary } \\
\text { Radionuclide }\end{array}$ & Short-Lived Decay Product ${ }^{\mathrm{c}}$ & $\begin{array}{c}\text { DC Value }{ }^{\mathrm{d}} \\
(\mathrm{Sv} / \mathrm{sec}) /(\mathrm{Bq} / \mathrm{m} 3)\end{array}$ & $\begin{array}{c}\text { Effective DC } \\
(\mathrm{Sv} / \mathrm{sec}) /(\mathrm{Bq} / \mathrm{m} 3)\end{array}$ \\
\hline Th-232 & (a) & $1.99 \mathrm{E}-20$ & $1.99 \mathrm{E}-20$ \\
\hline Ra-228D & Ac-228 (6.13 hr) & $\begin{array}{l}0.00 \mathrm{E}+00 \\
1.04 \mathrm{E}-16\end{array}$ & $1.04 \mathrm{E}-16$ \\
\hline $\mathrm{U}-232$ & - & $3.22 \mathrm{E}-20$ & $3.22 \mathrm{E}-20$ \\
\hline Th-228D & $\begin{array}{l}\text { Ra-224 (3.66 d) } \\
\text { Rn-220 (55.6 sec) } \\
\text { Po-216 (0.15 sec) } \\
\text { Pb-212 (10.64 hr) } \\
\text { Bi-212 (60.55 min) } \\
\text { Po-212 (64.07\%, } 0.305 \mu-\mathrm{sec}) \\
\text { Tl-208 (35.93\%, } 3.07 \mathrm{~min})\end{array}$ & $\begin{array}{l}2.05 \mathrm{E}-19 \\
1.03 \mathrm{E}-18 \\
4.03 \mathrm{E}-20 \\
1.80 \mathrm{E}-21 \\
1.52 \mathrm{E}-17 \\
2.00 \mathrm{E}-17 \\
0.00 \mathrm{E}+00 \\
3.84 \mathrm{E}-16\end{array}$ & 1.74E-16 \\
\hline Am-243D & Np-239 (2.355 d) & $\begin{array}{l}4.94 \mathrm{E}-18 \\
1.70 \mathrm{E}-17\end{array}$ & $2.19 \mathrm{E}-17$ \\
\hline Pu-239 & - & $9.60 \mathrm{E}-21$ & $9.60 \mathrm{E}-21$ \\
\hline U-235D & Th-231 (25.52 hr) & $\begin{array}{l}1.59 \mathrm{E}-17 \\
1.18 \mathrm{E}-18 \\
\end{array}$ & 1.71E-17 \\
\hline Pa-231 & - & $3.78 \mathrm{E}-18$ & $3.78 \mathrm{E}-18$ \\
\hline Ac-227D & $\begin{array}{l}\text { Th-227 (98.62\%, } 18.718 \mathrm{~d}) \\
\text { Fr-223 (1.38\%, } 21.8 \mathrm{~min}) \\
\text { Ra-223 (11.434 d) } \\
\text { Rn-219 (3.96 sec) } \\
\text { Po-215 (1.78 millisec) } \\
\text { Pb-211 (36.1 min) } \\
\text { Bi-211 (2.15 min) } \\
\text { Tl-207 (99.72\%, } 4.77 \mathrm{~min}) \\
\text { Po-211 (0.28\%, } 0.516 \mathrm{sec})\end{array}$ & $\begin{array}{l}1.30 \mathrm{E}-20 \\
1.07 \mathrm{E}-17 \\
5.11 \mathrm{E}-18 \\
1.35 \mathrm{E}-17 \\
5.85 \mathrm{E}-18 \\
1.84 \mathrm{E}-20 \\
5.41 \mathrm{E}-18 \\
4.85 \mathrm{E}-18 \\
3.38 \mathrm{E}-19 \\
8.27 \mathrm{E}-19 \\
\end{array}$ & $4.06 \mathrm{E}-17$ \\
\hline Am-241 & - & $1.88 \mathrm{E}-18$ & $1.88 \mathrm{E}-18$ \\
\hline Np-237D & $\mathrm{Pa}-233(27.0 \mathrm{~d})$ & $\begin{array}{l}2.32 \mathrm{E}-18 \\
2.05 \mathrm{E}-17\end{array}$ & $2.28 \mathrm{E}-17$ \\
\hline U-233 & - & $3.64 \mathrm{E}-20$ & $3.64 \mathrm{E}-20$ \\
\hline Th-229D & $\begin{array}{l}\text { Ra-225 (14.8 d) } \\
\text { Ac-225 (10.0 d) } \\
\text { Fr-221 (4.8 min) } \\
\text { At-217 (32.3 millisec) } \\
\text { Bi-213 (45.65 min) } \\
\text { Po-213 (97.84\%, } 4.2 \mu \text {-sec) } \\
\text { Tl-209 (2.16\%, 2.2 } \mathrm{min}) \\
\text { Pb-209 (3.253 hr) }\end{array}$ & $\begin{array}{l}8.56 \mathrm{E}-18 \\
6.49 \mathrm{E}-19 \\
1.61 \mathrm{E}-18 \\
3.22 \mathrm{E}-18 \\
3.22 \mathrm{E}-20 \\
1.39 \mathrm{E}-17 \\
0.00 \mathrm{E}+00 \\
2.22 \mathrm{E}-16 \\
1.57 \mathrm{E}-20\end{array}$ & $3.2 \mathrm{E}-17$ \\
\hline
\end{tabular}

a " $D$ " after a radionuclide denotes that it is treated together with its short half-life (less than $180 \mathrm{~d}$ ) decay product.

${ }^{\mathrm{b}}$ Indented radionuclides are long-lived decay products considered separately from the parents.

c Branching ratio and half-life from FGR-12 (Eckerman and Ryman 1993 [DIRS 107684], Table A.1).

d Dose coefficient values from FGR-12 (Eckerman and Ryman 1993 [DIRS 107684], Table III.2).

$\mathrm{DC}=$ dose coefficient 
Table 7.4-17. Effective Dose Coefficients for Infinite Depth Soil and Water Immersion

\begin{tabular}{|c|c|c|c|}
\hline $\begin{array}{c}\text { Primary } \\
\text { Radionuclide }\end{array}$ & $\begin{array}{l}\text { Infinite Depth Soil Effective DC }{ }^{\mathrm{c}} \\
(\mathrm{Sv} / \mathrm{sec}) /(\mathrm{Bq} / \mathrm{m} 3)\end{array}$ & $\begin{array}{c}\text { Water Immersion Effective DC }{ }^{d} \\
(\mathrm{~Sv} / \mathrm{sec}) /(\mathrm{Bq} / \mathrm{m} 3)\end{array}$ & $\begin{array}{c}\text { Ratio }^{\mathrm{e}} \\
\text { (Water/Soil) }\end{array}$ \\
\hline C-14 & $7.20 \mathrm{E}-23$ & $4.39 \mathrm{E}-22$ & 6.1 \\
\hline $\mathrm{Cl}-36$ & $1.28 \mathrm{E}-20$ & $4.48 \mathrm{E}-20$ & 3.5 \\
\hline Se-79 & $9.96 \mathrm{E}-23$ & $5.93 \mathrm{E}-22$ & 5.6 \\
\hline $\mathrm{Sr}-90 \mathrm{D}^{\mathrm{a}}$ & $1.32 \mathrm{E}-19$ & $3.78 \mathrm{E}-19$ & 2.9 \\
\hline Tc-99 & $6.72 \mathrm{E}-22$ & $3.14 \mathrm{E}-21$ & 4.7 \\
\hline Sn-126 & $6.34 \mathrm{E}-17$ & $2.10 \mathrm{E}-16$ & 3.3 \\
\hline $1-129$ & $6.93 \mathrm{E}-20$ & $8.91 \mathrm{E}-19$ & 12.9 \\
\hline Cs-135 & $2.05 \mathrm{E}-22$ & $1.10 \mathrm{E}-21$ & 5.4 \\
\hline Cs-137D & $1.83 \mathrm{E}-17$ & $5.92 \mathrm{E}-17$ & 3.2 \\
\hline Pu-242 & $6.85 \mathrm{E}-22$ & $9.35 \mathrm{E}-21$ & 13.6 \\
\hline $\mathrm{U}-238 \mathrm{D}$ & $8.13 \mathrm{E}-19$ & $2.96 \mathrm{E}-18$ & 3.6 \\
\hline Pu-238 & $8.10 \mathrm{E}-22$ & $1.14 \mathrm{E}-20$ & 14.1 \\
\hline $\mathrm{U}-234$ & $2.15 \mathrm{E}-21$ & $1.75 \mathrm{E}-20$ & 8.1 \\
\hline Th-230 & $6.47 \mathrm{E}-21$ & $3.94 \mathrm{E}-20$ & 6.1 \\
\hline Ra-226D & $5.99 \mathrm{E}-17$ & $1.93 \mathrm{E}-16$ & 3.2 \\
\hline $\mathrm{Pb}-210 \mathrm{D}$ & $3.27 \mathrm{E}-20$ & $1.95 \mathrm{E}-19$ & 6.0 \\
\hline Pu-240 & $7.85 \mathrm{E}-22$ & $1.11 \mathrm{E}-20$ & 14.1 \\
\hline $\mathrm{U}-236$ & $1.15 \mathrm{E}-21$ & $1.16 \mathrm{E}-20$ & 10.1 \\
\hline Th-232 & $2.79 \mathrm{E}-21$ & $1.99 \mathrm{E}-20$ & 7.1 \\
\hline $\mathrm{Ra}^{2} 228 \mathrm{D}^{\mathrm{b}}$ & $3.20 \mathrm{E}-17$ & $1.04 \mathrm{E}-16$ & 3.3 \\
\hline $\mathrm{U}-232$ & $4.83 \mathrm{E}-21$ & $3.22 \mathrm{E}-20$ & 6.7 \\
\hline Th-228D & $5.46 \mathrm{E}-17$ & 1.74E-16 & 3.2 \\
\hline Am-243D & $4.79 \mathrm{E}-18$ & $2.19 \mathrm{E}-17$ & 4.6 \\
\hline Pu-239 & $1.58 \mathrm{E}-21$ & $9.60 \mathrm{E}-21$ & 6.1 \\
\hline$U-235 D$ & 4.06E-18 & $1.71 \mathrm{E}-17$ & 4.2 \\
\hline $\mathrm{Pa}-231$ & $1.02 \mathrm{E}-18$ & $3.78 \mathrm{E}-18$ & 3.7 \\
\hline Ac-227D & $1.08 \mathrm{E}-17$ & $4.06 \mathrm{E}-17$ & 3.8 \\
\hline Am-241 & $2.34 \mathrm{E}-19$ & $1.88 \mathrm{E}-18$ & 8.0 \\
\hline Np-237D & $5.88 \mathrm{E}-18$ & $2.28 \mathrm{E}-17$ & 3.9 \\
\hline $\mathrm{U}-233$ & $7.48 \mathrm{E}-21$ & $3.64 \mathrm{E}-20$ & 4.9 \\
\hline Th-229D & $8.55 \mathrm{E}-18$ & $3.20 \mathrm{E}-17$ & 3.8 \\
\hline
\end{tabular}

a " $D$ " denotes that the radionuclide is treated together with its short half-life (less than $180 \mathrm{~d}$ ) decay product.

${ }^{\mathrm{b}}$ Indented radionuclides are long-lived decay products considered separately from the parents.

c Data from Table 6.4-2.

d Data from Table 7.4-16.

e Calculated from Column 3 divided by Column 2.

$\mathrm{DC}=$ dose coefficient

Table 7.4-18. Evaluation of Water Immersion Pathway

\begin{tabular}{|l|c|c|c|c|c|c|}
\hline $\begin{array}{c}\text { Primary } \\
\text { Radionuclide }\end{array}$ & $\begin{array}{c}\text { Effective } \\
\text { Removal } \\
\text { Constant } \\
\text { (lyr) }\end{array}$ & $\begin{array}{c}\text { Radionuclide } \\
\text { Concentration } \\
\text { Ratio } \\
\text { (Soil/Water) }\end{array}$ & $\begin{array}{c}\text { Effective } \\
\text { Exposure } \\
\text { Time for } \\
\text { Soil (hr/d) }\end{array}$ & $\begin{array}{c}\text { Effective } \\
\text { Exposure Time } \\
\text { Ratio } \\
\text { (Soil/Water) }\end{array}$ & $\begin{array}{c}\text { Dose } \\
\text { Coefficient } \\
\text { Ratio } \\
\text { (Water/Soil) }\end{array}$ & $\begin{array}{c}\text { Dose } \\
\text { Contribution } \\
\text { Ratio } \\
\text { (Soil/Water) }\end{array}$ \\
\hline C-14 & $1.13 \mathrm{E}-02$ & $1.77 \mathrm{E}+02$ & 5.5 & 19 & 6.1 & 560 \\
\hline Cl-36 & $6.10 \mathrm{E}-01$ & $3.28 \mathrm{E}+00$ & 9.0 & 32 & 3.5 & 30 \\
\hline Se-79 & $2.51 \mathrm{E}-03$ & $7.98 \mathrm{E}+02$ & 3.7 & 13 & 5.6 & 1849 \\
\hline Sr-90D & $3.40 \mathrm{E}-02$ & $5.89 \mathrm{E}+01$ & 9.0 & 32 & 2.9 & 645 \\
\hline Tc-99 & $6.10 \mathrm{E}-01$ & $3.28 \mathrm{E}+00$ & 5.5 & 19 & 4.7 & 13 \\
\hline Sn-126 & $1.71 \mathrm{E}-03$ & $1.17 \mathrm{E}+03$ & 9.0 & 32 & 3.3 & 11256 \\
\hline I-129 & $3.97 \mathrm{E}-02$ & $5.04 \mathrm{E}+01$ & 3.7 & 13 & 12.9 & 51 \\
\hline Cs-135 & $1.35 \mathrm{E}-03$ & $1.48 \mathrm{E}+03$ & 3.7 & 13 & 5.4 & 3567 \\
\hline
\end{tabular}


Table 7.4-18. Evaluation of Water Immersion Pathway (Continued)

\begin{tabular}{|c|c|c|c|c|c|c|}
\hline $\begin{array}{c}\text { Primary } \\
\text { Radionuclide }\end{array}$ & $\begin{array}{c}\text { Effective } \\
\text { Removal } \\
\text { Constant } \\
\text { (lyr) }\end{array}$ & $\begin{array}{c}\text { Radionuclide } \\
\text { Concentration } \\
\text { Ratio } \\
\text { (Soil/Water) }\end{array}$ & $\begin{array}{c}\text { Effective } \\
\text { Exposure } \\
\text { Time for } \\
\text { Soil (hr/d) }\end{array}$ & $\begin{array}{c}\text { Effective } \\
\text { Exposure Time } \\
\text { Ratio } \\
\text { (Soil/Water) }\end{array}$ & $\begin{array}{c}\text { Dose } \\
\text { Coefficient } \\
\text { Ratio } \\
\text { (Water/Soil) }\end{array}$ & $\begin{array}{c}\text { Dose } \\
\text { Contribution } \\
\text { Ratio } \\
\text { (Soil/Water) }\end{array}$ \\
\hline Cs-137D & 2.45E-02 & $8.18 \mathrm{E}+01$ & 9.0 & 32 & 3.2 & 812 \\
\hline Pu-242 & $1.46 \mathrm{E}-03$ & $1.37 \mathrm{E}+03$ & 3.7 & 13 & 13.6 & 1310 \\
\hline U-238D & 6.70E-03 & $2.99 \mathrm{E}+02$ & 9.0 & 32 & 3.6 & 2636 \\
\hline Pu-238 & 9.36E-03 & $2.14 \mathrm{E}+02$ & 3.7 & 13 & 14.1 & 197 \\
\hline U-234 & 6.70E-03 & $2.99 \mathrm{E}+02$ & 5.5 & 19 & 8.1 & 709 \\
\hline Th-230 & $1.38 E-03$ & $1.45 E+03$ & 7.2 & 26 & 6.1 & 6080 \\
\hline $\mathrm{Ra}-226 \mathrm{D}$ & $1.74 \mathrm{E}-03$ & $1.15 E+03$ & 9.0 & 32 & 3.2 & 11379 \\
\hline $\mathrm{Pb}-210 \mathrm{D}$ & $3.24 \mathrm{E}-02$ & $6.17 \mathrm{E}+01$ & 9.0 & 32 & 6 & 327 \\
\hline Pu-240 & $1.56 \mathrm{E}-03$ & $1.28 \mathrm{E}+03$ & 3.7 & 13 & 14.1 & 1179 \\
\hline U-236 & 6.70E-03 & $2.99 \mathrm{E}+02$ & 3.7 & 13 & 10.1 & 384 \\
\hline Th-232 & 1.37E-03 & $1.46 \mathrm{E}+03$ & 5.5 & 19 & 7.1 & 3967 \\
\hline Ra-228D & 1.22E-01 & $1.64 \mathrm{E}+01$ & 9.0 & 32 & 3.3 & 158 \\
\hline $\mathrm{U}-232$ & 1.63E-02 & $1.23 \mathrm{E}+02$ & 7.2 & 26 & 6.7 & 466 \\
\hline Th-228D & 3.64E-01 & $5.50 \mathrm{E}+00$ & 9.0 & 32 & 3.2 & 55 \\
\hline Am-243D & $1.49 \mathrm{E}-03$ & $1.34 \mathrm{E}+03$ & 9.0 & 32 & 4.6 & 9270 \\
\hline Pu-239 & 1.48E-03 & $1.35 \mathrm{E}+03$ & 7.2 & 26 & 6.1 & 5633 \\
\hline U-235D & 6.70E-03 & $2.99 \mathrm{E}+02$ & 9.0 & 32 & 4.2 & 2259 \\
\hline $\mathrm{Pa}-231$ & 1.43E-03 & $1.40 \mathrm{E}+03$ & 9.0 & 32 & 3.7 & 12033 \\
\hline Ac-227D & 3.33E-02 & $6.01 \mathrm{E}+01$ & 9.0 & 32 & 3.8 & 503 \\
\hline Am-241 & 3.00E-03 & $6.67 \mathrm{E}+02$ & 5.5 & 19 & 8 & 1604 \\
\hline Np-237D & 8.41E-03 & $2.38 \mathrm{E}+02$ & 9.0 & 32 & 3.9 & 1937 \\
\hline U-233 & 6.70E-03 & $2.99 \mathrm{E}+02$ & 9.0 & 32 & 4.9 & 1935 \\
\hline Th-229D & 1.46E-03 & $1.37 E+03$ & 9.0 & 32 & 3.8 & 11447 \\
\hline
\end{tabular}

NOTE: Column 2 from Table 7.4-4. Column 3 calculated using Equation 6.4.1-4, 6.4.1-5, and input values from Table 6.6-3. Column 4 calculated using part of Equation 6.4.7-1 (outdoor time + indoor time $\times$ external shielding factor). Column 5 calculated as the ratio of Column 4 to an average water immersion time of 17 minutes. Column 6 taken from Table 7.4-17. Column 7 calculated as Column $3 \times$ Column $5 \div$ Column 6. These calculations are performed using Excel spreadsheet, ERMYN validation.xIs, which is listed in Appendix A.

\subsubsection{Inhalation Submodel}

The ERMYN inhalation submodel uses human activity and environment-specific exposure times, breathing rates, and mass loading. The magnitude of other factors, such as resuspension enhancement factors, also are a function of human activities. Information on human activity and environments is used to obtain accurate results and to include uncertainty about the input parameters. The validation models use simpler methods than those used in the ERMYN, using, for example, average exposure times rather than population and activity related exposure times (Section 7.3.8). Therefore, a comparison of activity inhaled per day (Table 7.4-19), using average values, is done to evaluate differences between the two approaches.

Using input values from Table 6.6-3, the inhaled activity is $1.5 \times 10^{-5} \mathrm{~Bq} / \mathrm{d}$. Using the approach from the validation models and a method similar to that used for the biosphere model for the TSPA-SR, the dose is $1.2 \times 10^{-5} \mathrm{~Bq} / \mathrm{d}$. This result is based on a weighted average exposure time 
where indoor time is weighted at 50 percent of outdoor time, and other parameter values are shown in Table 7.4-19. The results of the two methods are comparable, and ACM 7 (Section 6.3.3) does not need further consideration.

Table 7.4-19. Evaluation of Population and Environmental Related Inhalation Submodel

\begin{tabular}{|c|c|c|c|c|c|c|c|c|}
\hline \multirow[b]{2}{*}{ Parameter } & \multirow[b]{2}{*}{ Units } & \multirow[b]{2}{*}{ Notation } & \multicolumn{5}{|c|}{ Environment } & \multirow{2}{*}{$\begin{array}{l}\text { Selected } \\
\text { Average } \\
\text { Value for } \\
\text { Simple } \\
\text { Inhalation } \\
\text { Submode }\end{array}$} \\
\hline & & & $\begin{array}{c}\text { Active } \\
\text { Outdoors }\end{array}$ & $\begin{array}{l}\text { Inactive } \\
\text { Outdoors }\end{array}$ & $\begin{array}{c}\text { Active } \\
\text { Indoors }\end{array}$ & Asleep & $\begin{array}{l}\text { Away } \\
\text { from } \\
\text { Area }\end{array}$ & \\
\hline $\begin{array}{l}\text { Exposure time, } \\
\text { commuter }\end{array}$ & hr/day & $t(n, m)$ & 0.3 & 1.4 & 6.0 & 8.3 & 8.0 & 8.9 \\
\hline $\begin{array}{l}\text { Exposure time, } \\
\text { outdoor worker }\end{array}$ & hr/day & $t(n, m)$ & 3.1 & 4.0 & 6.6 & 8.3 & 2 & 14.6 \\
\hline $\begin{array}{l}\text { Exposure time, } \\
\text { indoor worker }\end{array}$ & hr/day & $t(n, m)$ & 0.3 & 1.3 & 12.1 & 8.3 & 2 & 11.8 \\
\hline $\begin{array}{l}\text { Exposure time, } \\
\text { nonworker }\end{array}$ & hr/day & $t(n, m)$ & 0.3 & 1.2 & 12.2 & 8.3 & 2 & 11.8 \\
\hline $\begin{array}{l}\text { Exposure time, } \\
\text { weighted } \\
\text { average }\end{array}$ & hr/day & $t(n, m)$ & 0.45 & 1.45 & 9.45 & 8.30 & 4.35 & 10.8 \\
\hline Breathing rate & $\mathrm{m}^{3} / \mathrm{hr}$ & $B R(n)$ & 1.57 & 1.08 & 1.08 & 0.39 & 1.08 & 1.08 \\
\hline Mass loading & $\mathrm{kg} / \mathrm{m}^{3}$ & $S(n)$ & $5.0 \mathrm{E}-6$ & 6.0E-8 & 1.0E-7 & 3.0E-8 & 0 & 1.0E-06 \\
\hline $\begin{array}{l}\text { Enhancement } \\
\text { factor }\end{array}$ & none & $f_{\text {enhance, } n}$ & 4.0 & 0.7 & 0.7 & 0.7 & 0 & 1.0 \\
\hline $\begin{array}{l}\text { Calculated } \\
\text { inhaled activity } \\
\text { per day }\end{array}$ & $\mathrm{Bq} / \mathrm{d}$ & & $1.4 \mathrm{E}-05$ & 6.6E-08 & 7.1E-07 & $6.8 \mathrm{E}-08$ & $0.0 \mathrm{E}+00$ & \\
\hline Sum & $\mathrm{Bq} / \mathrm{d}$ & & 1.5E-05 & & & & & $1.2 \mathrm{E}-05$ \\
\hline
\end{tabular}

NOTE: Calculations based on population group fractions: commuters $=0.392$; outdoor workers $=0.055$; indoor workers $=0.161$; nonworkers $=0.392$. Calculation performed using an Excel spreadsheet, ERMYN validation.xIs, which is listed in Appendix $A$.

\subsubsection{Ingestion Submodel}

The ERMYN ingestion submodel is similar to the validation models (Section 7.3.9). There are no ACMs for the ingestion submodel (Section 6.3.3), and no complex numerical comparisons are required to validate the submodel.

\subsection{VALIDATION OF THE GOLDSIM OUTPUT}

To validate the GoldSim implementation, BDCFs generated with the ERMYN are compared to those from the previous biosphere model (implemented in GENII-S) to determine if they are similar. For the groundwater scenario, a direct comparison of BDCFs is possible; however, the conceptual models for the volcanic ash scenarios differ, so a conversion is required before the BDCFs can be compared. The comparisons are based on the results of stochastic runs using ${ }^{239} \mathrm{Pu}$ as an example radionuclide. 


\subsubsection{Validation of the GoldSim Output for the Groundwater Scenario}

For the groundwater scenario, the ${ }^{239} \mathrm{Pu}$ BDCF generated using the ERMYN is $(9.16 \pm 3.59) \times 10^{-3}(\mathrm{rem} / \mathrm{year}) /(\mathrm{pCi} / \mathrm{L}) \quad($ Section 6.10 .1 .2$)$, while that generated using the GENII-S model is $(5.78 \pm 2.14) \times 10^{-3}$ (rem/year)/(pCi/L) (CRWMS M\&O 2001 [DIRS 152539], Table 9, sixth irrigation period). In general, the ERMYN BDCFs are higher than the GENII-S BDCFs because several new pathways are added to the ERMYN (e.g., inhalation of aerosols from evaporative coolers). The use of radionuclide equilibrium concentrations in the soil also increases the BDCFs. The uncertainty associated with BDCFs for the two models is comparable, with one standard deviation equaling about 40 percent of the mean value.

\subsubsection{Validation of the GoldSim Output for the Volcanic Ash Scenario}

For the volcanic ash scenario, the ${ }^{239} \mathrm{Pu}$ BDCF generated using the ERMYN (Section 6.10.2.2) is $(1.24 \pm 1.58) \times 10^{-9}(\mathrm{rem} /$ year $) /\left(\mathrm{pCi} / \mathrm{m}^{2}\right)$ for external exposure and ingestion, $(9.12 \pm 5.52) \times 10^{-7}(\mathrm{rem} /$ year $) /\left(\mathrm{pCi} / \mathrm{m}^{2}\right)$ for inhalation under post-volcanic conditions, and $(1.84 \pm 1.13) \times 10^{-6}(\mathrm{rem} /$ year $) /\left(\mathrm{pCi} / \mathrm{m}^{2}\right)$ for inhalation under normal conditions. The ${ }^{239} \mathrm{Pu}$ BDCF from the GENII-S model (CRWMS M\&O (2001 [DIRS 152536], Table 11), is $(1.51 \pm 1.31) \times 10^{-7}(\mathrm{rem} /$ year $) /\left(\mathrm{pCi} / \mathrm{m}^{2}\right)$ for the transition phase, $1-\mathrm{cm}$ ash layer and annual average mass loading. To make the comparison, the BDCFs from the ERMYN are combined using Equation 6.5.8-2, converted to a 1-cm ash layer using Equation 6.5.1-5, and calculated for 1-year of decay using the mass loading function $f(t)$ defined for 1-cm initial ash depth in Table 6.6-3. The time function is $0.82\left(=\mathrm{e}^{-0.2 / \mathrm{yr} \times 1 \mathrm{yr}}\right)$, and the ash thickness function is $0.2(=2 \mathrm{~mm} / 1 \mathrm{~cm})$. Therefore, the combined BDCF from the ERMYN is about $5.19 \times 10^{-7}$ (rem/year) $/\left(\mathrm{pCi} / \mathrm{m}^{2}\right)$, which is comparable to the BDCF from the GENII-S model, $1.51 \times 10^{-7}$ (rem/year) $/\left(\mathrm{pCi} / \mathrm{m}^{2}\right)$. The BDCFs from the ERMYN are higher than the BDCFs from the GENII-S model because of increased overall mass loading concentrations from the environment-specific parameters, which makes inhalation an important pathway.

In conclusion, BDCFs generated with the ERMYN are similar to those from the GENII-S model, which validates the GoldSim implementation of the ERMYN.

\subsection{MODEL VALIDATION RANGE}

The previous detailed discussion has shown that the ERMYN is valid over the ranges of input parameter values which are reasonably expected to occur in the arid to semi-arid region surrounding Yucca Mountain. To reach this conclusion, it was not necessary to conduct detailed mathematical evaluations over the reasonable ranges of all parameters because the model is based primarily on linear equations for the proportional transfer of radionuclides from one medium to another. Accordingly, the equations produce proportional results across the entire ranges of the input parameters for which the model has been validated. For the few equations which include nonlinear calculations, (e.g., the weathering function in the calculation of the dust interception fraction, Equation 6.4.3-6, asymptotes to 1.0 at relatively low values of growing time), either the results asymptotically approach reasonable limits or are limited in GoldSim to prevent invalid results (e.g., the irrigation interception fraction, Equation 6.4.3-5, is limited to a maximum of 1.0; Section 6.8.4). 
Numerical comparisons for model validation and ACM evaluations (Section 7.4) use the mean, mode, or other single values representative of the input parameters distributions listed in Table 6.6-3. The same values are used for both methods being compared (unless the ERMYN does not use a parameter value). Using values representative of distributions other than those in Table 6.6-3 would cause the results of both methods to change proportionally. Therefore, conclusions of the validation analyses, generally, are independent of input parameter values.

The outputs of the ERMYN, BDCFs, represent the annual dose to the RMEI, a receptor with average characteristics of the Amargosa Valley population, and the use of input data for other time frames or for specific individuals and environments might produce invalid results. In particular, seven parameters might cause problems if unreasonable values are used (Table 7.6-1). Thus, input parameters representative of periods other than a year or representative of individual people, dwellings, cultivated fields, or other items may cause invalid results. For example, using a minimum daily wind speed rather than the average annual wind speed (Equation 6.4.6-3) would result in invalid output.

Table 7.6-1. Unreasonable Parameters Input Values

\begin{tabular}{|c|c|c|}
\hline $\begin{array}{c}\text { Parameter } \\
\text { name }\end{array}$ & $\begin{array}{l}\text { Used in } \\
\text { Equation }\end{array}$ & Discussion \\
\hline $\begin{array}{l}\text { Surface soil } \\
\text { depth }\end{array}$ & $\begin{array}{l}6.4 .1-5 \\
6.4 .1-6 \\
6.4 .1-11 \\
6.5 .1-2\end{array}$ & $\begin{array}{l}\text { Very low values of surface soil depth (i.e., } \mathrm{mm} \text { to few } \mathrm{cm} \text { ) would result in invalid, high } \\
\text { concentrations of radionuclides in the soil. Such low values are unreasonable } \\
\text { because crops cannot grow in soil irrigated or tilled to a very shallow depth. The } \\
\text { distribution of surface soil depths (Table 6.6-3 ; BSC } 2004 \text { [DIRS 169673], } \\
\text { Section 6.10) is based on typical tilling depths. }\end{array}$ \\
\hline $\begin{array}{l}\text { Evaporative } \\
\text { cooler air flow } \\
\text { rates }\end{array}$ & $6.4 .2-3$ & $\begin{array}{l}\text { Unreasonably low air flow rates would result in invalid, high concentrations of } \\
\text { radionuclides in indoor air. Typical air flow rates for evaporative coolers range from } \\
\left.\text { about } 2,000 \text { to } 10,000 \mathrm{~m}^{3} / \mathrm{h} \text { (BSC } 2004 \text { [DIRS } 169672 \text { ] Section } 6.5 .2\right) \text {. Much lower } \\
\text { rates are unreasonable because building cooling would be ineffective. In addition, } \\
\text { low rates would not transfer radionuclides from water to the entire volume of indoor } \\
\text { air. }\end{array}$ \\
\hline $\begin{array}{l}\text { House } \\
\text { ventilation } \\
\text { rate }\end{array}$ & $\begin{array}{l}6.4 .2-7 \\
6.4 .2-8\end{array}$ & $\begin{array}{l}\text { Unreasonably low house ventilation rates would result in an accumulation of indoor } \\
\text { radon and high estimates of indoor radon concentrations. Typical ventilation rates } \\
\text { are about } 0.5 \text { exchanges per hour or greater (BSC } 2004 \text { [DIRS 169672], } \\
\text { Section 6.6.2), and according to the Manufactured Home Construction and Safety } \\
\text { Standards, the whole house ventilation rate for manufactured homes should be at } \\
\text { least } 0.35 \text { air exchanges per hour (24 CFR 3280.103(b) [DIRS 160555]). }\end{array}$ \\
\hline $\begin{array}{l}\text { Crop wet } \\
\text { yield }\end{array}$ & $\begin{array}{l}6.4 .3-3 \\
6.4 \cdot 3-6 \\
6.5 \cdot 3-3\end{array}$ & $\begin{array}{l}\text { Very low values of crop yield would result in invalid, high estimates of radionuclide } \\
\text { concentrations in crops. Typical values of average annual yield for all production in a } \\
\text { region range from about } 1 \text { to } 8 \mathrm{~kg} / \mathrm{m}^{2} \text { for leafy vegetables to } 0.3 \text { to } 1.2 \mathrm{~kg} / \mathrm{m}^{2} \text { for grain } \\
\text { (BSC } 2004 \text { [DIRS 169673], Section } 6.11 \text { ). Much lower values are unreasonable } \\
\text { because few or no contaminated foodstuffs would be available for consumption. }\end{array}$ \\
\hline $\begin{array}{l}\text { Annual } \\
\text { average wind } \\
\text { speed }\end{array}$ & $6.4 .3-3$ & $\begin{array}{l}\text { Very low wind speeds, such as daily minimum wind speeds or wind speeds on very } \\
\text { calm days, would result in an invalid, high estimate of the annual average }{ }^{14} \mathrm{C} \\
\text { concentration in air. Parameter values must represent annual average speeds to } \\
\text { ensure that a reasonable annual average concentration is calculated. }\end{array}$ \\
\hline $\begin{array}{l}\text { Fraction of } \\
\text { stable carbon } \\
\text { in soil }\end{array}$ & $6.4 .6-4$ & $\begin{array}{l}\text { If the fraction of stable carbon in the soil is zero, calculations of root uptake of }{ }^{14} \mathrm{C} \text { in } \\
\text { crops would be invalid. This value is unreasonable because there is always some } \\
\text { stable carbon in the soil. }\end{array}$ \\
\hline $\begin{array}{l}\text { Concentration } \\
\text { of stable } \\
\text { carbon in air }\end{array}$ & $6.4 .6-5$ & $\begin{array}{l}\text { If the fraction of stable carbon in the air is zero, calculations of leaf uptake of }{ }^{14} \mathrm{C} \text { in } \\
\text { crops would be invalid. This value is unreasonable because there is always some } \\
\text { stable carbon in the air. }\end{array}$ \\
\hline
\end{tabular}




\subsection{EXTERNAL REVIEW OF THE ERMYN}

An external review was conducted by J. I. Daniels (D.Env.) of the Environmental Science Division in the Energy \& Environment Directorate of the LLNL in accordance with AP-SIII.10Q (see also Section 7.1.1). The finding of this review (Daniels 2003 [DIRS 163016]) adds to confidence in the model. The external review concluded:

The ERMYN model is a more complete biosphere modeling tool than its predecessor. It is well constructed, it conforms to design requirements prescribed by site-specific features, events, and processes, and it is appropriate for the specified human receptor (the reasonably maximally exposed individual). The model contains a thorough assembly of applicable multimedia, multipathway exposure-related processes, and it uses a credible dose-assessment strategy using reliably calculated, acceptable, and applicable biosphere dose conversion factors for the radionuclides of interest. The authors provide a more transparent framework and a better explanation of the applicability and validity of the submodels, and they better document the assumptions.

The ERMYN model contains several new exposure pathways, including inhalation of aerosols generated by residential evaporative cooling, animal soil ingestion, and radon-gas inhalation, particularly from surface deposited Ra-226. The outputs (biosphere dose conversion factors) are understandable and appropriate, and I agree that differences in biosphere dose conversion factors between those generated by the ERMYN model and those produced by the earlier model are reasonable and can be explained by changes in the input parameters necessary to accommodate the ERMYN model framework. Thus, the ERMYN model displays progress and commitment to applying a scientifically defensible approach to a comprehensive assessment of the biosphere as a consequence of a potential release of radionuclides from the proposed repository.

Accordingly, the ERMYN model is an improvement over the previous model. Furthermore, the modeling deficiencies noted in the Model Validation Status Review technical report have been addressed adequately through the construction and description of the ERMYN model.

\subsection{SUMMARY OF MODEL VALIDATION}

Requirements for confidence building during model development (Section 7.1.1) have been satisfied. The descriptions of the comparisons between various representations of the many biosphere processes in the previous sections demonstrate that there is great deal of commonality in the approaches used in the various models. Because there are few differences in the methods used in modeling the biosphere processes and their resulting predictions, the methods used in the ERMYN are considered valid and accepted by the scientific community, and the results are consistent with the results generated by other process-level models.

Also, the post-development validation requirement defined in Section 7.1.2 has been fulfilled, including corroboration of model results with the alternative conceptual models. The model 
development and post-development validation activities described establish the scientific bases for the biosphere model. No future activities need to be accomplished for model validation. Based on this, the biosphere model is considered to be adequate and sufficiently accurate for the intended purpose and validated to the level of confidence required by the model's relative importance to the potential performance of the repository system. 


\section{INTENTIONALLY LEFT BLANK}




\section{CONCLUSIONS}

This report documents the development of the ERMYN biosphere model, which supports the TSPA-LA. The reference biosphere, the human receptor, two exposure scenarios, and the primary radionuclides used in the model are described. The conceptual model incorporates site-specific FEPs, the site-specific reference biosphere, a theoretical human receptor, and justifiable approximations. The mathematical model incorporates the conceptual model by using site-specific information and other information from five published models. The mathematical model input parameters are described. Improvements made to the ERMYN over the predecessor model, GENII-S for the TSPA-SR, are identified. The GoldSim stochastic simulation software package is used for building the ERMYN implementation tool. The ERMYN implementation tool (i.e., the constructed ERMYN) is verified by comparing outputs with hand calculations to ensure that the GoldSim implementation works in the manner described by the mathematical model. The ERMYN is validated by corroboration of conceptual models, mathematical models, and the necessary numerical results. All of the NRC acceptance criteria (NRC 2003 [DIRS 163274], Section 2.2.1.3.14) listed in Section 4.2 are addressed in detail in Section 6. Model and model inputs are discussed in Sections 6.3 to 6.5. Uncertainty in the model and input parameters are discussed in Section 6.6, and they are considered in the model implementation in Sections 6.8 and 6.9 .

\subsection{MODEL OUTPUT}

As discussed in the description of the biosphere documentation structure (Section 1), the model outputs from this report are not numerical results; rather, they are the model implementation tools built with the GoldSim software. Two biosphere model implementation tools, one for the groundwater scenario (ERMYN_GW.gsm) and one for the volcanic ash scenario (ERMYN_VA.gsm) (both listed in Appendix A), are the model outputs to be used for generating BDCFs. These two model implementation tools are used to calculate BDCFs for individual radionuclides, as well as for conducting pathway, uncertainty, and sensitivity analyses. The output data tracking number for the model implementation tools is MO0306MWDBGSMF.001 [DIRS 163816].

\subsection{BIOSPHERE MODEL LIMITATIONS}

The ERMYN applies to the specific environments identified in 10 CFR 63.305 [DIRS 156605]. If used for other situations, the results generated by the ERMYN may not apply. The radionuclide sources for the biosphere model are specific to the two exposure scenarios considered (contaminated groundwater and contaminated volcanic ash deposited on the ground). Although the mathematical models described in Sections 6.4 and 6.5 apply to all radionuclides, the ERMYN focuses on the radionuclides that were screened for the TSPA LA (BSC 2002 [DIRS 160059]). The model output, the ERMYN GoldSim model, is built for 28 primary radionuclides (Section 6.1.3). This model applies to assessing chronic radiation doses (Section 6.1). This model is valid for all values of input parameters reasonably expected to occur in the arid to semi-arid region surrounding Yucca Mountain. Users should be aware that some unreasonable input parameters values will produce invalid results (Section 7.6). 
For the groundwater scenario, the ERMYN applies to an agricultural situation with long-term irrigation and soil contamination at long-term equilibrium conditions. If radionuclides in soils are not at equilibrium, the ERMYN might overestimate the radiation dose. The biosphere model applies to an arid or semi-arid climate (Section 6.1.1.1), and it is valid only for limited groundwater discharge to the surface and limited surface water transport, as long as the radionuclide concentration in the surface water is the same as in the groundwater, and the reference biosphere is not greatly altered. For example, if permanent surface waters such as rivers or lakes are present, the environment would be sufficiently different to change the reference biosphere, and other pathways would have to be added for the ERMYN to remain valid.

For the volcanic ash exposure scenario, the model applies to a layer of small ash particles that could be resuspended into the air. The ash could be deposited directly on the land surface or it could be redistributed by wind or water from other locations. The model does not apply to other volcanic media, such as contaminated gas, lava, or coarse tephra. Some approximations (Approximations 12 and 15) used in the model development are based on thin ash deposits. However, these are justified considering the distribution of ash thickness predicted by the TSPA-LA model to occur at the location of the RMEI (DTN: MO0410MWDTLSCM.000 [DIRS 172208]). In an unlikely outcome of the TSPA-LA model, if thick ash deposits occur, the model might overestimate the radiation dose. In addition, the ERMYN does not apply during a volcanic eruption when volcanic ash is still in the air (i.e., before initial settling on the ground). If radionuclide concentrations in the air and the exposure time are known, a dose assessment for the eruption period could be considered as a simple inhalation scenario

\subsection{HOW ACCEPTANCE CRITERIA WERE ADDRESSED}

The following information describes how this analysis addresses the acceptance criteria in the Yucca Mountain Review Plan (NRC 2003 [DIRS 163274], Sections 2.2.1.3.13 and 2.2.1.3.14). Only those acceptance criteria that are applicable to this report (see Section 4.2) are discussed. In most cases, the applicable acceptance criteria are not addressed solely by this report; rather, the acceptance criteria are fully addressed when this report is considered in conjunction with other analysis and model reports that describe the Yucca Mountain Biosphere. Where a subcriterion includes several components, only some of those components may be addressed. How these components are addressed is summarized below.

\section{Acceptance Criteria from Section 2.2.1.3.13, Redistribution of Radionuclides in Soil}

\section{Acceptance Criterion 1, System Description and Model Integration are Adequate}

Subcriterion (1): This model, which supports the TSPA-LA, adequately incorporates important features, physical phenomena, and couplings related to redistribution of radionuclides in soil. Section 6.2 describes how the FEPs related to the biosphere were identified and why they are important. The included FEPs and the reasons for their inclusion are in Table 6.2-1. Section 6.3.1 explains how connected sequences of FEPs were used to construct the exposure scenarios. Section 6.3.1.2 identifies the components of the biosphere model. Section 6.3.4 and Table 6.3-6 show how the FEPS were mapped into the submodel constituents of the biosphere 
model to ensure that it addresses all of the included FEPs. Section 6.7.1 describes how these FEPs are dispositioned in the base case model.

Sections 6.3.1.4 and 6.3.2.4 discuss the 16 approximations incorporated in the model. For each approximation, a rationale is provided for its basis and cross-references are provided to where it is used in the model. Because this model calculates BDCFs (the annual dose per unit concentration of radionuclides) that are independent of the concentration of the radionuclides, there are few approximations that are shared with other TSPA process models. Those that are shared, such as the density of ash (Section 6.5.1) and future climatic conditions, are consistent and traceable to a common source with those other process models.

Subcriterion (2): Section 6.3.1.6 describes the conceptual model for the groundwater scenario and includes the supporting technical bases. Section 6.3.2.6 provide the equivalent information for the volcanic ash scenario. Included are the groundwater scenario submodels required to account for the radionuclides accumulation and removal processes in surface soil and depth distribution of radionuclides. Important individual components are rates of irrigation with water containing radionuclides and the removal mechanisms of decay, leaching, and erosion. For the biosphere model, soil is divided into the surface soil layer encompassing the crop root zone and the deep soil where radionuclides are assumed inaccessible to plants and other redistribution mechanisms. Radionuclide concentrations in the surface soil layer are discussed in Section 6.4.1 for the groundwater scenario and Section 6.5.1 for the volcanic ash scenario.

Subcriterion (3): Section 6.2 describes how the important FEPs related to redistribution of radionuclides from surface processes were identified. The included FEPs and the reasons for their inclusion are noted in Table 6.2-1. Of these FEPs, many are associated with radionuclide redistribution in soil for the groundwater scenario. The models and their technical bases developed to predict the redistribution of radionuclides are discussed in Sections 6.4.1 and 6.4.2. For the volcanic scenario, the appropriate sections are 6.5.1 and 6.5.2.

\section{Acceptance Criterion 2, Data are Sufficient for Model Justification}

Subcriterion (1): Applicable behavioral, hydrological, and geochemical values used in developing this model are adequately justified in Section 6. Section 4.1 explains that this biosphere model report describes the development and validation of the base case model, but that this report does not include a description of the development of the hundreds of input parameters used in the model. Adequate descriptions of how the data with the attendant uncertainty were used, interpreted, and appropriately synthesized into the model are provided in Section 6. Justifications for the input parameter values used are developed in the five separate input analysis reports, which are related to this report as shown in Figure 1-1.

Acceptance Criterion 3, Data Uncertainty in Characterized and Propagated Through the Model Abstraction

Subcriterion (1): Section 4.1 explains that parameter values are developed in related reports, which show that assumed ranges, probability distributions, and bounding approximations are technically defensible; reasonably account for uncertainties; and are consistent with the definition of the RMEI. Technical review activities for the ERMYN included an international 
peer review of the previous biosphere model by an IAEA International Review Team (IAEA 2001 [DIRS 155188]), a model validation status review by experts at LLNL (BSC 2003 [DIRS 170030]), and a technical review of the ERMYN implementation by another expert at LLNL (Daniels 2003 [DIRS 163016]). These reviews and the empirical nature of the data and parameters that will be employed by the model ensure that use of the model presents an accurate representation of the processes by which radionuclides in the soil expose individuals to doses from radioactivity. This process also ensures that the model does not result in an under-representation of the risk estimate.

Subcriterion (2): Section 4.1 explains that parameter values and ranges in the abstractions are developed in related reports specific to Yucca Mountain, which show that the parameter values are consistent with site characterization data, applicable laboratory testings, and natural analogs and are technically defensible. Section 6.4.1 presents the model developed to predict the radionuclide accumulation in soil for the groundwater scenario. Section 6.5.1 provides the approach to modeling radionuclides in soil after an ashfall. The input parameters developed for these submodels include site specific information on soil (BSC 2004 [DIRS 169459]) and current farming practices. This model fulfills the requirements in 10 CFR 63.312 by calculating exposure using receptor specific external exposure times, inhalation exposure times, food consumption rates, and resuspension of soil particles and level of disturbance expected in the location of the RMEI. Among the factors considered are diet, gardens, employment, commute time, housing type, metabolism, and physiology.

Subcriterion (3): Uncertainty is adequately represented in parameter development for the conceptual model related to redistribution of radionuclides in soil through adoption of conservative approach/bounding values (e.g., assuming radionuclides are in equilibrium through the compliance period [Section 6.3.1.4, Approximation 1]). Also, the model developed in Section 6 has the capability to stochastically sample all of those parameters that are represented by statistical distributions. Section 6.6.3 identifies those parameters that are represented in the model by distributions and provides details of the uncertainty in Table 6.6-3 where the correlation between stochastic variables is identified. Thus, the model that is the product output for this effort generates BDCFs for the TSPA-LA with due consideration for parametric uncertainty inherent in the redistribution of radionuclides in soil. Uncertainty in the parameters developed in related process models that feed the TSPA is discussed in the process model reports. Parameters for alternate conceptual models discussed in this report were typically those used in the conceptual model, and the uncertainties would be the same as those for the parameters in the conceptual model (Section 6.6.3).

Subcriterion (4): Parameters that are important for determining redistribution of radionuclides in soil were identified and implemented in the surface soil submodel (Section 6.4.1). These parameters include those related to repository performance measure (e.g., groundwater concentrations) and time period of compliance (e.g., equilibrium concentrations of radionuclides in soil). 
Acceptance Criterion 4, Model Uncertainty is Characterized and Propagated Through the Model Abstraction

Subcriterion (1): As explained in Sections 6.3 .4 and 6.6.1, the biosphere model is based on the inclusion of all potentially significant FEPs. Alternative models for the FEPs considered are discussed in Section 6.3.3 (Table 6.3-5). The discussion in Table 6.3-5 indicates that the approaches are consistent with available data and current scientific understanding. Results of the alternative models in Table 6.3-5 are, typically, shown to be comparable to those of the conceptual model (ERMYN), but the alternative models were less consistent with available data and scientific understanding or do not have an important effect on the model results.

Subcriterion (2): Seven existing, published ACMs are addressed in Section 6.3.3 for various submodel and submodel components of aspects of features and processes. These ACMs are either conceptually or mathematically different from their base-case alternatives. The ACMs were screened and evaluated qualitatively and quantitatively, and results using mathematical representations of them are compared with the results for the base case model in Section 7.3. Justifications for not adopting the ACMs into the base case model are provided in Sections 7.3 and 7.4.

Subcriterion (3): Conceptual model uncertainty is discussed in general in Section 6.6.1. As stated in section 6.6.1, all applicable human exposure pathways were considered during development of the conceptual model (Sections 6.3.1 and 6.3.2), and only applicable pathways shown to have little influence on the results are excluded. Uncertainties in the conceptual model are consistent with those of available Yucca Mountain data, related laboratory experiments, field measurements, natural analog information, and process-level modeling studies (Section 6.6.1). Uncertainty in mathematical models is discussed in Section 6.6.2. Uncertainties in the eleven model approximations are discussed qualitatively and their magnitudes and impacts are conservative as shown in Table 6.6-1. Section 6.6.4 explains how uncertainty in model results is taken into account. This stochastic approach, which uses the reasonably conservative model approximations discussed in Section 6.6.2, produces results that do not under-represent the risk estimate.

\section{Acceptance Criterion 5, Evaluation Findings}

Subcriterion (1): The individual models developed in Section 6 to quantify the movement of radionuclides between the various biosphere compartments are based on empirical observations (e.g., transfer coefficients, accumulation factors, transfer coefficients, etc.) and, as such, are consistent with the laboratory testing, field measurements, and natural analogs from which they were derived.

\section{Acceptance Criteria from Section 2.2.1.3.14, Biosphere Characteristics}

\section{Acceptance Criterion 1, System Description and Model Integration are Adequate}

Subcriterion (1): The biosphere model adequately incorporates important site features, physical phenomena, and couplings as shown in Section 6.2, which describes how the FEPs related to the biosphere were identified. The included FEPs and the reasons for their inclusion are in Table 6.2-1. Section 6.3.1 explains how connected sequences of FEPs were used to construct the 
exposure scenarios. Section 6.3.1.2 identifies the components of the biosphere model. Section 6.3.4 and Table 6.3-6 show how the FEPS were mapped into the submodel constituents of the biosphere model to ensure that it addresses all of the included FEPs. Section 6.7.1 describes how these FEPs are dispositioned in the base case model.

Section 6.3.1.4 discusses the 16 approximations or assumptions that were determined to be appropriate and that were applied consistently throughout the biosphere modeling abstraction process. For each approximation, a rationale is provided for its basis, and cross-references are provided to where it is used in the model.

Subcriterion (2): Section 6.1.1 describes how the model abstraction identifies and describes aspects of the biosphere characteristics modeling that are important to repository performance, and includes the technical bases for these descriptions. Section 6.1.1.1 addresses site geography, geology, and physiology; Section 6.1.1.2 addresses site climate, flora, and fauna; and Section 6.1.1.3 addresses groundwater, human activities, and agriculture in the Yucca Mountain vicinity.

Section 6.3.1.6 describes the conceptual model for the groundwater scenario and includes the supporting technical bases consistent with arid and semi-arid conditions (Section 6.3). Included are the submodels for the groundwater source, surface soil, air, plant uptake, animal uptake, fish, ${ }^{14} \mathrm{C}$, external exposure, inhalation, and ingestion. Section 6.3.2.1 describes the conceptual model for the volcanic ash exposure scenario.

Subcriterion (3): Assumptions employed in the biosphere model are consistent with the assumptions made in other abstractions. Section 6.2 describes how the FEPs related to the biosphere were identified and included. Because this model calculates BDCFs (the annual dose per unit concentration of radionuclides) that are independent of the concentration of the radionuclides, many assumptions are specific to this model and are not shared with other TSPA process models. Those that are shared are, generally, characteristics of the reference biosphere [e.g., characteristics of ash in soil (Section 6.5.1), climate and climate change (Section 6.1.1.2), properties of radionuclides, and soil types] that are consistent across other process models incorporated in the TSPA-LA.

\section{Acceptance Criterion 2, Data are Sufficient for Model Justification}

Subcriterion (1): Applicable behavioral values used in developing this model are justified in Section 6 and are consistent with the definition of the RMEI. Section 4.1 explains that this biosphere model report describes the development and validation of the base case model, but that this report does not include a description of the development of the hundreds of input parameters used in the model. Descriptions of how the data with the attendant uncertainty were used, interpreted, and appropriately synthesized into the model are provided in Section 6 . Justifications for the input parameter values used are developed in the five separate input analysis reports, which are related to this report as shown in Figure 1-1.

Subcriterion (2): Section 6.2 describes the features, events, and processes that have been considered and incorporated in the biosphere model. Table 6.2-1 discusses each of the FEPs considered, their characteristics, and provides an explanation for their applicability within the 
model. This table also presents the basis for the FEPs, which indicates that they are consistent with present knowledge of conditions in the Yucca Mountain region.

Acceptance Criterion 3, Data Uncertainty is Characterized and Propagated Through the Model Abstraction

Subcriterion (1): Section 4.1 explains that parameter values are developed in related reports, which document that assumed ranges, probability distributions and bounding approximations are technically defensible, reasonably account for uncertainties, and are consistent with the definition of the RMEI. The mathematical model, as implemented in GoldSim (Section 6.8), has the capability of representing any input parameter by a predefined distribution. This approach allows a stochastic approach to be adopted where uncertainty and variability in the input parameters can be propagated to the end results (BDCFs) while retaining the correlation of inputs for each radionuclide so modeled. The five parameter-generating reports (Figure 1-1) focus on deriving site, climate, and receptor specific input parameter distributions from site data, scientific analysis, and natural analog information that are technically defensible.

Technical review activities for the ERMYN included an international peer review of the previous biosphere model by an IAEA International Review Team (IAEA 2001 [DIRS 155188]), a model validation status review by experts at LLNL (BSC 2003 [DIRS 170030]), and a technical review of the ERMYN implementation by another expert at LLNL (Daniels 2003 [DIRS 163016]). These reviews and the empirical nature of the data and parameters that will be employed by the model ensure that use of the model presents an accurate representation of the processes by which radionuclides in the soil expose individuals to doses from radioactivity. This process also ensures that the model does not result in an under-representation of the risk estimate.

Subcriterion (4): Uncertainty is adequately represented in parameter development for the conceptual model related to biosphere characteristics through adoption of a conservative approach and/or bounding values, e.g., approximating the radionuclide concentration in surface soil by the equilibrium value (Section 6.3.1.4, Approximation 5) based on a constant radionuclide concentration in groundwater (Section 6.3.1.4, Approximation 1). Also, the model developed in Section 6 has the capability to stochastically sample all parameters that are represented by statistical distributions. Section 6.6.3 identifies those parameters that are represented in the model by distributions and provides details of the uncertainty in Table 6.6-3, where the correlation, if any, between stochastic model parameters is identified. Thus, the model that is the product output for this effort is used to generate BDCFs for TSPA-LA with due consideration for parametric uncertainty inherent in the redistribution of radionuclides in soil.

Subcriterion (6): Parameters and models that are important for determining biosphere characteristics were identified and implemented in the various submodels (Sections 6.4 and 6.5). Section 6.3.3 discusses those alternate submodels and parameters that were considered to not be important in determining dose. These parameters include those related to repository performance measure (e.g., groundwater concentrations) and time period of compliance (e.g., equilibrium concentrations of radionuclides in soil). 
Acceptance Criterion 4, Model Uncertainty is Characterized and Propagated Through the Model Abstraction.

Subcriterion (1): Seven alternate modeling approaches of FEPs are analyzed in Section 6.3.3. Section 7.3 compares the current GoldSim model with other models and submodels, including those that are considered as alternate models. These approaches are consistent with current scientific understanding and characteristics of the Yucca Mountain region. In many cases, the fundamental models in use by the radiological protection community have general acceptance and, as a result, are not subject to having alternative approaches. Differences, where identified, are discussed in Section 7.3. The stochastic approach described in Sections 6.8 and 6.9 allow the defined uncertainty and variability in parameters (including those parameters necessary to characterize the RMEI from the available data on the Amargosa Valley residents) to be propagated to the model output.

Subcriterion (2): Seven alternate conceptual models are discussed in Section 6.3.3. Section 7.3 compares the model implemented in GoldSim to represent the Yucca Mountain biosphere with a suite of other models that have been independently developed to perform similar radiological assessments of doses from radionuclides introduced into the accessible environment. Processes considered include plant and animal uptake, human ingestion of water and locally produced food, soil resuspension, and inhalation dose for both scenarios. In many cases, each of the modeling capabilities identified employ the same model for a process. In these cases, it is considered that the models are accepted and no alternative conceptual model is required. In those cases where the modeling approaches were not identical, these other methodologies were evaluated and justification was provided for selection of the included method.

Subcriterion (3): Conceptual model uncertainty is discussed in general in Section 6.6.1. As stated in section 6.6.1, all applicable human exposure pathways were considered during development of the conceptual model (Sections 6.3.1 and 6.3.2), and only applicable pathways shown to have little influence on the results are excluded. For example, air submersion and water immersion pathways are not included because numerical comparisons made between dose coefficients and typical exposure times indicate that they are not important when compared with the included pathways. By virtue of the general acceptance of the individual submodels (Section 7.3) and the discussions of conceptual model uncertainty (Section 6.6.1) and mathematical model uncertainty (Section 6.6.2) with their appropriate conservative development, the dominant contribution to total uncertainty arises from parametric uncertainty and variability. Uncertainties in the conceptual model are consistent with those of available Yucca Mountain data, related laboratory experiments, field measurements, natural analog information, and process-level modeling studies (Sections 6.4 through 6.6). As a result, risk (dose) estimates based on the BDCFs provided to the TSPA for the LA compliance calculations do not result in an under representation of risk.

\section{Acceptance Criterion 5, Model Abstraction Output is Supported by Objective Comparisons}

Subcriterion (1): The biosphere model provides the total system performance assessment the capability of calculating dose arising from two sources of radionuclides in the biosphere. The model developed in Section 6 is based upon accepted radiological transport predictive practices. The parameters and submodels reflect many simple linear relationships that are based upon 
experimental data and, as such, have an empirical basis. Accordingly, this model provides a mechanism for the TSPA abstraction to determine dose levels based on empirical observations. 


\section{INTENTIONALLY LEFT BLANK}




\section{INPUTS AND REFERENCES}

The following is a list of the references cited in this document. Column 2 represents the unique six digit numerical identifier (the Document Input Reference System number), which is placed in the text following the reference callout (e.g., BSC 2002 [DIRS 160059]). The purpose of these numbers is to assist in locating a specific reference. Within the reference list, multiple sources by the same author (e.g., BSC 2002) are sorted alphabetically by title.

\subsection{DOCUMENTS CITED}

Anspaugh, L.R. 1987. Retention by Vegetation of Radionuclides Deposited in Rainfall:

123696 A Literature Summary. UCRL-53810. Livermore, California: Lawrence Livermore National Laboratory. TIC: 237907.

Baes, C.F., III and Sharp, R.D. 1983. “A Proposal for Estimation of Soil Leaching and 109606 Leaching Constants for Use in Assessment Models.” Journal of Environmental Quality, 12, (1), 17-28. Madison, Wisconsin: American Society of Agronomy. TIC: 245676.

BIOMASS (Biosphere Modelling and Assessment) 2000. Example Reference Biosphere 2A: Agricultural Well, Constant Biosphere. Draft TECDOC. BIOMASS/T1/WD08. Vienna, Austria: International Atomic Energy Agency, Division of Radiation and Waste Safety. TIC: 249456.

BIOMASS (The IAEA Programme on Biosphere Modelling and Assessment Methods) 159468 2001. "Themes for a New Co-ordinated Research Programme on Environmental Model Testing and Improvement: Theme 1: Radioactive Waste Disposal, Theme 2: Environmental Releases, Theme 3: Biospheric Processes.” Working Material, Limited Distribution, Biosphere Modelling and Assessment, Biomass Programme. Version $\beta 2$. Vienna, Austria: International Atomic Energy Agency. TIC: 252966.

BSC (Bechtel SAIC Company) 2002. Guidelines for Developing and Documenting Alternative Conceptual Models, Model Abstractions, and Parameter Uncertainty in the Total System Performance Assessment for the License Application.

TDR-WIS-PA-000008 REV 00 ICN 01. Las Vegas, Nevada: Bechtel SAIC Company. ACC: MOL.20020904.0002.

BSC 2002. Radionuclide Screening. ANL-WIS-MD-000006 REV 01. Las Vegas, Nevada: Bechtel SAIC Company. ACC: MOL.20020923.0177.

BSC 2002. Scientific Processes Guidelines Manual. MIS-WIS-MD-000001 REV 01. 
BSC 2002. Validation Test Report, GoldSim Version 7.50.100.

SDN: 10344-VTR-7.50.100-00. Las Vegas, Nevada: BSC (Bechtel SAIC Company).

ACC: MOL.20030312.0227.

BSC 2003. Agricultural and Environmental Input Parameters for the Biosphere

160976 Model. ANL-MGR-MD-000006 REV 01. Las Vegas, Nevada: Bechtel SAIC Company. ACC: DOC.20030624.0004.

BSC 2003. Biosphere Model Report. MDL-MGR-MD-000001 REV 00. Las Vegas, 164186 Nevada: Bechtel SAIC Company. ACC: DOC.20030721.0001.

BSC 2003. Biosphere Model Report. MDL-MGR-MD-000001 REV 00 Errata 002. Las Vegas, Nevada: Bechtel SAIC Company. ACC: DOC.20030721.0001; DOC.20031014.0002; DOC.20031028.0001.

BSC 2003. Characteristics of the Receptor for the Biosphere Model. ANL-MGR-MD-000005 REV 02. Las Vegas, Nevada: Bechtel SAIC Company. ACC: DOC.20030701.0001.

BSC 2003. Environmental Transport Input Parameters for the Biosphere Model. ANL-MGR-MD-000007 REV 01. Las Vegas, Nevada: Bechtel SAIC Company. ACC: DOC.20030701.0002.

BSC 2003. Inhalation Exposure Input Parameters for the Biosphere Model. ANL160965 MGR-MD-000001 REV 02 ICN 00. Las Vegas, Nevada: Bechtel SAIC Company. ACC: DOC.20030611.0002.

BSC 2003. Model Validation Status Review. TDR-WIS-MD-000005 REV 00 Errata 1. Las Vegas, Nevada: Bechtel SAIC Company. ACC: MOL.20011211.0095; DOC.20031027.0004.

BSC 2003. Soil-Related Input Parameters for the Biosphere Model. ANL-NBS-MD-000009 REV 01. Las Vegas, Nevada: Bechtel SAIC Company. ACC: DOC.20030707.0002.

BSC 2003. Technical Work Plan for: Biosphere Modeling and Expert Support. TWP-NBS-MD-000004 REV 01. Las Vegas, Nevada: Bechtel SAIC Company. ACC: DOC.20030604.0001.

BSC 2003. Total System Performance Assessment-License Application Methods and 166296 Approach. TDR-WIS-PA-000006 REV 00 ICN 01. Las Vegas, Nevada: Bechtel SAIC Company. ACC: DOC.20031215.0001. 
BSC 2003. Yucca Mountain Project Summary of Socioeconomic Data Analyses

168723

Conducted in Support of the Radiological Monitoring Program, During FY 2003. TDR-MGR-EV-000040 REV 00. Las Vegas, Nevada: Bechtel SAIC Company. ACC: DOC.20031203.0003.

BSC 2004. Agricultural and Environmental Input Parameters for the Biosphere Model. ANL-MGR-MD-000006 REV 02. Las Vegas, Nevada: Bechtel SAIC Company. ACC: DOC.20040915.0007.

BSC 2004. Characteristics of the Receptor for the Biosphere Model.

169671 ANL-MGR-MD-000005 REV 03. Las Vegas, Nevada: Bechtel SAIC Company. ACC: DOC.20040913.0004.

BSC 2004. Disruptive Event Biosphere Dose Conversion Factor Analysis. ANL-MGR-MD-000003 REV 03. Las Vegas, Nevada: Bechtel SAIC Company. ACC: DOC.20040908.0007.

BSC 2004. Environmental Transport Input Parameters for the Biosphere Model. 169672 ANL-MGR-MD-000007 REV 02. Las Vegas, Nevada: Bechtel SAIC Company. ACC: DOC.20040913.0003.

BSC 2004. Evaluation of Features, Events, and Processes (FEP) for the Biosphere 169826 Model. ANL-MGR-MD-000011 REV 04. Las Vegas, Nevada: Bechtel SAIC Company. ACC: DOC.20041028.0002.

BSC 2004. Future Climate Analysis. ANL-NBS-GS-000008 REV 01. Las Vegas, Nevada: Bechtel SAIC Company. ACC: DOC.20040908.0005.

BSC 2004. Inhalation Exposure Input Parameters for the Biosphere Model. ANL-MGR-MD-000001 REV 03. Las Vegas, Nevada: Bechtel SAIC Company. ACC: DOC.20040913.0001.

BSC 2004. Nominal Performance Biosphere Dose Conversion Factor Analysis. ANL-MGR-MD-000009 REV 03. Las Vegas, Nevada: Bechtel SAIC Company. ACC: DOC.20040909.0003.

BSC 2004. Q-List. 000-30R-MGR0-00500-000-000 REV 00. Las Vegas, Nevada: 168361 Bechtel SAIC Company. ACC: ENG.20040721.0007.

BSC 2004. Soil-Related Input Parameters for the Biosphere Model. ANL-NBS-MD-000009 REV 02. Las Vegas, Nevada: Bechtel SAIC Company. ACC: DOC.20040913.0002. 
BSC 2004. Technical Work Plan for Biosphere Modeling and Expert Support.

TWP-NBS-MD-000004 REV 03. Las Vegas, Nevada: Bechtel SAIC Company.

ACC: DOC.20040527.0004.

BSC 2004. Yucca Mountain Site Description. TDR-CRW-GS-000001 REV 02 ICN 01.

169734

Two volumes. Las Vegas, Nevada: Bechtel SAIC Company.

ACC: DOC.20040504.0008.

Bureau of the Census. 2001. Census 2000 Summary File 1 (ASCII) 2000, Census of

Population and Housing, California, Montana, Nebraska, Nevada, New Hampshire, New Jersey, and New Mexico. Washington, D.C.: Bureau of the Census.

TIC: 251242.

Bureau of the Census. 2002. "2000 Summary File 3 (SF 3) Sample Data, Amargosa Valley CCD, Nye County, Nevada." Washington, D.C.: U.S. Department of Commerce, Bureau of the Census. Accessed August 28, 2002. TIC: 253098. http://factfinder.census.gov/servlet/DTTable?_ts=48597952130

Canori, G.F. and Leitner, M.M. 2003. Project Requirements Document. TER-MGR-MD-000001 REV 02. Las Vegas, Nevada: Bechtel SAIC Company. ACC: DOC.20031222.0006.

Chow, J.C.; Watson, J.G.; Green, M.C.; Lowenthal, D.H.; DuBois, D.W.; Kohl, S.D.; Egami, R.T.; Gillies, J.; Rogers, C.F.; Frazier, C.A.; and Cates, W. 1999. "Middle- and Neighborhood-Scale Variations of PM10 Source Contributions in Las Vegas, Nevada." Journal of the Air \& Waste Management Association, 49, 641-654. Pittsburgh, Pennsylvania: Air \& Waste Management Association. TIC: 247013.

Chow, J.C.; Watson, J.G.; Lowenthal, D.H.; Solomon, P.A.; Magliano, K.L.; Ziman, 162999 S.D.; and Richards, L.W. 1993. " $\mathrm{PM}_{10}$ and $\mathrm{PM}_{2.5}$ Compositions in California's San Joaquin Valley.” Aerosol Science and Technology, 18, (2), 105-128. New York, New York: Elsevier. TIC: 251303.

CRWMS (Civilian Radioactive Waste Management System) M\&O (Management and 102235 Operating Contractor) 1996. The Vegetation of Yucca Mountain: Description and Ecology. B00000000-01717-5705-00030 REV 00. Las Vegas, Nevada:

CRWMS M\&O. ACC: MOL.19970116.0055.

CRWMS M\&O 1997. Engineering Design Climatology and Regional Meteorological Conditions Report. B00000000-01717-5707-00066 REV 00. Las Vegas, Nevada:

CRWMS M\&O. ACC: MOL.19980304.0028. 
CRWMS M\&O 1997. Yucca Mountain Site Characterization Project Summary of

101090

Socioeconomic Data Analyses Conducted in Support of the Radiological Monitoring Program First Quarter 1996 to First Quarter 1997. Las Vegas, Nevada:

CRWMS M\&O. ACC: MOL.19971117.0460.

CRWMS M\&O 1999. Environmental Baseline File for Biological Resources. B00000000-01717-5700-00009 REV 00. Las Vegas, Nevada: CRWMS M\&O. ACC: MOL.19990302.0181; MOL.19990330.0560.

CRWMS M\&O 1999. Evaluation of Soils in the Northern Amargosa Valley. B00000000-01717-5705-00084 REV 00. Las Vegas, Nevada: CRWMS M\&O. ACC: MOL.19990224.0268.

CRWMS M\&O 2000. Biosphere Process Model Report. TDR-MGR-MD-000002

REV 00 ICN 01. Las Vegas, Nevada: CRWMS M\&O. ACC: MOL.20000620.0341.

CRWMS M\&O 2000. Total System Performance Assessment for the Site Recommendation. TDR-WIS-PA-000001 REV 00 ICN 01. Las Vegas, Nevada: CRWMS M\&O. ACC: MOL.20001220.0045.

CRWMS M\&O 2001. Disruptive Event Biosphere Dose Conversion Factor Analysis. ANL-MGR-MD-000003 REV 01. Las Vegas, Nevada: CRWMS M\&O. ACC: MOL.20010125.0233.

CRWMS M\&O 2001. Distribution Fitting to the Stochastic BDCF Data. ANL-NBS-MD-000008 REV 00 ICN 01. Las Vegas, Nevada: CRWMS M\&O. ACC: MOL.20010221.0148.

CRWMS M\&O 2001. Evaluate Soil/Radionuclide Removal by Erosion and Leaching. ANL-NBS-MD-000009 REV 00 ICN 01. Las Vegas, Nevada: CRWMS M\&O. ACC: MOL.20010214.0032.

CRWMS M\&O 2001. Nominal Performance Biosphere Dose Conversion Factor Analysis. ANL-MGR-MD-000009 REV 01. Las Vegas, Nevada: CRWMS M\&O. ACC: MOL.20010123.0123.

D'Agnese, F.A.; Faunt, C.C.; Turner, A.K.; and Hill, M.C. 1997. Hydrogeologic 100131 Evaluation and Numerical Simulation of the Death Valley Regional Ground-Water Flow System, Nevada and California. Water-Resources Investigations Report 96-4300. Denver, Colorado: U.S. Geological Survey. ACC: MOL.19980306.0253. 
Daniels, J.I. 2003. “Summary of Review Actions for Development Drafts of Biosphere

Model Report MDL-MGR-MD-000001 Development Drafts of Rev 00A Dated between September-December 2002 Which Includes a Description of An Environmental Radiation Model for the Yucca Mountain, Nevada (ERMYN).” Letter from J.I. Daniels (LLNL) to A.J. Smith (BSC), March 20, 2003.

ACC: MOL.20030428.0001.

DOE (U.S. Department of Energy) 1997. The 1997 “Biosphere” Food Consumption

Survey Summary Findings and Technical Documentation. Las Vegas, Nevada: U.S. Department of Energy, Office of Civilian Radioactive Waste Management. ACC: MOL.19981021.0301.

DOE 2002. Final Environmental Impact Statement for a Geologic Repository for the Disposal of Spent Nuclear Fuel and High-Level Radioactive Waste at Yucca Mountain, Nye County, Nevada. DOE/EIS-0250. Washington, D.C.: U.S. Department of Energy, Office of Civilian Radioactive Waste Management. ACC: MOL.20020524.0314; MOL.20020524.0315; MOL.20020524.0316; MOL.20020524.0317; MOL.20020524.0318; MOL.20020524.0319; MOL.20020524.0320.

Dollarhide, W.E. 1999. "United States Department of Agriculture Soil Survey Data 159253 Lathrop Wells Area.” Letter from W.E. Dollarhide (USDA) to R. Aguilar (SNL), December 5, 1999, with attachments. ACC: MOL.19991217.0513.

Duan, N. 1982. "Models for Human Exposure to Air Pollution.” Environment 162466 International, 8, 305-309. New York, New York: Pergamon Press. TIC: 250558.

Eckerman, K.F. and Ryman, J.C. 1993. External Exposure to Radionuclides in Air, Water, and Soil, Exposure-to-Dose Coefficients for General Application, Based on the 1987 Federal Radiation Protection Guidance. EPA 402-R-93-081. Federal Guidance Report No. 12. Washington, D.C.: U.S. Environmental Protection Agency, Office of Radiation and Indoor Air. TIC: 225472.

Eckerman, K.F.; Wolbarst, A.B.; and Richardson, A.C.B. 1988. Limiting Values of 101069 Radionuclide Intake and Air Concentration and Dose Conversion Factors for Inhalation, Submersion, and Ingestion. EPA 520/1-88-020. Federal Guidance Report No. 11. Washington, D.C.: U.S. Environmental Protection Agency.

ACC: MOL.20010726.0072.

EPA (U.S. Environmental Protection Agency) 1997. Activity Factors. Volume III of 116135 Exposure Factors Handbook. EPA/600/P-95/002Fc. Washington, D.C.:

U.S. Environmental Protection Agency. TIC: 241062. 
EPA 1997. Food Ingestion Factors. Volume II of Exposure Factors Handbook.

EPA/600/P-95/002Fb. Washington, D.C.: U.S. Environmental Protection Agency.

TIC: 241061.

EPRI (Electric Power Research Institute) 2002. Evaluation of the Proposed High-Level Radioactive Waste Repository at Yucca Mountain Using Total System Performance Assessment, Phase 6. EPRI TR-1003031. Palo Alto, California: Electric Power Research Institute. TIC: 252239.

Golder Associates. 2000. GoldSim, Graphical Simulation Environment, User's Guide. Version 6.02. Manual Draft \#4 (March 17, 2000). Redmond, Washington: Golder Associates. TIC: 247347.

GoldSim Technology Group. 2002. GoldSim, Graphical Simulation Environment, 160643 User's Guide. Version 7.40. Redmond, Washington: Golder Associates. TIC: 253564.

Hoffman, F.O.; Frank, M.L.; Blaylock, B.G.; von Bernuth, R.D.; Deming, E.J.; Graham, R.V.; Mohrbacher, D.A.; and Waters, A.E. 1989. Pasture Grass Interception and Retention of (131)I, (7)BE, and Insoluble Microspheres Deposited in Rain. ORNL-6542. Oak Ridge, Tennessee: Oak Ridge National Laboratory. TIC: 237241.

Hoffman, F.O.; Thiessen, K.M.; and Rael, R.M. 1995. “Comparison of Interception and Initial Retention of Wet-Deposited Contaminants on Leaves of Different Vegetation Types.” Atmospheric Environment, 29, (15), 1771-1775. New York, New York: Pergamon Press. TIC: 243593.

Hoffman, F.O.; Thiessen, K.M.; Frank, M.L.; and Blaylock, B.G. 1992. "Quantification of the Interception and Initial Retention of Radioactive Contaminants Deposited on Pasture Grass by Simulated Rain.” Atmospheric Environment, 26A, (18), 3313-3321. New York, New York: Pergamon Press. TIC: 243594.

Horak, C. and Carns, D. 1997. Amargosa Focus Group Report. Biosphere Study. Las Vegas, Nevada: University of Nevada, Las Vegas. TIC: 241712.

IAEA (International Atomic Energy Agency) 1994. Handbook of Parameter Values 100458 for the Prediction of Radionuclide Transfer in Temperate Environments. Technical Reports Series No. 364. Vienna, Austria: International Atomic Energy Agency. TIC: 232035. 
IAEA 1996. Modelling of Radionuclide Interception and Loss Processes in Vegetation

160402 and of Transfer in Semi-Natural Ecosystems, Second Report of the VAMP Terrestrial Working Group. IAEA-TECDOC-857. Vienna, Austria: International Atomic Energy Agency. TIC: 253017.

IAEA 2001. An International Peer Review of the Biosphere Modelling Programme of 155188 the US Department of Energy's Yucca Mountain Site Characterization Project, Report of the IAEA International Review Team. Vienna, Austria: International Atomic Energy Agency. TIC: 250092.

IAEA 2001. Generic Models for Use in Assessing the Impact of Discharges of Radioactive Substances to the Environment. Safety Reports Series No. 19. Vienna, Austria: International Atomic Energy Agency. TIC: 251295.

ICRP (International Commission on Radiological Protection) 1975. Report of the Task Group on Reference Man: A Report Prepared by a Task Group of Committee 2 of the International Commission on Radiological Protection. ICRP Number 23. Tarrytown, New York: Elsevier. ACC: MOL.20000516.0124.

ICRP 1978. "Limits for Intakes of Radionuclides by Workers." Annals of the ICRP. 101076 ICRP Publication 30 Supplement to Part 1. New York, New York: Pergamon Press. TIC: 221575.

ICRP 1979. Limits for Intakes of Radionuclides by Workers. Volume 2, No. 3/4 of Annals of the ICRP. Sowby, F.D., ed. ICRP Publication 30 Part 1. New York, New York: Pergamon Press. TIC: 4939.

ICRP 1980. Limits for Intakes of Radionuclides by Workers. Volume 4, No. 3/4 of Annals of the ICRP. Sowby, F.D., ed. ICRP Publication 30 Part 2. Reprinted 1990. Elmsford, New York: Pergamon Press. TIC: 4941.

ICRP 1981. Limits for Intakes of Radionuclides by Workers. Volume 5, No. 1-6 of 153056 Annals of the ICRP. ICRP Publication 30, Supplement to Part 2. New York, New York: Pergamon Press. TIC: 4942.

ICRP 1981. Limits for Intakes of Radionuclides by Workers. Volume 6, No. 2/3 of 110352 Annals of the ICRP. Sowby, F.D., ed. ICRP Publication 30 Part 3, Including Addendum to Parts 1 and 2. New York, New York: Pergamon Press. TIC: 4943. 
ICRP 1982. Limits for Intakes of Radionuclides by Workers. Volume 8, No. 1-3 of Annals of the ICRP. ICRP Publication 30, Supplement B to Part 3 Including Addendum to the Supplements of Parts 1 and 2. New York, New York: Pergamon Press. TIC: 4945.

ICRP 1994. Human Respiratory Tract Model for Radiological Protection. Volume 24, Nos. 1-3 of Annals of the ICRP. Smith, H., ed. ICRP Publication 66. New York, New York: Pergamon. TIC: 249223.

ICRP 1996. Age-Dependent Doses to Members of the Public from Intake of 152446 Radionuclides: Part 5 Compilation of Ingestion and Inhalation Dose Coefficients. Volume 26, No. 1 of Annals of the ICRP. Smith, H., ed. ICRP Publication 72. New York, New York: Pergamon Press. TIC: 235870.

Jensen, M.E.; Burman, R.D.; and Allen, R.G., eds. 1990. Evapotranspiration and 160001 Irrigation Water Requirements. ASCE Manuals and Reports on Engineering Practice No. 70. New York, New York: American Society of Civil Engineers. TIC: 246697.

Karlsson, S.; Bergström, U.; and Meili, M. 2001. Models for Dose Assessments, Models Adapted to the SFR-Area, Sweden. SKB TR-01-04. Stockholm, Sweden: Svensk Kärnbränsleförsörjning A.B. TIC: 252806.

Kennedy, W.E., Jr. and Strenge, D.L. 1992. Technical Basis for Translating Contamination Levels to Annual Total Effective Dose Equivalent. Volume 1 of Residual Radioactive Contamination from Decommissioning. NUREG/CR-5512. Washington, D.C.: U.S. Nuclear Regulatory Commission. ACC: MOL.20010721.0030.

Klepeis, N.E. 1999. “An Introduction to the Indirect Exposure Assessment Approach: 160094 Modeling Human Exposure Using Microenvironmental Measurements and the Recent National Human Activity Pattern Survey." Environmental Health Perspectives, 107, (Supplement 2), 365-374. Research Park Triangle, North Carolina: National Institute of Environmental Health Sciences, National Institutes of Health. TIC: 250567.

Lamarsh, J.R. 1983. Introduction to Nuclear Engineering. 2nd Edition. Menlo Park, 149069 California: Addison-Wesley. TIC: 244841.

LaPlante, P.A. and Poor, K. 1997. Information and Analyses to Support Selection of Critical Groups and Reference Biospheres for Yucca Mountain Exposure Scenarios. CNWRA 97-009. San Antonio, Texas: Center for Nuclear Waste Regulatory Analyses. ACC: MOL.20010721.0035. 
Leigh, C.D.; Thompson, B.M.; Campbell, J.E.; Longsine, D.E.; Kennedy, R.A.; and

Napier, B.A. 1993. User's Guide for GENII-S: A Code for Statistical and Deterministic Simulations of Radiation Doses to Humans from Radionuclides in the Environment. SAND91-0561. Albuquerque, New Mexico: Sandia National Laboratories.

ACC: MOL.20010721.0031.

Lide, D.R. and Frederikse, H.P.R., eds. 1997. CRC Handbook of Chemistry and 103178 Physics. 78th Edition. Boca Raton, Florida: CRC Press. TIC: 243741.

Mage, D.T. 1985. "Concepts of Human Exposure Assessment for Airborne Particulate 162465 Matter." Environment International, 11, 407-412. New York, New York: Pergamon Press. TIC: 250582.

McKone, T.E. and Bogen, K.T. 1992. "Uncertainties in Health-Risk Assessment: An 160440 Integrated Case Study Based on Tetrachloroethylene in California Groundwater.” Regulatory Toxicology and Pharmacology, 15, 86-103. New York, New York: Academic Press. TIC: 253420.

McKone, T.E. and Daniels, J.I. 1991. "Estimating Human Exposure Through Multiple Pathways from Air, Water, and Soil.” Regulatory Toxicology and Pharmacology, 13, 36-61. New York, New York: Academic Press. TIC: 253419.

Mills, L.; Morris, B.; Roberts, A.; Robinson, M. L.; Hammond, B.; and Mandekic, J. n.d. Beginning Gardening in the Desert. Pahrump, Nevada: Southern Nye County Cooperative Extension. TIC: 243450.

NAHB Research Center. 1998. Factory and Site-Built Housing, a Comparison for the 160428 21st Century. Washington, D.C.: U.S. Department of Housing and Urban Development, Office of Policy Development and Research. TIC: 253431.

Napier, B.A.; Peloquin, R.A.; Strenge, D.L.; and Ramsdell, J.V. 1988. Conceptual 157927 Representation. Volume 1 of GENII - The Hanford Environmental Radiation Dosimetry Software System. PNL-6584. Richland, Washington: Pacific Northwest Laboratory. TIC: 252237.

NCRP (National Council on Radiation Protection and Measurements) 1984. Radiological Assessment: Predicting the Transport, Bioaccumulation, and Uptake by Man of Radionuclides Released to the Environment. Report No. 76. Bethesda, Maryland: National Council on Radiation Protection and Measurement. TIC: 223622. 
NCRP 1999. Recommended Screening Limits for Contaminated Surface Soil and

Review of Factors Relevant to Site-Specific Studies. NCRP Report No. 129. Bethesda, Maryland: National Council on Radiation Protection and Measurements.

TIC: 250396.

NRC (U.S. Nuclear Regulatory Commission) 2003. Yucca Mountain Review Plan,

Final Report. NUREG-1804, Rev. 2. Washington, D.C.: U.S. Nuclear Regulatory

Commission, Office of Nuclear Material Safety and Safeguards. TIC: 254568.

Pinnick, R.G.; Fernandez, G.; Hinds, B.D.; Bruce, C.W.; Schaefer, R.W.; and

Pendleton, J.D. 1985. "Dust Generated by Vehicular Traffic on Unpaved Roadways:

Sizes and Infrared Extinction Characteristics.” Aerosol Science and Technology, 4, (1), 99-121. New York, New York: Elsevier. TIC: 252364.

Rasmuson, K.E. 2004. "Summary of 2004 Agricultural Activities, Businesses, and Community Services and Organizations in Amargosa Valley.” Interoffice memorandum from K.E. Rasmuson (BSC) to K.R. Rautenstrauch, May 20, 2004, 0520041667, with enclosures. ACC: MOL.20040519.0033.

Reamer, C.W. 2001. "U.S. Nuclear Regulatory Commission/U.S. Department of Energy Technical Exchange and Management Meeting on Igneous Activity (June 21-22, 2001).” Letter from C.W. Reamer (NRC) to S. Brocoum (DOE/YMSCO), June 29, 2001, with enclosure. ACC: MOL.20010918.0001.

Reamer, C.W. 2001. “U.S. Nuclear Regulatory Commission/U.S. Department of 158380 Energy Technical Exchange and Management Meeting on Total System Performance Assessment and Integration (August 6 through 10, 2001).” Letter from C.W. Reamer (NRC) to S. Brocoum (DOE/YMSCO), August 23, 2001, with enclosure.

ACC: MOL.20011029.0281.

Reamer, C.W. 2001. “U.S. Nuclear Regulatory Commission/U.S. Department of Energy Technical Exchange and Management Meeting on Igneous Activity (September 5, 2001).” Letter from C.W. Reamer (NRC) to S. Brocoum (DOE/YMSCO), September 12, 2001, with enclosure. ACC: MOL.20011114.0008.

Rittmann, P.D. 1993. Verification Tests for the July 1993 Revision to the GENII Radionuclide and Dose Increment Libraries. WHC-SD-WM-TI-596. Richland, Washington: Westinghouse Hanford Company. TIC: 233965. 
Roe, L.K. 2002. "Summary of RDA Investigation ID: 4/10/02 Fish Farming in 160674 Amargosa Valley.” Interoffice memorandum from L.K. Roe (BSC) to File, November 5, 2002, 1105024986, with an attachment. ACC: MOL.20021107.0091;

MOL.20020821.0002.

Sheppard, M.I.; Sheppard, S.C.; and Amiro, B.D. 1991. "Mobility and Plant Uptake of 159545 Inorganic \{superscript 14\}C and ssuperscript 14\}C-Labelled PCB in Soils of High and Low Retention.” Health Physics, 61, (4), 481-492. New York, New York: Pergamon Press. TIC: 252687.

Shleien, B., ed. 1992. The Health Physics and Radiological Health Handbook. Revised 127299 Edition. Silver Spring, Maryland: Scinta. TIC: 9360.

Simmons, A.M. 2004. Yucca Mountain Site Description. TDR-CRW-GS-000001 166960 REV 02. Two volumes. Las Vegas, Nevada: Bechtel SAIC Company. ACC: DOC.20040120.0004.

Smith, G.M.; Watkins, B.M.; Little, R.H.; Jones, H.M.; and Mortimer, A.M. 1996. Biosphere Modeling and Dose Assessment for Yucca Mountain. EPRI TR-107190. Palo Alto, California: Electric Power Research Institute. TIC: 231592.

State of Nevada 1997. Ground Water Pumpage Inventory Amargosa Valley, No. 230 1997. Carson City, Nevada: State of Nevada, Department of Conservation and Natural Resources. ACC: MOL.19990329.0141.

Till, J.E. and Meyer, H.R. 1983. Radiological Assessment, A Textbook on Environmental Dose Analysis. NUREG/CR-3332. Washington, D.C.: U.S. Nuclear Regulatory Commission. TIC: 223809.

Troeh, F.R.; Hobbs, J.A.; and Donahue, R.L. 1980. Soil and Water Conservation for 110012 Productivity and Environmental Protection. Englewood Cliffs, New Jersey: Prentice-Hall. TIC: 246612.

UNSCEAR (United Nations Scientific Committee on the Effects of Atomic Radiation) 158644 2000. Sources and Effects of Ionizing Radiation, United Nations Scientific Committee on the Effects of Atomic Radiation, UNSCEAR 2000 Report to the General Assembly, with Scientific Annexes. Two volumes. New York, New York: United Nations. TIC: 249863. 
USDA (U.S. Department of Agriculture) 1999. 1997 Census of Agriculture, Nevada State and County Data. Volume 1, Geographic Area Series Part 28. AC97-A-28. Washington, D.C.: U.S. Department of Agriculture, National Agricultural Statistics Service. TIC: 246010.

Watt, J.R. and Brown, W.K. 1997. Evaporative Air Conditioning Handbook. 3rd Edition. Lilburn, Georgia: Fairmont Press. TIC: 252688.

YMP (Yucca Mountain Site Characterization Project) 1999. Yucca Mountain Site Characterization Project: Summary of Socioeconomic Data Analyses Conducted in Support of the Radiological Monitoring Program, April 1998 to April 1999. North Las Vegas, Nevada: Yucca Mountain Site Characterization Office.

ACC: MOL.19991021.0188.

Yu, C.; Zielen, A.J.; Cheng, J.-J.; LePoire, D.J.; Gnanapragasam, E.; Kamboj, S.; 159465 Arnish, J.; Wallo, A., III.; Williams, W.A.; and Peterson, H. 2001. User's Manual for RESRAD Version 6. ANL/EAD-4. Argonne, Illinois: Argonne National Laboratory. TIC: 252702.

Zach, R.; Amiro, B.D.; Bird, G.A.; Macdonald, C.R.; Sheppard, M.I.; Sheppard, S.C.; 103831 and Szekely, J.G. 1996. Biosphere Model. Volume 4 of The Disposal of Canada's Nuclear Fuel Waste: A Study of Postclosure Safety of In-Room Emplacement of Used CANDU Fuel in Copper Containers in Permeable Plutonic Rock. AECL-11494-4. Pinawa, Manitoba, Canada: Atomic Energy of Canada Limited. TIC: 226735.

\subsection{CODES, STANDARDS, REGULATIONS, AND PROCEDURES}

10 CFR 63. Energy: Disposal of High-Level Radioactive Wastes in a Geologic 156605 Repository at Yucca Mountain, Nevada. Readily available.

24 CFR 3280. Housing and Urban Development: Manufactured Home Construction 160555 and Safety Standards. Readily available

AP-2.22Q, Rev. 1, ICN 1. Classification Analyses and Maintenance of the Q-List. Washington, D.C.: U.S. Department of Energy, Office of Civilian Radioactive Waste Management.

AP-2.27Q, Rev. 1, ICN 4. Planning for Science Activities. Washington, D.C.: U.S. Department of Energy, Office of Civilian Radioactive Waste Management.

AP-SIII.2Q, Rev 1 ICN 2. Qualification of Unqualified Data. Washington, D.C.: U.S. Department of Energy, Office of Civilian Radioactive Waste Management.

AP-SIII.10Q, Rev. 2, ICN 7. Models. Washington, D.C.: U.S. Department of Energy, Office of Civilian Radioactive Waste Management. 
LP-SI.11Q-BSC Rev. 000. Software Management. Washington, D.C.:

U.S. Department of Energy, Office of Civilian Radioactive Waste Management.

\subsection{SOURCE DATA, LISTED BY DATA TRACKING NUMBER}

MO0407SEPFEPLA.000. LA FEP List. Submittal date: 07/20/2004.

MO0410MWDTLSCM.000. TSPA-LA Igneous Scenario Class Model Case. Submittal

172208 date: 10/19/2004.

\subsection{OUTPUT DATA, LISTED BY DATA TRACKING NUMBER}

MO0306MWDBGSMF.001. Biosphere GoldSim Model Files. Submittal date: 06/13/2003.

\subsection{SOFTWARE CODES}

BSC (Bechtel SAIC Company) 2003. Software Code: GoldSim. V7.50.100. PC.

SNL (Sandia National Laboratories) 1998. Software Code: GENII-S V1.4.8.5.

V1.4.8.5. PC, Windows NT. 30034 V1.4.8.5. 
APPENDIX A

ELECTRONIC FILES USED FOR THE MODEL REPORT 
This appendix lists all of the electronic files generated for this report. All of the files are stored on a data compact disk titled BioModFiles. All of the GoldSim files can be reproduced using the model output files for the groundwater (ERMYN_GW.gsm) and volcanic ash (ERMYN_VA.gsm) scenarios (Table A-1). Two Excel files containing various calculations using standard Excel functions also are on the compact disk (Table A-3). The Excel calculation methods are explained on the individual spreadsheets and need no further explanation in this appendix. The file names, purpose, date, time last modified, and volume size (file size in $\mathrm{kB}$ ) are given in the tables below for each electronic file.

Table A-1. GoldSim Model Files

\begin{tabular}{|c|c|c|c|}
\hline File Name & Purpose & Date and Time & Size (kB) \\
\hline ERMYN_GW.gsm & GoldSim model for the groundwater scenario & $6 / 10 / 200310: 58 a$ & 3,930 \\
\hline ERMYN_VA.gsm & GoldSim model for the volcanic ash scenario & $6 / 10 / 200310: 59 a$ & 2,705 \\
\hline
\end{tabular}

Table A-2. GoldSim Model Verification Files

\begin{tabular}{|l|l|c|c|}
\hline \multicolumn{1}{|c|}{ File Name } & \multicolumn{1}{|c|}{ Purpose } & \multicolumn{1}{|c|}{ Date and Time } & Size (kB) \\
\hline ERMYN_GW_Pu239verf.gsm & $\begin{array}{l}\text { Deterministic run for }{ }^{239} \text { Pu groundwater } \\
\text { scenario; used in Table 6.10-1 }\end{array}$ & $06 / 23 / 2003$ 03:29p & 3,931 \\
\hline ERMYN_GW_C14verf.gsm & $\begin{array}{l}\text { Deterministic run for }{ }^{14} \mathrm{C} \text { groundwater } \\
\text { scenario; used in Table 6.10-2 }\end{array}$ & $06 / 23 / 200303: 29 \mathrm{p}$ & 3,931 \\
\hline ERMYN_GW_Ra226verf.gsm & $\begin{array}{l}\text { Deterministic run for }{ }^{226} \text { Ra groundwater } \\
\text { scenario; used in Table 6.10-3 }\end{array}$ & $06 / 23 / 200303: 29 \mathrm{p}$ & 3,931 \\
\hline ERMYN_GW_Pu239stoc.gsm & $\begin{array}{l}\text { Stochastic run for }{ }^{239} \text { Pu groundwater } \\
\text { scenario; used in Table 6.10-4 }\end{array}$ & $06 / 23 / 200303: 32 \mathrm{p}$ & 11,865 \\
\hline ERMYN_VA_Pu239verf.gsm & $\begin{array}{l}\text { Deterministic run for }{ }^{239} \text { Pu volcanic ash } \\
\text { scenario; used in Table 6.10-5 }\end{array}$ & $06 / 23 / 200303: 33 p$ & 2,705 \\
\hline ERMYN_VA_Ra226verf.gsm & $\begin{array}{l}\text { Deterministic run for }{ }^{226} \text { Ra volcanic ash } \\
\text { scenario; used in Table 6.10-6 }\end{array}$ & $06 / 23 / 200303: 33 p$ & 2,705 \\
\hline ERMYN_VA_Pu239stoc.gsm & $\begin{array}{l}\text { Stochastic run for }{ }^{239} \mathrm{Pu} \text { volcanic ash } \\
\text { scenario; used in Table 6.10-7 }\end{array}$ & $06 / 23 / 200303: 34 p$ & 8,496 \\
\hline
\end{tabular}

Table A-3. Microsoft Excel Files

\begin{tabular}{|l|l|c|c|}
\hline \multicolumn{1}{|c|}{ File Name } & \multicolumn{1}{|c|}{ Purpose } & Date and Time & Size (kB) \\
\hline ERMYN verification.xls & $\begin{array}{l}\text { File contains five worksheets for model hand } \\
\text { calculations; used in Tables 6.10-1, 6.10-2, 6.10-3, } \\
6.10-5, \text { and 6.10-6 }\end{array}$ & 07/14/2003 09:44a & 194 \\
\hline ERMYN validation.xls & $\begin{array}{l}\text { File contains five worksheets for model validation } \\
\text { calculations; used in Tables 7.4-4, 7.4-7, 7.4-12, } \\
7.4-13,7.4-18, \text { and 7.4-19 }\end{array}$ & 07/14/2003 05:37p & 69 \\
\hline
\end{tabular}


Table A-4. GENII-S Files

\begin{tabular}{|c|c|c|c|}
\hline File Name & Purpose & Date and Time & Size (kB) \\
\hline RMDLIB.dat & $\begin{array}{l}\text { GENII-S radionuclide library file for model validation } \\
\text { used in Table } 7.4-5\end{array}$ & 11/29/1990 05:24p & 14 \\
\hline C14subroutine.txt & $\begin{array}{l}\text { GENII-S }{ }^{14} \mathrm{C} \text { Fortran subroutine taken from GENII-S } \\
\text { source code, referenced in Table } 7.3-21\end{array}$ & $12 / 24 / 200203: 44 p$ & 13 \\
\hline
\end{tabular}




\section{APPENDIX B}

ANALYTICAL SOLUTION FOR RADON CONCENTRATIONS IN THE AIR DUE TO RADIUM CONTAMINATED SOIL 
An analytical solution for the radon diffusion equation used in the RESRAD model (Yu et al. 2001 [DIRS 159465], Appendix C) and typical numerical results from this method are presented in this appendix. The purpose of this analytical solution is to evaluate radon concentrations in the air using the RESRAD method, which is considered an ACM for the ERMYN radon submodel (Section 6.3.3). The numerical results presented here are compared to the selected radon model, which is suggested by NCRP-129 (NCRP 1999 [DIRS 155894] Section 4.3.6). By using the analytical solution, use of the RESRAD software can be avoided, and, therefore, software qualification is unnecessary.

As discussed in the RESRAD manual (Yu et al. 2001 [DIRS 159465], p. C-9), the annual average radon concentration in outdoor air is calculated as

$$
C_{0}=\frac{J_{0} F_{a o}}{\lambda H_{0}}\left[1-e^{-\left(\frac{\lambda X}{2 U}\right)}\right]
$$

where

$$
\begin{array}{lll}
C_{0} & = & \text { annual average concentration of radon outdoors }\left(\mathrm{Bq} / \mathrm{m}^{3}\right) \\
J_{0} & = & \text { radon flux at the soil surface outdoors }\left(\mathrm{Bq} /\left(\mathrm{m}^{2} \mathrm{~s}\right)\right) \\
F_{a o} & = & \text { outdoor area factor (dimensionless), it is equal to } 1 \text { if } A>100 \mathrm{~m}^{2} \\
H_{0} & = & \text { height into which plume is uniformly mixed }(\mathrm{m}) \\
\lambda & = & \text { radon decay constant }(/ \mathrm{s}) \\
U & = & \text { annual average wind speed }(\mathrm{m} / \mathrm{s}) . \\
X & = & \text { effective length of the contaminated zone }(\mathrm{m}), \text { and can be calculated as }
\end{array}
$$

$$
X=\sqrt{A}
$$

where

$A=$ area of the contaminated zone, which can be estimated by dividing the total of water usage by the average annual irrigation rate defined in Equation 6.4.6-3 $\left(\mathrm{m}^{2}\right)$

In Equation B-1, everything on the right-hand side is known except $J_{0}$ (the radon flux at the soil surface outdoors), but solving for $J_{0}$ requires the solution to the applicable radon diffusion equation for radium contaminated soil (not given in text). Normally, this radon diffusion equation can only be solved by numerical methods, which are used in the RESRAD code, due to the complicated geometry of the contaminated soil and uncontaminated cover. However, considering only a special, but applicable, case (large area of radium contaminated soil, uniform contamination over depth, and equilibrium conditions), the radon diffusion equation can be solved analytically.

The radon concentration in a slab of contaminated soil is governed by the differential equation and boundary conditions discussed in the RESRAD manual (Yu et. al. 2001 [DIRS 159465], pp. C-5 to C-7). For steady state conditions, these equations are 


$$
\left\{\begin{array}{l}
-\frac{d}{d z}\left(D \frac{d C}{d z}\right)+\lambda C=Q \\
C\left(z_{a}\right)=0 \\
J(0)=\left.\frac{d C}{d z}\right|_{z=0}=0
\end{array}\right.
$$

where

$$
\begin{aligned}
C= & \text { radon concentration in the pore space of soil }\left(\mathrm{Bq} / \mathrm{m}^{3}\right) \\
D= & \text { diffusion coefficient of radon in soil }\left(\mathrm{m}^{2} / \mathrm{s}\right) \\
& \text { vertical axial distance in the direction of diffusion }(\mathrm{m}) . z=0 \text { is the bottom of the } \\
& \text { contaminated soil, while } z=z_{a} \text { is the interface between the ground and the air. } \\
C\left(z_{a}\right)= & \text { radon concentration at the upper boundary of the contaminated soil } \\
J(0)= & \text { radon flux at the bottom boundary of the contaminated soil. } \\
Q \quad= & \text { radon source term into the pore space }\left(\mathrm{Bq} /\left(\mathrm{m}^{3} \mathrm{~s}\right)\right)
\end{aligned}
$$

The radon source term into the pore space $\left(\mathrm{Bq} /\left(\mathrm{m}^{3} \mathrm{~s}\right)\right)$ is evaluated as

$$
Q=\frac{\varepsilon \rho_{b} S_{R a} \lambda}{p_{t}}
$$

where

$$
\begin{aligned}
\varepsilon \quad= & \text { radon emanation coefficient, which represents the fraction of radon } \\
& \begin{array}{l}
\text { generated by radium decay that escapes from the soil particles } \\
\end{array} \\
& \text { (dimensionless) } \\
\rho_{\mathrm{b}}= & \text { soil bulk density }\left(\mathrm{kg} / \mathrm{m}^{3}\right) \\
S_{R a}= & \text { radium concentration in the soil }(\mathrm{Bq} / \mathrm{kg}) \\
p_{t}= & \text { total porosity (dimensionless) } \\
\lambda \quad= & \text { radon decay constant }(/ \mathrm{s})
\end{aligned}
$$

Because the radon concentration and flux are continuous across the interface of the two media (i.e., air and ground), radon flux at the soil surface outdoors $J_{0}$ is numerically equal to radon flux at the upper boundary of the contaminated soil, but an opposite direction. It can be calculated as

$$
J_{0}=-J\left(z_{a}\right)=-\left.p_{t} D \frac{d C}{d z}\right|_{z=z_{a}}
$$

As the diffusion coefficient, $D$, does not change with distance $z$, the differential equation (Equation B-3) can be solved with a general solution of

$$
C=C_{1} e^{-z / k}+C_{2} e^{z / k}+C_{3}
$$


where

$$
\begin{aligned}
C_{1}, C_{2} \text {, and } C_{3}= & \text { constants to be determined by the differential equation and its boundary } \\
& \text { conditions (Equations B-3 to B-5) }\left(\mathrm{Bq} / \mathrm{m}^{3}\right) \\
k & \text { diffusion length }(\mathrm{m})
\end{aligned}
$$

which, by substituting Equation B-8 into Equation B-3, can be shown to be

$$
k=\sqrt{\frac{D}{\lambda}}
$$

Taking the first and second derivatives of Equation B-8 gives

$$
\begin{aligned}
& \frac{d C}{d z}=-\frac{C_{1}}{k} e^{-z / k}+\frac{C_{2}}{k} e^{z / k} \\
& \frac{d^{2} C}{d z^{2}}=\frac{C_{1}}{k^{2}} e^{-z / k}+\frac{C_{2}}{k^{2}} e^{z / k}
\end{aligned}
$$

By inserting Equations B-11 and B-8 into the differential equation (Equation B-3), the constant $C_{3}$ can be determined as

$$
C_{3}=\frac{Q}{\lambda}
$$

Using the second boundary condition (Equation B-5) within Equation B-10, the relationship between $C_{1}$ and $C_{2}$ can be determined as

$$
C_{1}=C_{2}
$$

Using the first boundary condition (Equation B-4) and Equation B-13 into Equation B-8, the constants $C_{1}$ and $C_{2}$ can be determined as

$$
\begin{aligned}
& C\left(z_{a}\right)=C_{1} e^{-z_{a} / k}+C_{2} e^{z_{a} / k}+C_{3}=0 \\
& C_{1}=C_{2}=\frac{-C_{3}}{e^{-z_{a} / k}+e^{z_{a} / k}}
\end{aligned}
$$


Then the radon flux at the soil surface outdoors $J_{0}$ can be calculated using Equations B-7, B-10, B-12, B-13, and B-15 as

$$
\begin{aligned}
J_{0} & =-J\left(z_{a}\right)=-\left.p_{t} D \frac{d C}{d z}\right|_{z=z_{a}} \\
& =p_{t} k Q\left(\frac{e^{z_{a} / k}-e^{-z_{a} / k}}{e^{z_{a} / k}+e^{-z_{a} / k}}\right)
\end{aligned}
$$

By inserting Equation B-13 into Equation B-1, the annual average radon concentration in the air $\left(C_{0}\right)$ can be calculated as a function of the contaminated soil depth $\left(z_{a}\right)$. When contaminated soil is very deep, $z_{a}=\infty$, radon flux at soil surface becomes independent of $z_{a}$ and reaches the maximum value

$$
J_{0}=p_{t} k Q
$$

The annual average radon concentration in the air can be calculated by combining Equations B-17, B-1, and B-6 as

$$
\begin{aligned}
C_{0} & =\frac{p_{t} k Q F_{a o}}{\lambda H_{0}}\left[1-e^{-\left(\frac{\lambda X}{2 U}\right)}\right] \\
& =\frac{k F_{a o} \varepsilon \rho_{\mathrm{b}} S_{R a}}{H_{0}}\left[1-e^{-\left(\frac{\lambda X}{2 U}\right)}\right]
\end{aligned}
$$

The radon concentration ratio between outdoor air and soil, which is the radon release factor used in the ERMYN, can be evaluated using Equation B-18.

$$
\frac{C_{0}}{S_{R a}}=\frac{k F_{a o} \varepsilon \rho_{\mathrm{b}}}{H_{0}}\left[1-e^{-\left(\frac{\lambda X}{2 U}\right)}\right]
$$

Using typical values from the ERMYN, or using default values from the RESRAD code if the parameters are unique to that radon model, the radon release factor can be calculated using Equation B-19. The inputs and calculated results are shown in Table B-1. 
Table B-1. Calculation of the Radon Release Factor

\begin{tabular}{|l|l|c|l|l|}
\hline \multicolumn{1}{|c|}{ Parameter name } & \multicolumn{1}{c|}{ Notation } & Value & \multicolumn{1}{c|}{ Units } & \multicolumn{1}{c|}{ Data Source } \\
\hline Outdoor area factor & $F_{\mathrm{ao}}$ & 1 & - & RESRAD default \\
\hline Radon emanation coefficient & $\varepsilon$ & 0.25 & - & RESRAD default \\
\hline Soil bulk density & $\rho_{\mathrm{b}}$ & 1500 & $\mathrm{~kg} / \mathrm{m}^{3}$ & ERMYN input \\
\hline Diffusion coefficient of radon in soil & $D$ & $2 \mathrm{E}-6$ & $\mathrm{~m}^{2} / \mathrm{s}$ & RESRAD default \\
\hline Radon decay constant & $\lambda$ & $2.1 \mathrm{E}-6$ & $/ \mathrm{s}$ & ERMYN input \\
\hline Diffusion length & $k$ & 0.976 & $\mathrm{~m}$ & Calculated \\
\hline Radon plume height & $H_{0}$ & 2 & $\mathrm{~m}$ & RESRAD default \\
\hline Area of the contaminated zone & $\mathrm{A}$ & $3.952 \mathrm{E} 6$ & $\mathrm{~m}^{2}$ & ERMYN input \\
\hline Effective length of contaminated zone & $\mathrm{X}$ & $1.988 \mathrm{E} 3$ & $\mathrm{~m}$ & Calculated \\
\hline Annual average wind speed & $U$ & 2 & $\mathrm{~m} / \mathrm{s}$ & RESRAD default \\
\hline Radon release factor & $C_{0} / S_{R a}$ & 0.19 & $\mathrm{~kg} / \mathrm{m}^{3}$ & Calculated \\
\hline
\end{tabular}

ERMYN=Environmental Radiation Model for Yucca Mountain, Nevada

The calculated radon release factor used by the RESRAD radon diffusion model, $0.19 \mathrm{~kg} / \mathrm{m}^{3}$, is close to the selected value, $0.25 \mathrm{~kg} / \mathrm{m}^{3}$, in the ERMYN. To calculate the radon release factor as a function of contaminated soil depth, $z_{a}$, the exponential term in Equation B-16 is applied to Equation B-19.

$$
\frac{C_{0}}{S_{R a}}=\frac{k F_{a o} \varepsilon \rho_{\mathrm{b}}}{H_{0}}\left[1-e^{-\left(\frac{\lambda X}{2 U}\right)}\right]\left(\frac{e^{z_{a} / k}-e^{-z_{a} / k}}{e^{z_{a} / k}+e^{-z_{a} / k}}\right)
$$

Using the data in Table B-1, the concentration of radon in the air can be calculated as shown in Table B-2.

Table B-2. Radon Release Factors Due to Radium Contaminated Soil

\begin{tabular}{|c|c|c|c|c|}
\hline $\begin{array}{c}\text { Depth of } \\
\text { Contaminant }(\mathbf{m})\end{array}$ & $\begin{array}{c}\text { Source } \\
\text { Exponential Term }\end{array}$ & $\begin{array}{c}\text { Rn-222 Release } \\
\text { Factor } \\
\left(\mathbf{B q} / \mathbf{m}^{3}\right) /(\mathbf{B q} / \mathbf{k g})\end{array}$ & $\begin{array}{c}\text { Surface Soil } \\
\text { Density }\left(\mathbf{k g} / \mathbf{m}^{2}\right)\end{array}$ & $\begin{array}{c}\text { Rn-222 Release } \\
\text { Factor } \\
\left(\mathbf{B q} / \mathbf{m}^{3}\right) /\left(\mathbf{B q} / \mathbf{m}^{2}\right)\end{array}$ \\
\hline 0.003 & 0.0031 & $5.86 \mathrm{E}-04$ & 4.5 & 0.00013 \\
\hline 0.01 & 0.0102 & $1.95 \mathrm{E}-03$ & 15 & 0.00013 \\
\hline 0.02 & 0.0205 & $3.91 \mathrm{E}-03$ & 30 & 0.00013 \\
\hline 0.05 & 0.0512 & $9.76 \mathrm{E}-03$ & 75 & 0.00013 \\
\hline 0.1 & 0.1021 & $1.95 \mathrm{E}-02$ & 150 & 0.00013 \\
\hline 0.2 & 0.2020 & $3.85 \mathrm{E}-02$ & 300 & 0.00013 \\
\hline 0.5 & 0.4716 & $9.00 \mathrm{E}-02$ & 750 & 0.00012 \\
\hline 1 & 0.7716 & $1.47 \mathrm{E}-01$ & 1500 & 0.00010 \\
\hline 2 & 0.9673 & $1.85 \mathrm{E}-01$ & 3000 & 0.00006 \\
\hline 5 & 0.9999 & $1.91 \mathrm{E}-01$ & 7500 & 0.00003 \\
\hline 10 & 1.0000 & $1.91 \mathrm{E}-01$ & 15000 & 0.00001 \\
\hline
\end{tabular}

NOTE: Column 1 is input for the calculations. Column 2 is the source exponential term in Equation B-20. Column 3 is the result of using Equation B-20 and input values from Table B-1. Column 4 is converted from Column 1 with soil density of $1,500 \mathrm{~kg} / \mathrm{m}^{3}$. Column 5 is calculated as Column 3 / Column 4 . 
INTENTIONALLY LEFT BLANK 
APPENDIX C

CALCULATION OF ABSOLUTE HUMIDITY FROM RELATIVE HUMIDITY 
In dry weather, a swamp cooler may deliver air at 60 percent relative humidity. However, the relative humidity of air discharged from the house is only about 40 percent because of the temperature increase upon mixing with the indoor air (Watt and Brown 1997 [DIRS 159497], p. 24). If the indoor temperature is $75^{\circ} \mathrm{F}\left(24^{\circ} \mathrm{C}\right)$, the absolute humidity corresponding to this temperature can be estimated from the ideal gas law as

$$
\frac{p \times V}{T}=n \times R
$$

where

$\begin{array}{lll}p & = & \text { water vapor pressure (partial pressure of water vapor) } \\ V & = & \text { volume }\left(\mathrm{m}^{3}\right) \\ T & = & \text { temperature }(\mathrm{K}) \\ n & = & \text { number of moles } \\ R & = & \text { universal gas constant }\end{array}$

After rearranging and multiplying Equation C-1 by the molecular weight of water, $m w$, the density of water vapor in the air, $\rho_{w}$, can be calculated as

$$
\frac{n \times m w}{V}=\frac{m w \times p}{R \times T}=\rho_{w}
$$

The molecular weight of water is $18.016 \mathrm{~g} / \mathrm{mole}$. The partial pressure of water vapor at saturation depends on temperature. For $24^{\circ} \mathrm{C}(297 \mathrm{~K})$, the partial water pressure is $2.9850 \mathrm{kPa}$ (Lide and Frederikse 1997 [DIRS 103178], p. 6-8). The numerical value of the universal gas constant, $R$, depends on the units of $p, V, n$, and $T$. For the units used in this calculation, $R=0.00831451\left(\mathrm{~m}^{3} \mathrm{kPa}\right) /($ mole K) (Lide and Frederikse 1997 [DIRS 103178], p. 1-40). The absolute humidity at saturation (the density of water vapor in the air), $\rho_{w}$, can be calculated as

$$
\rho_{w}=\frac{m w \times p}{R \times T}=\frac{18.016 \frac{\mathrm{g}}{\mathrm{mole}} \quad 2.985 \mathrm{kPa}}{0.008315 \frac{\mathrm{m}^{3} \mathrm{kPa}}{\mathrm{mole} K} 297 \mathrm{~K}}=21.8 \frac{\mathrm{g}}{\mathrm{m}^{3}}
$$

At 40 percent relative humidity, the concentration of water vapor in the air is about $8.7 \mathrm{~g} / \mathrm{m}^{3}$.

Average absolute humidity at Yucca Mountain Weather Station \#9 is calculated based on measured data in the report by CRWMS M\&O (1997 [DIRS 100117], Table A-9). From this table, monthly averaged relative humidity and temperature are given. Because evaporative coolers are used during the summer, average relative humidity and temperature from May to September are used to calculate the average absolute humidity (Table C-1). 
Table C-1. Average Relative Humidity and Temperature at the Weather Station 9

\begin{tabular}{|l|c|c|c|c|c|c|}
\hline & May & June & July & August & September & Average \\
\hline Temperature $\left({ }^{\circ} \mathrm{C}\right)$ & 21.3 & 27.6 & 31.4 & 30.5 & 25.4 & 27.2 \\
\hline Relative Humidity (\%) & & & & & & \\
\hline Time $=0400 \mathrm{hr}$ & 42.5 & 26.4 & 23.7 & 24.2 & 26.6 & 18.5 \\
\hline Time $=1000 \mathrm{hr}$ & 23.5 & 14.1 & 12.6 & 14.0 & 15.6 & 9.1 \\
\hline Time $=1600 \mathrm{hr}$ & 16.0 & 7.8 & 7.2 & 8.2 & 19.0 & \\
\hline Time $=2200 \mathrm{hr}$ & 30.1 & 17.0 & 15.4 & 16.6 & 2 \\
\hline
\end{tabular}

Using the same calculation method described above, the partial water pressure for $27.2^{\circ} \mathrm{C}(300.2 \mathrm{~K})$ is $3.6100 \mathrm{kPa}$ (Lide and Frederikse 1997 [DIRS 103178], p. 6-8). The absolute humidity at saturation (the density of water vapor in the air), $\rho_{w}$, can be calculated as $26.1 \mathrm{~g} / \mathrm{m}^{3}$. At 18.2 percent relative humidity, the concentration of water vapor in the air is about $4.8 \mathrm{~g} / \mathrm{m}^{3}$. 


\section{APPENDIX D}

\section{EVAPORATIVE COOLER PATHWAYS}




\section{D1. INTRODUCTION}

The biosphere model (Section 6.4.2.2) includes a submodel for the use of evaporative coolers fed by groundwater containing radionuclides. The model only considers the inhalation pathway arising from the transfer of radionuclides from the water to the air injected into the dwelling for the purpose of cooling. The model assumes that a fraction (represented by a uniform distribution over 0,1 ) of each radionuclide in the source is transferred to the cooling airflow.

There are other exposure pathways associated with evaporative coolers. These units are effective in cooling ambient air with a low relative humidity by inducing forced evaporation of water in water-saturated (evaporator) filter pads and, thereby, utilizing the latent heat of evaporation of the water to cool the air that is then ducted to the dwelling. The process of ongoing forced evaporation leaves behind a residue of solid matter originally in solution. Any radionuclides, if not transferred to the airflow as modeled, will accumulate with this residue. This process will continue through the period that cooling is used. The residue will persist until routine service and maintenance to the cooling unit is conducted. Such a service is usually rendered before the onset of cold weather at the time the cooling system is "winterized" to minimize corrosion in the dormant period and reduce heat loss when indoor heating is required. Part of this process is to drain the reservoir and water supply line to preclude damage caused by freezing. The cooling pads, with the attached residue scale, are, generally, replaced in the spring months when the unit is made ready for the next cooling season.

This appendix provides the scoping calculations to identify important exposure pathways arising from evaporative cooler operation for inclusion in the biosphere model.

\section{D2. THE INHALATION PATHWAY}

To model the pathway for the dose contribution from inhalation of the cooling system air, a simple conceptual model has to be defined. For a given radionuclide present in a known concentration in the groundwater used to feed the evaporative cooler, this model assumes that a known fraction of that radionuclide is transferred to the air flowing through the system. The receptor inhales this air while indoors, thus, receiving a committed dose from the exposure.

The formula representing the transfer of the radionuclide from the water to the air is given by Equation 6.4.2-3. For the purpose of this scoping calculation, it is conservative to allow all of the radionuclide present in the water to be transferred to the airflow. Equation 6.4.2-3 now reduces to

$$
C a_{e, i}=\frac{M_{\text {water }}}{F_{\text {air }}} C w_{i}
$$

where

$$
\begin{array}{ll}
C a_{e, i}= & \text { activity concentration of radionuclide } i \text { in the air resulting from the operation } \\
& \text { of an evaporative cooler }\left(\mathrm{Bq} / \mathrm{m}^{3}\right)
\end{array}
$$


Having determined the radionuclide concentration in the air during the period the cooling system is running, Equation 6.4.8-3 can then be used to calculate the annual dose contribution from evaporative coolers.

$$
D_{\text {inh,e, },}=E D C F_{\text {inh }, i} C a_{e, i} f_{\text {use }} \sum_{n} B R_{n} t_{n, \text { indoors }}
$$

where

$$
\begin{array}{ll}
D_{\text {inh,e,i }}= & \text { annual dose from inhalation of primary radionuclide } i \text { from evaporative } \\
& \text { cooler operation (Sv/year) } \\
E D C F_{\text {inh, } i}= & \text { effective dose conversion factor for inhalation of radionuclide } i(\mathrm{~Sv} / \mathrm{Bq}) \\
B R_{n}= & \text { breathing rate for environment } n\left(\mathrm{~m}^{3} / \mathrm{hr}\right) \\
t_{n, \text { indoors }}= & \text { population-weighted time spent in indoor environment } n \text { (hr/year) }
\end{array}
$$

The population-weighted time is the sum of the products of time spent in a given environment by a population group and the proportion of population constituting a given population group (see Equation 6.4.8-3). The values of the time spent in indoor environments and the corresponding breathing rates are summarized in Table D-1. The sum of the products of the breathing rate and the weighted time indoors (i.e., $\sum_{n} B R_{n} t_{n, \text { indoors }}$ ) gives the mean value of the volume of air inhaled per year indoors $(\bar{V})$.

Table D-1. Time Spent in Indoor Environments and Corresponding Breathing Rates

\begin{tabular}{|l|c|c|c|}
\hline \multicolumn{1}{|c|}{ Environment } & $\begin{array}{c}\text { Weighted time indoors, } \\
\boldsymbol{t}_{\boldsymbol{n} \text {, indoors }} \\
(\mathbf{h} / \mathbf{d})\end{array}$ & $\begin{array}{c}\text { Breathing rate, } \boldsymbol{B} \boldsymbol{R}_{\boldsymbol{n}} \\
\left(\mathbf{m}^{\mathbf{3}} / \mathbf{h}\right)\end{array}$ & $\begin{array}{c}\text { Volume of air inhaled } \\
\text { indoors, } \boldsymbol{B} \boldsymbol{R}_{\boldsymbol{n}} \boldsymbol{t}_{\boldsymbol{n} \text {, indoors }} \\
\left(\mathbf{m}^{\mathbf{3}} / \mathbf{d}\right)\end{array}$ \\
\hline Active indoors & 9.45 & 1.08 & 10.21 \\
\hline Asleep & 8.30 & 0.39 & 3.24 \\
\hline Total indoors & 17.75 & & 13.44 \\
\hline Annual values & $6482 \mathrm{~h} / \mathrm{yr}$ & $\bar{V}=4908 \mathrm{~m}^{3} / \mathrm{yr}$ \\
\hline
\end{tabular}

Source: Table 6.10-3 (time indoors) and Table 6.6-3 (breathing rates).

By combining Equations D-1 and D-2, the equation for annual dose from an evaporative cooler for groundwater containing a unit concentration of radionuclide $i$ (i.e., $C w_{i}=1.0 \mathrm{~Bq} / \mathrm{m}^{3}$ ) is

$$
D_{\text {inh,e,i }}=E D C F_{\text {inh }, i} \frac{M_{\text {water }}}{F_{\text {air }}} f_{\text {use }} \bar{V}
$$

The values of other radionuclide-independent parameters used in Equation D-3 are shown in Table D-2. 
Table D-2. Representative Parameter Values and Their Sources

\begin{tabular}{|l|lc|}
\hline \multicolumn{2}{|c|}{ Parameter Name, Symbol, and Units } & Value \\
\hline Water evaporation rate, $\mathrm{m}^{3} / \mathrm{hr}$ & $M_{\text {water }}$ & 0.017 \\
\hline Air flow rate for evaporative cooler, $\mathrm{m}^{3} / \mathrm{hr}$ & $F_{\text {air }}$ & 8300 \\
\hline $\begin{array}{l}\text { Evaporative cooler use factor, modern climate, } \\
\text { dimensionless }\end{array}$ & $f_{\text {use }}$ & 0.39 \\
\hline
\end{tabular}

Source: Table 6.6-3.

The evaporative cooler pathway dose contribution can be compared with the dose resulting from drinking 2 liters of water per day $\left(0.73 \mathrm{~m}^{3} /\right.$ year $)$, which establishes a lower dose limit for the RMEI. The drinking water dose is calculated as (from Equation 6.4.9-2).

$$
D_{\text {ing }, w, i}=E D C F_{\text {ing }, i} U w
$$

where

$$
\begin{aligned}
E D C F_{\text {ing }, i}= & \text { effective dose conversion factor for ingestion of radionuclide } i(\mathrm{~Sv} / \mathrm{Bq}) ; \\
& \text { calculation of effective dose conversion factors for ingestion is discussed in } \\
& \text { Section 6.4.9.6 } \\
= & \text { annual consumption rate of contaminated drinking water by humans } \\
& \text { (L/year) }
\end{aligned}
$$

The example calculations were performed for three primary radionuclides: two fission products $\left({ }^{126} \mathrm{Sn}\right.$ and $\left.{ }^{137} \mathrm{Cs}\right)$, known to have penetrating gamma ray emissions, and a radionuclide chain headed by ${ }^{227}$ Ac due to its high effective dose coefficient for external exposure (see the effective dose coefficients for external exposure to contaminated soil in Table 6.4-2). The effective dose conversion factors for inhalation and ingestion of these radionuclides are presented in Table D-3. Substitution of these values and values from Tables D-1 and D-2 in equations D-3 and D-4, give the annual doses for both pathways, also shown in Table D-3.

Table D-3. Effective Dose Conversion Factors and Annual Doses for Ingestion and Inhalation

\begin{tabular}{|l|c|c|c|c|}
\hline Radionuclide & $\begin{array}{c}\text { Effective DCF for } \\
\text { Inhalation } \\
\text { (Sv/Bq) }\end{array}$ & $\begin{array}{c}\text { Annual Inhalation } \\
\text { Dose } \\
\text { (Sv) }\end{array}$ & $\begin{array}{c}\text { Effective DCF for } \\
\text { Ingestion } \\
\text { (Sv/Bq) }\end{array}$ & $\begin{array}{c}\text { Annual Ingestion } \\
\text { Dose } \\
\text { (Sv) }\end{array}$ \\
\hline${ }^{126} \mathrm{Sn}$ & $2.74 \mathrm{E}-08$ & $1.07 \mathrm{E}-10$ & $5.70 \mathrm{E}-09$ & $4.16 \mathrm{E}-09$ \\
\hline${ }^{137} \mathrm{Cs}$ & $8.63 \mathrm{E}-09$ & $3.38 \mathrm{E}-11$ & $1.35 \mathrm{E}-08$ & $9.86 \mathrm{E}-09$ \\
\hline${ }^{227} \mathrm{AC}$ & $1.82 \mathrm{E}-03$ & $7.14 \mathrm{E}-06$ & $3.99 \mathrm{E}-06$ & $2.91 \mathrm{E}-06$ \\
\hline
\end{tabular}

a From Table 6.4-3.

b From Table 6.4-4.

$\mathrm{DCF}=$ dose conversion factor

The table above illustrates that the dose from inhalation of aerosols generated by evaporative coolers is, for some radionuclides, comparable to that from drinking water. 


\section{D3. EXTERNAL DOSE PATHWAY}

Most residential dwellings only require a single cooling unit. The cooled air output from the unit is generally ducted within the outer shell of the building to each room where cooling is required. The system is designed to reduce costs by keeping the ducting requirements close to the minimum to satisfy the cooling specification. Depending on the dwelling design, the centralized cooling unit may be located on the roof of the dwelling or against one wall.

The biosphere model already includes the external exposure pathway from radionuclides deposited in soil from irrigation. This section presents scoping calculations to compare the external dose contributions from soil to those from coolers.

\section{D3.1 ACTIVITY REMAINING IN EVAPORATIVE COOLERS}

Considering the case where radionuclides in the water system do not get transferred to the airflow, the continual evaporation of water gives rise to sediment that contains all of the radionuclides originally present in the water. For the case of this scoping calculation, the cooling unit considered will be one that does not have a built in flushing system. At the start of the cooling season, the cooling system is considered to be cleaned (no sediment) when new evaporation pads are installed. The sediment builds up during cooler use in the hot periods of the year and remains there until the owner closes the system down for winter. For this scoping calculation, it will be assumed that the pad contains all the activity accumulated during use and the pads remain in place until they are replaced the following spring.

Until the system is serviced, the only exposure pathway is direct radiation. This pathway will be assessed. The dose from the evaporative cooler pathway will be compared to the dose from external exposure to soil.

If the activity of radionuclide $i$ in the evaporative cooler residue is $A_{i}(\mathrm{~Bq})$, at the end of the cooling season it is given by

$$
A_{i}=C w_{i} M_{\text {water }} t_{e c}
$$

where

$$
t_{e c}=\text { time evaporative cooler is operated in a year (hr/year). }
$$

Over the cooling period of 3,419 hr/year (calculated as the product of number of hours in a year and the evaporative cooler use factor from Table D-2), the accumulation of activity in the cooler pads and water reservoir is $58.1 \mathrm{~Bq}$ per $\mathrm{Bq} / \mathrm{m}^{3}$ of activity in the groundwater. The cooler operates for a fraction of a year equal to, on average, 0.39 for the modern climate (for the future climate this fraction is less). During this time, the activity will accumulate in the cooler. This will be followed by the dormant period during which the activity is constant. If it were to be assumed that the activity were accumulating at a linear rate over the period of operation, the average activity over this period would be half of the total accumulated activity for the period of cooler operation combined with the rest of a year of fixed activity. If credit is taken for the time 
required for the accumulation of this activity to occur, the annual average activity in the cooler can be calculated as $46.8 \mathrm{~Bq}$.

\section{D3.2 ACTIVITY IN IRRIGATED SOIL}

The exposure to contaminated soil is discussed in Section 6.4.7.1. The conceptual model here is that all land on which the receptor lives is contaminated by ongoing irrigation. After a long period of irrigation, the rate of addition of activity is equal to the rate of loss, and the level of activity for the soil equilibrium contamination is determined by Equations 6.4.1-4.

$$
C s_{i}=\frac{C w_{i} I R}{\lambda_{e f f, i}}
$$

where

$$
\begin{aligned}
\lambda_{\text {eff }, i}= & \text { effective removal rate of the radionuclide } i \text { from soil by all mechanisms } \\
& \text { (see discussion following Equation 6.4.1-2). } \\
C s_{i}= & \text { equilibrium activity concentration in surface soil for long-lived radionuclide } \\
& i\left(\mathrm{~Bq} / \mathrm{m}^{2}\right)
\end{aligned}
$$

The effective removal constant, $\lambda_{\text {eff, }}$ is comprised of three removal components $\lambda_{d}+\lambda_{l}+\lambda_{e}$ where the subscript refers to decay, leaching, and erosion respectively. The first two of these are dependent upon the radionuclide. The decay removal constant is determined from the half life $\left(T_{1 / 2}\right)$ of the radionuclide, as given in the definition of terms to Equation 6.4.1-1 as $\ln (2) / T_{1 / 2}$.

\section{D3.2.1 Decay Removal Constant}

Using the values of radionuclide half-lives from Table 6.3-7 allows the construction of Table D-4, showing the values of the removal constant for radioactive decay. These values are fixed and are not subject to any significant degree of uncertainty.

Table D-4. Radionuclide Half-Lives and Removal Constants

\begin{tabular}{|c|c|c|}
\hline Radionuclide & Half-life $(\mathbf{y r})$ & Decay Removal Constant, $\boldsymbol{\lambda}_{\boldsymbol{d}}\left(\mathbf{y r} \mathbf{r}^{-1}\right)$ \\
\hline Sn-126 & $1.00 \mathrm{E}+05$ & $6.93 \mathrm{E}-06$ \\
\hline Cs-137 & 30 & $2.31 \mathrm{E}-02$ \\
\hline Ac-227 & 21.8 & $3.18 \mathrm{E}-02$ \\
\hline
\end{tabular}

\section{D3.2.2 Leaching Removal Constant}

Calculation of radionuclide removal by leaching uses the parameter of leaching removal constant, which is calculated in the biosphere model using Equation 6.4.1-10. The parameters of interest in the leaching process are the crop overwatering rate, $O W,(\mathrm{~m} / \mathrm{y})$; the partition coefficient, $K_{d},\left(\mathrm{~m}^{3} / \mathrm{kg}\right)$, which depends on element under consideration; the volumetric water content of soil, $\theta$ (dimensionless); the soil density, $\rho\left(\mathrm{kg} / \mathrm{m}^{3}\right)$; and the surface soil thickness, $d(\mathrm{~m})$. 
The average value of $K_{d}$ was calculated from the values of parameters describing its distribution, i.e., the geometric mean and standard deviation (Table 6.6.3).

The geometric mean and geometric standard deviation can be expressed as

$$
\begin{aligned}
& G M=e^{\lambda} \\
& G S D=e^{\zeta}
\end{aligned}
$$

with the arithmetic mean, $\mu$, given by (Golder Associates 2000 [DIRS 146973], p. B-3)

$$
\mu=\exp \left[\lambda+\frac{1}{2} \varsigma^{2}\right]
$$

The average $\mathrm{K}_{\mathrm{d}}$ values calculated using Equation D-8 are presented in Table D-5.

Table D-5. Partition Coefficient Values Used to Determine Radionuclide Concentration in Soils

\begin{tabular}{|l|c|c|c|}
\hline Radionuclide & $\begin{array}{c}\text { Geometric Mean }^{\mathbf{a}} \\
\left(\mathbf{m}^{\mathbf{3}} \mathbf{/ k g}\right)\end{array}$ & $\begin{array}{c}\text { Geometric Standard }^{\mathbf{a}} \\
\text { Deviation }\end{array}$ & $\begin{array}{c}\text { Arithmetic Mean }^{\mathbf{b}} \\
\left(\mathbf{m}^{\mathbf{3}} / \mathbf{k g}\right)\end{array}$ \\
\hline Sn-126 & 0.45 & 6 & 2.24 \\
\hline Cs-137 & 4.40 & 3.7 & 10.4 \\
\hline Ac-227 & 1.50 & 6 & 7.47 \\
\hline
\end{tabular}

${ }^{\text {a }}$ From Table $6.6-3$ converting $\mathrm{L} / \mathrm{kg}$ to $\mathrm{m}^{3} / \mathrm{kg}$.

${ }^{b}$ Calculated using Equation D-7.

The values of the other parameters used in Equation 6.4.1-10 to calculate leaching removal constant are given in Table D-6.

Table D-6. Other Parameter Values Used to Determine External Exposure to Soils

\begin{tabular}{|l|l|c|}
\hline \multicolumn{2}{|c|}{ Parameter Name, Symbol, and Units } & Value \\
\hline Overwatering rate, $(\mathrm{m})$ & OW & 0.079 \\
\hline Volumetric water content & $\theta$ & 0.23 \\
\hline Soil density, $\left(\mathrm{kg} / \mathrm{m}^{3}\right)$ & $\rho$ & 1500 \\
\hline Depth of surface soil, $(\mathrm{m})$ & $d(\mathrm{~m})$ & 0.25 \\
\hline
\end{tabular}

NOTE: From Table 6.6-3.

Substituting the parameter values above in Equation 6.4.1-10 gives the values for the leaching removal constant given in Table D-7.

Table D-7. Leaching Removal Constants

\begin{tabular}{|l|c|}
\hline \multicolumn{1}{|c|}{ Radionuclide } & Leaching Removal Constant $\left(\mathbf{y r}^{-1}\right)$ \\
\hline Sn-126 & $9.40 \mathrm{E}-05$ \\
\hline Cs-137 & $2.03 \mathrm{E}-05$ \\
\hline Ac-227 & $2.82 \mathrm{E}-05$ \\
\hline
\end{tabular}




\section{D3.2.3 Erosion Removal Constant}

The erosion removal rate is specified by Equation 6.4.1-11 and is independent of the radionuclide. To determine a value for this parameter, the erosion rate was selected at the higher end of the specified distribution, and the values of surface soil thickness and density given in Table D-6 were used. From Table 6.6-3, the erosion rate was set at $1.1 \mathrm{~kg} /\left(\mathrm{m}^{2} \mathrm{yr}\right)$. This resulted in an erosion removal constant of $2.93 \times 10^{-3} \mathrm{yr}^{-1}$.

\section{D3.2.4 Effective Removal Constant and Activity Concentration in Surface Soil}

The effective removal constant for the three mechanisms considered is, for a radionuclide, given by the sum of the three individual removal constants. Table D-8 provides the details. The radionuclide concentrations in surface soil calculated using Equation D-6 and the annual average value of irrigation rate for the modern climate of $0.94 \mathrm{~m} /$ year are also shown in Table D-8. In all three cases considered, the effective removal constant is determined primarily by the decay and erosion processes.

Table D-8. Summary of Individual Removal Constants, Effective Removal Constants, and Activity Concentration in Surface Soil

\begin{tabular}{|l|c|c|c|c|c|}
\hline Radionuclide & $\begin{array}{c}\text { Decay Removal } \\
\text { Constant } \\
\boldsymbol{\lambda}_{d}\left(\mathbf{y r}^{-1}\right)\end{array}$ & $\begin{array}{c}\text { Erosion } \\
\text { Removal } \\
\text { Constant } \\
\boldsymbol{\lambda}_{\boldsymbol{l}}\left(\mathrm{yr}^{-1}\right)\end{array}$ & $\begin{array}{c}\text { Leaching } \\
\text { Removal } \\
\text { Constant } \\
\boldsymbol{\lambda}_{\boldsymbol{e}}\left(\mathrm{yr}^{-1}\right)\end{array}$ & $\begin{array}{c}\text { Effective } \\
\text { Removal } \\
\text { Constant } \\
\boldsymbol{\lambda}_{\text {eff }}\left(\mathbf{y r}^{-1}\right)\end{array}$ & $\begin{array}{c}\text { Activity } \\
\text { Concentration } \\
\text { in Surface Soil } \\
\left(\mathbf{B q} / \mathbf{m}^{2}\right)\end{array}$ \\
\hline Sn-126 & $6.93 \mathrm{E}-06$ & $2.93 \mathrm{E}-03$ & $9.40 \mathrm{E}-05$ & $3.03 \mathrm{E}-03$ & $3.10 \mathrm{E}+02$ \\
\hline $\mathrm{Cs}-137$ & $2.31 \mathrm{E}-02$ & $2.93 \mathrm{E}-03$ & $2.03 \mathrm{E}-05$ & $2.61 \mathrm{E}-02$ & $3.61 \mathrm{E}+01$ \\
\hline Ac-227 & $3.18 \mathrm{E}-02$ & $2.93 \mathrm{E}-03$ & $2.82 \mathrm{E}-05$ & $3.48 \mathrm{E}-02$ & $2.70 \mathrm{E}+01$ \\
\hline
\end{tabular}

\section{D3.3 EXTERNAL EXPOSURE FROM ACTIVITY IN SOIL}

As discussed in Section 6.4.7.1, the exposure to contaminated soil is based on a uniform distribution of radionuclides over the depth of surface soil. For a given radionuclide, a fixed receptor (i.e., a single environment) Equation 6.4.7-1 reduces to

$$
D_{e x t, i}=E D C i_{\text {soil }, i} \frac{C s_{i}}{d} t
$$

where

$$
\begin{aligned}
D_{\text {ext }, i}= & \begin{array}{l}
\text { annual dose from external exposure to primary radionuclide } i \text { in soil } \\
\text { (Sv/year) }
\end{array} \\
E D C i_{\text {soil }, i}= & \begin{array}{l}
\text { effective dose coefficient for exposure to soil contaminated to an infinite } \\
\text { depth for a long-lived radionuclide } i\left(\mathrm{~Sv} / \mathrm{s} \text { per } \mathrm{Bq} / \mathrm{m}^{3}\right)
\end{array} \\
= & \text { depth of surface soil }(\mathrm{m}) \\
t & \text { exposure time }(\mathrm{s})
\end{aligned}
$$


The values for the effective dose coefficient for exposure to soil contaminated to an infinite depth from Table 6.4-2 are reproduced in Table D-9. Using these values, the areal radionuclide concentration is soil values from Tables D-8, the depth of soil of $0.25 \mathrm{~m}$, and, taking the exposure over a full year $\left(3.16 \times 10^{7} \mathrm{~s}\right)$, the annual dose from external exposure to soil was calculated from Equation D-9. The results are also shown in Table D-9.

Table D-9. Estimated Annual Dose from External Exposure to Irrigated Soils

\begin{tabular}{|l|c|c|}
\hline Radionuclide & $\begin{array}{c}\text { Effective Dose Coefficient for } \\
\text { Exposure to Soil } \\
(\text { Sv/s per Bq/m }\end{array}$ & $\begin{array}{c}\text { Annual dose } \\
\text { (Sv/yr) }\end{array}$ \\
\hline Sn-126 & $6.34 \mathrm{E}-17$ & $2.56 \mathrm{E}-06$ \\
\hline $\mathrm{Cs}-137$ & $1.83 \mathrm{E}-17$ & $8.34 \mathrm{E}-08$ \\
\hline Ac-227 & $1.08 \mathrm{E}-17$ & $3.69 \mathrm{E}-08$ \\
\hline
\end{tabular}

\section{D3.4 EXTERNAL RADIATION IN EVAPORATIVE COOLER FROM WATER EVAPORATION}

The data presented in The Health Physics and Radiological Health Handbook (Shleien 1992 [DIRS 127299], Table 6.1.2) can be used to estimate dose for the accumulation of activity in an evaporative cooler. These data provide, for a single radionuclide source, the parameter named "Specific gamma ray dose constants at 1 meter." The units used in the publication are $\mathrm{mSv} / \mathrm{hr}$ per MBq at 1 meter. No credit is taken for attenuation by the building.

Given that the units used in this analysis are $\mathrm{Bq}$ for the source term and Sv per year for the exposure, the equation implied in Shleien (1992 ([DIRS 127299], Table 6.1.2) can be written as,

$$
D_{p s, i}=E \Gamma_{i} \times S A_{i} \times \overline{d^{-2}}
$$

where

$$
\begin{array}{ll}
D_{p s, i}= & \text { dose from exposure to the source }(\mathrm{Sv} / \mathrm{hr}) \\
E \Gamma_{i} & \text { effective specific gamma ray constant for a point source } \\
& \left(\mathrm{Sv} / \mathrm{hr} \text { per } \mathrm{Bq} / \mathrm{m}^{2}\right) \\
S A_{i} & =
\end{array}
$$

\section{D3.4.1 Effective Gamma Ray Constant for a Point Source}

The values of gamma ray constants were taken from The Health Physics and Radiological Health Handbook (Shleien 1992 ([DIRS 127299], Table 6.1.2). The data provided in this reference are for individual radionuclides. The biosphere model combines the dose contributions of the primary radionuclides and their short-lived decay products. Therefore, the effective gamma ray constants were calculated to add the contribution from the decay products to that of the primary radionuclide. Table $\mathrm{D}-10$ shows the results. 
Table D-10. Effective Specific Gamma Ray Constants for a Point Source

\begin{tabular}{|c|c|c|c|c|}
\hline Primary Radionuclide & Decay Product & Branching Fraction & $\begin{array}{c}\text { Specific Gamma Ray } \\
\text { Constant } \Gamma^{\mathrm{a}} \\
\mathrm{mSv} / \mathrm{h} \mathrm{MBq} \mathrm{M}^{-1} \mathrm{~m}^{2}\end{array}$ & $\begin{array}{c}\text { Effective Specific } \\
\text { Gamma Ray } \\
\text { Constant } E \Gamma^{\mathrm{b}} \\
\mathrm{mSv} / \mathrm{h} \mathrm{MBq}^{-1} \mathrm{~m}^{2}\end{array}$ \\
\hline \multirow[t]{3}{*}{${ }^{126} \mathrm{Sn}$} & & 1 & $3.41 \mathrm{E}-05$ & $3.84 \mathrm{E}-04$ \\
\hline & $\mathrm{Sb}$ & 1 & $2.82 \mathrm{E}-04$ & \\
\hline & $\mathrm{Sb}$ & 0.14 & 4.86E-04 & \\
\hline \multirow[t]{2}{*}{${ }^{137} \mathrm{Cs}$} & & 1 & & 1.02E-04 \\
\hline & ${ }^{137 m} \mathrm{Ba}$ & 0.946 & $1.08 \mathrm{E}-04$ & \\
\hline \multirow[t]{10}{*}{${ }^{227} \mathrm{Ac}$} & & 1 & $2.36 \mathrm{E}-06$ & $2.42 \mathrm{E}-04$ \\
\hline & ${ }^{227} \mathrm{Th}$ & 0.9862 & 1.15E-04 & \\
\hline & ${ }^{223} \mathrm{Fr}$ & 0.0138 & 8.93E-05 & \\
\hline & ${ }^{223} \mathrm{Ra}$ & 1 & 8.79E-05 & \\
\hline & ${ }^{219} \mathrm{Rn}$ & 1 & $1.42 \mathrm{E}-05$ & \\
\hline & ${ }^{215} \mathrm{Po}$ & 1 & $2.86 \mathrm{E}-08$ & \\
\hline & ${ }^{211} \mathrm{~Pb}$ & 1 & $9.84 \mathrm{E}-06$ & \\
\hline & ${ }^{211} \mathrm{Bi}$ & 1 & 1.27E-05 & \\
\hline & ${ }^{207} \mathrm{TI}$ & 0.9972 & 3.52E-07 & \\
\hline & ${ }^{211} \mathrm{Po}$ & 0.0028 & 1.33E-06 & \\
\hline
\end{tabular}

a Shleien (1992 [DIRS 127299], Table 6.1.2).

${ }^{\mathrm{b}}$ Calculated as a weighted sum of gamma ray constants for a primary radionuclide and its decay products, with branching fractions being the weights.

\section{D3.4.2 Mean Inverse Square of Distance}

The 2000 Census data indicated that approximately 90 percent of the total Amargosa Valley population lived in manufactured homes (Bureau of the Census 2002 [DIRS 159728], Table H33). Therefore, manufactured homes are representative of a typical dwelling in Amargosa Valley.

Most manufactured homes are single- or doublewide. Single-wide homes are 12 to 18 feet wide and 30 to 80 feet long; double-wide houses are 24 to 28 feet wide and 40 to 80 feet long. According to the report prepared by the NAHB Research Center for the U.S. Department of Housing and Urban Development, the average square footage is $1,056 \mathrm{ft}^{2}$ for the single-wide (single-section) homes and 1,629 $\mathrm{ft}^{2}$ for double-wide (double-section) homes and 1,955 $\mathrm{ft}^{2}$ for multisection homes (NAHB Research Center 1998 [DIRS 160428], p. 35). Single-wide houses constitute 46.2 percent and double-wide homes 51.2 percent of the manufactured homes in the United States, with the remainder (2.6 percent) being multisection structures.

Evaporative cooler water usage in Table D-2 is based on the average for the region. It is, therefore, reasonable to use a weighted average for the area of a dwelling (i.e., the average dwelling has the average cooler water usage). From the numbers cited in the above paragraph, this value is $1,373 \mathrm{ft}^{2}$ (or $128 \mathrm{~m}^{2}$ ).

The time averaged mean inverse square distance from the cooler residue source will be dependent upon the layout of the dwelling (and time spent in each room) with respect to the 
location of the cooler. The cooler could be mounted against either the long or short wall or on the roof. To derive a simple mean value for this parameter, the dwelling will be taken to be the square with the cooler mounted outside against the middle of one wall. The effective source (point source) of the radiation will be taken to be one meter from the inside wall.

With these simplifying approximations, the wall length will be $11.3 \mathrm{~m}$ (square root of $128 \mathrm{~m}^{2}$ ). The receptor will be placed on the center line of the dwelling; i.e., no credit will be taken for the reduction in the mean value of the inverse square for a receptor off the center line.

The average value of a function $\mathrm{f}(\mathrm{x})$ over an interval from $x=a$ to $x=b$ is expressed as

$$
\overline{f(x)}=\frac{1}{b-a} \int_{a}^{b} f(x) d x
$$

For $f(x)=1 / x^{2}$ and a receptor located at a distance of $x$ from the wall adjacent to the cooler unit, the average value of the function of the distance from the cooler is given by

$$
\overline{d^{-2}}=\frac{1}{\mathrm{x}_{\mathrm{u}}-\mathrm{x}_{1}} \int_{x_{l}}^{x_{u}} \frac{d x}{(x+1)^{2}}
$$

where

$$
\begin{array}{lll}
x_{l} & = & \text { lower value of } x(0.0 \mathrm{~m}) \\
x_{u} & = & \text { upper value of } x(11.3 \mathrm{~m})
\end{array}
$$

Making the substitution, $y=x+1$, gives

$$
\begin{aligned}
& \overline{d^{-2}}=\frac{1}{\mathrm{x}_{\mathrm{u}}-\mathrm{x}_{1}} \int_{x_{i}+1}^{x_{\mathrm{u}}+1} \frac{d y}{y^{2}} \\
& \overline{d^{-2}}=\frac{1}{\mathrm{x}_{\mathrm{u}}-\mathrm{x}_{1}}\left[\frac{-1}{y}\right]_{x_{\mathrm{l}}+1}^{x_{\mathrm{u}}+1}
\end{aligned}
$$

Substitution of the values above gives $\overline{d^{-2}}$ (distance factor) to be $8.1 \times 10^{-2} \mathrm{~m}^{-2}$.

\section{D3.4.3 Estimated Annual External Exposure for an Evaporative Cooler from Radionuclides in Water}

Substitution of the values developed above along with the total activity in a cooler from water evaporation (46.8 Bq per $\mathrm{Bq} / \mathrm{m}^{3}$ from Section D.3.1) into equation D-10 allows an estimate to be obtained for the use of an evaporative cooler (Table D-11). Note that, as was done for the soil exposure, no credit has been taken for the building shielding factors. Dose per year was 
calculated by taking into consideration weighted time spent indoors per year (from Table D-1) and the cooler use factor (Table D-2).

Table D-11. Estimated Annual Dose from External Exposure to Evaporative Coolers

\begin{tabular}{|c|c|c|c|c|c|}
\hline Radionuclide & $\begin{array}{c}\text { Specific Gamma Ray } \\
\text { Constant, } \Gamma \\
\mathrm{mSv} \mathrm{h}^{-1} \mathrm{MBq}^{-1} \mathrm{~m}^{2} \\
\end{array}$ & $\begin{array}{l}\text { Specific Gamma } \\
\text { Ray Constant, } \Gamma \\
\mathrm{Sv} \mathrm{h}^{-1} \mathrm{~Bq}^{-1} \mathrm{~m}^{2} \\
\end{array}$ & \begin{tabular}{|c} 
Distance Factor, $\mathbf{d}^{-2}$ \\
$\mathbf{m}^{-2}$ \\
\end{tabular} & $\begin{array}{c}\text { Dose per Hour } \\
\text { Sv h }{ }^{-1}\end{array}$ & $\begin{array}{c}\text { Annual Dose } \\
\text { Sv } \mathrm{yr}^{-1}\end{array}$ \\
\hline Sn-126 & 3.84E-04 & $3.84 \mathrm{E}-13$ & 0.0813 & 1.46E-12 & $3.69 \mathrm{E}-09$ \\
\hline Cs-137 & 1.02E-04 & $1.02 \mathrm{E}-13$ & 0.0813 & 3.89E-13 & $9.82 \mathrm{E}-10$ \\
\hline Ac-227 & $2.42 \mathrm{E}-04$ & $2.42 \mathrm{E}-13$ & 0.0813 & $9.21 \mathrm{E}-13$ & 2.33E-09 \\
\hline
\end{tabular}

\section{D3.5 Estimated Annual External Exposure for an Evaporative Cooler from Radionuclides in Particulate Matter}

During operation of the cooling system, particulate matter (groundwater or volcanic scenario) present in the air will be entrained with air into the cooler intake. During this time, the cooler would behave as an external source as addressed in Section D.3.4. Assuming that all particles in the air are trapped by the cooler pads, the mass of particulate matter remaining in the cooler can be estimated from the following expression.

$$
M p m_{s w}=F_{a i r} C a_{e, i} \operatorname{TSP} t_{e c}
$$

where

$$
\begin{aligned}
& \mathrm{Mpm}_{s w} \quad=\text { mass of particulate matter in evaporative cooler at the end of the cooling } \\
& \text { season }(\mathrm{kg}) \\
& F_{\text {air }} \quad=\text { air flow rate for cooler }\left(\mathrm{m}^{3} / \mathrm{hr}\right)\left(8300 \mathrm{~m}^{3} / \mathrm{hr}\right. \text { from Table D-2) } \\
& C a_{e, i}=\text { activity concentration of radionuclide } i \text { in the air resulting from the } \\
& \text { operation of an evaporative cooler }\left(\mathrm{Bq} / \mathrm{m}^{3}\right) \\
& \text { TSP = atmospheric particulate matter mass loading }\left(\mathrm{kg} / \mathrm{m}^{3}\right)\left(6 \times 10^{-8} \mathrm{~kg} / \mathrm{m}^{3}\right. \text { from } \\
& \text { Table 6.6-3 entry for inactive outdoors) } \\
& t_{e c}=\text { time cooling system is operating in year }(\mathrm{hr})(3419 \mathrm{hr} / \text { year }- \text { see } \\
& \text { Section D.3.1) }
\end{aligned}
$$

Substitution of these values into Equation D-15 indicates that a cooler system will capture $1.7 \mathrm{~kg}$ of resuspended particulate matter over an operating season. To establish the external dose from the cooler requires knowledge of the activity in the system. To derive this value, the activity concentration in the particulate matter is needed. In the groundwater scenario Equation D-6 provides the areal activity density in surface soil. The activity per unit mass can be obtained from the relationship (taking the enhancement factor to be unity):

$$
C m_{i}=\frac{C s_{i}}{d \rho}
$$


where

$\begin{array}{ll}C m_{i} & =\text { activity concentration in surface soil per unit mass }(\mathrm{Bq} / \mathrm{kg}) \\ C s_{i} & =\text { areal activity concentration in surface soil (from Table D-8) } \\ d & =\text { surface soil depth }(0.25 \mathrm{~m}) \\ \rho & =\text { surface soil density }\left(1500 \mathrm{~km} / \mathrm{m}^{3}\right)\end{array}$

Substituting these values into Equation D-16 indicates that $1.7 \mathrm{~kg}$ of matter trapped in a cooler contain a total activity of between $0.12 \mathrm{~Bq}\left({ }^{227} \mathrm{Ac}\right)$ and $1.5 \mathrm{~Bq}\left({ }^{126} \mathrm{Sn}\right)$. This activity is significantly less than that predicted as being deposited directly from the water (46.8 Bq). Thus, this pathway can be ignored.

In the case of the volcanic eruption, the approach adopted involves comparing the external dose from ground shine to that from the cooler. A deposited activity $(A s)$ of $1 \mathrm{~Bq} / \mathrm{m}^{2}$ will be used for the comparison. From Table 6.6-3, ash has a density, $\rho$, of $1000 \mathrm{~kg} / \mathrm{m}^{3}$ and the mean value of the critical thickness, $d_{c}$, of ash is $2 \mathrm{~mm}(0.002 \mathrm{~m})$. This comparison will take the ash thickness to be equal to the critical thickness. For these conditions, the activity density is $0.5 \mathrm{~Bq} / \mathrm{kg}$ $\left(A s /\left(d_{c} \rho\right)\right)$. Thus, the cooler unit will contain $0.85 \mathrm{~Bq}$ of activity.

Annual dose estimates for this activity in a cooling system can be arrived at by scaling the prediction given in Table D-11 for a cooler activity of $46.8 \mathrm{~Bq}$ by the ratio of 0.85/46.8 (i.e., by multiplying the annual dose in Table D-11 by the ratio of activities accumulated in the cooler from volcanic particles and from groundwater). The estimates of external dose due to activity deposited on the ground can be generated from effective dose coefficients for contaminated ground surface given in Table 6.5-1 (converted to annual exposure) and the activity density of $1 \mathrm{~Bq} / \mathrm{m}^{2}$. The results are presented in Table D-12.

Table D-12. Estimated Annual Dose from Ground Shine and Evaporative Cooler Units for the Volcanic Scenario

\begin{tabular}{|l|c|c|}
\hline Radionuclide & $\begin{array}{c}\text { Annual external dose due to } \\
\text { ground shine } \\
\text { (Sv) }\end{array}$ & $\begin{array}{c}\text { Annual external dose due to } \\
\text { evaporative cooler } \\
\text { (Sv) }\end{array}$ \\
\hline Sn-126 & $6.19 \mathrm{E}-08$ & $6.71 \mathrm{E}-11$ \\
\hline $\mathrm{Cs}-137$ & $1.75 \mathrm{E}-08$ & $1.78 \mathrm{E}-11$ \\
\hline Ac-227 & $1.22 \mathrm{E}-08$ & $4.23 \mathrm{E}-11$ \\
\hline
\end{tabular}

These comparisons indicate that the additional annual dose due to the accumulation of ash in an evaporative cooler can be neglected.

\section{D3.6 ADDITIONAL PATHWAYS}

\section{Cooler Maintenance}

To maintain unit efficiency and to reduce heat loss during the winter months, some operations must be performed on the system. As mentioned in Section D.1, winterizing takes place in fall 
and evaporator pad replacement is generally performed in spring. Both operations for a domestic unit take about one hour. During this time the operator is close to the unit and, by virtue of the inverse square law, is subject to higher radiation levels.

An estimate of the exposure can be obtained by simple scaling of the in-house exposure. The exposure time is reduced from about 4,000 hours per year to two. If the operator is about three quarters of a meter away for the source (at this close range the sources of radiation, i.e., the pads and the water reservoir, are extended sources, but for the purposes of this estimate this will be overlooked), then the geometric factor increases from $0.08 \mathrm{~m}^{-2}$ to $(4 / 3)^{2}$, or 1.7 , an increase by a factor of 22. Thus it would be estimated that the cooling system annual maintenance would increase the external exposure dose from the evaporative cooler by about 1 percent. This pathway is inconsequential.

\section{D4. SUMMARY}

Estimating the dose (disregarding the shielding factor for the dwelling as this has the same value for a given radionuclide for both the cooler and soil irradiation sources) using parameter values given in Tables D-3, D-9, and D-11 yields the results shown in Table D-13 for unit activity in groundwater.

Table D-13. Annual Dose Estimates from External Exposure from Radionuclides in Soil and in Evaporative Coolers and from Drinking Water

\begin{tabular}{|l|l|c|c|c|}
\hline \multicolumn{2}{|c|}{ Pathway } & \multicolumn{2}{c|}{$\begin{array}{c}\text { Annual dose (Sv) from indicated pathway from unit } \\
\text { concentration of specified radionuclide in groundwater }\end{array}$} \\
\cline { 3 - 5 } \multicolumn{2}{|c|}{} & Sn-126 & Cs-137 & Ac-227 \\
\hline \multirow{2}{*}{ Inhalation } & A - Evaporative Cooler & $1.1 \mathrm{E}-10$ & $3.4 \mathrm{E}-11$ & $7.1 \mathrm{E}-06$ \\
\hline \multirow{2}{*}{ External } & B1 - Evaporative Cooler & $3.7 \mathrm{E}-09$ & $9.8 \mathrm{E}-10$ & $2.3 \mathrm{E}-09$ \\
\cline { 2 - 5 } & B3 - Surface Soil & $2.6 \mathrm{E}-06$ & $8.3 \mathrm{E}-08$ & $3.7 \mathrm{E}-08$ \\
\hline Ingestion & C - Drinking Water (2 liters per day) & $4.2 \mathrm{E}-09$ & $9.9 \mathrm{E}-09$ & $2.9 \mathrm{E}-06$ \\
\hline
\end{tabular}

The results show that external exposure from evaporative coolers is a relatively insignificant contributor to the all-pathway dose and, thus, can be neglected. 


\section{INTENTIONALLY LEFT BLANK}


APPENDIX E

FISH INGESTION DOSE IN THE VOLCANIC SCENARIO 
In developing the ERMYN biosphere, the ingestion pathway of eating contaminated fish raised on the local fish-farm was not included in the volcanic ash exposure scenario. The rationale for this decision was based on five factors. These are discussed below.

A. Unlike the groundwater release scenario, in the volcanic scenario the water used in the ponds is considered to contain no radionuclides and, as a result, will not be subject to concentration increase from replenishment of evaporated water.

B. Fish consumption by the local population is small compared to the intake of other locally grown foods (BSC 2004 [DIRS 169671], Table 7-5).

C. Fish farming efficiency is, generally, susceptible to rapid changes in the aqueous environment. This was substantiated by the fish farm operators in Amargosa Valley (BSC 2004 [DIRS 169672) who stated that a distant forest fire created sufficient pollution that the fish died. Ashfall would have a similar effect.

D. In the volcanic scenario, the radioactive waste deposited in Amargosa Valley would be in the form of small particles of waste that would be relatively insoluble. As such, the radionuclides would only slowly leach into the water where they would be available for uptake by the fish.

E. The pond water is changed on a regular basis as the fish mature and are harvested. BSC (2004 [DIRS 169672]) indicated that this drain/refill cycle is performed at least once every two years. Thus, if the waste form is solubility or dissolution limited, the activity available to the fish would be so reduced. If the waste form were to rapidly dissolve, the exposure duration of the fish eating pathway would be limited to a maximum of two years.

To evaluate the reasonableness of not considering the fish ingestion pathway in the volcanic ash scenario biosphere model, the following analysis was conducted with the conservative assumption that all available radionuclides in the waste deposited by the ashfall into the fishponds instantaneously became available for uptake by the fish.

The contribution to the volcanic ash BDCFs from ingestion of fish raised in a fishpond that has been contaminated by an ashfall event can be calculated as follows (Section 6.4.9.4):

$$
D_{\text {ing, } f, i}=E D C F_{\text {ing }, i} C f_{i} U f
$$

where

$$
\begin{array}{lll}
D_{\text {ing. }, \mathrm{fi}} & = & \text { annual dose contribution to BDCF from ingestion of primary radionuclide } \\
& & i \text { in fish }\left(\mathrm{Sv} / \text { year per } \mathrm{Bq} / \mathrm{m}^{2}\right) \\
C f_{i} & = & \text { activity concentration of primary radionuclide } i \text { in fish }\left(\mathrm{Bq} / \mathrm{kg} \text { per } \mathrm{Bq} / \mathrm{m}^{2}\right) \\
U f & = & \text { annual consumption rate of locally produced fish }(\mathrm{kg} / \text { year }) .
\end{array}
$$


The activity concentration in fish can be calculated as follows (Section 6.4.5):

$$
C f_{i}=C w_{f, i} B F_{i}
$$

where

$C w_{f, i}=$ activity concentration of radionuclide $i$ in fishpond water, at the time of harvest $\left(\mathrm{Bq} / \mathrm{m}^{3}\right.$ per $\left.\mathrm{Bq} / \mathrm{m}^{2}\right)$

$B F_{i}=$ bioaccumulation factor for radionuclide $i$ in freshwater fish $\left(\mathrm{m}^{3} / \mathrm{kg}\right)$.

The activity concentration in fishpond water from an ashfall event can be calculated as follows:

$$
C w_{f, i}=\frac{C s_{i}}{P D}
$$

$C s_{i}=$ activity concentration of radionuclide $i$ in ash deposited on the ground surface $\left(1 \mathrm{~Bq} / \mathrm{m}^{2}\right)$

$P D \quad=$ fishpond depth (m).

The ashfall source term used to derive the BDCFs for the volcanic ash scenario is given as $1 \mathrm{~Bq} / \mathrm{m}^{2}$. The average fishpond depth, $P D$, can be determined from the dimensions of fishponds reported by BSC (2004 [DIRS 169672], Table 6-66) and reproduced in Table E-1. The depth of the ponds is in the range from 0.8 to $1.7 \mathrm{~m}$.

Table E-1. Dimensions of the Grow-Out Ponds

\begin{tabular}{|l|c|c|c|c|l|}
\hline $\begin{array}{c}\text { Pond } \\
\text { No. }\end{array}$ & Length & Width & Depth & Surface Area & \multicolumn{1}{|c|}{ Volume } \\
\hline 1 & $192 \mathrm{ft}$ & $\begin{array}{c}70 \mathrm{ft} \\
(59.2 \mathrm{~m})\end{array}$ & $\begin{array}{c}2.5 \mathrm{ft} \\
(0.8 \mathrm{~m})\end{array}$ & $\begin{array}{c}13,440 \mathrm{ft}^{2} \\
\left(1,278 \mathrm{~m}^{2}\right)\end{array}$ & $\begin{array}{l}33,600 \mathrm{ft}^{3} \\
\left(986 \mathrm{~m}^{3}=9.86 \times 10^{5} \mathrm{~L}\right)\end{array}$ \\
\hline 2 & $200 \mathrm{ft}$ & $82 \mathrm{ft}$ & $5.5 \mathrm{ft}$ & $16,400 \mathrm{ft}^{2}$ & $90,200 \mathrm{ft}^{3}$ \\
& $(61.7 \mathrm{~m})$ & $(25.3 \mathrm{~m})$ & $(1.7 \mathrm{~m})$ & $\left(1,560 \mathrm{~m}^{2}\right)$ & $\left(2,646 \mathrm{~m}^{3}=2.65 \times 10^{6} \mathrm{~L}\right)$ \\
\hline 3 & $182 \mathrm{ft}$ & $82 \mathrm{ft}$ & $5.5 \mathrm{ft}$ & $14,924 \mathrm{ft}^{2}$ & $82,082 \mathrm{ft}^{3}$ \\
& $(56.1 \mathrm{~m})$ & $(25.3 \mathrm{~m})$ & $(1.7 \mathrm{~m})$ & $\left(1,419 \mathrm{~m}^{2}\right)$ & $\left(2,408 \mathrm{~m}^{3}=2.41 \times 10^{6} \mathrm{~L}\right)$ \\
\hline Total & & & & $44,764 \mathrm{ft}^{2}$ & $205,882 \mathrm{ft}^{3}$ \\
& & & & $\left(4,258 \mathrm{~m}^{2}\right)$ & $\left(6,039 \mathrm{~m}^{3}=6.04 \times 10^{6} \mathrm{~L}\right)$ \\
\hline
\end{tabular}

Source: BSC 2004 [DIRS 169672], Table 6-66.

The average fishpond depth is calculated as an area-weighted average by dividing the total volume by total surface area in Table E-1 as follows:

$$
P D(m)=\frac{6,039 m^{3}}{4,258 m^{2}}=1.42 m
$$

Inserting the result of Equation E-4 into Equation E-3 results in an activity concentration in fishpond water of $0.705 \mathrm{~Bq} / \mathrm{m}^{3}$ per $\mathrm{Bq} / \mathrm{m}^{2}$. The mean annual consumption rate of locally produced fish, $U f$, is $0.23 \mathrm{~kg} /$ year (BSC 2004 [DIRS 169671], Table 7-5). Since these two 
values are independent of the primary radionuclide, $i$, Equations E-1 and E-2 can be consolidated as follows:

$$
D_{\text {ing }, f, i}=0.705 \times 0.23 \times E D C F_{\text {ing }, i} B F_{i}=0.162 \times E D C F_{\text {ing }, i} B F_{i}
$$

Three representative principal radionuclides were selected for comparison based on the different pathways that dominate their BDCFs (dominant pathway in parenthesis): Tc-99 (ingestion), Cs-137 (external exposure), and Pu-239 (inhalation) (BSC 2004 [DIRS 167287], Table 6.2-7). Table E-2 lists the effective dose conversion factors for ingestion and the bioaccumulation factors for these three radionuclides, as well as the calculation of the fish ingestion dose using Equation E-5.

Table E-2. Fish Ingestion Dose for Representative Radionuclides Resulting from Ashfall Event

\begin{tabular}{|l|c|c|c|c|}
\hline Radionuclide & $\begin{array}{c}\text { Ingestion EDCF } \\
\text { Sv/Bq }\end{array}$ & $\begin{array}{c}\mathbf{B F}^{\mathbf{b}} \\
\mathbf{m}^{\mathbf{3}} / \mathbf{k g}\end{array}$ & $\begin{array}{c}\text { Fish Ingestion } \\
\text { Dose }^{\mathbf{c}} \\
\text { Sv/yr per } \mathbf{~ B q} / \mathbf{m}^{2}\end{array}$ & $\begin{array}{c}\text { Fish Ingestion } \\
\text { Dose }^{\mathbf{d}} \\
\text { rem/yr per } \mathbf{~ p C i} / \mathbf{m}^{2}\end{array}$ \\
\hline TC-99 & $3.95 \mathrm{E}-10$ & $2.00 \mathrm{E}-02$ & $1.28 \mathrm{E}-12$ & $4.74 \mathrm{E}-12$ \\
\hline Cs-137 & $1.35 \mathrm{E}-08$ & $3.50 \mathrm{E}+00$ & $7.66 \mathrm{E}-09$ & $2.84 \mathrm{E}-08$ \\
\hline Pu-239 & $9.56 \mathrm{E}-07$ & $4.10 \mathrm{E}-02$ & $6.36 \mathrm{E}-09$ & $2.35 \mathrm{E}-08$ \\
\hline
\end{tabular}

a Source: BSC 2004 [DIRS 169671], Table 6-24.

b Source: BSC 2004 [DIRS 169672], Table 7-12, converted from L/kg to $\mathrm{m}^{3} / \mathrm{kg}$ by factor of 1000 . These values are the geometric mean values; the geometric standard deviation for these three radionuclides are 2.0, 2.3, and 4.7, respectively, so the upper truncation limit recommended for the distribution are significantly higher than the values used in this analysis.

${ }^{c}$ Calculated using Equation E-5.

${ }^{\mathrm{d}} 1 \mathrm{~Sv}=100 \mathrm{rem} ; 1 \mathrm{~Bq}=27 \mathrm{pCi}$.

$\mathrm{EDCF}=$ effective dose conversion factor; $\mathrm{BF}=$ bioaccumulation factor

Table E-3 is a comparison between the total of the three volcanic ashfall scenario BDCFs and the fish ingestion dose calculated in Table E-2.

Table E-3. Comparison of Fish Ingestion Dose and BDCFs from Volcanic Ashfall Event

\begin{tabular}{|l|c|c|c|}
\hline Radionuclide & $\begin{array}{c}\text { Total BDCF }^{\mathbf{a}} \\
\text { rem/yr per pCi/m2 }\end{array}$ & $\begin{array}{c}\text { Fish Ingestion Pathway } \\
\text { BDCF }^{\mathbf{b}} \\
\text { rem/yr per pCi/m2 }\end{array}$ & $\begin{array}{c}\text { Ratio of Fish Pathway } \\
\text { BDCF to Total BDCF }\end{array}$ \\
\hline TC-99 & $6.98 \mathrm{E}-10$ & $4.74 \mathrm{E}-12$ & $6.80 \mathrm{E}-03$ \\
\hline Cs-137 & $2.70 \mathrm{E}-08$ & $2.84 \mathrm{E}-08$ & $1.05 \mathrm{E}+00$ \\
\hline Pu-239 & $2.76 \mathrm{E}-06$ & $2.35 \mathrm{E}-08$ & $8.52 \mathrm{E}-03$ \\
\hline
\end{tabular}

a Source: BSC 2004 [DIRS 167287], Tables 6.2-1 through 6.2-3 (sum of mean BDCFs). This approach provides the maximum BDCF, i.e., time of zero and ash thichness of the critical thickness

b Source: Last column of Table E-2.

$\mathrm{BDCF}=$ biosphere dose conversion factor

While the dose from the fish ingestion pathway is a small fraction (less than one percent) of the BDCF from all other pathways for ${ }^{99} \mathrm{Tc}$ and ${ }^{239} \mathrm{Pu}$, for ${ }^{137} \mathrm{Cs}$ it is comparable in magnitude to the total BDCF. This is due to a relatively high value of the bioaccumulation factor for ${ }^{137} \mathrm{Cs}$. In fact, if the fish ingestion pathway were included in the ERMYN for the volcanic ash scenario, it would double the total BDCF for Cs-137 for the period of time until the pond is drained and 
refilled. However, this estimate is an unlikely upper bound of the annual dose from fish consumption for the following reasons:

1. It is unlikely that a total dissolution of the waste form would occur during the time period it takes to produce full-grown fish (1 to 2 years), thus, reducing the activity concentration in the water available for fish uptake.

2. Bioaccumulation factors for fish were developed by using literature values that are not fully applicable for the farmed fish (BSC 2004 [DIRS 169672], Section 6.4.3). This is because the bioaccumulation factors reported in the literature were established for the natural aquatic systems. In natural aquatic systems, fish receive radionuclides directly from the water and the food. However, this is not the case for the fish farm, where the fish are fed commercial, uncontaminated feed. Therefore, bioaccumulation factors provide an upper bound of the estimated uptake.

3. If a volcanic eruption occurred with an ensuing ash deposition on fish ponds, fish would likely die and thus not contribute to the human food chain.

In addition, the fish consumption pathway, as described above, would only contribute to the annual dose for the first and, possibly, the second year after a volcanic eruption. Such a contribution would be negligible for the expected dose calculated in the TSPA model, which is weighted by the probability of an eruption occurring in a given time period. 